\title{
Materials Safety Data Sheets The Basis for Control of Toxic Chemicals Volume 1
}

\author{
E. E. Ketchen \\ W. E. Porter
}

\section{MASTER}




\section{DISCLAIMER}

This report was prepared as an account of work sponsored by an agency of the United States Government. Neither the United States Government nor any agency Thereof, nor any of their employees, makes any warranty, express or implied, or assumes any legal liability or responsibility for the accuracy, completeness, or usefulness of any information, apparatus, product, or process disclosed, or represents that its use would not infringe privately owned rights. Reference herein to any specific commercial product, process, or service by trade name, trademark, manufacturer, or otherwise does not necessarily constitute or imply its endorsement, recommendation, or favoring by the United States Government or any agency thereof. The views and opinions of authors expressed herein do not necessarily state or reflect those of the United States Government or any agency thereof. 


\section{DISCLAIMER}

Portions of this document may be illegible in electronic image products. Images are produced from the best available original document. 


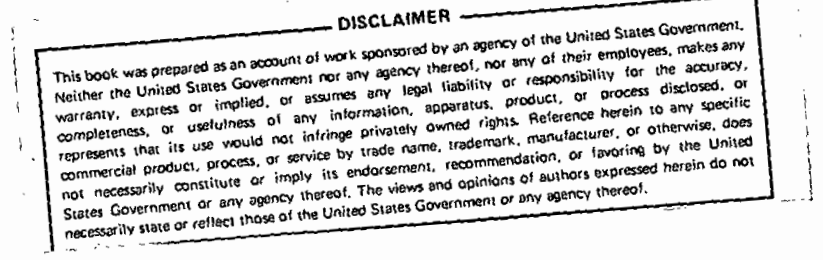

ORNL/TM-6981/V1

(Rev. Dec. 1979)

Contract No. W-7405-eng-26

HEALTH DIVISION

Industrial Hygiene Department

MATERIAL SAFETY DATA SHEETS

THE BASIS FOR CONTROL OF TOXIC CHEMICALS

VOLUME 1

E. E. Ketchen

W. E. Porter

Date Published: September 1979

OAK RIDGE NATIONAL LABORATORY

Oak Ridge, Tennessee 37830

operated by

UNION CARBIDE CORPORATION

for the

Department of Energy 
Printed in the United States of America. Available from National Technical Information Service

U.S. Department of Commerce

- 5285 Port Royal Road, Springfield, Virginia 22161

Price: Printed Copy $\$ 15.00$; Microfiche $\$ 3.00$

This report was prepared as an account of work sponsored by an agency of the United States Government. Neither the United States Government nor any agency thereof, nor any of their employees, contractors, subcontractors, or their employees, makes any warranty, express or implied, nor assumes any legal liability or responsibility for any third party's use or the results of such use of any information. apparatus. product or process disclosed in this report, nor represents that its use by such third party would not infringe privately owned rights. 
FOREWORD

The Material Safety Data Sheets contained in this volume are the basis for the Toxic Chemical Control Program developed by the Industrial Hygiene Department, Health Division, ORNL. The three volumes are the update and expansion of ORNL/TM-5721 and ORNL/TM-5722 'Material Safety Data Sheets The Basis for Control of Toxic Chemicals", Volume I and Volume IT. As such, they are a valuable adjunct to the data cards issued with specific chemicals. The chemicals are identified by name, stores catalog number where appropriate, and sequence numbers from the NIOSH Registry of Toxic Effects of Chemical Substances, 1977 Edition, if available.

The data sheets were developed and compiled to aid in apprising the employees of hazards peculiar to the handling and/or use of specific toxic chemicals. Space limitation necessitate the use of descriptive medical terms and toxicological abbreviations. A glossary and an abbreviation list were developed to define some of those sometimes unfamiliar terms and abbreviations. Questions concerning these data sheets should be referred to the Industrial Hygiene Department.

The three volumes are being printed in a looseleaf form to accommodate additional data sheets and updated sheets without the re-issue of new volumes. The page numbers are keyed to the catalog number in the chemical stores at ORNL. 
THIS PAGE

\section{WAS INTENTIONALLY LEFT BLANK}


(Rev. Dec. 1979)

\section{V \\ TABLE OF CONTENTS \\ FOREWORD}

1. Control of Toxic Materials

$$
\begin{aligned}
& \text { Introduction } \\
& \text { Mechanics of System } \\
& \text { Conclusion } \\
& \text { References }
\end{aligned}
$$

2. Glossary of Terms

3. Abbreviations

4. Material Safety Data Sheets

Page Index

Data Sheets

5. Appendix

Chemical Abstracts Service Registry Number (To be Continued in ORNL/TM-6981/V2) 


\section{CONTROL OF TOXIC MATERIALS}

\section{Introduction}

The widely divergent activities of Oak Ridge National Laboratory make it virtually impossible for the industrial hygiene group to anticipate the use of all toxic materials and to monitor each operation involving their use. Hence the user must have a knowledge of the toxicity of the material he is using in order to use it in a safe mamer.

More than five years ago a program (1) was developed to: 1) inform the material user of the health hazards and 2)inform Industrial Hyglene of the toxic material use. When a chemical is purchased from the chemical store room, the user receives a toxic material control card (TMCC). The front side of the card contains, information relative to potential health hazards. The user describes the proposed use of the material, the location, and identifies himself on the back side of the card before returning it to the Industrial Hygiene Department. With this information, appropriate follow-up action can be taken relative to the handling of the material. Attached to the card is a gummed label which gives the health, flammability and reactivity rating, along with other special information in abbreviated form. The user detaches the label from the card and affixes it to the container.

At the time the TMCC is prepared, a more detailed material safety data sheet (MSDS) is also prepared to provide additional information for both the user and the Industrial Hygiene Department. Toxicity may not bc a direct measurement of the health hazard. In some cases the 
material may be extremely toxic, but the physical properties may be such that it is easy to prevent exposure. Hence, the MSDS can be valuable in several ways to the user. An attempt is made to place the MSDS's in many locations throughout the Laboratory in order to have them available to all who may need them.

Mechanics of System

ORNL storcs chemicals ait classifted as to toxicity, flammability, reactivity, and special properties using a 0 through 4 index. $(1,2)$ At this point those materials which are extremely toxic or highly roxic (C1ass 3 and 4 ) have been classified and put into the system. Materials which are of importance primarily because of flammability and/or re activity have not yet been included. Through a joint effort with the ORNL Safety Department, materials with limited shelf life, such isopropyl ether, have also been included.

The toxicity rating appears on the tear-off label portion of the card. Descriptive terminology is given in Table I. The materials are placed in a 0 through 4 toxicity class based on the range of threshold limi.t. values (TLV' 8 ), $(1,2,3)$ also shown in Llis table. No claim is made as to the validity of the TLV ranges shown in Table I. They were selected somewhat arbitrarily by mutual agreement of respresentatives of the Industrial Hygiene Departments of the Nuclear Division plants. In cases where the TLV of a material is given both in parts per million parts air ( $p$ pill) and in̈ milligrams per cubic meter of air $\left(\mathrm{mg} / \mathrm{m}^{3}\right)$, the one which results in the more restrictive (higher toxicity) rating 
is preferentially used. This is, almost without exception, the ppm limit. In cases where no TLV's have been set, the oral $\mathrm{LD}_{50}$ for rats, the inhalation $L C 50$ for rats, or the skin $L_{50}$ for rabbits is used as shown in Table II. $(1,2)$ Many of the chemicals stocked at ORNL cannot be classified using Table I or II. These materials are classified on the basis of other toxicity data or by comparison with similar materials. Materials in class 2 are now being evaluated and are being placed in the system.

The TMCC is designed to inform the user of the potential health hazard(s) of stores chemicals (Fig. 1). The current.TLV's (if available) and the routes of absorption into the body are listed on the TMCC. "The " reader is informed of the acute symptoms of exposure and the effects of chronic exposure. Brief emergency procedures, which are to be used in the event of an exposure, and unusual hazards are also given. In the space designated as "carcinogen" on the TMCC, a "yes", "no", or "neoplastic" is entered, followed by "mutagen" and/or "teratogen" if applicable. (See Figs. 1,2). The health, flammability and reactivity hazard ratings are shown on the label as a 0-4 designation, as well as a special hazard notice (if any).

Recently, with the adoption of new rules on the release of toxic materials to the environment, ${ }^{(4)}$ the word "spills" has been added to the label, and the phrase "report all spills" has been added to the TMCC in the section for unusual hazards, as shown in Fig. 1. This alerts the employee to the fact that there are rules on the discharge of this material and that he must contact the Laboratory Environmental 
Coordinator before disposing of the material.

The MSDS's compiled in this volume were designed to provide additional information beyond the scope of TMCC's. The MSDS contains all the information from the TMCC, but in an expanded form, with the exception of the environmental control information. This will be included in a manual being developed by the Laboratory Environmental Cuordinator. In addition, physical properties information, toxicity data (such as LD50's and LC50's), and fire and explosion data are given. Ihe toxicological information is printed on the TMCC (shown in Fig. 1), which is about $71 / 2 \times 31 / 2$ inches. The shelf 1 ife is reported on both the TMCC and adhesive-backed label (shown in Fig. 3). These are found in the stores bin along with the chemical and the stores issue card. When the material is issued, the purchaser receives the TMCC. The empluyee, who uses the material or who is responsible for its use, is requested to read the information on the front side of the card and sign the card as an indication he understands the information.

The employee who uses the chemical(s) is requested to furnish information concerning its planned use. Space is availahle for this purpose on the back side of the TMCC (Fig. 4). Since in certain cases it is difficult to correlate the material purchase (by one employee) with the use (by another employee), the charge number and tata are requested. The description of use, which should include precautions to avoid exposure, is also an important part. This information helps to determine whether it is necessary for an industrial hygiene representative to contact the user of the material. The other information on the card is 


\section{$1-5$}

an aid to the location and identification of the worker. The employee then returns the completed card to the Industrial Hygiene Department, Bldg. 3550 .

The materials issued are checked monthly against the TMCC's returned (a sample reconciliation report sheet is shown in Fig. 5). To help reconcile the toxic material purchases with the returned TMCC's, employee badge number is also requested. Each Division Safecy Officer is given the tabulated results of the reconciliation report and asked to contact the employee(s) in his particular division who did not comply. CONCLUSION

The toxic material control card was designed to inform the user, in a brief descriptive format, of materials with toxic properties and to have the user inform Industrial Hygiene of plans for use of the material. The MSDS is designed to give the user more comprehensive information on the toxic material. 
TABLE I

Relative Tnxirity Criteria Based on TLV!s

\begin{tabular}{clcc}
\hline Rating & $\begin{array}{c}\text { Commonly Used } \\
\text { Term }\end{array}$ & ppm & mg/c.m. \\
\hline 4 & Extremely Toxic & $\leq 10$ & $\leq .25$ \\
3 & Highly Toxic & 11 to 250 & .26 to 2.5 \\
2 & $\begin{array}{l}\text { Moderately Toxic } \\
\text { Slightly Toxic or } \\
\text { Practically Non- } \\
\text { Toxic } \\
\text { Relatively Harmless }\end{array}$ & 251 to 500 & 2.51 to 5.0 \\
0 & 501 to 1000 & 5.1 to 10 \\
\hline
\end{tabular}


TABLE II

Relative Toxicity Criteria Based on LD50 and LC50 Data

\begin{tabular}{|c|c|c|c|}
\hline $\begin{array}{c}\text { Commonly Used } \\
\text { Term }\end{array}$ & $\begin{array}{l}\text { LD50 Single Oral } \\
\text { Dose Rats } \\
\text { mg/kg }\end{array}$ & $\begin{array}{l}\text { LC50 Inhalation } 4-\mathrm{hr} \text {. } \\
\text { Vapor Exposure } \\
\text { Rats } \\
\text { PPM }\end{array}$ & $\begin{array}{l}\text { LD50-Skin } \\
\text { Rabbits } \\
\mathrm{mg} / \mathrm{kg}\end{array}$ \\
\hline Extremely Toxic & $s_{1}$ & $\leq_{10}$ & $\leq 5$ \\
\hline Highly Toxic & 1.1 to 50 & 11 to 100 & 5.1 to 43 \\
\hline Moderately Toxic & 50.1 to 500 & 101 to 1,000 & 44 to 340 \\
\hline $\begin{array}{l}\text { Slightly Toxic or } \\
\text { Practically Non- } \\
\text { Toxic }\end{array}$ & 501 to 15,000 & 1,001 to 100,000 & 350 to $22 ; 600$ \\
\hline Relatively Harmless & $>15,000$ & $>100,000$ & $>22,600$ \\
\hline
\end{tabular}


CATALOG NO. 03-206-8801 MATERIAL

ACRYLONITRILE

OSHA THRESHOLO LIMIT VAL JE

2PPM

PRINCIPLE ROUTES O A ABSORPTIONABSCRPTICN

ORRL 13

CONTROL OF TOXIC MATERIALS

RELEVANT SYMPTOMS O EXPOSUREHEAUACHE, NAUSEA. VGMITING. ABDCMINAL CRAMFS. LUNONSIOUSNESS. CONVULSILAS

EFFETS OF CHRONIC EXPOSURE FluSHING CF FACE, NAUSEA. JALNDICE. TUMDRS CF C.h.S. G EAR CANAL.

EMERGENCY AND FIRST AID PROCEOUPESGT MEOICAL ÄTTENTICN, WASH SKIN WITH SCAP E WATER. INHALATION- INHALE AMYL NITRITE VAPOR: IF SWALLOWEO-GIVE EMETIC. UNUSUAL HAZAROS

$$
\text { REPORT ALL SPILLS. }
$$

TEAR OFF PERFORATEL LABEL AND FFFIX

TO CHEMICAL CONTAINER.

SIGNED -LSER OF MATERIALI

$\cup C_{N}-10736$

$\{1235 \quad 10-751$
( PLEASE COMPLETE REVERSE SIDE)
ACRYLCAITRILE

FLAMMABILITY

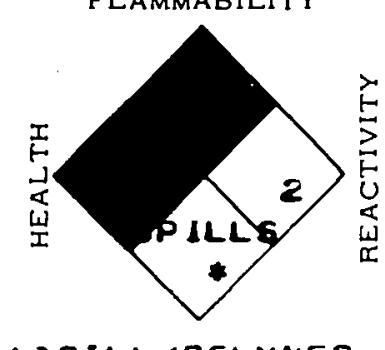

- jPLll p PCl ymer. $0=$ MARMLESS

$I=S L I G H T$ HAZARD $2=$ HAZAFCOUS $3=$ HIGH HAZAKO $4=E X T R E M \bullet H A \angle A R D$ 
INHALATION UK INUESTION OH DUST.

RELEVATT SYMPTOMS OF EXPOSURENAUSFA, VOMITING, DIARRHEA, HEAUACHE, SALIVATIUN, LIVER DAMA UEE. KENAL FAILURE. ANU SHORTNESS OF BKEATH.

EFFECTSOF CHRONIC EXPOSURE LUSS UF SENSE UF SMELL. COUGH. UYSPNEA. WEIGHT LOSS. a VILIHIA. ANO YELLUM-STAINED TEETH. EMEPEEMCY. AND FIRST AID PROCEDURES

REMUYE FROM EXPRISURE. CUTAIN MEOICAL AID.

RENASUAL HAZAROS

KEEPORT ALL SPILLS.

TEAR OFF PEPFORA TED LABEL AND AFFIX

TO CHENICAL CONTAINER.

UCN-10736

$\left(\begin{array}{ll}235 & 110.75\end{array}\right)$

( PLEASE COMFLETE REVERSE SIDE)

C.TUMIUM CHLCKIi

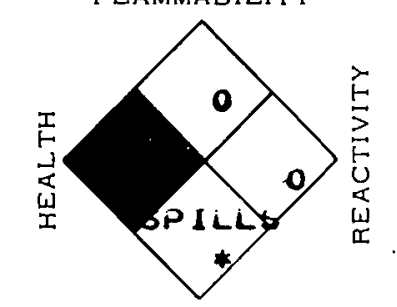

O=HATMLESS

$1=S L I G H T$ MAZARU

2: HALAROUUS

$3=$ HIGH HALAKO

$4=E X I R E M$ HAZARO

(PLEASE COMFLETE REVERSE SIDE)

Fig. 2 


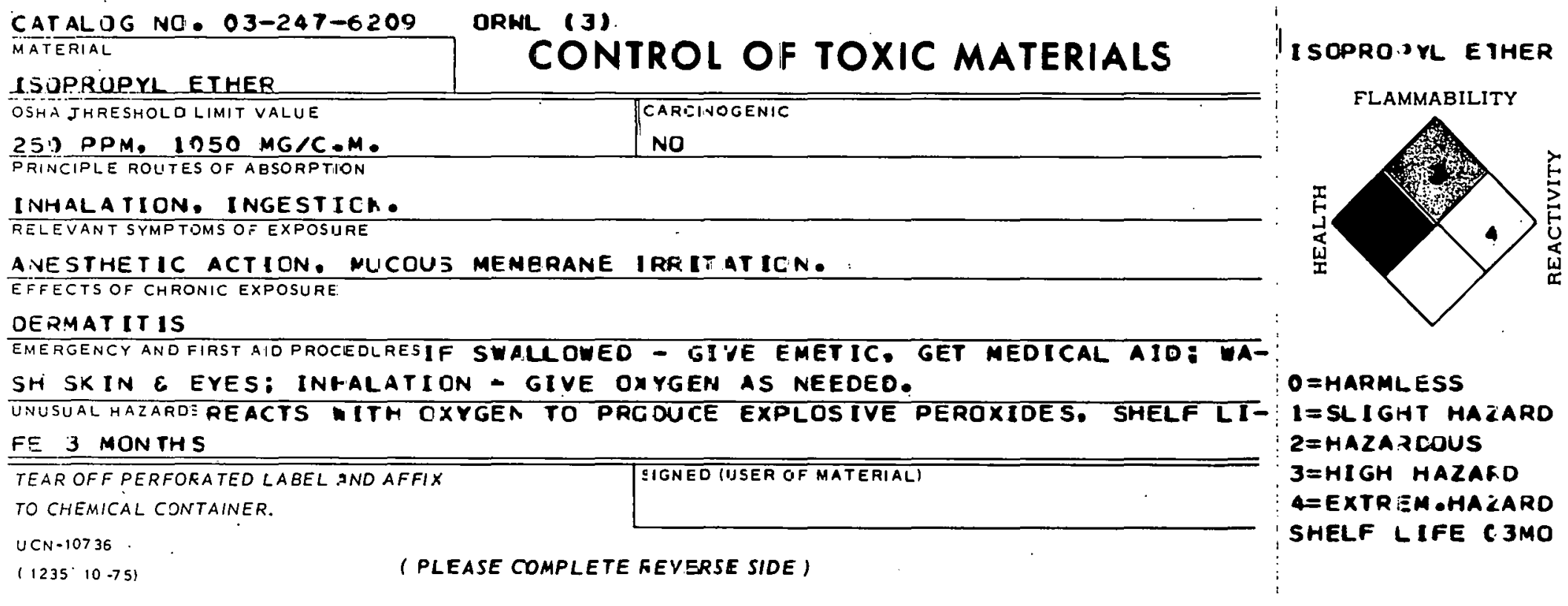


PLEASE

PRINT

\section{* THIS CARD MUST BE FILLED OUT BY PERSON. US ING CHEMICAL AND}

RETURNED TO YOUR PLANT INDUSTRIAL HYGIENE DEPARTMENT IMMEDIATELY.

NOTE: BIOLOGY DIVISION STAFF SEND CARDS TO INDUSTRIAL HYGIENE DEPARTMENTAT X.IO, BLDG. 3550

\begin{tabular}{l|l|l|l|l|l}
\hline CMAFEE DR WORK DRDER NUMBEF & DIVISION NAME & DATE OF PURCHASE \\
\hline BUILDING & ROCM & QUANTITY PURCHASED \\
\hline
\end{tabular}

EESCPIPTION OF USE, INCLUDING PRECAUTIOHS (e.g., OPERATION IN HOOD

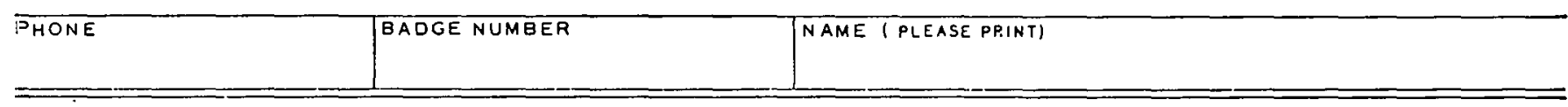

* Cinorge or Work Order for Identificotion only. * Only one completed card required for each furchose.
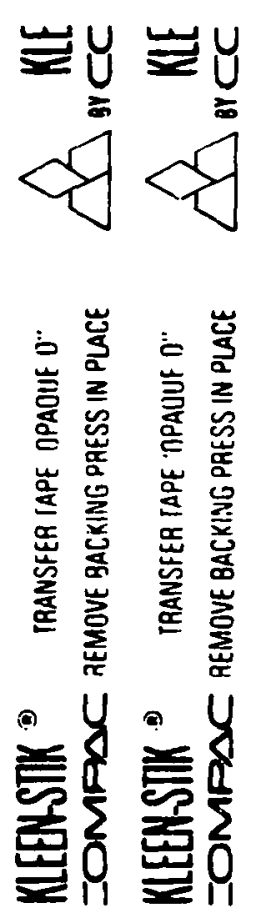


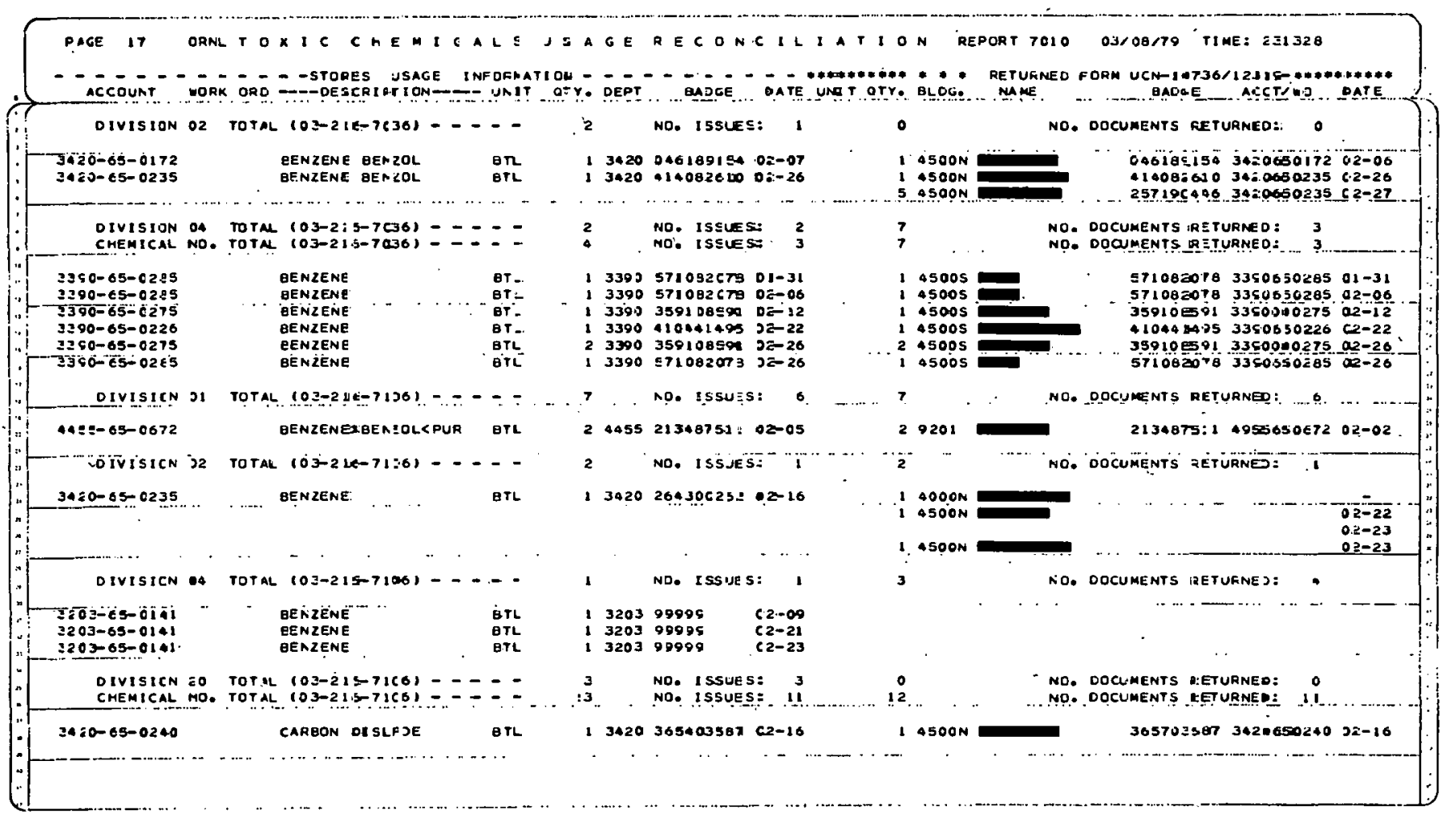


$1-13$

FIG. 1 CONTROL CARD FOR ACRYLONITRILE

FIG. 2 CONTROL CARD FOR CADMIUM CHLORIDE

FIG. 3 CONTROL CARD FOR ISOPROPYL ETHER

FIG. 4 REVERSE SIDE OF CONTROL CARD

FIG. 5 PRINTOUT SHEET FROM RECONCILIATION REPORT 


\section{REFERENCES}

1. Porter; W. E., C. L. Hunt and N. E. Bolton: A System for Labeling and Control of Toxic Materials in a Large Research Facility. Am. Ind. Hyg. Assoc. J. 36: 51-56 (1977).

2. U. S. Department of Health, Education and Welfare: A Recommended Standard .... An Identification System for Occupationally Hazardous Materials. HEW Publication No. (NIOSH) 75-126:13-23 (1974).

3. Threshold Limit Values for Chellical Substances in Work koom Air Adoptod by $\Lambda \mathrm{CG} 1 \mathrm{H}$ for 1978 . Am. Conf. nf rinv. Tnd. Hyg. Cincinnati (1978).

4. Federa1 Register 44(34): 10,280 - 10,283, Friday, Feb. 16, 1979. 


\section{PAGES 2-1 to 2-2 \\ WERE INTENTIONALLY \\ LEFT BLANK}




\begin{tabular}{|c|c|}
\hline Albumin & $\begin{array}{l}\text { A water-soluble protein excreted in } \\
\text { urine }\end{array}$ \\
\hline Anemia & $\begin{array}{l}\text { A condition in which the blood is } \\
\text { deficient in hemoglobin and/or numbers } \\
\text { of red cells compared with normal blood }\end{array}$ \\
\hline Anesthesia & $\begin{array}{l}\text { Loss of feeling or sensation, especially } \\
\text { the loss of tactile sensibility }\end{array}$ \\
\hline Anorexia & Lack or loss of the appetite for food \\
\hline Anosmia & Absence of the sense of smell \\
\hline Anuria & $\begin{array}{l}\text { Total suppression of urinary output by } \\
\text { the kidney }\end{array}$ \\
\hline Apathy & Lack of feeling or emotion, indifference \\
\hline Arthralgia & Neuralgia or paịn in a joint \\
\hline Asbestosis & $\begin{array}{l}\text { Lung disease caused by prolonged inhaling } \\
\text { of asbestos }\end{array}$ \\
\hline Asphyxia & Suffocation \\
\hline Bronchiolitis & Capillary bronchitis \\
\hline Bronchitis & Inflammation of the bronchial tubes \\
\hline Bronchopneumonia & $\begin{array}{l}\text { Inflammation of the lungs following } \\
\text { infection of the upper respiratory tract, } \\
\text { infectious fevers, or debilitating } \\
\text { diseases }\end{array}$ \\
\hline Bronchospasms & Bronchial spasm \\
\hline Carcinoma & $\begin{array}{l}\text { A malignant new growth made up of } \\
\text { epithelial cells, which give rise to } \\
\text { metastases }\end{array}$ \\
\hline Cataract & $\begin{array}{l}\text { An opacity of the crystalline eye lens } \\
\text { or its capsule }\end{array}$ \\
\hline Cirrhosis & $\begin{array}{l}\text { A disease of the liver marked by the } \\
\text { progressive destruction of liver cells }\end{array}$ \\
\hline
\end{tabular}


Conjunctivitis

Convulsion

Cornea

C.yannsis

Delirium

Dermatitis

Dyspena

Emphysema

Epigastric

Erosion

Euphoria

Gangrene

Gastroenteritis

Goiters

Hallucinations

Hematemesis

Hematuria

Hemoglobinuria

Hemolytic Anemia
Inflammation of the conjunctiva, the delicate membrane that lines the eyelids and covers the eyeballs

A violent contraction of the voluntary muscles

Transparent structure of the external layer of the eyeball

Rl,1eness of the skin raused by insuffieient oxygenation of the blood

$\Lambda$ mental disturbance marked by illusion, hallucinations, physical restlessness, and incoherence

Inflammation of the skin

Difficult or labored breathing

A swelling or inflation due to presence of air in the interstices of the connective tissues of the lungs

Pertaining to the belly or (epigastrium)

An eating or gnawing away

Exaggerated sense of well-being

Anemic necrosis of tissue

Inflammation of the stomach and intestines

Enlargement of the thyroid gland

A sense perception not founded upon objective reality

Vomiting of blood

Discharge of blood in the urine

Presence of hemoglobin in the urine

Anemia due to active destruction of red cells 


\section{$2-5$}

Hepatitis

Hyperactivity

Hyperemia

Hyperpnea

Hypotension

Hypothermia

Inebriation

Ingestion

Insomnia

Intoxication

Lacrimation

Laryngitis

Lassitude

Lethargy

Leukopenia

Malaise

Malignant tumors

Mottling

Myalgia

Narcosis
Inflammation of the liver

Overactivity

Excess of blood in any part of the body Abnormal exaggeration of the respiratory movements

Abnormally low blood pressure

Abnormally low temperature

The condition of being drunk

The act of taking food, medicines, etc. into the body by mouth

Inability to sleep

The condition produced by excessive use of alcohol; also, the state of being poisoned

The secretion and discharge of tears

Sore throat, hoarseness, and cough due to inflammation of the larynx

Weakness, exhaustion

A condition of drowsiness of mental origin

Reduction of white cells in the blood

Uneasiness, discomfort, and distress

Tumors that tend to become progressively worse and result in death

A condition of a tissue spotted with patches of color

Pain in a muscle or muscles

A state of profound unconsciousness produced by a drug 
Narcotic

Nausea

Necrosis

Nephritis

Neuritis

Neurotoxic

Oliguria

Opacity

Opaque

Pallor

Palpitation

Papilledema

Paralyris

Petechia

Pharyngitis

Pneumonitis

Polyneuritis

Prostration

Psychic disturbances

Pulmonary edema

Pulmonary fibrosis
Having the effect of producing sleep or stupor and at the same time relieving pain (adj.)

Tending to vomit, feeling of sickness at the stomach

Death of a circumscribed portion of tissue

Inflammation of the kidney (hemorrhagic)

Intlammation of a nerve

Poisonous or destructive to nerve tissue

Reduced daily output of urine

The condition of being opaque

Impervious to light rays

Paleness

Unduly rapid beating of the heart

Edema of the optic papilla

Loss or impairment duc to lcsion of muscular mechanism

A small area of discoloration formed by the effusion of blood

Inflammation of the pharynx

Localized inflammation of the lung

Inflammation of many nerves at once

Extreme exhaustion or powerlessiess

Mental disturbances

Fluid in the lungs

Fibrous tissues in the lungs 
Pyorrhea

Retching

Salivation

Somnolence

Stomatitis

Stupor

Thiocyanate Intoxication

Tinnitus

Ulceration

Vertigo
A discharge of pus, particularly from the gums

A strong involuntary effort to vomit

An excessive discharge of saliva

Sleepiness

Inflammation of the mouth

Partial or nearly complete unconsciousness

Characterized by central nervous system stimulation and depression, and muscular incoordination

Ringing in the ears

The formation of an ulcer

A sensation as if the external world were revolving around the patient or if he were revolving in space; the term is sometimes erroneously used as a synonym for dizziness 
3. Key to Abbreviations 
THIS PAGE

\section{WAS INTENTIONALLY}

LEFT BLANK 
KEY TO ABBREVIATIONS

$\begin{array}{ll}\text { cc } & \text { cubic centimeter } \\ \text { CNS } & \text { central nervous system effects } \\ \text { gm } & \text { gram } \\ \text { H } & \text { hour } \\ \text { IRR } & \text { irritant effects } \\ \text { kg } & \text { kilogram (one thousand grams) } \\ \text { LC50 } & \text { lethal concentration } 50 \text { percent kill } \\ \text { LCLo } & \text { lowest published lethal concentration } \\ \text { LD50 } & \text { lethal dose } 50 \text { percent kill } \\ \text { LDLo } & \text { lowest published lethal dose } \\ \text { C.M. } & \text { cubic meter } \\ \text { mg } & \text { milligram (one thousandth of a gram; } 10^{-3} \mathrm{gm} \text { ) } \\ \text { ml } & \text { milliliter } \\ \text { ppm } & \text { parts per million (v/v) } \\ \text { TCLo } & \text { lowest published toxic concentration } \\ \text { TDLo } & \text { lowest published toxic dose } \\ \text { TLV } & \text { threshold limit value } \\ \text { TWA } & \text { time weighted average } \\ \text { W } & \text { week } \\ \text { Y } & \text { year }\end{array}$


4. Material Safety Data Sheets 
THIS PAGE

\section{WAS INTENTIONALLY}

LEFT BLANK 
$\underline{\text { Inorganic }}$

Chromic Acid

Hydriodic Acid

Hydrobromic Acid

Hydrochloric Acid

Hydrofluoric Acid

Hydrofluosilicic Acid

Hypophosphorous Acid ( $30 \%$ )

Iodic Acid

Nitric Acid

Perchloric Acid

Periodic Acid

Phosphoric Anhydride

Phosphoric Acid

Phosphotungstic Acid

Sulfuric Acid

Sulfurous Acid

Aluminum Chloride

Ammonium Bichromate

Ammonium Bifluoride

Ammonium Fluoride

Ammonium Hydroxide

Ammonium Oxalate

Ammonium Sulfide Solution

Ammonium Vanadate

Antimony

Antimony Trichloride

Arsenic Metal

Barium Acetate

Barium Carbonate

Barium Chloride

Barium Hydroxide

Barium Metal

Barium Nitrate

Beryllium Nitrate

Bromine

Cadmium Chloride

Cadmium (Metal)

Calcium Fluoride

Calrium Oxide

Chromium Metal

Chromic Nitrate

Chromium Potassium Sulfate

Cobalt Metal

Cupric Carbonate
Page No.

$1-0721$

$1-0922$

$1-1113$

$1-1324$

$1-1500$

$1-1716$

1-1917

$1-2128$

1-2933

1-3703

$1-3904$

1-4506

$1-4516$

1-4807

$1-7212$

1-7262

6-1310

8-1102

$8-1503$

8-4401

8-5123

8-6527

8-7851.

8-9336

10-0360

10-0600

13-0401

15-0102

15-0903

15-1904

15-3706

15-4607

$15-5538$

17-6661

24-5204

26-2805

26-4805

$28-3516$

28-5926

40-3400

$40-4550$

$40-5600$

42-4001

46-2903 


\begin{tabular}{|c|c|}
\hline Cupric Chloride & $46-3433$ \\
\hline Cupric Nitrate & $46-4403$ \\
\hline Cupric Oxide & $46-4953$ \\
\hline Cupric Sulfate & $46-6403$ \\
\hline Cuprous Chloride & $46-8423$ \\
\hline Cuprous Cyanide & $46-8721$ \\
\hline Cuprous Oxide & $46-9303$ \\
\hline Ferric Chloride & $53-2624$ \\
\hline Fericic Nitrate & $53-3804$ \\
\hline Iodine & $74-1009$ \\
\hline Lead Acetate & $82-0102$ \\
\hline Leàd Chloride & $82-1802$ \\
\hline Lead Dioxide & $82-.300 ?$ \\
\hline Lead Meral & $82-1802$ \\
\hline Lead Nitrate & $82-5402$ \\
\hline Leàd Oxide & $82-6002$ \\
\hline I.ead sultato & $82-\overline{7} 202$ \\
\hline Lithium Aluminum Hydride & $84-1003$ \\
\hline Lithium Fluoride & $84-5003$ \\
\hline Lithium Hydroxide & $84-6003$ \\
\hline Lithium Metal & $84-8003$ \\
\hline Mercuric Chloride & $91-2006$ \\
\hline Mercuric Iodide & $91-3006$ \\
\hline Mercuric Nitrate & $91-4006$ \\
\hline Mercurous Nitrate & $91-8006$ \\
\hline Mercury Metal & $91-9026$ \\
\hline Nickel Carbonyl & $97-0001$ \\
\hline Nickel Metal & $97-2848$ \\
\hline Nickelous Ammoniuin Sulfate & $97-3508$ \\
\hline Nickclous Chloride & $87 \ldots 1.908$ \\
\hline Nickelous Nitrate & $97-7008$ \\
\hline Nickelous Sulfate & $97-8508$ \\
\hline Phosphorous Pentachloride & $106-5001$ \\
\hline Potassium Arsenate & $110-0452$ \\
\hline Potassium Bichromate & $110-0802$ \\
\hline Potassium Bifluoride & $110-1032$ \\
\hline Potassium Ghromato & $110-2902$ \\
\hline Potassium Cyanide & $110 \ldots .3502$ \\
\hline Putassium Fluoride & $110-4522$ \\
\hline Potassium Hydroxide & $110-5042$ \\
\hline Putassium Metà 1 & $110-5602$ \\
\hline Potassium Oxalate & $110-6402$ \\
\hline Potassium Periodate & $110-6802$ \\
\hline Ruthenium Chloride & $121-2905$ \\
\hline Selenium Metal. & $125-4006$ \\
\hline Sodium Arsenite & $131-1068$ \\
\hline Sodium Azide & $131-1108$ \\
\hline
\end{tabular}


Sodium Calcium Hydrate

$131-3158$

Sodium Chromate

$131-3608$

Sodium Cyanide

$131-4008$

Sodium Dichromate

$131-4108$

Sodium Fluoride

$131-4808$

Sodium Hydroxide

$131-5323$

Sodium Hypochlorite

$131-5508$

Sodium Metal

Sodium Oxalate

Sodiun Peroxide

$131-6028$

$131-6908$

$131-7338$

$131-8148$

Sodium Silicate

$131-8758$

Stannic Chloride

$133-3008$

$133-6008$

Stannous Chloride

$141-5902$

Tellurium Metal

$150-4003$

Titanium Chloride

$154-6035$

Vanadium Pentoxide

$159-5006$

Yttrium Metal

$159-8006$

Yttrium Oxide

$161-2007$

Gases

Ammonia (anhydrous)

$910-4030$

Carbon Monoxide

$927-5550$

Chlorine, liquid, compressed

$934-4000$

Hydrogen Sulfide

$950-9425$

Sulfur Dioxide

988-2020

Other

Asbestos Fiber

$609-2050$

Precious Metals

Gold Cyanide

Sel-Rex Bright Gold B1

$5201-1400$

$5201-4000$

Sel-Rex Bright Gold $\mathrm{M}^{2}$

$5201-4050$

Gold(ous) Potassium Cyanide

$5201-6200$

Osmic Acid Anhydride

5401-0401

Chloroplatinic Acid

$5601-0801$

Platinum Chloride

5601-1120

Diamino Platinum Nitrite

$5601-1510$

Rhodium Trichloride

$5801-1105$

Silver Carbonate

$5901-1101$

Silver Cyanide

5901-1251

Silver Nitrate

5.901-5621 
Organic Acids

$\begin{array}{ll}\text { Acetic Anhydride } & 201-0100 \\ \text { Acetic Acid } & 201-0200 \\ \text { P-Arsanilic Acid } & 201-2102 \\ \text { Bromoacetic Acid } & 201-4004 \\ \text { B-Bromopropionic Acid } & 201-4454 \\ \text { Chloroacetic Acid } & 201-5905 \\ \text { Chloromaleic Anhydride } & 201-6186 \\ \text { Chlorosulfonic Acid } & 201-6706 \\ \text { Formic Acid } & 202-1400 \\ 8 \text { Hydroxy-5-Quinoline Sulfonic Acid } & 202-2903 \\ \text { Maleic Acid } & 202-4405 \\ \text { Maleic Anhydride } & 202-4505 \\ \text { Malonic Acid } & 202-4805 \\ \text { Methoxyacetic Acid } & 202-5206 \\ \text { Nitrophthalic Anhydride } & 202-9610 \\ \text { Oxalic Acid } & 203-0302 \\ \text { Phenylarsonic Acid } & 203-1303 \\ \text { Phthalic Anhydride } & 203-2024 \\ \text { Picramic Acid } & 203-2504 \\ \text { Picric Acid } & 203-2704 \\ \text { Pyrogallic Acid } & 203-3455 \\ \text { Tannic Acid } & 203-7309 \\ \text { Thioglycolic Acid } & 203-8310 \\ \text { Trichloro Acetic Acid } & 203-9202\end{array}$

Organic

Acetal

$205-1550$

Acetaldehyde

205-2000

Acetanide

205-2500

Acetronitrile

205-5000

Acetonylacetone

205-6000

Acetyl Chloride

$206=3501$

Acetyl Methyl Amine

$206-4501$

Acetyl Methylurea

206-6001

Acety 1 Thiourea

206-6501

Acetyl Urea

206-7501

Acrylonitrilc

$206-8801$

Alcohols

Allyl Alcohol

208-0202

Iso-Amy1 Alcohol

208-0.332

n-Butyl Alcohol

208-0902

Isobutyl Al cohol

208-0942 
Sec Butyl Alcohol

208-0962

Tertiary Butyl Alcohol

208-0982

Diacetone Alcohol

208-1602

2 Di-N-Butylaminoethyl Alcohol

208-2202

B-Di Ethylaminoethyl Alcohol

$208-2752$

B-Dimethylaminoethyl Alcohol

208-3102

Furfuryl Alcohol

n-Heptyl Alcohol

Methyl Alcohol

208-5002

208-5202

N-Propy1 Alcohol

$208-6002$

208-8702

\section{Organic}

Allylamine

Allyl Bromide

21] -3103

Allyl Chloride

$211-3203$

$211-3303$

P-Aminoazobenzene

211-6503

P-Aminoazobenzene Hydrochloride

$211-6703$

2-Amino-1, 4-Dimethylbenzene

212-0104

4 amino - 1, 3-Dimethylbenzene

212-0134

5-amino-1, 3-Dimethylbenzene

212-0164

0 -aminodiphenyl

212-0204

2-Aminopyridine

$212-1704$

Iso amy 1 acetate

212-5004

n-Amyl Acetate

$212-5104$

Aniline

Aniline Hydrochloride

213-2005

Aniline Sulfatc

213-2205

o-Anisidine

213-2405

P-Anisidine

213-3205

Anthracene

213-3255

Azobenzene

213-4005

Azoxybenzene

213-9005

Benzalazine

213-9505

216-3006

1,2-Benzanthracene

$216-6006$

Benzene (Benzol)

Benzidine, Dihydrochloride

$216-7006$

Benzoyl Bromide

Benzoyl Chloride

$216-9146$

217-6107

217-7107

Benzyl Bromide

Benzyl Chloride

219-0708

Benzy1-M-Toluidine

219-1708

Benzyl-0-Toluidine

219-8208

Benzyl-P-Toluịdine

$219-8238$

$219-8268$

Bromal

220-0809

$\alpha$-Bromo-acetophenone

220-1803 
$4-8$

Bromoacetyl Bromide

220-2309

3 Bromo-4-Aminotoluene

220-2809

5-Bromo-2-Aminotoluene

$220-3309$

M-Bromoaniline

220-3809

o-Bromoaniline

$220-3839$

P-Bromoaniline

$220-3869$

Bromobenzene

$220-4859$

M-Bromobenzoyl Bromide

$220-5809$

Bromochloromethane

$220-6809$

P-BromoDiethylaniline

$220-7809$

P-Bromodimethylaniline

$220-8309$

220-8809

$220-9.309$

0 -Bromodipheny 1

$220 \quad 0359$

P-Brollodiphenyl 


\begin{tabular}{|l|}
\hline \multicolumn{1}{|c|}{ PRODUCT DESIGNATION } \\
$03-001-0721$ \\
$03-001-0741$ \\
Chromic Acid \\
\hline
\end{tabular}

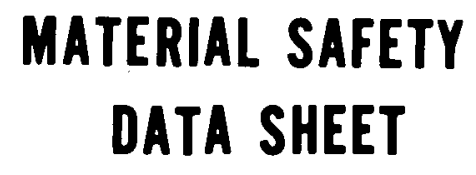

\section{SECTION 1 SOURCE AND NOMENCLATURE}

MANUFACTURER'S NAME
ADDRESS INUMBER, STREET, CITY,
TRADE NAME AND SYNONYMS
CHEMICAL NAME ANO SYNONYMS
ChYOMIUM TriOXIde

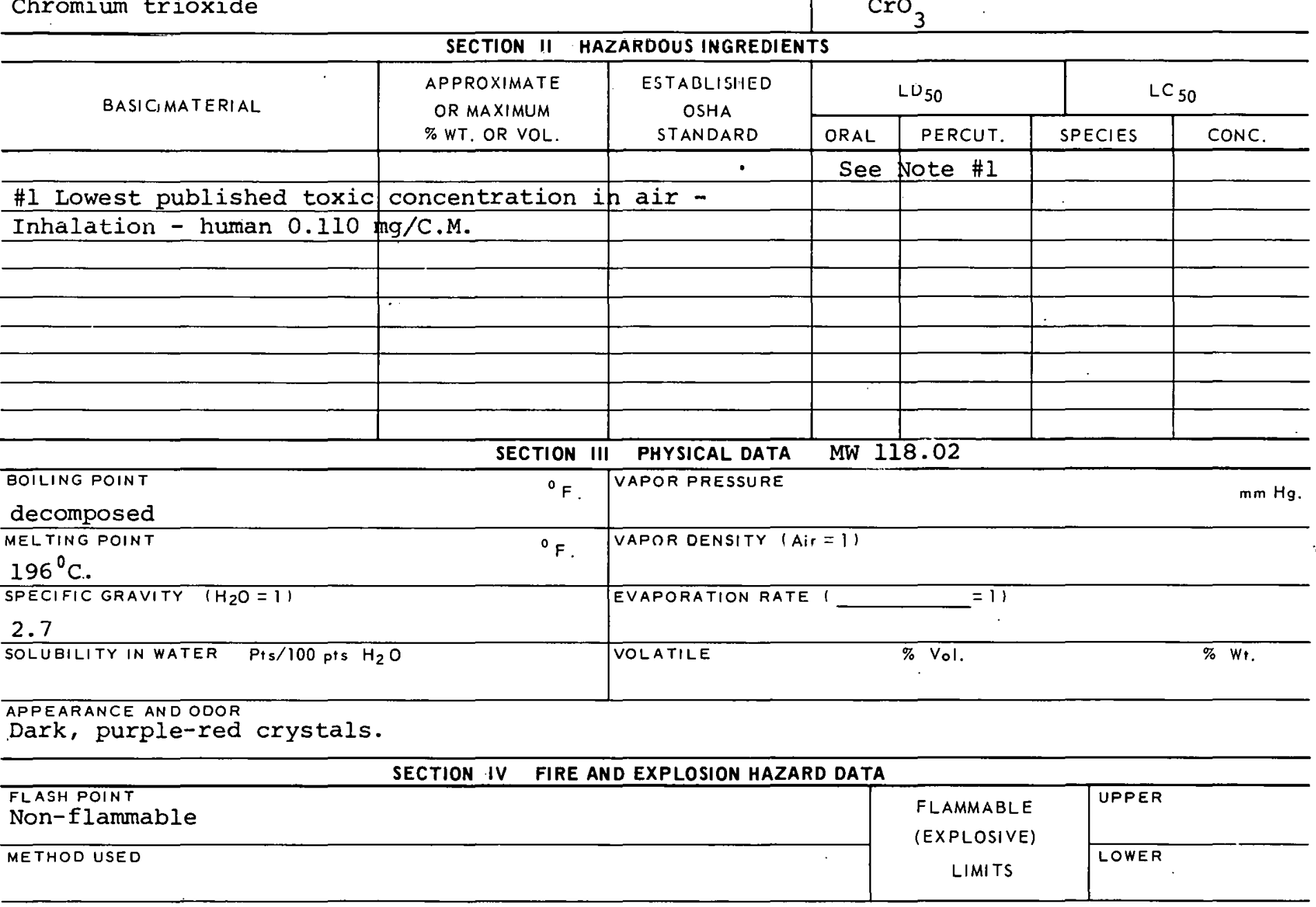

\section{EXTINGUISHING MEDIA \\ Water}

SPECIAL FIRE FIGHTING PROCEDURES

\footnotetext{
UNUSUAL FIRE AND EXPLOSION HAZARDS

Decomposing material will form hot viscous foam - caution against possibility

of steam explosion.
} 


TOXICLEVEL
TLV $0.05 \mathrm{mg} / \mathrm{m}^{3}$

PRINCIPLE ROUTES OF ABSORBTION

Inhalation of dust or mist.

REVELANT SYMOTOMS OF EXPOSURF, Respiratorv system irritant, severe skin \& eye burns. If ingested - dizziness, intense thirst, shock, oliguria and anuria. EFFECTS OF CHRONIC EXPOSURE Dermatitis with edema \& ulceration. If inhaled, ulceration, bleeding and nasal septum perforation. Acute hepatitis \& jaundice. EMERGENCY AND FIRST AID PROCEDURES

Remove from exposure, rest, keep warm and obtaiı medical aid.

CONOITIONS CONTRIBUTING TO INSTABILITY

SECTION VI REACTIVITY DATA

CONDITIONS CONTRIBUTING TO HAZARDQUS POLYMERIZATION

INCOMPATABILITY (MATERIALS TO AVOIDI

Alkali metals, organic matter, acetone, pyridine, benzene.

HAZARDOUS DECOMPOSITION PRODUCTS

SECTION VII SPILL OR LEAK PROCEDURES

STEPS TO BE TAKEN IN CASE MATERIAL IS RELEASED OR SPILLED

WASTE DISPOSAL METHOD

SECTION VIII SPECIAL PROTECTION INFORMATION

OTHER PROTECTIVE EQUIPMENT

Rubluer apron

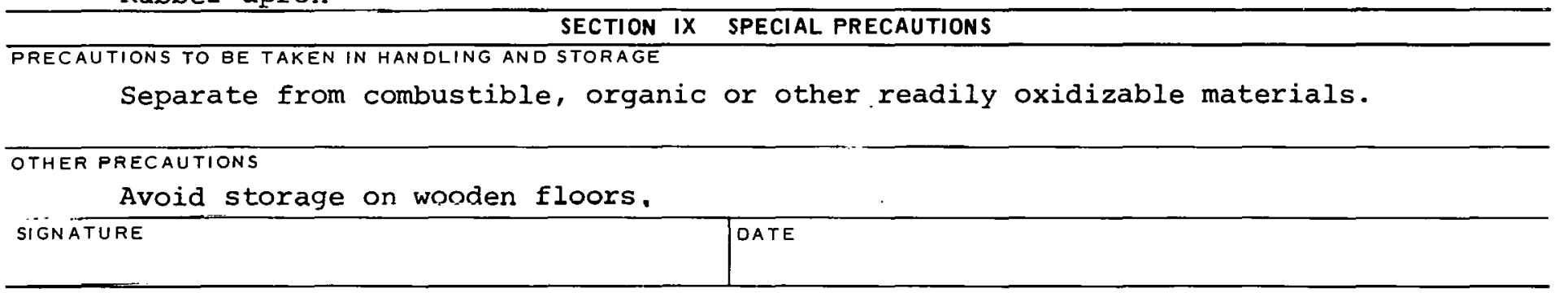


CAS : 010034852

MW- 37600

\section{PRODUCT DESIGNATION}

03-001-0922

Hydriodic Acid

\section{MATERIAL SAFETY \\ DATA SHEET}

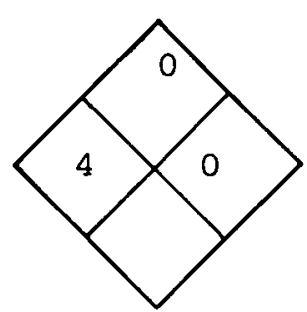

SECTION I SOURCE AND NOMENCLATURE

\begin{tabular}{l|l}
\hline MANUFACTURER'S NAME & EMERGENCY TELEPHONE NO \\
\hline ADDRESS (NUMBER, STREET, CITY, STATE, ZIP CODE) &
\end{tabular}

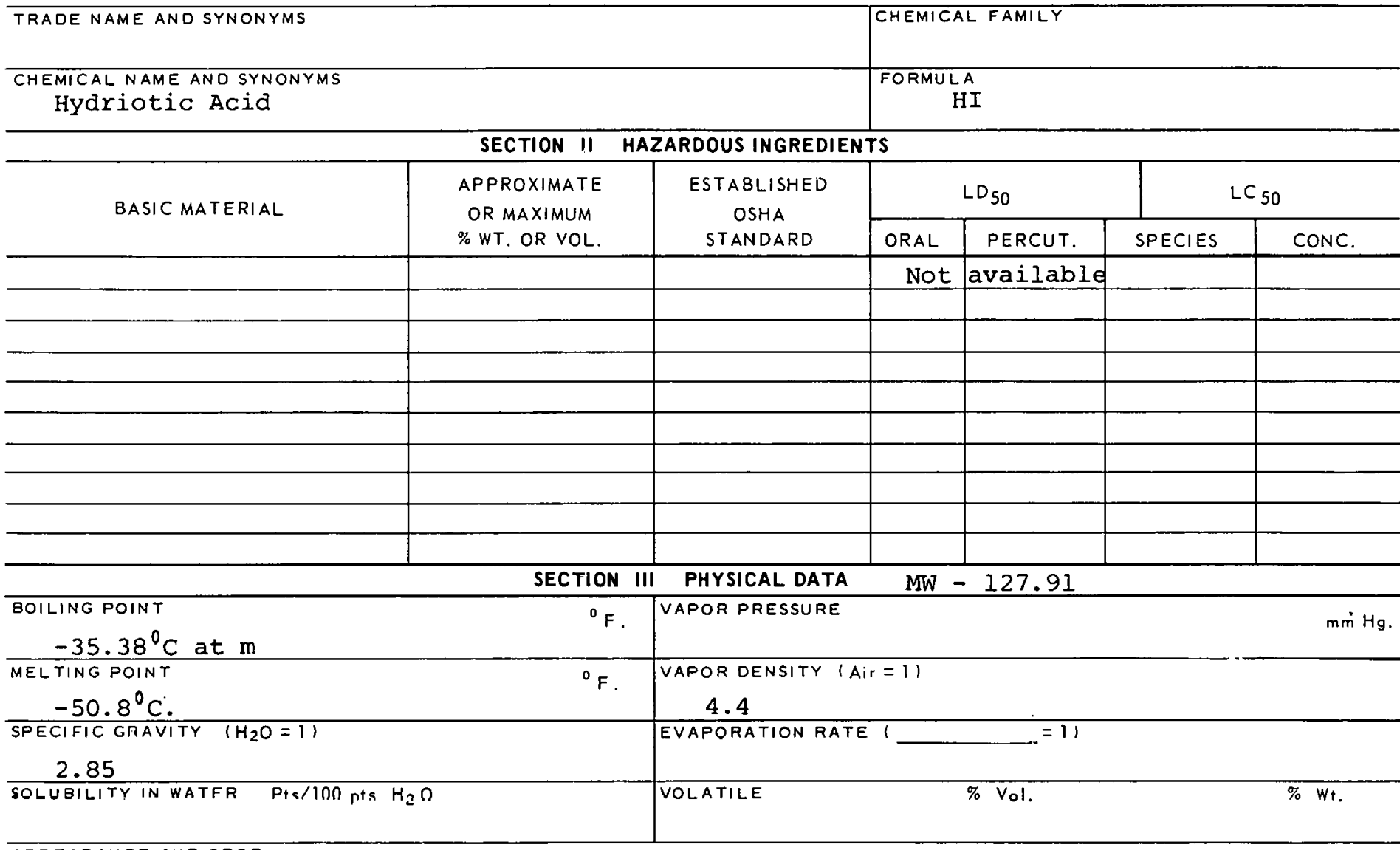

APPEARANCE AND ODOR

Pale yellow liguid.

\section{SECTION IV FIRE AND EXPLOSION HAZARD DATA}

FLASH POINT

None available

METHOD USED

EXTINGUISHING MEDIA

Use water, neutralize with chemically basic substances.

SPECIAL FIRE FIGHTING PROCEDURES

Wear full protective clothing.

UNUSUAL FIRE ANDEXPLOSION HAZARDS

\section{FLAMMABLE}

(EXPLOSIVE)

LIMITS
UPPER

LOWER 


\section{TOXIC LEVEL \\ Suggest value 10 PPM}

PRINCIPLE ROUTES OF ABSORETION

Inhalation of vapor
CARCINOGENIC NO

SKIN AND EYE

IRRITATION Severe

REVELANT SYMPTOMS OF EXPOSURE Conjunctivitis, pulmonary edema, coughing, choking, headache, dizziness, cyanosis.

EFFECTS OF CHRONIC EXPOSURE ' POSSible jaw necrosis, erosion of teeth, bronchial irritation

with chronic cough.

EMERGENCY AND FIRST AID PROCEDURES Ingestion - give milk of magnesia; get medical aid;

inhalation - respiratory resuscitation as needed, wash skin \& eyes - get medical aid.

\section{SECTION VI REACTIVITY DATA}

CONDITIONS CONTRIBUTING TO INSTABILITY

CONDITIONS CONTRIBUTING TO HAZARDOUS POLYMERIZATION

INCOMPATAEILITY (MATERIALS TO AVOID)

F'luorine, Perchloric Acid

HA7ARMNIIS RFTOMPQ̨SITION PRQDUGTE

SECTION VII SPILL OR LEAK PROCEDURES

STEPS TO BE TAKEN IN CASE MATERIAL IS RELEASED OR SPILLED

WASTE DISPOSAL METHOD

SECTION VIII SPECIAL PROTECTION INFORMATION

\begin{tabular}{l|l}
\hline VENTILATION REQUIREMENTS LOCAL EXHAIJST & $\begin{array}{l}\text { GROTECTIVE EQUIPMENT (SPECIFY TYPFS) EYE } \\
\text { Goggles }\end{array}$ \\
\hline MECHANICAL (GENERAL) & $\begin{array}{c}\text { GLOVES } \\
\text { Neoprene rubber }\end{array}$ \\
\hline SPECIAL & RESHIRAIUR \\
& W/appropriate filter \\
\hline
\end{tabular}

OTHER PROTECTIVF, EQUIPMENT

Rubber apron, full face shield. SECTION IX SPECIAL PRECAUTIONS

PRECAUTIONS TO BE TAKEN IN HANDLING AND STORAGE

Avoid physical damage.

OTHER PRECAUTIONS

SIGNATURE

DATE

1-0922B 


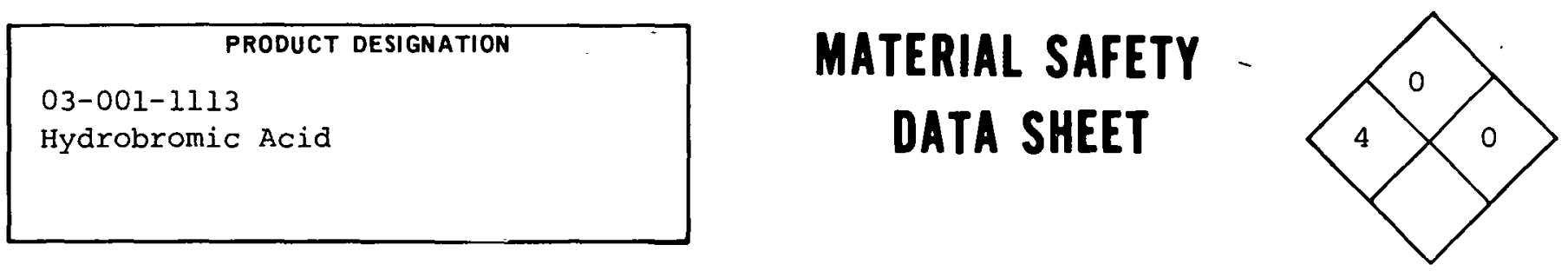

SECTION I SOURCE AND NOMENCLATURE

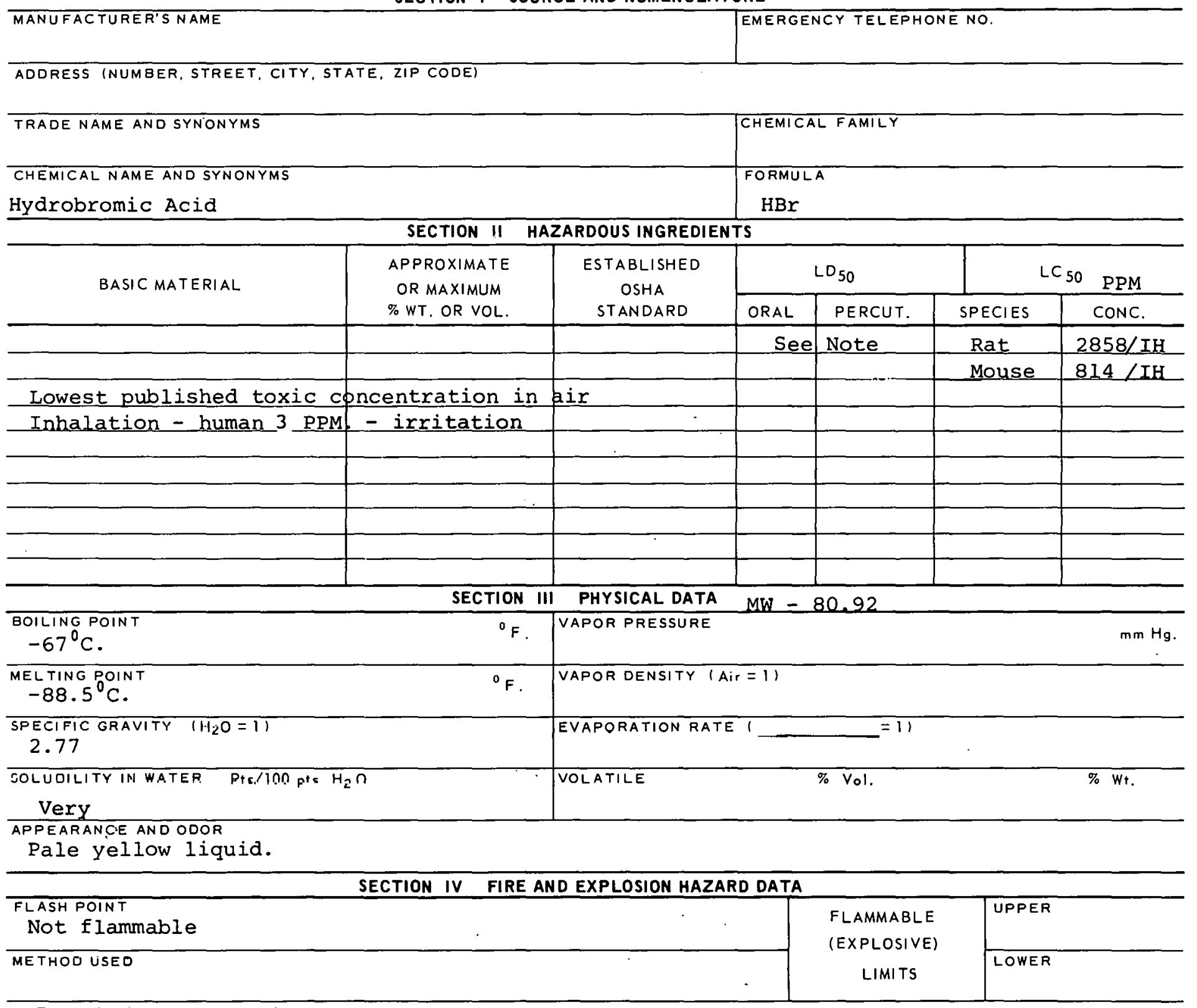

EXTINOUISHING MEDIA

Add water, then neutralize with chemically basic substances such as sodium bicarbonate, soda ash, or slaked lime.

SPECIAL FIRE FIGHTING PROCEDURES

Wear full protective clothing.

IINUSUAL FIRE AND EXPLOSION HAZARDS In contact with common metals and presence of moisture produces hydrogen which may form explosive mixtures in air. 
Hydrobromic Acid SECTION V HEALTH HAZARD DATA

TOXICLEVEL

TLV 3 PPM, $10 \mathrm{mg} / \mathrm{C.M}$.

CARCINOGENIC

ROUTES OF ABSORBTION

Inhalation of vapor, ingestion

SKIN AND ĖYE

IRRITATION Severe

REVELANT SYMPTOMS OF EXPOSURE Conjunctivitis, pulmonary edema, coughing, choking, headache, dizziness, cyanosis.

EFFECTS OF CHRONIC EXPOSURE POSSible jaw necrosis, erosion of teeth, bronchial irritation with chronic cough.

EMERGENCY AND FIRST AID PROCEDURES If ingested - give milk of magnesia, get medical aid;

Inhalation - resusitation measures if necessary. Wash skin \& eyes.

\section{SECTION VI REACTIVITY DATA}

CONDITIONS CONTRIBUTING TO INSTABILITY

CONDITIONS CONTRIBU TING TO HAZARDOUS POLYMERIZATION

INCOMPATABILITY IMATERIALSTQ AVOTI

Fluorine.

HAZARDOUS DECOMPOSITION PRODUCTS

SECTION VII SPILL OR LEAK PROCEDURES

STEPS TO BE TAKEN IN CASE MATERIAL IS RELEASED OR SPILLED

WASTE DISPOSAL METHOD

SECTION VIII SPECIAL PROTECTION INFORMATION

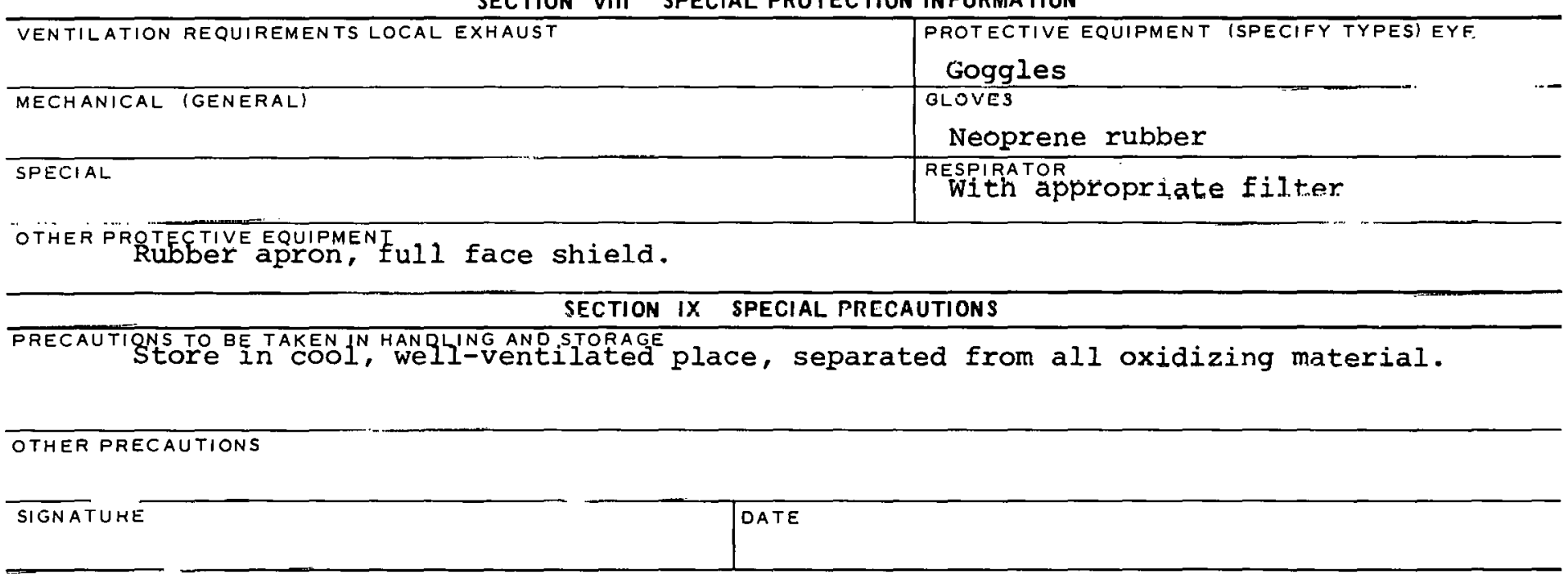

$1-1113 B$ 
CAS: 007647010

\section{PRODUCT DESIGNATION}

3-001-1324

Hydrochloric Acid

\section{MATERIAL SAFETY \\ DATA SHEET}

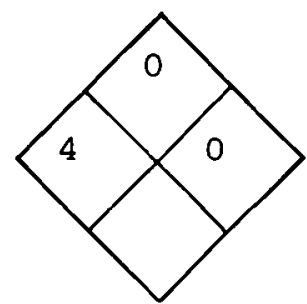

SECTION I SOURCE AND NOMENCLATURE

\begin{tabular}{l|l}
\hline MANUFACTURER'S NAME & EMERGENCY TELEPHONE NO. \\
\hline ADDRESS (NUMBER, STREET, CITY, STATE, ZIP CODE)
\end{tabular}

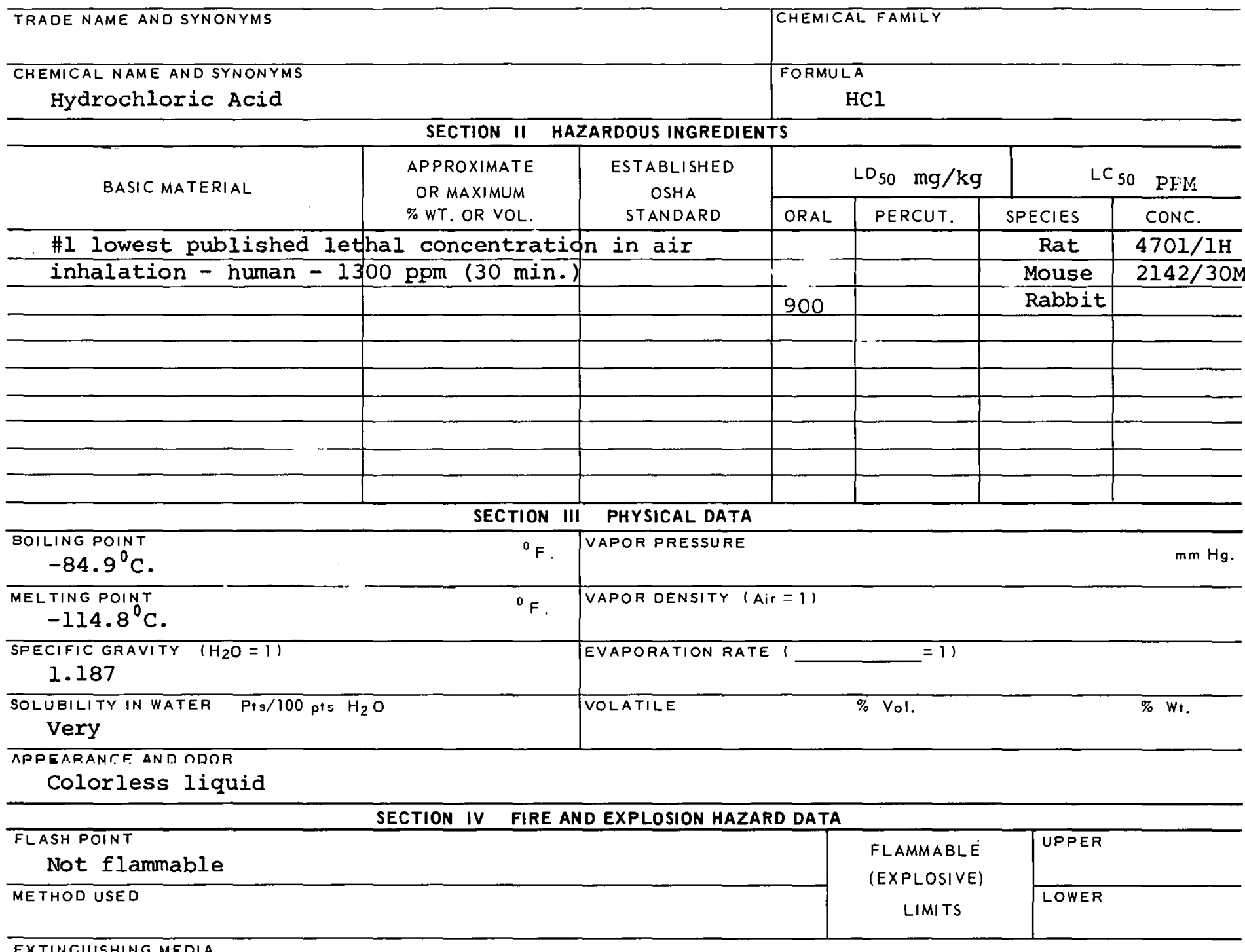

EXTINGUISHIAG MEDIA

Use water, neutralize with chemically basic substances

SPECIAL FIRE FIGHTING PROCEDURES

Wear full protective clothing

UNUSUAL FIRE AND EXPLOSION HAZARDS

On contact with common metals produces hydrogen which may form explosive mixtures in air. 
TOXIC LEVEL

TLV $5 \mathrm{ppm}, 7 \mathrm{mg} / \mathrm{C} . \mathrm{M}$. ceiling value PRINCIPLE ROUTES OF ABSORETION

Inhalation of vapor or mist. SKIN AND EYE

CARCINOGENIC

IRRITATION Severe

REVELANT SYMPTOMS OF EXPOSURE Conjunctivitis, pulmonary edema, choking, coughing, headache, dizziness, cyanosis, low blood pressure and high pulse pressure. Severe ulceration of skin. EFFECTS OF CHRONIC EXPOSURE

Possible jaw necrosis, erosion of teeth, bronchial irritation with chronic cough. EMERGENCY AND FIRST AID PROCEDURES Ingestion: dilute with large quantities of water or milk.

Then use milk of magnesia. Eye or skin contact: flood areas with large amounts of water for $15 \mathrm{~min}$. Inhalation: remove from exposure - use artificial respiration if necessary. CONDITIONS CONTRIBUTING TO INSTABILITY SECTION VI REACTIVITY DATA Obtain medical help.

CONOITIONS CONTRIBUTING TO HAZARDOUS POLYMERIZATION

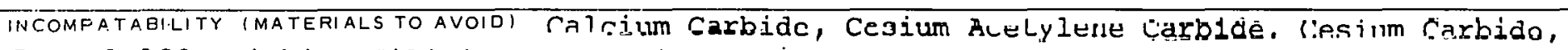
Doural 100, Lilliun Sil1cide, Magnesium Boride, Rubidium Acetylene Carbide. HAZARDOUS DECOMPOSITION PRODUCTS

\section{SECTION VII SPILL OR LEAK PROCEDURES}

STEPS TO BE TAKEN IN CASE MATERIAL IS REI, FASED OR SPILLED

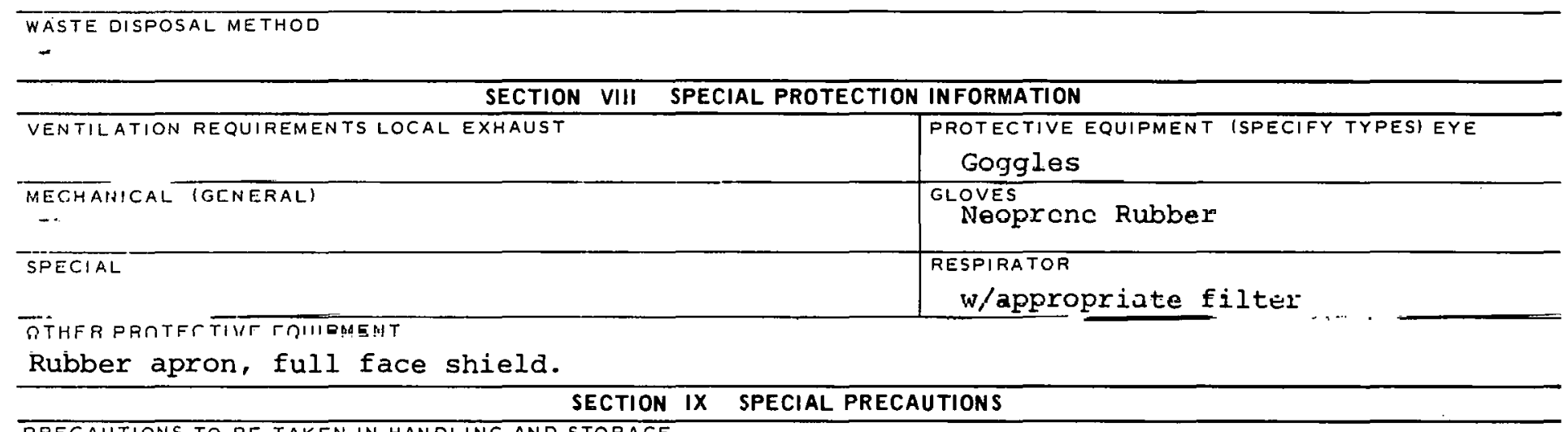

PRECAUTIONS TO BE TAKEN IN HANDLING AND STORAGE

Store in cool, well-ventilated place, separated from all oxidizing materials.

OTHER PRECAUTIONS

SITIN DTIIBS

DATE

$1-1324 \mathrm{~B}$ 

CAS - 007664393
PRODUCT DESIGNATION
MATERIAL SAFETY
DATA SHEET
Hydrofluoric Acid
03-001-1500

\section{SECTION I SOURCE AND NOMENCLATURE}

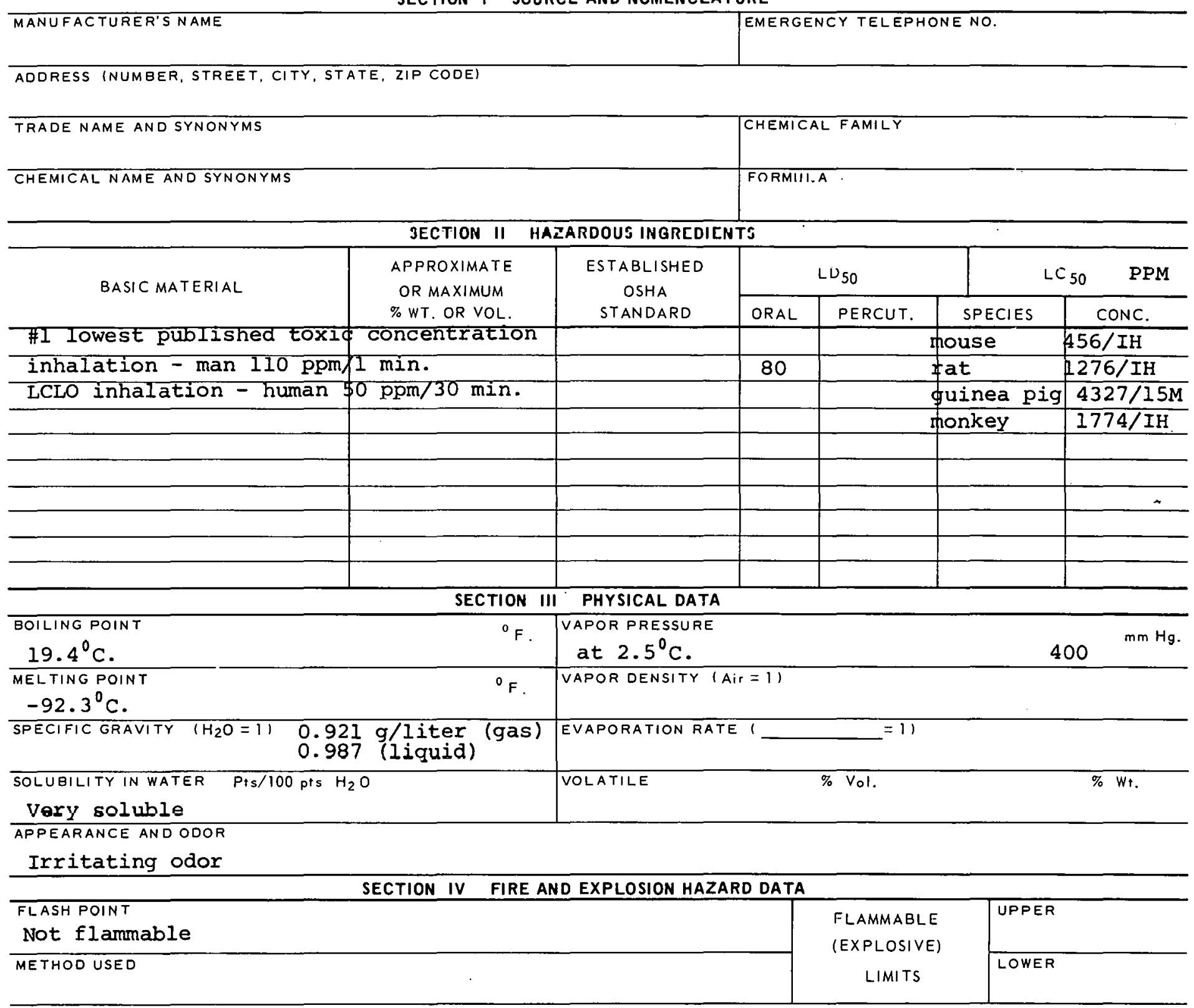

EXTINGUISHING MEDIA

Water

SPECIAL FIRE FIGHTING PROCEDURES

Wear full protective clothing

UNUSUAL FIRE ANO EXPLOSION HAZARDS

Hydrogen may be formed when in contact with certain metals. 


\section{Hydrofluoric Acid}

\section{SECTIION $V$ HEALTH HAZARD DATA}

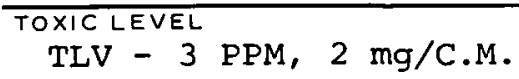


CAS: OOI309451e

03-001-1716

Hydrofluosilicic Acid
VV82250

MATERIAL SAFETY

DATA SHEET

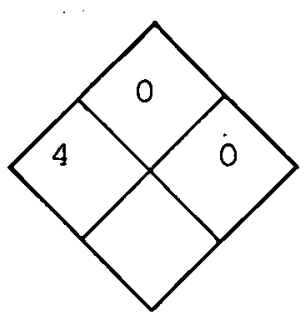

\section{SECTION I SOURCE AND NOMENCLATURE}

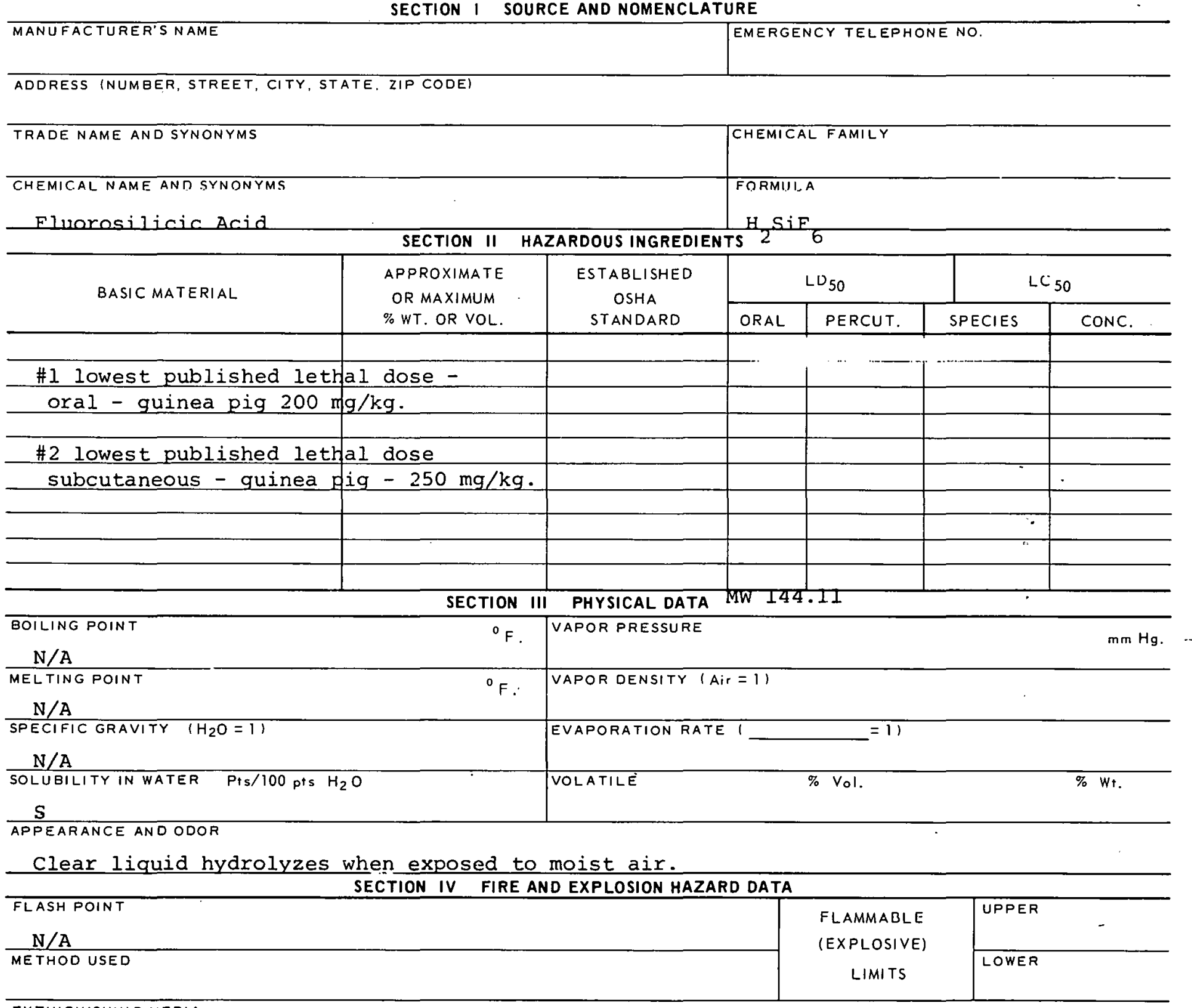

EXTINGUISHING MEDIA

Water

SPECIAL FIRE FIGHTING PROCEDURES

Wear full protective clothing.

UNUSUAL FIRE AND EXPLOSION HAZARDS

Hydrogen may be formed when in contact with certain metals. 


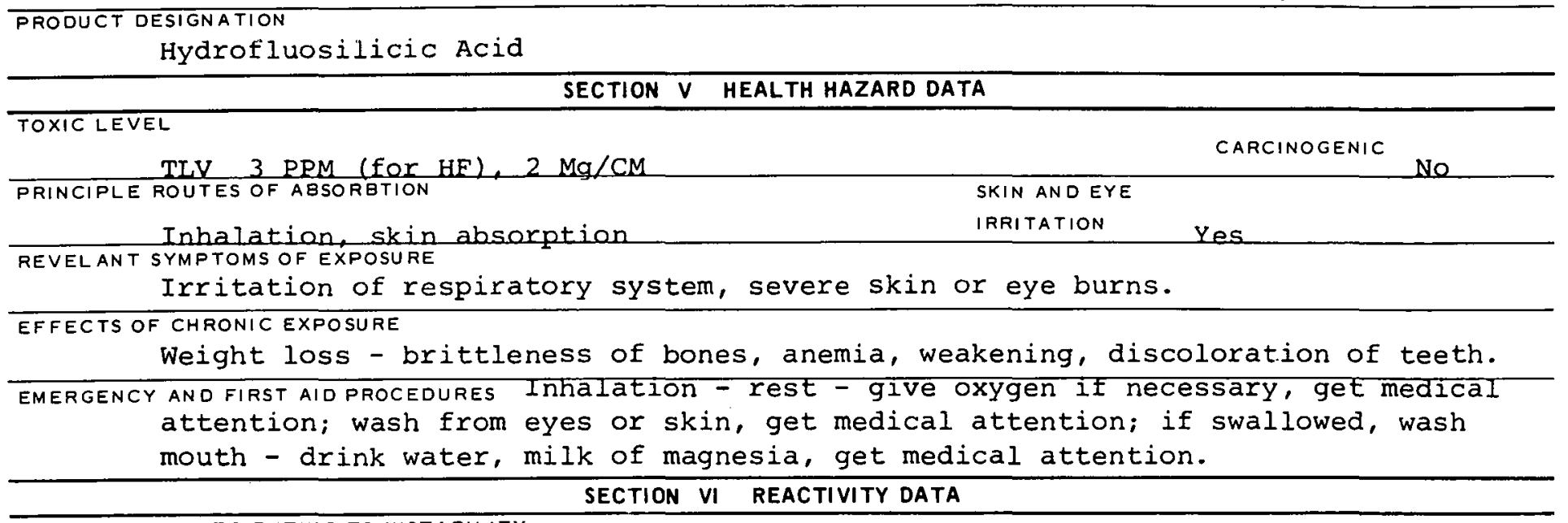

CONDITIONS CONTRIBUTING TO INSTABILITY

CONDITIONS CONTRIBUTING TO HAZARDOUS POLYMERI ZATION

INCOMPATABILITY (MATERIALS TO AVOIO)
May react exothermally with $\mathrm{As}_{2} \mathrm{O}_{3} \mathrm{cr} \mathrm{P}_{2} \mathrm{O} 5$

HAZARDOUS DECOMPOSITION PRODUCTS

SECTION VII SPILL OR LEAK PROCEDURES

STEPS TO BE TAKEN IN CASE MATERIAL IS RELEASED OR SPILLED

WASTE DISPOSAL METHOD

SECTION VIII SPECIAL PROTECTION INFORMATION

\begin{tabular}{|c|c|}
\hline VENTILATION REQUIREMENTS LOCAL EXHAUST & $\begin{array}{l}\text { PROTECTIVE EQUIPMENT (SPECIFY TYPES) EYE } \\
\text { GOggleS }\end{array}$ \\
\hline MECHANICAL (GENERAL) & $\begin{array}{l}\text { GLOVES } \\
\text { Rubber gloves }\end{array}$ \\
\hline EREGIAL & 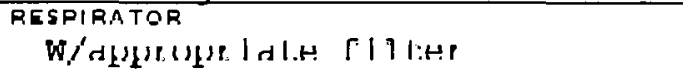 \\
\hline
\end{tabular}

OTHER PROTECTIVE EQUIPMENT

Full face shield

SECTION IX SPECIAL PRECAUTIONS

PRECAUTIONS TO BE TAKEN IN HANDLING AND STORAGE

OTHER PRECAUTIONS

SIGNATURE

DATE

$1-1716 B$ 


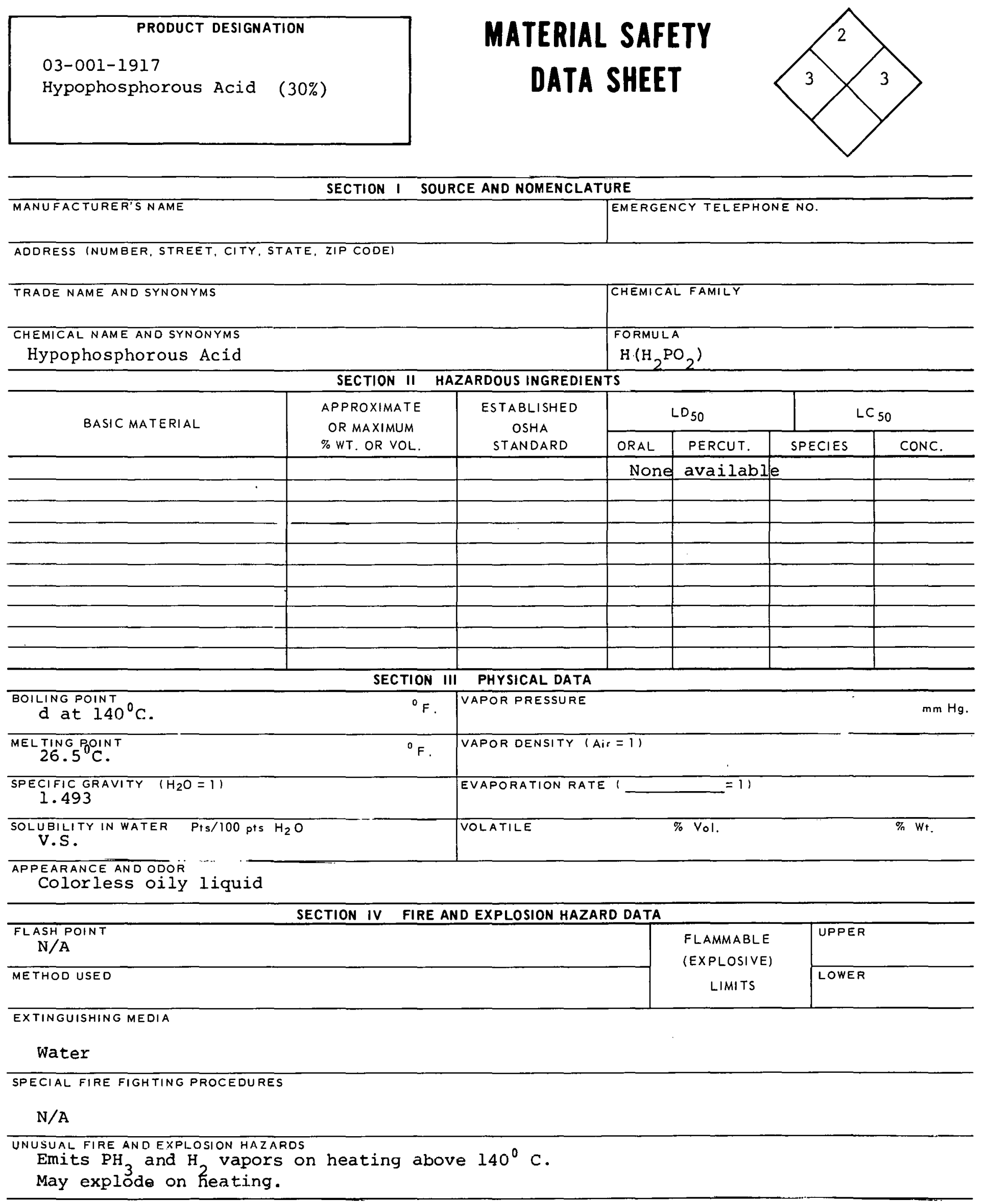


TOXIC LEVEL

Not available

CARCINOGENIC

No

PRINCIPLE ROUTES OF ABSORBTION

SKIN ANDEYE

Ingestion

IRRITATION YES

REVELANT SYMPTOMS OF EXPOSURE Slow attack on skin, attack on mucous membranes and

digestive tract.

EFFECTS OF CHRONIC EXPOSURE

Erosion of teeth.

EMERGENCY AND FIRST AID PROCEDURES Wash from SkIn and eyes; If swaIlowed - wash mouth, arink water, then milk of magnesia, get medical aid.

\section{SECTION VI REACTIVITY DATA}

\section{CONDITIONS CONTRIBUTING TO INSTABILITY \\ CONDITIONS CONTRIBUTING TO HAZARDOUS POLYMERIZATION \\ INCOMPATAEILITY (MATERIALS TO AVOID) \\ Reacts violently with oxidizing agents.}

HAZARDOIIS RFCOMPOGIT!ON PROQUETS

SECTION VII SPILL OR LEAK PROCEDURES

STEPS TO BE TAKEN IN CASE MATERIAL IS RELEASED OR SPILLED

WASTE DISPOSAL METHOD

\section{SECTION VIII SPECIAL PROTECTION INFORMATION}

\begin{tabular}{l|l}
\hline VENTILATION REQUIREMENTS LOCAL EXHAUST & FROTECTIVE EQUIPMENT (SPECIFY TYPES) EYE
\end{tabular}

Face shield

MECHANICAL (GENERAL) GLOVES

Rubber

SPECIAL REGPIRATUR

OTHER PROTECTIVE EQUIPMENT

Face shield and rubber apron.

SECTION IX SPECIAL PRECAUTIONS

PRECAUTIONS TO BE TAKEN IN HANDLING AND STORAGE

OTIIER PRECAUTIONS

SIGNATURE

DATE

$1-1917 \mathrm{~B}$ 


\section{PRODUCT DESIGNATION}

03-001-2128

Iodic Acid
MATERIAL SAFETY

DATA SHEET

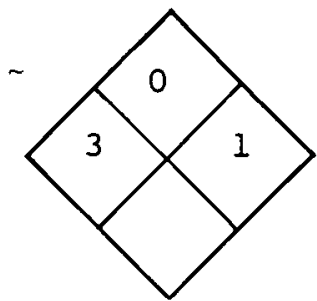

SECTION I SOURCE AND NOMENCLATURE

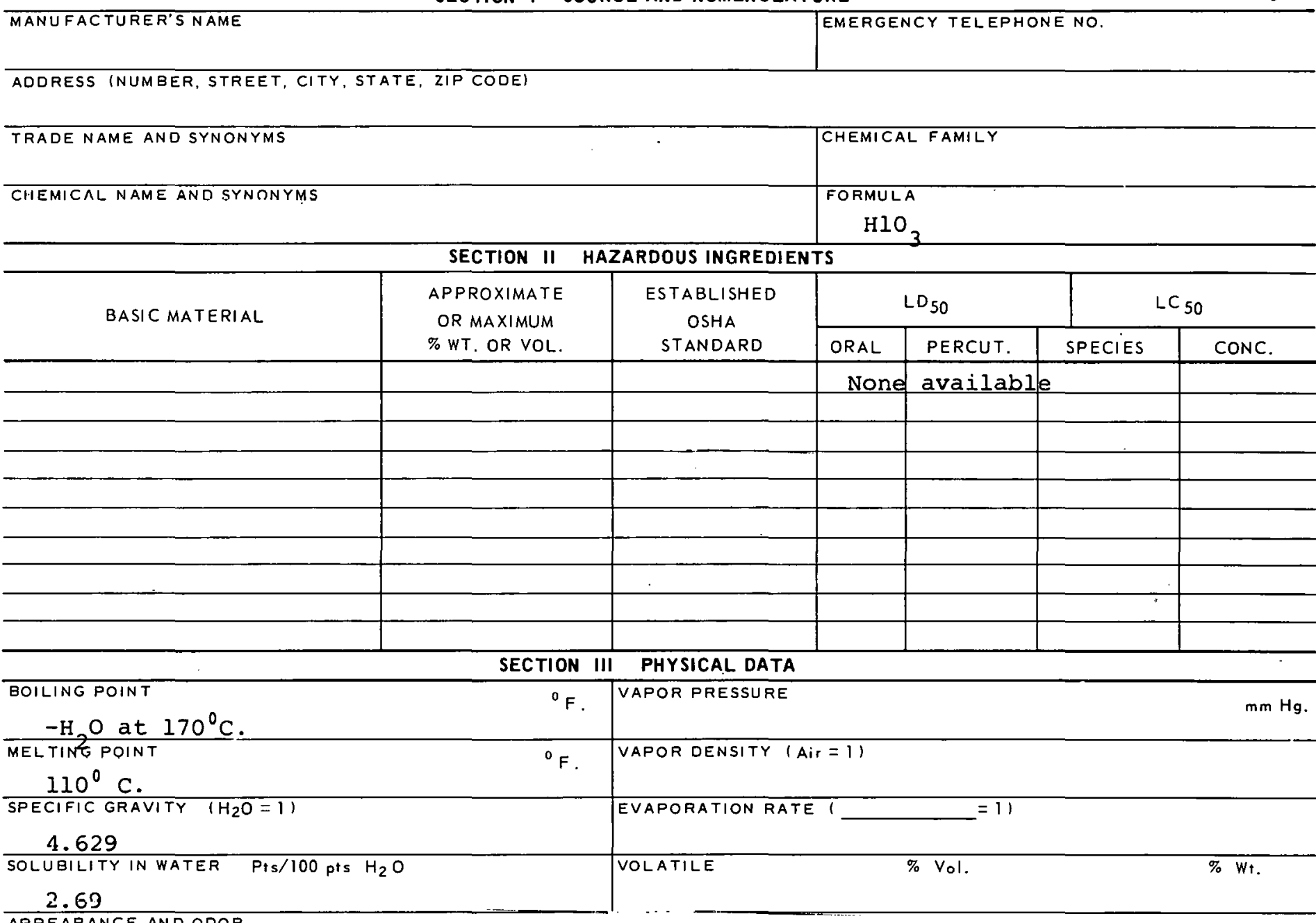

APPEARANCE AND ODOR

White crystal

SECTION IV FIRE AND EXPLOSION HAZARD DATA

FLASH POINT

None available

METHOD USED

EXTINGUISHING MEDIA

Water

SPECIAL FIRE FIGHTING PROCEDURES

UNUSUAL FIRE AND EXPLOSION HAZAROS

Strong oxidizing agent

\begin{tabular}{c|c}
$\begin{array}{c}\text { FLAMMABLE } \\
(\text { EXPLOSIVE) } \\
\text { LIMITS }\end{array}$ & UPPER \\
\cline { 2 - 2 } & LOWER
\end{tabular}

FLAMMABLE

LIMITS

LOWER 


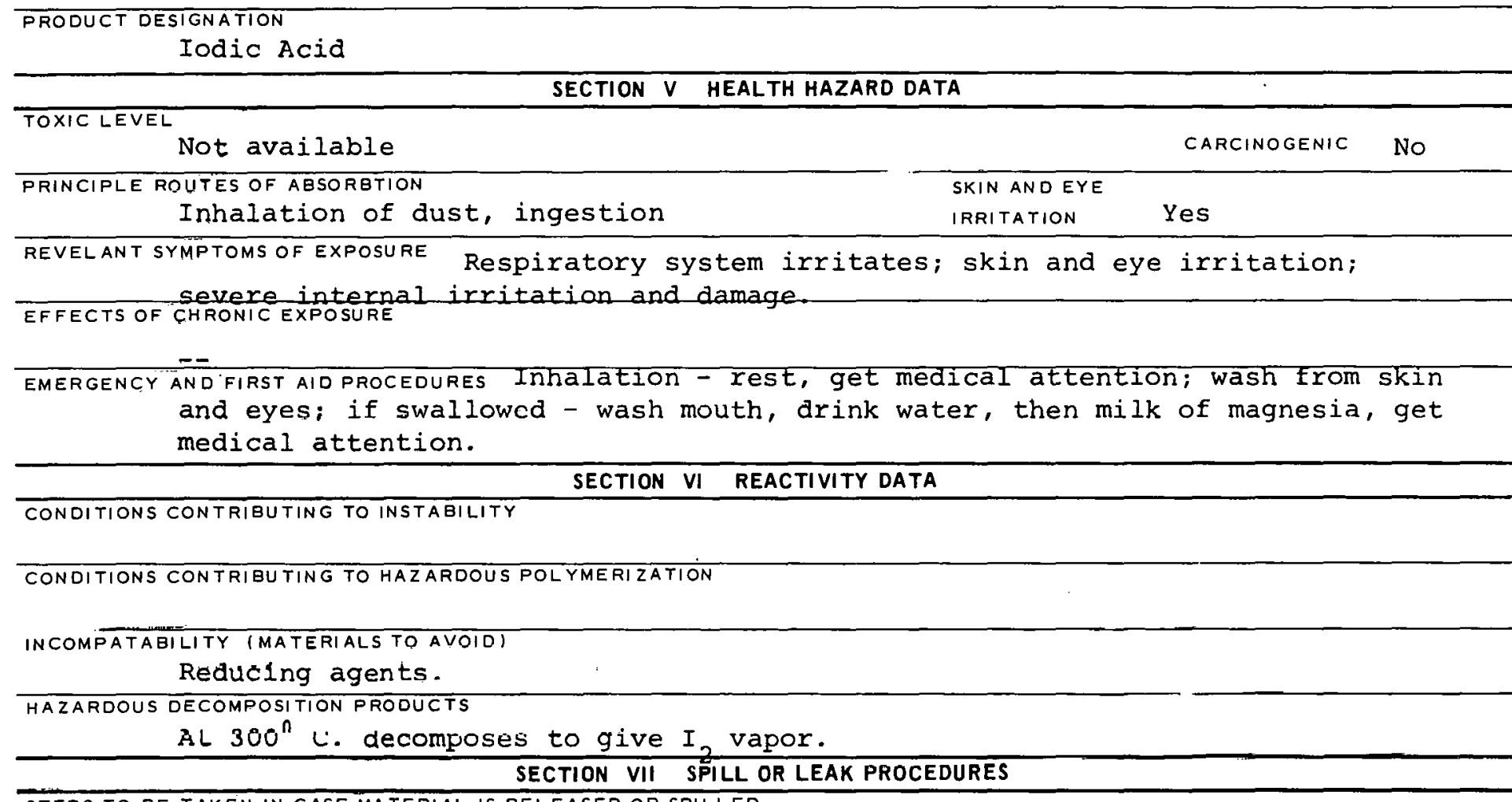

STEPS TO BE TAKEN IN CASE MATERIAL IS RELEASED OR SPILLED

WASTE DISPOSAL METHOD

SECTION VIII SPECIAL PROTECTION INFORMATION

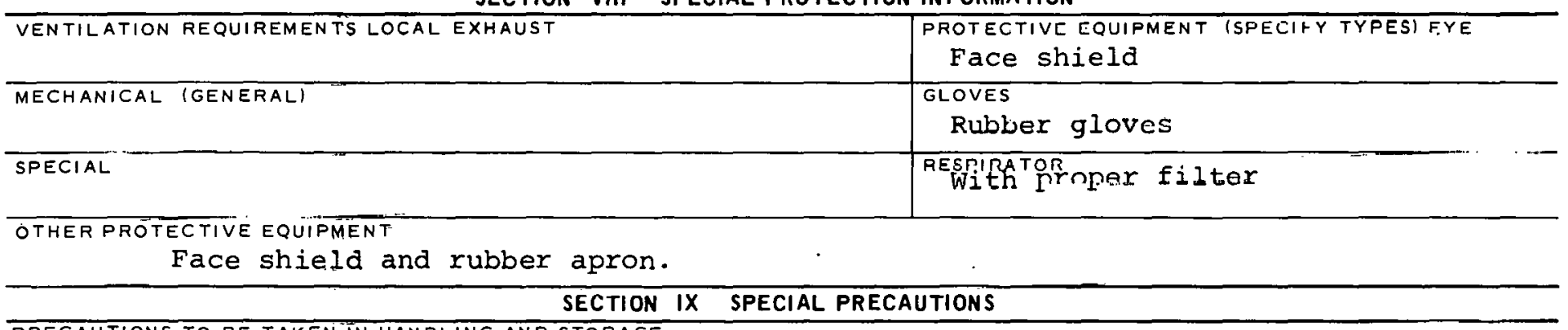

PRECAUTIONS TO BE TAKEN TIN HANDLING AND STORAGE

OTHER PRECAUTIONS

SIGNATURE

DATE

$1-2128 \mathrm{~B}$ 


\begin{tabular}{|c|}
\hline PRODUCT DESIGNATION \\
03-001-2933 \\
Nitiic ficic \\
\hline
\end{tabular}

\section{MATERIAL SAFETY DATA SHEET}

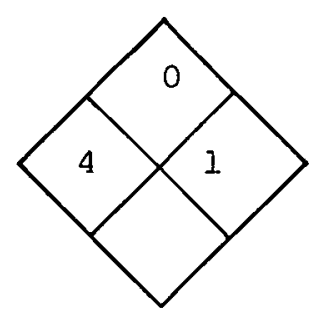

SECTION I SOURCE AND NOMENCLATURE

\begin{tabular}{l|l}
\hline MANUFAC TURER'S NAME & EMERGENCY TELEPHONE NO. \\
\hline ADDRESS INUMBER, STREET, CITY, STATE, ZIP CODE)
\end{tabular}

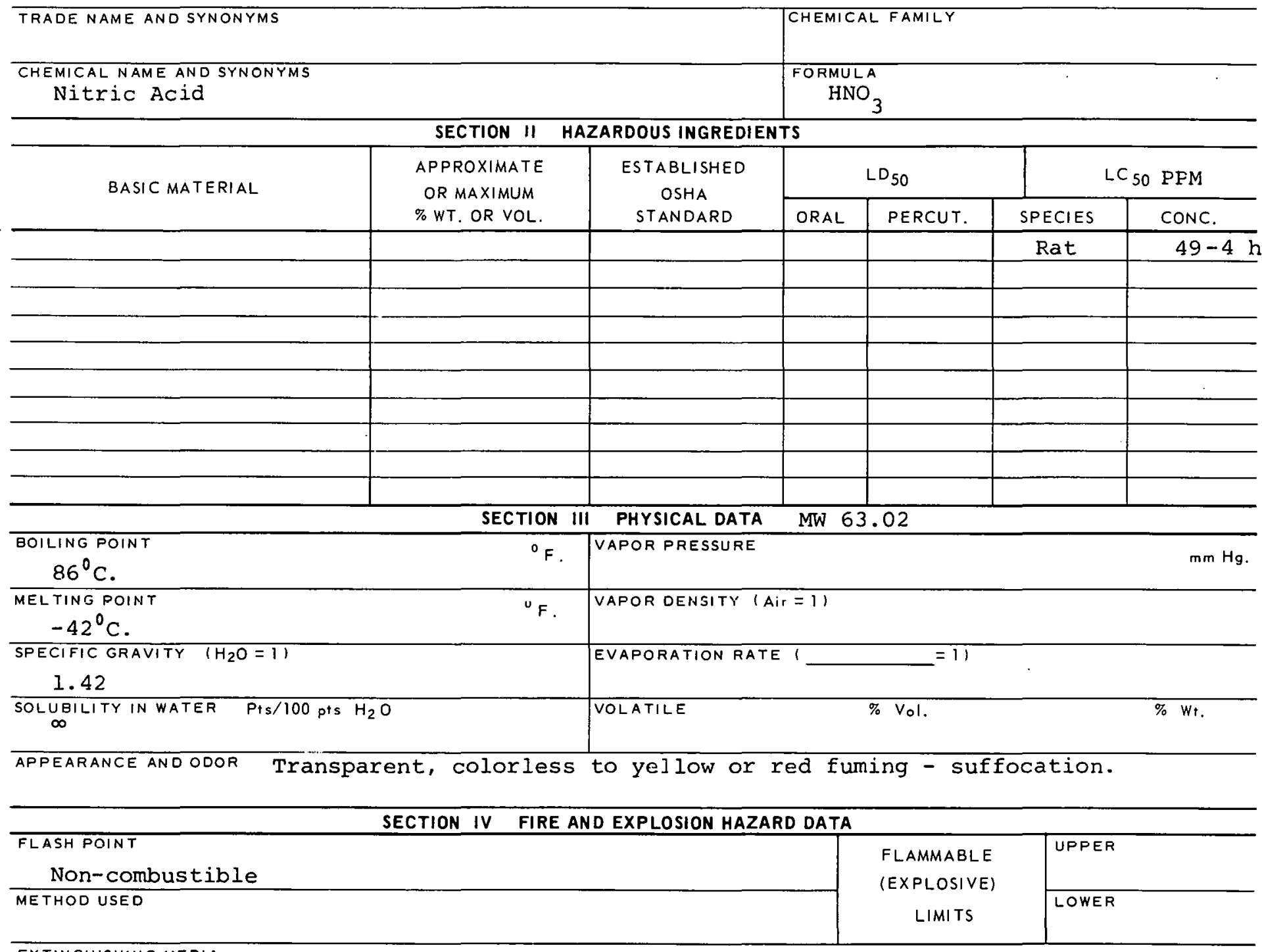

EXTINGUISHING MEDIA

Water spray

SPECIAL FIRE FIGHTING PROCEDURES

Use water spray, wear full-protective clothing.

UNUSUAL FIRE AND EXPLOSION HAZARDS

Reacts explosively with metallic powders, carbides, hydrogen sulfide and turpentine. 


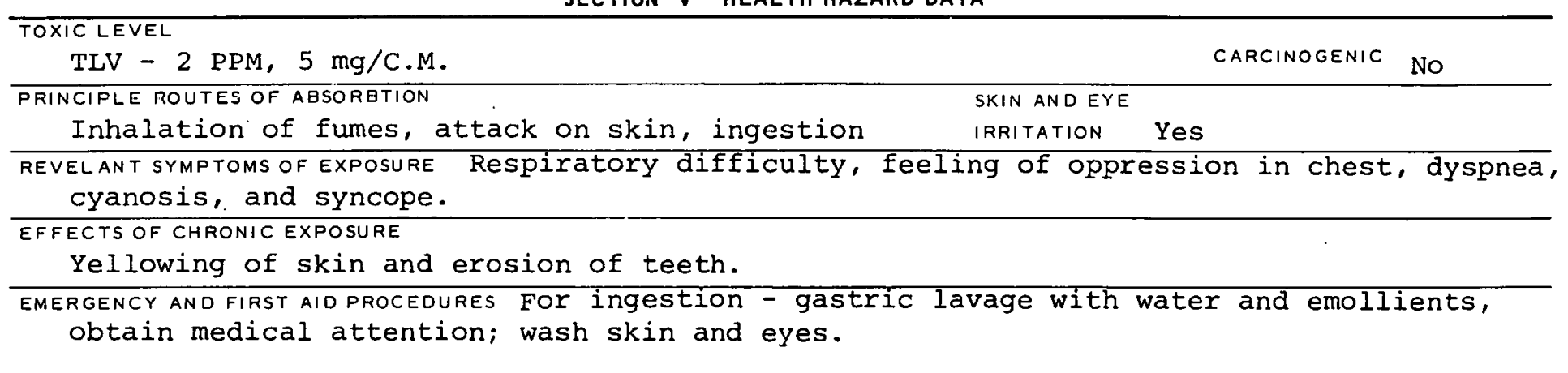

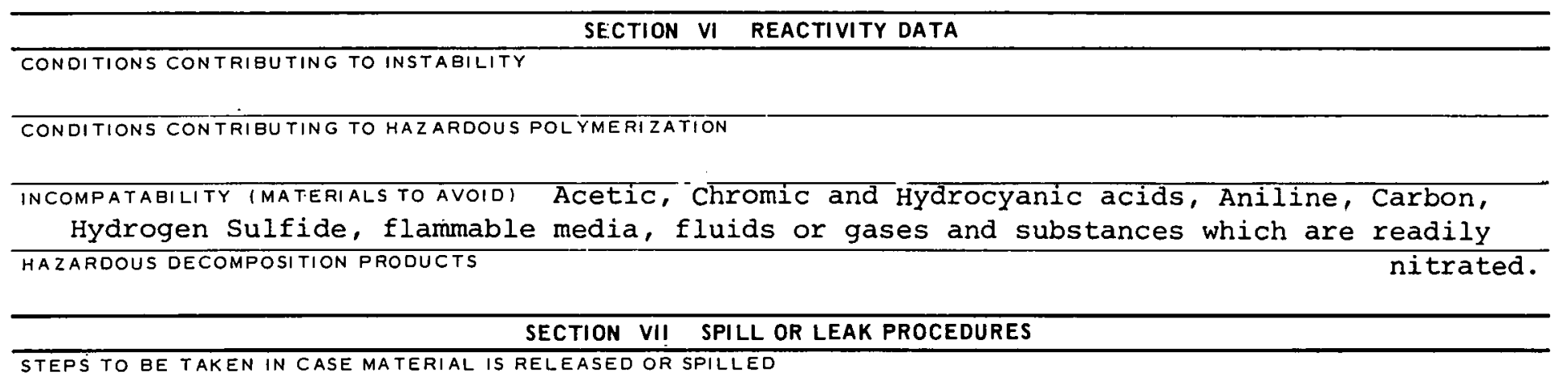

WASTE DISPOSAL METHOD

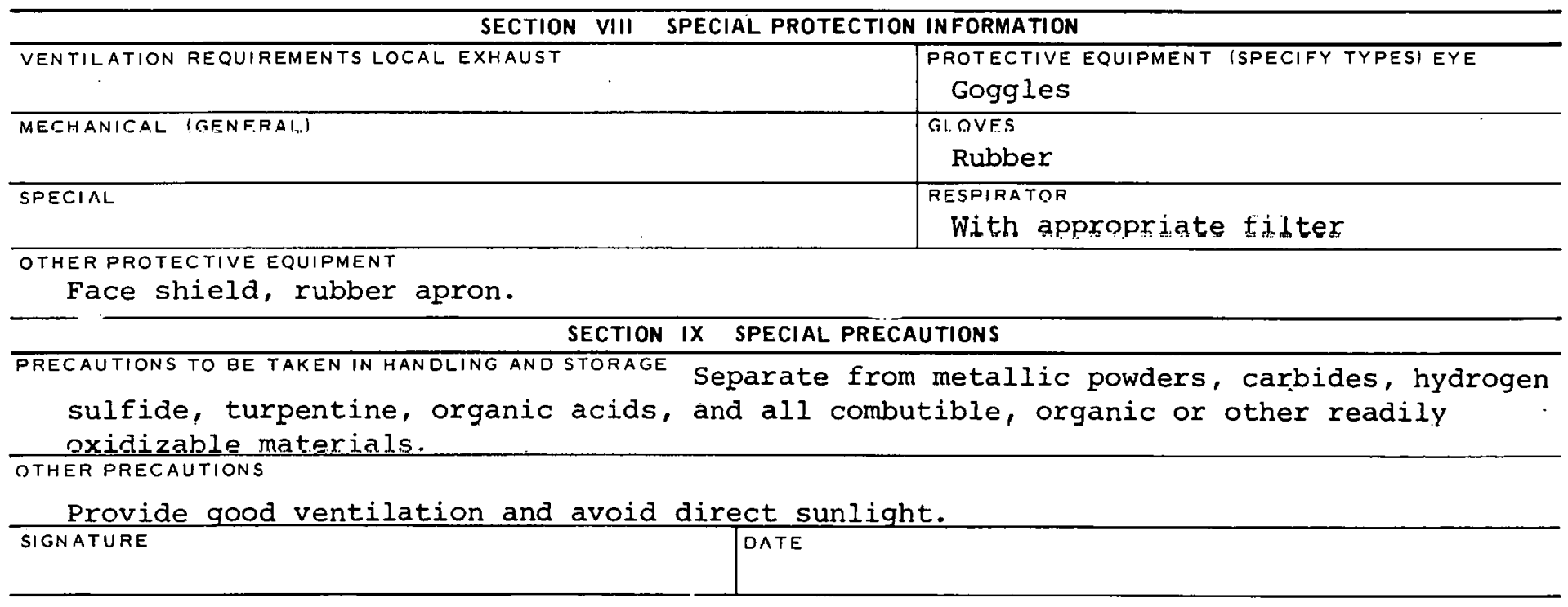




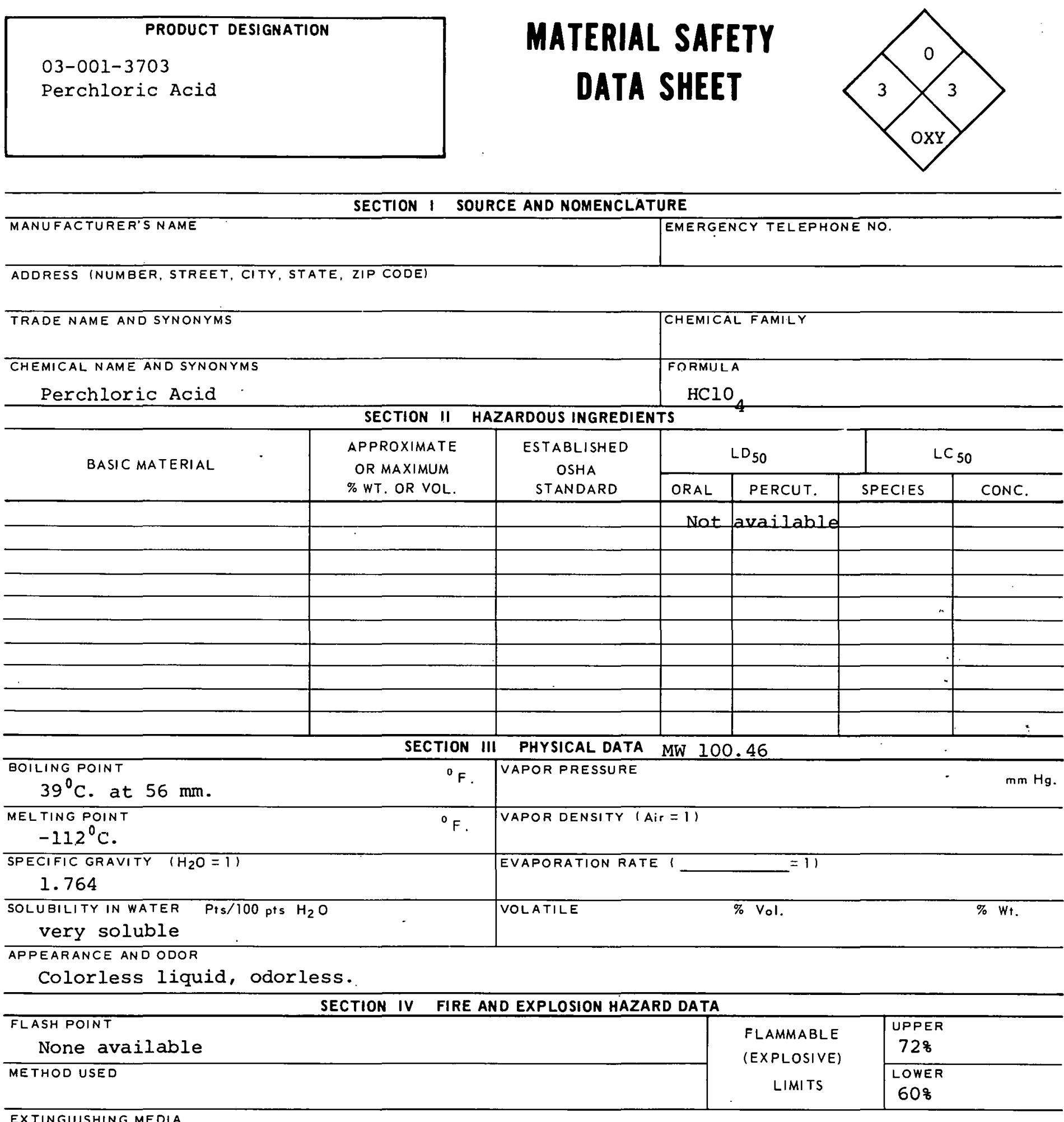

EXTINGUISHING MEDIA

Water spray

SPECIAL FIRE FIGHTING PROCEDURES

Wear full protective clothing.

UNUSUAL FIRE AND EXPLOSION HAZARDS

Explodes on contact with most organic materials.
(3) $10-74)$ 


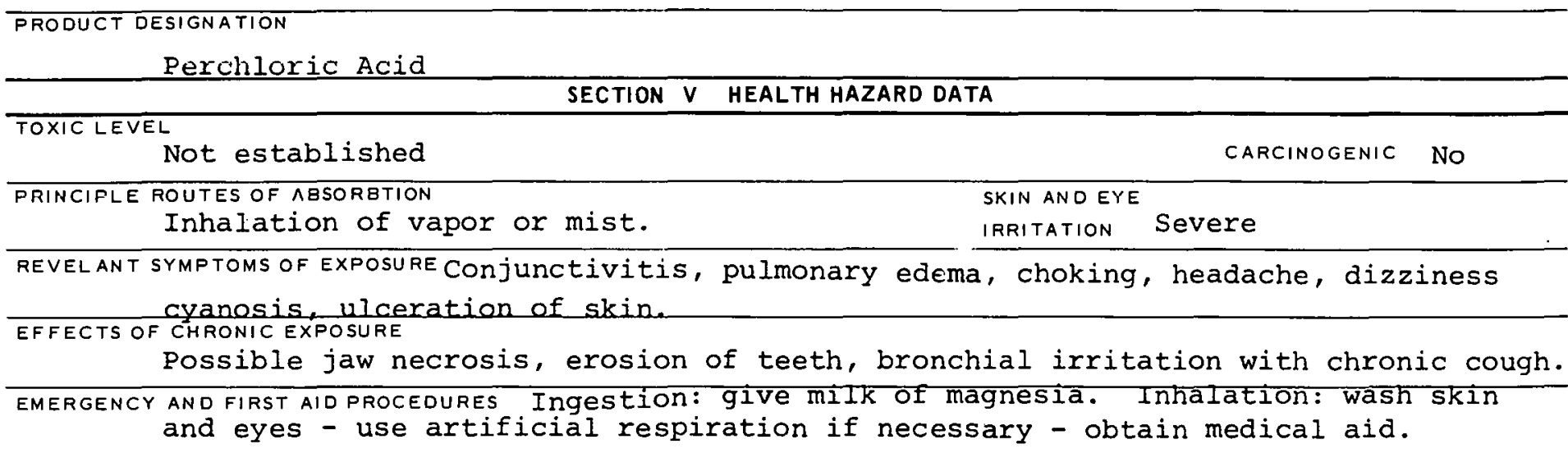

\section{SECTION VI REACTIVITY DATA}

CONDITIONS CONTRIBUTING TO INSTABILITY

CONDITIONS CONTRIBU TING TO HAZARDOUS POLYMERI ZATION

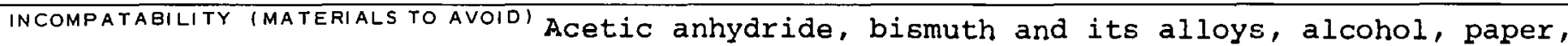
WoOd.

HAZARDOUS DECOMPOSITION PRODUCTS

SECTION VII SPILL OR LEAK PROCEDURES

STEPS TO BE TAKEN IN CASE MATERIAL IS RELEASED OR SPILLED

Cover with reducing agents such as bisulfites, transfer sludge into a

large container of water for disposal.

WASTE DISPOSAL METHOD

SECTION VIII SPECIAL PROTECTION INFORMATION

\begin{tabular}{l|l}
\hline VENTILATION REQUIREMENTS LOCAL EXHAUST & $\begin{array}{l}\text { PROTECTIVE EQUIPMENT (SPECIFY TYPES) EYE } \\
\text { GOggles }\end{array}$ \\
\hline MECHANICAL IGENERAL) & GLOVES \\
& NeOprene rubber \\
\hline SPECIAL & RESPIRATOR \\
W/appropriate filter
\end{tabular}

Rubber apron, full face shield.

SECTION IX SPECIAL PRECAUTIONS

PRECAUTIONS TO BE TAKEN IN HANDLING AND STORAGE
Protect glass container from breaking. Keep from freezing and heat.

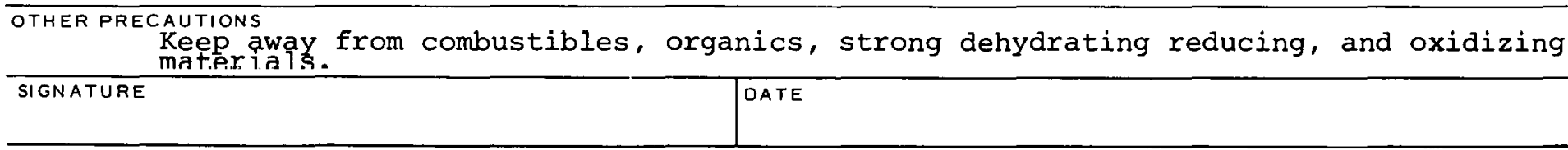

$1-3703 B$ 


\begin{tabular}{l|}
\multicolumn{1}{|c|}{ PRODUCT DESIGNATION } \\
$\begin{array}{l}\text { 3-001-3904 } \\
\text { Periodic Acid }\end{array}$ \\
DATA SHEET
\end{tabular}

SECTION I SOURCE AND NOMENCLATURE

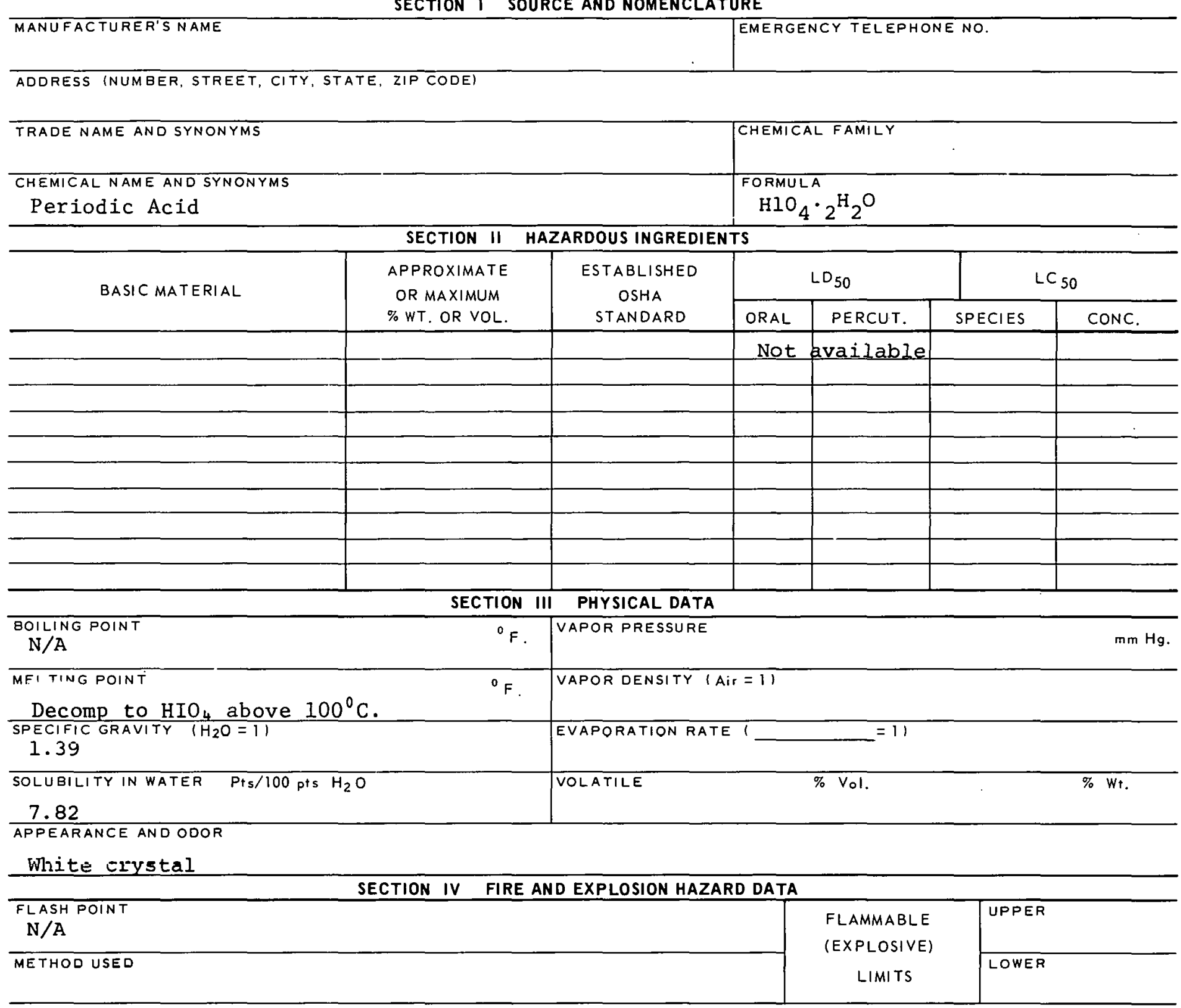

EXTINGUISHING MEDIA

Water spray

SPECIAL FIRE FIGHTING PROCEDURES

$\mathrm{N} / \mathrm{A}$

UNUSUAL FIRE AND EXPLOSION HAZARDS

Strong oxidizing agent - explosive properties unknown but assumed to be like perchloric acid.
$\left(\begin{array}{ll}3 & 10-74\end{array}\right)$ 


\section{SECTION $V$ HEALTH HAZARD DATA}

TOXIC LEVEL

Not available

CARCINOGENIC

PRINCIPLE ROITES OF ABSORBTION

Inhalation of dust, ingestion

SKIN AND EYE

IRRITATION SEVERe REVELANT SYMPTOMS OF EXPOSURE Respiratory system irritated; skin and eye irritation; severe
internal irritation and dăage.

EFFECTS OF CHRONIC EXPOSURE

$--$

EMERGENCY AND FIRST AID PROCEDURES Inhalation - rest, get medical attention; wash from skin and eyes; if swallowed - wash from skin and eyes; if swallowed - wash mouth, drink water, then milk of magnesia, get medical attention. SECTION VI REACTIVITY DATA

CONDITIONS CONTRIBUTING TO INSTABILITY

It is a stronger oxidizing agent than $\mathrm{K}$ MnO

CONDITIONS CONTRIQUTING TO HAZARDOUS POLYMERIZATION

INCOMPATABILITY (MATERIALS TO AVOIO)

Avoid contact with strong reducing agents.

HAZARDOUS DECOMPOSITION PRODUCTS

Dccompoocd to oxido of iodino.

SECTION VII SPILL OR LEAK PROCEDURES

STEPS TO BE TAKEN IN CASE MATERIAL IS RELEASED OR SPILLED

WASTE DISPOSAL METHOD.

\begin{tabular}{l|l}
\hline \multicolumn{1}{c}{ SECTION VIII SPECIAL PROTECTION INFORMATION } \\
\hline VENTILATION REQUIREMENTS LOCAL EXHAUST & $\begin{array}{l}\text { PROTECTIVE EQUIPMENT (SPECIFY TYPESI EYE } \\
\text { FaCe shield }\end{array}$ \\
$\because \because$ & GLOVES \\
\hline MECHANICAL IGENERAL) & RESPIRATOR \\
SPECIAL & With appropriate filter \\
\hline
\end{tabular}

OIHER HKUIELIIVE EÜUIPMENT

SECTION IX SPECIAL PRECAUTIONS

PRECAUTIONS TO BE TAKEN IN HANDLING AND STORAGE

OTHER PRECAUTION3

SIGNATURE 


\begin{tabular}{|c|}
\hline PRODUCT DESIGNATION \\
$\mathrm{P}_{2} \mathrm{O}_{5}$ Phosphoric Anhydride \\
$03-001-4506$ \\
\hline
\end{tabular}

\section{MATERIAL SAFETY DATA SHEET}

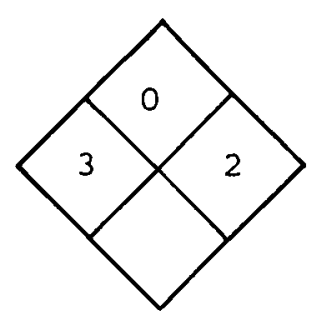

\section{SECTION I SOURCE AND NOMENCLATURE}

MANUFACTURER'S NAME

ADDRESS INUMBER, STREET, CITY, STATE, ZIP CODE)

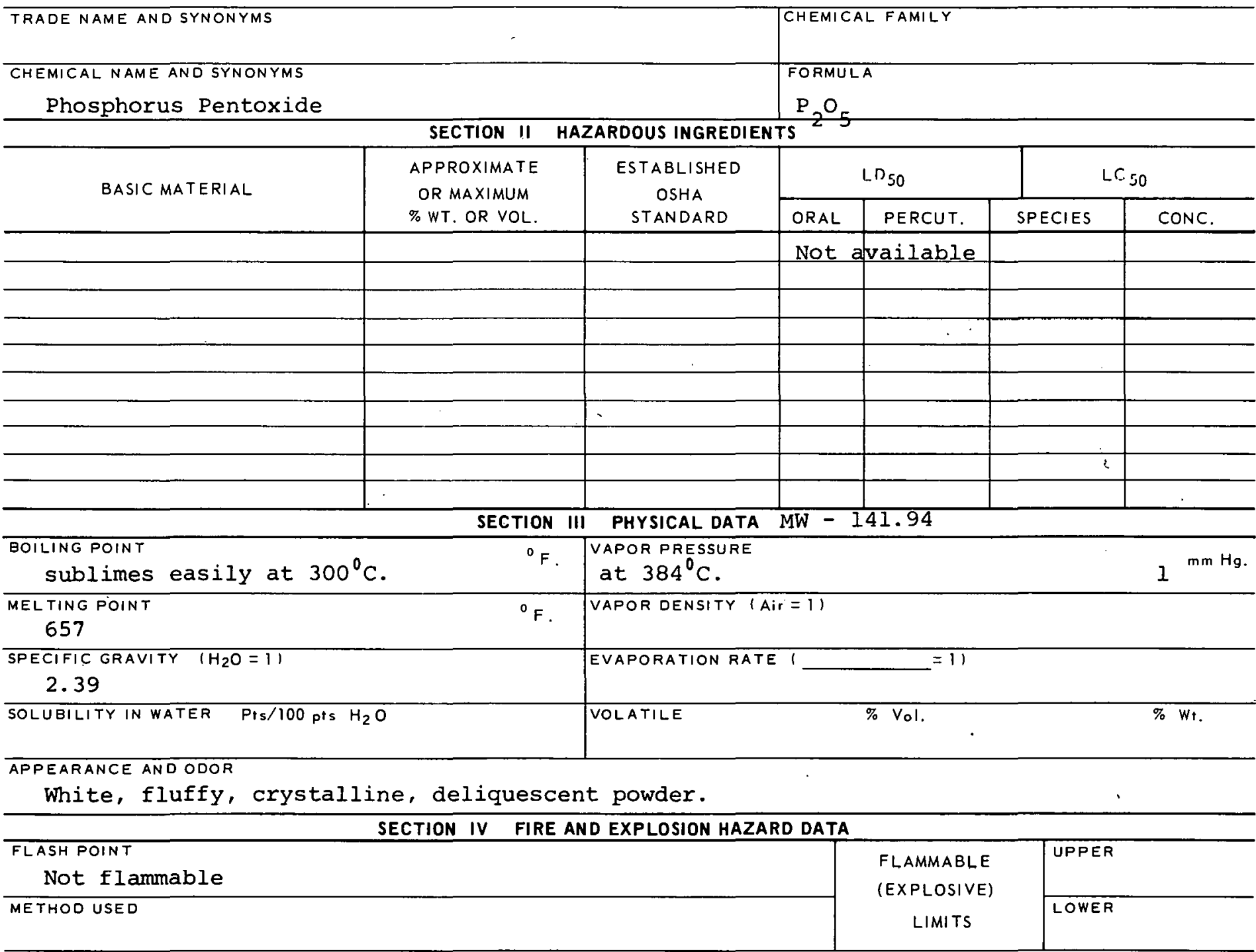

EXTINGUISHING MEDIA

Avoid water

SPECIAL FIRE FIGHTING PROCEDURES

Wear self-contained respirator.

UNUSUAL FIRE AND EXPLOSION HAZARDS 
Phosphoric Anhydride

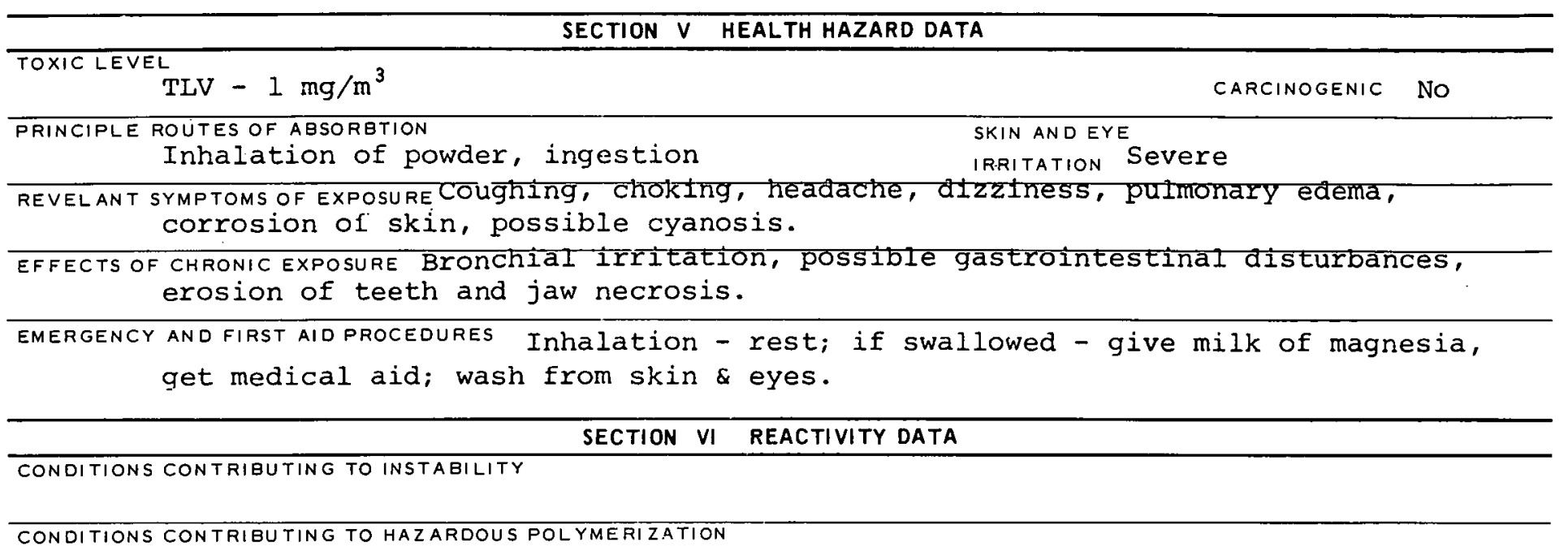
HAZARDOUS DECOMPOSITION PRODUCTS

\section{SECTION VII SPILL OR LEAK PROCEDURES}

STEPS TO BE TAKEN IN CASE MATERIAL IS RELEASED OR SPILLED

Add soda ash to spill. Add Water.

WASTE DISPOSAL METHOD

SECTION VIII SPECIAL PROTECTION INFORMATION FUIl protection

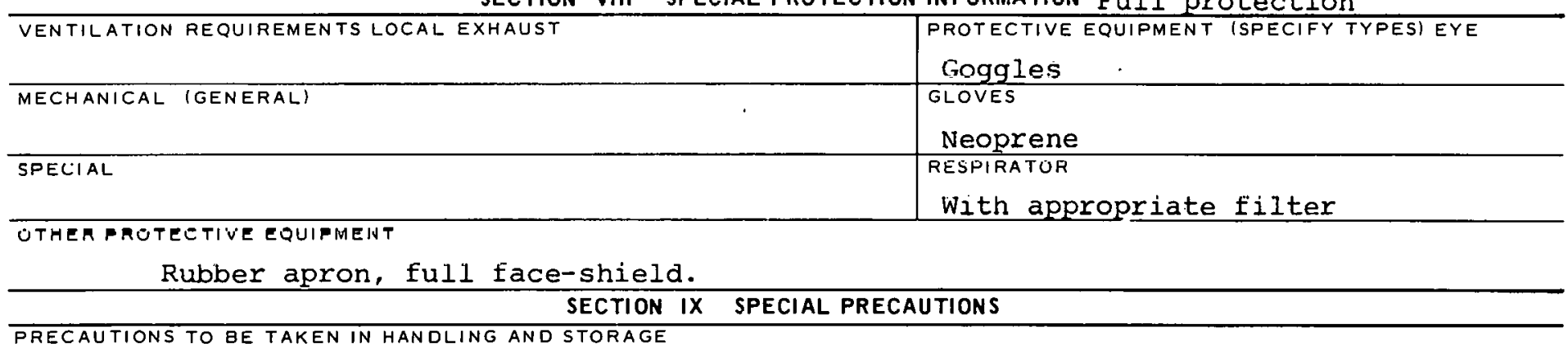

OTHER PR̈ECÁUT̈IONS 


\begin{tabular}{|l|}
\hline PRODUCT DESIGNATION \\
03-001-4516 \\
Phosphoric Acid \\
\hline
\end{tabular}

\section{MATERIAL SAFETY \\ DATA SHEET}

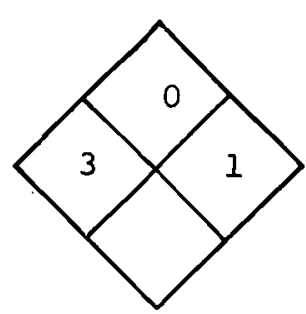

SECTION I SOURCE AND NOMENCLATURE

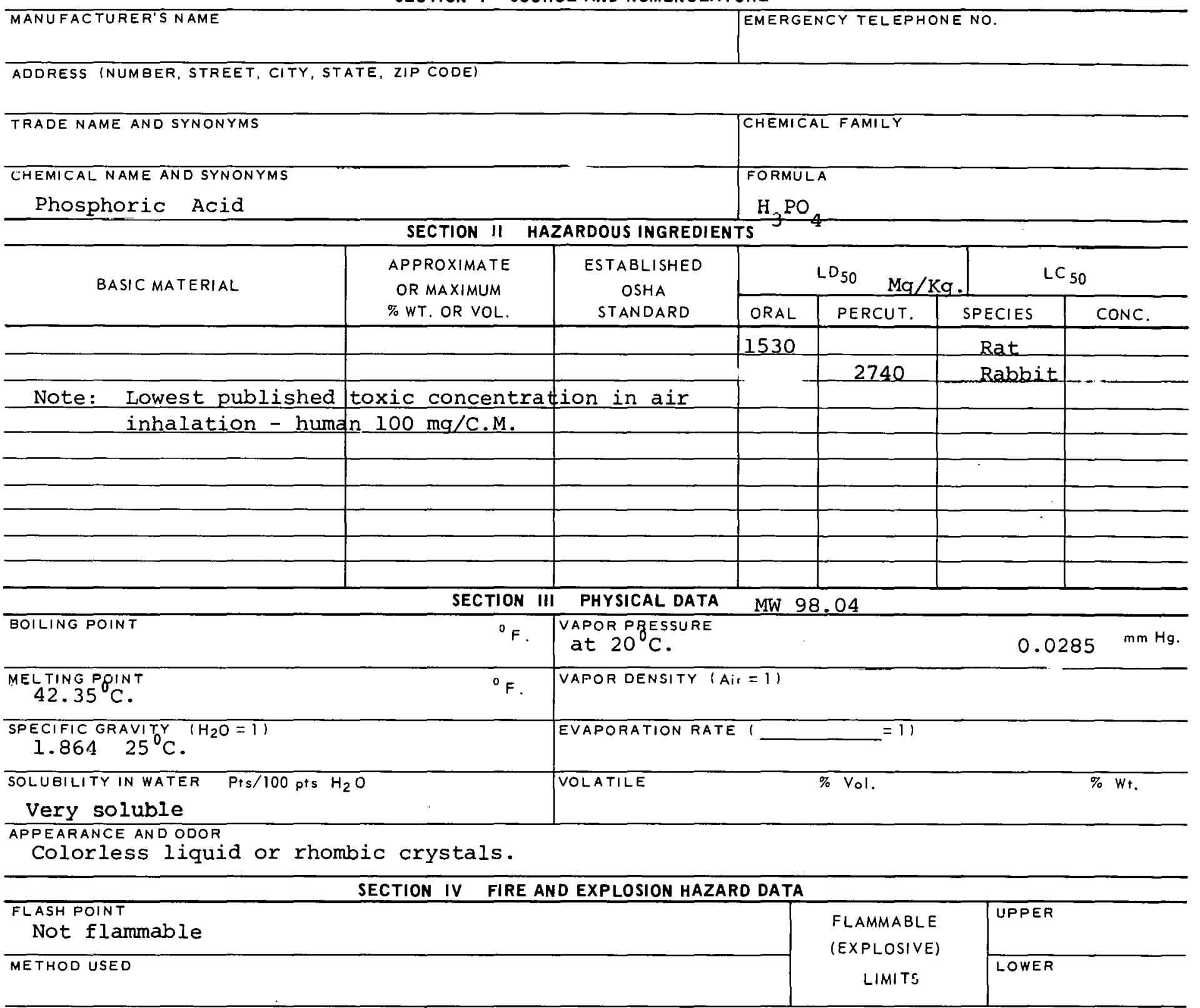

EXTINGUISHING MEDIA

Water

SPECIAL FIRE FIGHTING PROCEDURES

$\mathrm{N} / \mathrm{A}$

UNUSUAL FIRE AND EXPLOSION HAZARDS

$\mathrm{N} / \mathrm{A}$ 


\section{TOXIC LEVEL \\ $1 \mathrm{mg} / \mathrm{m}^{3}$}

PRINCIPLE ROUTES OF ABSORBTION

Inhalation
CARCINOGENIC

No

SKIN AND EYE

IRRITATION SEVETE

REVELANT SYMPTOMS OF EXPOSURE

Coughing, choking, headache, pulmonary edema, weakness. .

EFFECTS OF CHRONIC EXPOSURE

Erosion of teeth, jaw necrosis. Bronchial irritation. Chronic cough.

EMERGENCY AND FIRST AID PROCEDURES

Use respiratory resuscitation, treat shock and pulmonary edema. Obtain medical aid.

SECTION VI REACTIVITY DATA

\section{CONDITIONS CONTRIBUTING TO INSTABILITY \\ CONDITIONS CONTRIBUTING TO HAZARDOUS POLYMERIZATION \\ INCOMPATABILITY (MATERIALS TO AVOID) \\ HAZARDOUS OECOMPOSITION PRODUCTS \\ SECTION VII SPILL OR LEAK PROCEDURES}

STEPS TO $8 E$ TAKEN IN CASE MATERIAL IS RELEASED OR SPILLED

Cover with sodium carbonate.

WASTE DISPOSAL METHOD

SECTION VIII SPECIAL PROTECTION INFORMATION

\begin{tabular}{|c|c|}
\hline VENTILATION REQUIREMENTS LOCAL EXHAUST & $\begin{array}{l}\text { PROTECTIVE EQUIPMENT (SPECIFY TYPES) EYE } \\
\text { GOggleS }\end{array}$ \\
\hline MECHANICAL (GENERAL) & $\begin{array}{l}\text { GLOVES } \\
\text { Rubber }\end{array}$ \\
\hline SPECIAL & $\begin{array}{l}\text { RESPIRATOR - } \\
\text { w/proper filter }\end{array}$ \\
\hline
\end{tabular}

OTIICR RROTECTIVE EQUINMEHT

Rubber apron, full face-shield.

SECTION IX SPECIAL PRECAUTIONS

PRECAUTIONS TO BE TAKEN IN HANDLING AND STORAGE

WIHEK HHE: MITIIINS 


\begin{tabular}{|l|}
\hline \multicolumn{1}{|c|}{ PRODUCT DESIGNATION } \\
03-001-4807 \\
Phosphotungstic Acid
\end{tabular}

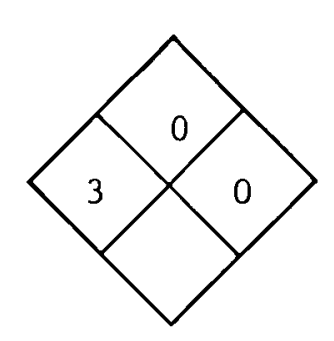

\section{SECTION I SOURCE AND NOMENCLATURE}

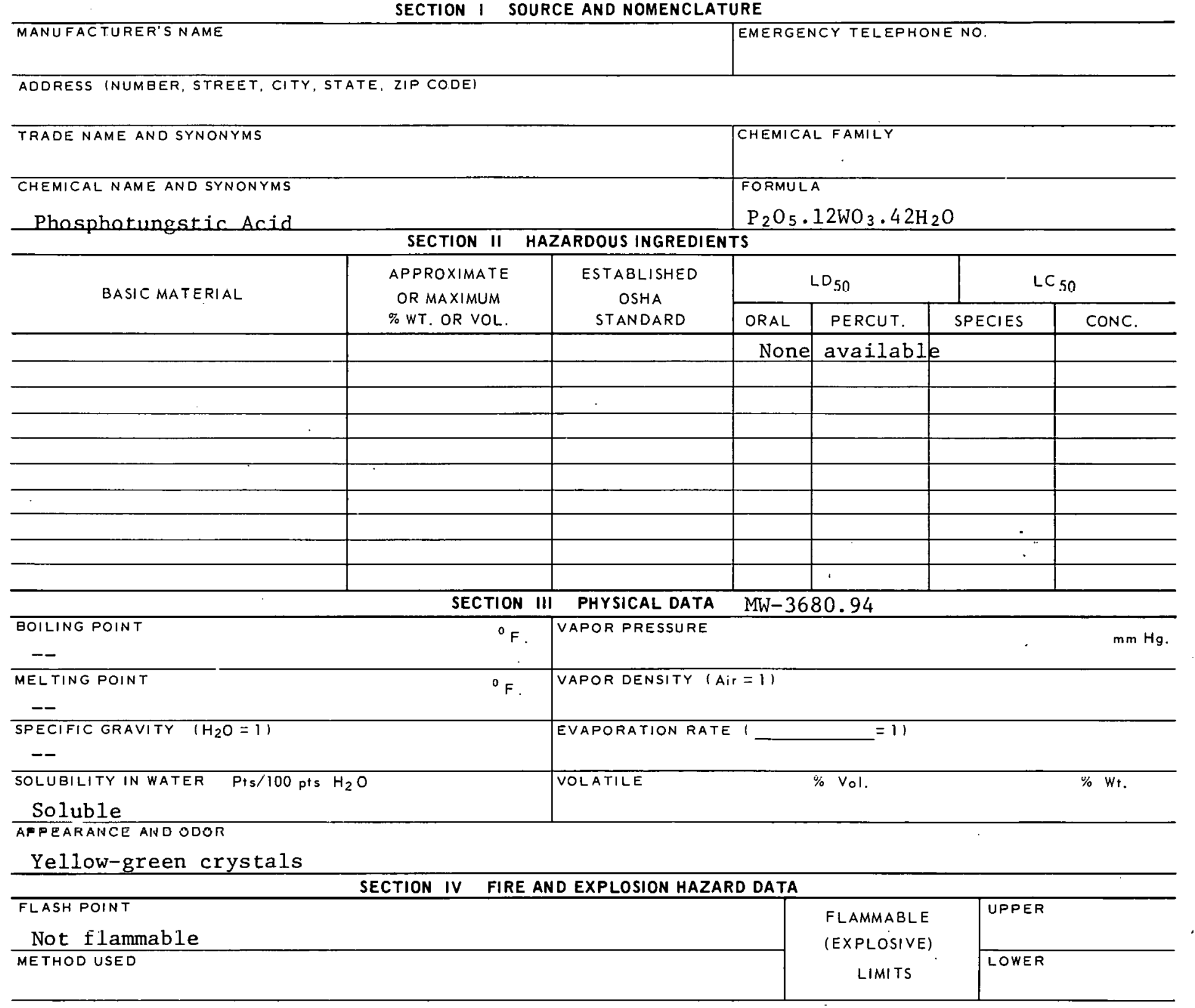

EXIINGUISHING MEUIA

SPECIAL FIRE FIGHTING PROCEDURES

UNUSUAL FIRE AND EXPLOSION HAZARDS

$\begin{array}{lll}13 & 10-74) & 1-4807 \mathrm{~A}\end{array}$ 
TOXIC LEVEL

$1.7 \mathrm{mg} / \mathrm{C} . \mathrm{M}$.

PRINCIPLE ROUTES OF ABSORBTION

Ingestion (mainly), inhalation of dust

REVELANT SYMPTOMS OF EXPOSURE LOSS of appetite, colic, loss of coordination, trembling, difficulty in breathing, weight loss.

EFFECTS OF CHRONIC EXPOSURE -

EMERGENCY AND FIRST AID PROCEDURES If swallowed - wash mouth, induce vomiting, get medical aid; inhalation - rest; wash skin and eyes.

SECTION VI REACTIVITY DATA

CARCINOGENIC

No

SKIN AND EYE

IRRITATION Severe

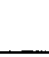

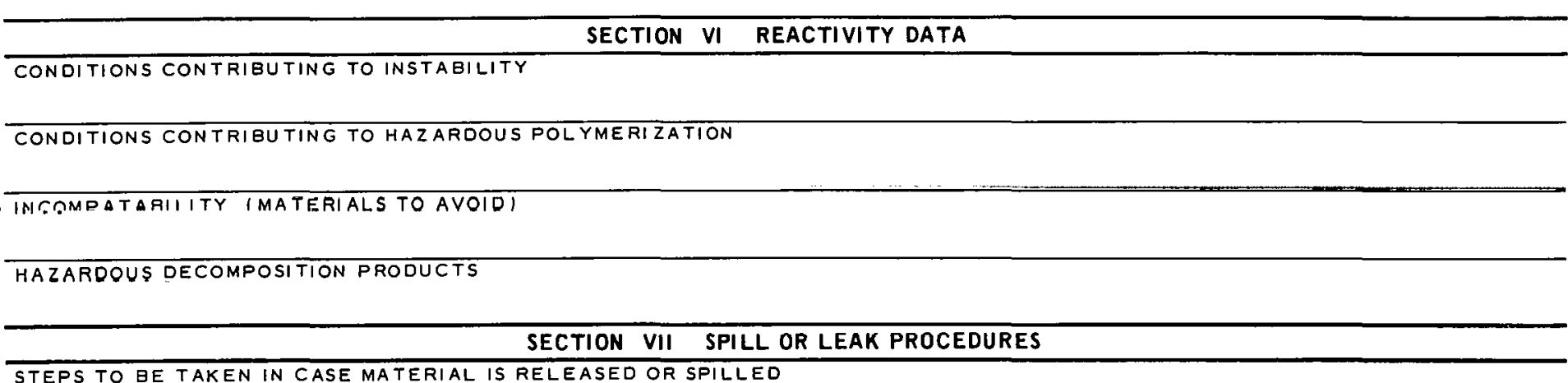

SECTION VIII SPECIAL PROTECTION INFORMATION

\begin{tabular}{l|l}
\hline \multicolumn{1}{c}{ SECTION VIII SPECIAL PROTECTION INFORMATION } \\
\hline VENTILATION REQUIREMENTS LOCAL EXHAUST & $\begin{array}{l}\text { PROTECTIVE EQUIPMENT ISPECIFY TYPESI EYE } \\
\text { GOggIES }\end{array}$ \\
\hline MECHANICAL IGENERALI & GLOVES \\
\hline SPECIAL & חLETIMATOA \\
\hline OTHER PROTECTIVE EQUIPMENT
\end{tabular}

\section{SECTION IX SPECIAL PRECAUTIONS}

PRECAUTIONS TO BE TAKEN IN HANOLING AND STORAGE 
CAS : 007664939

\begin{tabular}{|l|}
\hline \multicolumn{1}{c|}{ PRODUCT DESIGNATION } \\
Sulfuric Acid \\
$03-001-7212$ \\
$03-001-7247$ \\
$03-001-7242$ \\
\hline
\end{tabular}

WS 56000

\section{MATERIAL SAFETY \\ DATA SHEET}

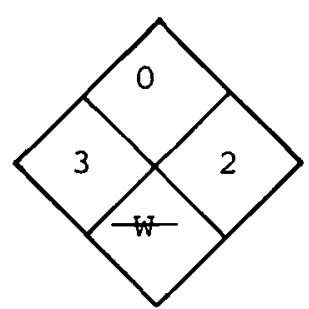

\section{SECTION I SOURCE AND NOMENCLATURE}

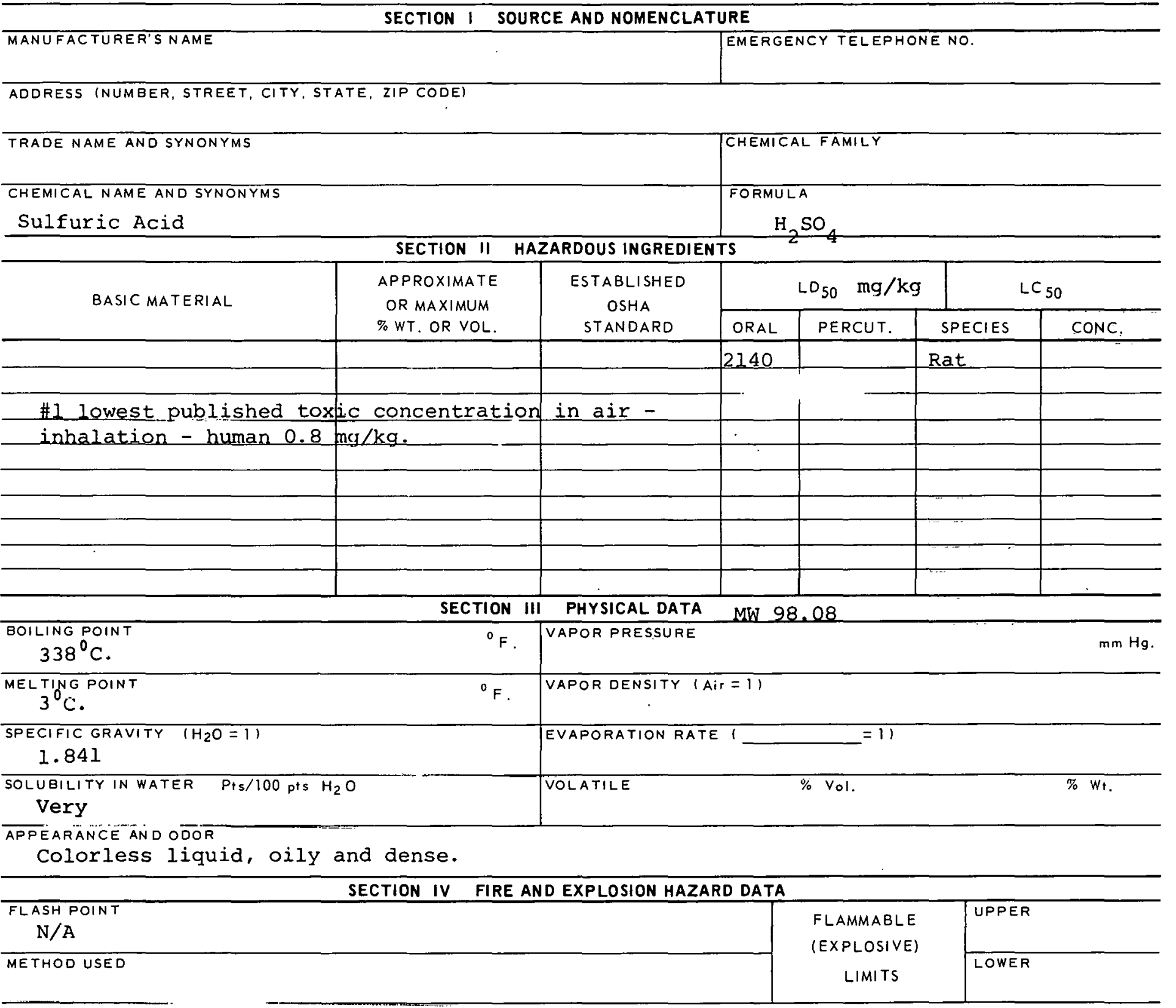

EXTINGUISHING MEDIA

Dry chemical

SPECIAL FIRE FIGHTING PROCEDURES

Wear full protective clothing - keep water away from the acid. UNUSUAL FIRE AND EXPLOSION HAZARDS 
TOXIC LEVEL

TLV $1 \mathrm{mg} / \mathrm{m}^{3}$

CARCINOGENIC

no

PRINCIPLE ROUTES OF ABSORBTION

Inhalation of vapor or mist.

SKIN AND EYE

IRRITATION SEvere

REVELANT SYMPTOMS OF EXPOSURE Choking, coughing, headache, dizziness, cyanosis. Severe ulceration of skin.

EFFECTS OF CHRONIC EXPOSURE

Possible jaw necrosis, erosion of teeth, bronchial irritation with chronic cough.

EMERGENCY AND FIRST AID PROCEDURES Ingestion: give milk or milk of magnesia. Inhalation:

artificial respiration if necessary - wash from skin $\alpha$ eyes.

\section{SECTION VI REACTIVITY DATA}

CONDITIONS CONTRIBUTING TO INSTABILITY

CONDITIONS CONTRIBUTING TO HAZARDOUS POLYMERIZATION

INCOMPATABILITY (MATERIALS TO AVOIO) Water, chlorates, perchlorates, permangantes, picrates, carbidcs, powdered metals, phosphorous.

HAZARDOUS DECOMPOSITION PRODUCTS

SECTION VII SPILL OR LEAK PROCEDURES

STEPS TO BE TAKEN IN CASE MATERIAL IS RELEASED OR SPILLED

WASTE DISPOSAL METHOD

SECTION VIII SPECIAL PROTECTION INFORMATION

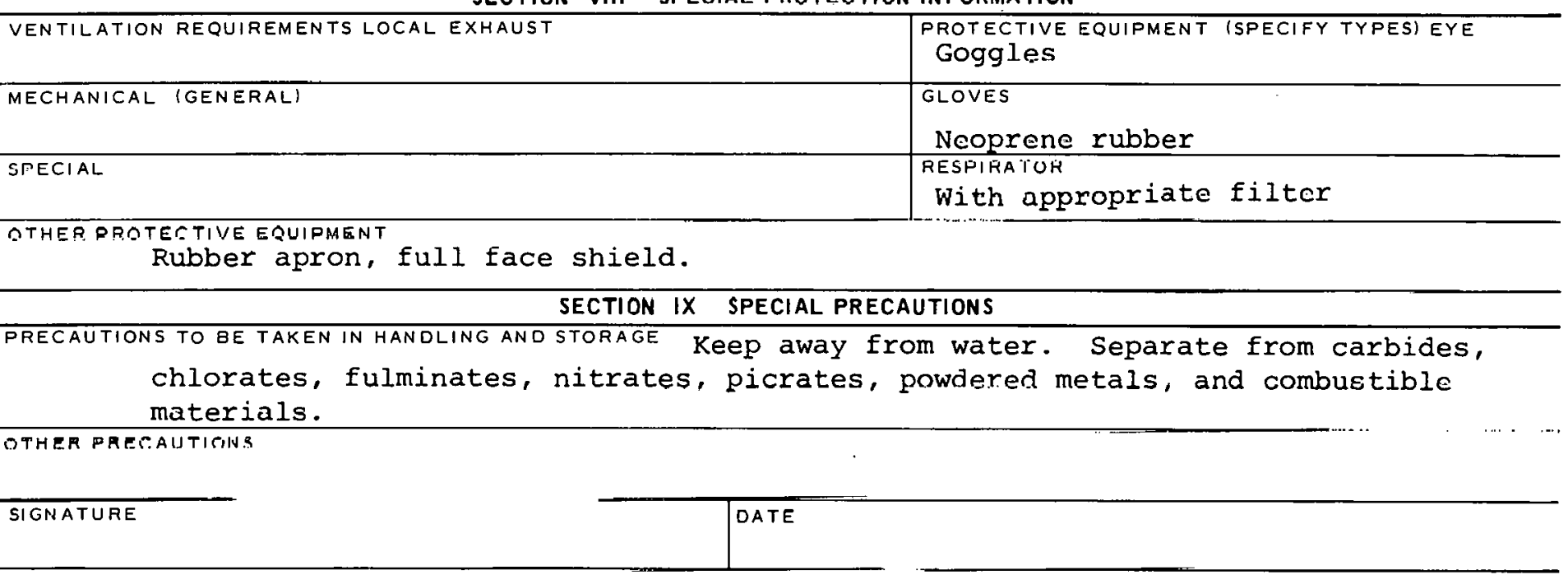

$1-7212 \mathrm{~B}$ 
CAS : 007782992

PRODUCT DESIGNATION

WT 27750

\begin{tabular}{l}
\multicolumn{1}{c|}{ PRODUCT DESIGNATION } \\
Sulfurous Acid \\
$03-001-7262$
\end{tabular}$\quad \begin{array}{r}\text { WT27750 } \\
\text { MATERIAL SAFETY }\end{array}$

SECTION I SOURCE AND NOMENCLATURE

MANUFACTURER'S NAME

TIRET, CITY, STATE, ZIPCODI

ADDRESS (NUMBER, STREET, CITY, STATE, ZIP CODE)

EMERGENCY TELEPHONE NO.

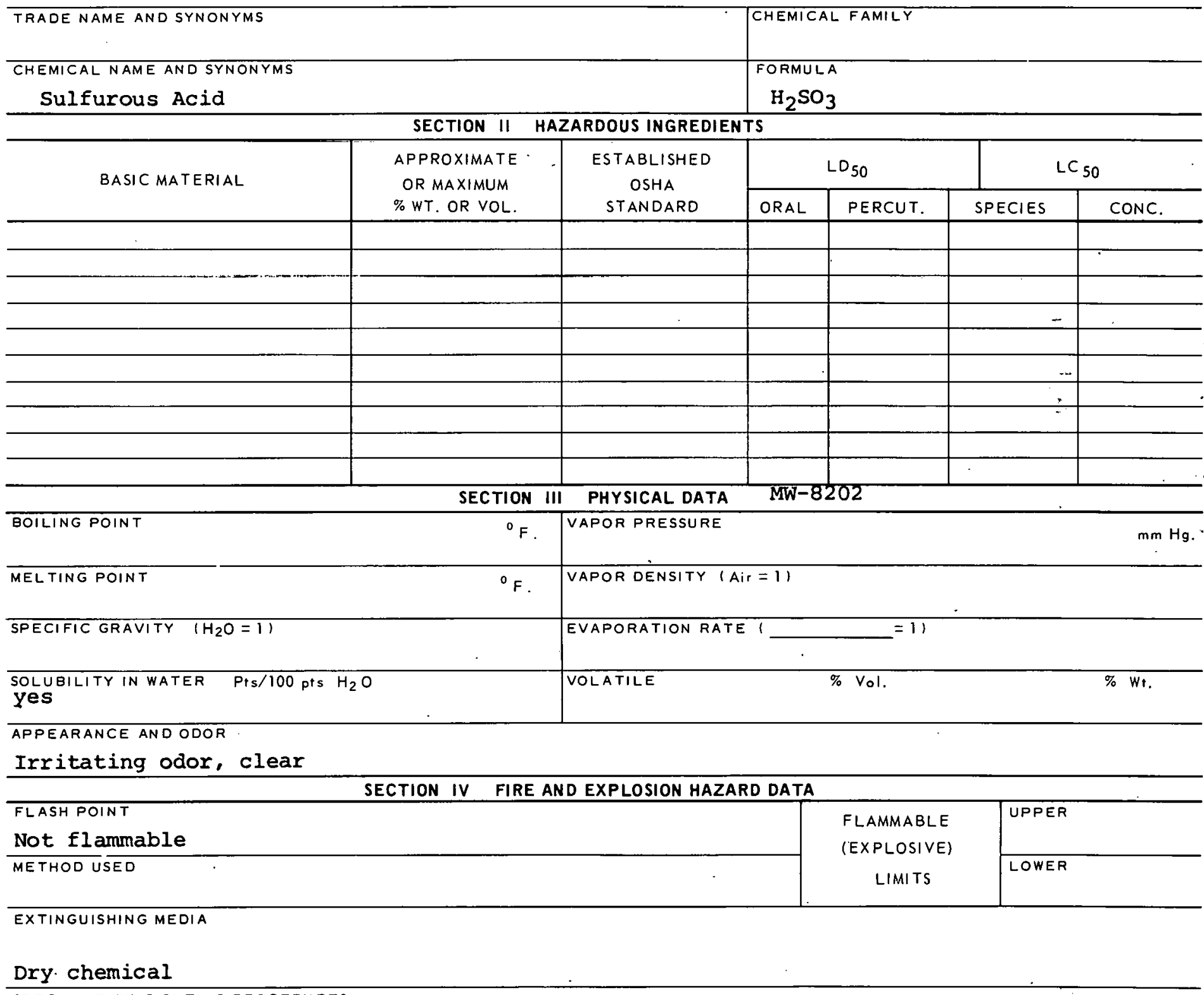

SPECIAL FIRE FIGHTING PROCEDURES

UNUSUAL FIRE AND EXPLOSION HAZARDS

$11 \mathrm{~N}, \mathrm{~N}+1137 ?$

$13 \quad 10-741$

$1-7262 A$ 


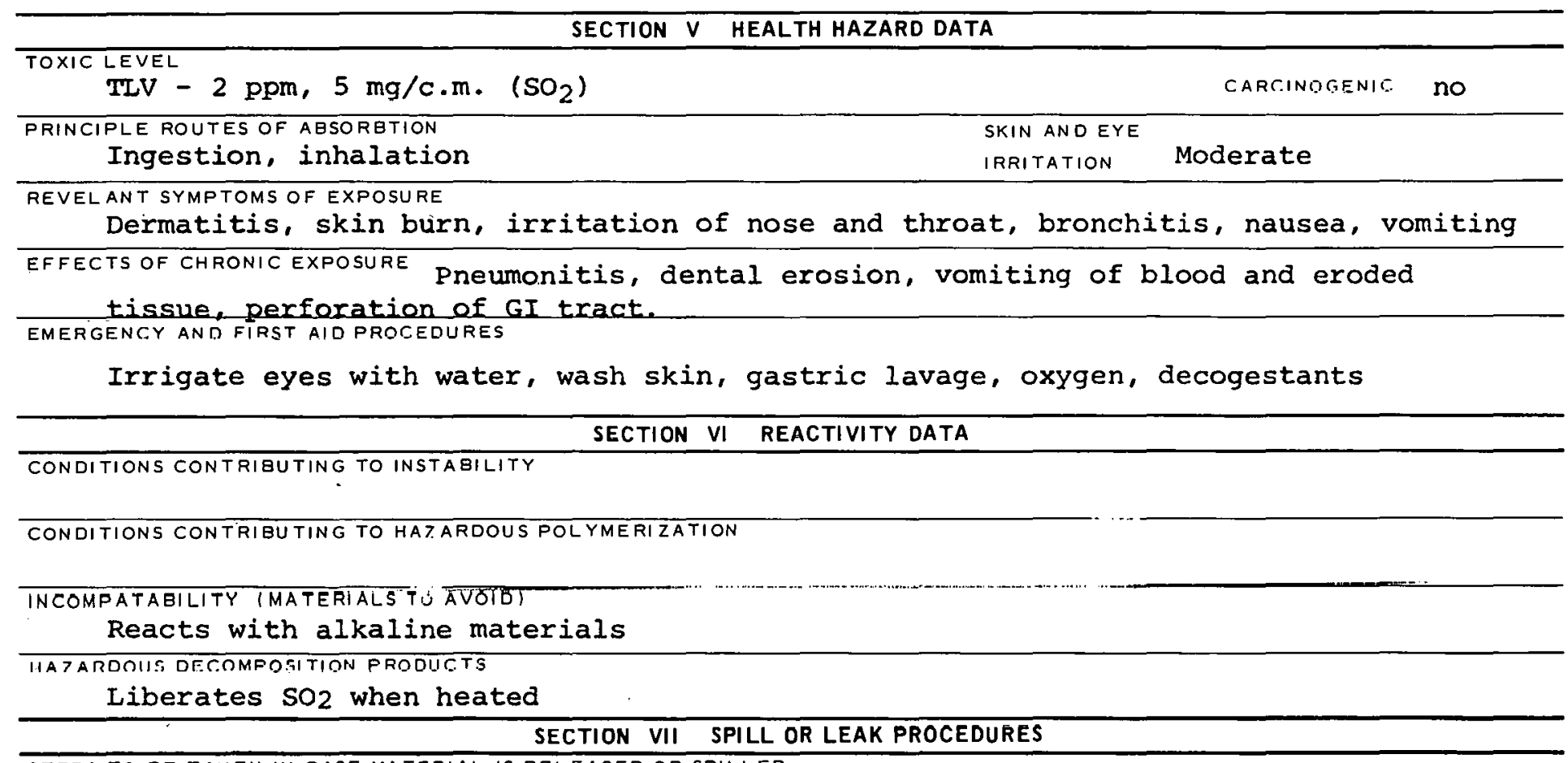

STEPS TO BE TAKEN IN CASE MATERIAL IS RELEASED OR SPILLED

WASTE DISPOSAL METHOD

SECTION VIII SPECIAL PROTECTION INFORMATION

\begin{tabular}{l|l}
\hline VENTILATION REQUIREMENTS LOCAL EXHAUST & $\begin{array}{l}\text { PROTECTIVE EQUIPMENT (SHECIFY IYPES) EYE } \\
\text { Safety gOgGles }\end{array}$ \\
\hline MECHANICAL IGENERAL) & $\begin{array}{c}\text { GLOVES } \\
\text { Rubber }\end{array}$ \\
\hdashline SPECIAL & nEERIRATON \\
\hline
\end{tabular}

OTHER PROTECTIVE EQUIPMENT

\section{SECTION IX SPECIAL PRECAUTIONS}

PRECAUTIONS TO BE TAKENN IN HANDLING AND STORAGE

store in well-ventilated area.

OTHER PRECAUTIONS 
CAS: 007446700

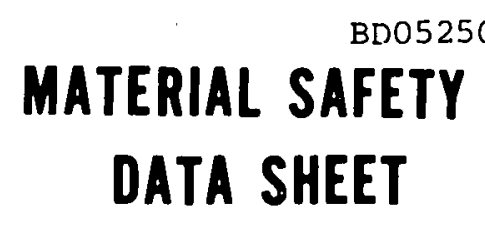

PRODUCT DESIGNATION

3-006-1310 - (Anhydrous)

Aluminum Chloride

SECTION I SOURCE AND NOMENCLATURE

MANUFACTURER'S NAME
ADDRESS INUMBER, STREET, CI TY,
TRADE NAME AND SYNONYMS
CHEMICAL NAME AND SYNONYMS

Aluminum Chloride

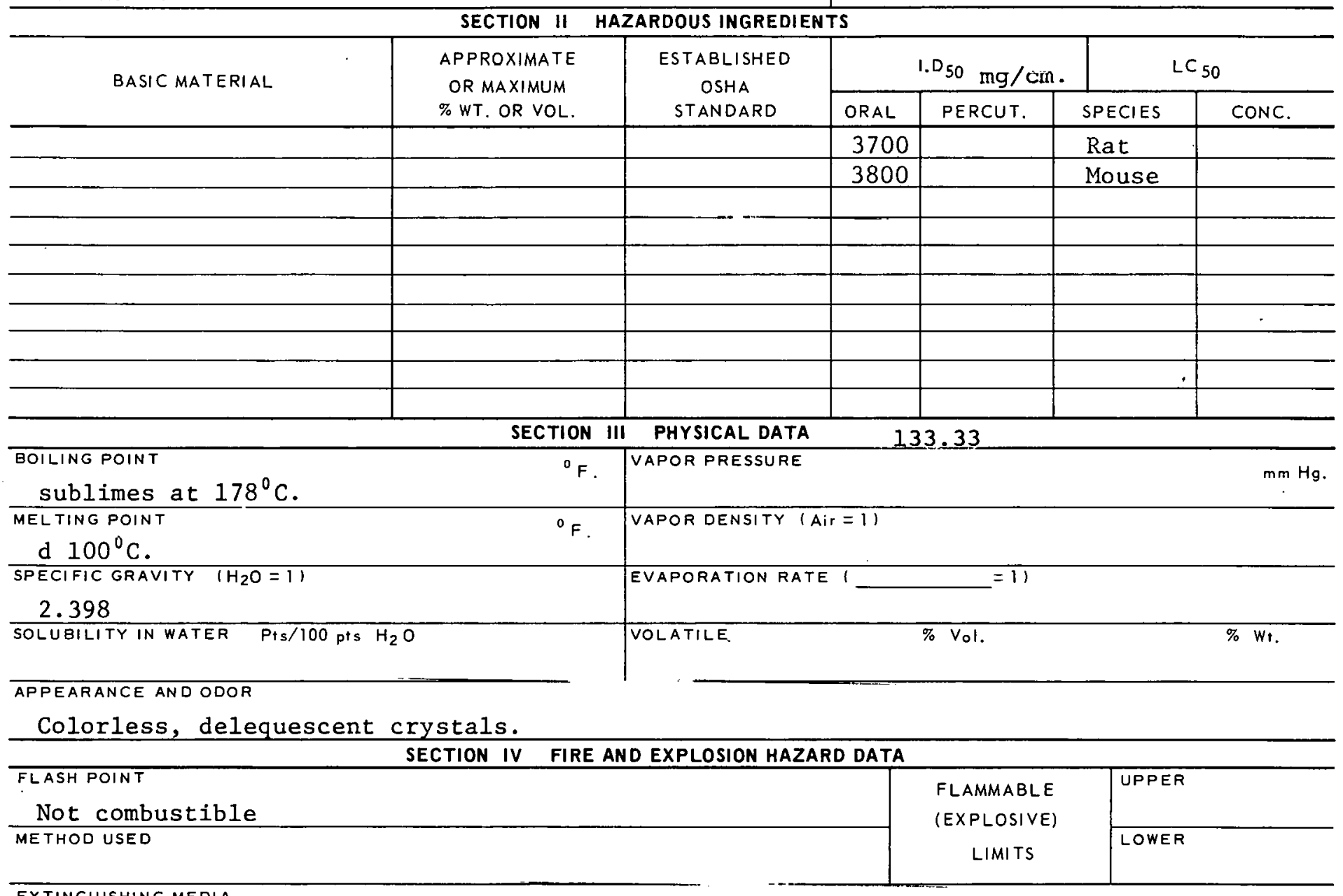

EXTINGUISHING MEDIA

Dry rhemicall or foam.

SPECIAL FIRE FIGHTING PROCEDURES

UNUSUAL FIRE AND EXFLOSION HAZARDS

Violent reaction if streams of water hit large quantities of chemical. 


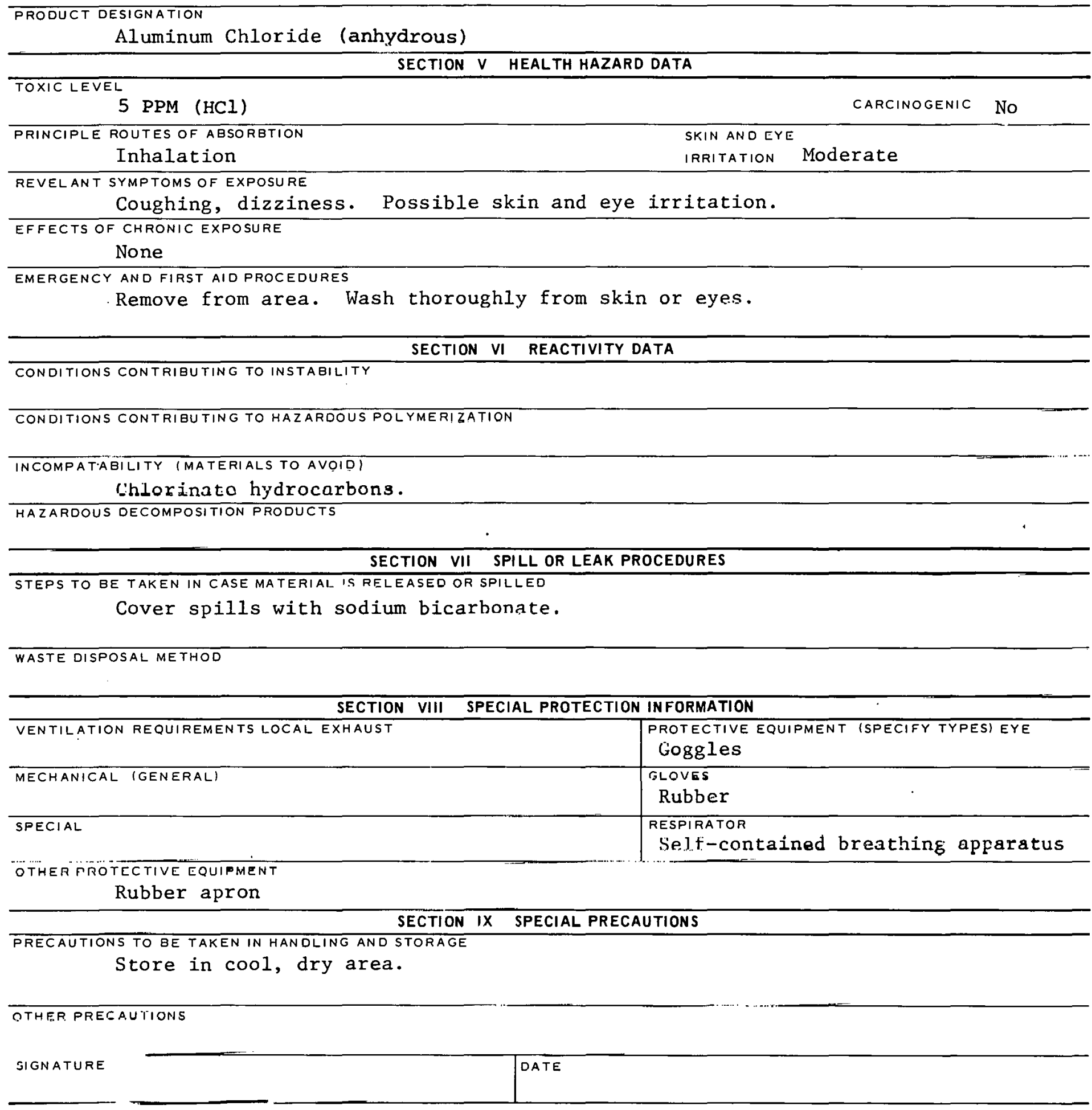

$6-1310 B$ 
CAS: 007789095

$\mathrm{HX76500}$

\begin{tabular}{|l|}
\hline PRODUCT DESIGNATION \\
03-008-1102 \\
Ammonium Bichromate \\
\hline
\end{tabular}

MATERIAL SAFETY

DATA SHEET

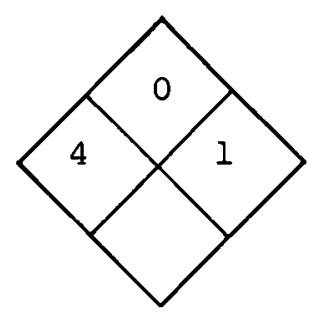

SECTION I SOURCE AND NOMENCLATURE

MANUFAC TURER'S NAME
ADDRESS INUMBER, STREET, CI
TRADE NAME AND SYNONYMS
CHEMICAL NAME AND SYNONYMS
AMMONIUM DiChYOMate

EMERGENCY TELEPHONE NO.

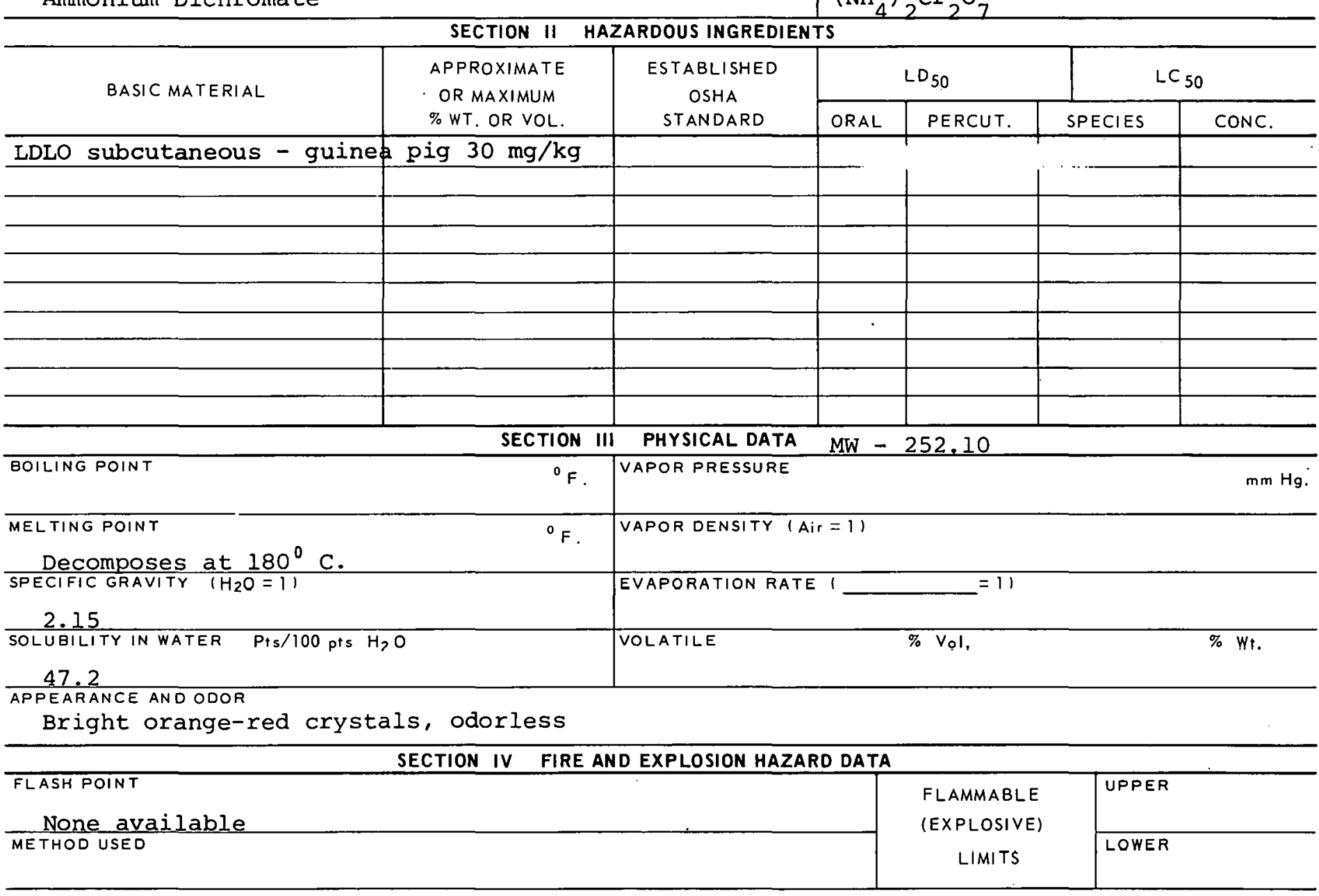

EXTINGUISHING MEDIA

Water

SPECIAL FIRE FIGHTING PROCEDURES

Use water but caution should be exercised against possibility of steam explosion. UNUSUAL FIRE AND EXPLOSION HAZAROS 
TOXIC LEVEL

$$
\text { TLV }=0.13 \mathrm{mg} / \mathrm{m}^{3}
$$

PRINCIPLE ROUTES OF ABSORBTION

Inhalation of dust, ingestion

REVELANT SYMPTOMS OF EXPOSURE Irritation of skin and eyes;

irritation and internal damage.

CARCINOGENIC SKIN AND EYE

IRRITATION Yes respiratory tract EFFECTS OF CHRONIC EXPOSURE

Asthmatic symptoms - liver and kidney damage. EMERGENCY AND FIRST AID PROCEDURES Wash from skin and eyes; if swallowed - wash mouth, give emetic, get medical attention; inhalation - rest and keep warm.

\section{SECTION VI REACTIVITY DATA}

CONDITIONS CONTRIBUTING TO INSTABILITY

Powerful oxidizing agent. CONDITIONS CONTRIBUTING TO HAZARDOUS POLYMERIZATION INCOMPATÁËILITY (MATERIALS IU AVUIU)

Reducing materials

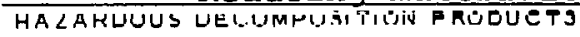

SECTION VII SPILL OR LEAK PROCEDURES

STEPS TO BE TAKEN IN CASE MATERIAL IS RELEASED OR SPILLED

WASTE OISPOSAL METHOD

SECTION VIII SPECIAL PROTECTION INFORMATION

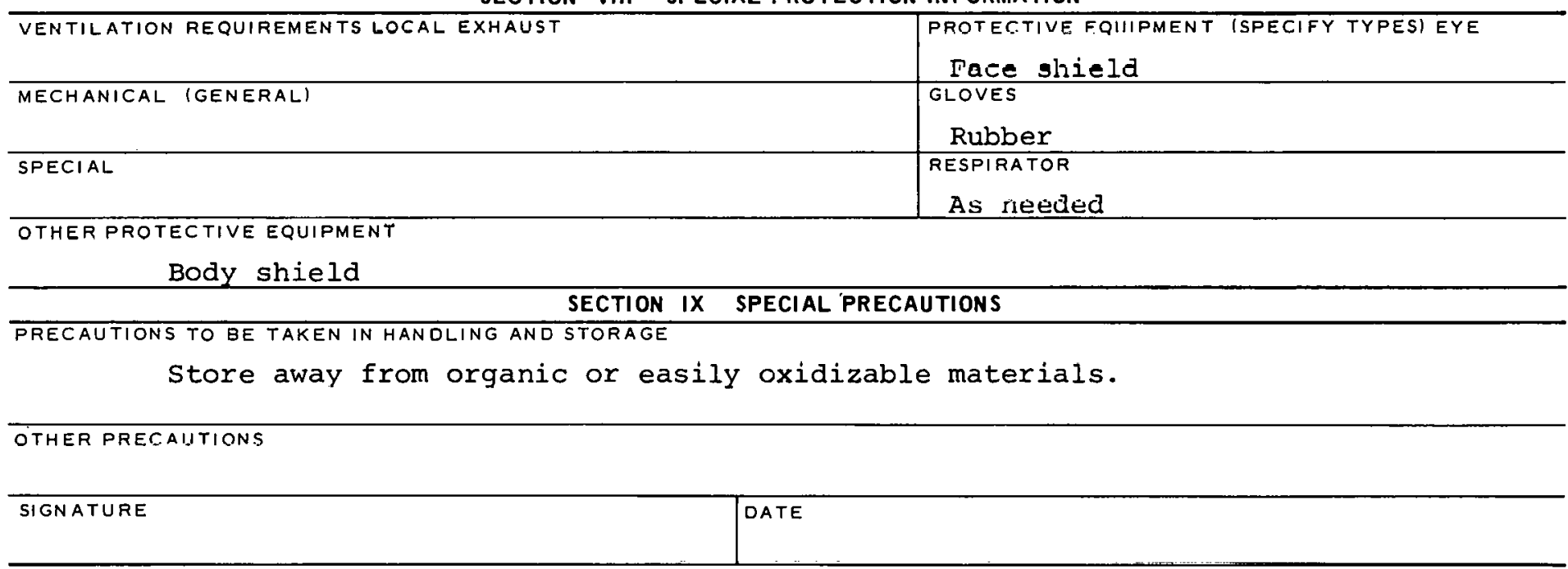


CAS : 001341497

\section{PRODUCT DESIGNATION}

03-008-1503

Ammonium Bifluoride

\section{MATERIAL SAFETY \\ DATA SHEET}

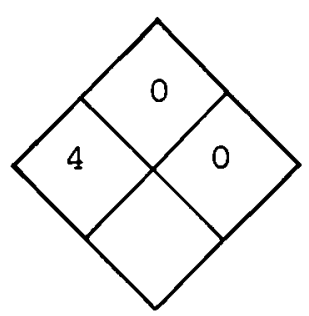

\section{SECTION I SOURCE AND NOMENCLATURE}

\section{MANUFACTURER'S NAME \\ ADDRESS (NUMBER, STREET, CITY, STATE, ZIP CODE)

\begin{tabular}{l} 
TRADE NAME AND SYNONYMS \\
Ammonium Hydrogen Fluoride \\
CHEMICAL NAME AND SYNONYMS \\
Ammonium Difluoride \\
\hline
\end{tabular}

EMERGENCY 'TELEPHONE NO.

\begin{tabular}{l|l} 
CHEMICAL FAMILY \\
& $\begin{array}{c}\text { FORMULA } \\
\left(\mathrm{NH}_{4}\right)^{\mathrm{HF}}{ }_{2}\end{array}$ \\
\hline
\end{tabular}

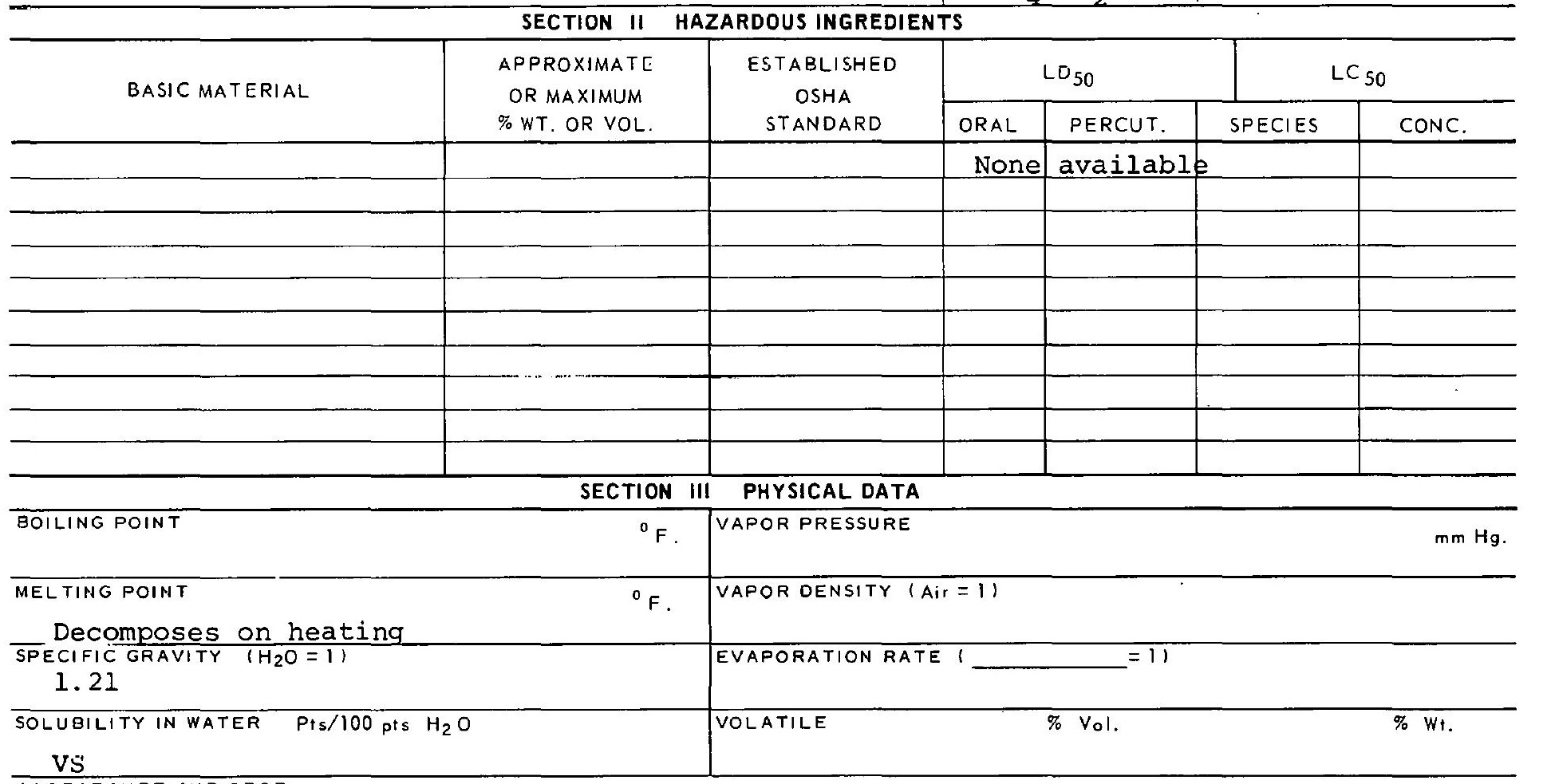

APPEARANCE AND ODOR

White solid - produces HF when heated. SECTION IV FIRE AND EXPLOSION HAZARD DATA FLASH POINT

ot flammable METHOD USED

EXTINGUISHING MEDIA

Water

SPECIAL FIRE FIGHTING PROCEDURES

Wear full protective clothing and self-contained mask. UNUSUAL FIRE AND EXPLOSION HAZARDS 


\section{TOXIC LEVEL}

$T L Y$ - 3 PPM (for HF), $2 \mathrm{mg} / \mathrm{C} . \mathrm{M}$.

PRINCIPLE ROUTES OF ADSORBTION
Inhalation of HF, skin absorption REVELANT SYMPTOMS OF EXPOSURE

Irritation of respiratory system; severe skin or eye burns.

EFFECTS OF CHRONIC EXPOSURE Weight loss, brittleness of bones, anemia, weakness, discoloration of teeth.

EMERGENCY AND FIRST AID PROCEDURES Inhalation - rest; wash from skin or eyes; if swallowed wash mouth, drink water, then milk of magnesia, get medical attention.

\section{SECTION VI REACTIVITY DATA}

CONDITIONS CONTRIBUTING TO INSTABILITY

CONDITIONS CONTRIQUTING TO HAZARDOUS POI.YMERIZATION

INLUMHAIABILIIY (MA IERIALS IO AVUID)

Bismuthic Acid, Fluorine, sodium

HAZARDOUS DECOMPOSITION PRODUCTS

SECTION VII SPILL OR LEAK PROCEDURES

STEPS TO BE TAKEN IN CASE MATERIAL IS RELEASED OR SPILLED

WASTE DISPOSAL METHOD

SECTION VIII SPECIAL PROTECTION INFORMATION

\begin{tabular}{|l|l}
\hline VENTILATION REQUIREMENTS LOCAL EXHAUST & PROTECTIVE
\end{tabular}

MEEHAINICAL IOEINERALI Goggles

GLOVES

Rubber gloves

SHELIAL

-.

OTHER PROTECTIVE EQUIPMENT

RESPIRATOR

W/appropriate filter

PRECAUTIONS TO BE TAKEN IN HANDLING AND STORAGE 
CASO12125018

PRODUCT DESIGNATION

03-008-4401

Ammonium Fluoride

BQ63000

MATERIAL SAFETY

DATA SHEET

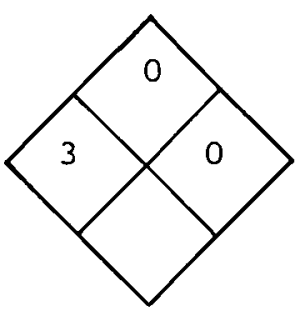

SECTION I SOURCE AND NOMENCLATURE

\section{MANUFACTURER'S NAME}

\begin{tabular}{l|l}
\hline & EMERGENCY TELEPHONE NO.
\end{tabular}

ADDRESS (NUMBER, STREET, CITY, STATE, ZIP CODE)

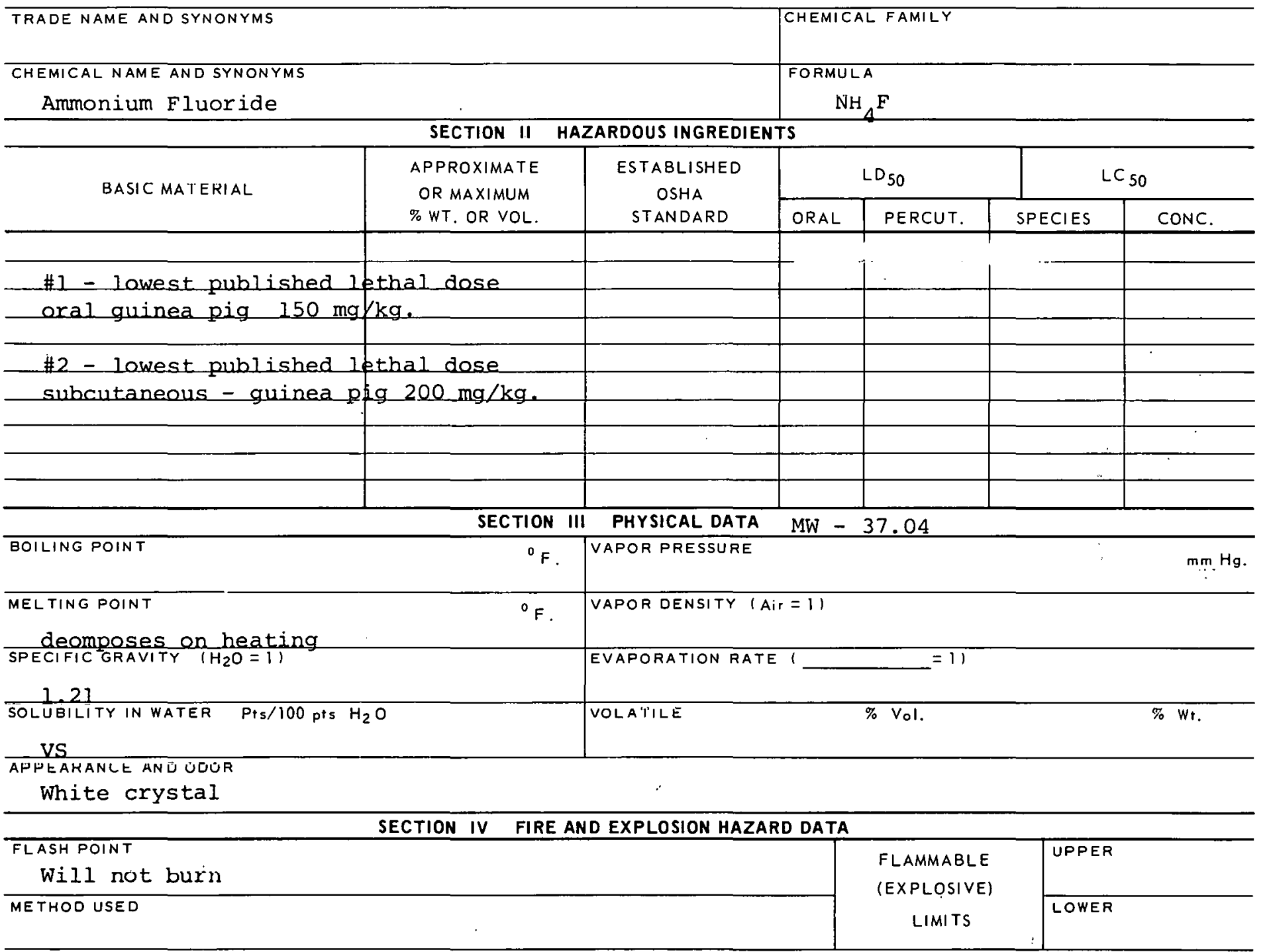

EXTINGUISHING MEDIA

SPECIAL FIRE FIGHTING PROCEDURES

Wear protective clothing.

UNUSUAL FIRE ANDEXPLOSION HAZARDS

May react with arsenic, trioxide and phosphorous pentoxide. 
Ammonium Fluoride

\section{SECTION $V$ HEALTH HAZARD DATA}

TOXIC LEVEL

$\mathrm{TLV}-2.5 \mathrm{mg} / \mathrm{m}^{3}$

PRINCIPLE ROUTES OF ABSORBTION

Inhalation, ingestion, skin absorption.

CARCINOGENIC no

SKIN AND EYE

IRRITATION Moderate

REVELANT SYMPTOMS OF EXPOSURE Irritation of respiratory system; severe internal irritation and damage; skin and eye burns -

EFFECTS OF CHRONIC EXPOSURE

Increase in fluoride content of teeth.

EMERGENCY AND FIRST AID PROCEDURES Inhalation - rest, keep warm, get medical attention; if

swallowed - wash mouth, drink water, then milk of magnesia, get medical attention; wash from skin and eyes, get medical attention.

SECTION VI REACTIVITY DATA

CONDITIONS CONTRIBUTING TO INSTABILITY

CONDITIONS CONTRIBUTING TO HAZARDOUS POLYMERIZATION

INCOMPATAEILITY (MATERIALE TO AVQID)

$\mathrm{As}_{2} \mathrm{O}_{3}$ and $\mathrm{P}_{2} \mathrm{O}_{5}$

HAZARDOUS DECOMPOSITION PROOUCTS

SECTION VII SPILL OR LEAK PROCEDURES

STEPS TO BE TAKEN IN CASE MATERIAL IS RELEASED OR SPILLED

Store away from acids.

WASTE DISPOSAL METHOD

SECTION VIII SPECIAL PROTECTION IN FORMATION

\begin{tabular}{l|l}
\hline VENTILATION REQUIREMENTS LOCAL EXHAUST & $\begin{array}{l}\text { PROTECTIVE EQUIPMENT (SPECIFY TYPESI EYE } \\
\text { SafetY glasSES }\end{array}$ \\
\hline MECHANICAL (GENERAL) & $\begin{array}{c}\text { GLOVES } \\
\text { Rubber gloves }\end{array}$ \\
\hline SPECIAL & RESPIRATOR \\
\hline $\begin{array}{l}\text { OIHFR PROTFCTIVF FRIIIPMENT } \\
\text { Wear faCe maSk and coveralls. }\end{array}$ \\
\hline
\end{tabular}

PRECAUTIONS TO BE TAKEN IN HANDLING AND STORAGE

OTIILR FRECAUTIONS

SIGNAIUKE

\begin{tabular}{l|l|l|}
\hline DATt \\
\hline
\end{tabular}

$8-4401 B$ 
CAS : OC 1336216

\begin{tabular}{|c|}
\hline PRODUCT DESIGNATION \\
$03-008-5123$ \\
$03-008-5154$ \\
Ammonium Hydroxide
\end{tabular}

\section{MATERIAL SAFETY \\ DATA SHEET}

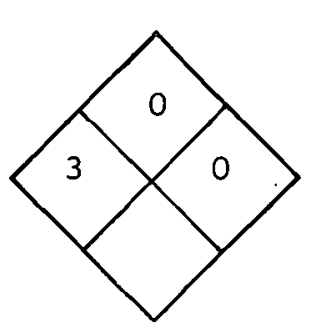

SECTION 1 SOURCE AND NOMENCLATURE

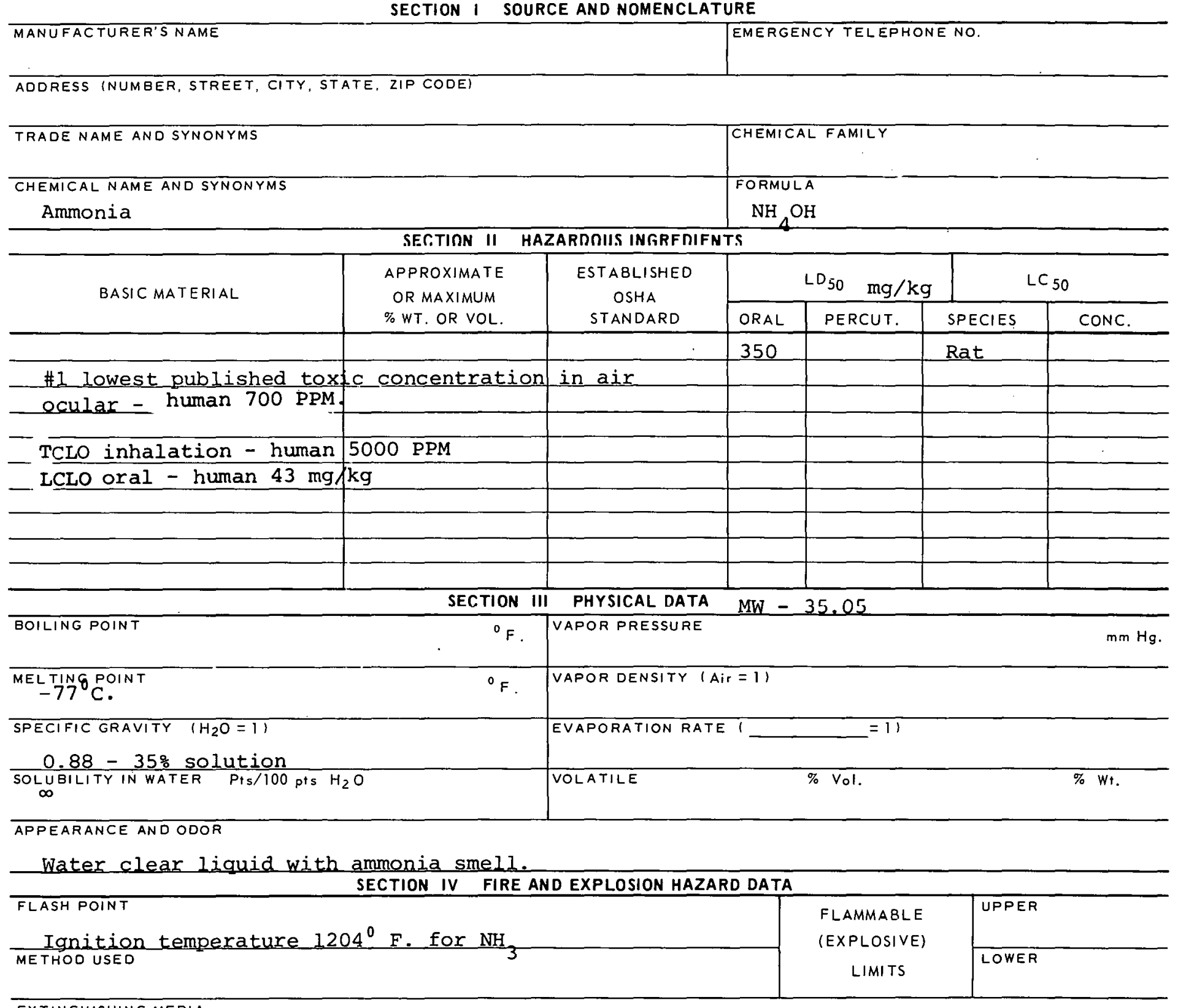

EXTINGUISHING MEDIA

water, dry rhemiral, rarhon dioxide

SPECIAL FIRE FIGHTING PROCEDURES

Use self-contained breathing apparatus.

UNUSUAL FIRE AND EXPLOSION HAZARDS
(3 10-74) 


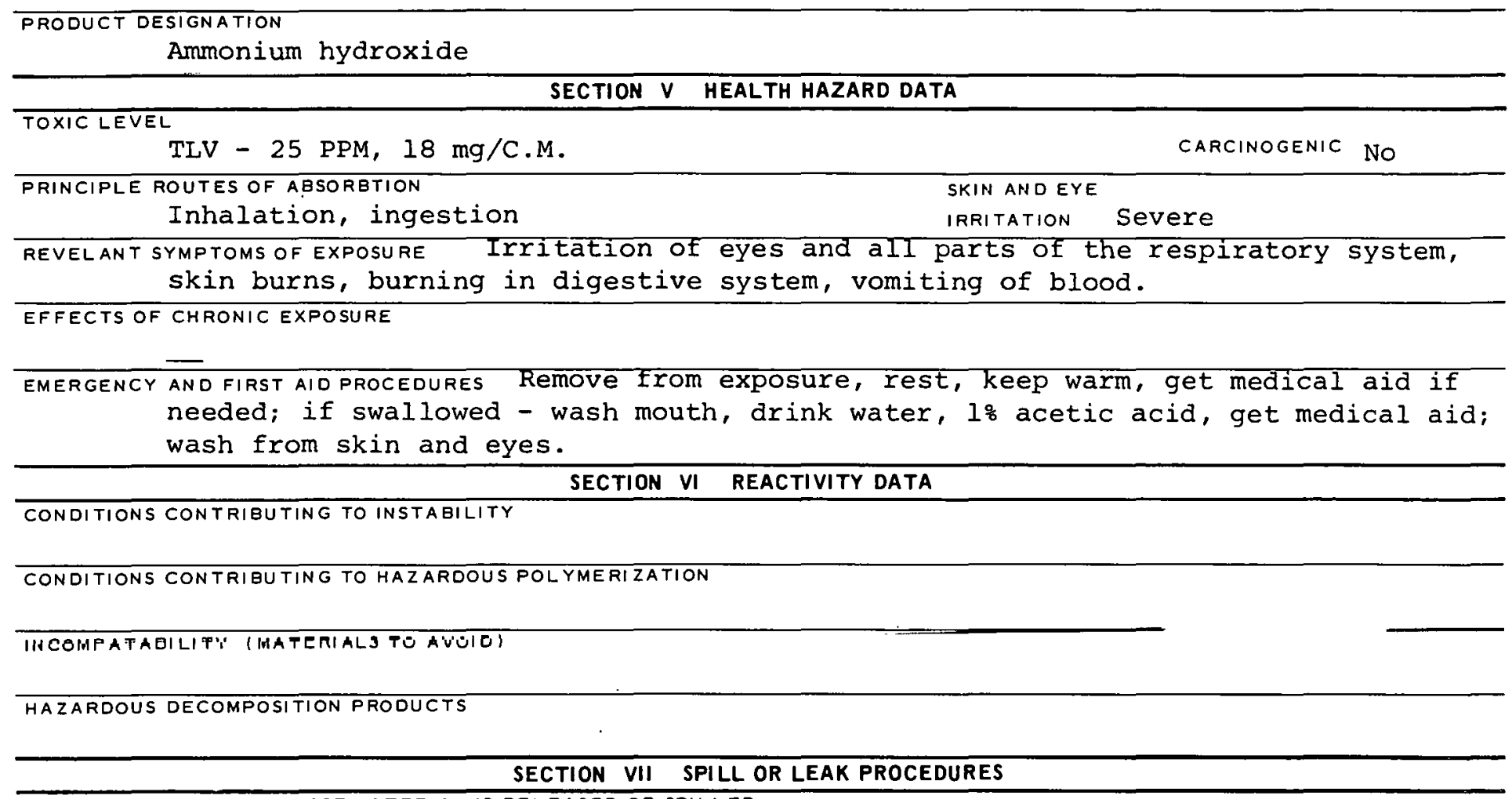
STEPS TO BE TAKEN IN CASE MATERIAL IS RELEASED OR SPILLED

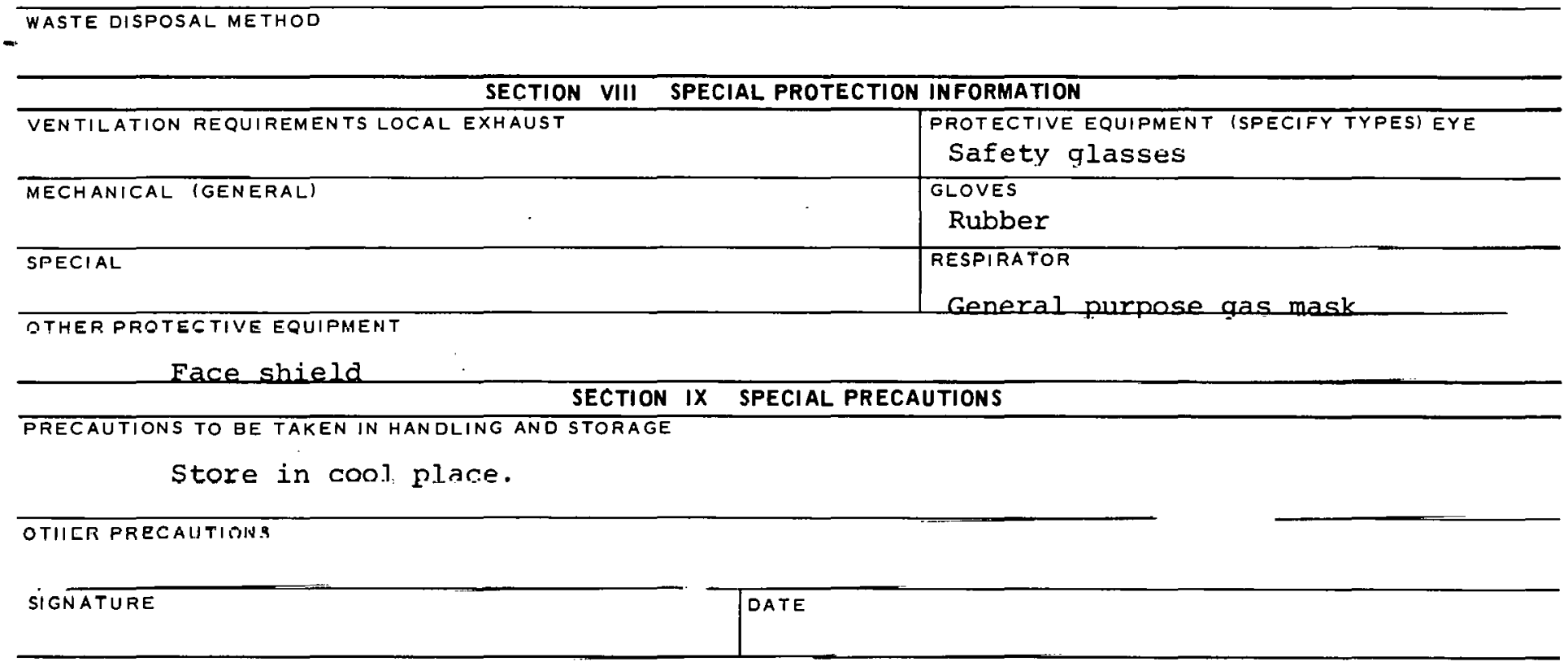


PRODUCT DESIGNATION

03-008-6527

Ammonium Oxalate
MATERIAL SAFETY

DATA SHEET

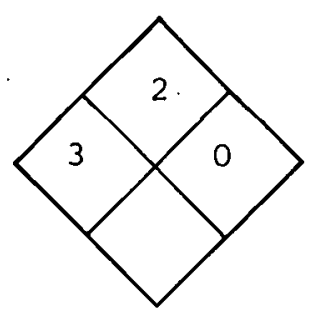

SECTION I SOURCE AND NOMENCLATURE

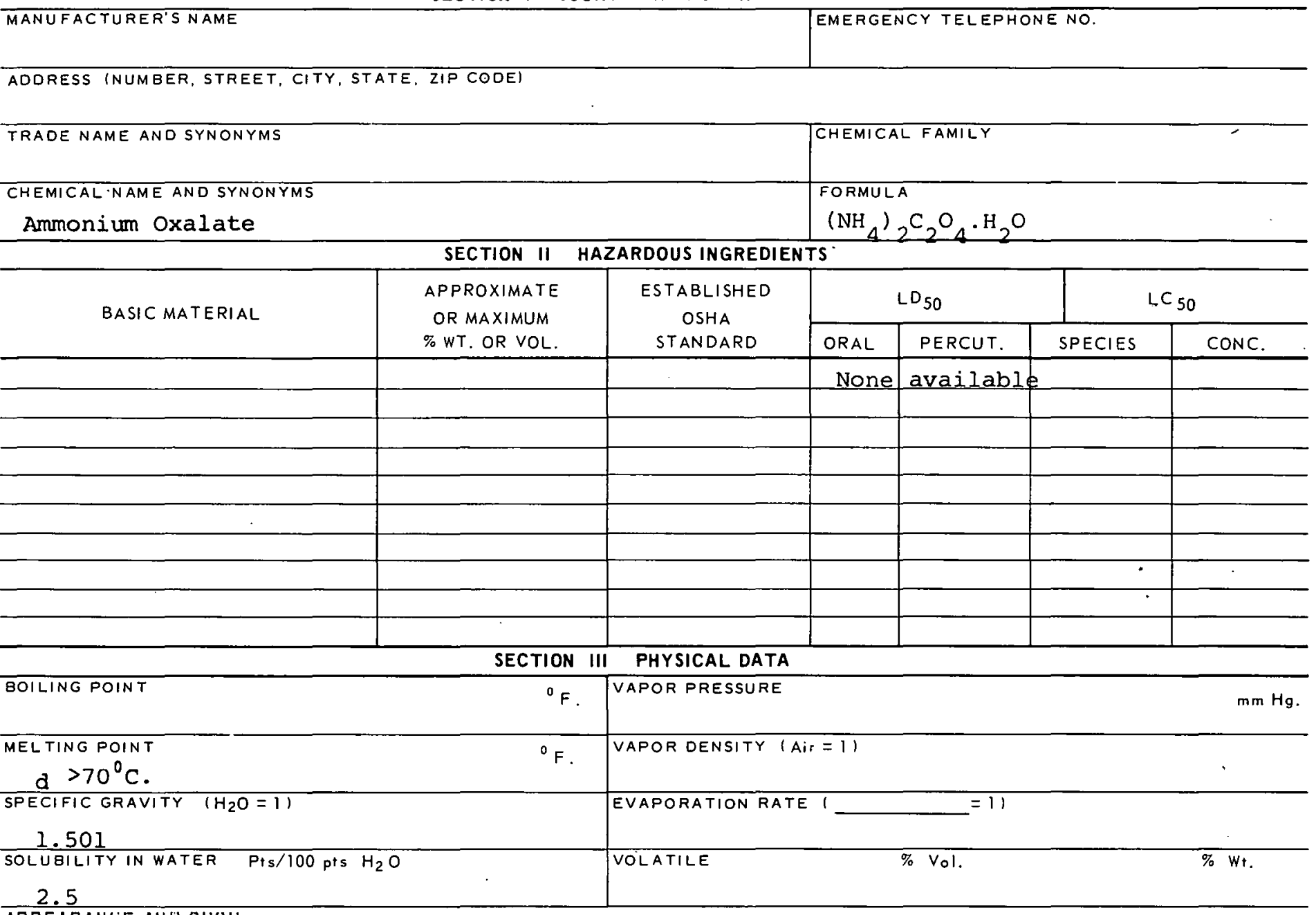

APPEARANCE AND OUUK

White crystals

\begin{tabular}{|c|c|c|c|c|}
\hline & SECTION IV & FIRE AND EXPLOSION HAZARD DATA & & \\
\hline $\begin{array}{l}\text { FLASH POINT } \\
\mathrm{N} / \mathrm{A} \\
\end{array}$ & & & \multirow{2}{*}{$\begin{array}{c}\text { FLAMMABLE } \\
\text { (EXPLOSIVE) } \\
\text { LIMITS }\end{array}$} & UPPER \\
\hline METHO'D USED & & & & LOWER \\
\hline
\end{tabular}

EXTINGUISHING MEDIA

Water spray, dry chemical, carbon dioxide, dry chemical.

SPECIAL FIRE FIGHTING PROCEDURES

Wear goggles and self-contained breathing apparatus.

UNUSUAL FIRE AND EXPLOSION HAZAROS

toxic fumes including $\mathrm{NH}_{3}$.

When heated to decomposition temperature may emit 
TOXIC LEVEL

TLV - $1 \mathrm{mg} / \mathrm{C} . \mathrm{M}$. oxalic acid

PRINCIPLE ROUTES OF ABSORETION Ingestion
CARCINOGENIC NO

SKIN AND EYE

IRRITATION YES

REVELANT SYMPTOMS OF EXPosure Severe internal pain, collapse, kidney damage, lung edema.

EFFECTS OF CHRONIC EXPOSURE

May produce calcium oxalate crystals in the bladder.

EMERGENCY AND FIRST AID PROCEDURES If SWaLlowed - wash mouth, give salt water to induCe vomiting, get medical aid; wash from skin or eyes.

\section{SECTION VI REACTIVITY DATA}

CONDITIONS CONTRIBUTING TO INSTABILITY

CONDITIONS CONTRIBUTING TO HAZARDOUS POLYMERIZATION

TIUEUMPATADILIT' (MATLRIALS TO AVOID)

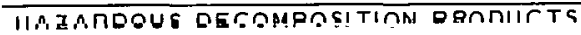

SECTION VII SPILL OR LEAK PROCEDURES

STEPS TO BE TAKEN IN CASE MATERIAL IS RELEASED OR SPILLED

WASTE DISPOSAL METHOD

\section{SECTION VIII SPECIAL.PROTECTION INFORMATION}

\begin{tabular}{|c|c|}
\hline VENTILATION REQUIREMENTS LOCAL EXHAUST & $\begin{array}{l}\text { PROTECTIVE EQUIPMENT (SPECIFY TYPES) EYE } \\
\text { Safety glasses. }\end{array}$ \\
\hline MECHANICAL (GENERAL) & GLOVES \\
\hline SPECIAL & RESPIRATOR \\
\hline \multicolumn{2}{|l|}{ OTHER PROTECTIVE EQUIPMENT } \\
\hline \multicolumn{2}{|c|}{ SECTION IX SPECIAL PRECAUTIONS } \\
\hline \multicolumn{2}{|c|}{ PRECAUTIONS TO BE TAKEN IN HANDLING AND STORAGE } \\
\hline \multicolumn{2}{|l|}{ OTHER PRECAUTIONS } \\
\hline SIGNATURE & DATE \\
\hline
\end{tabular}

$8-6527 B$ 
CAS: 012124991

BS49000

\section{PRODUCT DESIGNATION}

03-008-7851

Ammonium Sulfide Solution

MATERIAL SAFETY

DATA SHEET

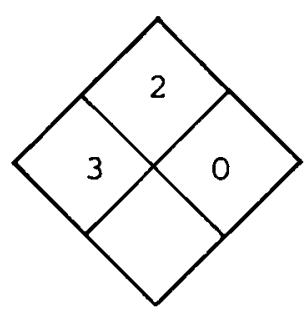

\section{SECTION I SOURCE AND NOMENCLATURE}

\begin{tabular}{l|l}
\hline MANUFACTURER'S NAME & EMERGENCY TELEPHONE NO. \\
\hline ADDRESS (NUMBER, STREET, CITY, STATE, ZIP CODE)
\end{tabular}

TRADE NAME AND SYNONYMS
CHEMICAL NAME AND SYNONYMS
AmmOnium Sulfide

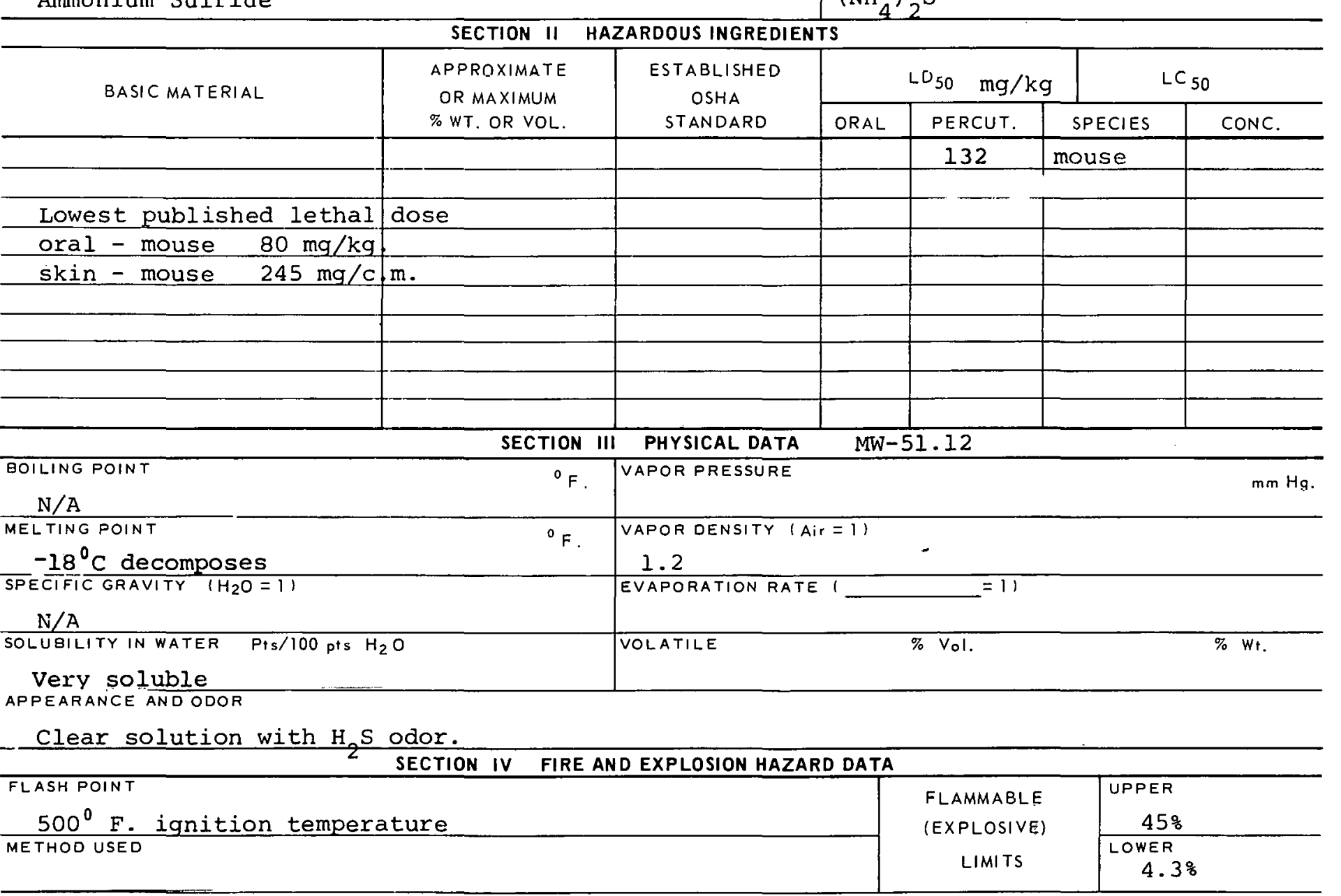

EXTINGUISHING MEDIA

Use water spray alcohol foam, carbon dioxide or dry chemical.

SPECIAL FIRE FIGHTING PROCEDURES

Wear goggles and self-contained breathing apparatus.

UNUSUAL FIRF, AND EXPLOSION HAZAROS

Produces inflammable gas upon heating. 
Ammonium Sulfide Solution SECTION $V$ HEALTH HAZARD DATA

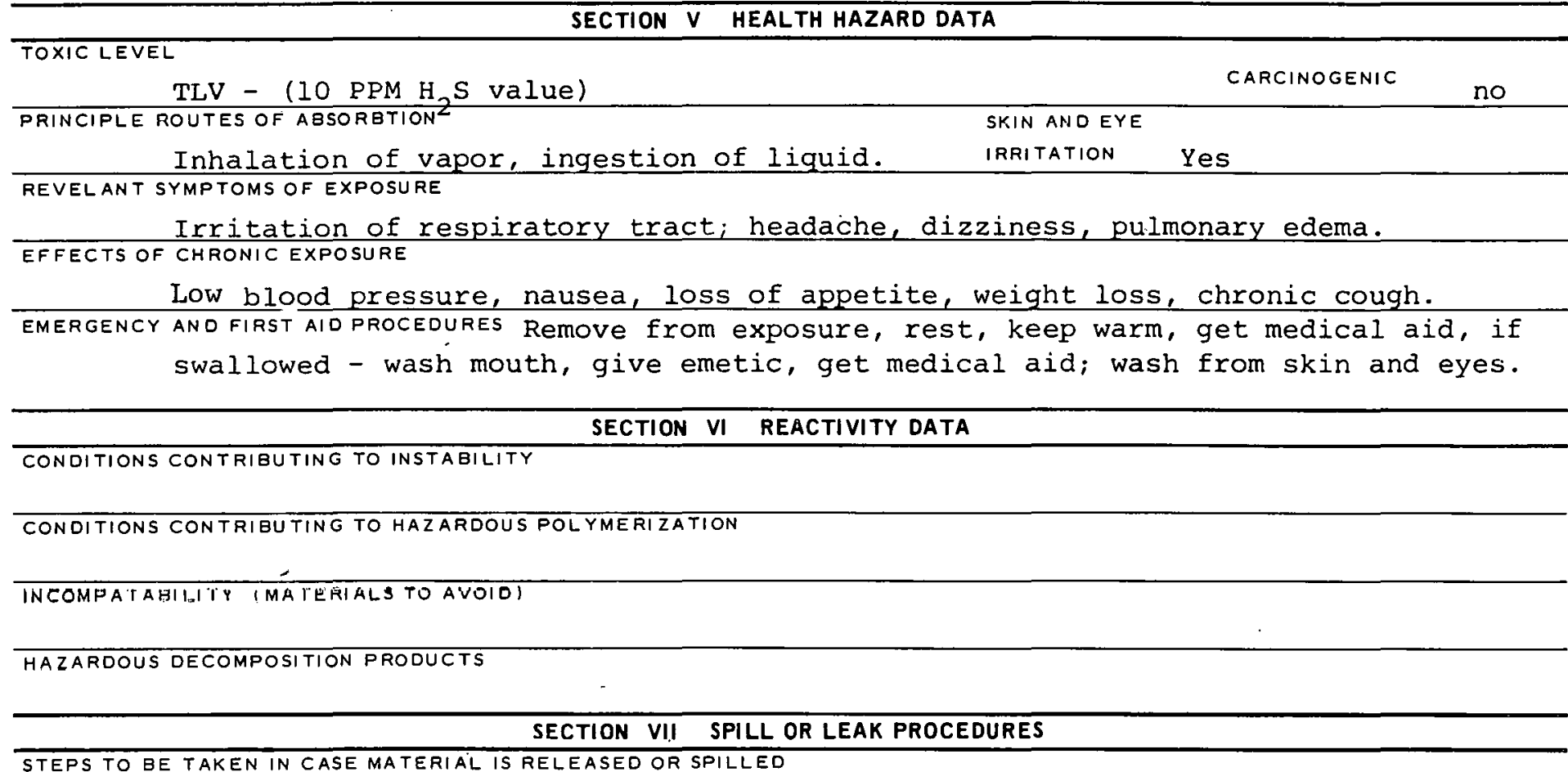
STEPS TO BE TAKEN IN CASE MATERIAL IS RELEASED OR SPILLED

WASTE DISPOSAL METHOD

SECTION VIII SPECIAL PROTECTION INFORMATION

\begin{tabular}{l|l}
\hline VENTILATION REQUIREMENTS LOCAL EXHAUST & $\begin{array}{l}\text { PROTECTIVE EQUIPMENT ISPECIFY TYPESI EYE } \\
\text { Safety glasSES }\end{array}$ \\
\hline MECHANICAL IGENERALI & $\begin{array}{c}\text { SLOVES } \\
\text { Rubber gloves }\end{array}$ \\
\hline SPECIAL & RESPIRATOR \\
Self-contained breathing apparatus.
\end{tabular}

OTHER PROTECTIVE EQUIPMENT

SECTION IX SPECIAL PRECAUTIONS

PRECAUTIONS TO BE TAKEN IN HANDLING AND STORAGE

OTHER PRECAUTIONS

SIGNATURE

DATE

$8-7851 B$ 


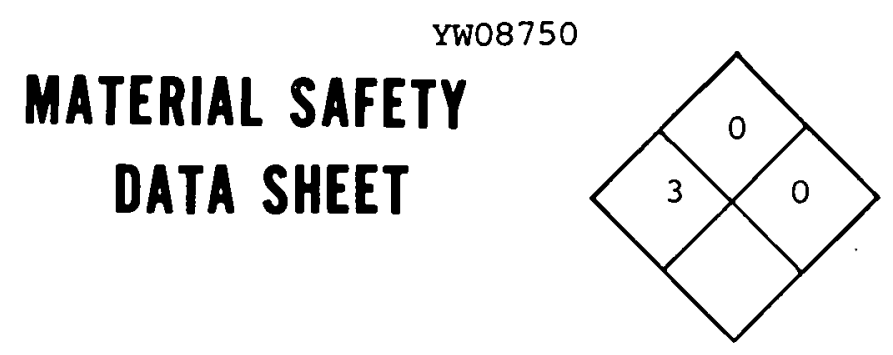

03-008-9336

Ammonium Vanadate

SECTION I SOURCE AND NOMENCLATURE

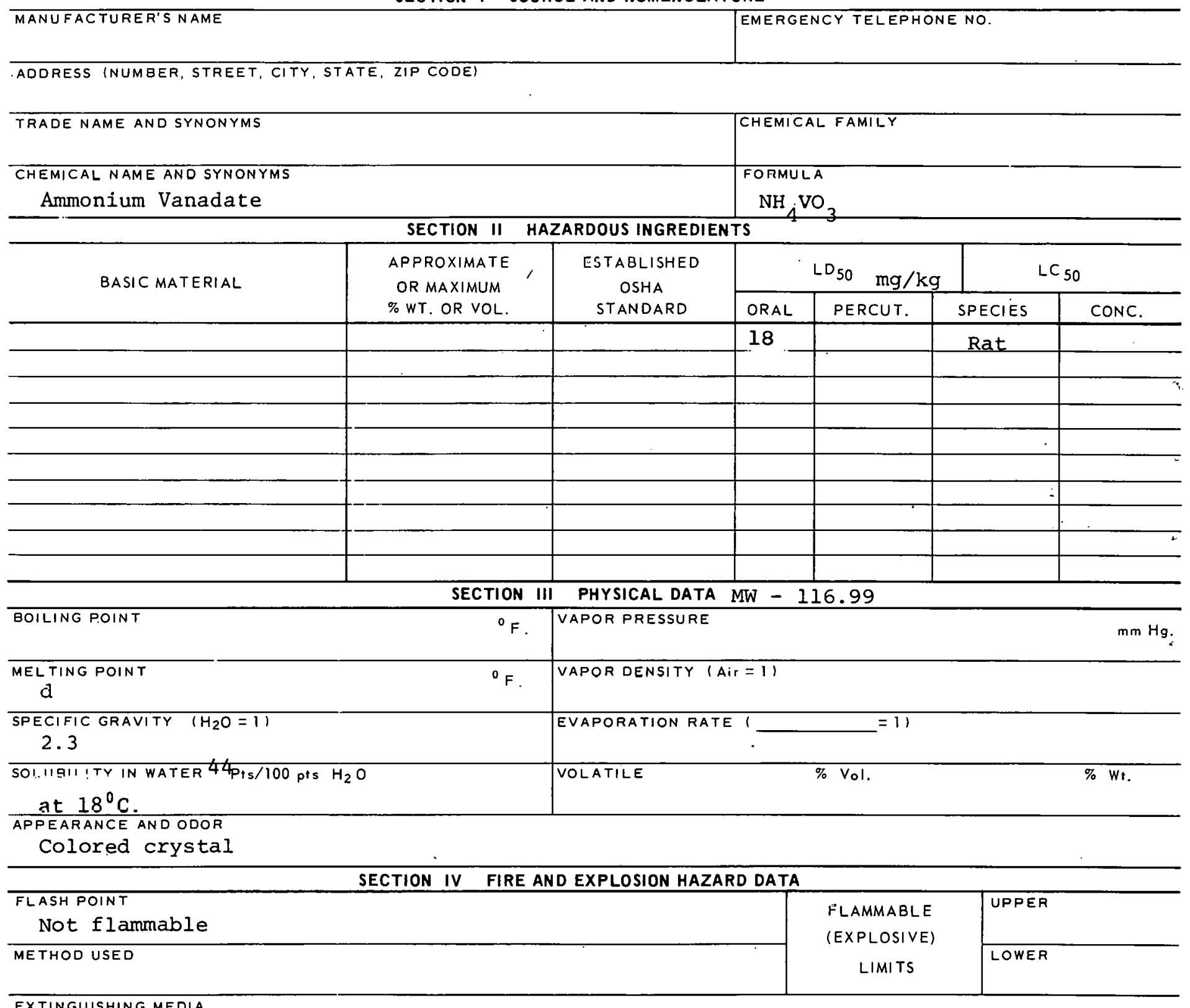

EXTINGUISHING MEDIA

SPECIAL FIRE FIGHTING PROCEDURES

UNUSUAL FIRE AND EXPLOSION HAZARDS 
Ammonium Vanadate

SECTION $V$ HEALTH HAZARD DATA

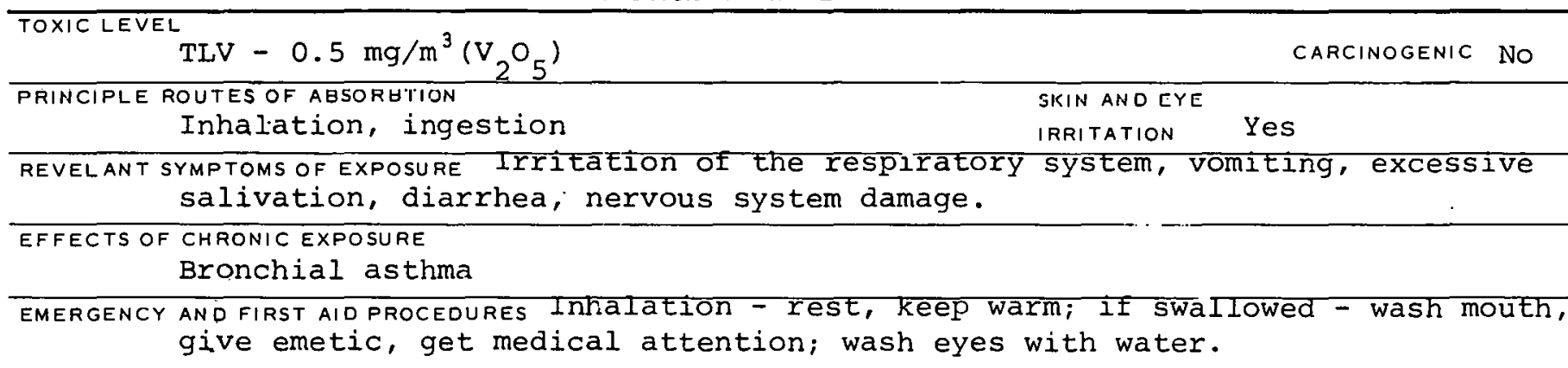

SECTION VI REACTIVITY DATA

CONDITIONS CONTRIBUTING TC TSTABILITY

CONDITIONS CONTRIBU TING TO HAZARDOUS POLYMERIZATION

INCOMPATAEILITI IMATEFIALS TO AVOIDI

HAZARDOUS DECOMPOSITION PRODUCTS

SECTION VII SPILL OR LEAK PROCEDURES

STEPS TO BE TAKEN IN CASE MATERIAL IS RELEASED OR SPILLEO

Vacuum or wet clean; do not dry sweep.

WASTE DISPOSAL METHOD

In seated plastic bag.

SECTION VIII SPECIAL PROTECTION INFORMATION

\begin{tabular}{l|l}
\hline VENTILATION REQUIREMENTS LOCAL EXHAUST & $\begin{array}{c}\text { PROTECTIVE EQUIPMENT (SPECIFY TYPES) EYE } \\
\text { SafetY glasSeS }\end{array}$ \\
\hline MECHANICAL IGENERAL) & GLOVES \\
\hline SPECIAL & $\begin{array}{c}\text { RESPIRATOR } \\
\text { Wi.th appropri.ate filter }\end{array}$ \\
\hline
\end{tabular}

OTHER PROTECTIVE EQUIPMENT

SECTION IX SPECIAL PRECAUTIONS

PRECAUTIONS TO BE TAKEN IN HANDLING AND STORAGE

OTHER PRECAUTIONS

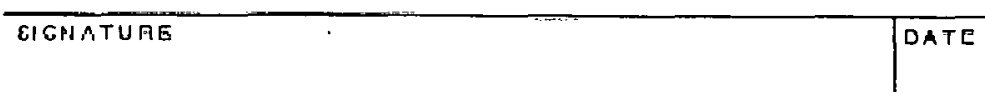

8-9336B 
03-010-0360

03-010-0390

MATERIAL SAFETY

DATA SHEET

Antimony

\section{SECTION I SOURCE AND NOMENCLATURE}

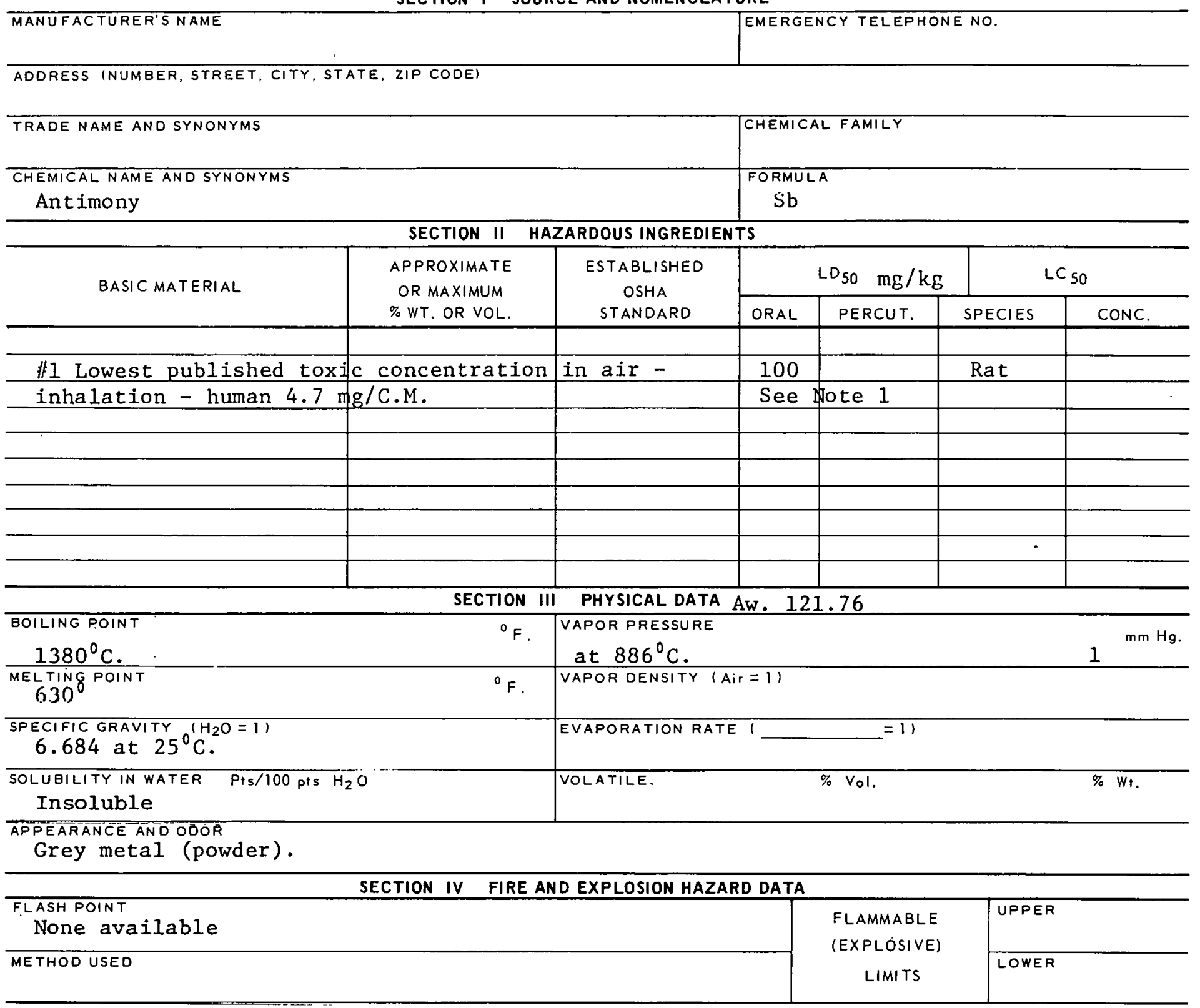

EXTINGUISHING MEDIA

Carbon dioxide, dry chemicals.

SPECIAL FIRE FIGHTING PROCEDURES

UNUSUAL FIRE AND EXPLOSION HAZARDS 
TOXIC LEVEL

TLV-TWA $0.5 \mathrm{mg} / \mathrm{m}^{3}$

PRINCIPI,F, ROUTES OF ABSUREIION

Inhalation
CARCINOGENIC No SKIN AND EYE IRRITATION SkIn

\section{REVELANT SYMPTOMS OF EXPOSURE}

Acute pneumonitis, nausea, vomiting

\section{EFFECTS OF CHRONIC EXPOSURE}

Bleeding gums, conjunctivitis, laryngitis, headache, weight loss and anemia EMERGENCY AND FIRST AID PROCEDURES

Remove from exposure - if ingested give gastric lavage, emesis and catharsis.

(Give dimercaprol). Administer oxygen if necessary.

\section{CONDITIONS CONTRIBUTING TO INSTABILITY \\ INCOMPATAOILITY IMATERIALS TO AVUIOU \\ OXIdizing materials}

CONDITIONS CONTRIBU TING TO HAZARDOUS POLYMERIZATION

HAZARDOUS DECOMPOSITION PRODUCTS

SECTION VII SPILL OR LEAK PROCEDURES

STEPS TO BE TAKEN IN CASE MATERIAL IS RELEASED OR SPILLEO

WASTE DISPOSAL METHOD

SECTION VIII SPECIAL PROTECTION INFORMATION

\begin{tabular}{|c|c|}
\hline VENTILATION REQUIREMENTS LOCAL EXHAUST & $\begin{array}{l}\text { PROTECTIVE EQUIPMENT (SPECIFY TYPES) FYE } \\
\text { Goggles or safety glasses }\end{array}$ \\
\hline MESHAMIIGAL (GEMERAL) & $\begin{array}{l}\text { GLOVES } \\
\text { Rubber }\end{array}$ \\
\hline SPECIAL & $\begin{array}{l}\text { RESPIRATOR } \\
\text { With approjlidile I1.titer. }\end{array}$ \\
\hline
\end{tabular}

OTHER PROTECTIVE EQUIPMENT

Rubber apron

\section{SECTION IX SPECIAL PRECAUTIONS}

PRECAUTIONS TO BE TAKEN IN HANDLING AND STORAGE 
03-010-0600

Antimony Trichloride

SECTION I SOURCE AND NOMENCLATURE

ADDRESS (NUMBER, STREET, CITY, STATE, ZIP CODE)

\section{TRADE NAME AND SYNONYMS \\ CHEMICAL NAME AND SYNONYMS \\ Antimony Butter}

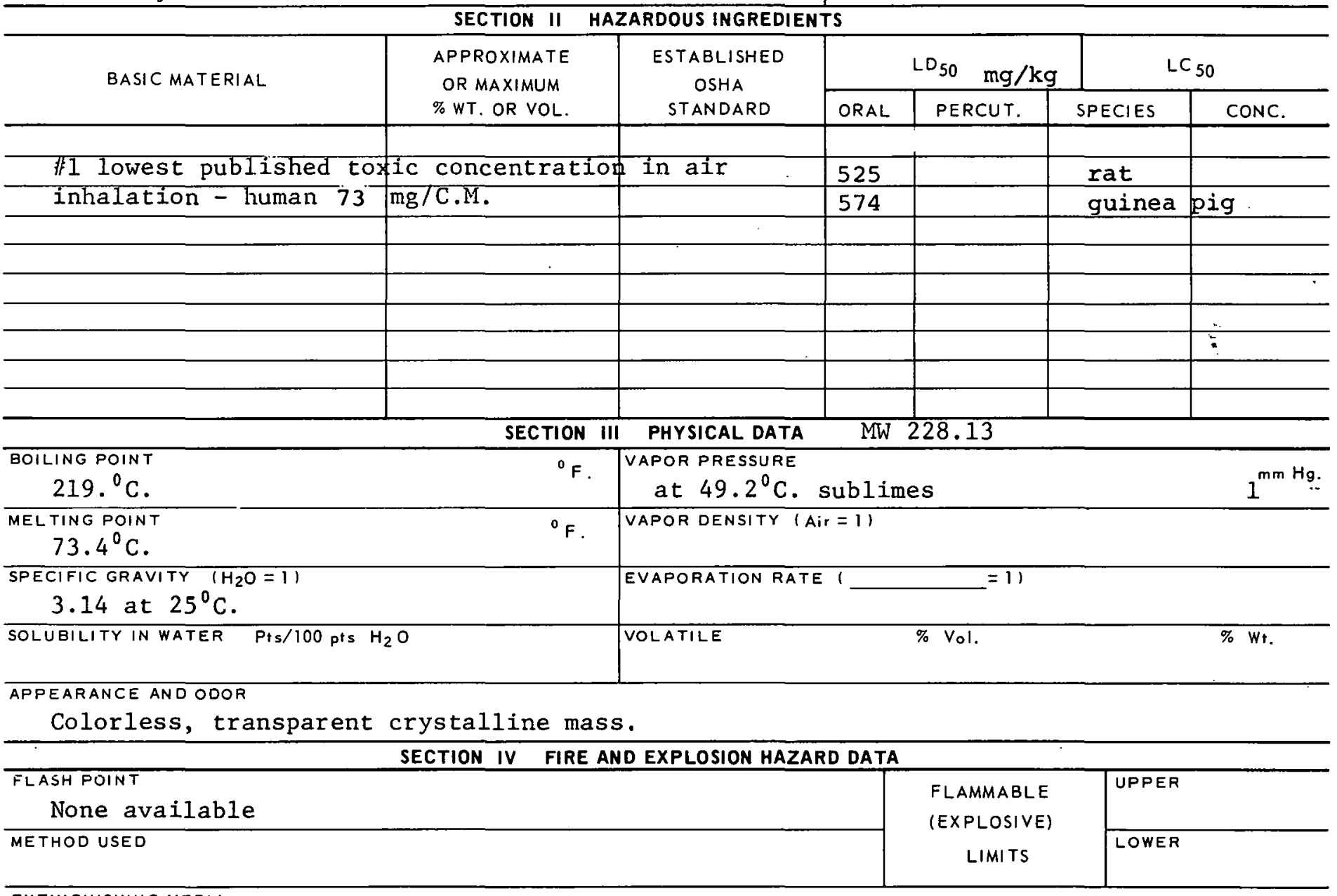

EXTINGUISHING MEDIA

Carbun diuxide, dry cliémical.
CHEMICAL FAMILY

FORMULA

$\mathrm{SbCl}_{3}$

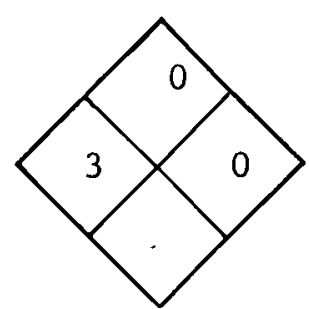

EMERGENCY TELEPHONE NO 


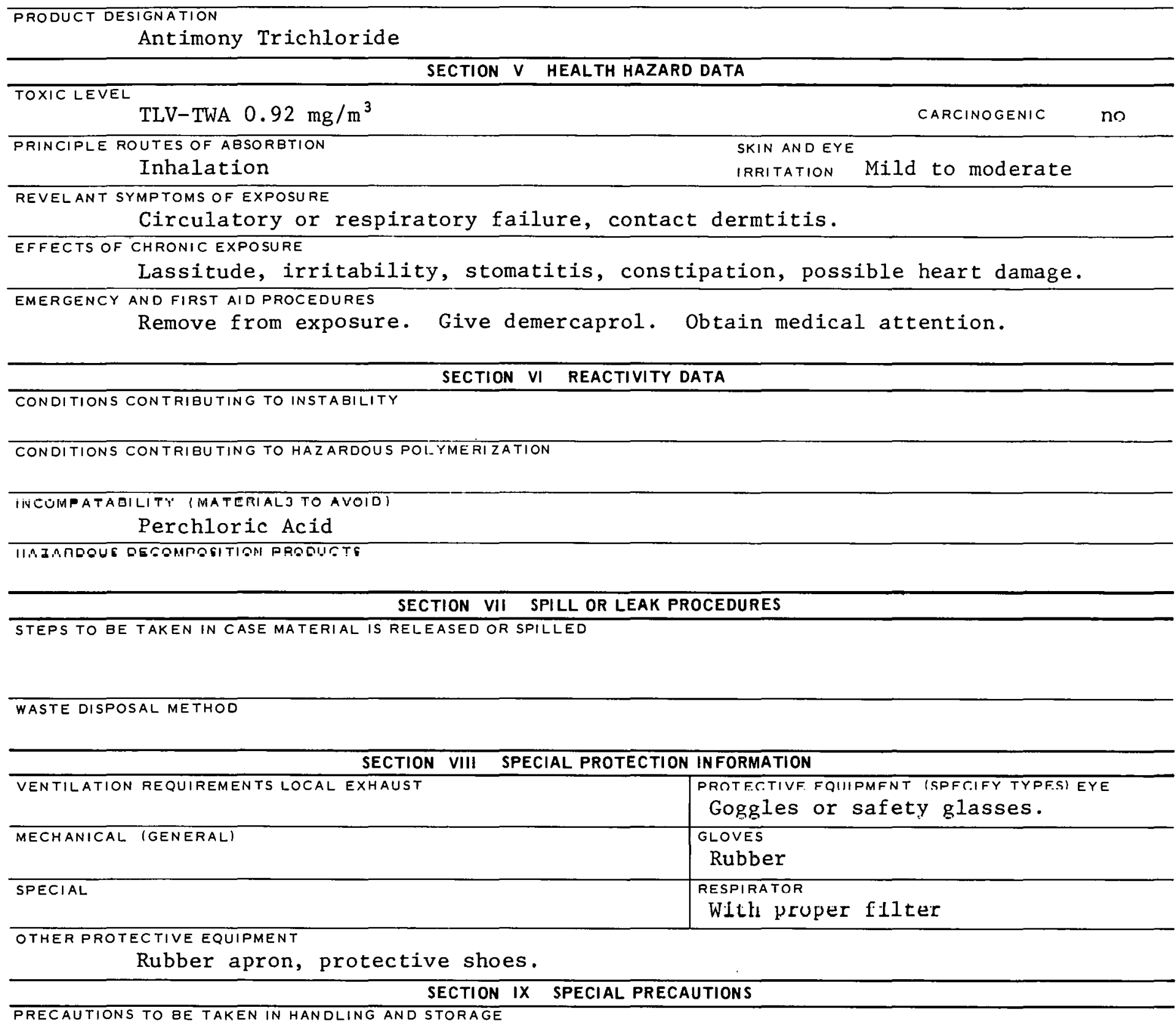

\begin{tabular}{l|l|l|}
\hline OTHER PRECAUTIONS & DATE \\
\hline SIGNATURE & \\
\hline
\end{tabular}




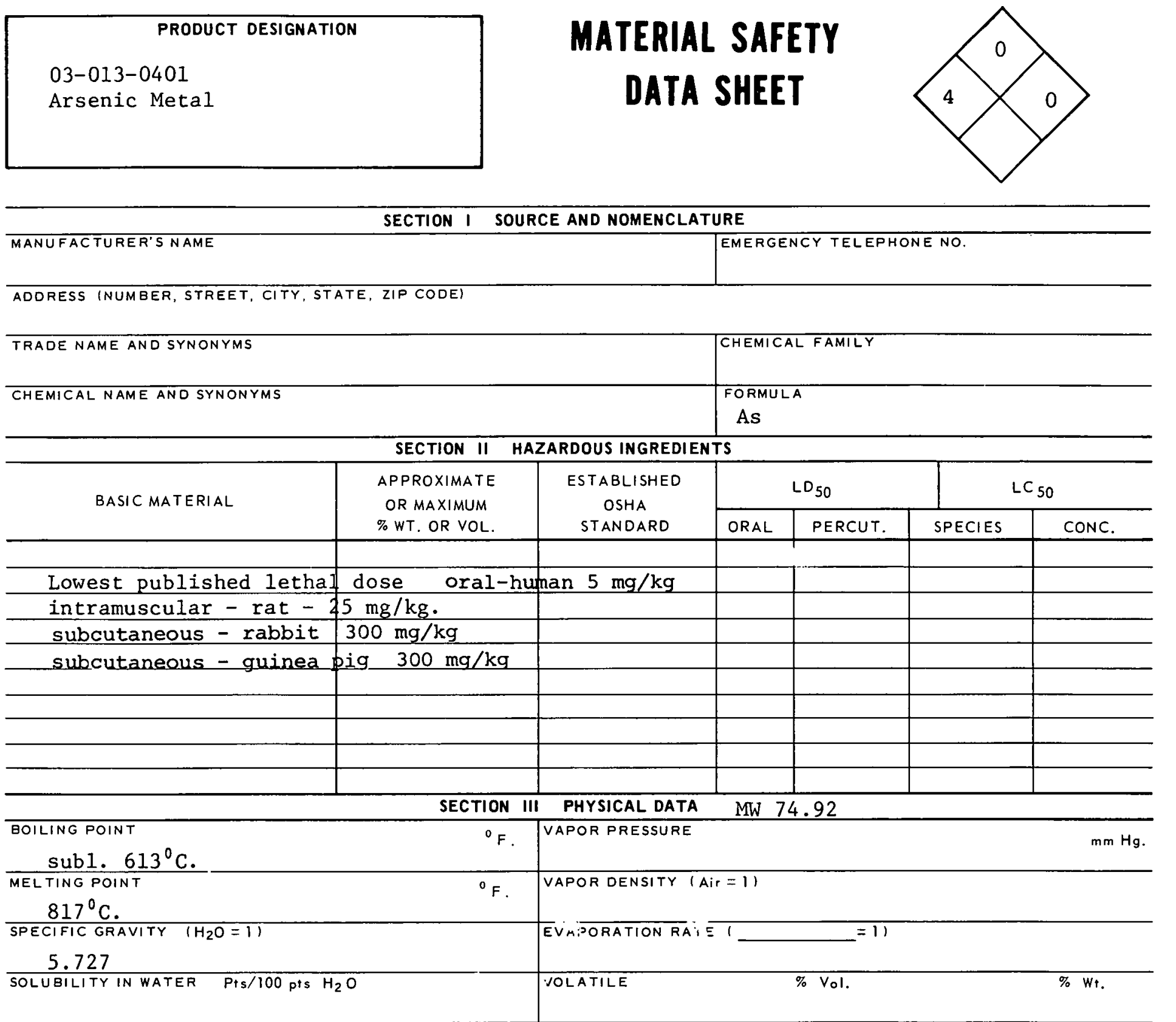

APPEARANCE AND ODOR

Grey metal

\begin{tabular}{l|c|l}
\hline & SECTION IV FIRE AND EXPLOSION HAZARD DATA & \\
\hline FLASH POINT & FLAMMABLE & UPPER \\
None available & (EXPLOSIVE) & LIMITS \\
\hline METHOD USED & LOWER & \\
\hline
\end{tabular}

EXTINGUISHING MEDIA

Carbon dioxide

SPECIAL FIRE FIGHTING PROCEOURES

UNUSUAL FIRE AND EXPLOSION HAZARDS 


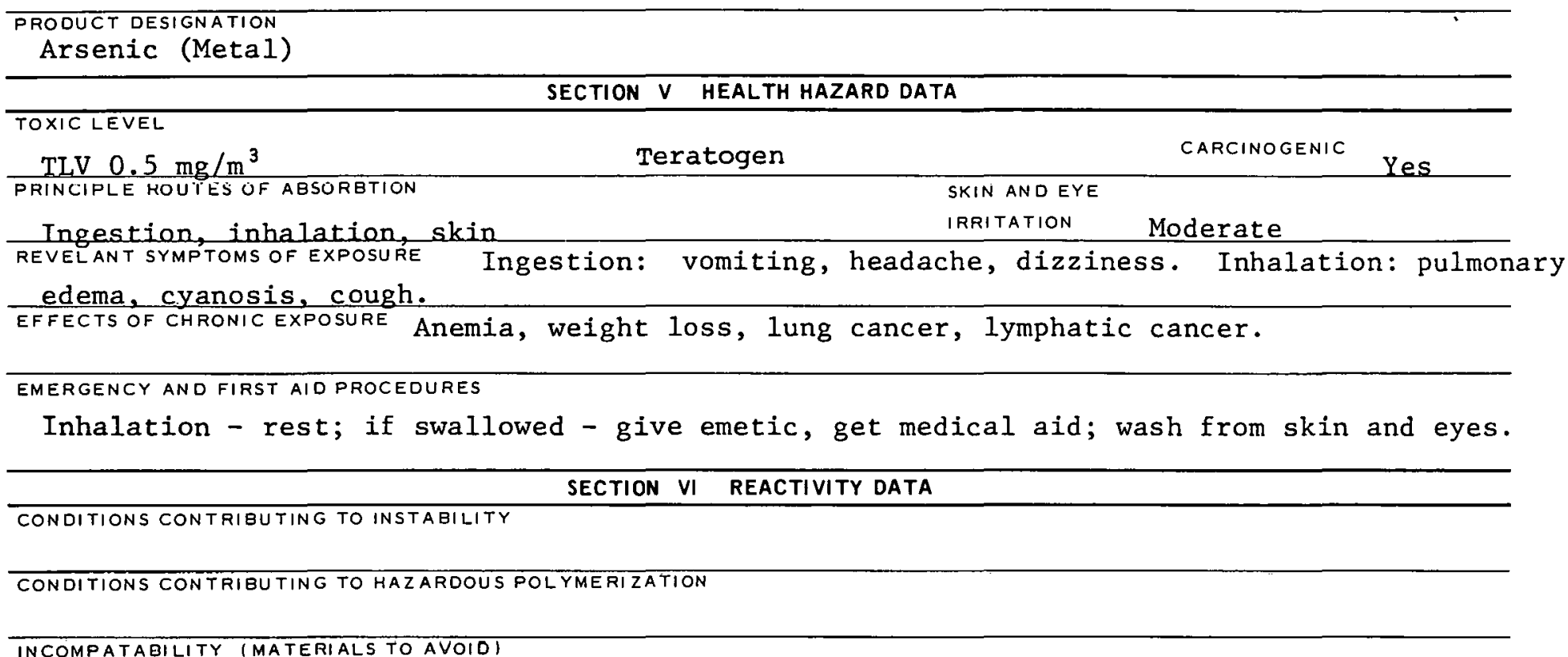

Avoid bromates, chlorates and iodates, also fluorides, nitrates and peroxides. HAZARDOUS DEROMPOSITION PRODIICTS

SECTION VII SPILL OR LEAK PROCEDURES

STEPS TO BE TAKEN IN CASE MATERIAL IS RELEASED OR SPILLED

WASTE DISPOSAL METHOD

\begin{tabular}{l|l}
\hline \multicolumn{1}{c}{ SECTION VIII SPECIAL PROTECTION INFORMATION } \\
\hline VENTILATION REQUIREMENTS LOCAL EXHAUST & $\begin{array}{l}\text { PROTECTIVE EQUIPMENT (SPECIFY TYPESI EYE } \\
\text { Goggles }\end{array}$ \\
\hline MECHANICAL IGENERALI & GLUVES \\
& Rubber \\
\hline SPECIAL- & RESPIRATOR \\
& With proper filter \\
\hline
\end{tabular}

OTHER PROTECTIVE EQUIPMENT

Long sleeve coveralls.

SECTION IX SPECIAL PRECAUTIONS

PRECAUTIONS TO BE TAKEN IN HANDLING AND STORAGE

Store in we11-ventilated area.

OTHER PRECAUTIONS

SIGNATURE

DATE

$13-0401 B$ 
EAS:00054306 AF45500

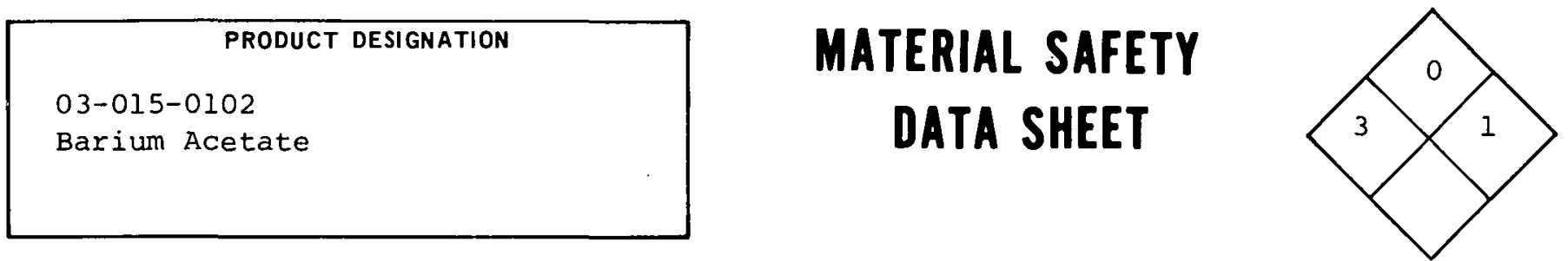

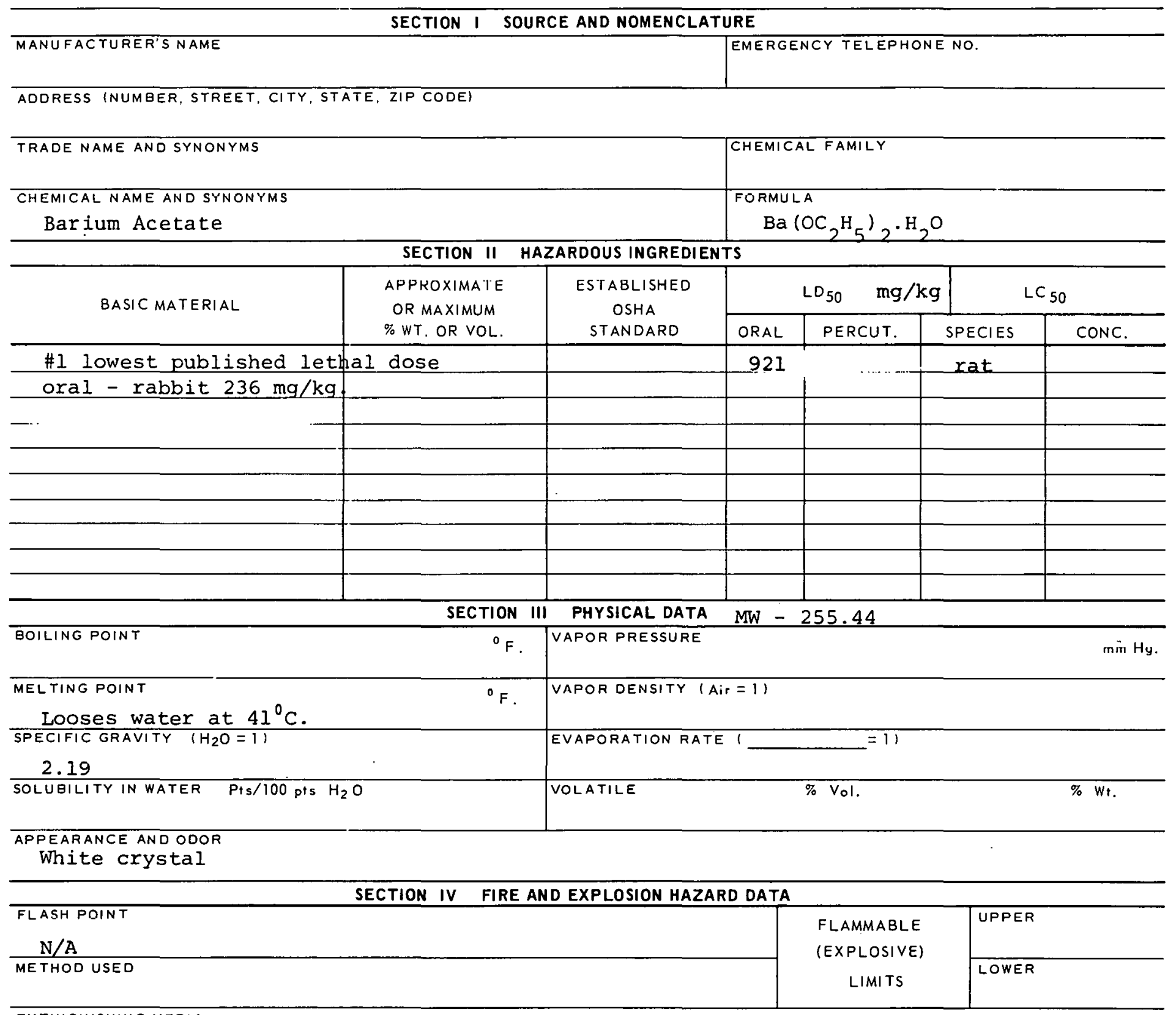

EXTINGUISHING MEDIA

$\mathrm{N} / \mathrm{A}$

SPECIAL FIRE FIGHTING PROCEDURES

$\mathrm{N} / \mathrm{A}$

UNUSUAL FIRE AND EXPLOSION HAZARDS

$N / A$ 
TOXIC LEVEL

TLV - $0.93 \mathrm{mg} / \mathrm{C} . \mathrm{M}$.

PRINCIPLE ROUTES OF ABSORBTION

Ingestion, inhalation of dust

REVELANT SYMPTOMS OF EXPOSURE Nausea, vomiting, stomach pains, diarrhea, headache, weaknesS, paralysis of central nervous system, digitalis like action on the heart.

EFFECTS OF CHRONIC EXPOSURE

-

EMERGENCY AND FIRST AID PROCEDURES If swallowed - wash mouth, drink 2 tablespoons of epSOM salts in water, then emetic; rest, keep warm, get medical aid.

\section{SECTION VI REACTIVITY DATA}

CONDITIONS CONTRIBUTING TO INSTABILITY

CONDITIONS CONTRIBUTING TO HAZARDOUS POLYMERIZATION

INCOMPATABILITY (MATERIALSTO AVOID)

HAZARDOUS DECOMPOSITION PRODUCTS

SECTION VII SPILL OR LEAK PROCEDURES

STEPS TO BE TAKEN IN CASE MATERIAL IS RELEASED OR SPILLED

SECTION VIII SPECIAL PROTECTION INFORMATION

\begin{tabular}{l|l}
\hline VENTILATION REQUIREMENTS LOCAL EXHAIIST & $\begin{array}{l}\text { PROTECTIVE EQUIPMENT (SPPCIFY TYPESI EYE } \\
\text { GOggleS }\end{array}$ \\
\hline MECHANICAL IGENERAL) & $\begin{array}{l}\text { GLOVES } \\
\text { Rubber gloves }\end{array}$ \\
\hline SPECIAL & $\begin{array}{l}\text { RESPIRATOR } \\
\text { With proper filter }\end{array}$ \\
\hline
\end{tabular}

OTHER PROTECTIVE EQUIPMENT

\section{SECTION IX SPECIAL PRECAUTIONS}

PRECAUTIONS TO BE TAKEN IN HANDLING AND STORAGE

OTHER PRECAUTIONS

SIGNATURE

DATE

15-0102B

CARCINOGENIC NO

SKIN ANO EYE

IRRITATION Moderate

WASTE DISPOSAL METHOD

TI

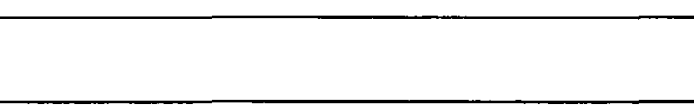

s 
CAS : 000513779

$\mathrm{CQ86000}$

PRODUCT DESIGNATION

Barium Carbonate

03-015-0903

MATERIAL SAFETY

DATA SHEET

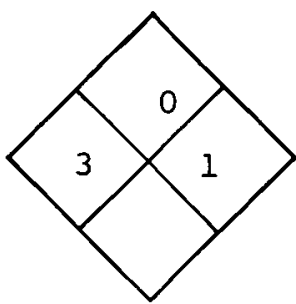

SECTION I SOURCE AND NOMENCLATURE

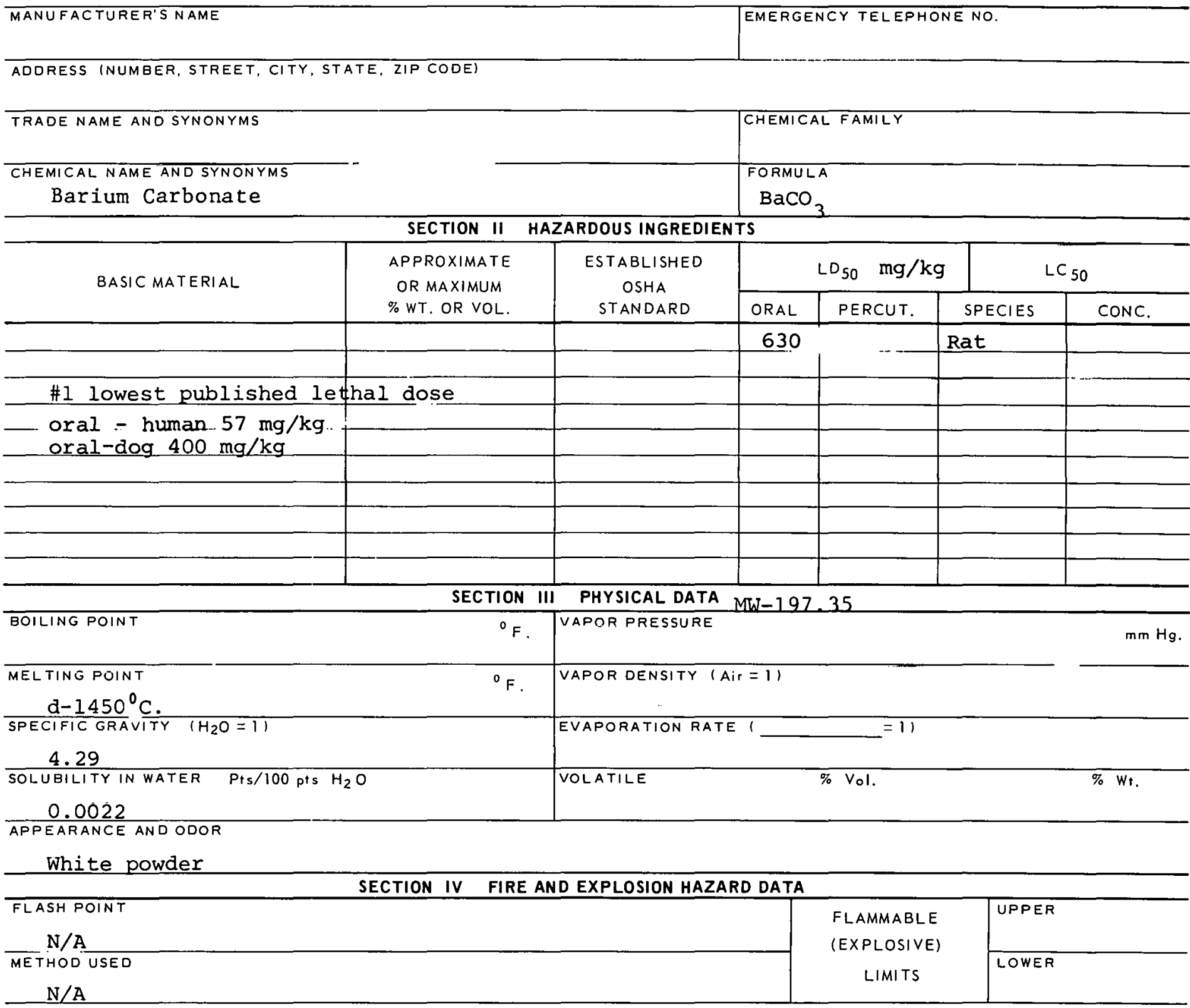

EXTINGUISHING MEDIA

$\mathrm{N} / \mathrm{A}$

SPECIAL FIRE FIGHTING PROCEDURES

$\mathrm{N} / \mathrm{A}$

UNUSUAL FIRE AND EXPLOSION HAZARDS

$N / A$ 


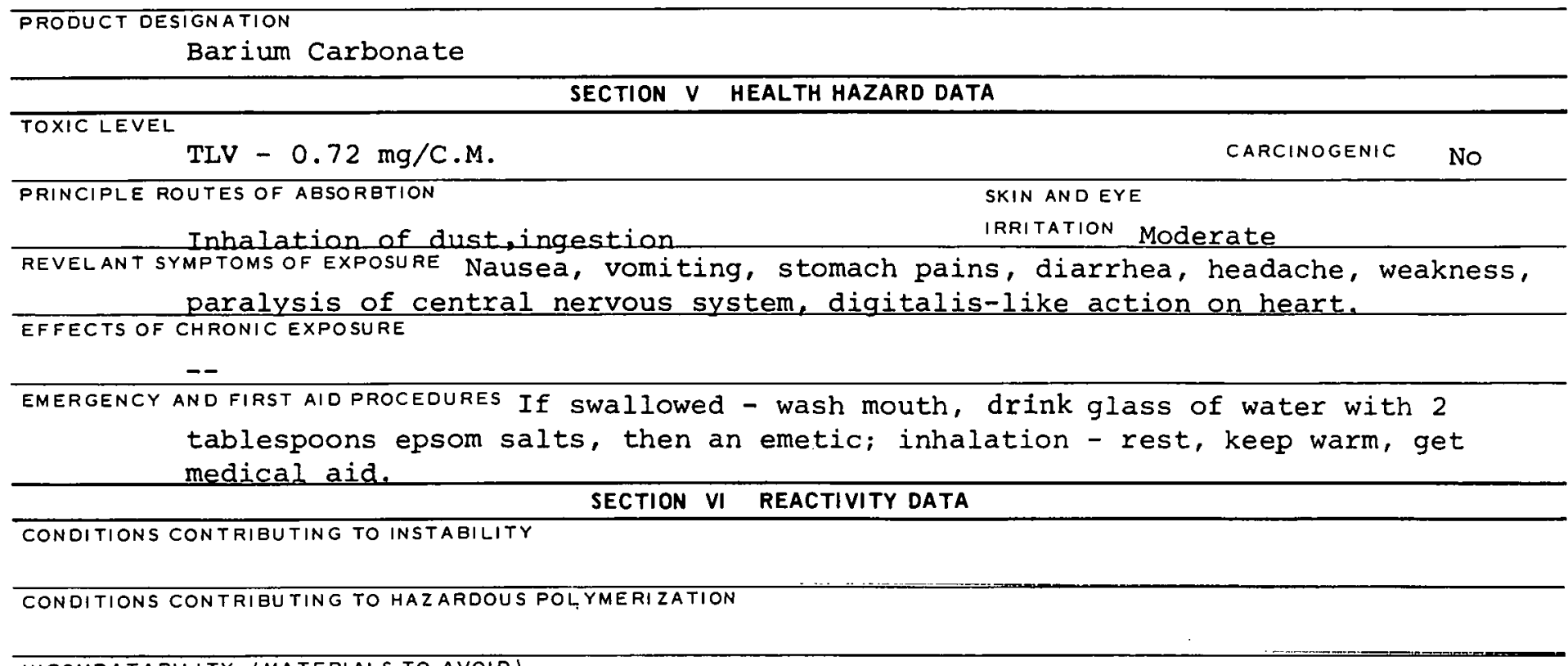

IPIGOMPATAA!L!TY (MATERIALS TO AVOID)
HAZARDOUS OECUMHUSIIIUN FRUDUETS

\section{SECTION VII SPILL OR LEAK PROCEDURES}

STEPS TO BE TAKEN IN CASE MATERIAL IS RELEASED OR SPILLED

\section{WASTE DISPOSAL METHOD}

\begin{tabular}{l|l}
\hline \multicolumn{2}{c}{ SECTION VIII SPECIAL PROTECTION INFORMATION } \\
\hline VENTILATION REQUIREMENTS LOCAL EXHAUST & $\begin{array}{l}\text { PROTECTIVE EQUIPMENT } \\
\text { SafEtY glaSSES }\end{array}$ \\
\hline MECHANICAL IGENERALI & GLOVES \\
\hline SPECIAL & $\begin{array}{l}\text { RESPIRATOR } \\
\text { With proper filter }\end{array}$ \\
\hline
\end{tabular}

SECTION IX SPECIAL PRECAUTIONS

\begin{tabular}{l|l}
\hline PRECAUTIONS TO BE TAKEN IN HANDLING AND STORAGE \\
\hline OTHER PRECAUTIONS \\
\hline SIGNATURE & DATE
\end{tabular}


CAS : 010361372

CQ87500

PRODUCT DESIGNATION

MATERIAL SAFETY

Barium Chloride

03-015-1904

DATA SHEET

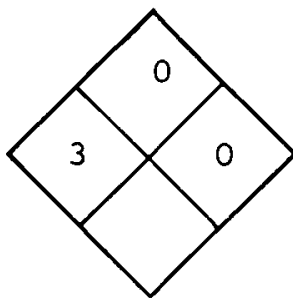

SECTION I SOURCE AND NOMENCLATURE

\begin{tabular}{l|l}
\hline MANUFACTURER'S NAME & EMERGENCY TELEPHONE NO. \\
\hline ADDRESS (NUMBER, STREET, CITY, STATE, ZIP CODE) & \\
\hline
\end{tabular}

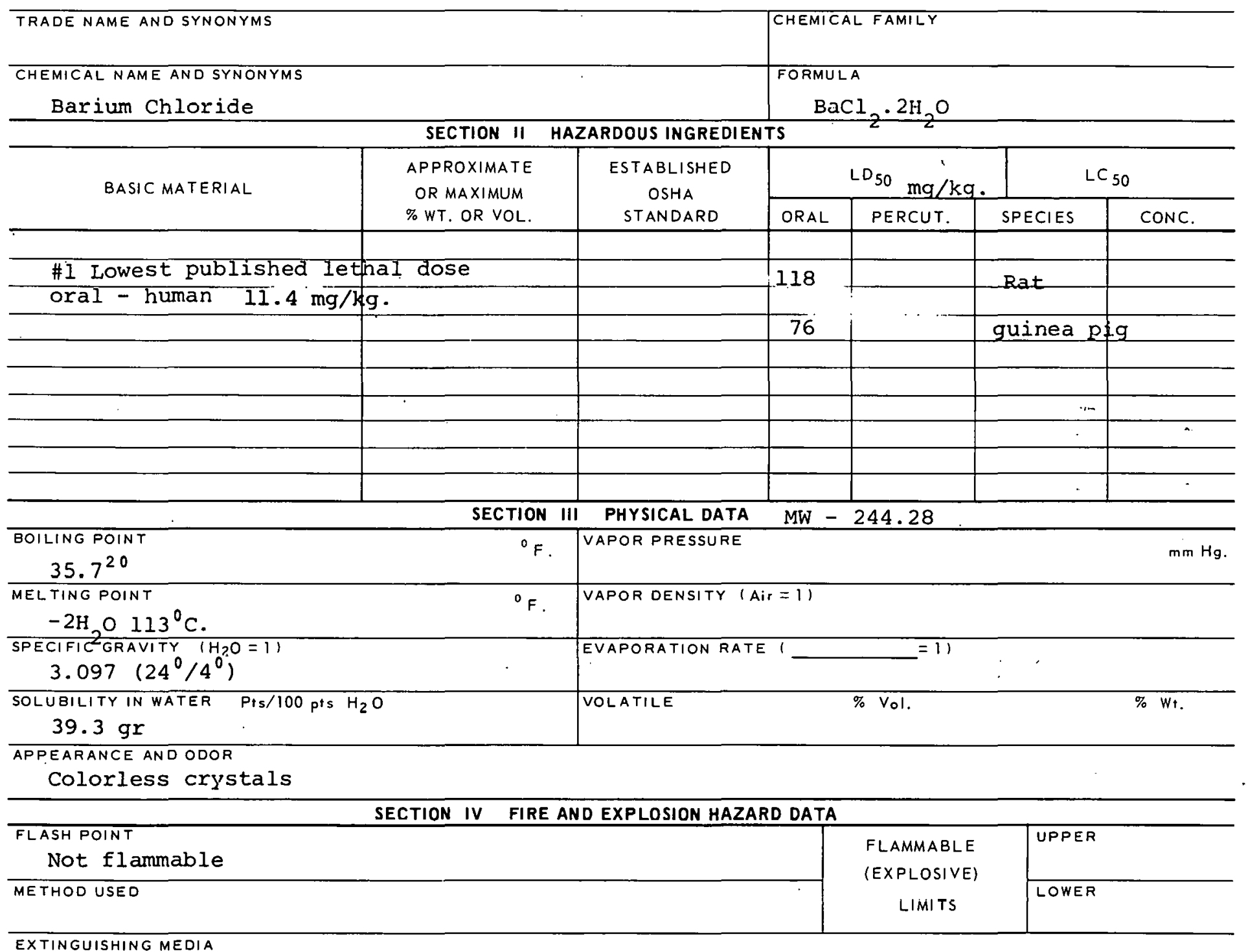

EXTINGUISHING MEDIA

SPECIAL FIRE FIGHTING PROCEDURES

UNUSUAL FIRE AND EXPLOSION HAZARDS 


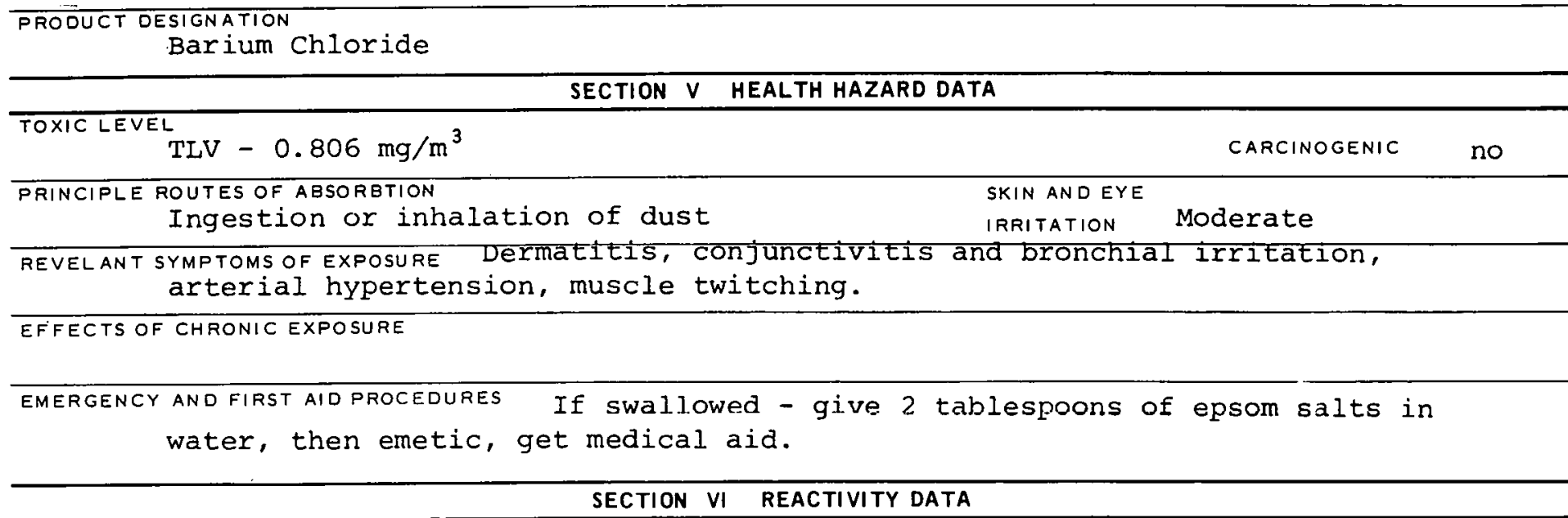

CONDITIONS CONTRIBUTING TO INSTABILITY
CONDITIONS CONYRIBUTING TO HAZARDOUS POLYMERIZATION

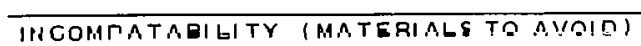

HATARMOIIS RFROMPRSITION PRODIIS.TS

SECTION VII SPILL OR LEAK PROCEDURES

STEPS TO BE TAKEN IN CASE MATERIAL IS RELEASED OR SPILLED

WASTE DISPOSAL METHOD

SECTION VIII SPECIAL PROTECTION INFORMATION

\begin{tabular}{l|l}
\hline VENTILATION REQUIREMENTS LOCAL EXHAUST & $\begin{array}{l}\text { PROTECTIVE EQUIPMENT (SPECIFY TYPES) EYE } \\
\text { GOggles }\end{array}$ \\
\hline MECHANICAL (GENERAL) & $\begin{array}{l}\text { GLOQVES } \\
\text { Rubber }\end{array}$ \\
\hline SPECIAL & $\begin{array}{l}\text { RESPIRATOR } \\
\text { W/proper tilter }\end{array}$ \\
\hline
\end{tabular}

OTHER PROTECTIVE EQUIPMENT

SECTION IX SPECIAL PRECAUTIONS

PRECAUTIONS TO BE TAKEN IN HANDLING AND STORAGE

OTHER PRECAUTIONS

SIGNATURE

DATE

$15-1904 B$ 


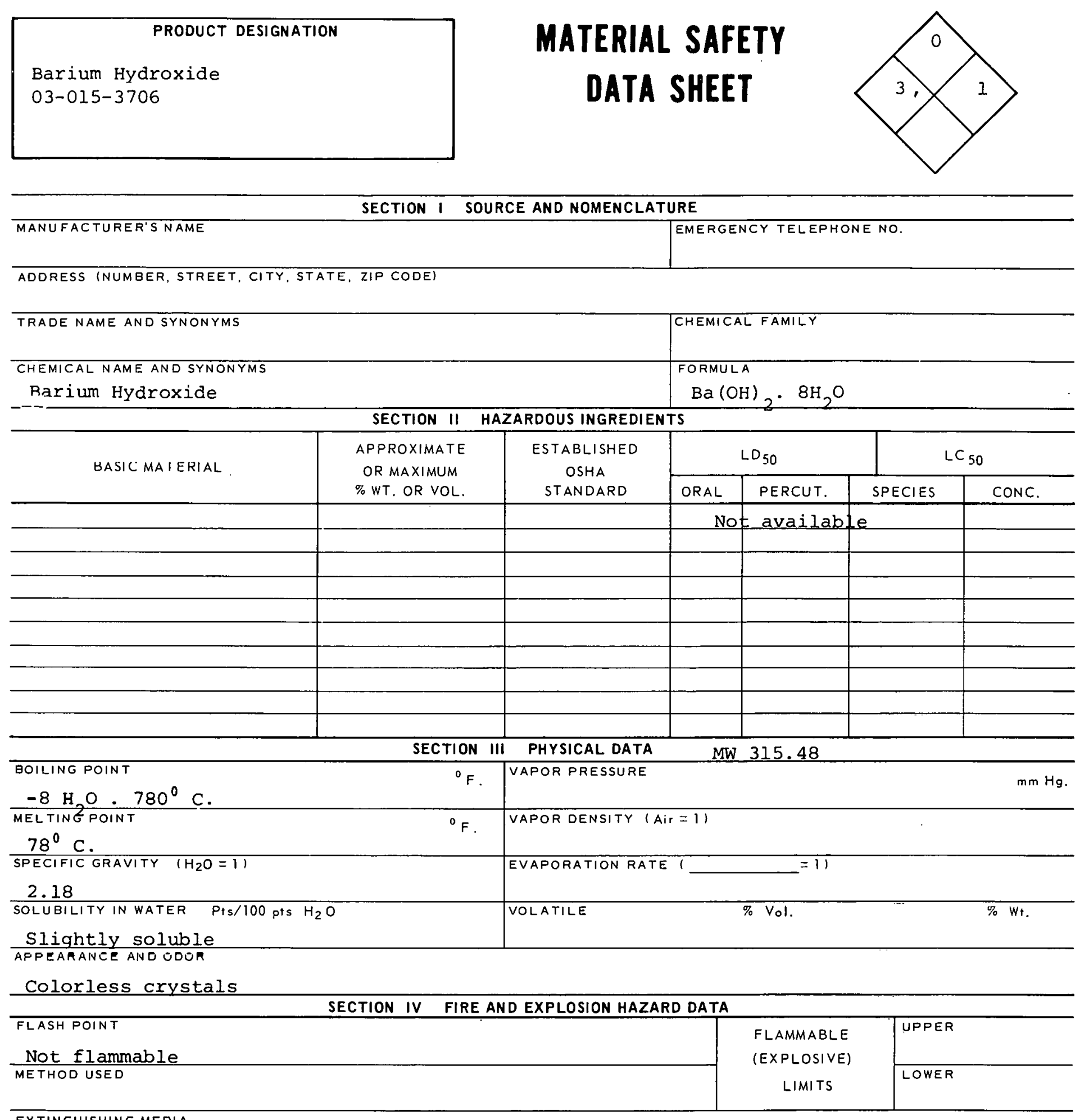

EXTINGUISHING MEDIA

SPECIAL FIRE FIGHTING PROCEDURES

UNUSUAL FIRE AND EXPLOSION HAZARDS 


\begin{tabular}{|c|}
\hline $\begin{array}{l}\text { PRODUCT DESIGNATION } \\
\text { Barium HYdroxide }\end{array}$ \\
\hline SECTION $V$ HEALTH HAZARD DATA \\
\hline $\begin{array}{l}\text { TOXIC LEVEL } \\
\text { TLV } 1.15 \mathrm{mg} / \mathrm{m}^{3}\end{array}$ \\
\hline $\begin{aligned} \text { PRINCIPLE ROUTES OF ABSORBTION } & \text { SKIN ANDEYE } \\
\text { Ingestion or inhalation of dust } & \text { IRRITATION MOderate }\end{aligned}$ \\
\hline $\begin{array}{l}\text { REVELANT SYMPTOMS OF EXPOSURE Dermatitis, conjunctivis and bronchial irritation, tightness of } \\
\text { face and neck muscles, vomiting, diarrhea, weakness, difficulty in breathing. }\end{array}$ \\
\hline EFFECTS OF CHRONIC EXPOSURE \\
\hline $\begin{array}{l}\text { EMERGENCY AND FIRST AID PROCEDURES Give artificial respiration if necessary, specifically with } \\
\text { oxygen if available. Antidote to be administered as soon as possible } 10 \% \text { sodium } \\
\text { sulfate slowly } \mathrm{I.V} \text {. - repeat every } 15 \mathrm{~min} \text {. until symtom subsides. } 30 \mathrm{Gm} \text { of sodium }\end{array}$ \\
\hline CONDITIONS CONTRIBUTING TO INSTABILITY water OraIIY. \\
\hline CONDITIONS CONTRIBUTING TO HAZARDOUS POLYMERIZATION \\
\hline INCOMPAIABILITY (MATERIALS TO AVOID) \\
\hline HALAREOUS DECBHFOJTIOII RROQUGTE \\
\hline SECTION VII SPILL OR LEAK PROCEDURES \\
\hline
\end{tabular}

STEPS TO BE TAKEN IN CASE MATERIAL IS RELEASED OR SPILLED

WASTE DISPOSAL METHOD

SECTION VIII SPECIAL PROTECTION INFORMATION

\begin{tabular}{l|l}
\hline VENTILATION REQUIREMENTS LOCAL EXHAUST & $\begin{array}{l}\text { PROTECTIVE EQUIPMEN'I (SPECIFY TYPESI EYE } \\
\text { GOggleS }\end{array}$ \\
\hline MECHANICAL IGENERAL) & $\begin{array}{l}\text { GLOVES } \\
\text { Rubber }\end{array}$ \\
\hline GPFSIAI. & $\begin{array}{c}\text { RESPIRATOR } \\
\text { With appropriate filter }\end{array}$ \\
\hline
\end{tabular}

OTHER PROTECTIVE EQUIPMENT

\section{SECTION IX SPECIAL PRECAUTIONS}

PRECAUTIONS TO BE TAKEN IN HANDLING AND STORAGE

Store in cloṣed containers.

OTHER PRECAUTIONS

SIGNATURE

DATE

$15-3706 B$ 

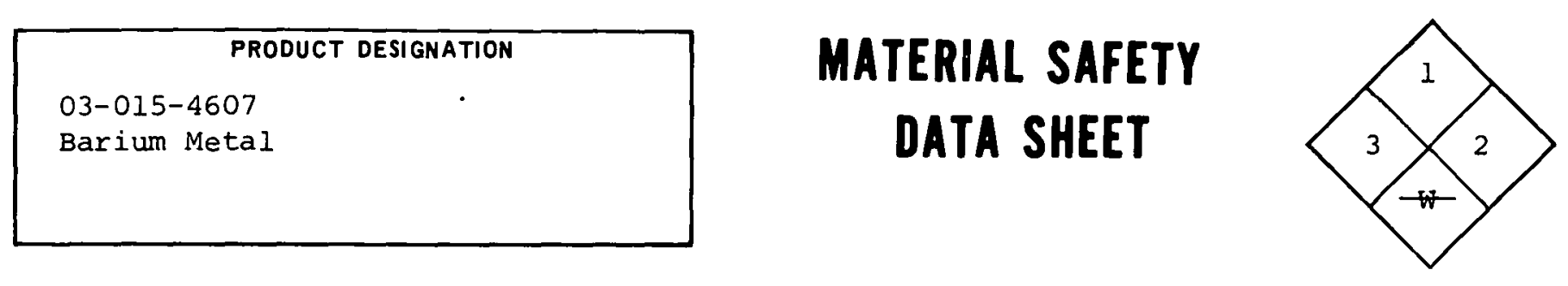

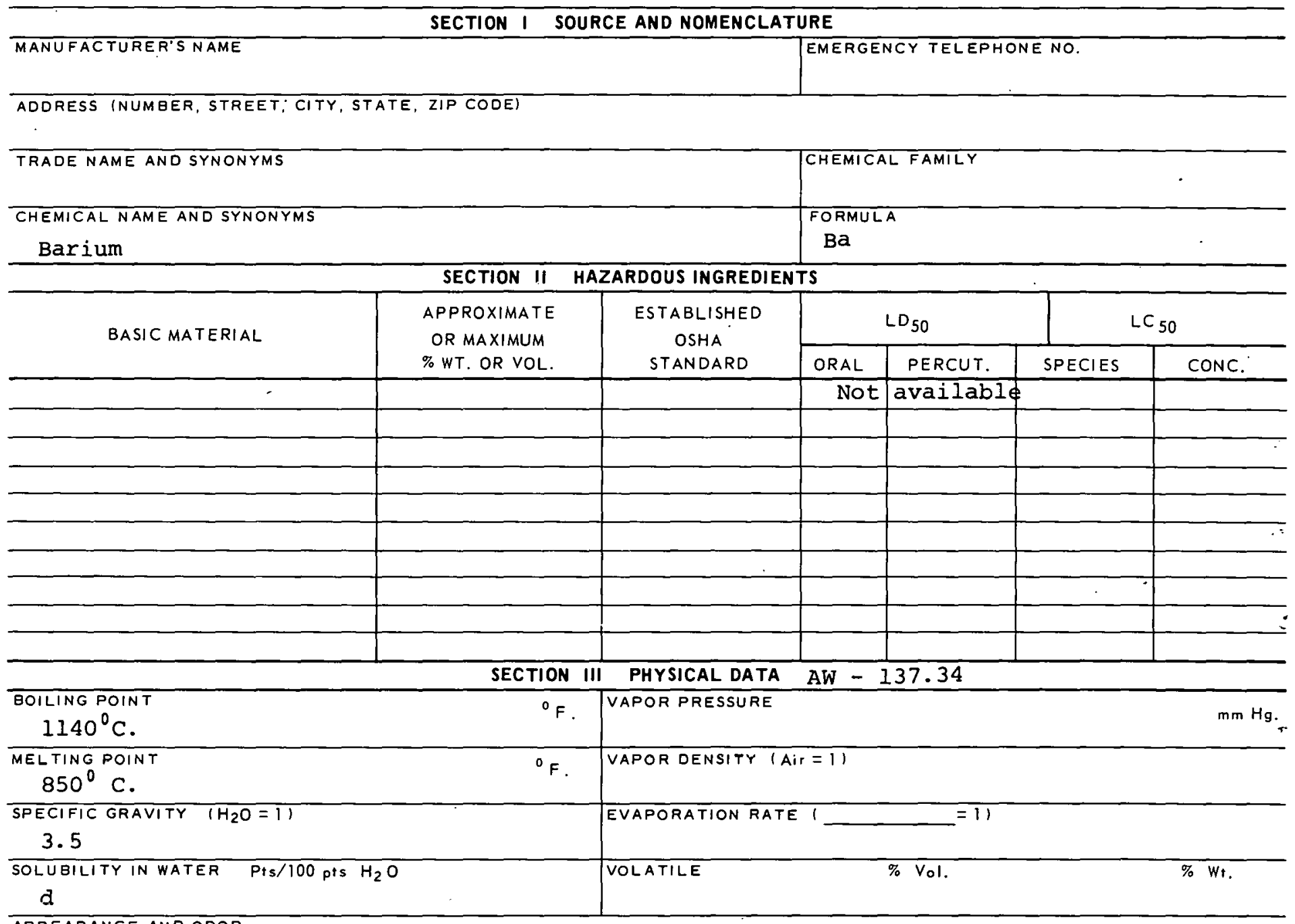

APPEARANCE AND ODOR

Silver metal

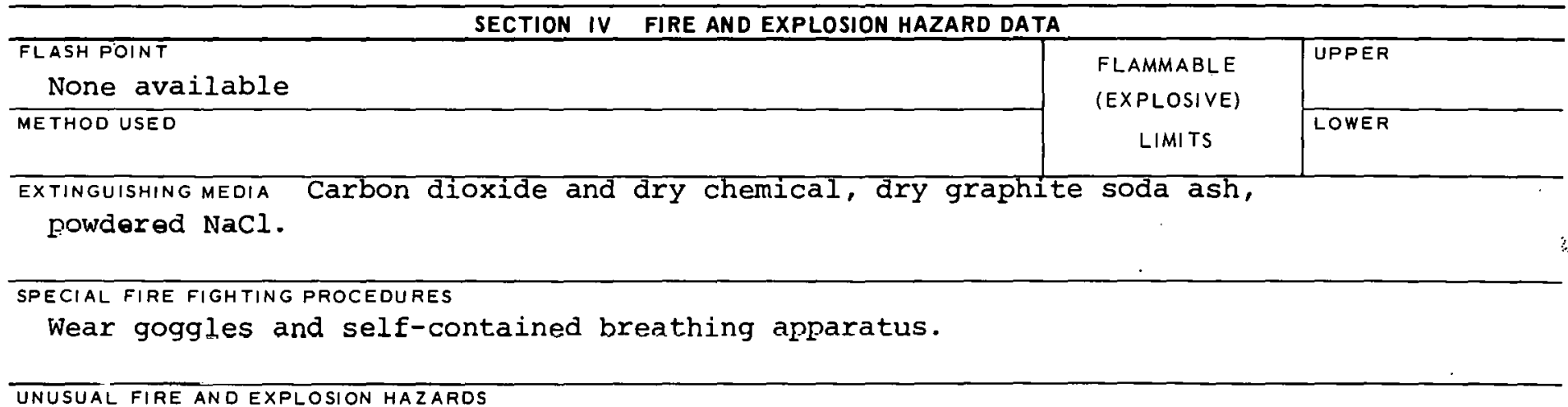

UNUSUAL FIRE AND EXPLOSION HAZARDS 
TOXIC LEVEL

$\mathrm{TLV}-0.5 \mathrm{mg} / \mathrm{m}^{3}$

PRINCIPLE ROUTES OF ABSORBTION

REVELANT SYMPTOMS OF EXPOSURE paralysis of central

EFFECTS OF CHRONIC EXPOSURE

- -

EMERGENCY AND FIRST AID PROCEDURES If swallowed - wash mouth, drink glass of water with 2 tablespoons of epsom salt; inhalation - rest, keep warm; get medical attention; wash from eyes and skin with water.

\section{SECTION VI REACTIVITY DATA}

CONDITIONS CONTRIBUTING TO INSTABILITY

CONDITIQNS CQNTR!BUTING TO HAZARDOUS POLYMERIZATION

INCOMPATABILITY (MATERIALS TO AVOID)

Water, acids

$\overline{H A} Z \overline{Z A R D O U S}$ DECOMPOSITION PRODUCTS

SECTION VII SPILL OR LEAK PROCEDURES

STEPS TO BE TAKEN IN CASE MATERIAL IS RELEASED OR SPILLED

WASTE UISPOSAL METHOD

SECTION VIII SPECIAL PROTECTION INFORMATION

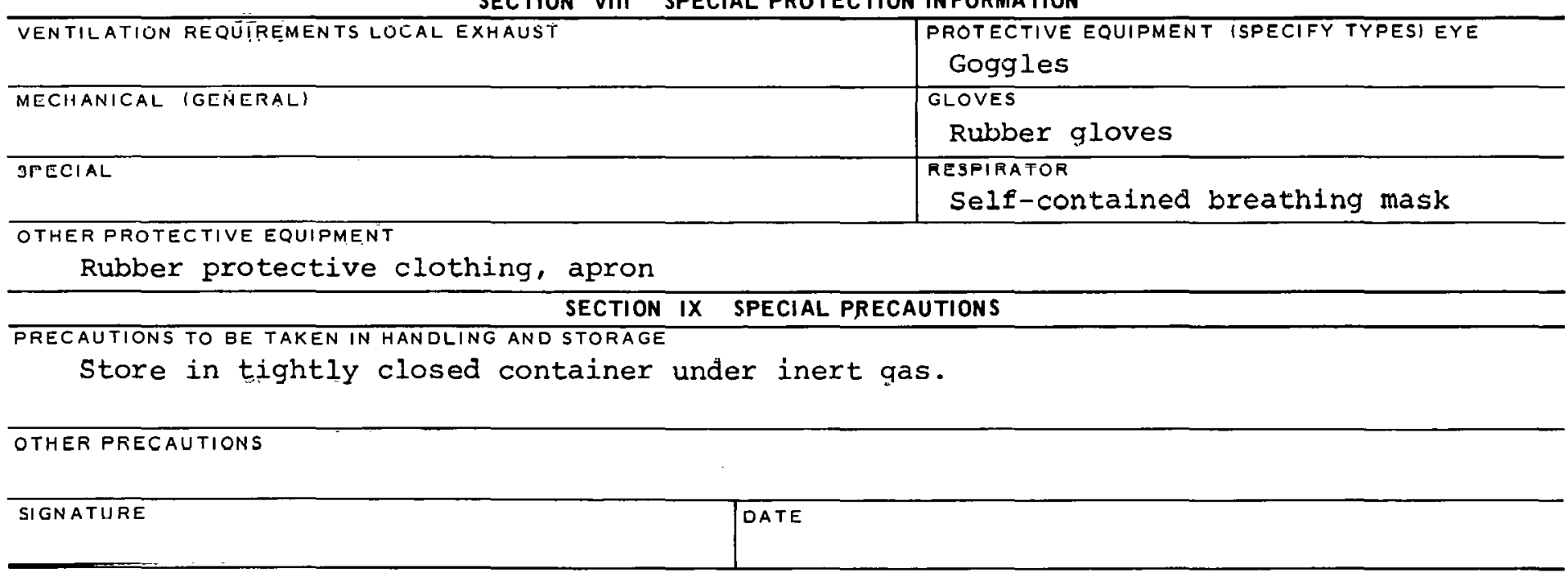

15-4607B 
CAS: 010022318

\section{PRODUCT DESIGNATION}

03-015-5538

Barium Nitrate
MATERIAL SAFETY

DATA SHEET

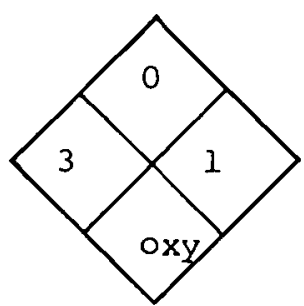

\section{SECTION I SOURCE AND NOMENCLATURE}

\begin{tabular}{l} 
MANUFACTURER'S NAME \\
\hline ADDRESS INUMBER, STREET, CI \\
TRADE NAME AND SYNONYMS \\
CHEMICAL NAME AND SYNONYMS \\
BaYIUM Niträte
\end{tabular}

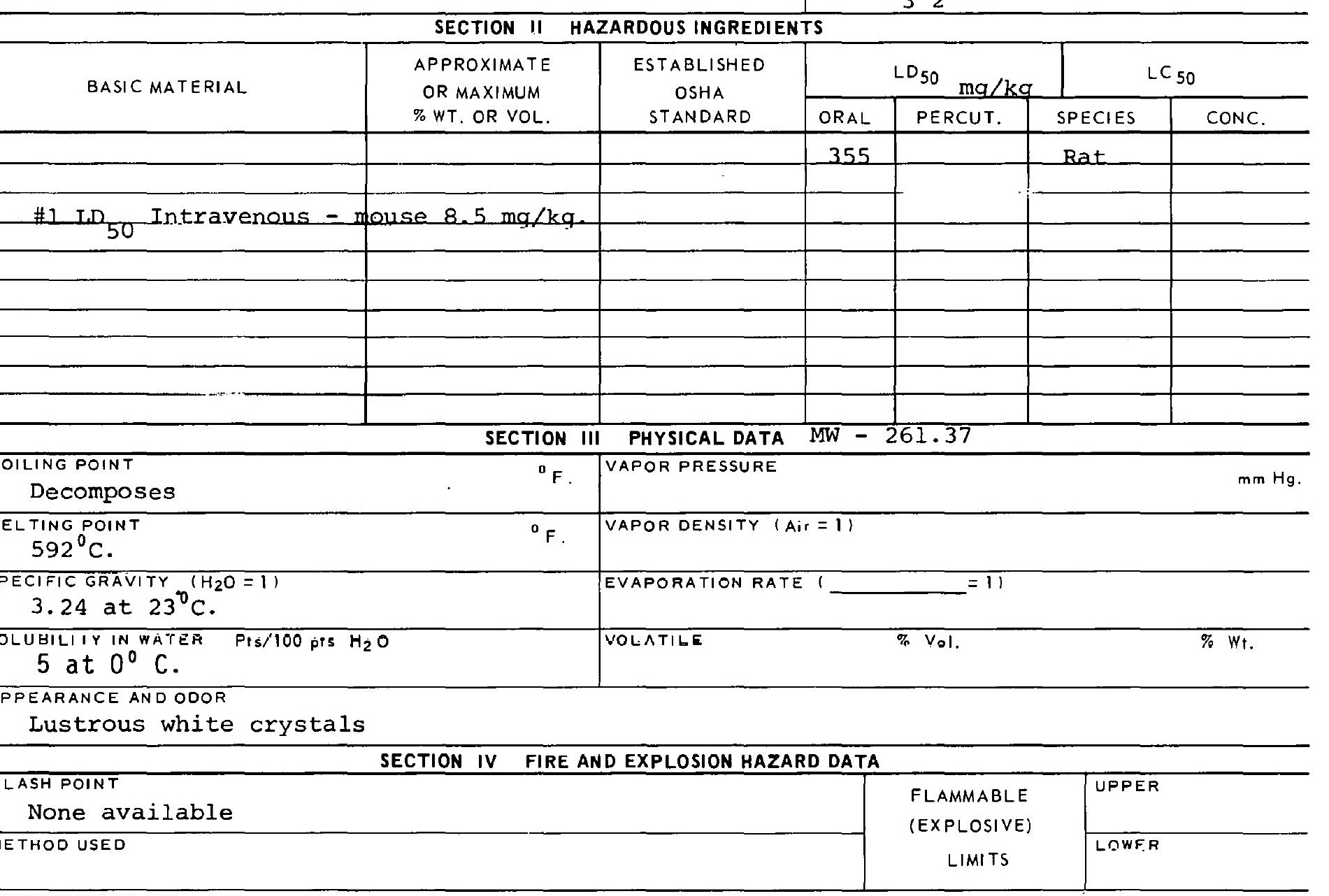

EXTINGUISHING MEUIA

Water

SPECIAL FIRE FIGHTING PROCEDURES

Wear self-contained breathing apparatus.

UNUSUAL FIRE AND EXPLOSION HATAROS

In contact with easily oxidizable substances - may react rapidly enough to cause ignition, violent combustion, or explosion. 
TOXIC LEVEL

$T L V-0.925 \mathrm{mg} / \mathrm{C} . \mathrm{M}$.

CARCINOGENIC NO

PRINCIPLE ROUTES OF ABSORBTION

Ingestion or inhalation dust

SKIN AND EYE

IRRITATION MOderate

REVELANT SYMPTOMS OF EXPOSURE Dermatitis conjunctivitis ang boonchial irritation tightaps of pecistalsis, arterial hypertension, muscle twitching and cardiac dysfunction. EFFECTS OF CHRONIC EXPOSURE

$--$

EMERGENCY AND FIRST AID PROCEDURES If necessary give artificial respiration - if ingested give

2 tablespoonfuls of magnesium sulfate in water then an emetic, rest and keep warm obtain medical attention.

\begin{tabular}{|c|c|c|}
\hline 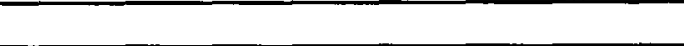 & SECTION VI & REACTIVITY DATA \\
\hline \multicolumn{3}{|l|}{ CONDITIONS CONTRIBUTING TO INSTABILITY } \\
\hline \multicolumn{3}{|c|}{ CONDITIONS CONTRIBU TING TO HAZARDOUS POLYMERIZATION } \\
\hline \multicolumn{3}{|c|}{ IMCOMPATAEILITY IMATERIALS TO AVOID) } \\
\hline \multicolumn{3}{|c|}{$\begin{array}{l}\text { HATARDSIIS MFR.MMPNSITINN RRAMIISTS } \\
\text { Yields toxic gaseous oxides of nitrogen }\end{array}$} \\
\hline \multicolumn{3}{|c|}{ SECTION VII SPILL OR LEAK PROCEDURES } \\
\hline \multicolumn{3}{|c|}{$\begin{array}{l}\text { STEPS TO BE TAKEN IN CASE MATERIAL IS RELEASED OR SPILLED } \\
\text {.. }\end{array}$} \\
\hline \multicolumn{3}{|l|}{ WASTE DISPOSAL METHOD } \\
\hline SECTION & VIII SPECI & IL PROTECTION INFORMATION \\
\hline VENTILATION REQUIREMENTS LOCAL EXHAUST & & $\begin{array}{l}\text { PROTECTIVE EQUIPMENT (SPECIFY TYPES) EYE } \\
\text { Gogeles }\end{array}$ \\
\hline MECHANICAL IGENERAL) & & $\begin{array}{l}\text { GLOVES } \\
\text { Rubber }\end{array}$ \\
\hline SPECIAL & & $\begin{array}{l}\text { OESPIRATOA } \\
\text { With proper filter }\end{array}$ \\
\hline
\end{tabular}

OTHER PROTECTIVE EQUIPMENT

\section{SECTION IX SPECIAL PRECAUTIONS}

PRECAUTIONS TO BE TAKEN IN HANDLING AND STORAGE.

Separate from combustible, organic or other readily oxidizable materials. 


PRODUCT DESIGNATION
Beryllium Nitrate
$03-017-6661$

\section{MATERIAL SAFETY DATA SHEET}

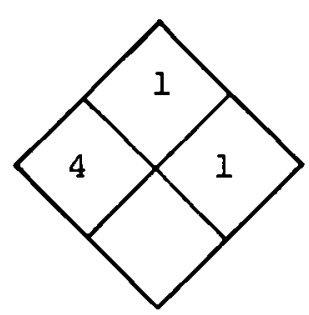

SECTION I -SOURCE AND NOMENCLATURE

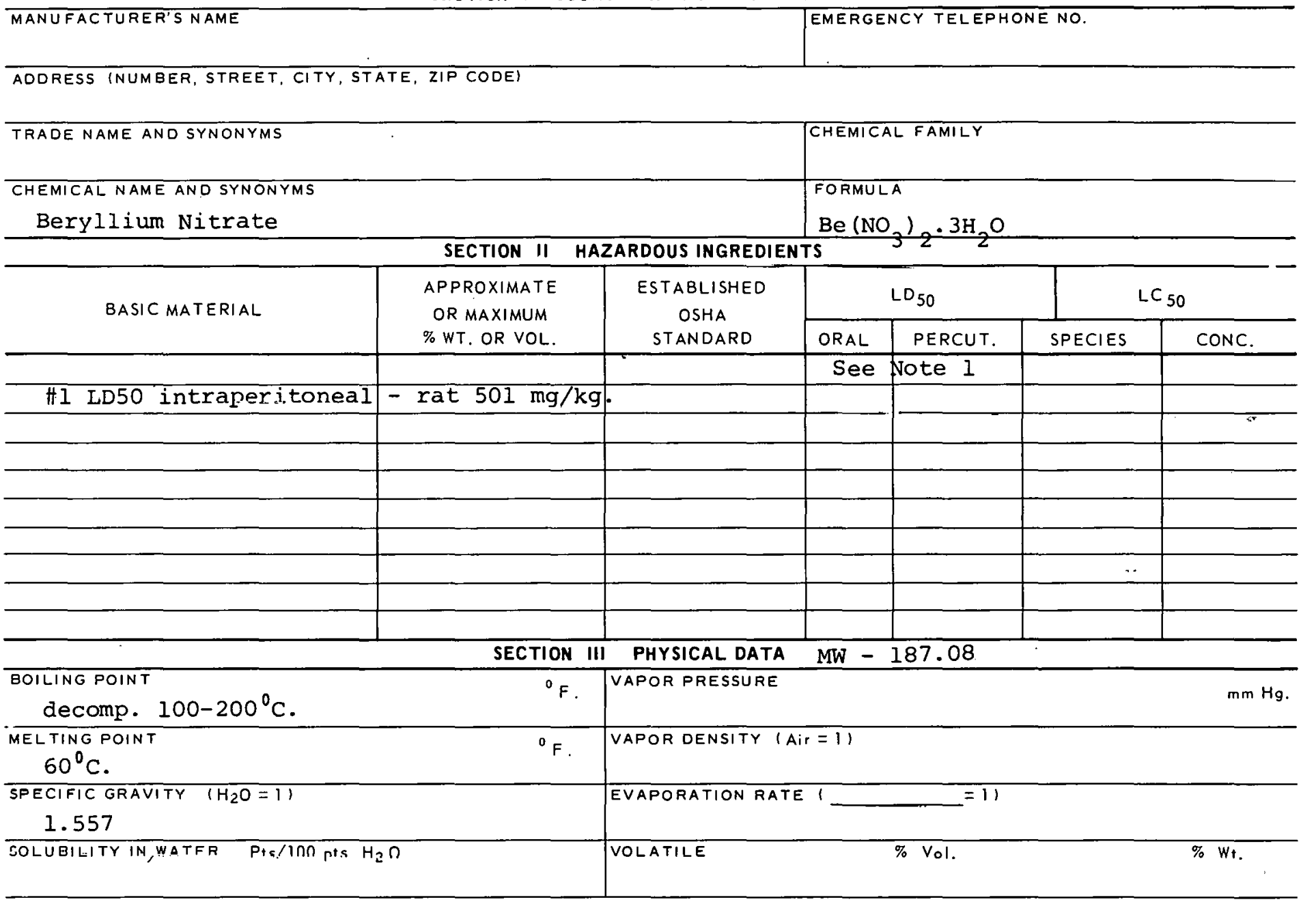

APPEARANCE AND ODOR

White, yellowish crystals.

\begin{tabular}{|c|c|c|c|c|}
\hline & SECTION IV & FIRE AND EXPLOSION HAZARD DATA & & \\
\hline $\begin{array}{c}\text { FLASH POINT } \\
\text { N/A }\end{array}$ & & & \multirow{2}{*}{$\begin{array}{c}\text { FLAMMABLE } \\
\text { (EXPLOSIVE) } \\
\text { LIMITS }\end{array}$} & UPPER \\
\hline METHOD USED & & & & LOWER \\
\hline
\end{tabular}

EXTINGUISHING MEDIA

$\mathrm{N} / \mathrm{A}$

SPECIAL, FIRE FIGHTING PROCEDURES

Wear protective clothing and self-contained respirator.

UNUSUAL FIRE AND EXPLOSION HAZARDS 


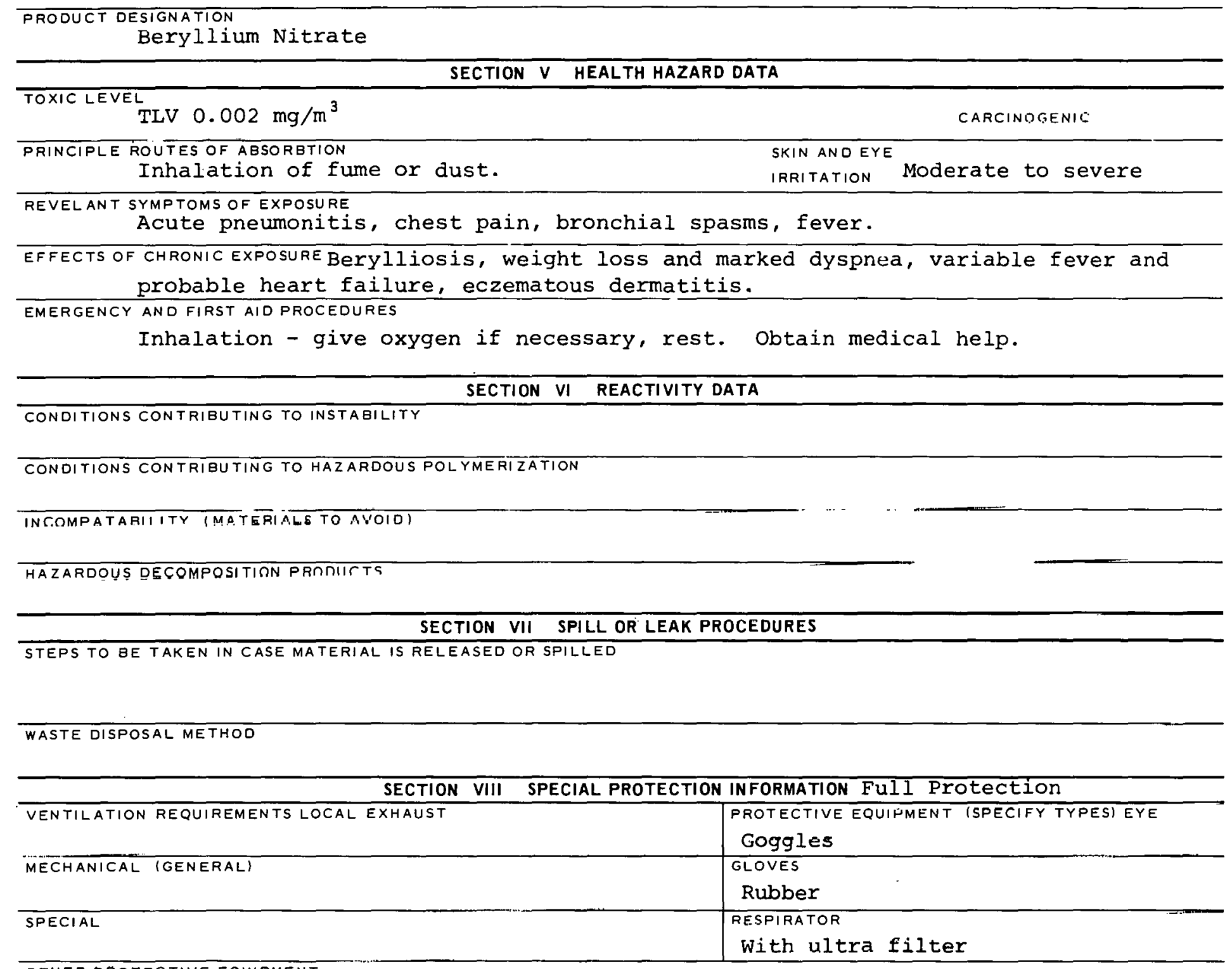

OTHER PROTECTIVE EQUIPMENT

SECTION IX SPECIAL PRECAUTIONS

PRECAUTIONS TO BE TAKEN IN HANDLING AND STORAGE

OTHER PRECAUTIONS

$\overline{\text { SIGNATURE }}$

DATE

17-6661B 
CAS : 007726956

PRODUCT DESIGNATION

03-024-5204

Bromine

\section{MATERIAL SAFETY DATA SHEET}

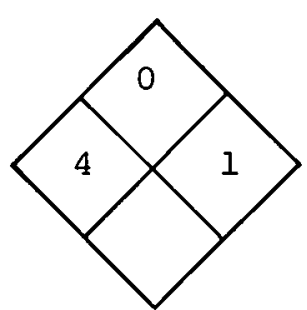

\section{SECTION I SOURCE AND NOMENCLATURE}

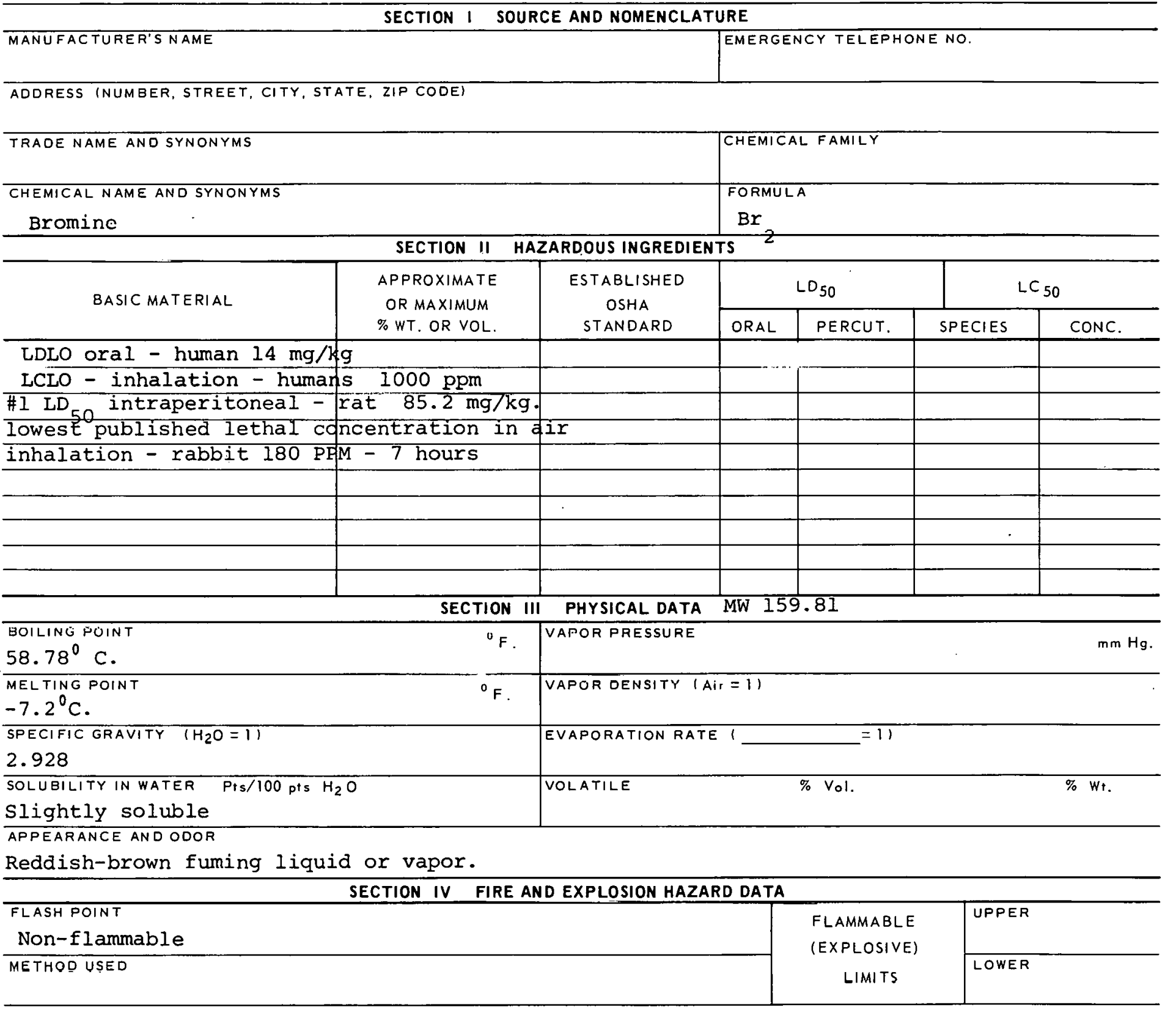

EXTINGUISHING MEDIA

Large amounts of water

SPECIAL FIRE FIGHTING PROCEDURES

Wear special protective clothing and gas mask.

UNUSUAL FIRE ANDEXPLOSION HAZAROS

Strong oxidizing materials.
(3) 10-74) 


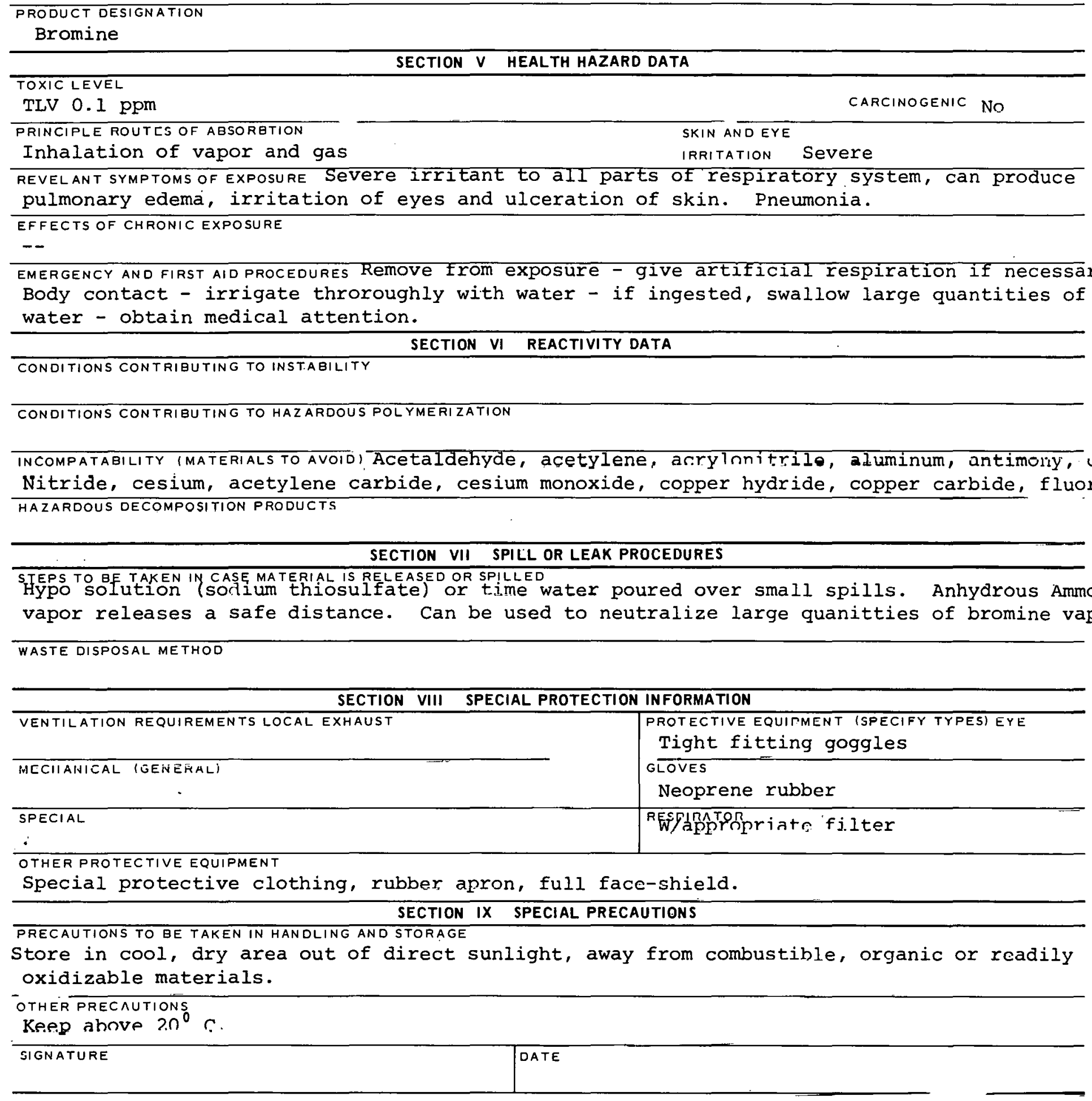




\begin{tabular}{|l|l|l|}
\hline \multicolumn{1}{|c}{ PROOUCT DESIGNATION } \\
03-026-2805 \\
Cadmium Chloride
\end{tabular}

SECTION I SOURCE AND NOMENCLATURE

\section{MANUFACTURER'S NAME \\ TRADE NAME AND SYNONYMS \\ CHEMICAL NAME AND SYNONYMS \\ Cadmium Chloride}

ADORESS (NUMBER, STREET, CITY, STATE, ZIP CODE)

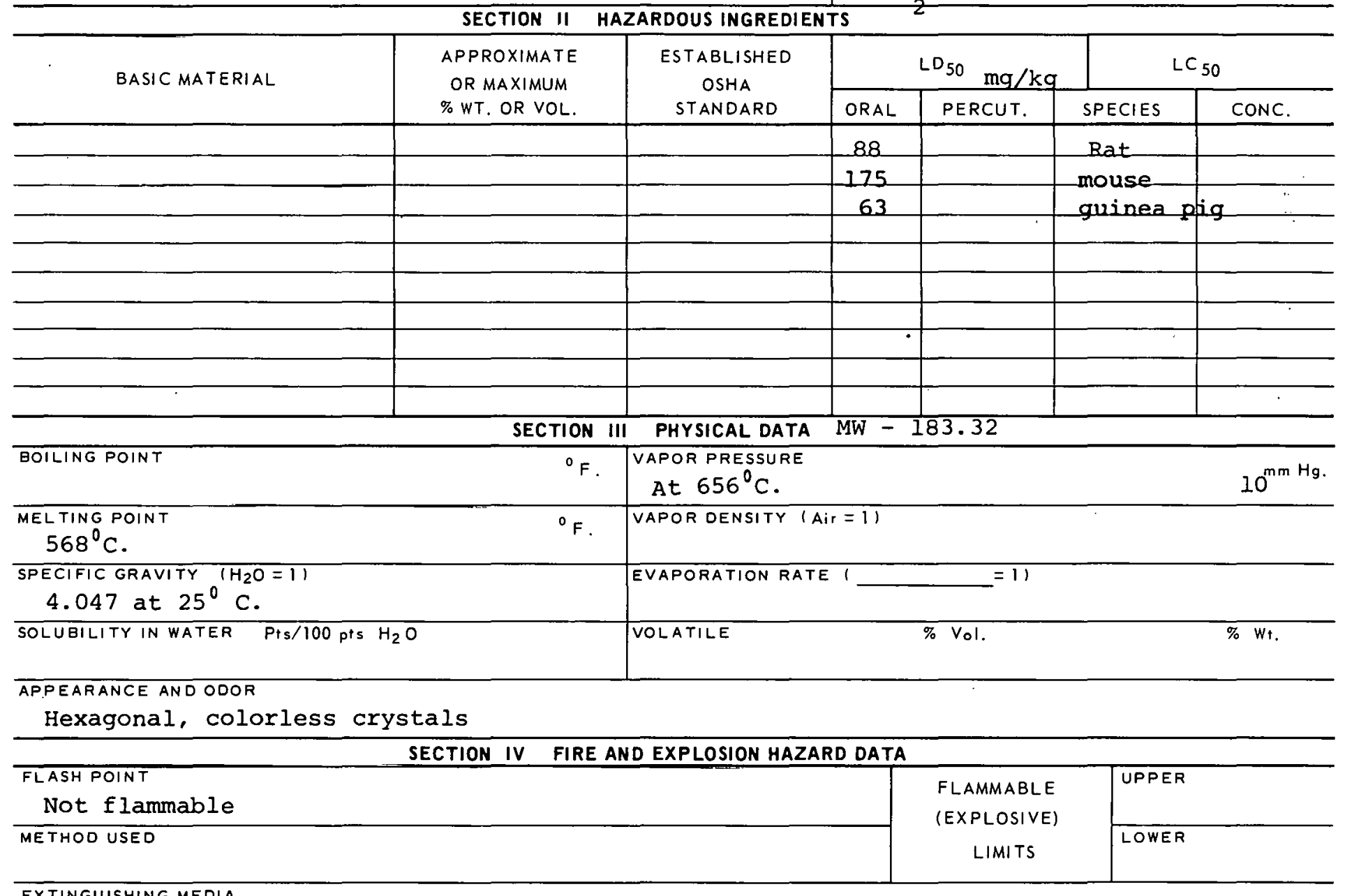

$$
\mathrm{N} / \mathrm{A}
$$

SPECIAL FIRE FIGHTING PROCEDURES

$\mathrm{N} / \mathrm{A}$

EMERGENCY TELEPHONE NO.

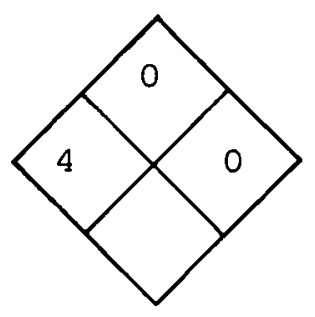




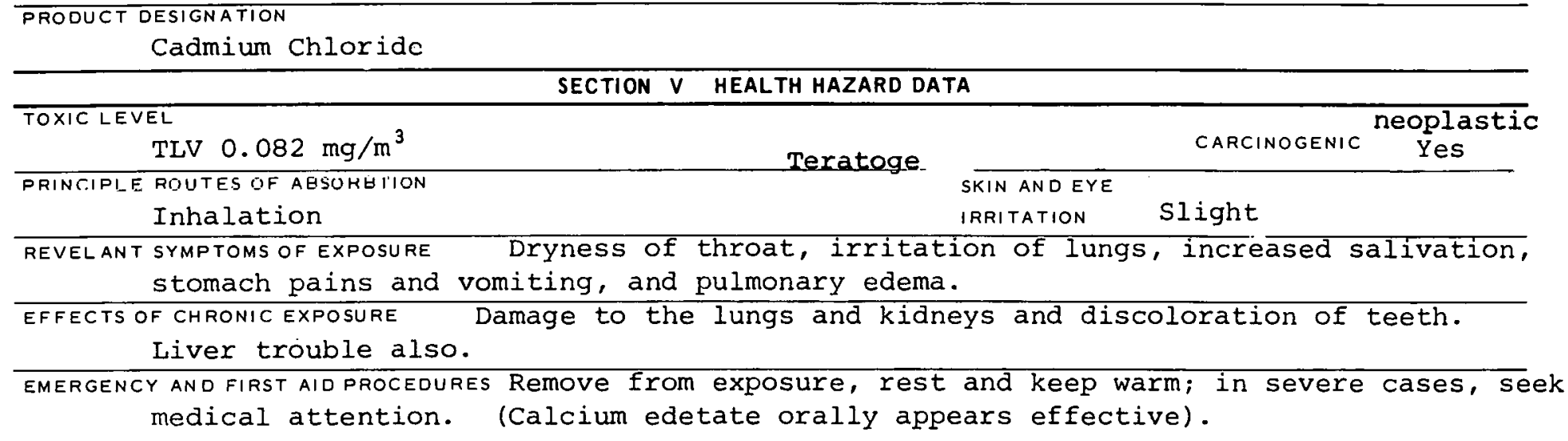

SECTION IX SHECIAL PRECAUTIONS

PRECAUTIONS TO BE TAKEN IN HANOLING AND STORAGE 


\begin{tabular}{|l|l|l|l|}
\hline \multicolumn{1}{|c|}{ PRODUCT DESIGNATION } \\
03-026-4805 Granular \\
03-026-4865 Sticks \\
Cadmium (Metal)
\end{tabular}

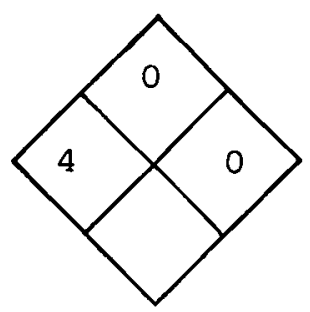

\section{SECTION I SOURCE AND NOMENCLATURE}

MANUFACTURER'S NAME

ADDRESS (NUMBER, STREET, CITY, STATE, ZIP CODE)

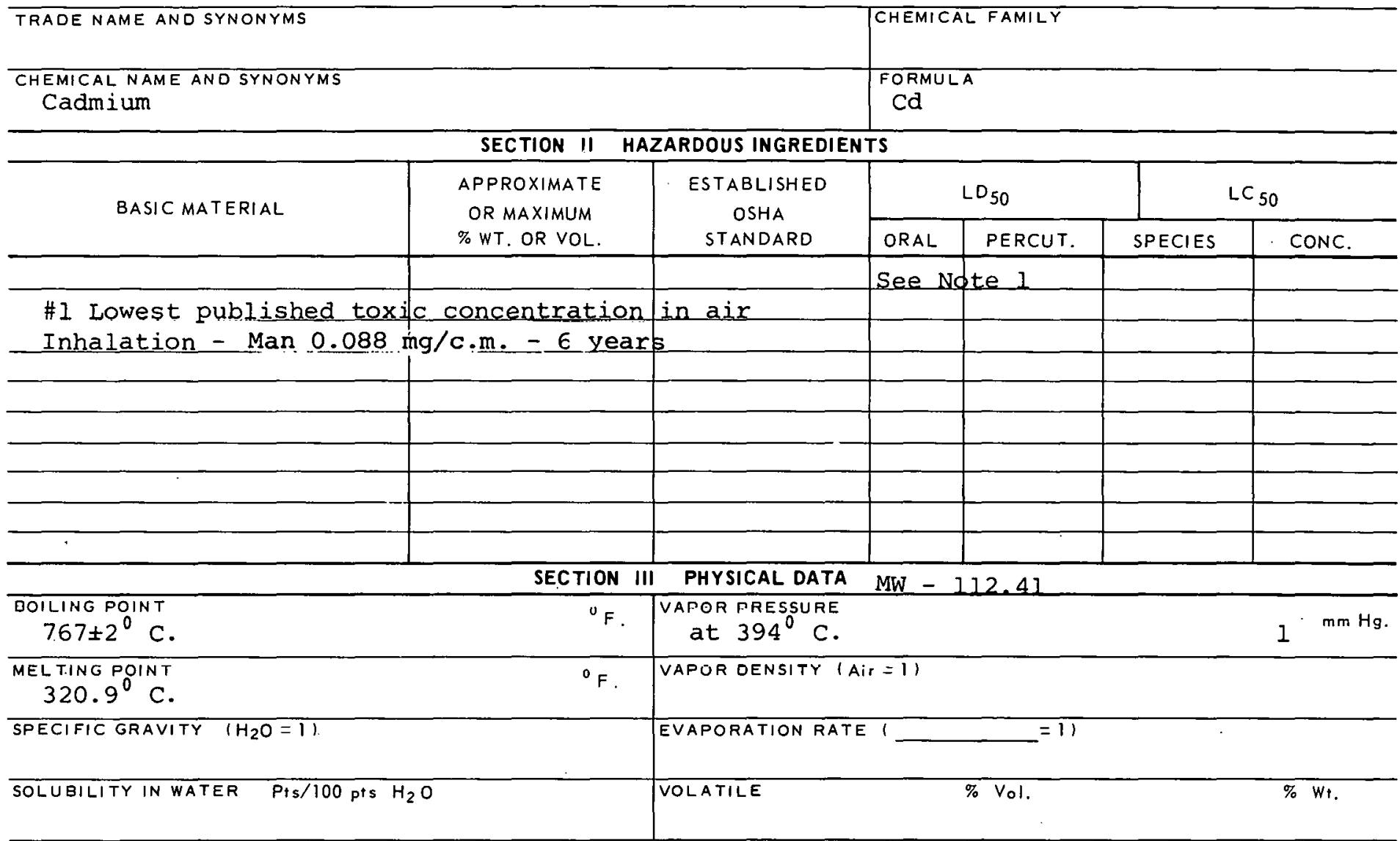

APPEARANCE AND ODOR

Hexagonal crystals or silver-white malleable metal.

\begin{tabular}{l|c|c}
\hline \multicolumn{2}{c}{ SECTION IV FIRE AND EXPLOSION HAZARD DATA } \\
\hline \begin{tabular}{l|l} 
FLASH POINT \\
NOne available
\end{tabular} & FLAMMABLE \\
\hline METHOD USED & (EXPLOSIVE) \\
& LIMITS & LOWER \\
\hline
\end{tabular}

EXTINGUISHING MEDIA

None available

SPECIAL FIRE FIGHTING PROCEDURES

Use self-contained breathing apparátus.

UNUSUAL FIRE AND EXPLOSION HAZARDS 


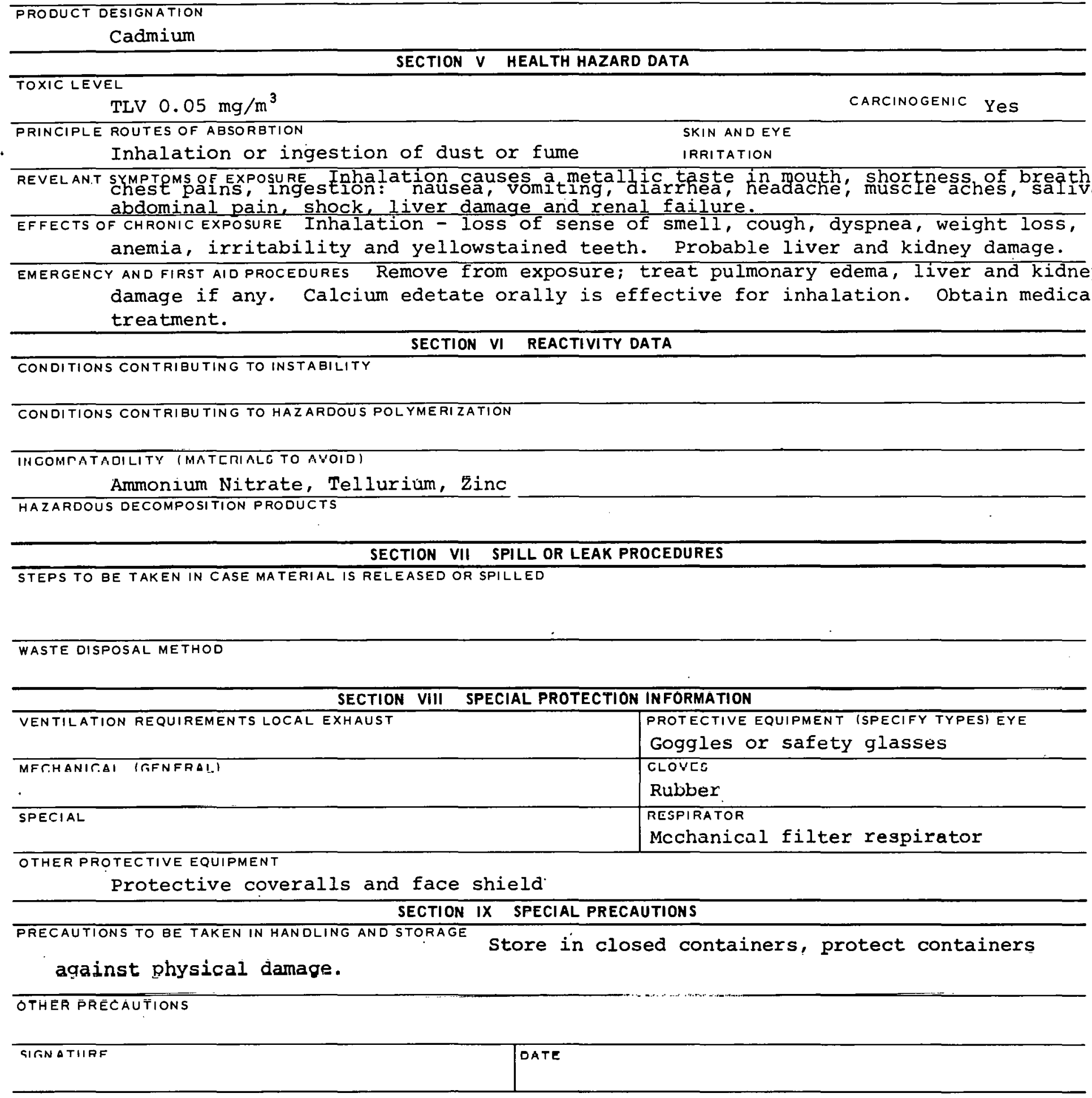




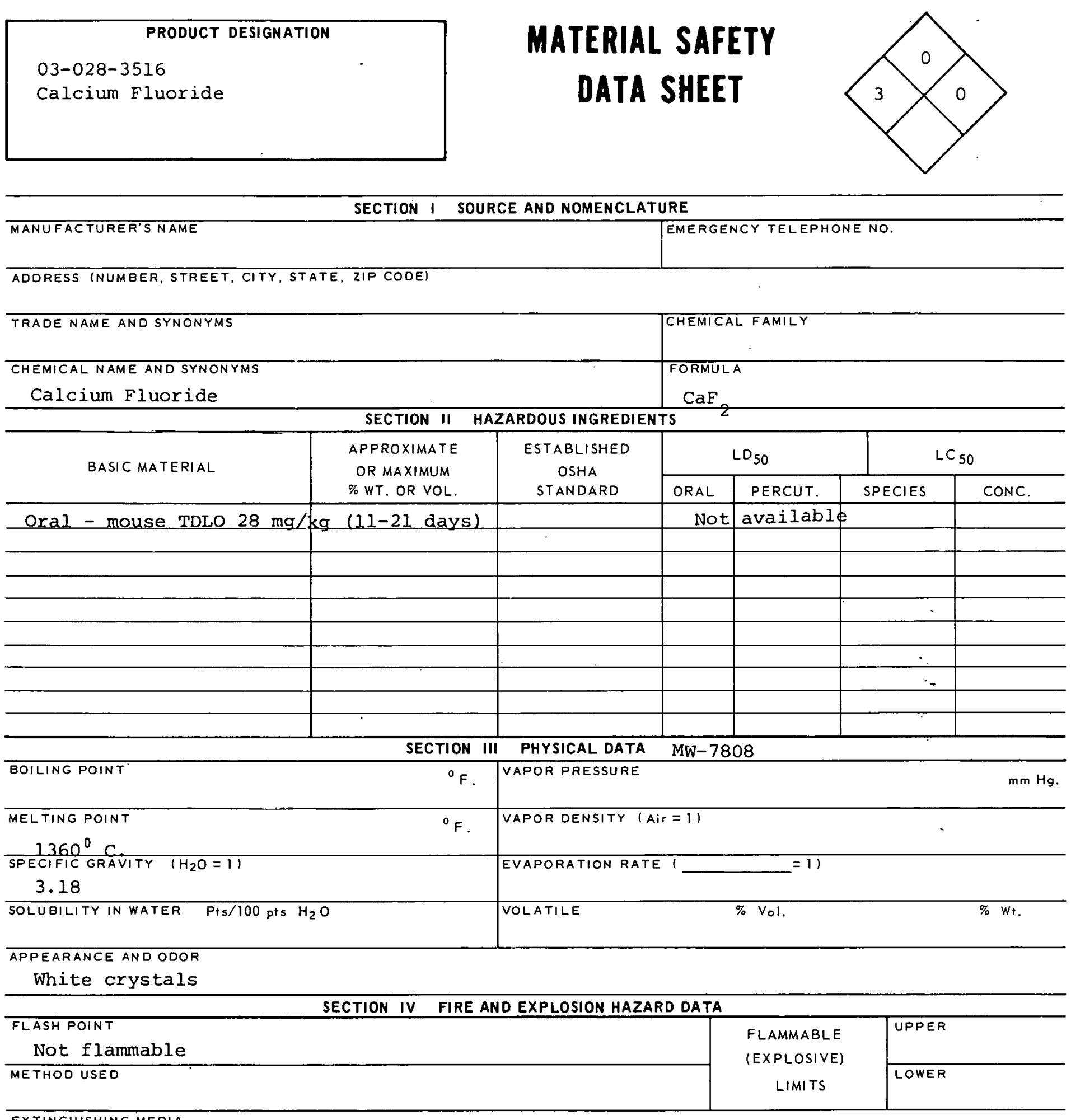

\section{EXTINGUISHING MEDIA}

$N / A$

SPECIAL FIRE FIGHTING PROCEDURES

$$
N / A
$$

UNUSUAL FIRE AND EXPLOSION HAZARDS

$\mathrm{N} / \mathrm{A}$ 


\section{TOXIC LEVEL}

$$
T L V \text { - TWA - } 2.5 \mathrm{mg} / \mathrm{M}^{3}
$$

FRINCIFLE ROUTLS OF ABSORBTION

Inhalation of dust, ingestion

Teratogen

CARCINOGENIC NO SKIN AND EYE

IRRITATION

Light

REVELANT SYMPTOMS OF EXPOSURE Irritation of nasal passages, nausea, vomiting, diarrhea, abdominal pains, irritation of eyes and skin.

EFFECTS OF CHRONIC EXPOSURE

Pulmonary fibrosis, increase radiographic density of bones.

EMERGENCY AND FIRST AID PROCEDURES Inhalation - rest, keep warm, get medical attention; if swallowed - wash mouth, give emetic, get medical attcntion; wash from skin ur eyes, get medical attention.

SECTION VI REACTIVITY DATA

CONDITIONS CONTRIBUTING TO INSTABILITY

CONDITIONS CONTRIBUTING TO HAZARDOUS POLYMERIZATION

INCOMPATABILITY (MATERIALS TO AVOIO)

HAZARDOUS DECOMPOSITION PRODUCTS

SECTION VII SPILL OR LEAK PROCEDURES

STEPS TO BE TAKEN IN CASE MATERIAL IS RELEASED OR SPILLED

WASTE DISPOSAL METHOD

SECTION VIII SPECIAL PROTECTION INFORMATION

\begin{tabular}{l|l}
\hline VENTILATION REQUIREMENTS LOCAL EXHAUST & $\begin{array}{l}\text { PROTECTIVE EQUIPMENT (SPECIFY TYPES) EYE } \\
\text { SafetY gláses }\end{array}$ \\
\hline MECHANICAL IGENERAL) & GLOVES \\
SPECIAL & $\begin{array}{l}\text { RESPIRATOR } \\
\text { With appropriate filter }\end{array}$ \\
\hline
\end{tabular}

OTHER PROTECTIVE EQUIPMENT

SECTION IX SPECIAL PRECAUTIONS

PRECAUTIONS TO BE TAKEN IN HANDLING AND STORAGE

OTHER PRECAUTIONS

SIGNATURE

DATE

$28-3516 B$ 


\section{PRODUCT DESIGNATION}

03-028-5926

Calcium Oxide
MATERIAL SAFETY DATA SHEET

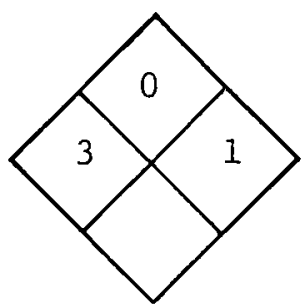

SECTION I SOURCE AND NOMENCLATURE

\begin{tabular}{l} 
MANUFACTURER'S NAME \\
ADORESS INUMBER, STREET, CITY, STATE \\
TRADE NAME AND SYNONYMS \\
Uns laked 1ime, quick lime \\
CHEMICAL NAME AND SYNONYMS \\
CalCium OXide \\
\hline
\end{tabular}

SECTION 1 SOURCE AND NOMENCLATURE

EMERGENCY TELEPHONE NO.

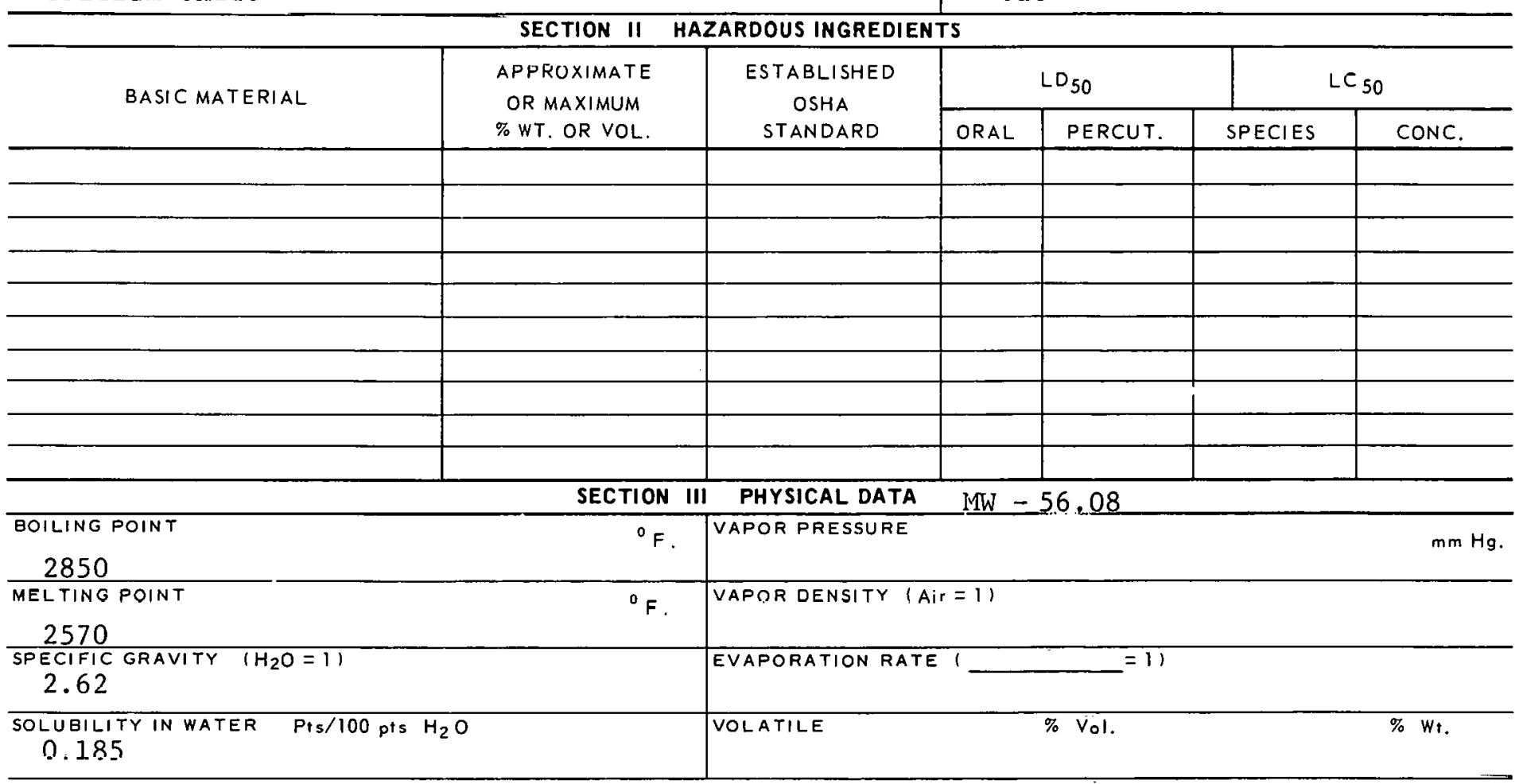

APPEARANCE AND ODOR

White powder

\begin{tabular}{l|c|c}
\hline \multicolumn{2}{c}{ SECTION IV FIRE AND EXPLOSION HAZARD DATA } \\
\hline FLASH POINT & FLAMMABLE & UPPER \\
Wi11 not burn & (EXPLOSIVE) & LIMITS \\
\hline METHOD USED & LOWER & \\
\hline
\end{tabular}

\section{EXTINGUISHING MEDIA}

$$
--
$$

SPECIAL FIRE FIGHTING PROCEDURES Avoid water unless necessary to use on other burning materials, in that case flood with water.

UNUSUAL FIRE AND EXPLOSION HAZARDS 


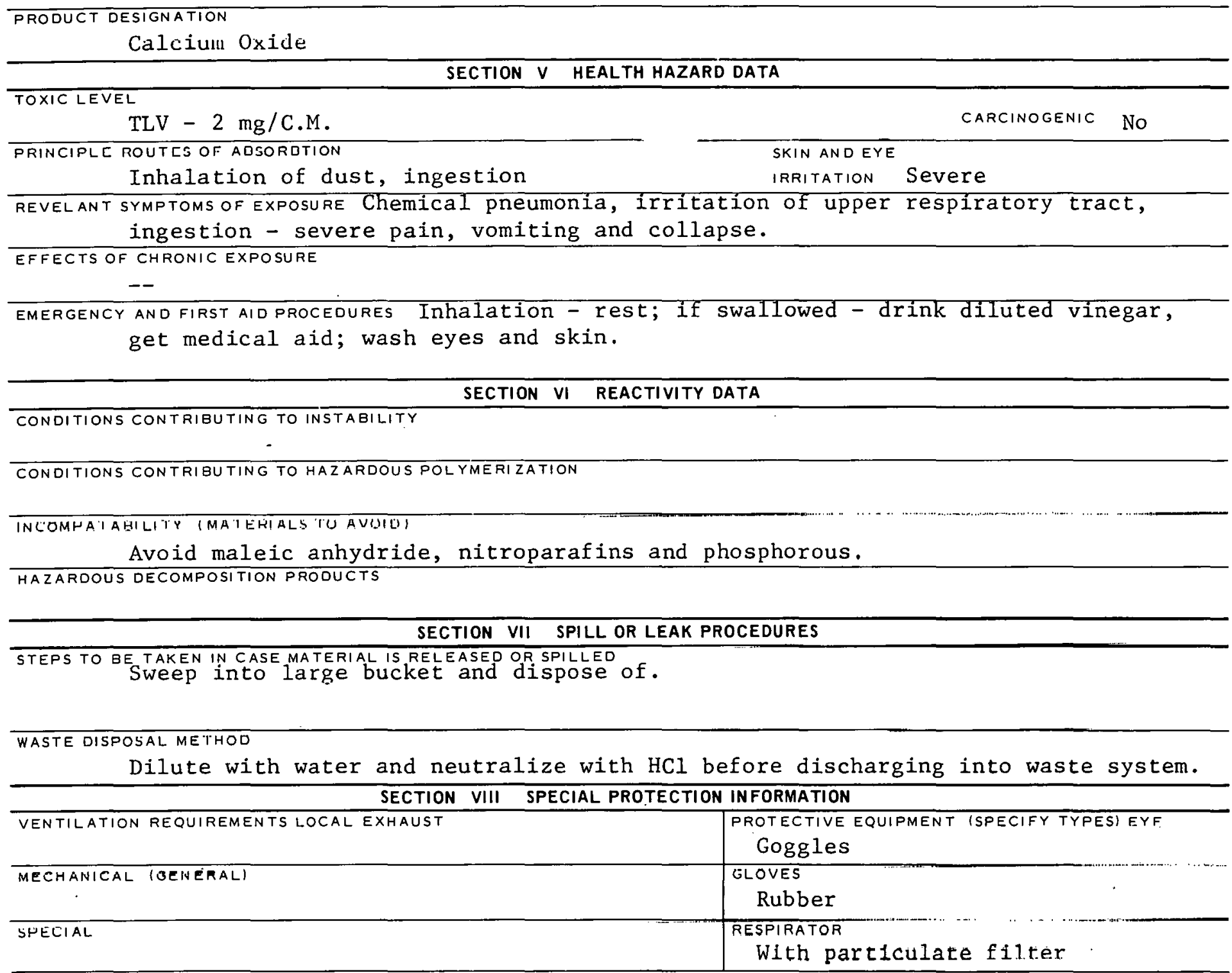

OTHER PROTECTIVE EQUIPMENT

\section{SECTION IX SPECIAL PRECAUTIONS}

PRECAUTIONS TO BE TAKEN IN HANDLING AND STORAGE

Store in cool, dry place away from acids and oxidizing materials. 


\begin{tabular}{c} 
PRODUCT DESIGNATION \\
03-040-3400 \\
Chromium Metal \\
\hline
\end{tabular}

\section{MATERIAL SAFETY DATA SHEET}

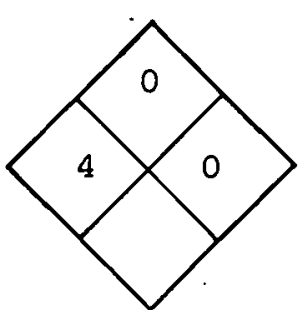

SECTION I SOURCE AND NOMENCLATURE

\section{MANUFACTURER'S NAME \\ Chromium}

ADDRESS (NUMBER, STREET, CITY, STATE, ZIP CODE)

\begin{tabular}{|l|l|} 
& CHEMICAL FAMILY \\
\hline & FORMULA \\
Cr
\end{tabular}

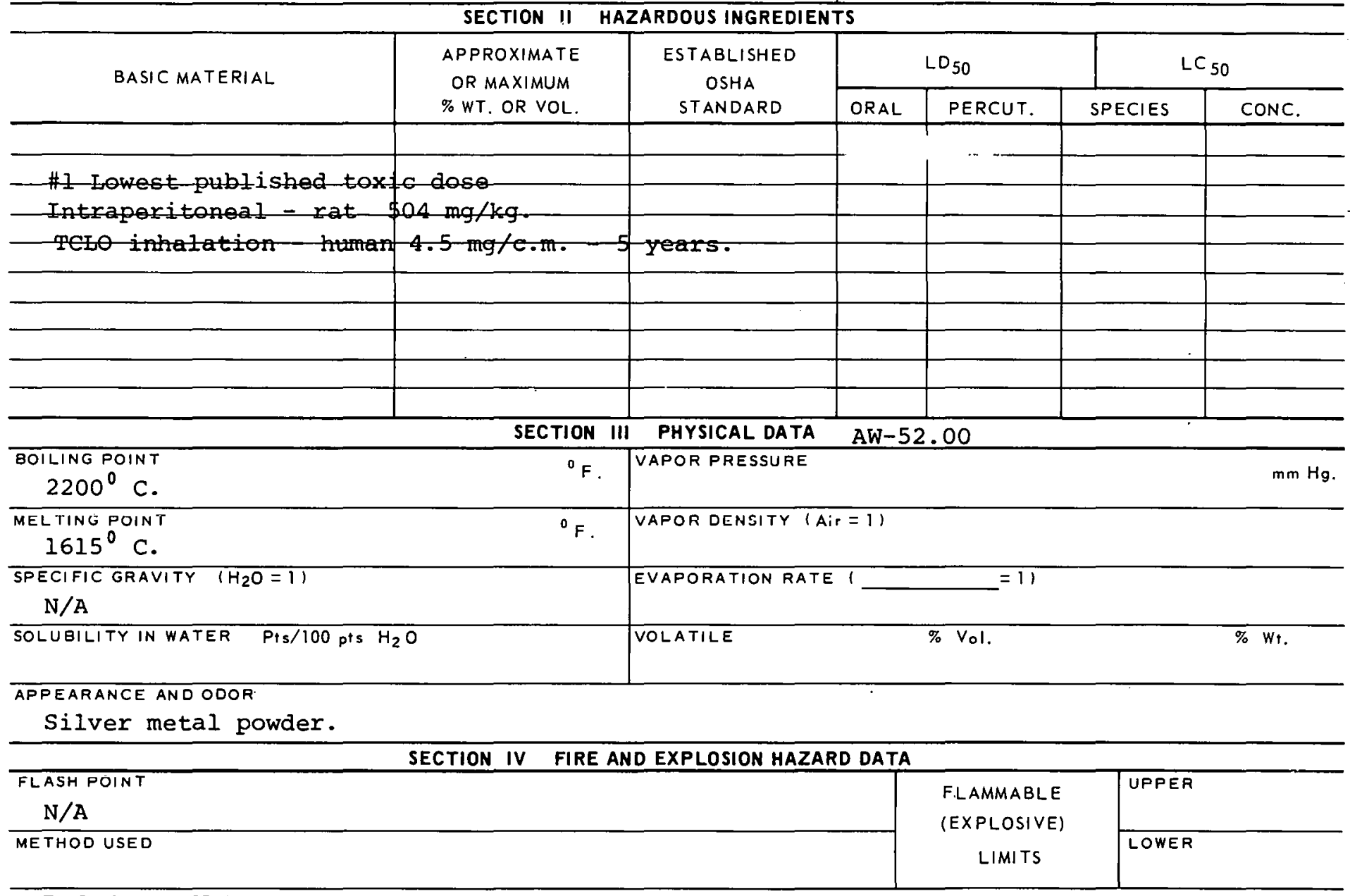

$\mathrm{N} / \mathrm{A}$

SPECIAL FIRE FIGHTING PROCEDURES

$\mathrm{N} / \mathrm{A}$

UNUSUAL FIRE AND EXPLOSION HAZARDS

$\mathrm{N} / \mathrm{A}$ 


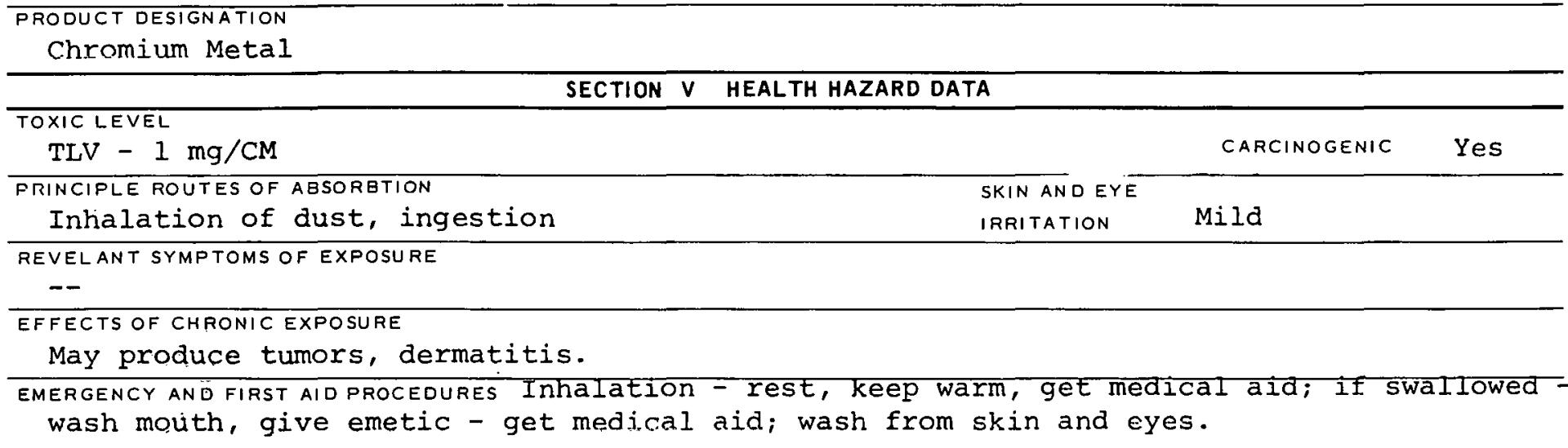

\begin{tabular}{l}
\hline SECTION VI REACTIVITY DATA \\
\hline CONDITIONS CONTRIBUTING TO INSTABILITY \\
\hline CONDITIONS CONTRIBUTING TO HAZARDOUS POLYMERIZATION \\
HACOMPATARILITY IMATERIALS TO AVOIOI \\
HAZARDOUS DECOMPOSITION PRODUCTS
\end{tabular}

SECTION VII SPILL OR LEAK PROCEDURES

STEPS TO BE TAKEN IN CASE MATERIAL IS RELEASED OR SPILLED

WASTE DISPOSAL METHOO

SECTION VIII SPECIAL PROTECTION INFORMATION

\begin{tabular}{|c|c|}
\hline VENTILATION REQUIREMENTS LQCAL EXHAUST & $\begin{array}{l}\text { PROTECTIVE EQUIPMENT (SPECIFY TYPES) EYE } \\
\text { Safety glasses }\end{array}$ \\
\hline MECHANICAL (GFNERAI) & ऊLUVẼS \\
\hline SPECIAL & $\begin{array}{l}\text { RESPIRATOR } \\
\text { With proper filter }\end{array}$ \\
\hline
\end{tabular}

SECTION IX SPECIAL PRECAUTIONS

PRECAUTIONS TO BE TAKEN IN HANDLING AND STORAGE

\begin{tabular}{l|l|l|l|}
\hline OTHER PRECAUTIONS & DATE \\
\hline SIGNATURE & \\
\hline
\end{tabular}


CAS : 007789028

\begin{tabular}{|c|}
\hline PRODUCT DESIGNATION \\
03-040-4550 \\
Chromic Nitrate \\
\hline
\end{tabular}

\section{GB-63000 \\ MATERIAL SAFETY \\ DATA SHEET}

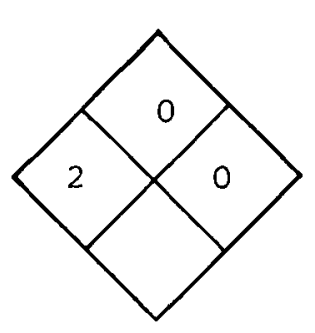

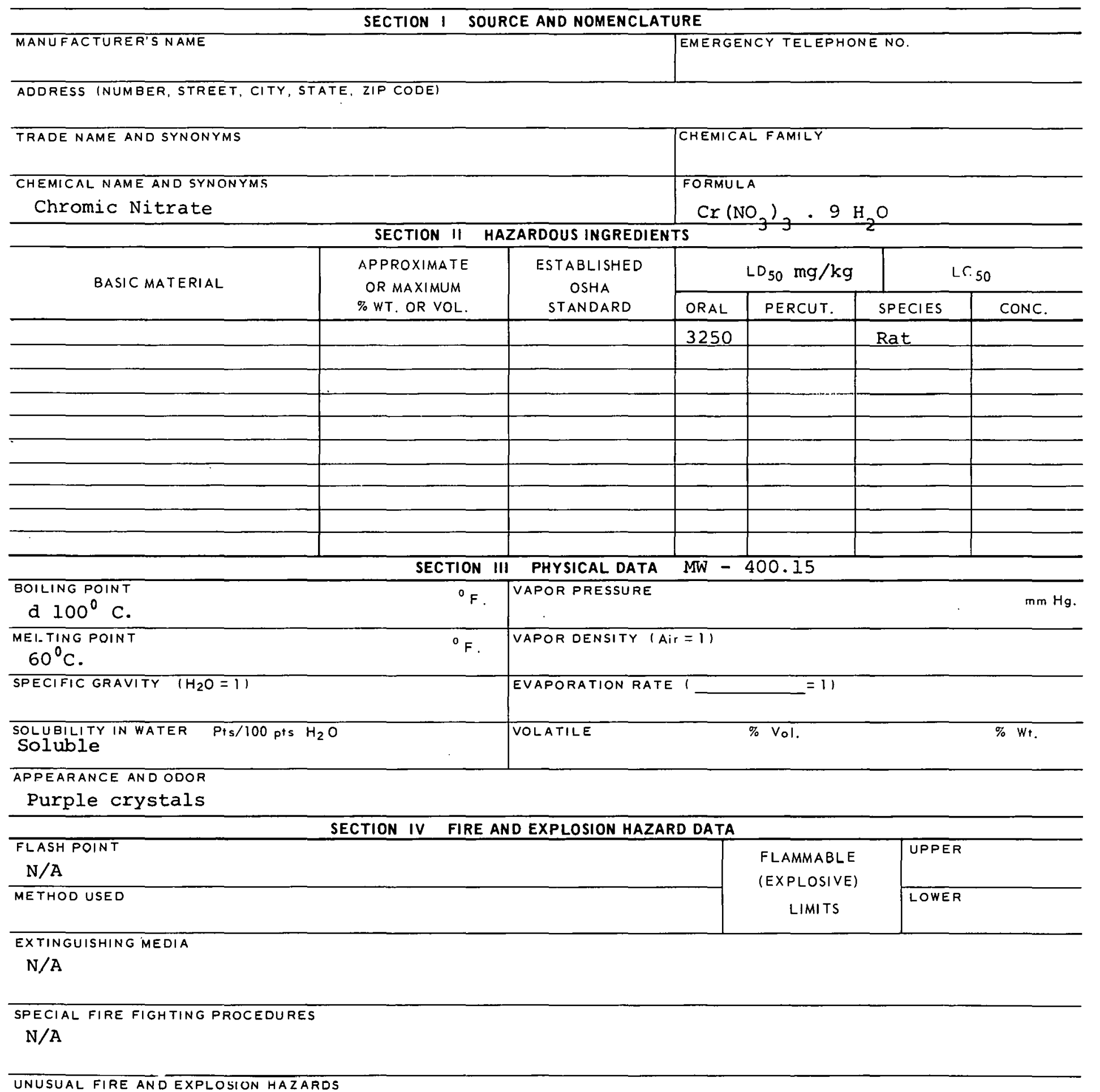


TOXICLEVEL

$T L V-3.84 \mathrm{mg} / \mathrm{m}^{3}$

CARCINOGENIC NO

PRINCIPLE ROUTES OF ABSORBTION

Inhalation, ingestion

REVELANT SYMPTOMS OF EXPOSURE

Lesions of the skin and mucous membrane.

EFFECTS OF CHRONIC EXPOSURE

Possible hepatitis, jaundice and kidney lesions.

EMERGENCY AND FIRST AID PROCEDURES After ingestion; induce vomiting or give gastric lavage;

if inhaled - rest, keep warm, get medical.attention.

\section{SECTION VI REACTIVITY DATA}

CONDITIONS CONTRIBUTING TO INSTABILITY

CONDITIONS CONTRIBUTING TO HAZARDOUS POLYMERIZATION

- -

INCOMPATABILITY (MATERIALS TO AVOIO)

Avoid contact with strong reducing agents.

HAZARDOUS DECOMPOSITION PRODUCTS

lixides nt nitrogen.

SECTION VII SPILL OR LEAK PROCEDURES

STEPS TO BE TAKEN IN CASE MATERIAL IS RELEASED OR SPILLED

WASTE DISPOSAL METHOD

SECTION VIII SPECIAL PROTECTION INFORMATION

\begin{tabular}{l|l}
\hline VENTILATION REQUIREMENTS LOCAL EXHAUST & $\begin{array}{l}\text { PROTECTIVE EQUIPMENT (SPECIFY TYPESI EYE } \\
\text { Safety glaSSES }\end{array}$ \\
\hline MECHANICAL IGENERAL) & SLRVES \\
\hline SPECIAL & $\begin{array}{c}\text { RESPIRATOR } \\
\text { With appropriate filter }\end{array}$ \\
\hline
\end{tabular}

OTHER PROTECTIVE EQUIFMENT

SECTION IX SPECIAL PRECAUTIONS

PRECAUTIONS TO BE TAKEN IN HANDLING AND STORAGE

OTHER PRECAUTIONS

SIGNATURE

DATE

$40-4550 B$ 


\section{arascaso \\ MATERIAL SAFETY DATA SHEET}

03-040-5600

PRODUCT DESIGNATION

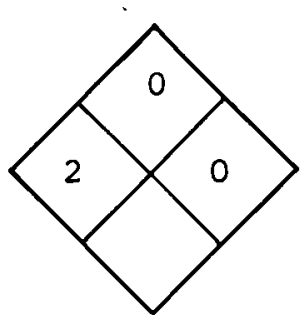

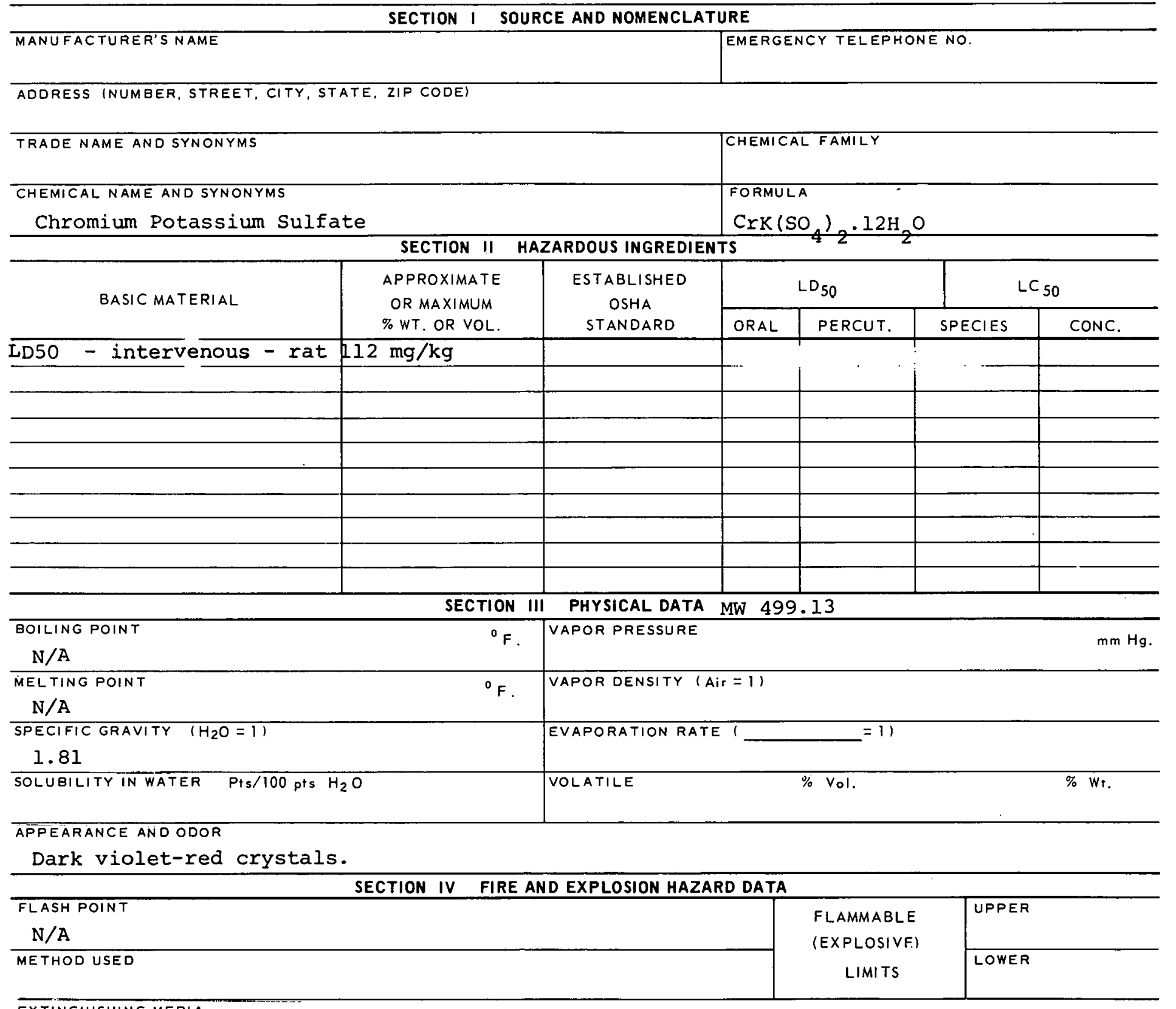

EXTINGUISHING MEDIA

$\mathrm{N} / \mathrm{A}$

SPECIAL FIRE FIGHTING PROCEDURES

$N / A$

UNUSUAL FIRE AND EXPLOSION HAZAROS

$\mathrm{N} / \mathrm{A}$ 


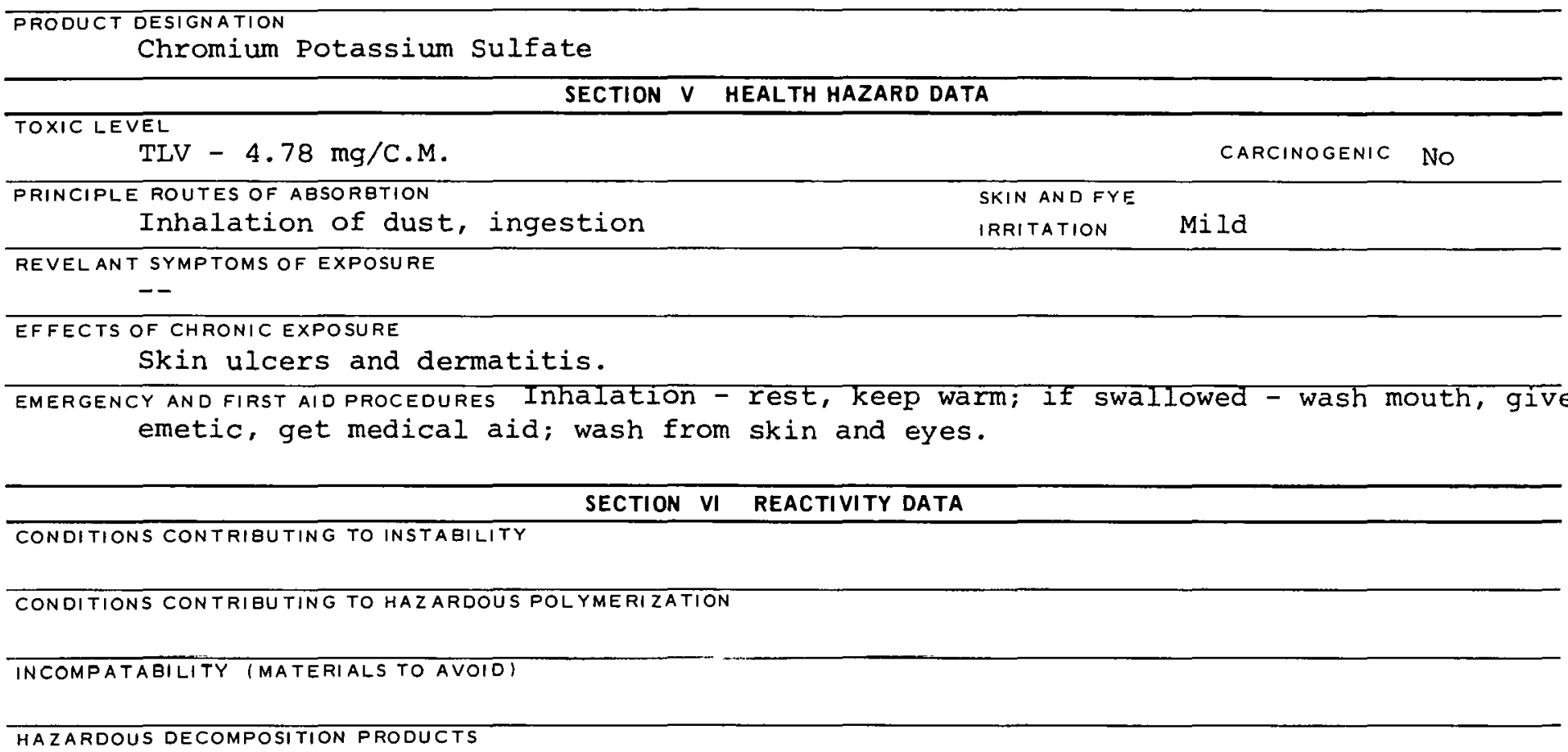

SECTION VII SPILL OR LEAK PROCEDURES

STEPS TO BE TAKEN IN CASE MATERIAL IS RELEASED OR SPILLED

\begin{tabular}{l|l}
\hline \multicolumn{1}{|c|}{ SASTE DISPOSAL METHOD } \\
\hline VENTILATION REQUIREMENTS LOCAL EXHAUST & SPECIAL PROTECTION INFORMATION \\
\hline MECHANICAL IGENERAL) & $\begin{array}{c}\text { PROTECTIVE EQUIPMENT (SPECIFY TYPES) EYE } \\
\text { SafETY' glaSSES }\end{array}$ \\
\hline SPECIAL & GLOVES \\
\hline
\end{tabular}

OTIIRRROTECTIVE EQUIPMENT

SECTION IX SPECIAL PRECAUTIONS

PRECAUTIONS TO BE TAKEN IN HANDLING AND STORAGE

DTHER, PREEAUTIONE 


\begin{tabular}{c} 
PRODUCT DESIGNATION \\
03-042-4001 \\
Cobalt Metal \\
\hline
\end{tabular}

\section{MATERIAL SAFETY DATA SHEET}

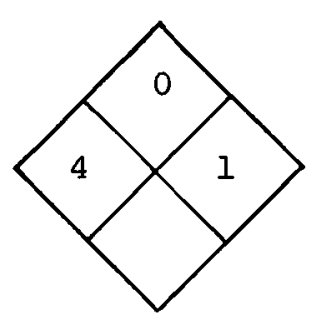

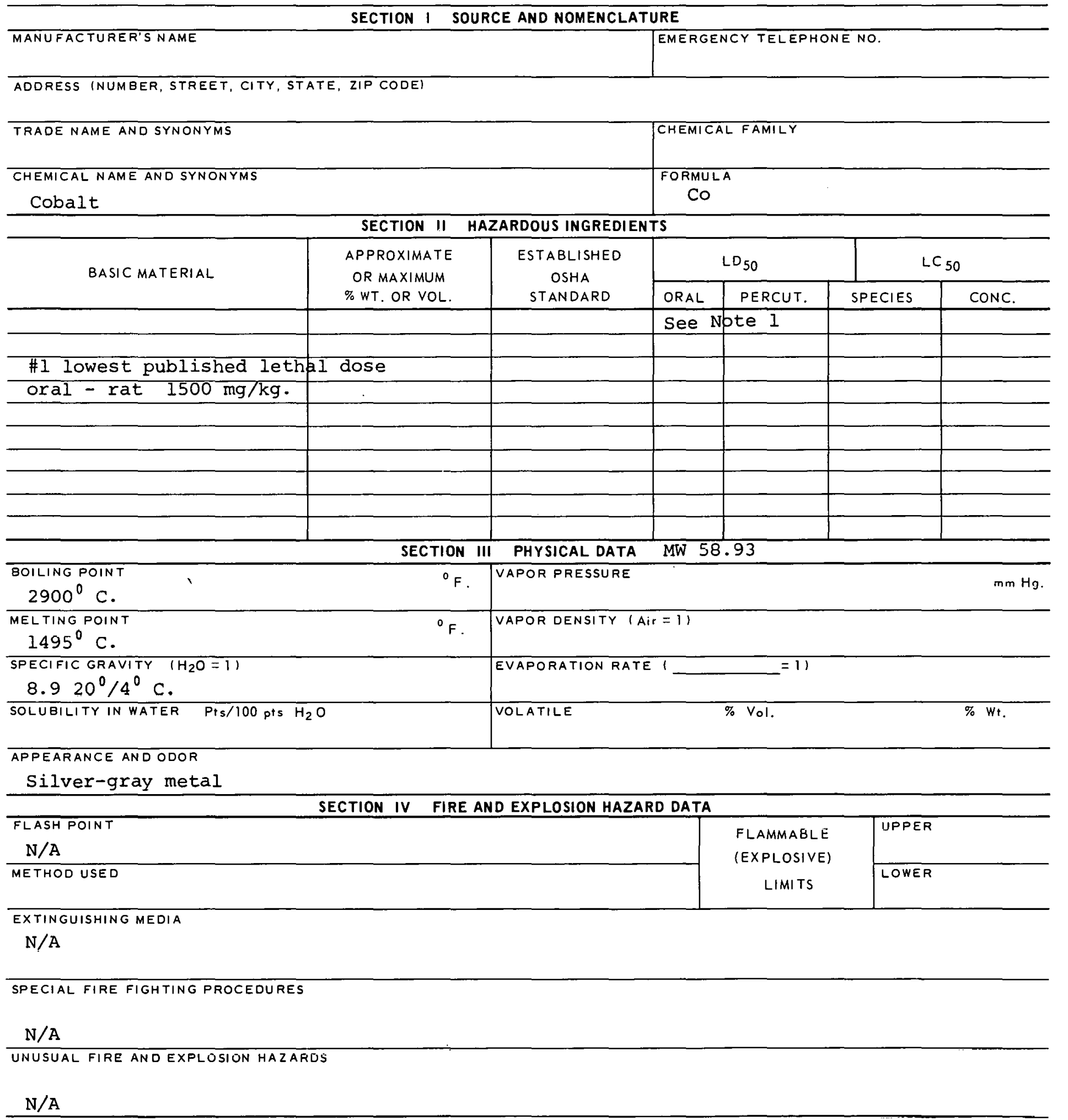




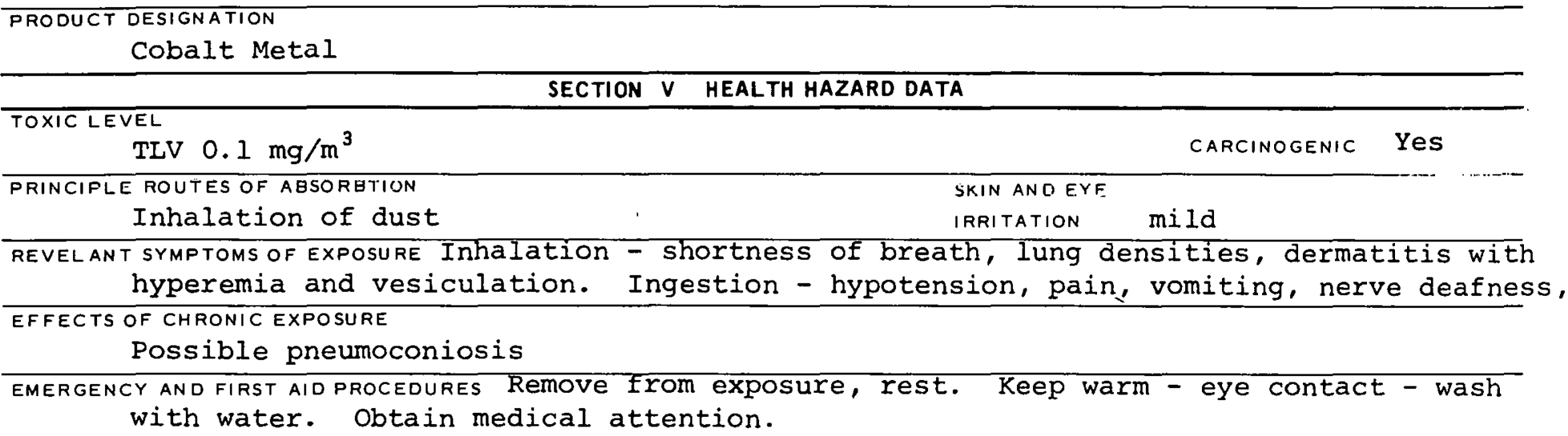

SECTION VII SPILL OR LEAK PROCEDURES

STEPS. TO BE TAKEN IN CASE MATERIAL IS RELEASED OR SPILLED

WASTE DISPOSAL METHOD

\section{SECTION VIII. SPECIAL PROTECTION INFORMATION}

\begin{tabular}{l|l}
\hline VENTILATION REQUIREMENTS LOCAL EXHAUST & $\begin{array}{l}\text { PROTECTIVE EQUIPMENT (SPECIFY TYPES) EYE } \\
\text { SafetY glaSSES }\end{array}$ \\
\hline MECIIANIEAL IOEINERALI & $\begin{array}{l}\text { ri OVFG } \\
\text { Rubber }\end{array}$ \\
\hline SPECIAL & $\begin{array}{l}\text { RESPIRATOR } \\
\text { W/appropriate filter }\end{array}$ \\
\hline
\end{tabular}

OTHER PROTECTIVE EQUIPMENT

SECTION IX SPECIAL PRECAUTIONS

PRECAUTIONS TO BE TAKEN IN HANDLING AND STORAGE 
CAS : 012069691

\section{PRODUCT DESIGNATION}

03-046-2903

Cupric Carbonate
GL- -69100

MATERIAL SAFETY

DATA SHEET

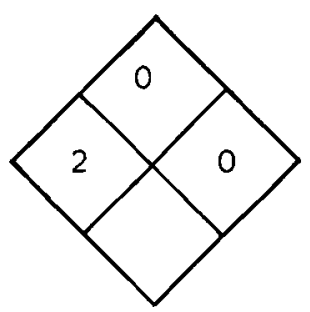

\section{SECTION I SOURCE AND NOMENCLATURE}

\section{MANUFACTURER'S NAME

(1) \\ TRADE NAME AND SYNONYMS \\ CHEMICAL NAME AND SYNONYMS \\ Cupric Carbonate}

SECTION I SOURCE AND NOMENCLATU

ADDRESS (NUMBER, STREET, CITY, STATE, ZIP CODE)

EMERGENCY TELEPHONE NO.

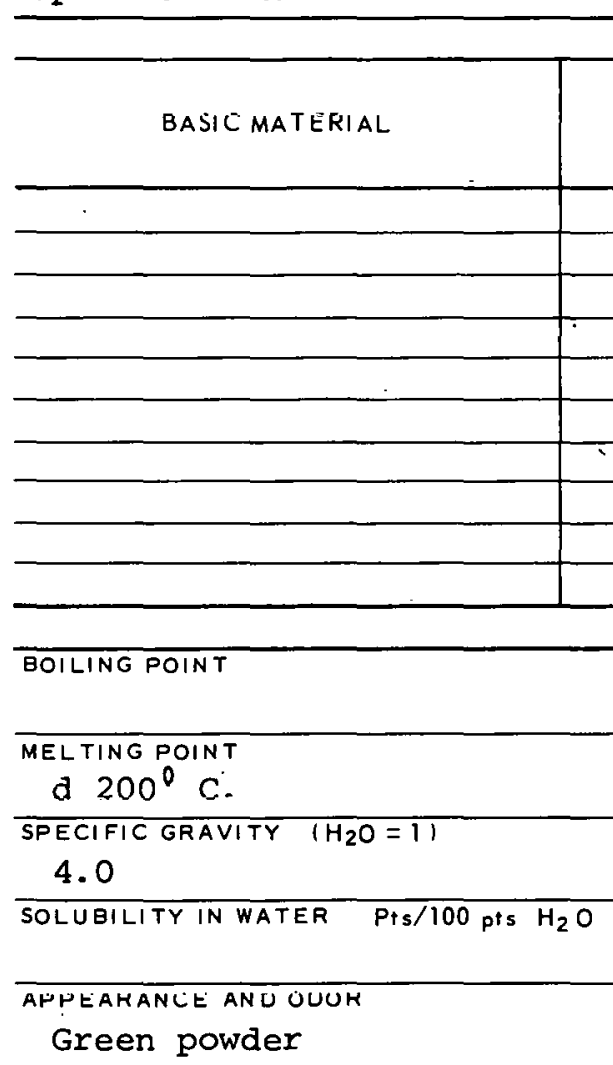

\begin{tabular}{l|l} 
& CHEMICAL FAMILY \\
& $\begin{array}{l}\text { FORMULA } \\
\text { CUCO }, \mathrm{Cu}(\mathrm{OH})_{2}\end{array}$ \\
\hline
\end{tabular}
APPROXIMATE ESTABLISHED

\begin{tabular}{|l|l|l|l|}
\hline \multicolumn{2}{|c|}{ LD $_{50} \mathrm{mg} / \mathrm{kg}$} & \multicolumn{2}{c|}{$\mathrm{LC}_{50}$} \\
\hline ORAL & PERCUT. & SPECIES & CONC. \\
\hline 159 & & Rat & \\
\hline
\end{tabular}

SECTION IV FIRE AND EXPLOSION HAZARD DATA

\begin{tabular}{l}
\hline FLASH POINT \\
N/A \\
\hline METHOD USED \\
\hline
\end{tabular}

MW -221.17

SECTION III

${ }^{\circ} \mathrm{F}$. VAPOR DENSITY (Air $=1$ ) EVAPORATION RATE VOLATILE

$\%$ Vol. $\% w_{+}$.

\section{FLAMMABLE}

(EXPLOSIVE)

LIMITS
UPPER

LOWER

EXTINGUISHING MEDIA

$N / A$

SPECIAL FIRE FIGHTING PROCEDURES

$\mathrm{N} / \mathrm{A}$

UNUSUAL FIRE AND EXPLOSION HAZARDS

$\mathrm{N} / \mathrm{A}$ 
TOXIC LEVEL

TLV $3.35 \mathrm{mg} / \mathrm{C} . \mathrm{M}$.

CARCINOGENIC

No

PRINCIPLE ROUTES OF A ASORBTION

Inhalation of dust (mainly), ingestion

SKIN AND EYE

IRRITATION Moderate

REVELANT SYMPTOMS OF EXPOSURE Irritation of eyes and mucous membranes, vomiting, diarrhea, abdominal pain, Collapse.

EFFECTS OF CHRONIC EXPOSURE

Livex and kidney damage.

EMERGENCY AND FIRST AID PROCEDURES Inhalation - rest, keep warm; if swallowed - wash mouth, give emetic, get medical aid; wash from skin and eyes.

\section{SECTION VI REACTIVITY DATA}

CONDITIONS CONTRIBUTING TO INSTABILITY

CONDITIONS CONTRIBUTING TO HAZARDOUS POLYMERIZATION

INCOMHAIABILITY (MAIERIMLS TU A'VOID)

With acids

HAZARDOUS DECOMPOSITION PRODUCTS

SECTION VII SPILL OR LEAK PROCEDURES

STEPS TO BE TAKEN IN CASE MATERIAL IS RELEASED OR SPILLED

WASTE DISPOSAL METHOD

\begin{tabular}{l|l}
\hline \multicolumn{2}{c}{ SECTION VIII SPECIAL PROTECTION INFORMATION } \\
\hline VENTILATION REQUIREMENTS LOCAL EXHAUST & $\begin{array}{l}\text { PROTECTIVE EQUIPMENT (SPECIFY TYPES) EYE } \\
\text { SafEtY glaSSES }\end{array}$ \\
\hline MECHANICAL IGENERALI & GLOVES \\
\hline SPECIAL & RESFIRATOR \\
\hline
\end{tabular}

OTHER PROTECTIVE EQUIPMENT

SECTION IX SPECIAL PRECAUTIONS

PRECAUTIONS TO BE TAKEN IN HANDLING AND STORAGE

OTMER PRECAUTIONS

SIGNATURE

DATE

$46-2903 B$ 
CAS : 001344678

GL70000

\section{PRODUCT DESIGNATION}

03-046-3433

Cupric Chloride

MATERIAL SAFETY

DATA SHEET

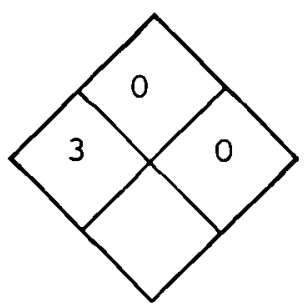

SECTION I SOURCE AND NOMENCLATURE

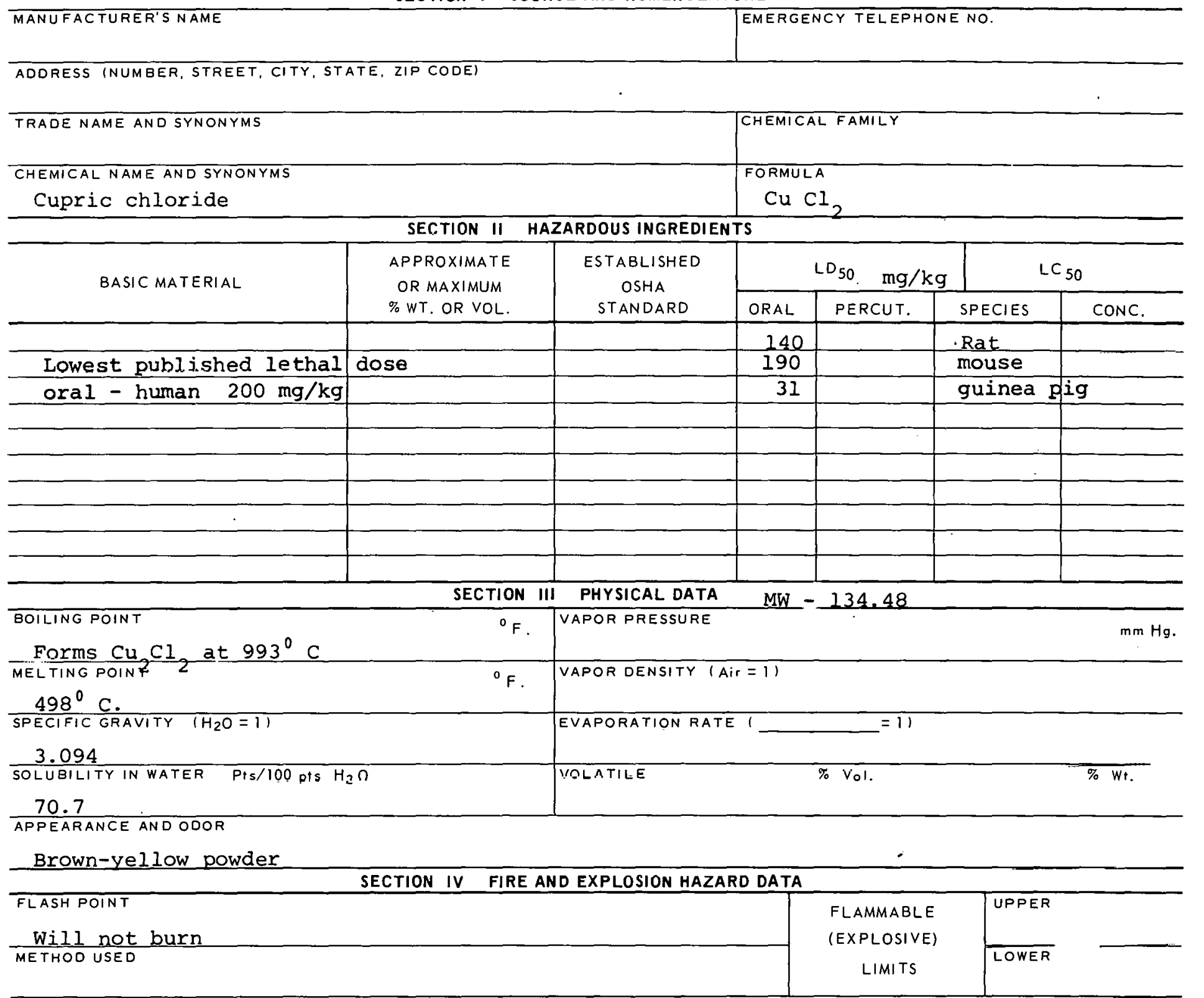

EXTINGUISHING MEDIA

$\mathrm{N} / \mathrm{A}$

SPECIAL FIRE FIGHTING PROCEDURES

$\mathrm{N} / \mathrm{A}$

UNUSUAL FIRE AND EXPLOSION HAZARDS

$\mathrm{N} / \mathrm{A}$ 


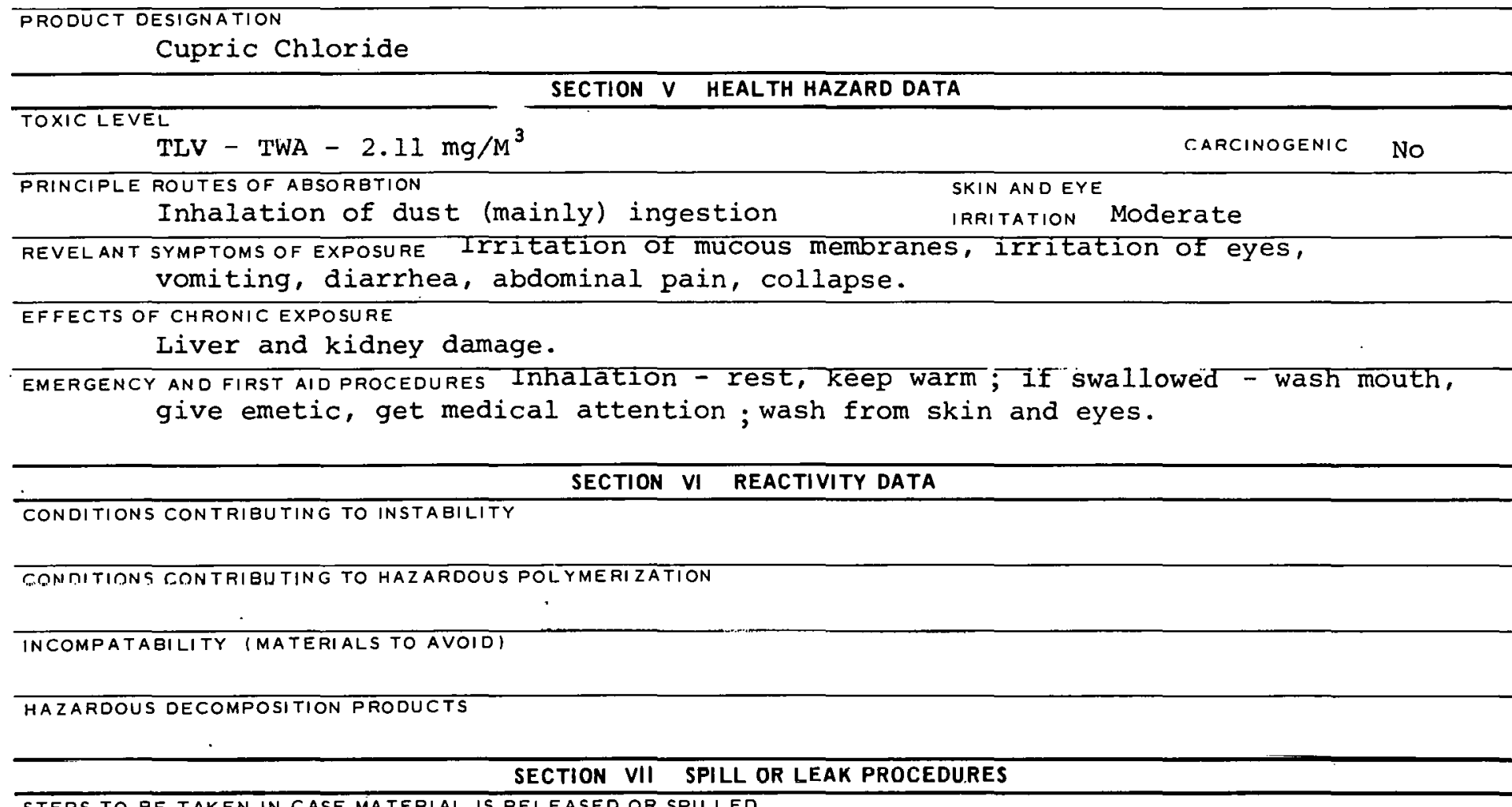

\section{WASTE DISPOSAL METHOD}

SECTION VIII SPECIAL PROTECTION INFORMATION

\begin{tabular}{l|l}
\hline VENTILATION REQUIREMENTS LOCAL EXHAUST & $\begin{array}{c}\text { PROTECTIVE EQUIPMENT (SPECIFY TYPES) EYE } \\
\text { SafETYY glaSSES }\end{array}$ \\
\hline MECHANICAL IGENERAL) & GLOVES \\
\hline SPECIAL & RFGPIRATOR \\
\hline
\end{tabular}

OTHER PROTECTIVE EQUIPMENT

\section{SECTION IX SPECIAL PRECAUTIONS}

UTHER PREGAUTIONS

MIONATURE
DATE

$46-3433 B$ 


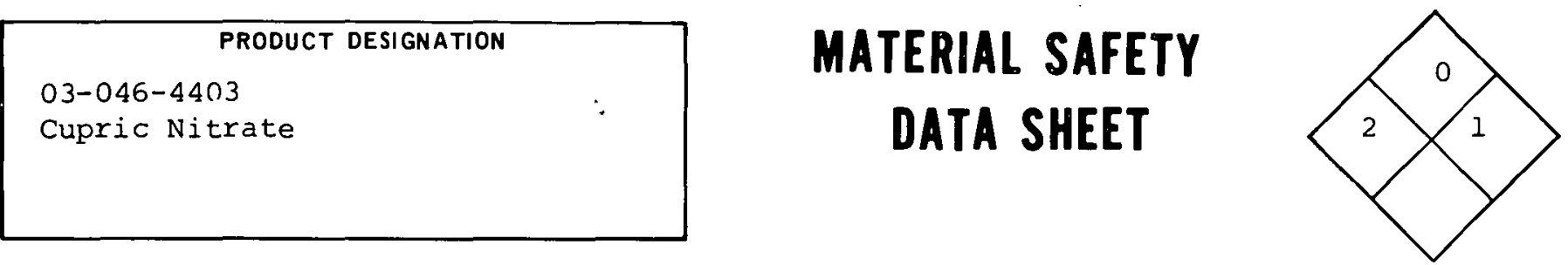

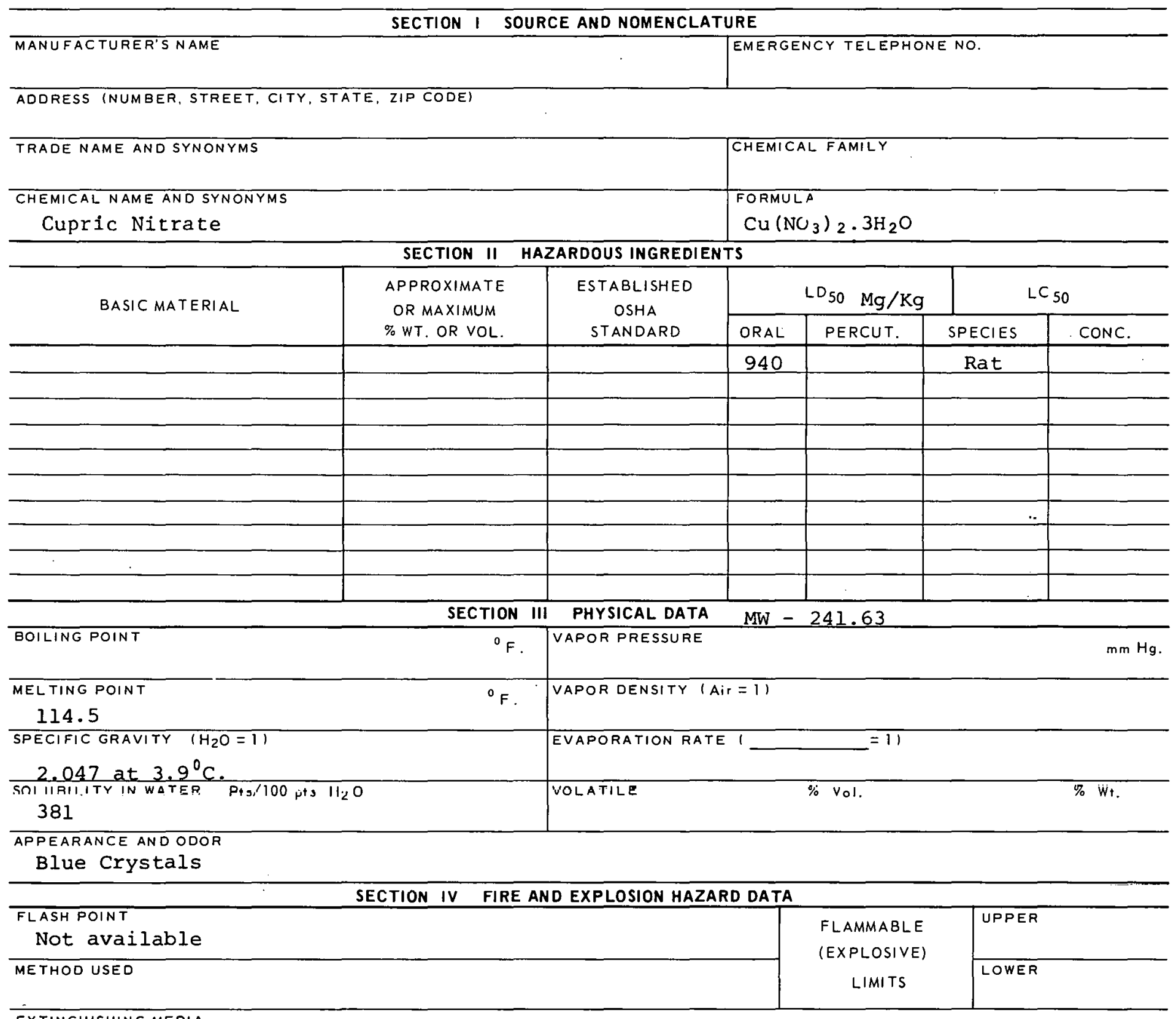

FXTINGUISHING MEDIA

Use flooding of water in early stages of fire.

SPECIAL FIRE FIGHTING PROCEDURES

Wear self-contained breathing apparatus.

UNUSUAL FIRE AND EXPLOSION HAZARDS

Gives off toxic nitrogen oxides when heated. 
TOXIC LEVEL

$\mathrm{TLV}$ - TWA $3.40 \mathrm{mg} / \mathrm{M}^{3}$

CARCINOGENIC NO

PRINCIPLE ROUTES OF ABSORBIION

Inhalation of dust (mainly) ingestion

SKIN ANOEYE

IRRITATION Moderate

REVELANT SYMPTOMS OF EXPOSURE Irritation of mucous membranes, irritation of eyes,

vomiting, diarrhea, abdominal pain, collapse.

EFFECTS OF CHRONIC EXPOSURE

Liver and kidney damage.

EMERGENCY AND FIRST AID PROCEDURES Inhalation - rest, keep warm. If swallowed - wash mouth,

give emetic, get medical attention, wash from skin and eyes.

\section{SECTION VI REACTIVITY DATA}

CONDITIONS CONTRIBUTING TO INSTABILITY

Strong oxidizing agent.

CONDITIONS CONTRIBU TING TO HAZARDOUS POLYMERIZATION

INCOMPATABILITY (MATERIALS TO AVOID)

May react violently with reducing agent.

HAZARDOUS DECOMPOSITION PRODUCTS

Uxides of nitrogën.

SECTION VII SPILL OR LEAK PROCEDURES

STEPS TO BE TAKEN IN CASE MATERIAL IS RELEASED OR SPILLED

WASTE DISPOSAL METHOD

SECTION VIII SPECIAL PROTECTION INFORMATION

\begin{tabular}{l|l}
\hline VENTILATION REQUIREMENTS LOCAL EXHAUST & PROTECTIVE EQUIPMENT (SPECIFY TYPES) EYE
\end{tabular}

MECHANICAL IGENERAL)

Safety glasses

SPECIAL

GI.OVES

Rubber gloves

SPECIAL

RESPIRATOR

OTHER PROTECTIVE EQUIPMENT

SECTION IX SPECIAL PRECAUTIONS

PRECAUTIONS TO BE, TAKEN IN HANDLING AND STORAGE

OTHER PRECAUTIONS

SIGNATURE

DATE

46-4403B 

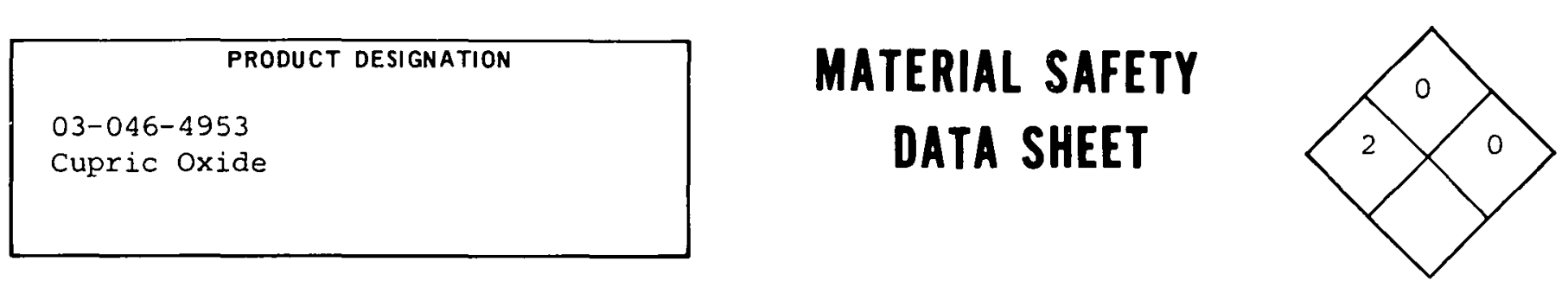

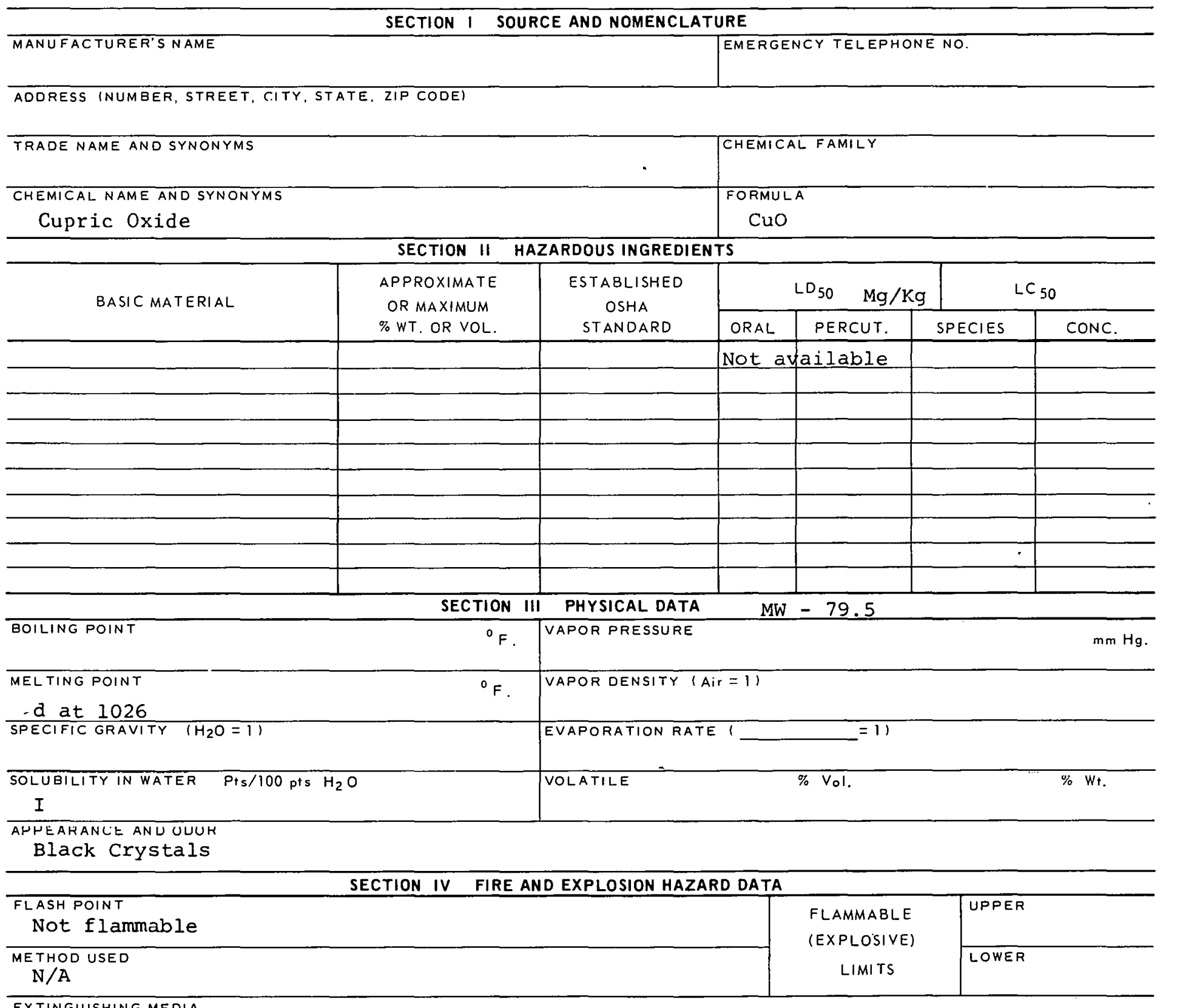

EXTINGUISHING MEDIA

$\mathrm{N} / \Lambda$

SPECIAL FIRE FIGHTING PROCEDURES

$\mathrm{N} / \mathrm{A}$

UNUSUAL FIRE AND EXPLOSION HAZARDS 


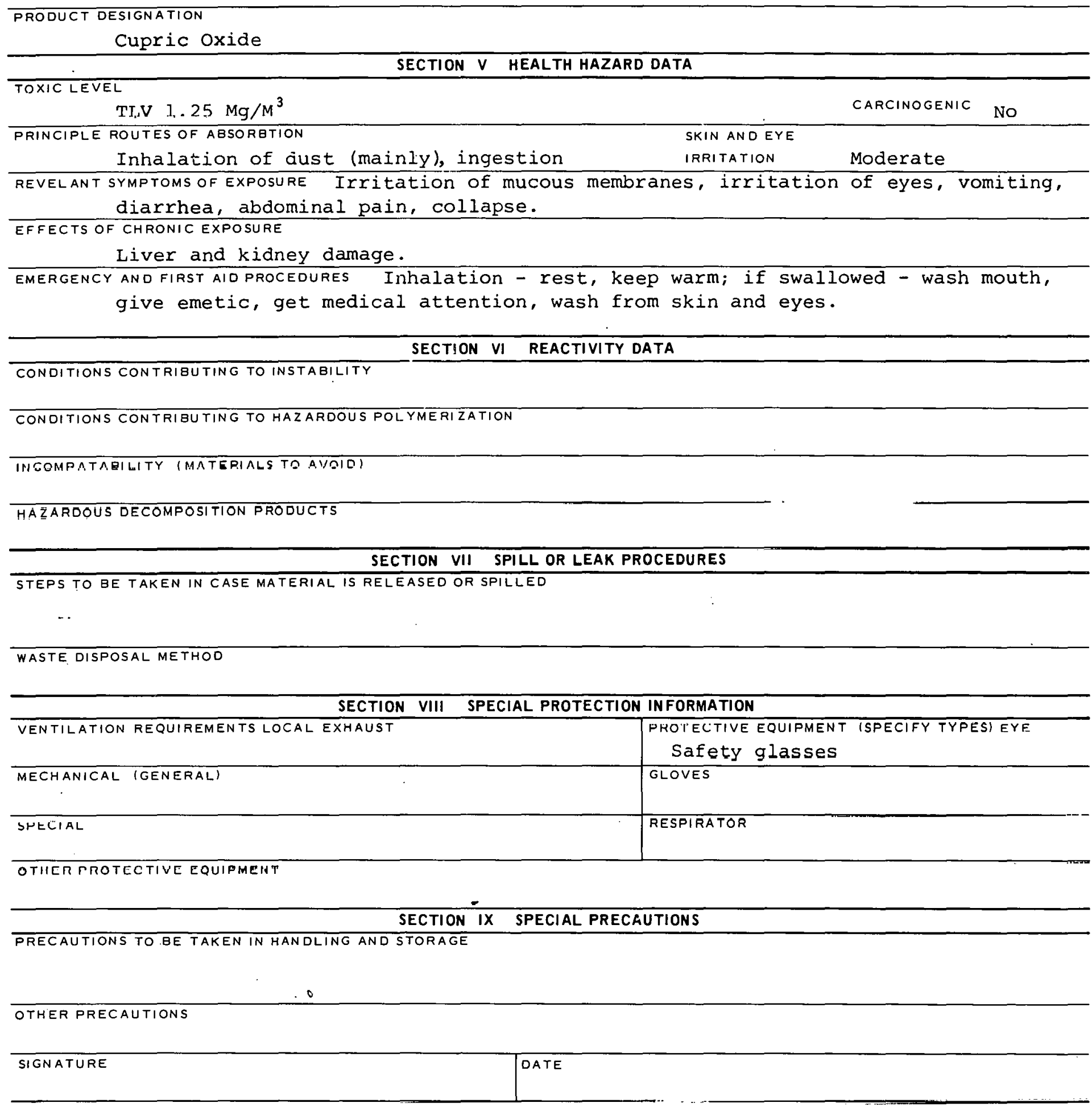

\section{6-4953B}


CAS : 007758987

\begin{tabular}{|c|}
\hline PRODUCT DESIGNATION \\
03-046-6403 \\
Cupric Sulfate \\
\hline
\end{tabular}

GL88000

\section{MATERIAL SAFETY \\ DATA SHEET}

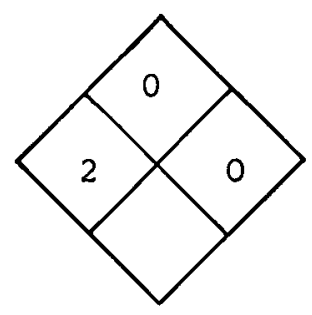

SECTION I SOURCE AND NOMENCLATURE

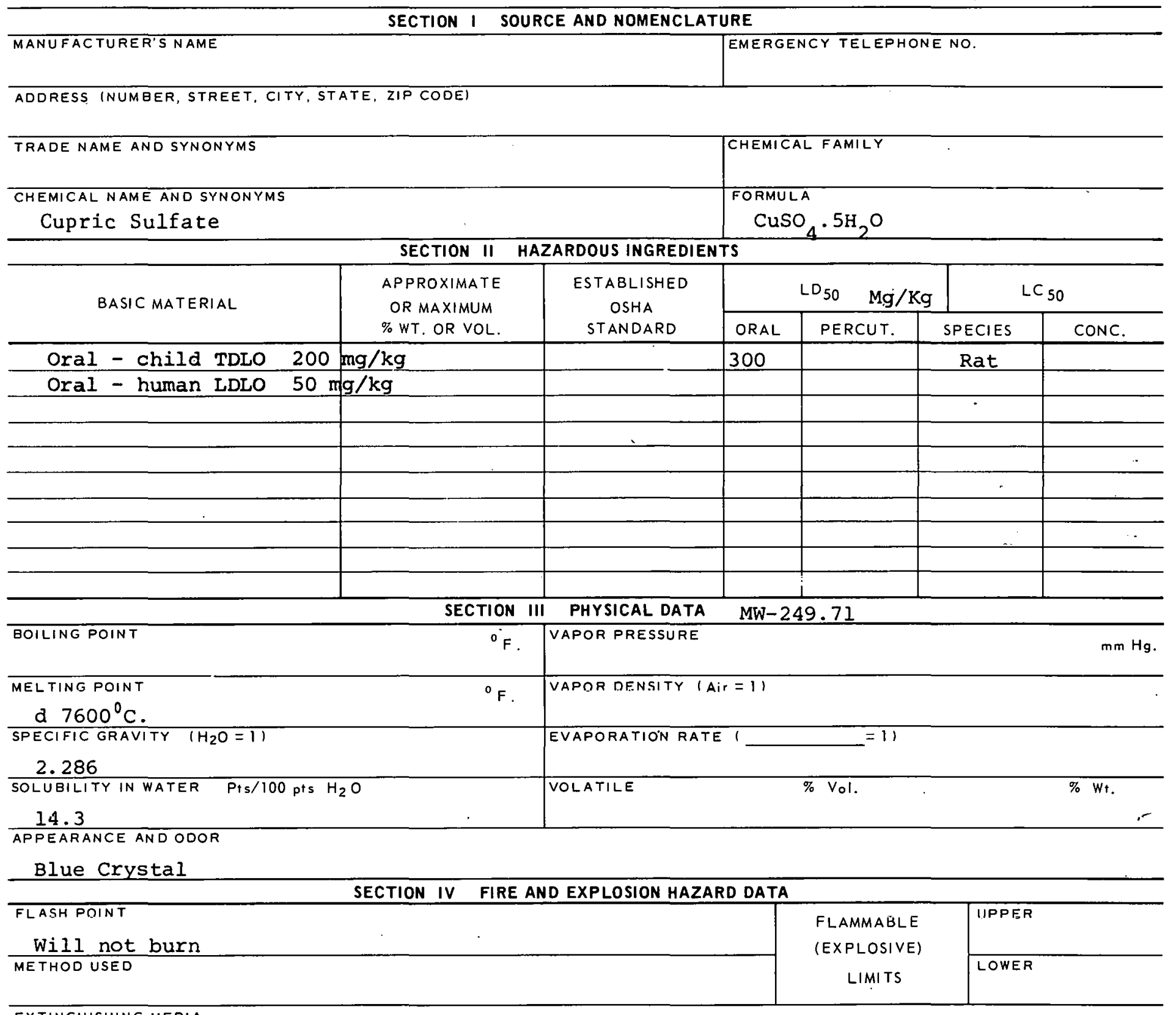

EXTINGUISHING MEOIA

$\mathrm{N} / \mathrm{A}$

SPECIAL FIRE FIGHTING PROCEDURES

$\mathrm{N} / \mathrm{A}$

UNUSUAL FIRE AND EXPLOSION HAZARDS

$\mathrm{N} / \mathrm{A}$ 

vomiting, aiarrhea, abdominal pain, collapse.

EFFECTS OF CHRONIC EXPOSURE

Liver and kidney damage.

EMERGENCY AND FIRST AID PROCEDURES Inhalation - rest, keep warm, if swallowed - wash mouth, give emetic, get medical aid, wash from skin and eyes.

\section{SECTION VI REACTIVITY DATA}

CONDITIONS CONTRIBUTING TO INSTABILITY

CONDITIONS CONTRIBUTING TO HAZARDOUS POLYMERIZATION

INCOMPATABILITY (MATERIALS TO AVOIO)

HAZARDOUS DECOMPOSITION PRODUCTS

SECTION VII SPILL OR LEAK PROCEDURES

STEPS TO BE TAKEN IN CASE MATERIAL IS RELEASED OR SPILLED

WASTE DISPOSAL METHOD

\section{SECTION VIII SPECIAL PROTECTION INFORMATION}

VENTILATION REQUIREMENTS LOCAL EXHAUST

MECHANICAL (GENERAL)

$\overline{\text { SPECIAL }}$

0 OTHER PROTECTIVE EQUIPMENT

\section{SECTION IX SPECIAL PRECAUTIONS}

PROTECTIVE EQUIPMENT (SPECIFY TYPES) EYE Safety glasses rLOVES RESPIRATOR

PRECAUTIONS TO $B E$ TAKEN IN HANDLING AND STORAGE 
CAS : 007758896

\section{PRODUCT DESIGNATION}

03-046-8423

Cuprous Chloride
GL69900

\section{MATERIAL SAFETY \\ DATA SHEET}

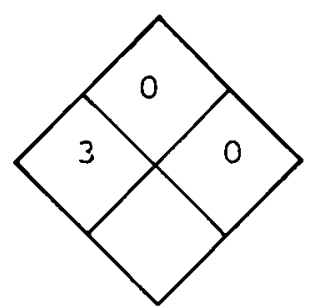

SECTION I SOURCE AND NOMENCLATURE

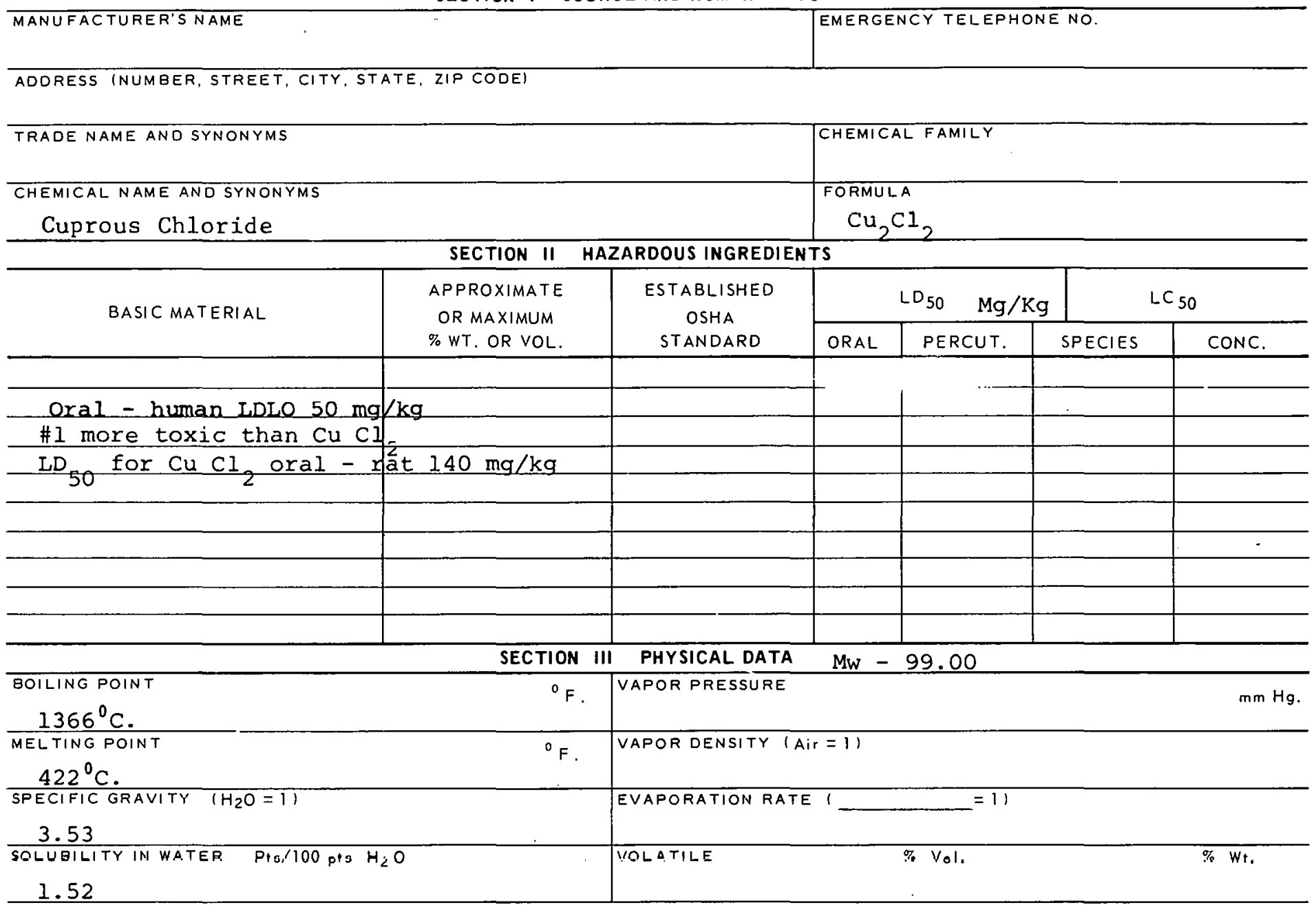

APPEARANCE AND ODOR

White crystals

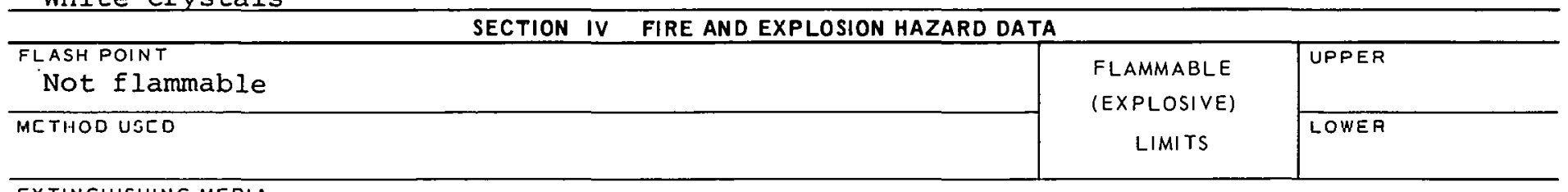

EXTINGUISHING MAEDIA

$\mathrm{N} / \mathrm{A}$

SPECIAL FIRE FIGHTING PROCEDURES

$\mathrm{N} / \mathrm{A}$

UNUSUAL FIRE AND EXPLOSION HAZARDS

$\mathrm{N} / \mathrm{A}$ 


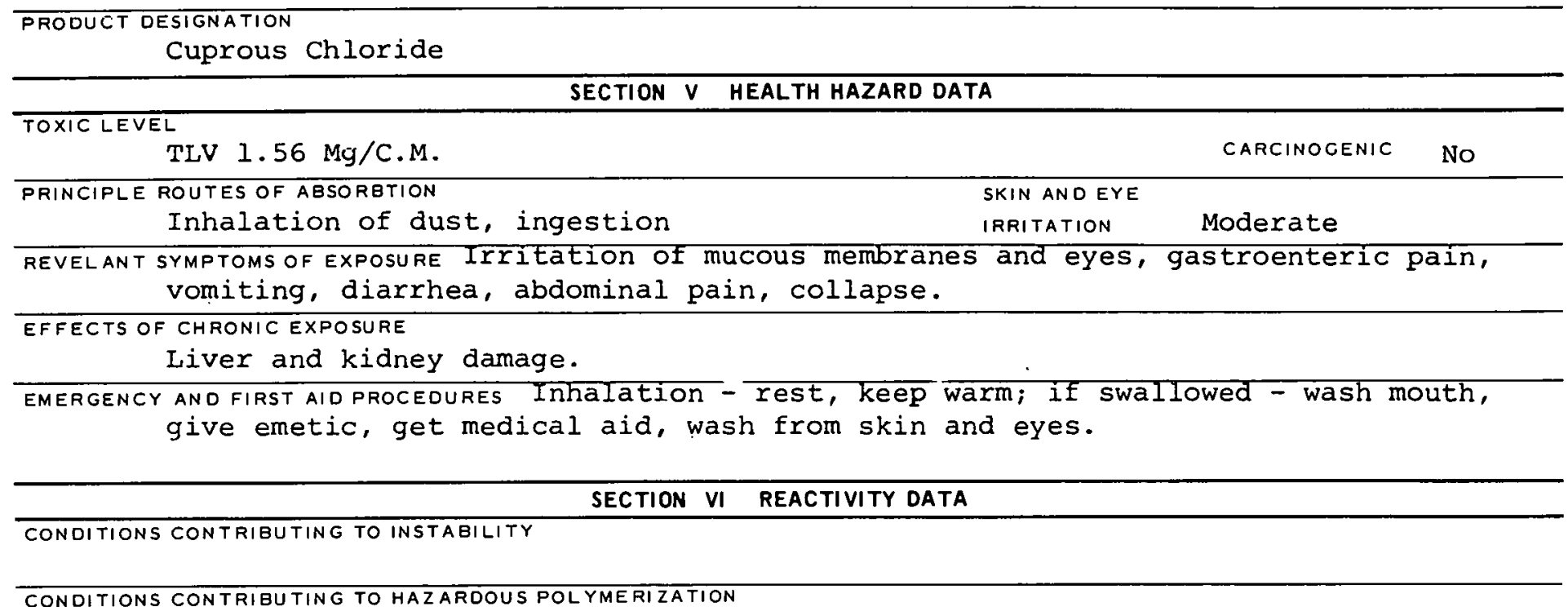

INCOMPATABILITY (MATERIALS TO AVOID)

HAZARDOUS DECOMPOSITION PRODUCTS

SECTION VII SPILL OR LEAK PROCEDURES

STEPS TO BE TAKEN IN CASE MATERIAL IS RELEASED OR SPILLED

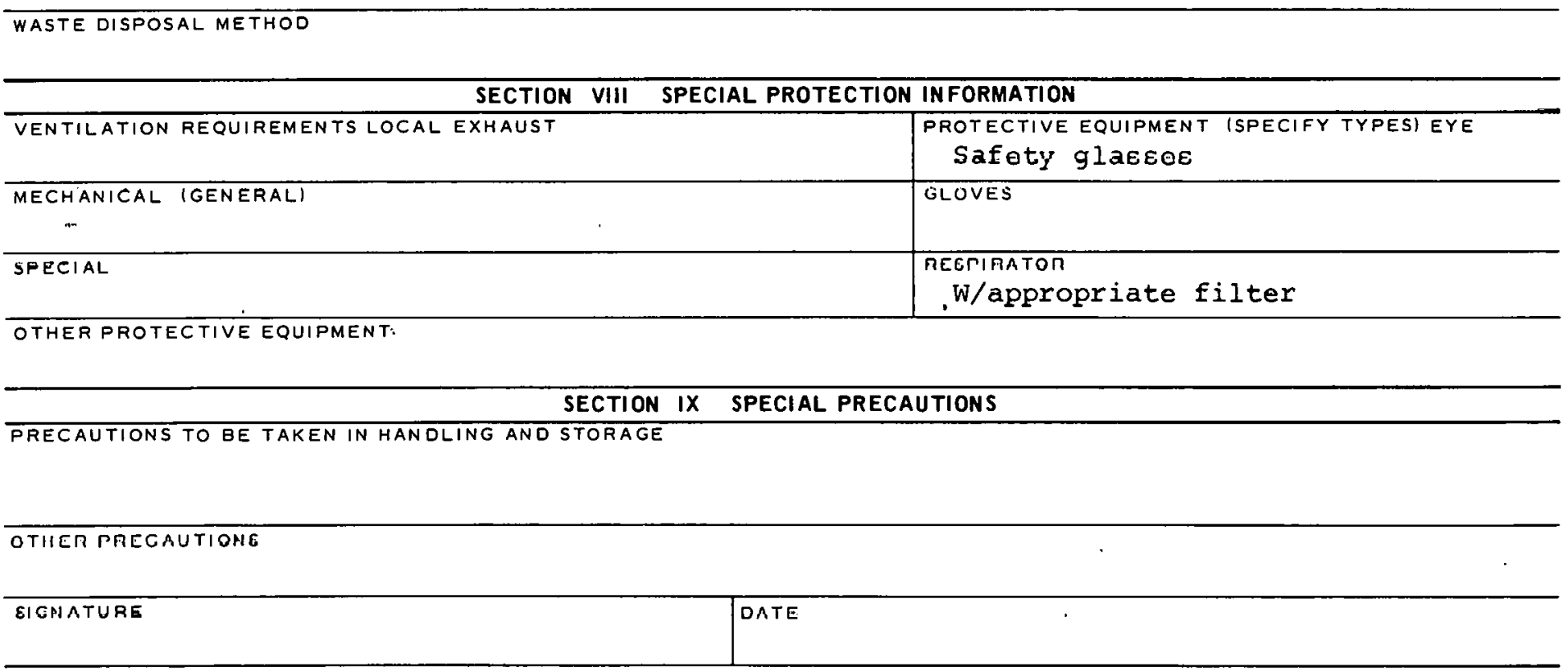


CAS : 000544923

PRODUCT DESIGNATION

Cuprous Cyanide

03-046-8721
GL71500

MATERIAL SAFETY

DATA SHEET

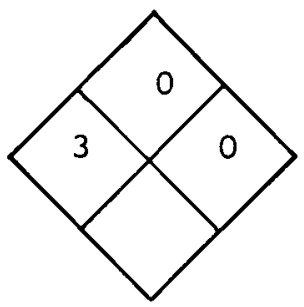

\section{SECTION I SOURCE AND NOMENCLATURE}

\begin{tabular}{l} 
MANUFACTURER'S NAME \\
ADDRESS INUMBER, STREET, CITY, \\
\hline TRADE NAME AND SYNONYMS \\
CHEMICAL NAME AND SYNONYMS \\
CUPYOUS CYANIdP \\
\hline
\end{tabular}

IEMERGENCY TELEPHONE NO.

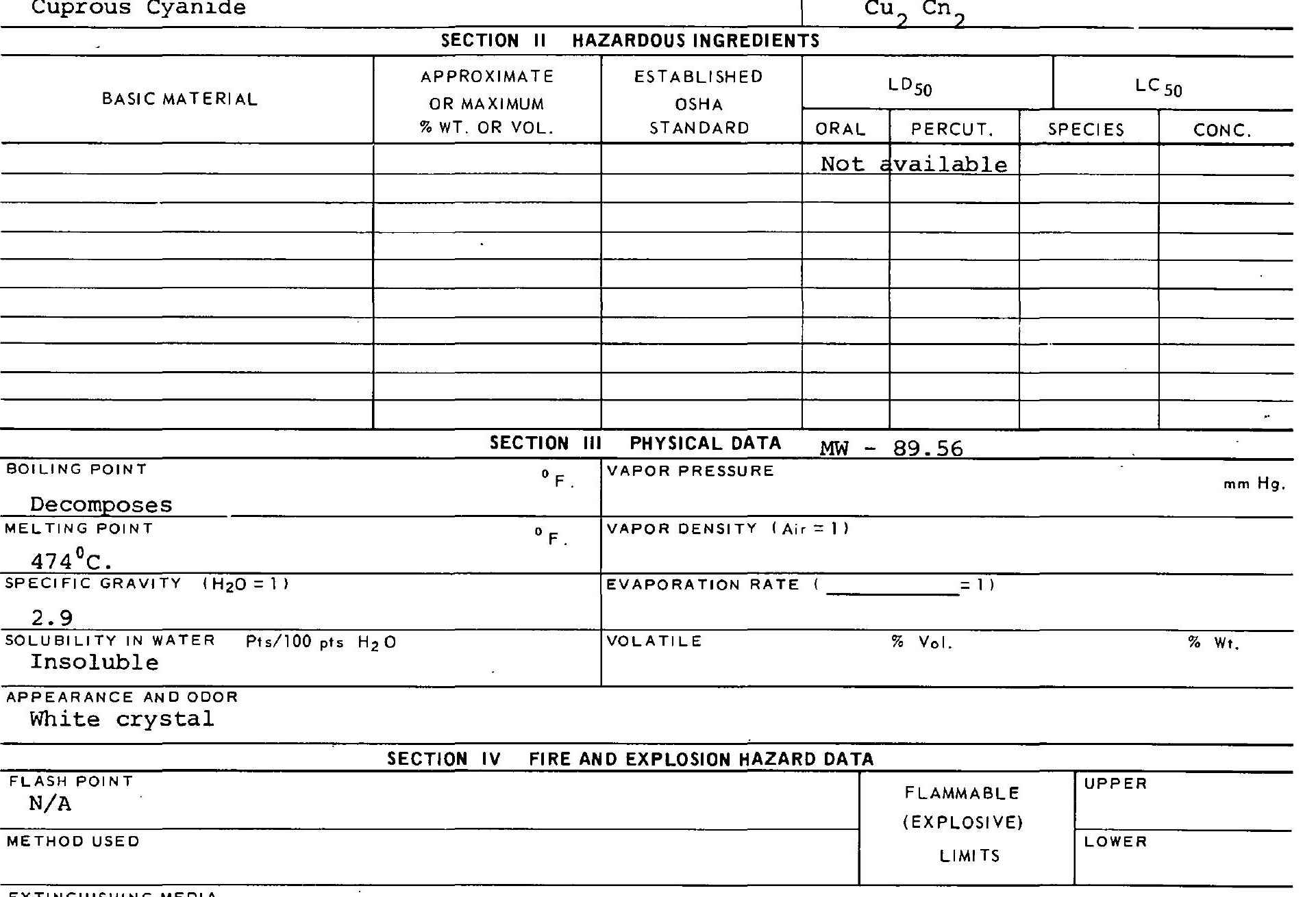

EXTINGUISHING MEDIA

$\mathrm{CO}_{2}$, Nonalkaline dry chemical or foam.

SPECIAL FIRE FIGHTING PROCEDURES

$\mathrm{H} \mathrm{CN}$ from $\mathrm{Cu}_{2}(\mathrm{CN})_{2}$, produces an explosive mixture with air. UNUSUAL FIRE AND EXPLOSION HAZAROS

$\mathrm{CO}_{2}$ from air may cause acid hydrolyses to form $\mathrm{HCN}$. 


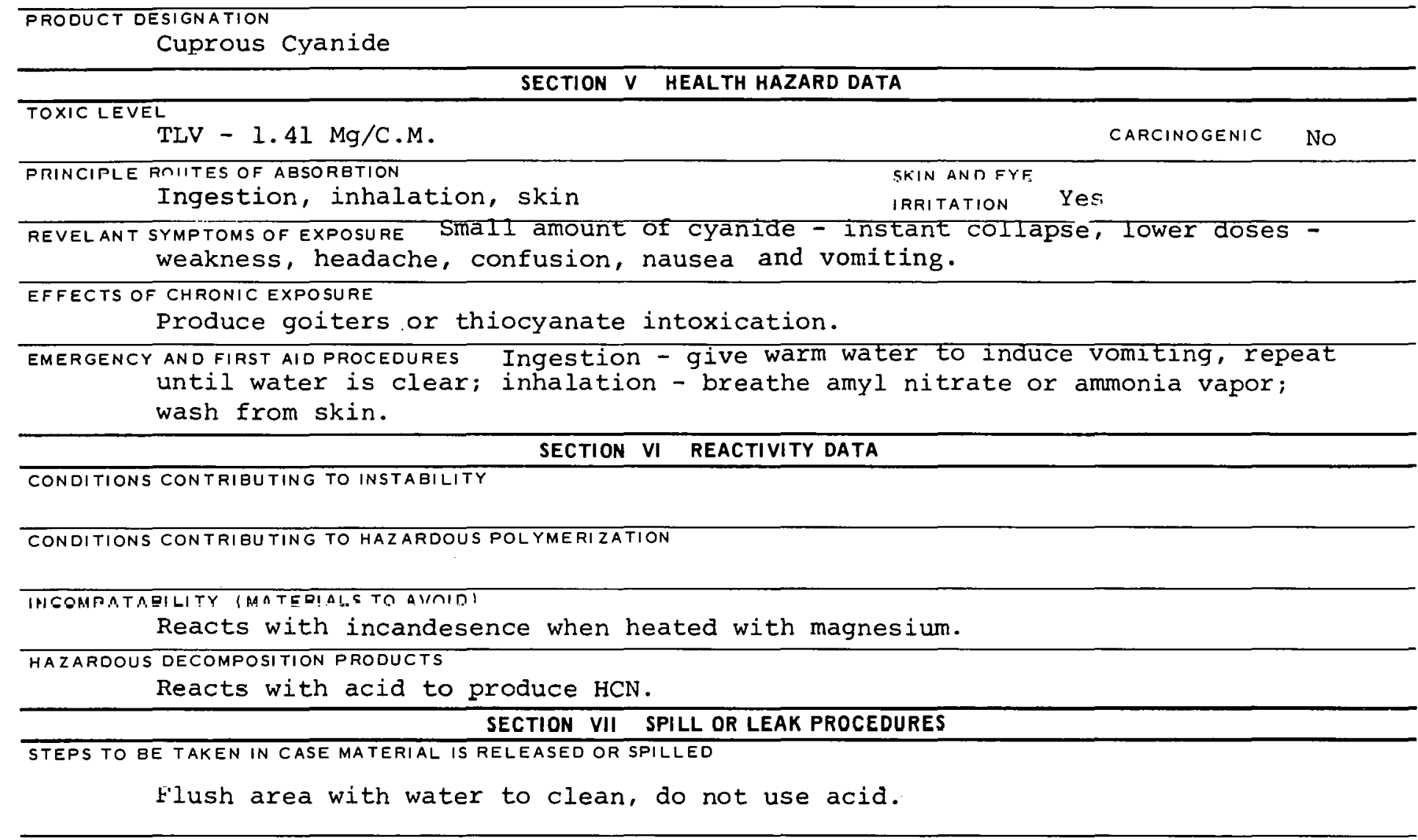

\section{WASTE DISPOSAL METHOD}

\section{SECTION VIII SPECIAL PROTECTION INFORMATION}

\begin{tabular}{|c|c|}
\hline VENTILATION REQUIREMENTS LOCAL EXHAUST & $\begin{array}{l}\text { PROTECTIVE EQUIPMENT (SPECIFY TYPES) EYE } \\
\text { Safety glasses }\end{array}$ \\
\hline MECHANICAL (GENERAL) & $\begin{array}{l}\text { GL.SVES } \\
\text { Rubber }\end{array}$ \\
\hline SPECIAL & $\begin{array}{l}\text { RESPIRATOR } \\
\text { W'siepar filter: }\end{array}$ \\
\hline $\begin{array}{l}\text { OTHER PROTECTIVE EQUIPMENT } \\
\text { DO not store near acids. }\end{array}$ & \\
\hline
\end{tabular}

PRECAUTIONS TO BE TAKEN IN HANDLING AND STORAGE

Store in cool, well-ventilated area. 
CAS : 001317391

GL80500

PRODUCT DESIGNATION
$03-046-9303$
Cuprous Oxide

\section{MATERIAL SAFETY DATA SHEET}

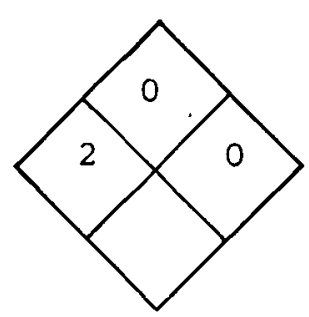

\section{SECTION I SOURCE AND NOMENCLATURE}

MANUFACTURER'S NAME

ADORESS (NUMBER, STREET, CITY, STATE, ZIP CODE)

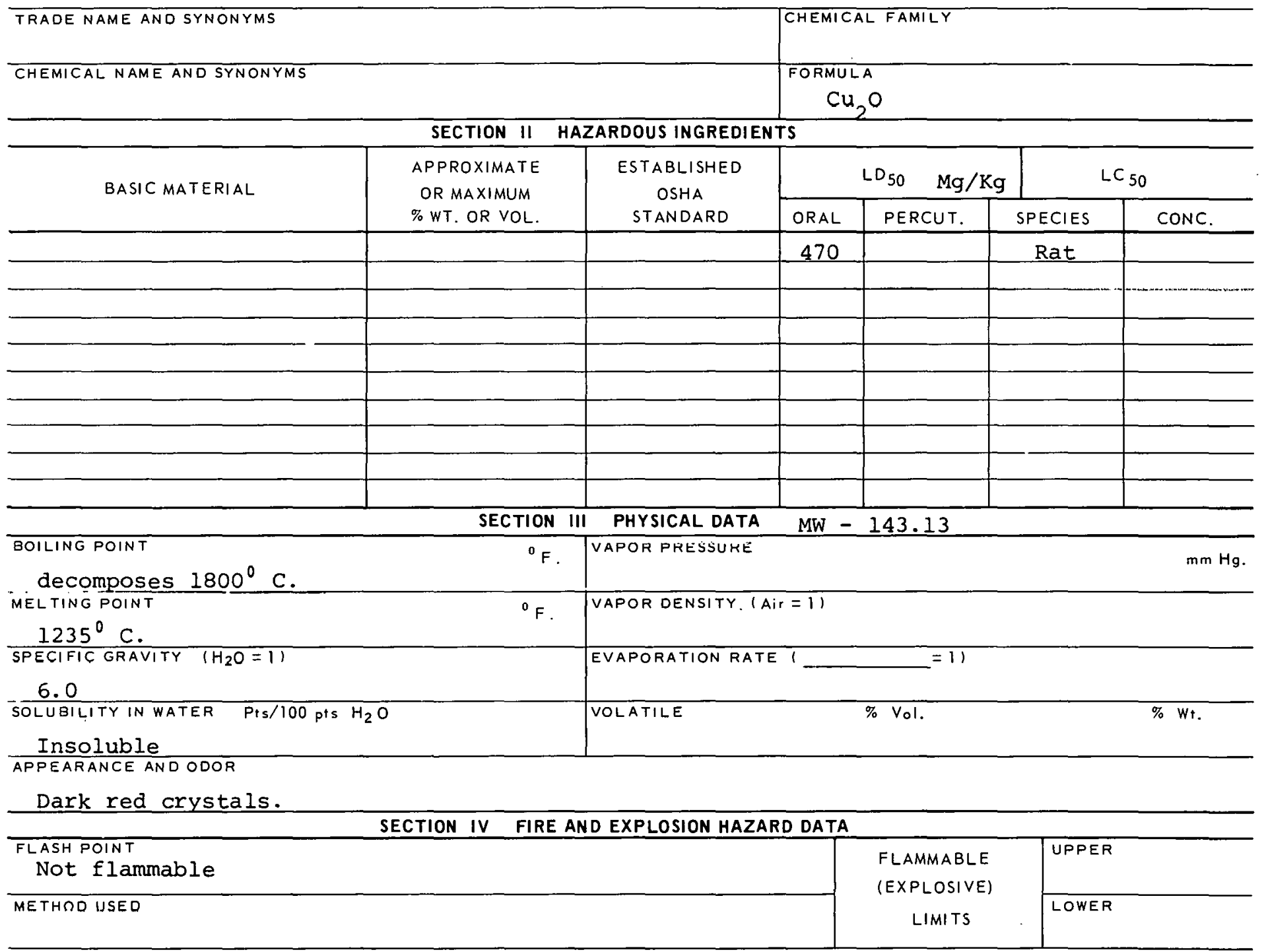

EXTINGUISHING MEDIA

$\mathrm{N} / \mathrm{A}$

SPECIAL FIRE FIGHTING PROCEDURES

$\mathrm{N} / \mathrm{A}$

UNUSUAL FIRE AND EXPLOSION HAZARDS

$\mathrm{N} / \mathrm{A}$ 


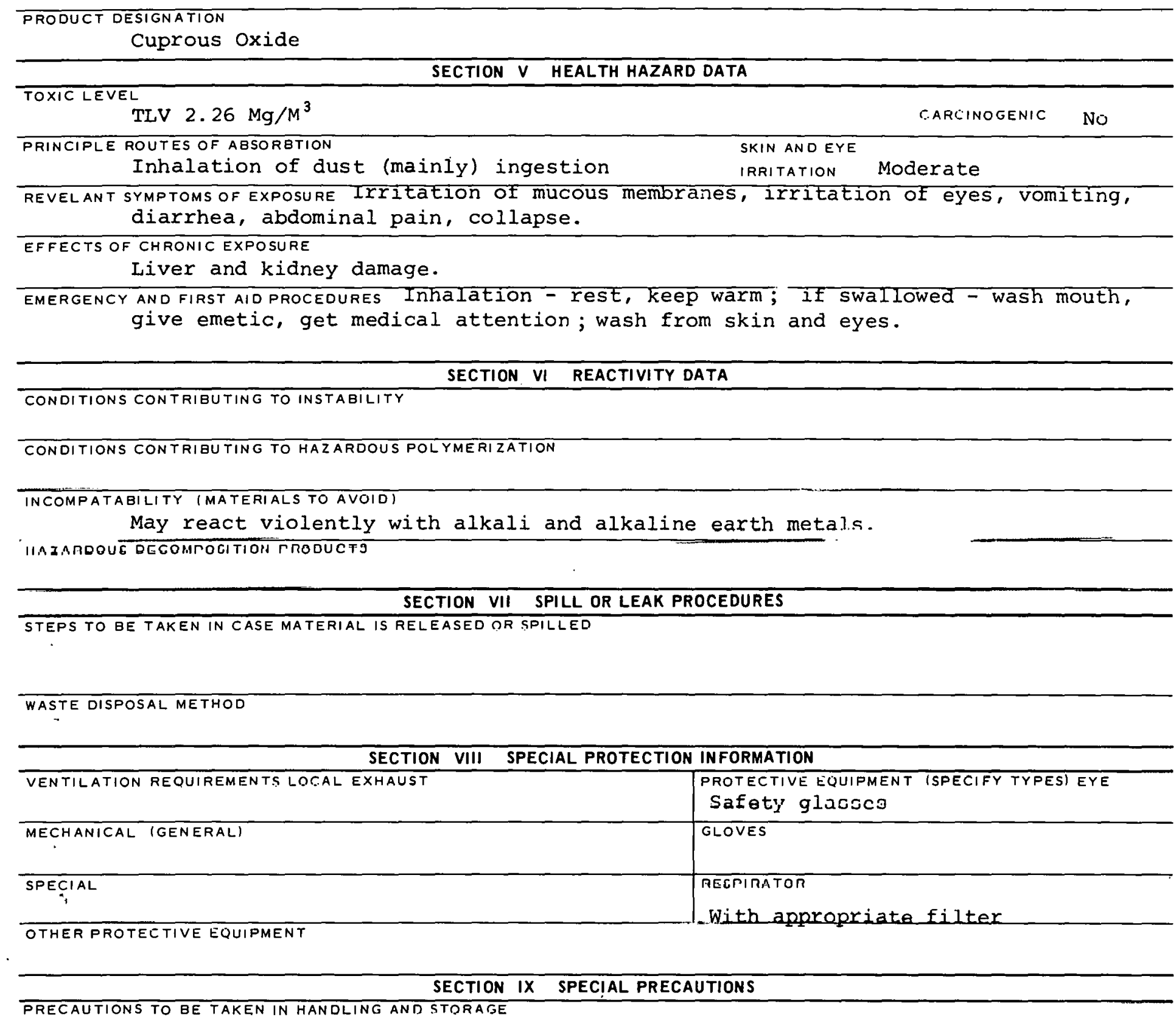

PRECAUTIONS TO BE TAKEN IN HANDLING AND STORAGE

\begin{tabular}{l|l|l|}
\hline OTHER PRECAUTIONS & \\
\hline SIGNATURE & DATF \\
\hline
\end{tabular}


CAS : 010025771 NO5 4250

\section{PRODUCT DESIGNATION}

03-053-2624

Ferric Chloride

\section{MATERIAL SAFETY DATA SHEET}

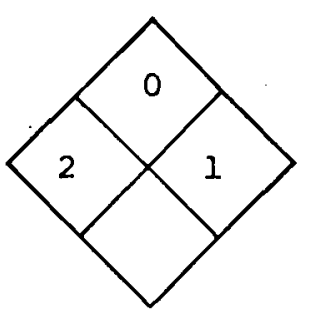

SECTION I SOURCE AND NOMENCLATURE

MANUFAC TURER'S NAME
ADDRESS INUMBER, STREET, CITY
TRADE NAME AND SYNONYMS
CHEMICAL NAME AND SYNONYMS
FEIYIC ChIOYIDE

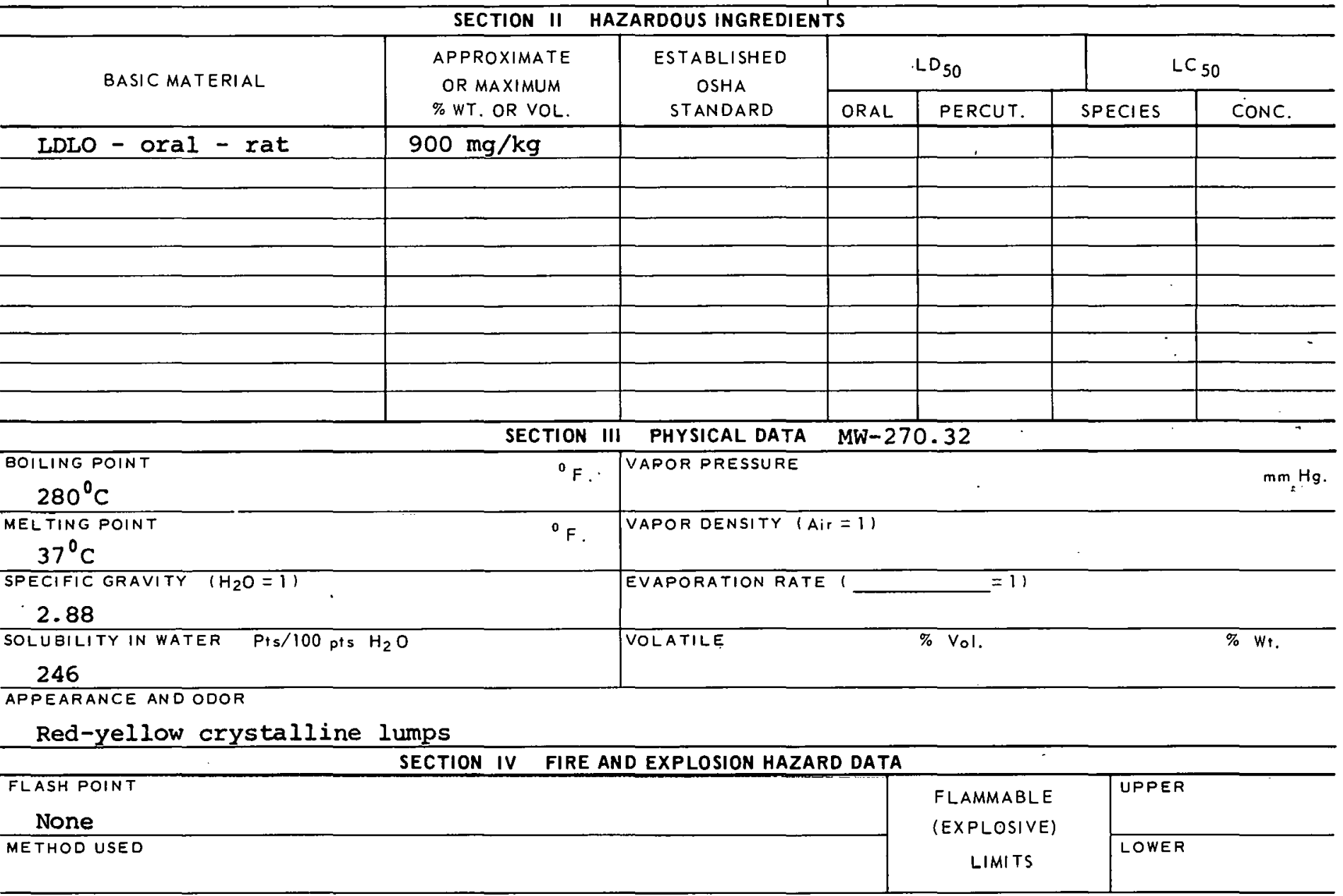

EXTINGUISHING MEDIA

Water spray

SPECIAL FIRE FIGHTING PROCEDURES

Wear self-contained mask.

UNUSUAL FIRE ANDEXPLOSION HAZARDS

When heated, gives off $\mathrm{H}$ Cl fumes. 


\section{TOXIC LEVEL \\ $4.8 \mathrm{mg} / \mathrm{c} . \mathrm{m}$. (Fe)}

PRINCIPLE ROUTES OF ABSORBTION

Inhalation and ingestion
CARCINOGENIC

No SKIN AND EYE IRRITATION YeS

REVELANT SYMPTOMS OF EXPOSURE Respiratory tract irritation, nausea, vomittng, gastric hemorrhage, shortness of breath, eye and skin irritation

EFFECTS OF CHRONIC EXPOSURE None indicated

EMERGENCY AND FIRST AID PROCEDURES

rest; wash skin and eyes.

Ingestion - get medical aid, gastric lavage, inhalation SECTION VI REACTIVITY DATA

\section{CONDITIONS CONTRIBUTING TO HAZARDOUS POLYMERIZATION}

IINCOMRATADILITY (MATERIALS TO AVOID)

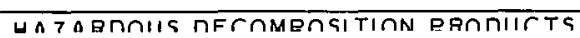

Heating to decomposition produces HCl fumes

SECTION VII SPILL OR LEAK PROCEDURES

STEPS TO BE TAKEN IN CASE MATERIAL IS RELEASED OR SPILLEO

WASTE DISPOSAL METHOD
Contact environmental control officer

SECTION VIII SPECIAL PROTECTION INFORMATION

\begin{tabular}{l|l}
\hline VENTILATION REQUIREMENTS LOCAL EXHAUST & $\begin{array}{l}\text { PROTECTIVE EQIIIPMFNT (SPFCIFY TYPES) EYE } \\
\text { SafEty glaSSES }\end{array}$ \\
\hline MECHANICAL (GENERAL) & GLOVES \\
\hline SPECIAL & RESPIRATOR \\
\hline
\end{tabular}

OTHER PROTECTIVE EQUIPMENT

\section{SECTION IX SPECIAL PRECAUTIONS}

PRECAUTIONS TO BE TAKEN IN HANDLING AND STORAGE

store in cool, dry place.

OTHER PRECAUTIONS

SIGNATURE

DATE

$53-2624 B$ 


PRODUCT DESIGNATION
03-053-3804
Ferric Nitrate

\section{MATERIAL SAFETY \\ DATA SHEET}

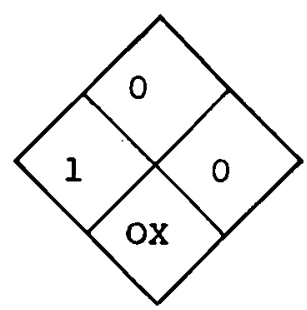

SECTION I SOURCE AND NOMENCLATURE

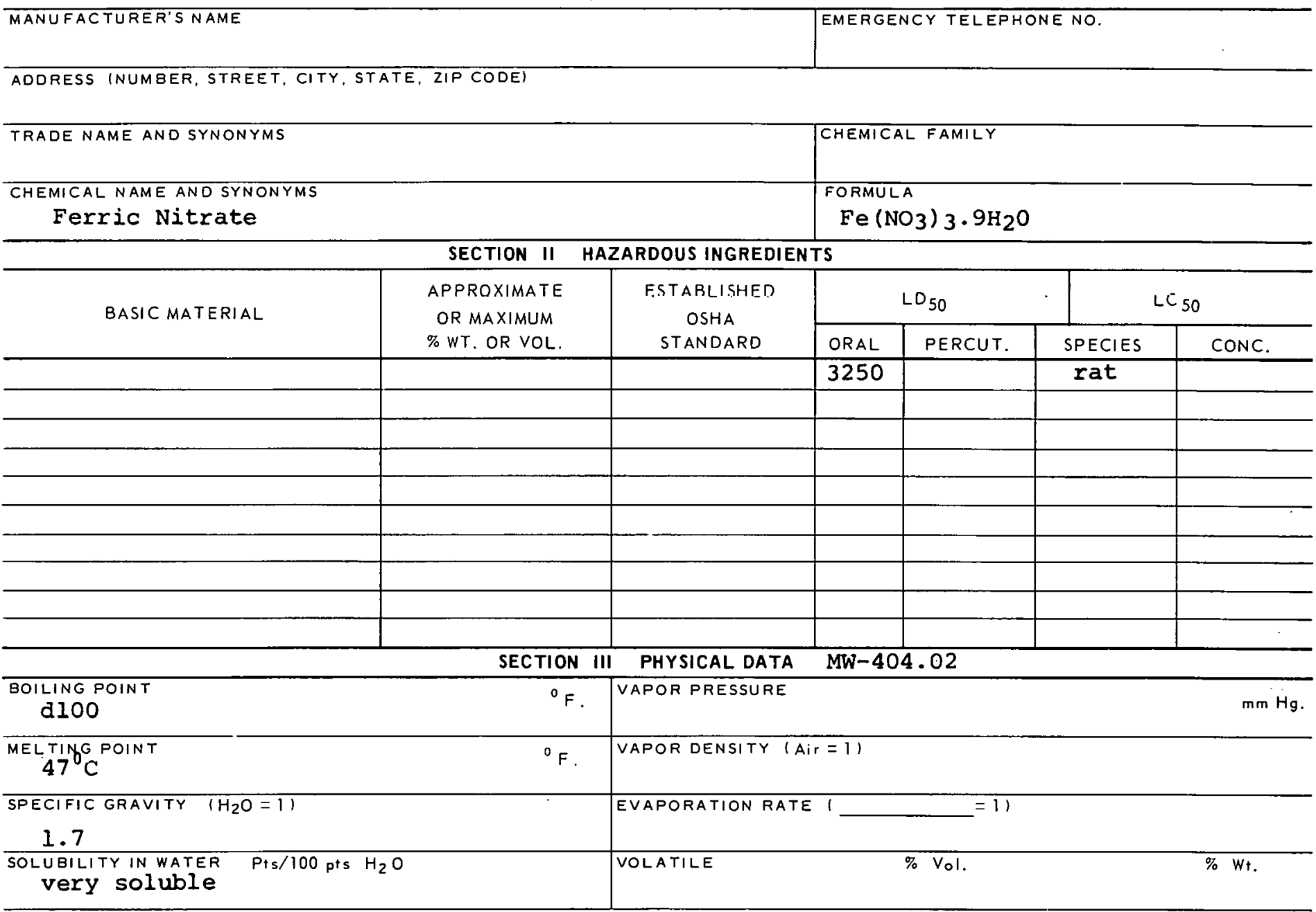

APPEARANCE AND ODOR

Pale violet deliquescent crystals

\begin{tabular}{l|c|c}
\hline \multicolumn{2}{c}{ SECTION IV FIRE AND EXPLOSION HAZARD DATA } \\
\hline \begin{tabular}{l|l} 
FLASH POINT \\
NONE
\end{tabular} & FLAMMABLE \\
\hline METHOD USED & UPPER & LIMITS \\
& LOWER & \\
\hline
\end{tabular}

EXTINGUISHING MEDIA

Water spray

SPECIAL FIRE FIGHTING PROCEDURES

Wear self-contained breathing apparatus.

UNUSUAL FIRE AND EXPLOSION HAZARDS

When heater gives off toxic nitrogen oxide fumes. 


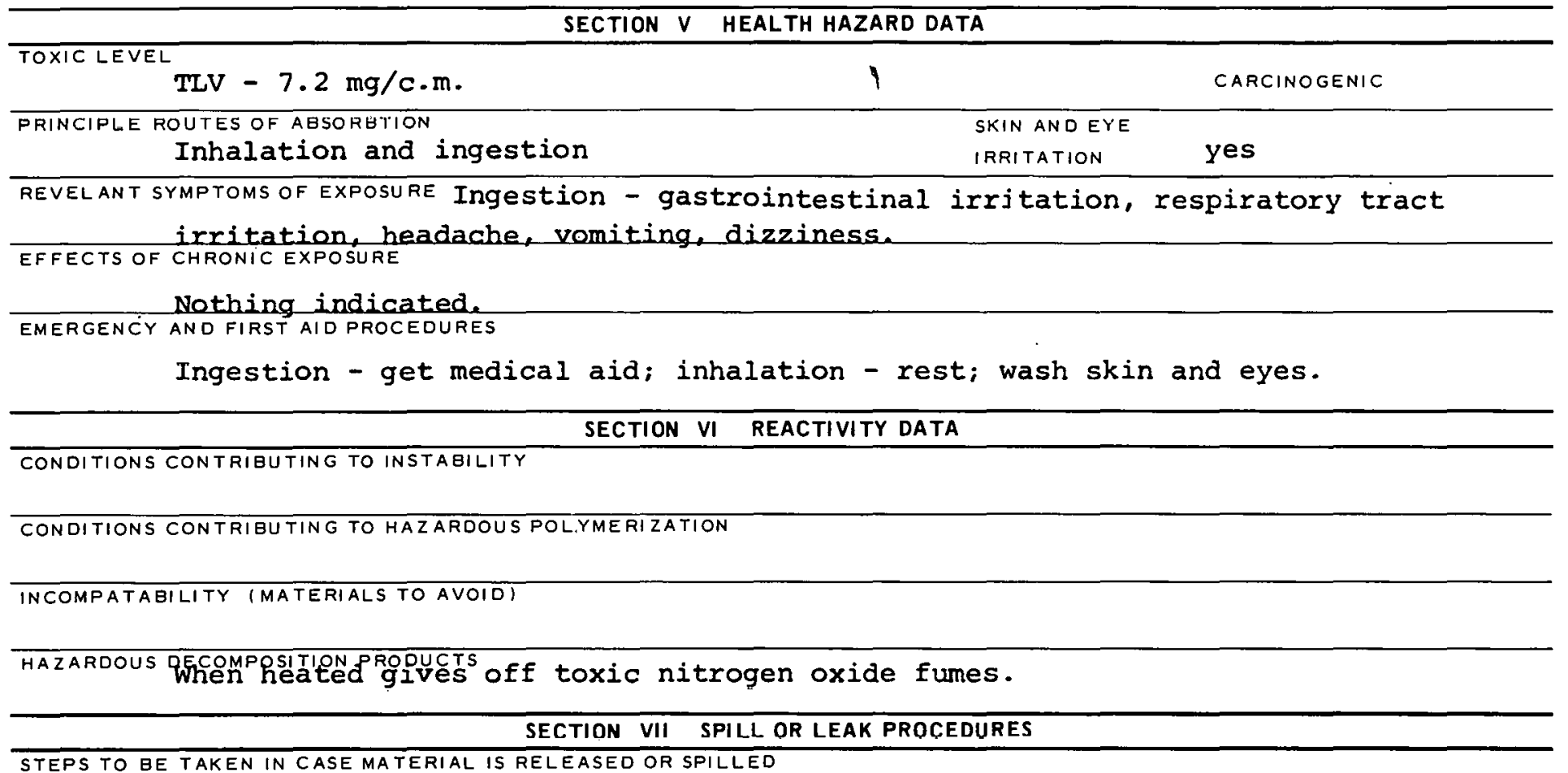

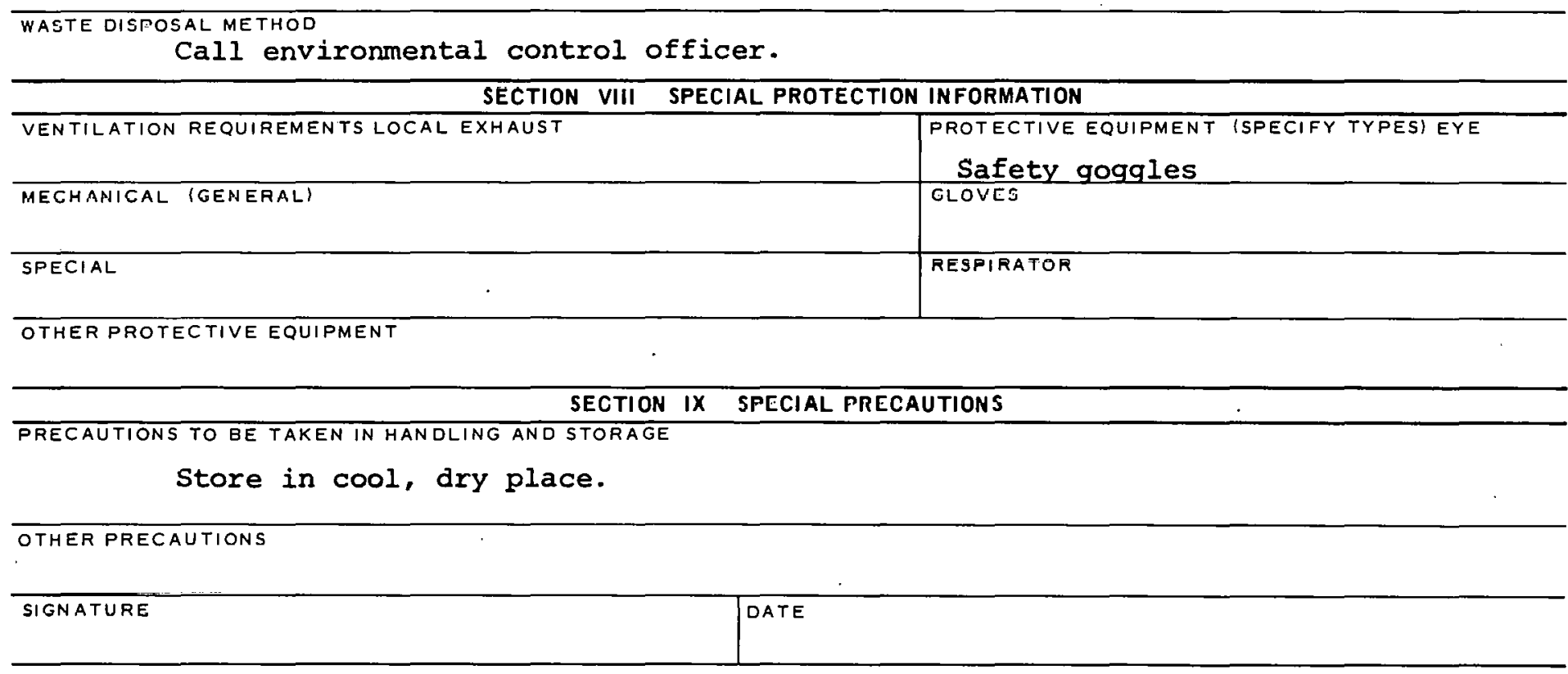


CAS:007790990e

\section{PRODUCT DESIGNATION}

Iodine

03-074-1009
MATERIAL SAFETY

DATA SHEET

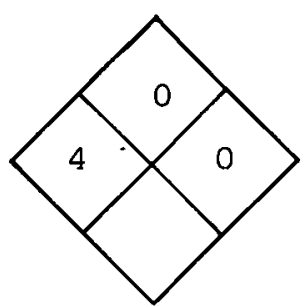

\section{SECTION I SOURCE AND NOMENCLATURE}

MANUFACTURER'S NAME
AODRESS INUMBER, STREET, CI
TRADE NAME AND SYNONYMS
CHEMICAL NAME AND SYNONYMS
IOdinE

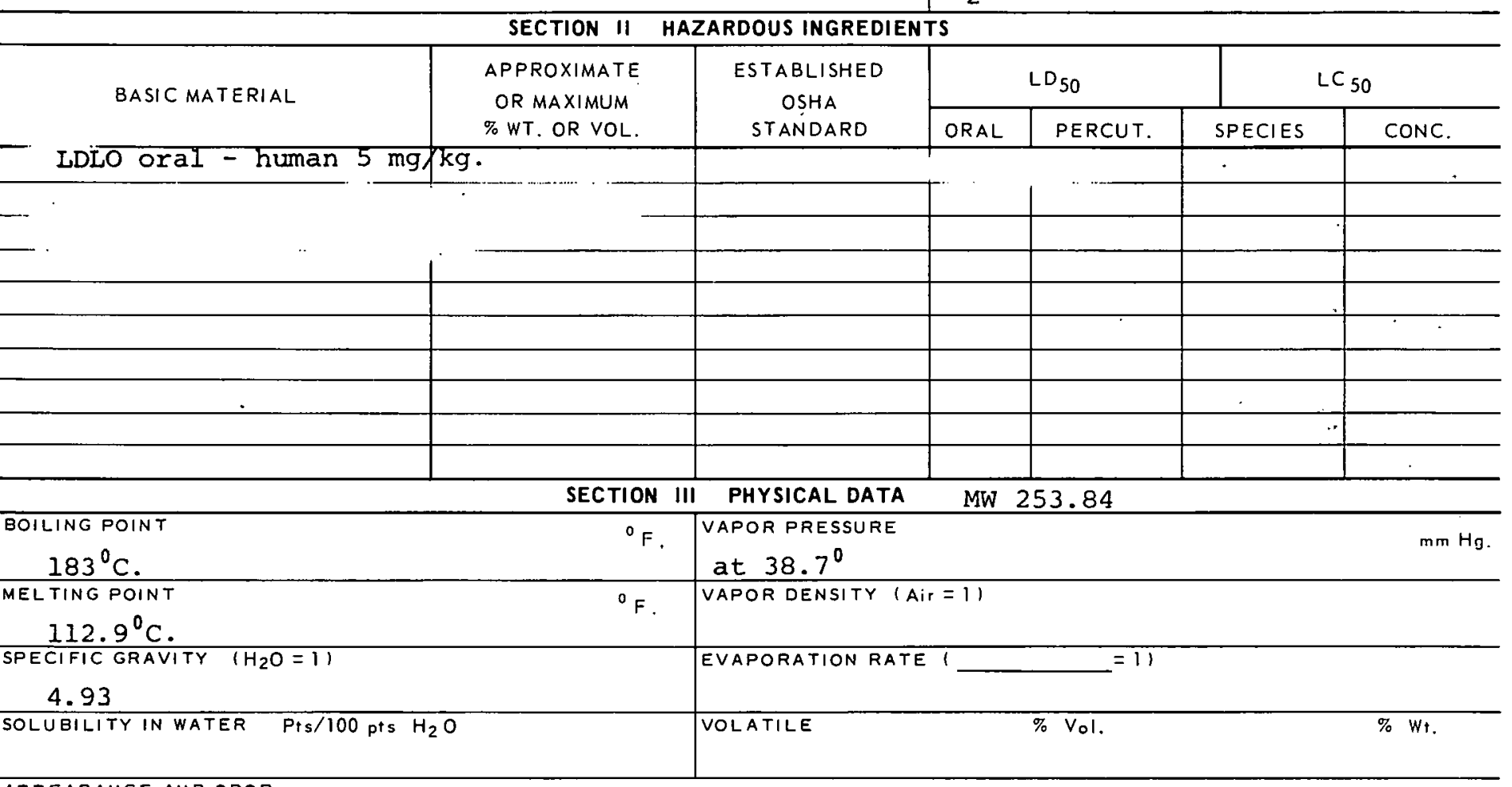

\section{APPEARANCE AND ODOR}

Rhombic, violet-black crystals, metallic luster - characteristic odor.

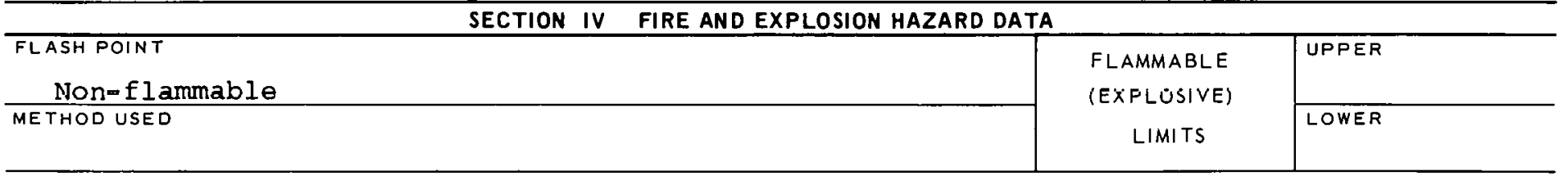

\section{EXTINGUISHING MEDIA}

Water

SPECIAL FIRE FIGHTING PROCEOURES

Wear full protective clothing.

UNUSUAL FIRE ANDEXPLOSION HATARDS

Emits toxic fumes. 


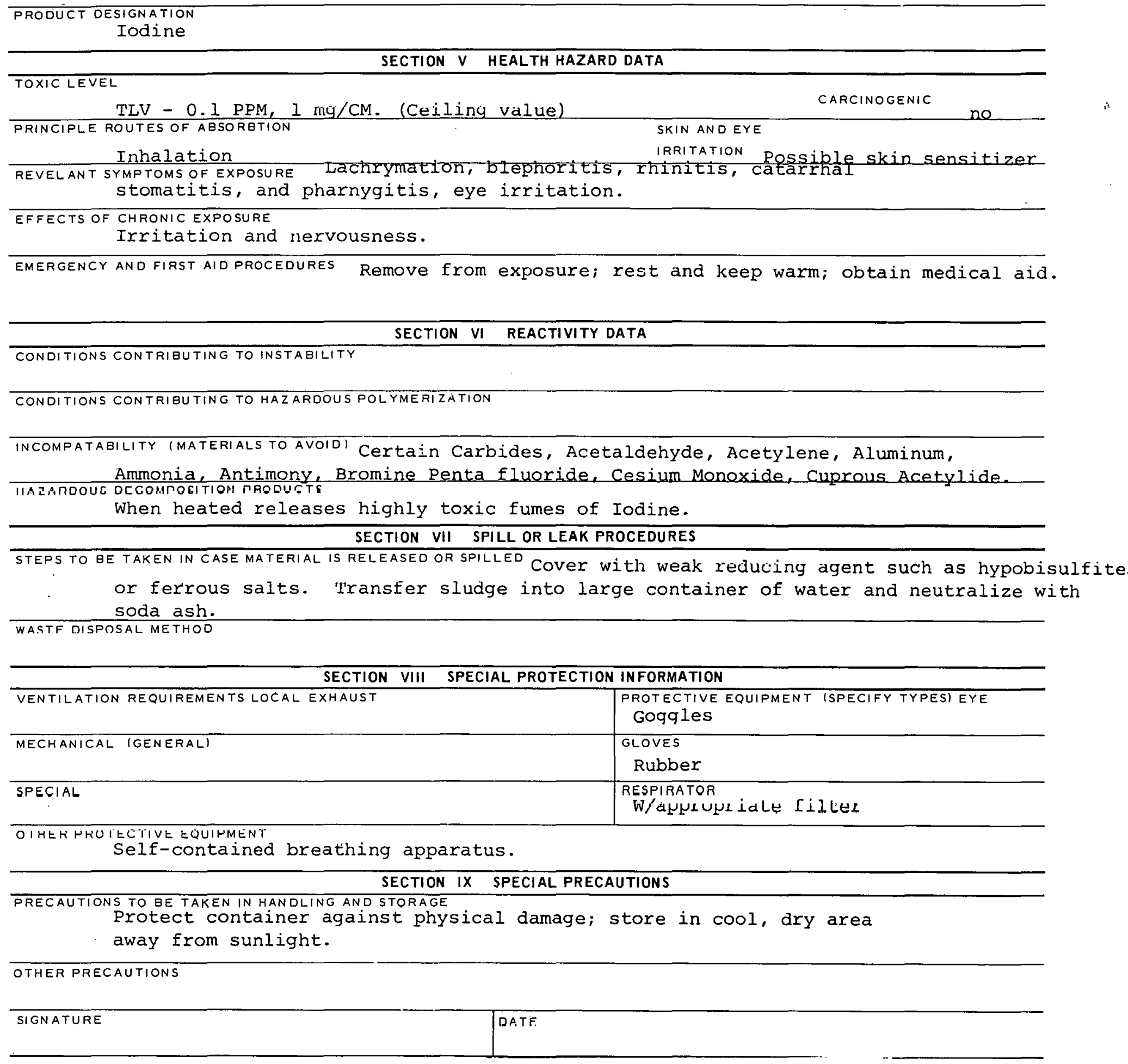


CAS: 000301042

\section{MATERIAL SAFETY DATA SHEET}

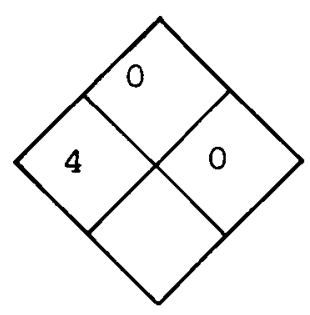

\section{SECTION I SOURCE AND NOMENCLATURE}

\begin{tabular}{l} 
MANUFACTURER'S NAME \\
\hline ADDRESS INUMBER, STREET, CITY \\
TRADE NAME AND SYNONYMS \\
CHEMICAL NAME ANO SYNONYMS \\
Lead ACetate
\end{tabular}

Lead Acetate

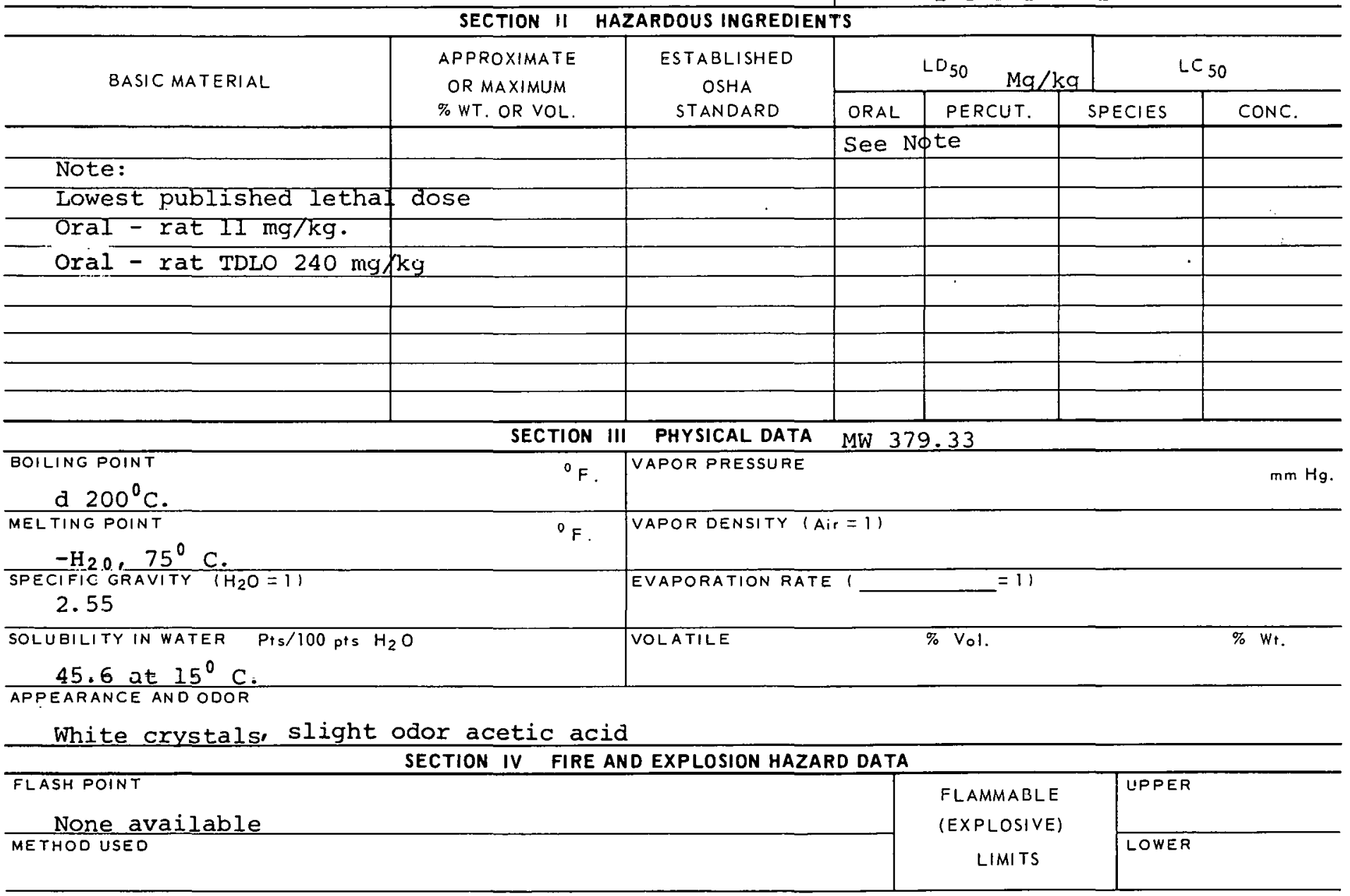

EXTINGUISHING MEDIA

\section{None available}

SPECIAL FIRE FIGHTING PROCEDURES

UNUSUAL FIRE AND EXPLOSION HALARUS

When heated, it emits acrid fumes. 


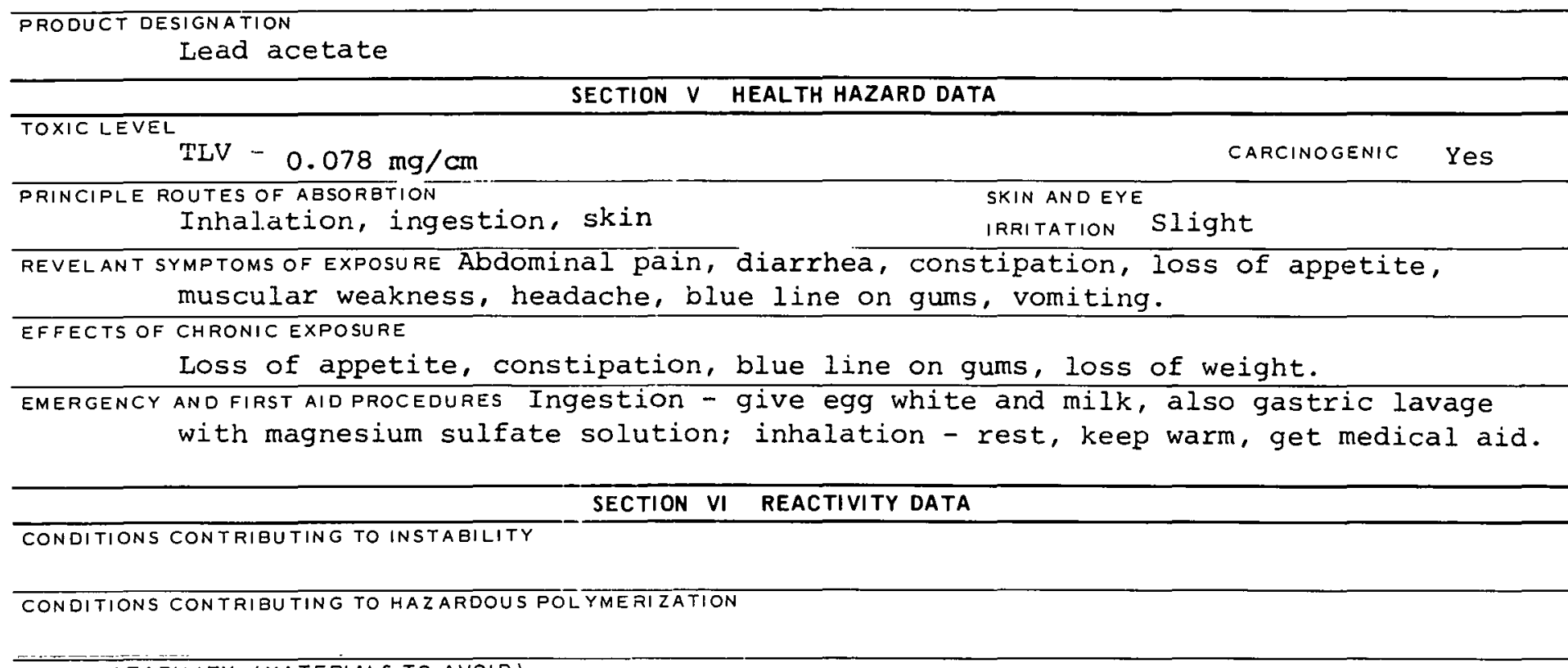

IIACOMCATADILITY (MATCMIALC TO A, OOID)

HAZARDOUS DECOMPOSITION PRODUCTS

SECTION VII SPILL OR LEAK PROCEDURES

STEPS TO BE TAKEN IN CASE MATERIAL IS RELEASED OR SPILLED

Add soda ash and sweep up into box for disposal.

WASTE DISPOSAL METHOD

SECTION VIII SPECIAL PROTECTION INFORMATION

\begin{tabular}{l|l}
\hline VENTILATION REQUIREMENTS LOCAL EXHAUST & $\begin{array}{l}\text { PROTECTIVE EQUIPMENT (SPECIFY TYPES) EYE } \\
\text { FaCe shield }\end{array}$ \\
\hline MEGHANISAL (GEMERAL) & $\begin{array}{l}\text { GLOVIS } \\
\text { Rubber }\end{array}$ \\
\hline STECIAL & $\begin{array}{l}\text { RESPIRATOR } \\
\text { With proper filter }\end{array}$ \\
\hline
\end{tabular}

OTHER PROTECTIVE EQUIPMENT

Self-contained respirator.

SECTION IX SPECIAL PRECAUTIONS

PRECAUTIONS TO BE TAKEN IN HANDLING AND STORAGE

\begin{tabular}{l|l}
\hline OTHER PRECAUTIONS & OATE \\
\hline SIGNATURE & OT
\end{tabular}

82-0102B 
CAS : 007758954

03-082-1802

PRODUCT DESIGNATION

Lead Chloride
OF 94500

MATERIAL SAFETY

DATA SHEET

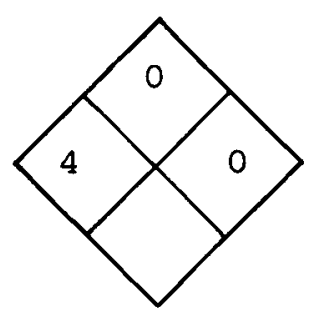

\section{SECTION I SOURCE AND NOMENCLATURE}

MANUFACTURER'S NAME

ADDRESS (NUMBER, STREET, CITY, STATE, ZIP CODE)

TRADE NAME AND SYNONYMS

CHEMICAL NAME AND SYNONYMS

Lead chloride

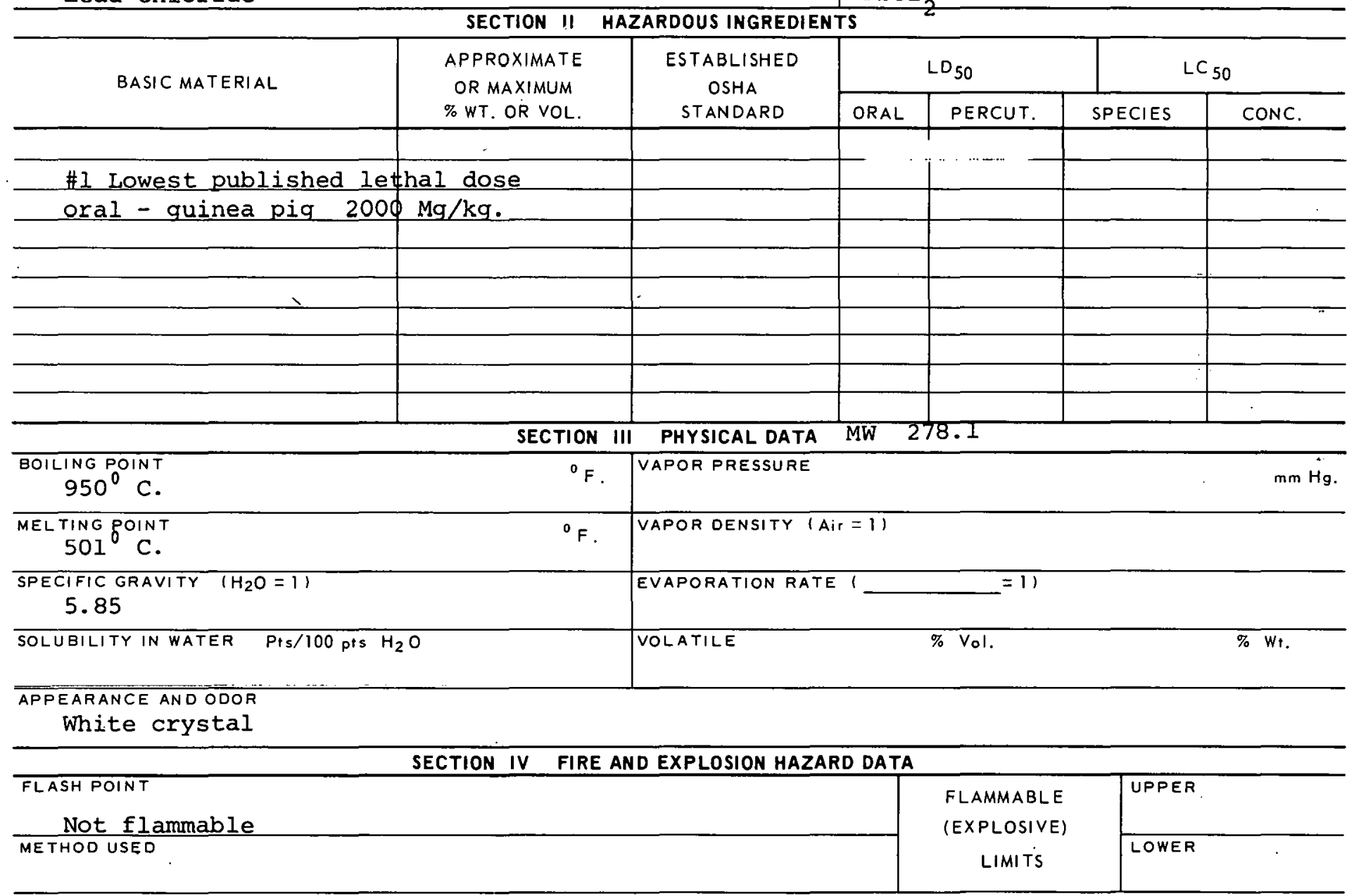

\begin{tabular}{l|l} 
& CHEMICAL FAMILY \\
& FORMULA \\
& $\mathrm{PbCl}_{2}$ \\
\hline
\end{tabular}
SECTION II HAZARDOUS INGREDIENTS

OR MAXIMUM

EXTINGUISHING MEDIA

$N / A$

SPECIAL FIRE FIGHTING PROCEDURES

$\mathrm{N} / \mathrm{A}$

UNUSUAL FIRE AND EXPLOSION HAZARDS

$\mathrm{N} / \mathrm{A}$ 


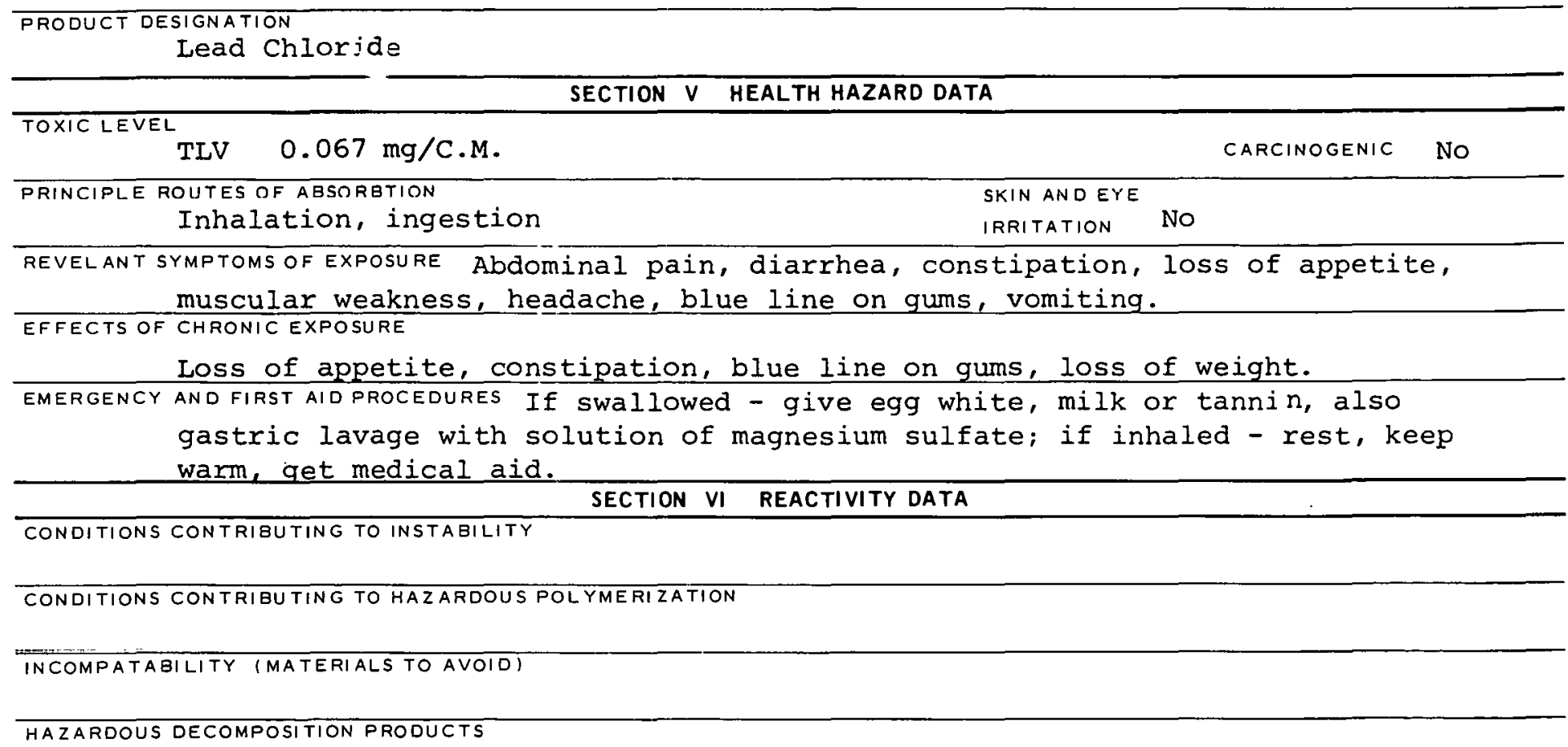

SECTION VII SPILL OR LEAK PROCEDURES

STEPS TO BE TAKEN IN CASE MATERIAL IS RELEASED OR SPILLED

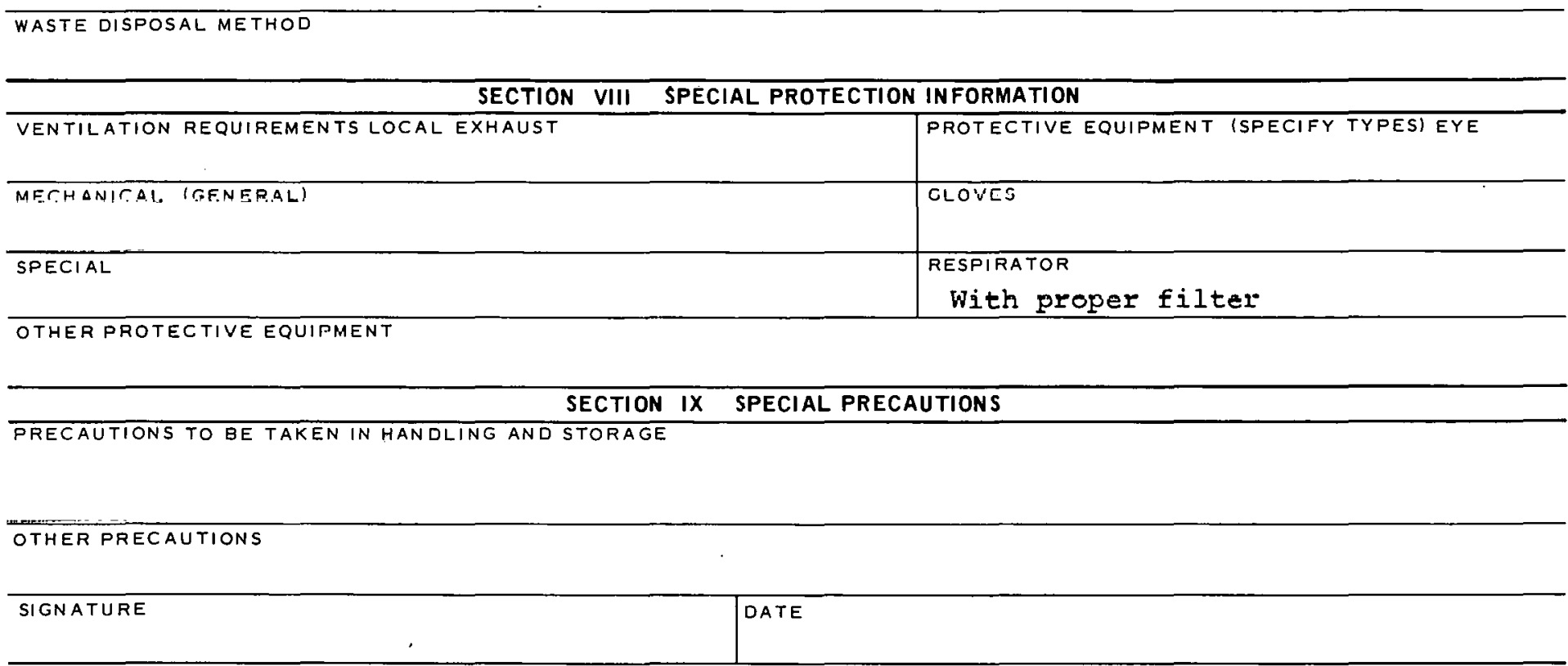


CAS: 001309600

\begin{tabular}{|l|}
\hline PRODUCT DESIGNATION \\
03-082-3002 \\
Lead Dioxide \\
\end{tabular}

OG 07000

\section{MATERIAL SAFETY \\ DATA SHEET}

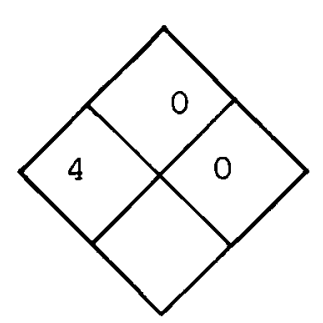

\section{SECTION I SOURCE AND NOMENCLATURE}

\section{MANUFACTURER'S NAME \\ Lead Dioxide}

ADDRESS (NUMBER, STREET, CITY, STATE, ZIP CODE)

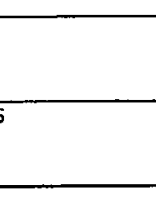

\begin{tabular}{l|l} 
& CHEMICAL FAMILY \\
& $\begin{array}{l}\text { FORMULA } \\
\mathrm{PbO}_{Z}\end{array}$ \\
\hline SECTION II HAZARDOUS INGREDIENTS
\end{tabular}
SECTION II HAZARDOUS INGREDIENTS
BASIC MATERIAL

$\mid$

APPROXIMATE

OR MAXIMUM \% WT. OR VOL.

EMERGENCY TELEPHONE NO.

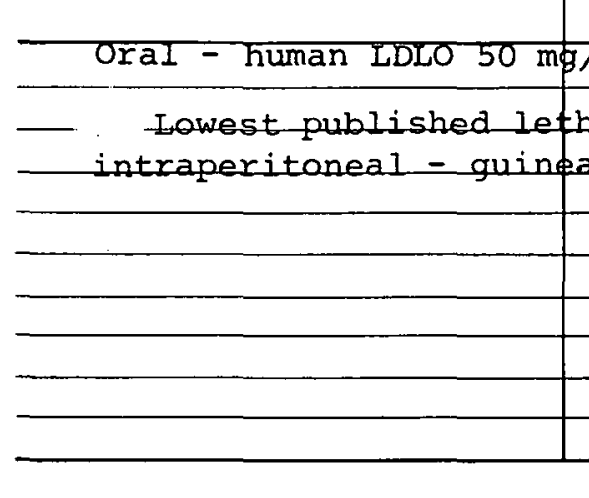

BOILING POINT

1 dose

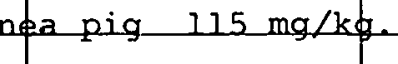

ESTABLISHED
OSHA
STANDARD

\begin{tabular}{|l|l|l|l|}
\hline \multicolumn{2}{|c|}{ LD $_{50}$} & \multicolumn{2}{c|}{ LC $_{50}$} \\
\hline ORAL & PERCUT. & SPECIES & CONC. \\
\hline
\end{tabular}

MELTING POINT

d $290^{\circ} \mathrm{C}$.

SPECIFIC GRAVITY $\left(\mathrm{H}_{2} \mathrm{O}=1\right)$

9.375

SOLUBILITY IN WATER Pts/100 pts $\mathrm{H}_{2} \mathrm{O}$

\section{Prs/ 100 pts $\mathrm{H}_{2} \mathrm{O}$}

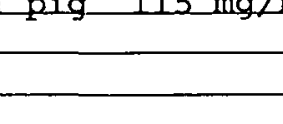

APPEARANCE AND ODOR

Brown crystals

\section{SECTION IV FIRE AND EXPLOSION HAZARD DATA}

\section{FLASH POINT}

Not flammable

\section{METHOD USED}

EXTINGUISHING MEDIA

\begin{tabular}{c|l} 
SECTION III PHYSICAL DATA MW 239.19 \\
\hline${ }^{\circ} \mathrm{F}$. & VAPOR PRESSURE. \\
\hline${ }^{\circ} \mathrm{F}$. & VAPOR DENGITY (Air $=11$ \\
& EVAPORATION RATE \\
& VOLATILE \\
&
\end{tabular}

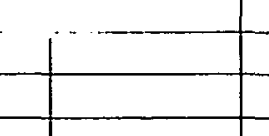

\begin{tabular}{l|l}
\hline & \\
\hline &
\end{tabular}

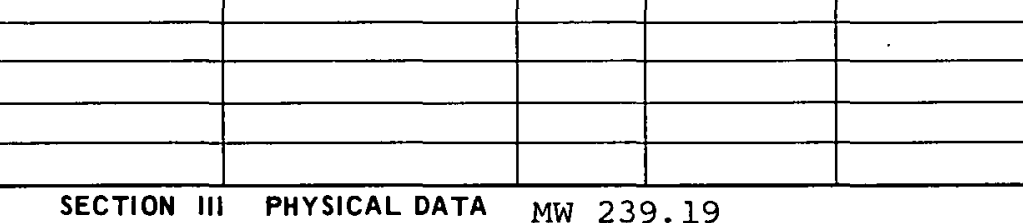




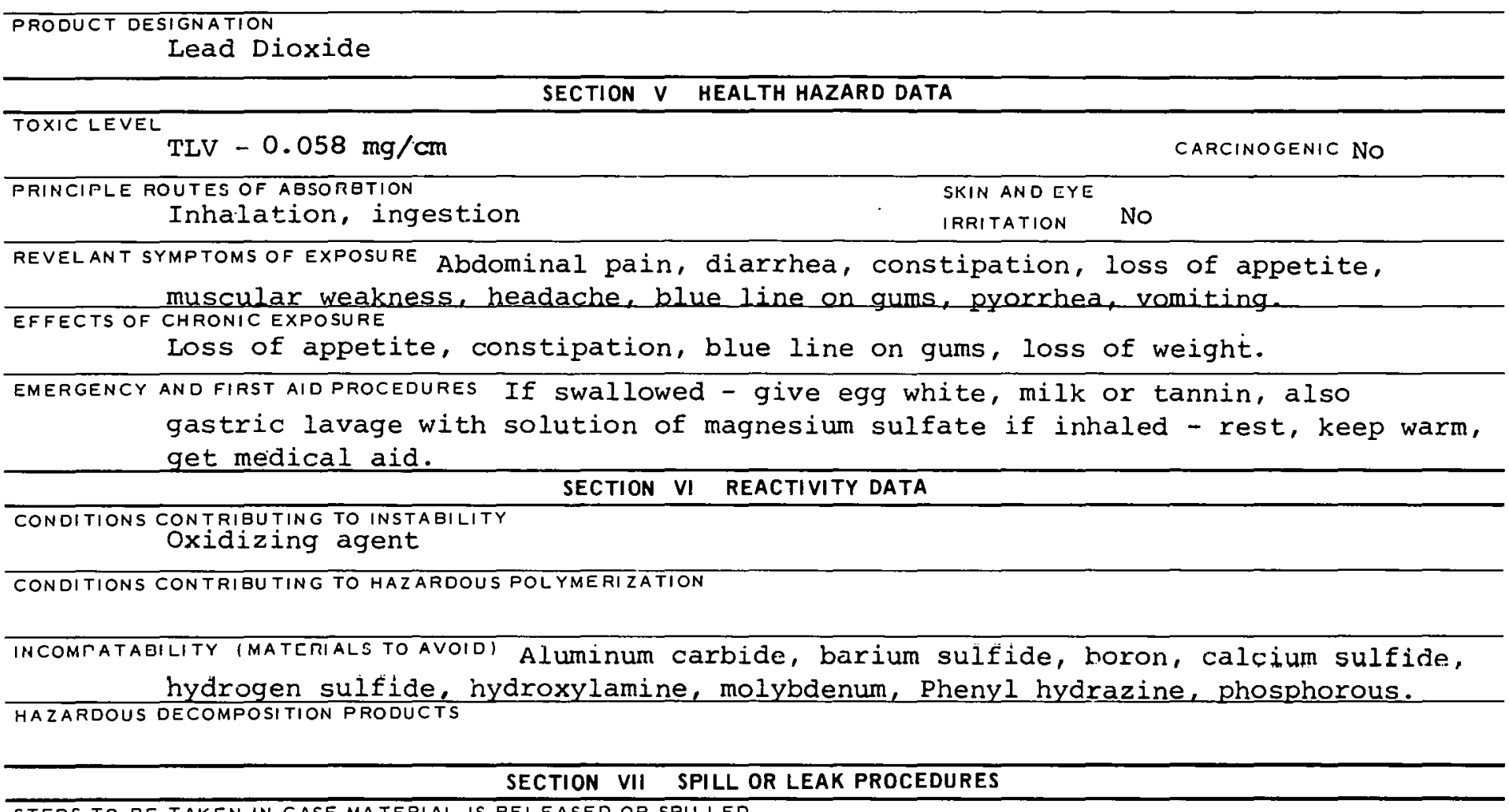
STEPS TO BE TAKEN IN CASE MATERIAL IS RELEASED OR SPILLED

\section{WASTE DISPOSAL METHOD}

SECTION VIII SPECIAL PROTECTION INFORMATION

\begin{tabular}{l|l}
\hline VENTILATION REQUIREMENTS LOCAL EXHAUST & PROTECTIVE EQUIPMENT (SPECIFY TYPES) EYE \\
\hline MFSHANITAI. IGFNFRAL! & GLOVLS \\
\hline SPEGIAL & $\begin{array}{c}\text { RESPIRATOR } \\
\text { With PIOPEI FIILEI }\end{array}$ \\
\hline
\end{tabular}

OTHER PROTECTIVE EQUIPMENT

SECTION IX SPECIAL PRECAUTIONS

PRECAUTIONS TO BE TAKEN IN HANDLING AND STORAGE 
CAS : 007439921

OF75250

\begin{tabular}{|c|} 
PRODUCT DESIGNATION \\
$03-082-4802$ \\
Lead Metal \\
\end{tabular}

\section{MATERIAL SAFETY \\ DATA SHEET}

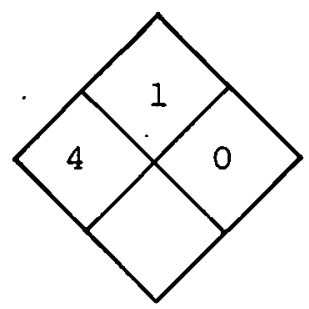

\section{SECTION I SOURCE AND NOMENCLATURE}

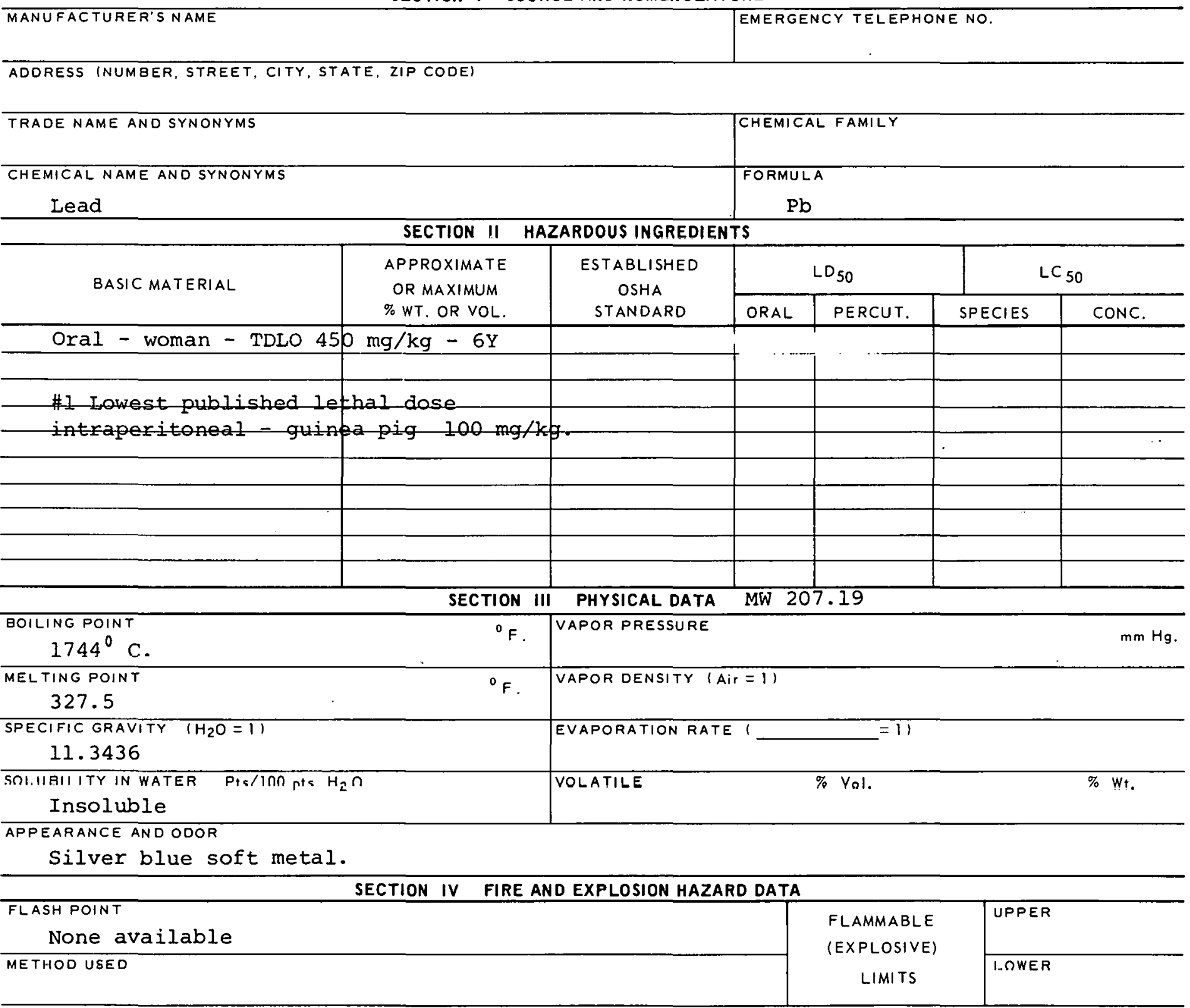

EXTINGUISHING MERIA

Not available

SPECIAL FIRE FIGHTING PROCEDURES

UNUSUAL FIRE AND EXPLOSION HAZARDS 
Lead Metal

\section{SECTION $V$ HEALTH HAZARD DATA}

\section{TOXIC LEVEL}

$\mathrm{TLV}-0.05 \mathrm{mg} / \mathrm{C} . \mathrm{M}$. PRINCIPLE ROUTES OF AESORBTION

Inhalation of dust or fume, ingestion SKIN AND EYE

IRRITATION
CARCINOGENIC $\mathrm{NO}$ No REVELANT SYMPTOMS OF EXPOSURE Abdominal pain, constipation, diarrhea, loss of appetite, muscular weakness, headache, line on gumes, pyorrhea. EFFECTS OF CHRONIC EXPOSURE

Loss of appetite, muscular weakness, loss of weight, blue line on gums. EMERGENCY AND FIRST AID PROCEOURES If swallowed - give egg white, milk or tannin, also gastric lavage with $1 \%$ solution of magnesium sulfate if inhaled - rest, keep warm, get medical aid. SECTION VI REACTIVITY DATA

CONDITIONS CONTRIBUTING TO INSTABILITY

CONDITIONS CONTRIQUY TING TO HAZARQQUS PQLYYMERIZATIQN

IN COMPATAARILITY (MATERIALS TO AVOID)

Ammonium nitrate, hydrogen pweroxide, zirconium. HAZARDOUS DECOMPOSITION PRODUCTS

SECTION VII SPILL OR LEAK PROCEDURES

STEPS TO BE TAKEN IN CASE MATERIAL IS RELEASED OR SPILLED

WASTE DISPOSAL METHOD

SECTION VIII SPECIAL PROTECTION INFORMATION

\begin{tabular}{l|l}
\hline $\begin{array}{l}\text { VENTILATIUN KEQUIREMENTS LOCAL EXHAUST } \\
\text { MECHANICAL IGENERAL) }\end{array}$ & $\begin{array}{l}\text { PROTECTIVE EQUIPMENT (SPECIFY TYPES) EYE } \\
\text { GUYYIES }\end{array}$ \\
\hline SFECIAL & $\begin{array}{l}\text { GI.NVFS } \\
\text { Rubber }\end{array}$ \\
& $\begin{array}{l}\text { PESPIRATOR } \\
\text { With proper fiIter }\end{array}$ \\
\hline
\end{tabular}

OTHER PROTECTIVE EQUIPMENT

SECTION IX SPECIAL PRECAUTIONS

PRECAUTIONS TO BE TAKEN IN HANOLING AND STORAGE

QTHER MAEGAUTIOHE

SIFN DTURE

UATE

$82-4802 B$ 


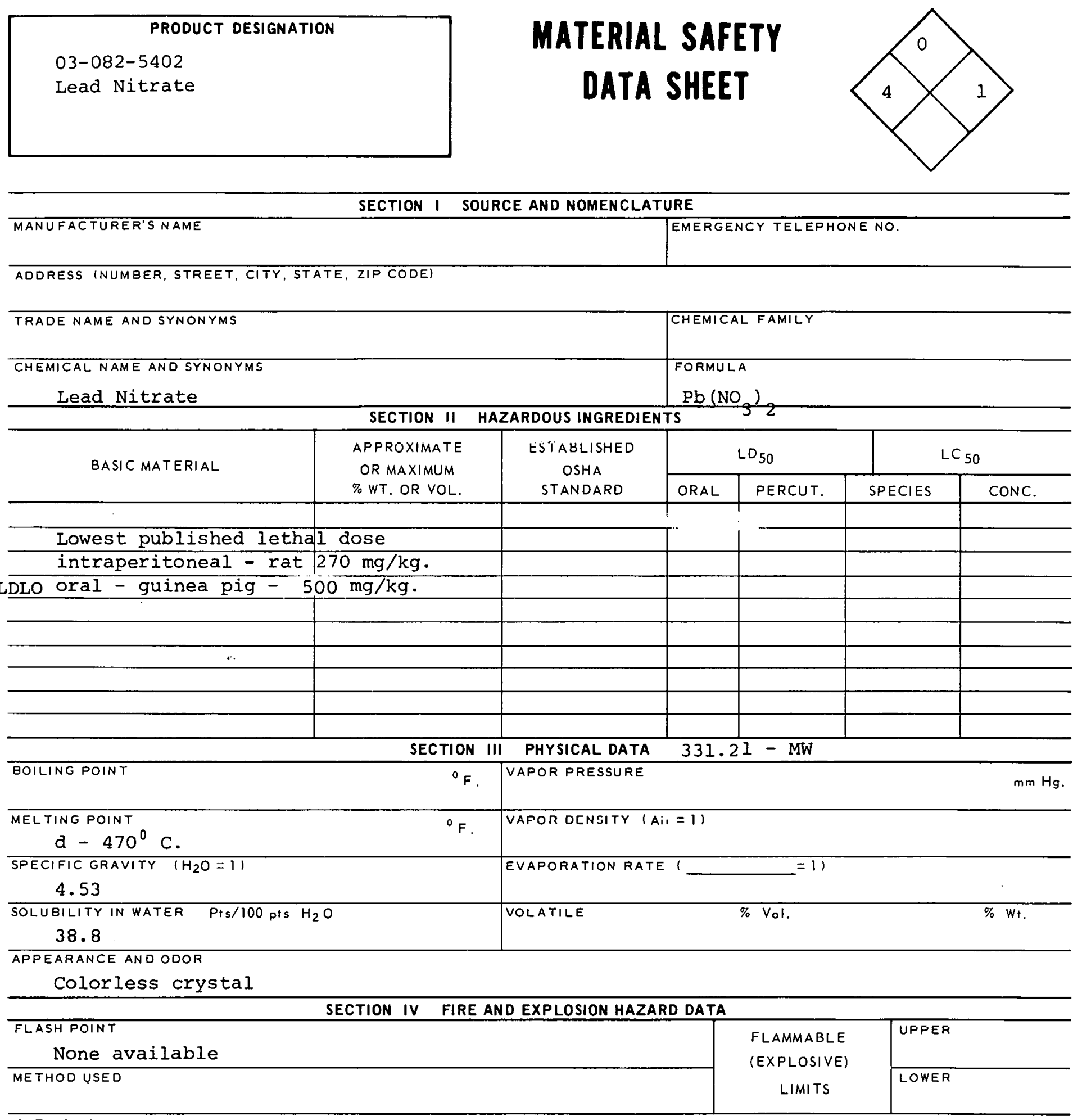

EXTINGUISHING MEDIA

Watcr

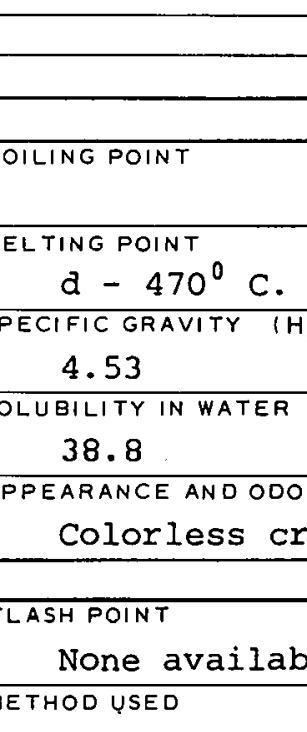

SPECIAL FIRE FIGHTING PROCEDURES breathing apparatus.

Flood with water early in fire. Wear self-contained UNUSUAL FIRE AND EXPLOSION HAZARDS

Decomposes at $410^{\circ} \mathrm{F}$. to give off toxic nitrogen oxide fumes. 


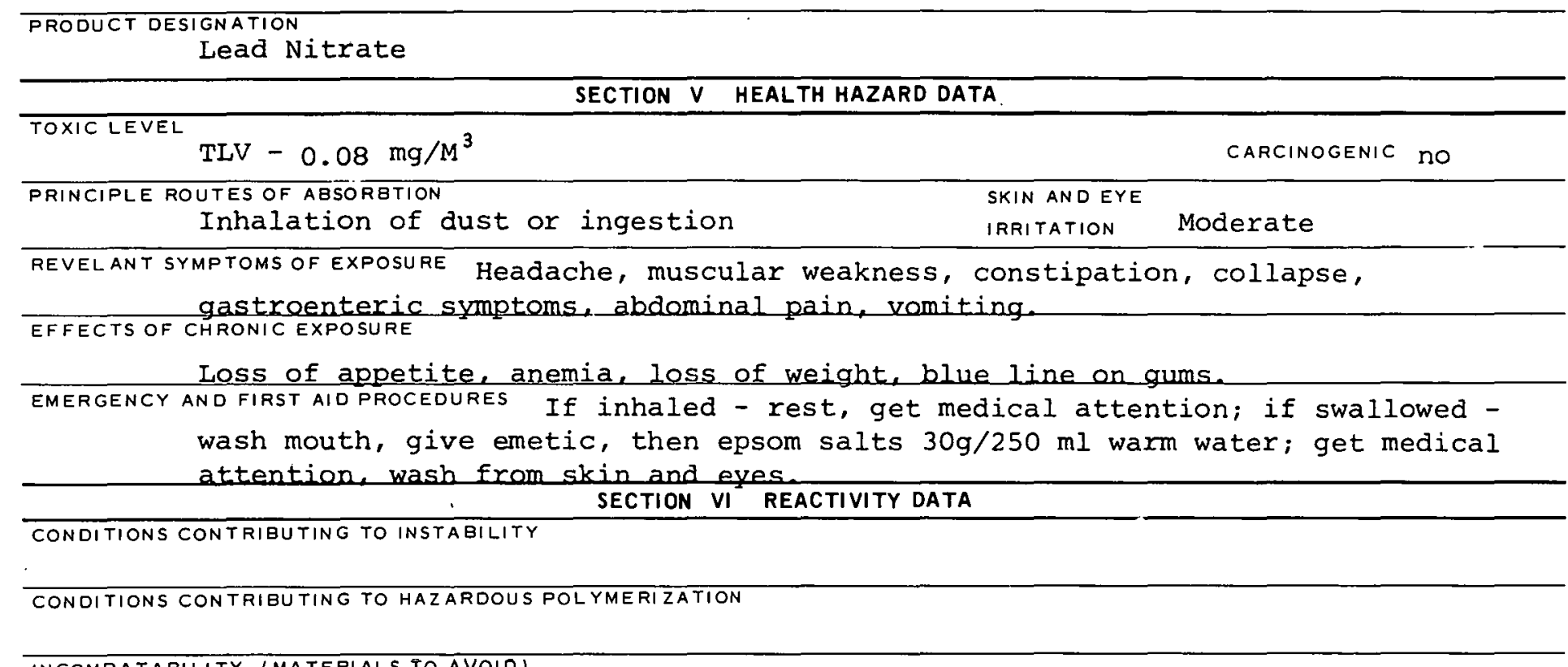

INTOMPATARII ITY (MATERIALS TO AVOIO)

Reacts with sparks on red hot carbon. HALARUUUS ÜELUMFUSITIUN PRUDUETS

SECTION VII SPILL OR LEAK PROCEDURES

STEPS TO BE TAKEN IN CASE MATERIAL IS RELEASED OR SPILLED

WASTE DISPOSAL'METHOD

SECTION VIII SPECIAL PROTECTION INFORMATION

\begin{tabular}{l|l}
\hline VENTILATION REQUIREMENTS LOCAL EXHAUST & $\begin{array}{l}\text { PROTECTIVE EQUIPMENT (SPECIFY TYPES) EYE } \\
\text { SafetY glaSSES }\end{array}$ \\
\hline MECHANICAL IGENERALI & GLOVES \\
\hline SPECIAL & $\begin{array}{l}\text { RESPIRATOR } \\
\text { W1th approprtate f1Iter }\end{array}$ \\
\hline
\end{tabular}

OTHER PROTECTIVE EQUIPMENT

SECTION IX SPECIAL PRECAUTIONS

PRECAUTIONS TO BE TAKEN IN HANDLING AND STORAGE

OTHER PRECAUTIONS

SIGNATURE

DATE

$82-5402 B$ 
CAS : 001317368

PRODUCT DESIGNATION

03-082-6002

Lead oxide (mono)
OGI 7500

MATERIAL SAFETY

DATA SHEET

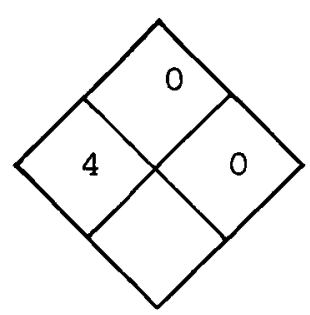

SECTION I SOURCE AND NOMENCLATURE

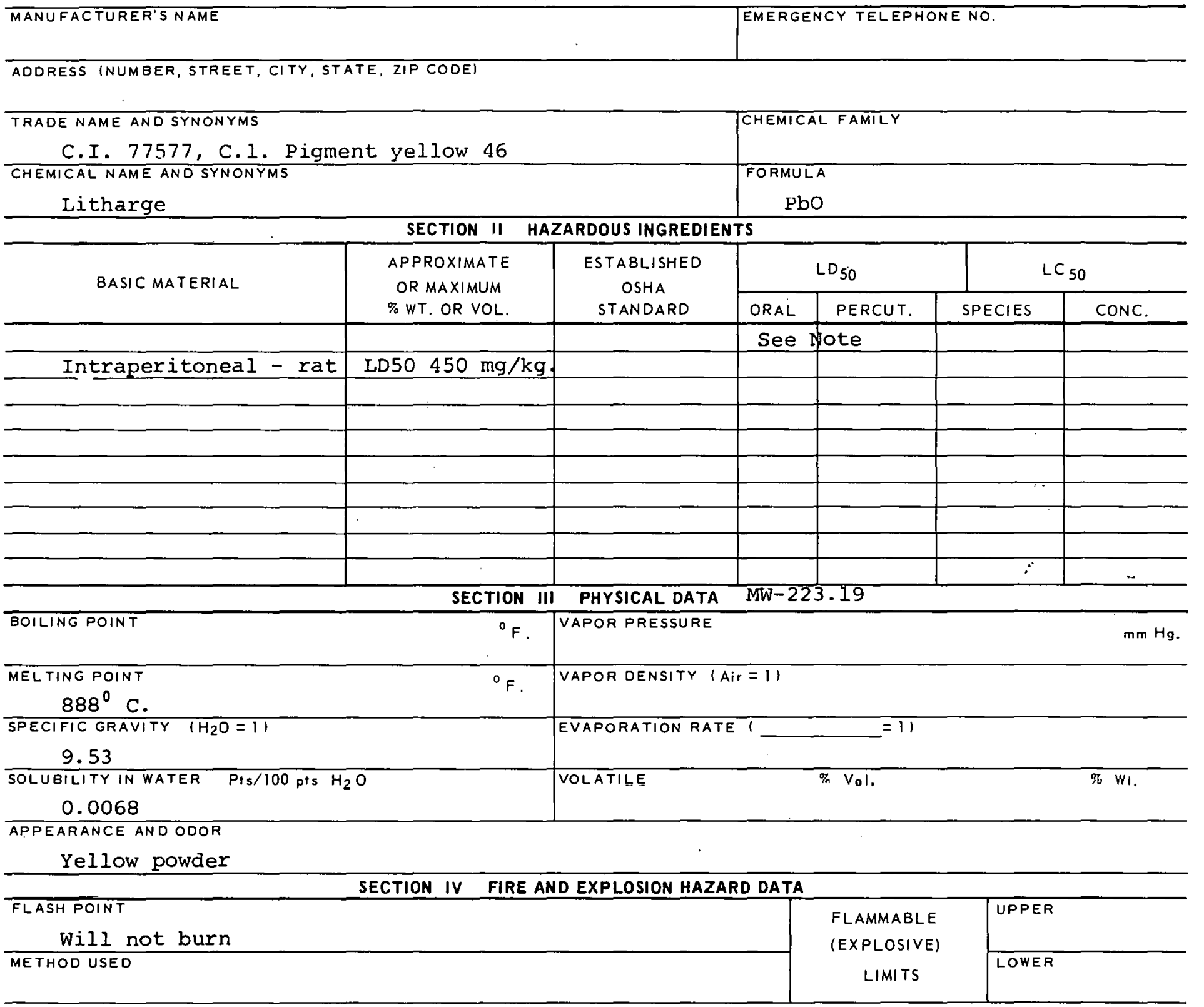

EXTINGUISHING MEDIA

Flood with water

SPECIAL FIRE FIGHTING PROCEDURES

UNUSUAL FIRE AND EXPLOSION HAZARDS 


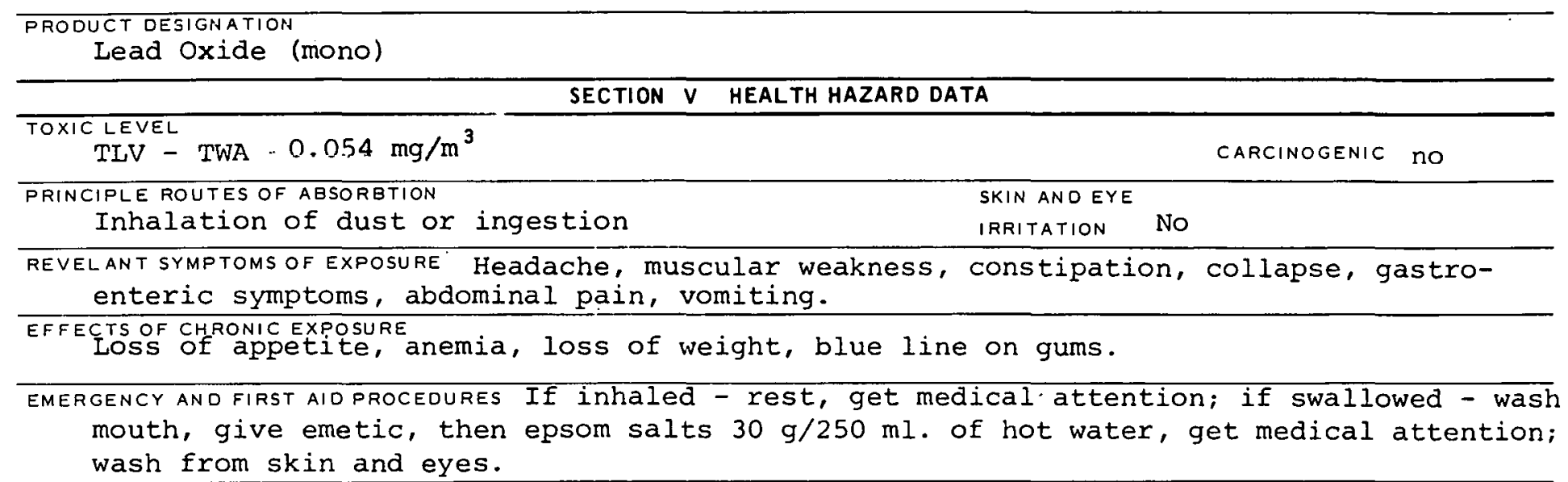

\begin{tabular}{l}
\hline SECTION VI REACTIVITY DATA \\
\hline CONDITIONS CONTRIBUTING TO INSTABILITY \\
\hline INCOMPITIONS CONTRIBUTING TO HAZARDOIIS POLYMERIZATION
\end{tabular}

INCOMPATABILITY (MATERIALS TO AVOID)

HAZAROOUS DECOMPOSTTTON PHOUUL I

SECTION VII SPILL OR LEAK PROCEDURES

STEPS TO BE TAKEN IN CASE MATERIAL IS RELEASED OR SPILLED

WASTE DISPOSAL METHOD

\begin{tabular}{l|l}
\hline \multicolumn{1}{|c|}{ SECTION VIII SPECIAL PROTECTION INFORMATION } \\
\hline $\begin{array}{l}\text { VENTILATION REQUIREMENTS LOCAL EXHAUST } \\
\text { MECHANICAL IGENERALI }\end{array}$ & $\begin{array}{c}\text { PROTECTIVE EQUIPMENT ISPECIFY TYPFS! EYE } \\
\text { FaGE shIOLA }\end{array}$ \\
\hline $\begin{array}{l}\text { SPECIAL } \\
\text { OTHER FROTFCTIVE EQUIPMENT } \\
\text { FaCE ShIEId }\end{array}$ & $\begin{array}{l}\text { RFSPIRATRR } \\
\text { With appropriate filter }\end{array}$ \\
\hline
\end{tabular}

PRECAUTIONS TO BE TAKEN IN HANDLING AND STORAGE

OTHER PRECAUTIONS

\begin{tabular}{l|l}
\hline GLNATURE & DATC
\end{tabular}

$82-6002 B$ 
CAS: 007446142

OG43750

\section{PRODUCT DESIGNATION}

03-082-7202

Lead Sulfate

MATERIAL SAFETY

DATA SHEET

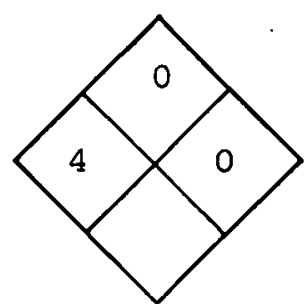

SECTION I SOURCE AND NOMENCLATURE

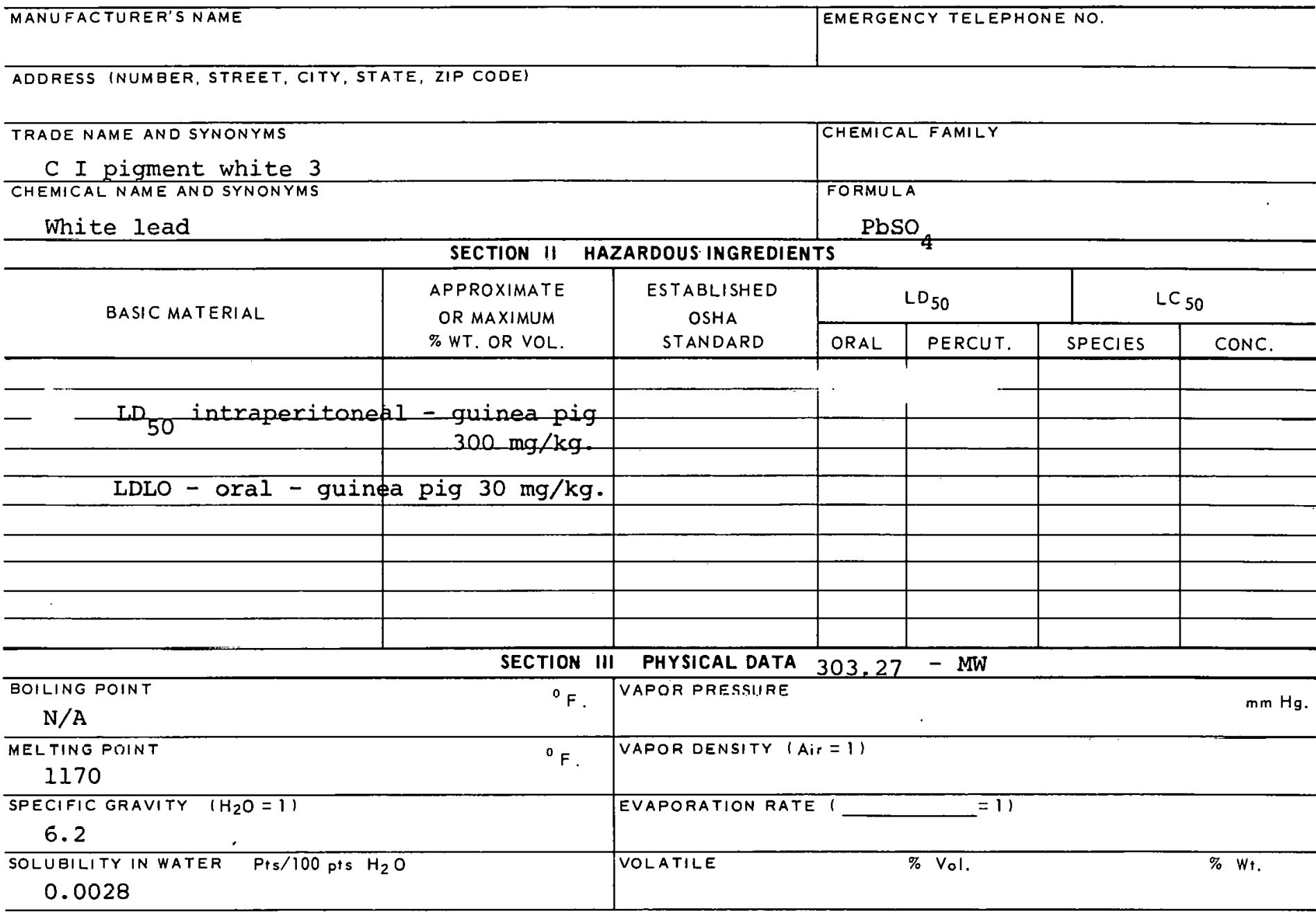

APPEARANCE AND ODOR

White powder

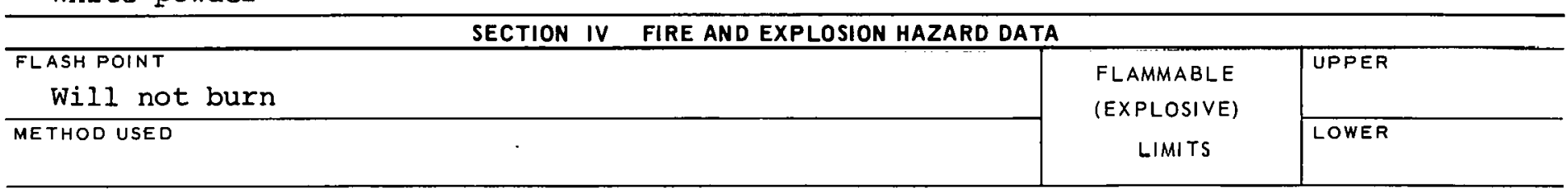

EXTINGUISHING MEDIA

Water

SPECIAL FIRE FIGHTING PROCEDURES

$N / A$

UNUSUAL FIRE AND EXPLOSION HAZARDS

$\mathrm{N} / \mathrm{A}$ 


$$
\text { TLV }-0.073 \mathrm{mg} / \mathrm{m}^{3}
$$

PRINCIPLE ROUTES OF ABSORBTION

Inhalation of dust or ingestion
CARCINOGENIC

no

SKIN AND EYE

IRRITATION NO

REVELANT SYMPTOMS OF EXPOSURE Headache, muscular weakness, constipation, collapse, gastroenteric symptoms, abdominal pain, vomiting

CHRONIC EXṔOSURE

Loss of appetite, anemia, loss of weight, blue line on gums.

ANO FIRST AID PROCEDURES If inhaled - rest, get medical attention.

EMERGENCY AND FIRST AID PROCEDURES If inhaled - rest, get medical attention. If swallowed wash mouth, give emetic, then epsom salts $30 \mathrm{~g} / 250 \mathrm{ml}$. hot water, get medical attention: wash from skin and eyes. SECTION VI REACTIVITY DATA

CONDITIONS CONTRIBUTING TO INSTABILITY

CONDITIONS CONTRIBUTING TO HAZARDOUS POLYMERIZATION

INISOMPATAR!L!TY IMATFRIAI S TO AVRIRI

Reacts with lithium carbide exothermally.

HAZARDOUS OECOMNUSIIIUN RHUUUETS

SECTION VII SPILL OR LEAK PROCEDURES

STEPS TO BE TAKEN IN CASE MATERIAL IS RELEASED OR SPILLED

WASTE DISPOSAL METHOD

\section{SECTION VIII SPECIAL PROTECTION INFORMATION}

\begin{tabular}{|c|c|}
\hline VẼNTILATION REQUIREMENTS LOCAL EXHAUST & $\begin{array}{l}\text { PROTECTIVE EQUIPMENT (SPECIFY TYPES) EYE } \\
\text { Face shield }\end{array}$ \\
\hline $\begin{array}{l}\text { MECHANICAL (GENERAL) } \\
\text { - }\end{array}$ & GLOVES \\
\hline SHECIAL & $\begin{array}{l}\text { REJPIRATOR } \\
\text { With appropriate filter }\end{array}$ \\
\hline
\end{tabular}

OTHER PROTECTIVE EQUIPMENT

SECTION IX SPECIAL PRECAUTIONS

PRECAUTIONS TO BE TAKEN IN HANDLING AND STORAGE 
CAS:001302303 $\Omega$

\begin{tabular}{|c|}
\hline PRODUCT DESIGNATION \\
$03-084-1003$ \\
Lithium Aluminum Hydride \\
\hline
\end{tabular}

BD01000

\section{MATERIAL SAFETY \\ DATA SHEET}

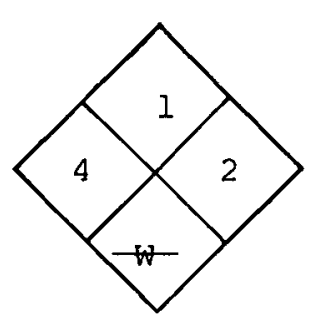

\section{SECTION I SOURCE AND NOMENCLATURE}

ADDRESS (NUMBER, STREET, CITY, STATE, ZIP CODE)

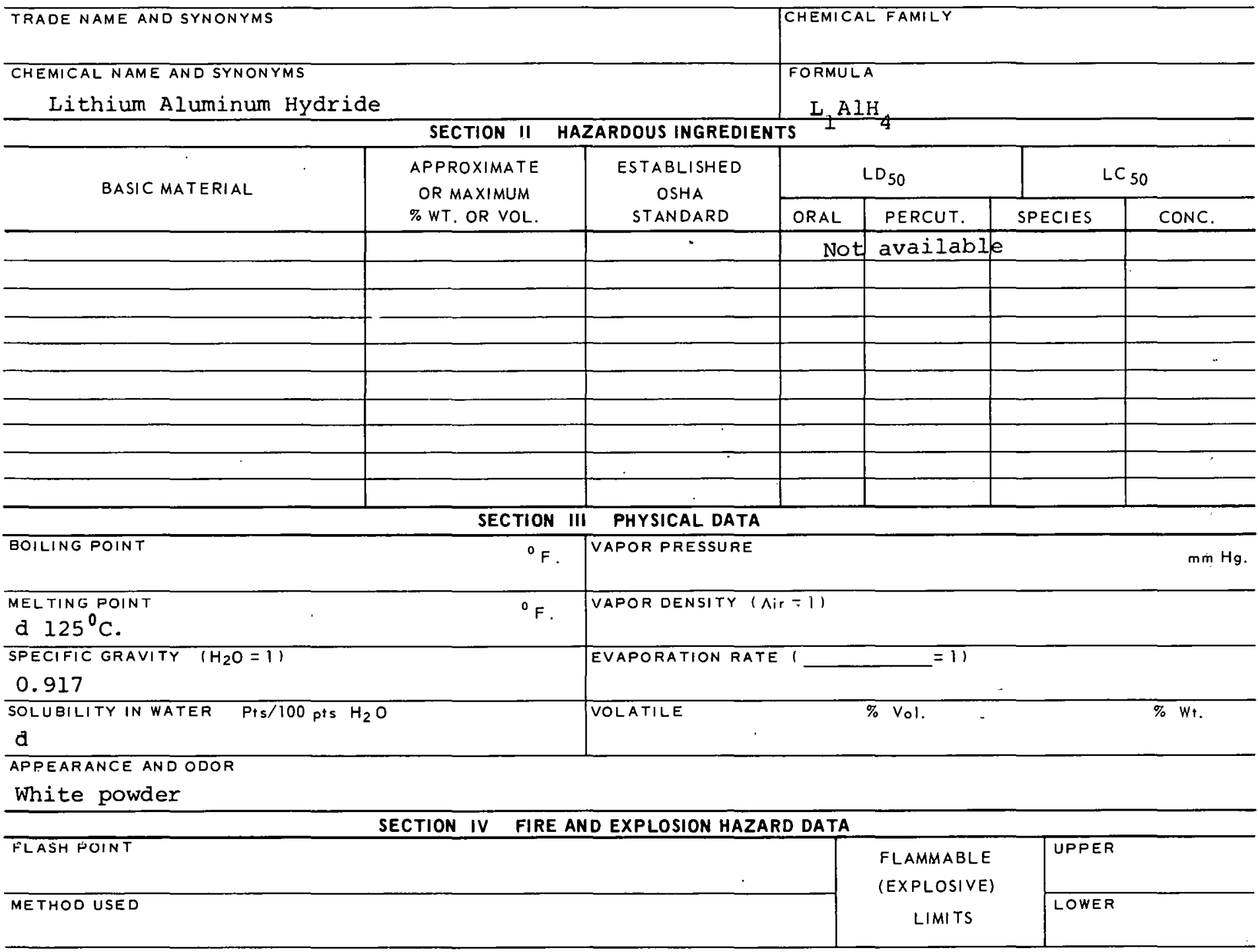

EXTINGUISHING MEDIA

Suitable dry chemical

SPECIAL FIRE FIGHTING PROCEDURES

Do not use water. Wear full protective clothing.

UNUSUAL FIRE AND EXPLOSION HAZARDS

Decomposes at 257 to form LiH, aluminum and hydrogen gas. 
Lithium Aluminum Hyaride SECTION $V$ HEALTH HAZARD DATA

\section{TOXIC LEVEL}

TLV - TWA $0.119 \mathrm{mg} / \mathrm{M}^{3}$

PRINCIPLE ROUTES OF ABSORBTION

Inhalation of dust, ingestion

SKIN AND EYE

IRRITATION

Salivation, nausea, vomiting, diarrhea, loose or bloody stools, irritation of skin and eyes.

EFFECTS OF CHRONIC EXPOSURE

Emphysema, kidney damage.

EMERGENCY AND FIRST AIO PROCEDURES Inhalation - rest, keep warm, get medical attention. If swallowed - wash mouth, drink water, then 1\% acetic acid, get medical attention. Wash skin and eyes.

SECTION VI REACTIVITY DATA

CONDITIONS CONTRIBUTING TO INSTABILITY

CONDITIONS CONTRIBUTING TO HAZARDOUS POLYMERIZATION

INCOMPATABILITYY (MATERIALS TO AVUIO)

Violent reaction with oxidizing agents,

HAZAHUUUS UELUMMUSH IIN F MEDUETJ

Produces hydrogen.

SECTION VII SPILL OR LEAK PROCEDURES

STEPS TO BE TAKEN IN CASE MATERIAL IS RELEASED OR SPILLED

SECTION VIII SPECIAL PROTECTION INFORMATION

\begin{tabular}{l|l}
\hline VENTILATION REQUIREMENTS LOCAL EXHAUST & $\begin{array}{l}\text { PROTECTIVE EQUIIPMFNT (SPECIFY TYPES) EYE } \\
\text { FaCO EhIQId }\end{array}$ \\
\hline MECHANICAL IGENERAL) & $\begin{array}{l}\text { GLOVES } \\
\text { Rubber }\end{array}$ \\
\hline GPEGIAL & RESPIRATOR \\
With appropriate filter
\end{tabular}

OTHER PROTECTIVE EQUIPMEN T

Face shield and protective clothing.

\section{SECTION IX SPECIAL PRECAUTIONS}

PRECAUTIONS TO BE TAKEN IN HANDLING AND STORAGE

OTHER PRECAUTIONS

SIGNATURE

DATE

$84-1003 B$ 


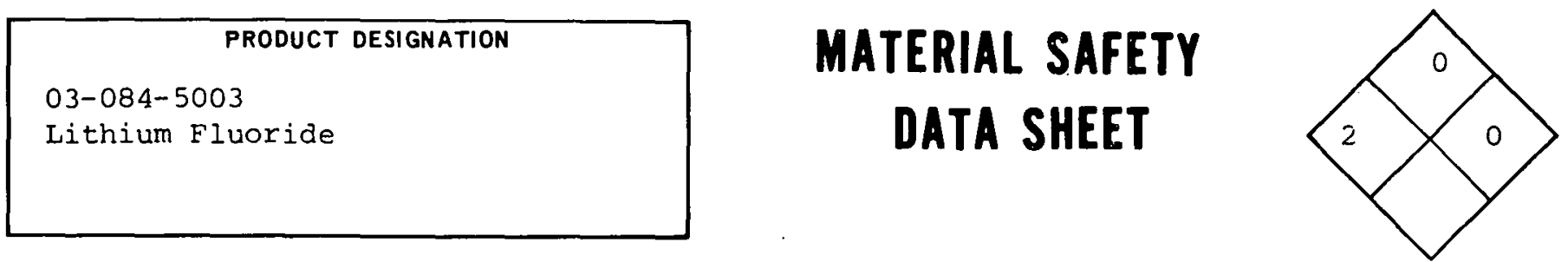

\section{SECTION I SOURCE AND NOMENCLATURE}

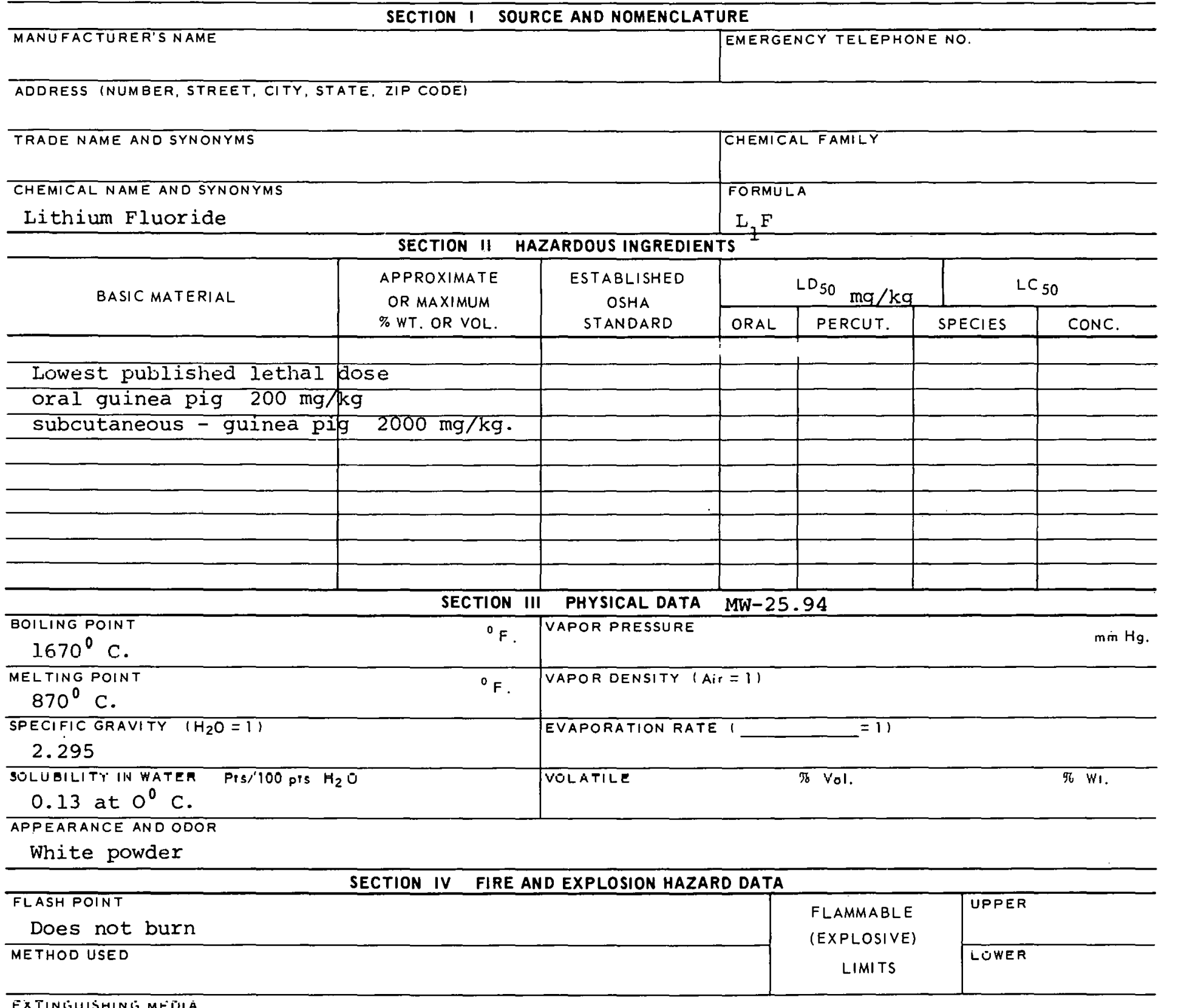

EXTINGUISHING MEDIAA

SPECIAL FIRE FIGHTING PROCEDURES

May produce HF with water.

IJNIJSUALI. FIRE AND EXPLOSION HATARDS

Wear self-contained mask. 


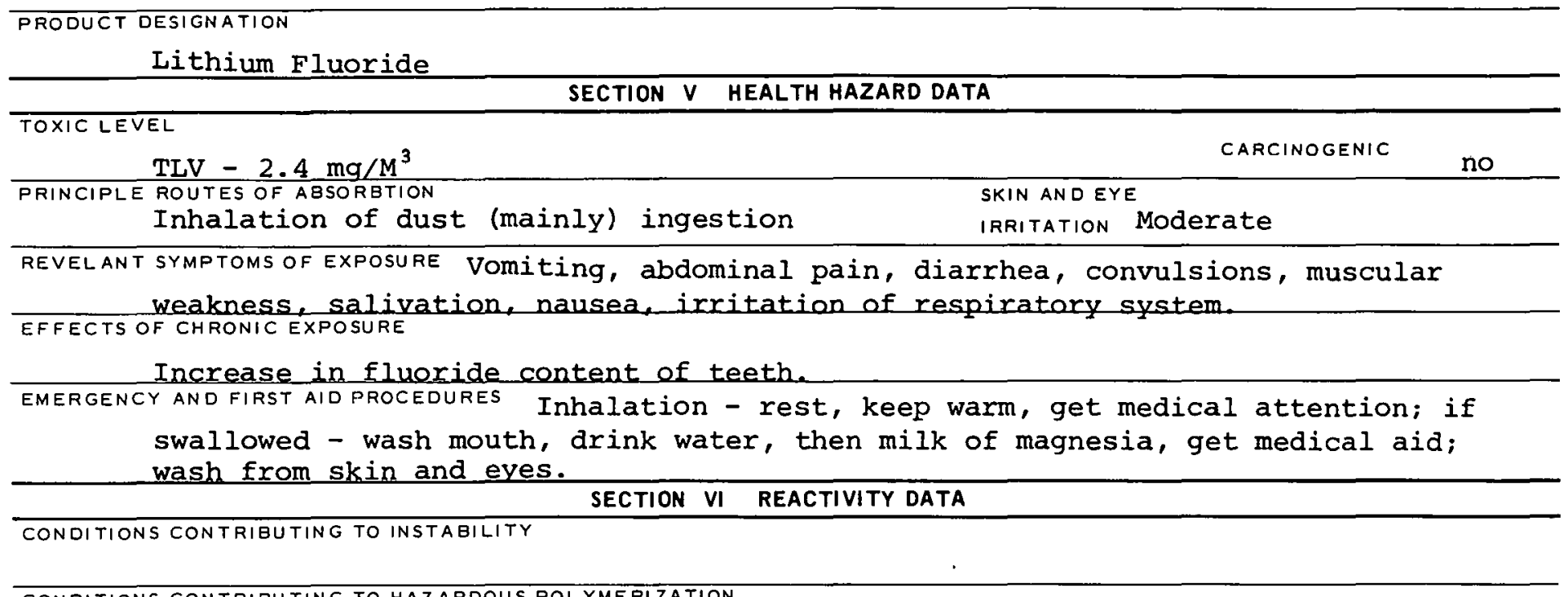

CONDITIONS CONTRIRUTING TO HAZARDOUS POLYMERIZATION

INCOMPATABILITY (MATERIALS TO AVOID)

May prodince HF with water.

IIAZARDOUS UECUMHUSIIIUN RRULUITS

SECTION VII SPILL OR LEAK PROCEDURES

STEPS TO BE TAKEN IN CASE MATERIAL IS RELEASED OR SPILLED

WASTE DISPOSAL METHOD

SECTION VIII SPECIAL PROTECTION IN FORMATION

\begin{tabular}{l|l}
\hline VENTILATION REQUIREMENTS LOCAL EXHAUST & $\begin{array}{l}\text { PROTECTIVE EQUIPMENT (SPECIFY TYPESIEYE } \\
\text { FaOC chICld }\end{array}$ \\
\hline MECHANICAL IGENERAL) & GLOVES \\
\hline ECECIAL.. & $\begin{array}{l}\text { RESPIRATOR } \\
\text { With proper filter }\end{array}$ \\
\hline
\end{tabular}

OTHER PROTECTIVE EQUIPMENT

Face shield and protective clothing. SECTION IX SPECIAL PRECAUTIONS

PRECAUTIONS TO BE TAKEN IN HANDLING AND STORAGE

OTHER PRECAUTIQNS

SIGNATURE

DATE

84-5003B 


\section{PRODUCT DESIGNATION \\ 03-084-5953 \\ 03-084-6003 \\ Lithium Hydroxide

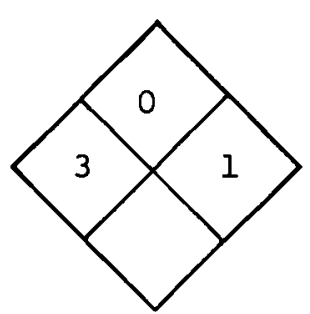

SECTION I SOURCE AND NOMENCLATURE

\begin{tabular}{l} 
MANUFACTURER'S NAME \\
\hline AODRESS INUMBER, STREET, CITY, \\
TRADE NAME AND SYNONYMS \\
CHEMICAL NAME AND SYNONYMS \\
LithiUM HYdYOXIde
\end{tabular}

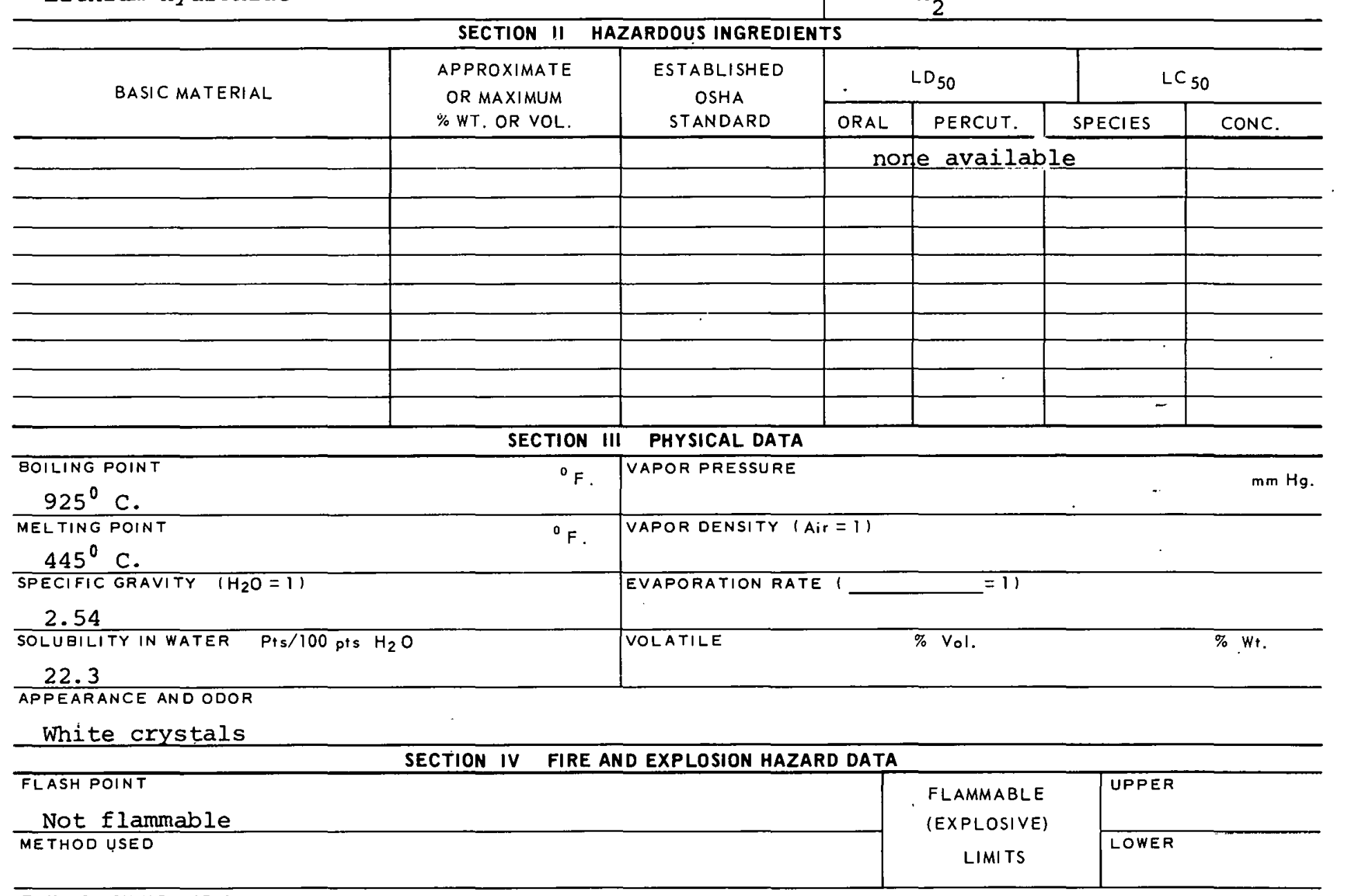

EXTINGUISHING MEDIA

Water

SPECIAL FIRE FIGHTING PROCEDURES

$\mathrm{N} / \mathrm{A}$

UNUSUAL FIRE AND EXPLOSION HAZARDS

$\mathrm{N} / \mathrm{A}$ 
Not available $2 \mathrm{mg} / \mathrm{C} . \mathrm{M}$. (NaOH) PRINCIPLE ROUTES OF ABSORETION

PRINCIPLE ROUTES OF ABSORATION
Inhalation, ingestion

CARCINOGENIC nO

REVELANT SYMPTOMS OF EXPOSURE Severe attack of membranes in digestive tract and stomach, anorexia, nausea, vomiting, diarrhea, salivation, irritation of skin and eyes. EFFECTS OF CHRONIC EXPOSURE

Kidney damage. EMERGENCY AND FIRST AIDPROCEDURES Wash from skin and eyes - if swallowed - wash mouth, drink water, then li vinegar, get medical aid, if inhaled - rest, keep warm, get medical aid.

\section{SECTION VI REACTIVITY DATA}

CONDITIONS CONTRIBUTING TO INSTABILITY

CONDITIONS CONTRIEUTING TO HAZARDOUS POLYMERIZATION

INCOMPATABILITY (MATERIALS TO AVOIU)

Reacts with acials

HAZARDOUS DECOMPOSITION PRODUCTS

SECTION VII SPILL OR LEAK PROCEDURES

STEPS TO BE TAKEN IN CASE MATERIAL IS RELEASED OR SPILLED

WASTE OISPOSAL METHOD

SECTION VIII SPECIAL PROTECTION INFORMATION

\begin{tabular}{l|l}
\hline VENTILATION REQUIREMENTS LOCAL EXHAUST & $\begin{array}{l}\text { PROTECTIVE EQUIPMENT (SPECIFY TYPES) EYE } \\
\text { FaCe shield }\end{array}$ \\
\hline MECHANICAL IGENERAL) & $\begin{array}{l}\text { GLOVES } \\
\text { RUbber }\end{array}$ \\
\hline SPECIAL & RESPIRATOR \\
& With proper filter \\
\hline
\end{tabular}

OTHER PROTECTIVE EQUIPMENT

Protective clothing

SECTION IX SPECIAL PRECAUTIONS

PRECAUTIONS TO BE TAKEN IN HANDLING AND STORAGE

OIHER PRECAUIIONS

SIGNATURE

$84-6003 B$ 


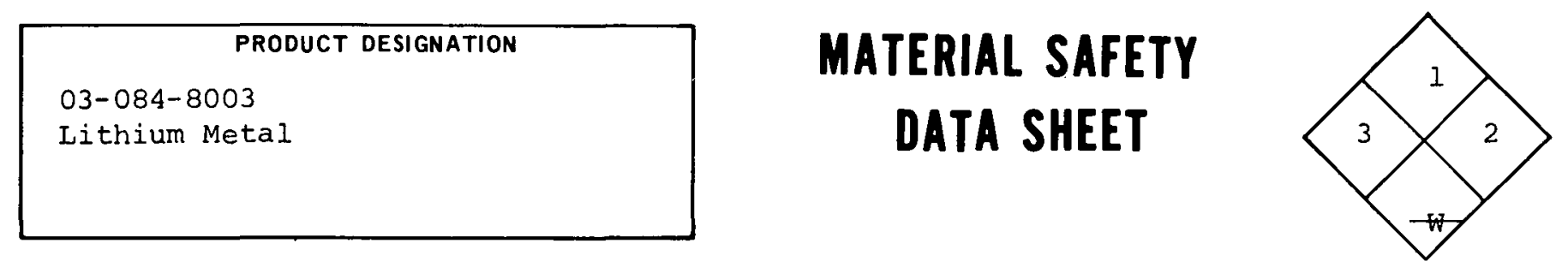

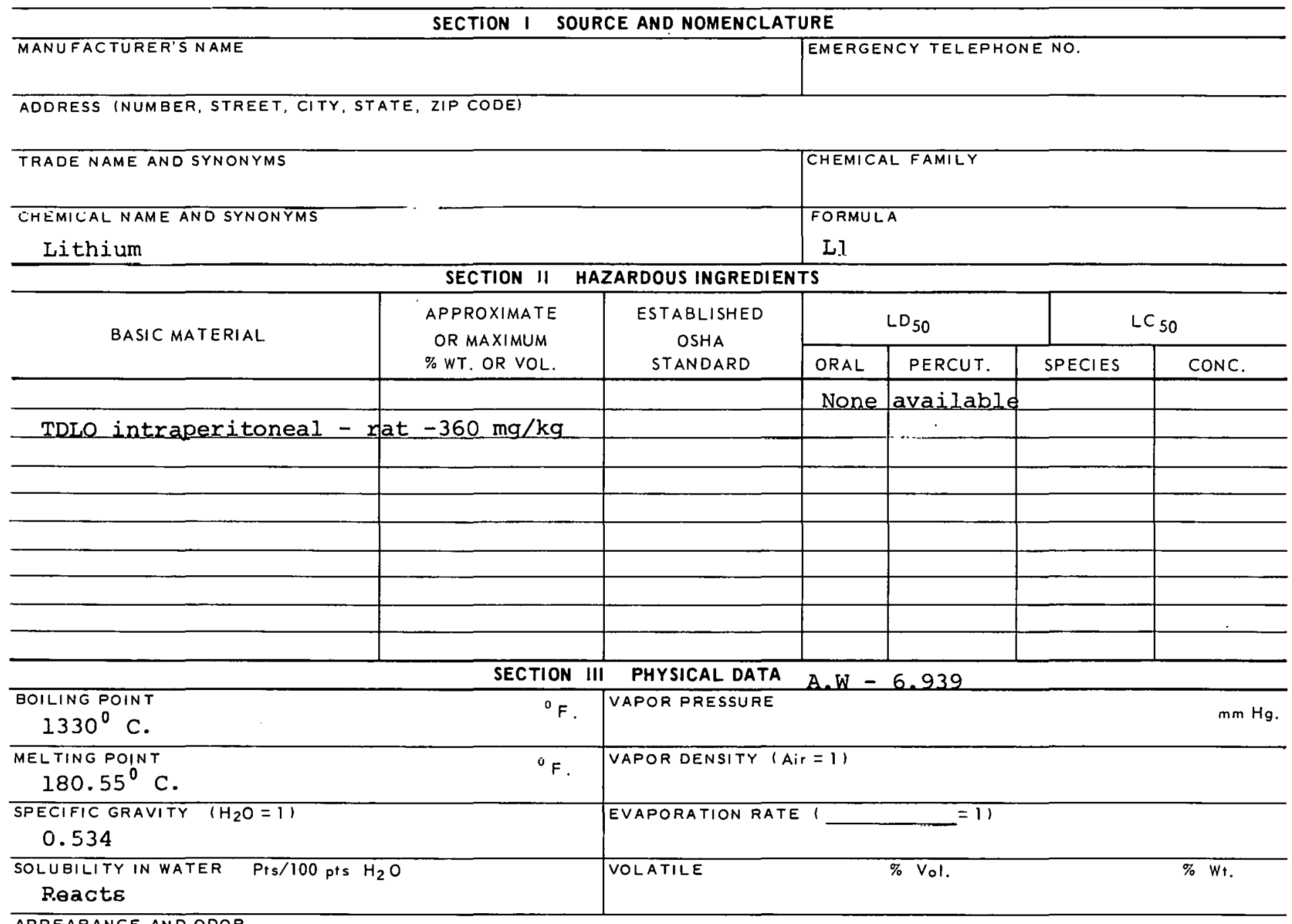
APPEARANCE AND ODOR

Silver metal

\begin{tabular}{l|c|c}
\hline \multicolumn{2}{c}{ SECTION IV } & FIRE AND EXPLOSION HAZARD DATA \\
\hline FLASH FOINT & FLAMMABLE & UPPER \\
\hline METHOD USED & (EXPLOSIVE) & LIMITS \\
& LOWER & \\
\hline
\end{tabular}

EXTINGUISHING MEDIA

Use dry graphite, soda ash or sodium chloride.

SPECIAL FIRE FIGHTING PROCEDURES

Wear protective clothing.

UNUSUAL FIRE AND EXPLOSION HAZARDS

Reacts with water to produce hydrogen gas. 
TOXIC LEVEL

Not available

PRINCIPLE ROUTES OF ABSORBTION

Inhalation of fumes, ingestion (mainly)
Teratogen

SKIN AND EYE

IRRITATION
CARCINOGENIC NO

Severe

REVELANT SYMPTOMS OF EXPOSURE Salivation, nausea, vomiting, diarrhea, severe internal attack, extreme irritation of respiratory system, severe skin and eye attack.

EFFECTS OF CHRONIC EXPOSURE Emphysema, kidney damage.

EMERGENCY AND FIRST AID PROCEDURES Inhalation - rest, keep warm, get medical aid; if swallowed wash mouth, drink water, then lo vinegar, get medical aid; wash skin and eyes, get medical aid.

\section{SECTION VI REACTIVITY DATA}

CONDITIONS CONTRIBUTING TO INSTABILITY

CONDITIONS CONTRIBUTING TO HAZARDOUS POLYMERIZATION

INCOMPATABILITY (MATERIALSTOAVOIO)

Reacts rapidly with water, fluorinated hydrocarbons.

AAZARDOUS DECOMPOSITION PRODUCTS

Produces hydrogen gas when it reacts with water. SECTION VII SPILL OR LEAK PROCEDURES

STEPS TO BE TAKEN IN CASE MATERIAL IS RELEASED OR SPILLED

WASTE DISPOSAL METHOO

SECTION VIII SPECIAL PROTECTION INFORMATION

VENTII,ATION REQUIREMENTS LOCAL EXHAUST TECTIVE EQUIPMENT (SPECIFY TYPES) EYE Face shield

MECHANICAL (GENERAL) GLOVES

Leather

SRECIAL RESPIRATOR

OTHER PROTECTIVE EQUIPMENT With proper filter

Protective clothing. SECTION IX SPECIAL PRECAUTIONS

PRECAUTIONS TO BE TAKEN IN HANDLING AND STORAGE

Store in moisture free place; protect against physical damage. 


\begin{tabular}{|c|}
\hline PRODUCT DESIGNATION \\
03-091-2006 \\
Mercuric Chloride \\
\hline
\end{tabular}

\section{MATERIAL SAFETY \\ DATA SHEET}

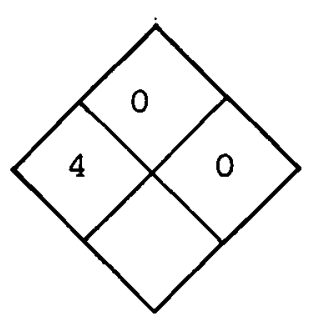

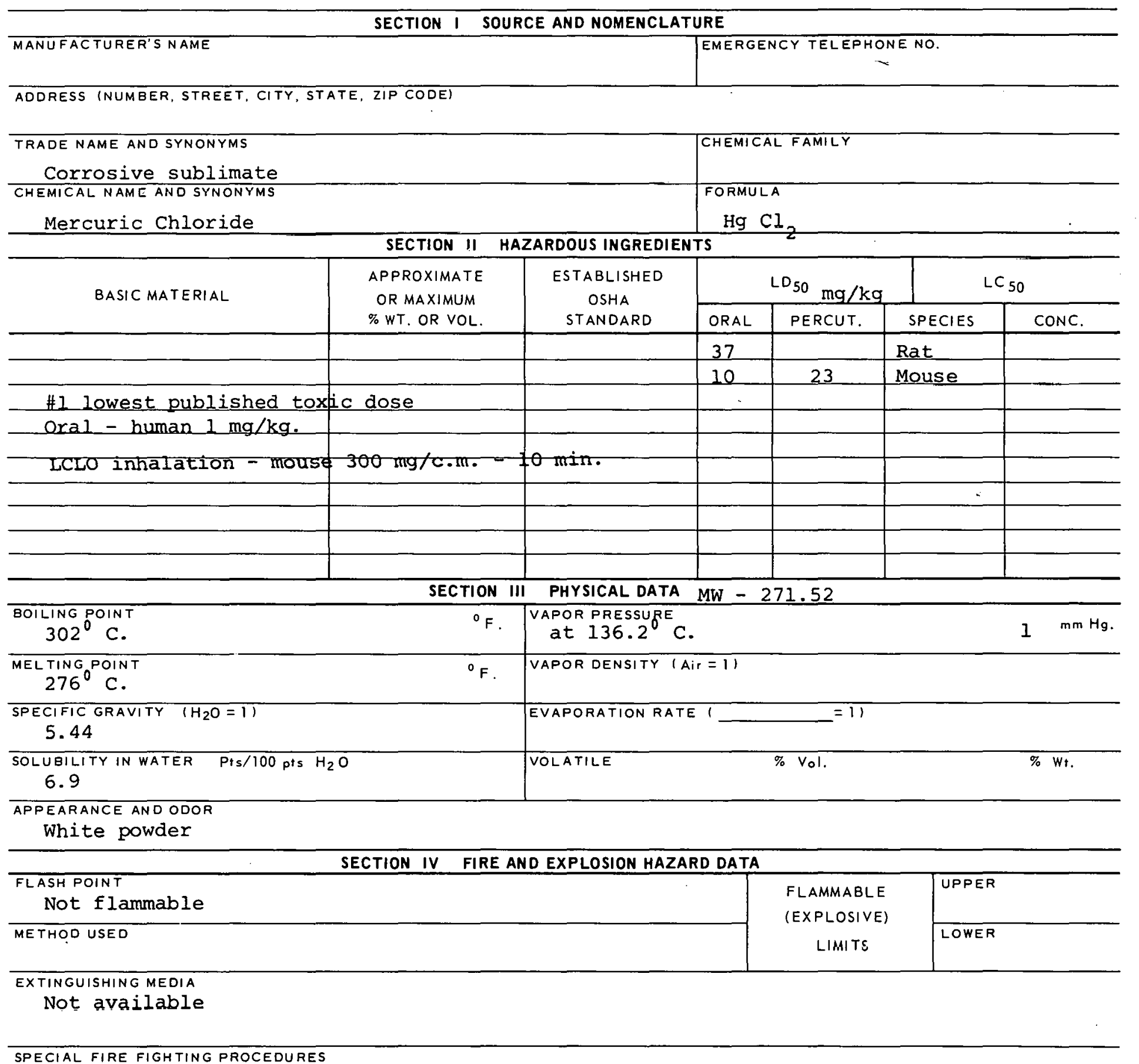

UNUSUAL FIRE AND EXPLOSION HAZARDS 


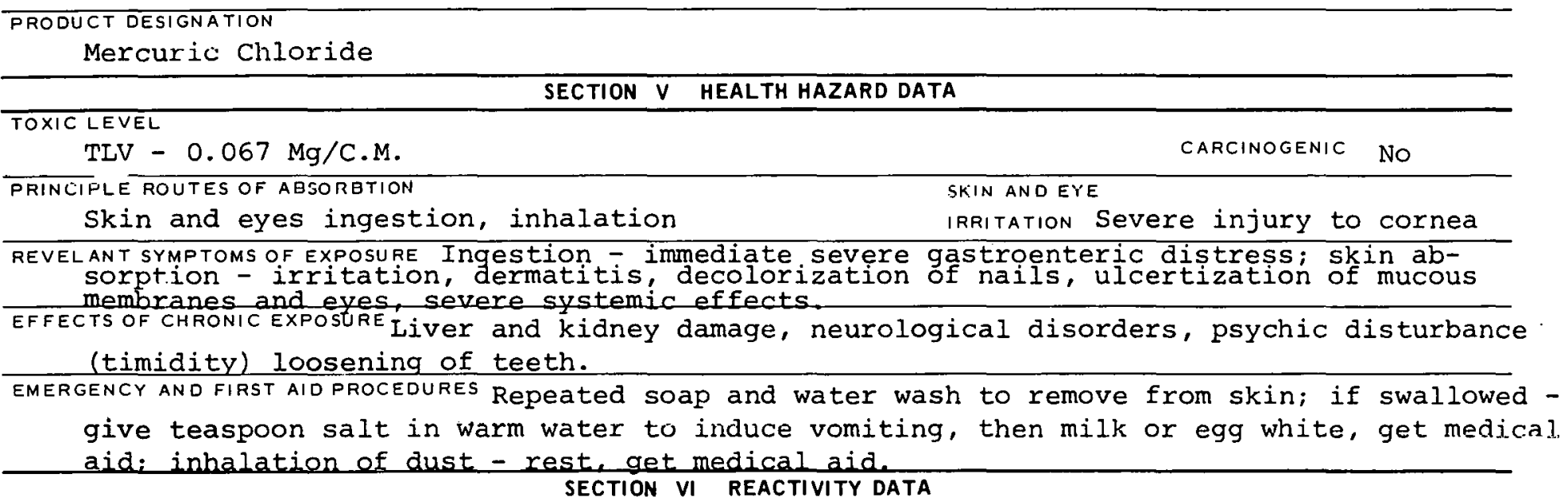

\section{SECTION VIII SPECIAL PROTECTION INFORMATION}

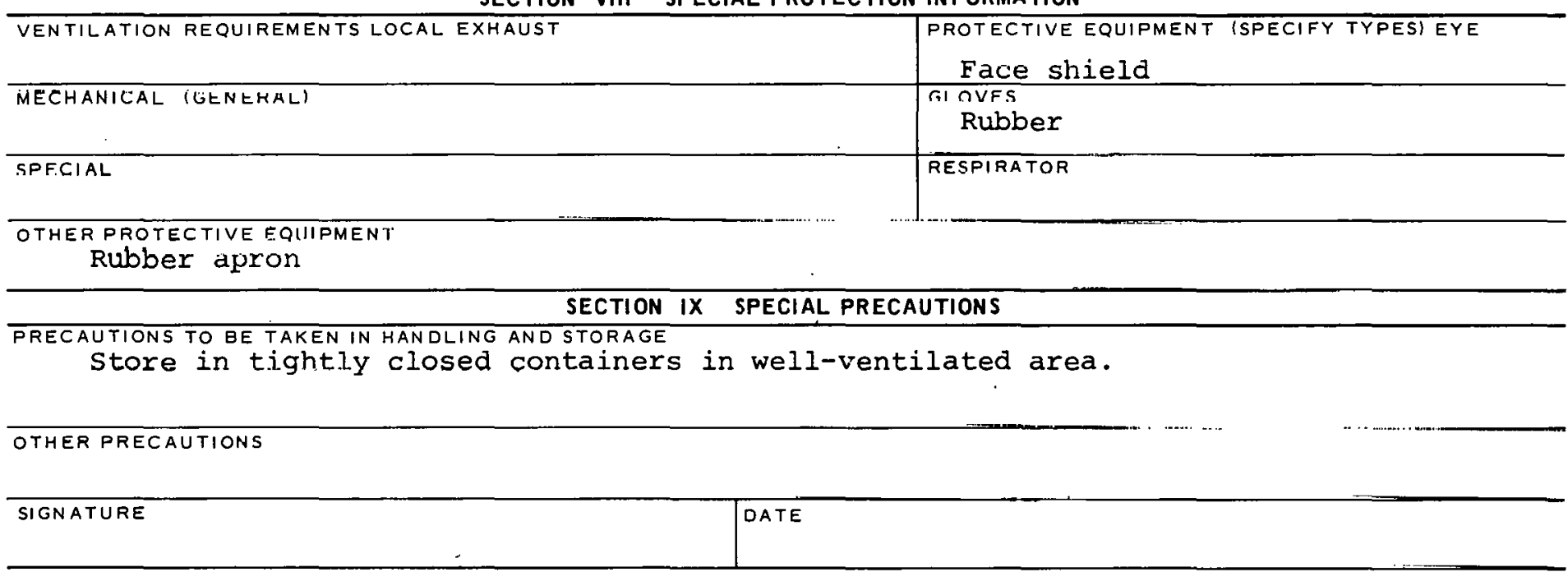

$91-2006 \mathrm{~B}$ 


\section{PRODUCT DESIGNATION}

Mercuric Iodide

03-091-3006

\section{MATERIAL SAFETY DATA SHEET}

\section{SECTION I SOURCE AND NOMENCLATURE}

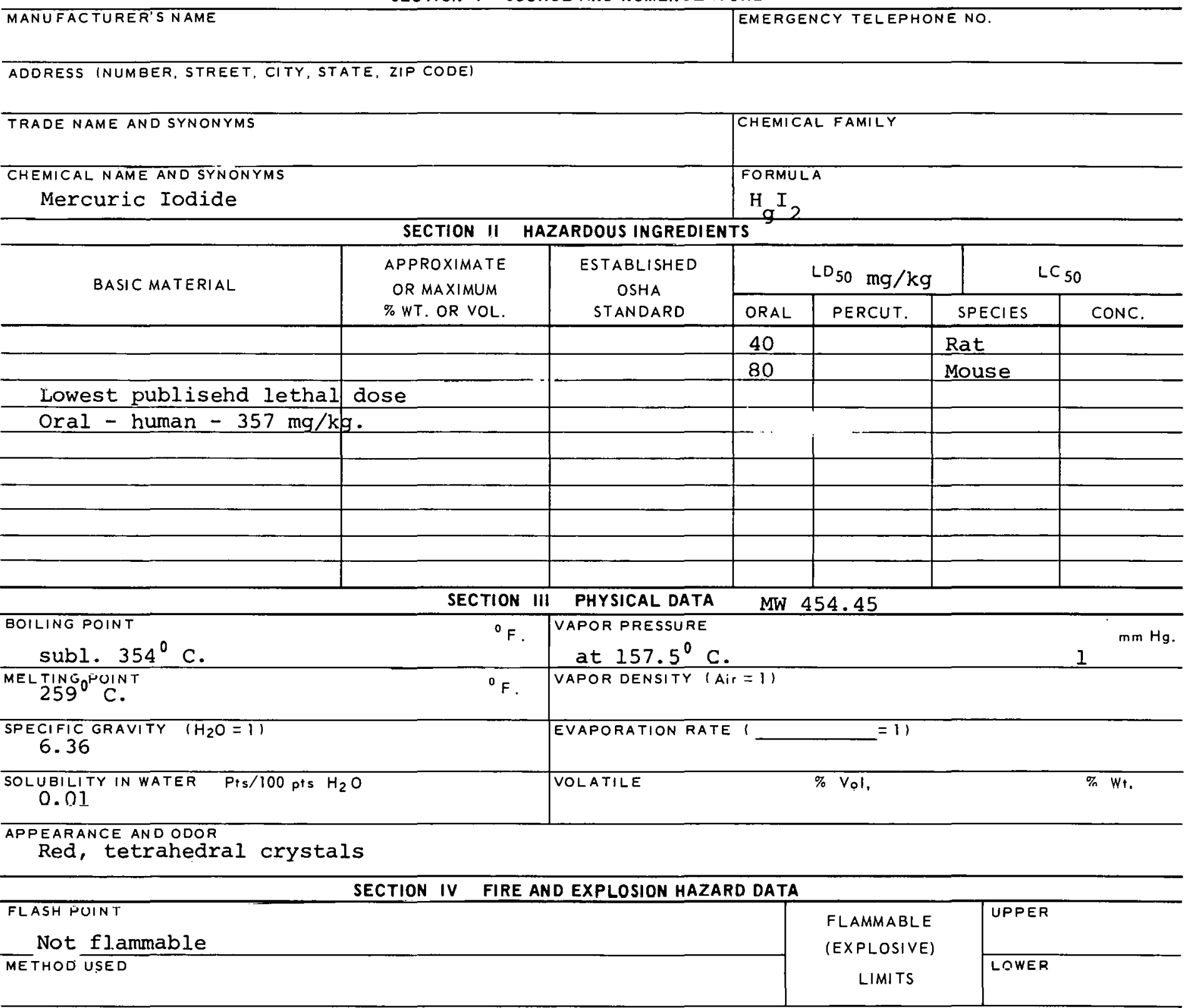

EXTINGUISHING MEDIA

$N / A$

SPECIAL FIRE FIGHTING PROCEDURES

$\mathrm{N} / \mathrm{A}$

UNUSUAL FIRE AND EXPLOSION HAZARDS

$\mathrm{N} / \mathrm{A}$ 
TOXIC LEVEL

TLV $0.113 \mathrm{mg} / \mathrm{m}^{3}$

PRINCIPLE ROUTES OF ABSORBTION

Skin absorption, ingestion, inhalation

CARCINOGENIC

no

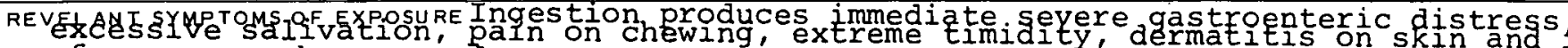
of mucous membranes and eyes.

EFFECTS OF CHRONIC EXPOSURE Liver and kidney. damage, neurological disorders. Psychic disturbances (timidity), dermatitis, and inflammation of mucous membranes, loosening of teeth. EMERGENCY AND FIRST AIDPROCEDURES If CONSCious, give teaspoon salt in glass warm water. To induce vomiting, give milk or egg white afterward. If on skin, remove by washing with soap and water.

\section{SECTION VI REACTIVITY DATA}

CONDITIONS CONTRIBUTING TO INSTABILITY

CONDITIONS CONTRIBUTING TO HAZARDOUS POLYMERIZATION

INCOMPATABILITY (MATERIALSTO MVOIO)

HAZARDOUS RFROMPOSITION PRODUCTS

SECTION VII SPILL OR LEAK PROCEDURES

STEPS TO BE TAKEN IN CASE MATERIAL IS RELEASED OR SPILLED

WASTE DISPOSAL METHOD

SECTION VIII SPECIAL PROTECTION INFORMATION

VENTILATION REQUIREMENTS LOCAL EXHAUST

MECHANICAL (GENERAL)

SPES.IAL

OTHER PROTECTIVE EQUIPMENT

Rubber apron

PRECAUTIONS TO BE TAKEN IN HANDLING AND STORAGE
PROTECTIVE EQUIPMENT (SPECIFY TYPES) EYE

Face shield

GLOVES

Rubber

RESPIRATQR

with proper filter 


\begin{tabular}{|c|}
\hline PRODUCT DESIGNATION \\
$03-091-4006$ \\
$03-091-4016$ \\
Mercuric Nitrate \\
\hline
\end{tabular}

\section{MATERIAL SAFETY DATA SHEET}

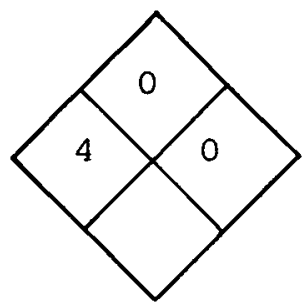

\section{SECTION I SOURCE AND NOMENCLATURE}

MANUFACTURER'S NAME

ADDRESS (NUMBER, STREET, CITY, STATE, ZIP CODE)

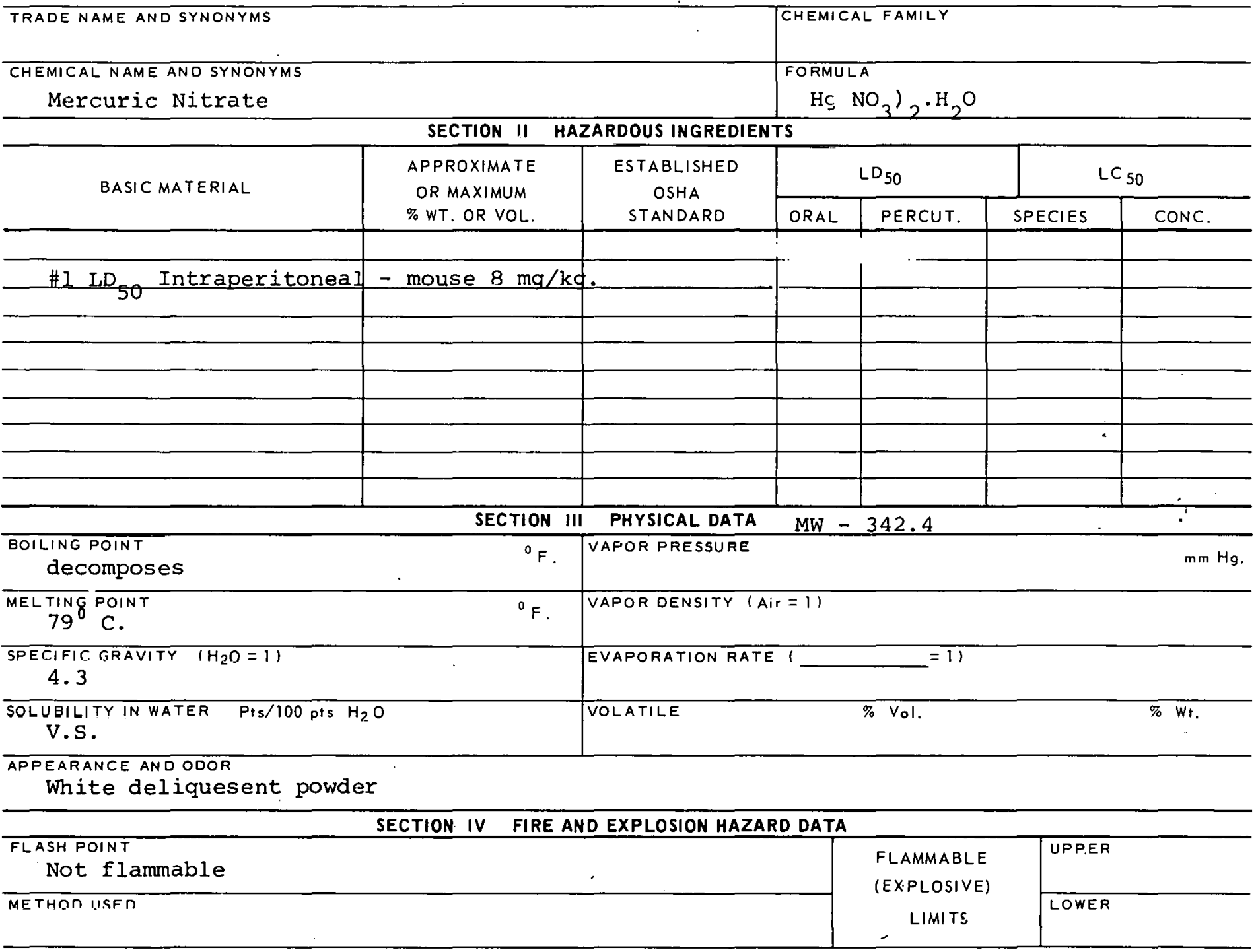

EXTINGUISHING MEDIA

Water

SPECIAL FIRE FIGHTING PROCEOURES

Wear mask to protect from $\mathrm{NO}_{2}$ fumes.

UNUSUAL FIRE AND EXPLOSION HAZARDS

Decomposes to produce $\mathrm{NO}_{2}$ fumes. 


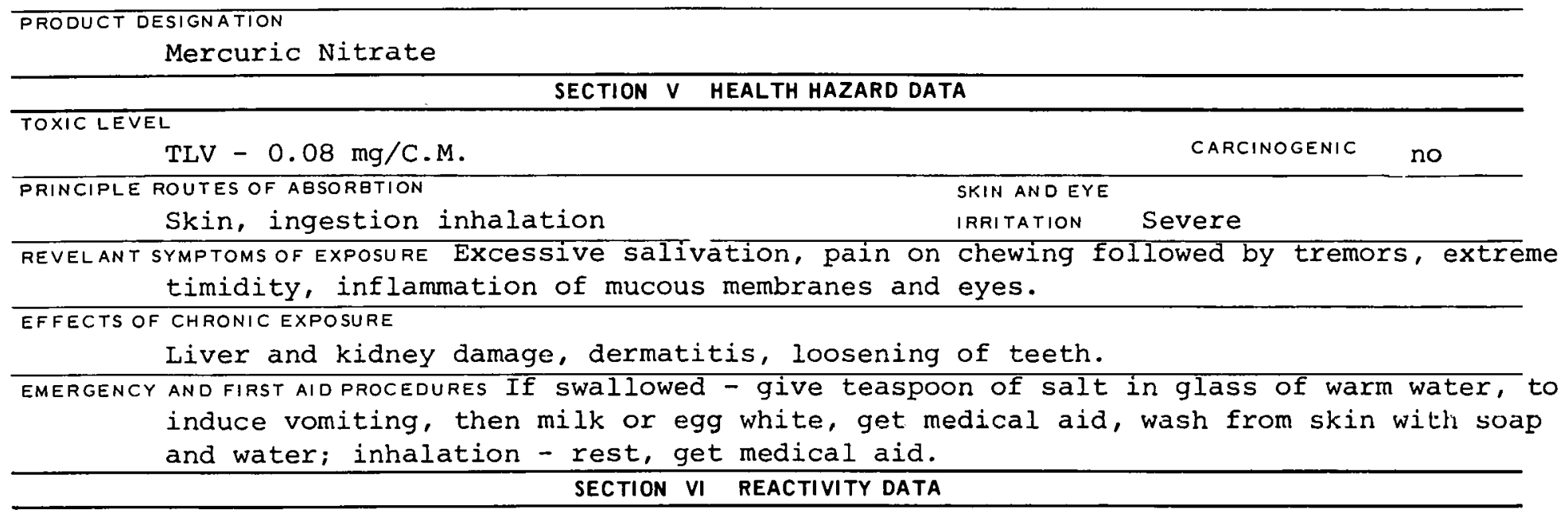

CONDITIONS CONTRIBUTING TO INSTABILITY

CONDITIONS CONTRIBUTING TO HAZARDOUS POLYMERIZATION

INCOMPATABILITY (MATER!ALSTO AVOIDI

Acetylene, ethyl alcohol, phosphene, sulfur, unsaturated aromatics.

HAZARDOUS DECOMPOSITION PRODUCTS

SECTION VII SPILL OR LEAK PROCEDURES

STEPS TO BE TAKEN IN CASE MATERIAL IS RELEASED OR SPILLED

\begin{tabular}{|c|c|}
\hline SECTION VIII & SPECIAL PROTECTION INFORMATION \\
\hline VENTILATION REQUIREMENTS LOCAL EXHAUST & $\begin{array}{l}\text { PROTECTIVE EQUIPMENT (SPECIFY TYPES) EYE } \\
\text { Face shield }\end{array}$ \\
\hline MECHANICAL (GENERAL) & $\begin{array}{l}\text { GLOVEE } \\
\text { Rubber }\end{array}$ \\
\hline SPECIAL & $\begin{array}{l}\text { RESPIRATOR } \\
\text { With proper filtor }\end{array}$ \\
\hline
\end{tabular}

OTIIER PROTECTIVE EQUIPMENT

SECTION IX SPECIAL PRECAUTIONS

PRECAUTIONS TO BE TAKEN IN HANDLING AND STORAGE

OTHER FRECAUTIONS

SIGNATURE

DATE

$91-4006 B$ 


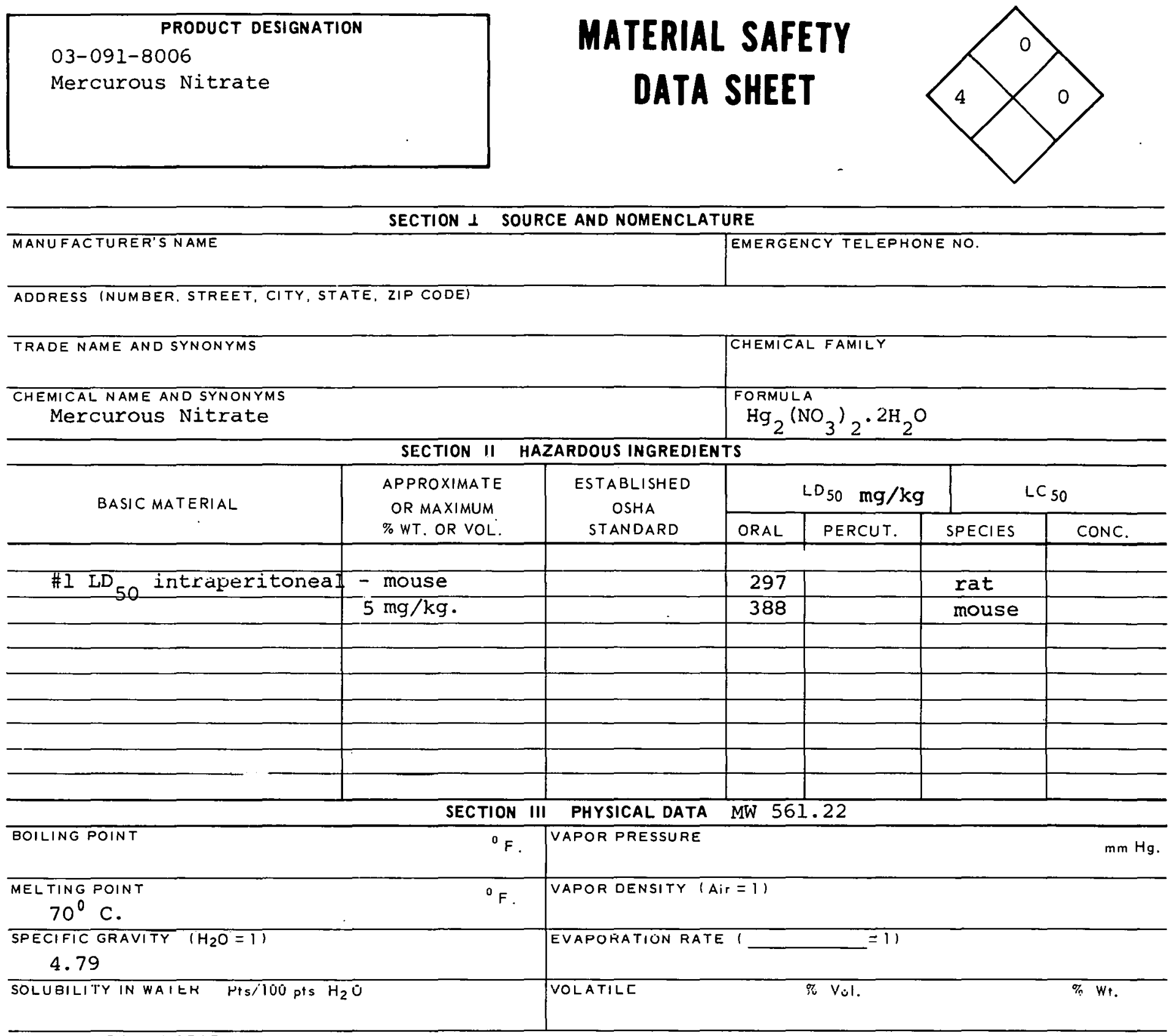

APPEARANCE AND ODOR

Crystalline, colorless

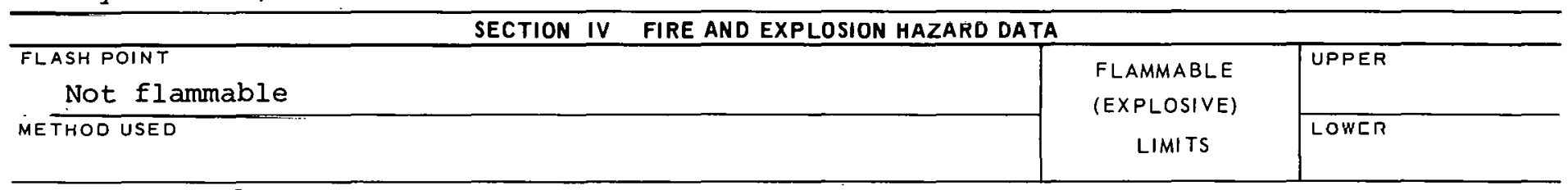

EXTINGUISHING MEDIA

Water - best

SPECIAL FIRE FIGHTING PROCEOURES

Water in form of fine droplets or spray.

UNUGUAL TIRC AND EXRLOEION HAZARDS

Strong oxidizing agent - moderate fire hazard by chemical reaction with reducing agents. 


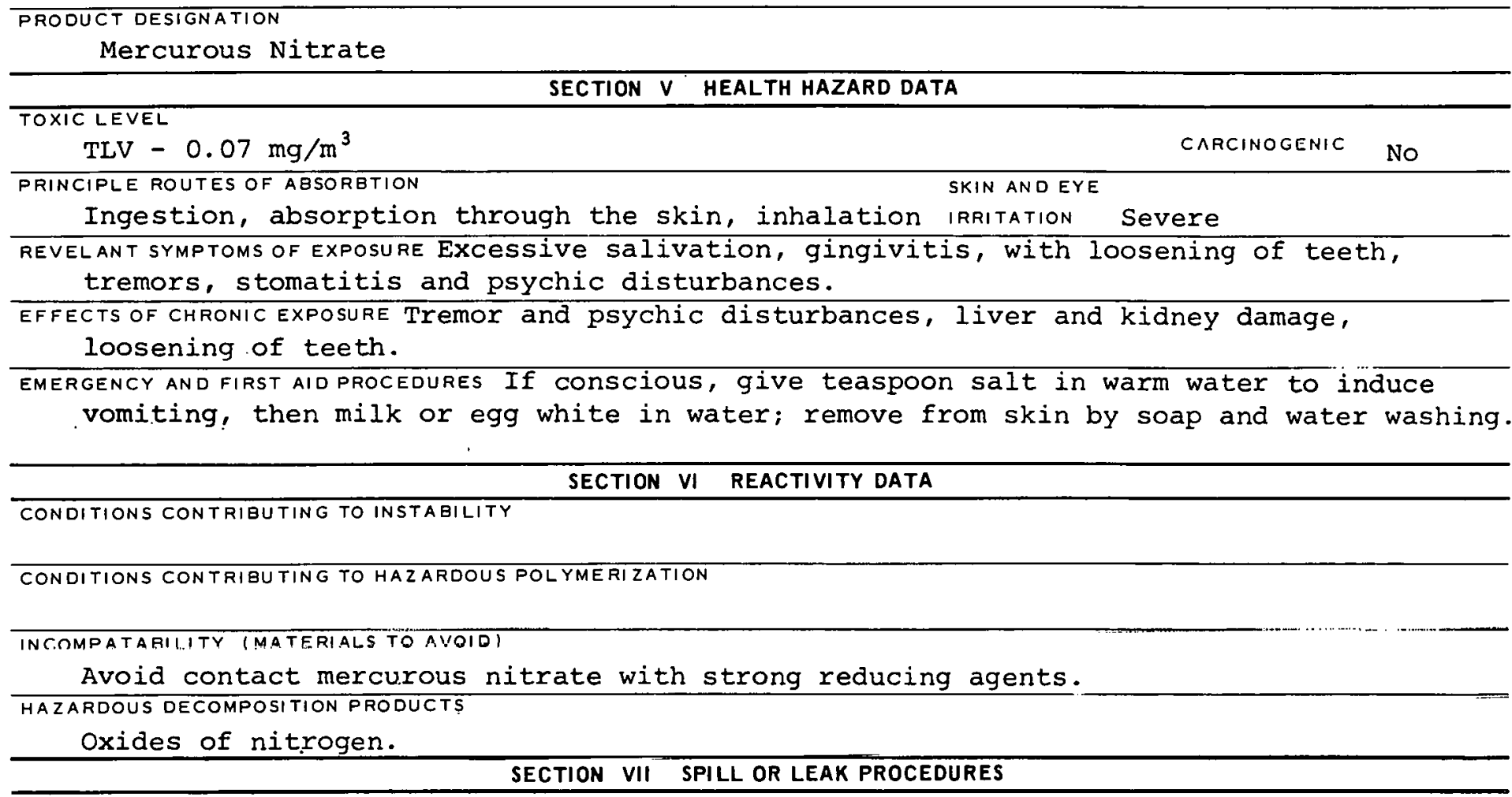

STEPS TO BE TAKEN IN CASE MATERIAL IS RELEASED OR SPILLED

WASTE DISPOSAL METHOD

SECTION VIII SPECIAL PROTECTION INFORMATION

\begin{tabular}{|c|c|}
\hline VENTILATION REQUIREMENTS LOCAL EXHAUST & $\begin{array}{l}\text { PROTECTIVE EQUIPMENT (SFCCIFY TYPESI EYE } \\
\text { Safety glasses or goggles }\end{array}$ \\
\hline $\begin{array}{l}\text { MECHANICAL (GENERAL) } \\
\text { - }\end{array}$ & $\begin{array}{l}\text { GLOVES } \\
\text { Rubber }\end{array}$ \\
\hline SPECIAL & $\begin{array}{l}\text { RESPIRATOR } \\
\text { W/appropriate filter }\end{array}$ \\
\hline
\end{tabular}

OTHFR PROTTECTIVE EQUIPMENT

Rubber apron

SECTION IX SPECIAL PRECAUTIONS

PRECAUTIONS TO BE TAKEN IN HANDLING AND STORAGE

OTHER PRECAUTIONS

SIGNATURE

DATE

91-8006B 


\begin{tabular}{|l|}
\hline \multicolumn{1}{|c|}{ PRODUCT DESIGNATION } \\
$03-091-9026$ \\
Mercury Metal
\end{tabular}

\section{MATERIAL SAFETY DATA SHEET}

\section{SECTION I SOURCE AND NOMENCLATURE}

MANUFAC TURER'S NAME
AOORESS INUMBER, STREET, CI
TRADE NAME AND SYNONYMS
CHEMICAL NAME AND SYNONYMS
QUICKSIIVEX

BASIC MATERIAL

\begin{tabular}{l|l} 
& CHEMICAL FAMILY \\
\hline & $\begin{array}{c}\text { FORMULA } \\
\text { Hg }\end{array}$ \\
\hline
\end{tabular}
SECTION II HAZARDOUS INGREDIENTS

\begin{tabular}{c|c}
\hline APPROXIMATE & ESTABLISHED \\
OR MAXIMUM & OSHA \\
$\%$ WT OR VOL. & STANDARD
\end{tabular}

\begin{tabular}{|c|c|c|c|}
\hline \multicolumn{2}{|c|}{$\operatorname{LD}_{50}$} & \multicolumn{2}{|c|}{$\operatorname{LC}_{50}$} \\
\hline ORAL & PERCUT. & SPECIES & CONC. \\
\hline & & & \\
\hline & & & \\
\hline & & & \\
\hline & & & \\
\hline & & & \\
\hline & & & \\
\hline & & & \\
\hline & & & \\
\hline & & & \\
\hline
\end{tabular}

BOILING POINT

$356.73^{\circ} \mathrm{C}$.

MELTING POINT

$-38.87^{\circ} \mathrm{C}$.

SPECIFIC GRAVITY $\left(\mathrm{H}_{2} \mathrm{O}=1\right)$

13.645

SOLUBILITY IN WATER Pts/100 pts $\mathrm{H}_{2} \mathrm{O}$

insoluble

APPEARANCE AND ODOR

Silver liguid.

\section{FLASH POINT}

none available

METHOD USED

EXTINGUISHING MEDIA

none available
EMERGENCY TELEPHONE NO.

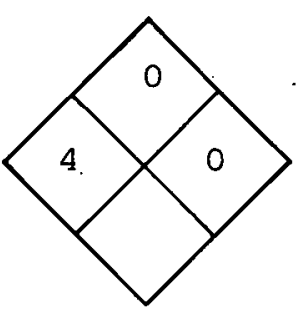

\begin{tabular}{l|l}
${ }^{\circ} \mathrm{F}$. & VAPOR PRESSURE \\
\hline
\end{tabular}

${ }^{\circ} \mathrm{F}$.
$=11$

EVAPORATION RATE I

VOLATILE

$\%$ Vol.

$\% W_{t}$.

SPECIAL FIRE FIGHTING PROCEDURES

FLAMMABLE

(EXPLOSIVE)

LIMITS
UPPER

LOWER 


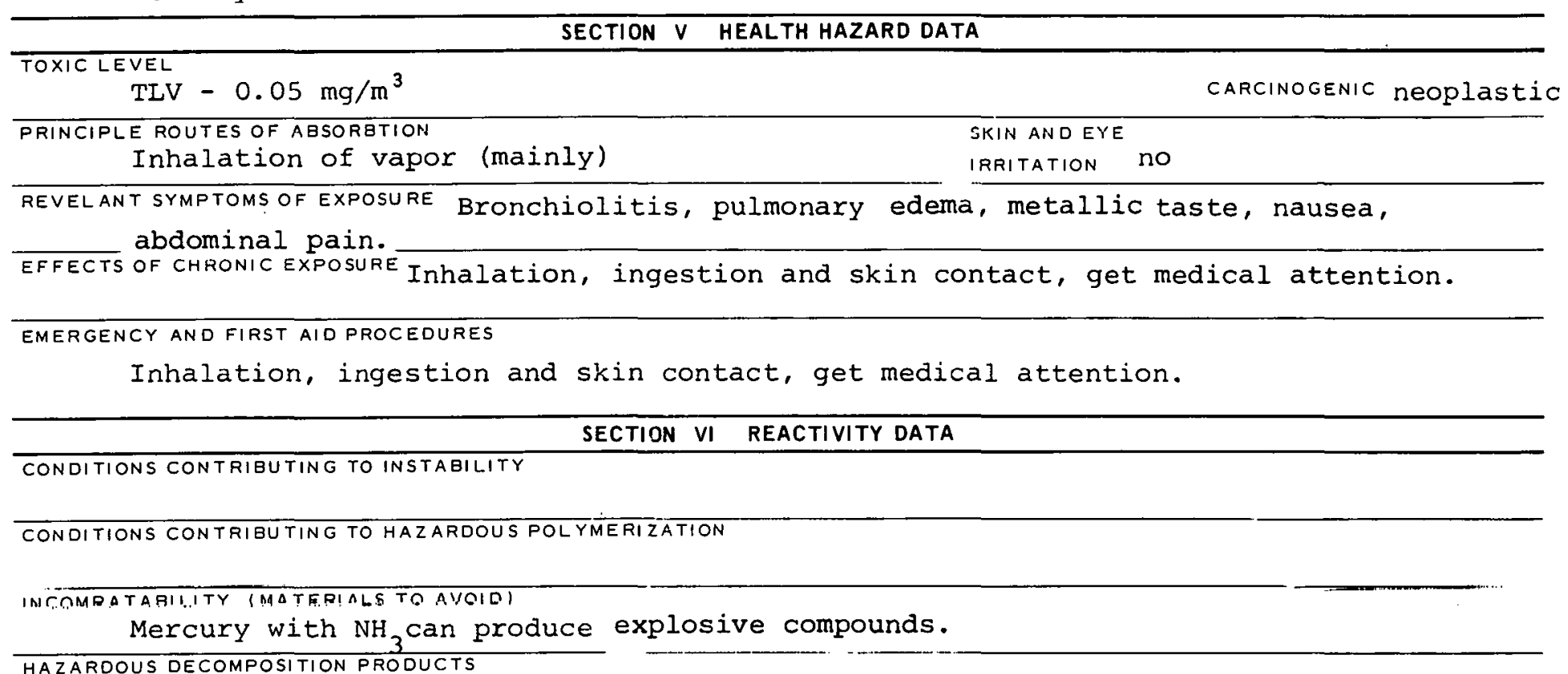

\section{SECTION VII SPILL OR LEAK PROCEDURES}

STEPS TO BE TAKEN IN CASE MATERIAL IS RELEASED OR SPILLED

Add powdered Cā metal. Sweep up cd metal for disposal.

\section{WASTE DISPOSAL METHOD}

SECTION VIII SPECIAL PROTECTION INFORMATION

\begin{tabular}{l|l}
\hline VENTILATION REQUIREMENTS LOCAL EXHAUST & $\begin{array}{c}\text { PROTECTIVE EQUIPMENT ISPECIFY TYPES) EYE } \\
\text { Safety glasSES }\end{array}$ \\
\hline MECHANICAI. (GENERAI) & $\begin{array}{c}\text { GLOVE3 } \\
\text { Gloves }\end{array}$ \\
\hline SPECIAL & $\begin{array}{c}\text { RESPIRATOR } \\
\text { With proper fj. t ter }\end{array}$ \\
\hline
\end{tabular}

OTHER PROTECTIVE EQUIPMENT

SECTION IX SPECIAL PRECAUTIONS

PRECAUTIONS TO BE TAKEN IN HANDLING ANO STORAGE

Store in closed container in well-ventilated area.

OTHER PRECAUTIONS 
CAS-013463393

PRODUCT DESIGNATION

Nickel Carbonyl
QR63000

MATERIAL SAFETY

DATA SHEET

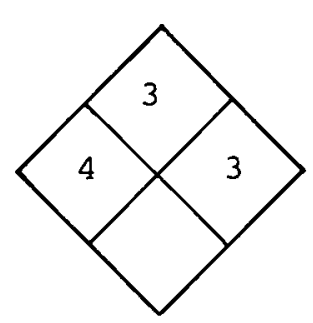

\section{SECTION I SOURCE AND NOMENCLATURE}

MANUFACTURER'S NAME

EMERGENCY TELEPHONE NO.

ADDRESS (NUMBER, STREET, CITY, STATE, ZIP CODE)

TRADE NAME AND SYNONYMS

\begin{tabular}{l|l}
\hline CHEMICAL FAMILY \\
\hline
\end{tabular}

CHEMICAL NAME AND SYNONYMS

Nickel Carbonyl FORMULA SECTION II HAZARDOUS INGREDIENTS

EXTINGUISHING MEDIA

Water, Foam, Carhnn Dioxide, Dry Chemical

SPECIAL FIRE FIGHTING PROCEDURES

Wear full protective clothing, self contained breathing apparatus 
TOXIC LEVEL

TLV - 0.001 PPM, 0.007 MG/C.M.

CARCINOGENIC

Yes

PRINCIPLE ROUTES OF ABSORBTION

Inhalation (mainly)

SKIN AND EYE

IRRITATION

No

REVELANT SYMPTOMS OF EXPOSURE Induced gastroenteric symptoms; respiratory distress due to

delayed pulmonary edema; headache, nausea, weakness, convulsions.

EFFECTS OF CHRONIC EXPOSURE

May produce cancer in lungs - lung inflamation

EMERGENCY AND FIRST AID PROCEOURES COmplete bed rest and oxygen for pulmonary edema; for

intoxication--give dimercaprol ( $4 \mathrm{mg} / \mathrm{kg}$ every $4 \mathrm{hrs}$. first day) washcontaminated

areas of body with soap and water.

SECTION VI REACTIVITY DATA

CONDITIONS CONTRIBUTING TO INSTABILITY

CONDITIONS CONTRIBUTING TO HAZARDOUS POLYMERI ZATION

INCOMPATABILITY (MATERIALS TO AVOID) May decompose and ignite in presence of air may explode with butane-air mixture at $\left(20-40^{\circ} \mathrm{C}\right)$.

HAZARDOUS DECOMPOSITION PRODUCTS

SECTION VII SPILL OR LEAK PROCEDURES

STEPS TO BE TAKEN IN CASE MATERIAL IS RELEASED OR SPILLED

WASTE DISPOSAL METHOD

SECTION VIII SPECIAL PROTECTION INFORMATION

\begin{tabular}{l|l}
\hline VENTILATION REQUIREMENTS LOCAL EXHAUST & $\begin{array}{c}\text { PROTECTIVE EQUIPMENT (SPECIFY TYPES) EYE } \\
\text { SafetY GOggles }\end{array}$ \\
\hline MECHANICAL (GENERAL) & GLOVES \\
\hline SPECIIAL & RESPIRATOR \\
Self contained
\end{tabular}

OTHER PROTECTIVE EOUIPMENT

Wear full protective clothing

SECTION IX SPECIAL PRECAUTIONS

PRECAUTIONS TO BE TAKEN IN HANDLING AND STORAGE

Protect cylinders against physical damage.

OTHER PRECAUTIONS

SIGNATURE

UAIE

$97-0001 B$ 


\section{PRODUCT DESIGNATION \\ 03-097-2848 \\ Nickel Metal \\ MATERIAL SAFETY \\ DATA SHEET}

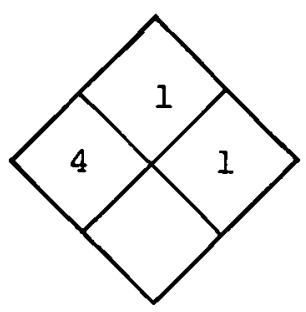

\section{SECTION I SOURCE AND NOMENCLATURE}

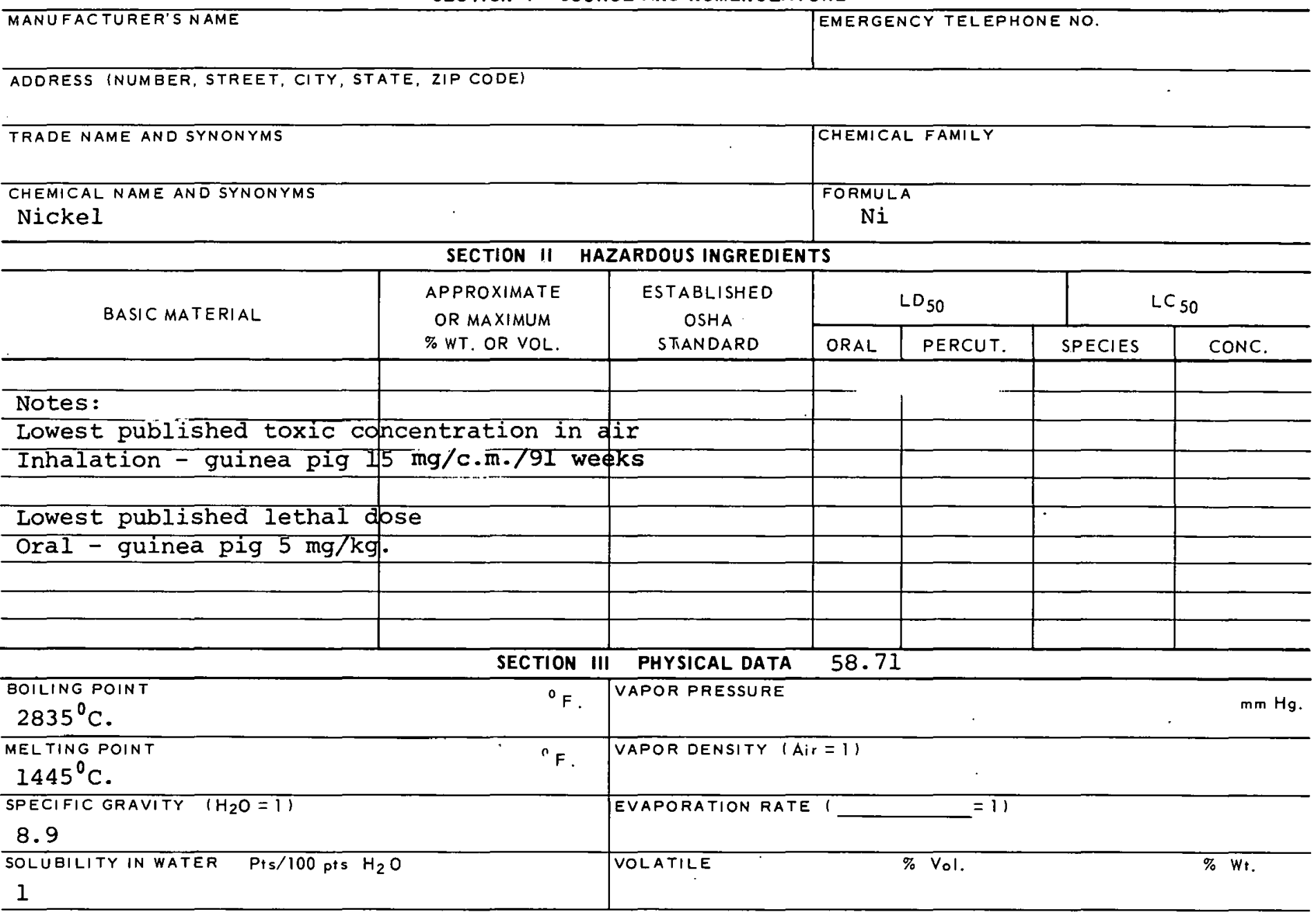

APPEARANCE AND ODOR

Silver colored metal.

SECTION IV FIRE AND EXPLOSION HAZARD DATA

\begin{tabular}{l|c}
\hline FLASH POINT & FLAMMABLE \\
N/A & UPPËR \\
\hline METHOD USED & (EXPLOSIVE) \\
N/A & LIMITS LOWER \\
\hline
\end{tabular}

EXTINGUISHING MEDIA

$\mathrm{N} / \mathrm{A}$

SPECIAL FIRE FIGHTING PROCEDURES

UNUSUAL FIRE AND EXPLOSION HAZARDS

Powder may burn. 


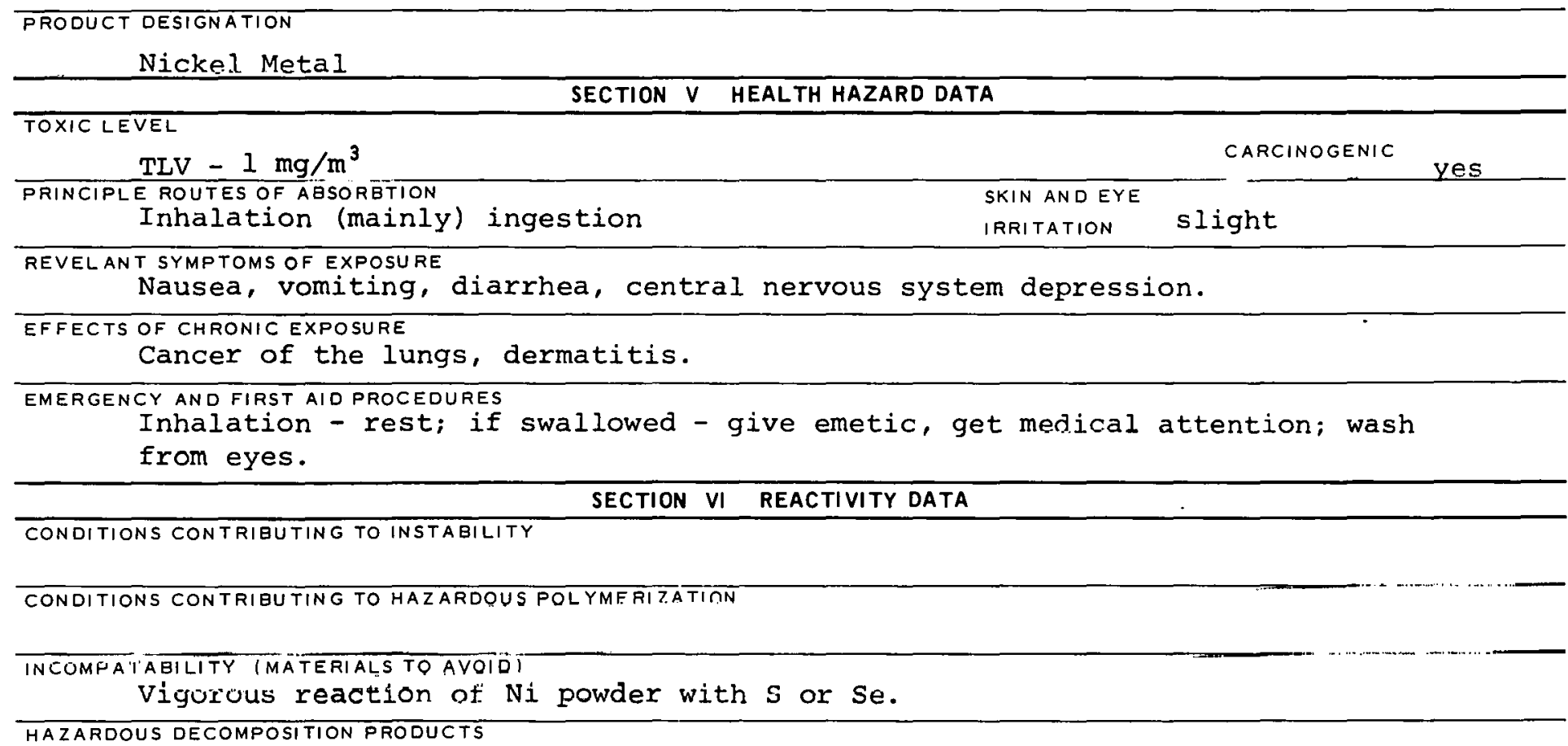

\section{SECTION VII SPILL OR LEAK PROCEDURES}

STEPS TO BE TAKEN IN CASE MATERIAL IS RELEASED OR GPILLED

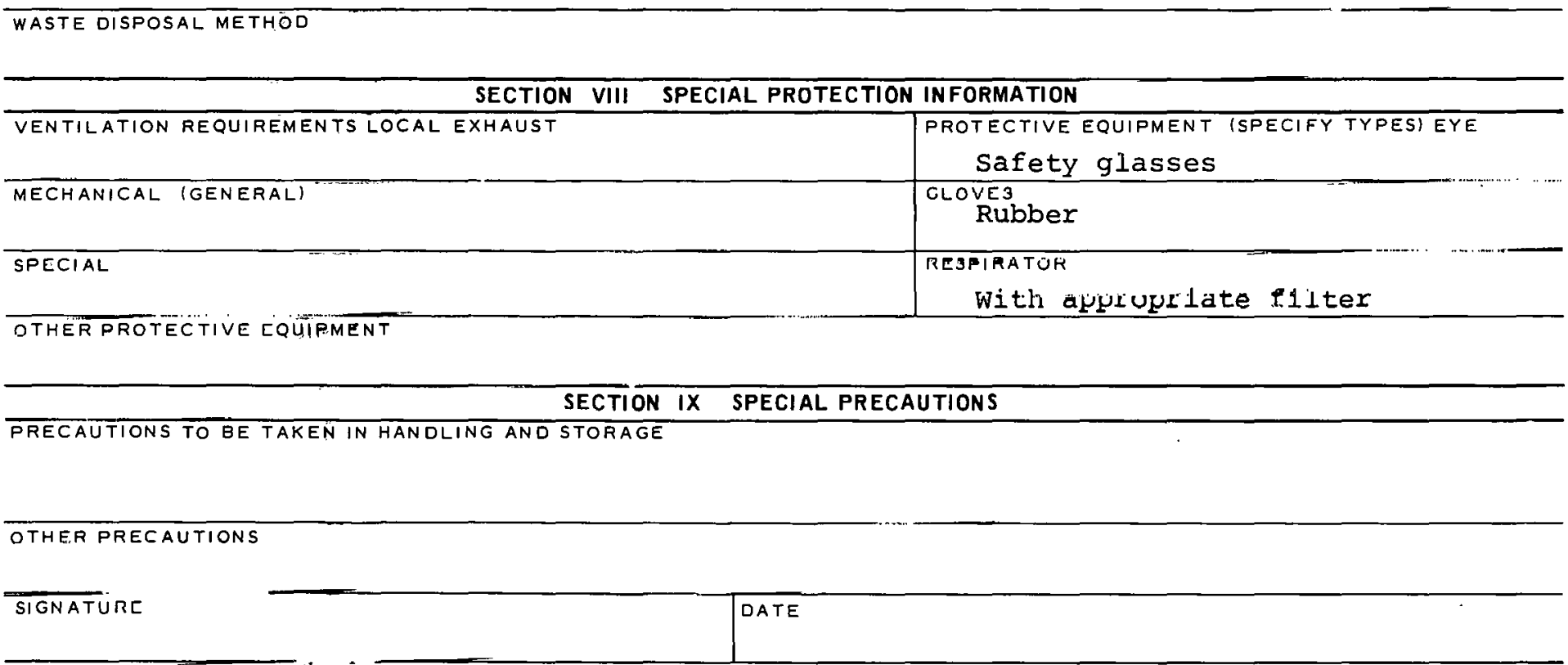




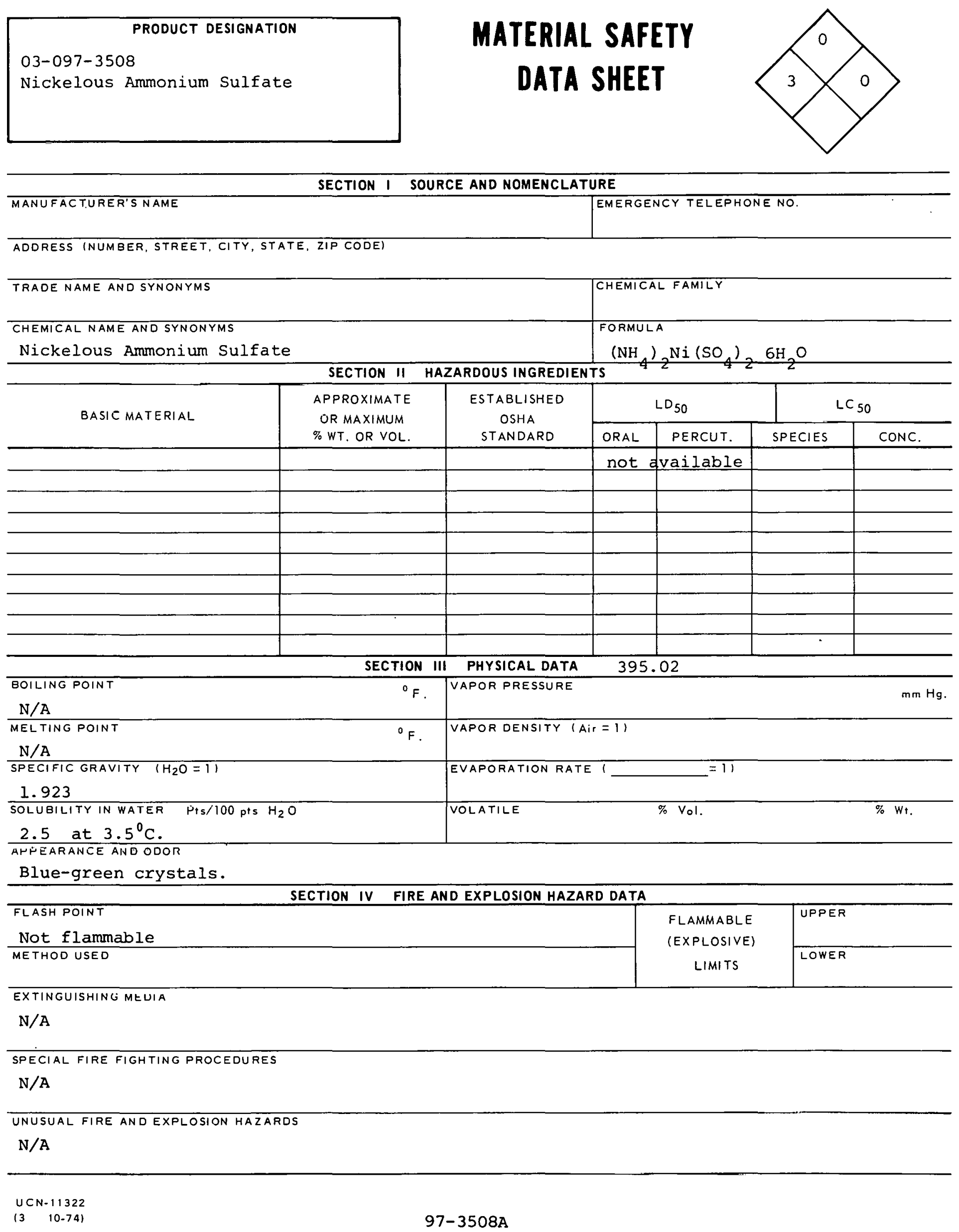


CAS : 007791200

QR64800

PRODUCT DESIGNATION

03-097-4908

Nickelous Chloride

MATERIAL SAFETY

DATA SHEET

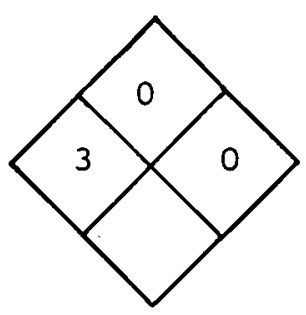

\section{SECTION I SOURCE AND NOMENCLATURE}

MANUFACTURER'S NAME

EMERGENCY TELEPHONE NO.

ADDRESS (NUMEER, STREET, CITY, STATE, ZIP CODE)

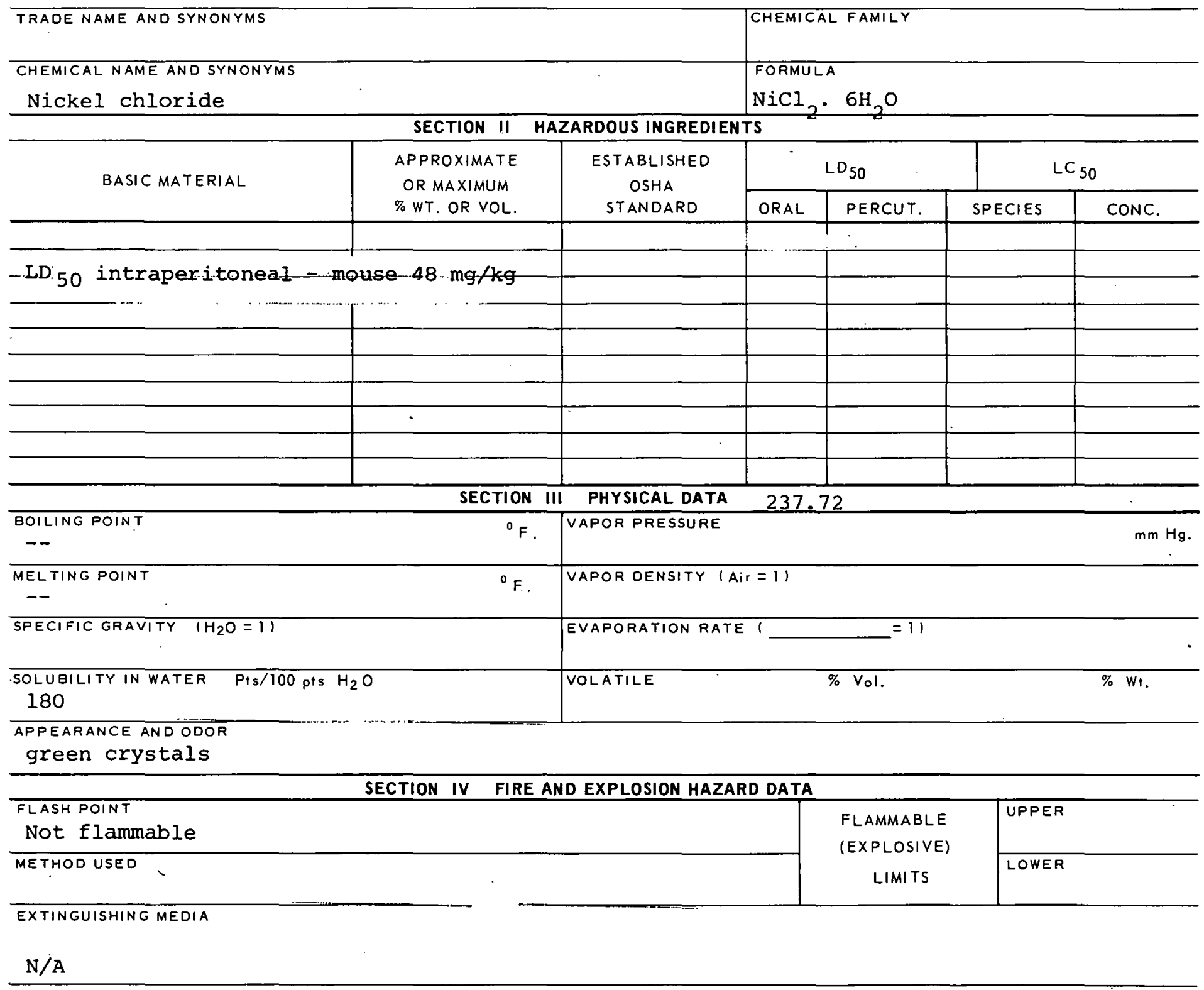

SPECIAL FIRE FIGHTING PROCEDURES

$\mathrm{N} / \mathrm{A}$

UNUSUAL FIRE AND EXPLOSION HAZARDS

$\mathrm{N} / \mathrm{A}$ 


\begin{tabular}{|l|}
\hline \multicolumn{1}{|c|}{ PRODUCT DESIGNATION } \\
03-097-6958 \\
03-097-7008 \\
Nickelous Nitrate
\end{tabular}$\quad$ DATERIAL SAFETY

SECTION I SOURCE AND NOMENCLATURE

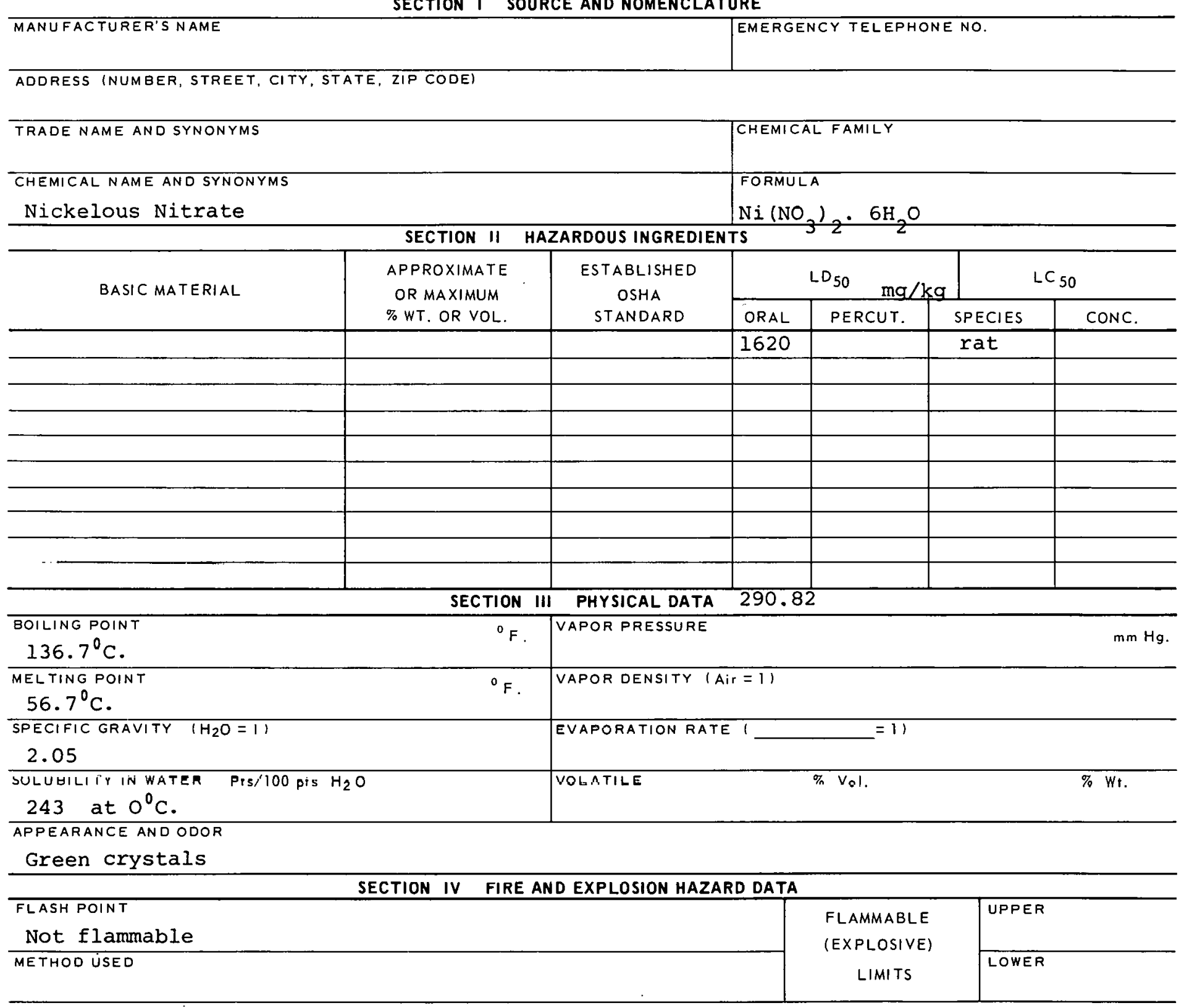

EXTINGUISHING MEUIA

Flood with water

SPECIAL FIRE FIGHTING PROCEDURES

Wear self-contained breathing apparatus.

UNUSUAL FIRE ANO EXPLOSION HATARTS

Oxidizing material. 


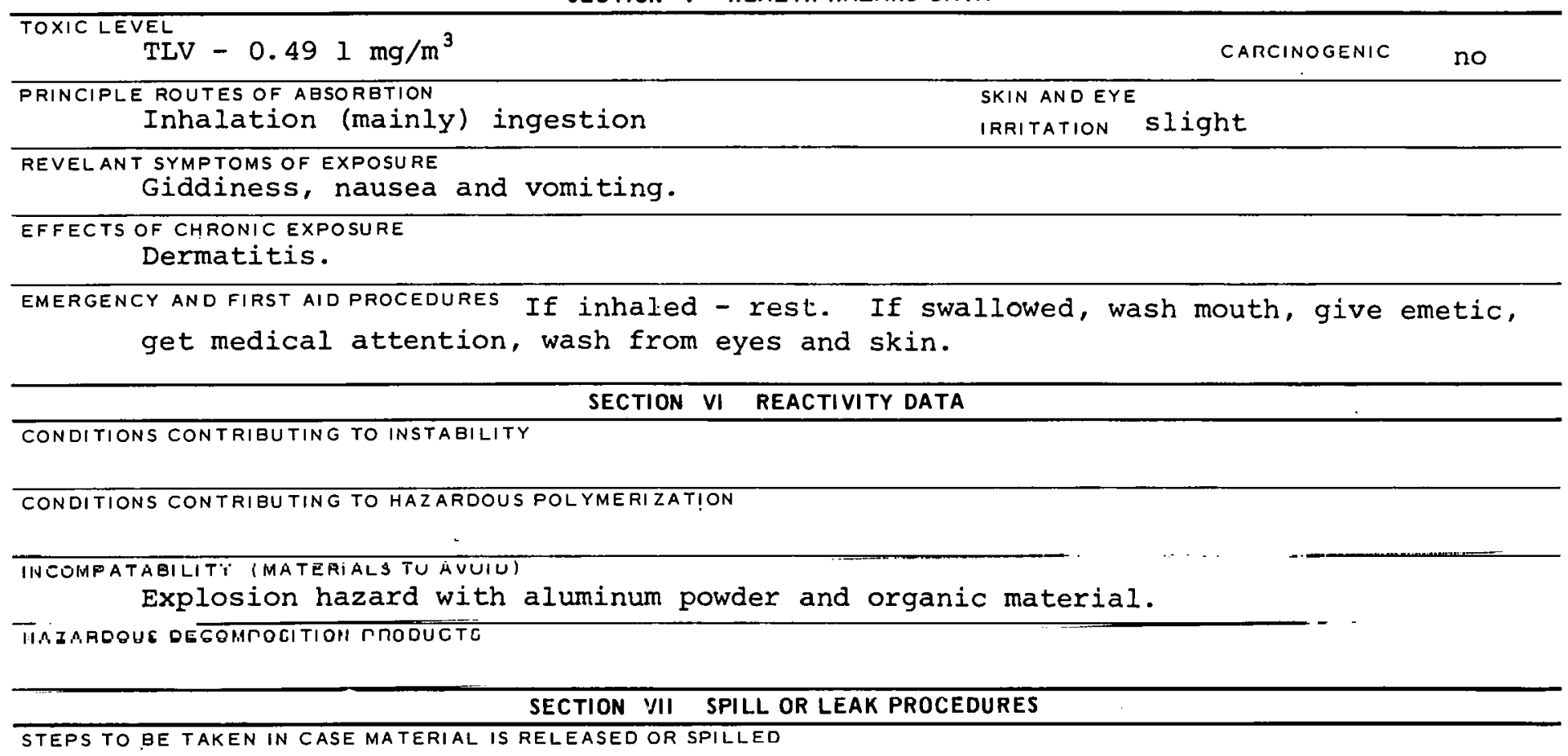

STEPS TO BE TAKEN IN CASE MATERIAL IS RELEASED OR SPILLED

WASTE DISPOSAL METHOD

\section{SECTION VIII SPECIAL PROTECTION INFORMATION}

\begin{tabular}{|c|c|}
\hline VENTILATION REQUIREMENTS I.OCAL EXHAUST & $\begin{array}{l}\text { PROTECTIVE EQUIPMENT (SPECIFY 'IYYPES) EYE } \\
\text { 'H'ace shield }\end{array}$ \\
\hline MECHANICAL (GENERAL) & GLOVES \\
\hline SPECIAL & REERIRATON \\
\hline & W1th appropriate filter \\
\hline OTHER PROTECTIVE EQUIPMENT & \\
\hline SECTION IX & SPECIAL PRECAUTIONS \\
\hline PRECAUTIONS TO BE TAKEN IN HANDLING AND STORAGE & (1) \\
\hline OTHER PRECAUTIONS & \\
\hline SIGNATURE & nATF. \\
\hline
\end{tabular}

\section{7-7008B}




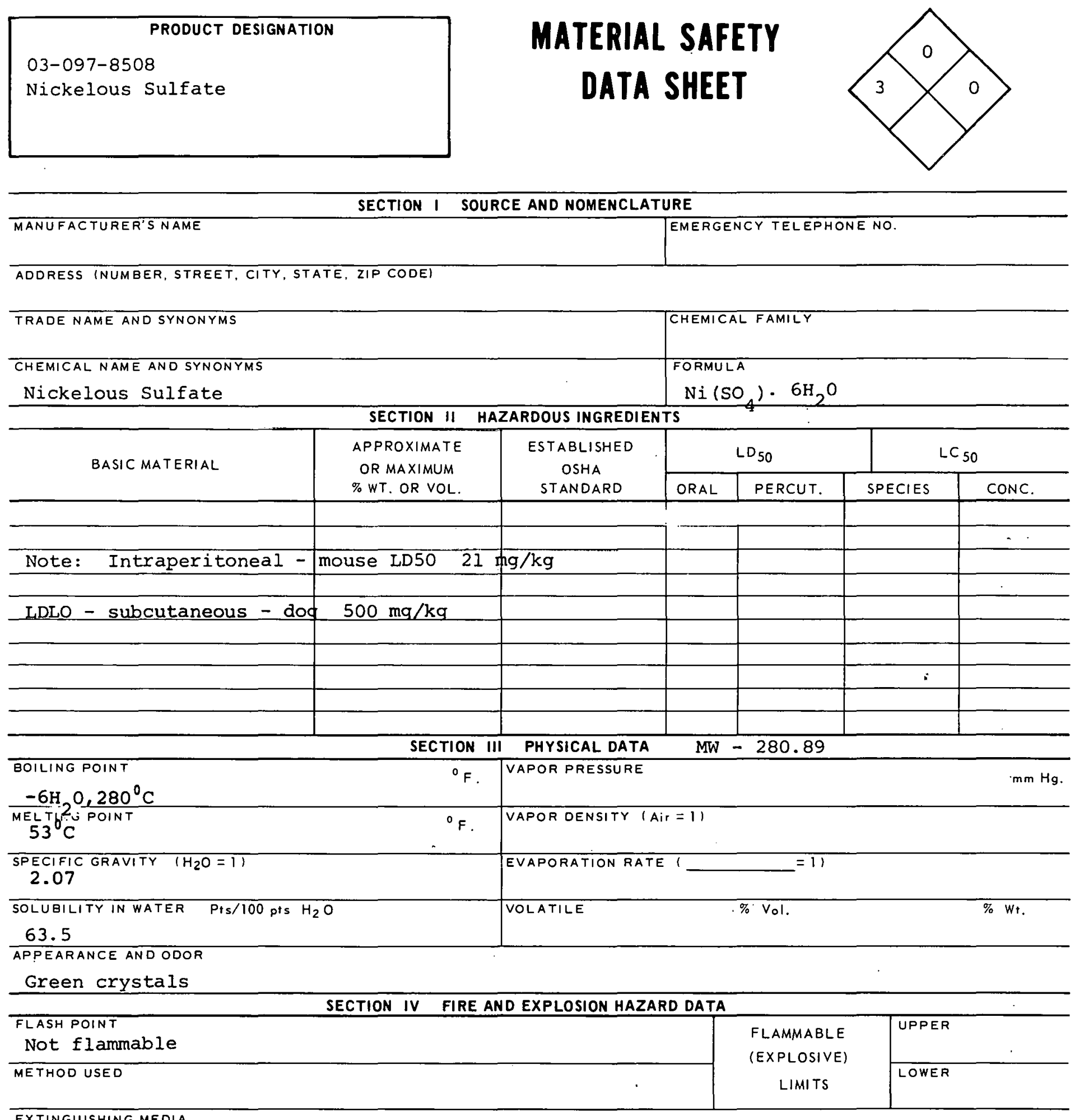

EXTINGUISHING MEDIA

Water

SPECIAL FIRE FIGHTING PROCEDURES

$\mathrm{N} / \mathrm{A}$

UNUSUAL FIRE AND EXPLOSION HAZARDS

$N / A$ 


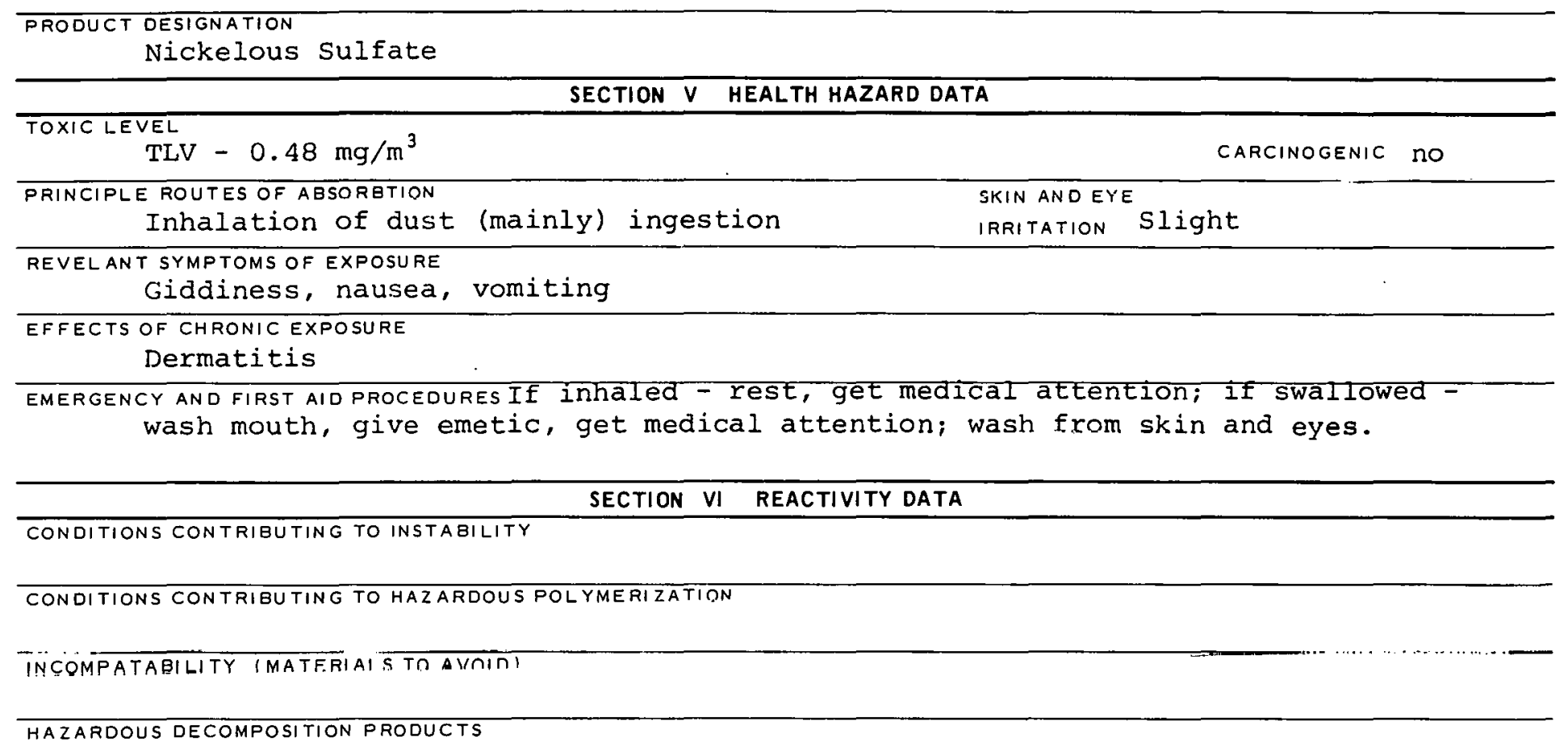

SECTION VII SPILL OR LEAK PROCEDURES

STEPS TO BE TAKEN IN CASE MATERIAL IS RELEASED OR SPILLED

\begin{tabular}{|c|c|c|c|}
\hline \multicolumn{4}{|l|}{ WASTE DISPOSAL METHOD } \\
\hline \multicolumn{4}{|c|}{ SPECIAL PROTECTION INFORMATION } \\
\hline \multicolumn{3}{|l|}{ VENTILATION REQUIREMENTS LOCAL EXHAUST } & $\begin{array}{l}\text { PROTECTIVE EQUIPMENT (SPECIFY TYPES) EYE } \\
\text { Face shield }\end{array}$ \\
\hline \multicolumn{3}{|l|}{ MECHANICAL (GENERAL) } & OLOVES \\
\hline \multicolumn{3}{|l|}{ SPECIAL } & $\begin{array}{l}\text { RESPIRATOK } \\
\text { With àppropriate filter }\end{array}$ \\
\hline \multicolumn{4}{|l|}{ OTHER PROTECTIVE EQUIPMENT } \\
\hline \multicolumn{4}{|c|}{ SECTION IX SPECIAL PRECAUTIONS } \\
\hline \multicolumn{4}{|c|}{ PRECAUTIONS TO BE TAKEN IN HANDLING AND STORAGE } \\
\hline \multicolumn{4}{|l|}{ OTHER PRECAUTIONS } \\
\hline \multicolumn{4}{|c|}{\begin{tabular}{l|l} 
SIGNATURE & DATE
\end{tabular}} \\
\hline
\end{tabular}




\section{PRODUCT DESIGNATION}

03-106-5001

Phosphorous Pentachloride
MATERIAL SAFETY DATA SHEET

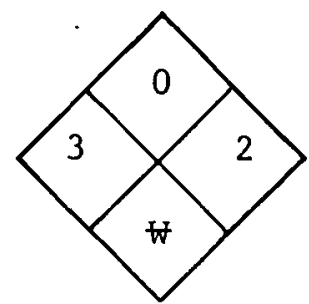

\section{SECTION I SOURCE AND NOMENCLATURE}

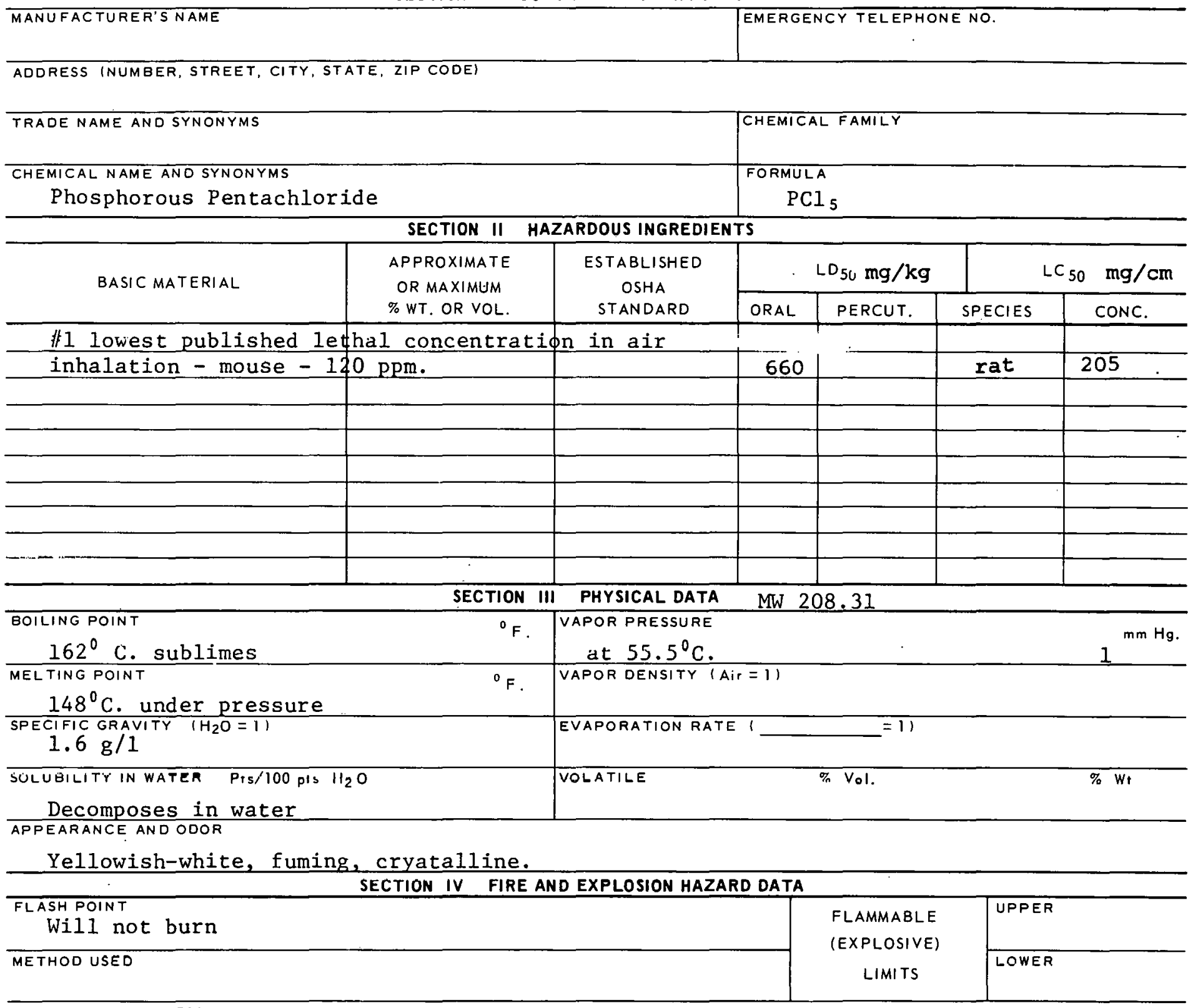

EXTINGUISHING MËBIA

Avoiid water

SPECIAL FIRE FIGHTING PROCEDURES

Wear self-contained resplrator.

UNUSUAL FIRE $\triangle N$ R XPI OSION HAZARDS 


\section{TOXIC LEVEL}

$\mathrm{TLV} 1 \mathrm{mg} / \mathrm{m}^{3}$

PRINCIPLE ROUTES OF ABSORBTION

Inhalation of dust, ingestion, skin.

REVELANT SYMPTOMS OF EXPOSURE Irritant to mucous membranes and all parts of respiratory

system. Coughing, choking, etc.

EFFECTS OF CHRONIC EXPOSURE

Possible damage to lungs, pulmonary edema.

EMERGENCY AND FIRST AID PROCEDURES

Inhalaed - rest, wash skin and eyes.

SECTION VI REACTIVITY DATA

CONDITIONS CONTRIBUTING TO INSTABILITY

CONDITIONS CONTRIBUTING TO HAZARDOUS POLYMERIZATION

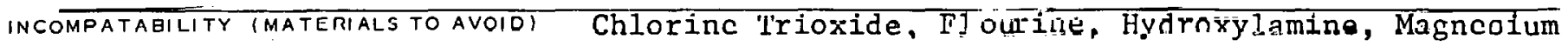
OXIde, Thosphorous

HAZARDOUS DECOMPOSITION PRODUCTS

Healed iu decomposition, emits highly toxic fumes of chlorides.

SECTION VII SPILL OR LEAK PROCEDURES

STEPS TO BE TAKEN IN CASE MATERIAL IS RELEASED OR SPILLED

Cover with sodium bicarbonate, then water spray, transfer into large container of water for disposal.

WASTE DISPOSAL METHOD

SECTION VIII SPECIAL PROTECTION INFORMATION

\begin{tabular}{|c|c|}
\hline VENTILATION REQUIREMENTS LOCAL EXHAUST & $\begin{array}{l}\text { PROTECTIVE EQUIPMENT (SPECIFY TYFES) EYE } \\
\text { COggles }\end{array}$ \\
\hline MECHANIS.AI (SEENERAL) & $\begin{array}{l}\text { GLoves } \\
\text { Rubbex (Neoprene) }\end{array}$ \\
\hline SPFCIAL & $\begin{array}{l}\text { RESPIRATOR } \\
\text { W/pIUper fifleer }\end{array}$ \\
\hline
\end{tabular}

OTHERPPROTECTIVE EQUIPMENT

Rubber apron

SECTION IX SPECI'AL PRECAUTIONS

PRECAUTIONS TO BE TAKEN IN HANDLING AND STORAGE

Protect the sealed glass bottles in which-material is stored.

OTHER PRECAUTIONS

SIGNATIIRF

OATE

106-5001B 
CAS : 007784410

CG11000

PRODUCT DESIGNATION
Potassium Arsenate
$03-110-0452$

\section{MATERIAL SAFETY DATA SHEET}

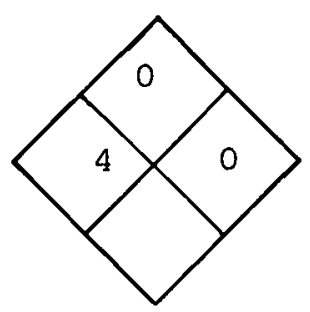

\section{SECTION I SOURCE AND NOMENCLATURE}

\section{MANUFACTURER'S NAME}

ADDRESS (NUMBER, STREET, CITY, STATE, ZIP CODE)
EMERGENCY TELEPHONE NO.

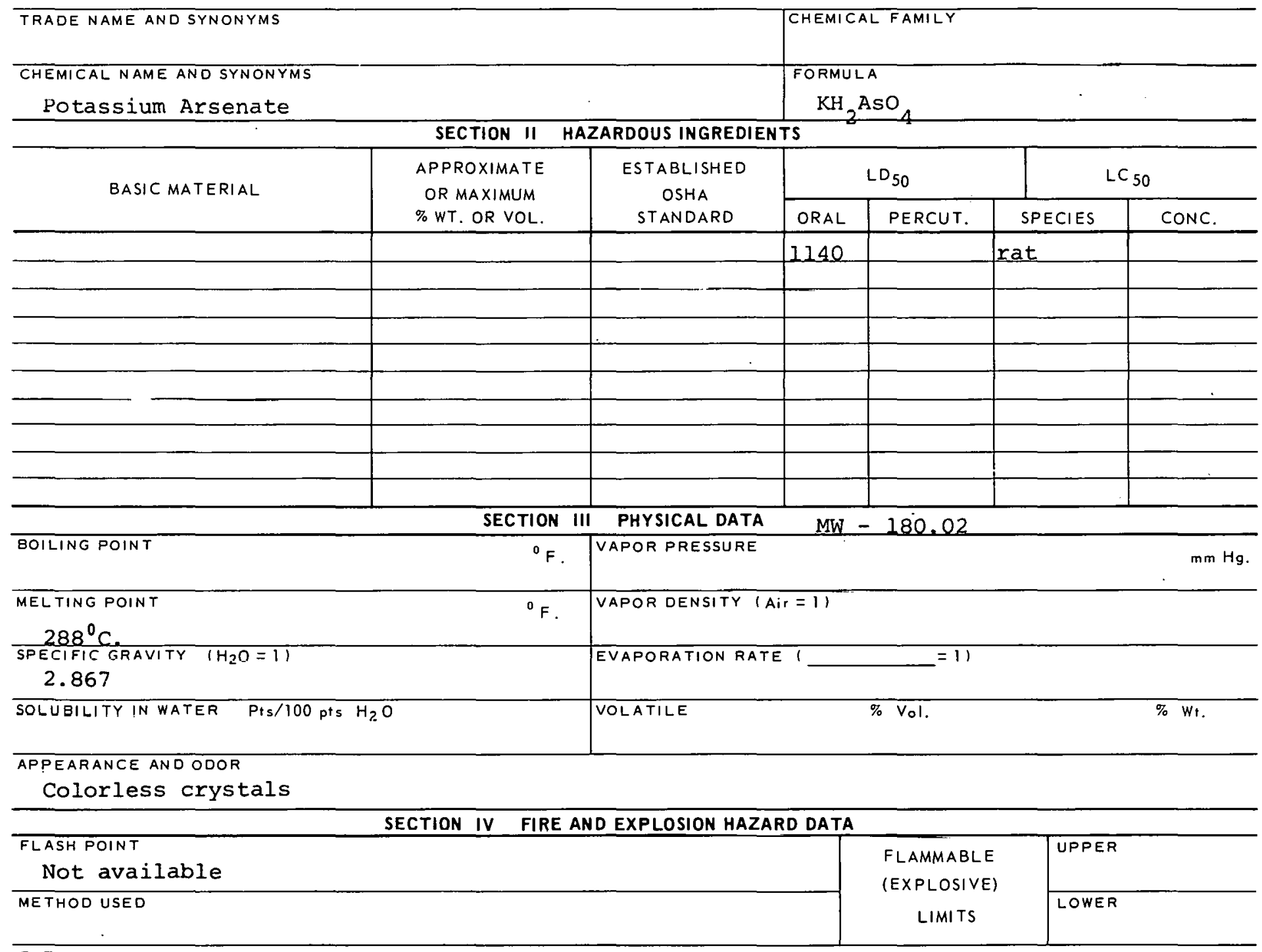

EXTINGUISHING MEDIA

None available

SPECIAL FIRE FIGHTING PROCEDURES

UNUSUAL FIRE AND EXPLOSION HAZARDS 
TOXIC LEVEL

TLV $0.25 \mathrm{mg} / \mathrm{m}^{3}$

PRINCIPLEE RUUIESS OF ABSORETION

Ingestion or inhalation of dust or fume.
CARCINOGENIC

no

SKIN AND EYE

IRRITATION Moderate

REVELANT SYMPTOMS OF EXPOSURE ACute pulmonary edema, restlessiiess, dyspnea, cyanosis. If ingested - gastroenteritis, vomiting diarrhea, drop in blood pressure. of weight, nausea, salivation.

EMERGENCYIAKa ILRI OHD PRQEESURESf ingested - give emetic (antidote - dimer caprol)obtain medical aid.

SECTION VI REACTIVITY DATA

CONDITIONS CONTRIBUTING TO INSTABILITY

CONDITIONS CONTRIBU TING TO HAZARDOUS POLYMERIZATION

INCOMPATABILITY (MATERIALS TO AVUID)

HAZARDOUS DECOMPOSITION PRODUCTS

SECTION VII SPILL OR LEAK PROCEDURES

STEPS TO BE TAKEN IN CASE MATERIAL IS RELEASED OR SPILLED

\section{WASTE DISPOSAL METHOD}

SECTION VIII SPECIAL PROTECTION INFORMATION

\begin{tabular}{l|l}
\hline VENTILATION REQUIREMENTS LOCAL EXHAUST & PRIO \\
\hline MECHANICAL IGENERALI & \\
\hline SPECIAL &
\end{tabular}

safety glasses or goggles

OTHER PROTECTIVE EOUIPMENT

GLOVES

Rubber

RFSPIP.ATAR

With partílulate rI १+er.

SECTION IX SPECIAL PRECAUTIONS

PRECAUTIONS TO BE TAKEN IN HANDLING AND STORAGE 


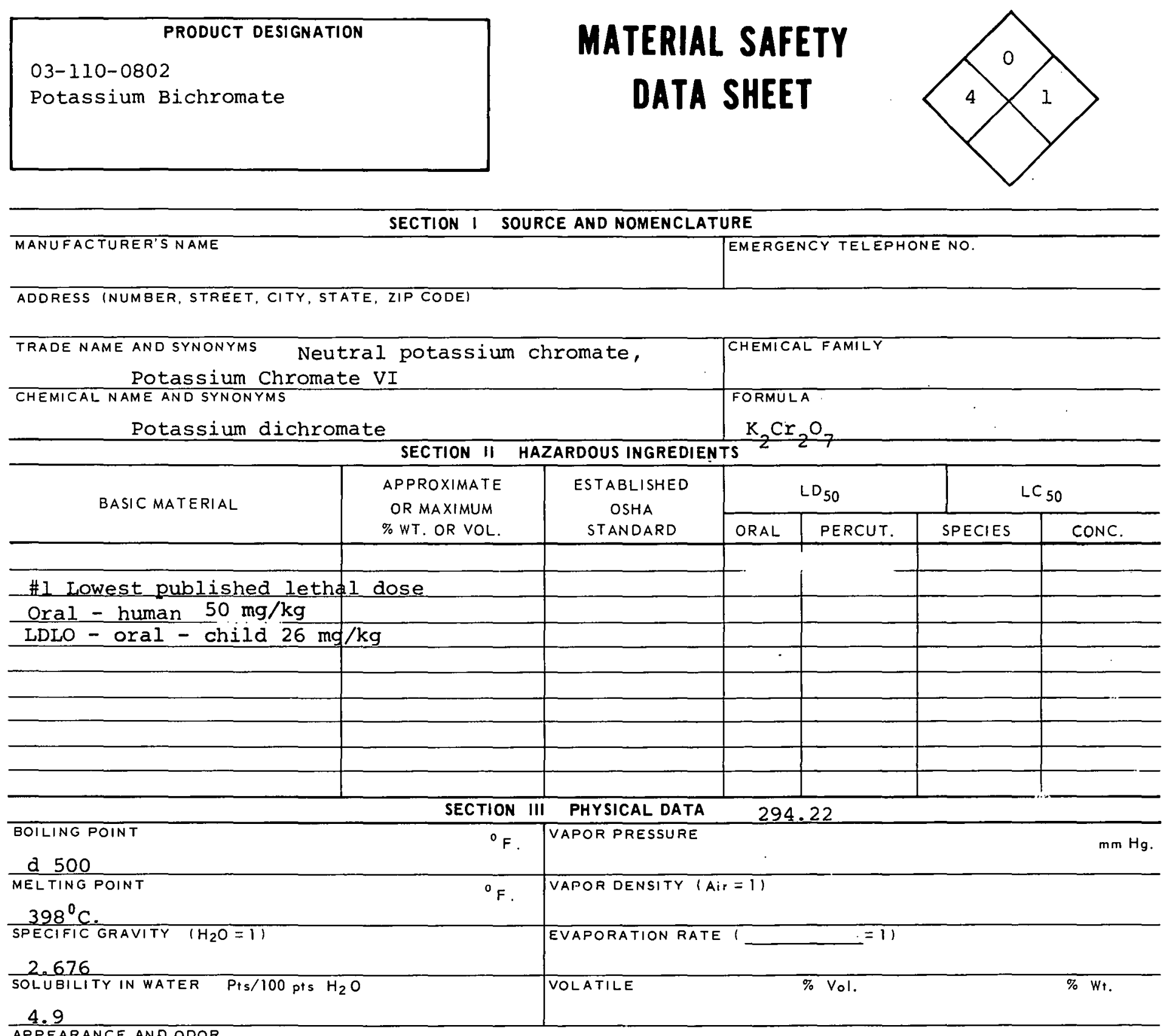

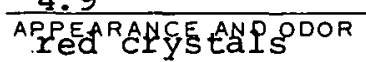

SECTION IV. FIRE AND EXPLOSION HAZARD DATA

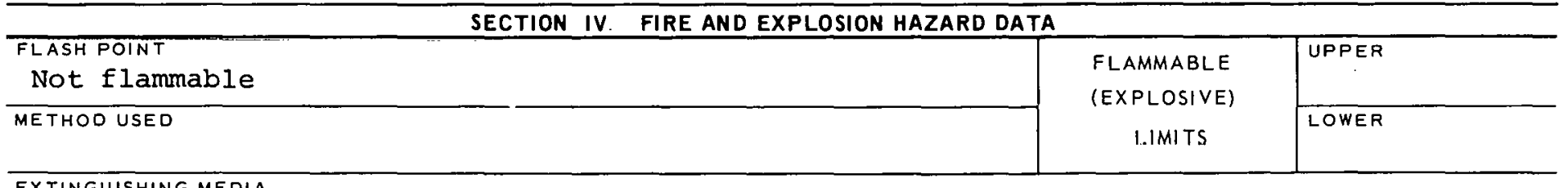

EXTINGUISHING MEDIA

Wälèr

SPECIAL FIRE FIGHTING PROCEDURES

Material may form hot viscous foam. Possible steam explosion.

UNUSUAL FIRE AND EXPLOSION HAZAROS

Furnishes oxygen - container may explode in fire. 


\section{TOXIC LEVEL \\ TLV $-0.15 \mathrm{mg} / \mathrm{m}^{3}$}

PRINCIFLE ROUTES OF ABSORBTION

Inhalation of dust by mouth.
CARCINOGENIC

yes
SKIN ANU EYE

IRRITATION Moderate

REVELANT SYMPTOMS OF EXPOSURE Irritation of skin and eyes, irritation of respiratory tract; internal irritation and damage.

EFFECTS OF CHRONIC EXPOSURE Asthmatic symptoms - liver and kidney damage; lung cancer.

EMERGENCY AND FIRST AID PROCEDURES Wash from skin and eyes; if swallowed - wash mouth, give emetic, get medical attention; inhalation - rest and keep warm.

\section{SECTION VI REACTIVITY DATA}

CONDITIONS CONTRIBUTING TO INSTABILITY

Powerful oxidizing agent.

CONDITIONS CONTRIBUTING TO HAZARDOUS POLYMERIZATION

INCOMPATAEILITY (MATERIALS TO AVOID) May react with organic materials to produce a fire

(acetic acid, acetic anhydride, anthracene, ethyl alcohol)

HAZARDOUS DECOMPOSITION PRODUCTS

SECTION VII SPILL OR LEAK PROCEDURES

STEPS TO BE TAKEN IN CASE MATERIAL IS RELEASED OR SPILLED

Cover with weak reducing agent such as sodium bisulfite. Transfer slurry

to large water container for disposal.

WASTE DISPOSAL METHOD

SECTION VIII SPECIAL PROTECTION INFORMATION

\begin{tabular}{l|l}
\hline VENTILATION REQUIREMENTS LOCAL EXHAUST & $\begin{array}{l}\text { PROTECTIVE EQUIPMENT (SPECIFY TYPES) EYE } \\
\text { GOggleS }\end{array}$ \\
\hline MELHANILAL (GENERAL) & $\begin{array}{l}\text { GLOVES } \\
\text { Rubber }\end{array}$ \\
\hline SPECIAL & $\begin{array}{l}\text { RESPIRATOR } \\
\text { With PYOPEX filtor }\end{array}$ \\
\hline
\end{tabular}

OTHER PROTECTIVE EQUIPMENT

SECTION IX SPECIAL PRECAUTIONS

PRECAUTIONS TO BE TAKEN IN HANDLING AND STORAGE

Store in cool, dry place away from easily oxidizable substances.

OTHER PRECAUTIONS

SIGNATURE

DATE

110-0802B 
CA5:007789229

PRODUCT DESIGNATION

03-110-103.2

Potassium Bifluoride
TS66500

MATERIAL SAFETY

DATA SHEET

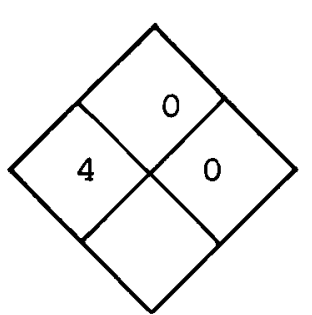

\section{SECTION I SOURCE AND NOMENCLATURE}

MANUFACTURER'S NAME

EMERGENCY TELEPHONE NO.

ADDRESS (NUMBER, STREET, CITY, STATE, ZIP CODE)

TRADE NAME AND SYNONYMS

Potassium acid fluoride

CHEMICAL NAME AND SYNONYMS

Potassium difluoride

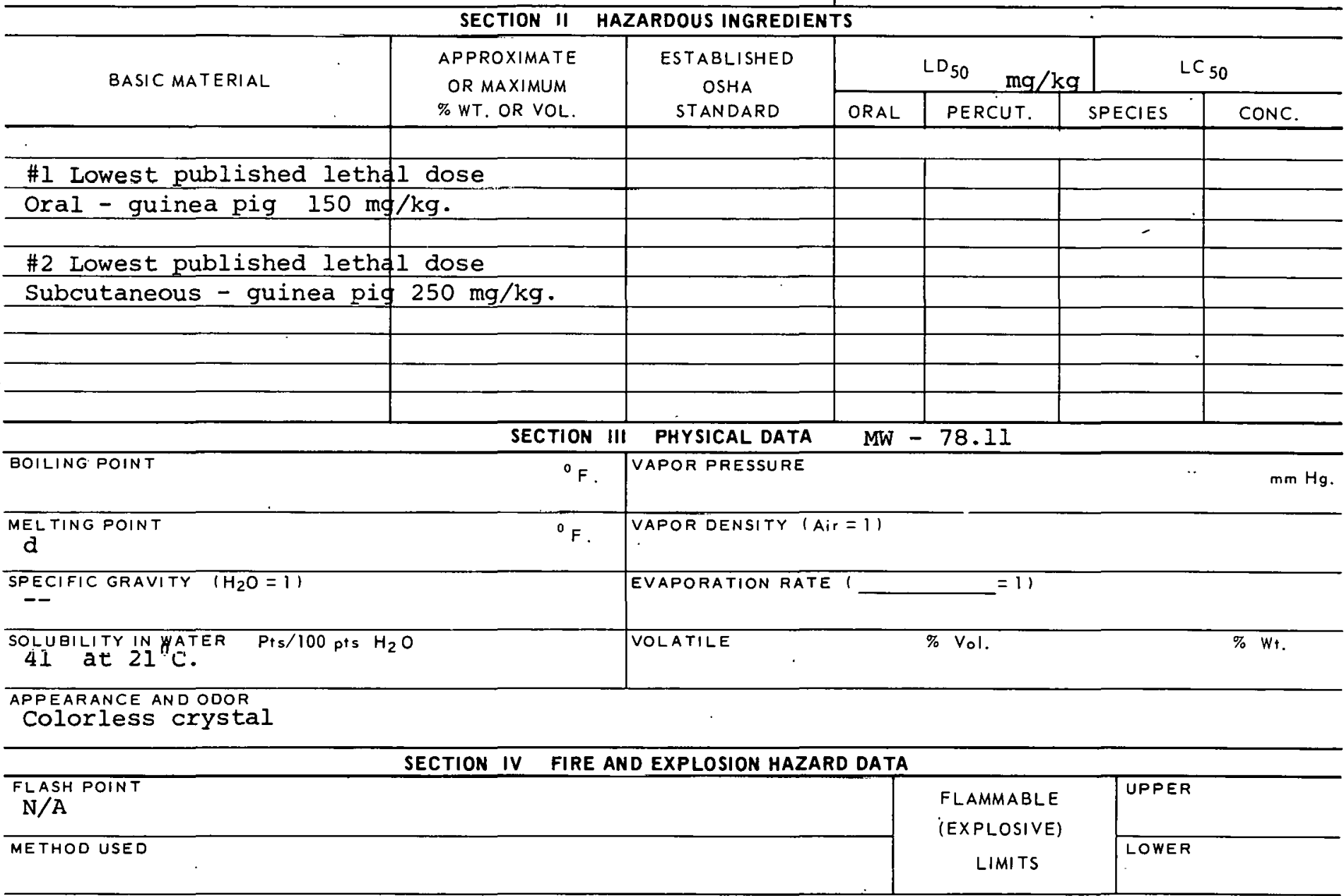

EXTINGUISHING MEDIA

\section{Water}

SPECIAL FIRE FIGHTING PROCEDURES

Wear full protective clothing and respirator.

UNUSUAL FIRE AND EXPLOSION HAZARDS

$\mathrm{N} / \mathrm{A}$ 


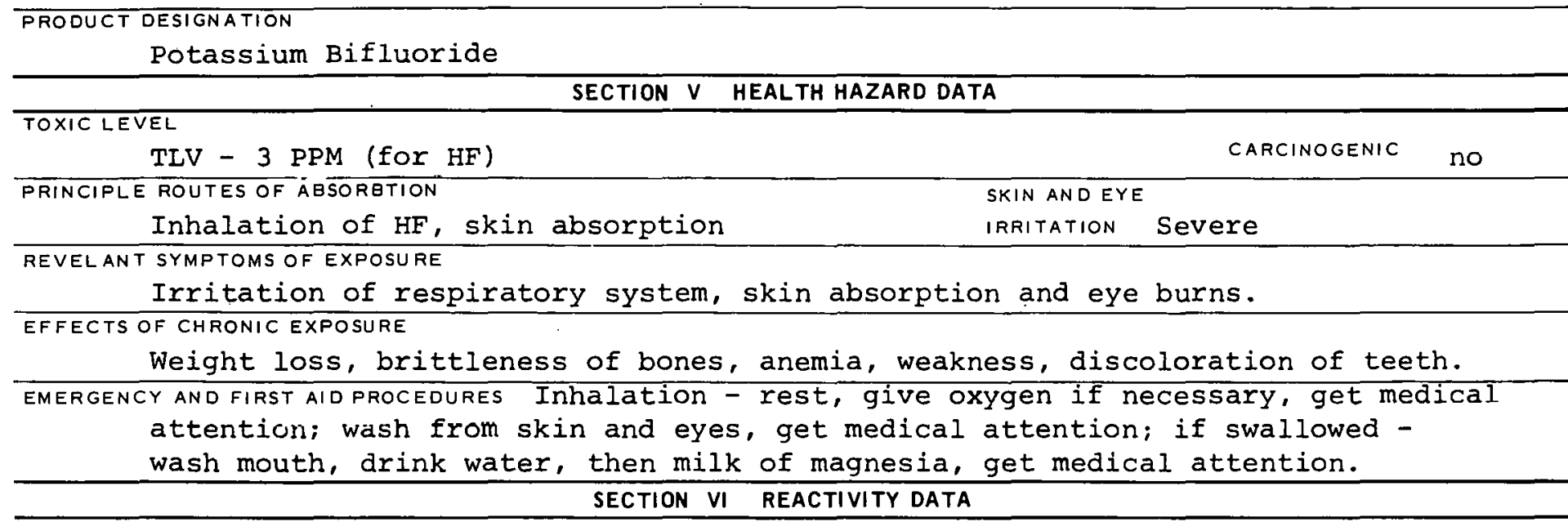

CONDITIONS CONTRIBUTING TO INSTABILITY

CONDITIONS CONTRIBUTING TO HAZARDOUS POLYMERIZATION

INCOMPATABILITY (MATERIALS TO AVOID)

HAZAROOUS DECOMPOSITION PRODUCTS

SECTION VII SPILL OR LEAK PROCEDURES

STEPS TO BE TAKEN IN CASE MATERIAL IS RELEASED OR SPILLED

WASTE DISPOSAL METHOD

SECTION VIII SPECIAL PROTECTION INFORMATION

\begin{tabular}{l|l}
\multicolumn{1}{c}{ SECTION VIII SPECIAL PROTECTION INFORMATION } \\
\hline VENTILATION REQUIREMENTS LOCAL EXHAUST & $\begin{array}{l}\text { PROTECTIVE LQUIPMENT ISPECIFY TYPESI EYE } \\
\text { GOggles }\end{array}$ \\
\hline MECHANICAL IGENERAL) & $\begin{array}{l}\text { GLOVES } \\
\text { Rubber gloves }\end{array}$ \\
\hline SPECIAL & $\begin{array}{l}\text { RESPIRATOR } \\
\text { W/appIOpridL filter }\end{array}$ \\
\hline
\end{tabular}

OTHER PROTECTIVE EQUIPMENT

\section{SECTION IX SPECIAL PRECAUTIONS}

PRECAUTIONS TO BE TAKEN IN HANDLING AND STORAGE

OTHER PRECAUTIONS

SIGNATURE

DATE

$110-1032 B$ 
CAS: 007789006

\begin{tabular}{|} 
PRODUCT DESIGNATION \\
$03-110-2902$ \\
Potassium Chromate
\end{tabular}

\section{MATERIAL SAFETY DATA SHEET}

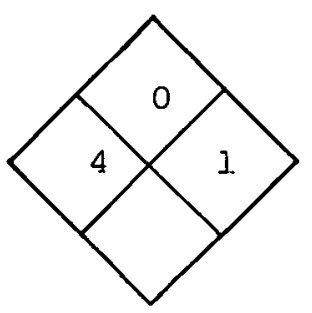

SECTION I SOURCE AND NOMENCLATURE

\begin{tabular}{l|l|l}
\hline MANUFACTURER'S NAME & EMERGENCY TELEPHONE NO. \\
\hline ADDRESS (NUMBER, STREET, CITY, STATE, ZIP CODE) &
\end{tabular}

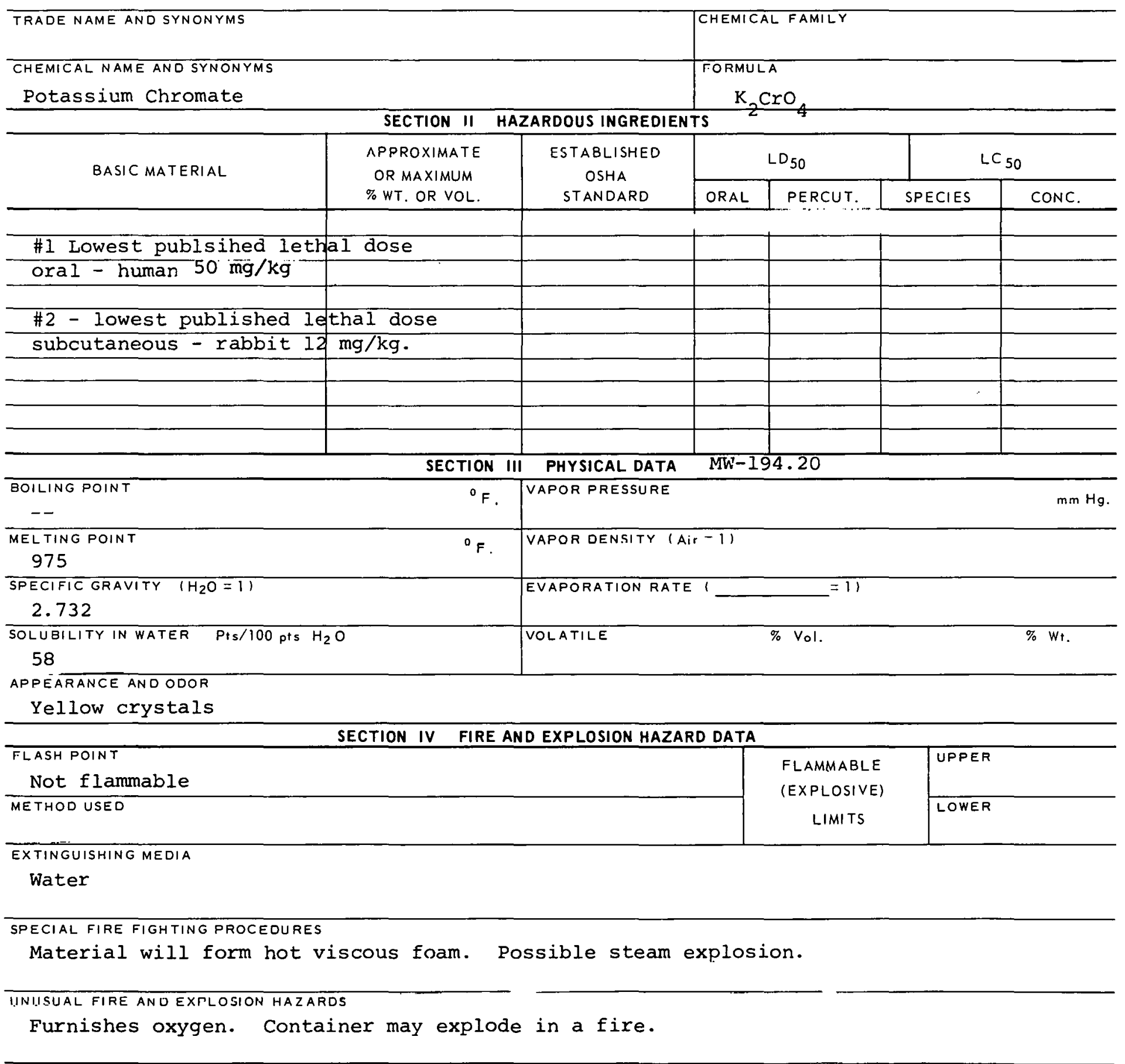


CAS : 000151508

$03-110-3532$

PRODUCT DESIGNATION

03-110-3502

Potassium Cyanide
TS87500

MATERIAL SAFETY

DATA SHEET

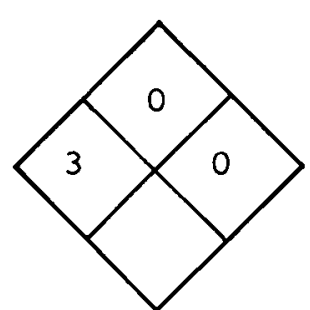

SECTION I SOURCE AND NOMENCLATURE

MANUFACTURER'S NAME

TEMERGENCY TELEPHONENO

ADDRESS (NUMBER, STREET, CITY, STATE, ZIP CODE)

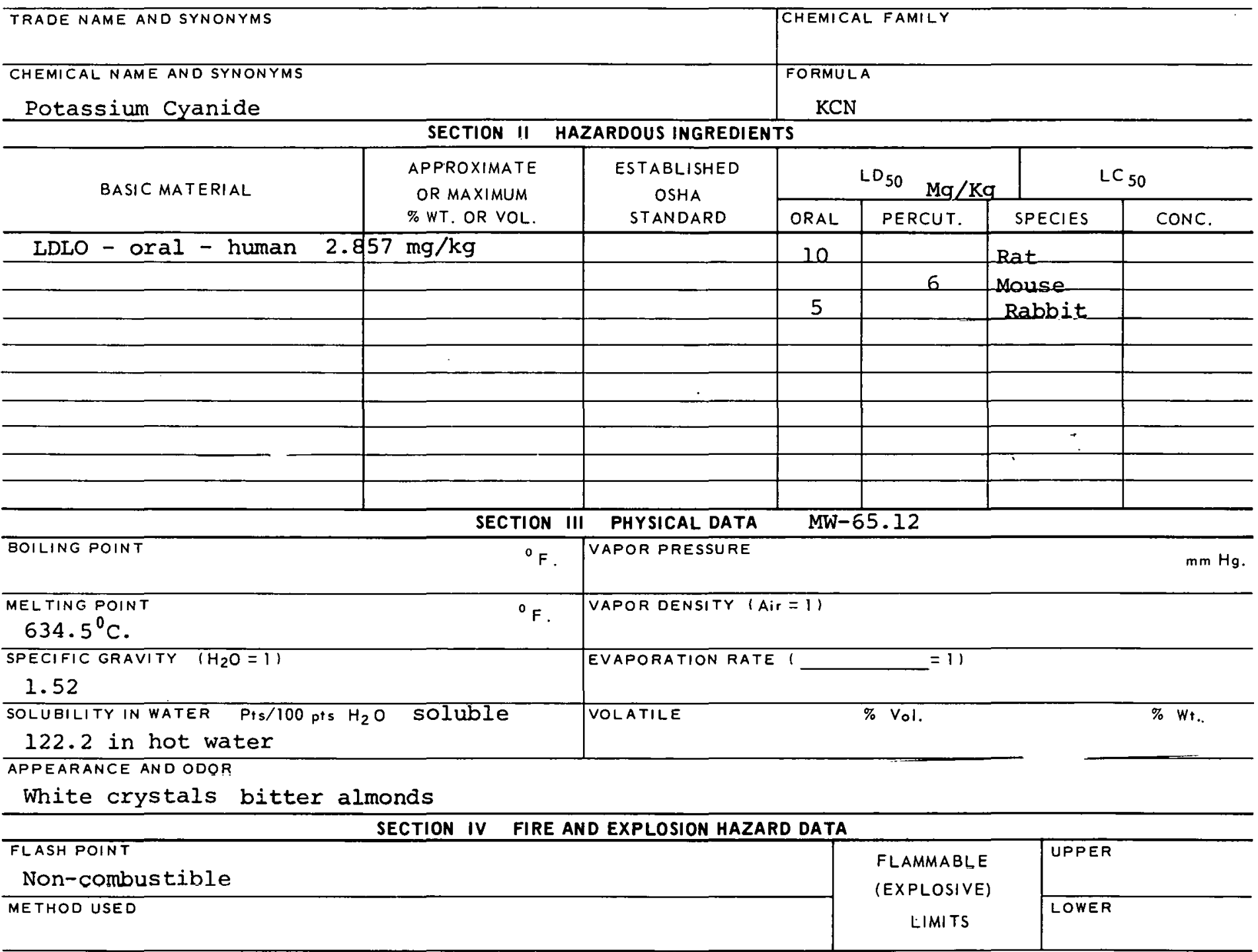

EXTINGUISHING MEDIA

None available

SPECIAL FIRE FIGHTING PROCEDURES

Wear full protective clothing.

UNUSUAL FIRE AND EXPLOSION HAZARDS

Releases HCN with water or acids. 
REVELANT SYMPTOMS OF EXPOSURE Gidainess, dizziness, rapid heart action followed by unconsciousness; if swallowed - rapid respiration - becoming slow - unconsciouness and death. EFFECTS OF CHRONIC EXPOSURE Dizziness, weakness, congestion of lungs, loss of appetite, loss of weight.

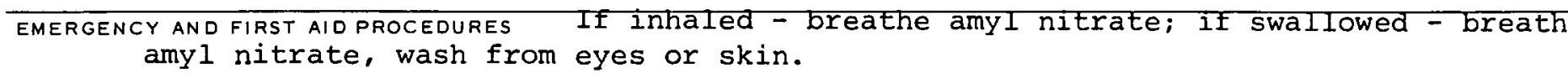

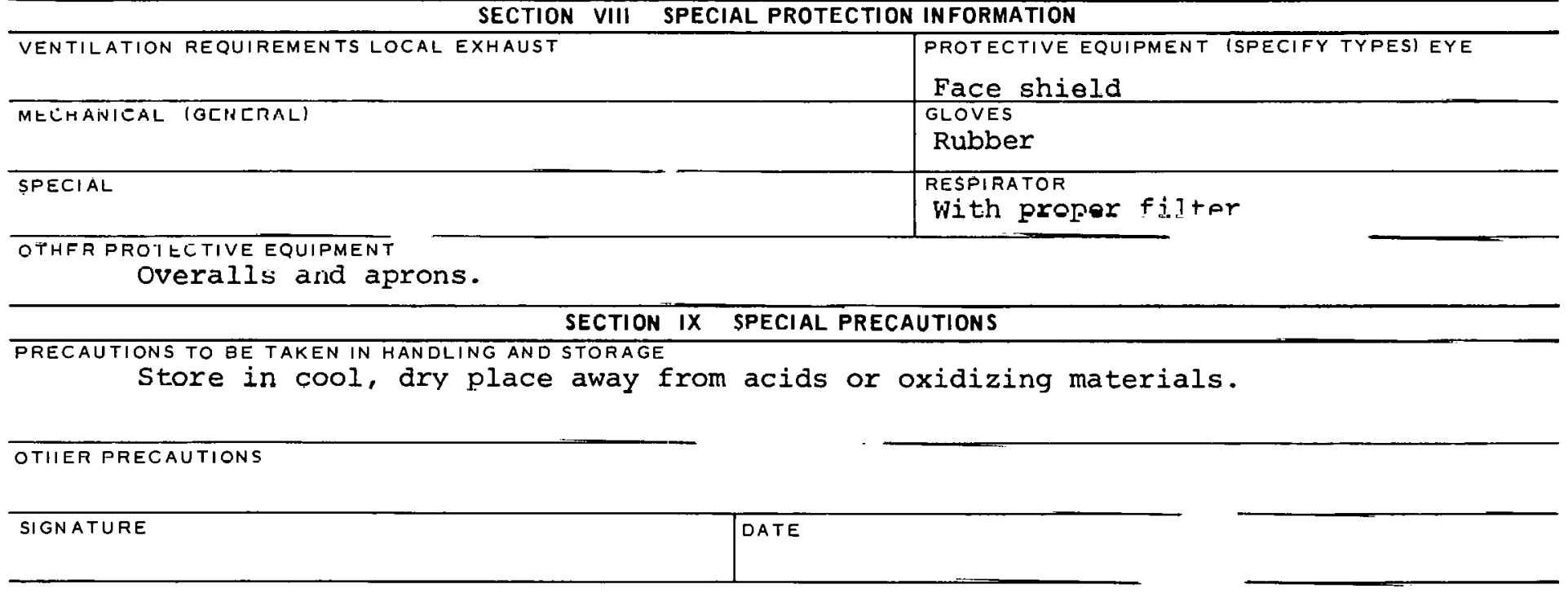




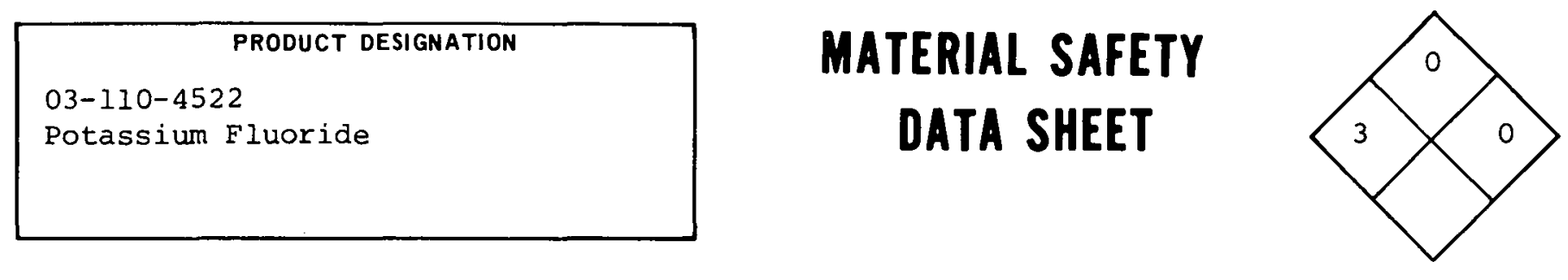

\section{SECTION I SOURCE AND NOMENCLATURE}

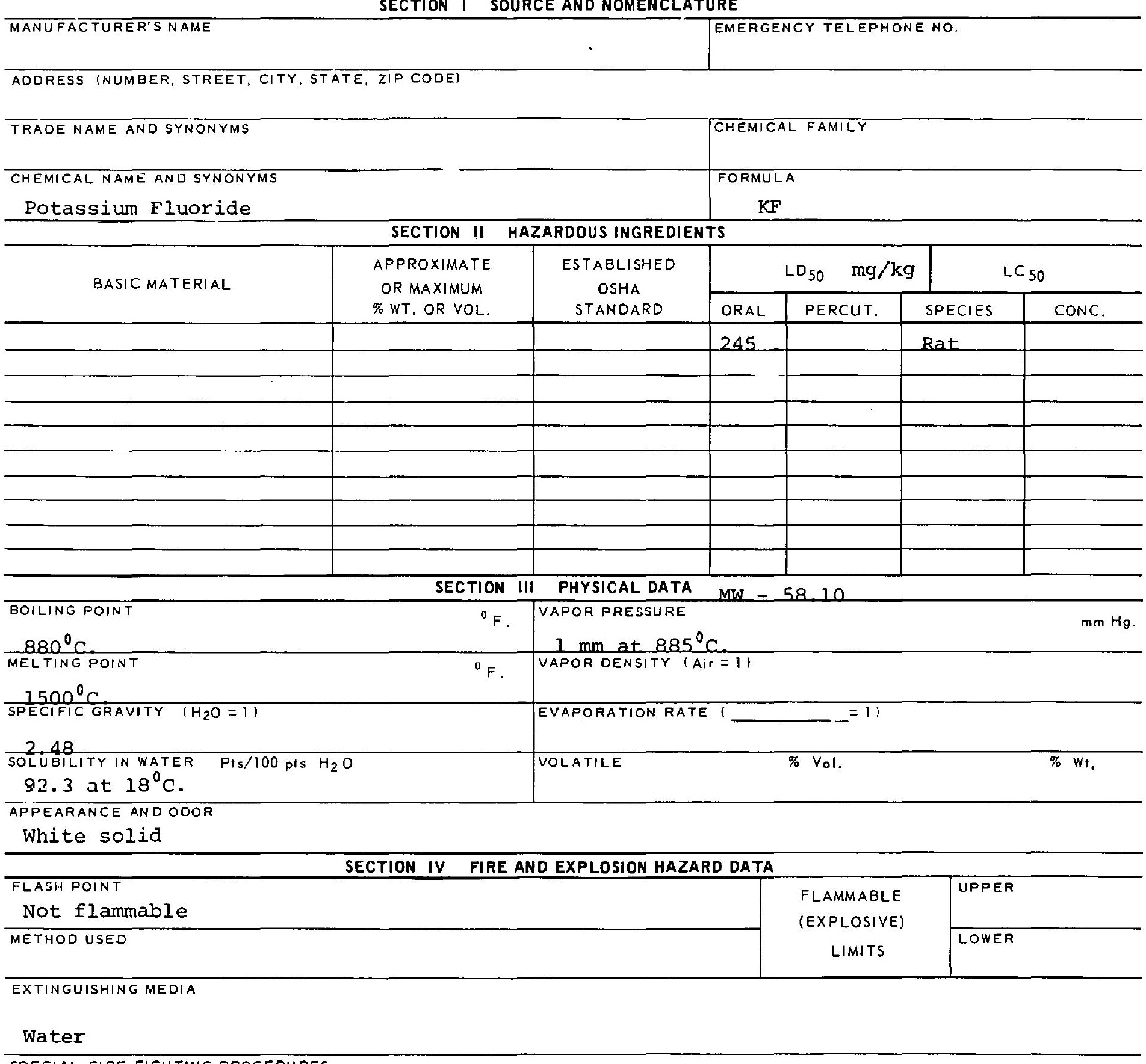

SPECIAL FIRE FIGHTING PROCEOURES

UNUSUAL FIRE AND EXPLOSION HAZARDS 


\section{SECTION V HEALTH HAZARD DATA}

TOXIC LEVEL

$$
2.5 \mathrm{mg} / \mathrm{m}^{3}
$$

PRINCIPLE ROUTES OF ABSORBTION

Inhalation of dust, ingestion skin.
CARCINOGENIC NO

SKIN AN D EYE

IRRITATION Moderate REVELANT SYMPTOMS OF EXPOSURE Irritation of respiratory system; severe internal irritation and damage; skin and eye burns. and damage; skin

Increase in fluoride content of teeth.

EMERGENCY AND FIRST AID PROCEDURES Inhalation - rest, keep warm, get medical aid; if swallowed wash mouth, drink water, then milk of magnesia, get medial aid, wash from skin and eyes, get medical aid.

\section{SECTION VI REACTIVITY DATA}

CONDITIONS CONTRIBUTING TO INSTABILITY

CONDITIONS CONTRIBUTING TO HAZARDOUS POLYMERIZATIION

IIGOMPATARI, ITY IMATERIALS TO AVOID)

HÄZ̈ARDOUS UELUMHUSITIUN PRODUCTS

SECTION VII SPILL OR LEAK PROCEDURES

STEPS TO BE TAKEN IN CASE MATERIAL IS RELEASED OR SPILLED

Sweep into container for disposal.

WASTE DISPOSAL METHOD

SECTION VIII SPECIAL PROTECTION INFORMATION

VENTILATION REQUIREMENTS LOCAL EXHAUST

ROTECTIVE EQUIPMENT (SPECIFY TYPES) EYE

Face shield

$\overline{\text { MECHANICAL (GENERAL) }}$

GLOVES

Rubber

SPECIAL

OTHER PROTECTIVE EQUIPMENT

RESPIRATOR

with proper Filter

\section{SECTION IX SPECIAL PRECAUTIONS}

PRECAUTIONS TO BE TAKEN IN HANDLING AND STORAGE

store away from contacts with acids.

\section{OTHER PRECAUTIONS}




PRODUCT DESIGNATION
Potassium Hydroxide
$03-110-5042$

\section{MATERIAL SAFETY DATA SHEET}

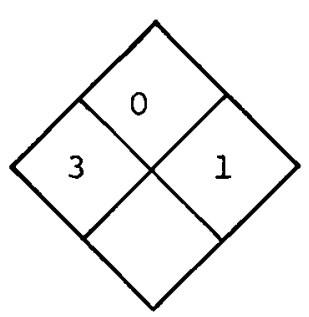

SECTION I SOURCE AND NOMENCLATURE

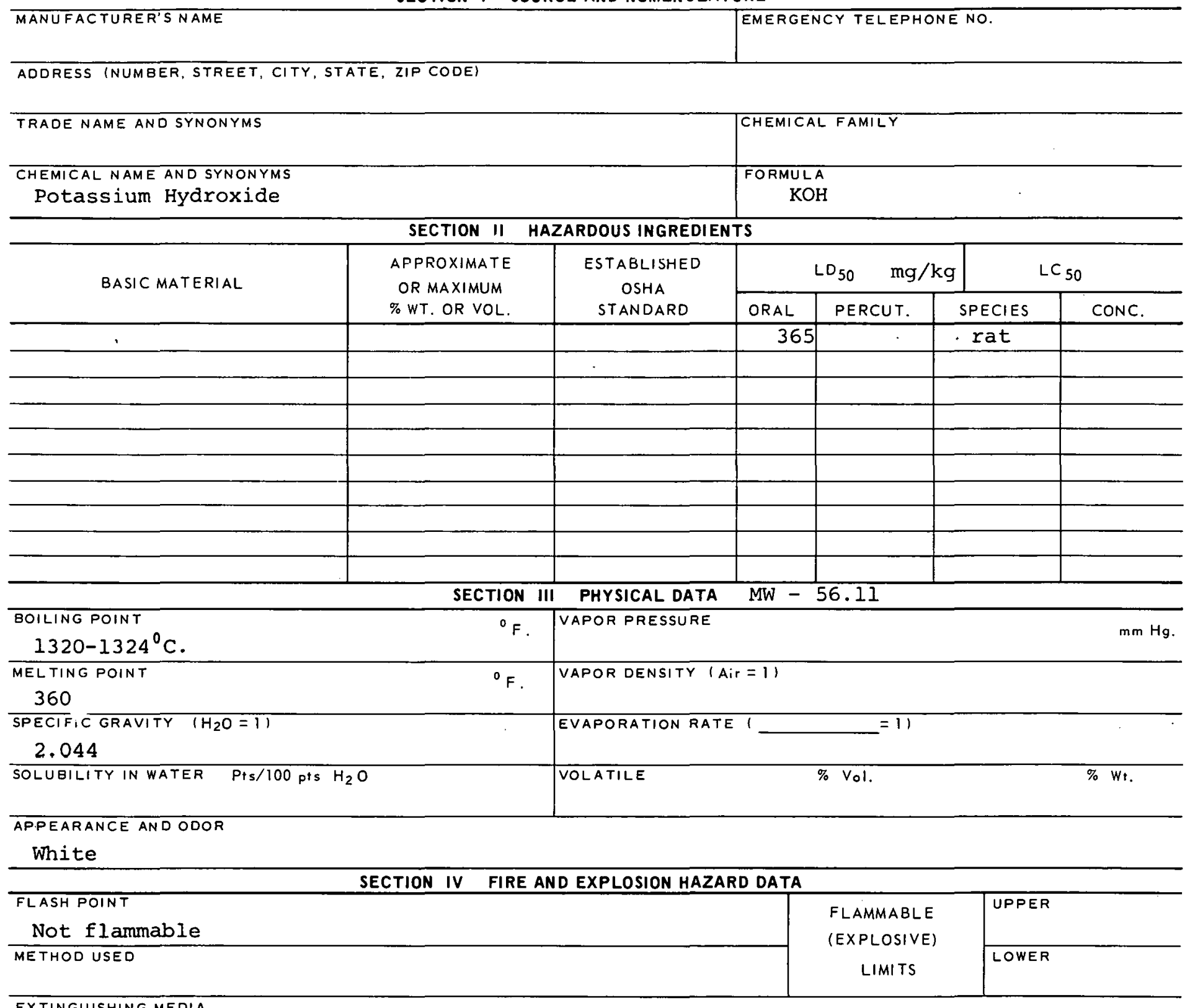

EXTINGUISHING MEDIA

Flood with water

SPECIAL FIRE FIGHTING PROCEDURES

Wear full protective clothing - do not splash or splatter material.

UNUSUAL FIRE AND EXPLOSION HAZARDS 


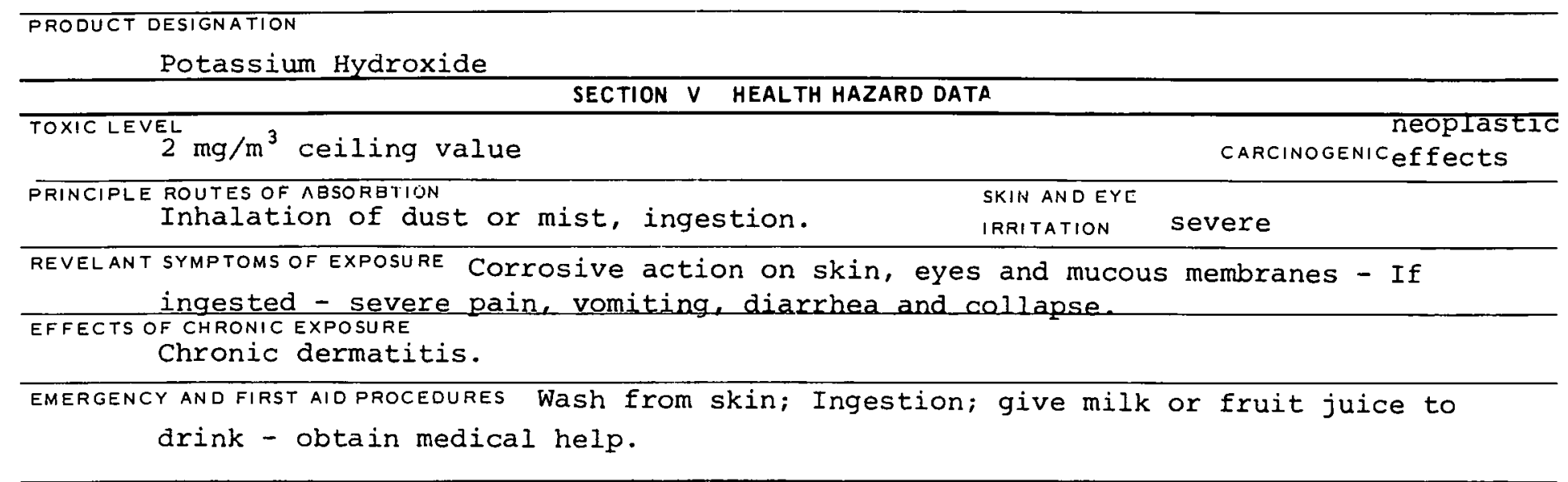

\section{SECTION VI REACTIVITY DATA}

CONDITIONS CONTRIBUTING TO INSTABILITY

CONDITIONS CONTRIBUTING TO HAZARDOUS POLYMERIZATION

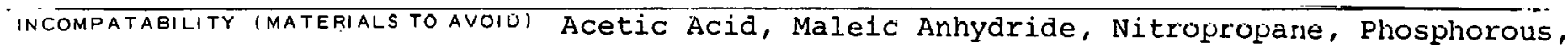

Trichloruethylene.

HAZARDOUS DECOMPOSITION PRODUCTS

SECTION VII SPILL OR LEAK PROCEDURES

STEPS TO BE TAKEN IN CASE MATERIAL IS RELEASEO OR SPILLED

WASTE DISPOSAL METHOD

SECTION VIII SPECIAL PROTECTION INFORMATION

VENTILATION REQUIREMENTS LOCAL EXHAUS

MECHANICAL (GENERAL)

Goggles

si.nVFs

Rubber

SPFC.IAI.

RESPIRATOR

OTIIER PROTECTIVE EQUIPMENT

w'appropriate filter

Rubber apron and full face shield.

SECTION IX SPECIAL PRECAUTIONS

PRECAUTIONS TO BE TAKEN IN HANDLING AND STORAGE

Store in dry place; protect against moisture and water. Separate from acids,

metals, explosives, organic peroxides, and easily ignitable materials.

OTHER PRECAUTIONS 


PRODUCT DESIGNATION
Pot-110-5602
Potassium Metal

\section{SECTION I SOURCE AND NOMENCLATURE}

MANUFACTURER'S NAME

EMERGENCY TELEPHONE NO.

ADDRESS (NUMBER, STREET, CITY, STATE, ZIP CODE)

TRADE NAME AND SYNONYMS

CHEMICAL FAMILY

CHEMICAL NAME AND SYNONYMS

FORMULA

Potassium, Kalium

K SECTION II HAZARDOUS INGREDIENTS

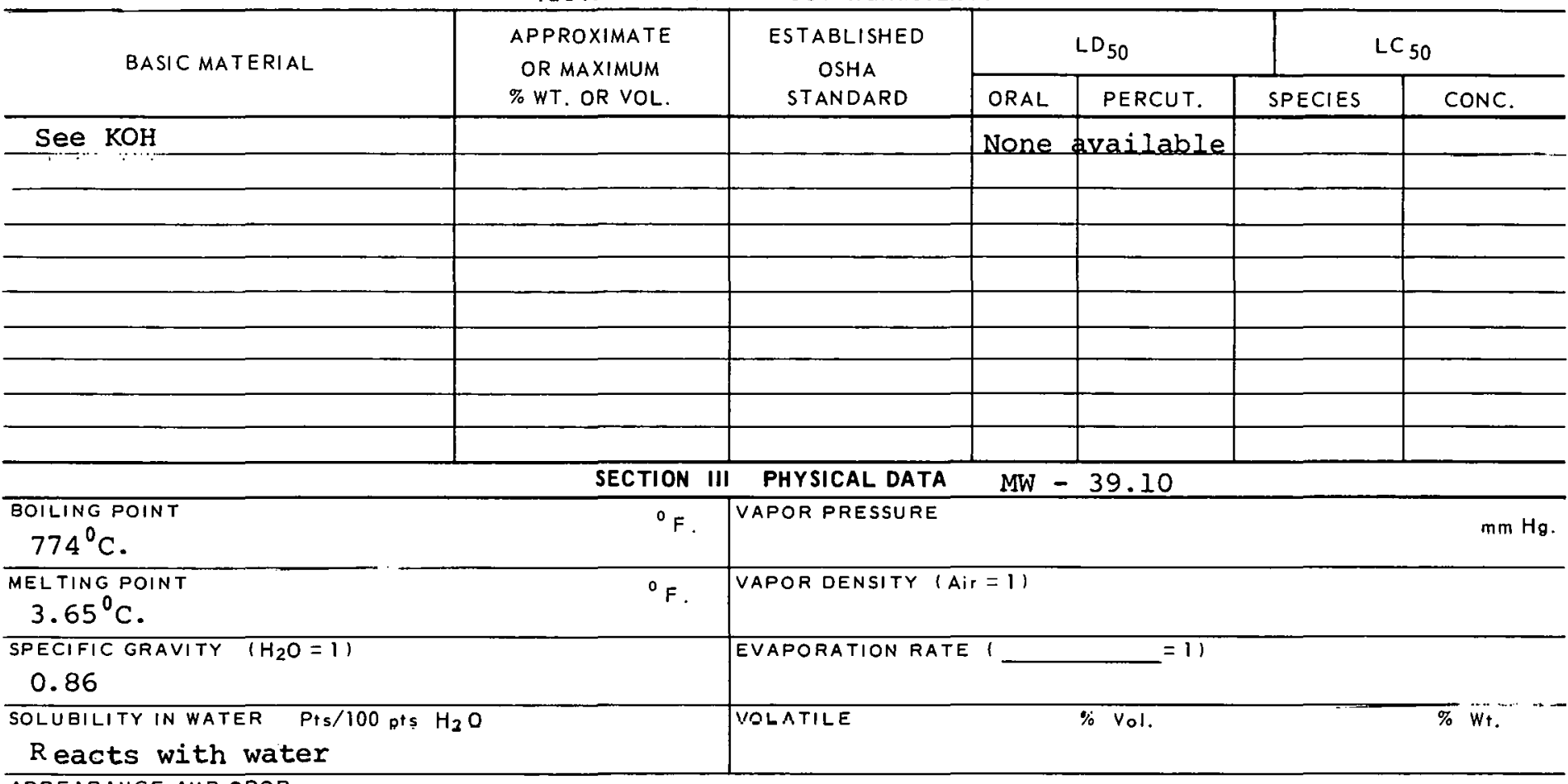

APPEARANCE AND ODOR

Silver metal

SECTION IV FIRE AND EXPLOSION HAZARD DATA

FLASH POINT

$\mathrm{N} / \mathrm{A}$

METHOD USED

FLAMMABLE

(EXPLOSIVE)

LIMITS
UPPER

LOWER

EXTINGUISHING MEDIA

Use metal " $\mathrm{X}$ " extinguisher.

SPECIAL FIRE FIGHTING PROCEDURES

Smother fire with powder (no water).

UNUSUAL FIRE AND EXPLOSION HAZARDS

Explosive action with water, forms $\mathrm{KO}_{2}$ or $\mathrm{K}_{2} \mathrm{O}$ (yellow) with oxygen which explodes on contact with organics. 
Potassium Metal

SECTION $V$ HEALTH HAZARD DATA

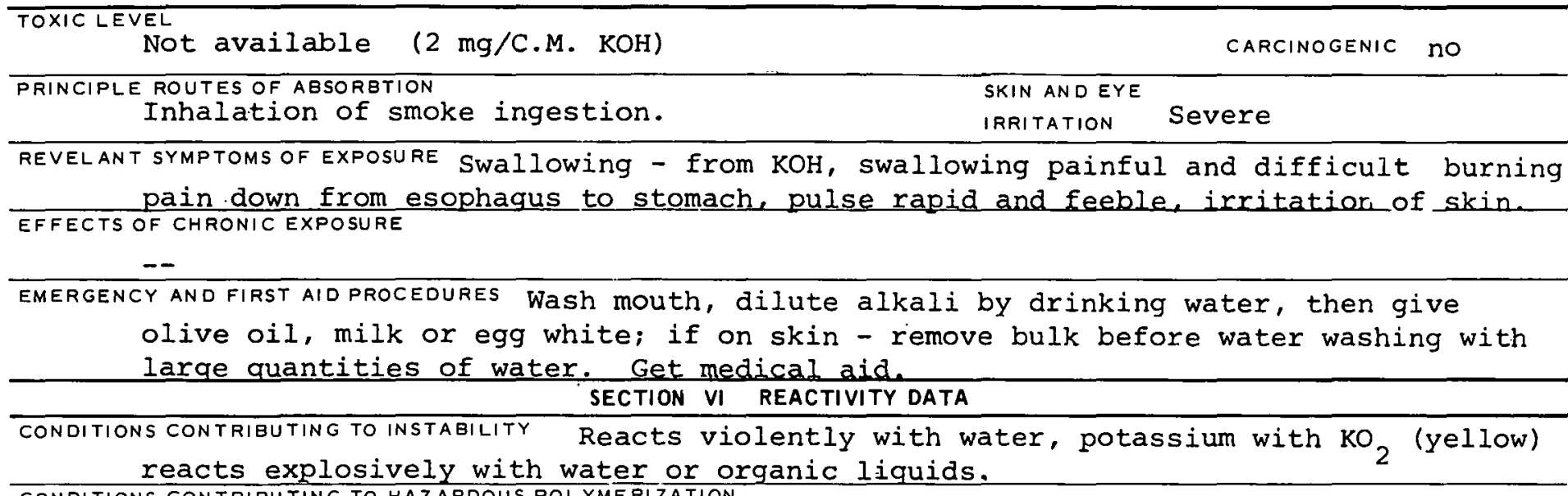
CONDITIONS CONTRIBUTING TO HAZARDOUS POLYMERIZATION

INCOMPATABILITY (MATERIALS TOAVOIO) Fluorocarbons, halogens, air, oxygen, potassium nitrate, water, potassium superoxide, chlorinated hydrocarbons. FÄTAROOUg DEOOMROGITIOM PPQNIICTS

\section{SECTION VII SPILL OR LEAK PROCEDURES}

STEPS TO BE TAKEN IN CASE MATERIAL IS RELEASED OR SPILLED

WASTE DISPOSAL METHOD

SECTION VIII SPECIAL PROTECTION INFORMATION

\begin{tabular}{l|l}
\hline VENTILATION REQUIREMENTS LOCAL EXHAUST & $\begin{array}{l}\text { PROTECTIVE EQUIPMENT (SPECIFY TYPES) EYE } \\
\text { FaCe ShIEld }\end{array}$ \\
\hline MECHANICAL IGENERAL) & $\begin{array}{l}\text { GLOVES } \\
\text { Rubber }\end{array}$ \\
\hline SPFCIAI.. & $\begin{array}{l}\text { RESPIRATOR } \\
\text { Wi.th proper filter }\end{array}$ \\
\hline
\end{tabular}

OTHER PROTECTIIVE EQUIPMENT

Face shield with protective clothing. SECTION IX SPECIAL PRECAUTIONS

PRECAUTIONS TO BE TAKEN IN HANDLING AND STORAGE

store under kerosens or mineral oil. Keep away from water.

OTHER PRECAUTIONS 

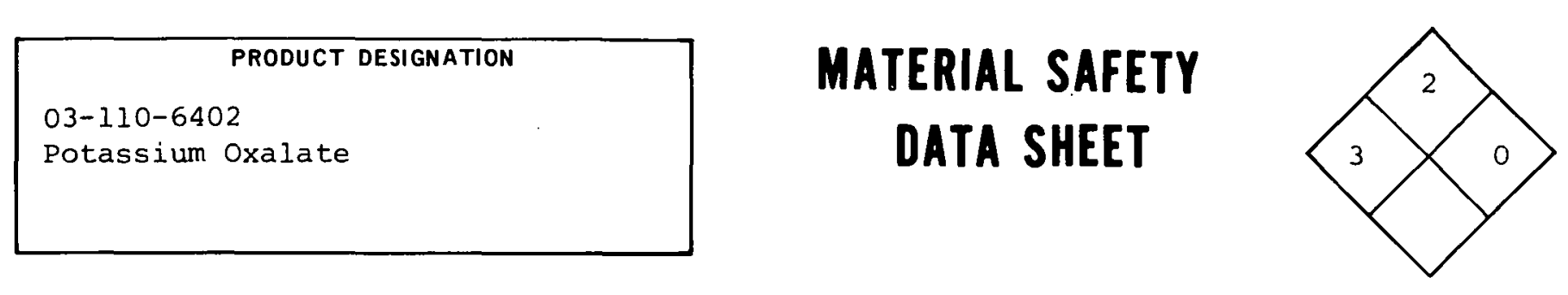

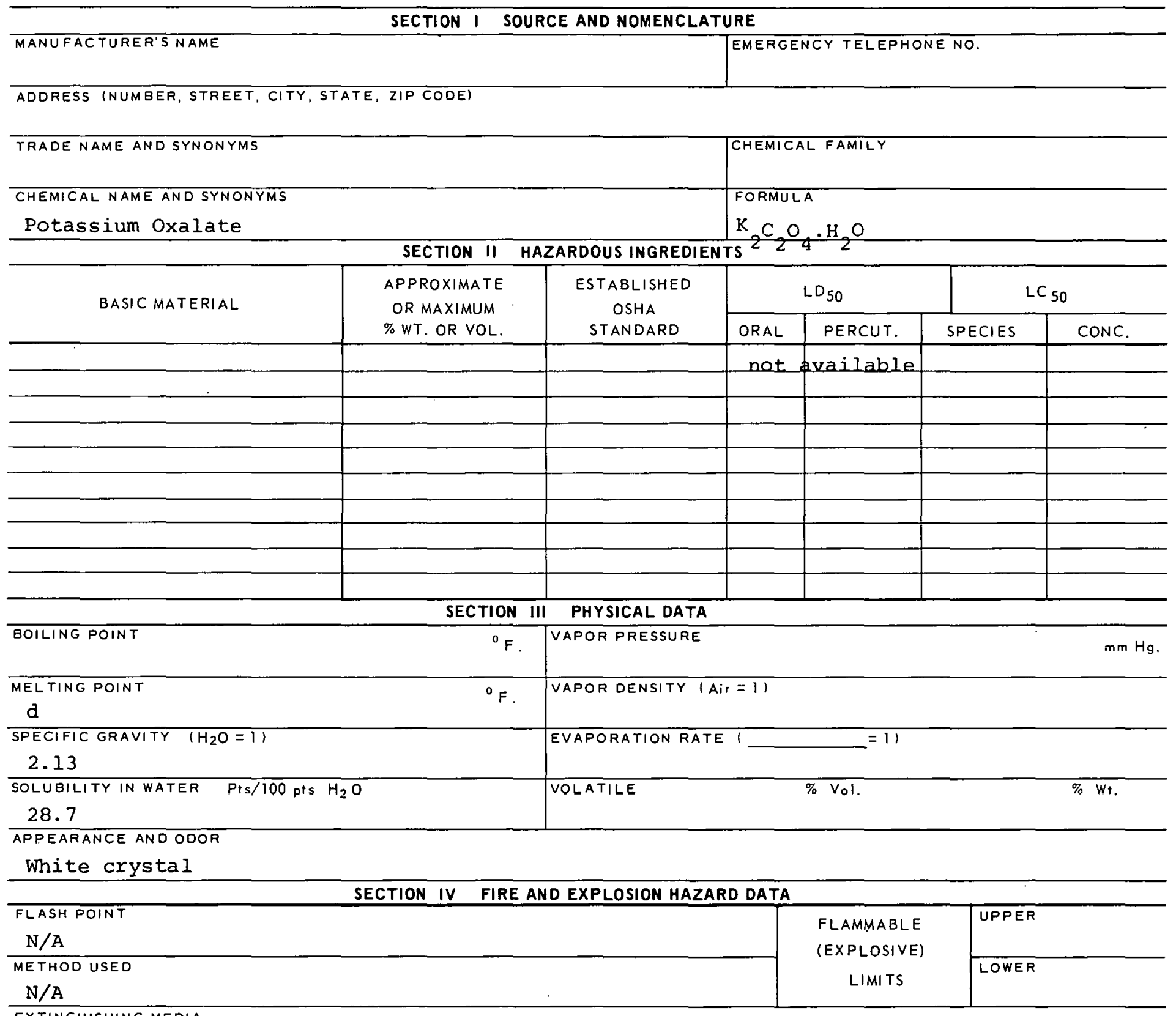

EXTINGUISHING MEDIA

$N / A$

SPECIAL FIRE FIGHTING PROCEDURES

$\mathrm{N} / \mathrm{A}$

UNUSUAL FIRE AND EXPLOSION HAZARDS

When heated to decomposition temperature may emit toxic fumes. 


\section{PRODUCT DESIGNATION \\ Potassium Oxalate}

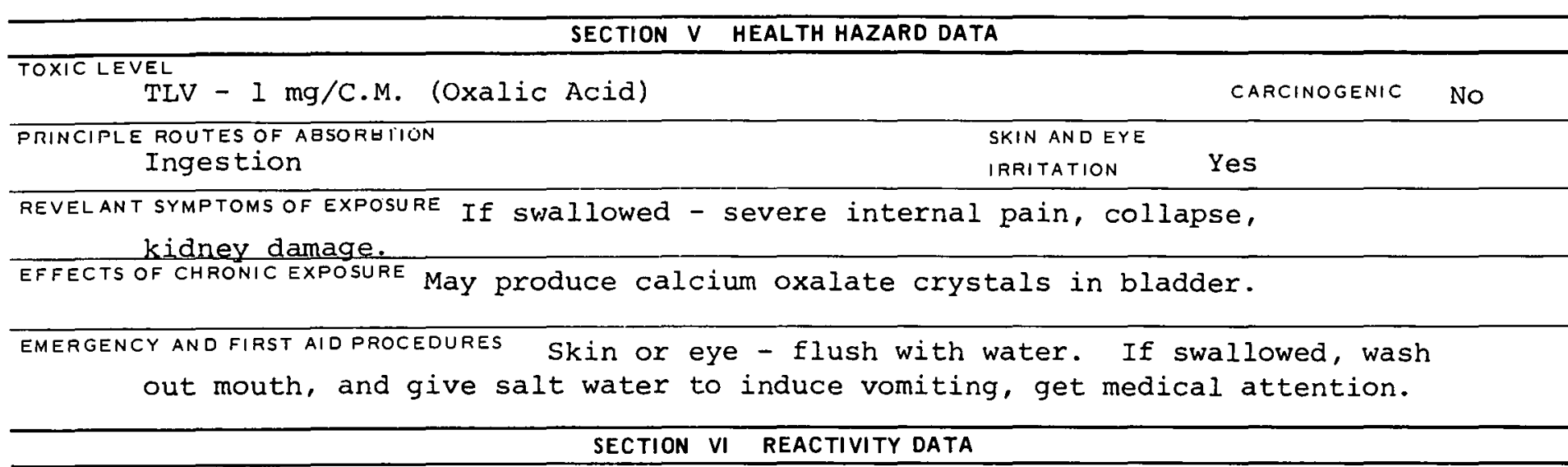

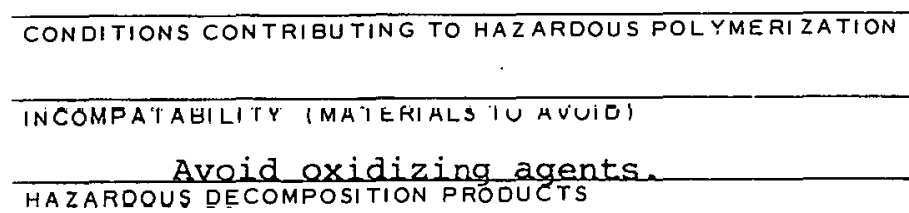

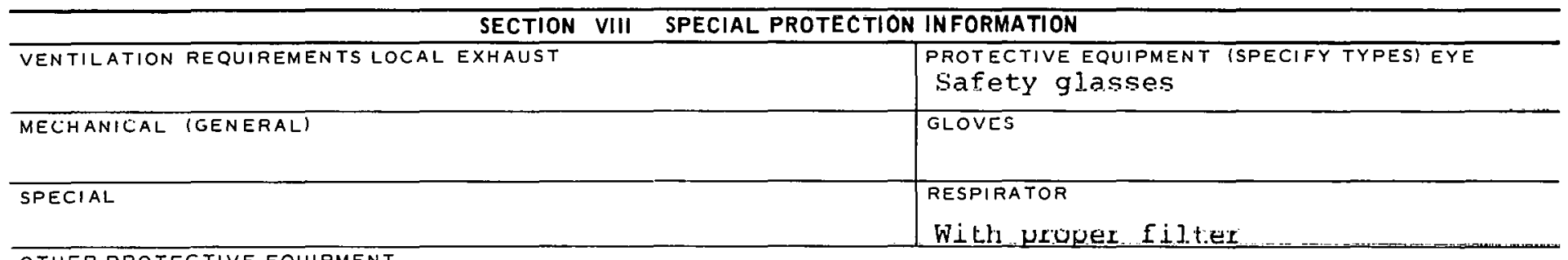



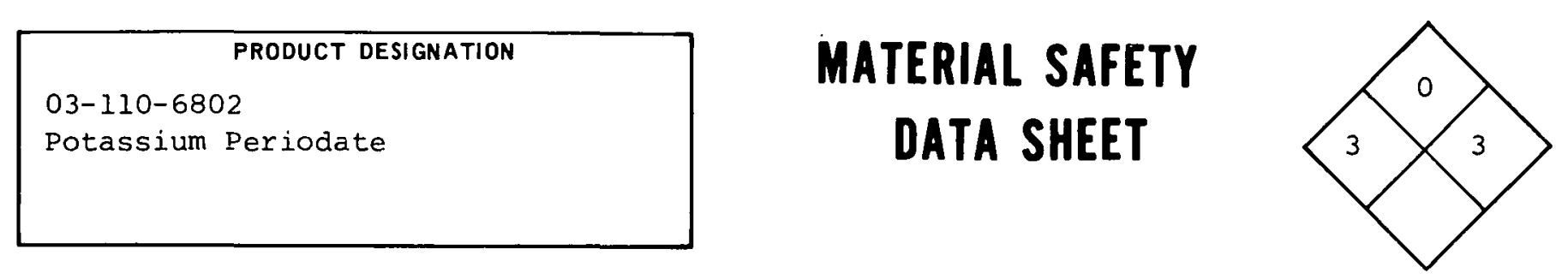

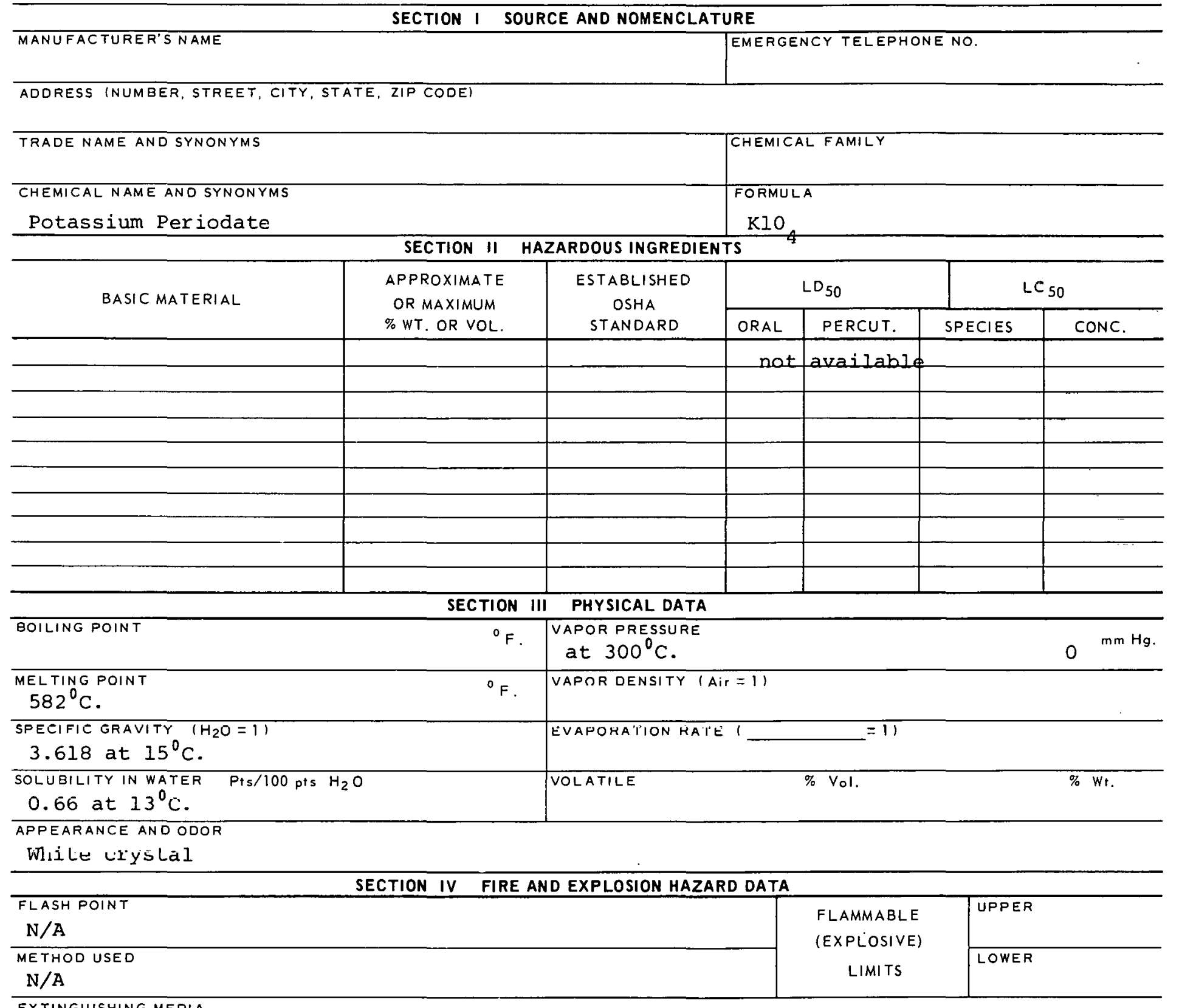

EXTINGUISHING MEDIA

$N / A$

SPECIAL FIRE FIGHTING PROCEDURES

$\mathrm{N} / \mathrm{A}$

UNUSUAL FIRE AND EXPLOSION HAZARDS

$\mathrm{N} / \mathrm{A}$ 


\section{PRODUCT DESIGNATION \\ Potassium Periodate}

\section{TOXIC LEVEL}

Not available

PRINCIPLE ROUTES OF ABSORBTION

\section{SECTION $V$ HEALTH HAZARD DATA}

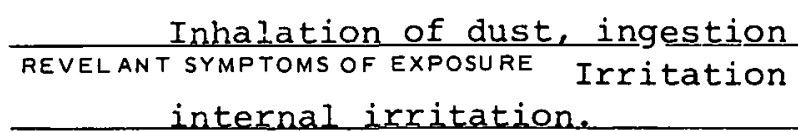

\section{SECTION VI REACTIVITY DATA}

CONDITIONS CONTRIBUTING TO INSTABILITY

Strong oxidizing agent. CONDITIONS CONTRIBUTING TO HAZARDOUS POL YMERIZATION

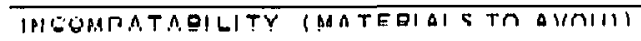

Reducing matcrialo. HAZARDOUS DECOMPOSITION PRODUCTS

SECTION VII SPILL OR LEAK PROCEDURES

STEPS TO BE TAKEN IN CASE MATERIAL IS RELEASED OR SPILLED

\section{WASTE DISPOSAL METHOD}

\section{SECTION VIII SPECIAL PROTECTION INFORMATION}

\begin{tabular}{l|l}
\hline VENTILATION REQUIREMENTS LOCAL EXHAUST & $\begin{array}{c}\text { PROTECTIVE EQUIPMENT (SPECIFY TYPES) EYE } \\
\text { SafEtY glaSSES }\end{array}$ \\
\hline MECHANICAL IGENERAL) & GLOVES \\
\hline SPECIAL & $\begin{array}{c}\text { RESPIRATOR } \\
\text { With prOpEY filLEY }\end{array}$ \\
\hline
\end{tabular}

OIHFK PHIIIL:IIVE EQUIHMEN I

SECTION IX SPECIAL PRECAUTIONS

PRECAUTIONS TO BE TAKEN IN HANDLING AND STORAGE 


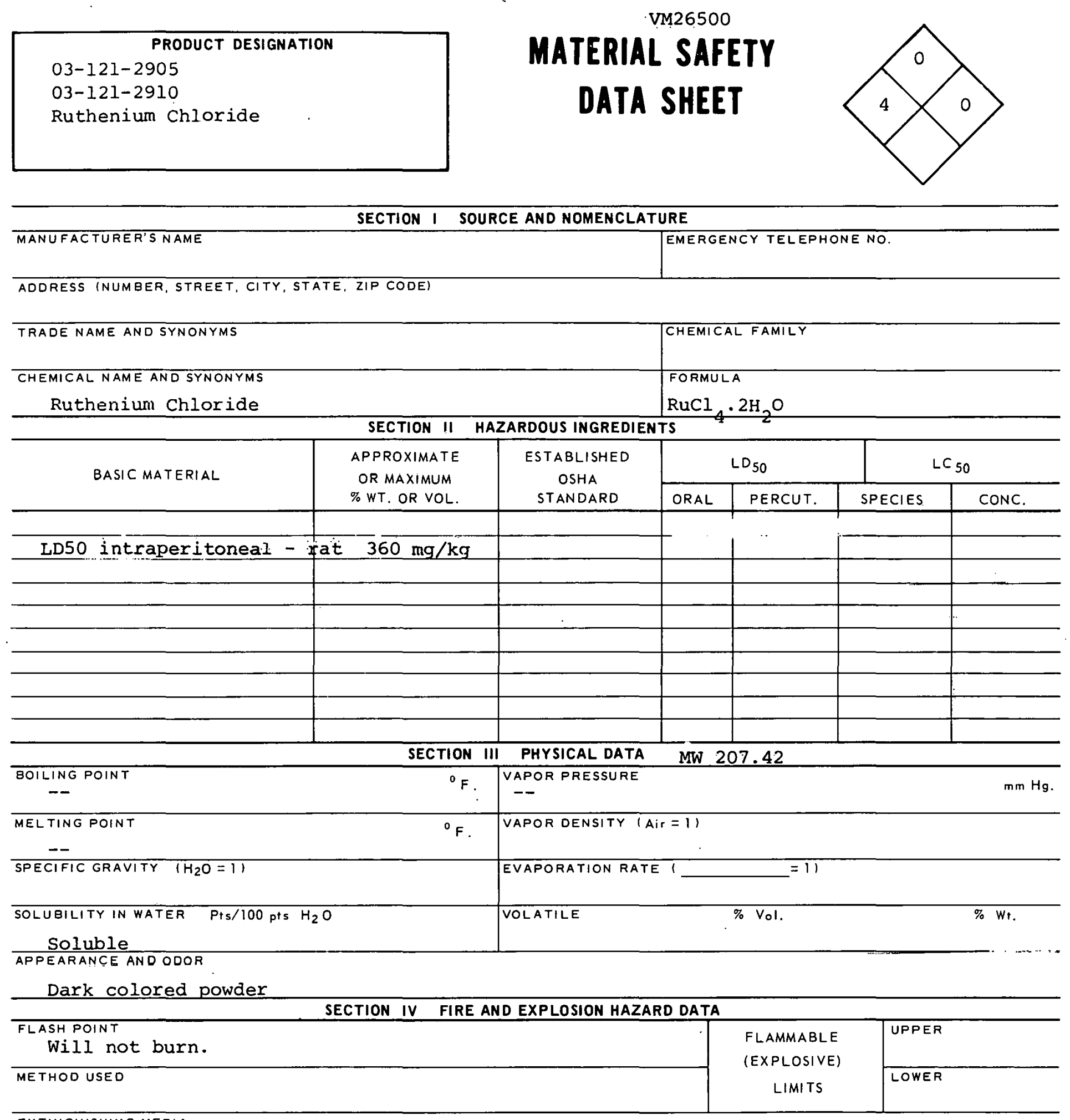

EXTINGUISHING MEDIA

SPECIAL FIRE FIGHTING PROCEDURES

UNUSUAL FIRE AND EXPLOSION HAZAROS 
Ruthenium Chloride

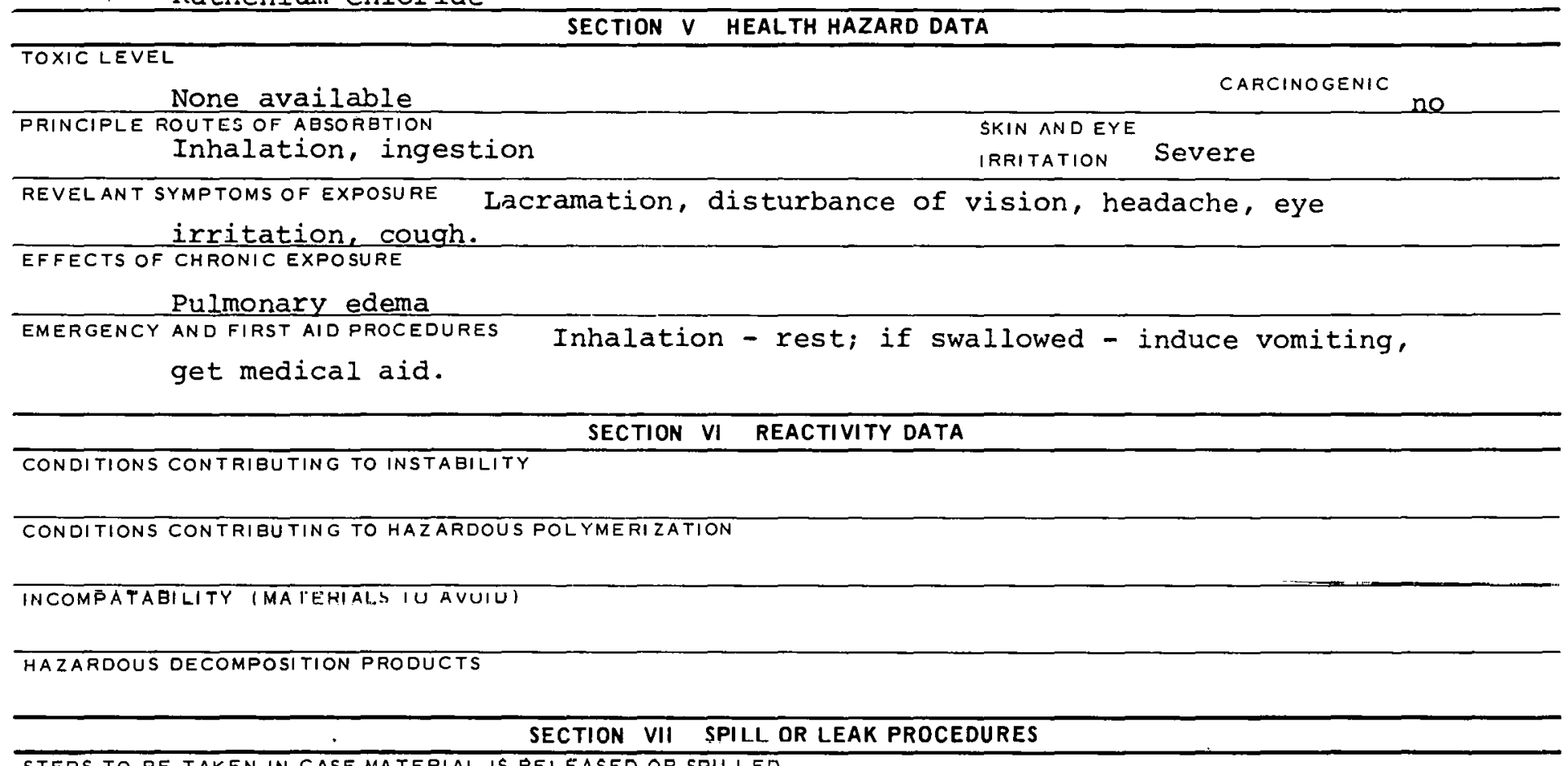
STEPS TO BE TAKEN IN CASE MATERIAL IS RELEASEO OR SPILLED

WASTE DISPOSAL METHOD

SECTION VIII SPECIAL PROTECTION INFORMATION

\begin{tabular}{|c|c|}
\hline VENTILATION REQUIREMENTS LOCAL EXHAUST & $\begin{array}{l}\text { PROTECTIVE EQUIPMENT (SPECIFY TYPES) EYE } \\
\text { GOgGleS }\end{array}$ \\
\hline MECHANICAL IGENERAL! & GLOVES \\
\hline SPECIAL & $\begin{array}{l}\text { RESPIRATOR } \\
\text { With proper filter }\end{array}$ \\
\hline
\end{tabular}

OTHER PROTECTIVE EQUIPMENT

\section{SECTION IX SPECIAL PRECAUTIONS}

PRECAUTIONS TO BE TAKEN IN HANDLING AND STORAGE

OTHER PRECAUTIONS

SIGNATURE

DATE

$121-2905 B$ 
Selenium Metal

\section{PRODUCT DESIGNATION}

Powder 99\% Pure

03-125-4006

\section{MATERIAL SAFETY DATA SHEET}

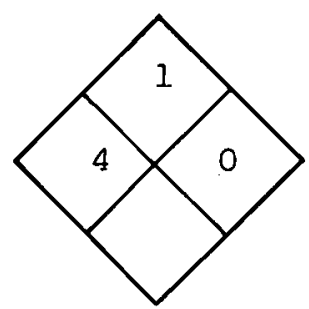

\section{SECTION I SOURCE AND NOMENCLATURE}

MANU FAC TURER'S NAME
AODRESS INUMBER, STREET, CITY
TRADE NAME AND SYNONYMS
CHEMICAL NAME AND SYNONYMS
SElENIUM

BASIC MATERIAL

\begin{tabular}{l}
\hline Note: \\
\hline Lowest published lethal \\
\hline inhalation - rat $33 \mathrm{mg} / \mathrm{C}$ \\
\hline \\
\hline
\end{tabular}

BOILING POINT

$688^{\circ} \mathrm{C}$.

MELTING POINT

$170-217^{\circ} \mathrm{C}$.

SPECIFIC GRAVITY $\left\{\mathrm{H}_{2} \mathrm{O}=11\right.$

$4.26-4.79$

SOLUBILITY IN WATER Pts/100 pts $\mathrm{H}_{2} \mathrm{O}$

insoluble

APPEARANCE AND ODOR

steel grey, non-metallic element.

SECTION IV FIRE AND EXPLOSION HAZARD DATA

${ }^{\circ} \mathrm{F}$.

APOR PRESSURE

at $356^{\circ} \mathrm{C}$.

EVAPORATION RATE

VOLATILE
SECTION II HAZARDOUS INGREDIENTS

\begin{tabular}{|c|c|}
\hline $\begin{array}{l}\text { APPROXIMATE } \\
\text { OR MAXIMUM } \\
\% \text { WT. OR VOL. }\end{array}$ & $\begin{array}{c}\text { LSTABLISHED } \\
\text { OSHA } \\
\text { STANDARD }\end{array}$ \\
\hline & \\
\hline concentration in & air \\
\hline M. & \\
\hline & \\
\hline & \\
\hline & \\
\hline & \\
\hline & \\
\hline & \\
\hline
\end{tabular}

SECTION III PHYSICAL DATA

${ }^{\circ} \mathrm{F} . \quad$ VAPOR DENSITY (Air $\left.=1\right)$ $=11$

$\%$ Vol.

$\% W_{+}$.
1

\begin{tabular}{|c|c|c|c|}
\multicolumn{2}{c|}{ LD $_{50}$} & \multicolumn{2}{c}{ LC $_{50}$} \\
\hline ORAL & PERCUT. & SPECIES & CONC. \\
\hline See NOTe & & \\
\hline
\end{tabular}

CHEMICAL FAMILY

ORMULA

Se

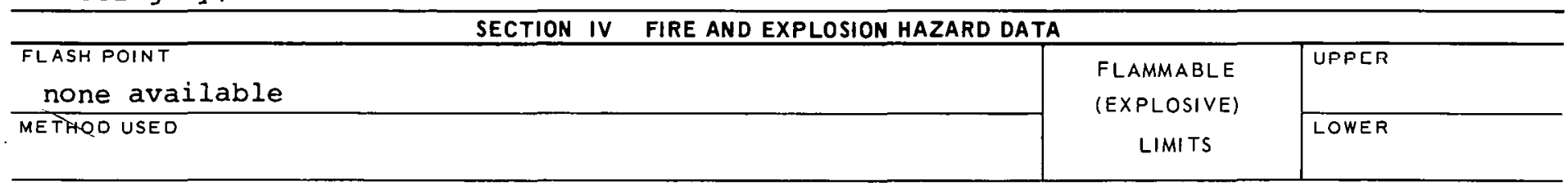

EXTINGUISHING MEDIA

Use water spray.

SPECIAL FIRE FIGHTING PROCEDURES

Small fires extinguished with sand. Self-contained breathing apparatus.

UNUSUAL FIRE AND EXPLOSION HAZARDS

Same as sulfur. Burning $\mathrm{Se}$ produces $\mathrm{SeO}_{2}$. 


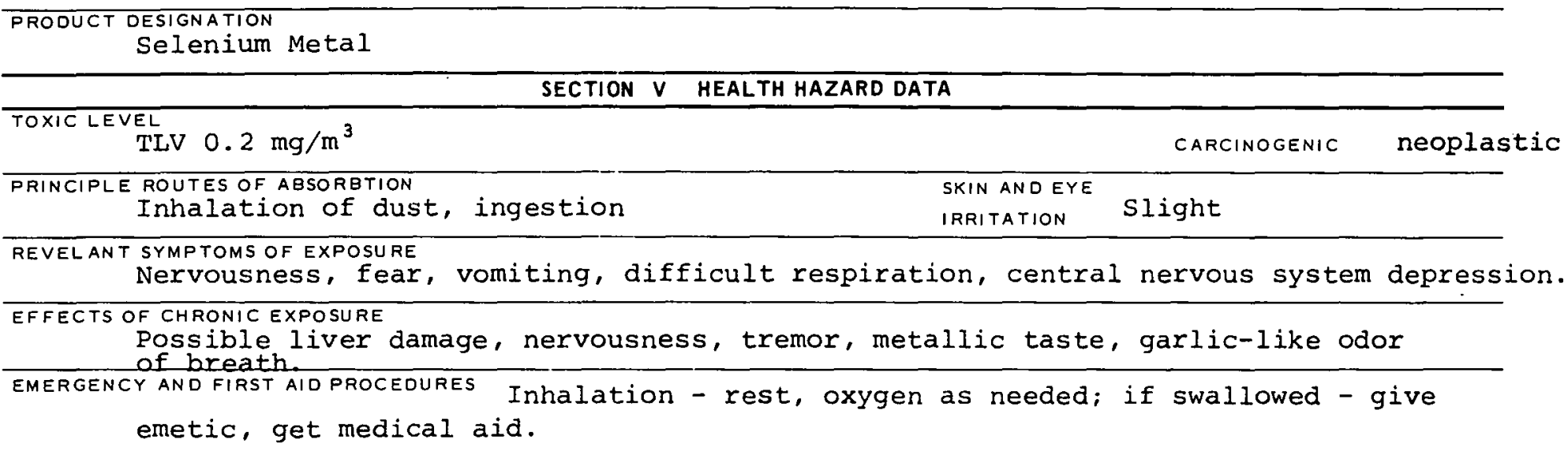

\begin{tabular}{l|l}
\hline VENTILATION REQUIREMENTS LOCAL EXHAUST & $\begin{array}{l}\text { PRTECTIVE EQUIPMENT (SPECIFY TYPES) EYE } \\
\text { GOggleS }\end{array}$ \\
\hline MECHANICAL IGENERAL) & $\begin{array}{l}\text { GLOVES } \\
\text { PlastiC or rubber }\end{array}$ \\
\hline SPECIAL & $\begin{array}{l}\text { RESPIRATOR } \\
\text { With proper filtor }\end{array}$ \\
\hline
\end{tabular}




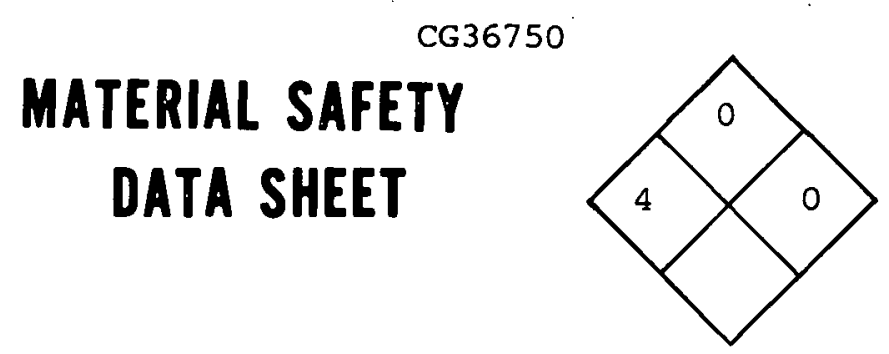

\begin{tabular}{|c|c|c|c|c|c|c|}
\hline & SECTION I SC & CE AND NOMENCL & URE & & & \\
\hline MANUFACTURER'S NAME & & & EMERG & CY TELEPH & $\overline{E N O}$ & \\
\hline ADDRESS INUMBER, STREET, CITY, STA & ATE, ZIP CODEI & & & & & \\
\hline TRADE NAME AND SYNONYMS & & & CHEMIC & FAMILY & & \\
\hline $\begin{array}{l}\text { CHEMICAL NAME AND SYNONYMS } \\
\text { Sodium Metaarsenite }\end{array}$ & & & $\begin{array}{l}\text { FORMU } \\
\mathrm{Na} A \mathrm{~s}\end{array}$ & & & \\
\hline & SECTION II & ZARDOUS INGREDII & TSS & & & \\
\hline BASIC MATERIAL & $\begin{array}{l}\text { APPROXIMATE } \\
\text { OR MAXIMUM }\end{array}$ & $\begin{array}{l}\text { ESTABLISHED } \\
\text { OSHA }\end{array}$ & & $\mathrm{mg}$ & $L^{\prime}-$ & \\
\hline & $\%$ WT. OR VOL. & STANDARD & ORAL & PERCUT. & SPECIES & CONC. \\
\hline LDIC - oral - hum!an 5 nig! & -9 & & 41 & & rat & \\
\hline & & & & 150 & rabbit & \\
\hline & & & $1 . ?$ & & Miguimas & \\
\hline & & & & & & \\
\hline & & & & & & \\
\hline & & & & & & \\
\hline & & & & & & \\
\hline & & & & & $\cdot$ & - \\
\hline & SECTION & PHYSICAL DATA & $\mathrm{MW} 129$ & 91 & & \\
\hline $\begin{array}{l}\text { BOILING POINT } \\
--\end{array}$ & ${ }^{0} \mathrm{~F}$ & VAPOR PRESSURE & & & . & $\mathrm{mm} \mathrm{Hg}$. \\
\hline $\begin{array}{l}\text { MELTING POINT } \\
--\end{array}$ & ${ }^{0} \mathrm{~F}$ & VAPOR DENSITY & $\mathrm{ir}=11$ & & & \\
\hline SPECIFIC GRAVITY $\left(\mathrm{H}_{2} \mathrm{O}=1\right)$ & & EVAPORATION RA & $=1$ & $=11$ & & \\
\hline SOLtBULITY IN WATER Pts/100 pts $\mathrm{H}_{2}$ & & VOLATILE & & $\% \mathrm{Vol}$ & & $\% W_{+}$. \\
\hline
\end{tabular}

Colorless or grayish-white powder.

SECTION IV FIRE AND EXPLOSION HAZARD DATA

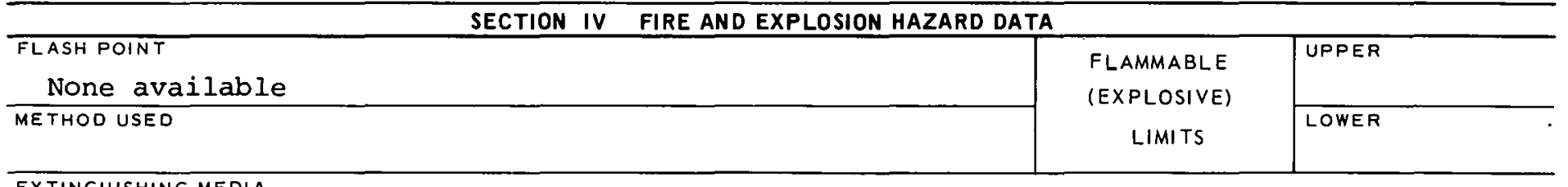

EXTINGUISHING MEDIA

None available

SPECIAL FIRE FIGHTING PROCEDURES

UNUSUAL FIRE AND EXPLOSION HAZARDS 


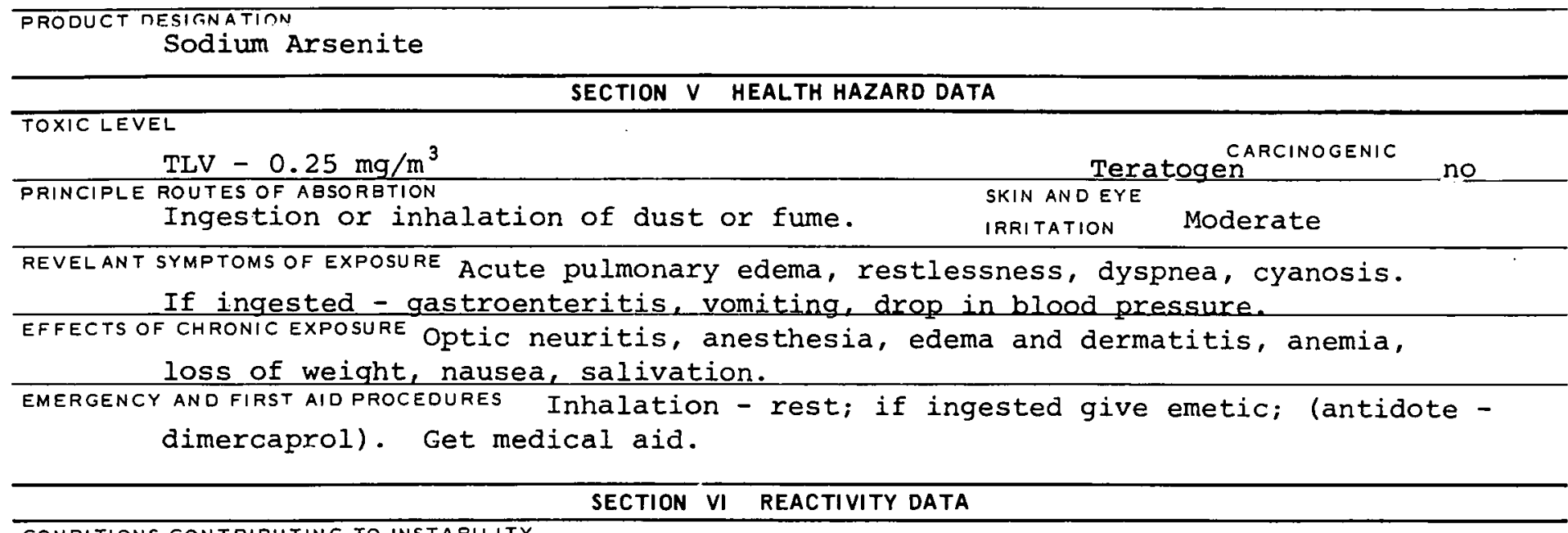

CONDITIONS CONTRIBUTING TO INSTABILITY

CONDITIONS CONTRIBUTING TO HAZAROOUS POLYMERIZATION

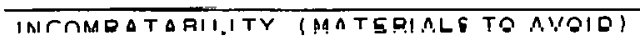

HAZARDOUS DECOMPOSITION PRODUCTS

SECTION VII SPILL OR LEAK PROCEDURES

STEPS TO BE TAKEN IN CASE MATERIAL IS RELEASED OR SPILLED

WASTE DISPOSAL METHOD

SECTION VIII SPECIAL PROTECTION INFORMATION

\begin{tabular}{l|l}
\hline VENTILATION REQUIREMENTS LOCAL EXHAUST & $\begin{array}{l}\text { PROTECTIVE EQUIPMENT (SPECIFY TYPES) EYE } \\
\text { Safety glasSES }\end{array}$ \\
\hline MECHANICAL (GENERAL) & GLOVES \\
\hline SPECIAL & RESPIRATOR \\
& WLth proper IILter \\
\hline
\end{tabular}

OTHER PROTECTIVE EQUIPMENT

SECTION IX SPECIAL PRECAUTIONS

PRECAUTIONS TO BE TAKEN IN HANDLING AND STORAGE

OTHER PRECAUTIONS

SIGNATURE

DATE

$131-1068 B$ 
CAS : 026628228

\section{PRODUCT DESIGNATION}

03-131-1108

Sodium Azide
VY80500

MATERIAL SAFETY

DATA SHEET

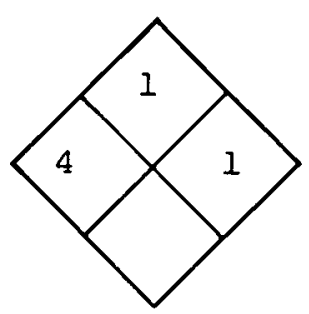

\section{SECTION I SOURCE AND NOMENCLATURE}

EXTINGUISHING MEDIA

$\mathrm{N} / \mathrm{A}$

SPECIAL FIRE FIGHTING PROCEOURES

UNUSUAL FIRE AND EXPLOSION HAZARDS 


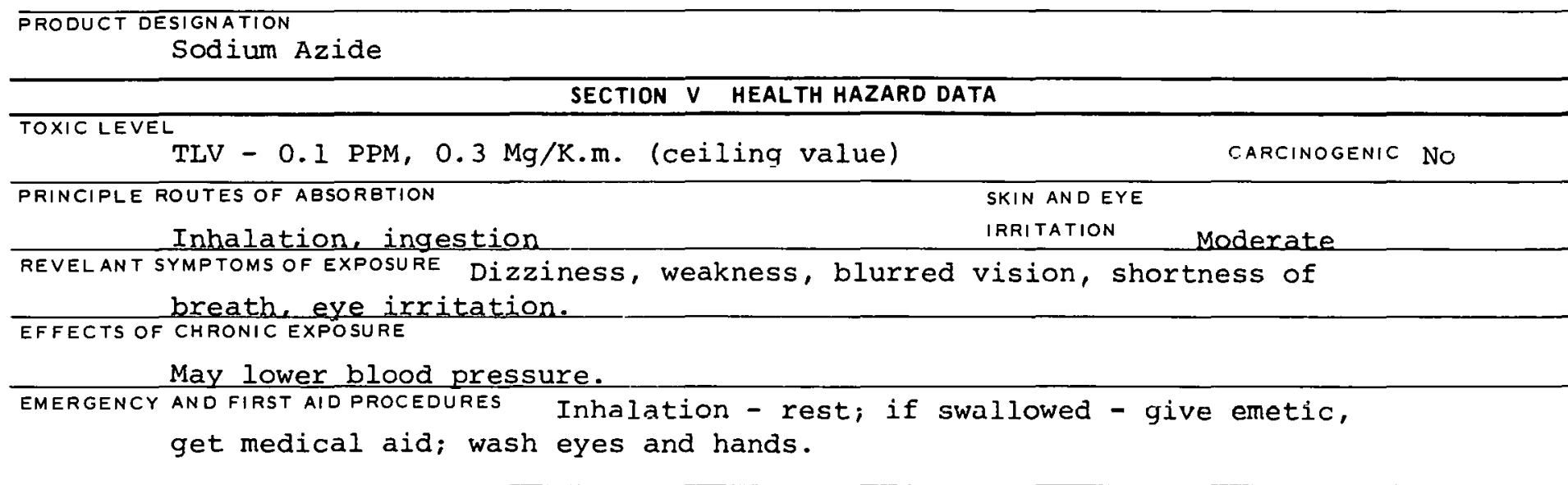

\begin{tabular}{l} 
SECTION VI REACTIVITY DATA \\
\hline CONDITIONS CONTRIBUTING TO INSTABILITY
\end{tabular}

CONDITIONS CONTRIBUTING TO HAZARDOUS POLYME:RIZATION

INCOMPATABILITY (MATERIALS TO AVOID) May react violently with bromine, carbon disulfide

dibromomalononitrile, and dimetbyl sulfate. HAZARDOUS DECOMPOSSITION PRODUCTS

SECTION VII SPILL OR LEAK PROCEDURES

STEPS TO BE TAKEN IN CASE MATERIAL IS RELEASED OR SPILLED

.

WASTE DISPOSAL METHOD

SECTION VIII SPECIAL PROTECTION INFORMATION

\begin{tabular}{l|l}
\hline VENTILATION REQUIREMENTS LOCAL EXHAUST & $\begin{array}{l}\text { PROTEGTIVE EQUIPMENT (SFECIFY TYPES) EYE } \\
\text { JaFEtY gOGgleS }\end{array}$ \\
\hline MECHANICAL IGENERAL) & GLOVES \\
\hline SPEGIAL & $\begin{array}{l}\text { RESPIRATOR } \\
\text { WIth proper filter }\end{array}$ \\
\hline
\end{tabular}

OTHER PROTECTIVE EQIIIPMENT

SECTION IX SPECIAL PRECAUTIONS

PRECAUTIONS TO BE TAKEN IN HANDLING AND STORAGE

OTHER PRECAUTIONS

SIGNATURE

DATE

$131-1108 B$ 
$03-131-3158$

\section{PRODUCT DESIGNATION}

03-131-3168

Sodium Calcium Hydrate
MATERIAL SAFETY

DATA SHEET

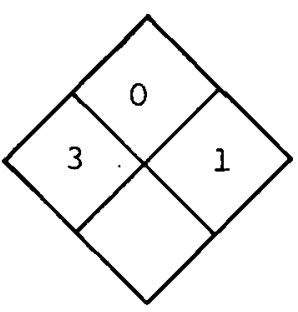

SECTION I SOURCE AND NOMENCLATURE

\section{MANUFACTURER'S NAME}

EMERGENCY TELEPHONE NO.

ADDRESS (NUMBER, STREET, CITY, STATE, ZIP CODE)

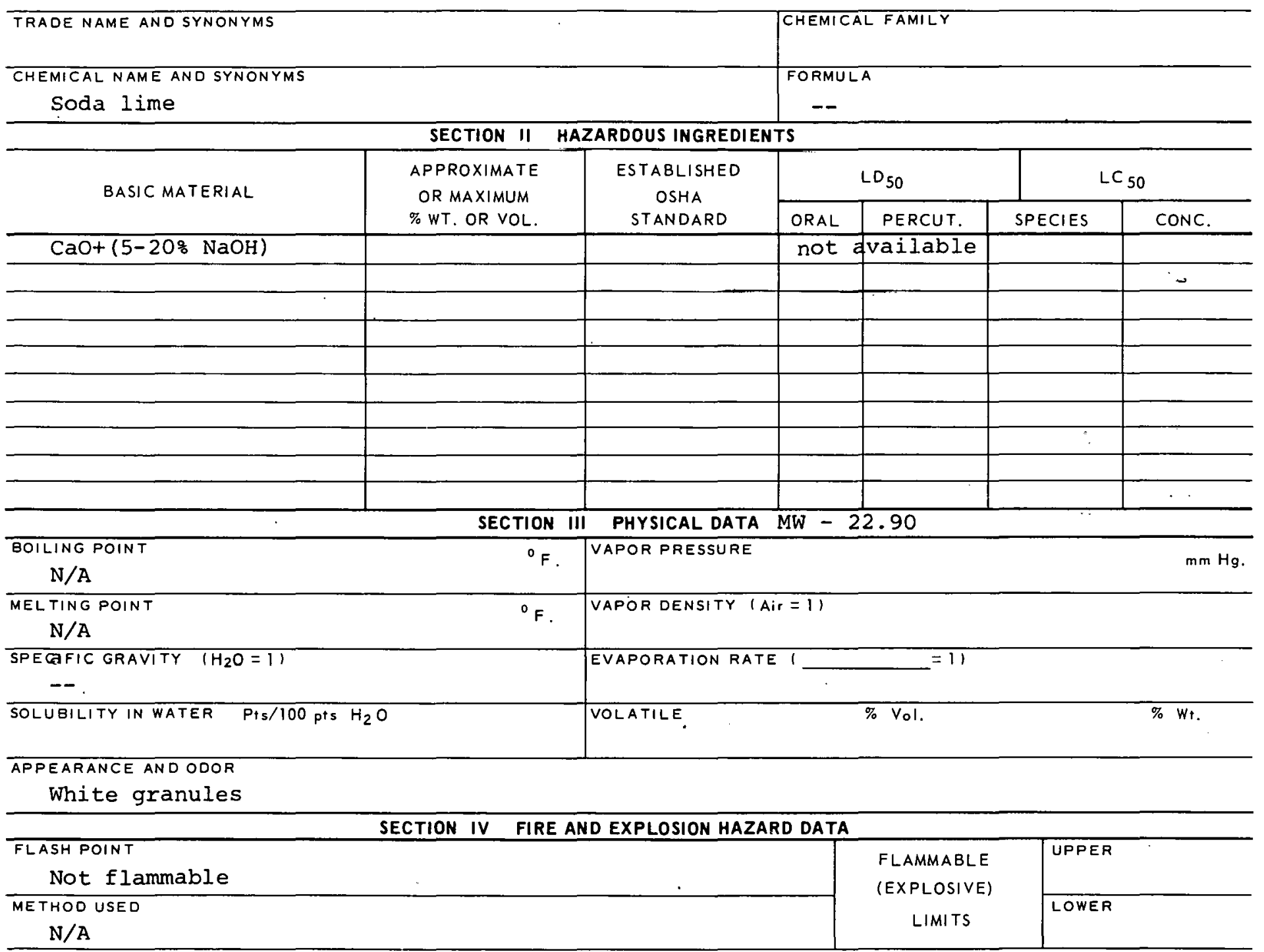

EXTINGUISHING MEDIA

$N / A$

SPECIAL FIRE FIGHTING PROCEDURES

$\mathrm{N} / \mathrm{A}$

UNUSUAL FIRE ANO EXPLOSION HAZARDS

$\mathrm{N} / \mathrm{A}$ 
TOXIC LEVEL

$\mathrm{TLV}-2 \mathrm{mg} / \mathrm{C} . \mathrm{M}$. (from NaOH)

CARCINOGENIC NO

FRINCIPLE ROUTES OF ABSORBTION

Ingestion

SKIN AND EYE

IRRITATION

Yes

REVELANT SYMPTOMS OF EXPOSURE Severe irritation of skin and eyes, severe internal irritation and damage, vomiting, diarrhea and collapse.

EFFECTS OF CHRONIC EXPOSURE

$--$

EMERGENCY AND FIRST AID PROCEDURES Flush with water and vinegar; if swallowed, wash mouth and give water to drink, then vinegar, get medical attention.

SECTION VI REACTIVITY DATA

CONDITIONS CONTRIBUTING TO INSTABILITY

CONDITIONS CONTRIBUTING TO HAZARDOUS POLYMERIZATION

INCOMPATABILITY (MATERIALS TO AVOIO)

Acids

HAZARDOUS DECOMPOSITION PRODUCTS

SECTION VII SPILL OR LEAK PROCEDURES

STEPS TO BE TAKEN IN CASE MATERIAL IS RELEASED OR SPILLED

$\overline{\text { WASTE DISPOSAL METHOD }}$

SECTION VIII SPECIAL PROTECTION INFORMATION

\begin{tabular}{l|l}
\hline \multicolumn{1}{c}{ SECTION VIII SPECIAL PROTECTION INFORMATION } \\
\hline VENTILATION REQUIREMENTS LOCAL EXHAUST & $\begin{array}{l}\text { FROTECTIVE EQUIPMENT (SPECIFY TYPES) EYE } \\
\text { SafETY glaSSES }\end{array}$ \\
\hline MECHANICAL (GENERAL) & GLOVES \\
\hline SPECIAL & RESPIRATOR \\
\hline
\end{tabular}

OTHER PROTECTIVE EQUIFMENT

\section{SECTION IX SPECIAL PRECAUTIONS}

PRECAUTIONS TO BE TAKEN IN HANDLING AND STORAGE

OTHER PRECAUTIONS

SIGNATURE

DATE

$131-3158 B$ 
CAS : 007775113

\begin{tabular}{|l|}
\hline \multicolumn{1}{|c|}{ PRODUCT DESIGNATION } \\
Sodium Chromate \\
$03-131-3608$ \\
\end{tabular}

\section{casasso \\ MATERIAL SAFETY \\ DATA SHEET}

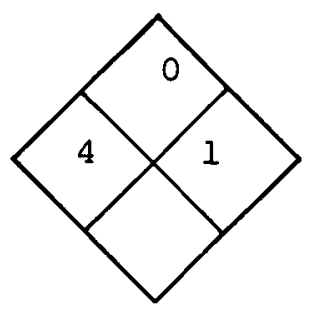

\section{SECTION I SOURCE AND NOMENCLATURE}

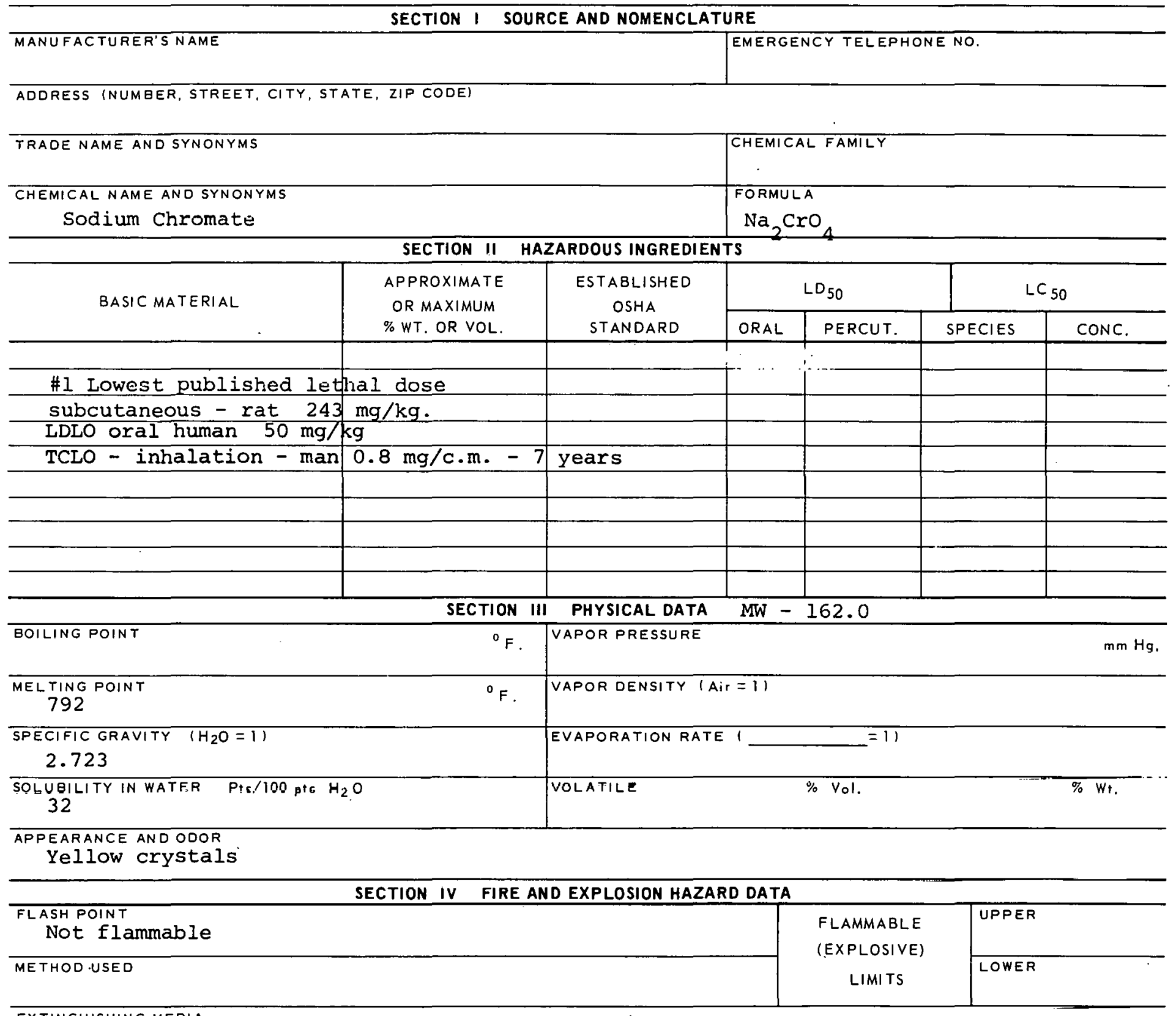

EXTINGIIISHINGS MEDIA

Water

SPECIAL FIRE FIGHTING PROCEOURES Material may form hot viscous foam. possible

steam explosion.

UNUSUAL FIRE AND EXPLOSION HAZARDS

Source of oxygen (container may explode in fire). 


\section{PRODUCT DESIGNATION \\ Sodium Chromate}

\begin{tabular}{ll}
\hline & SECTION $V$ HEALTH HAZARD DATA
\end{tabular}

TLV - $0.1 \mathrm{mg} / \mathrm{C} . \mathrm{M}$.

PRINCIPLE ROUTES OF ABSORBTION

Inhalation of dust (mainly) ingestion

CARCINOGENIC Yes
SKIN ANDEYE
IRRITATION Moderate

IRRITATION Moderate

REVELANT SYMPTOMS OF EXPOSURE IrTIEation of respiratory tract; internal irritation and

damage; irritation of skin and eyes.

EFFECTS OF CHRONIC EXPOSURE Asthmatic symptoms; liver and kidney damage, lung cancer.

EMERGENCY AND FIRST AID PROCEDURES If inhaled - rest keep warm; if swallowed - wash mouth, give emetic, get medical aid; wash from skin and eyes.

\section{SECTION VI REACTIVITY DATA}

CONDITIONS CONTRIBUTING TO INSTABILITY

Powerful oxidizing agent.

CONDITIONS CONTRIBUTING TO HAZARDOUS POLYMERIZATION

INCOMPATABILITY (MATERIALS TO AVOID) May react with organic materials to produce a fire

(acetic acid, acetic anhydride, anthoracene and ethyl alcohol).

HAZARDOUS DECOMPOSIIIUN HRUUUL Is

SECTION VII SPILL OR LEAK PROCEDURES

STEPS TO BE TAKEN IN CASE MATERIAL IS RELEASED OR SPILLED

WASTE DISPOSAL METHOD

SECTION VIII SPECIAL PROTECTION INFORMATION

\begin{tabular}{l|l}
\hline VENTILATION REQUIREMENTS LOCAL EXHAUST & $\begin{array}{l}\text { PROTECTIVE EQUIPMENT (SPECIFY TYPES) EYE } \\
\text { SafetY glaSSES }\end{array}$ \\
\hline MECHANICAL (GENERAL) & $\begin{array}{l}\text { GLOVES } \\
\text { Rubber }\end{array}$ \\
\hline SPECIAL & $\begin{array}{l}\text { PESPIRATOR } \\
\text { With proper filter }\end{array}$ \\
\hline
\end{tabular}

OTHER PROTECTIVE EQUIPMENT

SECTION IX SPECIAL PRECAUTIONS

PRECAUTIONS TO BE TAKEN IN HANDLING AND STORAGE

OTHER PRECAIITIONS

SIGN $\triangle T U R F$

DATE

,

$131-3608 \mathrm{~B}$ 
Sodium Cyanide

03-131-4008
MATERIAL SAFETY DATA SHEET

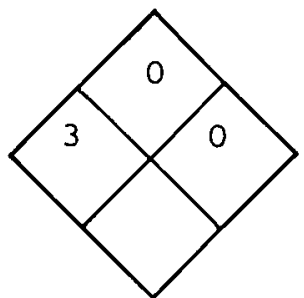

SECTION I SOURCE AND NOMENCLATURE

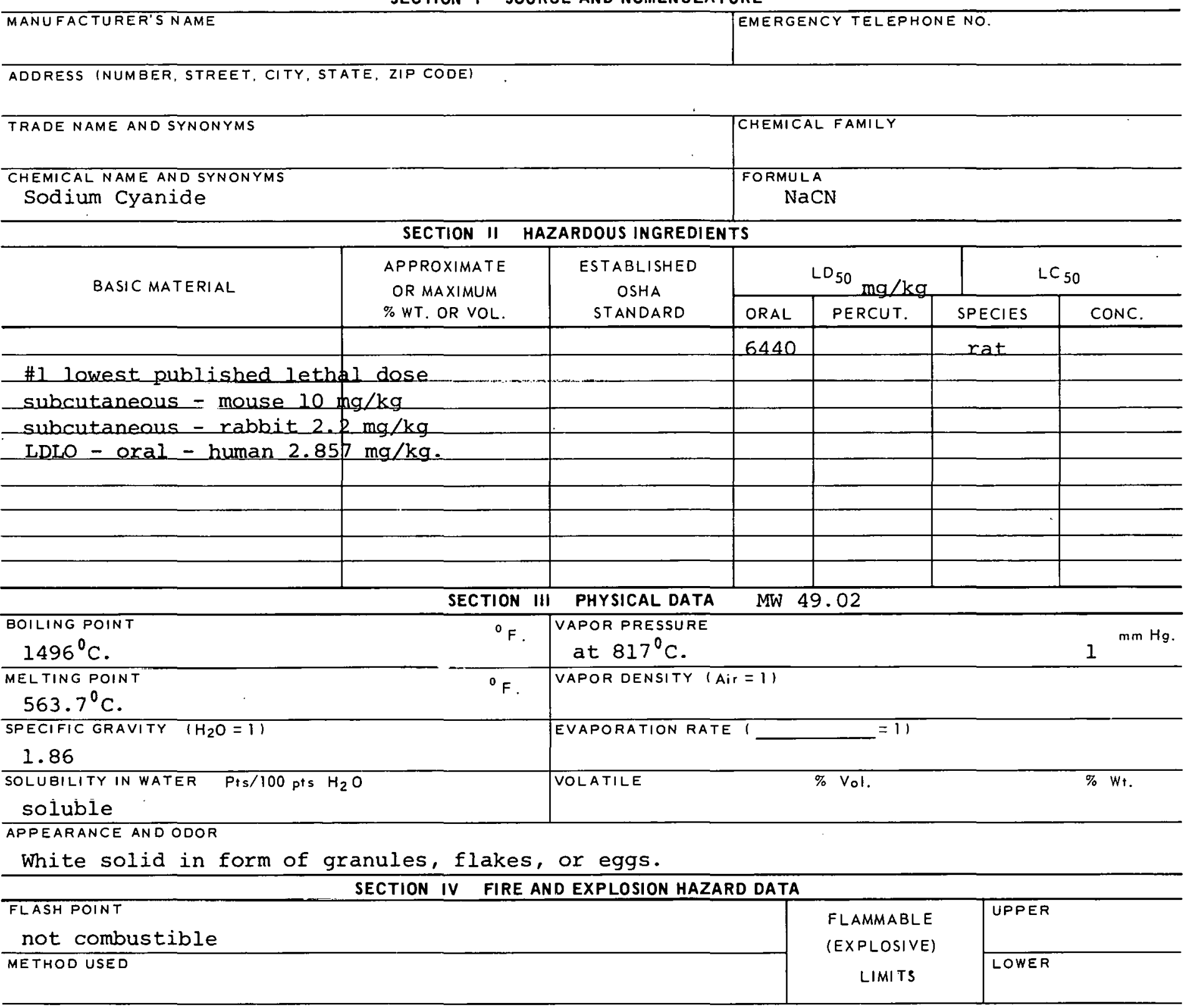

EXTINGUISHING MEDIA

Water

SPECIAL FIRE FIGHTING PROCEDURES

Wear full protective clothing.

UNUSUAL FIRE AND EXPLOSION HAZARDS

In cuntact with actas releases highly flammable hydrogen cyanide gas. 
Inhalation of dust, ingestion, skin IRRITATION

REVELANT SYMPTOMS OF EXPOSURE Giddiness, dizziness, rapid heart action, shortness of breath, drop in blood pressure.

EFFECTS OF CHRONIC EXPOSURE POssible dizziness, weakness, congestion of lungs, hoarseness, loss of appetite, loss of weight.

EMERGENCY AND FIRST AID PROCEDURES Give amyl nitrate inhalation, $0.2 \mathrm{ml} 3 \mathrm{~min}$. $-\mathrm{give}$ artificial. respiration with 1008 oxygen to maintain high blood oxygen tension wash skin and eyes - obtain medical aid.

\section{CONDITIONS CONTRIBUTING TO INSTABILITY \\ SECTION VI REACTIVITY DATA}

CONDITIONS CONTRIQUITING TO HAZAROOUS POLYMERIZATION

INCOMPATA ABILITY (MATERIALS TO AVOID)

Nitrates, nitrites

HAZAROOUS DECUMNUSIIIUN RRULUL.13

In contact with acids, releases $\mathrm{HCN}$ gas.

\section{SECTION VII SPILL OR LEAK PROCEDURES}

STEPS TO BE TAKEN IN CASE MATERIAL IS RELEASEO OR SPILLED

Collect by sweeping with a broom on to a sheet of paper.

WASTE DISPOSAL METHOD

SECTION VIII SPECIAL PROTECTION INFORMATION

\begin{tabular}{|c|c|}
\hline VENTILATION REQUIREMENTS LOCAL EXHAUST & $\begin{array}{l}\text { PROTECTIVE EQUIPMENT (SPECIFY TYPES) EYE } \\
\text { GNggl leS. }\end{array}$ \\
\hline MECHANICAL (GENERAL) & $\begin{array}{l}\text { GLOVES } \\
\text { Rubber }\end{array}$ \\
\hline BPECIAL & $\begin{array}{l}\text { RESPIRATOR } \\
\text { W/appropriate filter }\end{array}$ \\
\hline
\end{tabular}

OTHER PROTECTIVE EQUIPMENT

Rubber apron

SECTION IX SPECIAL PRECAUTIONS

PRECAUTIONS TO BE TAKEN IN HANDLING AND STORAGE

Store in cool, dry place separated from acids and oxidizing materials.

DTHER PRECAUTIONS

EIOMNTIIDE

nATF

$131-4008 B$ 
Sodium Dichromate

03-131-4108

$03-131-4138$
MATERIAL SAFETY

DATA SHEET

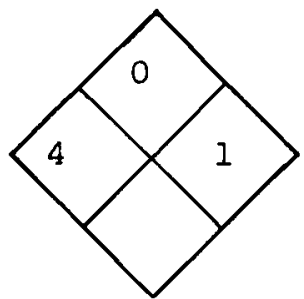

\section{SECTION I SOURCE AND NOMENCLATURE}

ADDRESS (NUMBER, STREET, CITY, STATE, ZIP CODE)

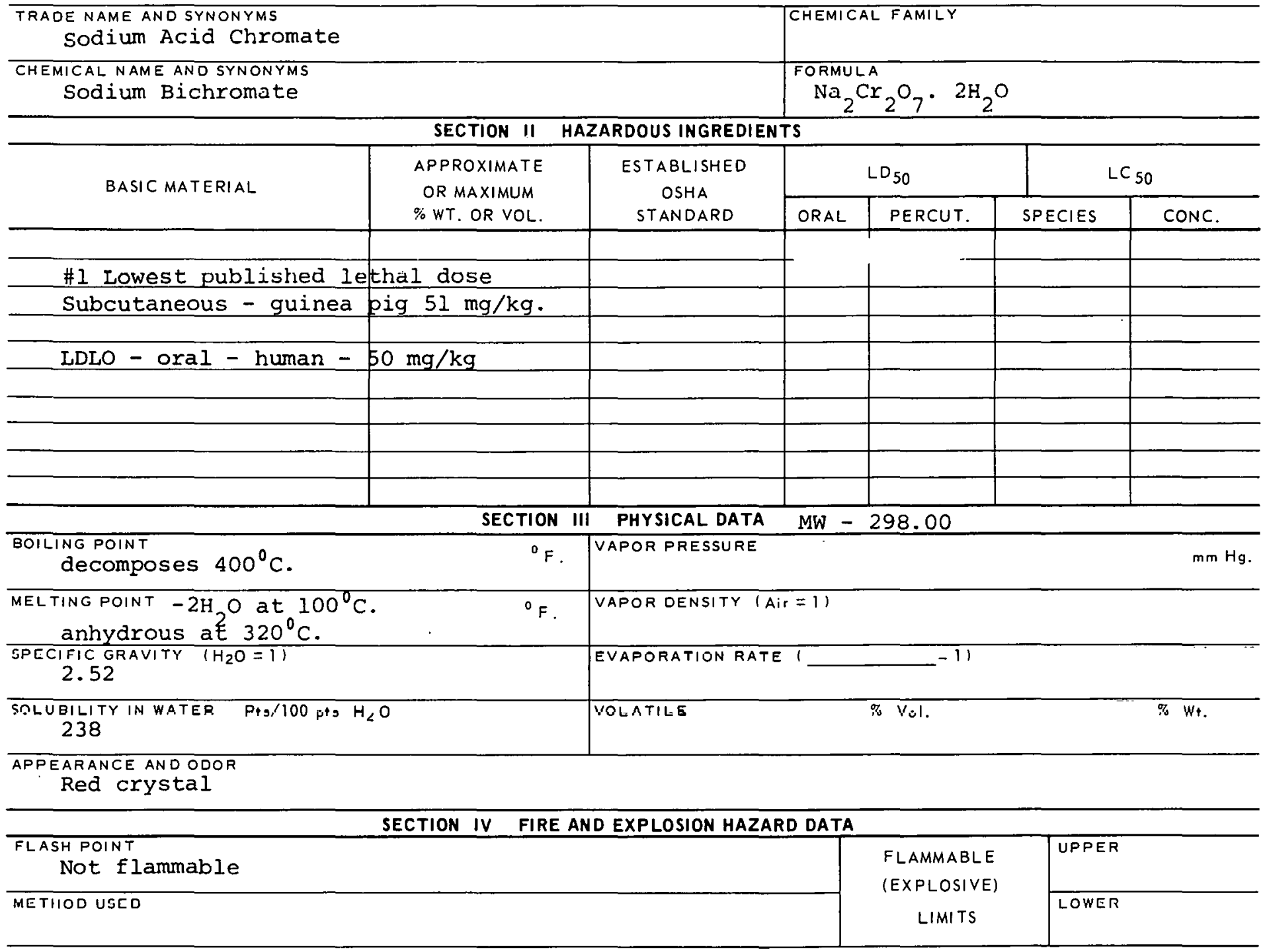

EXTINGUISHING MEDIA

Water

SPECIAL FIRE FIGHTING PROCEOURES Material may form hot viscous foam.

Possible steam explosion.

UNUSUAL FIRE AND EXPLOSION HAZARDS

Source of oxygen (container may explode in fire). 
TLV - $0.30 \mathrm{mg} / \mathrm{C} . \mathrm{M}$.

CARCINOGENIC

PRINCIPLE ROUTES OF ABSORBTION

Inhalation of dust (mainly) ingestion

SKIN AND EYE

IRRITATION moderate

REVELANT SYMPTOMS OF EXPOSURE Irritation of respiratory tract; internal irritation and

damage: irritation of skin and eyes.

EFFECTS OF CHRONIC EXPOSURE

Astbmatic symptoms, Iiver and kidney damage, lung cancer.

EMERGENCY AND FIRST AID PROCEDURES Inhalation - rest, keep warm, get medical aid; if swallowed wash mouth, give emetic, get medical aid; wash from skin and eyes.

\section{SECTION VI REACTIVITY DATA}

CONDITIONS CONTRIBUTING TO INSTABILITY

Powerful oxidizing agent.

CONOITIONS CONTRIBUTING TO HAZARDOUS POLYME $\overline{R I Z A T I O N}$

INCOMPATABILITY IMATERIALS IU AVUIUI

May react with organic materials to produce a fire (acetic anhydride and acid,

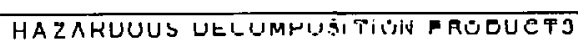
anthxacone, othyl adrohn!)

\section{SECTION VII SPILL OR LEAK PROCEDURES}

STEPS TO BE TAKEN IN CASE MATERIAL IS RELEASED OR SPILLED

WASTE DISPOSAL METHOD

SECTION VIII SPECIAL PROTECTION INFORMATION

\begin{tabular}{l|l}
\hline \multicolumn{1}{c}{ SECTION VIII SPECIAL PROTECTION INFORMATION } \\
\hline VENTILATION REQUIREMENTS LOCAL EXHAUST & $\begin{array}{l}\text { PROTFGTIVE EQUIPMENT (SPECIFY TYPESI EYE } \\
\text { Safety glagse3 }\end{array}$ \\
\hline MECHANICAL IGENERAL) & $\begin{array}{l}\text { GLOVES } \\
\text { Rubber }\end{array}$ \\
\hline GCEGIAL & $\begin{array}{l}\text { RESP!RATOR } \\
\text { With proper filter }\end{array}$ \\
\hline
\end{tabular}

OTHER PROTECTIVE EQUIPMENT

Protective work clothing.

SECTION IX SPECIAL PRECAUTIONS

PRECAUTIONS TO BE TAKEN IN HANDLING AND STORAGE

Keep container away from combustibles and easily oxidized materials.

OTHFR PRFTAIITIONS

SIGNATIIRE

DATE 


\section{PRODUCT DESIGNATION}

Sodium Fluoride

03-131-4808

$03-131-4848$
MATERIAL SAFETY DATA SHEET

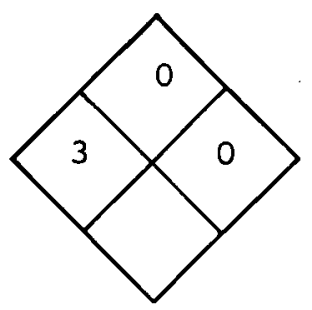

SECTION I SOURCE AND NOMENCLATURE

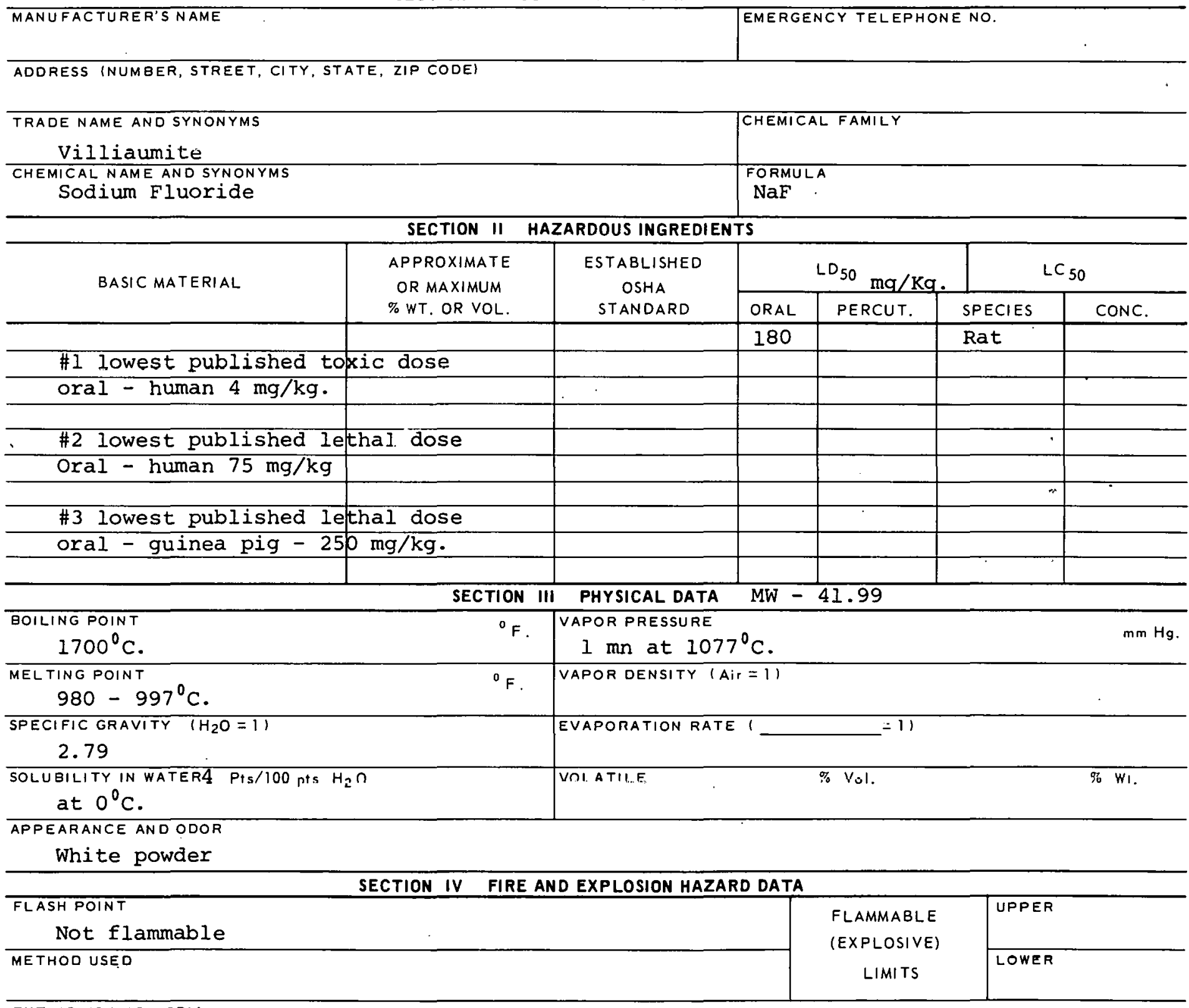

EXTINGUISHING MEDIA

Water

SPECIAL FIRE FIGHTING PROCEDURES

$N / A$

UNUSUAL FIRE AND EXPLOSION HAZAROS

- 
CAS : 001310732

WB49000

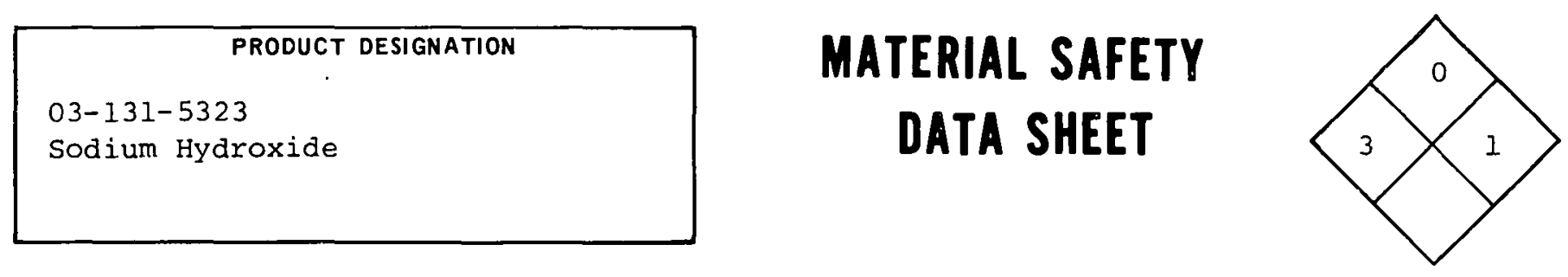

SECTION I SOURCE AND NOMENCLATURE

MANUFACTURER'S NAME
AODRESS INUMBER, STREET, CI
TRADE NAME AND SYNONYMS
LYE, CaUSTIC SOdA
CHEMICAL NAME AND SYNONYMS
SOdIUM hYdYOXIde

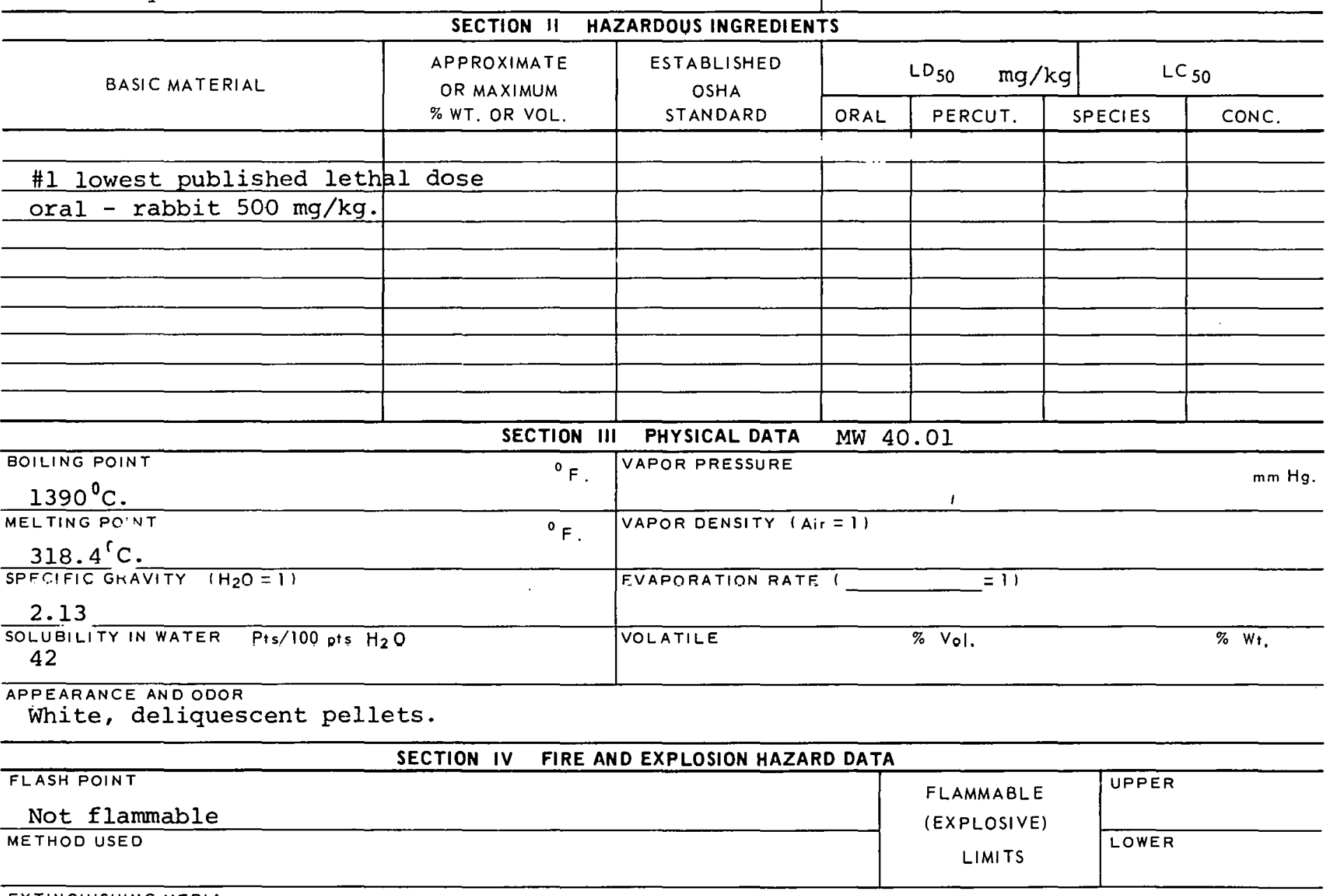

EXTINGUISHING MEDIA

Flood with water

SPECIAL FIRE FIGHTING PROCEDURES

Don't splash or splatter material.

UNUSUAL FIRE AND EXPLOSION HAZARDS

$--$ 


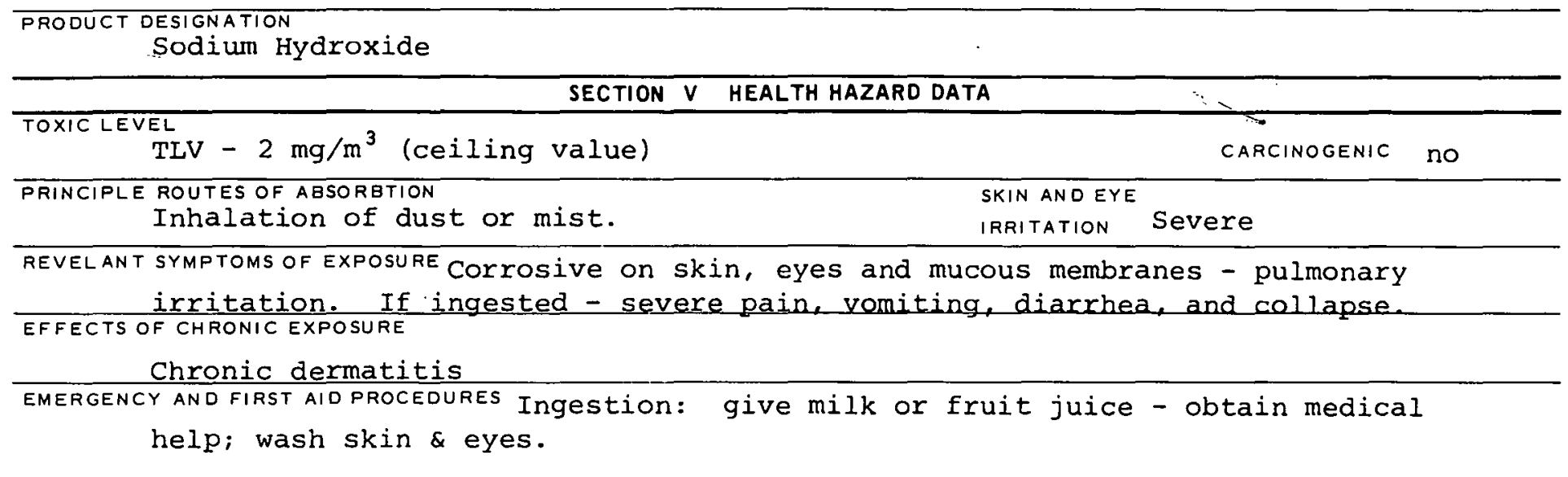

\begin{tabular}{l} 
SECTION VI REACTIVITY DATA \\
\hline CONDITIONS CONTRIBUTING TO INSTABILITY \\
\hline CONDITIONS CONTRIBUTING TO HAZARDOUS POLYME RIZATION \\
INCOMPATABILITY (MATERIALS TO AVOID) Acetaldehyde, ACrolein, ACrylonitrile, AlIyl AlCohol, \\
Aluminum, Chloronitrotoluenes, Maleic Anhydride, Nitroethane, Nitromethane \\
\hline HAZAROOUS DECOMPOSITION PRODUCT3 \\
\hline STEPS TO BE TAKEN IN CASE MATERIAL IS RELEASEO OR SPILLED
\end{tabular}
STEPS TO BE TAKEN IN CASE MATERIAL IS RELEASEO OR SPILLED

WASTE DISPOSAL METHOD

SECTION VIII SPECIAL PROTECTION INFORMATION FUII protection

\begin{tabular}{l|l}
\hline VENTILATION REQUIREMENTS LOCAL EXHAUST & $\begin{array}{c}\text { FUII PROTECtION } \\
\text { GOgGles }\end{array}$ \\
\hline MECHANICAL IGENERAL) & GLOVES \\
& Rubber \\
\hline SPECIAL & RESPIRATOR \\
& W/appropriate filter \\
\hline
\end{tabular}

OTHER PROTECTIVE EQUIPMENT

Rubber apron and full face shield.

\section{SECTION IX SPECIAL PRECAUTIONS}

PRECAUTIONS TO BE TAKEN IN HANDLING AND STORAGE

Store in dry place; protect against moisture and water. Separate from acids,

metals, explosives, organic peroxides, and easily ignitable materials. 


\begin{tabular}{|c|}
\hline PRODUCT DESIGNATION \\
$03-131-5508$ \\
Sodium HyPOChlorite \\
\end{tabular}

\section{MATERIAL SAFETY DATA SHEET}

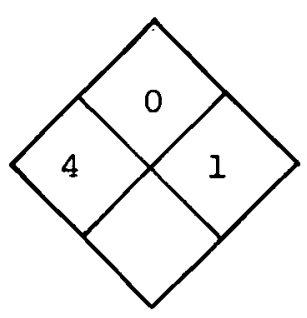

\section{SECTION I SOURCE AND NOMENCLATURE}

MANUFAC TURER'S NAME
ADDRESS INUMBER, STREET, CI TY
TRADE NAME AND SYNONYMS
CHEMICAL NAME AND SYNONYMS
SOdium HYPOChlOrite

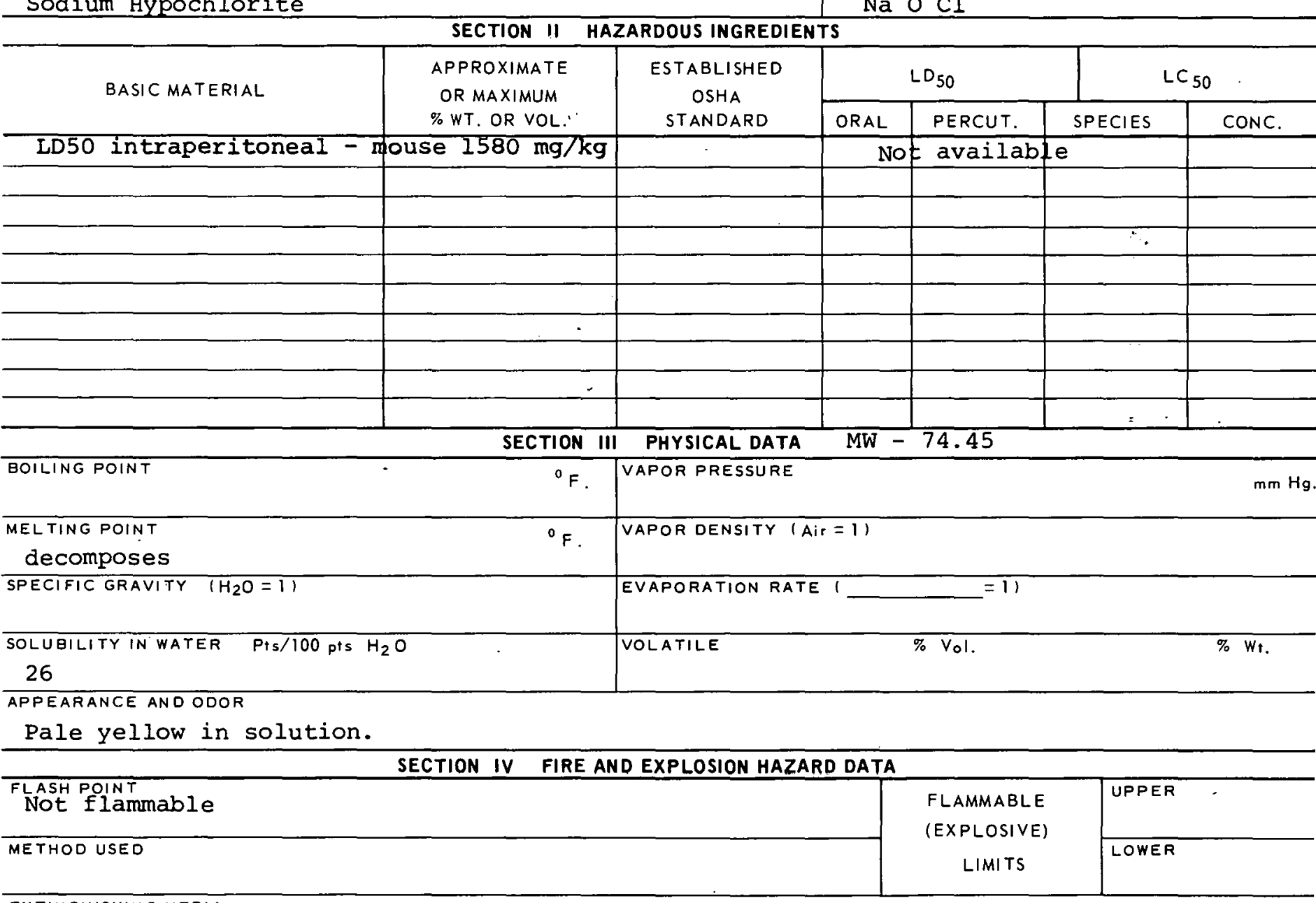

EXTINGUISHING MEDIA

\begin{tabular}{|l|l} 
& CHEMICAL FAMILY \\
& FORMULA \\
$\mathrm{NaOCl}$
\end{tabular}

EMERGENCY TELEPHONE NO.

\section{Y, STATE, ZIP CODEI}




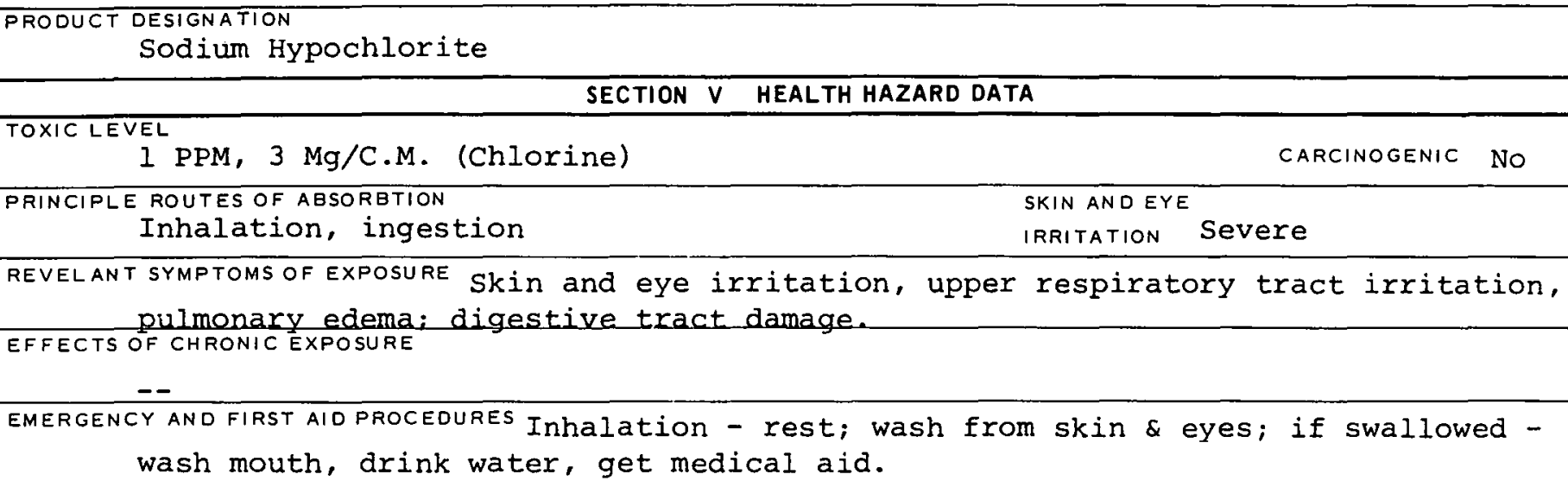

Sodium Hypochlorite

SECTION V HEALTH HAZARD DATA

TOXIC LEVEL

1 PPM, 3 Mg/C.M. (Chlorine)

CARCINOGENIC NO

PRINCIPLE ROUTES OF ABSORBTION

Inhalation, ingestion

SKIN ANDEYE

IRRITATION SEvere

REVELANT SYMPTOMS OF EXPOSURE Skin and eye irritation, upper respiratory tract irritation, pulmonary edema; digestive tract damage. EFFECTS OF CHRONIC EXPOSURE

$--$

EMERGENCY AND FIRST AIO PROCEDURES Inhalation - rest; wash from skin \& eyes; if swallowed wash mouth, drink water, get medical aid.

\section{SECTION VI REACTIVITY DATA}

CONDITIONS CONTRIBUTING TO INSTABILITY

CONDITIONS CONTRIBUTING TO HAZARDOUS POLYMERIZATION

INCOMPATABILITY (MATERIALS TO AVOIO) Mmines end oxidizable malerial such as ixalic acid.

HAZARDOUS DECOMPOSITION PRODUCTS

May produce explosive chloramines with amines.

SECTION VII SPILL OR LEAK PROCEDURES

STEPS TO BE TAKEN IN CASE MATERIAL IS RELEASED OR SPILLED

Cover with weak reducing agent such as sodium bisulfite. Transfer slurry

into water and dispose of.

WASTE DISPOSAL METHOD

SECTION VIII SPECIAL PROTECTION INFORMATION

VENTILATION REQUIREMENTS LOCAL EXHAUST

ROTECTIVE EQUIPMENT (SPECIFY TYPES) EYE

Goggles

$\overline{M E C H A N I C A L ~(G E N E R A L)}$

GLOVES

Rubber

$\overline{\text { SFECIAL }}$

OTHER PROTECTIVE EQUIPMENT

REEPIRATOR

With proper fileer

Coveralls

SECTION IX SPECIAL PRECAUTIONS

PRECAUTIONS TO BE TAKEN IN HANDLING AND STORAGE

Store in cool, dry place away from combustible materials. 


\begin{tabular}{|c|}
\hline PRODUCT DESIGNATION \\
$03-131-6028$ \\
Sodium Metal \\
\end{tabular}

\section{MATERIAL SAFETY DATA SHEET}

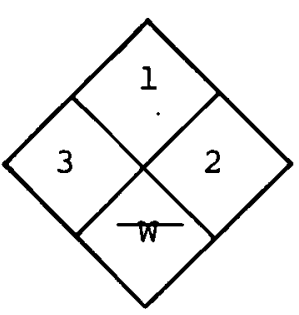

SECTION I SOURCE AND NOMENCLATURE

MANUFACTURER'S NAME
ADDRESS INUMBER, STREET, CI
TRADE NAME AND SYNONYMS
CHEMICAL NAME ANO SYNONYMS
SOdiUUH

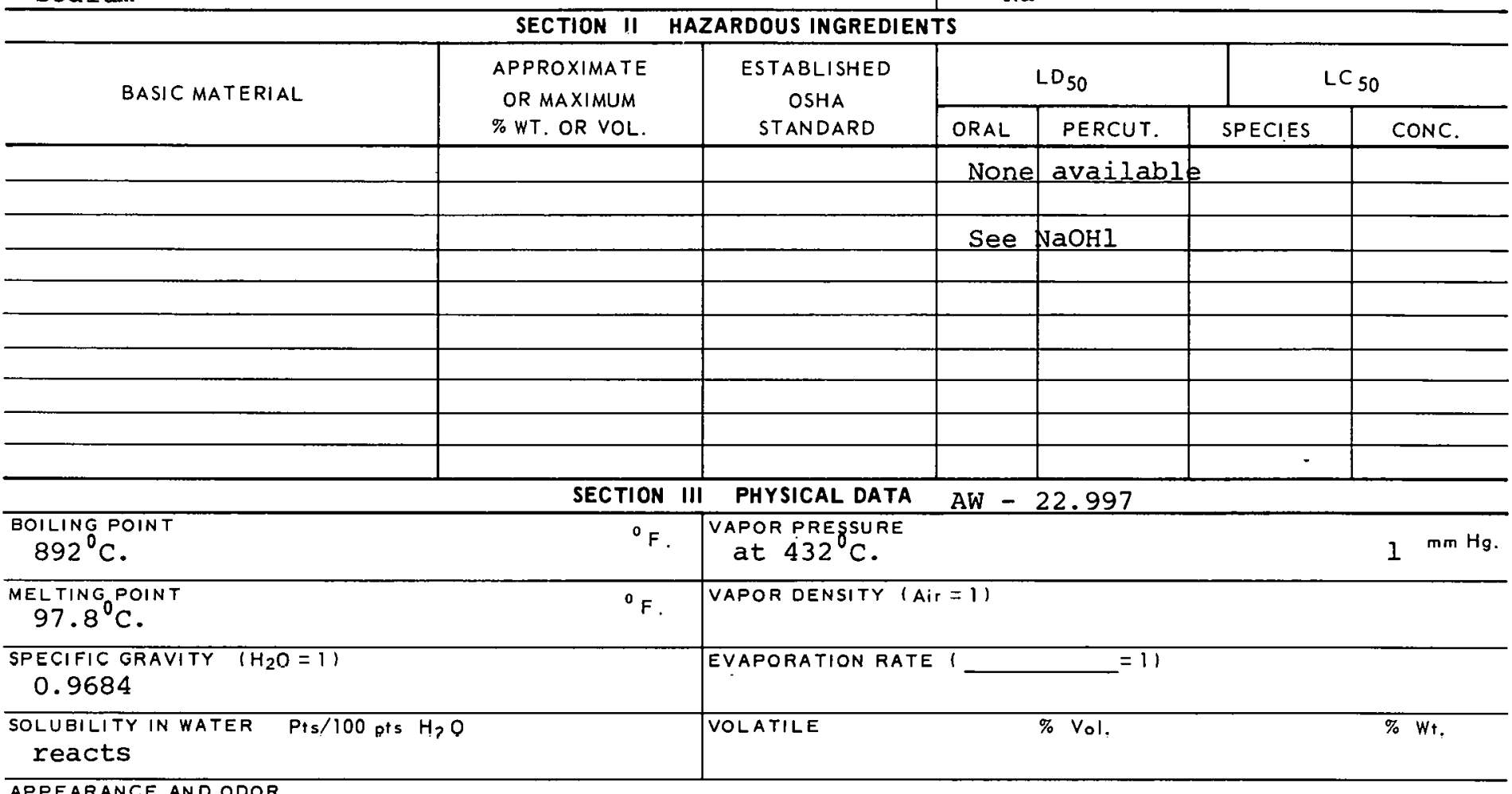

APPEARANCE AND ODOR

Silver white metal

SECTION IV FIRE AND EXPLOSION HAZARD DATA

FLASH POINT
Ignites above $115^{\circ} \mathrm{C}$. in air

METHOD IHSED

\begin{tabular}{|l|l} 
& CHEMICAL FAMILY \\
& FORMULA \\
$\mathrm{Na}$ \\
\hline
\end{tabular}

SECTION II HAZARDOUS INGREDIENTS APPROXIMATE OR MAXIMUM $\%$ WT. OR VOL.
EMERGENCY TELEPHONE NO. 
TOXIC LEVEL

Not available - TLV (2 mg/C.M. NaOH)

CARCINOGENIC

no

PRINCIPLE ROUTES OF ABSORBTION

Inhalation of smoke ingestion

SKIN AND EYE

IRRITATION SEvere

REVELANT SYMPTOMS OF EXPOSURE Severe skin and eye burns; extreme irritation of nose, throat

and respiratory passages; ingestion - severo pain, vomiting an eol lapse. EFFECTS OF CHRONICEXPOSURE

Dermatitis may follow repeated skin contact with alkalies. EMERGENCY AND FIRST AID PROCEDURES

dilute by giving water to aid.

Wash from skin with large amounts of water. If swallowed SECTION VI REACTIVITY DATA

CONDITIONS CONTRIBUTING TO INSTABILITY

CONDITIONS CONTRIBUTING TO HAZARDOUS POLYMERIZATION

INCOMPÁTÁAILITY IMATERIALS TO AVOIOI

Reacts violently with water and hydrocarbons

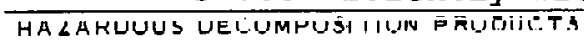

Sodium Hydroxide

SECTION VII SPILL OR LEAK PROCEDURES

STEPS TO BE TAKEN IN CASE MATERIAL IS RELEASED OR SPILLED

WASTE DISPOSAL METHOD

SECTION VIII SPECIAL PROTECTION INFORMATION

VENTILATION REQUIREMENTS LOCAL EXHAUST

FROTECTIVE EQUIFMENT (SFECIFY TYPES) EYE

Face shield

MECHANICAL (GENERAL)

GLOVES

Rubber

SPECIAL

RESPIRATOR

With proper filter

OTHER PROTECTIVE EQUIPMENT

Protective clothing

SECTION IX SPECIAL PRECAUTIONS

PRECAUTIONS TO BE TAKEN IN HANDLING AND STORAGE

Protect from physical damage, keep from water, store under nitrogen,

kerosene or mineral oil. OTHE.R PRES.ALITIONS 


\begin{tabular}{|l|}
\hline \multicolumn{1}{|c|}{ PRODUCT DESIGNATION } \\
03-131-6908 \\
Sodium Oxalate \\
\end{tabular}

\section{MATERIAL SAFETY \\ DATA SHEET}

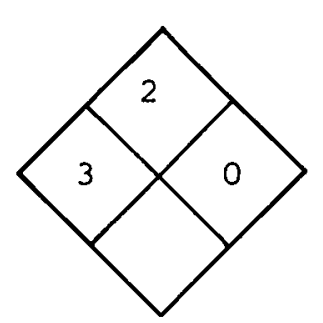

\section{SECTION I SOURCE AND NOMENCLATURE}

\section{MANUFACTURER'S NAME} ADDRESS INUMBER, STREET, CITY, STATE, ZIP CODE
CHEMICAL FAMILY

FORMULA

$\mathrm{Na}_{2} \mathrm{C}_{2} \mathrm{O}_{4}$

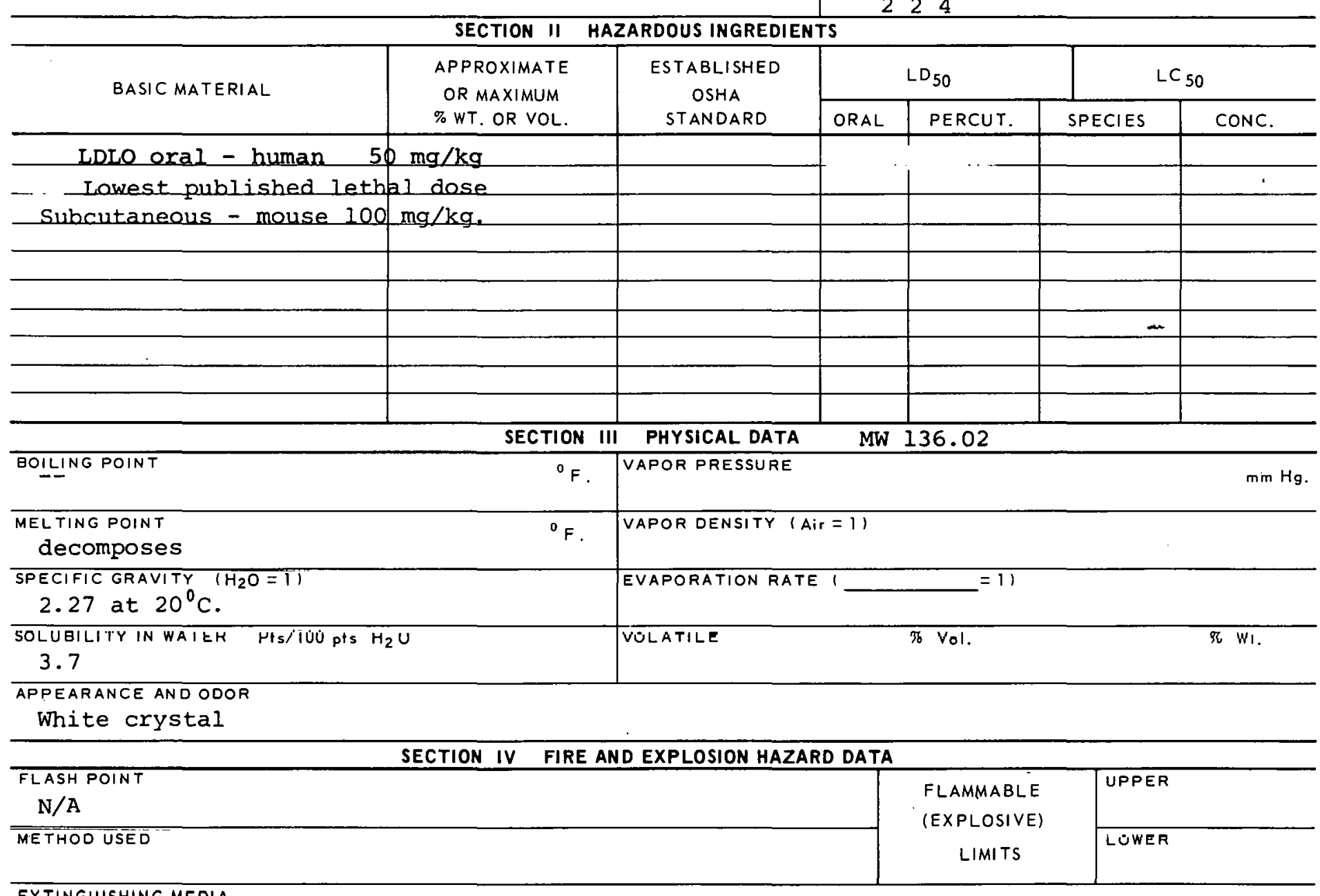

EXTINGUISHING MEDIA

$\mathrm{N} / \mathrm{A}$

SPECIAL FIRE FIGHTING PROCEOURES

$\mathrm{N} / \mathrm{A}$

UNUSUAL FIRE AND EXPLOSION HAZARDS

When heated to decomposition temperature may emit toxic fumes. 


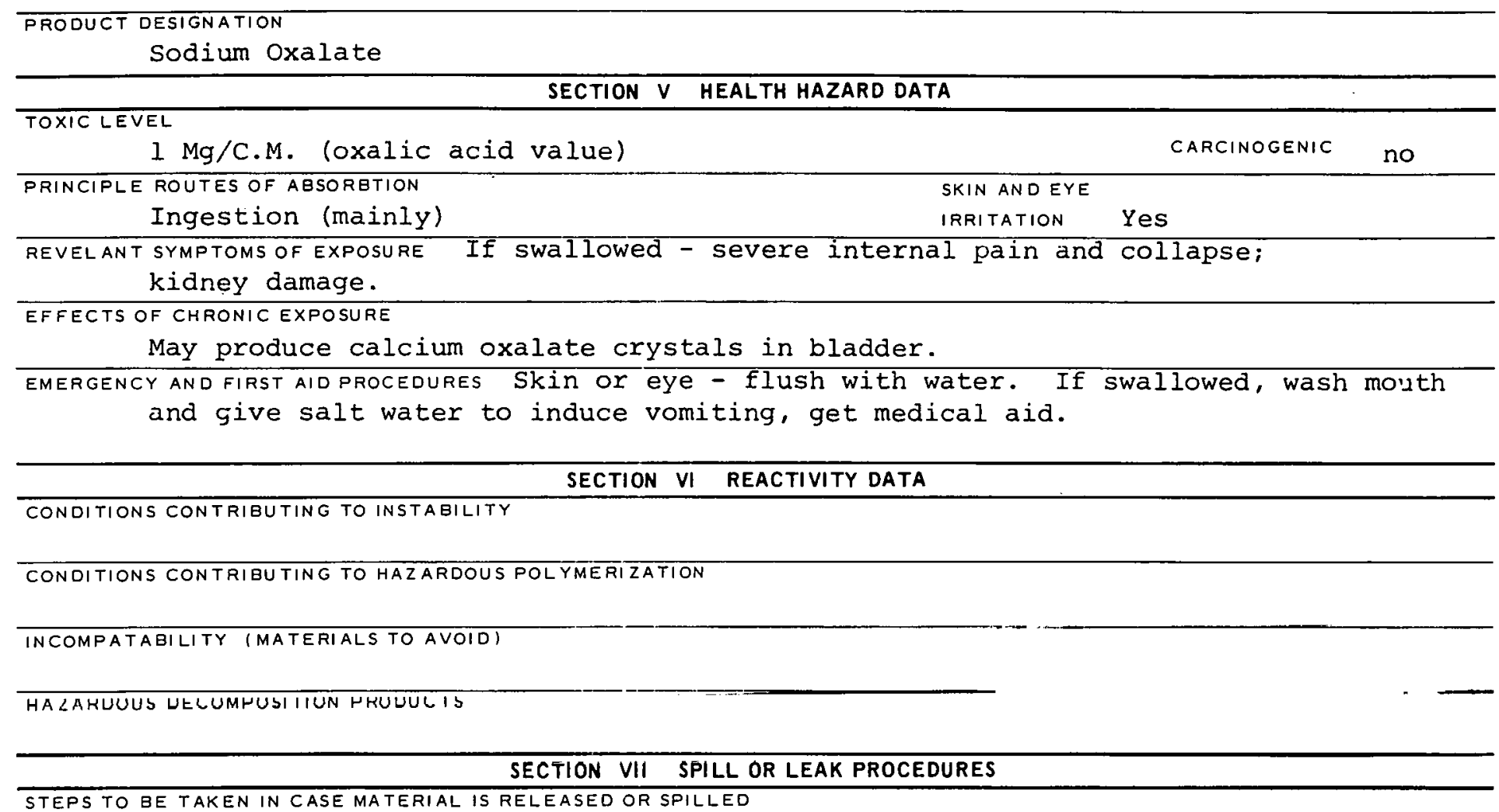

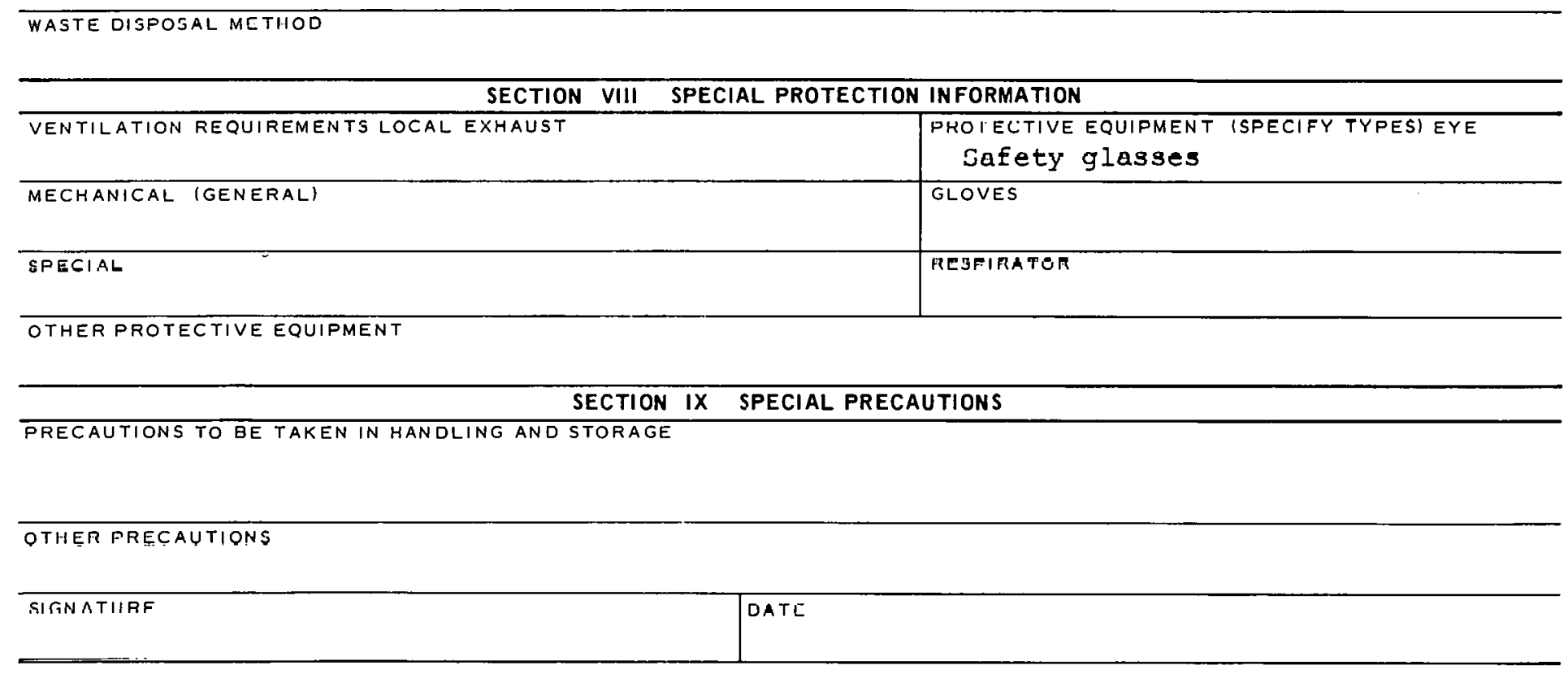




\section{PRODUCT DESIGNATION}

$03-131-7338$

Sodium Peroxide
MATERIAL SAFETY

DATA SHEET

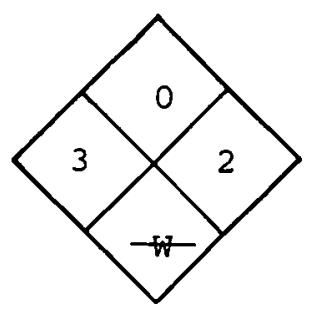

SECTION I SOURCE AND NOMENCLATURE

\section{MANUFACTURER'S NAME \\ TRADE NAME AND SYNONYMS \\ CHEMICAL NAME AND SYNONYMS \\ Sodium Peroxide}

ADORESS (NUMBER, STREET, CITY, STATE, ZIP CODE)
BASIC MATERIAL

\begin{tabular}{l|l|l} 
& CHEMICAL FAMILY \\
& & FORMULA \\
$\mathrm{Na}$ & $\mathrm{O} 2$ \\
\hline
\end{tabular}

SECTION II HAZARDOUS INGREDIENTS

\begin{tabular}{l|l}
\hline APPROXIMATE & ESTABLISHED
\end{tabular}

OR MAXIMUM $\%$ WT, OR VOL.
EMERGENCY TELEPHONE NO.

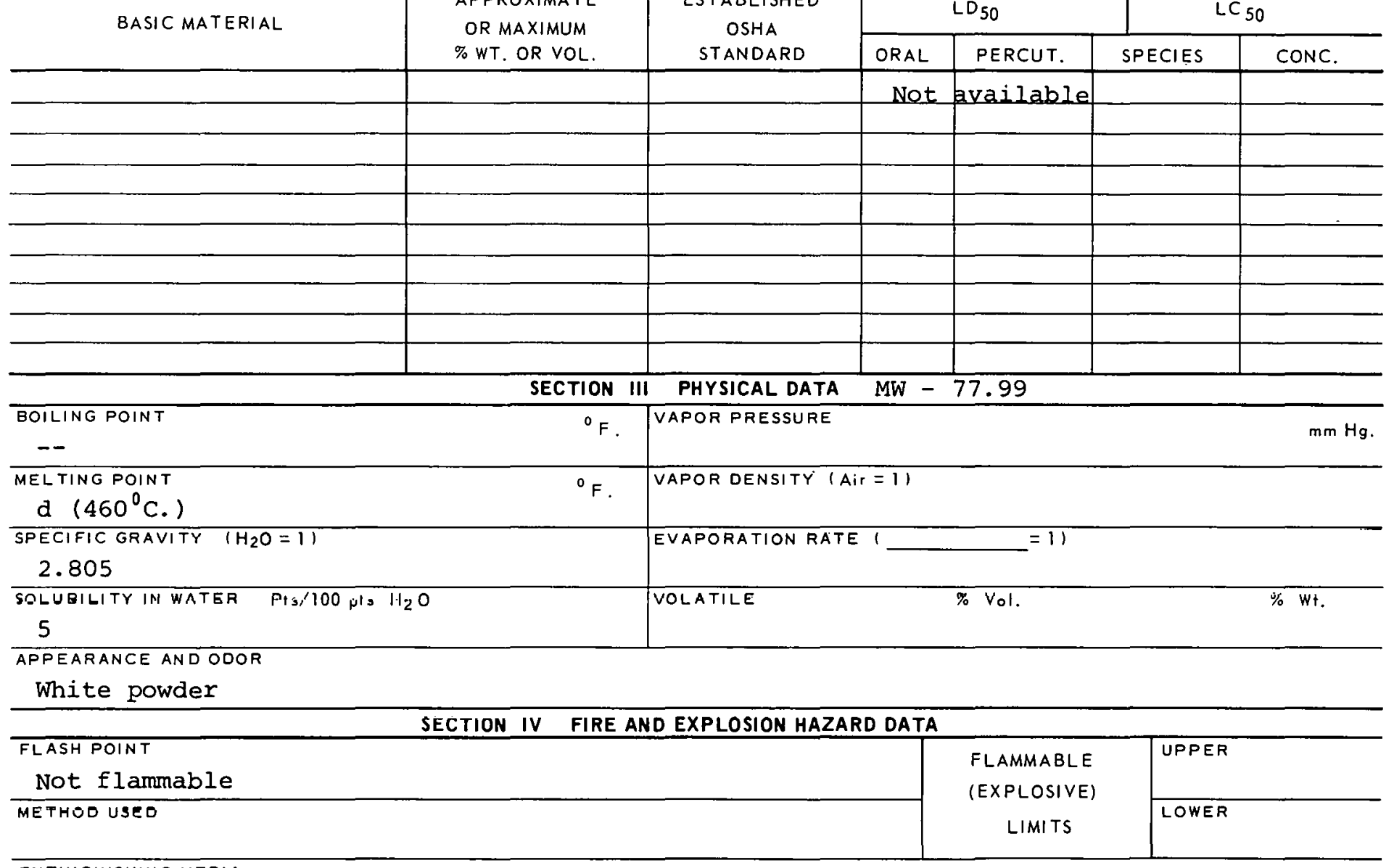

EXTINCUISIIING MEDIA

SPECIAL FIRE FIGHTING PROCEDURES

Wear full protective clothing.

UNUSUAL FIRE AND EXPLOSION HAZARDS

Reacts with water to produce oxygen. 


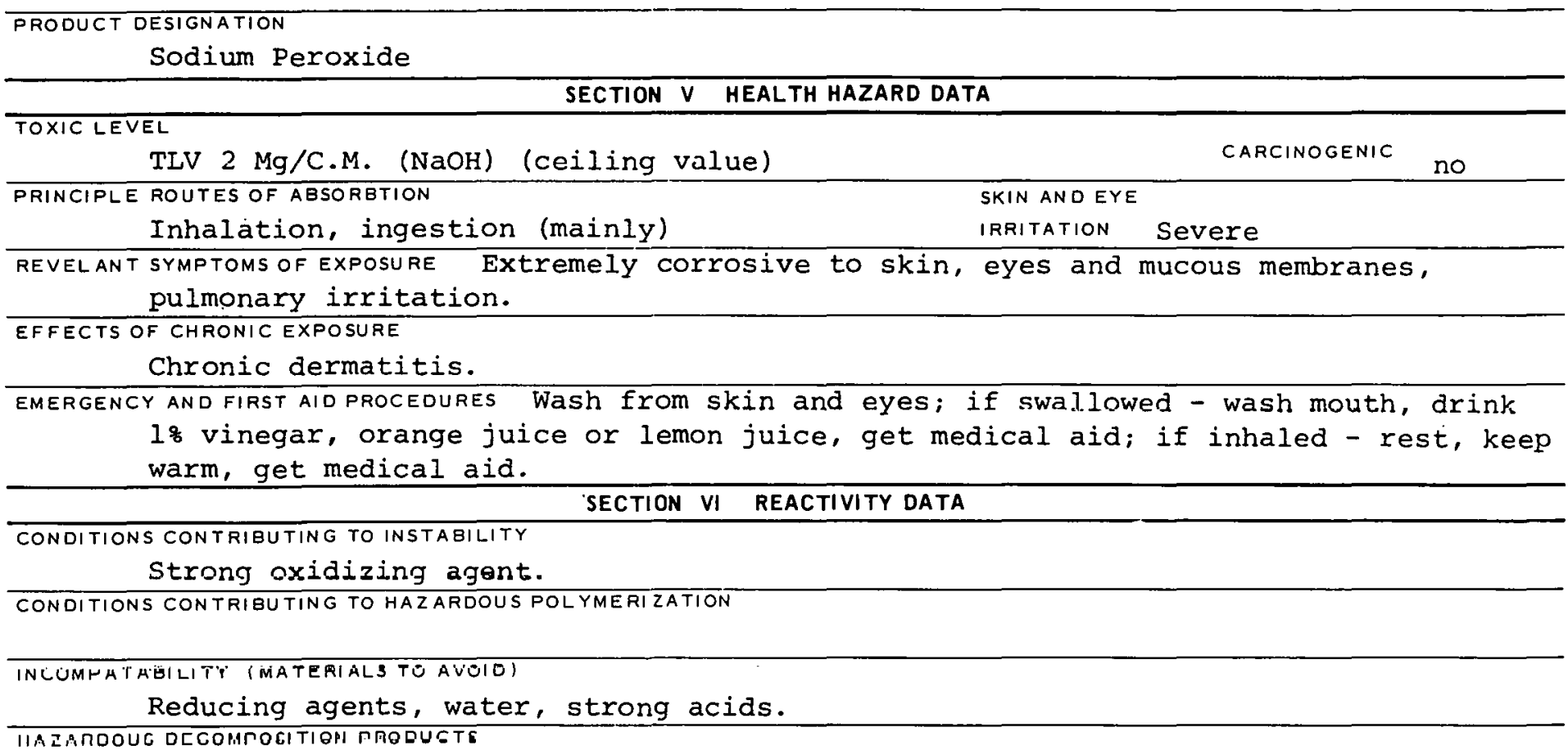

SECTION VII SPILL OR LEAK PROCEDURES

STEPS TO 日E TAKEN IN CASE MATERIAL IS RELEASED OR SPILLED

Cover with (9.1) sand to soda ash

WASTE DISPOSAL METHOD

\begin{tabular}{|c|c|}
\hline \multicolumn{2}{|c|}{ SECTION VIII SPECIAL PROTECTION INFORMATION } \\
\hline VENTILATION REQUIREMENTS LOCAL EXHAUST & $\begin{array}{l}\text { PROTECTIVE EQUIPMENT (SPECIFY TYPES) EYE } \\
\text { Safety glasses }\end{array}$ \\
\hline MECHANICAL (GENERAL) & $\begin{array}{l}\text { GLOVES } \\
\text { Rubber }\end{array}$ \\
\hline SPEC!|A! & RESPIRATQR \\
\hline \multicolumn{2}{|l|}{ OTHER PROTECTIVE EQUIPMENT } \\
\hline \multicolumn{2}{|c|}{ SECTION IX SPECIAL PRECAUTIONS } \\
\hline \multicolumn{2}{|l|}{ Store away from sources of water. } \\
\hline \multicolumn{2}{|l|}{ OTHER PRECAUTIONS } \\
\hline SIGNATURE & DATE \\
\hline
\end{tabular}


$03-131-8148$

03-131-8188

Sodium Silicate

MATERIAL SAFETY

DATA SHEET

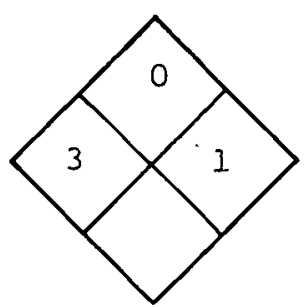

\begin{tabular}{l} 
MANUFACTURER'S NAME \\
\hline ADDRESS INUMBER, STREET, CI \\
TRADE NAME AND SYNONYMS \\
WatEY GlaSS \\
CHEMICAL NAME AND SYNONYMS \\
SOdIUM SIIICate
\end{tabular}

\section{SECTION I SOURCE AND NOMENCLATURE}

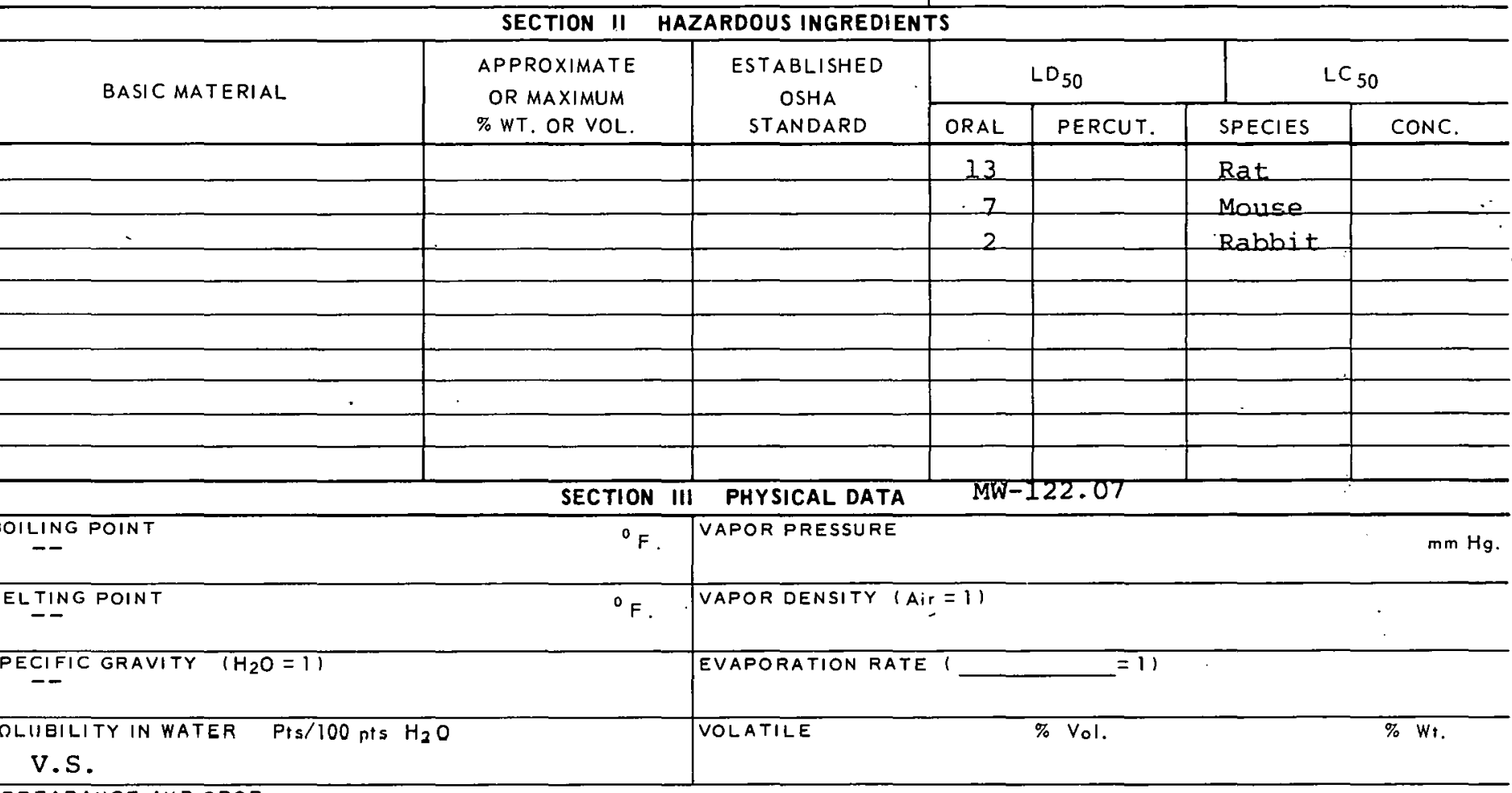

APPEARANCE AND ODOR

Clear solution

SECTION IV FIRE AND EXPLOSION HAZARD DATA

FLASH POINT
Not flammable

METHOO USED

EXTINGIIISHING MEDIA

Water

SPECIAL FIRE FIGHTING PROCEDURES

FLAMMABLE

(EXPLOSIVE)

LIMITS 
TOXIC:EVEL

$\mathrm{TLV}-2 \mathrm{Mg} / \mathrm{C} . \mathrm{M} . \quad(\mathrm{NaOH})$

CARCINOGENIC NO

PRINCIPLE ROUTES OF ABSORBTION

Inhalation of mist, ingestion

SKIN ANDEYE

IRRITATION SEVERE

REVELANT SYMPTOMS OF EXPOSURE EXtremely corrosive to skin, eyes and mucous membranes; also pulmonary ircitation.

EFFECTS OF CHRONIC EXPOSURE

Chronic dermatitis

EMERGENCY AND FIRST AID PROCEDURES Inhalation - rest, keep warm, get medical aid; if swallowed wash mouth, drink lo vinegar, get medical aid; wash from skin and eyes.

\section{SECTION VI REACTIVITY DATA}

CONDITIONS CONTRIBUTING TO INSTABILITY

CONDITIONS CONTRIBUTING TO HAZARDOUS POLYMERI $\bar{Z} \overline{A T I O N}$

IICOMTATADILITY (MATCRIALS TO AVOID)

Strong Acids

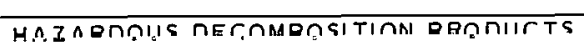

SECTION VII SPILL OR LEAK PROCEDURES

STEPS TO BE TAKEN IN CASE MATERIAL IS RELEASED OR SPILLED

WASTE DISPOSAL METHOD

\section{SECTION VIII SPECIAL PROTECTION INFORMATION}

\begin{tabular}{l|l}
\hline VENTILATION REQUIREMENTS LOCAL EXHAUST & $\begin{array}{l}\text { PROTECTIVE EQUIPMENT (SPECIFY TYPESI EYE } \\
\text { FaCe ShIELd }\end{array}$ \\
\hline MECHANICAL IGENERAL) & $\begin{array}{c}\text { GLOVES } \\
\text { Rubber }\end{array}$ \\
\hline SPECIAL & RESPIRATOR \\
With proper filter
\end{tabular}

\section{SECTION IX SPECIAL PRECAUTIONS}

PRECAUTIONS TO BE TAKEN IN HANDLING AND STORAGE

OTHER PRECAUTIONS

SIGNATURE

DATE

$131-8148 B$ 
CAS : 010102202

WY 24500

\section{PRODUCT DESIGNATION}

03-131-8758

Sodium Tellurite

SECTION I SOURCE AND NOMENCLATURE

\begin{tabular}{l} 
MANUFACTURER'S NAME \\
\hline ADDRESS INUMBER, STREET, CITY, STA \\
TRADE NAME AND SYNONYMS \\
DISOdIUM TEIIOYUS ACId \\
CHEMICAL NAME AND SYNONYMS \\
SOdiUm telIurite
\end{tabular}

MATERIAL SAFETY DATA SHEET

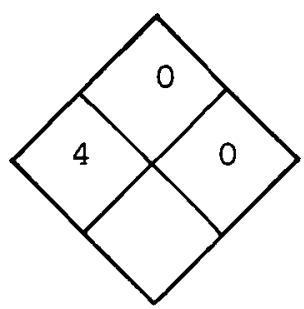

EMERGENCY TELEPHONE NO.

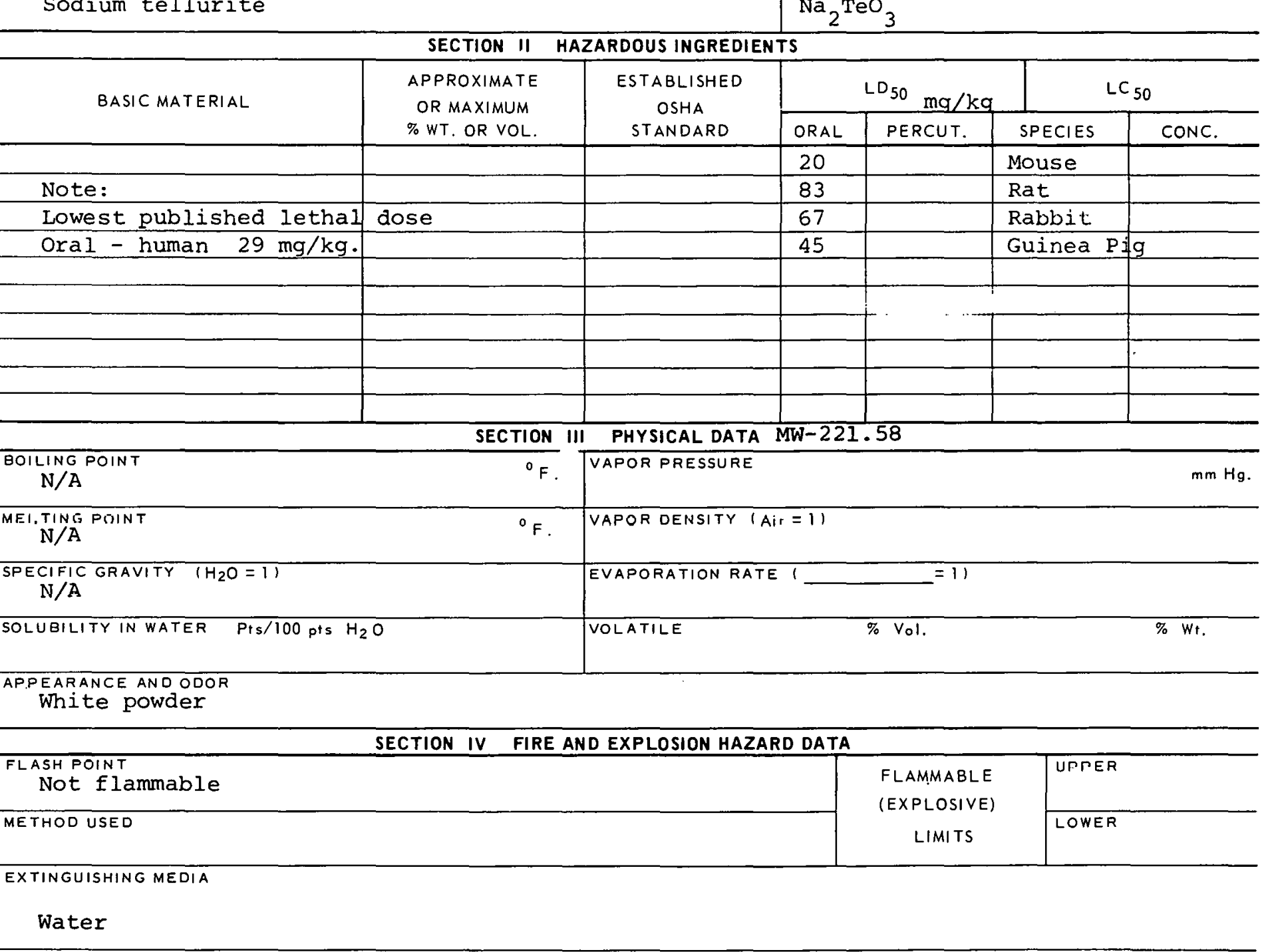

SPECIAL FIRE FIGHTING PROCEDURES

$\mathrm{N} / \mathrm{A}$

UNUSUAL FIRE AND EXPLOSION HAZARDS

$\mathrm{N} / \mathrm{A}$ 
TOXIC LEVEL

$0.1 \mathrm{Mg} / \mathrm{C} . \mathrm{M}$.

PRINCIPLE ROUTES OF ABSORETION

Inhalation, ingestion
CARCINOGENIC NO

SKIN AND EYE

IRRITATION
Moderate

REVELANT SYMPTOMS OF EXPOSURE Sleepiness, loss of appetite, nausea, metallic taste, garlic odor to breath.

EFFECTS OF CHRONIC EXPOSURE

Fatigue, loss of appetite, bronchitis, dermatitis.

EMERGENCY AND FIRST AID PROCEDURES If inhaled - rest, keep warm, get medical ald. If swallowed, wash mouth, give emetic, get medical aid; wash from skin and eyes.

\section{SECTION VI REACTIVITY DATA}

CONDITIONS CONTRIBUTING TO INSTABILITY

CONDITIONS CONTRIBUTING TO HAZARDOUS POLYMERI ZATION

INCOMPATABILITY (MATERIALS TO AVOIO)

HA ZARDOUS DECOMPOSITION PROOUCTS

SECTION VII SPILL OR LEAK PROCEDURES

STEPS TO BE TAKEN IN CASE MATERIAL IS RELEASED OR SPILLED

WASTE UISPOSAL MEIHUU

\section{SECTION VIII SPECIAL PROTECTION INFORMATION}

\begin{tabular}{|c|c|}
\hline SECTION VIII & SPECIAL PROTECTION INFORMATION \\
\hline VENTILATION REQUIREMENTS LOCAL EXHAUST & $\begin{array}{l}\text { PROTECTIVE EQUIPMENT (SPECIFY TYPES) EYE } \\
\text { Safety glasses }\end{array}$ \\
\hline MELHANILAL (UENLKAL) & $\begin{array}{l}\text { GLOVES } \\
\text { Rubber }\end{array}$ \\
\hline SPECIAL & $\begin{array}{l}\text { RESPIRATOR } \\
\text { With proper filtor }\end{array}$ \\
\hline
\end{tabular}

OTHER PROTECTIVE EQUIPMENT

SECTION IX SPECIAL PRECAUTIONS

PRECAUTIONS TO BE TAKEN IN HANDLING AND STORAGE

OTHER PRECAUTIONG

SIGNA I UHE

DATE

$131-8758 B$ 


\begin{tabular}{l} 
PRODUCT DESIGNATION \\
Stannic Chloride \\
$03-133-3008$ \\
\hline
\end{tabular}

\section{MATERIAL SAFETY DATA SHEET}

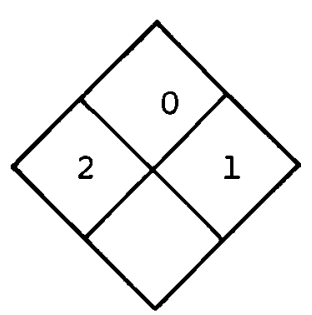

SECTION I SOURCE AND NOMENCLATURE

MANUFACTURER'S NAME
ADDRESS INUMBER, STREET, CITY
TRADE NAME AND SYNONYMS
CHEMICAL NAME AND SYNONYMS
Tin TetraChloride

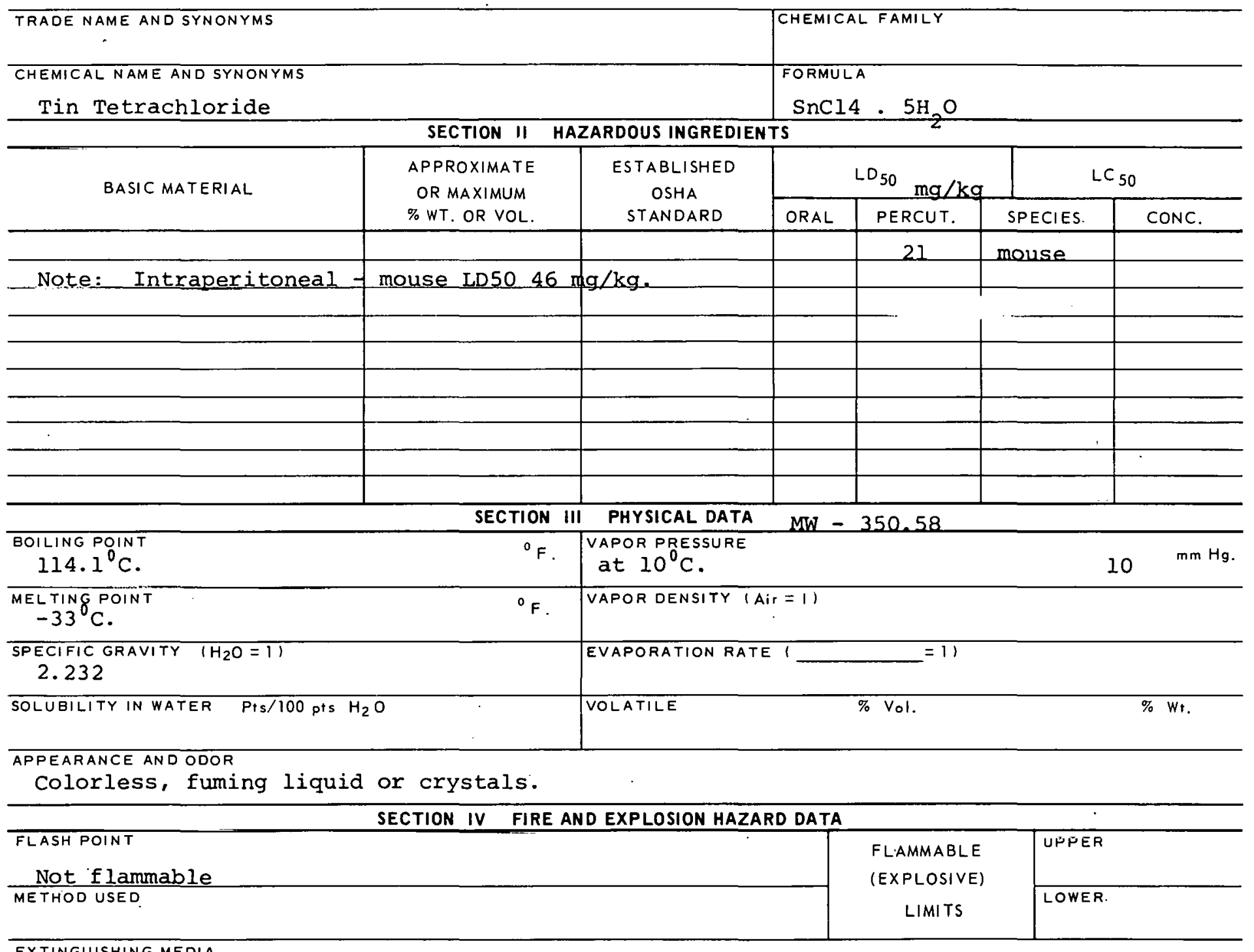

EXTINGUISHING MEDIA

Do not use hand-held water-base extinguishers.

SPECIAL FIRE FIGHTING PROCEDURES

Full protective clothing.

UNUSUAL FIRE AND EXPLOSION HAZARDS

-. 


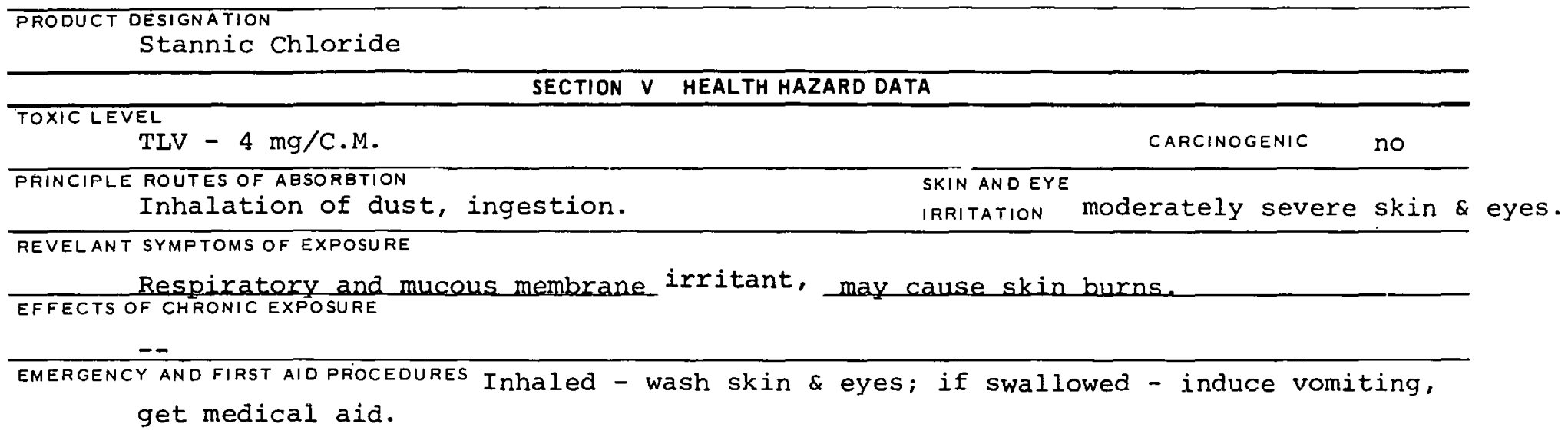

\section{SECTION VIII SPECIAL PROTECTION INFORMATION}

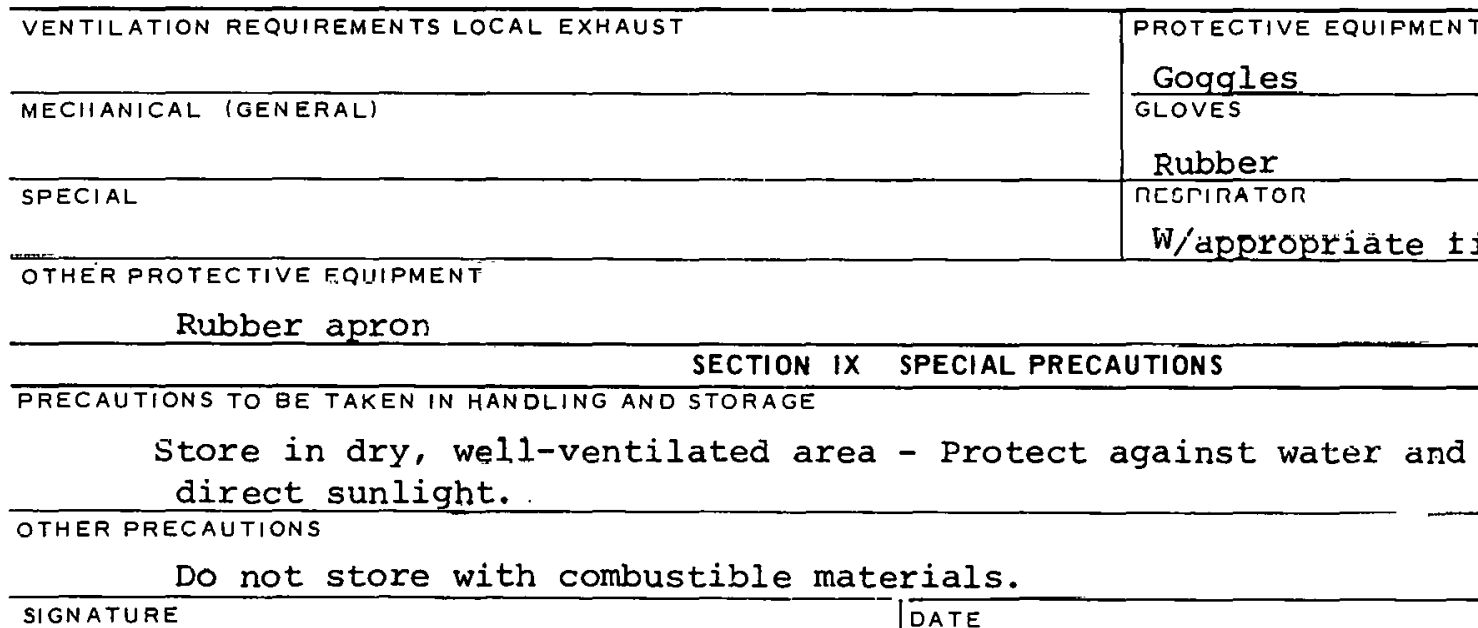




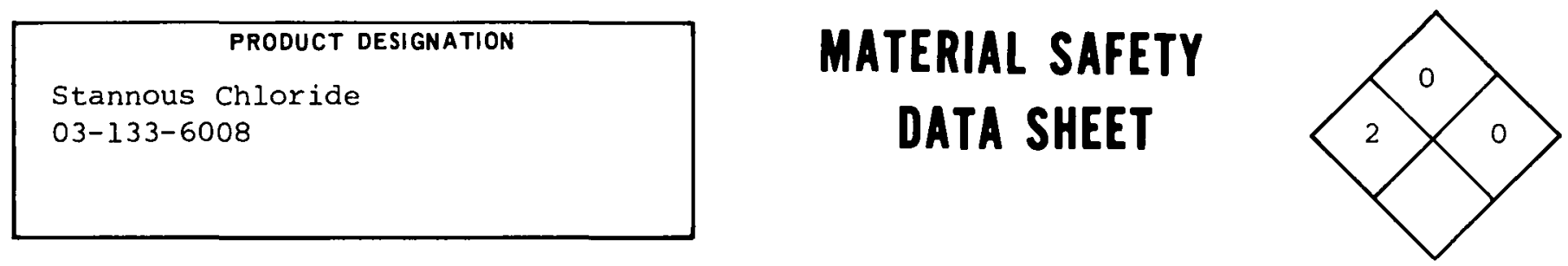

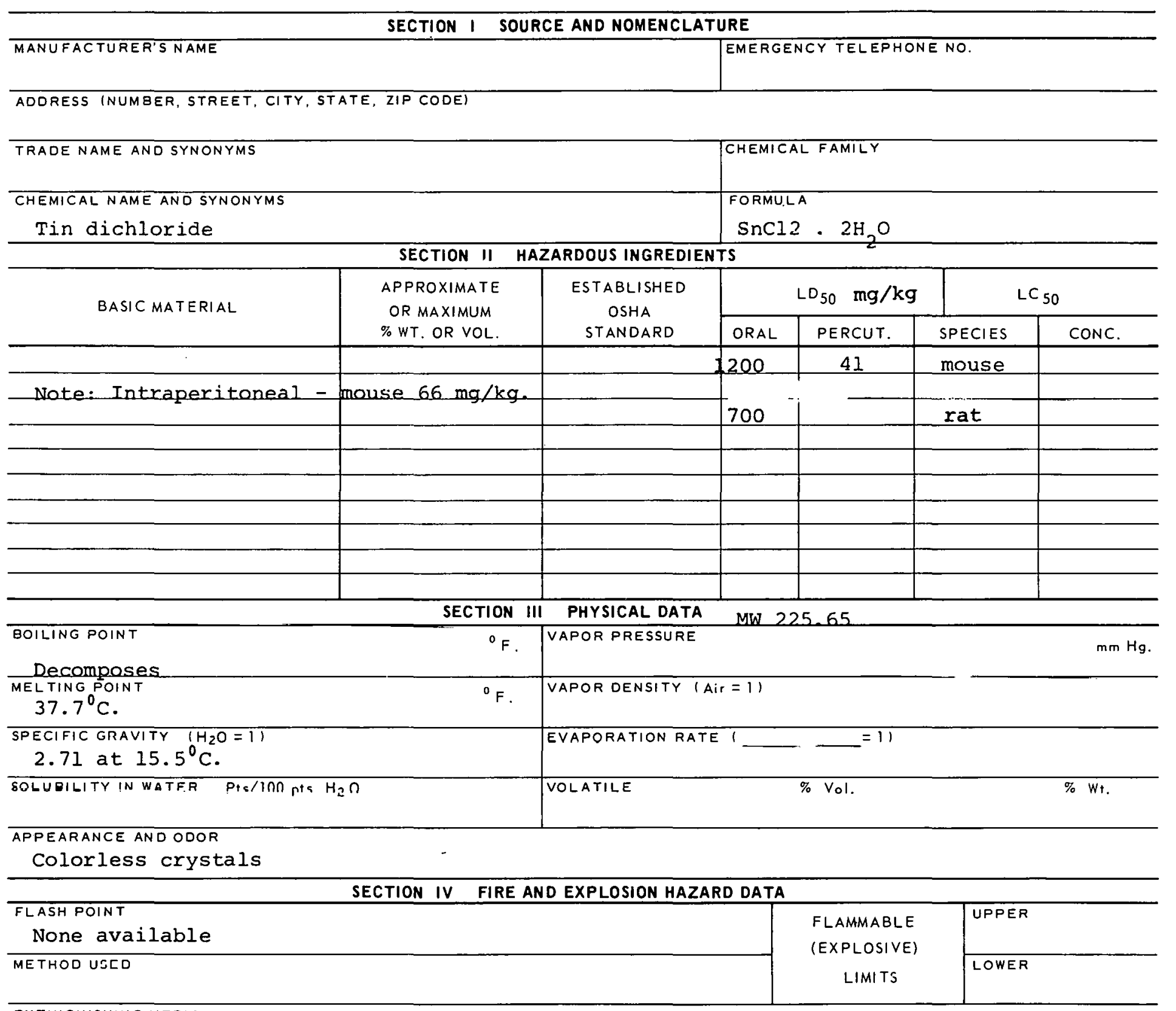

EXTINGUISHING MEOIA

None available

SPECIAL FIRE FIGHTING PROCEOURES

UNUSUAL FIRE AND EXPLOSION HAZARDS 


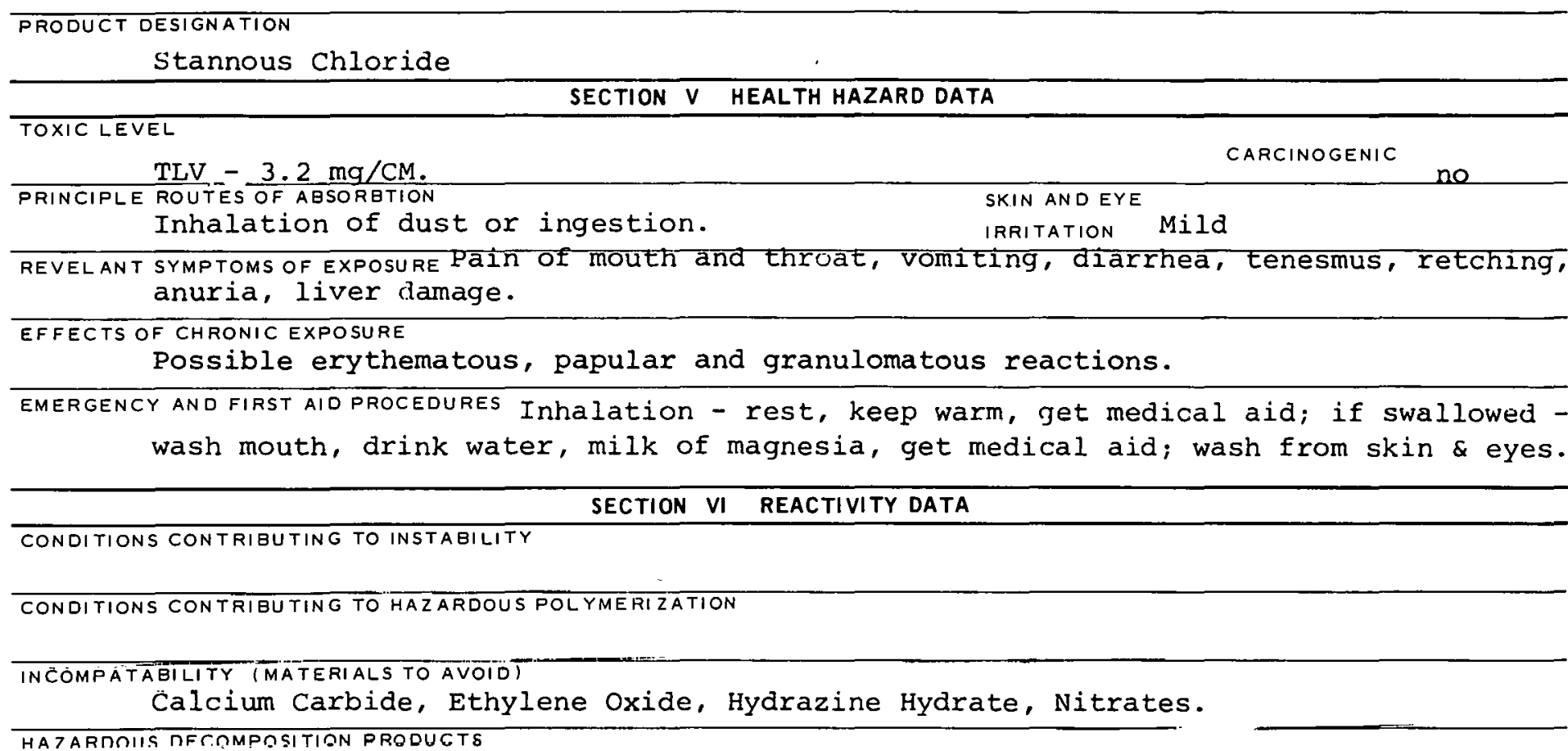

SECTION VII SPILL OR LEAK PROCEDURES

STEPS TO BE TAKEN IN CASE MATERIAL IS RELEASED OR SPILLED

WASTE DISPOSAL METHOD

SECTION VIII. SPECIAL PROTECTION INFORMATION

\begin{tabular}{l|l}
\hline VENTILATION REQUIREMENTS LOCAL EXHAUST & $\begin{array}{l}\text { PROTECTIVE EQUIPMENT (SPECIFY TYPES) EYE } \\
\text { Safety glasses or gOGQles }\end{array}$ \\
\hline MECHANICAL (GENERAL) & $\begin{array}{l}\text { GLOVES } \\
\text { Rubber }\end{array}$ \\
\hline SPECIAL & RESHIRATUR \\
W/appropriate filter
\end{tabular}

OTHER PROTECTIVE EQUIPMENT

SECTION IX SPECIAL PRECAUTIONS

PRECAUTIONS TO BE TAKEN IN HANDLING AND STORAGE

OTHER PRECAUTIONS

SIGNATURE

DATE

$133-6008 B$ 


\begin{tabular}{|l|}
\hline PRODUCT DESIGNATION \\
03-141-5902 \\
Tellurium Metal
\end{tabular}

\section{MATERIAL SAFETY DATA SHEET}

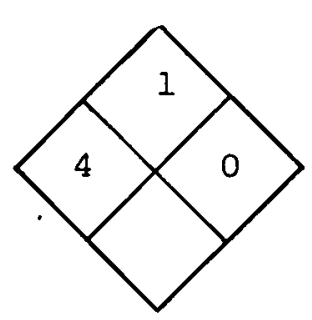

\section{SECTION I SOURCE AND NOMENCLATURE}

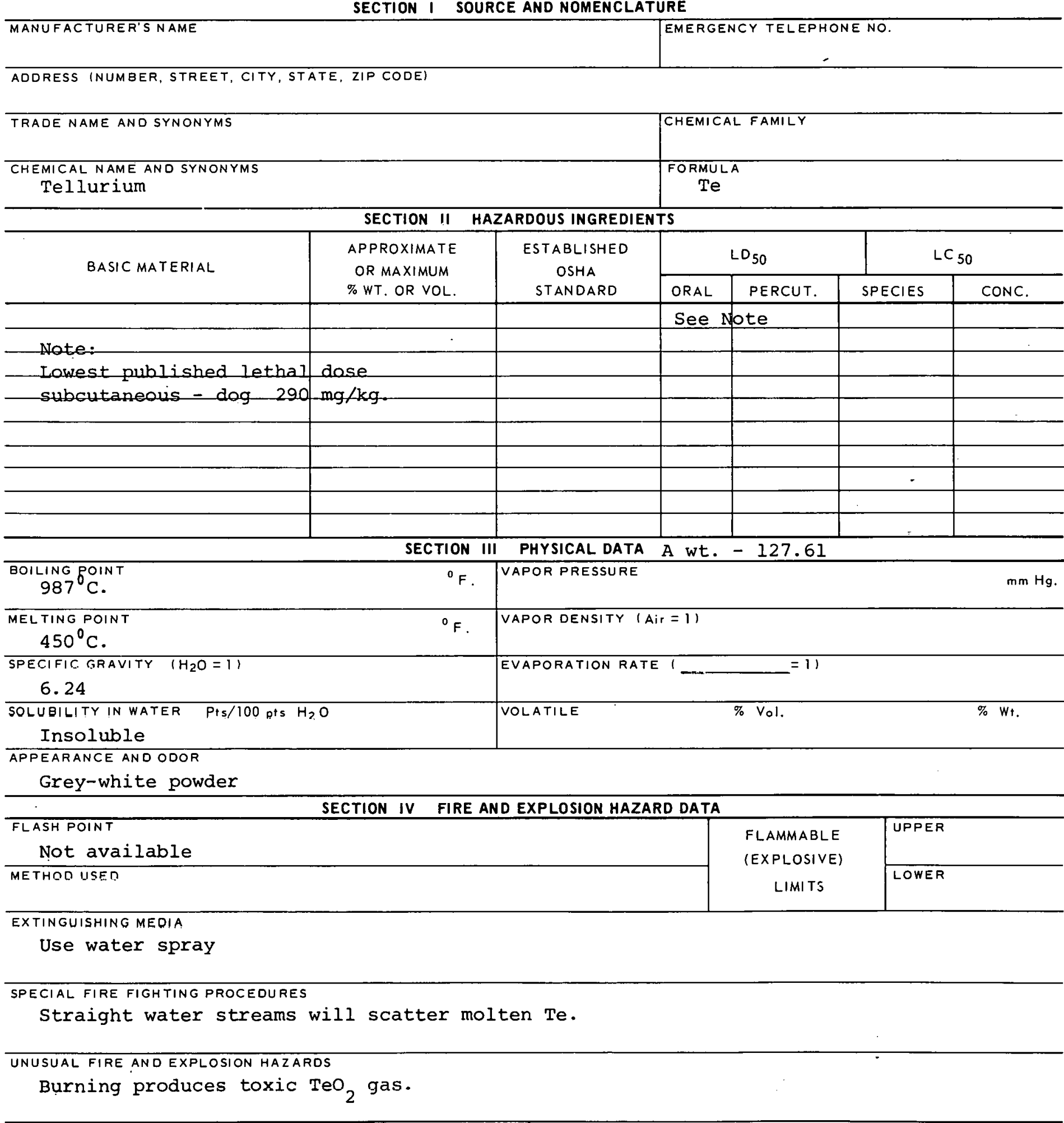


Tellurium Metal

SECTION $V$ HEALTH HAZARD DATA

TOXIC LEVEL

TLV - $0.1 \mathrm{mg} / \mathrm{CM}$.
CARCINOGENIC NO

SKIN AND EYE

IRRITATION MOderate

Inhalation, ingestion

REVELANT SYMPTOMS OF EXPOSURE Sleepiness, loss of appetite, nausea, metallic taste and garlic odor to breath. EFFECTS OF CHRONIC EXPOSURE

Fatigue, loss of appetite, bronchitis, dermatitis.

EMERGENCY AND FIRST AID PROCEDURES If inhaled - keep warm; if swallowed - wash mouth, give emetic, get medical aid; wash from skin and eyes.

SECTION VI REACTIVITY DATA

CONDITIONS CONTRIBUTING TO INSTABILITY

CONDITIONS CONTRIBUTING TO HAZARDOUS POLYMERIZATION

INCUMPATABILIIY IMATERIALS TO AVOIOI

HAZARDOUS DECOMPOSITION PRODUCTS

SECTION VII SPILL OR LEAK PROCEDURES

STEPS TO BE TAKEN IN CASE MATERIAL IS RELEASED OR SPILLED

SECTION VIII SPECIAL PROTECTION INFORMATION

\begin{tabular}{l|l}
\hline VENTILATION RFQUIREMENTS LOCAL EXHAUST & PROTECTIVE EQUIPMENT (SPECIFY TYPES) EYE
\end{tabular}

MECHANICAL (GENERAL)

Goggles

GLOVES

Rubber

SPECIAL

OTHER PROTECTIVE EQUIFMEN

RESPIRAIOR

With proper filter

\section{SECTION IX SPECIAL PRECAUTIONS}

PRECAUTIONS TO BE TAKEN IN HANDLING AND STORAGE

OTHER PRECAUTIONS

$\overline{\text { SIGNATURE }}$

DATE

$141-5902 B$ 
03-150-4003

PRODUCT DESIGNATION

Titanium Chloride
MATERIAL SAFETY

DATA SHEET

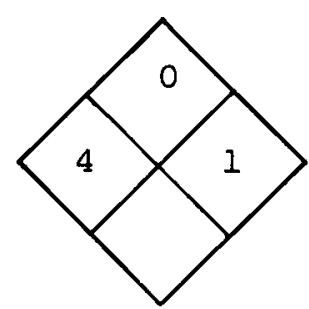

\section{SECTION I SOURCE AND NOMENCLATURE}

\begin{tabular}{l} 
MANUFAC TURER'S NAME \\
\hline ADDRESS INUMBER, STREET, CITY, STA \\
TRAOE NAME AND SYNONYMS \\
CHEMICAL NAME AND SYNONYMS \\
Titanium TetraChloride
\end{tabular}

EMERGENCY TELEPHONE NO.

BASIC MATERIAL

Lowest published lethal concentration in air

inhalation - mouse $10 \mathrm{mg} / \mathrm{C} . \mathrm{M}$. -2 hours. SECTION II HAZARDOUS INGREDIENTS

CHEMICAL FAMILY

\begin{tabular}{l|l} 
& CHEMICAL FAMILY \\
& FORMULA \\
& $\mathrm{T}_{2} \mathrm{Cl}_{4}$ \\
\hline
\end{tabular}

\begin{tabular}{l|c|c} 
BASIC MATERIAL & $\begin{array}{c}\text { OR MAXIMUM } \\
\% \text { WT. OR VOL. }\end{array}$ & $\begin{array}{c}\text { OSHA } \\
\text { STANDARD }\end{array}$ \\
\hline Lowest published lethal & concentration in air \\
\hline inhalation - mouse 10 mg/C.M. -2 hours.
\end{tabular}

APPROXIMATE ESTABLISHED

(20)

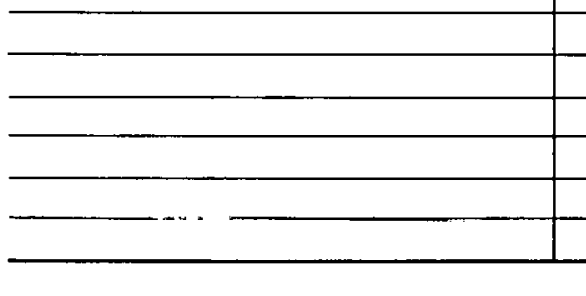

BOILING POINT

$136^{\circ} \mathrm{C}$.

MELTING POINT

$-30^{\circ} \mathrm{C}$.

SPECIFIC GRAVITY $\left(\mathrm{H}_{2} \mathrm{O}=1\right)$

1.726

SULUUILIIY IN WATTER PIS/100 PTS $\mathrm{H}_{2} \mathrm{O}$

\begin{tabular}{l|l|l}
$\mathrm{H}_{2} \mathrm{O}$ & VOLATILE
\end{tabular}

SECTION III PHYSICAL DATA

\begin{tabular}{l|l}
${ }^{\circ} \mathrm{F}$. & VAPOR PRESSURE \\
\hline
\end{tabular}

\begin{tabular}{|l|l}
\hline$L_{50}$ & LC \\
50
\end{tabular}

\begin{tabular}{|l|l|l|l|}
\hline ORAL & PERCUT. & SPECIES & CONC. \\
\hline
\end{tabular}

$S$

APPEARANCE AND ODOR

Colorless to light yellow liquid - fumes in moist air.

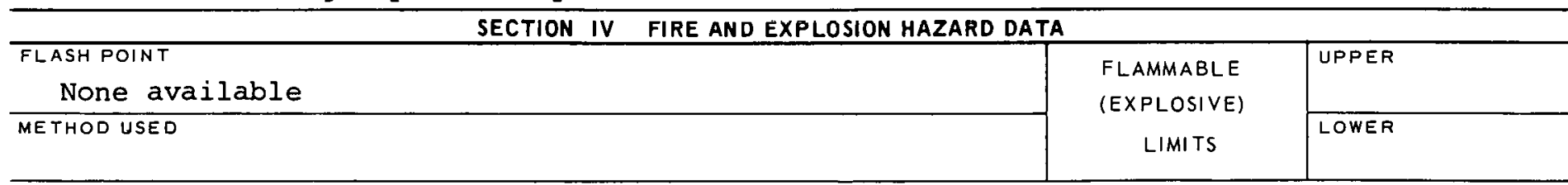

EXTINGUISHING MEDIA

SPECIAL FIRE FIGHTING PROCEDURES

Water spray if necessary to keep container cool - full protective clothing.

UNUSUAL FIRE ANO EXPLOSION HAZARDS

Reacts with water to produce $\mathrm{HCl}$. 


SECTION $V$ HEALTH HAZARD DATA

TOXIC LEVEL

Not available TLV (5 PPM HCl)

CARCINOGENIS NO

PRINCIPLE ROUTES OF ABSORBTION

Inhalation, ingestion

SKIN AND EYE

IRRITATION YES

REVELANT SYMPTOMS OF EXPOSURE Vapor irrjtating to skin and respixatory system, severe

irritation to digestive tract.

EFFECTS OF CHRONIC EXPOSURE Repeated exposure can cause symptoms similar to silicosis.

EMERGENCY AND FIRST AID PROCEDURES Wash from skin immediately; inhalation - artificial

respiration and shock treatment if needed; if swallowed drink water or milk

immediately, give milk of magnesia, get medical aid. SECTION VI REACTIVITY DATA

CONDITIONS CONTRIBUTING TO INSTABILITY

Reacts with moisture of the air to produce $\mathrm{HCl}$ fumes. CONDITIONS CONTRIBUTING TO HAZARDOUS POLYMERI ZATION

INCOMPATADILITU (MATERIALS TO AVUIOU)

Water

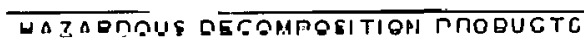

SECTTION VII SPILL OR LEAK PROCEDURES

STEPS TO BE TAKEN IN CASE MATERIAL IS RELEASED OR SPILLED

Cover spills with sodium bicarbonate. Remove to container for disposal.

WASTE DISPOSAL METHOD

SECTION VIII SPECIAL PROTECTION INFORMATION

\begin{tabular}{|c|c|}
\hline $\begin{array}{c}\text { VENTILATION REQUIREMENTS LOCAL EXHAUST } \\
\text { HandIe in hood }\end{array}$ & $\begin{array}{l}\text { PROTECTIVE EQUIPMENT (SPECIFY TYPES) EYE } \\
\text { Fäle Sllield }\end{array}$ \\
\hline MECHANICAL (GENERAL) & $\begin{array}{l}\text { GLOVES } \\
\text { Rubber gloves }\end{array}$ \\
\hline SPECIAL & $\begin{array}{l}\text { RESPIRATOR } \\
\text { WitH pIUper filter }\end{array}$ \\
\hline
\end{tabular}

OTHER PROTECTIVE EQUIPMENT'

TECTIVE EQUIPMENT
Wear protective clothing.

SECTION IX SPECIAL PRECAUTIONS

PRECAUTIONS TO BE TAKEN IN HANDLING AND STRRAGE.

Keep bottle tightly closed.

OTHER PRECAUTIONS

SIGNATURE

DATE

$150-4003 \mathrm{~B}$ 


\section{PRODUCT DESIGNATION}

03-.154-6.035

Vanadium Pentoxide

\section{MATERIAL SAFETY DATA SHEET}

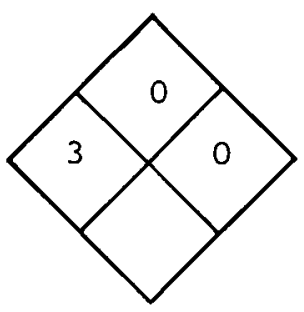

\section{SECTION I SOURCE AND NOMENCLATURE}

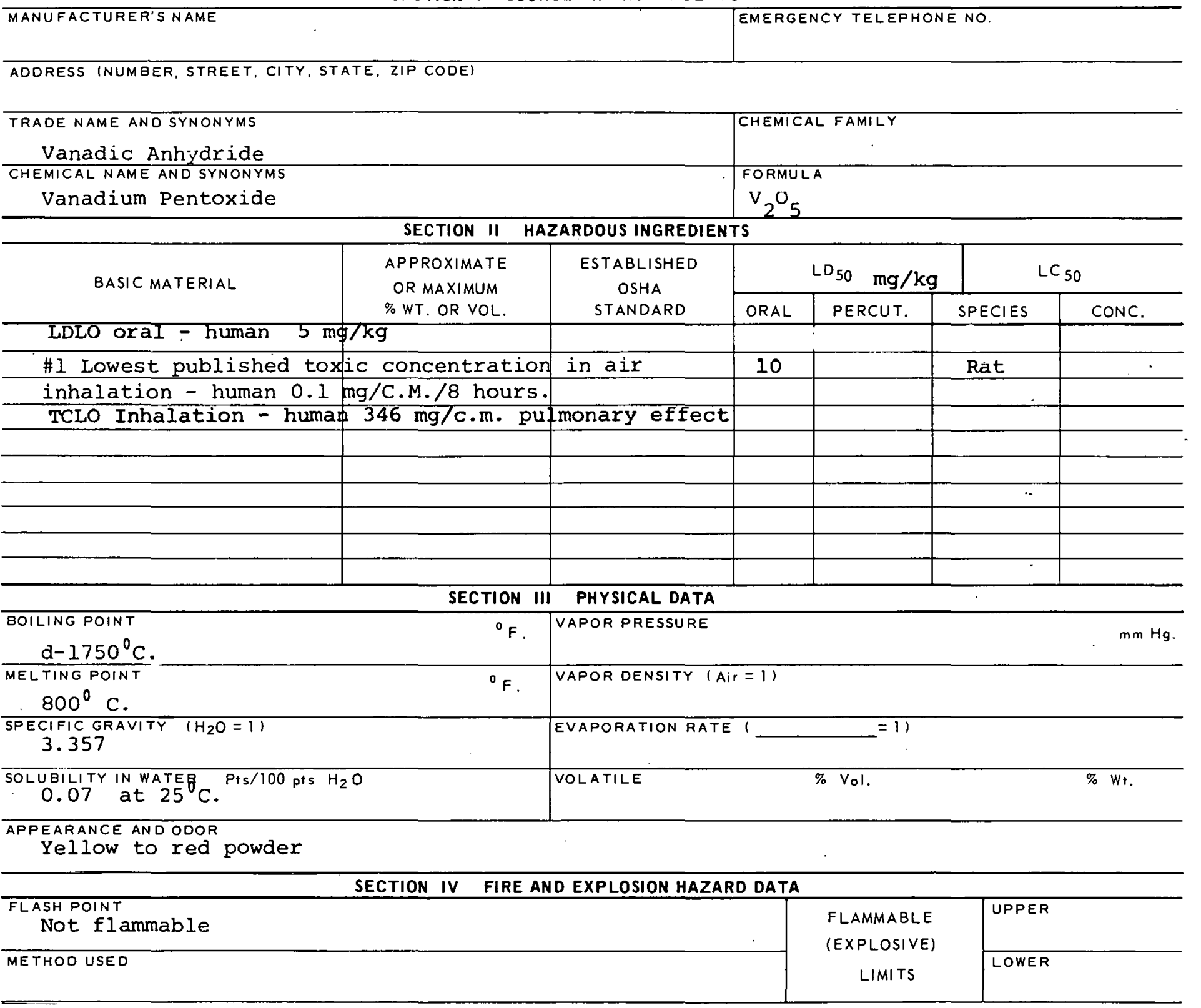

EXTINGUISHING MEDIA

Water

SPECIAL FIRE FIGHTING PROCEDURES

$\mathrm{N} / \mathrm{A}$

UNUSUAL FIRE AINO EXPLOSIOH IIAZAROS

$\mathrm{N} / \mathrm{A}$ 


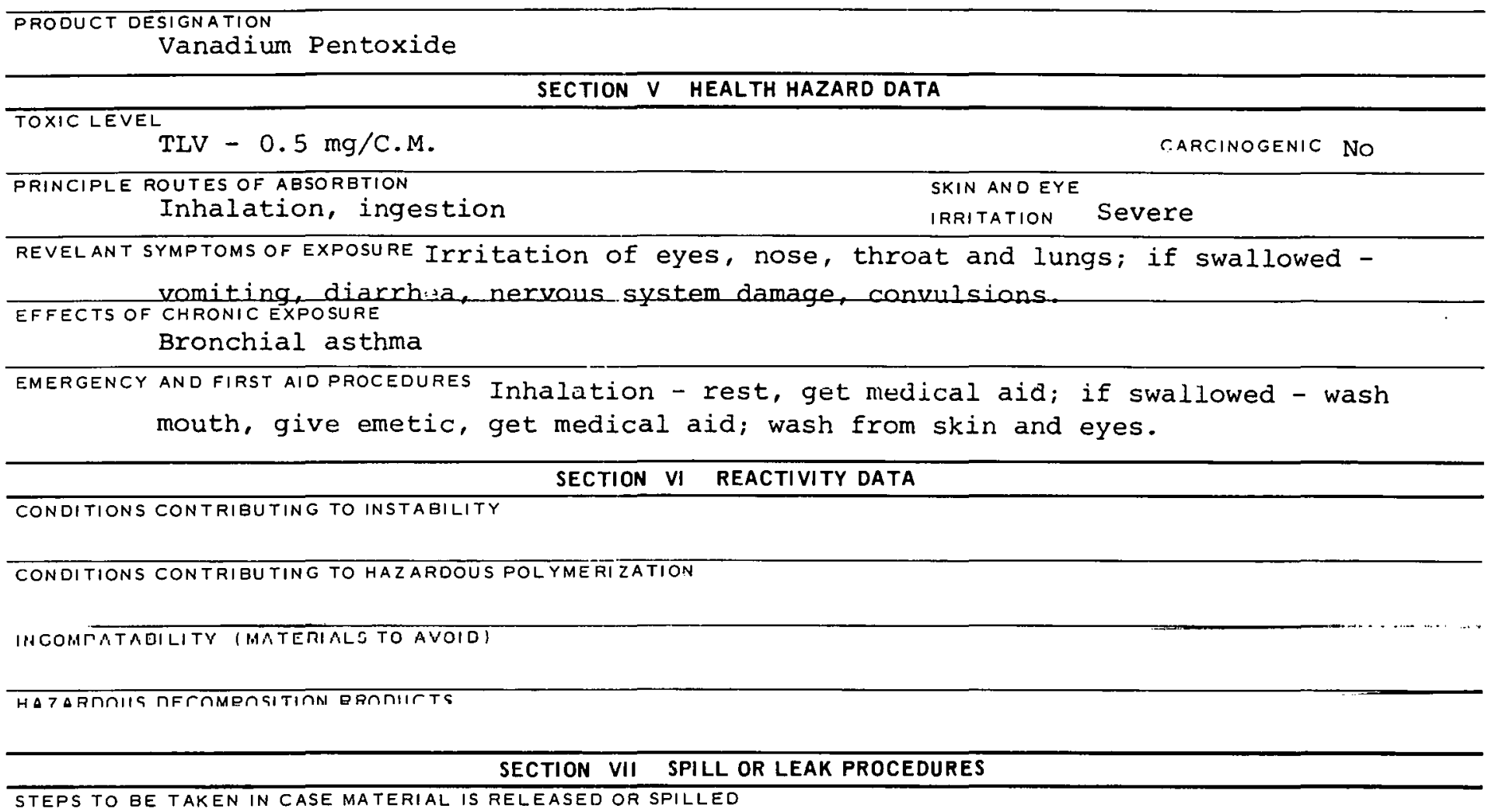

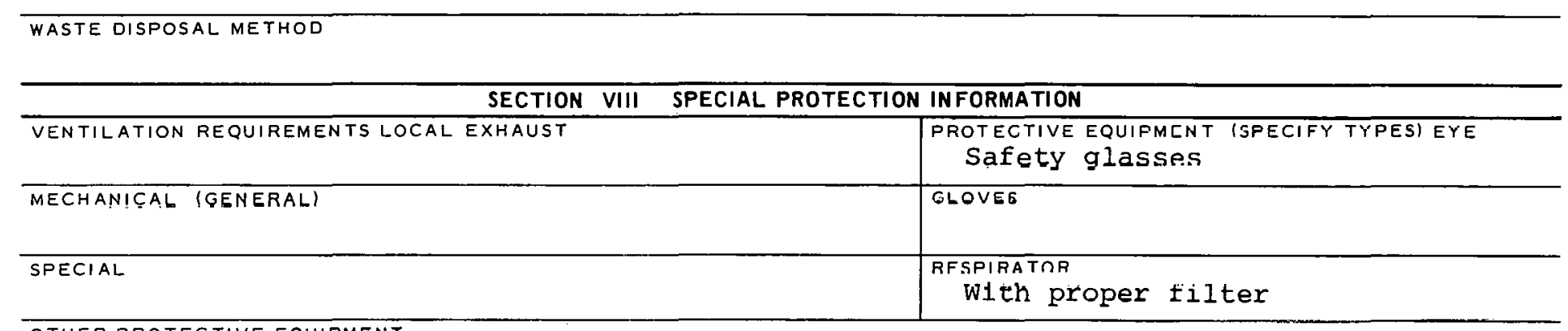

OTHER PROTECTIVE EQUIPMENT

\section{SECTION IX SPECIAL PRECAUTIONS}

PRECAUTIONS TO BE TAKEN IN HANOLING AND STORAGE

\begin{tabular}{l|l|l}
\hline OTHER PRECAUTIONS & RATF \\
\hline SIGNATURE & & \\
\hline
\end{tabular}


Yttrium Metal

03-159-5006
MATERIAL SAFETY

DATA SHEET

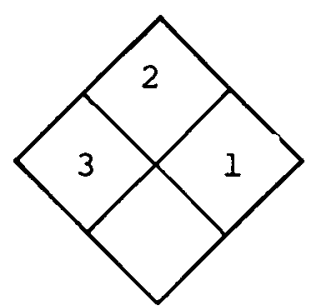

\section{SECTION I SOURCE AND NOMENCLATURE}

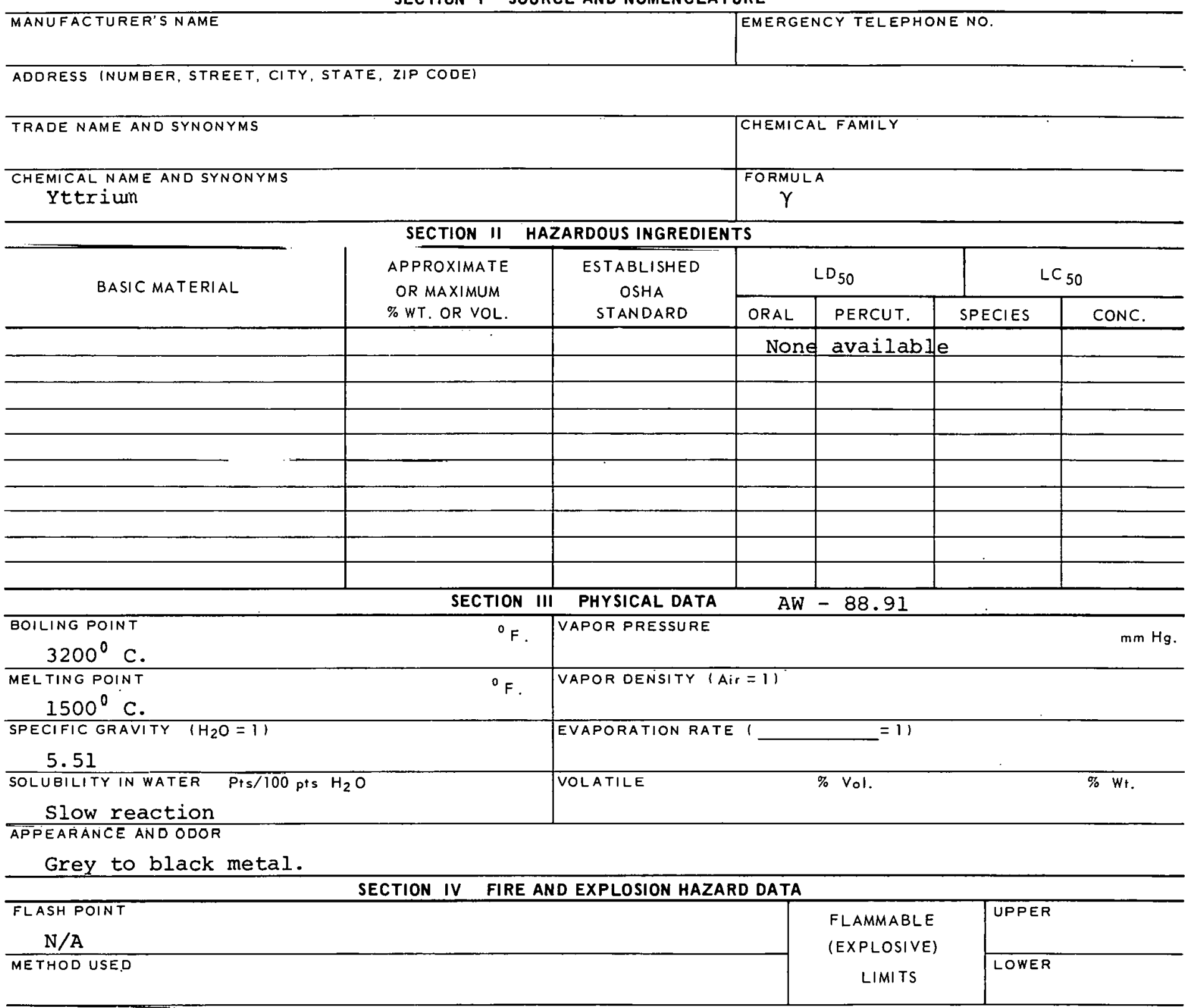

EXTINGUISHING MEDIA

Sand, dry powder.

SPECIAL FIRE FIGHTING PROCEDURES

Smother with suitable dry powder, no water.

UNUSUAL FIRE AND EXPLOSION HAZARDS

Moderate fire hazard. 
Yttrium Metal

\section{SECTION $V$ HEALTH HAZARD DATA}

TOXIC LEVEL

$\mathrm{TLV}-1 \mathrm{Mg} / \mathrm{C} . \mathrm{M}$.

GARCINOGENIC NO

PRINCIPLE ROUTES OF ABSORBTION

Inhalation, ingestion

SKIN AND EYE

IRRITATION MOderate

REVELANT SYMPTOMS OF EXPOSURE Irritation to respiratory system and eyes; kidney damage,

internal damage if swallowed.

EFFECTS OF CHRONIC EXPOSURE

--

EMERGENCY AND FIRST AID PROCEDURES If inhaled - rest, get medical aid; if swallowed - wash mouth, give emetic, get medical aid; wash eyes as needed.

SECTION VI REACTIVITY DATA

CONDITIONS CONTRIBUTING TO INSTABILITY

CONDITIONS CON TRIBU TING TO HAZARDOUS POLYMERI ZATION

INCOMPATABILITY IMATERIAI S Tn AIOIO!

Oxidizing materials

IIAZAROOUS DECOMPOSITION PRODUCTS

SECTION VII SPILL OR LEAK PROCEDURES

STEPS TO BE TAKEN IN CASE MATERIAL IS RELEASED OR SPILLED

WASTE DISPOSAL METHOD

SECTION VIII SPECIAL PROTECTION INFORMATION

\begin{tabular}{l|l}
\hline VENTILATION REQUIREMENTS LOCAL EXHAUST & $\begin{array}{l}\text { PROTECTIVE EQUIPMENT (SFECIFY TYPESI EYE } \\
\text { Eafety glaSSES }\end{array}$ \\
\hline MECHANICAL (GENERAL) & $\begin{array}{l}\text { GLOVES } \\
\text { Rubber }\end{array}$ \\
\hline SPECIAL & $\begin{array}{l}\text { RESPIRATOR } \\
\text { With proper filter }\end{array}$ \\
\hline
\end{tabular}

OTHER PROTECTIVE EQUIPMENT

SECTION IX SPECIAL PRECAUTIONS

PRECAUTIONS TO BE TAKEN IN HANOLING AND STORAGE

OTHER PRECAUTIONS

SIGNATURE

DATE

$159-5006 \mathrm{~B}$ 


PRODUCT DESIGNATION
03-159-8006
Yttrium Oxide

\section{MATERIAL SAFETY DATA SHEET}

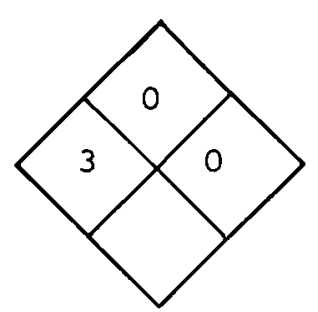

SECTION I SOURCE AND NOMENCLATURE

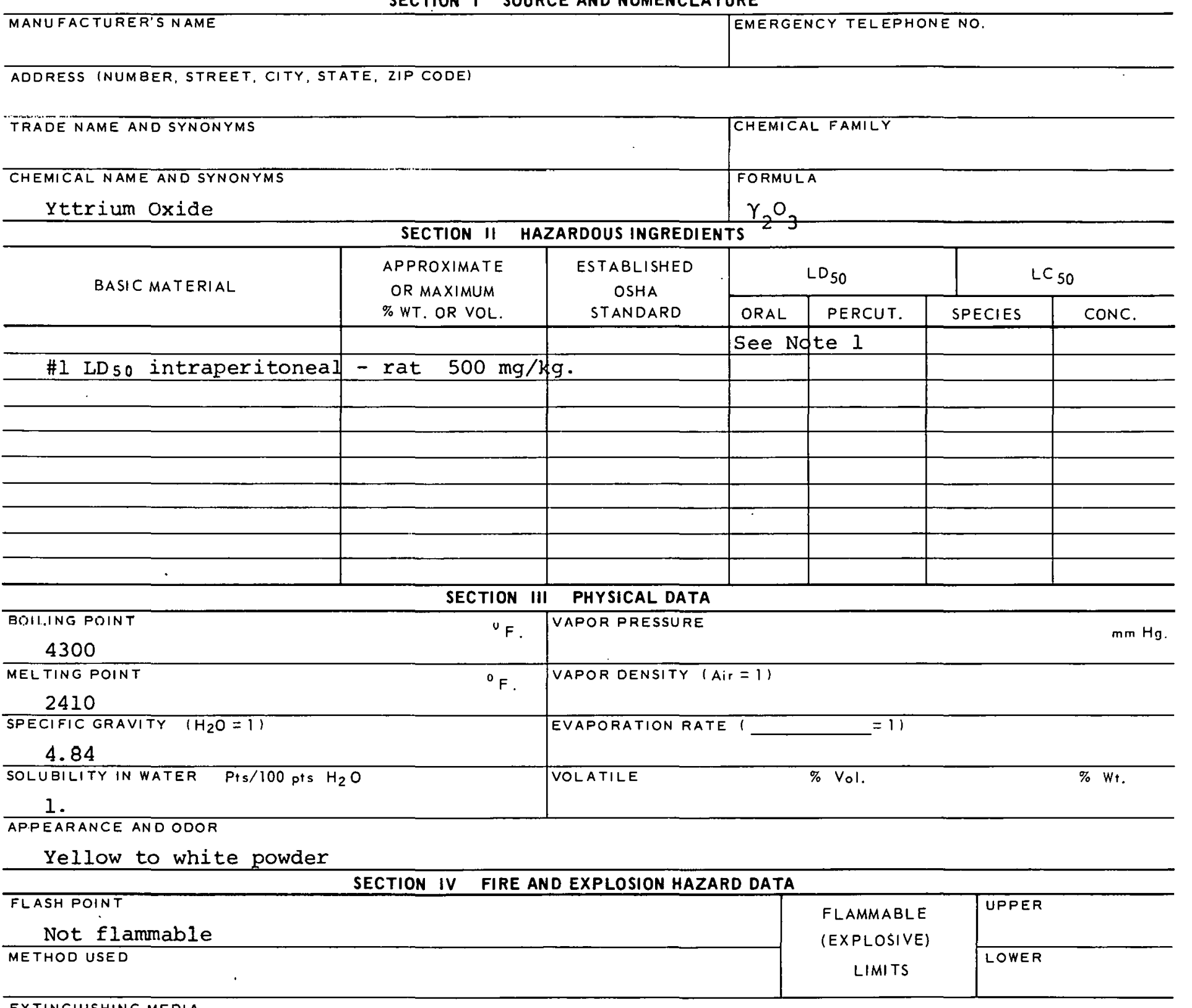

EXTINGUISHING MEDIA

Water

SPECIAL FIRE FIGHTING PROCEDURES

$N / A$

UNUSUAL FIRE ANDEXPLOSION HAZARDS

$N / A$ 
TOXIC LEVEL

$\mathrm{TLV}-1 \mathrm{Mg} / \mathrm{C} \cdot \mathrm{M}$. PRINCIPLE ROUTES OF ABSORBTIUN

Inhalation, ingestion

CARCINOGENIC SKIN AND EYE IRRITATION Moderate

REVELANT SYMPTOMS OF EXPOSURE Irritation of eyes and respiratory tract; kidney damage; internal damage if swallowed. EFFECTS OF CHRONIC EXPOSURE $--$

EMERGENCY AND FIRST AID PROCEDURES If inhaled - rest; wash from skin and eyes; if swallowed wash mouth, give emetic, get medical aid.

\section{SECTION VI REACTIVITY DATA}

CONDITIONS CONTRIBUTING TO INSTABILITY

CONDITIONS CONTRIBUTING TO HAZARDOUS POLYMERIZATION

INCOMPATABILITY (MATERIALS TO AVOID)

HAZARDOUS DECOMPOSITION PRODUCTS

SECTION VII SPILL OR LEAK PROCEDURES

STEPS TO BE TAKEN IN CASE MATERIAL IS RELEASED OR SPILLED

.

WASTE DISPOSAL METHOD

SECTION VIII SPECIAL PROTECTION INFORMATION

\begin{tabular}{|c|c|}
\hline VENTILATION REQUIREMENTS LOCAL EXHAUST & $\begin{array}{l}\text { PROTECTIVE EQUIPMENT (SPECIFY TYPES) EYE } \\
\text { Safety glasses }\end{array}$ \\
\hline MECHANICAL (GENERAL) & $\begin{array}{l}\text { GLOVES } \\
\text { Rubher }\end{array}$ \\
\hline SPECIAL & RESPIRATOR \\
\hline
\end{tabular}

DTHER PROTECTIVE EQUIPMENT

SECTION IX SPECIAL PRECAUTIONS

PRECAUTIONS TO BE TAKEN IN HANDLING AND STORAGE

OTHER PRECAUTIONS

SIGNATURE

DATE

$159-8006 \mathrm{~B}$ 
CAS : 007646857

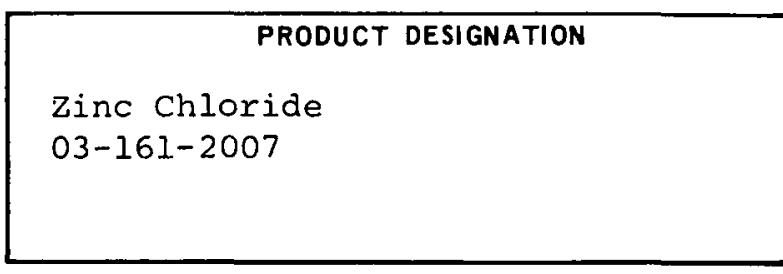

\section{SECTION 1 SOURCE AND NOMENCLATURE}

\section{MANUFACTURER'S NAME}

ADORESS (NUMBER, STREET, CITY, STATE, ZIP CODE)

Cubic, white, deliquescent crystals.

SECTION IV FIRE AND EXPLOSION HAZARD DATA

\section{FLASH NOINT \\ not flammable}

METHOO USED

EXTINGUISHING MEDIA

Water
MATERIAL SAFETY

DATA SHEET

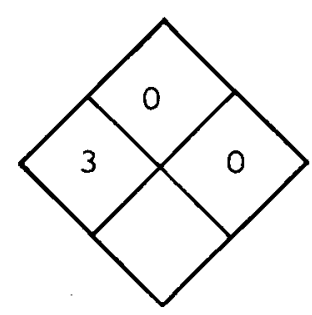

EMERGENCY TELEPHONE NO.

\section{SECTION II HAZARDUUS INGKEUIEN IS} APPROXIMATE

OR MAXIMUM OSHA

\% WT. OR VOL. STANDARD

\begin{tabular}{|l|l|l|l}
\hline \multicolumn{3}{|c|}{ LD $_{50} \mathrm{mg} / \mathrm{kg}$} & \multicolumn{2}{c}{ LC $_{50}$} \\
\hline ORAL & PERCUT. & SPECIES & CONC. \\
\hline & & & \\
\hline 350 & & rat & \\
\hline 350 & & mouse & \\
\hline & & & \\
\hline & & & \\
\hline & & & \\
\hline & & & \\
\hline & & & \\
\hline & & & $1^{\mathrm{mm} \mathrm{Hg} .}$ \\
\hline & & & \\
\hline
\end{tabular}

VAPOR PRESSURE

at $428^{\circ} \mathrm{C}$.

CHEMICAL FAMILY

Lowest published toxic doncentration in air

inhalation - man

$4800 \mathrm{mg} / \mathrm{C.M.}-30$ months

est publsihed lethal dose

LTINGS POIN

${ }^{\circ} \mathrm{F}$.

EVAPORATION RATE

VOLATILE $\%$ Vol.

SPECIAL FIRE FIGHTING PROCEDURES

UNUSUAL FIRE AND EXPLOSION HAZARDS

FLAMMABLE

(EXPLOSIVE)

LIMITS
UPPER

LOWER 


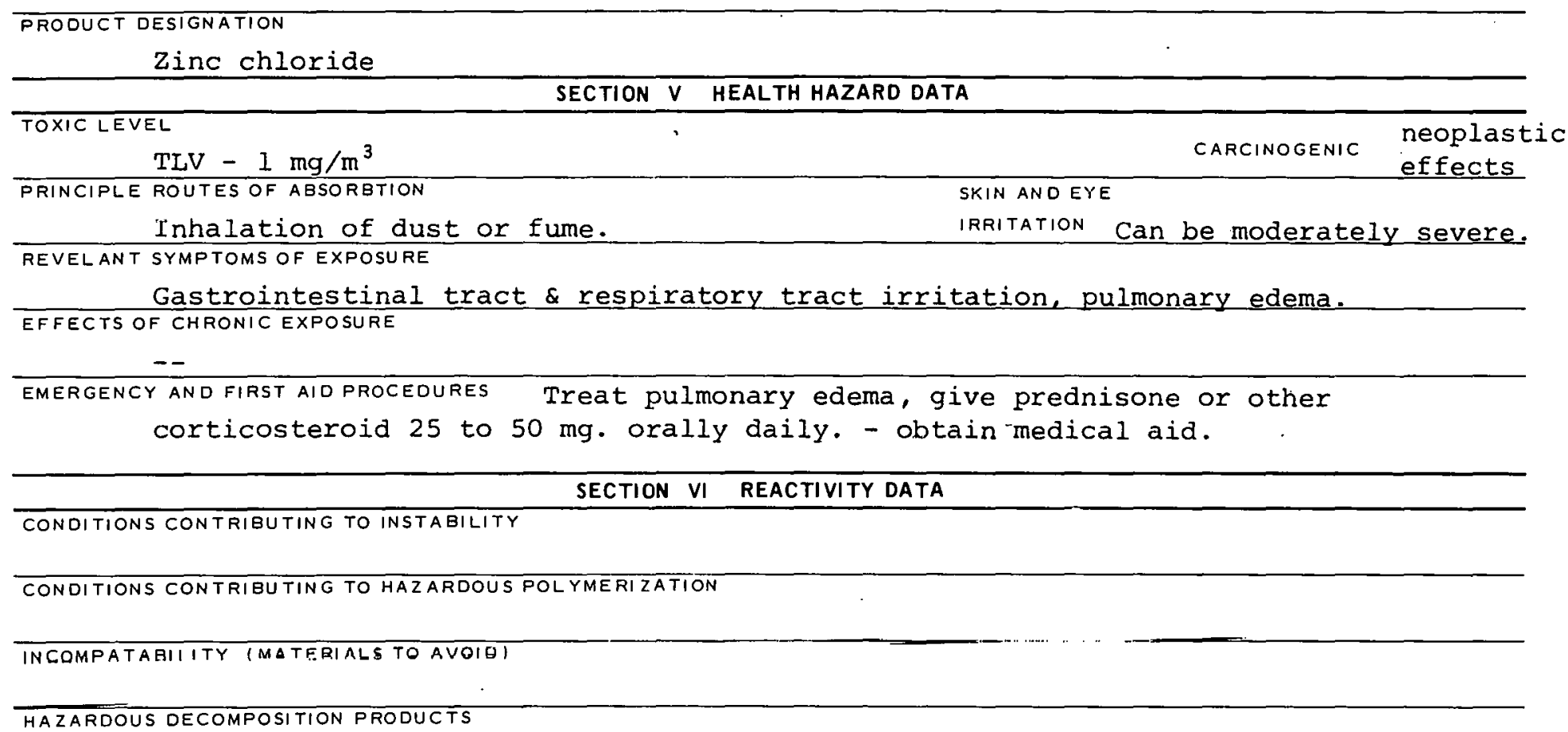

\section{SECTION VII SPILL OR LEAK PROCEDURES}

STEPS TO BE TAKEN IN CASE MATERIAL IS RELEASED OR SPILLED

\section{WASTE DISPOSAL METHOD}

\begin{tabular}{l|l}
\hline \multicolumn{1}{c}{ SECTION VIII. SPECIAL PROTECTION INFORMATION } \\
\hline VENTILATION REQUIREMENTS LOCAL EXHAUST & $\begin{array}{l}\text { PROTECTIVE EQUIPMENT } \\
\text { GlaSSEG }\end{array}$ \\
\hline MECHANICAL IGENERAL) & $\begin{array}{l}\text { GLOVES } \\
\text { Rubber }\end{array}$ \\
\hline SPECIAL & $\begin{array}{l}\text { RESPIRATOR } \\
\text { Dust mask. }\end{array}$ \\
\hline OTHER PROTECTIVEEQUIPMENT &
\end{tabular}

SECTION IX SPECIAL PRECAUTIONS

\section{PRECAUTIONS TO BE TAKEN IN HANDLING AND STORAGE
Store in cool, well-ventilated place.}

OTHER PRECALITIONS

SUIVA I UHE

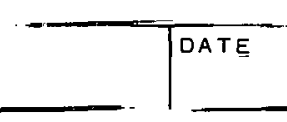

$161-2007 \mathrm{~B}$ 
CAS : 007664417

PRODUCT DESIGNATION

Ammonia (anhydrous)

03-910-4030

03-910-4080

03-910-4150

\section{MATERIAL SAFETY \\ DATA SHEET}

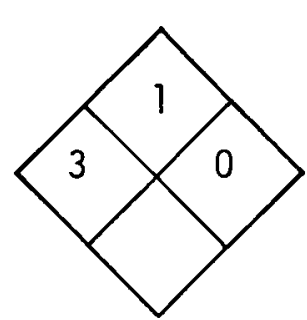

SECTION I SOURCE AND NOMENCLATURE

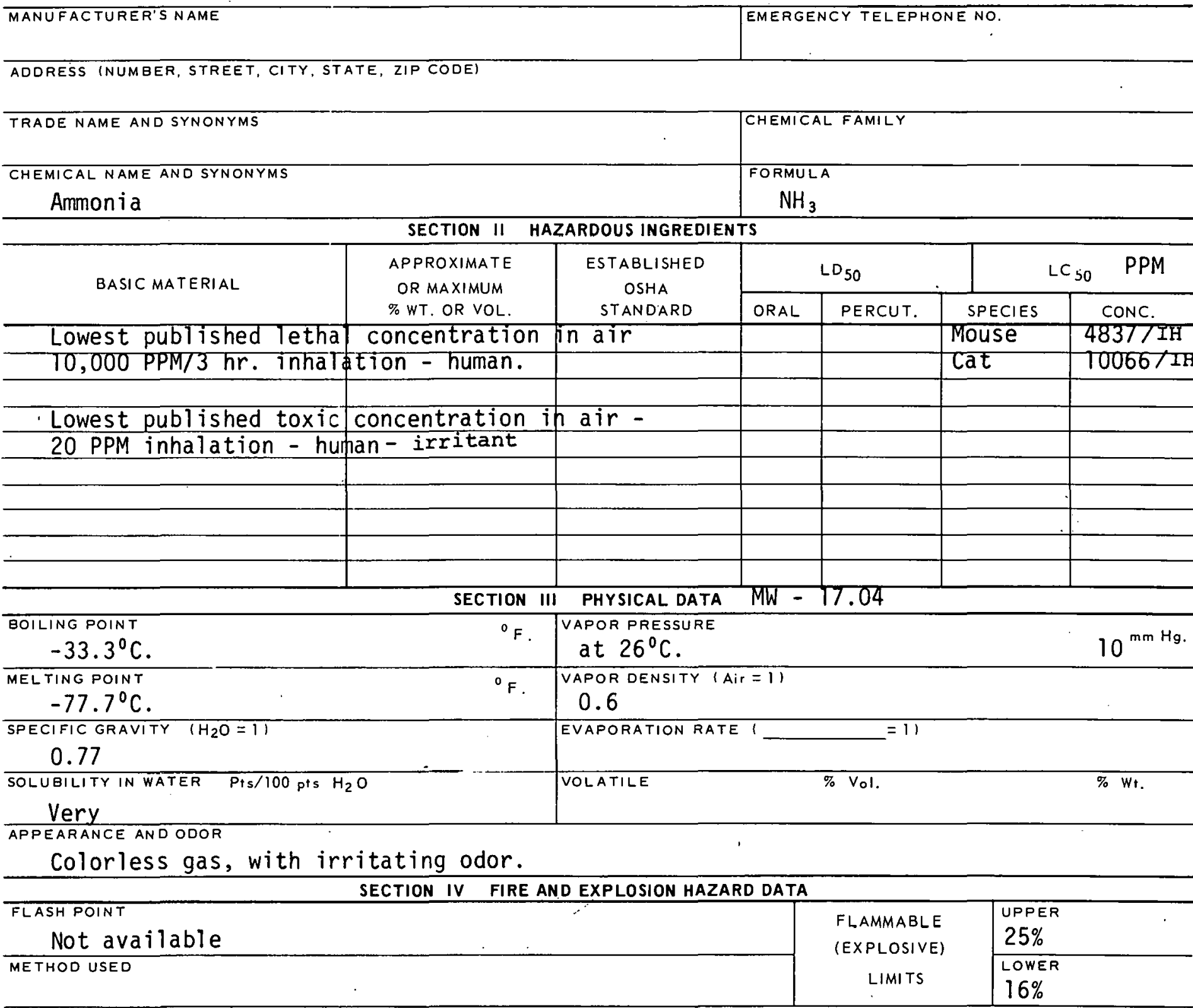

EXTINGUISHING MEDIA

Carbon dioxide, dry chemical.

SPECIAL FIRE FIGHTING PROCEDURES

Stop flow of gas, cool tank with water.

UNUSUAL FIRE AND EXPIOSION HAZAROS 


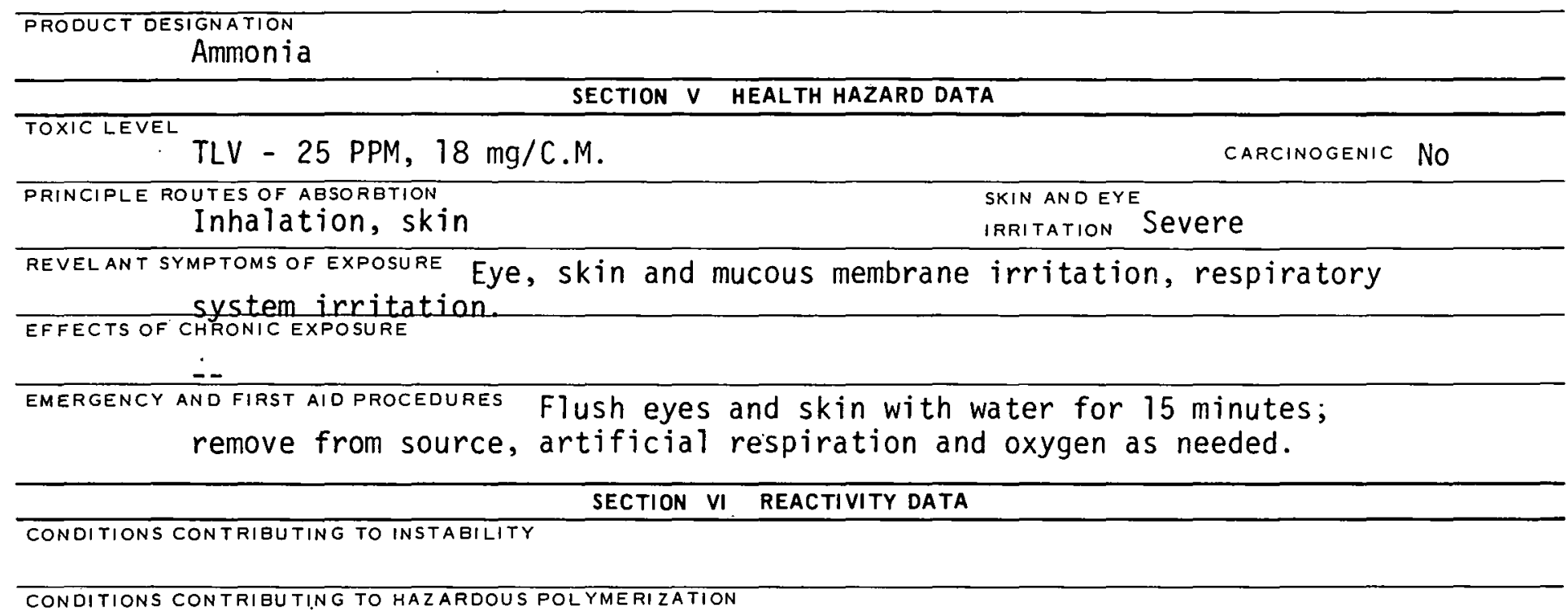
INCOMPATABILITY (MATERIALS TO AVOIO) ACETdTdehyde, acroteth, bromthe, chiorlne, chromic acid, ethylene dichloride, fluorine, iodine. HAZARDOUS DECOMPOSITION PRODUCTS

SECTION VII SPILL OR LEAK PROCEDURES

STEPS TO BE TAKEN IN CASE MATERIAL IS RELEASED OR SPILLED

\section{WASTE DISPOSAL METHOD}

\section{SECTION VIII SPECIAL PROTECTION INFORMATION}

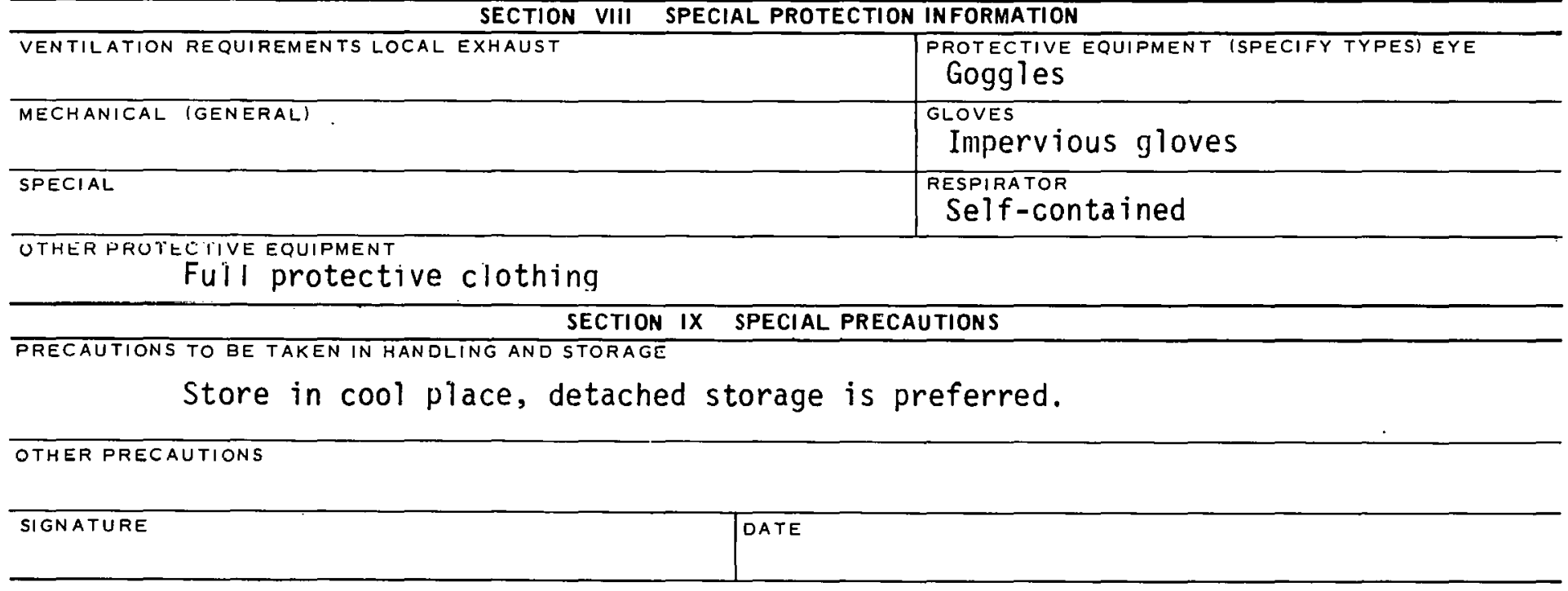

$910-4030 B$ 
CAS - 000630080

FG-35000

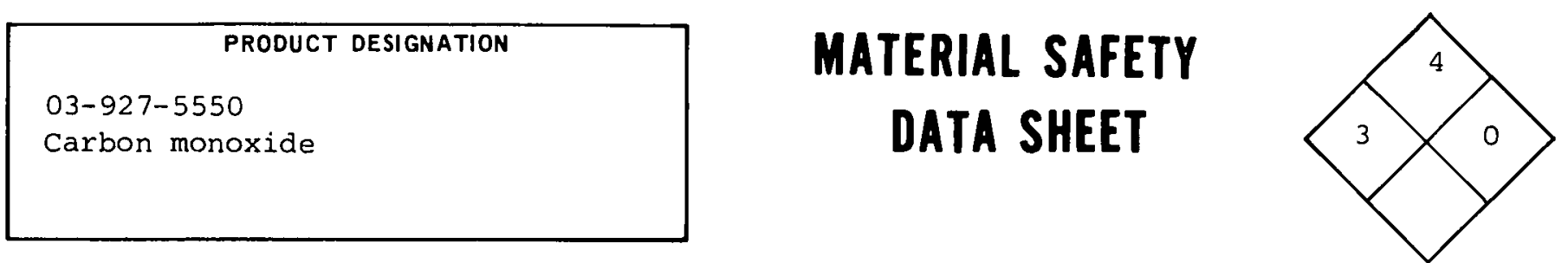

\section{SECTION I SOURCE AND NOMENCLATURE}

\begin{tabular}{l|l|l}
\hline SECTION I SOURCE AND NOMENCLATURE \\
\hline MANUFACTURER'S NAME & EMERGENCY TELEPHONE NO. \\
\hline
\end{tabular}

ADDRESS (NUMBER, STREET, CITY, STATE, ZIP CODE)

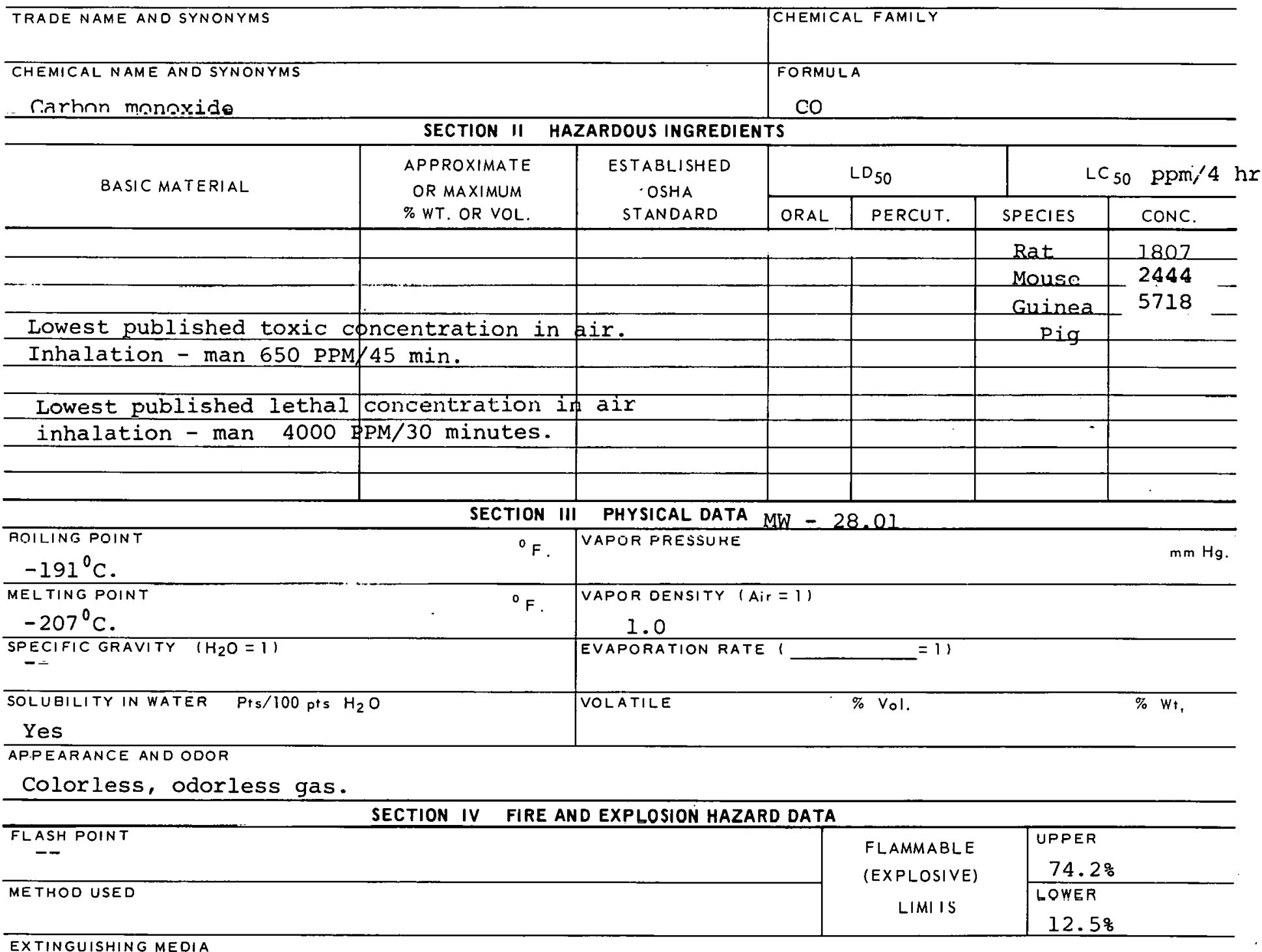

Carbon dioxide, dry chemical, water spray. SPECIAL FIRE FIGHTING PROCEDURES 


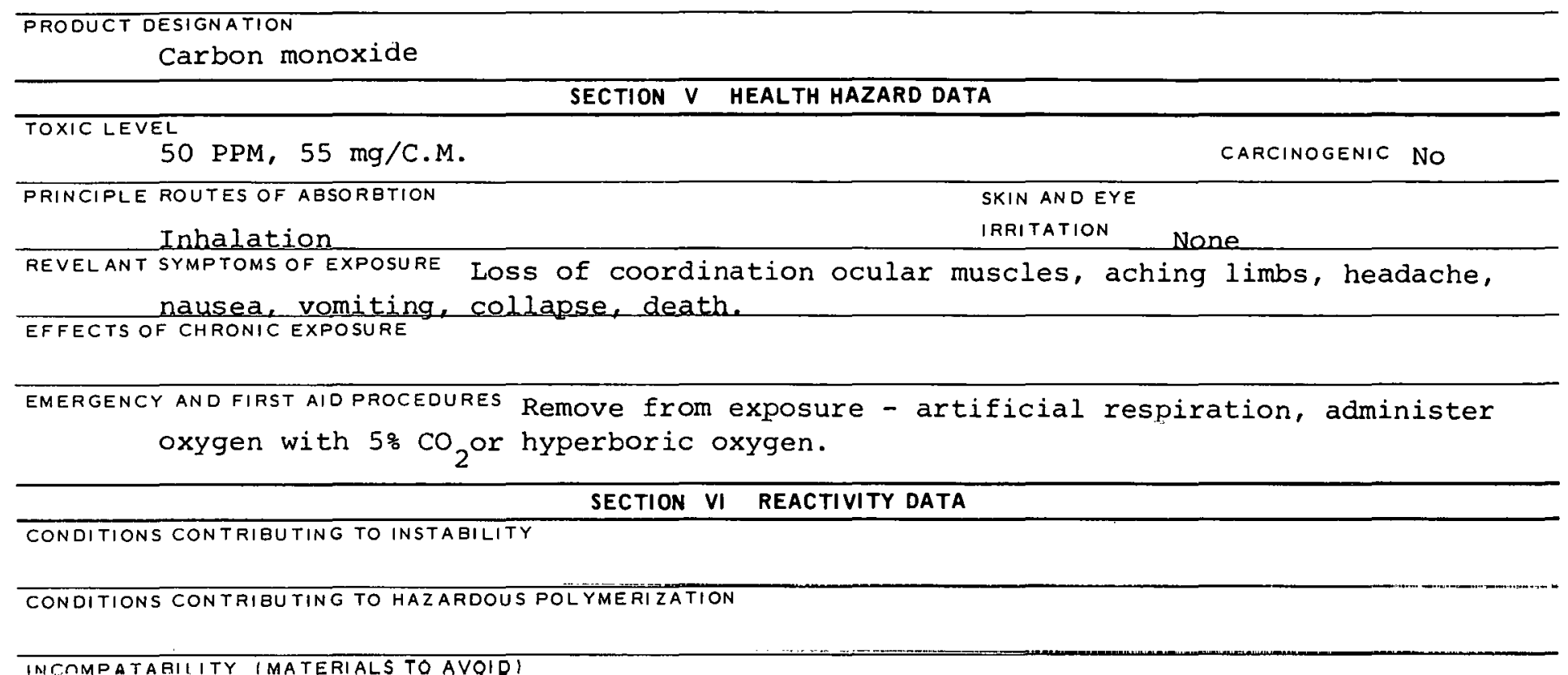

\begin{tabular}{l|l}
\hline \multicolumn{1}{c}{ SECTION VIII SPECIAL PROTECTION INFORMATION } \\
\hline VENTILATION REQUIREMENTS LOCAL EXHAUST & PROTECTIVE EQUIPMENT (SPECIFY TYPES) EYE \\
\hline MECHANICAL IGENERAL) & GLOVES \\
\hline SFECIAL & $\begin{array}{c}\text { RESTIRATOR } \\
\text { Self-COntained }\end{array}$ \\
\hline
\end{tabular}

OTHER PROTECTIVE EQUIPMENT

SECTION IX SPECIAL PRECAUTIONS

PRECAUTIONS TO BE TAKEN IN HANDLING AND STORAGE

OTHER PRECAUTIONS 
03-934-4000

PRODUCT DESIGNATION

03-934-4040

03-934-4080

Chlorine, liquid, compressed
MATERIAL SAFETY DATA SHEET

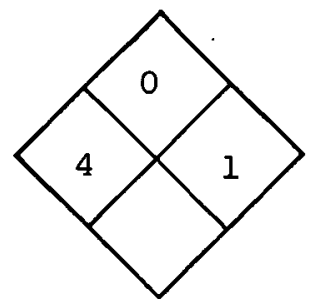

SECTION I SOURCE AND NOMENCLATURE

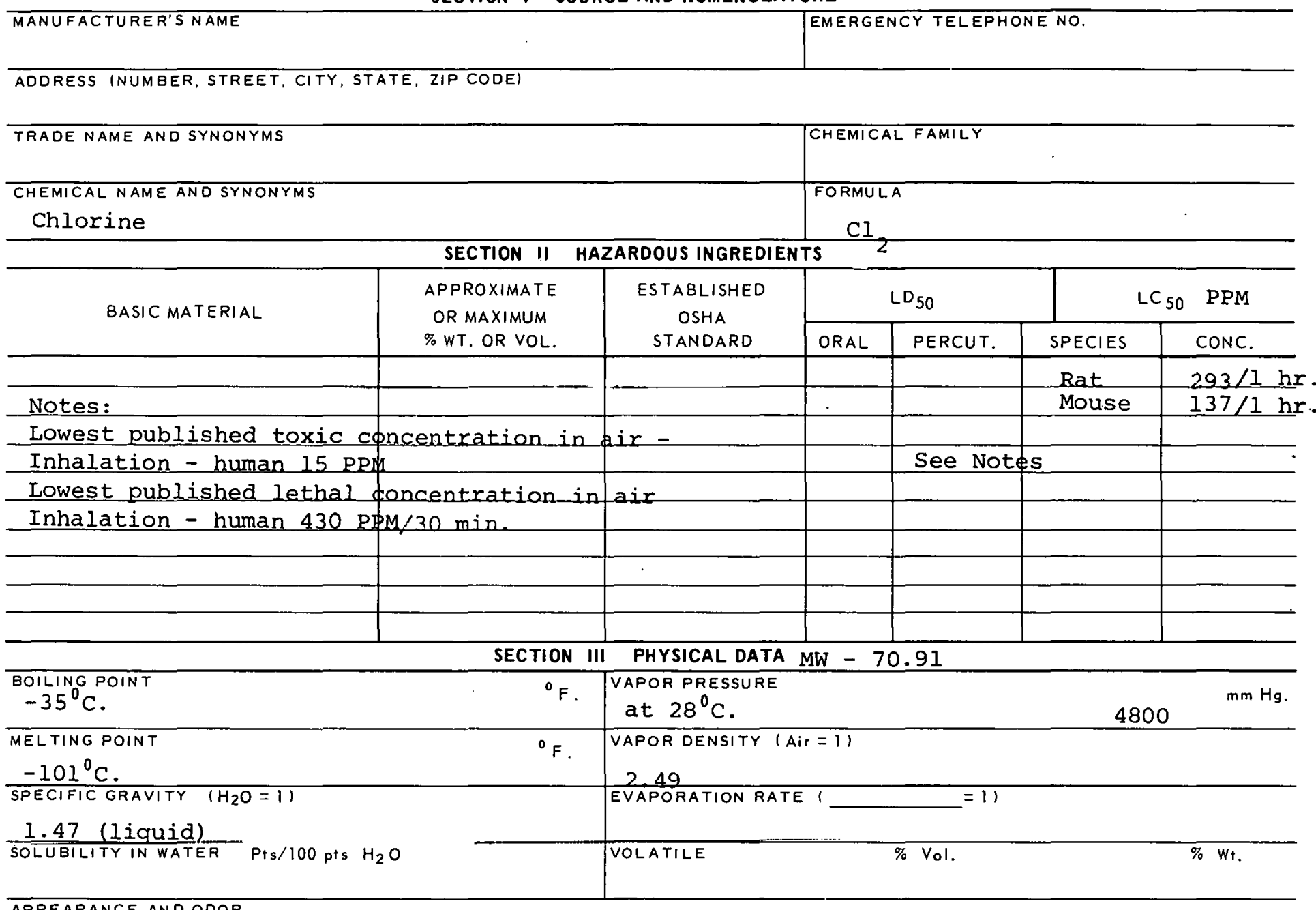

APPEARANCE ANO ODOR

Pungent, irritating, greenish-vellow gas (inhalation threshold 0.2 ppm) SECTION IV FIRE AND EXPLOSION HAZARD DATA

FLASH POIN T

Not comubustible (supports combustion)

METHOO USED

FLAMMABLE

(EXPLOSIVE)

LIMITS

EXTINGUISHING MEDIA Apply water to keep surrounding or tanks cool. Do not apply directly to leaks.

SPECIAL FIRE FIGHTING PROCEDURES

Wear full protective clothing.

UNUSUAL FIRE AND EXPLUSION HAZARDS 


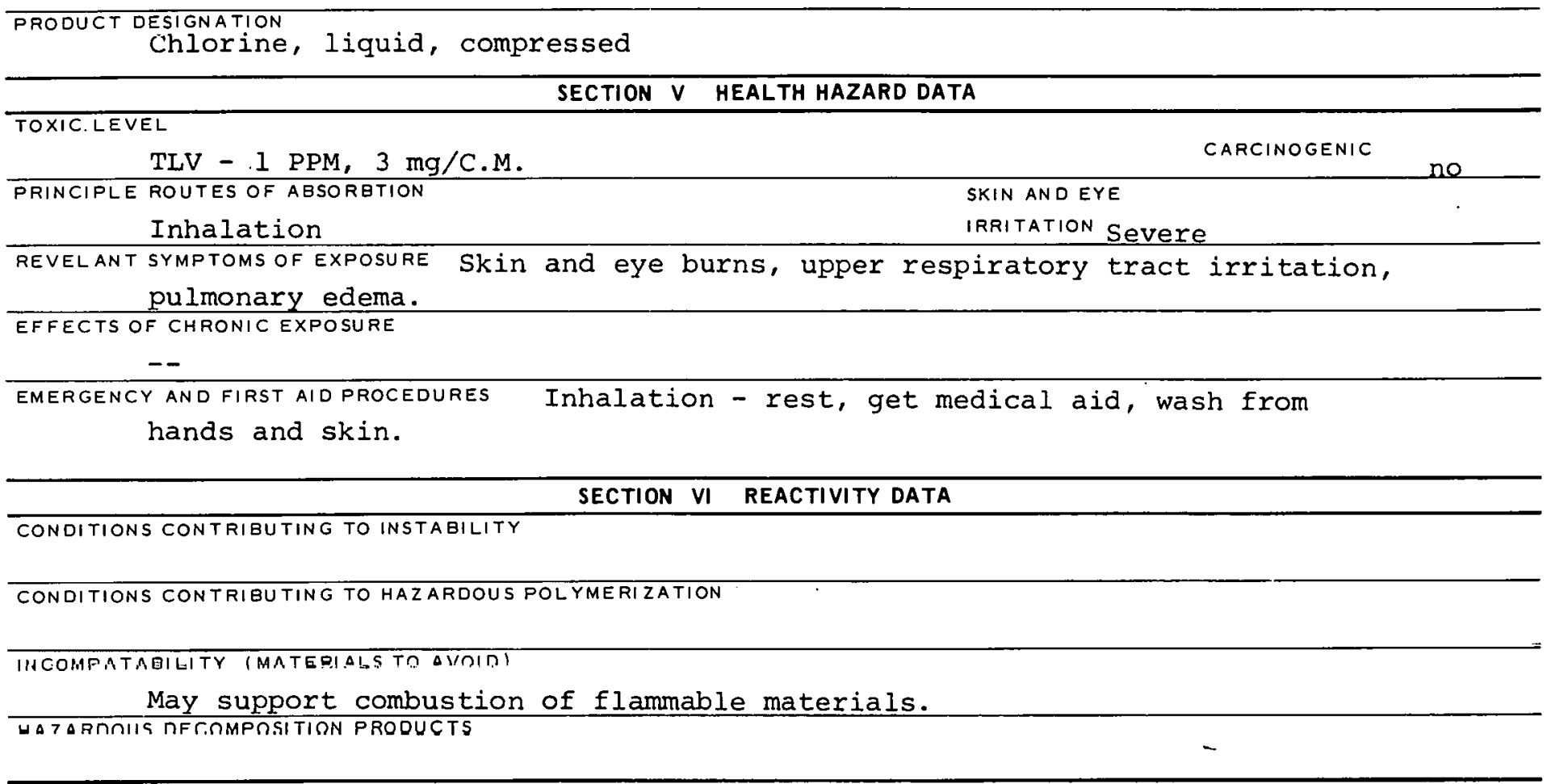

\section{SECTION VII SPILL OR LEAK PROCEDURES}

STEPS TO BE TAKEN IN CASE MATERIAL IS RELEASED OR SPILLED

$-$

WASTE DISPOSAL METHOD

\begin{tabular}{l|l}
\hline \multicolumn{1}{c}{ SECTION VIII SPECIAL PROTECTION INFORMATION } \\
\hline VENTILATION REQUIREMENTS LOCAL EXHAUST & $\begin{array}{l}\text { PROTECTIVE EQUIPMENT ISPECIFY TYPESI EYE } \\
\text { GOggleS }\end{array}$ \\
\hline MECHANICAL IGENERAL) & $\begin{array}{l}\text { GLOVES } \\
\text { Impervious }\end{array}$ \\
\hline SPECIAL & RESFIRATOR \\
& Self-contained \\
\hline
\end{tabular}

OTHER PROTECTIVE EQIIIPMFNT

Far less than 25 ppm - chemical cartridge respirators SECTION IX SPECIAL PRECAUTIONS

PRECAUTIONS TO BE TAKEN IN HANDLING AND STORAGE

Store in well-ventilated room shielded from direct rays of sun. OTHER PRECAUTIONS 


\section{PRODUCT DESIGNATION}

Hydrogen Sulfide

03-950-9405

$03-950-9425$

$03-950-9445$
MATERIAL SAFETY

DATA SHEET

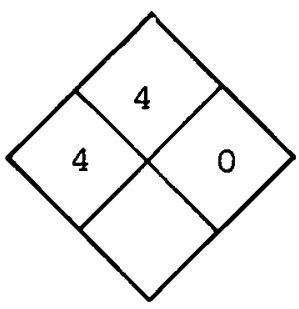

\section{SECTION I SOURCE AND NOMENCLATURE}

\section{MANUFACTURER'S NAME \\ TRADE NAME AND SYNONYMS \\ CHEMICAL NAME ANO SYNONYMS \\ Hydrogen Sulfide}

ADDRESS (NUMBER, STREET, CITY, STATE, ZIP CODE)

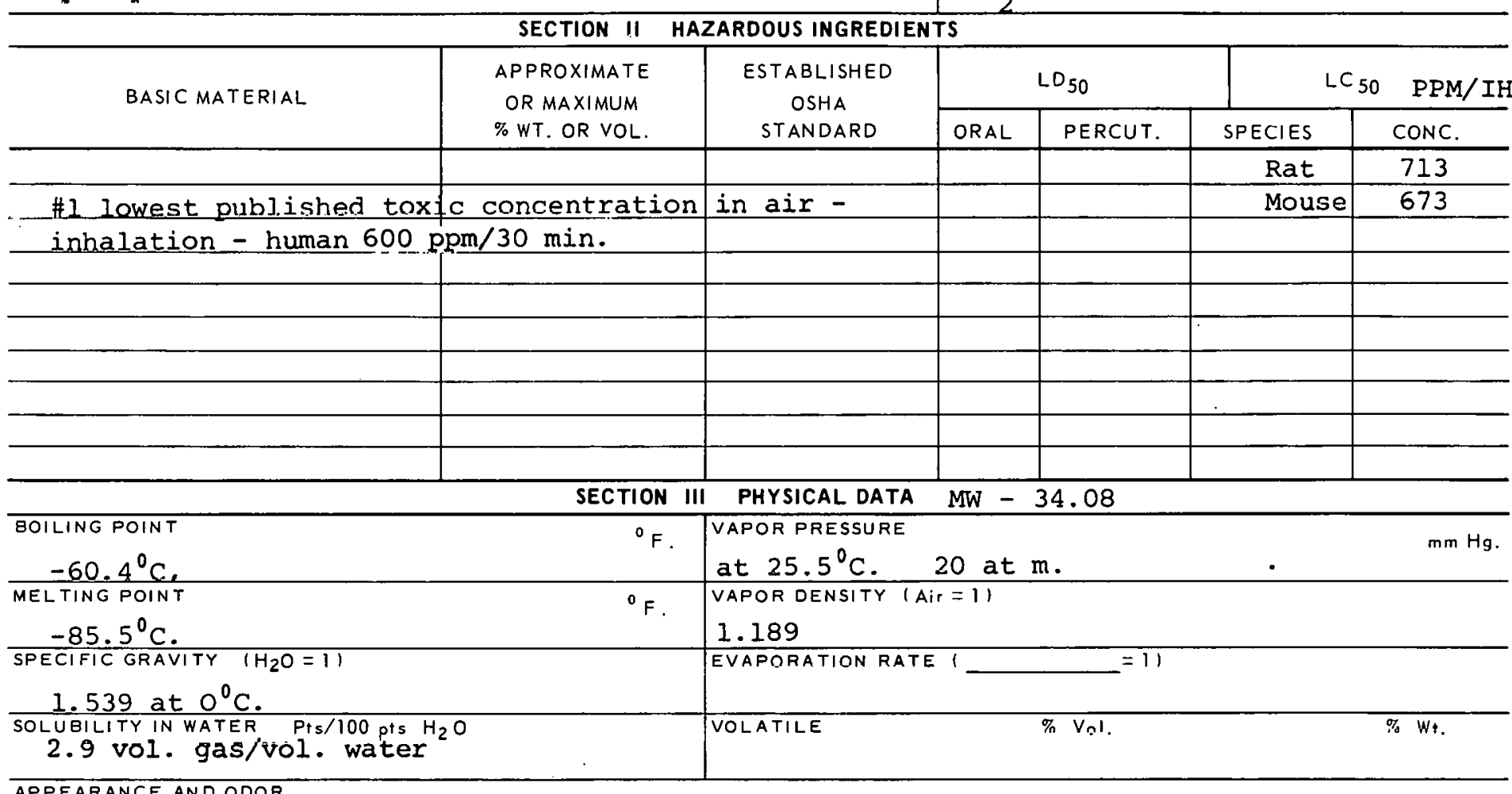

Colorless; flammable gas, offensive odor. (odor threshold 0.02 ppm) SECTION IV FIRE AND EXPLOSION HAZARD DATA क bY VOI.

FLASH POINT

None available METHOD USEO

EXTINGUISHING MEDIA

Stop flow of gas. Dry chemical, carbon dioxide, water spray.

\section{FLAMMABLE}

(EXPLOSIVE)

LIMITS

\begin{tabular}{l} 
UPPER \\
45 \\
\hline LOWCR \\
4.3
\end{tabular}

SPECIAL FIRE FIGHTING PROCEDURES

Wear goggles and self-contained breathing apparatus.

UNIIGIIAL FIRE AND EXPLOSION HAZARDS

Forms explosive mixtures with air. 


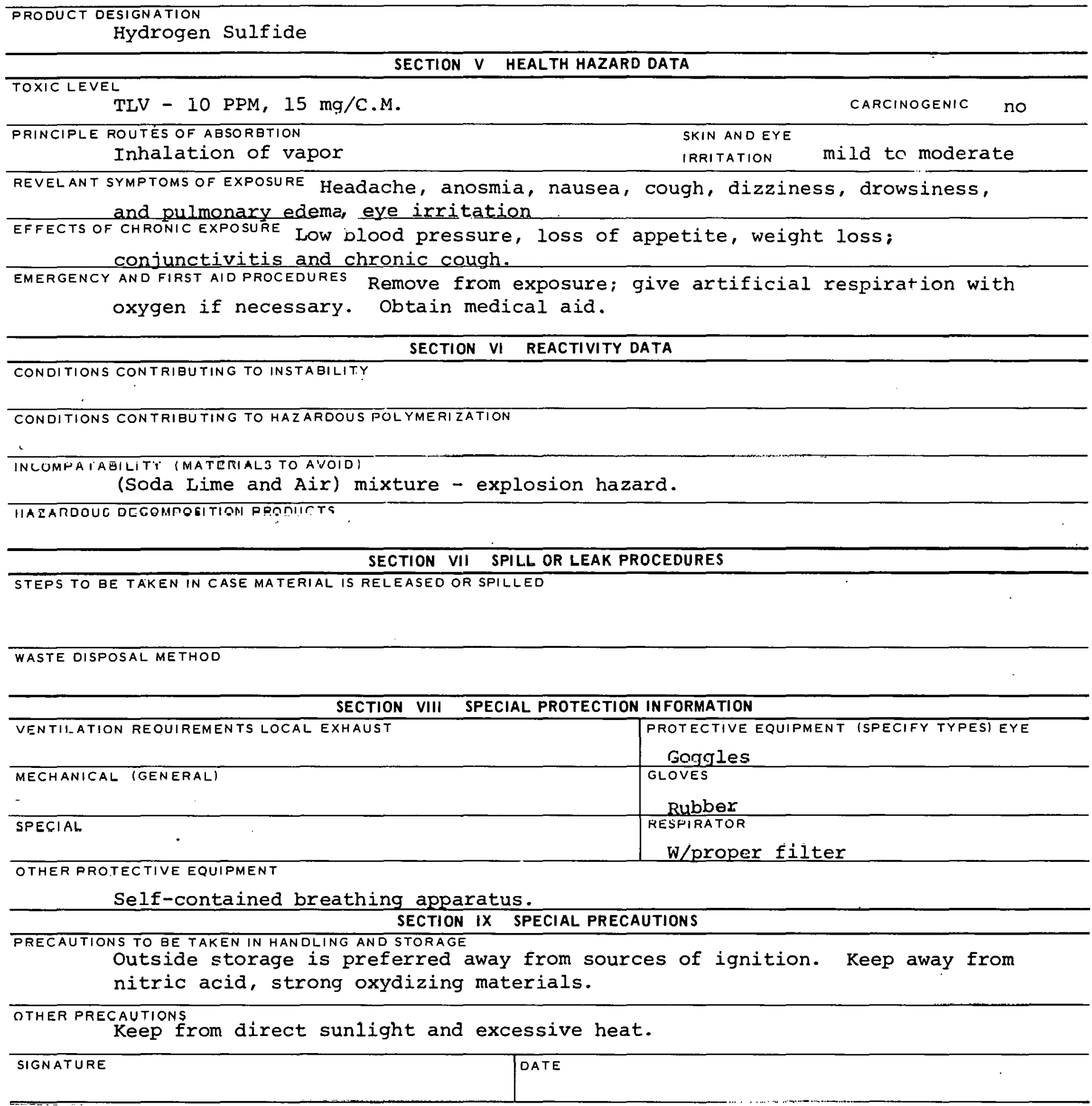




\begin{tabular}{|l|}
\hline \multicolumn{1}{c|}{ PRODUCT DESIGNATION } \\
$03-988-2020$ \\
$03-988-2130$ \\
Sulfur Dioxide \\
\end{tabular}

\section{MATERIAL SAFETY \\ DATA SHEET}

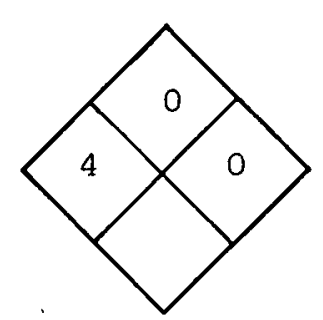

\section{SECTION I SOURCE AND NOMENCLATURE}

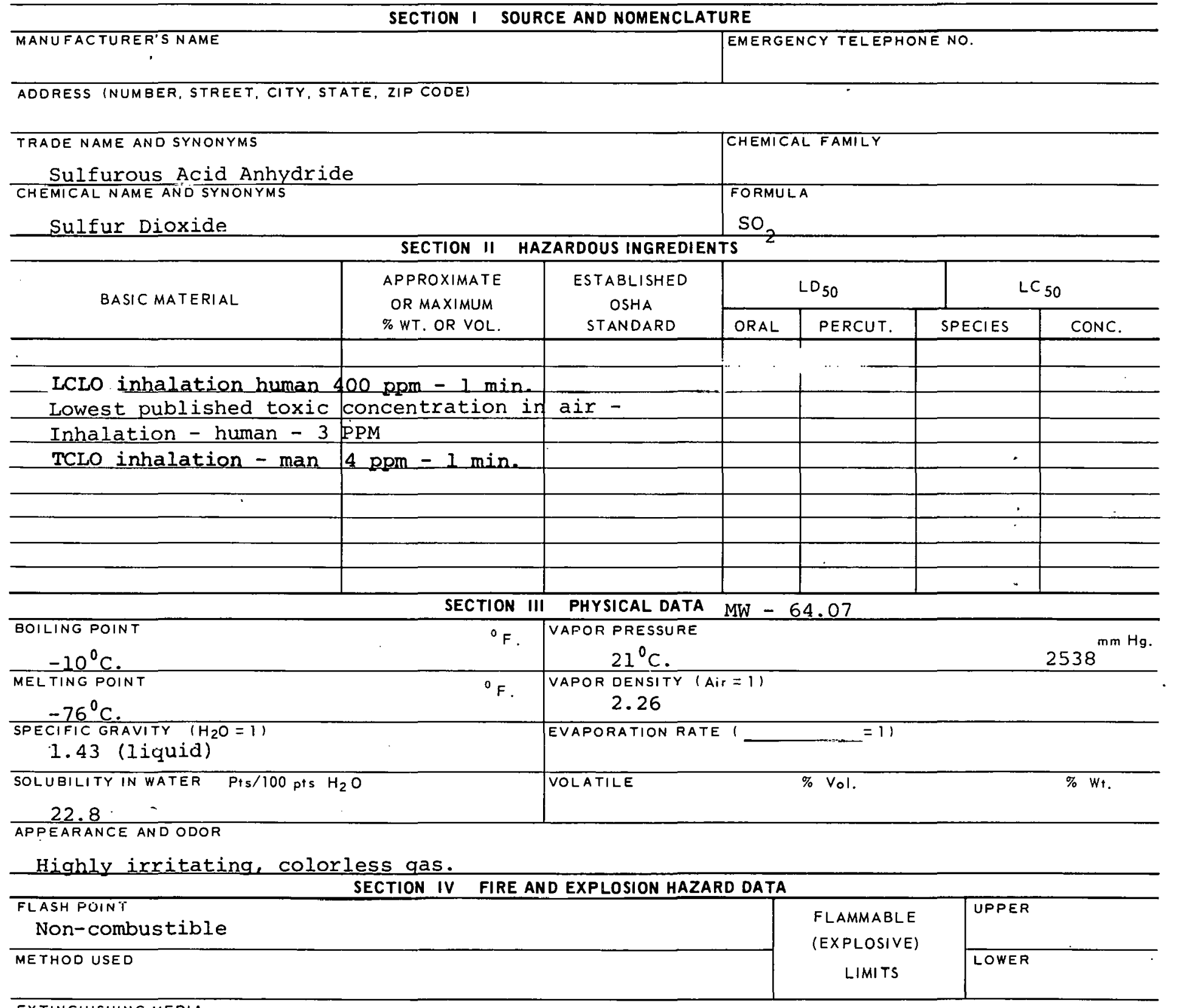

EXTINGUISHING MEDIA

SPECIAL FIRE FIGHTING PROCEOURES

UNUSUAL FIRE AND EXPLOSION HAZARDS 
Sulfur Dioxide

SECTION $V$ HEALTH HAZARD DATA

TOXIC LEVEL

$\mathrm{TLV}-5 \mathrm{PPM}, 13 \mathrm{mg} / \mathrm{C} . \mathrm{M}$.

CARCINOGENIC

SKIN ANO EYE

IRRITATION moderate - severe

Inhalation

Eye irritation, nose and throat irritation, bronchitis, REVELANT SYMPTOMS OF EXPOSURE asphyxia.

EFFECTS OF CHRONIC EXPOSURE $--$

EMERGENCY AND FIRST AIOPROCEDURES Inhalation - rest, get medical aid, wash from eyes.

\section{SECTION VI REACTIVITY DATA}

CONDITIONS CONTRIBUTING TO INSTABILITY

CONDITIONS CONTRIBUTING TO HAZARDOUS POLYMERI ZATION

IMCOMPATARII.ITY (MATERIALS TO AVOIO)

HAZARDOUS DECOMPOSITION PROOUCTS

SECTION VII SPILL OR LEAK PROCEDURES

STEPS TO BE TAKEN IN CASE MATERIAL IS RELEASEO OR SPILLED

WASTE DISPOSAL METHOD

SECTION VIII SPECIAL PROTECTION INFORMATION

\begin{tabular}{|c|c|}
\hline VENTILATION REQUIREMENTS LOCAL EXHAUST & $\begin{array}{l}\text { PROTECTIVE EQUIPMENT (SPECIFY TYPES) EYE } \\
\text { GOggleS }\end{array}$ \\
\hline MECHANICAL (GENERAL) & GLOVES \\
\hline SHECIAL & $\begin{array}{l}\text { RESPIRATAR } \\
\text { Self́-contalned }\end{array}$ \\
\hline
\end{tabular}

OTHER PROTECTIVE ERUIPMFNT

SECTION IX SPECIAL PRECAUTIONS

PRECAUTIONS TO BE TAKEN IN HANDLING AND STORAGE

Store in well-ventilated area free of combustibles.

OTHER PRECAUTIONS 


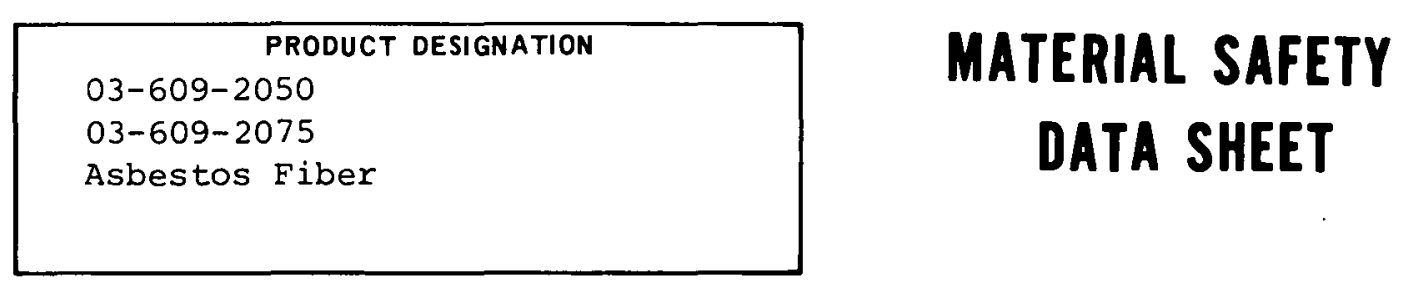

SÉCTION I SOURCE AND NOMENCLATURE

\begin{tabular}{l|l|l}
\hline MANUFACTURER'SNAME & EMERGENCY TELEPHONE NO. \\
\hline ADDRESS INUMEER, STREET, CITY, STATE, ZIP CODE) & \\
\hline
\end{tabular}

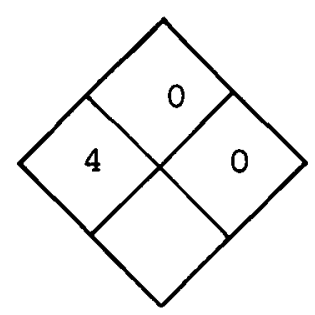

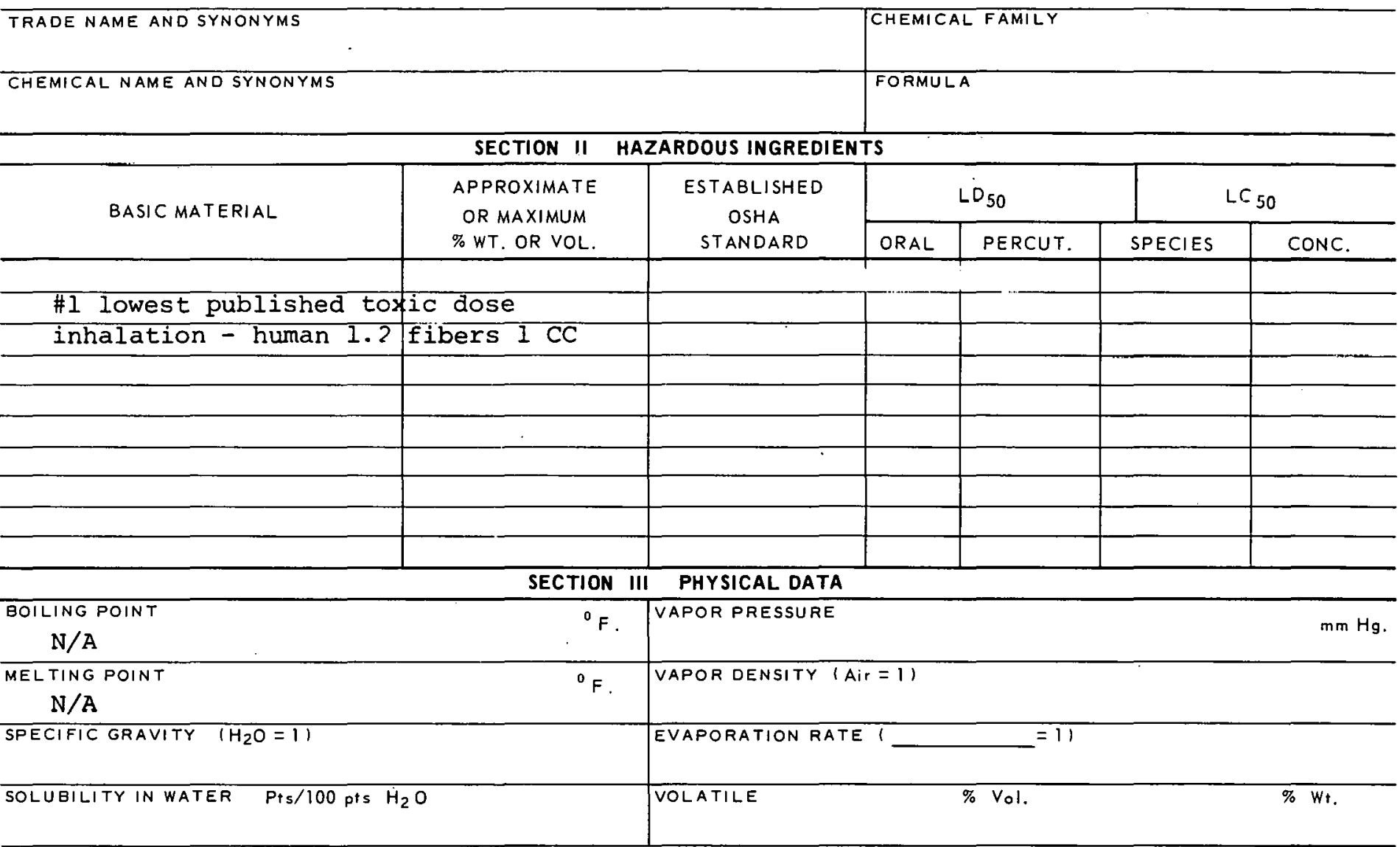

AFPCARANCE AND ODOR

Grey-white fibers

\begin{tabular}{|c|c|c|c|c|}
\hline & SECTION IV & FIRE AND EXPLOSION HAZARD DATA & & \\
\hline $\begin{array}{l}\text { FLASH POINT } \\
\text { Not flammable }\end{array}$ & & & \multirow{2}{*}{$\begin{array}{c}\text { FLAMMABLE } \\
\text { (EXPLOSIVE) } \\
\text { LIMITS }\end{array}$} & UPPER \\
\hline METHOD USED & & & & LOWER \\
\hline
\end{tabular}

EXTINGUISHING MEDIA

$\mathrm{N} / \mathrm{A}$

SPECIAL FIRE FIGHTING PROCEDURES

$\mathrm{N} / \mathrm{A}$

UNUSUAL FIRE AND EXPLOSION HAZARDS

$\mathrm{N} / \mathrm{A}$ 
TOXIC LEVEL

5 fibers/CC >5H long PRINCIPLE ROUTES OF ABSORBTION

Inhalation

CARCINOGENIC

CARCINOGENIC ye
SKIN ANDEYE
IRRITATION NO

REVELANT SYMPTOMS OF EXPOSURE

None

EFFECTS OF CHRONIC EXPOSURE

Asbestosis, lung cancer

EMERGENCY AND FIRST AID PROCEDURES

Not available

SECTION VI REACTIVITY DATA

CONDITIONS CONTRIBUTING TO INSTABILITY

$N / A$

CONDITIONS CONTRIBUTING TO HAZARDOUS POLYMERIZATION

ivis

INCOMPATABILIT' IMATERIALS TU AVUUIUI

$\mathrm{N} / \mathrm{A}$

TIAEAROOUS DCGOMTOOTTIOH TRODUETC

$\mathrm{N} / \mathrm{A}$

SECTION VII SPILL OR LEAK PROCEDURES

STEPS TO BE TAKEN IN CASE MATERIAL IS RELEASED OR SPILLED

Vacuum or wet clean only. No dry sweeping allowed.

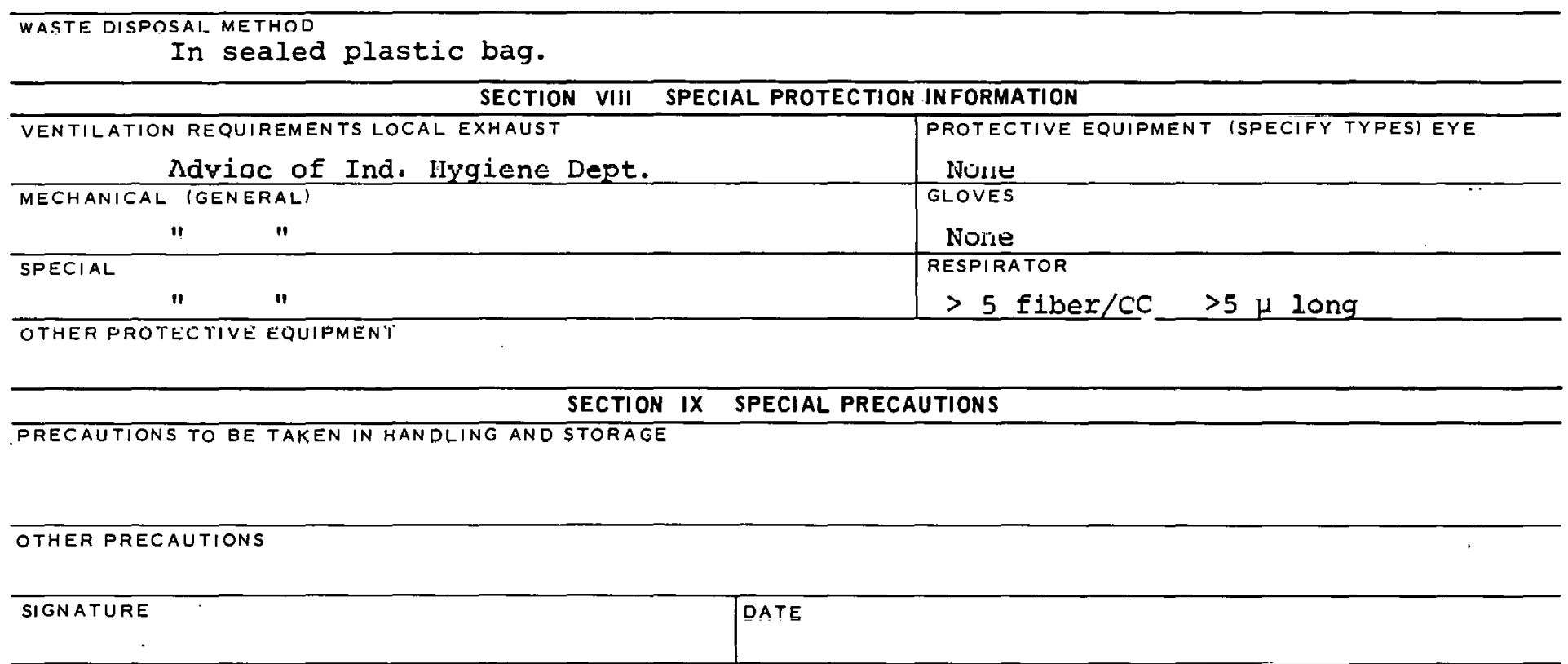




\begin{tabular}{|c|}
\hline PRODUCT DESIGNATION \\
$50-201-1400$ \\
Gold Cyanide \\
\end{tabular}

\section{MATERIAL SAFETY \\ DATA SHEET}

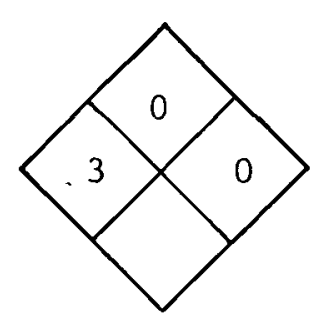

\section{SECTION I SOURCE AND NOMENCLATURE}

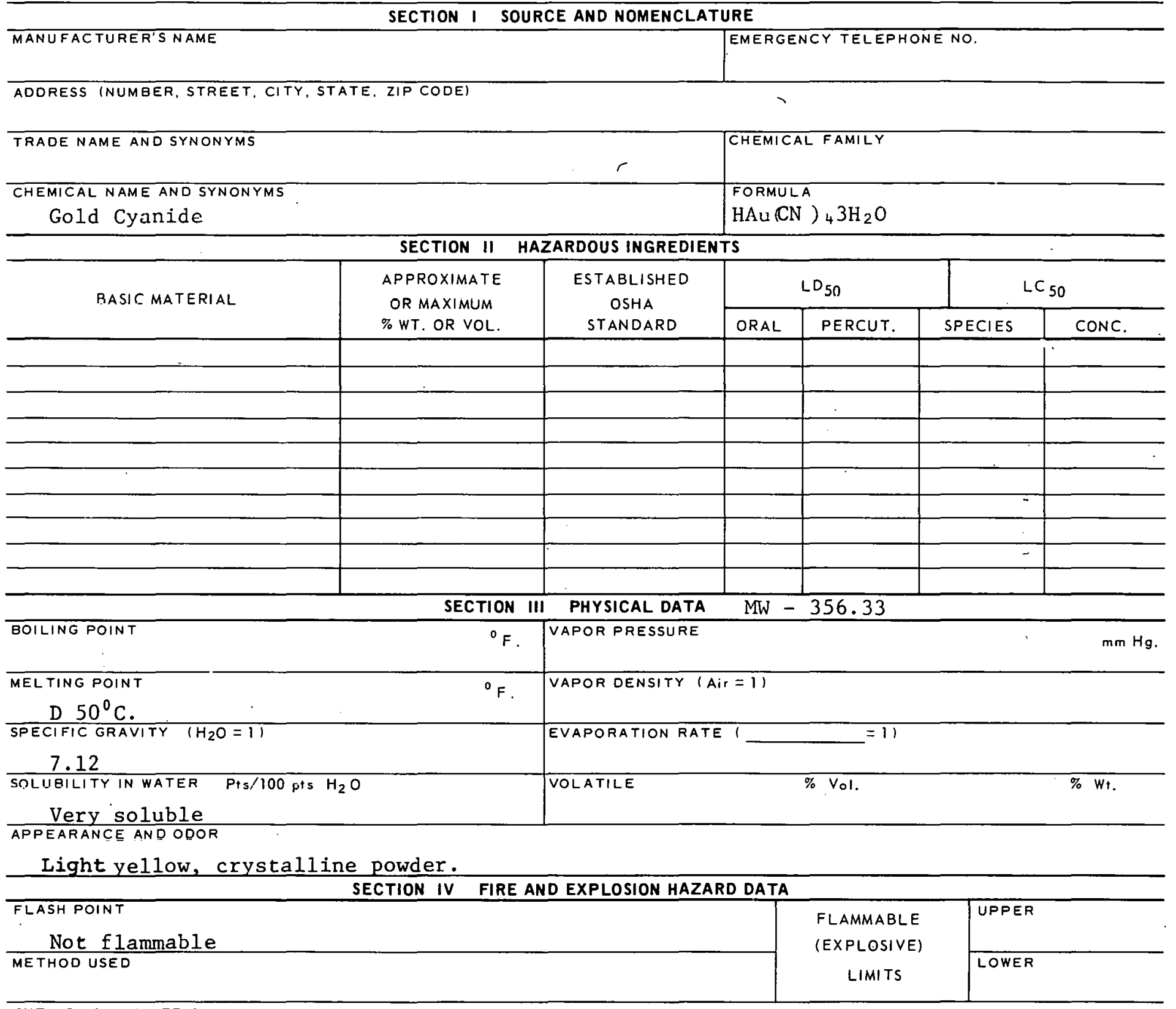

EXTINGIIISHING MEMIA

SPECIAL FIRE FIGHTING PROCEDURES

UNUSUAL FIRE AND EXPLOSION HAZARDS 
TOXIC LEVEL

TLV $5 \mathrm{m!} / \mathrm{C} . \mathrm{M} . \quad$ (CN)

PRINCIPLE ROUTES OF ABSORBTION

Inhalation, skin and ingestion
CARCINOGENIC No

SKIN AND EYE

IRRITATION Moderate

\section{REVELANT SYMPTOMS OF EXPOSURE Giddiness, dizziness, rapid heart action followed by}

unconsciousness; if swallowed - rapid respiration becoming slow.

EFFECTS OF CHRONIC EXPOSURE Dizziness, weakness, lung congestion, appetite loss,

Weight loss.

EMERGENCY AND FIRST AID PROCEDURES If inhaled - breathe amyl nitrite, artificial respiration and oxygen as needed; if swallowed same; wash from skin \& eyes.

\section{SECTION VI REACTIVITY DATA}

CONDITIONS CONTRIBUTING TO INSTABILITY

CONDITIONS CONTRIBUTING TO HAZARDOUS POLYMERI ZATION

INCOMNATABILITYY (MATERIALS TO AVOID)

Avoid acids.

HAZARDOUS DECOMPOSITION PRODUCTS

SECTION VII SPILL OR LEAK PROCEDURES

STEPS TO BE TAKEN IN CASE MATERIAL IS RELEASED OR SPILLED

WASTE DISPOSAL METHOD

SECTION VIII SPECIAL PROTECTION INFORMATION

\begin{tabular}{|c|c|}
\hline VFNTIILATION REQUIREMENTS LOCAL EXHAUST & $\begin{array}{l}\text { PROTECTIVE EQUIPMENT (SPECIFY TYPES) EYE } \\
\text { Face shield }\end{array}$ \\
\hline MECHANICAL (GENERAL) & $\begin{array}{l}\text { GLOVES } \\
\text { liubber }\end{array}$ \\
\hline SPECIAL & $\begin{array}{l}\text { RESPIRATOR } \\
\text { WILW pruper I } 11 \text { cER }\end{array}$ \\
\hline
\end{tabular}

OTHER PROTECTIVE EQUIPMENT

Overal1s and aprons

SECTION IX SPECIAL PRECAUTIONS

PRECAUTIONS TO BE TAKEN IN HANDLING AND STORAGE

Store in cool, dry place away from acids, OTHER PRECAUTIONS 

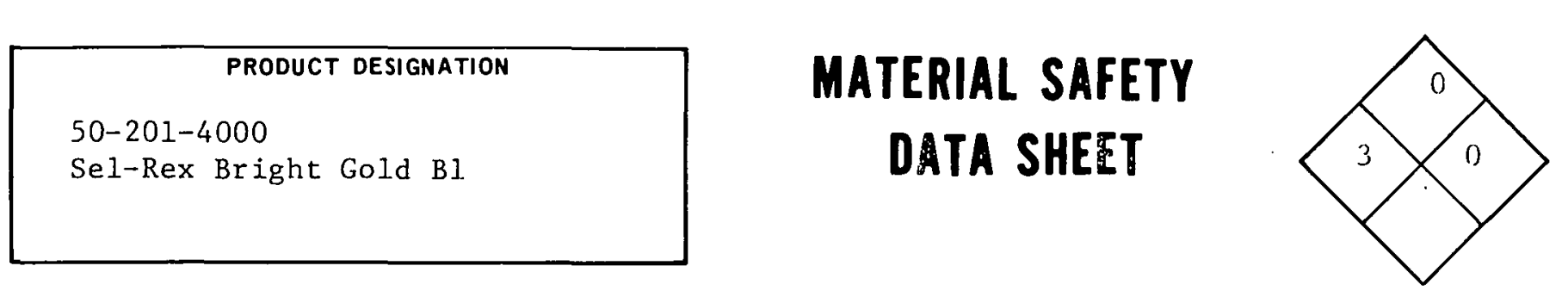

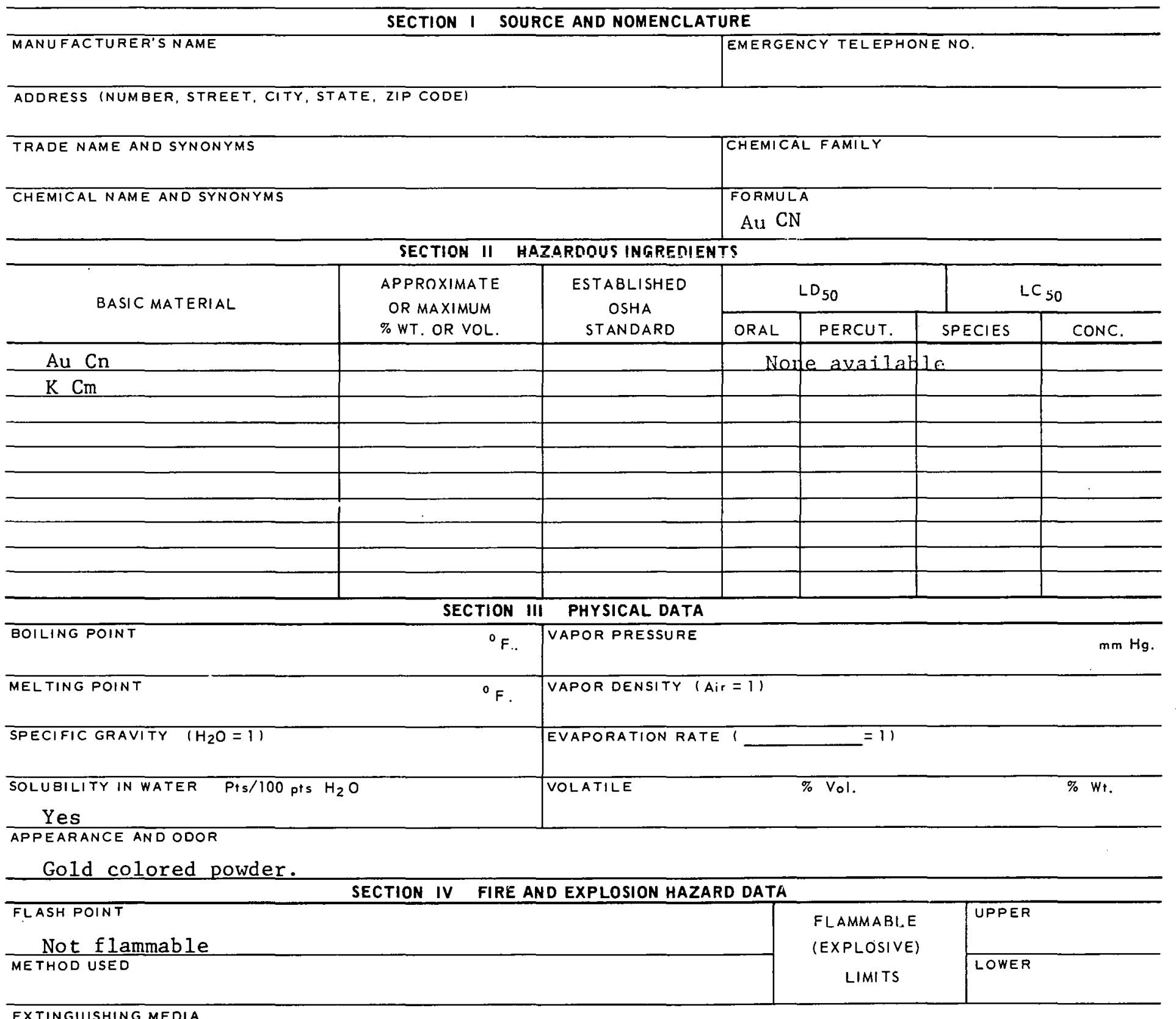

EXTINGUISHING MEDIA

SPECIAL FIRE FIGHTING PROCEDURES

UNUSUAL FIRE AND EXPLOSION HAZAROS

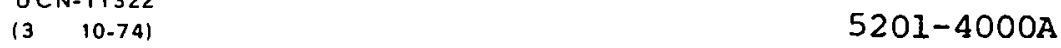


Sel-Rex bright gold $B-1$ SECTION $V$ HEALTH HAZARD DATA

\section{TOXICLEVEL}

$5 \mathrm{mg} / \mathrm{CM} \mathrm{M}$ (CN)

CARCINOGENIC

PRINCIPLE ROUTESOF ABSORETION

SKIN AND EYE

Inhalation, ingestion, skin IRRITATION Moderate

REVELANT SYMPTOMS OF EXPOSURE Dizziness, rapid heart action, unconsciousness and death; rapid respiration - becoming slow, coma and death.

EFFECTS OF CHRONIC EXPOSUREDizziness, weakness, lung congestion, loss of appetite, weight loss.

EMERGENCY AND FIRST AID PROCEDURES If inhaled or swallowed - breathe amyl nitrite

$30 \mathrm{sec}$. every 2 minutes; artificial respiration as needed.

\section{SECTION VI REACTIVITY DATA}

CONDITIONS CONTRIBUTING TO INSTABILITY

CONDITIONS CONTRIBUTING TO HAZARDOUS POLYMERIZATION

INCOMPATABILITY (MATERIALS TO AVOOIO)

Avoid contact with acids. HAZARDOUS DECOMPOSITION PRODUCTS

SECTION VII SPILL OR LEAK PROCEDURES

STEPS TO BE TAKEN IN CASE MATERIAL IS RELEASED OR SPILLED

$-$

WASTE DISPOSAL METHOD

SECTION VIII SPECIAL PROTECTION INFORMATION

\begin{tabular}{l|l}
\hline VENTILATION REQUIREMENTS LOCAL EXHAUST & $\begin{array}{l}\text { PROTECTIVE EQUIPMENT (SPECIFY TYPESIEYE } \\
\text { FaCe shield }\end{array}$ \\
\hline MECHANICAL IGENERALI & $\begin{array}{l}\text { GLOVES } \\
\text { Rubber }\end{array}$ \\
\hline SRCCIAL & $\begin{array}{l}\text { REGCIRATOR } \\
\text { WiLh proper filter }\end{array}$ \\
\hline
\end{tabular}

OTHEA PROTECTIVE EQUIT'MENT

SECTION IX SPECIAL PRECAUTIONS

Apron and clothes

PRECAUTIONS TO BE TAKEN IN HANDLING AND STORAGE 

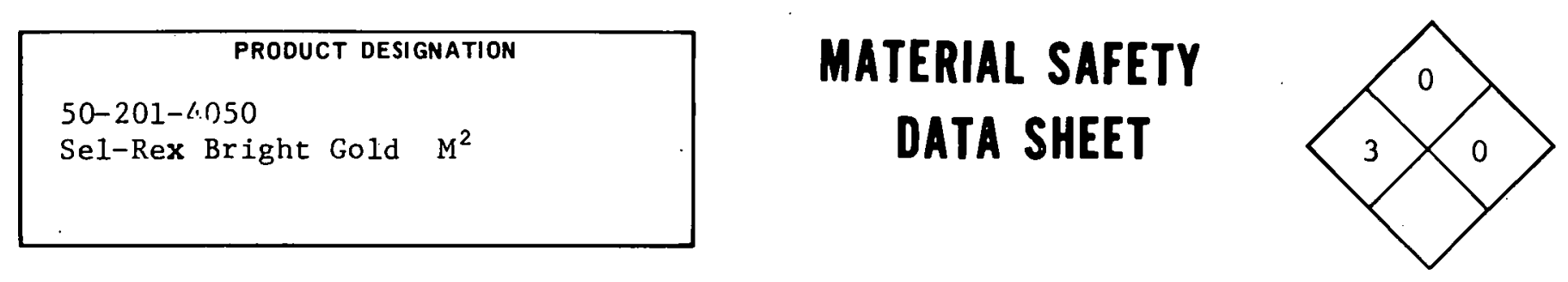

SECTION I SOURCE AND NOMENCLATURE

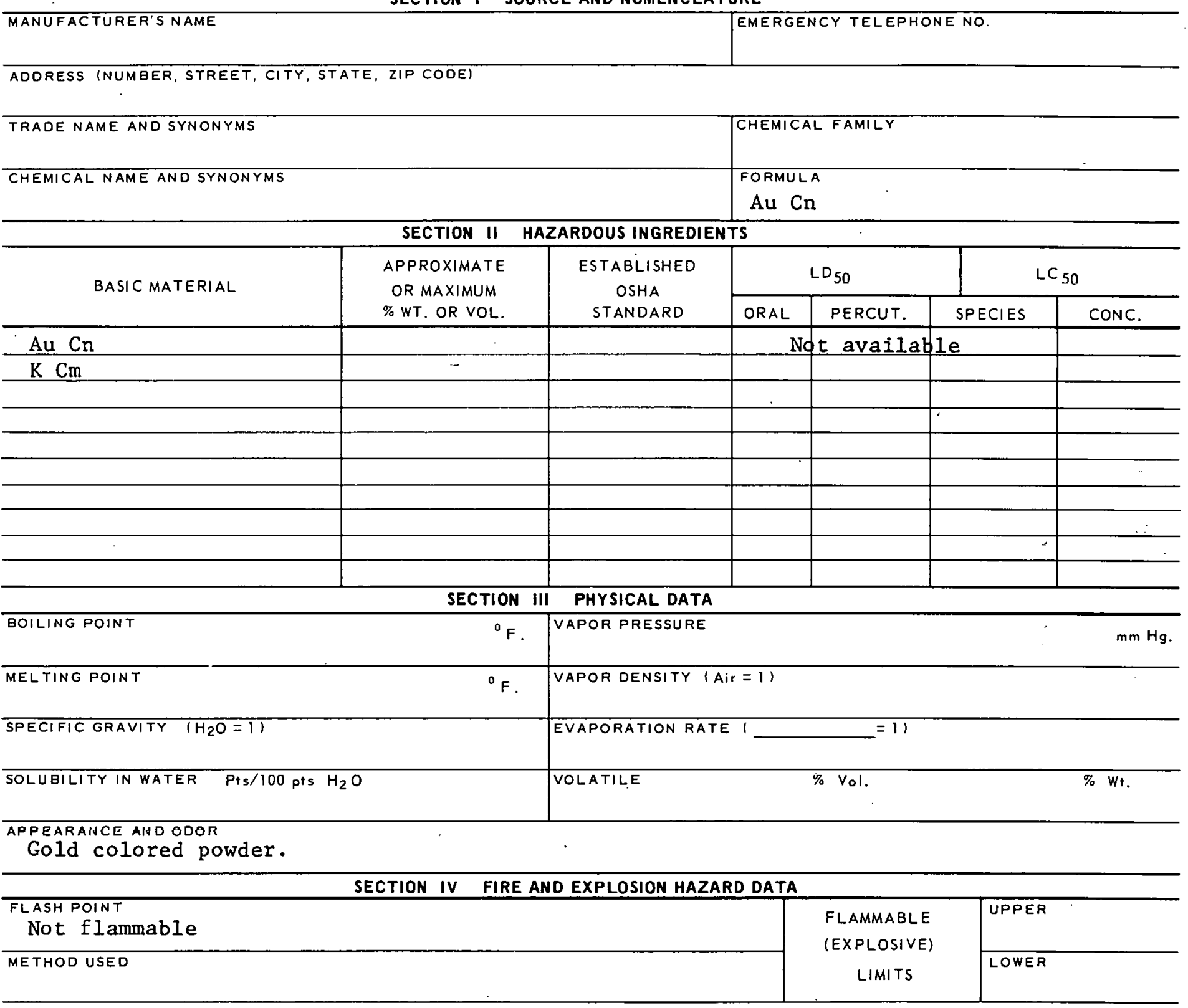

EXIINGUISHING MEUIA

SPECIAL FIRE FIGHTING PROCEDURES

UNUSUAL FIRE AND EXPLOSION HAZARDS 
Sel-Rex Bright Gold $M^{2}$ SECTION $V$ HEALTH HAZARD DATA

TOXIC LEVEL

$5 \mathrm{mg} / \mathrm{C} . \mathrm{M} .(\mathrm{CN})$

PRINCIPLE ROUTES OF ABSORETION

Inhalation, ingestion, skin

REVELANT SYMPTOMS OF EXPOSURE Dizziness, rapid heart action, unconsciousness and death. Rapid

respiration - hecoming slow, coma and death.

EFFECTS OF CHRONIC EXPOSURE

Dizziness, weakness, lung congestion, appetite loss, weight less. EMERGENCY AND FIRST AID PROCEDURES

Inhalation or ingestion - breathe amyl nitrate, every 2 min., artificial respiration as needed. SECTION VI REACTIVITY DATA
CARCINOGENIC No
Moderate 


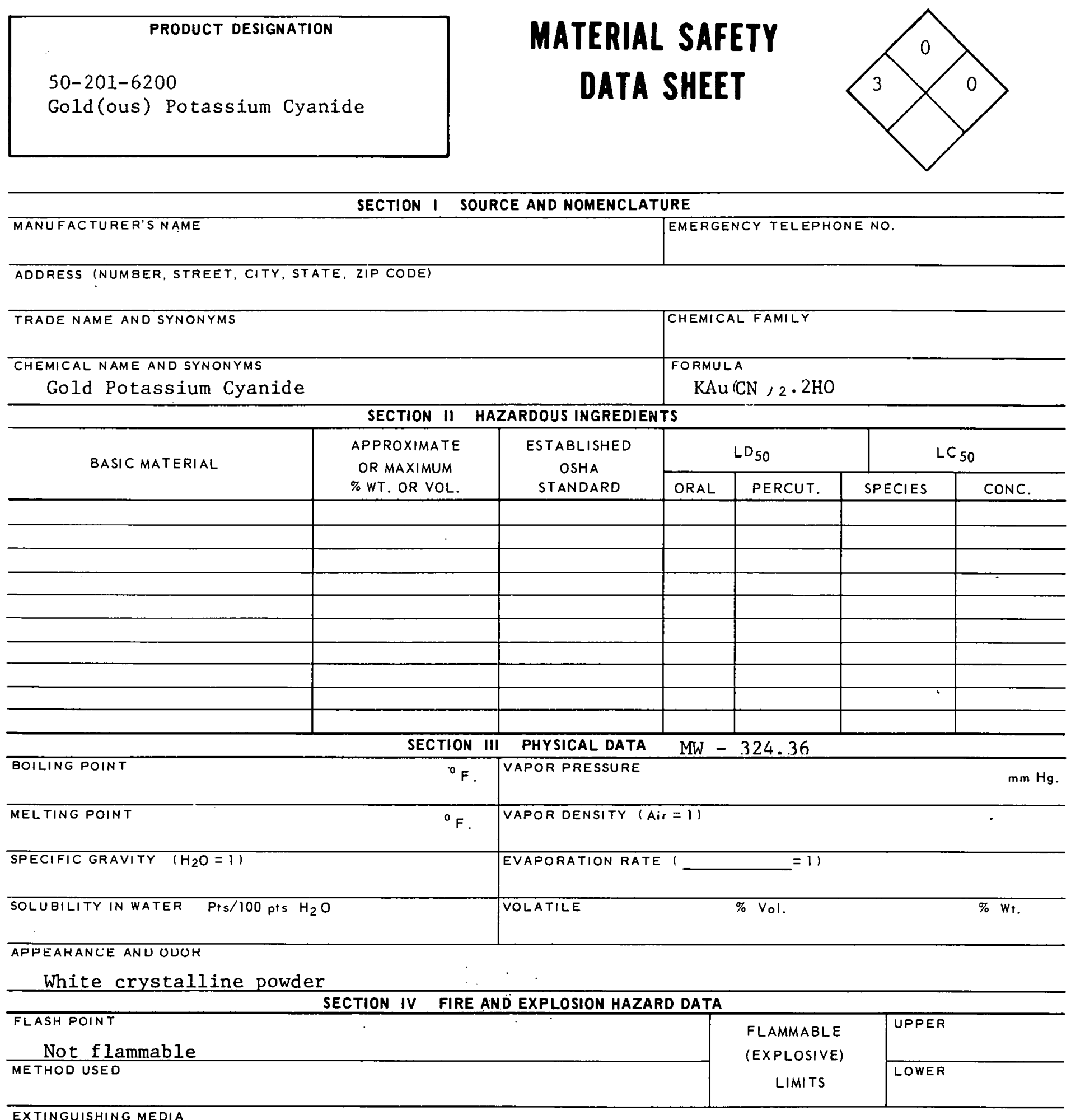

EXTINGUISHING MEDIA

SPECIAL FIRE FIGHTING PROCEDURES

UNUSUAL FIRE AND EXPI_OSION HAZARDS 
Gold Potassium Cyanide

\section{SECTION V HEALTH HAZARD DATA}

TOXIC LEVEL

$\mathrm{TLV}-5 \mathrm{mg} / \mathrm{C} . \mathrm{M} .(\mathrm{CN})$

CARCINOGENIC No

PRINCIPLE ROUTES OF ABSORBTION

Inhalation, ingestion, skin

SKIN AND EYE

IRRITATION Moderate

REVELANT SYMPTOMS OF EXPOSURE Giddiness, dizziness, rapid heart action followed by

unconsciousness; if swallowed - rapid respiration - becoming slow, coma.

EFFECTS OF CHRONIC EXPOSURE Dizziness, weakness, lung congestion, appetite loss,

weight loss.

EMERGENCY AND FIRST AID PROCEDURES

If inhaled or ingested - breathe amyl nitrite - artificial respiration and oxygen as needed.

\section{SECTION VI REACTIVITY DATA}

CONDITIONS CONTRIBUTING TO INSTABILITY

CONDITIONS CONTRIBUTING TO HAZARDOUS POLYMERIZATION

INGOAPATAEILITY (MATEPIALSTOAVOIO)

HAZARDOUS DECOMPOSITION PRODUCTS

Avoid contact with acids, produces HCN.

SECTION VII SPILL OR LEAK PROCEDURES

STEPS TO BE TAKEN IN CASE MATERIAL IS RELEASED OR SPILLED

WASTE DISPOSAL METHOD

SECTION VIII SPECIAL PROTECTION INFORMATION

\begin{tabular}{l|l}
\hline VENTILATION REQUIREMENTS LOCAL EXHAUST & $\begin{array}{c}\text { PROTECTIVE EQUIPMENT (SPECIFY TYPES) EYE } \\
\text { FaCe shield }\end{array}$ \\
\hline MECHANICAL (GENERAL) & $\begin{array}{c}\text { GLOVES } \\
\text { Rubber }\end{array}$ \\
\hline SHECIIAL & $\begin{array}{c}\text { RESPIRATOR } \\
\text { W1th proper filter }\end{array}$ \\
\hline
\end{tabular}

UIHEK HROTECTIVE EUUIPMENI

Overalls and aprons.

SECTION IX SPECIAL PRECAUTIONS

PRECAUTIONS TO BE TAKEN IN HANDLING AND STORAGE

Store in cool, dry place away from acids.

OTHER PRECAUTIONS

SIGNATURE

DATE

$5201-6200 B$ 
50-401-0401

Osmic Acid Arhydride

DATA SHEET

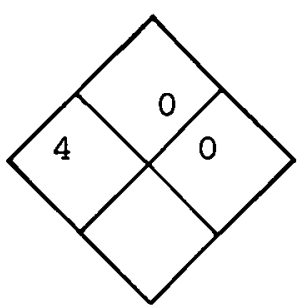

\section{SECTION I SOURCE AND NOMENCLATURE}

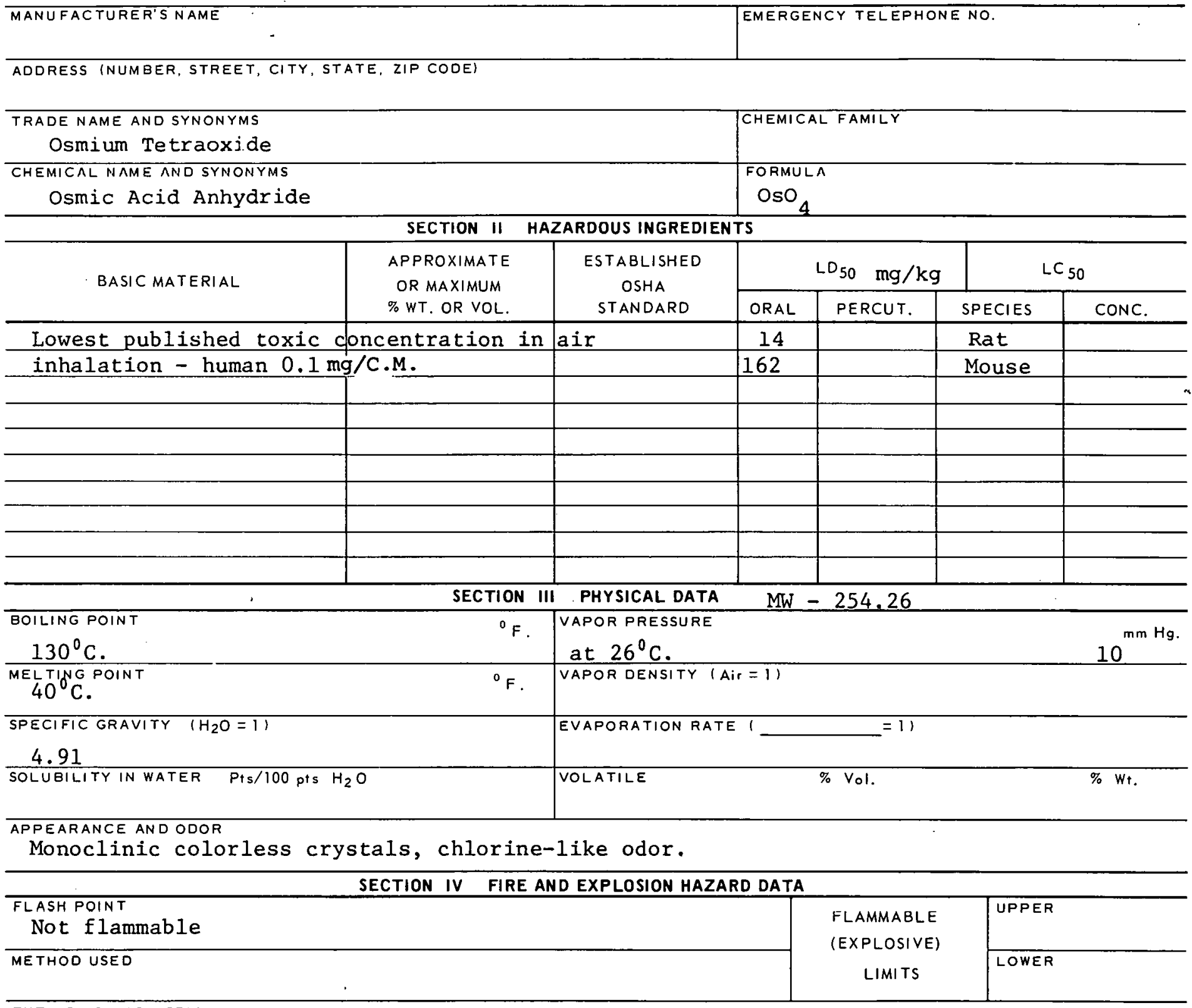

EXTINGUISHING MEDIA

SPECIAL FIRE FIGHTING PROCEDURES

UNUSUAL FIRE AND EXPLOSION HAZARDS 


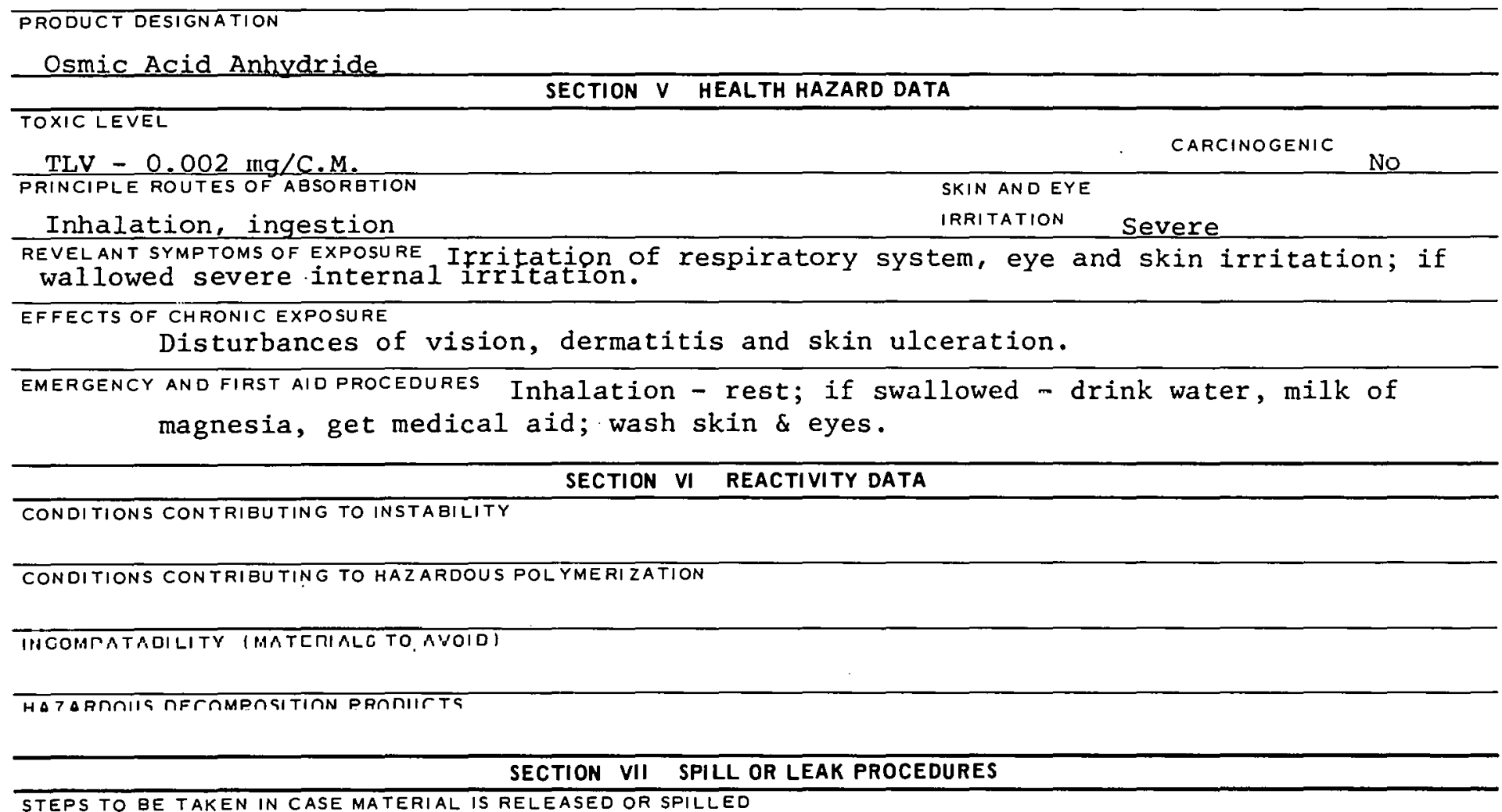

\begin{tabular}{|c|c|c|}
\hline SECTION & SPECIAL PROTECTION & INFORMATION \\
\hline VENTILATION REQUIREMENTS LOCAL EXHAUST & & $\begin{array}{l}\text { PROTECTIVE EQUIPMENT (SPECIFY TYPES) EYE } \\
\text { Safety goggles }\end{array}$ \\
\hline MECHANICAL (GENERAL) & & GLOVES \\
\hline SPECIIAL & & $\begin{array}{l}\text { RESPIRATOR } \\
\text { With chemical filter }\end{array}$ \\
\hline
\end{tabular}

\begin{tabular}{l}
\hline OTHER PROTECTIVE EQUIPMENT \\
\hline SECTION IX SPECIAL PRECAUTIONS \\
\hline
\end{tabular}

PRECAUTIONS TO BE TAKEN IN HANDLING AND STORAGE

OTHER PRECAUTIONS

SIGNATURE

DATE

5401-0401B 


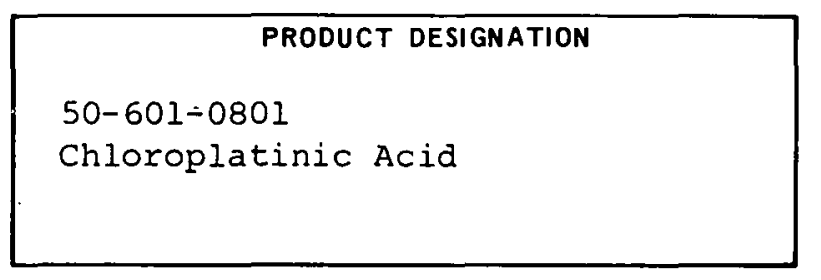

\section{MATERIAL SAFETY \\ DATA SHEET}

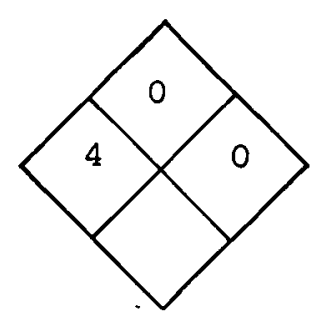

SECTION I SOURCE AND NOMENCLATURE

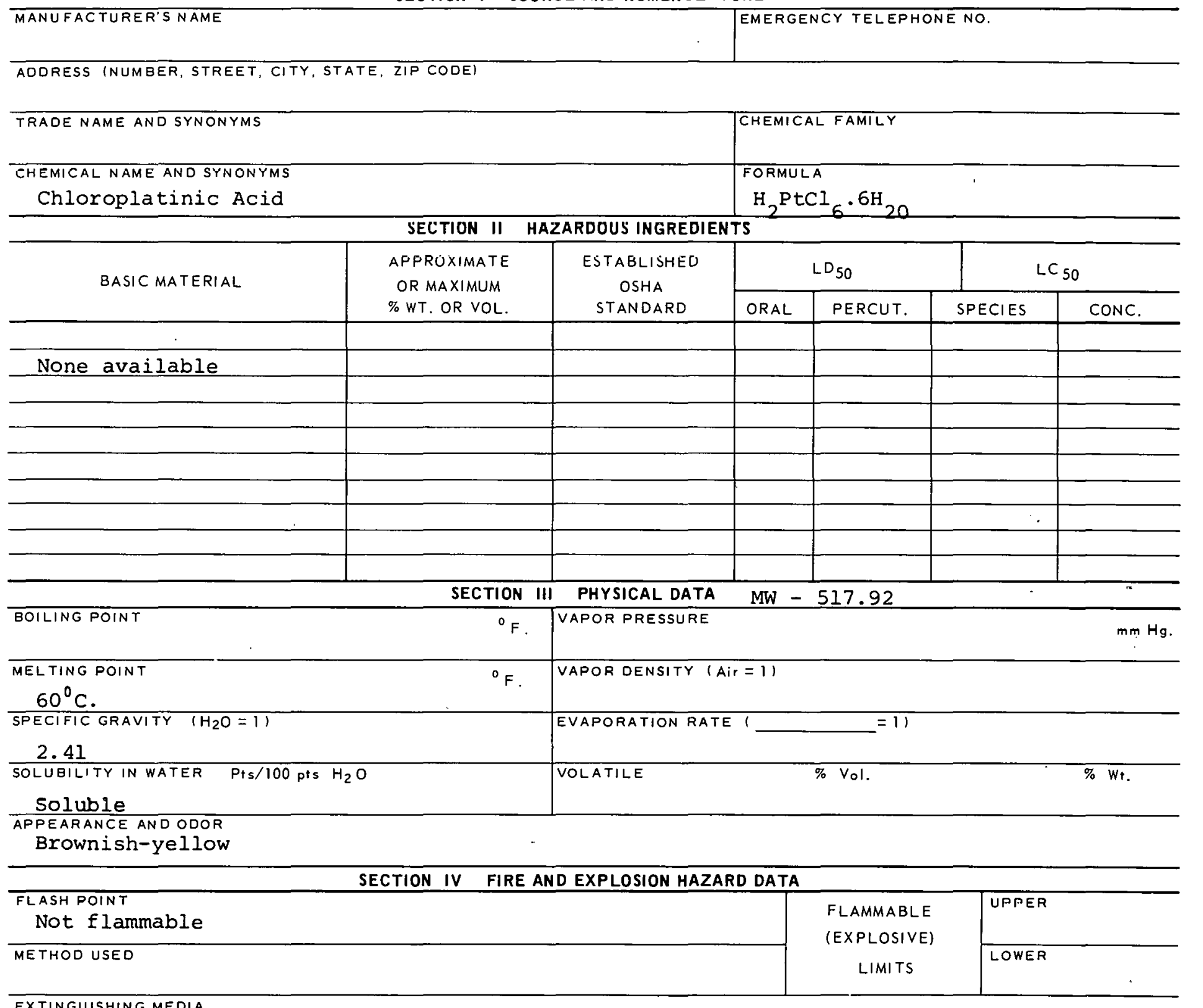

EXTINGUISHING MEDIA

SPECIAL FIRE FIGHTING PROCEDURES

UNUSUAL FIRE AND EXPLOSION HAZAROS 
Chloroplatinic Acid

SECTION V HEALTH HAZARD DATA

TOXIC LEVEL TLV - $0.002 \mathrm{Mg} / \mathrm{CM}$

PRINCIPLE ROUTES OF ABSURBIION Inhalation, ingestion

CARCINOGENIC

No SKIN AN DEYE

IRRITATION SEVERE

REVELANT SYMPTOMS OF EXPOSURE Inflammation of mucous membranes in the nose, lacramation and burning in the eyes.

EFFECTS OF CHRONIC EXPOSURE Running of the nose, sneezing, tightness of chest, cyanosis, wheezing, cough, dermatitis.

EMERGENCY AND FIRST AID PROCEDURES Inhalation - remove to fresh air, rest, treat asthmatic attack; ingestion - drink water, get medical aid.

\section{SECTION VI REACTIVITY DATA}

CONDITIONS CONTRIBUTING TO INSTABILITY

CONDITIONS CONTRIBUTING TO HAZARDOUS POLYMERIZATION

INCOMPATABILITY (MATERIALS TO AVOID)

HATARMOIIS RFROMPRSITION PROMIITTS

SECTION VII SPILL OR LEAK PROCEDURES

STEPS TO BE TAKEN IN CASE MATERIAL IS RELEASED OR SPILLED

WASTE DISPOSAL METHOD

SECTION VIII SPECIAL PROTECTION INFORMATION

\begin{tabular}{l|l}
\hline VENTILATION REQUIREMENTS LOCAL EXHAUST & PROTECTIVE
\end{tabular}

MECHANICAL (GENERAL)

Goggles

SPECIAL

GLOVES

OTHER PROTECTIVE EQUIPMENT

RESPIRATOA

SECTION IX SPECIAL PRECAUTIONS

PRECAUTIONS TO BE TAKEN IN HANDLING AND STORAGE

OTHER PRCCAUTIONS

SIGNATURE

DATE

5601-0801B 
CAS:010035657=

\section{PRODUCT DESIGNATION}

50-601-1120

Platinum Chloride
TP22750

MATERIAL SAFETY

DATA SHEET

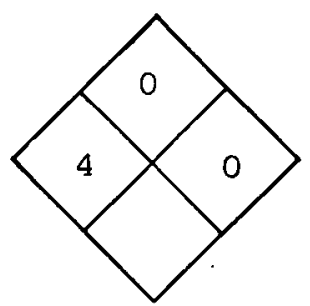

\section{SECTION I SOURCE AND NOMENCLATURE}

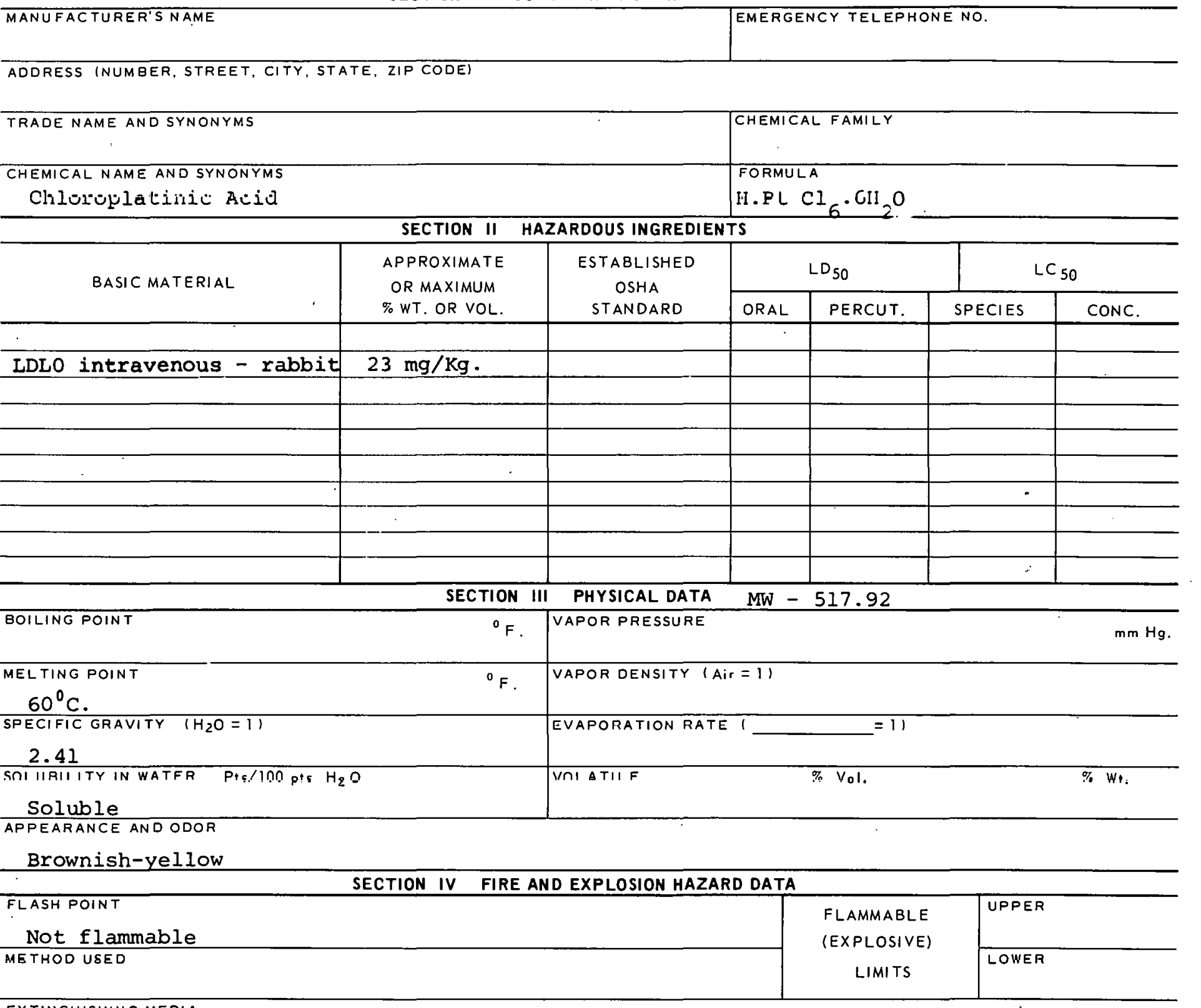

FXTINGIIISHING MFRIA

SPECIAL FIRE FIGHTING PROCEDURES

UNUSUAL FIRE AND EXPLOSION HAZARDS 
TOXIC LEVEL

TLV $0.002 \mathrm{mg} / \mathrm{CM}$

PRINCIPLE ROUTES OF ABSORBTION

Inhalation (dust)

REVELANT SYMPTOMS OF EXPOSURE

burning in the eyes

EFFECTS OF CHRONICEXPOSURE Running of the nose, sneezing, tightness of chest, cyanosis, wheezing, cough, dermatitis

EMERGENCY AND FIRSTAID PROCEDURES Inhalation - remove to fresh air, rest, treat asthmatic symptoms; drink water, get medical aid.

SECTION VII SPILL OR LEAK PROCEDURES

STEPS TO BE TAKEN IN CASE MATERIAL IS RELEASED OR SPILLED

WASTE DISPOSAL METHOD

SECTION VIII SPECIAL PROTECTION INFORMATION

\begin{tabular}{l|l}
\hline VENTILATION REQUIREMENTS LOCAL EXHAUST & $\begin{array}{l}\text { PROTECTIVE EQUIPMENT (SPECIFY TYPESI EYE } \\
\text { GOGglES }\end{array}$ \\
\hline MECHANICAL IGENERAL) & GLOVES \\
\hline SPECIAL & RESPIRATRR \\
\hline
\end{tabular}

UTHER PROTECTIVE EQUIHMENT

\section{SECTION IX SPECIAL PRECAUTIONS}

PRECAUTIONS TO BE TAKEN IN HANDLING AND STORAGE 


\begin{tabular}{|l} 
PRODUCT DESIGNATION \\
50-601-1510 \\
Diamino Platinum Nitrite \\
\hline
\end{tabular}

\section{SECTION I SOURCE AND NOMENCLATURE}

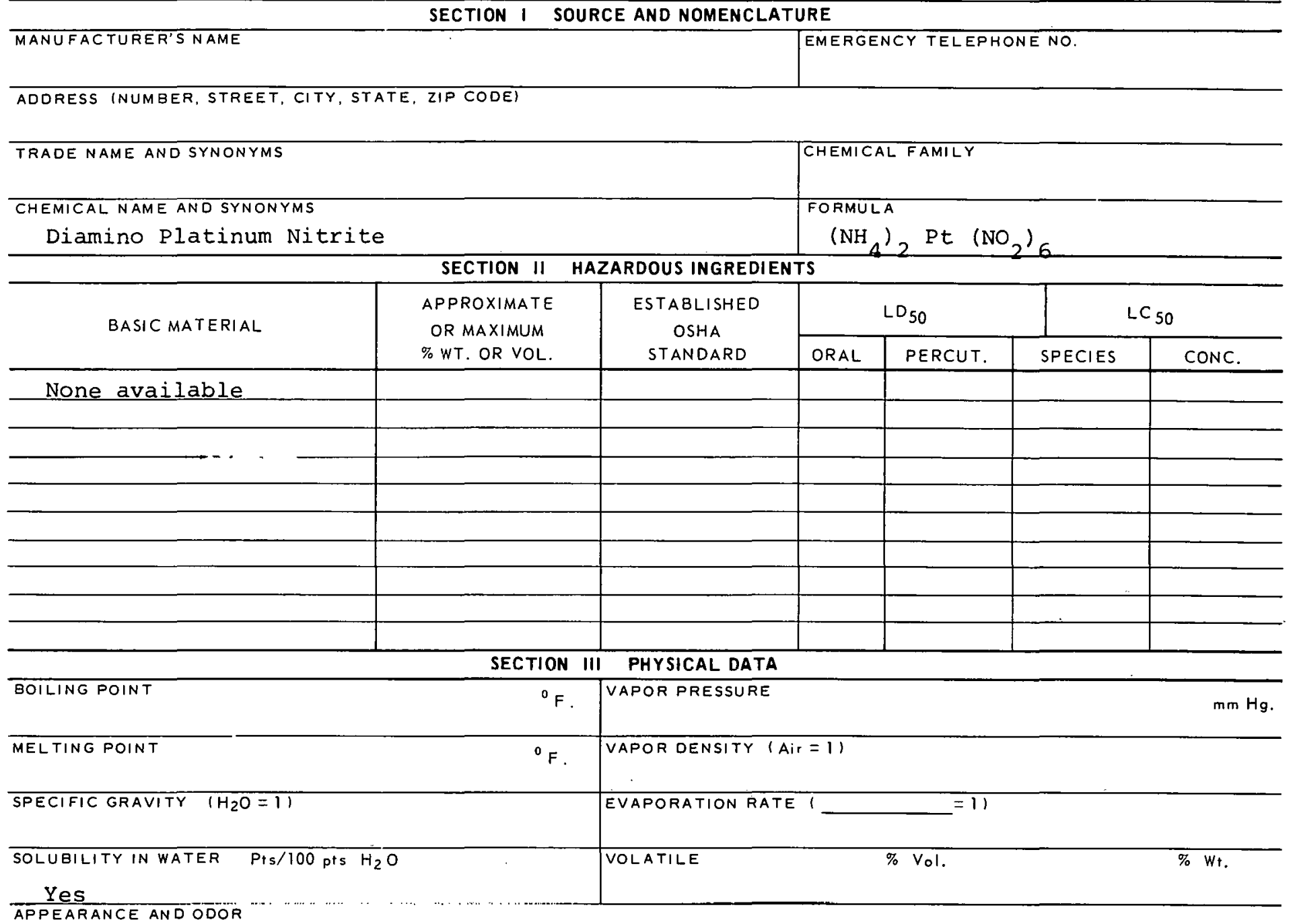

SECTION IV FIRE AND EXPLOSION HAZARD DATA

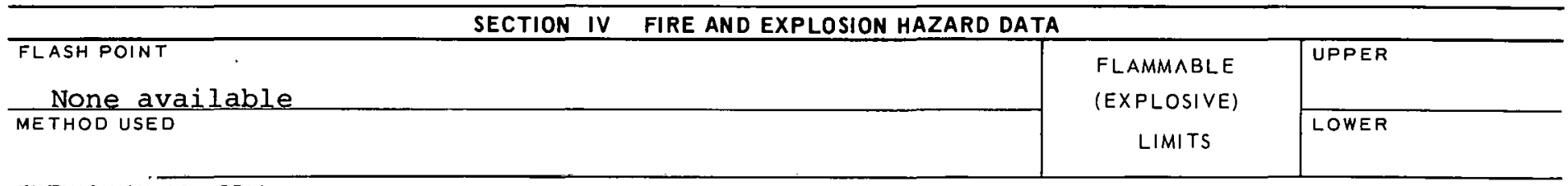

EXTINGUISHING MEDIA

SPECIAL FIRE FIGHTING PROCEDURES

UNUSUAL FIRE AND EXPI.OSION HAZARDS 


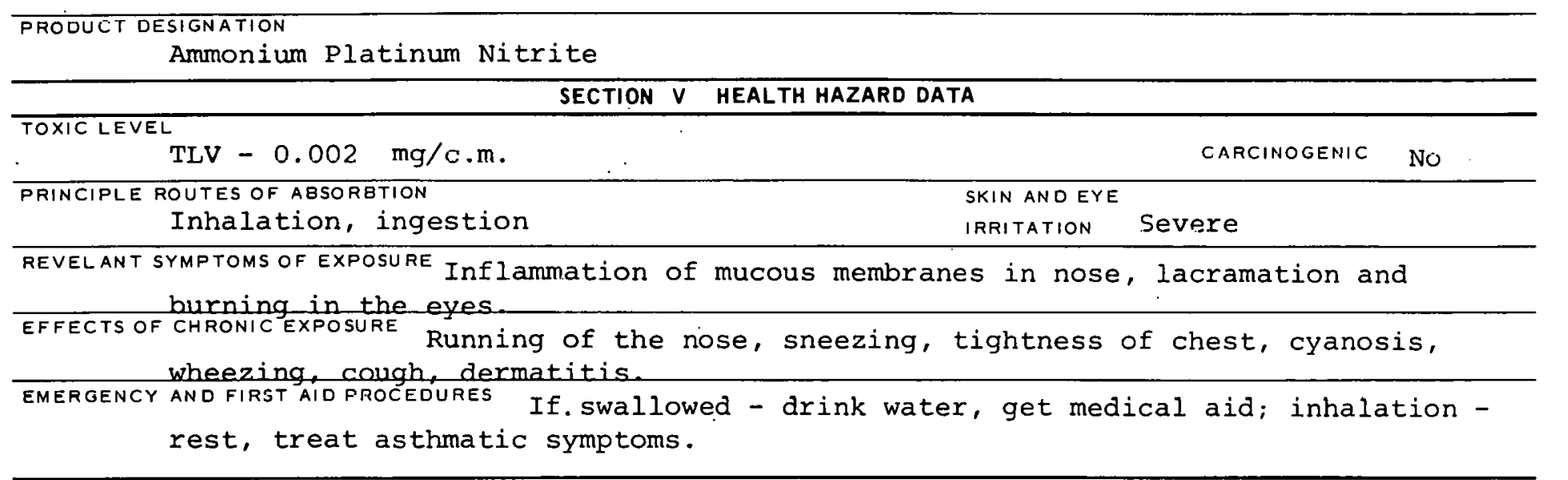

\section{SECTION VI REACTIVITY DATA}

CONDITIONS CONTRIBUTING TO INSTABILITY

CONQI TIONS CONTRIBUTING TO HAZARDOUS POLYMERIZATION

INCOMPATABILITY (MATERIALS TO AVOID)

HAËARDOUS DECOMPOSITIUN F'HUUUL IS

SECTION VII SPILL OR LEAK PROCEDURES

STEPS TO BE TAKEN IN CASE MATERIAL IS RELEASED OR SPILLED

WASTE DISPOSAL METHOD

SECTION VIII SPECIAL PROTECTION INFORMATION

VENTILATION REQUIREMENTS LOCAL EXHAUST PROTECTIVE EQUIPMENT (SFECIFY TYPES) EYE Goggles

MECHANICAL (GENERAL) GLOVES

SHËCIAL

RESPIRATOR

OTHER PROTECTIVE EQUIPMENT

SECTION IX SPECIAL PRECAUTIONS

PRECAUTIONS TO BE TAKEN IN HANDLING AND STORAGE

OTHER PRECAUTIONS

SITNATIIRE

DDTE

5601-1510B 


\section{PRODUCT DESIGNATION}

50-801-1105

Rhodium Trichloride

MATERIAL SAFETY

DATA SHEET

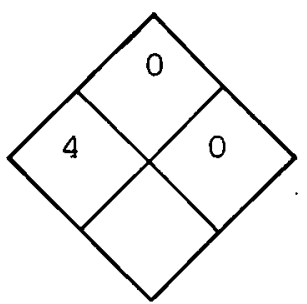

SECTION I SOURCE AND NOMENCLATURE

\section{MANUFACTURER'S NAME \\ TRADE NAME AND SYNONYMS \\ CHEMICAL NAME AND SYNONYMS \\ Rhodium Trichloride}

\begin{tabular}{l|l} 
& EMERGENCY TELEPHONE NO.
\end{tabular}

ADDRESS (NUMBER, STREET, CITY, STATE, ZIP CODE)

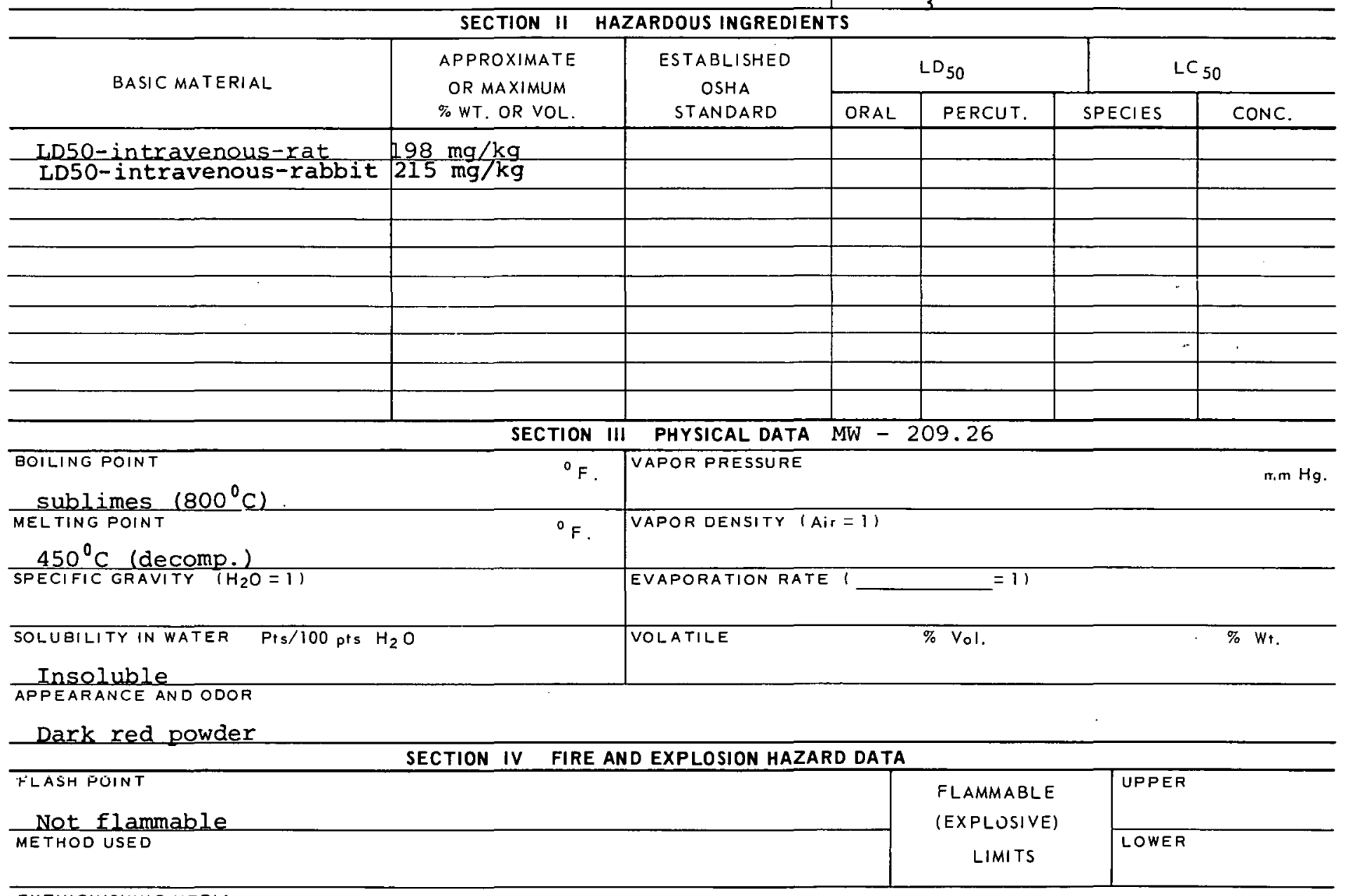

EXTINGUISHING MEDIA

Use water on fire

SPECIAL FIRE FIGHTING PROCEDURES

UNUSUAL FIRE ANDEXPLOSION HAZARDS 


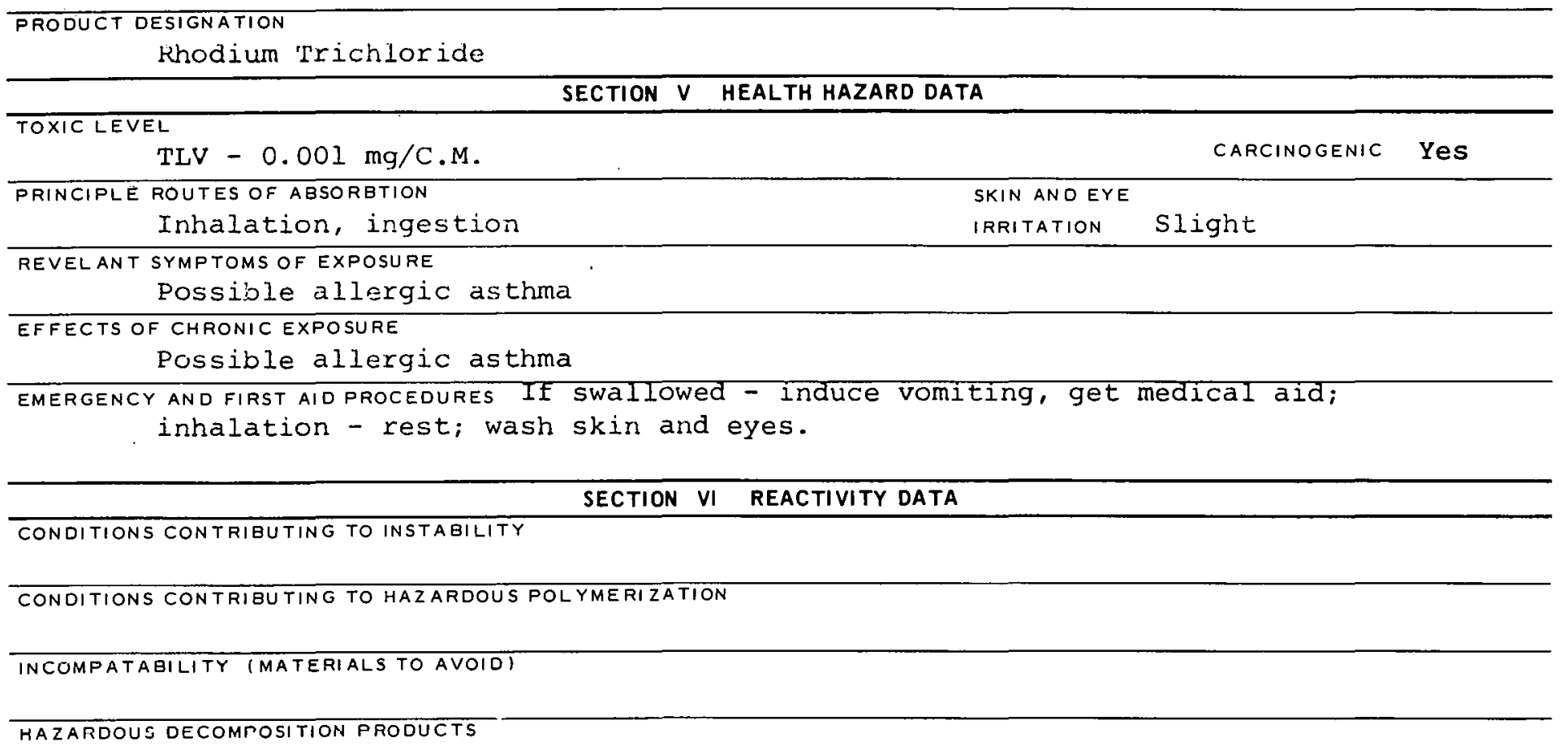

\section{SECTION VII SPILL OR LEAK PROCEDURES}

STEPS TO BE TAKEN IN CASE MATERIAL IS RELEASED OR SPILLED

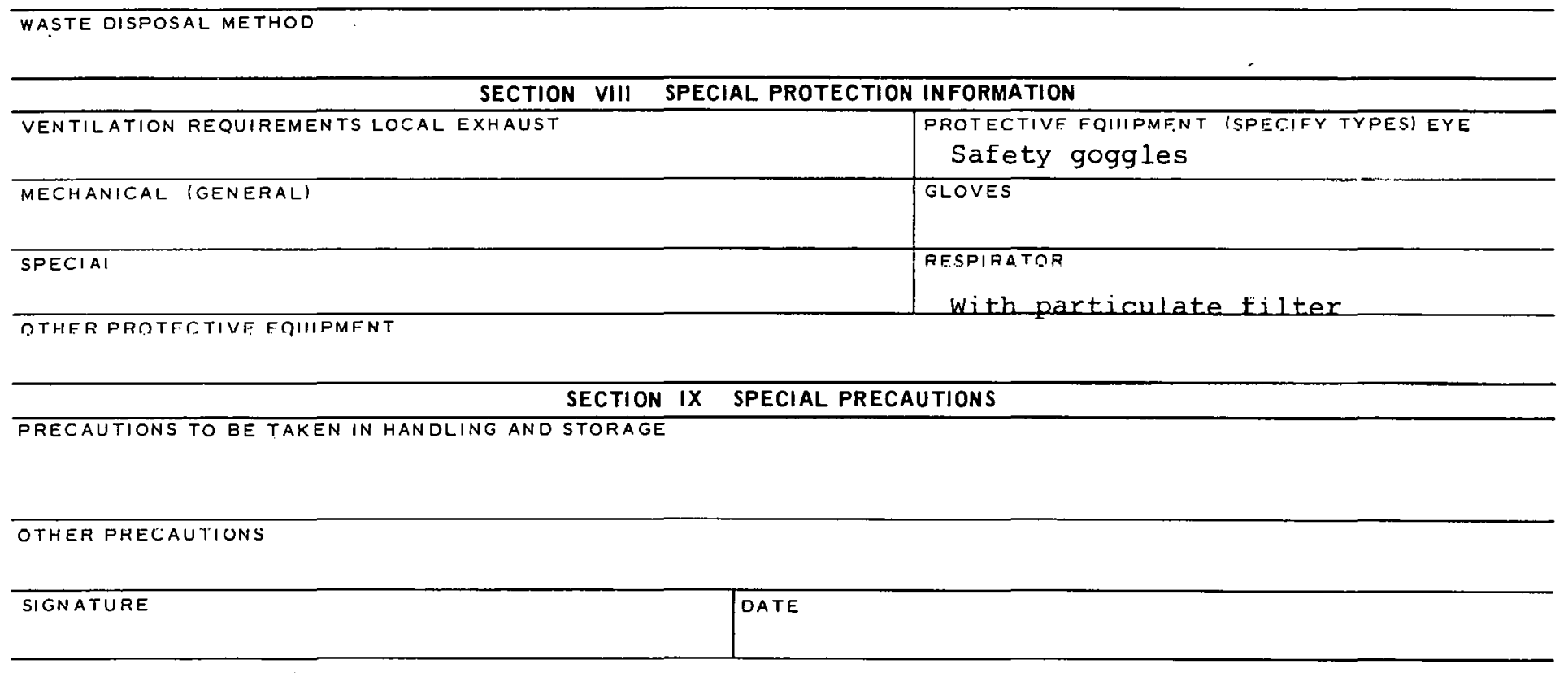




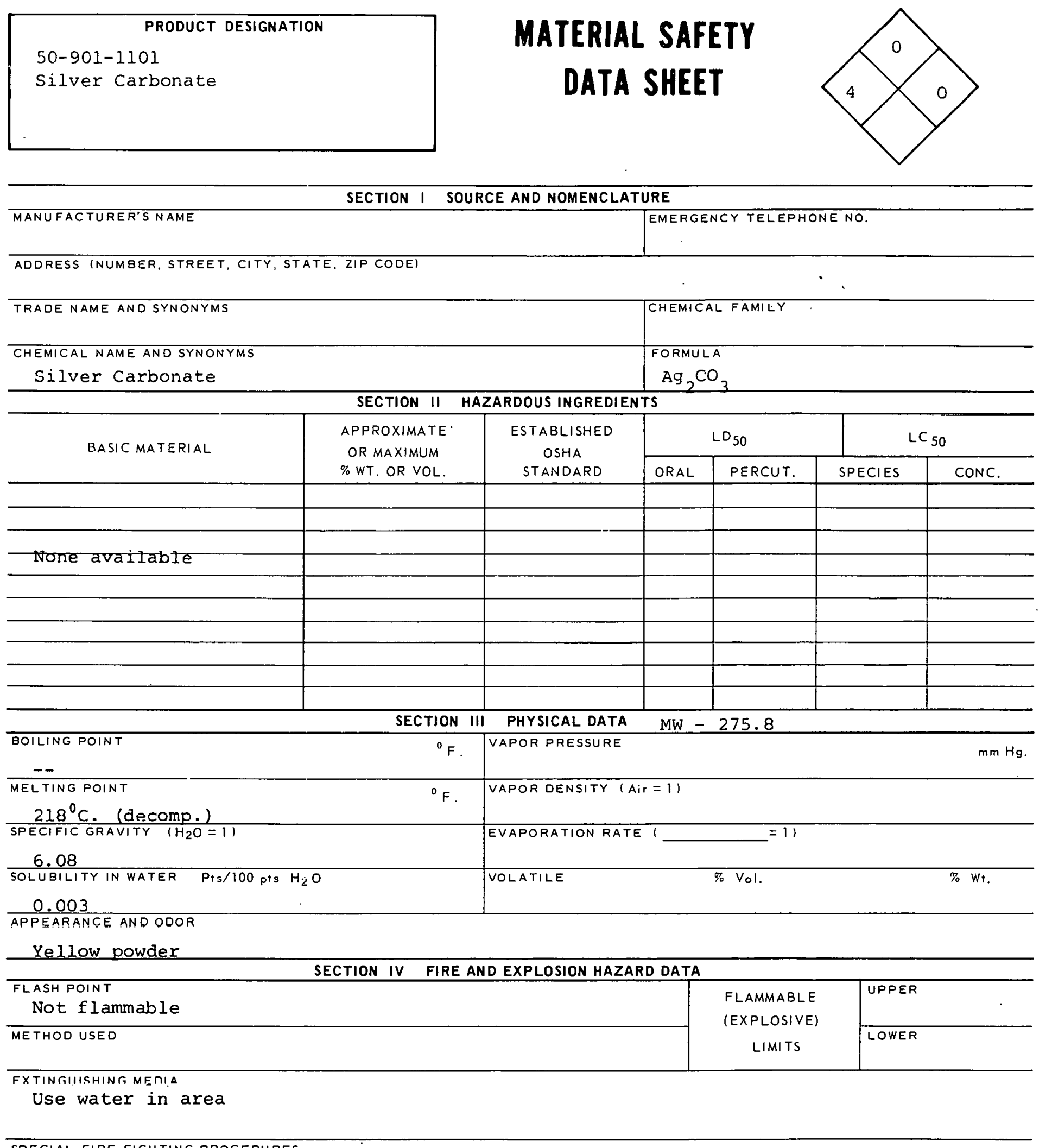

SPECIAL FIRE FIGHTING PROCEDURES

UNUSUAL FIRE ANO EXPLOSION HAZARDS 


\section{Silver Carbonate} SECTION $V$ HEALTH HAZARD DATA

\section{TOXIC LEVEL}

TLV - $0.05 \mathrm{mg} / \mathrm{CM}$

PRINCIPLE ROUTES OF ABSORETION

Inhalation, ingestion
CARCINOGENIC No
SKIN AND EYE

IRRITATION MOderate

REVELANT SYMPTOMS OF EXPOSURE Ingestion - abdominal pain; skin and respiratory

system irritation.

EFFECTS OF CHRONIC EXPOSURE Blue-grey discoloration of skin, mucous membrane and eyes.

EMERGENCY AND FIRST AID PROCEOURES

wash from skin \& eyes.

If swallowed - give emetic, get medical aid;

SECTION VI REACTIVITY DATA

CONDITIONS CONTRIBUTING TO INSTABILITY

CONDITIONS CONTRIBUTING TO HAZARDOUS POLYMERIZATION

INEUMPATADILITÍ (MATERIALS TO AVOIO)

HAZARDOUS DECOMPOSITION PRODUCTS

SECTION VII SPILL OR LEAK PROCEDURES

STEPS TO BE TAKEN IN CASE MATERIAL IS RELEASED OR SPILLED

WAGTE DISPOSAL METHOD

SECTION VIII SPECIAL PROTECTION INFORMATION

\begin{tabular}{l|l}
\hline VENTILATION REQUIREMENTS LOCAL EXHAUST & PROTECTIVE EQUIPMENT (SPECIFY TYPES) EYE
\end{tabular} Safety glasses

MECHANIGAL (GENERAL) GLOVES

SPECIAL

OTHER PROTECTIVE EQUIPMENT

MESPIRATOR

With particulate filter

SECTION IX SPECIAL PRECAUTIONS

PRECAUTIONS TO BE TAKEN IN HANOLING AND STORAGE

OTHER PRECAUTIONS

SIGNATURE

DATE

5901-1101B 
CAS - 000506649

\section{PRODUCT DESIGNATION}

50-901-1251

Silver Cyanide
VW38500

MATERIAL SAFETY

DATA SHEET

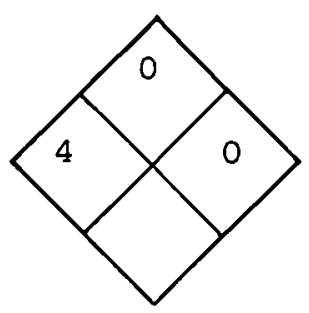

SECTION I SOURCE AND NOMENCLATURE

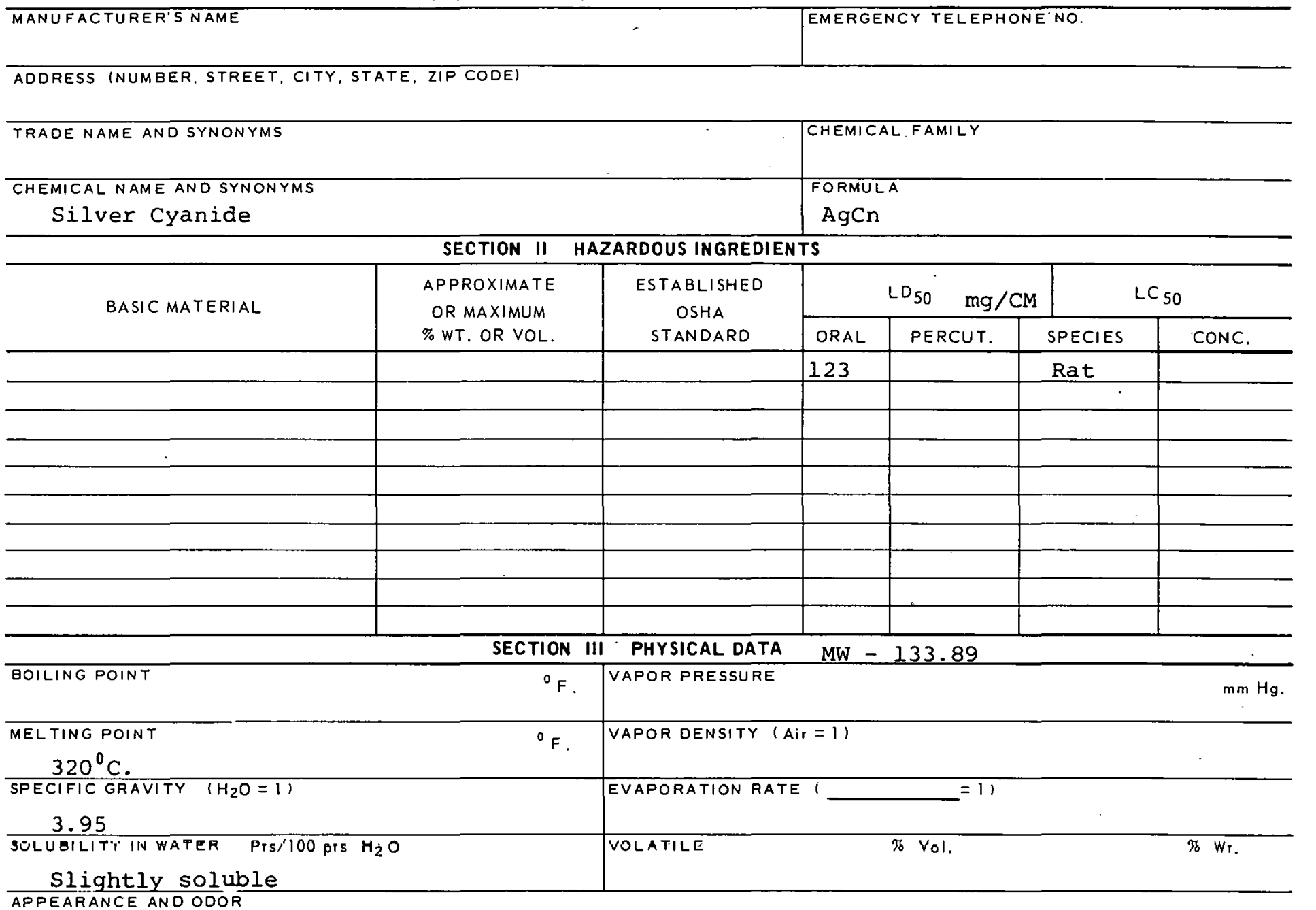

SECTION IV FIRE AND EXPLOSION HAZARD DATA

FLASH POINT

Not flammable

METHOD USED

EXTINGUISHING MEDIA

Water

SPECIAL FIRE FIGHTING PROCEDURES

IINUISIIAL FIRE AND EXPI OSION HAZARDS

UPPER

LOWER

\section{FLAMMABLE}

(EXPLOSIVE)

LIMITS
UPPER

Low 


\section{PRODUCT DESIGNATION \\ Silver Cyanide}

\section{SECTION $V$ HEALTH HAZARD DATA}

TOXIC LEVEL

$T L V-0.016$

CARCINOGENIC NO

PRINCIPLE ROUTES OF ABSORBTION

Inhalation, ingestion, skin

SKIN AND EYE

IRRITATION Moderate

REVELANT SYMPTOMS OF EXPOSURE Violent abdominal pain on ingestion, rapid respiration, then

gasping; dizziness, shortness of breath.

EFFECTS OF CHRONIC EXPOSURE Blue-grey discoloration of skin, mucous membranes

and eyes.

EMERGENCY AND FIRST AID PROCEDURES If swallowed - amyl nitrite inhalation, followed by

immediate vomiting or gastric lavage.

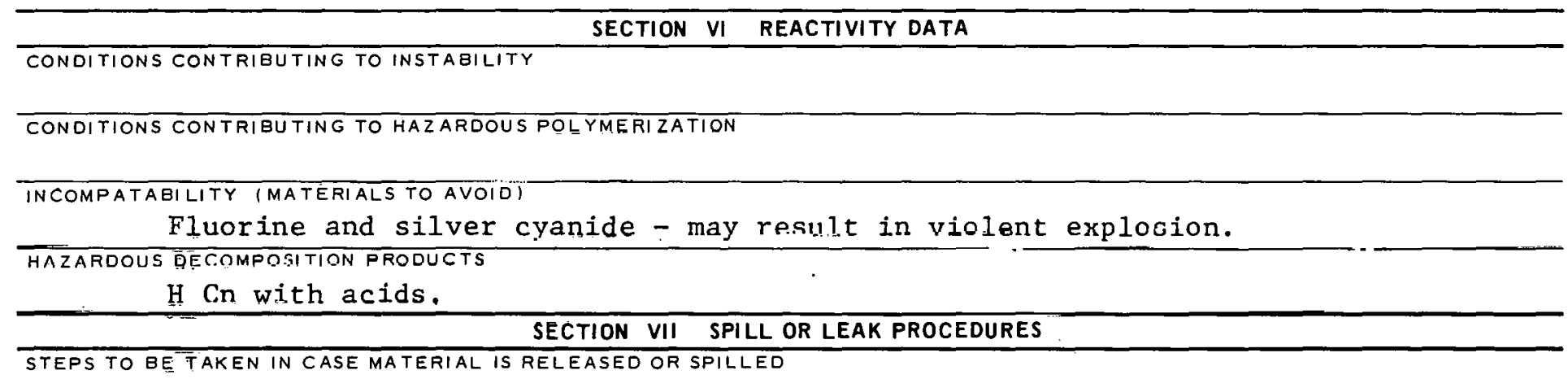

STEPS TO BE TAKEN IN CASE MATERIAL IS RELEASED OR SPILLED

WASTE DISPOSAL METHOD

SECTION VIII SPECIAL PROTECTION INFORMATION

\begin{tabular}{l|l}
\hline VFNTILATION REQUIREMENTS LOCAL EXHAUST & $\begin{array}{l}\text { PROTECTIVE EQUIPMENT (SPECIFY TYPES) EYE } \\
\text { GafELY glaSSES }\end{array}$ \\
\hline MECHANICAL. (GENERAL) & $\begin{array}{c}\text { GLOVES } \\
\text { Rubber }\end{array}$ \\
\hline SPECIAL' & $\begin{array}{l}\text { RESPIRAIUR } \\
\text { With particulate filter }\end{array}$ \\
\hline
\end{tabular}

OTHER PROTECTIVE EQUIPMENT

SECTION IX SPECIAL PRECAUTIONS

PRECAUTIONS TO BE TAKEN IN HANDLING AND STORAGE

Store away from acids.

OTHER PRECAUTIONS

SIGNATURE

BATE

$5901-1251 B$ 
CAS-007761888

PRODUCT DESIGNATION

50-901-56?. 1

Silver Nitrate
VW4 7250

\section{MATERIAL SAFETY \\ DATA SHEET}

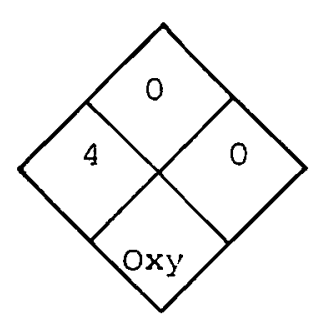

\section{SECTION I SOURCE AND NOMENCLATURE}

MANUFACTURER'S NAME
ADDRESS INUMBER, STREET, CI
TRADE NAME AND SYNONYMS
CHEMICAL NAME ANO SYNONYMS
SIIVEY Nitrate

SECTION II HAZARDOUS INGREDIENTS

EMERGENCY TELEPHONE NO.

BASIC MATERIAL

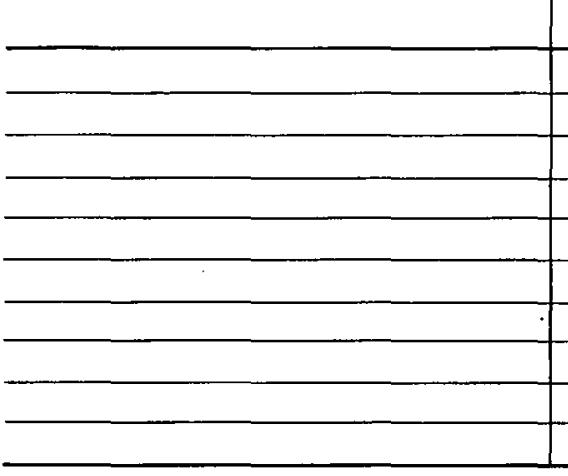

APPROXIMATE
OR MAXIMUM

$\%$ WT. OR VOL.

ESTABLISHED
OSHA
STANDARD

CHEMICAL FAMILY
FORMIII.A
AgNO $_{3}$ TS

BOILING POINT

$444^{\circ} \mathrm{C}$. (Decomp.) MELTING POINT

$212^{\circ} \mathrm{C}$.

SPECIFIC GRAVITY $\left(\mathrm{H}_{2} \mathrm{O}=11\right.$

4.35

SOLUBILITY IN WATER Pts/100 pts $\mathrm{H}_{2} \mathrm{O}$

122

APPEARANCE AND ODOR

White crystaline solid

\section{SECTION IV FIRE AND EXPLOSION HAZARD DATA}

FLASH POINT

Non inflammable

METHOD USED

EXTINGUISHING MEDIA

Use water

SPECIAL FIRE FIGHTING PROCEDURES

Wear goggles for fire fighting.

UNUSUAL FIRE AND EXPLOSION HAZARDS May increase flammability of combustible, organic

or other readily oxidizable materials. 


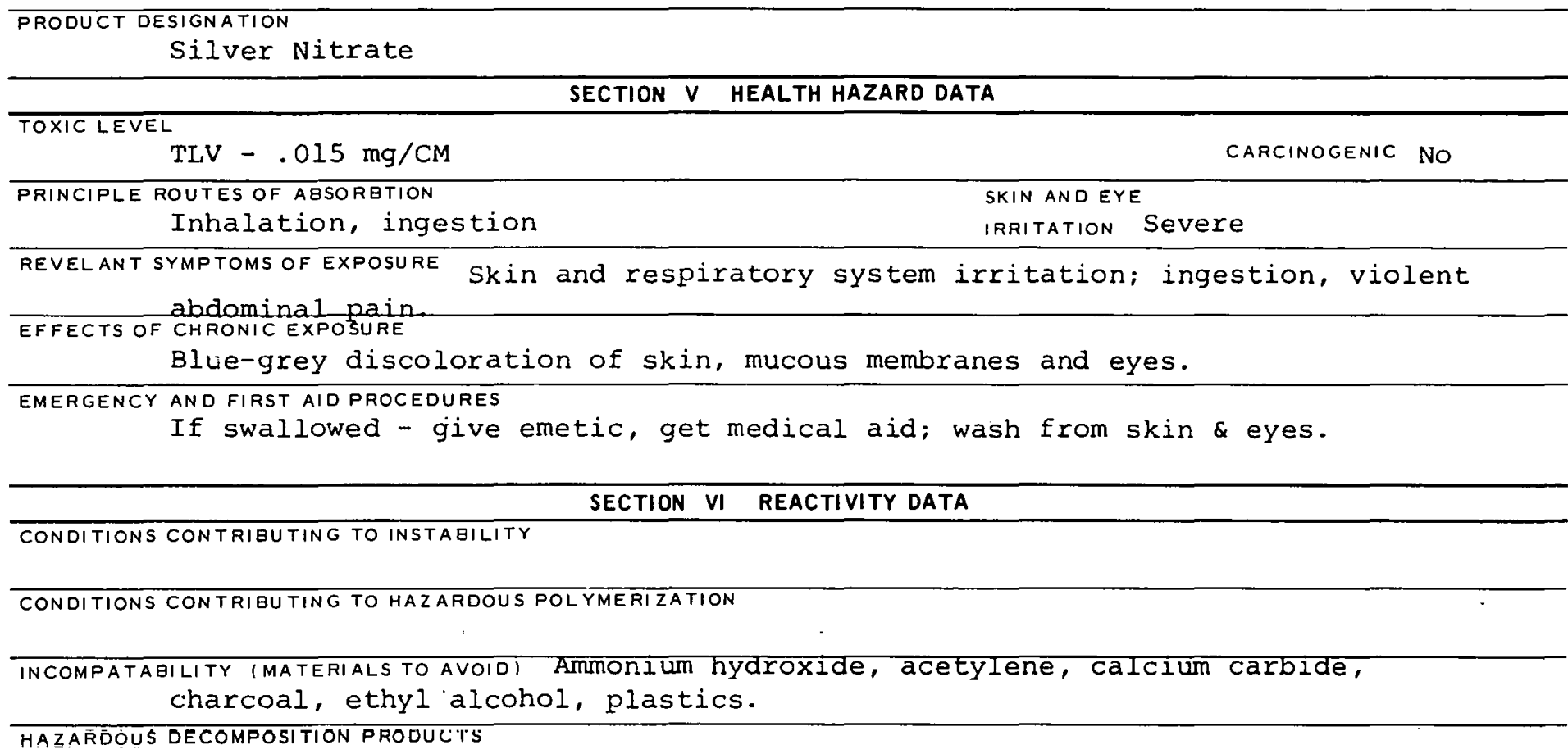

SECTION VII SPILL QR LEAK PROCEDURES

STEPS TO BE TAKEN IN CASE MATERIAL IS RELEASED OR SPILLED

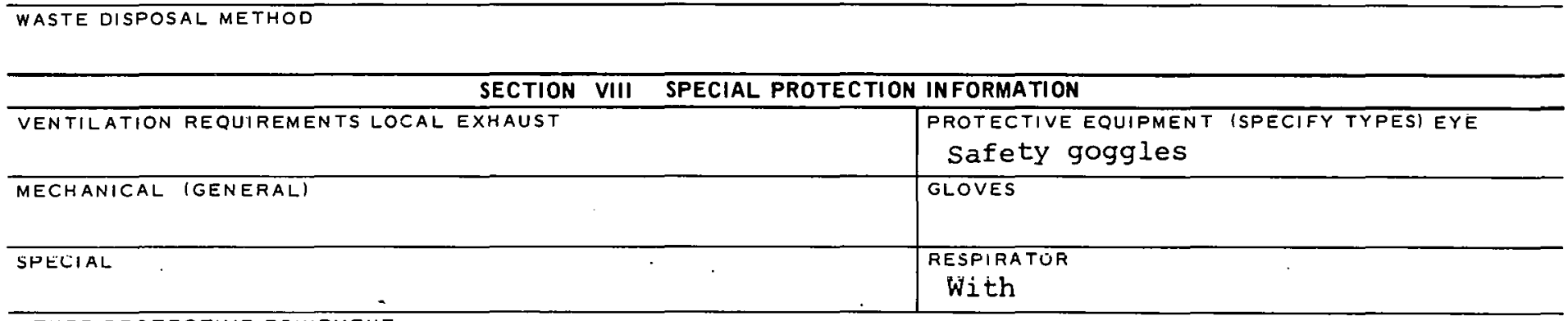

OTHER PROTECTIVE EQUIPMENT

SECTION IX SPECIAL PRECAUTIONS

PRECAUTIONS TO BE TAKEN IN HANDLING AND STORAGE
DO not store with combustible, organic or other readily oxidizable materials.

OTHER PRECAUTIONS

SIGNATURE

DATE

5901-5621B 


PRODUCT DESIGNATION
Acetic Anhydride
$03-201-0100$

\begin{tabular}{ll|l}
\hline \multicolumn{3}{|c|}{ SECTION I SOURCE AND NOMENCLATURE } \\
\hline MANUFACTURER'S NAME & EMERGENCY TELEPHONE NO. \\
\hline
\end{tabular}

ADORESS (NUMBER, STREET, CITY, STATE, ZIP CODE)

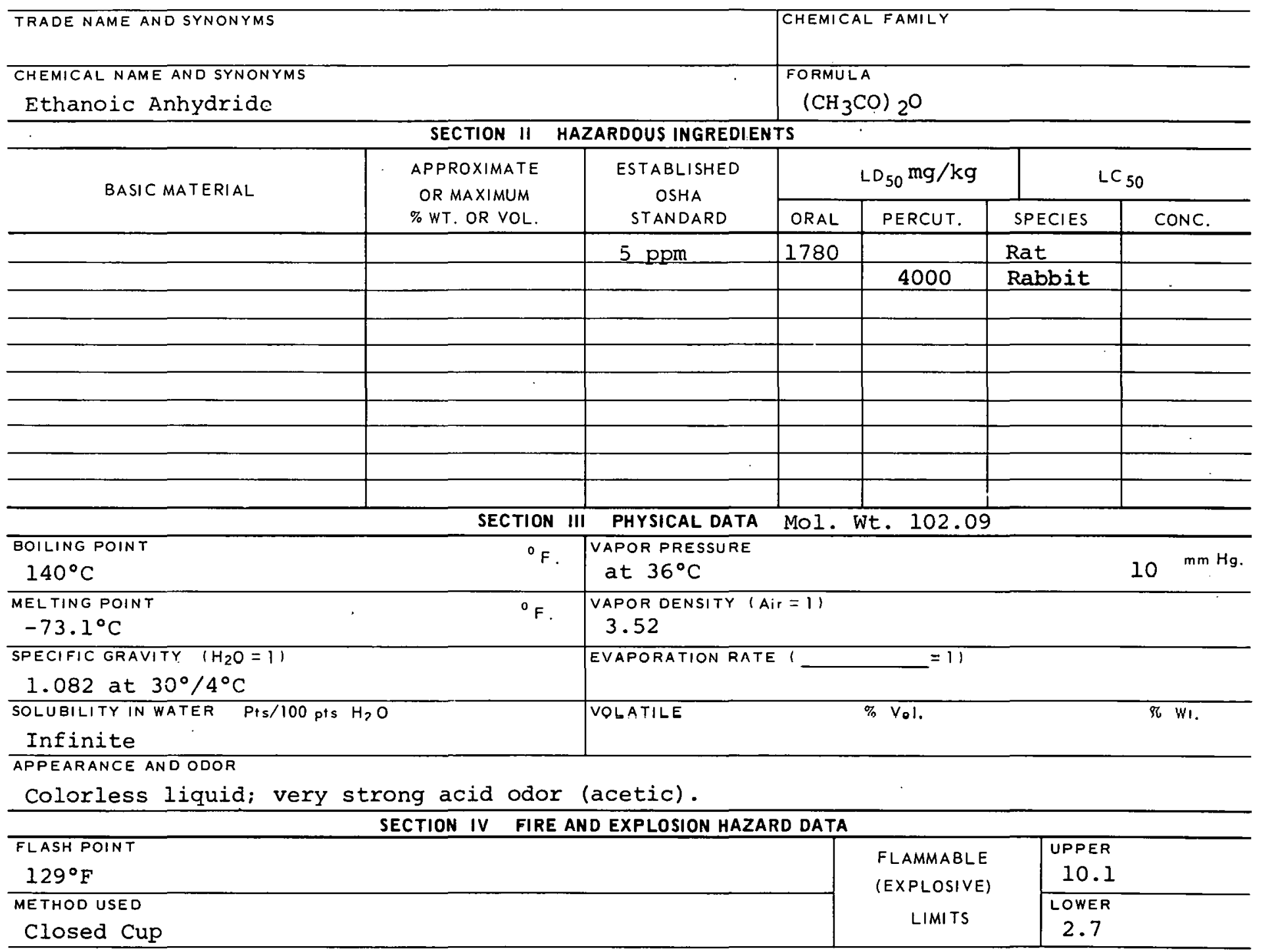

EXTINGUISHING MEOIA

Water spray, carbon dioxide, dry chemical, "alcohol" foam.

SPECIAL FIRE FIGHTING PROCEDURES

In advanced fires, fight from a distance or protected position.

UNUSUAL FIRE AND EXPLOSION HAZARDS

Vapors may form explosive mixture with air. 


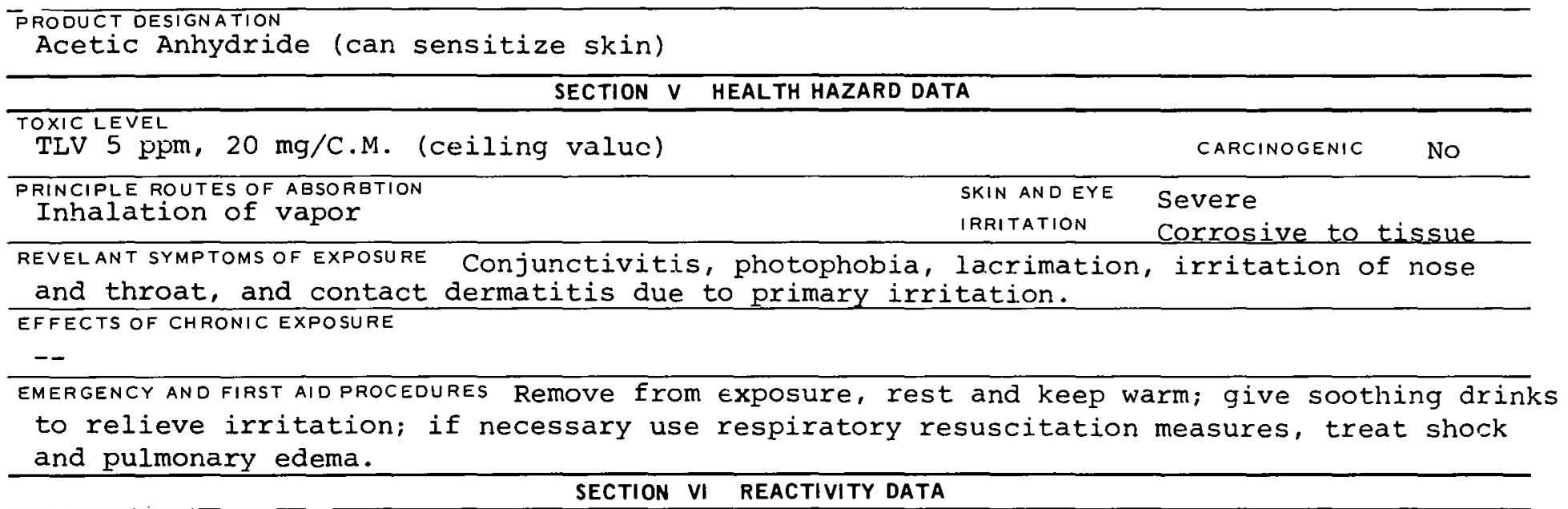

CONDITIONS CONTRIBUTING TO INSTABILITY

GQHDITIONS SINTRIRIITING TO HAZARDOUS POLYMERIZATION

INCOMPATABILITY (MATERIALS TO AVOID) Chromic acid, chromic anhydride, glycerol, water, nitric acid, nitrogen tetroxide, perchloric acid, sodium peroxide and permanganates.

HAZARDOUS DECOMPOSIIIUN PRODUCTS

Heated to decomposition, releases toxic fumes.

\begin{tabular}{l} 
SECTION VII SPILL OR LEAK PROCEDURES \\
\hline STEPS TO BE TAKEN IN CASE MATERIAL IS RELEASED OR SPILLED \\
Flush with water - water spray.
\end{tabular}

\section{WASTE DISPOSALMETHOD
Burial - CaII Safety Department.}

\begin{tabular}{|c|c|}
\hline SECTION VIII & SPECIAL PROTECTION INFORMATION \\
\hline $\begin{array}{l}\text { VENTILATION REQUIREMENTS LOCAL EXHAUST } \\
\text { Call Industrial Hygiene Department. }\end{array}$ & $\begin{array}{l}\text { FROTECTIVE EQUIPMENT (SPECIFY TYPES) EYE } \\
\text { GOggles }\end{array}$ \\
\hline MECHANICAL (GENERAL' Hygiene Department. & $\begin{array}{l}\text { GLOVES } \\
\text { Rubber, neoprene }\end{array}$ \\
\hline $\begin{array}{l}\text { SPECIAL } \\
\text { Call Industrial Hygiene Department. }\end{array}$ & $\begin{array}{l}\text { RESPIRATOR } \\
\text { Organic filter }\end{array}$ \\
\hline
\end{tabular}

OTHER PROTECTIVE EQUIPMENT

Self-contained breathing apparatus

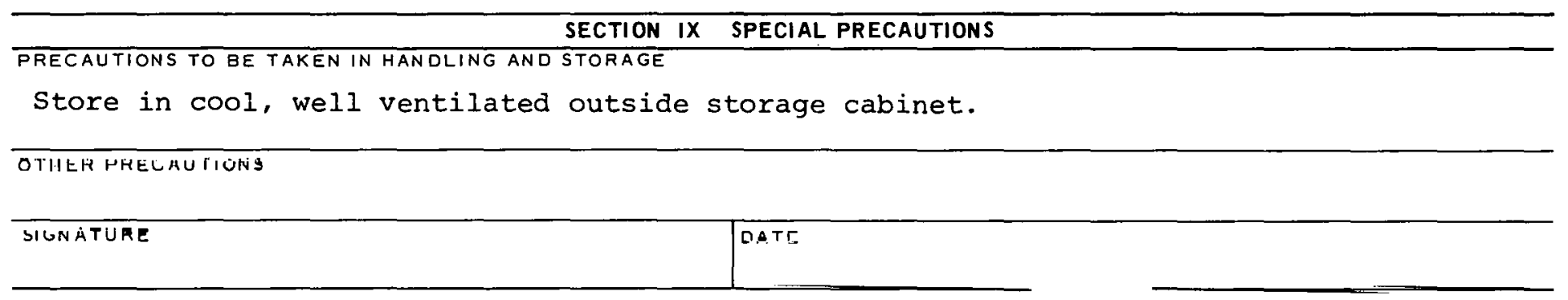


CAS : 000064197

PRODUCT DESIGNATION

Acetic Acid

03-201-0200
MATERIAL SAFETY

DATA SHEET

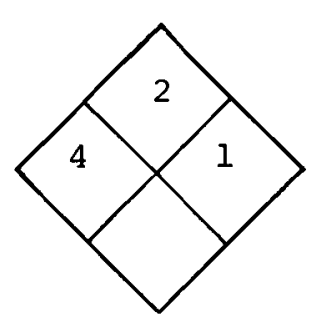

\section{SECTION I SOURCE AND NOMENCLATURE}

MANUFACTURER'S NAME

AODRESS INUMBER, STREET, CITY, STATE, ZIP CODE

TRADE NAME AND SYNONYMS

Vinegar acid Etharoic Acid

CEE TICAL NAME AND SYNONYMS

Acetic acid

CHEMICAL FAMILY

Organic acid

FORMULA

$\mathrm{CH}_{3} \mathrm{COOH}$

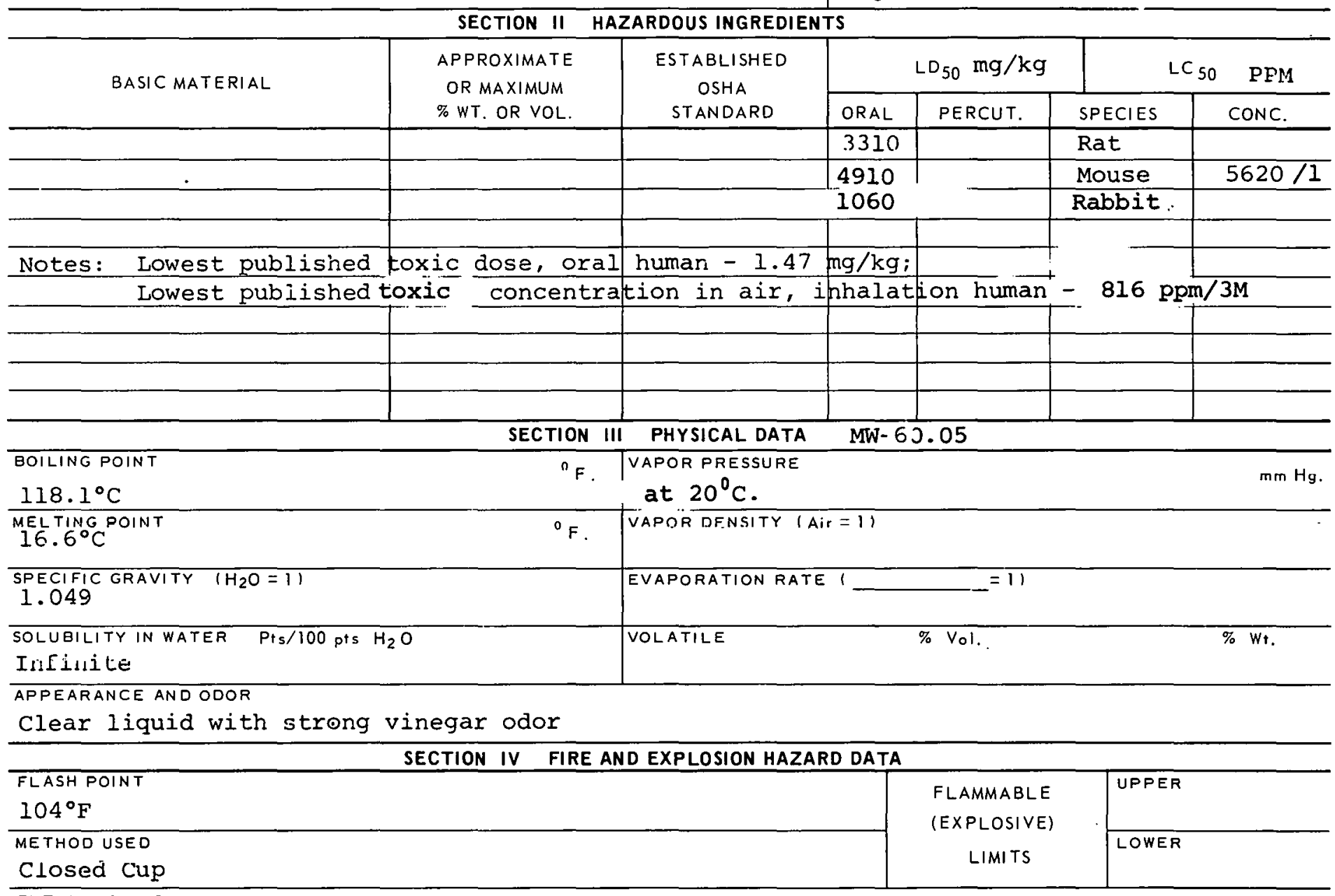

EXTINGUISHING MEDIA

Water spray, dry chenical, casbön aioxide or alcohol foam.

SPECIAL FIRE FIGHTING PROCEDURES

UNUSUAL FIRE AND EXPLOSION HAZAROS

Emits flammable vapors above $104^{\circ} \mathrm{F}$. May form explosive mixture with air. 


\begin{tabular}{|c|}
\hline $\begin{array}{l}\text { PRODUCT DESIGNATION } \\
\text { Acetic ACId }\end{array}$ \\
\hline SECTION $V$ HEALTH HAZARD DATA \\
\hline \\
\hline TLV $10 \mathrm{pPm}, 25 \mathrm{mg} / \mathrm{C.M}$ \\
\hline $\begin{array}{l}\text { PRINCIPLE ROUTES OF ABSORBTION } \\
\text { Inhalation, ingestion }\end{array}$ \\
\hline $\begin{array}{l}\text { REVELANT SYMPTOMS OF EXPOSURE Irritation of eyes, skin and respiratory system; destruction of } \\
\text { mucous membranes of the digestive tract. }\end{array}$ \\
\hline EFFECTS OF CHRONIC EXPOSURE \\
\hline $\begin{array}{l}\text { EMERGENCY AND FIRST AID PROCEDURES } \\
\text { Inhalation - remove from exposure, rest, keep warm, get medical aid; if swallowed - wash } \\
\text { mouth, drink water, milk of magnesia, get medical aid; wash from skin and eyes. }\end{array}$ \\
\hline SECTION VI REACTIVITY DATA \\
\hline CONDITIONS CONTRIBUTING TO INSTABILITY \\
\hline CONDITIONS CONTRIBUTING TO HAZARDOUS POLYMERIZATION \\
\hline \multirow{2}{*}{$\begin{array}{l}\text { INCOMPATABILITY (MATERIALS TO AVOID) } \\
\text { ChYOMIC acid, SOdIUM perOXide, nitric acid. } \\
\text { HAZAROOUS DECOMPOSITIOH RROOUETS }\end{array}$} \\
\hline \\
\hline SECTION VII SPILL OR LEAK PROCEDURES \\
\hline
\end{tabular}

STEPS TO BE TAKEN IN CASE MATERIAL IS RELEASED OR SPILLED

WASTE DISPOSAL METHOD

\begin{tabular}{|c|c|c|}
\hline \multicolumn{3}{|c|}{ SECTION VIII SPECIAL PROTECTION INFORMATION } \\
\hline $\begin{array}{l}\text { VENTILATION REQUIREMENTS LOCAL EXIIAUST } \\
\text { Consult Industrial HYgiene Department }\end{array}$ & & $\begin{array}{l}\text { PRUTECIIVE EQUIPMENT (SPECIFY TYPES) EYE } \\
\text { Goggles }\end{array}$ \\
\hline $\begin{array}{l}\text { MECHANICAL (GENERAL) } \\
\text { Consult Industrial Hygiene Department }\end{array}$ & c & $\begin{array}{l}\text { GLOVES } \\
\text { Rubber gloves }\end{array}$ \\
\hline $\begin{array}{l}\text { Consult Industrial Hygiene Department } \\
\text { Consul }\end{array}$ & & $\begin{array}{l}\text { REGHIRAlor } \\
\text { with organic filter }\end{array}$ \\
\hline \multicolumn{3}{|l|}{ OTHER PROTECTIVE EQUIPMENT } \\
\hline \multicolumn{3}{|c|}{$\begin{array}{l}\text { PRECAUTIONS TO BE TAKEN IN HANDLING AND STORAGE } \\
\text { Outside or detached storage is preferred, Keep from oxidizing materials. Keep } \\
\text { above freezing point. }\end{array}$} \\
\hline \multicolumn{3}{|l|}{ OTHER PRFSALTIONS } \\
\hline & & \\
\hline
\end{tabular}


CAS : 000098500

PRODUCT DESIGNATION

P-Arsanilic Acid

03-201-2102
CF78750

\section{MATERIAL SAFETY \\ DATA SHEET}

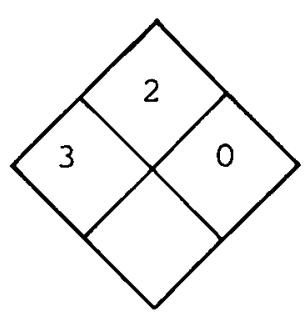

SECTION I SOURCE AND NOMENCLATURE

\section{TRADE NAME AND SYNONYMS \\ CHEMICAL NAME AND SYNONYMS \\ P-Aminobenzenearsonic acid}

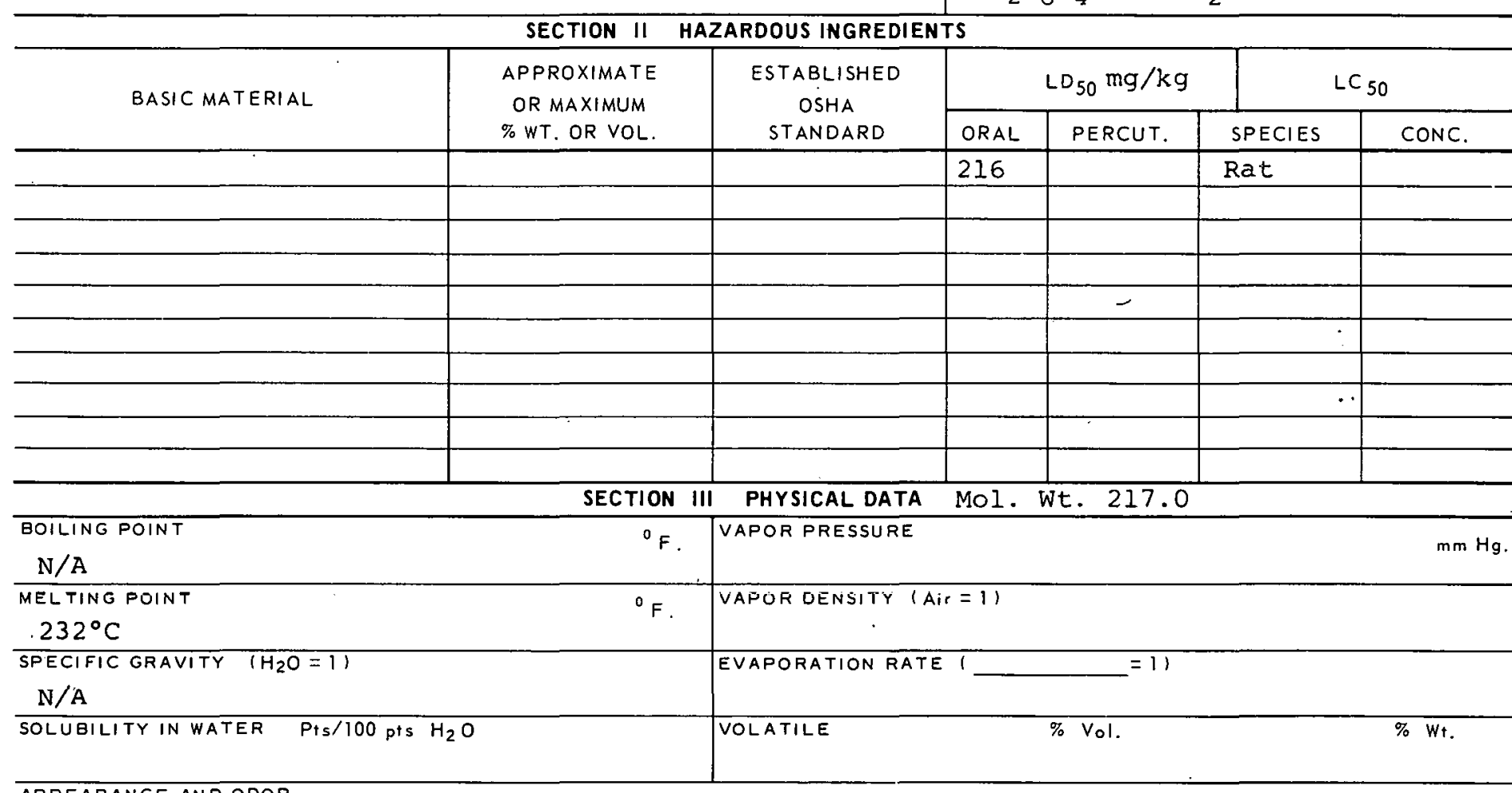

\section{APPEARANCE AND ODOR}

White crystaline powder

SECTION IV FIRE AND EXPLOSION HAZARD DATA

FLASH POINT
N/A
METHOD USED
N/A
EXTINGUISHING MEDIA
$N / A$
SPECIAL FIRE FIGHTING PROCEDURES
N/A

UNUSUAL FIRE AND EXPLOSION HAZARDS

Decomposed by heat to yield flammable vapors 


\section{TOXIC LEVEL}

TLV $0.72 \mathrm{mg} / \mathrm{C} . \mathrm{M}$.

PRINCIPLE ROUTES OF ABSORBTION

Inhalation, ingestion, skin absorption
CARCINOGENIC

No

SKIN AND EYE

IRRITATION Moderate

REVELANT SYMPTOMS OF EXPOSURE Burning of mouth and throat, gastroenteric pain, vomiting, diarrhea, hematuris, dehydration, oliguria and collapse

EFFECTS OF CHRONIC EXPOSURE

Arsenic poisoning and skin ulceration, falling out of hair, loss of appetite

EMERGENCY AND FIRST AID PROCEDURES Inhalation - rest, get medical attention; if swall lowed wash mouth, gastric lavage with $1 \%$ sodium thiosulfate; wash skin and eyes, get medical attention.

\section{SECTION VI REACTIVITY DATA}

CONDITIONS CONTRIBUTING TO INSTABILITY

CONDITIONS CONTRIBUTING TO HAZARDOUS POLYMERIZATION

TPIGOMPATARILITY (MATERIALSTOAVOIO)

HA ZARMOIIS DFROMPRSITION PRODUGTS

SECTION VII SPILL OR LEAK PROCEDURES

STEPS TO BE TAKEN IN CASE MATERIAL IS RELEASED OR SPILLED

WASTE DISPOSAL METHOD

SECTION VIII SPECIAL PROTECTION INFORMATION

\begin{tabular}{l|l}
\hline VENTILATION REQUIREMENTS LOCAL EXHAUST & $\begin{array}{l}\text { HHUTECIIVE EQUIPMENT (SPECIFY TYPESI EYE } \\
\text { GOgGl P.S }\end{array}$ \\
\hline MECHANICAL IGENERAL) & $\begin{array}{l}\text { GLOVES } \\
\text { Rubber }\end{array}$ \\
\hline SPECIAL & RESPIRATOR \\
WIth appropriate filter
\end{tabular}

OTHER PROTECTIVE EQUIPMENT

\section{SECTION IX SPECIAL PRECAUTIONS}

PRECAUTIONS TO BE TAKEN IN HANDLING AND STORAGE

OTHER PRECAUTIONS

SIGNATURE

DATE

$201-2102 B$ 


\begin{tabular}{|l|}
\hline PRODUCT DESIGNATION \\
Bromoacetic Acid \\
$03-201-4004$ \\
\end{tabular} \\ MATERIAL SAFETY DATA SHEET}

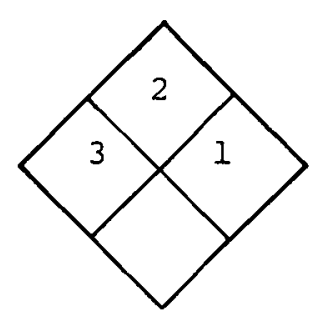

SECTION I SOURCE AND NOMENCLATURE

MANUFAC TURER'S NAME
ADDRESS INUMBER, STREEY, CITY,
TRADE NAME AND SYNONYMS
CHEMICAL NAME AND SYNONYMS
BrOMaCETIC ACIA

\section{SECTION II HAZARDOUS INGREDIENTS}

BASIC MATERIAL

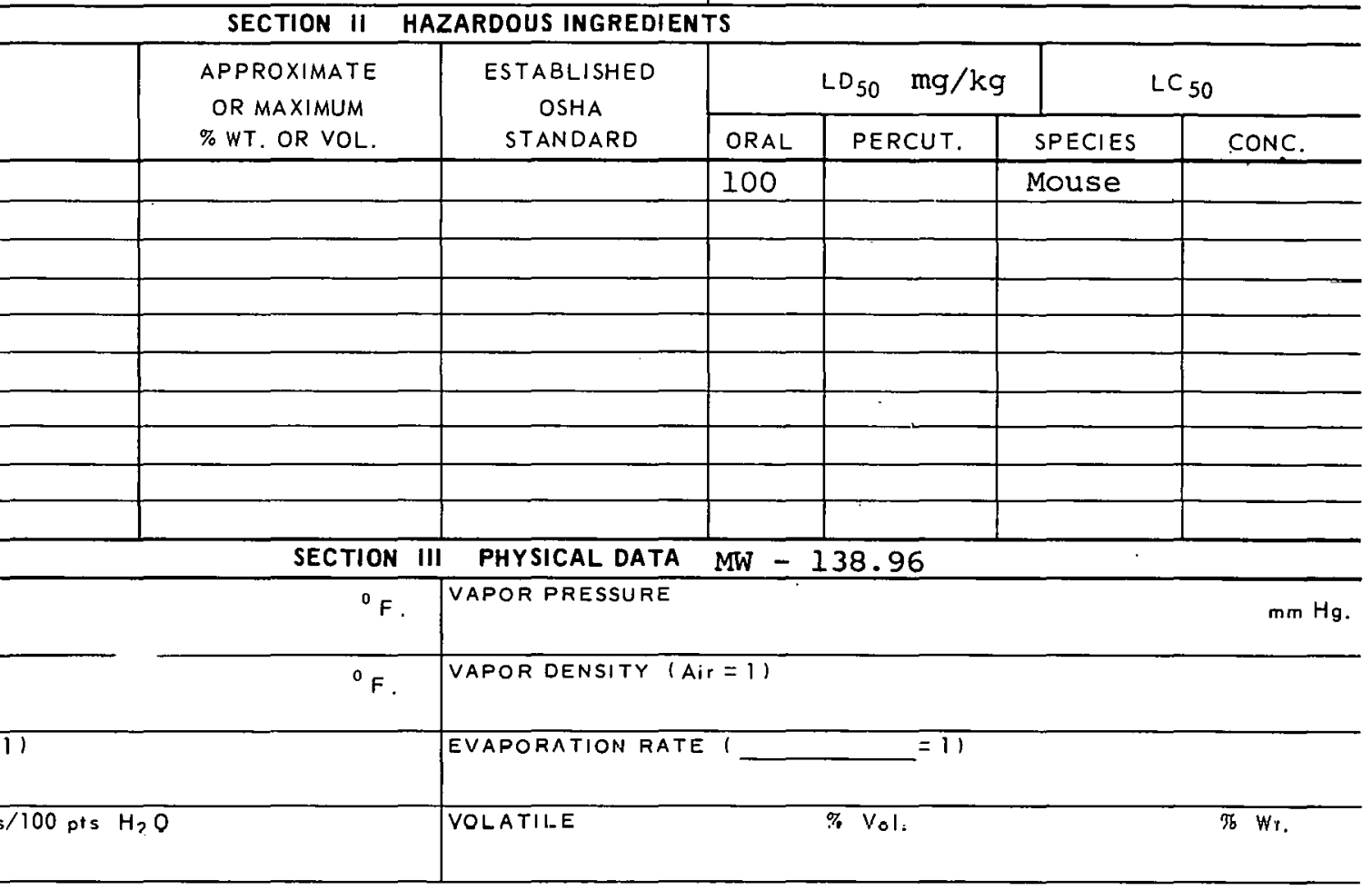

\section{BOILING POINT}

$208^{\circ} \mathrm{C}$

MEL TING POINT

$50^{\circ} \mathrm{C}$

SPECIFIC GRAVITY $\left(\mathrm{H}_{2} \mathrm{O}=1\right)$

1.94

SOLUBILITY IN WATER Pts/100 pts $\mathrm{H}_{2} \mathrm{O}$

Very soluble

APPEARANCE AND ODOR

Colorless crystals

SECTION IV FIRE AND EXPLOSION HAZARD DATA

FLASH POINT

None available

METHOD USED

EXTINGUISHING MEDIA

Use water spray, dry chemical, carbon dioxide alcohol foam.

SPECIAL FIRE FIGHTING PROCEDURES

Wear goggles \& self-contained breathing apparatus.

UNUSUAL FIRE AND EXPLOSION HAZARDS 


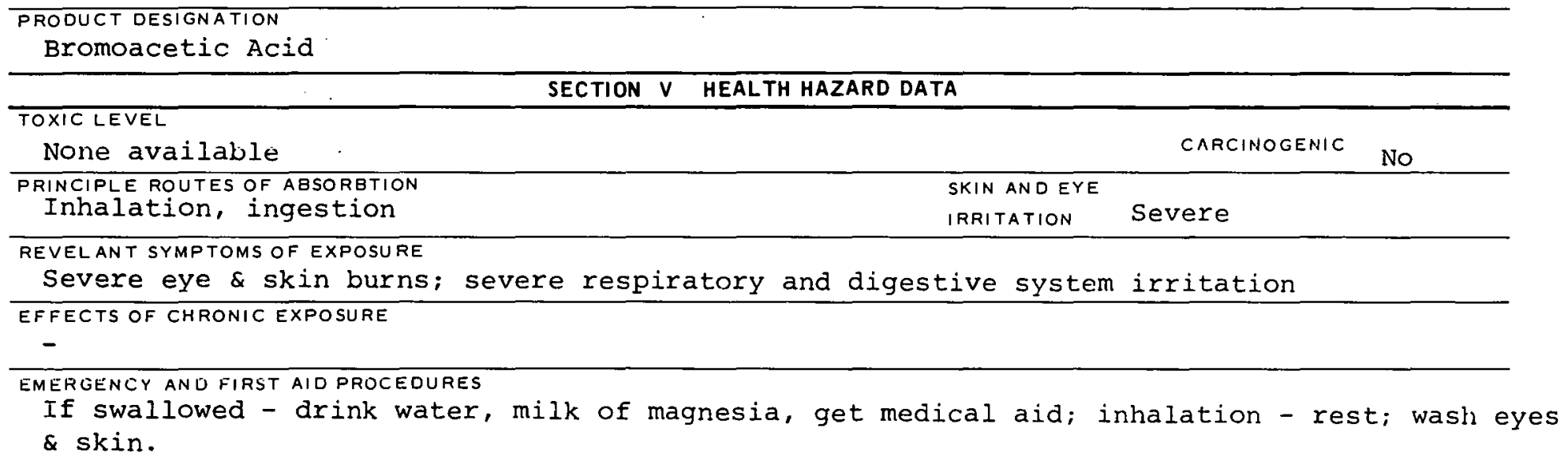


CAS - 000590921

\section{PRODUCT DESIGNATION}

R-Bromopropionic Acid

03-201-4454

\section{MATERIAL SAFETY \\ DATA SHEET}

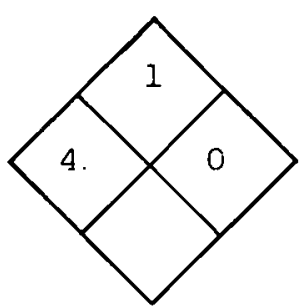

\section{SECTION I SOURCE AND NOMENCLATURE}

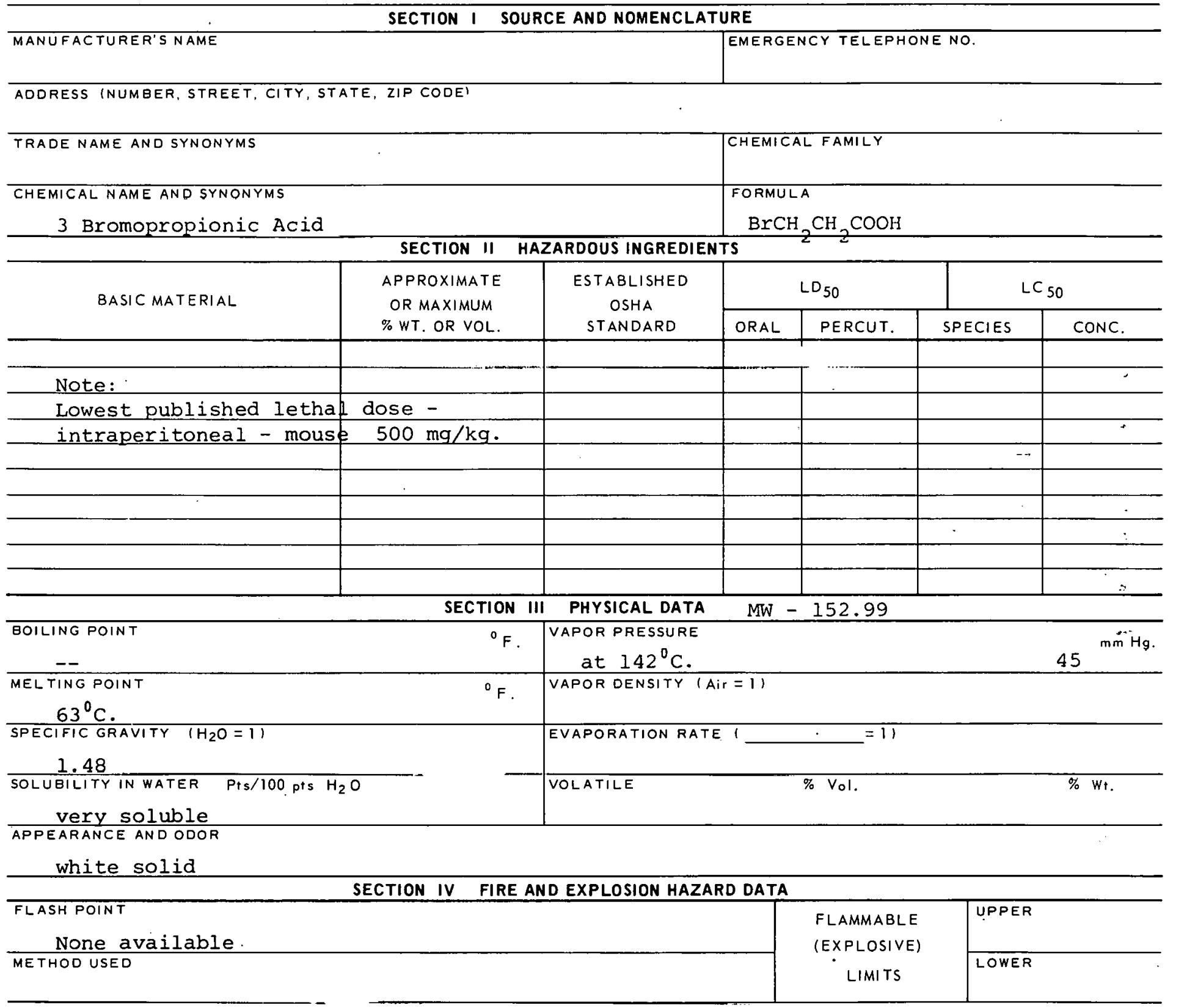

EXTINGUISHING MEDIA

Water, foam, carbon dioxide or dry chemical.

SPECIAL FIRE FIGHTING PROCEDURES

UNUSUAL FIRE AND EXPLOSION HAZARDS 
B-Bromopropionic Acid

SECTION $V$ HEALTH HAZARD DATA

\section{TOXIC LEVEL}

Nune available

PRINCIPLE ROUTES OF ABSORBTION

Inhalation, ingestion

CARCINOGENIC yes

SKIN AND EYE

IRRITATION SEvere

REVELANT SYMPTOMS OF EXPOSURE

Severe eye or skin burns, irritation of respiratory and digestive systems. EFFECTS OF CHRONIC EXPOSURE

$--$

EMERGENCY AND FIRST AID PROCEDURES If swallowed - drink water, milk of magnesia, get mediCal

aid; inhalation - rest; wash skin \& eyes.

CONDITIONS CONTRIBUTING TO INSTABILITY

CONDITIONS CONTRIBUTING TO HAZARDOUS POLYMERI ZATION

INCUMPAIABILITY IMAIERIALS IO AVUIUI

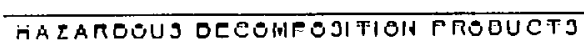

SECTION VII SPILL OR LEAK PROCEDURES

STEPS TO BE TAKEN IN CASE MATERIAL IS RELEASED OR SPILLED

WASTE DISPOSAL METHOD

SECTION VIII SPECIAL PROTECTION INFORMATION

\begin{tabular}{|c|c|}
\hline VENTILATION REQUIREMENTS LOCAL EXHAUST & $\begin{array}{l}\text { FROTLCTIVE EQUIPMENT ISPECIFY TYPES) EYE } \\
\text { GUg̈y̆lES }\end{array}$ \\
\hline MECHANICAL (GENERAL) & $\begin{array}{l}\text { GLOVES } \\
\text { Rubber }\end{array}$ \\
\hline SPFC.IAI. & $\begin{array}{l}\text { RFSBIRATOB } \\
\text { With proper filter }\end{array}$ \\
\hline
\end{tabular}

OTHER PROTECTIVE EQUIPMENT

SECTION IX SPECIAL PRECAUTIONS

PRECAUTIONS TO BE TAKEN IN HANDLING AND STORAGE

OTHER PRF, C.AIITIONS

SIGNATURE

DATE

$201-4454 B$ 


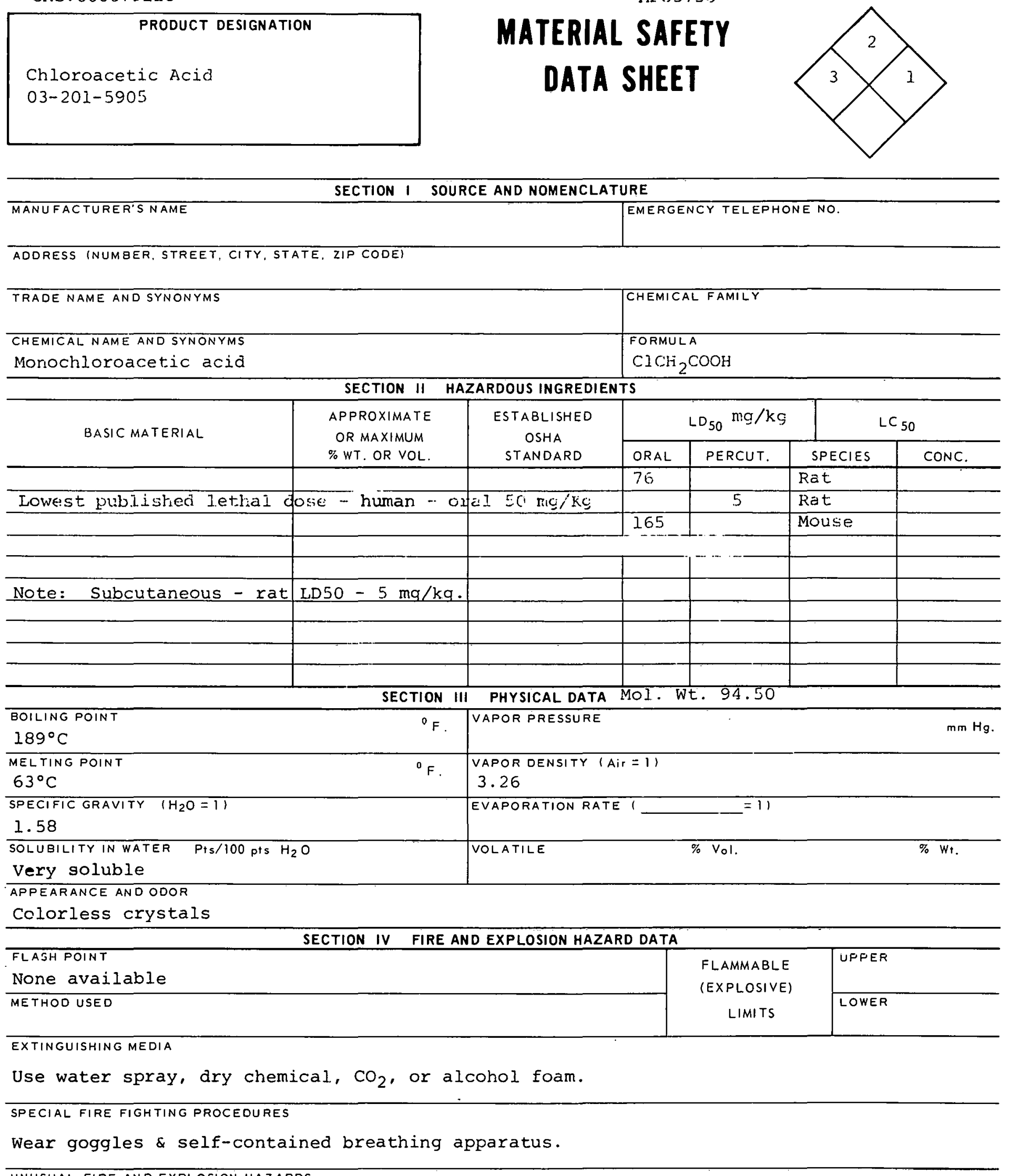




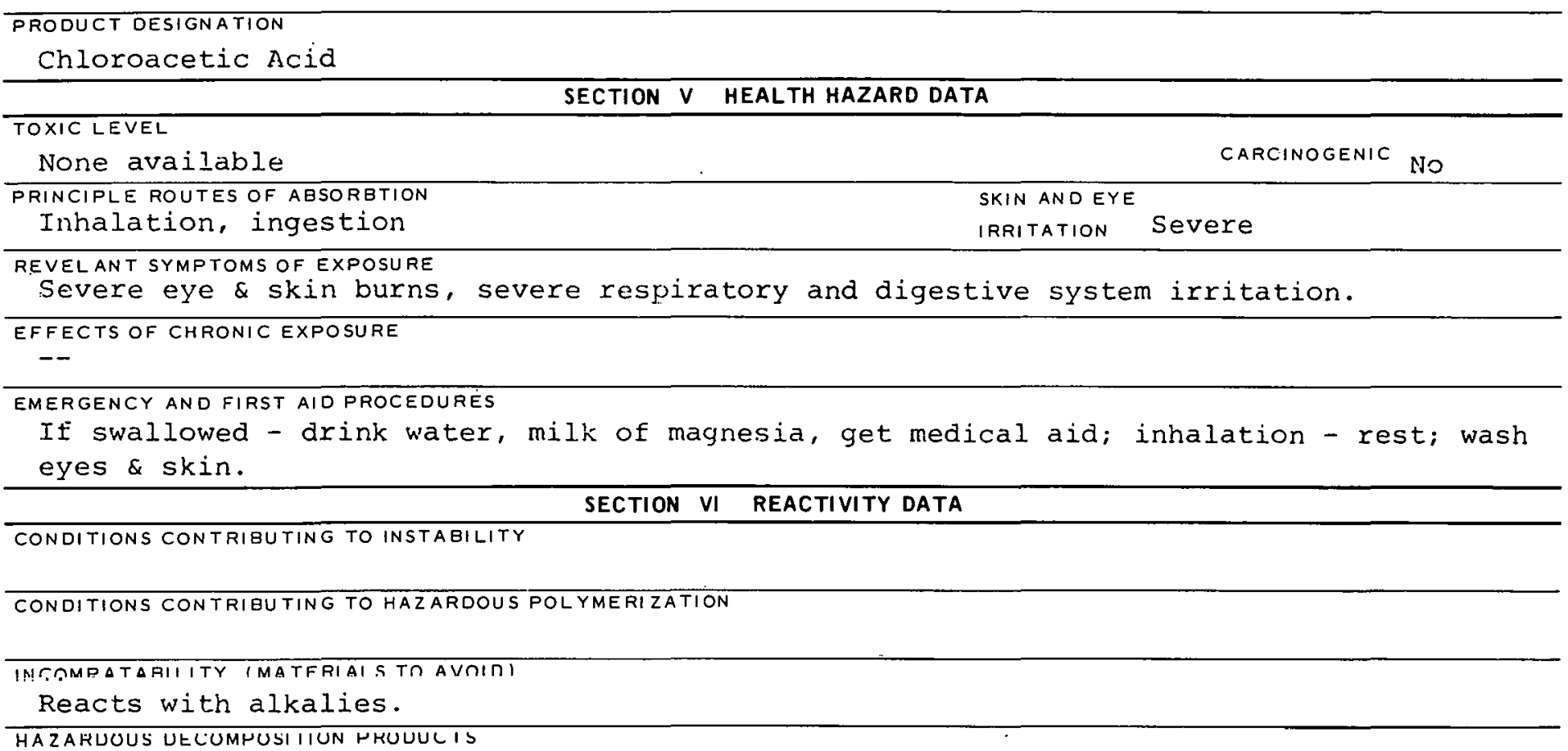

SECTION VII SPILL OR LEAK PROCEDURES

STEPS TO BE TAKEN IN CASE MATERIAL IS RELEASED OR SPILLED

WASTE DISPOSAL METHOD

Dissolve in combustible liquid and burn.

\section{SECTION VIII SPECIAL PROTECTION INFORMATION}

\begin{tabular}{l|l}
\hline VENTILATION REQUIREMENTS LOCAL EXHAUST & $\begin{array}{l}\text { PROTECTIVE EQUIPMENT (SPECIFY TYPES) EYE } \\
\text { GOggleS }\end{array}$ \\
\hline MECHANICAL IGENERAL) & $\begin{array}{l}\text { GLOVES } \\
\text { Rubber }\end{array}$ \\
\hline SPECIAL & $\begin{array}{l}\text { RESPIRATUR } \\
\text { W/proper filter }\end{array}$
\end{tabular}

OTHER PROTECTIVE EQUIPMENT

SECTION IX SPECIAL PRECAUTIONS

PRECAUTIONS TO BE TAKEN IN HANDLING AND STORAGE

Keep containers closed, store in a cool place.

OTHER PRECAUTIONS 


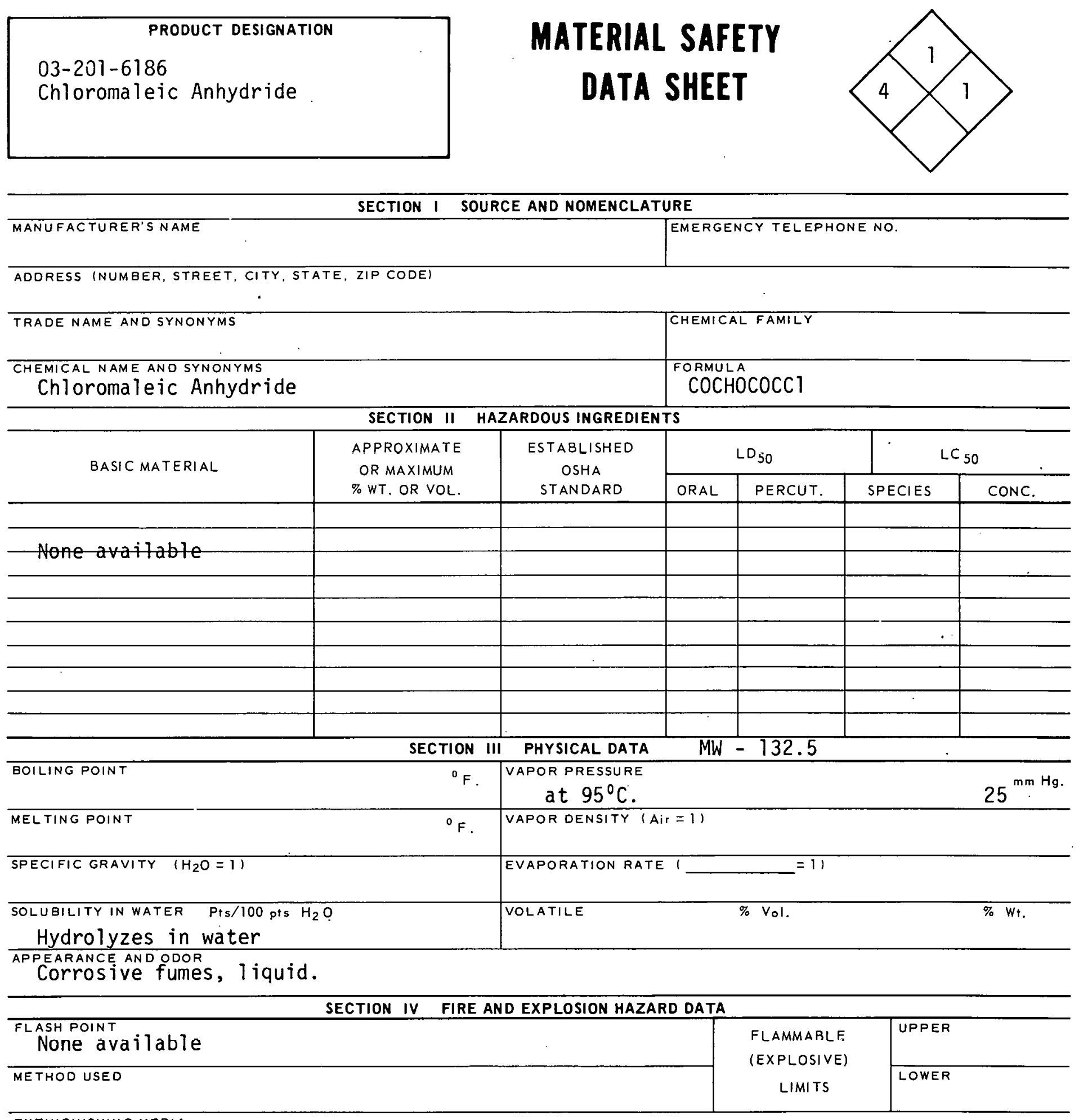

EXTINGUISHING MEDIA

Alcohol foam, dry chemical, carbon dioxide.

SPECIAL FIRE FIGHTING PROCEDURES

UNUSUAL FIRE AND EXPLOSION HAZAROS 


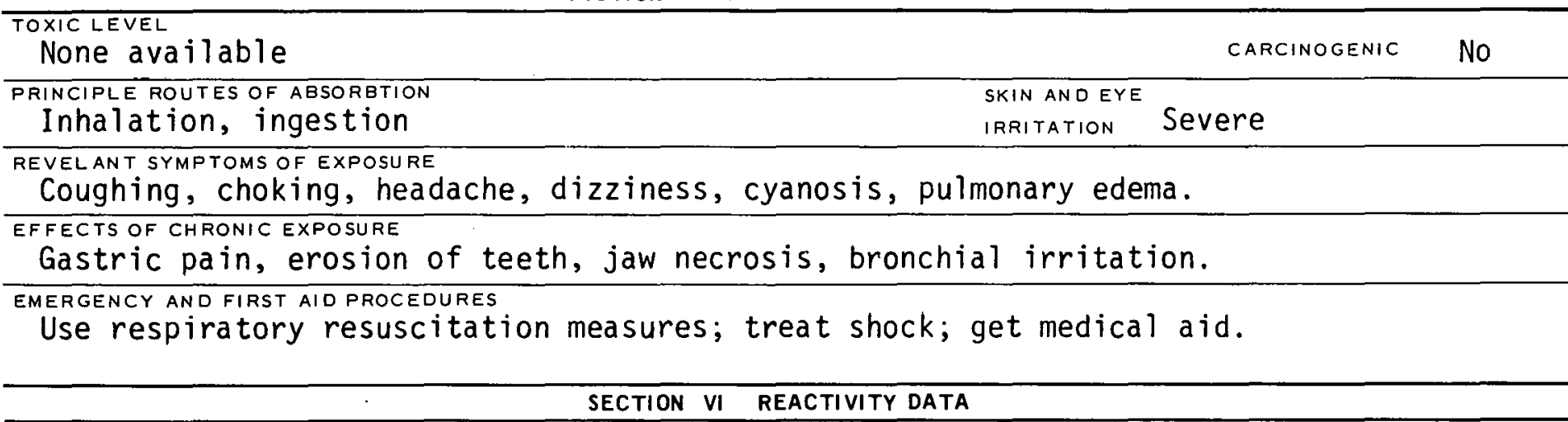

CONDITIONS CONTRIBUTING TO INSTABILITY

CONDITIONS CONTRIBUTING TO HAZARDOUS POLYMERIZATION

INCOMPATABILITY (MATERIALS TOAVOIO) ATkali metals, amines, calcium hydroxide, potasSiUm hydroxide, sodium hydroxide, pyridine, tertiary amines. HAZARDOUS DECOMPQS!T!QN PRQDUCTS

SECTION VII SPILL OR LEAK PROCEDURES

STEPS TO BE TAKEN IN CASE MATERIAL IS RELEASED OR SPILLED

WASTE DISPOSAL METHOD

SECTION VIII SPECIAL PROTECTION INFORMATION

\begin{tabular}{l|l}
\hline VENTILATION REQUIREMENTS LOCAL EXHAUST & $\begin{array}{l}\text { PROTECTIVE EQUIPMENT (SPECIFY TYPES) EYE } \\
\text { GOgg.les }\end{array}$ \\
\hline MECHANICAL (GENERAL) & $\begin{array}{l}\text { GLOVES } \\
\text { NeOPrene }\end{array}$ \\
\hline SPECIAL & $\begin{array}{c}\text { RESPIRATOR } \\
\text { W/chemical filter }\end{array}$ \\
\hline
\end{tabular}

CTHEH PROTECTIVE EQUITMENT

Rubber apron, full-face shield

SECTION IX SPECIAL PRECAUTIONS

PRECAUTIONS TO BE TAKEN IN HANDLING AND STORAGE

Protect from moisture and oxidizing materials. Store in cool, dry, well-ventilated area where fire hazard is not acute.

OTHER PRECAUTIONS 


\section{PRODUCT DESIGNATION}

$03-201-6706$

Chlorosulfonic Acid
MATERIAL SAFETY

DATA SHEET

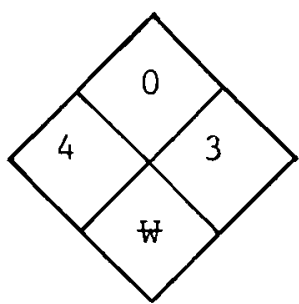

\section{SECTION I SOURCE AND NOMENCLATURE}

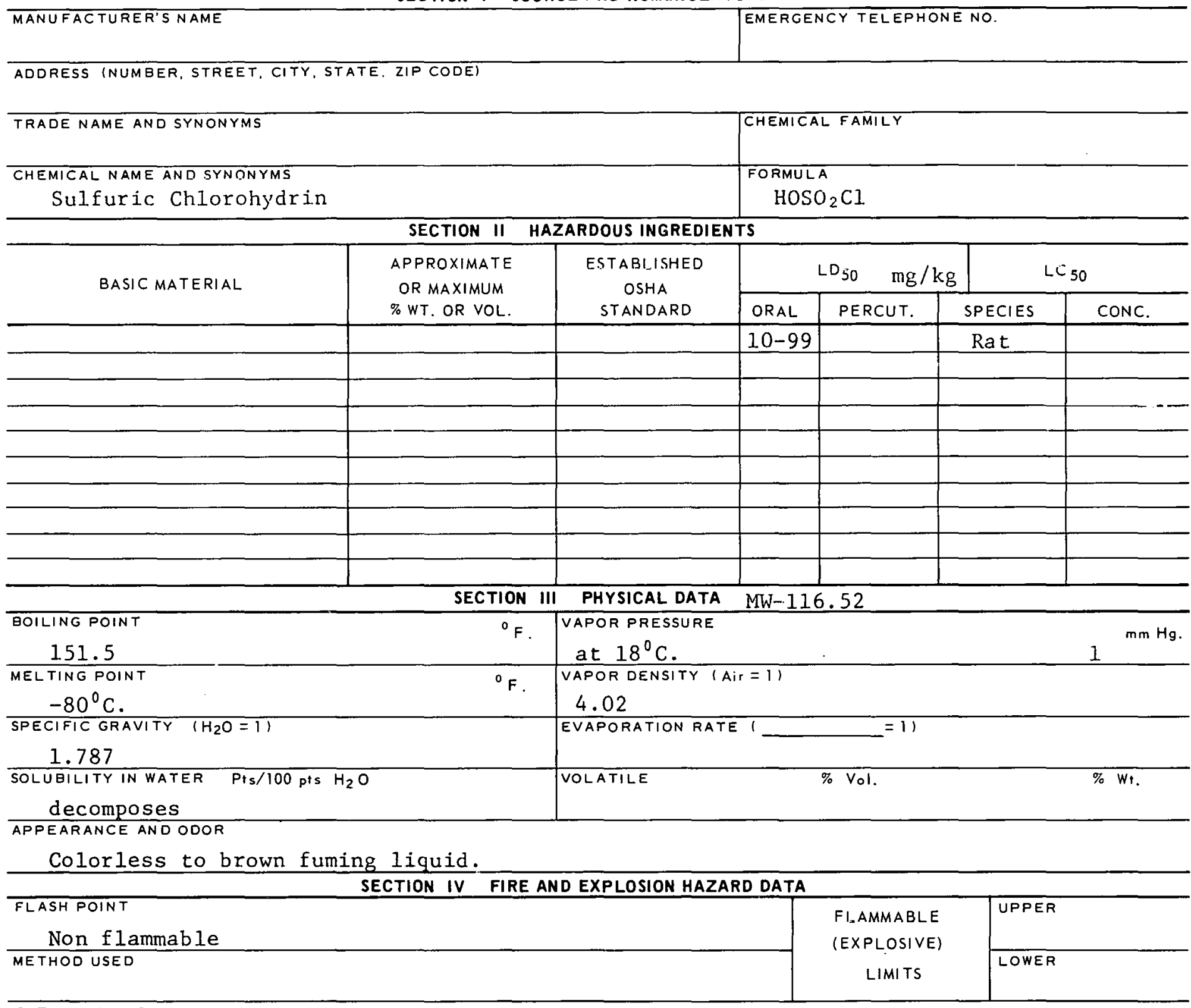

EXTINGUISHING MEDIA

Water may be used in an area but not directly on the chemical.

SPECIAL FIRE FIGHTING PROCEDURES

Avoid water contact with chlorosulfonic acid since reaction can proceed with

explosive violence.

UNUSUAL FIRE AND EXPLOSION HAZARDS 
Chlorosulfonic Acid

SECTION $V$ HEALTH HAZARD DATA

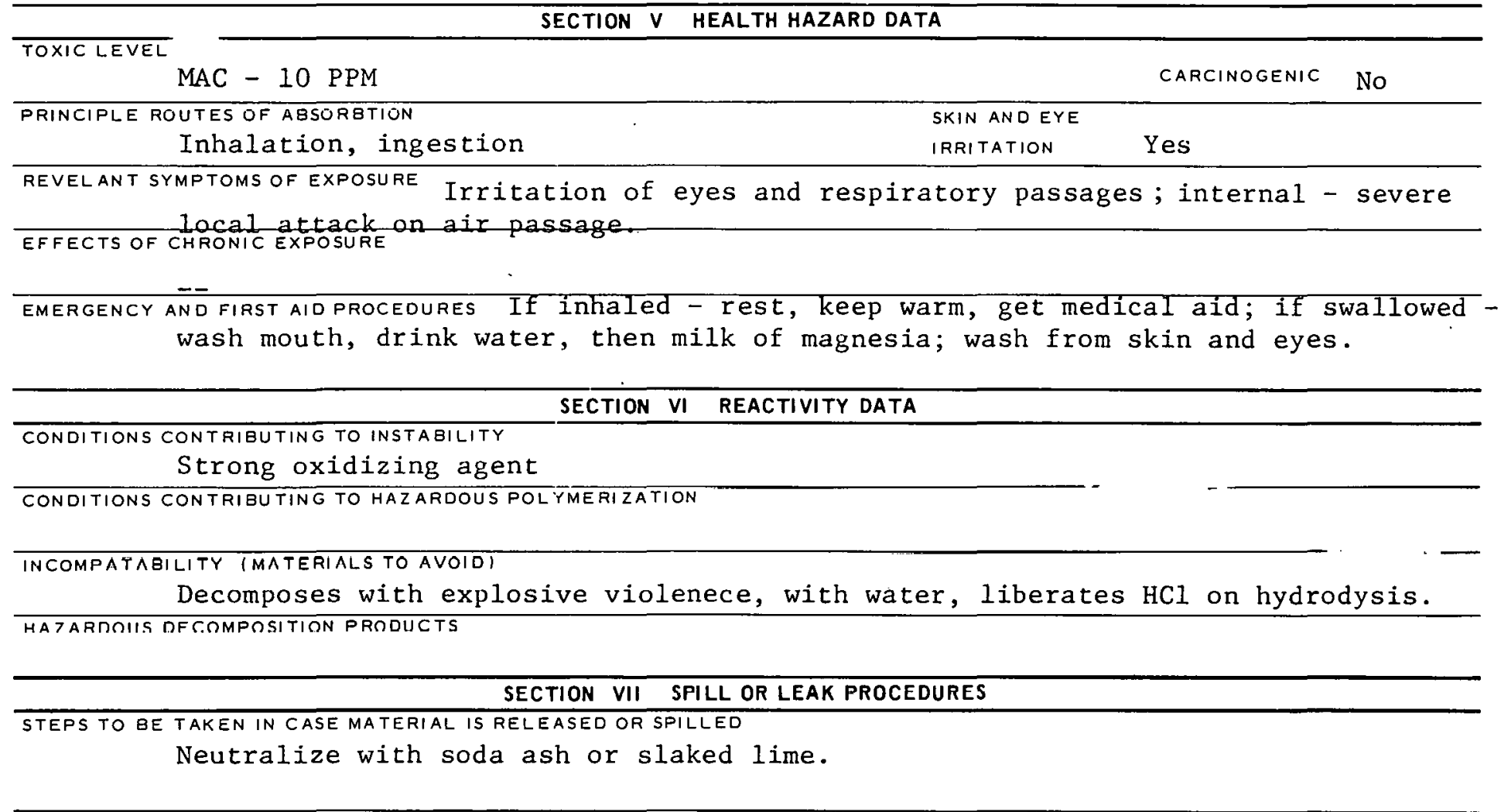

WASTE DISPOSAL METHOD

SECTION VIII SPECIAL PROTECTION INFORMATION

\begin{tabular}{l|l}
\multicolumn{1}{c}{ SECTION VIII SPECIAL PROTECTION INFORMATION } \\
\hline VENTILATION REQUIREMENTS LOCAL EXHAUST & $\begin{array}{c}\text { PROTECTIVE EQUIPMENT (SPECIFY TYPES) EYE } \\
\text { SafETY glaSSES }\end{array}$ \\
\hline MELHANILAL IGENERALI & GLOVES \\
\hline SPECIAL & $\begin{array}{c}\text { RESPIRATOR } \\
\text { WIth proper f11ter }\end{array}$ \\
\hline
\end{tabular}

OTHER PROTECTIVE EQUIPMENT

SECTION IX SPECIAL PRECAUTIONS

PRECAUTIONS TO BE TAKEN IN HANDLING AND STORAGE 


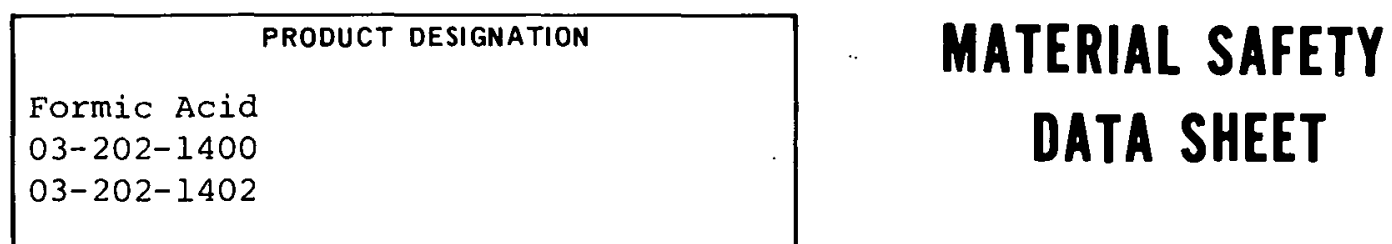

SECTION I SOURCE AND NOMENCLATURE

MANUFACTURER'S NAME

ADORESS (NUMBER, STREET, CITY, STATE, ZIP CODE)

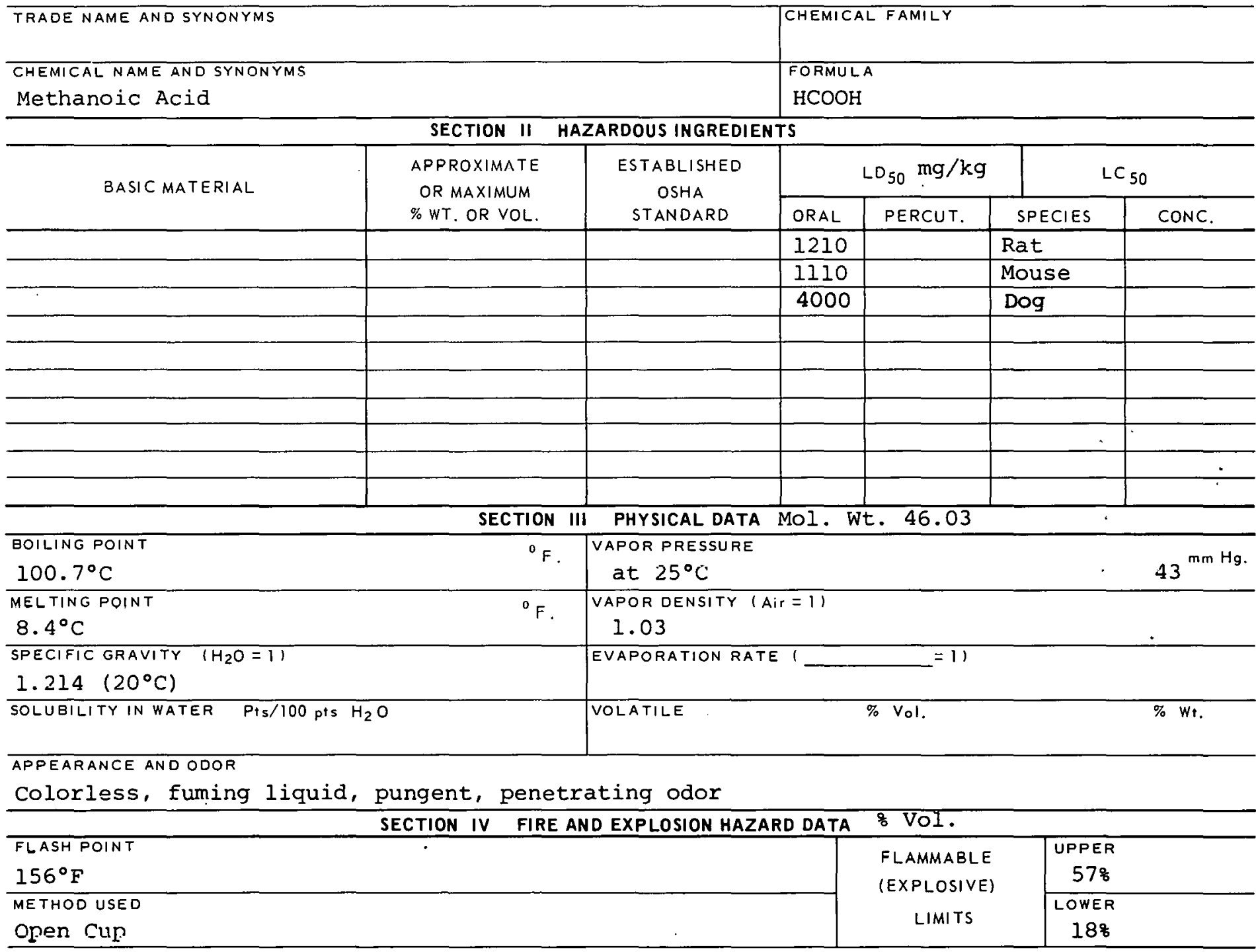

EXTINGUISHING MEDIA

water spray, dry chemical, "alcohol" foam, or carbon dioxide.

SPECIAL FIRE FIGHTING PROCEDURES

UNUSUAL FIRE AND EXPLOSION HAZARDS 
TOXIC LEVEL

TLV $5 \mathrm{ppm}, 9 \mathrm{mg} / \mathrm{C} . \mathrm{M}$.

PRINCIPLE ROUTES OF ABSORBTION Percutaneous absorption of

liquid or inhalation of vapor, ingestion

REVELANT SYMPTOMS OF EXPOSURE Irritating to mucous membranes, salivation, burning sensation in mouth and pharynx, vomiting, hematemesis, diarrhea, severe abdominal pain.

EFFECTS OF CHRONIC EXPOSURE conjunctival edema.

Erosion of teeth, jaw neurosis, bronchial irritation, possible gastrointestinal disturbances. EMERGENCY AND FIRST AID PROCEOURES Remove from exposure - rest, keep warm, obtain medical attention. Use respiratory resuscitation measure if necessary. For skin contact - flood effected area with $\mathrm{H}_{2} \mathrm{O}$, treat shock if necessary.

\section{SECTION VI REACTIVITY DATA}

CONDITIONS CONTRIBUTING TO INSTABILITY

CONDITIONS CONTRIBU TING TO HAZARDOUS POLYMERIZATION

INCOMPATABILITY (MATERIALS TO AVOID)

Furfuryl alcohol, hydrogen peroxide.

HAZARDOUS DECOMPOSITION PRODUCTS

SECTION VII SPILL OR LEAK PROCEDURES

STEPS TO BE TAKEN IN CASE MATERIAL IS RELEASED OR SPILLED

Use water spray.

WASTE DISPOSAL METHOD

SECTION VIII SPECIAL PROTECTION INFORMATION

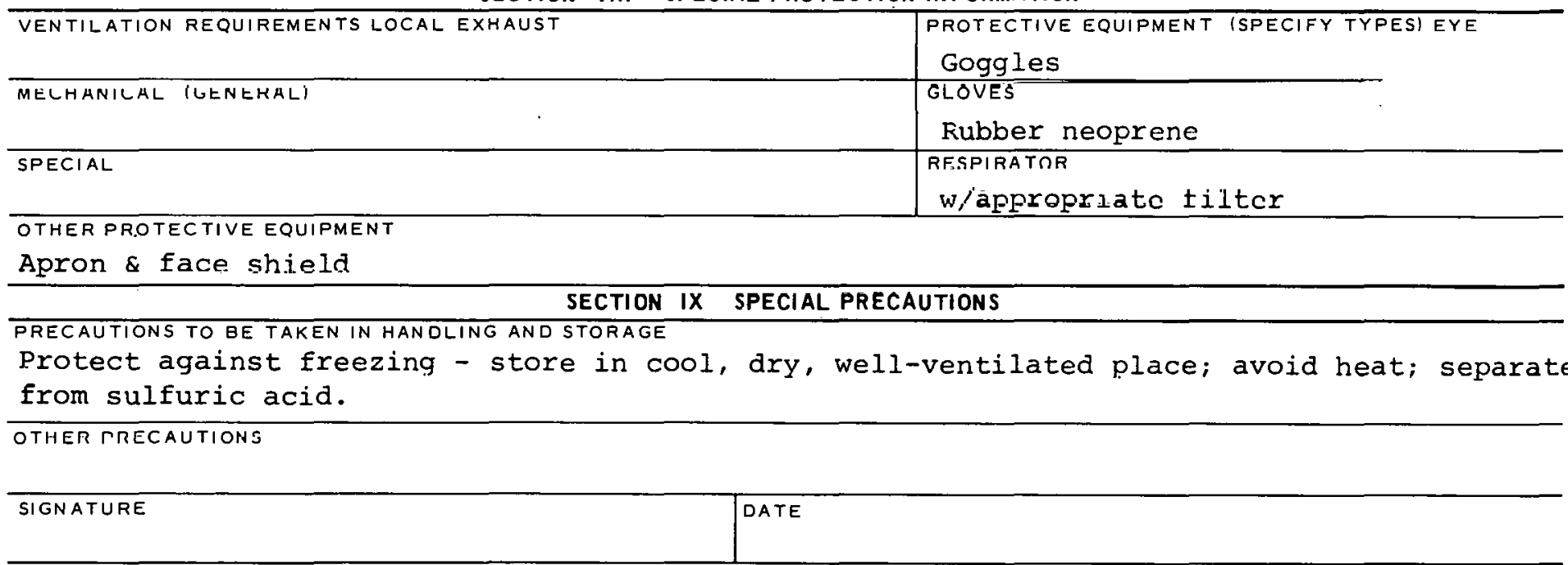


03-202-2903

8 Hydroxy-5-Quinoline

Sulfonic Acid
MATERIAL SAFETY

DATA SHEET

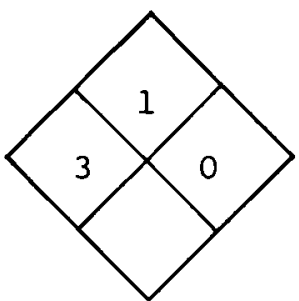

SECTION I SOURCE AND NOMENCLATURE

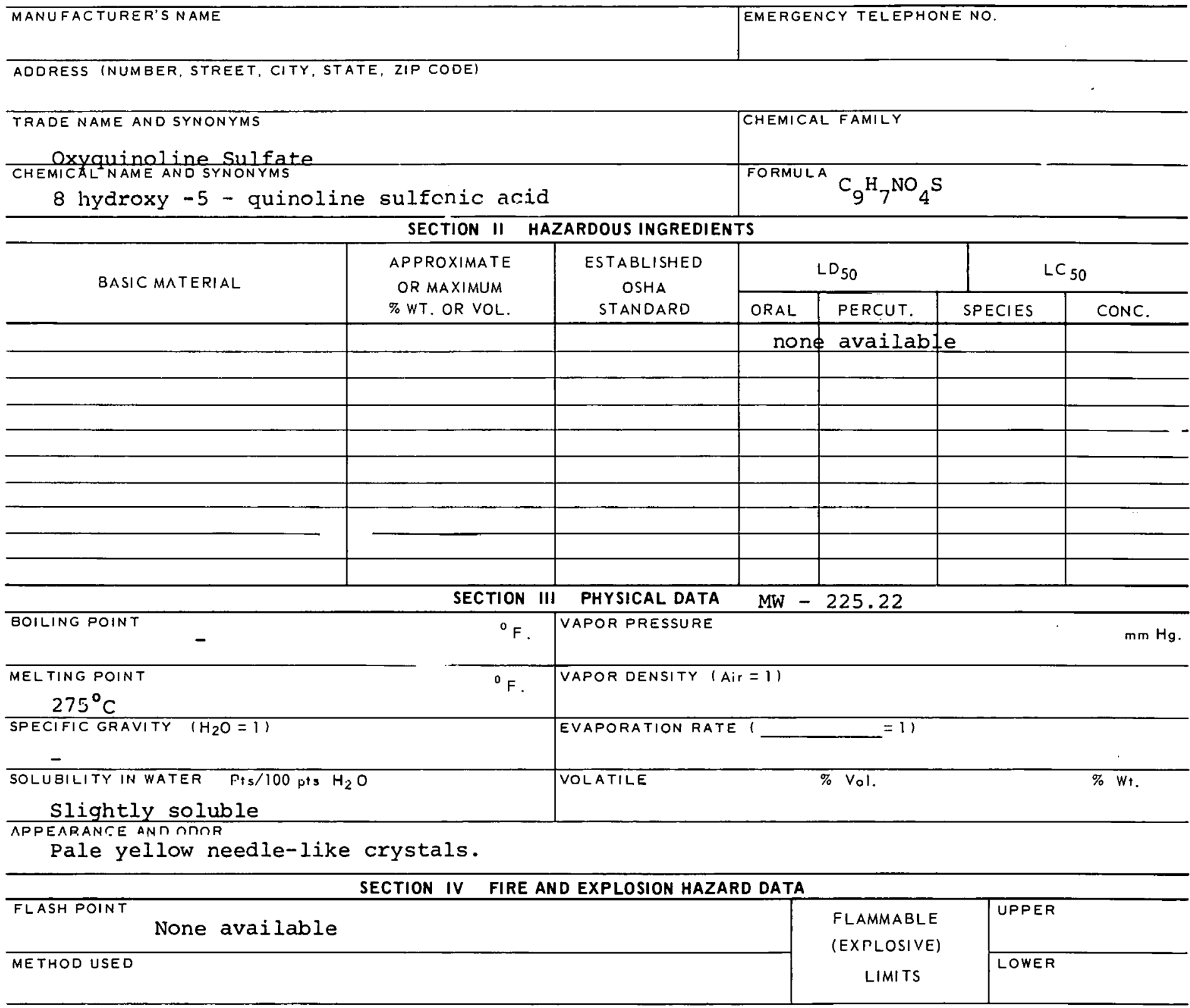

ENTINOUISHING MEDIA

Carbon dioxide, dry chemical

SPECIAL FIRE FIGHTING PROCEDURES

UNUSUAL FIRE ANDEXPLOSION HAZARDS 
8 hydroxy - 5 Quinoline Sulfonic Acid SECTION $V$ HEALTH HAZARD DATA

TOXIC LEVEL

None available

PRINCIPLE ROUTES OF ABSORETION

Inhalation, ingestion

REVELANT SYMPTOMS OF EXPOSURE Retina damage, eye \& mucous membrane irritation, cyanosis,

headache, dizziness.

EFFECTS OF CHRONIC EXPOSURE

Possible liver damage

EMERGENCY AND FIRST AID PROCEDURES If swallowed - induce vomiting, get medical aid; inhalation rest; wash from skin and eyes.

CONDITIONS CONTRIBUTING TO INSTABILITY

CONDITIONS CONTRIBUTING TO HAZARDOUS POLYMERIZATION

INCOMPATABII ITY (MATFBIALS TO AVQID)

HAZARDOUS DECOMPOSITION PRODUCTS

SECTION VII SPILL OR LEAK PROCEDURES

STEPS TO BE TAKEN IN CASE MATERIAL IS RELEASED OR SPILLED

\section{WASTE DISPOSAL METHOD}

\begin{tabular}{|c|c|c|c|}
\hline & SECTION VIII & SPECIAL PROTECTION & INFORMATION \\
\hline VENTILATION & REQUIREMENTS LOCAL EXHAUST & & $\begin{array}{l}\text { PROTECTIVE EQUIPMENT (SPECIFY TYPES) EYL } \\
\text { Goggles }\end{array}$ \\
\hline MECHANICAL & (GENERAL) & & GLUVES \\
\hline SPECIAL & & & $\begin{array}{l}\text { With proper filter } \\
\text { WiRATOR }\end{array}$ \\
\hline
\end{tabular}

\section{SECTION IX SPECIAL PRECAUTIONS}

PRECAUTIONS TO BE TAKEN IN HANDLING AND STORAGE

OTHER PRECAUTIONS

SIGNATURE

UAIE

$202-2903 B$ 


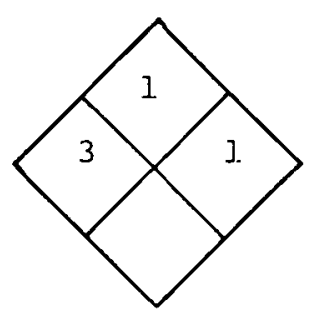

\section{SECTION I SOURCE AND NOMENCLATURE}

\section{MANUFACTURER'S N'AME ADDRESS (NUMBER, STREET, CITY, STATE, ZIP CODE)

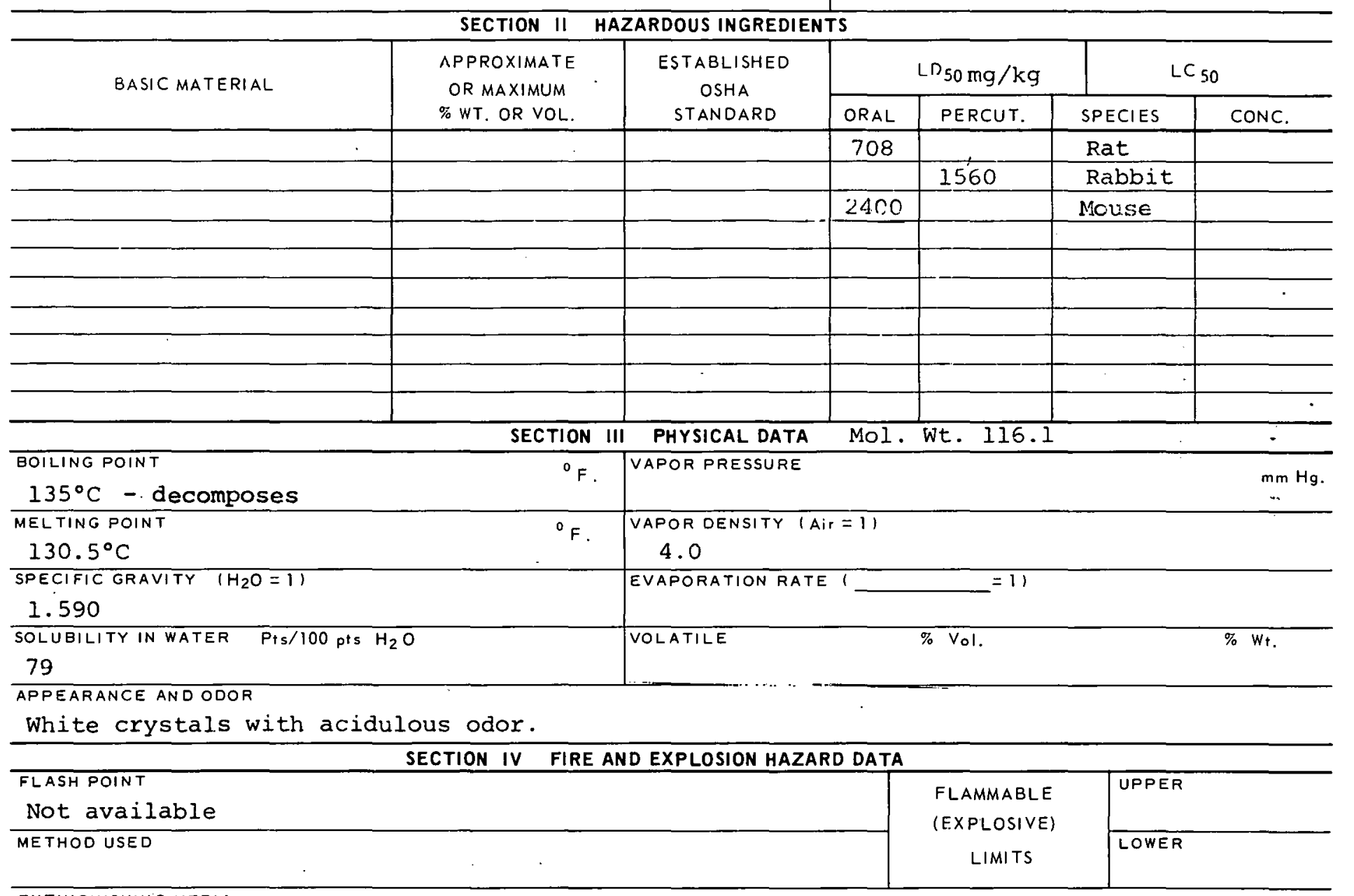

EXTINGUISHING.MEDIA

Ure alcohol fnam, carbon dioxide or dry chemical.

SPECIAL FIRE FIGHTING PROCEOURES

Wear full protective clothing.

UNUSUAL FIRE AND EXPLOSION HAZARDS

Volatile solid gives off flammable vapor when solid. 


\section{SECTION $V$ HEALTH HAZARD DATA}

TOXIC LEVEL

Not available

PRINCIPLE ROUTLS OF ABSORBTION

Inhalation, ingestion

REVELANT SYMPTOMS OF EXPOSURE

respiratory depression.

EFFECTS OF CHRONIC EXPOSURE

Errosion of teeth, bronchial irritation, gastrointestinal disturbances

EMERGENCY AND FIRST AID PROCEDURES

Inhalation - rest, get medical aid if necessary; ingestion - wash mouth, drink water, get medical aid; wash from skin \& eyes.

\section{SECTION VI REACTIVITY DATA}

CARCINOGENIC NO

SKIN AND EYE

IRRITATION SEvere
Gastric pain, headache, nausea and vomiting, cough, tearing and

CONDITIONS CONTRIBUTING TO INSTABILITY

CONDITIONS CONTRIBUTING TO HAZARDOUS POLYMERIZATION

INCOMPATABILITY (MATERIALS TO AVOID) Alkali metale, aminco, caleium liyuluxide, potassium

hydroxide, sodium hydroxide and pyridine

HAZARDQUS DECOMPOSITION PRONIIT TS

Gives ofr Flammable vapors when heated

SECTION VII SPILL OR LEAK PROCEDURES

STEPS TO BE TAKEN IN CASE MATERIAL IS RELEASED OR SPILLED

WASTE DISPOSAL METHOD

SECTION VIII SPECIAL PROTECTION INFORMATION

\begin{tabular}{l|l}
\hline VENTILATION REQUIREMENTS LOCAL EXHAUST & $\begin{array}{l}\text { PROTECTIVE EQUIPMENT ISPECIFY TYPESI FYE } \\
\text { Safety gOggles }\end{array}$ \\
\hline MECHANICAL IGENERALI & $\begin{array}{l}\text { GLOVES } \\
\text { Rubber }\end{array}$ \\
\hline SPECIAL & $\begin{array}{l}\text { RE3FIRATUR } \\
\text { w/proper Eilter }\end{array}$ \\
\hline
\end{tabular}

OTHER PROTECTIVE EQUIPMENT

SECTION IX SPECIAL PRECAUTIONS

PRECAUTIONS TO BE TAKEN IN HANDLING AND STORAGE

Store in a cool, dry, well-ventilated location away from fire hazards. 
CAS : 000108316

ON36750

PRODUCT DESIGNATION

MATERIAL SAFETY

DATA SHEET

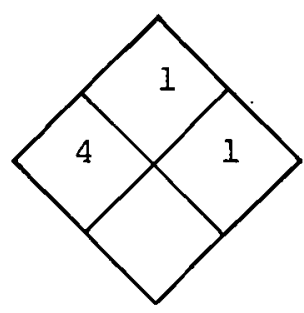

SECTION I SOURCE AND NOMENCLATURE

MANUFACTURER'S NAME
ADDRESS INUMBER, STREET, CITY, STA
TRADE NAME AND SYNONYMS
CHEMICAL NAME AND SYNONYMS
TOXIIIC ANhYdride

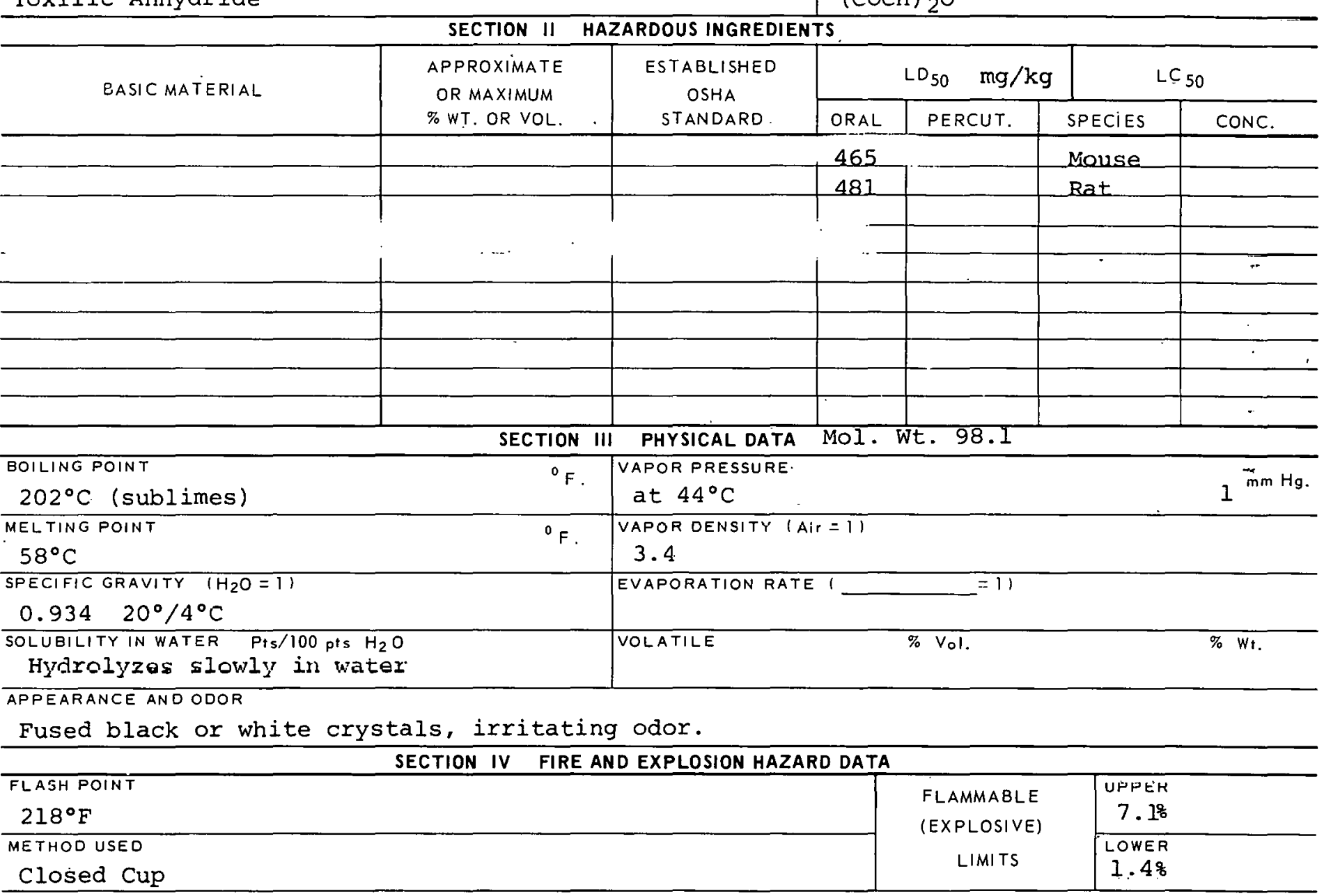

EXTINGUISHING MEDIA

"Alcohol" foam, carbon dioxide, or dry chemical. SPECIAL FIRE FIGHTING PROCEDURES

Wear full protective clothing.

UNUSUAL FIRE AND EXPLOSION HAZARDS

Dust cloud may be exploded by flame or spark. 


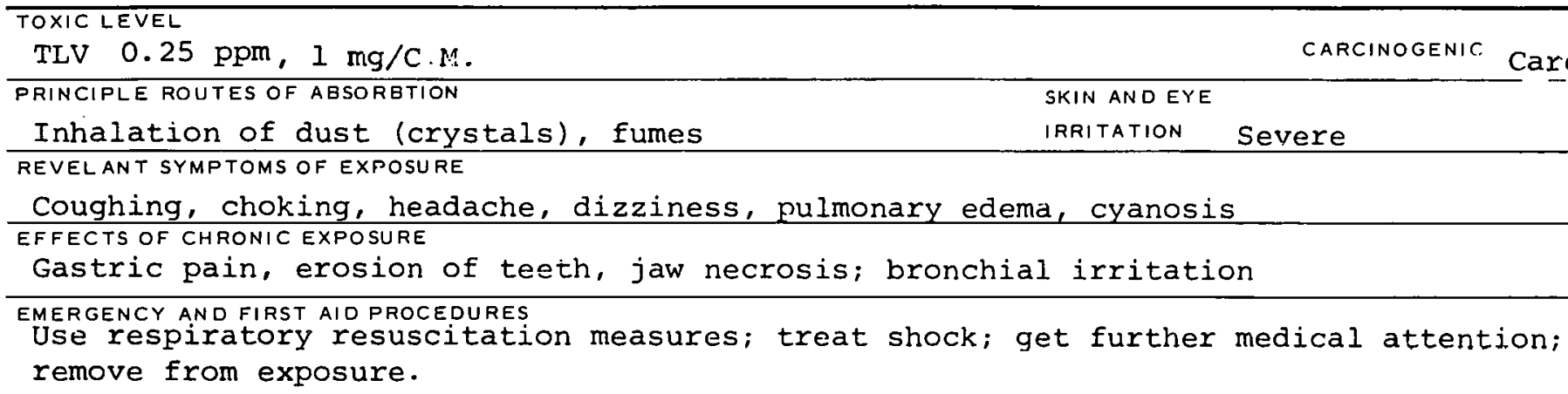


CAS : 000141822

\begin{tabular}{|c|}
\hline PRODUCT DESIGNATION \\
Malonic Acid \\
$03-202-4805$ \\
\hline
\end{tabular}

\section{$00-01750$ \\ MATERIAL SAFETY \\ DATA SHEET}

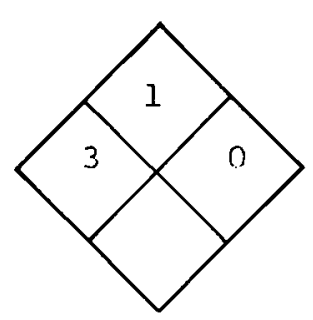

SECTION I SOURCE AND NOMENCLATURE

\begin{tabular}{l|l}
\hline MANUFACTURER'S NAME & EMERGENCY TELEPHONE NO. \\
\hline ADDRESS (NUMBER, STREET, CITY, STATE, ZIP CODE)
\end{tabular}

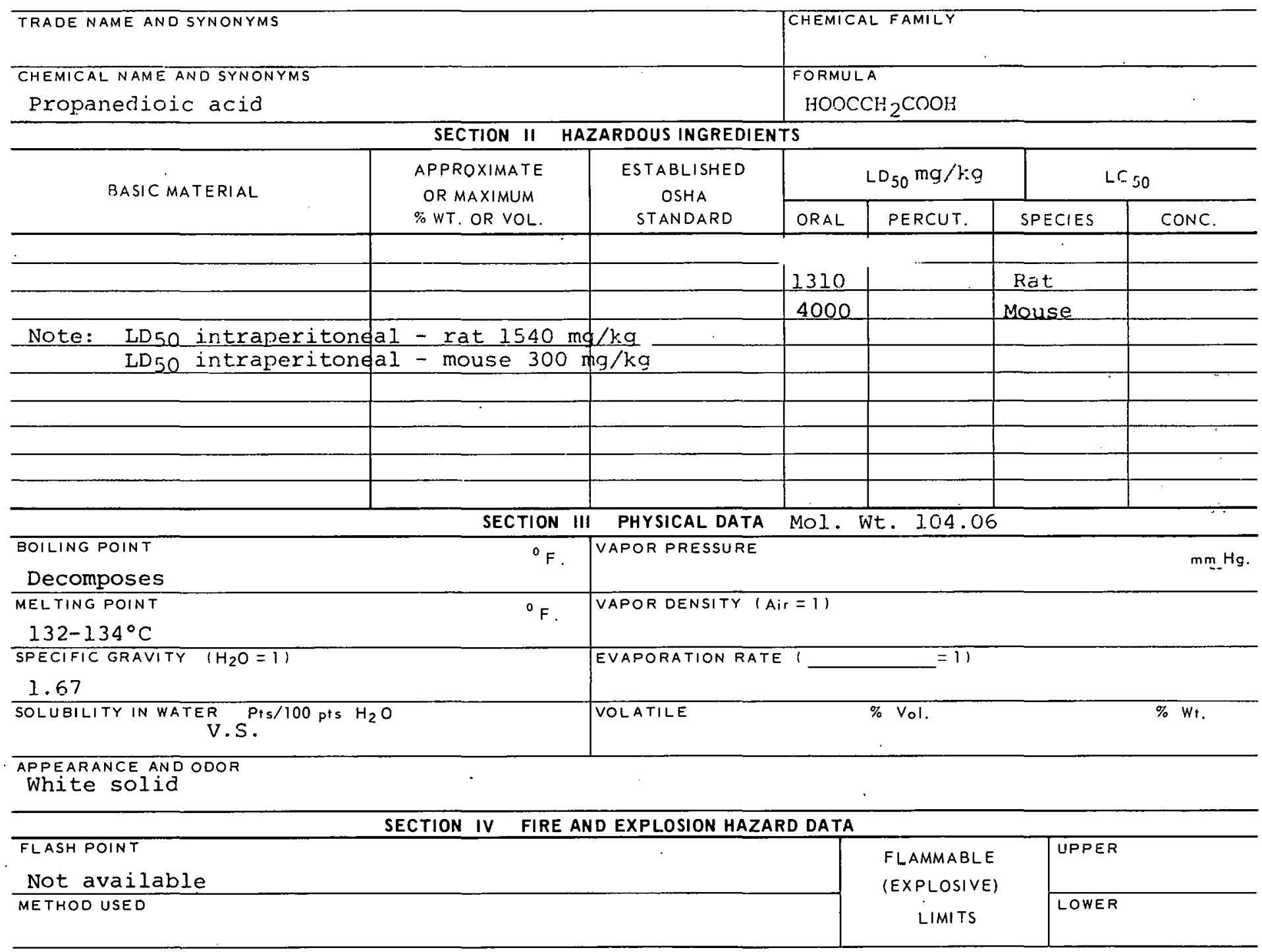

EXTINGUISHING MEDIA

Alcohol foam, carbon dioxide, dry chemical.

SPECIAL FIRE FIGHTING PROCEDURES

Wear full protective clothing.

UNUSUAL FIRE AND EXFLOSION HAZARUS 


\section{TOXIC LEVEL \\ Not available}

PRINCIPLE ROUIES UF AESORETION Inhalation, ingestion

REVELANT SYMPTOMS OF EXPOSURE and respiratory oppression

EFFECTS OF CHRONIC EXPOSURE

Errosion of teeth, bronchial irritation, gastrointestinal disturbance.

EMERGENCY AND FIRST AID PROCEDURES Inhalation - rest, get medical aid if needed; ingestion wash mouth, drink water, get medical aid; wash from skin and eyes.

\section{SECTION VI REACTIVITY DATA}

CARCINOGENIC NO

SKIN AND EYE

IRRITATION SEVERE

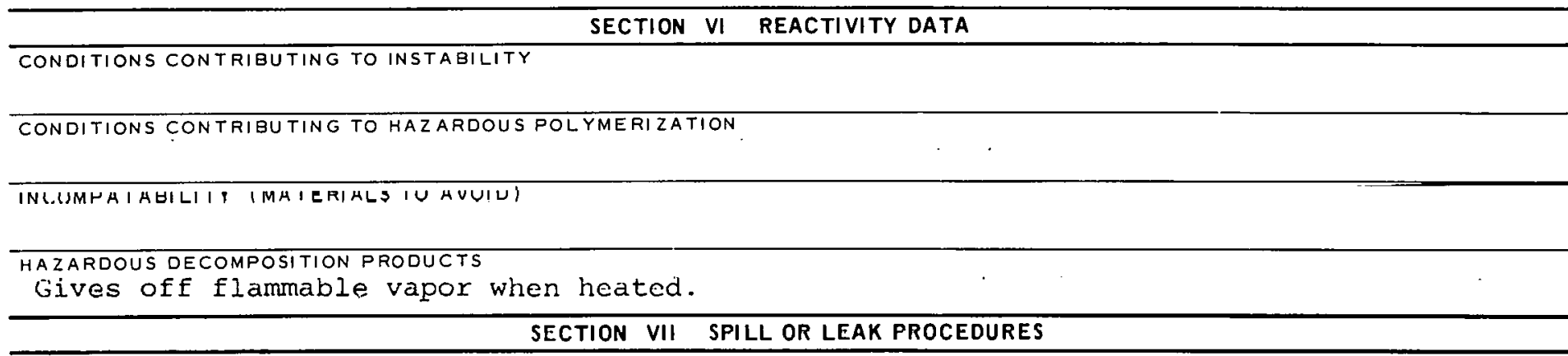

STEPS TO BE TAKEN IN CASE MATERIAL IS RELEASED OR SPILLED

\section{WASTE DISPOSAL METHOD}

\section{SECTION VIII SPECIAL PROTECTION INFORMATION}

\begin{tabular}{|c|c|}
\hline VENTILATION REQUIREMENTS LOCAL EXHAUST & $\begin{array}{l}\text { PROTECTIVE EQUIPMENT (SPECIFY TYPES) EYE } \\
\text { Satety glasses }\end{array}$ \\
\hline MECHANICAL (GENERAL) & $\begin{array}{l}\text { GLOVES } \\
\text { Rubber }\end{array}$ \\
\hline SPECIAL & $\begin{array}{l}\text { RESPIRATOR } \\
\text { WIPYORAY I1LEAY }\end{array}$ \\
\hline
\end{tabular}

OTHER PROTECTIVE EQUIPMENT

SECTION IX SPECIAL PRECAUTIONS

PRECAUTIONS TO BE TAKEN IN HANDLING ANO STORAGE 


\begin{tabular}{|} 
PRODUCT DESIGNATION \\
03-202-5206 \\
Methoxyacetic Acid \\
\hline
\end{tabular}

\section{MATERIAL SAFETY DATA SHEET}

\section{SECTION I SOURCE AND NOMENCLATURE}

MANUFACTURER'S N.AME

ADDRESS (NUMBER, STREET, CITY, STATE, ZIP CODE)

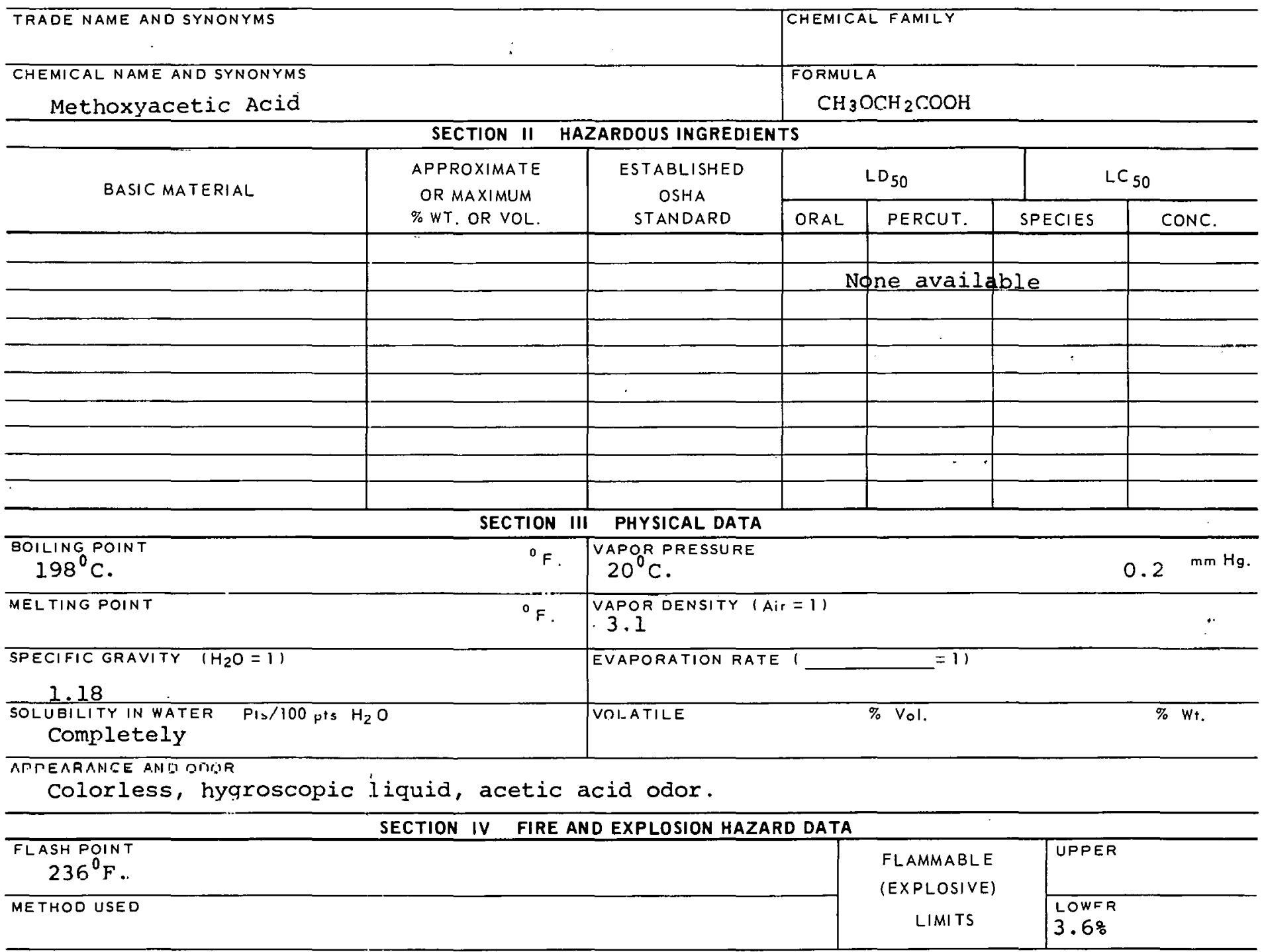

EXTINGUIGHING MLDIA

carbon dioxide, dry chemical, water.

SPECIAL FIRE FIGHTING PROCEDURES

Air mask should be worn.

UNUSUAL FIRE AND EXPLOSION HAZAROS 
Methoxyacetic Acid

SECTION $V$ HEALTH HAZARD DATA

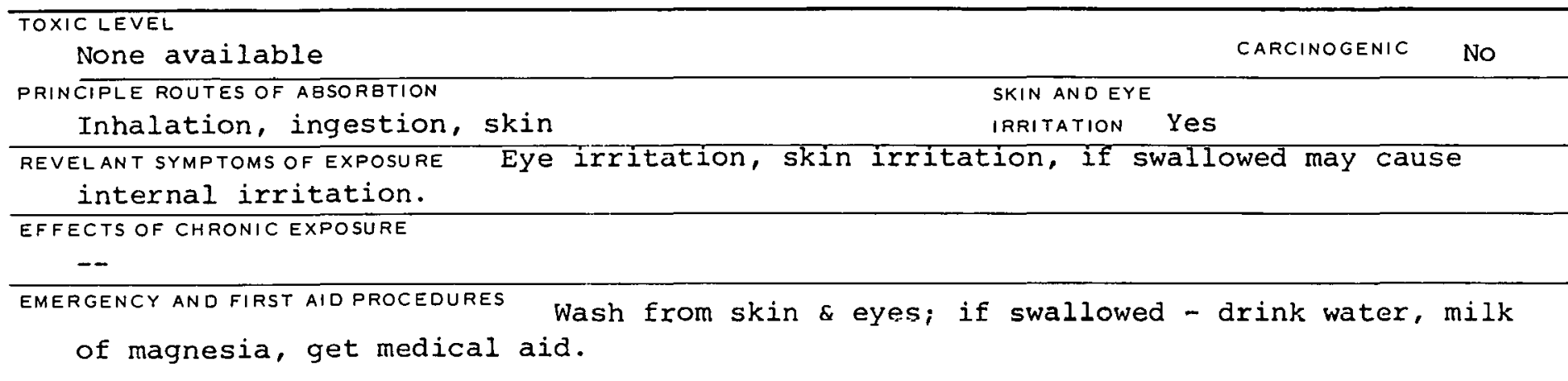

SECTION VI REACTIVITY DATA

CONDITIONS CONTRIBUTING TO INSTABILITY

CONDITIONS CONTRIBUTING TO HAZARDOUS POLYMERIZATION

Nring

INCOMFATAEILITY (MATERIALS TO AVOID)

Strong oxidizers

HAZARDOUS DECOMPOSITION PRODUCTS

SECTION VII SPILL OR LEAK PROCEDURES

STEPS TO BE TAKEN IN CASE MATERIAL IS RELEASED OR SPILLED REmOVe sourCe of ignition, abSOrb spilled material on vermiculite. Place in fiber carton and burn.

WASTE DISPOSAL METHOD

Incinerate

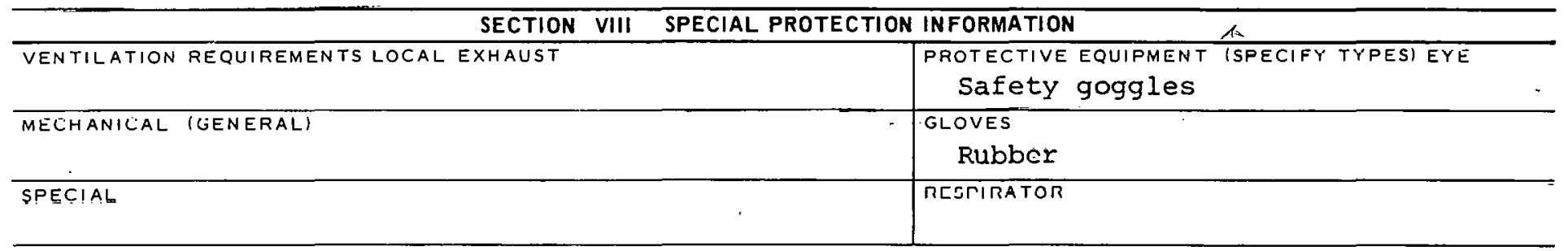

OTHER PROTECTIVE EQUIPMENT

SECTION IX SPECIAL PRECAUTIONS

PRECAUTIONS TO BE TAKEN IN HANDLING AND STORAGE

OTHER PRECAUTIONS

Wash after handling

SIGNAIURE

DATE

$202-5206 B$ 


\begin{tabular}{|c|}
\hline PRODUCT DESIGNATION \\
03-202-9610 \\
Nitrophthalic Anhydride \\
\hline
\end{tabular}

\section{MATERIAL SAFETY DATA SHEET}

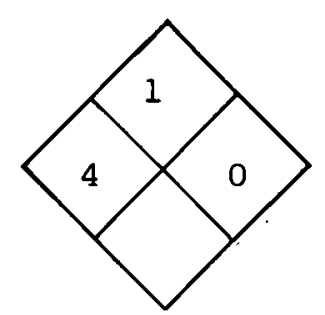

SECTION I SOURCE AND NOMENCLATURE

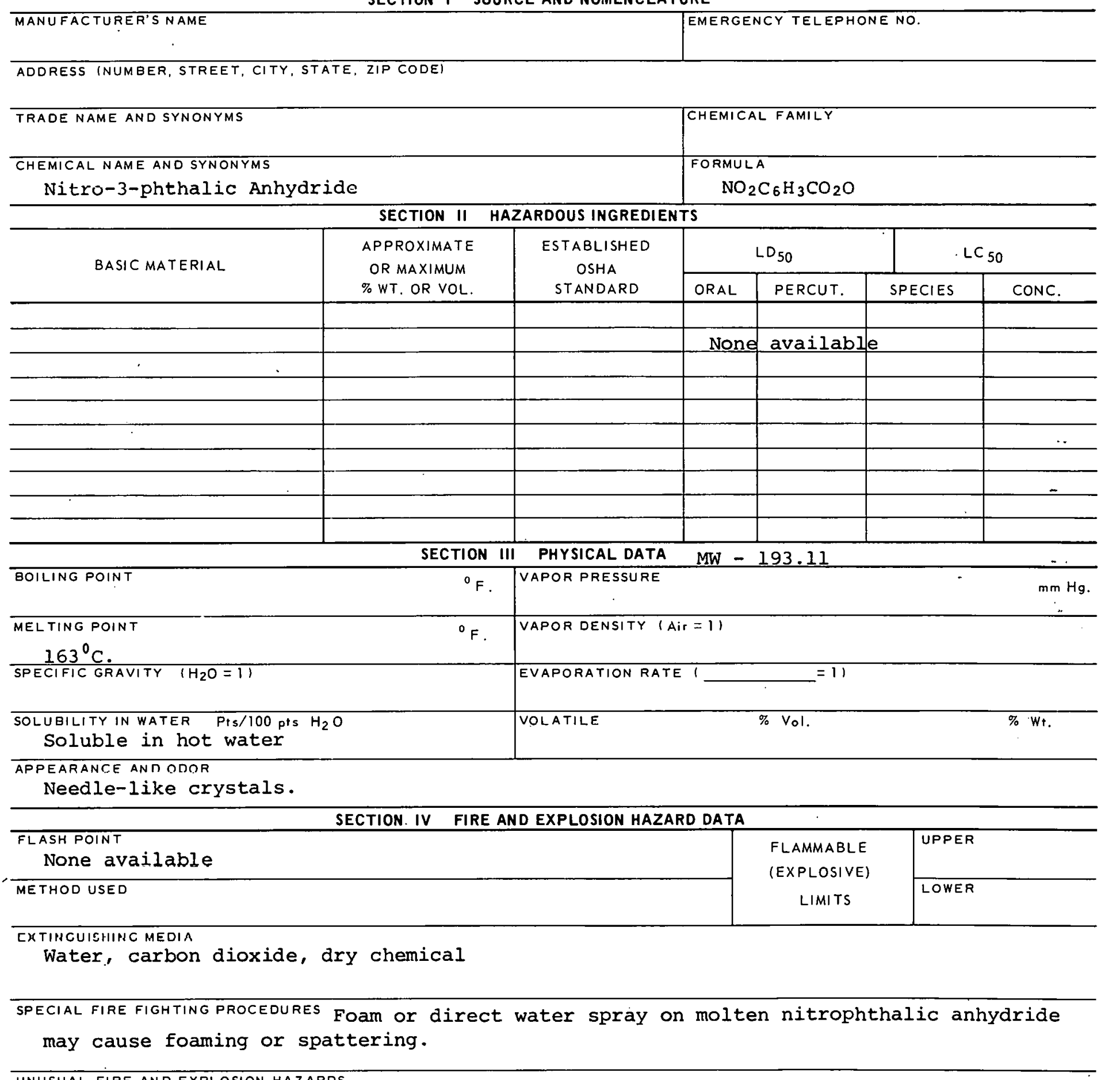

UNUSUAL FIRE AND EXPLOSION HAZARDS 


\section{TOXIC LEVEL}

None available PRINCIPLE ROUTES OF ABSORBTION

Inhalation, ingestion REVELANT SYMPTOMS OF EXPOSURE
CARCINOGENIC

SKIN ANO EYE

IRRITATION SEvere

Coughing, sneezing, burning in respiratory system, dermatitis, skin ixritation, if swallowed - digestive tract irritation. EFFECTS OF CHRONIC EXPOSURE Bronchitis, eye irritation, inflammation of respiratory tract; bloody nasal discharge. EMERGENCY AND FIRST AID PROCEDURES Inhalation - rest; if swallowed - give milk of magnesia, get medical aid; wash skin \& eyes.

SECTION VI REACTIVITY DATA

CONDITIONS CONTRIEUTING.TO HAZARDOUS POLYMERIZATION

INCOMPATABILITY (MATERIALS TO AVOID)

HAZARDOUS DECOMPOSITION PRODUCTS

SECTION VII SPILL OR LEAK PROCEDURES

STEPS TO BE TAKEN IN CASE MATERIAL IS RELEASED OR SPILLED

Cover with soda ash, mix and add water.

WASTE DISPOSAL METHOO

SECTION VIII SPECIAL PROTECTION INFORMATION

\begin{tabular}{l|l}
\hline VENTILATION REQUIREMENTS LOCAL EXHAUST & $\begin{array}{c}\text { PROTECTIVE EQUIPMENT (SPECIFY TYPES) EYE } \\
\text { GOggles }\end{array}$ \\
\hline MECHANICAL IGENERAL) & $\begin{array}{c}\text { GLOVES } \\
\text { Rubber }\end{array}$ \\
\hline SPECIAL & RESPIRATOR \\
gelf-contained breathing apparatuS
\end{tabular}

OTHER PROTECTIVE EOUIPMENT

SECTION IX SPECIAL PRECAUTIONS

PRECAUTIONS TO BE TAKEN IN HANDLING AND STORAGE
Protect against physical damage, store in cool, well-ventilated location.

OTHER PRECAUTIONS

SIGNATU $\overline{R E}$

DATE

$202-9610 B$ 
Oxalic Acid

\section{PRODUCT DESIGNATION}

03-203-0302

$03-203-0312$

03-203-0372

$03-203-0382$
MATERIAL SAFETY

DATA SHEET

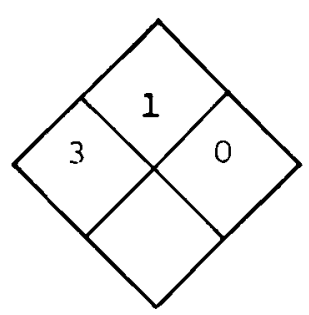

\section{SECTION I SOURCE AND NOMENCLATURE}

\section{MANUFACTURER'S NAME}

ADDRESS (NUMBER, STREET, CITY, STATE, ZIP CODE)

EMERGENCY TELEPHONE NO.

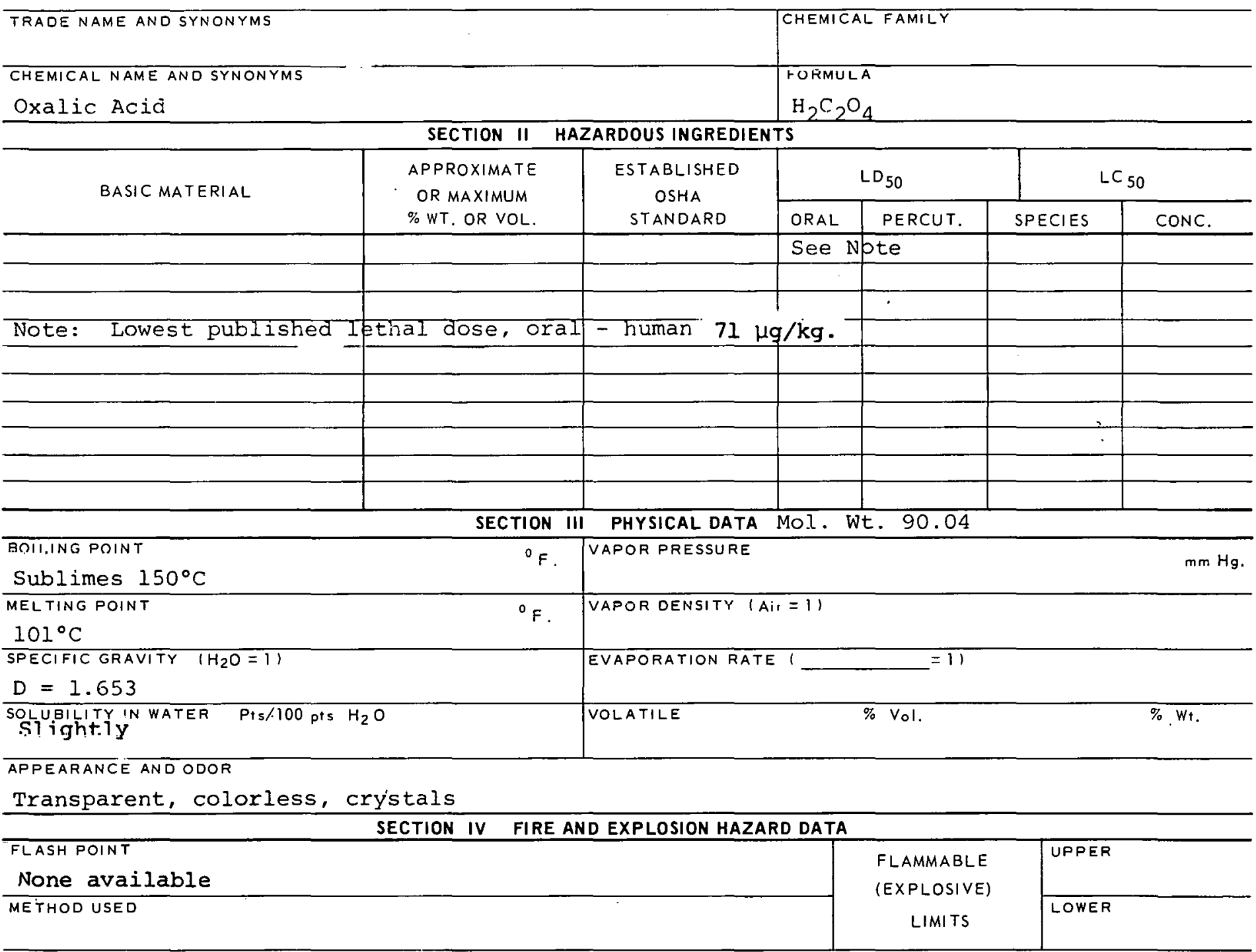

EXTINGUISHING MEDIA

Use water spray, carbon dioxide, dry chemical, ur alcohol foam.

SPECIAL FIRE FIGHTING PROCEDURES

Wear self-contained breathing apparatus and goggles.

UNUSUAL FIRE AND EXPLOSION HAZARDS

May produce carbon monoxide when heated. 


\section{TOXIC LEVEL}

TLV $1 \mathrm{mg} / \mathrm{C} . \mathrm{M}$.

PRINCIPLE ROUTES OF ABSORBTION

Inhalation of mist or powder

REVELANT SYMPTOMS OF EXPOSURE

Irritation of eyes and upper respiratory tract, ulceration of mucous membranes, headache, EFFECTS OF CHRONIC EXPOSURE irritability \& nervousness.

Inhalation of fumes - oxalic acid poisoning; skin contact - cyanosis \& gangrene by local EMERGENCY AND FIRST AID PROCEDURES COYYOSIVE effect.

Remove from exposure, rest \& keep warm; obtain medical help; if ingested - give emetic, large quantities of water, get medical aid.

\section{SECTION VI REACTIVITY DATA}

CONDITIONS CONTRIBUTING TO INSTABILITY

CONDITIONS CONTRIBUTING TO HAZARDOUS POLYMERIZATION

IHGOMPATADILITV (MATLFIALE TO AVOID)

Silver, sodium chlorite, sodium hypochlorite.

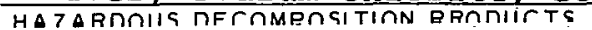

SECTION VII SPILL OR LEAK PROCEDURES

STEPS TO BE TAKEN IN CASE MATERIAL IS RELEASED OR SPILLED

Cover with soda ash or sodium bicarbonate to neutralize.

WASTE DISPOSAL METHOD

SECTION VIII SPECIAL PROTECTION INFORMATION

VENTILATION REQUIREMENTS LOCAL EXHAUST

MECHANICAL (GENERAL)

SPECIIAL

OTHER FROTECTIVE EQUIPMENT

SECTION IX SPECIAL PRECAUTIONS

PRECAUTIONS TO BE TAKEN IN HANDLING AND STORAGE

store in cool, dry well-ventilated area away from fire hazard. Outside

sturage preferred.

OTHER PRECAUTIONS 


\section{CY 31500 \\ MATERIAL SAFETY \\ DATA SHEET}

PRODUCT DESIGNATION

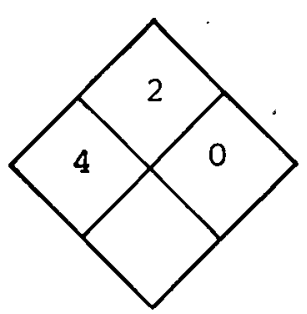

\section{SECTION I SOURCE AND NOMENCLATURE}

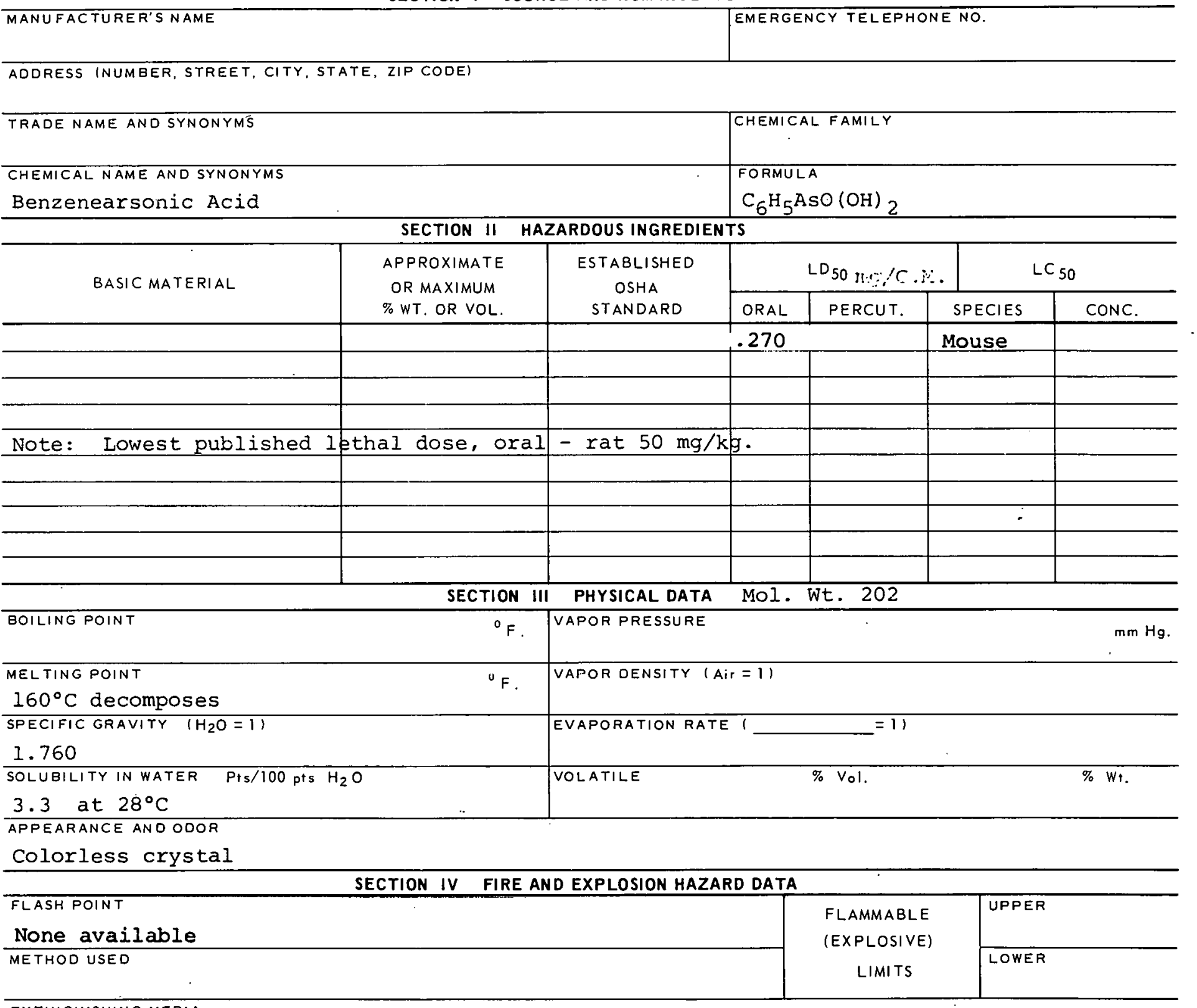

EXTINGUISHING MEDIA

Carbon dioxide, dry chemical, foam

SPECIAL FIRE FIGHTING PROCEDURES

UNUSUAL FIRE AND EXPLOSION HAZARDS

Decompuses to yield rlammable vapor. 
TOXIC LEVEL

TLV $0.68 \mathrm{mg} / \mathrm{C} . \mathrm{M}$.

PRINCIPLE ROUTES OF ABSORBTION

Teratogen

CARCINOGENIC

Yes

Inhalation, ingestion SKIN AND EYE

IRRITATION YES

REVELANT SYMPTOMS OF EXPOSURE Burning of mouth and throat, gastroentric pain, vomiting, diarrhea, hematuria, dehydration, oliguria and collapse.

EFFECTS OF CHRONIC EXPOSURE Arsenic poisoning and skin ulceration, falling out of hair, loss of appetite.

EMERGENCY AND FIRST AID PROCEDURES Inhalation - rest, keep warm, get medical aid; if swallowed wash mouth, gastric lavage with $1 \%$ sodium thiosulfate; wash skin and eyes.

\section{SECTION VI REACTIVITY DATA}

CONDITIONS CONTRIBUTING TO INSTABILITY

CONDITIONS CONTRIBU TING TO HAZARDOUS POLYMERIZATION

INTOMPATARIIITY (MATFRIAI S TO AVIIII)

HAZARDOUS DECOMPOSITION PRODUCTS

SECTION VII SPILL OR LEAK PROCEDURES

STEPS TO BE TAKEN IN CASE MATERIAL IS RELEASED OR SPILLED

WASTE DISPOSAL METHOD

SECTION VIII SPECIAL PROTECTION INFORMATION

\begin{tabular}{l|l}
\hline \multicolumn{1}{|c|}{ SECTION VIII SPECIAL PROTECTION INFORMATION } \\
\hline MECHANICAL IGENERALI & $\begin{array}{l}\text { PROTECTIVE EQUIPMENT ISPECIFY TYPESI EYE } \\
\text { GOggleS }\end{array}$ \\
\hline SPECIAL & GLOVES \\
\hline
\end{tabular}

OTHER PROTECTIVE EQUIPMENT

SECTION IX SPECIAL PRECAUTIONS

PRECAUTIONS TO BE TAKEN IN HANDLING AND STORAGE

OTHER PRECAUTIONS

SIGNATURE

DATE

203-1303B 
CAS : 000085449

PRODUCT DESIGNATION

Phthalic Anhydride

03-203-2024

\section{MATERIAL SAFETY DATA SHEET}

\section{SECTION I SOURCE AND NOMENCLATURE}

MANUFACTURER'SNAME

ADDRESS (NUMBER, STREET, CITY, STATE, ZIP CODE)

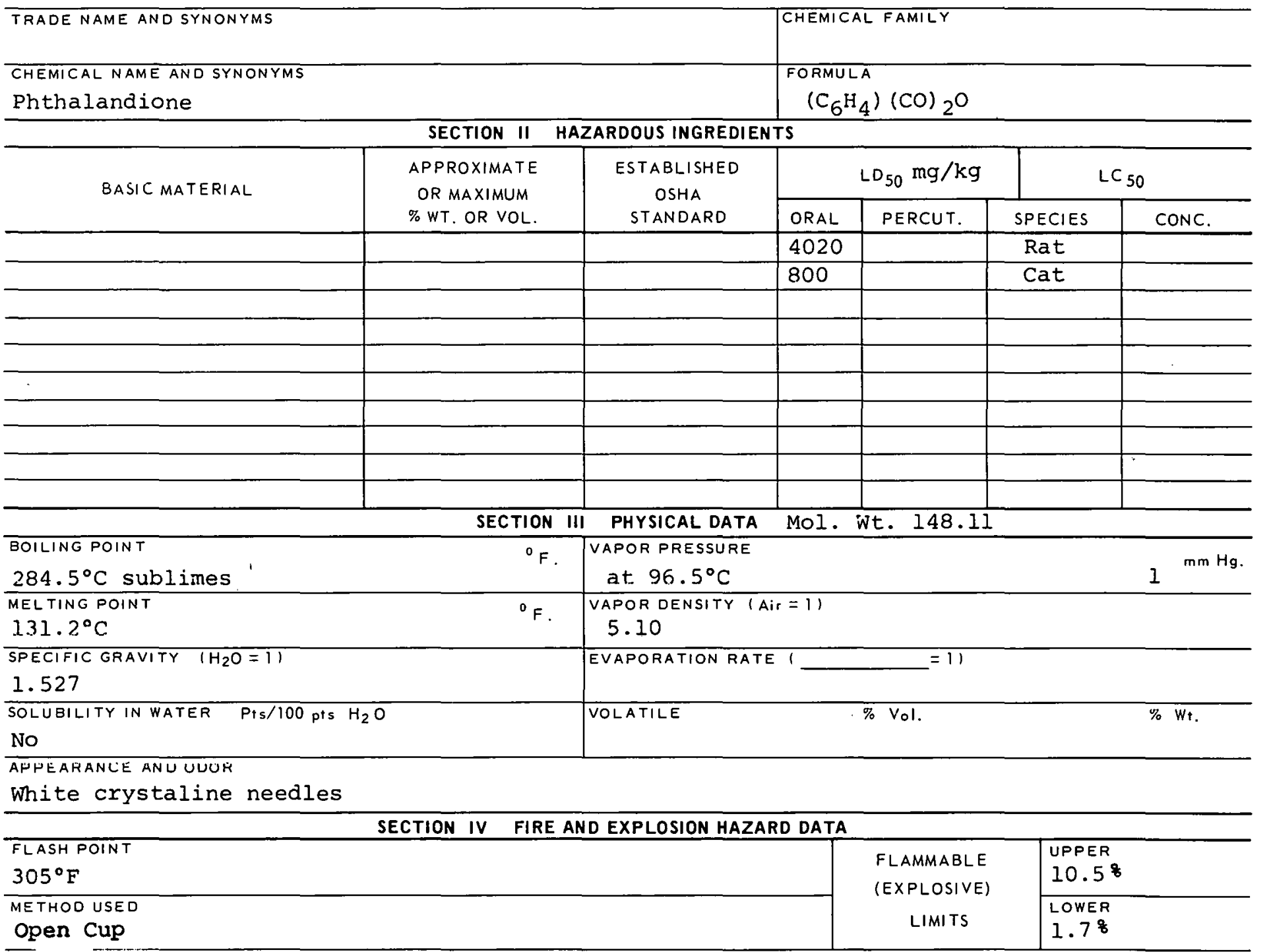

EXTINGUISHING MEDIA

Water, carbon dioxide, dry chemical. Foam or direct water spray on molten phthalic anhydric may cause foaming or spattering.

SPECIAL FIRE FIGHTING PROCEDURES

$\mathrm{N} / \mathrm{A}$

UNUSUAL FIRE AND EXPLOSION HAT.ARDS 


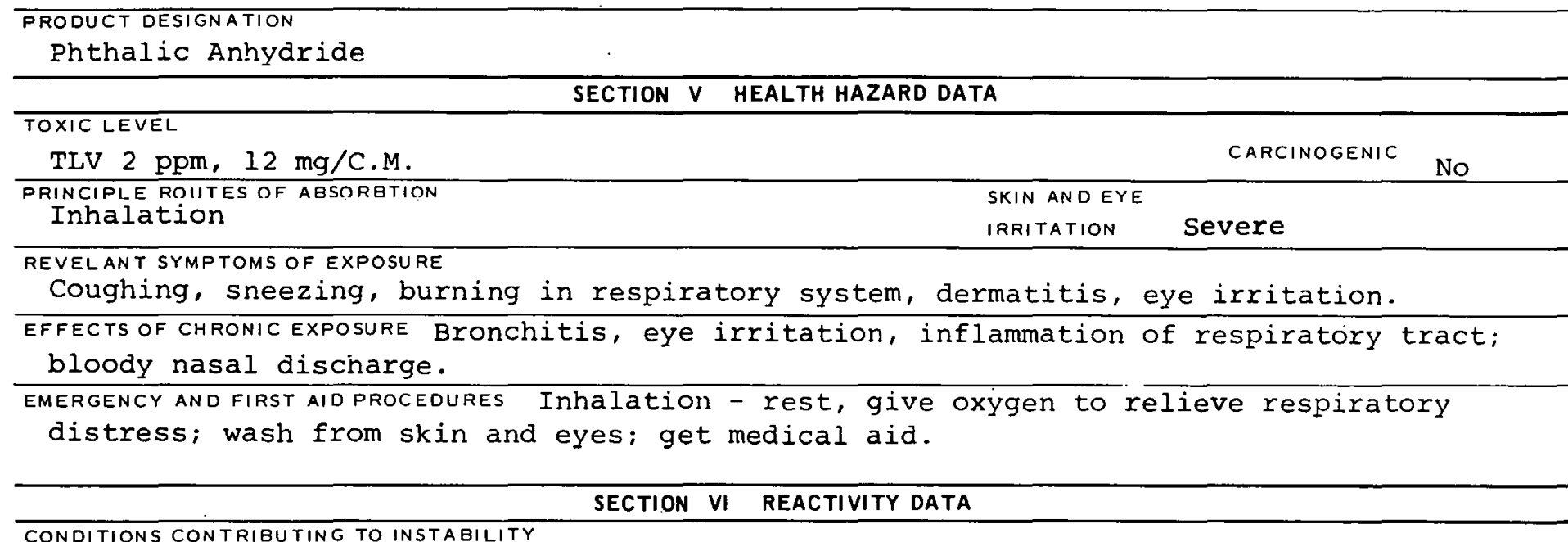

CONDITIONS CONTRIBUTING TO INSTABILITY

CONDITIONS CONTRIBUTING TO HAZARDOUS POLYMERIZATION

IN RIMPATARII ITY IMATFRIAI S TO AVOIRI

HAZARDOUS DECOMPOSITION PRODUCTS

SECTION VII SPILL OR LEAK PROCEDURES

STEPS TO BE TAKEN IN CASE MATERIAL IS RELEASED OR SPILLED

Cover with soda ash, $\operatorname{mix}$ and add water.

WASTE DISPOSAL METHOD

SECTION VIII SPECIAL PROTECTION INFORMATION

\begin{tabular}{l|l}
\hline \multicolumn{2}{c}{ SECTION VIII SPECIAL PROTECTION INFORMATION } \\
\hline VENTILATION REQUIREMENTS LOCAL EXHAUST & $\begin{array}{c}\text { PROTECTIVE EQUIPMENT ISPECIFY TYPESI EYE } \\
\text { Goggles }\end{array}$ \\
\hline MECHANICAL IGENERALI & $\begin{array}{c}\text { GLOVES } \\
\text { Rubber }\end{array}$ \\
\hline SPECIAL & $\begin{array}{c}\text { RESPIRATOR SClf-contained breathing apparatus } \\
\text { SPIS }\end{array}$ \\
\hline
\end{tabular}

OTHER PROTECTIVE EQUIPMENT

SECTION IX SPECIAL PRECAUTIONS

PRECAUTIONS TO BE TAKEN IN HANDLING AND STORAGE

Protect against physical damage. Store in bags in cool, dry, well-ventilated location.

UTHER PRECAUTIUNS

SIGNATURE

DATE 


\begin{tabular}{|c|}
\hline PRODUCT DESIGNATION \\
03-203-2504 \\
Picramic ACid \\
\hline
\end{tabular}

\section{MATERIAL SAFETY \\ DATA SHEET}

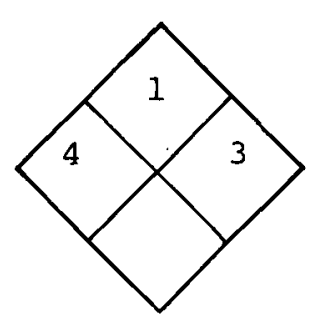

\section{SECTION I SOURCE AND NOMENCLATURE}

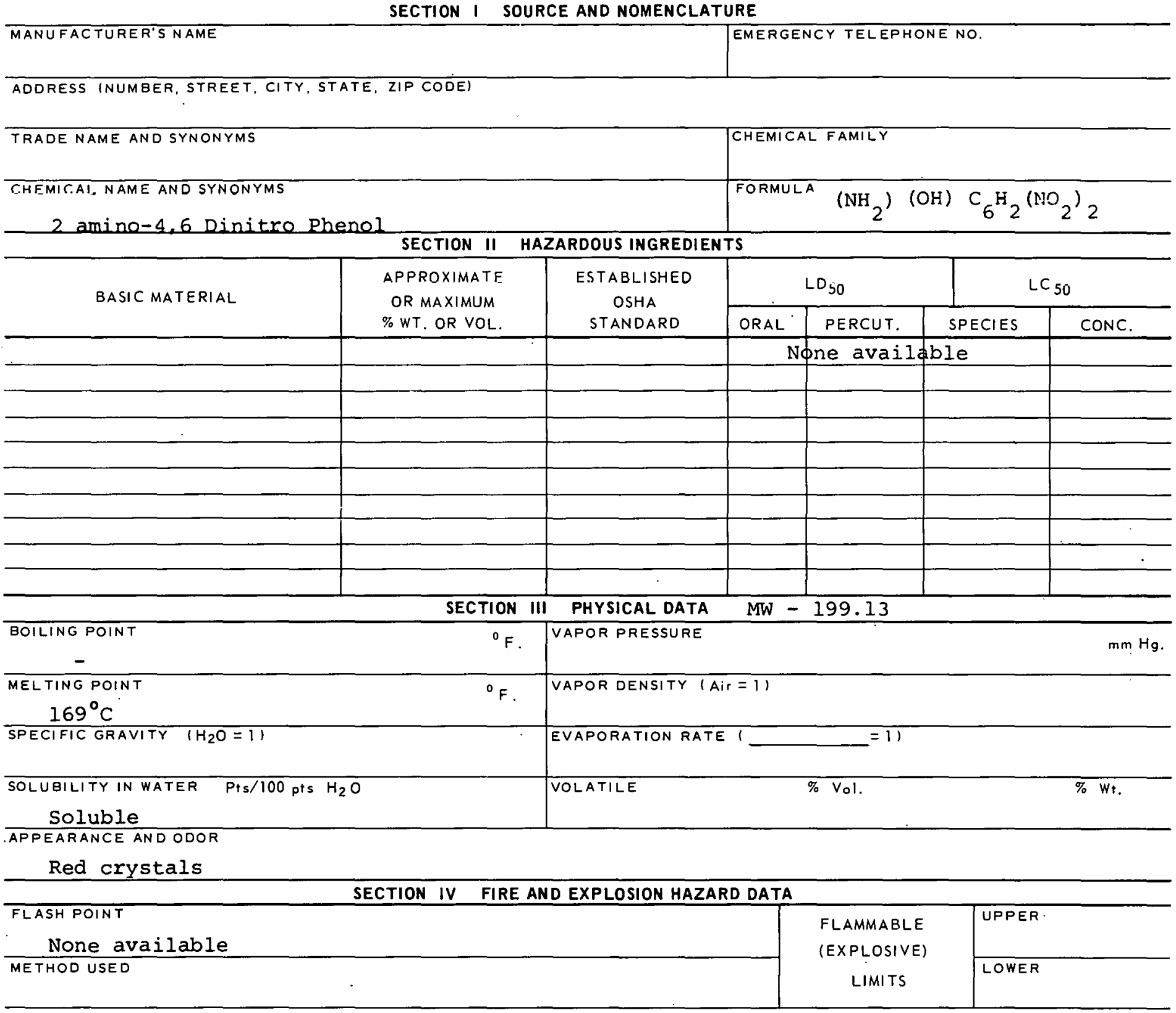

EXTINGUISHING MEDIA

Explosive - do not fight fire except for unmanned equipment - use water.

SPECIAL FIRE FIGHTING PROCEDURES

Wear full protective clothing for rescue operation.

UNUSUAL FIFE AND EXPLOSION HAZARDS

Severe explosion hazard 


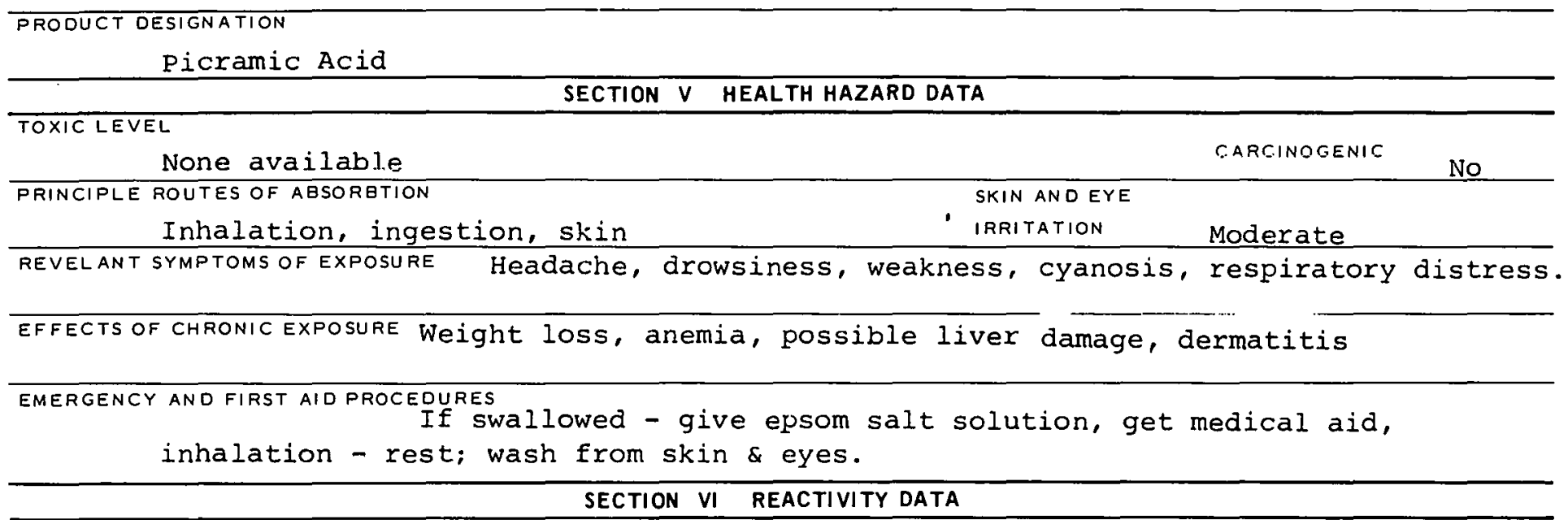

CONDITIONS CONTRIBUTING TO INSTABILITY

CONDITIONS CONTRIBUTING TO HAZARDOUS POLYMERIZATION

INCOMHAIAB̈ILITY IMAIEHIALS IU AVUIDI

Explosive (keep from oxidizing materials)

IIAEARBOUJ DECOMrOMTIOH RRODUCTJ

SECTION VII SPILL OR LEAK PROCEDURES

STEPS TO BE TAKEN IN CASE MATERIAL IS RELEASED OR SPILLED

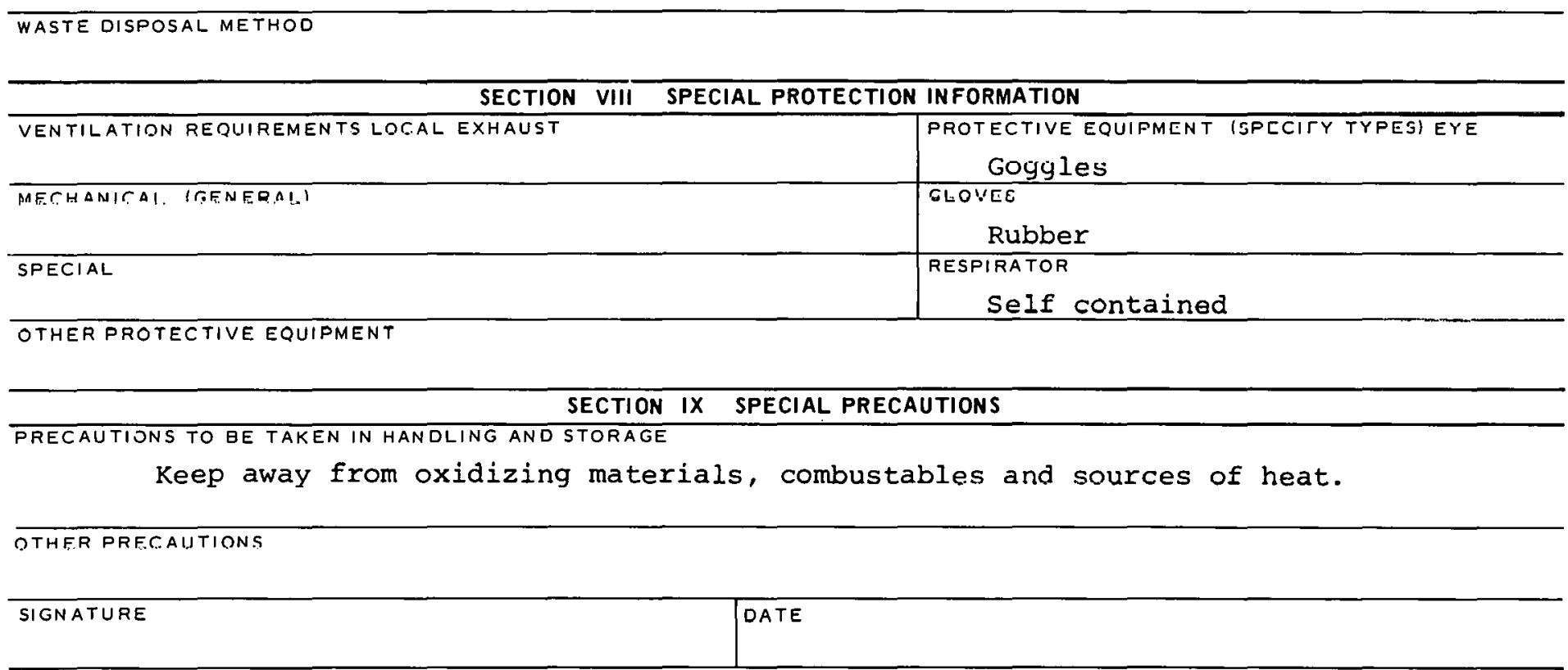


CAS : 000088891

\begin{tabular}{|c|}
\hline PRODUCT DESIGNATION \\
Picric Acid \\
03-203-2704
\end{tabular}

\section{MATERIAL SAFETY DATA SHEET}

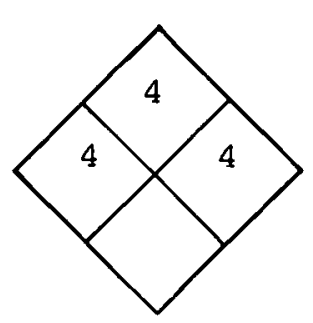

\section{SECTION I SOURCE AND NOMENCLATURE}

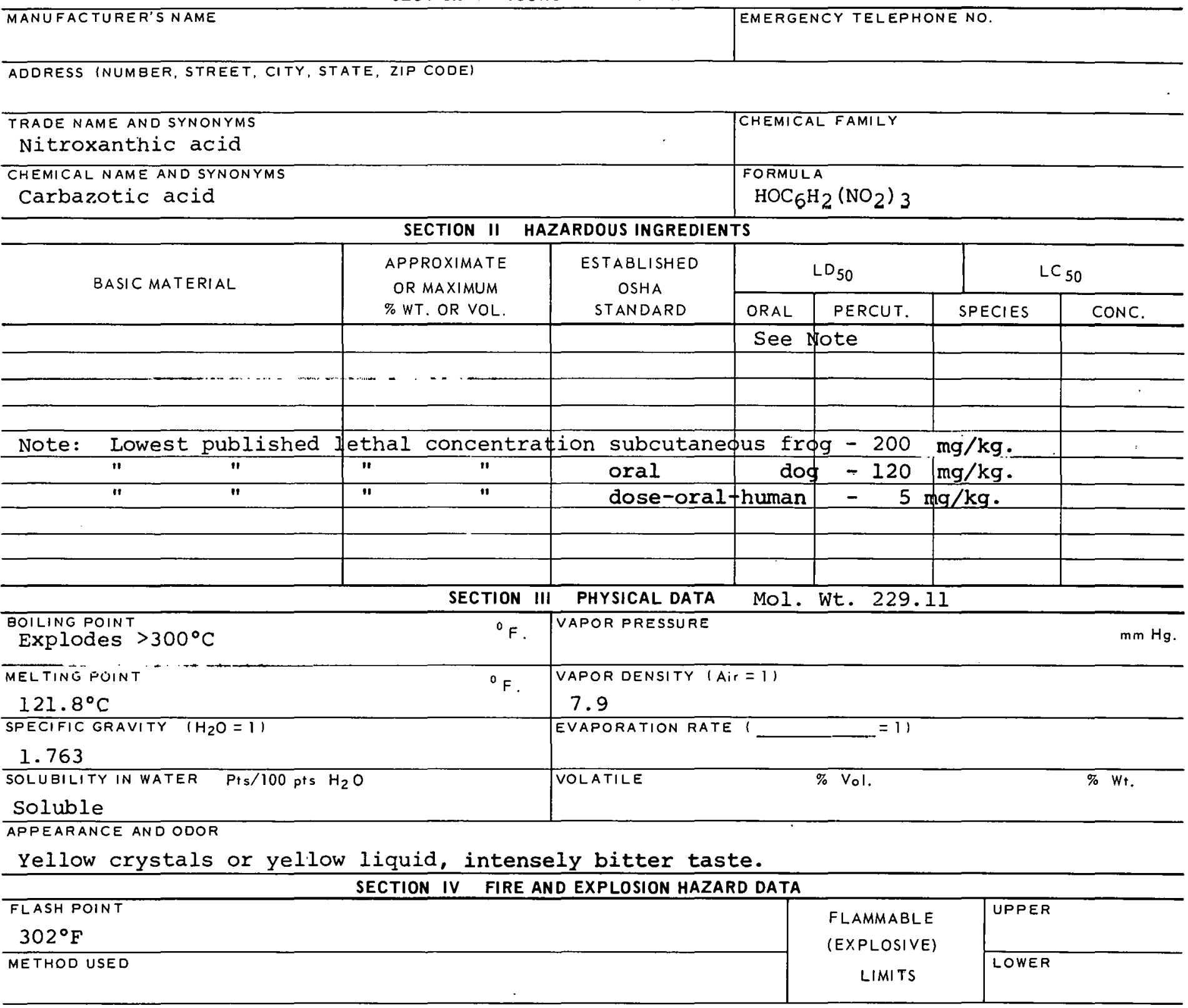

EXTINGUISHING MEDIA

Water

SPECIAL FIRE FIGHTING PROCEDURES

Fight from explosion-resistant location

UNUSUAL FIRE AND EXPLOSION HAZARDS

Highly explosive 


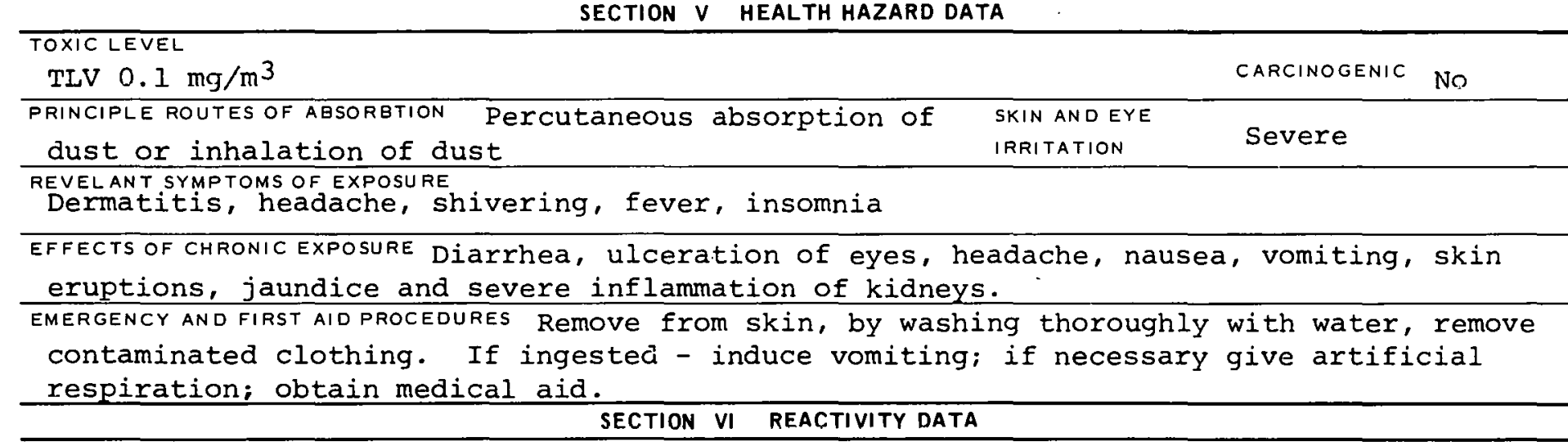

CONDITIONS CONTRIBUTING TO INSTABILITY

CONDITIONS CONTRIBU TING TO HAZARDOUS POLYMERIZATION

INCOMPATÁBILITY (MATERIALS T'O AVOIU)

Copper, lead, zinc, ammonia, bases, concrete

HALARUUUS UELUMPUSTiOHA PMOUETS

SECTION VII SPILL OR LEAK PROCEDURES

STEPS TO BE TAKEN IN CASE MATERIAL IS RELEASED OR SPILLED

Mix sodium bicarbonate or a mixture of 9:1 sand - soda ash and transfer to cardboard carton for disposal. WASTE DISPOSAL METHOD

SECTION VIII SPECIAL PROTECTION INFORMATION

\begin{tabular}{l|l}
\hline VENTILATION REQUIREMENTS LOCAL EXHAUST & $\begin{array}{l}\text { PROTEGTIVE EQUIPMENT (SPECIFY TYPES) EYE } \\
\text { GOgGIES }\end{array}$ \\
\hline MECHANICAL IGENERAL) & $\begin{array}{l}\text { GLOVES } \\
\text { Rubber (butYl) }\end{array}$ \\
\hline SPECIAL & $\begin{array}{l}\text { RESPIRATOR } \\
\text { w/appropriate filter }\end{array}$ \\
\hline
\end{tabular}

OTHER PROTECTIVE EQUIPMENT

Apron, face shield, self-contained breathing apparatus

PRECAUTIONS TO BE TAKEN IN HANDLING AND STORAGE

SECTION IX SPECIAL PRECAUTIONS

Protect againgt physical damage

OTHER PRECAUTIQNS 
CAS : 000087661

\section{PRODUCT DESIGNATION}

03-203-3455

Pyrogallic Acid
MATERIAL SAFETY

DATA SHEET

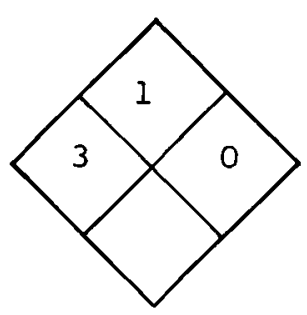

SECTION I SOURCE AND NOMENCLATURE

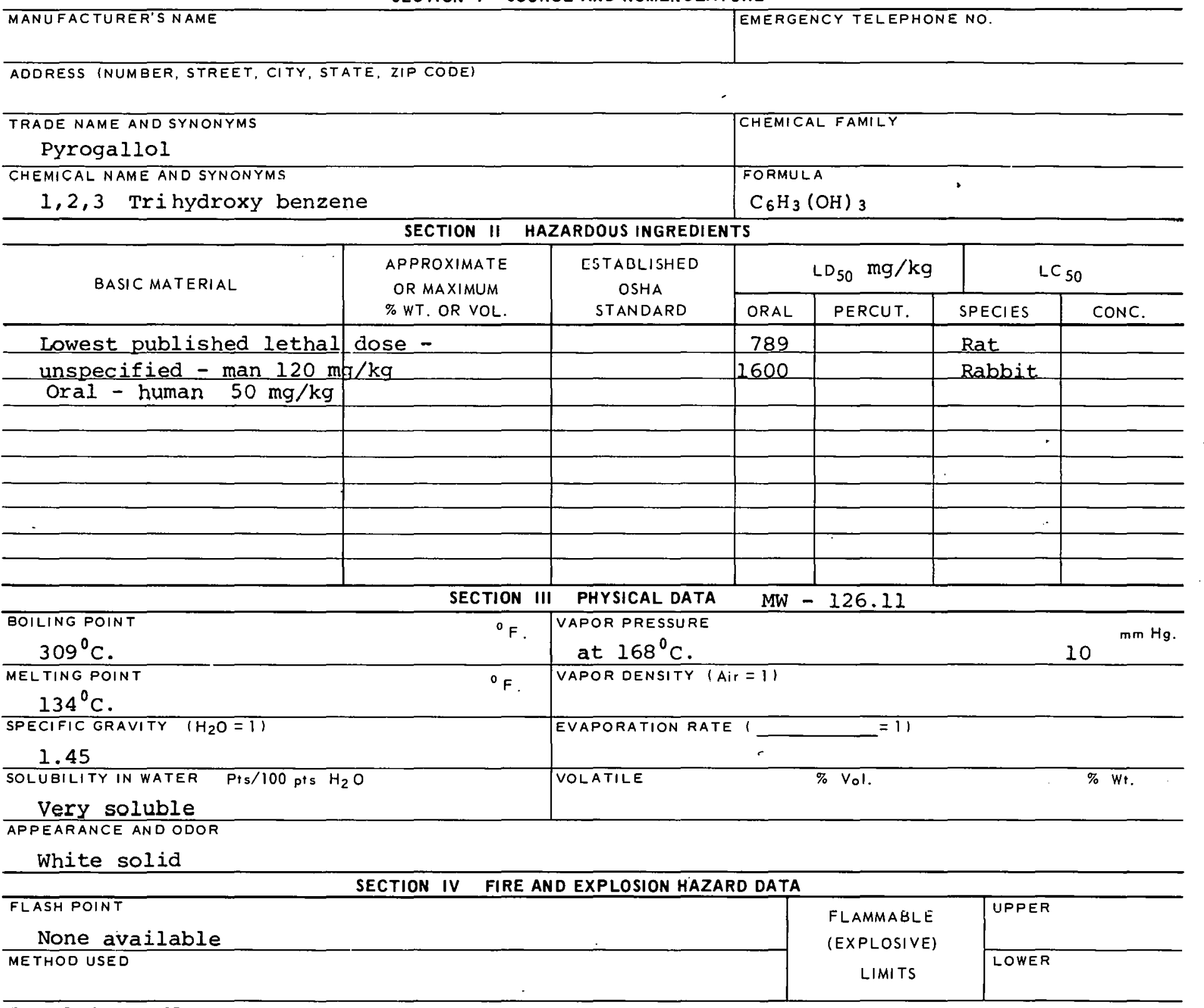

EXTINGUISHING MEDIA

Alcohol foam, rarhon dioxide, water

SPECIAL FIRE FIGHTING PROCEDURES

UNUSUAL FIRE AND EXPLOSION HAZARDS 
Pyrogallic Acid SECTION $\checkmark$ HEALTH HAZARD DATA

\section{TOXIC LEVEL}

None available

CARCINOGENIC NO

PRINCIPLE ROUTES OF ABSORBTION

Inhalation, ingestion, skin SKIN AND EYE

IRRITATION SEvere

REVELANT SYMPTOMS OF EXPOSURE Vomiting, diarrhea, convulsions, circulatory collapse, sensitizes, pulmonary edema.

EFFECTS OF CHRONIC EXPOSURE

Kidney and liver damage.

get medical aid; wash skin \& eyes.

SECTION VI REACTIVITY DATA

\section{CONDITIONS CONTRIBUTING TO INSTABILITY \\ CONDITIONS CONTRIBUTING TO HAZARDOUS POL YMERIZATION}

INCOMPATADILITY (BA.ATERIALSTO AVOID)

H.IIERIIIG RFR.NMPRSITIQN PRQDUCTS

SECTION VII SPILL OR LEAK PROCEDURES

STEPS TO BE TAKEN IN CASE MATERIAL IS RELEASED OR SPILLED

Cover with soda ash, then add water.

WASTE DISPOSAL METHOD

Dissolve in flammable solvent and burn. SECTION VIII SPECIAL PROTECTION INFORMATION

\begin{tabular}{|c|c|}
\hline VENTILATION REQUIREMENTS LOCAL EXHAUST & PROTECTIVE EQUIPMENT (SPECIFY TYPES) EYE \\
\hline MECHANICAL (GENERAL) & $\begin{array}{l}\text { GLOVES } \\
\text { Rubber }\end{array}$ \\
\hline SPECIAL & $\begin{array}{l}\text { RESPIRATOR } \\
\text { With proper tilter }\end{array}$ \\
\hline
\end{tabular}

UTHER PROTLCTIVE EQUITHENT

SECTION IX SPECIAL PRECAUTIONS

PRECAUTIONS TO BE TAKEN IN HANDLING AND STORAGE 
CAS-001401554

03-203-7229

PRODUCT DESIGNATION

03-203-7309

Tannic Acid

MATERIAL SAFETY

DATA SHEET

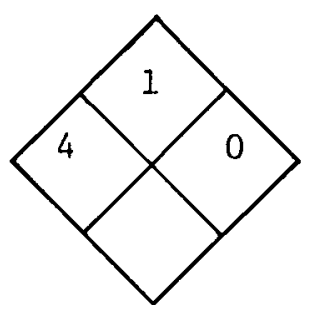

SECTION I SOURCE AND NOMENCLATURE

\begin{tabular}{l} 
MANUFACTURER'S NAME \\
AOORESS INUMEER, STREET, CI Y \\
TRADE NAME AND SYNONYMS \\
TanNII \\
CHEMICAL NAME AND SYNONYMS \\
TanNIC ACId \\
\hline
\end{tabular}

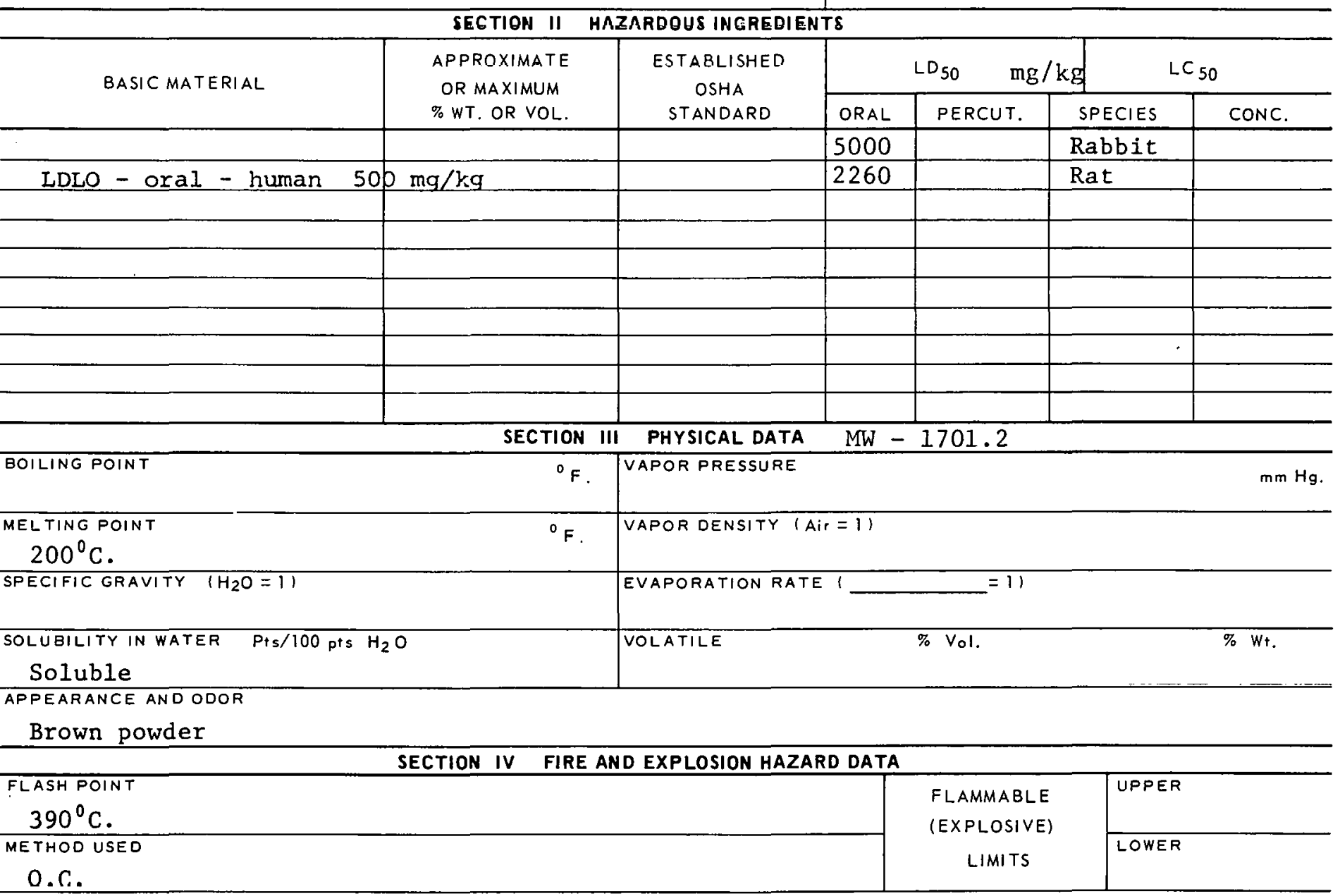

EXTINGUISHING MEDIA

Water, carbon dioxide, dry chemical,

SPECIAL FIRE FIGHTING PROCEDURES

UNUSUAL FIRE AND EXPLOSION HAZARDS 
Tannic Acid SECTION V HEALTH HAZARD DATA

TOXIC LEVEL

None Available

FRINEIFLE ROUTES OF ABSORBTION

Inhalation, ingestion SKIN AND EYE IRRITATION Moderate

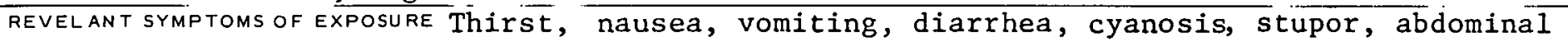
pain, convulsions

EFFECTS OF CHRONIC EXPOSURE

Dermatitis, Liver damage, liver cancer. EMERGENCY AND FIRST AID PROCEDURES

Inhalation - rest; ingestion - drink milk, emetic, get medical aid; wash from skin ana eyes. SECTION VI REACTIVITY DATA

CONDITIONS CONTRIBUTING TO INSTABILITY

CONOITIONS CONTRIBUTING TO HAZARDOUS POLYMEFIZATION

INCOMFATABILITY (MATERIALS TO AVOID)

HAZARDOUS DECOMPOSITION PRODUCTS

SECTION VII SPILL OR LEAK PROCEDURES

STEPS TO BE TAKEN IN CASE MATERIAL IS RELEASED OR SPILLED

WASTE DISPOSAL METHOD

SECTION VIII SPECIAL PROTECTION INFORMATION

\begin{tabular}{l|l}
\hline VENTILATION REQUIREMENTS LOCAL EXHAUST & $\begin{array}{l}\text { PROTECTIVE EQUIPMENT (SPECIFY TYPESI EYE } \\
\text { GOggleS }\end{array}$ \\
\hline MECHANILAL IGENEHALI & GLOVES \\
\hline SPECIAL & RESPIRATOR \\
\hline
\end{tabular}

OTHER PROTECTIVE EQUIPMENT

\section{SECTION IX SPECIAL PRECAUTIONS}

PRECAUTIONS TO BE TAKEN IN HANDLING AND STORAGE 
CAS : 000068111

Al59500

PRODUCT DESIGNATION

03-203-8310

Thioglycolic Acid

MATERIAL SAFETY

DATA SHEET

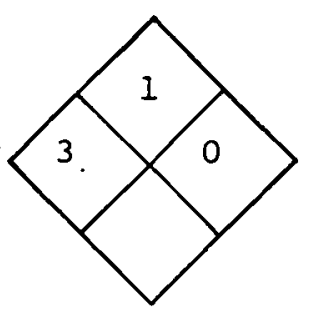

\section{SECTION I SOURCE AND NOMENCLATURE}

MANUFACTURER'S NAME

EET, CITY, STATE, ZIP CODE)

ADDRESS (NUMBER, STREET, CITY, STATE, ZIP CODE)

TRADE NAME ANO SYNONYMS

Mercaptoacetic Acid

CHEMICAL NAME AND SYNONYMS

Thioglycolic Acid

BASIC MATERIAL
SECTION II HAZARDOUS INGREDIENTS

\begin{tabular}{|c|c|c|c|c|c|}
\hline \multirow{2}{*}{$\begin{array}{c}\text { APPROXIMATE } \\
\text { OR MAXIMUM } \\
\% \text { WT. OR VOL. }\end{array}$} & \multirow{2}{*}{$\begin{array}{c}\text { ESTABLISHED } \\
\text { OSHA } \\
\text { STANDARD }\end{array}$} & \multicolumn{2}{|c|}{$L D_{50}$} & \multicolumn{2}{|c|}{$\mathrm{LC}_{50}$} \\
\hline & & ORAL & PERCUT. & SPECIES & CONC. \\
\hline & & 250 & & Rat & \\
\hline & & 126 & & Rabbit & \\
\hline & & 126 & & Guinea & \\
\hline & & & & & \\
\hline & & & & & \\
\hline & & & & & \\
\hline & & & & & \\
\hline & & & & & \\
\hline & & & & & \\
\hline & & & & & \\
\hline SECTIO & PHYSICAL DAT & MW & 92.11 & & \\
\hline
\end{tabular}

BOILING POINT

$123^{\circ} \mathrm{C}$ at $29 \mathrm{~mm}$.

MELTING POINT

$-16.5^{\circ} \mathrm{C}$

SPECIFIC GRAVITY $\left(\mathrm{H}_{2} \mathrm{O}=1\right)$

1.33

SOLUBILITY IN WATER Pts/100 pts $\mathrm{H}_{2} \mathrm{O}$

Soluble

APPEARANCE AND ODOR

Colorless liquid, disagreeable odor

${ }^{\circ} \mathrm{F} . \quad$ VAPOR PRESSURE

$106^{\circ} \mathrm{C}$

$\mathrm{mm} \mathrm{Hg}$.

${ }^{0} \mathrm{~F}$.

VAPOR DENSITY (Air $=1$ )

EVAPORATION RATE

$=11$

VOLATILE

$\%$ vol.

$\% W_{t}$

SECTION IV FIRE AND EXPLOSION HAZARD DATA

FLASH POINT

None available

METHOD USED

EXTINGUISHING MEDIA

Water

SPECIAL FIRE FIGHTING PROCEDURES

UNUSUAL FIRE AND EXPLOSION HAZARDS 
Thioglycolic Acid

SECTION $V$ HEALTH HAZARD DATA

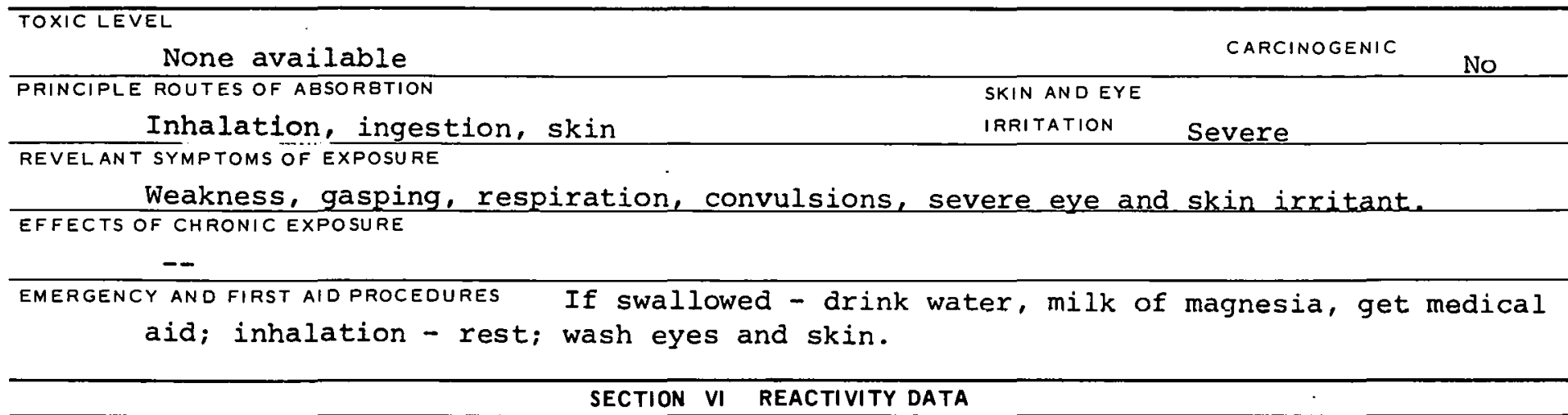

\section{CONDITIONS CONTRIBUTING TO INSTABILITY \\ CONDITIONS CONTRIBUTING TO HAZARDOUS POLYMERIZATION}

IPCOMPATAEILITY (MATERIALS TO AVQIO)

HA7ARANIIS RFROMPRSITION PRODIIS.TS

SECTION VII SPILL OR LEAK PROCEDURES

STEPS TO BE TAKEN IN CASE MATERIAL IS RELEASED OR SPILLED

WASTE DISPOSAL METHOD

SECTION VIII SPECIAL PROTECTION INFORMATION

\begin{tabular}{|c|c|}
\hline VENTILATION REQUIREMENTS LOCAL EXHAUST & $\begin{array}{l}\text { PROTECTIVE EQUIPMENT (SPECIFY TYPES) EYE } \\
\text { GOggIES }\end{array}$ \\
\hline MECHANICAL IGENERAL) & GLOVES \\
\hline SPECIAL & $\begin{array}{l}\text { RESPIRATOR } \\
\text { With proper filter. }\end{array}$ \\
\hline
\end{tabular}

OTHER PROTECTIVE EQUIPMENT

Coveral1s

SECTION IX SPECIAL PRECAUTIONS

PRECAUTIONS TO BE TAKEN IN HANDLING AND STORAGE

OTHER PRECAUTIONS

SIGNATURE

DATE

$203-8310 B$ 


\section{PRODUCT DESIGNATION}

Trichloro Acetic Acid

03-203-9202

03-203-9262

\section{MATERIAL SAFETY DATA SHEET}

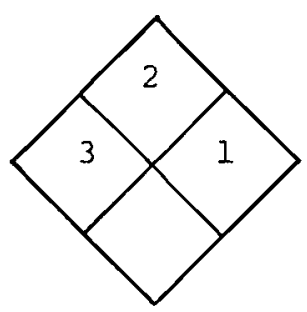

SECTION I SOURCE AND NOMENCLATURE

\section{MANUFACTURER'S NAME \\ TRADE NAME AND SYNONYMS \\ CHEMICAL NAME AND SYNONYMS \\ Trichloroacetic}

ADDRESS (NUMBER, STREET, CITY, STATE, ZIP CODE)

EMERGENCY TELEPHONE NO

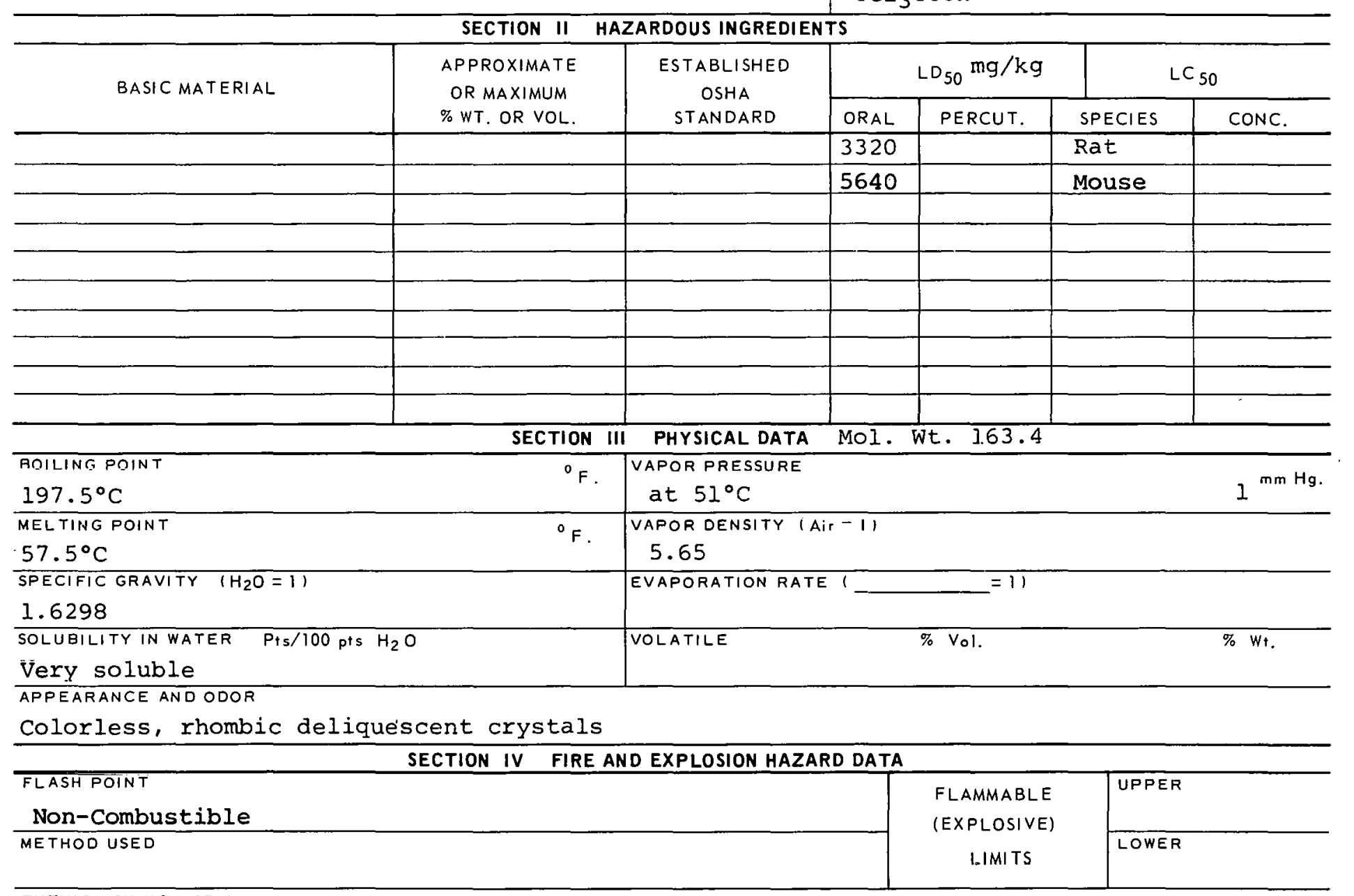

EXTINGUISHING MEDIA

Use water spray, dry chemical, $\mathrm{CU}_{2}$ or alcohol foam.

SPECIAL FIRE FIGHTING PROCEDURES

Wear goggles and self-contained breathing apparatus.

UNUSUAL FIRE ANO EXPLOSION HAZAROS 


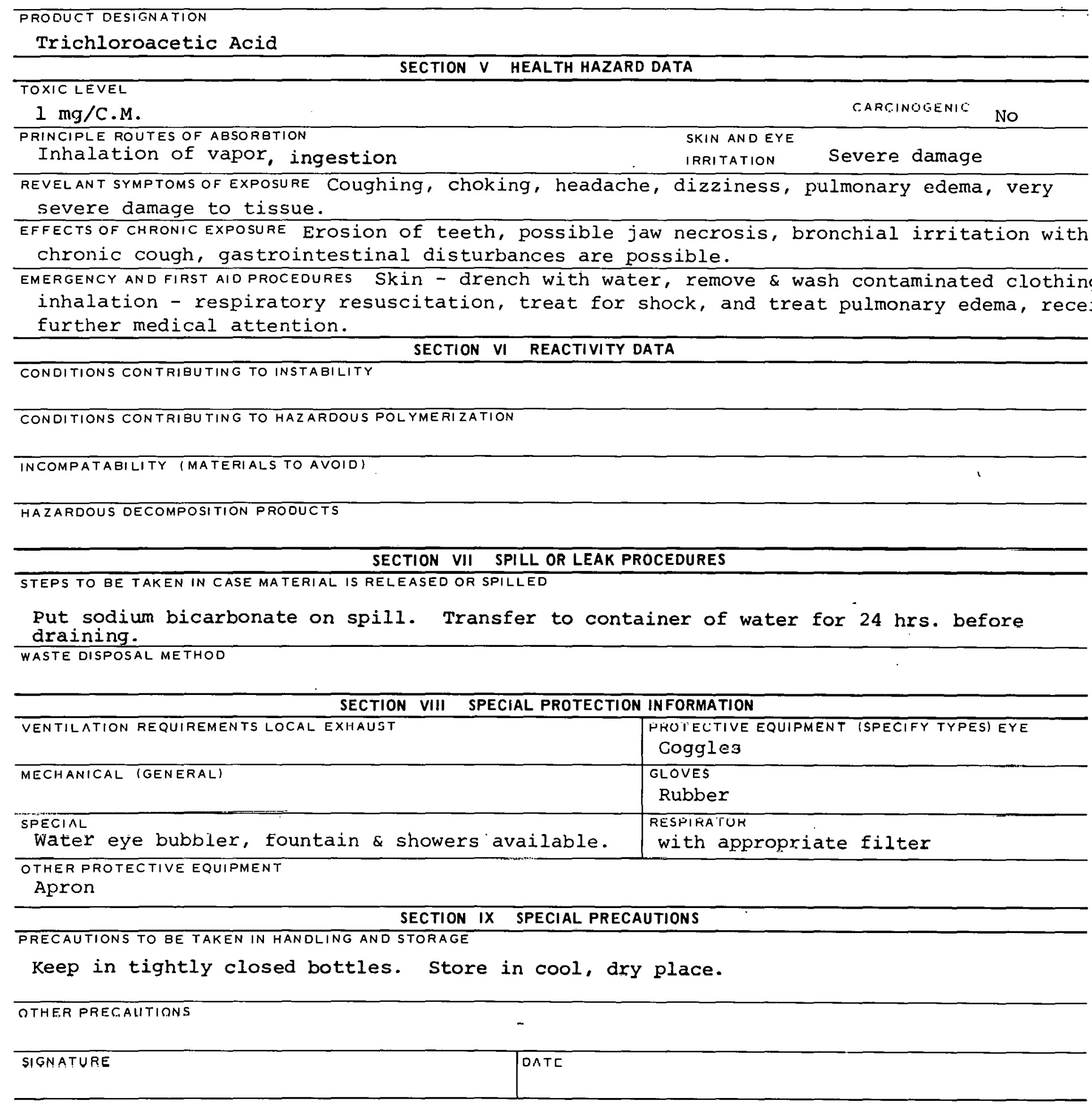




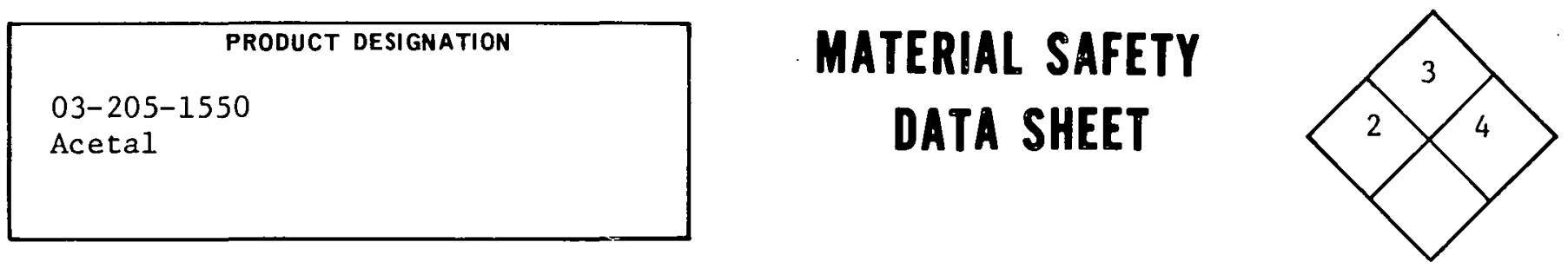

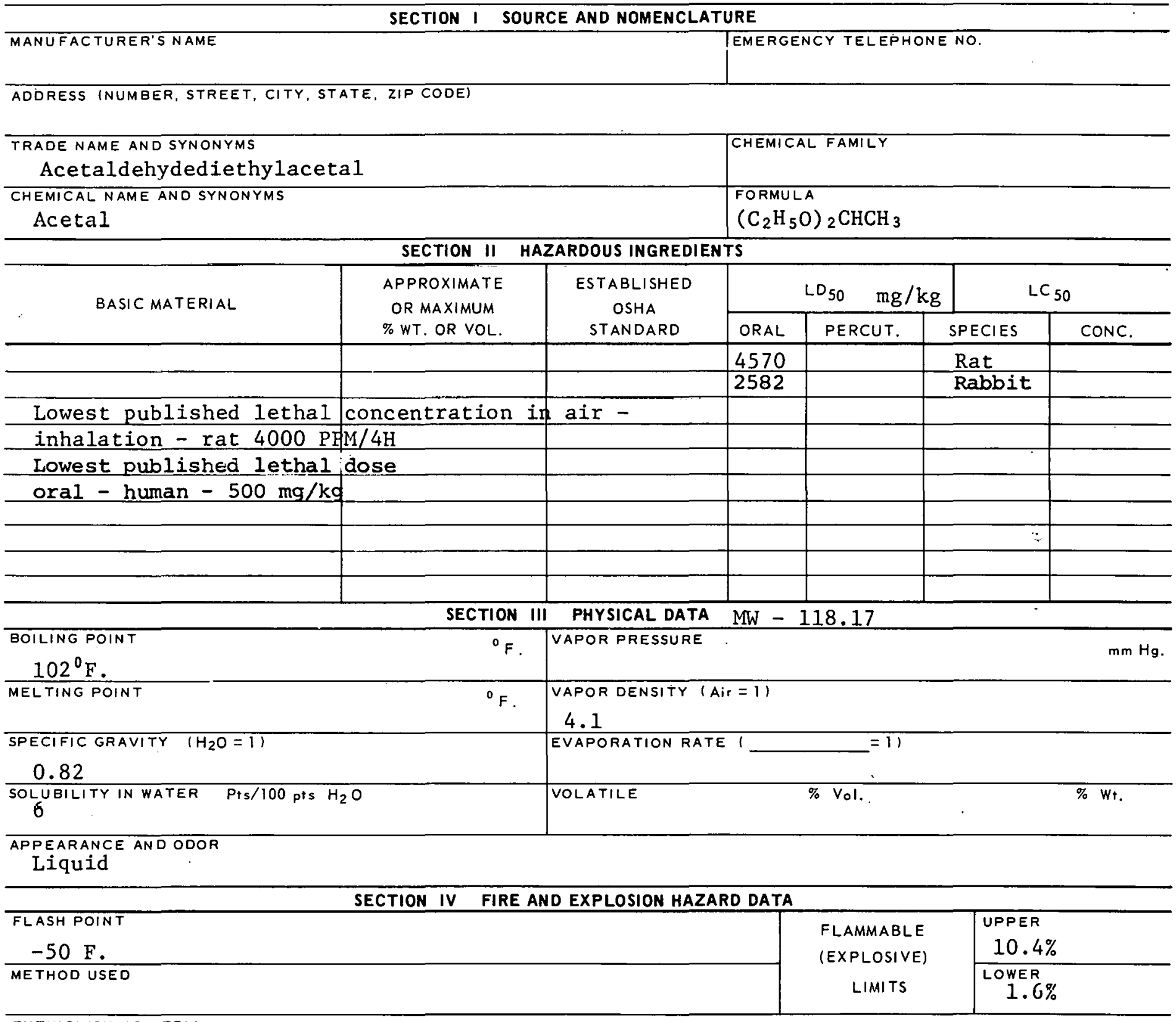

EXTINGUISHING MEDIA

Carbon dioxide, dry chemical, alcuhul fuam.

SPECIAL FIRE FIGHTING PROCEDURES

Water may be ineffective. UNUSUAL FIRE AND EXPLOSION HAZARDS

Former explosive peroxides 


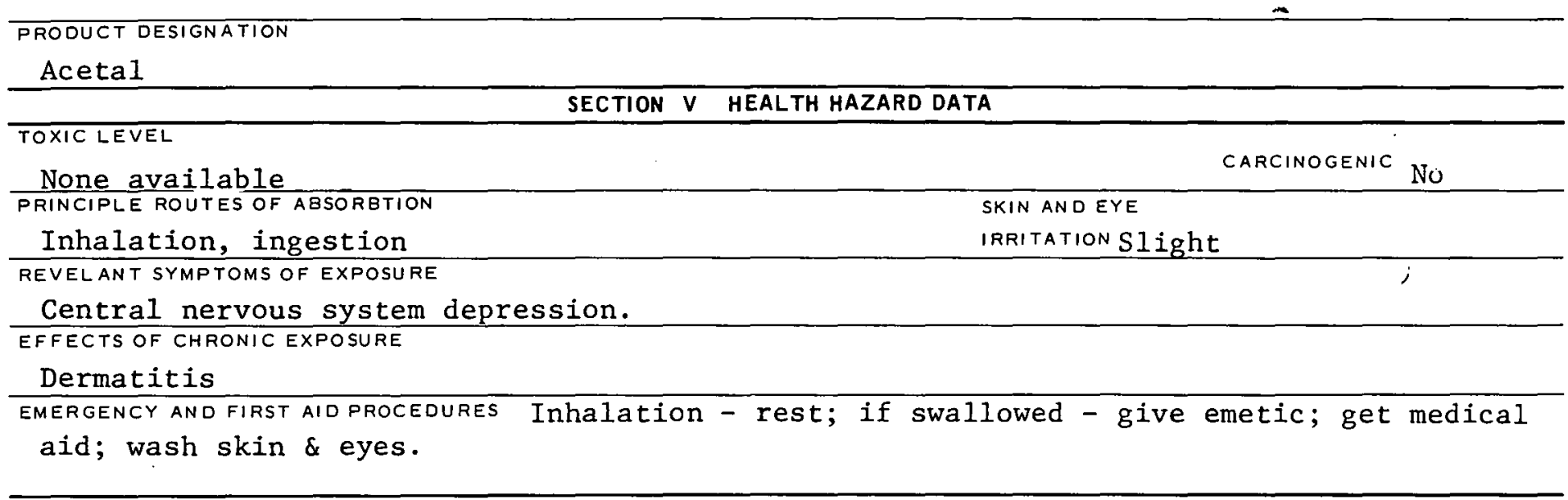

\section{SECTION VI REACTIVITY DATA}

CONDITIONS CONTRIBUTING TO INSTABILITY

Reacts with oxygen to produce exflosive peroxides shelf life - 12 months. CONDITIONS CONTRIGUTING TO HAZARDOUS POLYMERIZATION

INCOMPATABILITY (MATERIALS TO AVOOOOI

HAZARDOUS DECOMPOSITION PRODUCTS

SECTION VII SPILL OR LEAK PROCEDURES

STEPS TO BE TAKEN IN CASE MATERIAL IS RELEASED OR SPILLED

WASTE DISPOSAL METHOD

SECTION VIII SPECIAL PROTECTION IN FORMATION

\begin{tabular}{l|l}
\hline VENTILATION REQUIREMENTS LOCAL EXHAUST & $\begin{array}{l}\text { PROTECTIVE EQUIPMENT (SPECIFY TYPESIFYE } \\
\text { Safety glaSseS }\end{array}$ \\
\hline MECHANICAL (GENERAL) & GLOVES \\
\hline SPECIAL & $\begin{array}{l}\text { RESPIRAIUH } \\
\text { Canister type }\end{array}$ \\
\hline OTHER PROTECTIVE EQUITMENT & -
\end{tabular}

SECTION IX SPECIAL PRECAUTIONS

PRECAUTIONS TO BE TAKEN IN HANDLING AND STORAGE

OTIILR PRECAUTIONS

SIGNATURE

DATE

$205-1550 B$ 


\begin{tabular}{|c|}
\hline \multicolumn{1}{|c|}{ PRODUCT DESIGNATION } \\
Acetaldehyde \\
$03-205-2000$ \\
\end{tabular}

\section{MATERIAL SAFETY DATA SHEET}

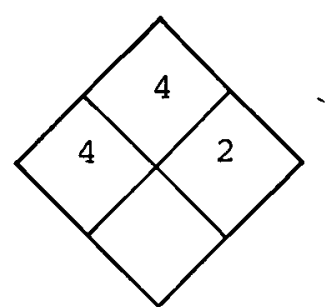

SECTION I SOURCE AND NOMENCLATURE

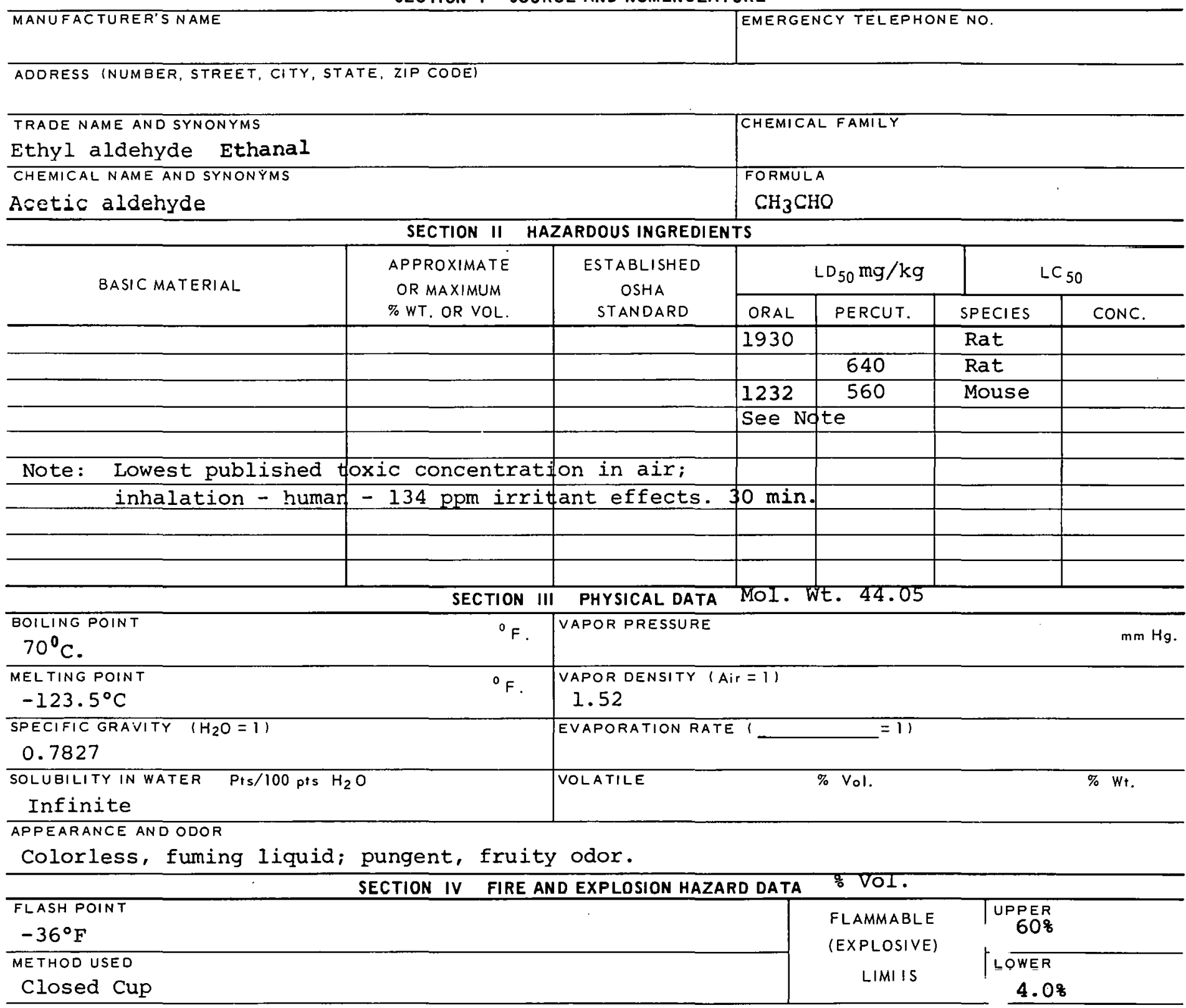

EXTINGUISHING MEDIA

Dry chemical, "alcohol" foam, or carbon dioxide.

SPECIAL FIRE FIGHTING PROCEDURES

In advanced or large fires, fight from a distance, wear goggles and self-contained breathing apparatus.

UNUSUAL FIRE ANO EXPLOSION HAZARDS

May cause explosion; flash backs. 


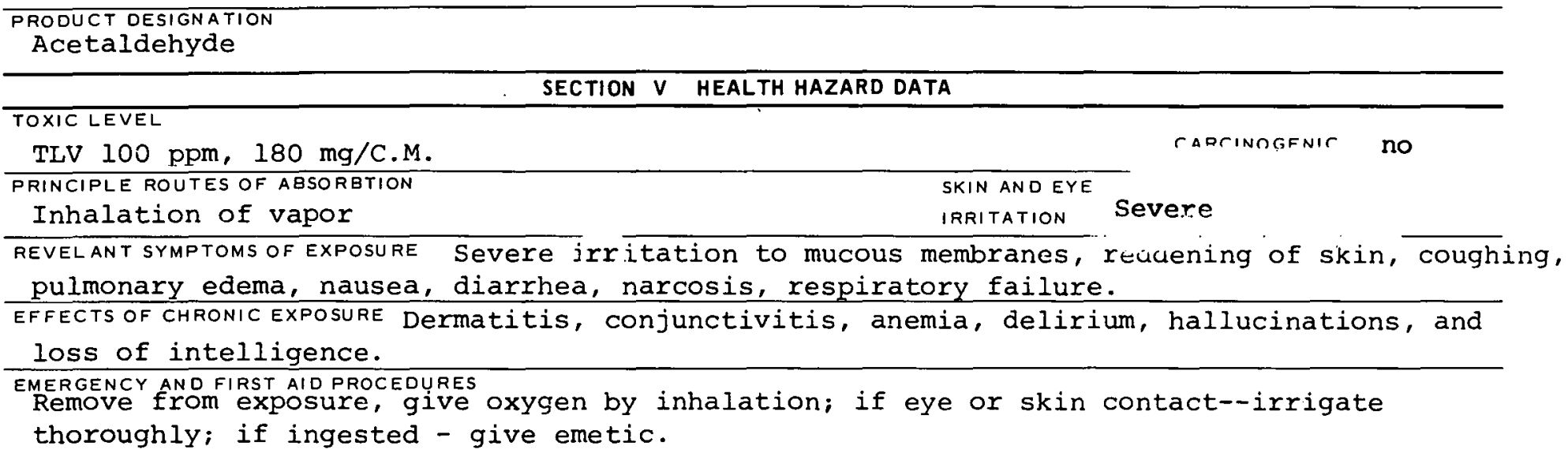




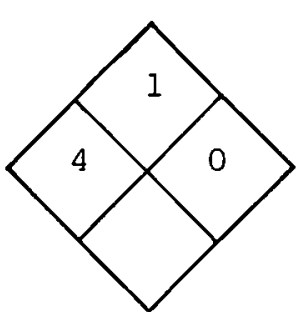

Acetamide

03-205-2500

SECTION I SOURCE AND NOMENCLATURE

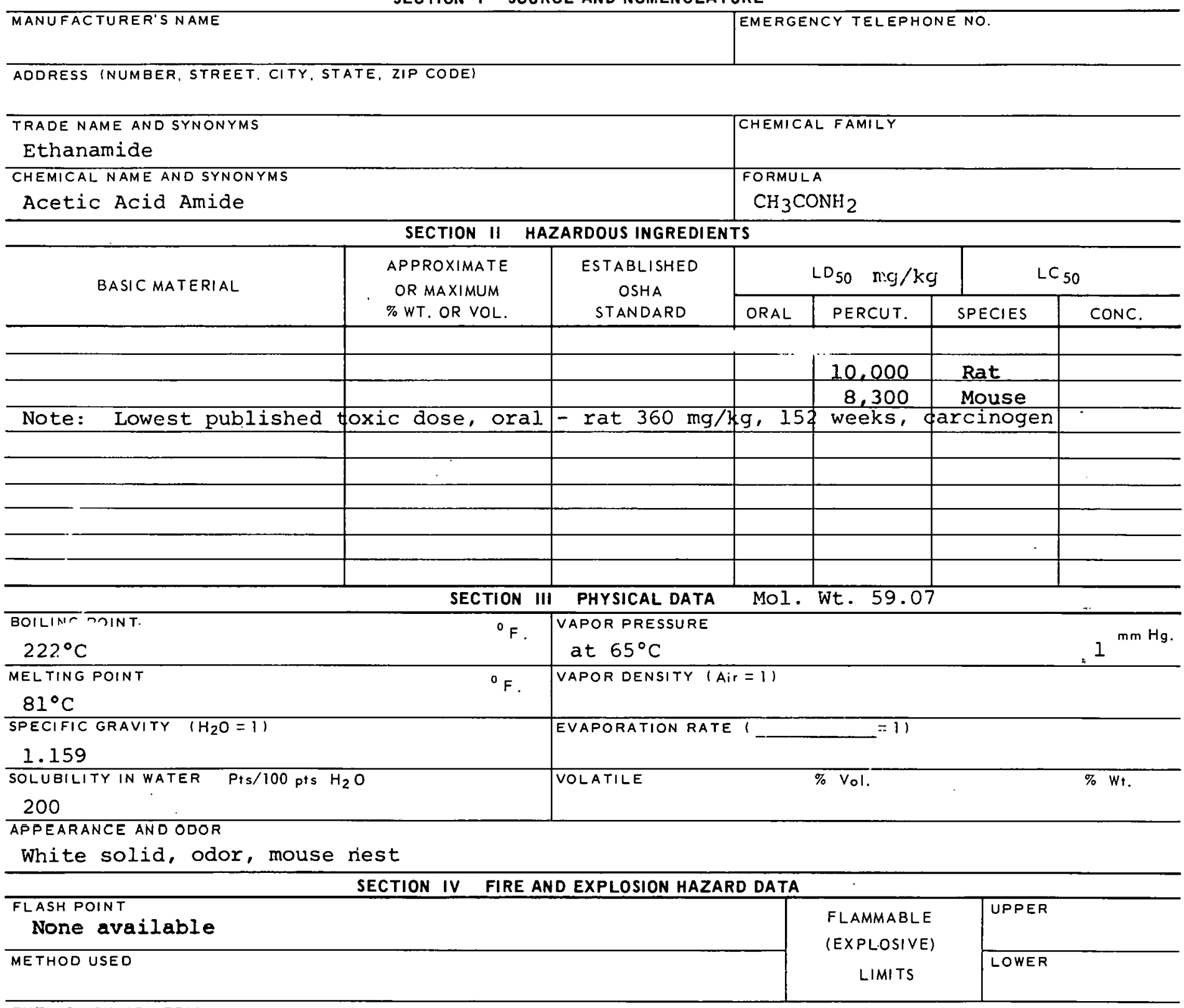

EXTINGUISHING MEDIA

Carbon dioxide, dry chemical

SPECIAL FIRE FIGHTING PROCEDURES

UNUSUAL FIRE AND EXPLOSION HAZAROS 


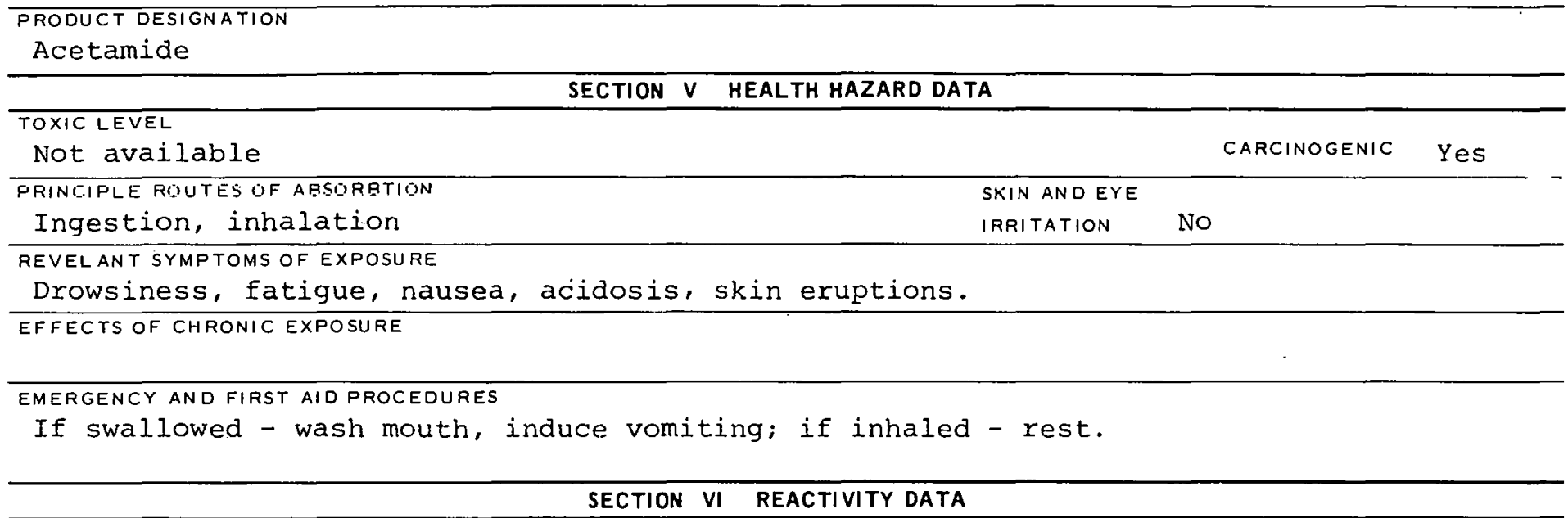

CONDITIONS CONTRIBUTING TO INSTABILITY

CONDITIONS CONTRIBUTING TO HAZARDOUS POLYMERIZATION

IIYUUMPAIAOILIII IMMIERIALS IV AVUIU)

HAZARDOUS DECOMPOSITION PRODUCTS

SECTION VII SPILL OR LEAK PROCEDURES

STEPS TO BE TAKEN IN CASE MATERIAL IS RELEASED OR SPILLED

WASTE DISPOSAL METHOD

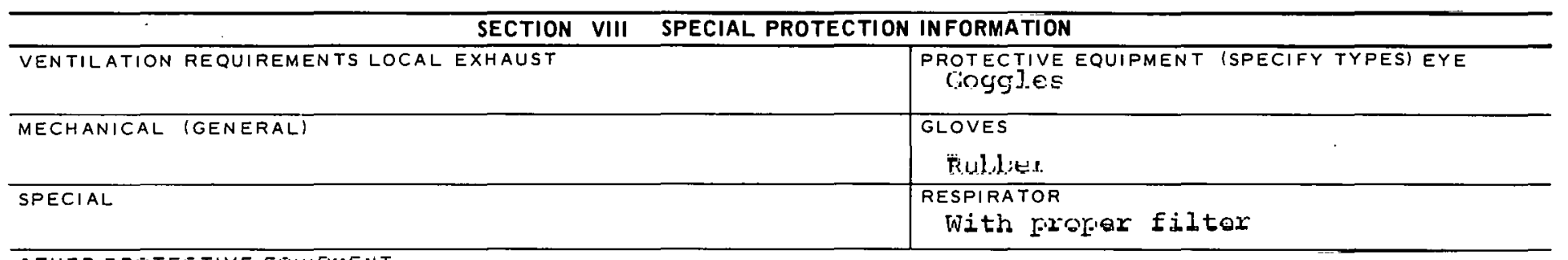

OTHER PRUTECTIVE EUUIFMENT

SECTION IX SPECIAL PRECAUTIONS

PRECAUTIONS TO BE TAKEN IN HANDLING AND STORAGE

OTHER PRECAUTIONS

SIGNATURE

DATE

$205-2500 B$ 
CAS:-000079058C

\begin{tabular}{|l|} 
PRODUCT DESIGNATION \\
Acetonitrile \\
$03-205-5000$
\end{tabular}

\section{AL 77000 \\ MATERIAL SAFETY \\ DATA SHEET}

\section{SECTION I SOURCE AND NOMENCLATURE}

\begin{tabular}{l} 
SECTION I SOURCE AND NOMENCLATURE \\
\hline MANUFACTURER'S NAME \\
\hline AODRESS (NUMBER, STREET, CITY, STATE, ZIP CODE)
\end{tabular}

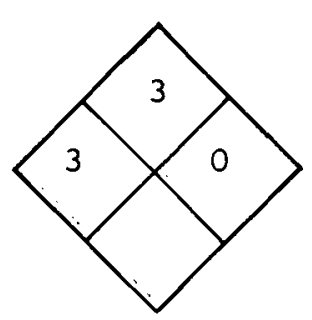

TRADE NAME AND SYNONYMS

Methyl Cyanide, Ethanenitrile

CHEMICAL NAME AND SYNONYMS

Ethyl Nitrile, Acetonitrile

\begin{tabular}{l|l} 
& CHEMICAL FAMILY \\
& $\begin{array}{r}\text { FORMULA } \\
\mathrm{CH}_{3} \mathrm{CN}\end{array}$ \\
\hline
\end{tabular}

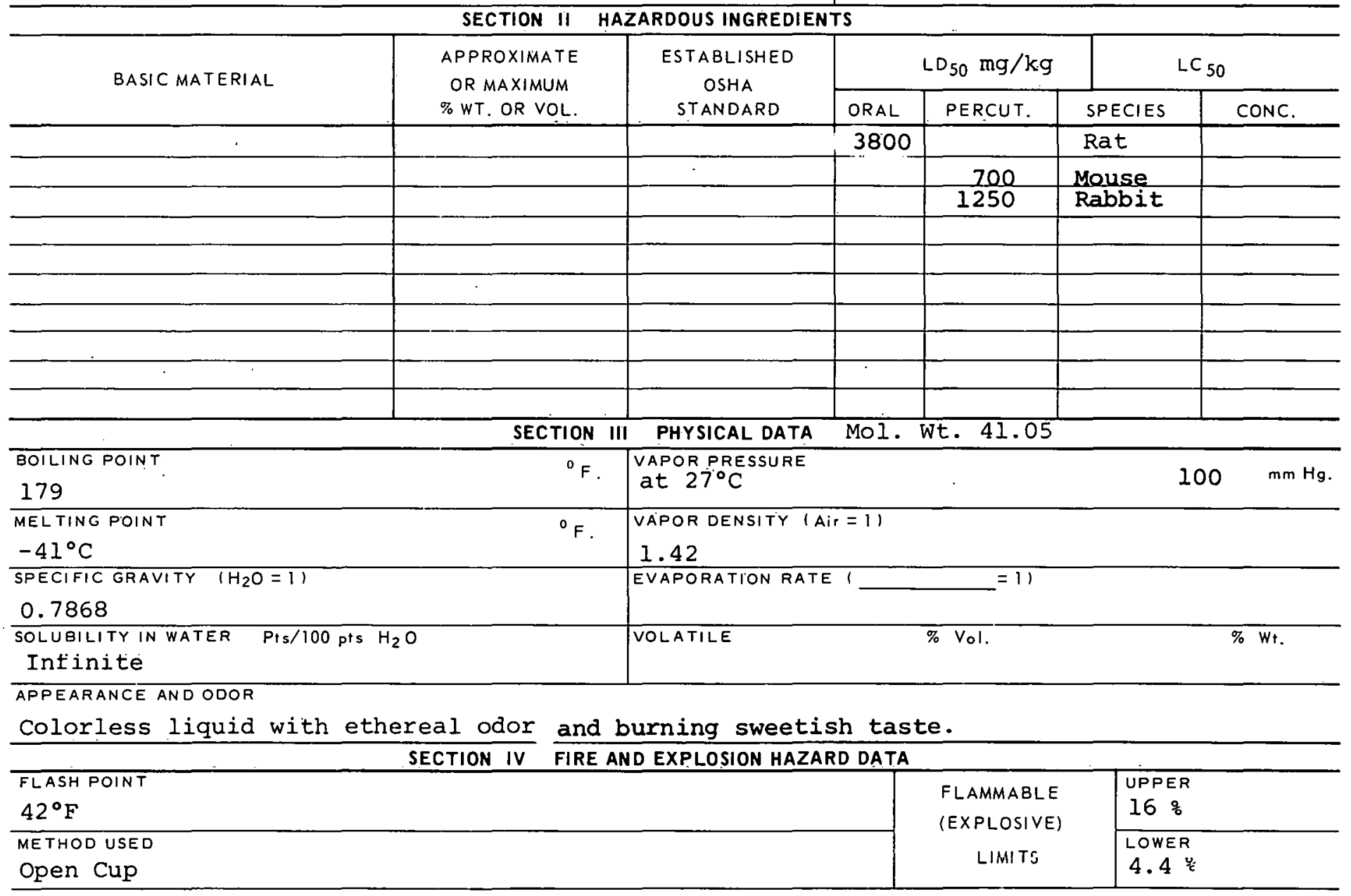

EXTINGUISHING MEDIA

Dry chemical, "alcohol" foam, or carbon dioxide

water spray - ineffective.

SPECIAL FIRE FIGHTING PROCEDURES

Wear self-contained breathing ápuarátus.

UNUSUAL FIRE AND EXPLOSION HAZARDS

Flash Lä̀ks 


\section{TOXIC LEVEL}

TLV $40 \mathrm{ppm}, 70 \mathrm{mg} / \mathrm{C} . \mathrm{M}$. PRINCIPLE ROUTES OF ABSORETION Inhalation and skin absorption, ingestion
CARCINOGENIC No SKIN AND EYE IRRITATION Mild to moderate

REVELANT SYMPTOMS OF EXPOSURE Dizziness, rapid respiration, headache, drowsiness, drop in blood pressure, rapid pulse, unconsciousness. EFFECTS OF CHRONIC EXPOSURE Dizziness, weakness, hoarseness, congestion of lungs, conjunctivitis, loss of weight and appetite, and mental deterioration. EMERGENCY AND FIRST AID PROCEDURES Remove from area; give amyl nitrite inhalation every 5 min.

$(0.2 \mathrm{ml} / 3 \mathrm{min.})$; give artificial respiration $\mathrm{w} / 100 \% \mathrm{O}_{2}$; $10 \mathrm{ml}$ of $3 \%$ sodium nitrite; skin or eye contact - wash thoroughly; Obtain medical attention.

SECTION VI REACTIVITY DATA

CONDITIONS CONTRIBUTING TO INSTABILITY

CONDITIONS CONTRIBU TING TO HAZARDOUS POLYMERIZATION

INCOMPAIAHILIIY (MATERIALS TO AVOID)

HAZARDOUS DECOMPOSITION PRODUCTS

Cyanıde

SECTION VII SPILL OR LEAK PROCEDURES

STEPS TO BE TAKEN IN CASE MATERIAL IS RELEASED OR SPILLEO

Use water spray.

WASTE DISPOSAL METHOD

SECTION VIII SPECIAL PROTECTION INFORMATION

\begin{tabular}{l|l}
\hline VENTILATION REQUIREMENTS LOCAL EXHAUST & $\begin{array}{c}\text { PROTECTIVE EQUIPMENT (SPECIFY TYPES) EYE } \\
\text { GoggleS }\end{array}$ \\
\hline MECHANICAL IGENERAL) & $\begin{array}{c}\text { GLOVES } \\
\text { Rubber }\end{array}$ \\
\hline SPECIAL & $\begin{array}{c}\text { RESPIRATOR } \\
\text { Self-contained breathing apparatus }\end{array}$ \\
\hline
\end{tabular}

OTHER PROTECTIVE EQUIPMENT

Rubber apron

SECTION IX SPECIAL PRECAUTIONS

PRECAUTIONS TO BE TAKEN IN HANDLING AND STORAGE

Store in standard flammable liquids storage room. Protect against physical damage.

OTHER PRECAUTIONS 
CAS : 000110134

PRODUCT DESIGNATION

03-205-6000

Acetorylacetone
MO 31500

MATERIAL SAFETY

DATA SHEET

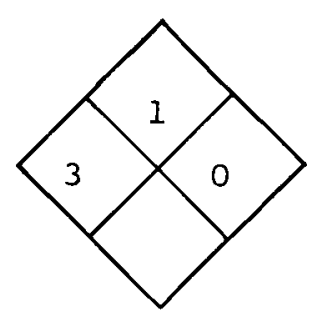

SECTION I SOURCE AND NOMENCLATURE

MANUFACTURER'S N-AME

EMERGENCY TELEPHONE NO.

ADDRESS (NUMBER, STREET, CITY, STATE, ZIP CODE)

TRADE NAME AND SYNONYMS

CHEMICAL NAME AND SYNONYMS

2,5 Hexenedione

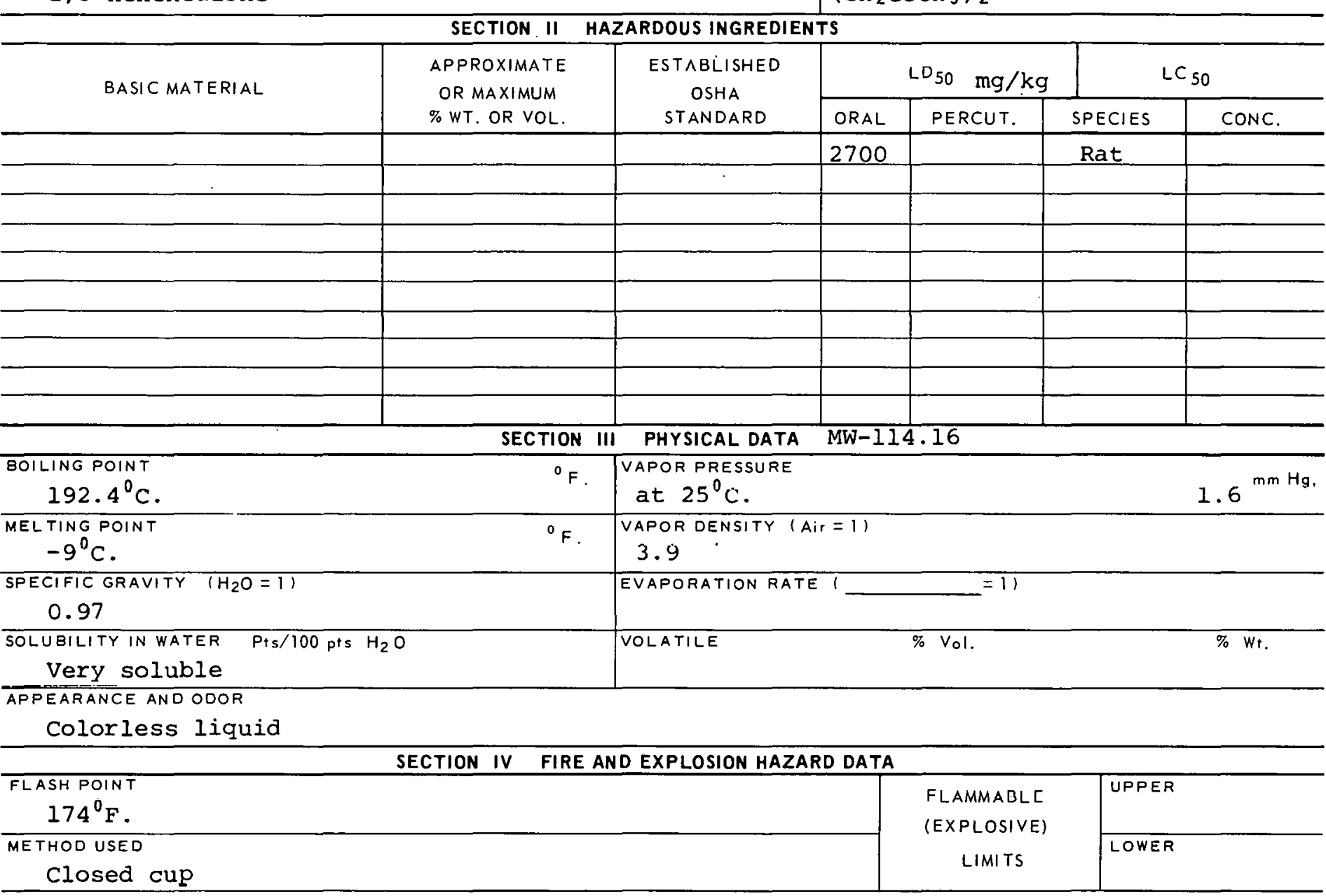

EXTINGUISHING MEDIA

Alcohol foam, carbon dioxide, dry chemical.

SPECIAL FIRE FIGHTING PROCEDURES

UNUSUAL FIRE AND EXFLOSION HALAHDS 


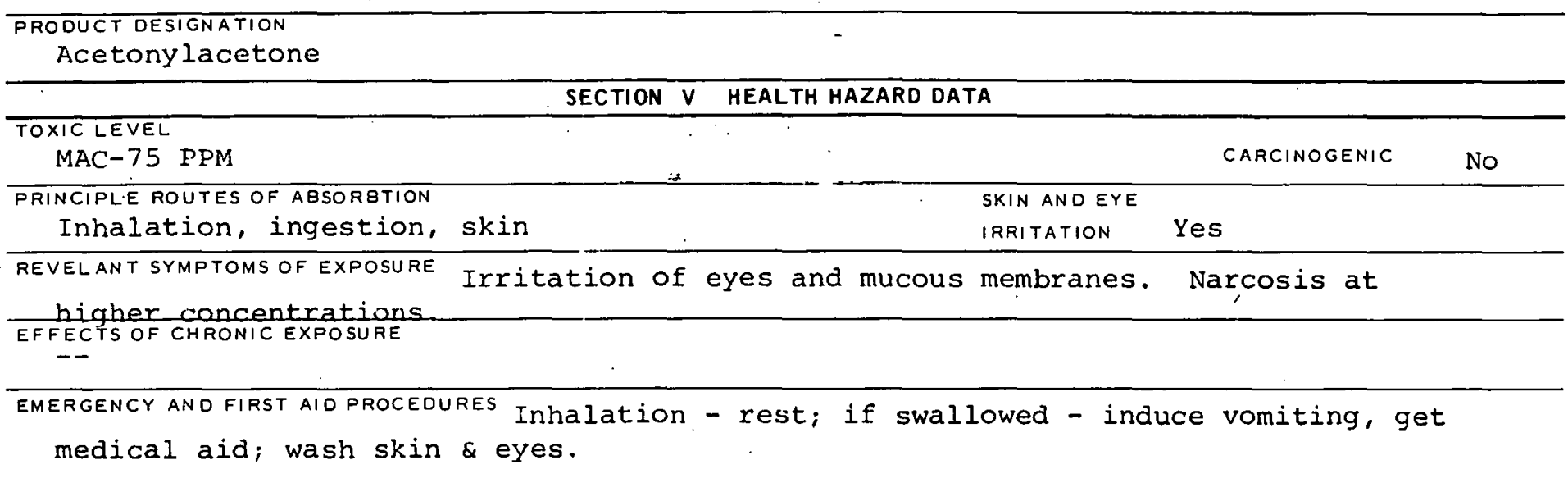

\begin{tabular}{|c|c|c|}
\hline SECTION VI & REACTIVITY DATA & . \\
\hline \multicolumn{3}{|l|}{ CONDITIONS CONTRIBUTING TO INSTABILITY } \\
\hline \multicolumn{3}{|l|}{ CONDITIONS CONTRIBUTING TO HAZARDOUS POLYYMERIZATION } \\
\hline IISCOAFATADILITY (AAATER!ALSTO AVOIR) & & \\
\hline can react with oxidizing materials. & . & \\
\hline
\end{tabular}

\section{SECTION VII SPILL OR LEAK PROCEDURES}

STEPS TO BE TAKEN IN CASE MATERIAL IS RELEASED OR SPILLED

WASTE DISPOSAL METHOD

,

\begin{tabular}{|c|c|}
\hline SECTION VIII & SPECIAL PROTECTION INFORMATION \\
\hline VENTILATION REQUIREMENTS LOCAL EXHAUST & $\begin{array}{l}\text { PROTECTIVE EQUIPMENT (SPECIFY TYPES) EYE } \\
\text { GoggleS }\end{array}$ \\
\hline MECHANICAL IGENERAL) & $\begin{array}{l}\text { GLOVES } \\
\text { Rubber }\end{array}$ \\
\hline SPECIAL & $\begin{array}{l}\text { RESPIRATOR } \\
\text { With chemlical cartriage Ilicer }\end{array}$ \\
\hline
\end{tabular}

OTHER PROTECTIVE EQUIPMENT

SECTION IX SPECIAL PRECAUTIONS

PRECAUTIONS TO BE TAKEN IN HANDLING ANO STORAGE

Store in well-ventilated area.

OTHER PRECAUTIONS

SIGNATURE

DATE

$205-6000 B$ 


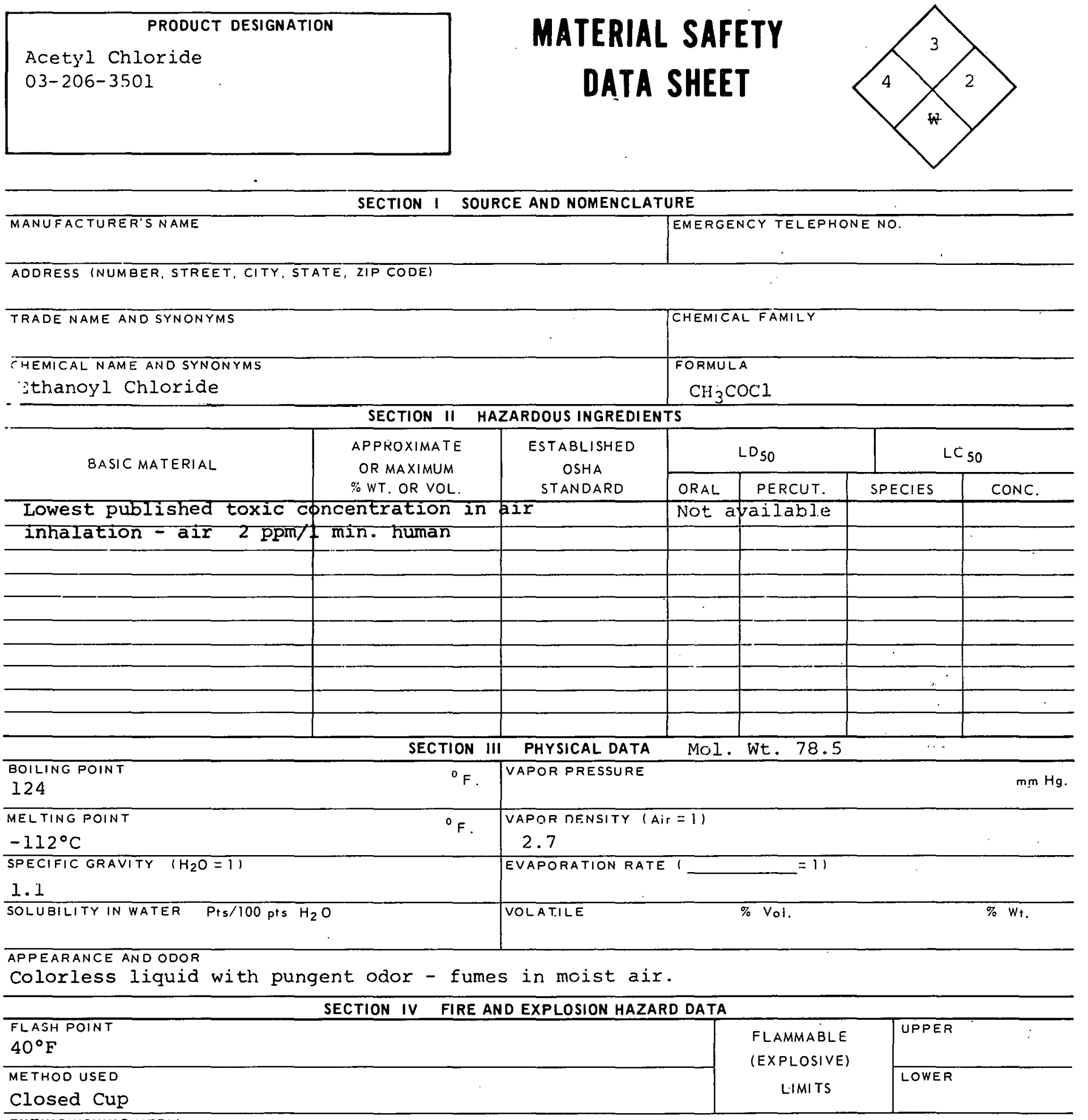

EXTINGUISHING MEDIA

Do not use watcr or foam = use carbon dioxide or dry chemical.

SPECIAL FIRE FIGHTING PROCEDURES

Wear. full protective clothing.

UNUSUAL FIRE AND EXPLOSION HAZARDS

Reacts violently with water - forms explosive mixtures in air. May flash back. 
TOXIC LEVEL

MAC (5 pPm)

PRINCIPLE ROUTES OF ABSORBTION

Ininalation

SKIN AND EYE

CARCINOGENIC

IRRITATION Moderate to severe

REVELANT SYMPTOMS OF EXPOSURE Inhalation - cyanosis, headache, coughing, dizziness, weakness, pulmonary edema; skin contact - severe pain, brownish stains, burns penetrating full thickEFFECTS OF CHRONIC EXPOSURE ness of skin.

Inhalation - errosion of teeth, jaw nerosis, broncinial irritation and chronic cough. EMERGENCY AND FIRST AID PROCEDURES Skin contact - flood area w/water for $15 \mathrm{~min}$, treat burns; inhalation - remove from area, use respiratory resuscitation, treat shock, pulmonary edena; obtain medical aid.

\section{SECTION VI REACTIVITY DATA}

CONDITIONS CONTRIBUTING TO INSTABILITY

CONDITIONS CONTRIBUTING TO HAZARDOUS POLYMERIZATION

INCUMHATABIIITY IMATERIALS TOAVOIDT

Water, ethyl alcohol, dimethyl sulfoxide.

HAZARDOUS OECOMPOSITION PRODUCTS

Healed - lyyduyen chlorlde and phosgene evolved.

SECTION VII SPILL OR LEAK PROCEDURES

STEPS TO BE TAKEN IN CASE MATERIAL IS RELEASED OR SPILLED

WASTE DISPOSAL METHOD

\section{SECTION VIII SPECIAL PROTECTION INFORMATION}

\begin{tabular}{|c|c|}
\hline VENTILATION REQUIREMENTS LOCAL EXHAUST & $\begin{array}{l}\text { PROTECTIVF. EQUIPMENT (SPECIFY TYPES) EYE } \\
\text { Goggles (tight fitting) }\end{array}$ \\
\hline MECIIANICAL IOENERALI & $\begin{array}{l}\text { GLOVES } \\
\text { Rubber }\end{array}$ \\
\hline SPECIAL & $\begin{array}{l}\text { RESPIRATOR } \\
\text { w/anPYOPYlate falter }\end{array}$ \\
\hline \multicolumn{2}{|l|}{$\begin{array}{l}\text { OTHER PROTECTIVE EQUIPMENT } \\
\text { Rubber apron, full face shield }\end{array}$} \\
\hline \multicolumn{2}{|c|}{ SECTION IX SPECIAL PRECAUTIONS } \\
\hline \multicolumn{2}{|c|}{$\begin{array}{l}\text { PRECAUTIONS TO BE TAKEN IN HANDLING ANDSTORAGE } \\
\text { Store in coOl, dry, well-ventilated, preferably detached location, free of sources of } \\
\text { ignition in a standard flammable liquids storage room or cabinet. }\end{array}$} \\
\hline \multicolumn{2}{|l|}{ OTHER PRECAUTIONS } \\
\hline SIGNATURË & DATE \\
\hline
\end{tabular}


See dimethyl acetamide

03-206-4501

Acetyl Methyl Amine

\section{MATERIAL SAFETY DATA SHEET}

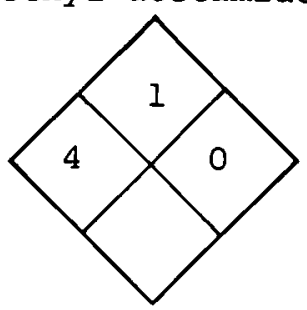

\section{SECTION I SOURCE AND NOMENCLATURE}

MANUFACTURER'S NAME

ADDRESS (NUMBER, STREET, CITY, STATE, ZIP CODE)

EMERGENCY TELEPHONE NO.

TRADE NAME AND SYNONYMS

Methyl Acetamide

CHEMICAL NAME AND SYNONYMS

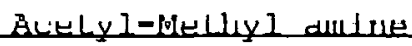

\begin{tabular}{l|l}
\hline & CHEMICAL FAMILY \\
& $\begin{array}{l}\text { FORMULA } \\
\text { GH }_{3} \text { SONHGH }_{3}\end{array}$ \\
\hline
\end{tabular}

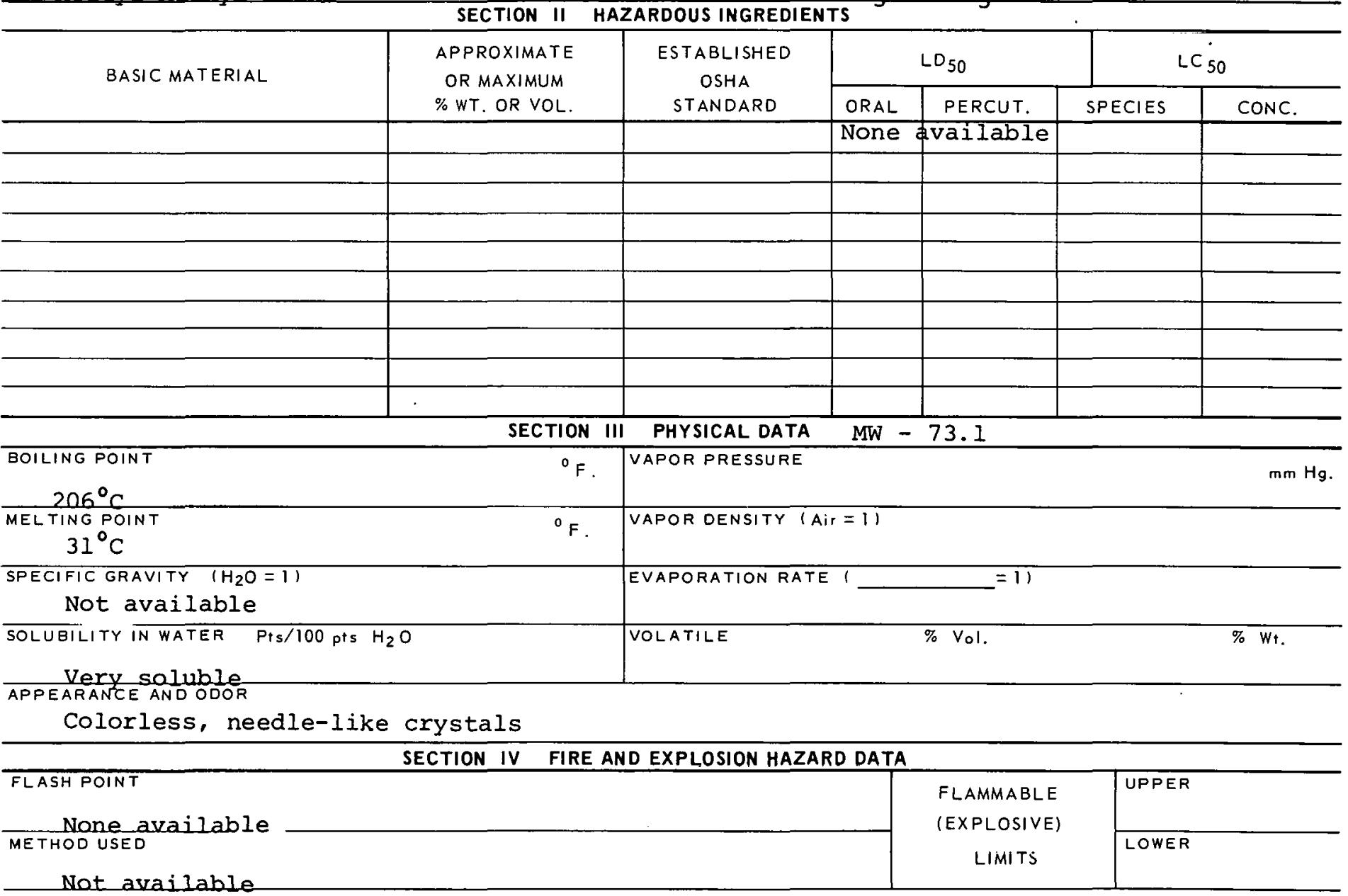

EXTINGUISHING MEDIA

SPECIAL FIRE FIGHTING PROCEDURES

UNUSUAL FIRE AND EXPLOSION HAZAROS 


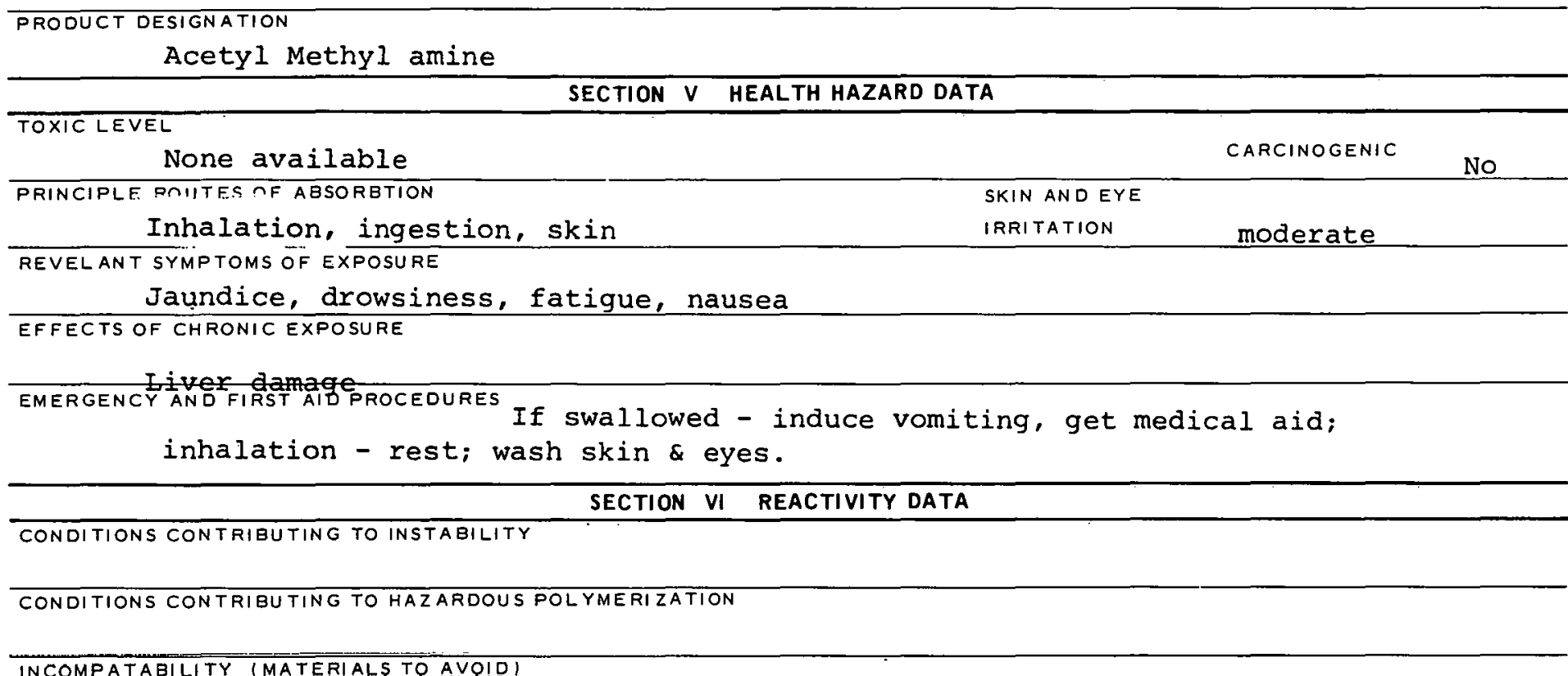

HAEARDOUS DECOMPOBITION PRODUCTS

SECTION VII SPILL OR LEAK PROCEDURES

STEPS TO BE TAKEN IN CASE MATERIAL IS RELEASED OR SPILLED

WASTE DISPOSAL METHOD

\begin{tabular}{l|l}
\hline \multicolumn{2}{c}{ SECTION VIII SPECIAL PROTECTION INFORMATION } \\
\hline VENTILATION REQUIREMENTS LOCAL EXHAUST & $\begin{array}{c}\text { PROTECTIVE EQUIPMENT (SPECIFY TYPESI EYE } \\
\text { Goggles }\end{array}$ \\
\hline MECHANICAL IGENERAL) & $\begin{array}{c}\text { GLOVES } \\
\text { Rubber gloves }\end{array}$ \\
\hline SPECIAL & $\begin{array}{c}\text { FESFIFATOF } \\
\text { With chemical filter }\end{array}$ \\
\hline
\end{tabular}

OTHER PROTEC.TIVE EQUIPMENT

SECTION IX SPECIAL PRECAUTIONS

PRECAUTIONS TOO $8 E$ TAKEN IN HANDLING AND STORAGE

\begin{tabular}{l|l|l}
\hline OTHER PRECAUTIONS & DATE \\
\hline SIGNATURE & \\
\hline
\end{tabular}




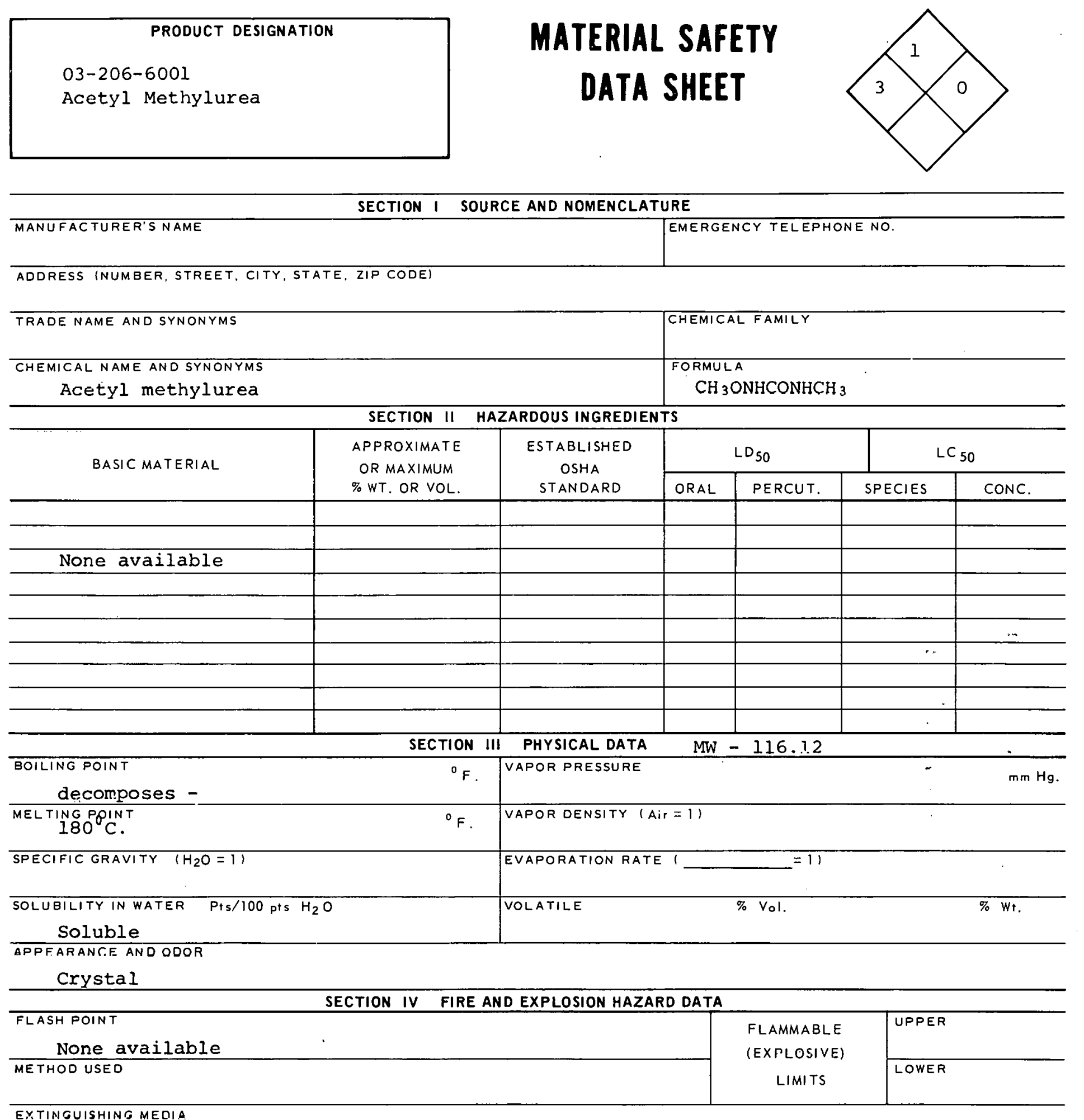

EXTINGUISHING MEOIA

Alcohol foam, carbon dioxide, dry chemical.

SPECIAL FIRE FIGHTING PROCEDURES

UNUSUAL FIRE AND EXPLOSION HAZARDS 


\section{TOXIC LEVEL \\ None available}

PRINCIPLE ROUTES OF ABSORBTION

Inhalation, ingestion

REVELANT SYMPTOMS OF EXPOSURE

Dermatitis

EFFECTS OF CHRONIC EXPOSURE Depression of bone marrow, anemia, allergic skin eruptions.

EMERGENCY AND FIRST AID PROCEDURES If swallowed - induce vomiting, get medical aid; inhalation - rest; wash from eyes \& skin.

CONDITIONS CONTRIBUTING TO INSTABILITY

CONDITIONS CONTRIBUTING TO HAZARDOUS POLYMERIZATION

INCOMPATABILITY (MATFR!ALS TQ AVQID)

HAZARDOUS DECOMPOSITION PRODUCTS

SECTION VII SPILL OR LEAK PROCEDURES

STEPS TO BE TAKEN IN CASE MATERIAL IS RELEASED OR SPILLED

Cover with weak calcium hypochlorite solution, neutralize with dilute HCl alter

24 hours and wash into drain.

WASTE DISPOSAL METHOD

SECTION VIII SPECIAL PROTECTION INFORMATION

\begin{tabular}{l|l}
\hline VENTILATION REQUIREMENTS LOCAL EXHAUST & $\begin{array}{l}\text { PROTECTIVE EQUIPMFNT (SPECIFY TYPESI EYE } \\
\text { GogqLES }\end{array}$ \\
MECHANICAL IGENERAL) & $\begin{array}{c}\text { GLOVES } \\
\text { Rubber }\end{array}$ \\
\hline SPECIAL & $\begin{array}{l}\text { RESPIRATOR } \\
\text { With proper Eilter }\end{array}$ \\
\hline
\end{tabular}

OTHER PROTECTIVE EQUIPMENT

SECTION IX SPECIAL PRECAUTIONS

PRECAUTIONS TO BE TAKEN IN HANDLING AND STORAGE

OTHER PRECAUTIONS

SIGNATURE

DATE

206-6001B 
CAS-000591082

\begin{tabular}{|c|}
\hline PRODUCT DESIGNATION \\
03-206-6501 \\
Acetyl Thiourea \\
\\
\end{tabular}

YR77000

MATERIAL SAFETY DATA SHEET

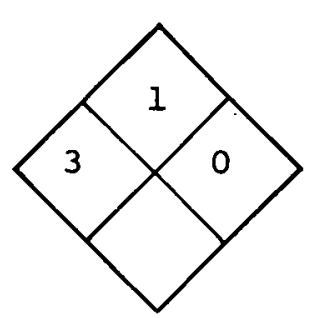

SECTION I SOURCE AND NOMENCLATURE

MANUFACTURER'S NAME

ADDRESS (NUMBER, STREET, CITY, STATE, ZIP CODE)

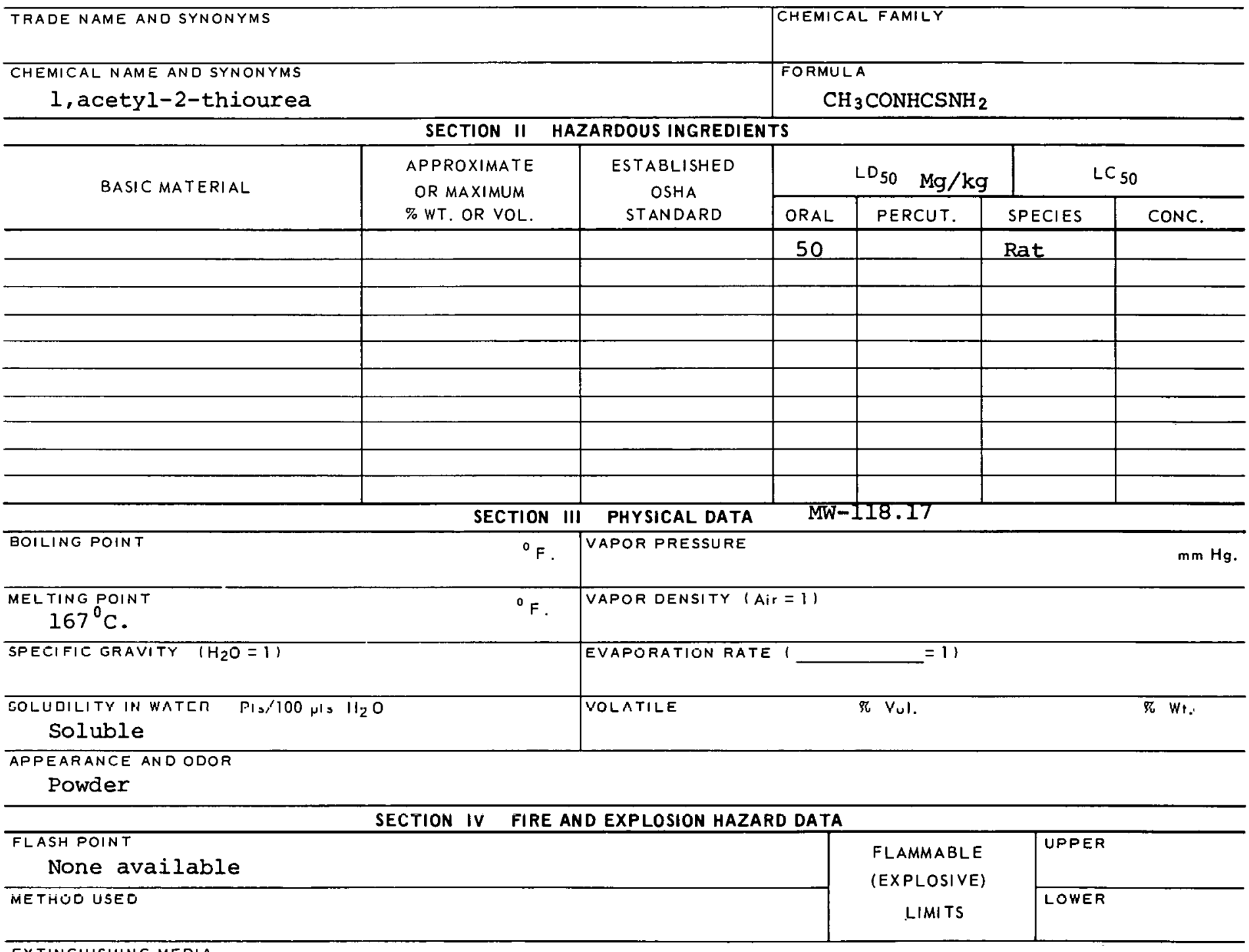

CXTINGUIGIING MEDIA

Alcohol foam, carbon dioxide, dry chemical.

SPECIAL FIRE FIGHTING PROCEDURES

UNUSUAL FIRE AND EXPLOSION HAZARDS 


\section{Acetylthiorurea}

\section{SECTION $V$ HEALTH HAZARD DATA}

TOXIC LEVEL

None available

PRINCIPLE ROUTES OF ABSORETION

Inhalation, ingestion

CARCINOGENIC

No

REVELANT SYMPTOMS OF EXPOSURE

Dermatitis

EFFECTS OF CHRONIC EXPOSURE

Depression of bone marrow, anemia, allergic skin eruptions.

EMERGENCY AND FIRST AID PROCEDURES If swallowed - induce vomiting, get medical aid;

wash from eyes.

SKIN AND EYE

IRRITATION Slight

SECTION VI REACTIVITY DATA

CONDITIONS CONTRIBUTING TO INSTABILITY

CONDITIONS CONTRIBUTING TO HAZARDOUS POLYMERIZATION

INCOMPA 9 A HILITY IMATERIALS TO AVOIO)

IIAZAROOUS DECOMROGITIOH TRODUCTE

SECTION VII SPILL OR LEAK PROCEDURES

STEPS TO BE TAKEN IN CASE MATERIAL IS RELEASED OR SPILLED

Cover with weak solution calcium hypochlorite. Neutralize with dilute HCl and wash into drain.

WASTE DISPOSAL METHOD

SECTION VIII SPECIAL PROTECTION INFORMATION

\begin{tabular}{|c|c|}
\hline VENTILATION REQUIREMENTS LOCAL EXHAUST & $\begin{array}{l}\text { PROTECTIVE EQUIPMENT (SPECIFY TYPES) EYE } \\
\text { GOgglCE }\end{array}$ \\
\hline MECHANICAL (GENERAL) & $\begin{array}{l}\text { GLOVES } \\
\text { Rubber }\end{array}$ \\
\hline SPES.IAL & $\begin{array}{l}\text { Wropinatro } \\
\text { With proper filter }\end{array}$ \\
\hline
\end{tabular}

OTHER PROT̈ECTIVE EQUIPMENT

SECTION IX SPECIAL PRECAUTIONS

PRECAUTIONS TOBE TAKEN IN HANDLING AND STORAGE

OTHER PRECAUTIONS

SIGNATURE

NATE

206-6501B 
CAS:-000596071

PRODUCT DESIGNATION

03-206-7501

Acetyl urea
YR53000

MATERIAL SAFETY

DATA SHEET

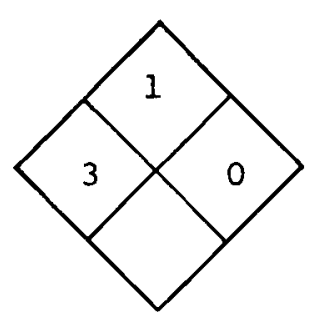

SECTION I SOURCE AND NOMENCLATURE

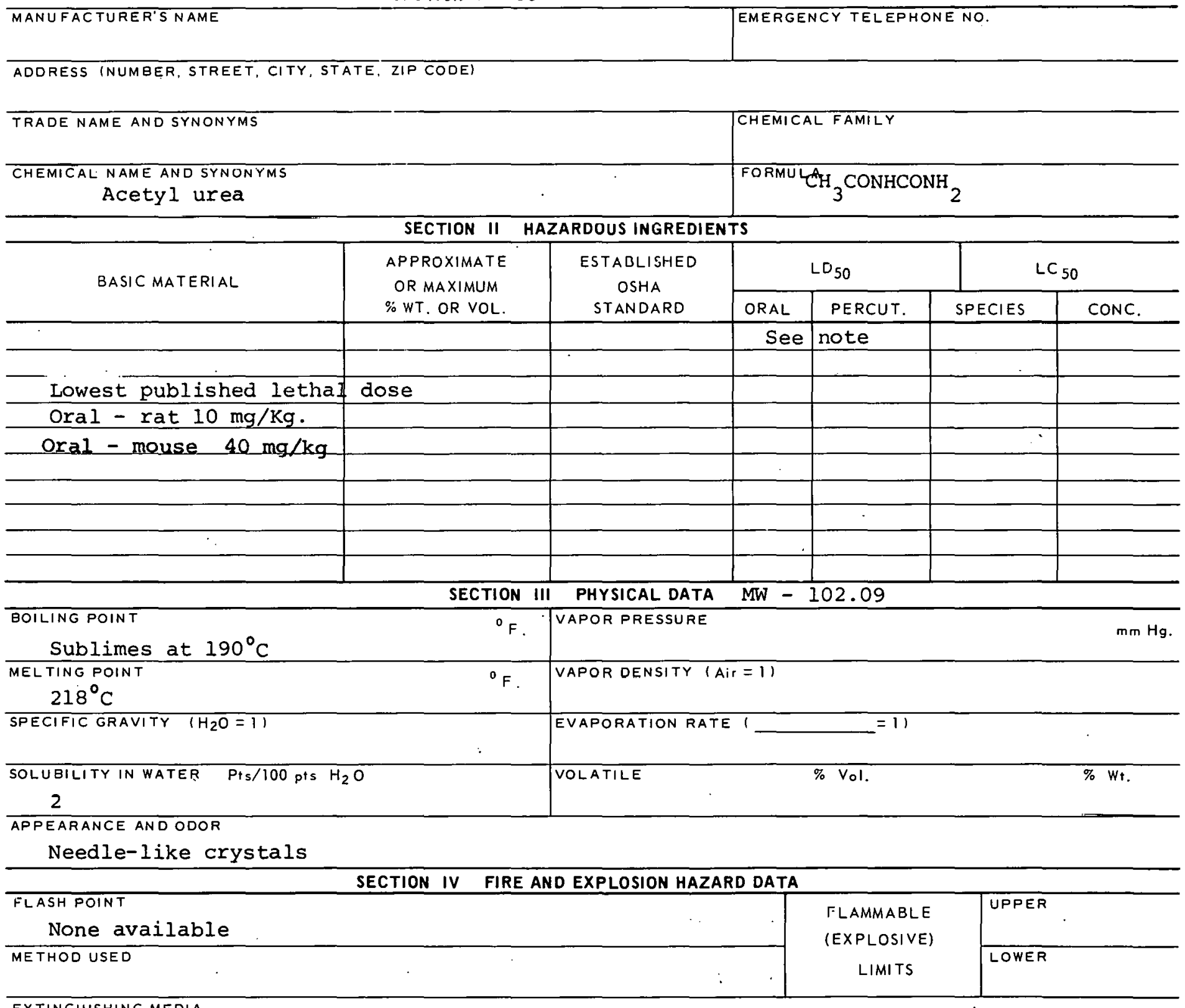

EXTINGUISHING MEDIA

Carbon dioxide, dry chemical

SPECIAL FIRE FIGHTING PROCEDURES

UNUSUAL FIRE AND EXPLOSION HAZARDS 
Acetyl Urea

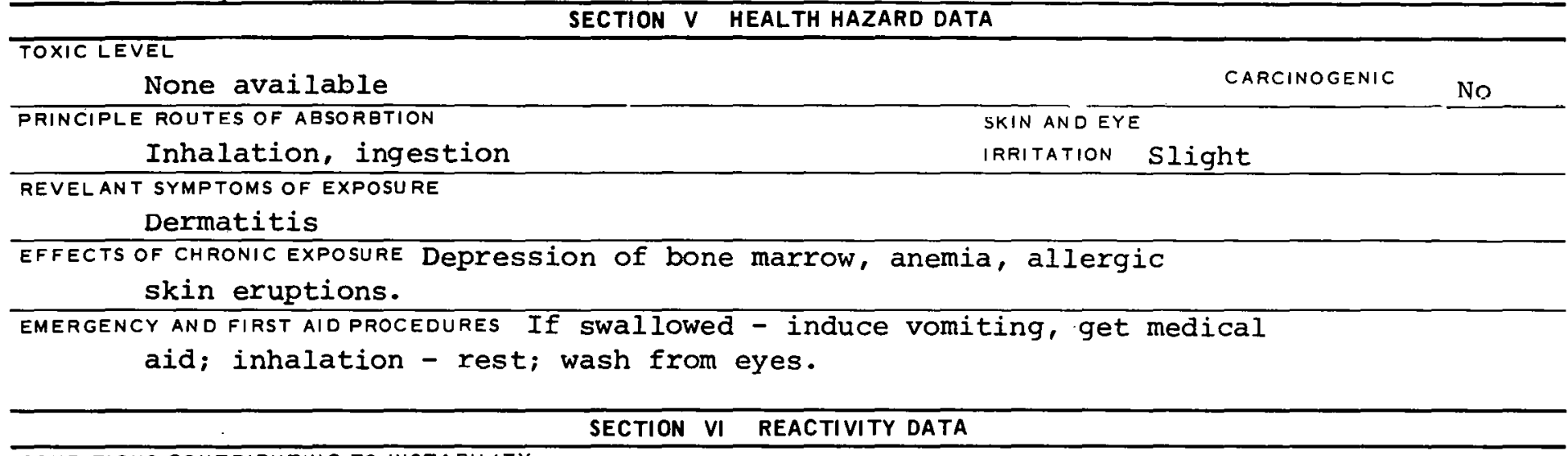

CONOITIONS CONTRIBUTING TO INSTABILITY

CONDITIONS CONTRIBUTING TO HAZARDOUS POLYMERIZATION

INCOMPATABILITY (MATERIALS TO AVOID)

HAZARDOUS DECOMPOSITION PRODUCTS

SECTION VII SPILL OR LEAK PROCEDURES

STEPS TO BE TAKEN IN CASE MATERIAL IS RELEASED OR SPILLED

WASTE DISPOSAL METHOD

SECTION VIII SPECIAL PROTECTION INFORMATION

\begin{tabular}{l|l}
\hline VENTILATION REQUIREMENTS LOCAL EXHAUST & $\begin{array}{l}\text { PROTECTIVE EQUIPMENT (SPECIFY TYPES) EYE } \\
\text { GOggleS }\end{array}$ \\
\hline MECHANICAL IGENERAL) & GLUVES \\
\hline SPECIAL & RESPIRATOR \\
With_proper_filter
\end{tabular}

OTHER PROTECTIVE EQUIPMENT

\section{SECTION IX SPECIAL PRECAUTIONS}

PRECAUTIONS TO BE TAKEN IN HANDLING AND STORAGE

OTHER PRECAUTIONS

SIGNATURE

DATE

-

206-7501B 
CAS : 000107131

\section{PRODUCT DESIGNATION}

Acrylonitrile

03-206-8801
AT5 2500

MATERIAL SAFETY

DATA SHEET

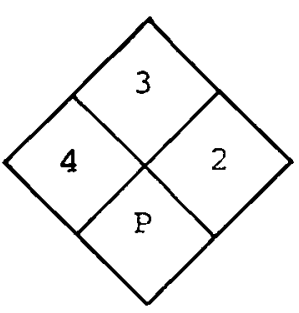

SECTION I SOURCE AND NOMENCLATURE

MANUFACTURER'S NAME

ADDRESS (NUMBER, STREET, CITY, STATE, ZIP CODE)

TRADE NAME AND SYNONYMS

CHEMICAL FAMILY

CHEMILAL NAME AND SYNONYMS

Propenenitrile Vinylcyanide, Cyanoethylene

$\mathrm{CH}_{2}=\mathrm{CHCN}$

SECTION II HAZARDOUS INGREDIENTS

BASIC MATERIAL

APPROXIMATE

OR MAXIMUM

$\%$ WT. OR VOL.

OSHA STANDARD

\begin{tabular}{|c|c|c|c|}
\hline \multicolumn{2}{|c|}{$\mathrm{LD}_{50} \mathrm{mg} / \mathrm{kc}$} & \multicolumn{2}{|c|}{$\mathrm{LC}_{50} \mathrm{ppm}$} \\
\hline ORAL & PERCUT. & SPECIES & CONC. \\
\hline 82 & 96 & Rat & \\
\hline 93 & 280 & Rabbit & \\
\hline 50 & 250 & Guinea & 576 \\
\hline 27 & & Mouse & \\
\hline & & & \\
\hline & & & \\
\hline & & & \\
\hline & & & \\
\hline & & & \\
\hline & & & \\
\hline
\end{tabular}

TCLO inhalation - man $16 \mathrm{ppm} / 20 \mathrm{~min}$. system effects

\section{BOILING POINT}

77.3

MELTING POINT

$-84^{\circ} \mathrm{C}$.

SPECIFIC GRAVITY $\left(\mathrm{H}_{2} \mathrm{O}=1\right)$

0.823

SOLUBILITY IN WATER $.3^{\text {Pts/100 pts } \mathrm{H}_{2} \mathrm{O}}$

APPEARANCE ANO ODOR

Colorless liquid with ethereal odor.

SECTION IV FIRE AND EXPLOSION HAZARD DATA

FLASH POIN

$30^{\circ} \mathrm{F}$

METHOD USED

Closed Cup Method

EXTINGUISHING MEDIA

Alcohol foam or carbon dioxide

SPECIAL FIRE FIGHTING PROCEDURES

Water. may be ineffective

UNUSUAL FIRE ANDEXPLOSION HAZARDS
FLAMMABLE

(EXPLOSIVE)

LIMITS
UPPER

178

LOWER

$3^{8}$ 


PRODUCT DESIGNATION
A1lyl Alcohol
$03-208-0202$

\section{MATERIAL SAFETY \\ DATA SHEET}

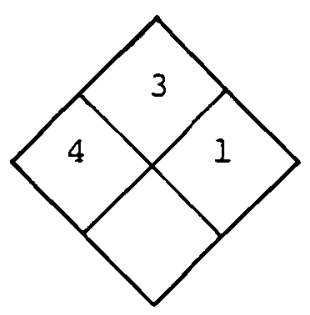

\section{SECTION I SOURCE AND NOMENCLATURE}

MANUFAC TURER'S NAME
ADDRESS INUMBER, STREET, CITY
TRADE NAME AND SYNONYMS
VINYI CarbinOI
CHEMICAL NAME ANO SYNONYMS
PXOPE $O 1$

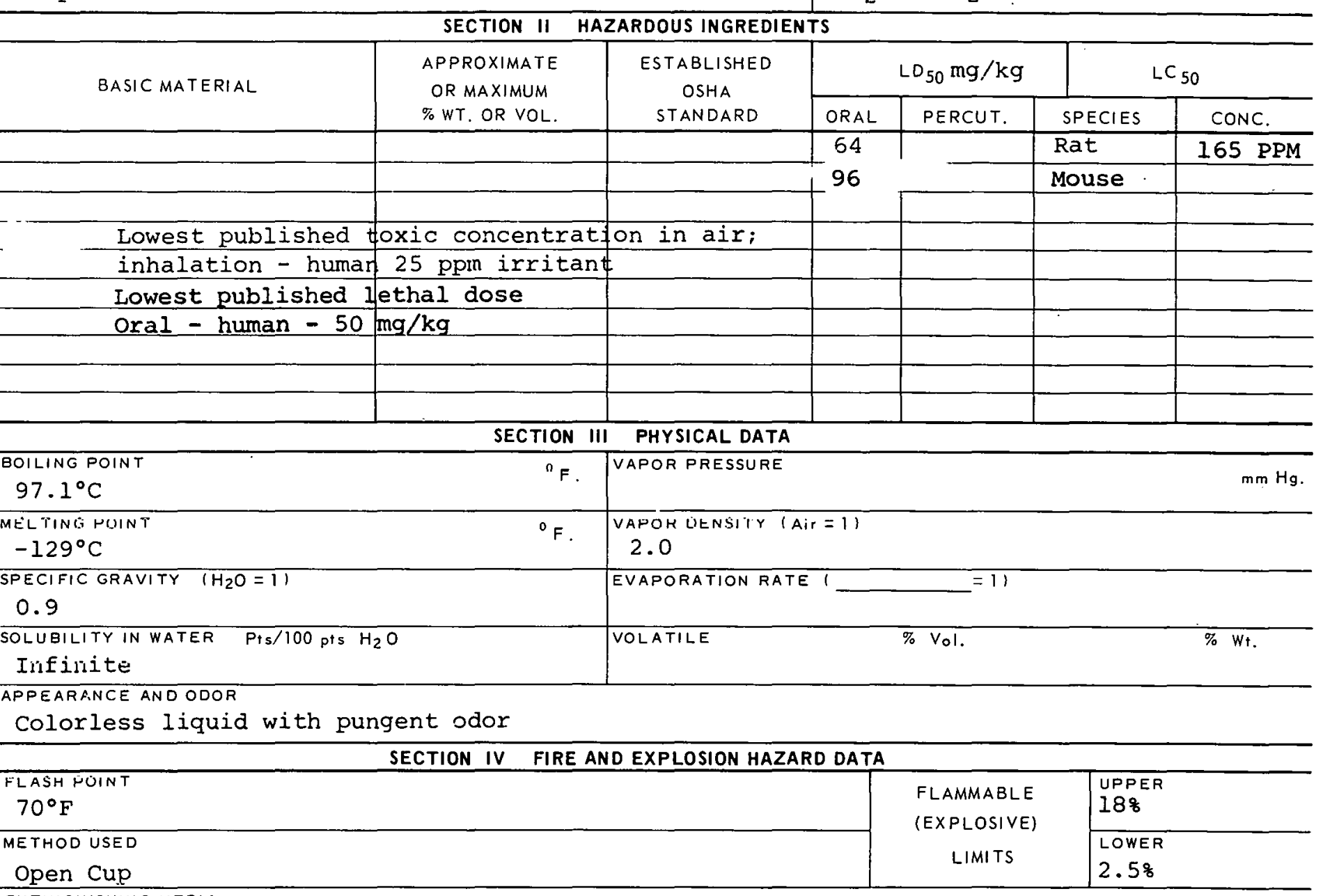

EXTINGUISHING MEDIA

Alcohol foam, carbon dloxide or dry chemical

\section{SPECIAL FIRE FIGHTING PROCEDURES}

Water may be ineffective

UNUSUAL FIRE AND EXPLOSION HAZARDS 
SECTION $V$ HEALTH HAZARD DATA

TOXIC LEVEL

TLV $2 \mathrm{ppm}, 5 \mathrm{mg} / \mathrm{C} . \mathrm{M}$.

PRINCIPLE ROUTES OF ABSORBTION

Inhalation, ingestion, skin

REVELANT SYMPTOMS OF EXPOSURE

deep bone ache.

EFFECTS OF CHRONIC EXPOSURE

Pulmonary Edema

EMERGENCY AND FIRST AID PROCEDURES

Remove from exposure; if severe - get medical aid; if swallowed - wash mouth, give emetic get medical aid; wash from skin and eyes. \begin{tabular}{l}
\hline SECTION VI REACTIVITY DATA \\
\hline CONDITIONS CONTRIBUTING TO INSTABILITY \\
\hline CONDITIONS CONTRIBUTING TO HAZARDOUS POLYMERIZATION \\
INCOMPATABILITY (MATERIALS TO AVOID) \\
AVOId SOdIUM hYdYOXIde, Tri-n-BrOMOMElamine \\
IIAZARDOUS DECOMPOSITION PRODUCTS
\end{tabular}

SECTION VII SPILL OR LEAK PROCEDURES

STEPS TO BE TAKEN IN CASE MATERIAL IS RELEASED OR SPILLED

WASTE DISPOSAL METHOD

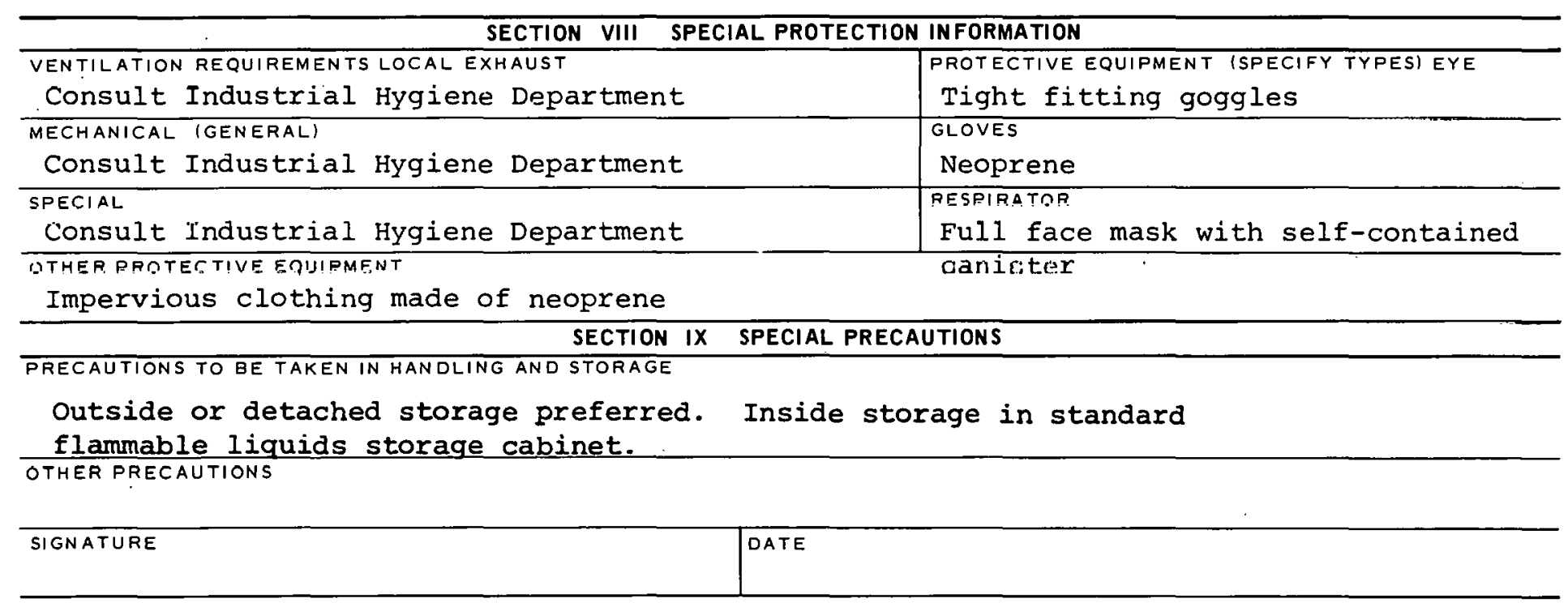


CAS : 000123513

PRODUCT DESIGNATION

Iso-Amyl Alcohol

03-208-0332

\section{MATERIAL SAFETY \\ DATA SHEET}

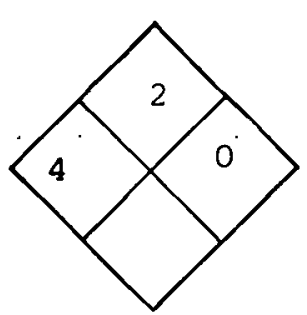

SECTION I SOURCE AND NOMENCLATURE

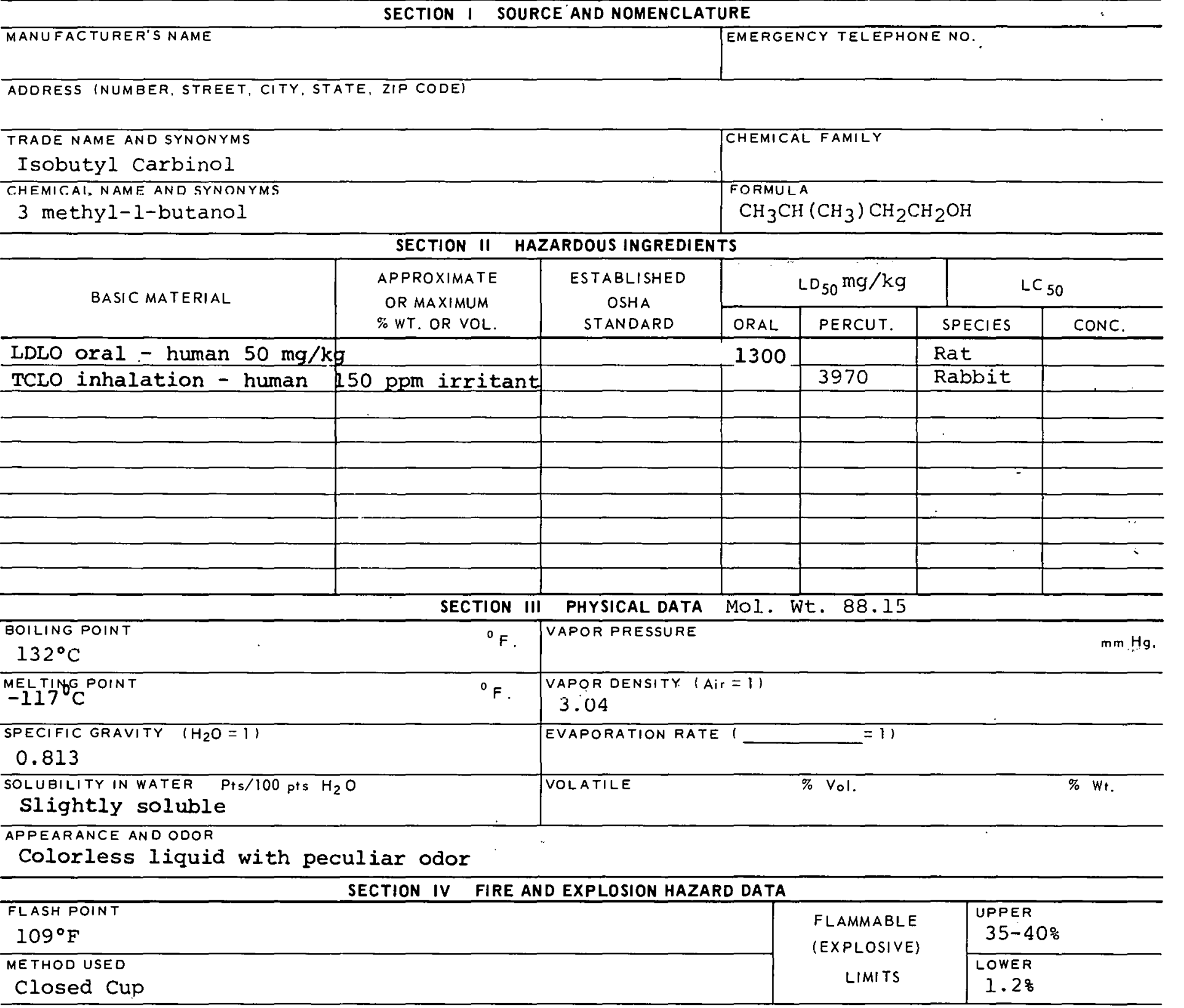

EXTINGUISHING MEDIA

Alcohol foam, carbon dioxide, dry chemical.

SPECIAL FIRE FIGHTING PROCEDURES

Self-contained gas mask.

UNUSUAL FIRE AND EXPLOSION HAZARDS

slight explosion hazard when exposed to flame. 


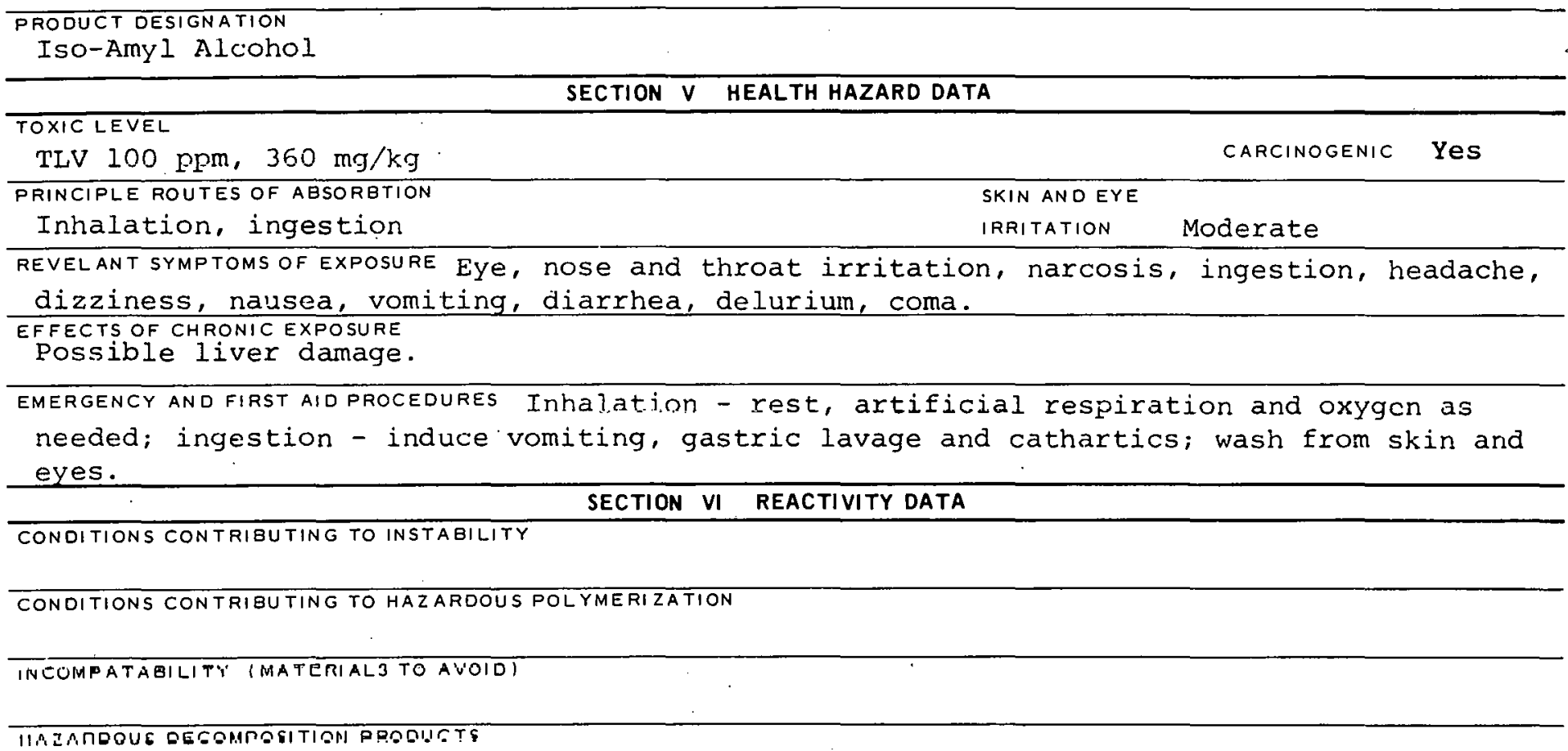

SECTION VII SPILL OR LEAK PROCEDURES

STEPS TO BE TAKEN IN CASE MATERIAL IS RELEASED OR SPILLED

Absorb on paper towel; evaporate in hood.

WASTE DISPOSAL METHOD

\begin{tabular}{|c|c|c|}
\hline \multicolumn{3}{|c|}{ SECTION VIII SPECIAL PROTECTION INFORMATION } \\
\hline VENTILATION REQUIREMENTS LOCAL EXHAUST & & $\begin{array}{l}\text { PROTEC.TIVE EQUIPMENT (SPECIFY TYPES) EYE } \\
\text { Safety goggles }\end{array}$ \\
\hline MEGHANICAI. (GENERAI.) & & $\begin{array}{l}\text { FInvf nos } \\
\text { Rubber }\end{array}$ \\
\hline SPECIAL & & $\begin{array}{l}\text { RESPIRATQR } \\
\text { Cartridge respirator }\end{array}$ \\
\hline $\begin{array}{l}\text { OTHER PROTECTIVE EQUIPMENT } \\
\text { Face shield, coveralls }\end{array}$ & & \\
\hline SECTION IX & SPECIAL PRECA & UTIONS \\
\hline $\begin{array}{l}\text { PRECAUTIONS TO BE TAKEN IN HANDLING AND STORAGE } \\
\text { Store tightIy closed container in flamm }\end{array}$ & ble liquid & storage cabinet. \\
\hline OTHER PRECAUTIONS & & \\
\hline SIGNATURE & DATE & \\
\hline
\end{tabular}


CAS $: 000071363$

\section{PRODUCT DESIGNATION}

n-Butyl Alcohol

$03-208-0902$
EO- 14000

\section{MATERIAL SAFETY DATA SHEET}

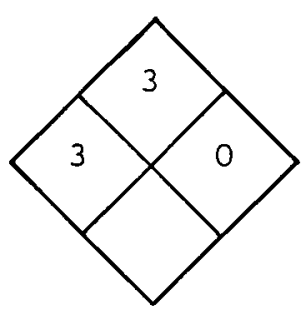

SECTION I SOURCE AND NOMENCLATURE

\section{MANU FAC TURER'S NAME \\ TRADE NAME AND SYNONYMS \\ CHEMICAL NAME AND SYNONYMS \\ $\mathrm{N}$-butanol}

ADDRESS (NUMBER, STREET, CITY, STATE, ZIP CODE)
EMERGENCY TELEPHONE NO.

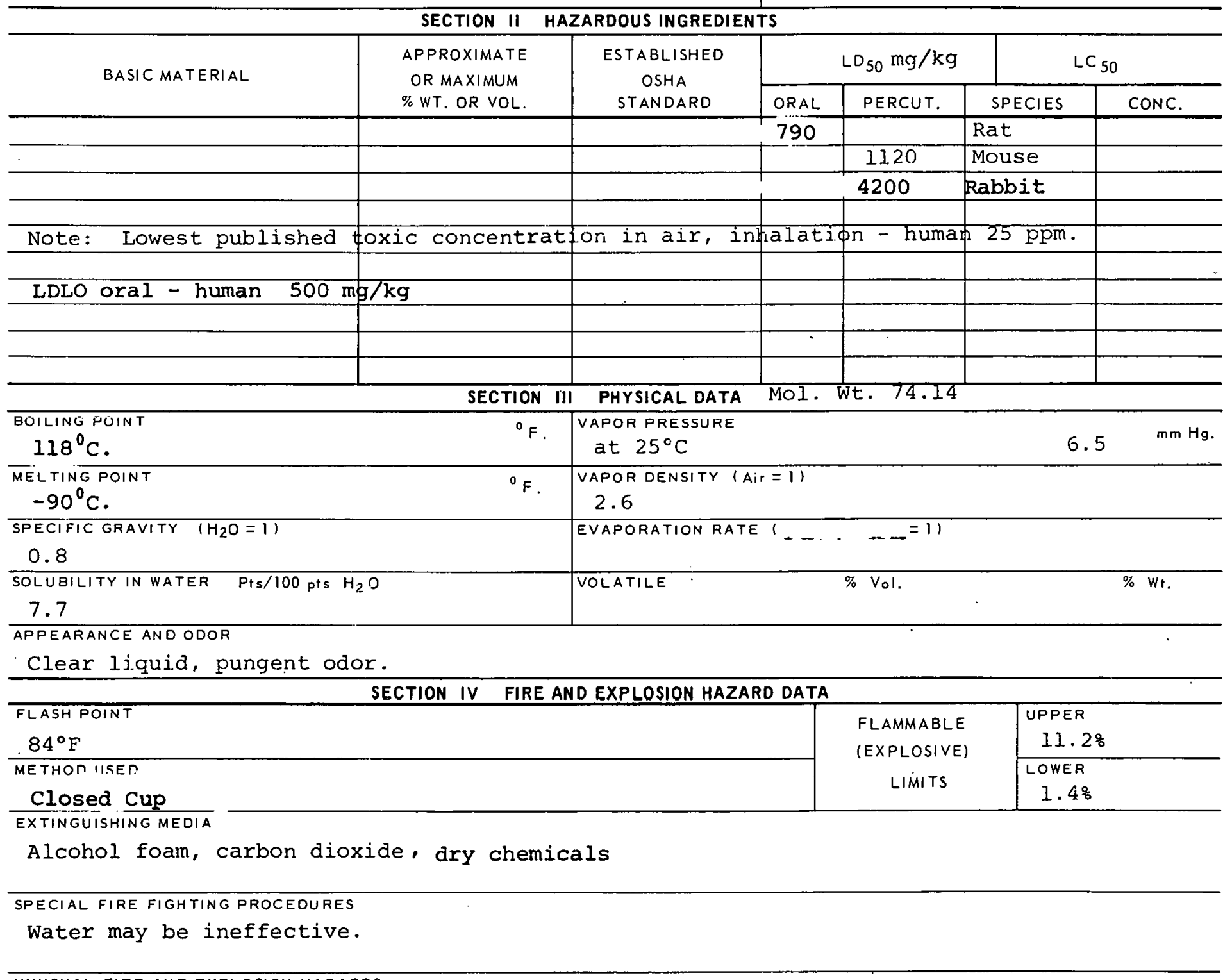

UNUSUAL FIRE AND EXPLOSION HAZAROS 


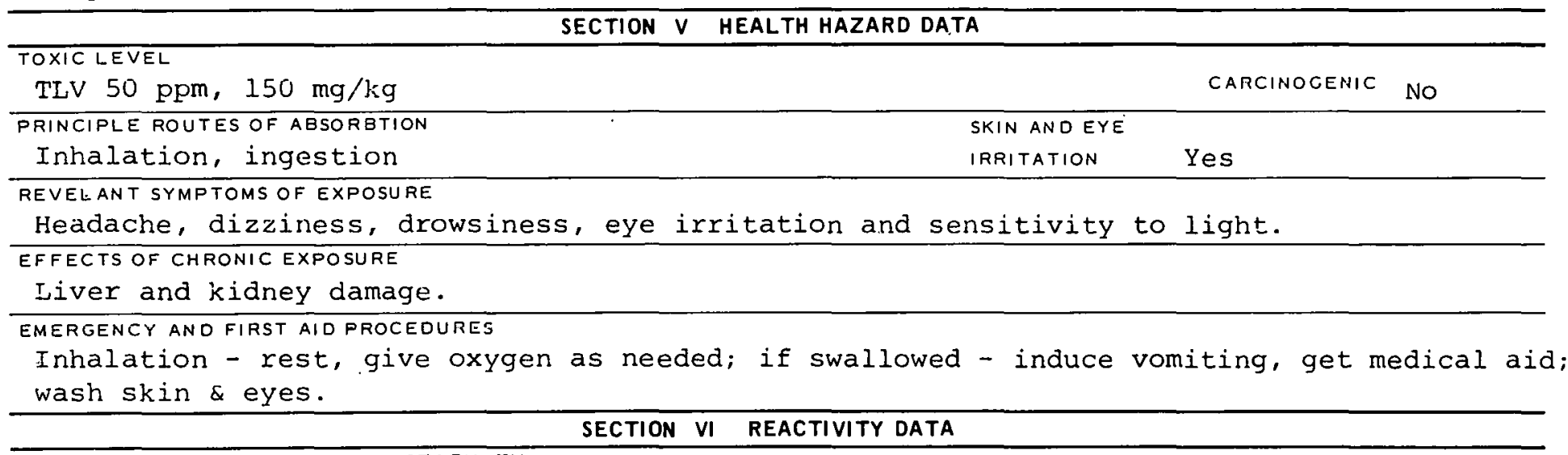

CONDITIONS CONTRIBUTING TO INSTABILITY SECTION VI REACTIVITY DATA

\section{CQND! TIONS CONTRIBUTING TO HAZARDOUS POLYMERIZATION}

INCOMPATABILITY (MATERIALS TO AVOIO)
ExplOSives, OXidizing materials, organic peroxides.

HAZARDOUS DECOMPOSITION PROOUCTS

SECTION VII SPILL OR LEAK PROCEDURES

STEPS TO BE TAKEN IN CASE MATERIAL IS RELEASED OR SPILLED

Absorb on paper, evaporate from paper in hood.

WASTE DISPOSAL METHOD

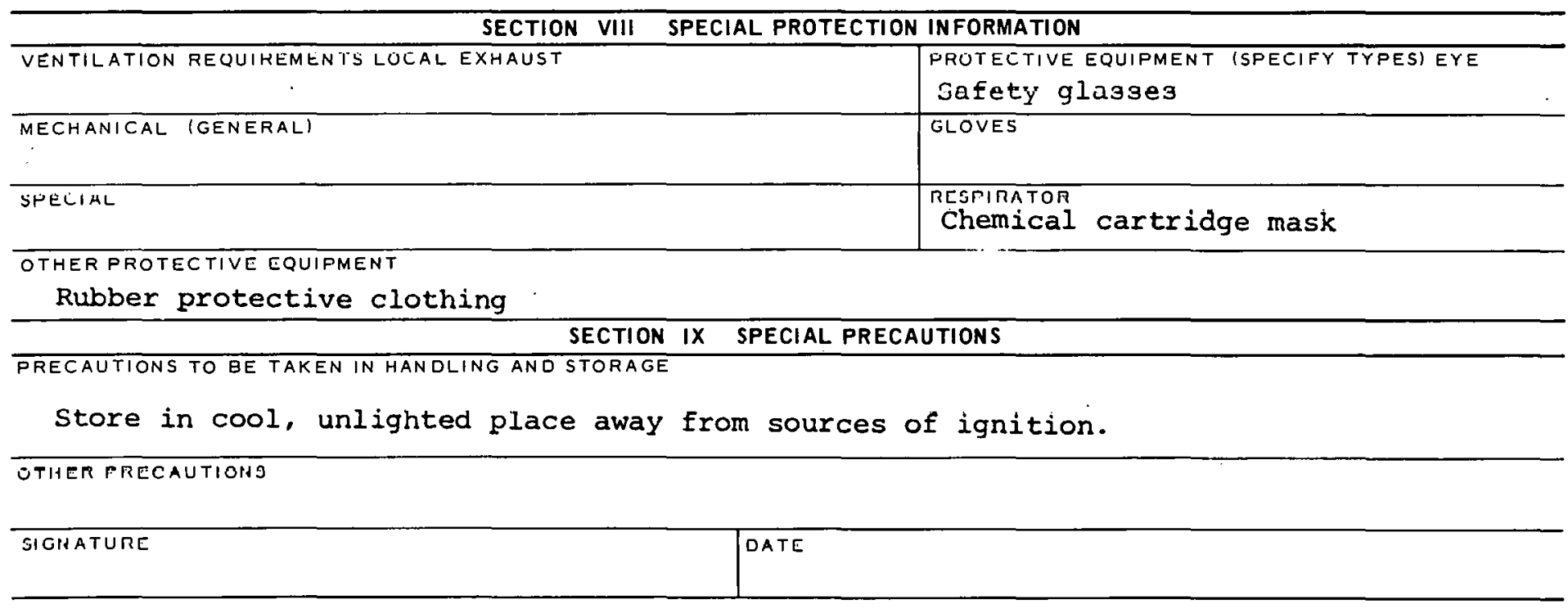




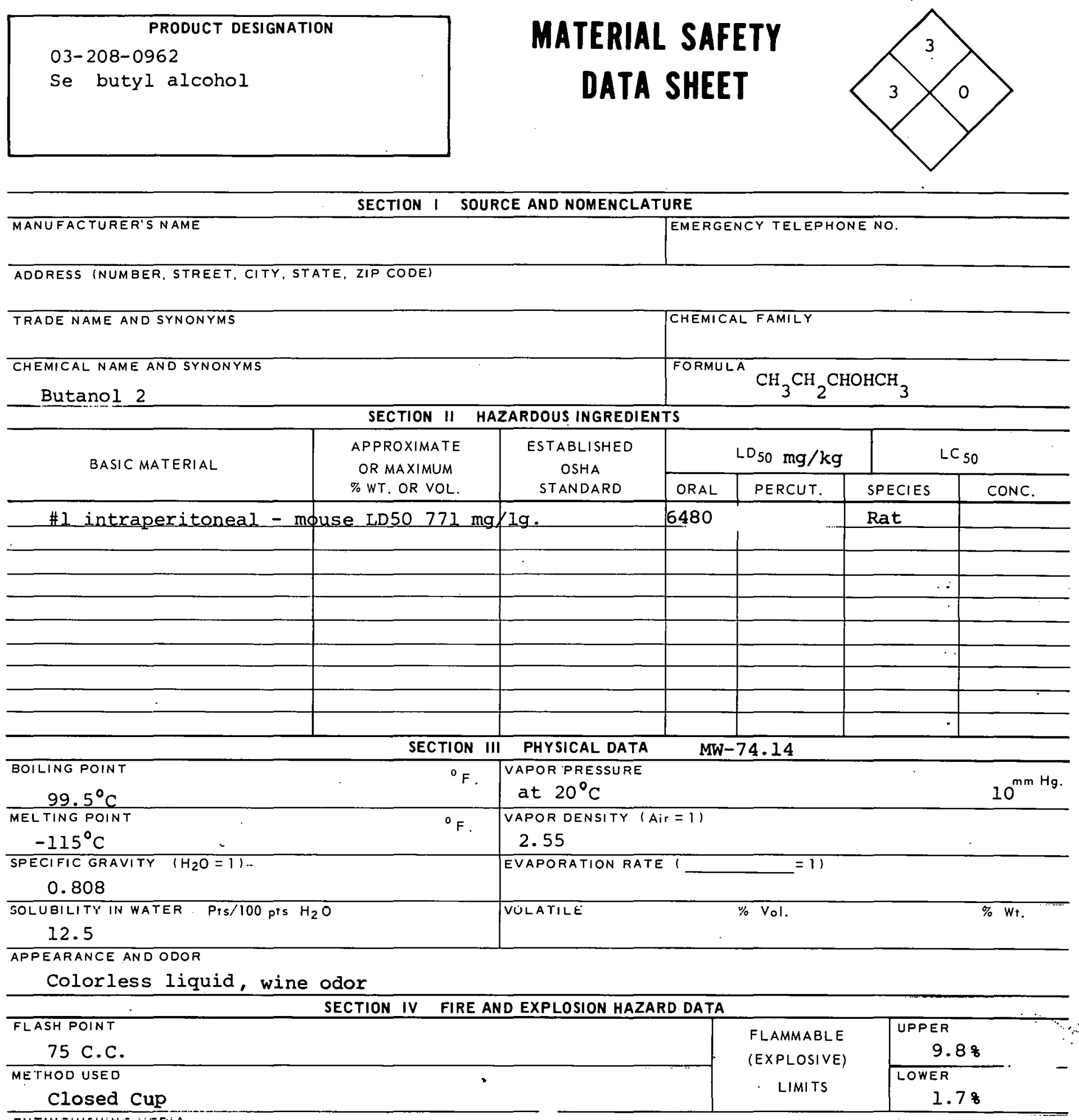

EXTINGUISHING MEEIA

Alcohol foam, water spray, carbon dioxide, dry chemical. SPECIAL FIRE FIGHTING PROCEDURES

IINIISIIAI, FIRE ANDEXPLOSION HAZARDS

May react with oxidizing materials 


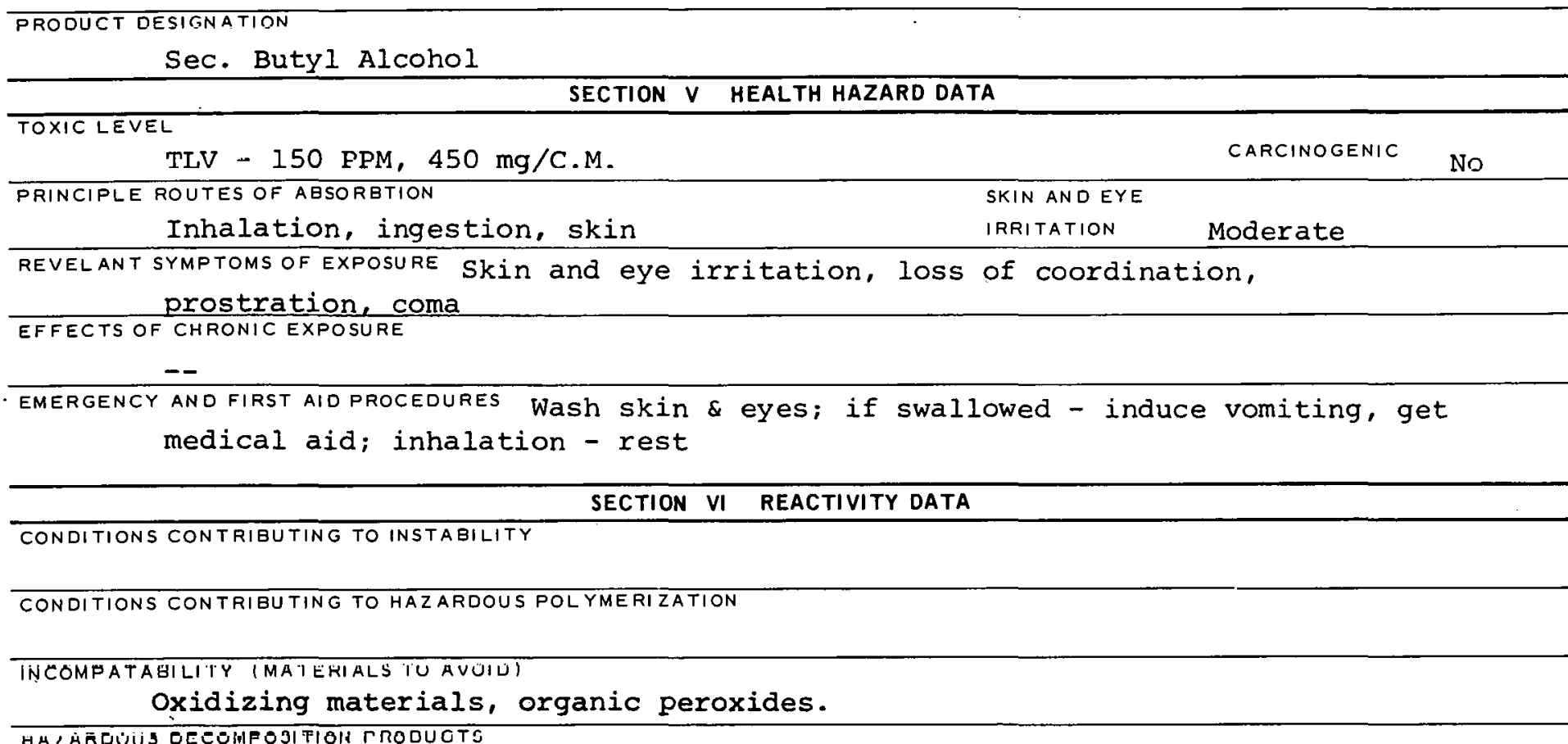

\section{SECTION VII SPILL OR LEAK PROCEDURES}

STEPS TO BE TAKEN IN CASE MATERIAL IS RELEASED OR SPILLED

Absorb on paper towel, evaporate in hood.

WASTE DISPOSAL METHOD
Burn in hoOd

\begin{tabular}{l|l}
\hline \multicolumn{1}{c}{ SECTION VIII SPECIAL PROTECTION INFORMATION } \\
\hline VENTILATION REQUIREMENTS LOCAL EXHAUST & $\begin{array}{c}\text { PROTECTIVE EQUIPMENT } \\
\text { SafETY gOggleS }\end{array}$ \\
\hline MECHANICAL IGENERALI & $\begin{array}{c}\text { GLOVES } \\
\text { Rubber }\end{array}$ \\
\hline SPECIAI. & $\begin{array}{l}\text { RESPIRATOR } \\
\text { With proper filter }\end{array}$ \\
\hline
\end{tabular}

OTHER PROTECTIVE EQUIPMENT

Protective clothing

SECTION IX SPECIAL PRECAUTIONS

PRECAUTIONS TO BE TAKEN IN HANDLING AND STORAGE

Store in cool, dark place away from sources of ignition.

OTHER PRECAUTIONS

\begin{tabular}{l|l}
\hline SIGNATURE & DATE
\end{tabular} 
CAS : 000078831

\section{NP96250 \\ MATERIAL SAFETY \\ DATA SHEET}

PRODUCT DESIGNATION

03-208-0942

Isobutyl Alcohol

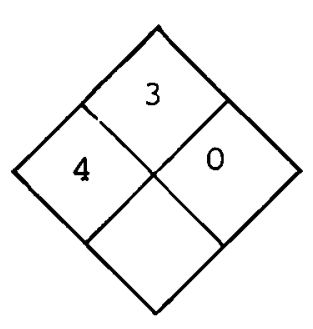

\begin{tabular}{l|l|l}
\hline MANUFACTURER'S NAME & SECTION I SOURCE AND NOMENCLATURE \\
\hline ADDRESS (NUMBER, STREET, CITY, STATE, ZIP CODE) & EMERGENCY TELEPHONE NO.
\end{tabular}

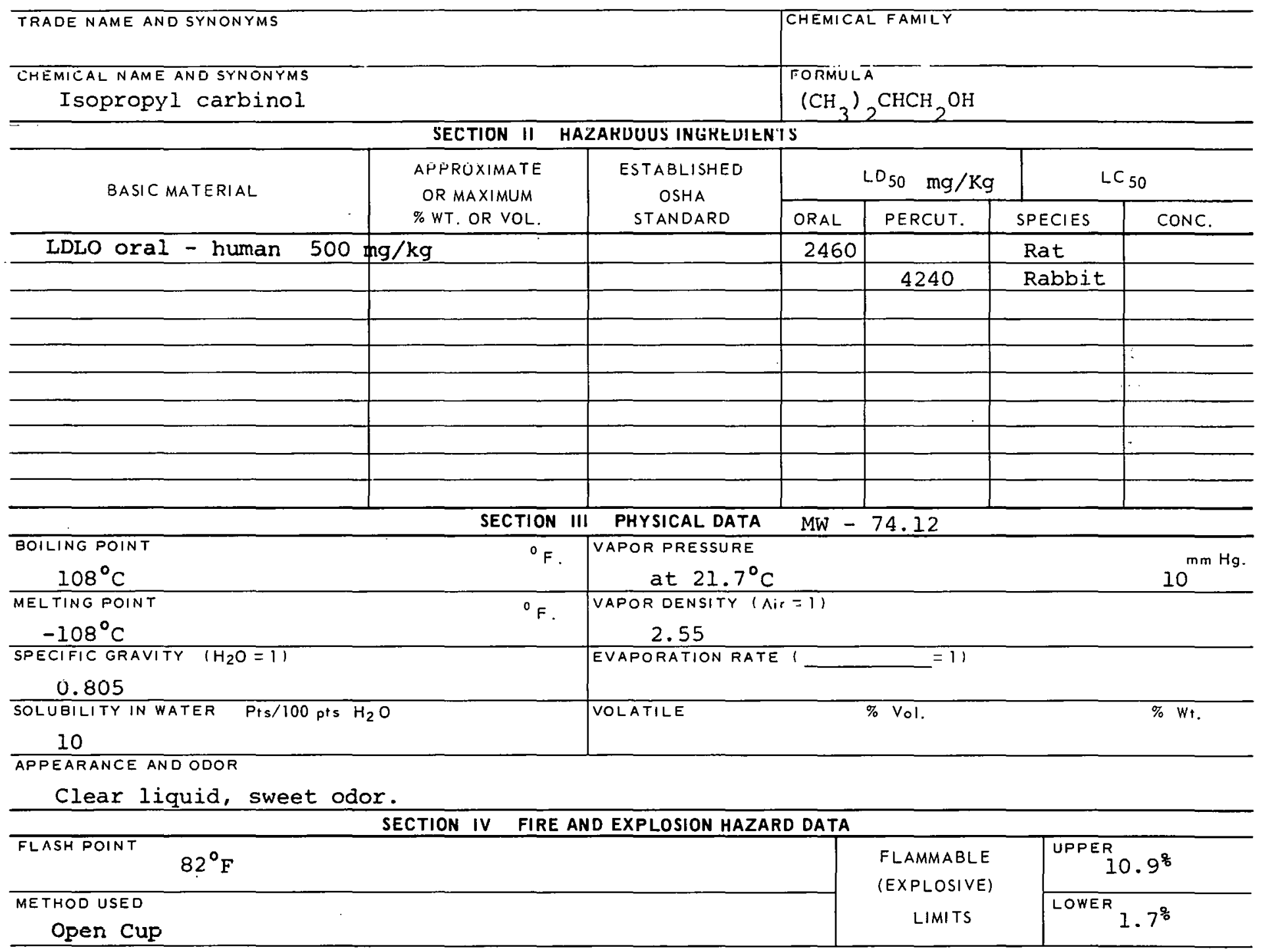

EXTINGUISHING MEDIA

Alcohol foam, carbon dioxide, dry chemical

SPECIAL FIRE FIGHTING PROCEDURES

UNUSUAL FIRE AND EXPLOSION HAZARDS 
Isobutyl alcohol

TOXIC LEVEL

TLV - $50 \mathrm{PPM}, 150 \mathrm{mg} / \mathrm{C} . \mathrm{M}$.

CARCINOGENIC YES

PRINCIPLE ROUTES OF ABSORETION

Inhalation, ingestion, skin

SKIN AND EYE

IRRITATION

Some

REVELANT SYMPTOMS OF EXPOSURE

Slight liver \& kidney damage, eye irritation, narcosis

EFFECTS OF CHRONIC EXPOSURE

$--$

EMERGENCY AND FIRST AID PROCEDURES

Wash skin \& eyes; if swallowed - induce vomiting, get

medical aidi inhalation .. rest.

SECTION VI REACTIVITY DATA

CONDITIONS CONTRIBUTING TO INSTABILITY

CONDITIONS CONTRIBUTING TO HAZARDOUS POLYMERIZATION

INCOMPATABILITY (MA TERIALLS TO AVOID)

HAZAHUUUS ULLUMPUSIIIUN H HULIUL. IS

SECTION VII SPILL OR LEAK PROCEDURES

STEPS TO BE TAKEN IN CASE MATERIAL IS RELEASED OR SPILLED

Absorb on paper towels, evaporate in hood.

WASTE DISPOSAL METHOD

SECTION VIII SPECIAL PROTECTION INFORMATION

\begin{tabular}{|c|c|}
\hline VENTILATION REQUIREMENTS LOCAL EXHAUST & $\begin{array}{l}\text { PROTECTIVE EQUIPMENT (SPECIFY TYPES) EYE } \\
\text { Safety gogyles }\end{array}$ \\
\hline MECHANICAL (GENERAL) & $\begin{array}{l}\text { GLOVES } \\
\text { Rubluer }\end{array}$ \\
\hline CחECIAL & $\begin{array}{l}\text { RFGPIRATAR } \\
\text { With proper filter }\end{array}$ \\
\hline
\end{tabular}

OTHER PROTECTIVE EQUIPMENT

Coveralls

SECTION IX SPECIAL PRECAUTIONS

PRECAUTIONS TO BE TAKEN IN HANDLING AND STORAGE

store in closed container in flammable liquids storage cabinet.

OTHER DRFTAIITIONS 


\section{PRODUCT DESIGNATION}

Tertiary Butyl Alcohol 03-208-0982
MATERIAL SAFETY

DATA SHEET

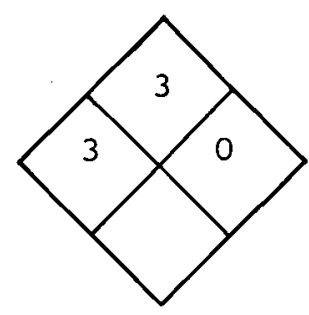

SECTION I SOURCE AND NOMENCLATURE

EMERGENCY TELEPHONE NO.

ADDRESS (NUMBER, STREET, CITY, STATE, ZIP CODE)

TRADE NAME AND SYNONYMS

2 Methyl-2-Propanol

CHEMICAL NAME AND SYNONYMS

Trimethyl Carbinol

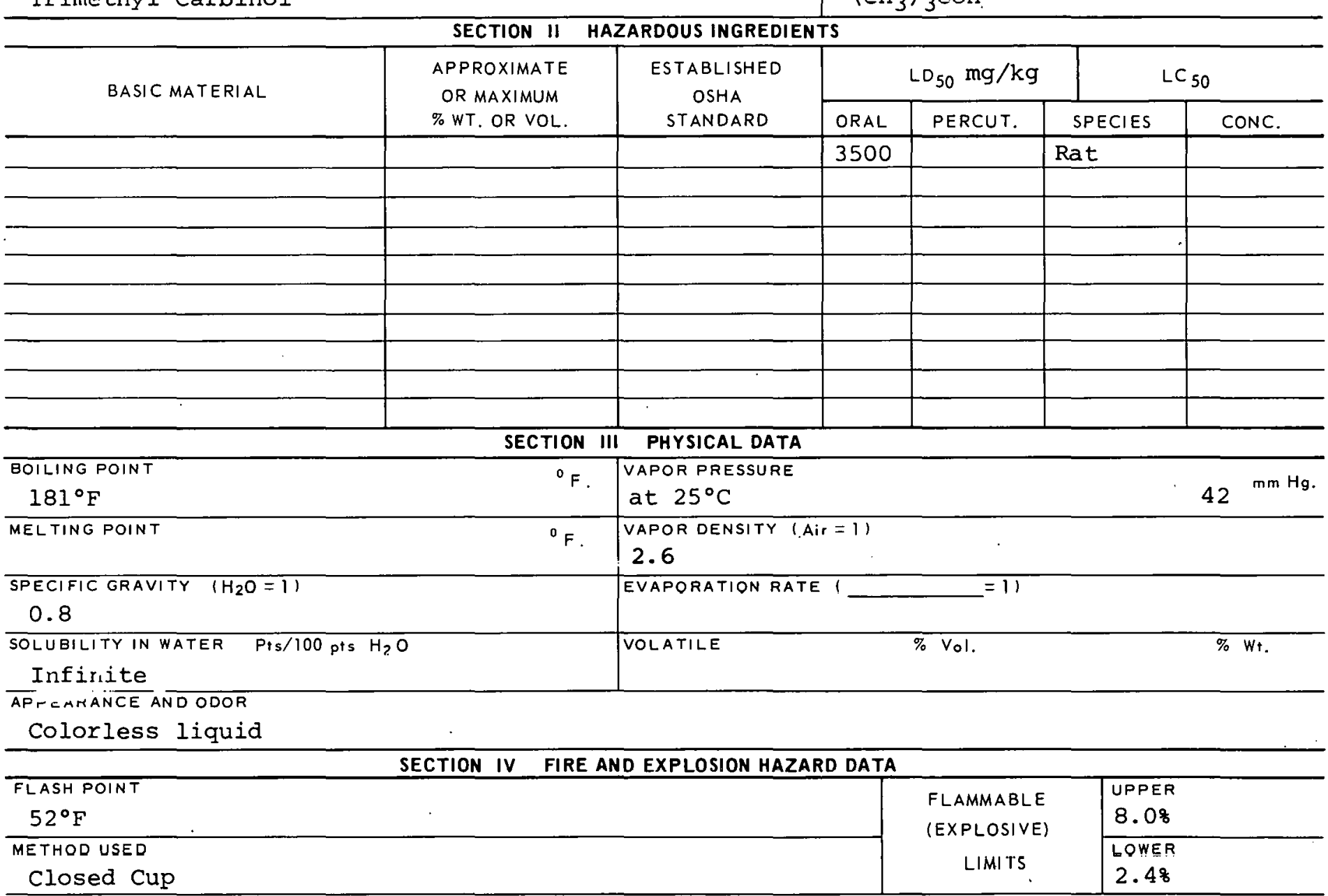

EXTINGUISHING MEDIA

Alcohol foam, carbon dioxide or dry chemical.

SPECIAL FIRE FIGHTING PROCEDURES

Water may be ineffective.

UNUSUAL FIRE AND EXPLOSION HAZARDS 


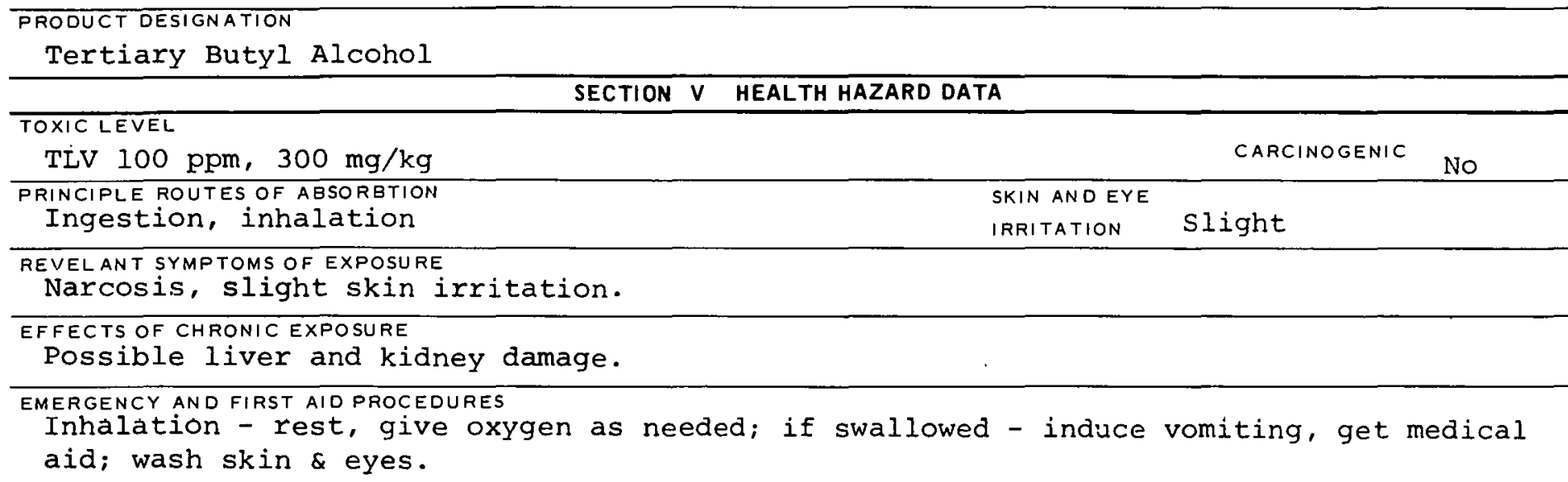

\section{SECTION VI REACTIVITY DATA}

CONDITIONS CONTRIBUTING TO INSTABILITY

CONDITIONS CONTRIBUTING TO HAZARDOUS POLYMERIZATION

IICOMNATAEILITY (MATERIALS TO AVOIO)

HA7ARחIIS RFROMPRSITION PRODIISTS

SECTION VII SPILL OR LEAK PROCEDURES

STEPS TO BE TAKEN IN CASE MATERIAL IS RELEASED OR SPILLED

WASTE DISPOSAL METHOD

SECTION VIII SPECIAL PROTECTION INFORMATION

\begin{tabular}{l|l}
\hline VENTILATION REQUIREMENTS LOCAL EXHAUST & $\begin{array}{l}\text { PROTECTIVE EQUIPMENT (SPECIFY TYPES) EYE } \\
\text { SafetY gOggles }\end{array}$ \\
\hline MECHANICAL IGENERAL) & $\begin{array}{l}\text { GLOVES } \\
\text { Rubber glOVES }\end{array}$ \\
\hline SPECIAL & RESPIRAIOR \\
\hline
\end{tabular}

OTHER PROTECTIVE EQUIPMENT

SECTION IX SPECIAL PRECAUTIONS

PRECAUTIONS TO BE TAKEN IN HANDLING AND STORAGE

OTHER PRECAUTIONS

SIGNATURE

DATE

208-0982B 
CAS : 000123422

PRODUCT DESIGNATION

03-208-1602

Diacetone Alcohol

\section{MATERIAL SAFETY \\ DATA SHEET}

SA91000

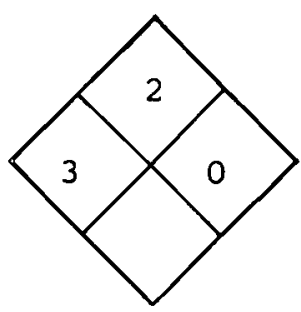

\section{SECTION I SOURCE AND NOMENCLATURE}

\begin{tabular}{l|l}
\hline MANUFACTURER'S NAME & EMERGENCY TELEPHONE NO. \\
\hline ADDRESS (NUMBER, STREET, CITY, STATE, ZIP CODE)
\end{tabular}

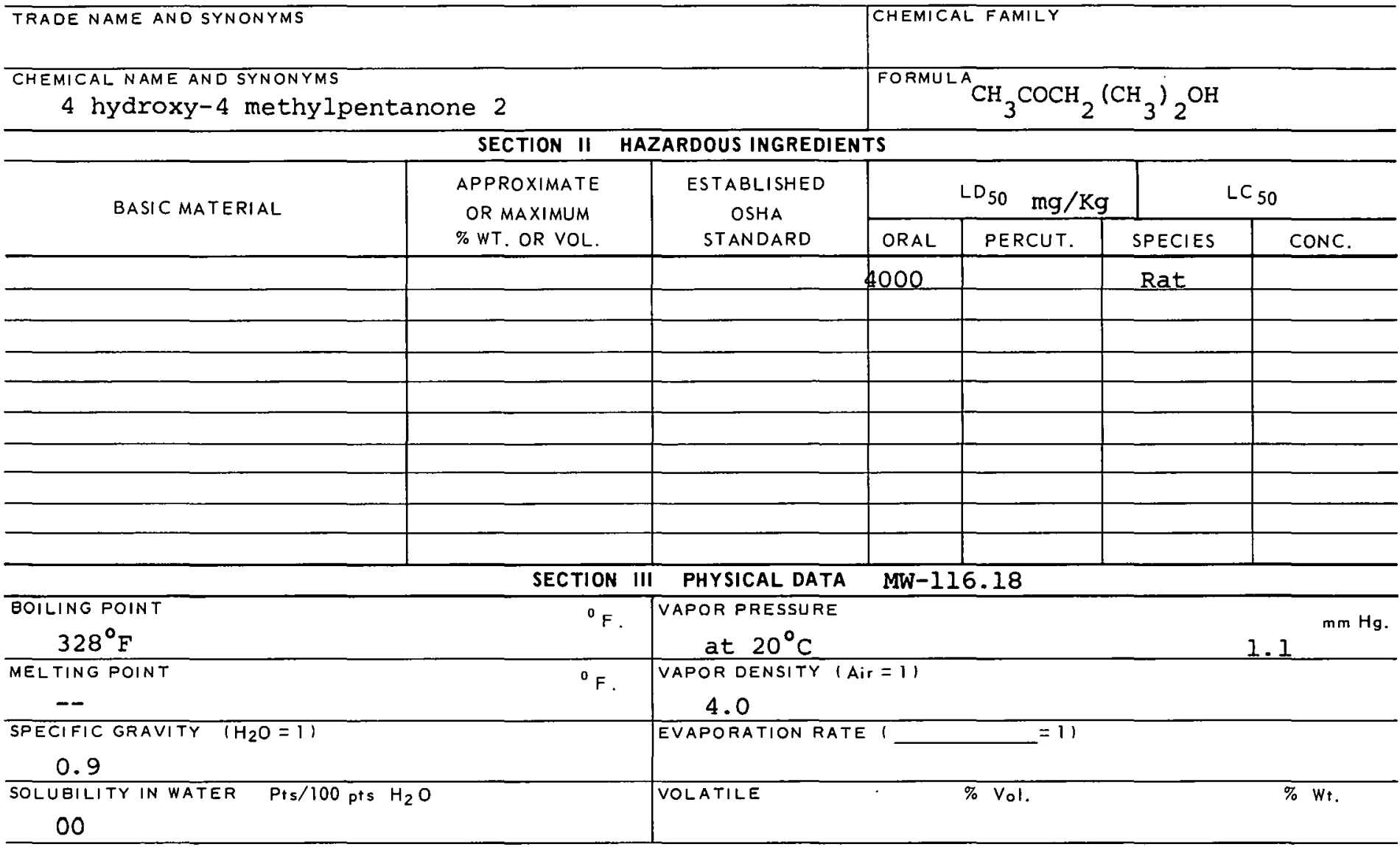

APPEARANCE AND ODOR

Liquid with faint pleasant odor

SECTION IV FIRE AND EXPLOSION HAZARD DATA

\begin{tabular}{l|c|c}
\hline \multicolumn{2}{c}{ SECTION IV FIRE AND EXPLOSION HAZARD DATA } \\
\hline FLASH POINT & FLAMMABLE \\
$151^{\circ} \mathrm{F}$ & UPPER \\
METHOD USED & $6.9 \%$ \\
& LOWER \\
\hline
\end{tabular}

EXTINGUISHING MEDIA

Alcohol foam, carbon dioxide or dry chemical

SPECIAL FIRE FIGHTING PROCEDURES

UNUSUAL FIRE AND EXPLOSION HAZARDS 


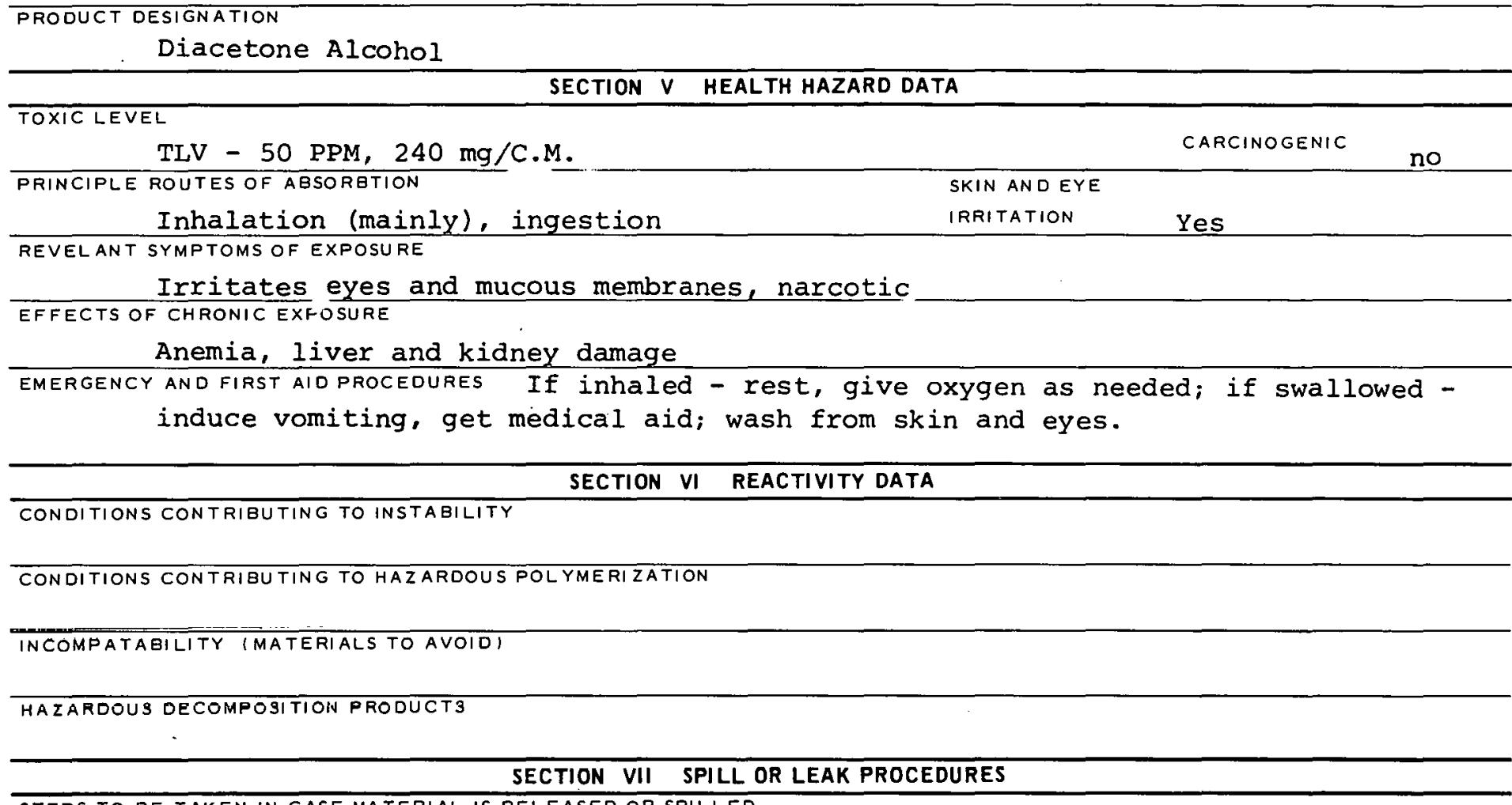

STEPS TO BE TAKEN IN CASE MATERIAL IS RELEASED OR SPILLED

WASTE DISPOSAL METHOD

SECTION VIII SPECIAL PROTECTION INFORMATION

\begin{tabular}{l|l}
\multicolumn{1}{c}{ SECTION VIII SPECIAL PROTECTION INFORMATION } \\
\hline VENTILATION REQUIREMENTS LOCAL EXHAUST & $\begin{array}{c}\text { PROTECTIVE EQUIPMENT (SPECIFY TYPES) EYE } \\
\text { Safety glasSES }\end{array}$ \\
\hline MECHANICAL (GENERAL) & GLOVES \\
\hline STECIAL & RESPIRATOR \\
WIth proper filter
\end{tabular}

OTHER PROTECTIVE EQUIPMENT

SECTION IX SPECIAL PRECAUTIONS

PRECAUTIONS TO BE TAKEN IN HANDLING AND STORAGE

OTHER PRECAUTIONS

SIGNATURE

DATE

208-1602B 
CAS : 000102818

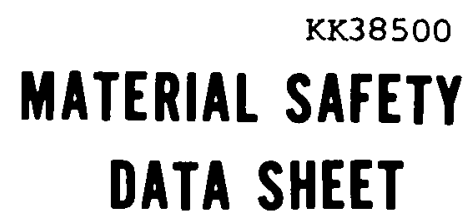

SECTION I SOURCE AND NOMENCLATURE

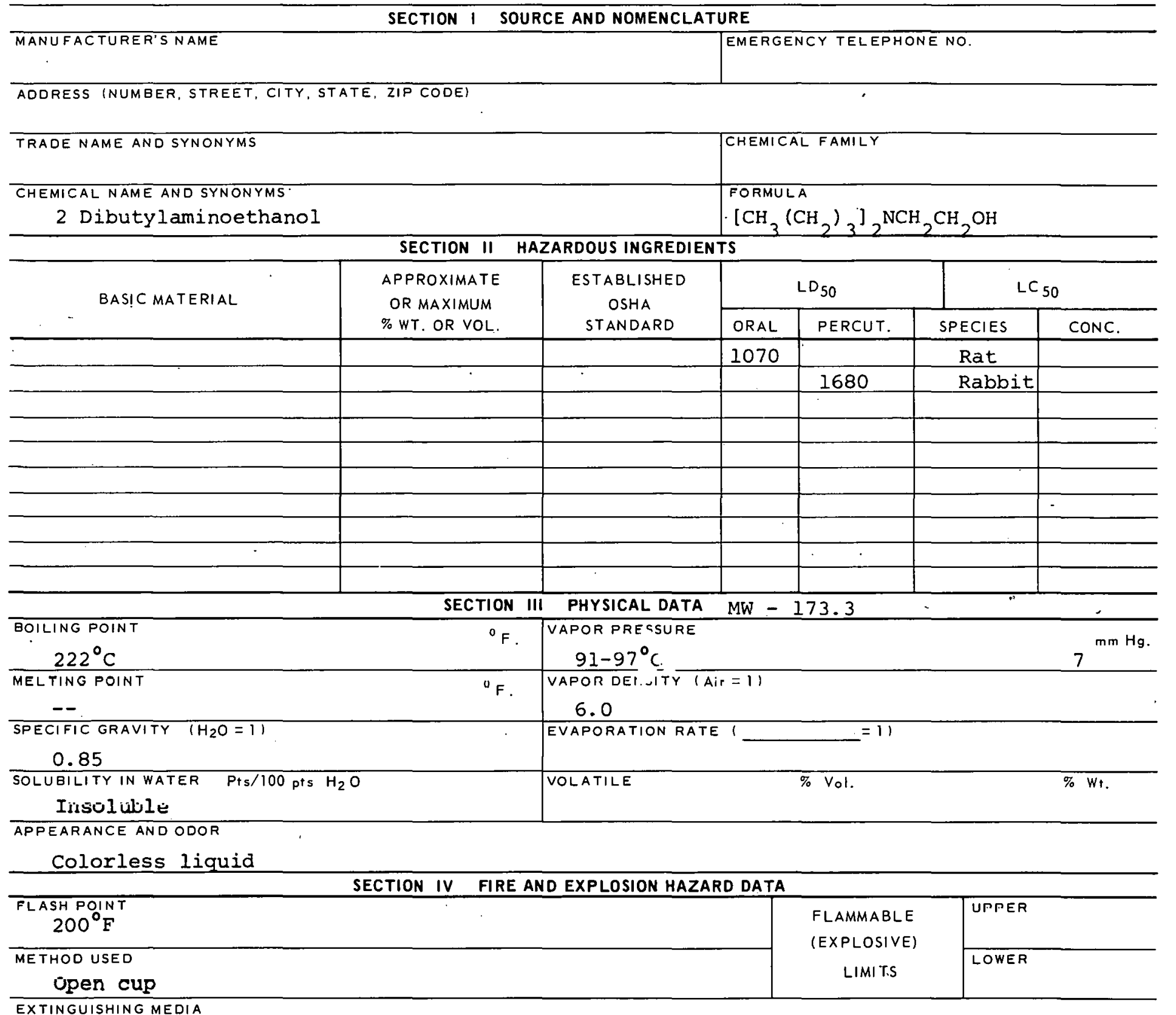

EXTINGUISHING MEDIA

Carbon dioxide, dry chemical

SPECIAL FIRE FIGHTING PROCEDURES

UNUSUAL FIRE AND EXPLOSION HAZARDS 
2 - Di - N - butylaminoethyl alcohol

\section{SECTION $\checkmark$ HEALTH HAZARD DATA}

TOXIC LEVEL

TLV - 2 PPM, $14 \mathrm{mg} / \mathrm{C.M}$.

CARCINOGFNIS No PRINCIPL'E ROUTES OF ABSORBTION

Skin, ingestion, inhalation SKIN AND EYE REVELANT SYMPTOMS OF EXPOSURE IRRITATION moderate

Dermatitis, weight loss, slight increase in clotting time EFFECTS OF CHRONIC EXPOSURE

Dermatitis EMERGENCY AND FIRST AID PROCEOURES If swallowed - drink fruit juice, get medical aid; inhalation rest. Wash from skin \& eyes.

\section{SECTION VI REACTIVITY DATA}

CONDITIONS CONTRIBUTING TO INSTABILITY

CONDITIONS CONTRIBU TING TO HAZARDOUS POLYMERIZATION

IN EUMPATABILIT'i' (MATERIALS TO A'V'OIO)

IIAZAROQUG DEGOMROEITIOH RMODUGTE

SECTION VII SPILL OR LEAK PROCEDURES

STEPS TO BE TAKEN IN CASE MATERIAL IS RELEASED OR SPILLED

\section{WASTE DISPOSAL METHOD}

\section{SECTION VIII SPECIAL PROTECTION INFORMATION}

\begin{tabular}{|c|c|}
\hline VENTILATION REQUIREMENTS LOCAL EXHAUST & $\begin{array}{l}\text { PROTECTIVE EQUIPMENT (SPECIFY TYPESI EYE } \\
\text { SafetY goggles }\end{array}$ \\
\hline MECHANICAL (GENERAL) & $\begin{array}{l}\text { GLOVES } \\
\text { Rubber }\end{array}$ \\
\hline SPECIAL & RESPIRATQR \\
\hline
\end{tabular}

OTMER FROTECTIVE EQUINMENP

SECTION IX SPECIAL PRECAUTIONS

PRECAUTIONS TO BE TAKEN IN HANDLING AND STORAGE 


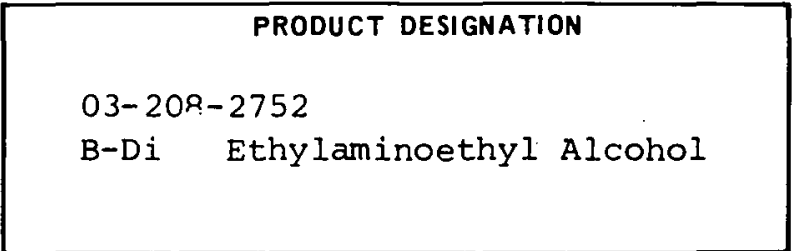

\section{MATERIAL SAFETY \\ DATA SHEET}

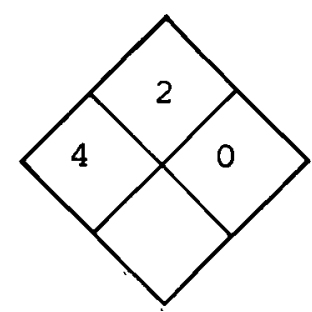

\section{SECTION I SOURCE AND NOMENCLATURE}

MANU FACTURER'S NAME

EMERGENCY TELEPHONE NO.

ADDRESS (NUMBER, STREET, CITY, STATE, ZIP CODE)

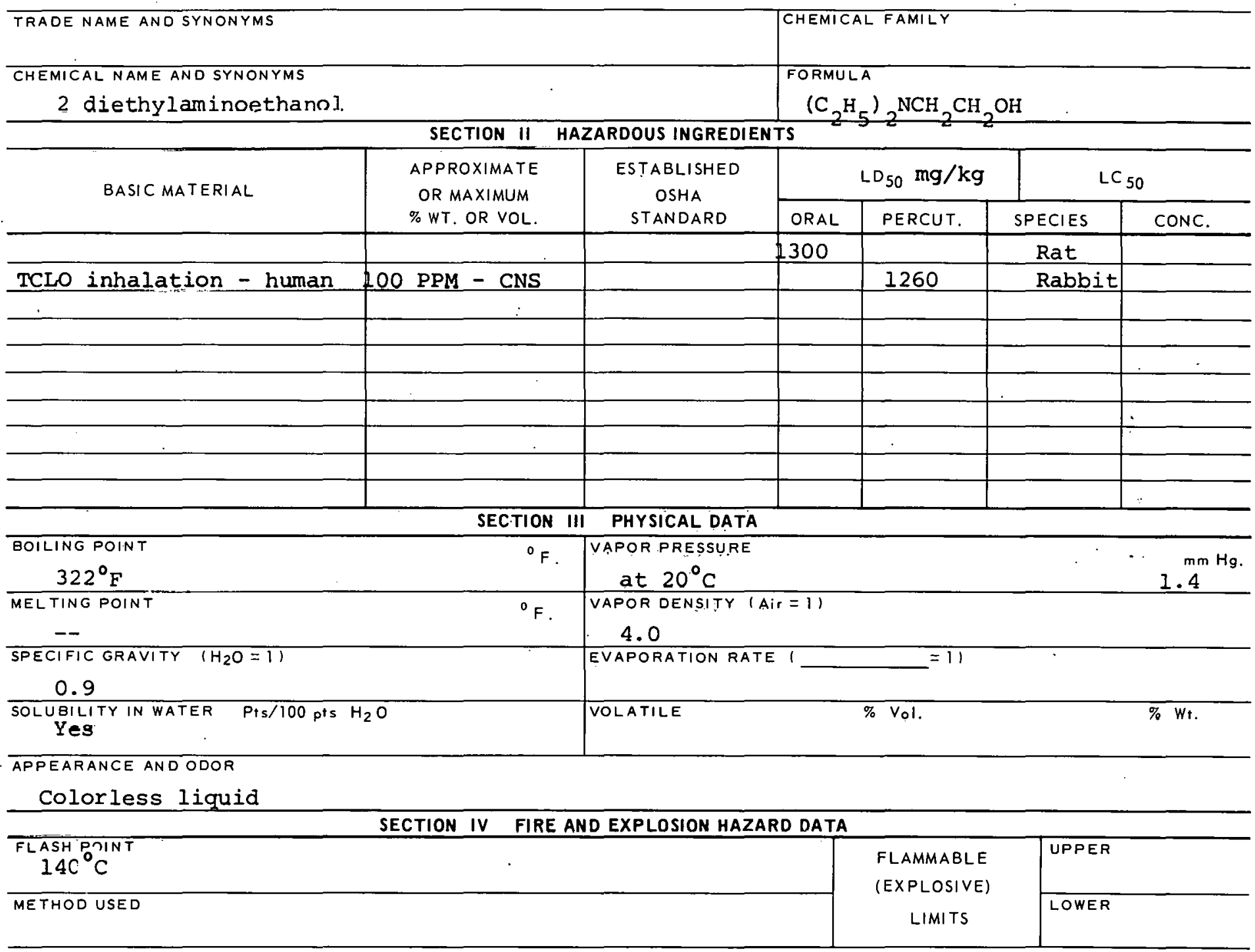

EXTINGUISHING MEDIA

Alcohol foam, carbon dioxide, dry chemical

SPECIAL FIRE FIGHTING PROCEDURES

UNUSUAL FIRE AND. EXPLOSION HAZAROS 
B-Di-N - ethyl aminoethyl alcohol

\section{TOXIC LEVEL}

TLV - $10 \mathrm{PPM}, 50 \mathrm{mg} / \mathrm{C.M}$.

CARCINOGENIC

PRINCIPLE ROUTES OF ABSORBTION

Skin, inhalation, ingestion

SKIN AND EYE

IRRITATION Severe

REVELANT SYMPTOMS OF EXPOSURE Dermatitis, weight loss, slight increase in clotting time

EFFECTS OF CHRONIC EXPOSURE

Dermatitis

EMERGENCY AND FIRST AID PROCEDURES

Wash from skin and eyes; if swallowed - drink fruit juice, get medical aid; inhalation - rest.

\section{SECTION VI REACTIVITY DATA}

CONDITIONS CONTRIBUTING TO INSTABILITY

CONDITIONS CONTRIBUTING TO HAZARDOUS POLYMERIZATION

INCOMPATABILITY (MATERIALS TO AVOID)

HAZARDOUS DECOMPOSITION PRODUCTS

SECTION VII SPILL OR LEAK PROCEDURES

STEPS TO BE TAKEN IN CASE MATERIAL IS RELEASED OR SPILLED

WASTE DISPOSAL METHOD

SECTION VIII SPECIAL PROTECTION INFORMATION

\begin{tabular}{l|l}
\hline VENTILATION REQUIREMENTS LOCAL EXHAUST & $\begin{array}{c}\text { PROTECTIVE EQUIPMENT (SPECIFY TYPES) EYE } \\
\text { Safety goggles }\end{array}$ \\
\hline MECHANICAL IGENERAL) & $\begin{array}{c}\text { RLOVES } \\
\text { Rubber }\end{array}$ \\
\hline SPECIAL & $\begin{array}{c}\text { RESPIRATOR } \\
\text { With proper filter }\end{array}$ \\
\hline
\end{tabular}

OTHER PROTECTIVE EQUIFMENT

SECTION IX SPECIAL PRECAUTIONS

PRECAUTIONS TO BE TAKEN IN HANDLING AND STORAGE 


\section{PRODUCT DESIGNATION}

03-208-3102

B-Dimethylaminoethyl Alcohol
MATERIAL SAFETY

DATA SHEET

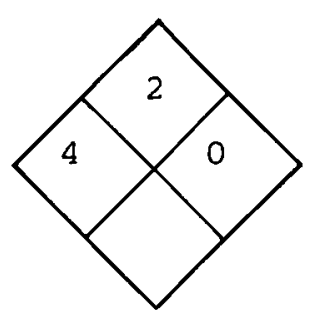

SECTION I SOURCE AND NOMENCLATURE

ADDRESS (NUMBER, STREET, CITY, STATE, ZIP CODE)

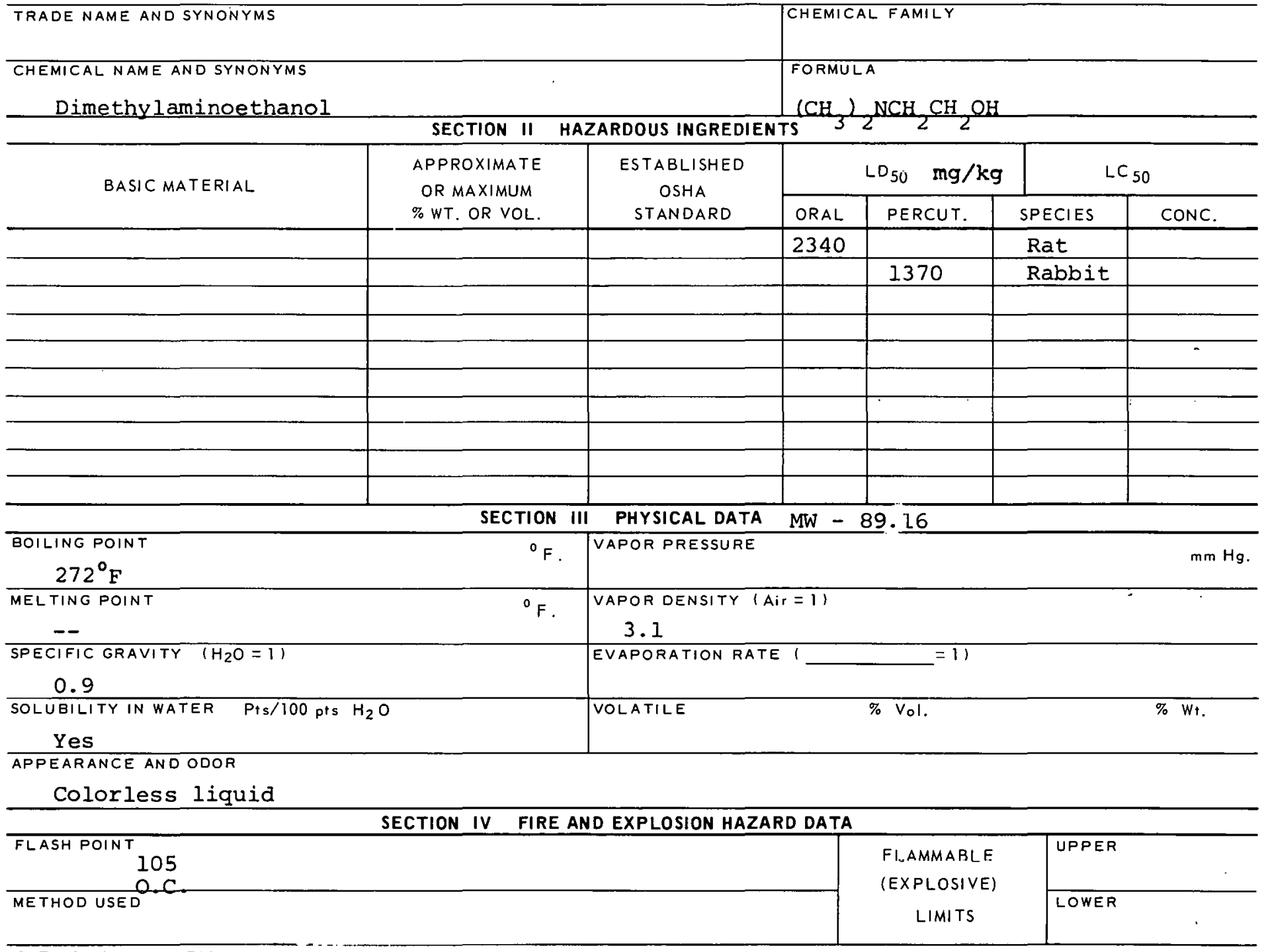

EXTINGUISHING MEDIA

Alcohol foam, carbon dioxide, dry chemical

SPECIAL FIRE FIGHTING PROCEOURES

Water may be ineffective

UNUSUAL FIRE AND EXPLOSION HAZARDS 
B-Dimethylaminoethyl Alcohol SECTION $V$ HEALTH HAZARD DATA

TOXIC LEVEL

Not available

CARCINOGENIC

PRINCIPLE ROUTES OF ABSORBTION

SKIN AND EYE

Skin, inhalation, ingestion IRRITATION Severe REVELANT SYMPTOMS OF EXPOSURE:

Dermatitis, weight loss, slight increase in clotting time.

EFFECTS OF CHRONIC EXPOSURE

Dermatitis

EMERGENCY AND FIRST AIDPROCEDURES Wash from skin and eyes; if swallowed - drink fruit juice, get medical aid; inhalation - rest.

\section{SECTION VI REACTIVITY DATA}

CONDITIONS CONTRIBUTING TO INSTABILITY

CONDITIONS CONTRIBUTING TO HAZARDOUS POLYMERIZATION

INCOMPATABILITY (MATERIALS TO AVOID)

HAZARDOUS OELUMPUSIIIUN FRULULIS

SECTION VII SPILL OR LEAK PROCEDURES

STEPS TO BE TAKEN IN CASE MATERIAL IS RELEASED OR SPILLED

SECTION VIII SPECIAL PROTECTION INFORMATION

\begin{tabular}{|c|c|}
\hline VENTILATION REQUIREMENTS LOCAL EXHAUST & $\begin{array}{l}\text { PROTECTIVE EQUIPMENT (SPECIFY TYPES) EYE } \\
\text { Safety goggles }\end{array}$ \\
\hline $\begin{array}{l}\text { MECHANICAL (GENERAL) } \\
\end{array}$ & $\begin{array}{l}\text { GLOVES } \\
\text { Rubber }\end{array}$ \\
\hline SPECIAL & $\begin{array}{l}\text { PESPIRATOR } \\
\text { With proper filter }\end{array}$ \\
\hline
\end{tabular}

OTHER PROTECTIVE EQUIPMENT

SECTION IX SPECIAL PRECAUTIONS

PRECAUTIONS TO BE TAKEN IN HANDLING AND STORAGE

OTHEARRECALITIOMS

SIGNATURE

กATF

208-3102B 
CAS : 000098000

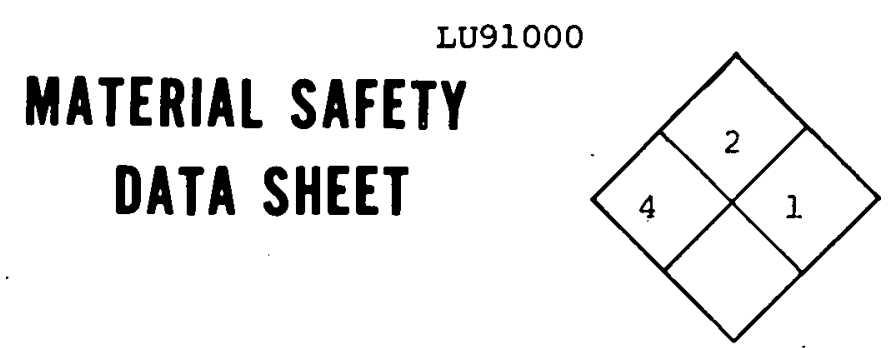

03-208-5002

PRODUCT DESIGNATION

Furfuryl alcohol

\section{SECTION I SOURCE AND NOMENCLATURE}

MANUFACTURER'S NAME

ADDRESS (NUMBER, STREET, CITY, STATE, ZIP CODE)

TRADE NAME AND SYNONYMS

Furfural Alcohol

CHEMICAL NAME AND SYNONYMS

2-Furyl carbinol

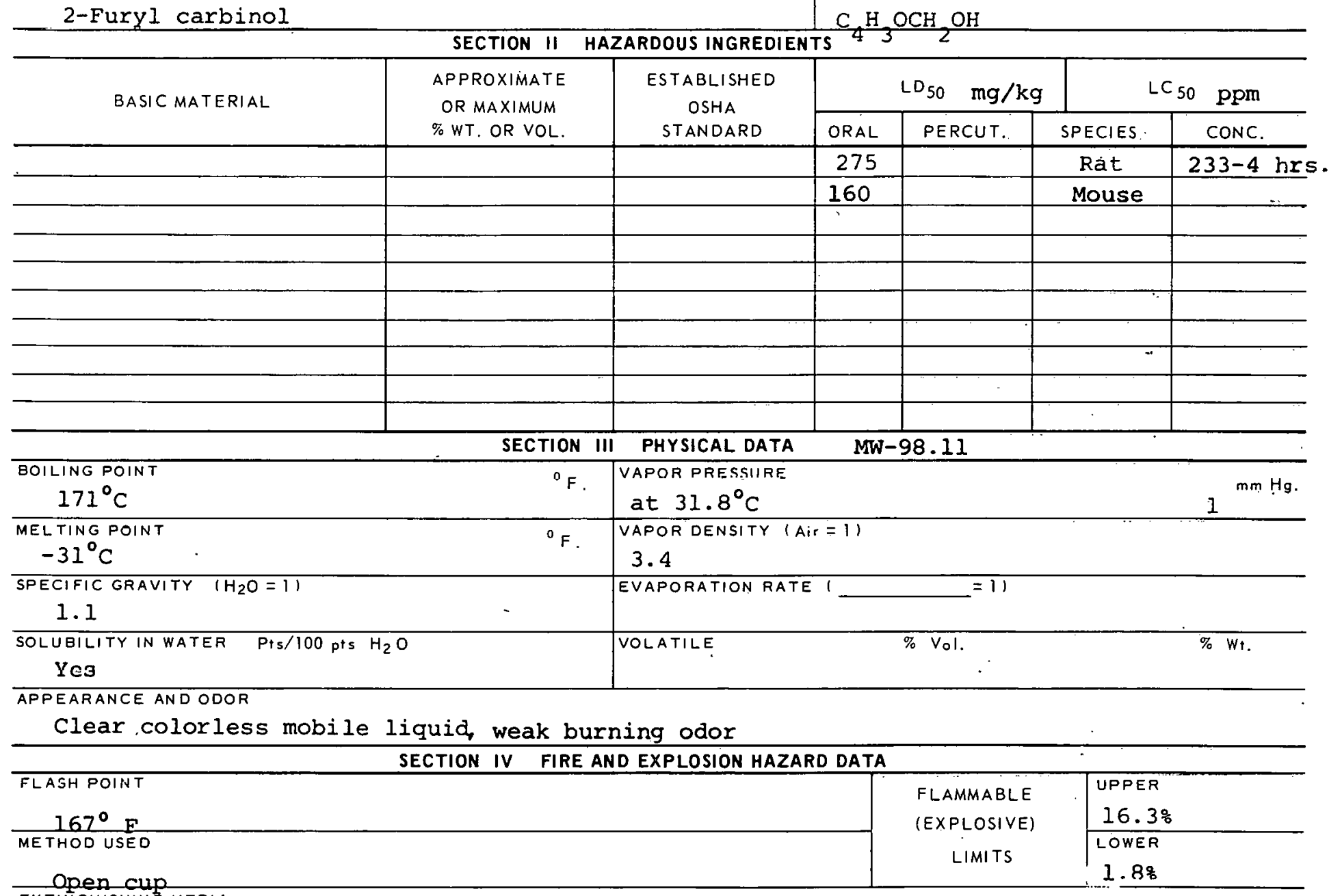

EXTINGUISHING MEDIA

Alcohol foam, carbon dioxide, dry chemical

SPECIAL FIRE FIGHTING PROCEDURES

UNUSUAL FIRE AND EXPLOSION HAZARDS 


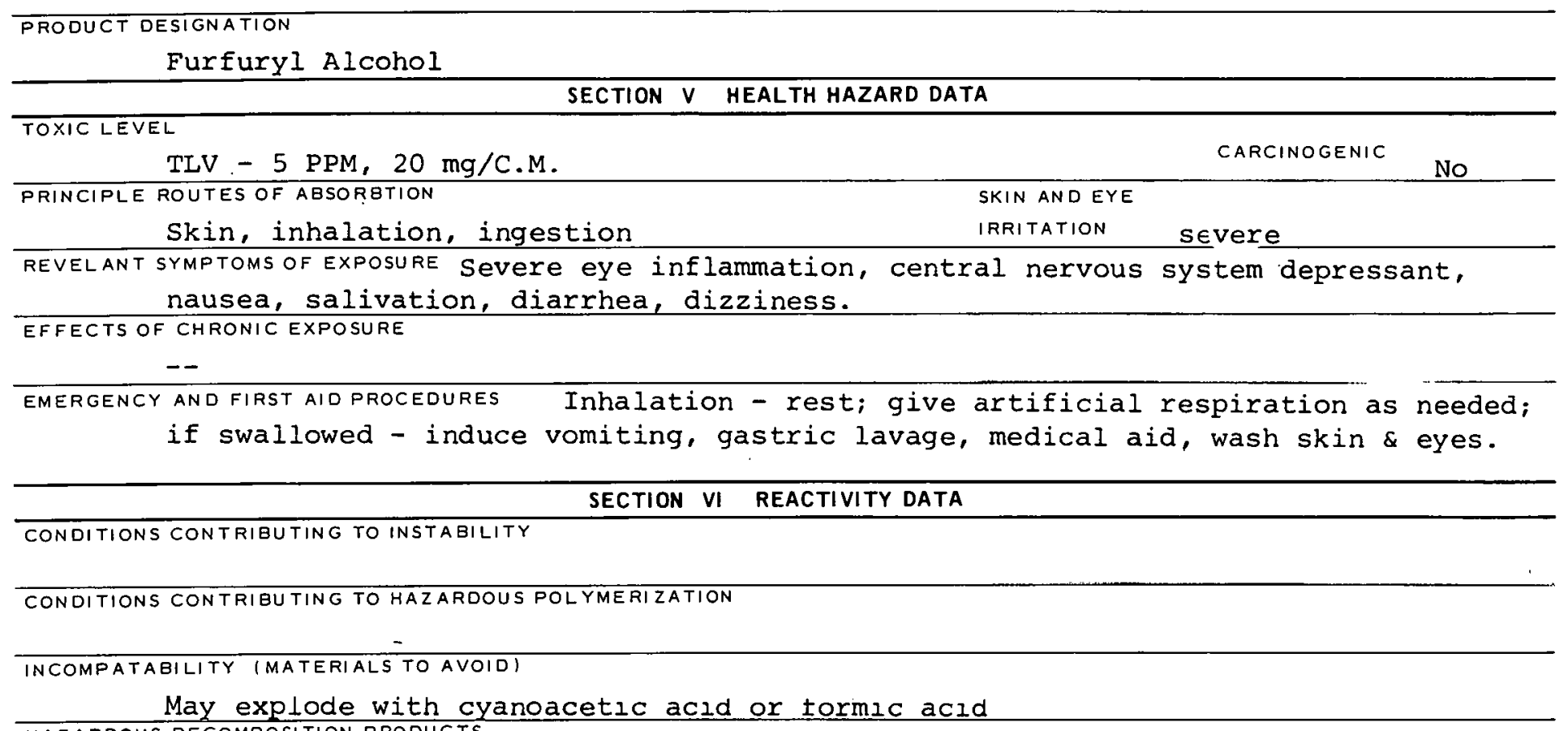
HAZARDOUS DECOMPOSITION PRODUCTS

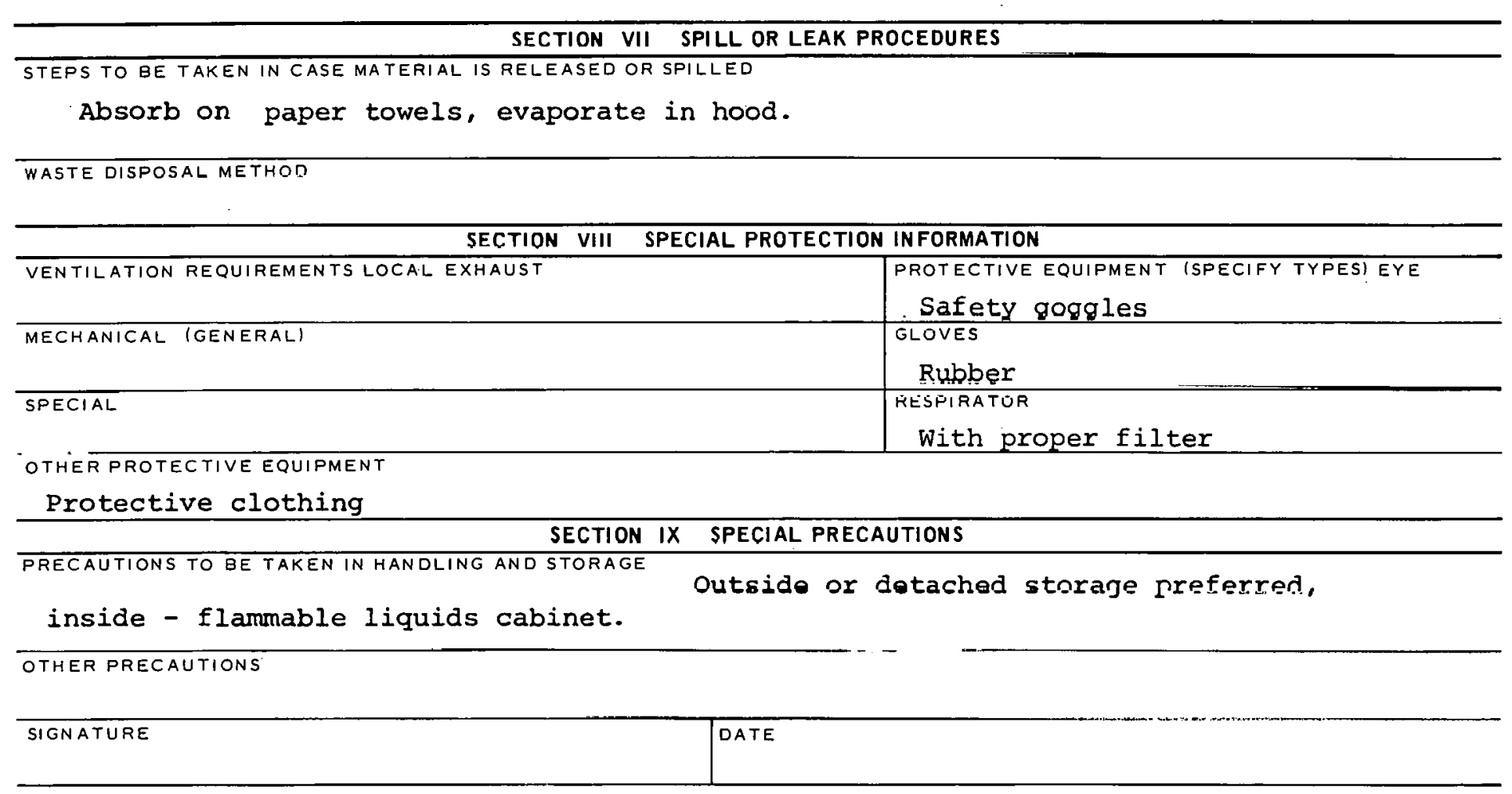


CAS - 000111706

\section{PRODUCT DESIGNATION}

n-Heptyl Alcohol

03-208-5202
MK03500

MATERIAL SAFETY

DATA SHEET

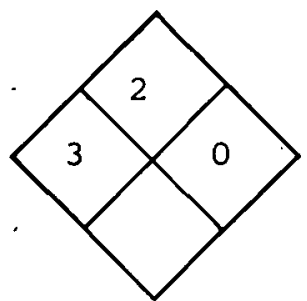

\section{SECTION I SOURCE AND NOMENCLATURE}

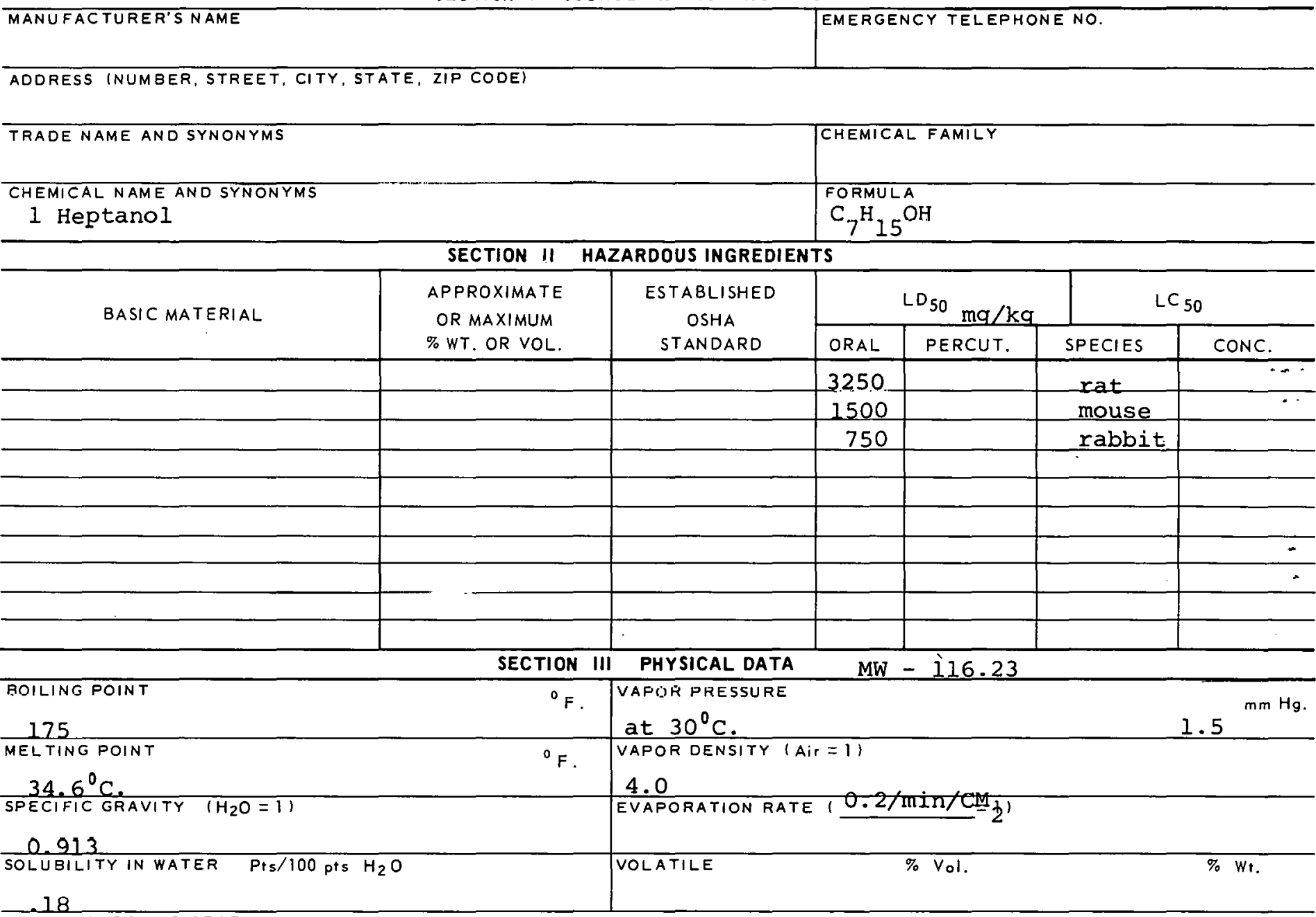

$\frac{18}{18}$

colorless liquid

FLASH POIN

$154^{\circ} \mathrm{F}$.

SECTION IV FIRE AND EXPLOSION HAZARD DATA

METHOD USED

EXTINGUISHING MEDIA

Foam, carbon dioxide, dry chemical

SPECIAL FIRE FIGHTING PROCEOURES

Water may be ineffective.

UNUSUAL FIRE AND.EXPLOSION HAZARDS 


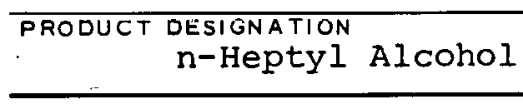

SECTION $V$ HEALTH HAZARD DATA

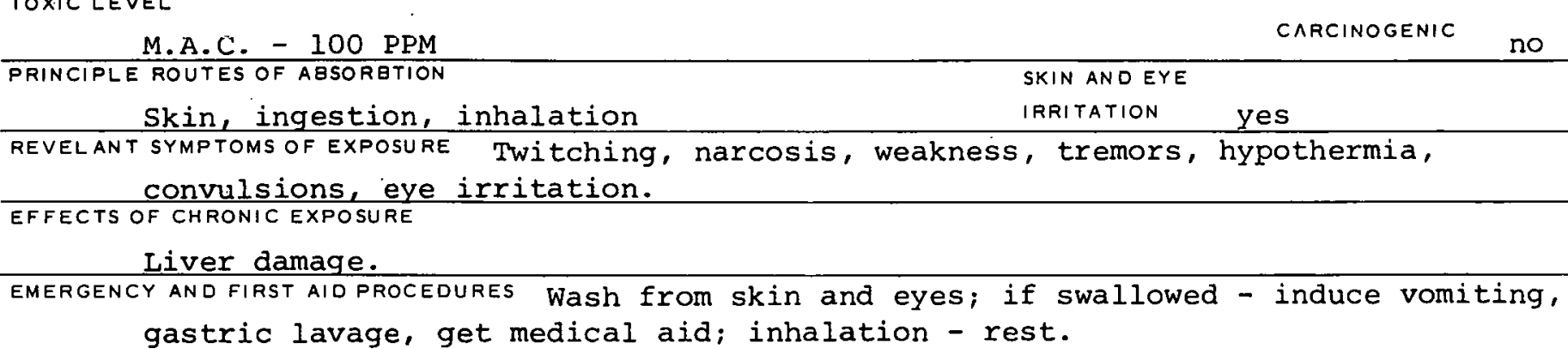

\section{SECTION VI REACTIVITY DATA}

SECTION VII SPILL OR LEAK PROCEDURES

STEPS TO BE TAKEN IN CASE MATERIAL IS RELEASED OR SPILLED

WASTE DISPOSAL METHOD

\section{SECTION VIII SPECIAL PROTECTION INFORMATION}

\begin{tabular}{|c|c|}
\hline VENTILATION REQUIREMENTS LOCAL EXHAUST & $\begin{array}{l}\text { PROTECTIVE EQUIPMENT (SPECIFY TYPES) EYE } \\
\text { Safety yldsises }\end{array}$ \\
\hline MECHANICAL (GENERAL) & $\begin{array}{l}\text { GLOVES } \\
\text { Rubber qloves }\end{array}$ \\
\hline SPECII & 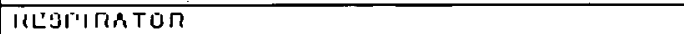 \\
\hline
\end{tabular}

OTHER PROTECTIVE EQUIPMENT

SECTION IX SPECIÁL PRECAUTIONS

PRECAUTIONS TO BE TAKEN IN HANDLING AND STORAGE

OTIIER RRLOAUTIOHO

EICANTURE

DATC

208-5202B 
CAS: 000067561

03-208-6502

PRODUCT DESIGNATION

$03-208-6552$

03-208-6002

03-208-6052

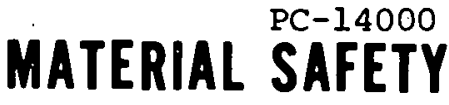 \\ DATA SHEET}

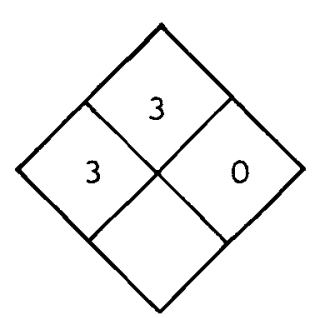

SECTION I SOURCE AND NOMENCLATURE

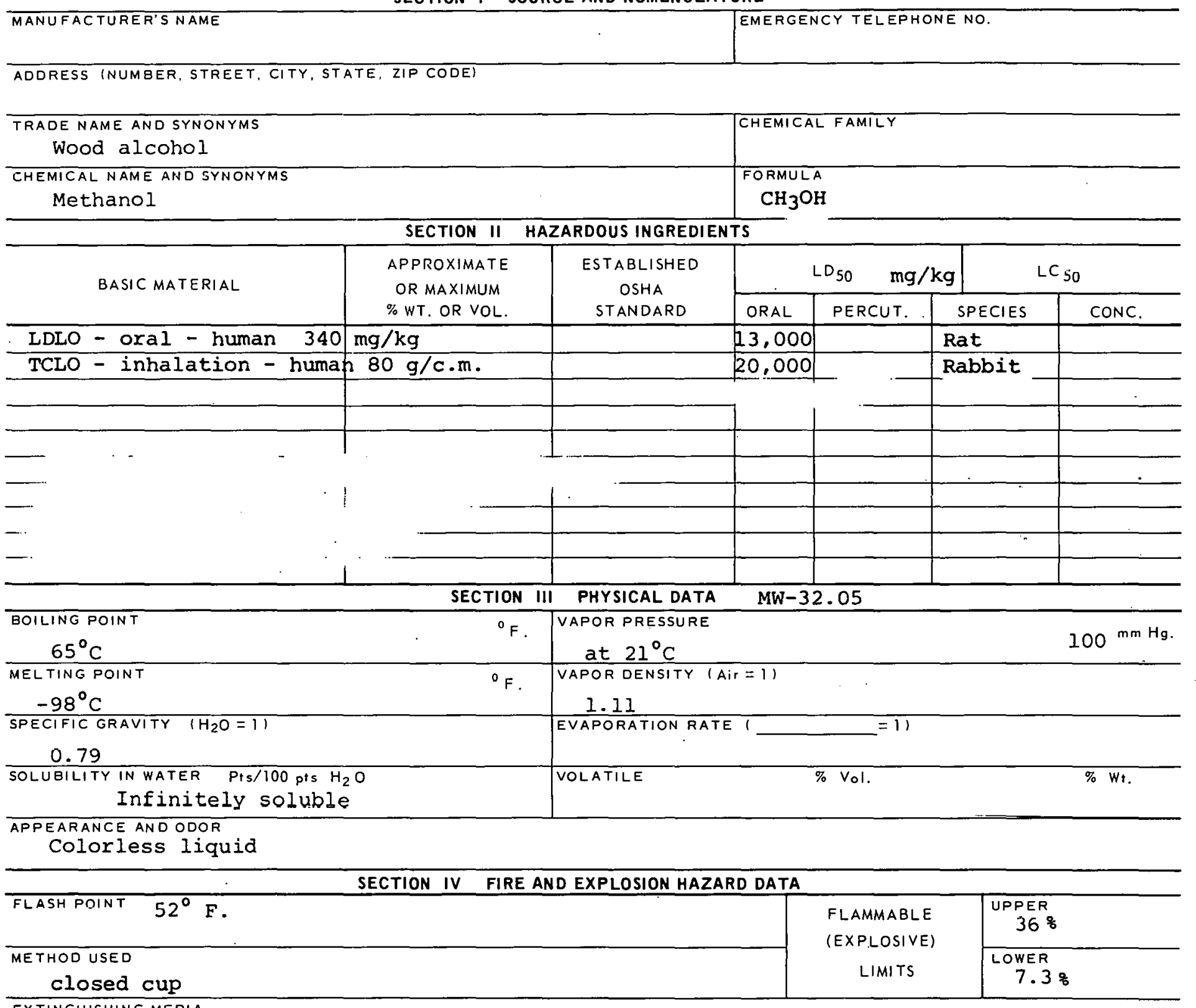

EXTINGUISHING MEDIA

Alcohol foam, dry chemical, carbon dioxide

SPECIAL FIRE FIGHTING PROCEOURES

Water may be ineffective.

UNUSUAL FIRE AND EXPLOSION HAZARDS 


\section{TOXIC LEVEL}

TLV - $200 \mathrm{PPM}, 260 \mathrm{mg} / \mathrm{C} . \mathrm{M}$. PRINCIPLE ROUTES OF ABSORBTION

Inhalation, ingestion, skin REVELANT SYMPTOMS OF EXPOSUREDisturbs vision, dizziness, stupor, cyanosis, abdominal cramps.

EFFECTS OF CHRONIC EXPOSURE Irritation of mucous membranes, headache, tremors, neuritis, vision failure.

EMERGENCY AND FIRST AID PROCEDURES If swallowed - induce vomiting, get medical aid; inhalation - rest; wash skin \& eyes.

\section{SECTION VI REACTIVITY DATA}

CARCINOGENIC

No SKIN AND EYE IRRITATION
Moderate

CONDITIONS CONTRIBUTING TO INSTABILITY

CONDITIONS CONTRIBUTING TO HAZARDOUS PQLYMERIZATION

INCOMPATABILITY (MATERIALS TO AVOID)

Perchloric acid, sodium hydroxide and chloroform HAZARDOUS DECOMPOSITION PRODUCTS

SECTION VII SPILL OR LEAK PROCEDURES

STEPS TO BE TAKEN IN CASE MATERIAL IS RELEASED OR SPILLED

Absorb on paper towels, evaporate in hood.

WASTE DISPOSAL METHOD

Burn

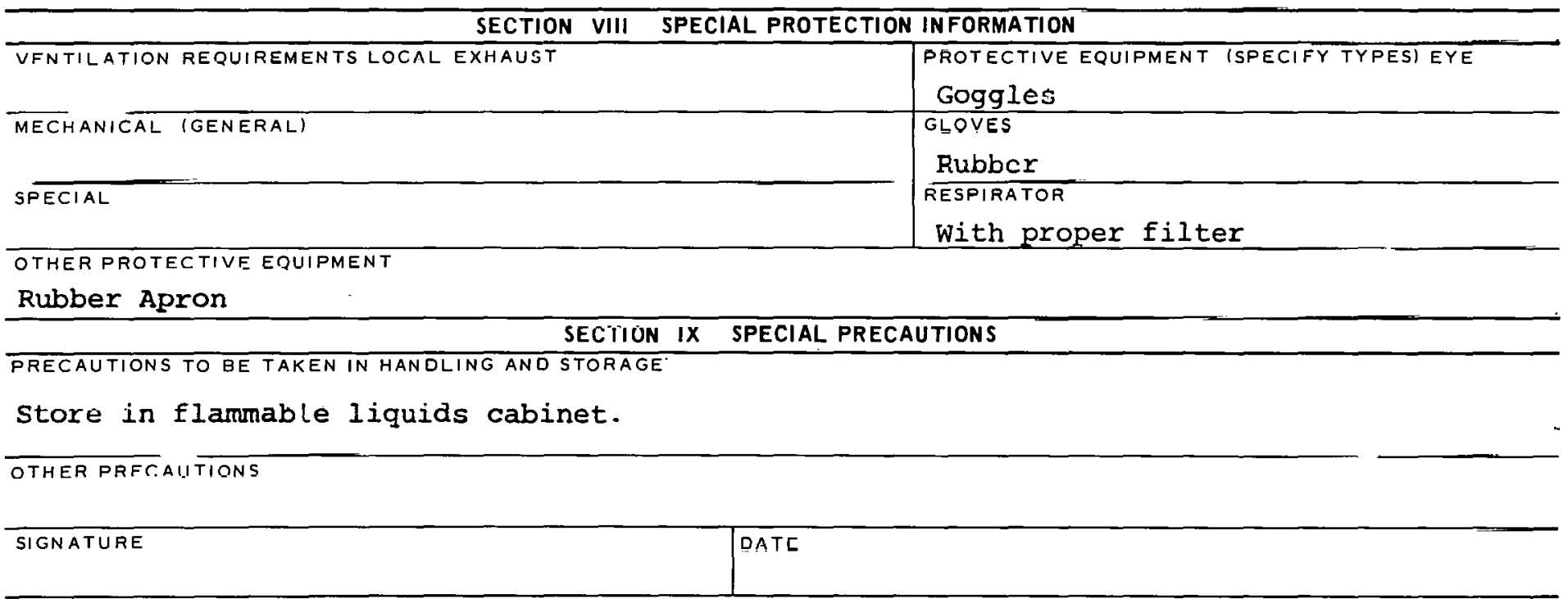


CAS : 000071238

OH82250

\begin{tabular}{|c|c|c|}
\hline PRODUCT DESIGNATION & MATERIAL SAFETY \\
03-208-8702 & DATA SHEET
\end{tabular}

SECTION I SOURCE AND NOMENCLATURE

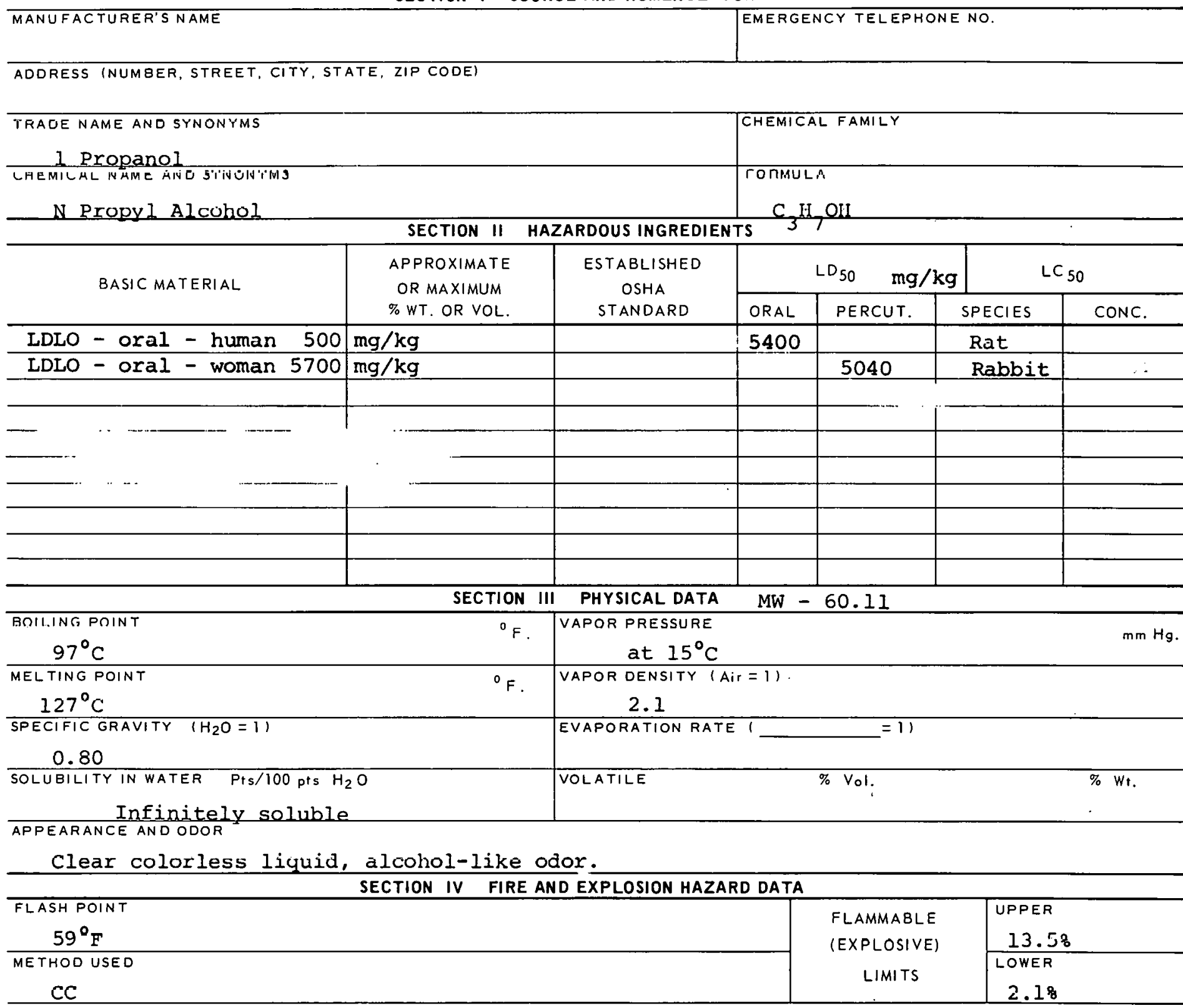

EXTINGUISHING MEDIA Alcohol foam, carbon dioxide, dry chemical - water may be ineffective.

SPECIAL FIRE FIGHTING PROCEDURES

UNUSUAL rIRE ANU EXPLOSION HAZARDS 


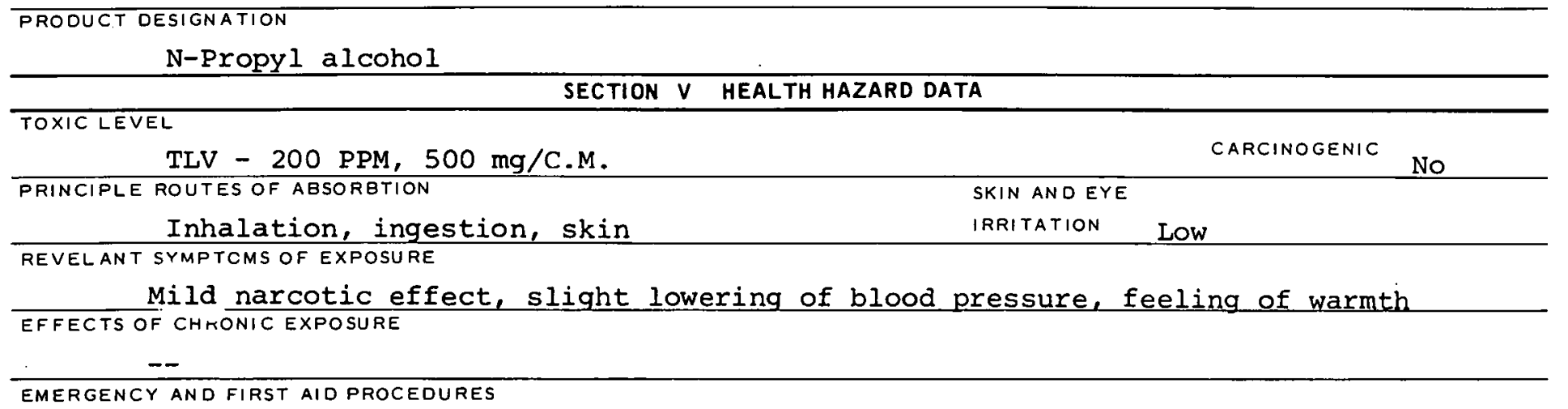

If swallowed - induce vomiting, get medical aid, gastric lavage; inhalation - rest; wash skin \& eyes.

\begin{tabular}{l}
\hline SECTION VI REACTIVITY DATA \\
\hline CONDITIONS CONTRIBUTING TO INSTABILITY \\
\hline INCOMPATABILITY (MATERIALS TO AVOIO) \\
\hline HAZARDUUS DECOMPOSITION PRODUCTS
\end{tabular}

\section{SECTION VII SPILL OR LEAK PROCEDURES}

STEPS TO BE TAKEN IN CASE MATERIAL IS RELEASED OR SPILLED

Absorb on paper towel, evaporate in hood.

WASTE DISPOSAL METHOD

Burn

\begin{tabular}{l|l}
\hline \multicolumn{1}{c}{ SECTION VIII SPECIAL PROTECTION INFORMATION } \\
\hline VENTILATION REQUIREMENTS LOCAL EXHAUST & $\begin{array}{l}\text { PROTECTIVE EQUIPMENT ISPECIFY TYPESI EYE } \\
\text { GOggles }\end{array}$ \\
\hline MECHANICAL IGENERAL) & $\begin{array}{c}\text { GLOVES } \\
\text { Rubber }\end{array}$ \\
\hline SFCCIAL & RESPIRATOR \\
With chemicad filter
\end{tabular}

OTHER PROTECTIVE EQUIPMENT

SECTION IX SPECIAL PRECAUTIONS

PRECAUTIONS TO BE TAKEN IN HANDLING AND STORAGE

Store in cool, well-ventilated place away from sources of ignition.

OTHER PRECAUTIONS 
CAS:00010711T

BA 54250

PRODUCT DESIGNATION

$03-211-3103$

Ally lamine

MATERIAL SAFETY

DATA SHEET

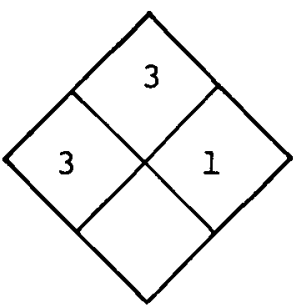

SECTION I SOURCE AND NOMENCLATURE

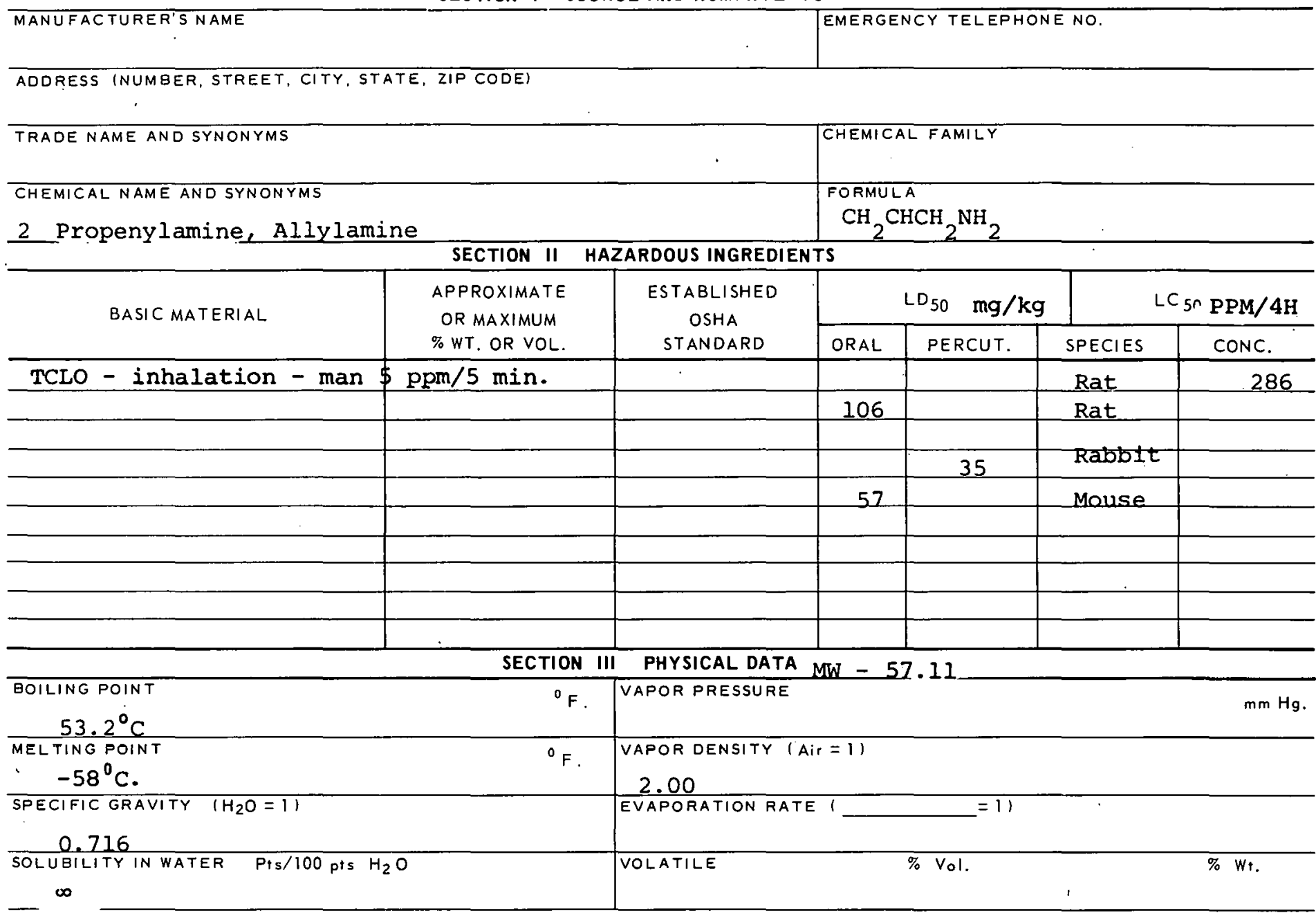

APPEARANCE AND ODOR

Colorless liquid, with strong ammonical odor.

SECTION IV FIRE AND EXPLOSION HAZARD DATA

\begin{tabular}{l|c|c}
\hline FLASH POINT & FLAMMABLE & UPPER \\
$-20^{\circ} \mathrm{F}$ & (EXPLOSIVE) & $22 \%$ \\
& LOWER & LIMITS \\
\hline
\end{tabular}

EXTINGUISHING MEDIA

Alcohol foam, carbon dioxide, dry chemical; water spray

SPECIAL FIRE FIGHTING PROCEDUPSS

Water may be ineffective - wear full protective clothing.

UNUSUAL FIRE AND EXPLOSION HAZARDS

When heated to decomposition temperature, emits fumes. 


\section{TOXIC LEVEL}

Not available

PRINCIPLE ROUTES OF ABSORBTION

Inhalation, ingestion, skin
CARCINOGENIC

SKIN ANO EYE

IRRITATION

Severe REVELANT SYMPTOMS OF EXPOSURE

Irritation of mucous membranes of eyes, nose and mouth

EFFECTS OF CHRONIC EXPOSURE

Liver and kidney damage

EMERGENCY AND FIRST AIDPROCEDURES Wash from eyes and skin; if swallowed - wash mouth, drink water, get medical aid; inhalation - rest.

\section{SECTION VI REACTIVITY DATA}

CONDITIONS CONTRIBUTING TO INSTABILITY

CONDITIONS CONTRIBUTING TO HAZARDOUS POLYMERIZATION

TINEOMPATADILIT'i I HATERIALJ TO A'UOOOO

kearts with acids.

HAZARDOUS DECOMPOSITION PRODUCTS

SECTION VII SPILL OR LEAK PROCEDURES

STEPS TO BE T.AKEN IN CASE MATERIAL IS RELEASED OR SPILLEO

Spread sodium bisulfate over area and sprinkle with water.

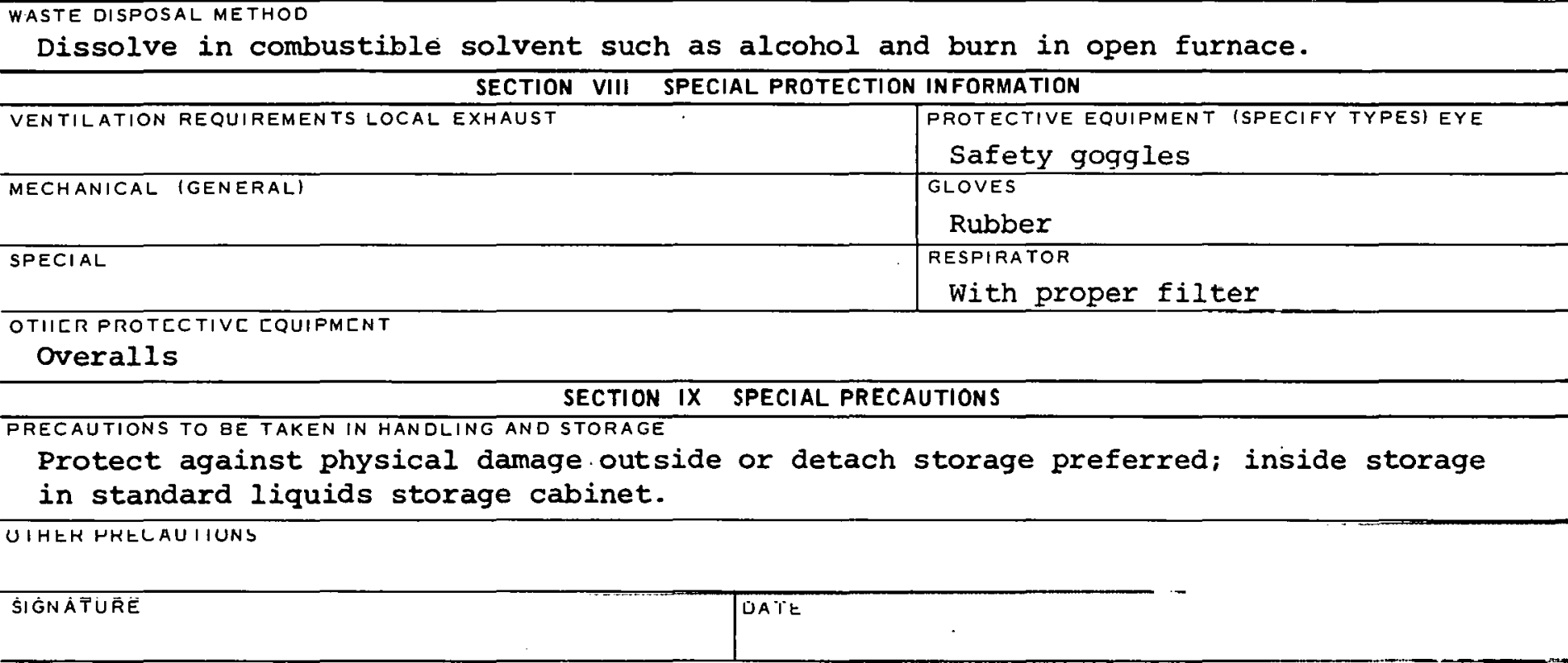


CAS : 000106956

\section{UC70900 \\ MATERIAL SAFETY \\ DATA SHEET}

PRODUCT DESIGNATION

03-211-3203

Allyl bromide

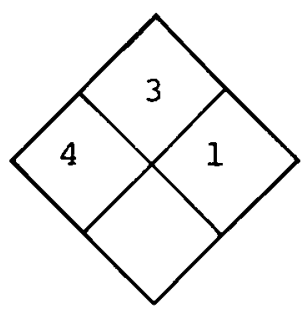

\section{SECTION I SOURCE AND NOMENCLATURE}

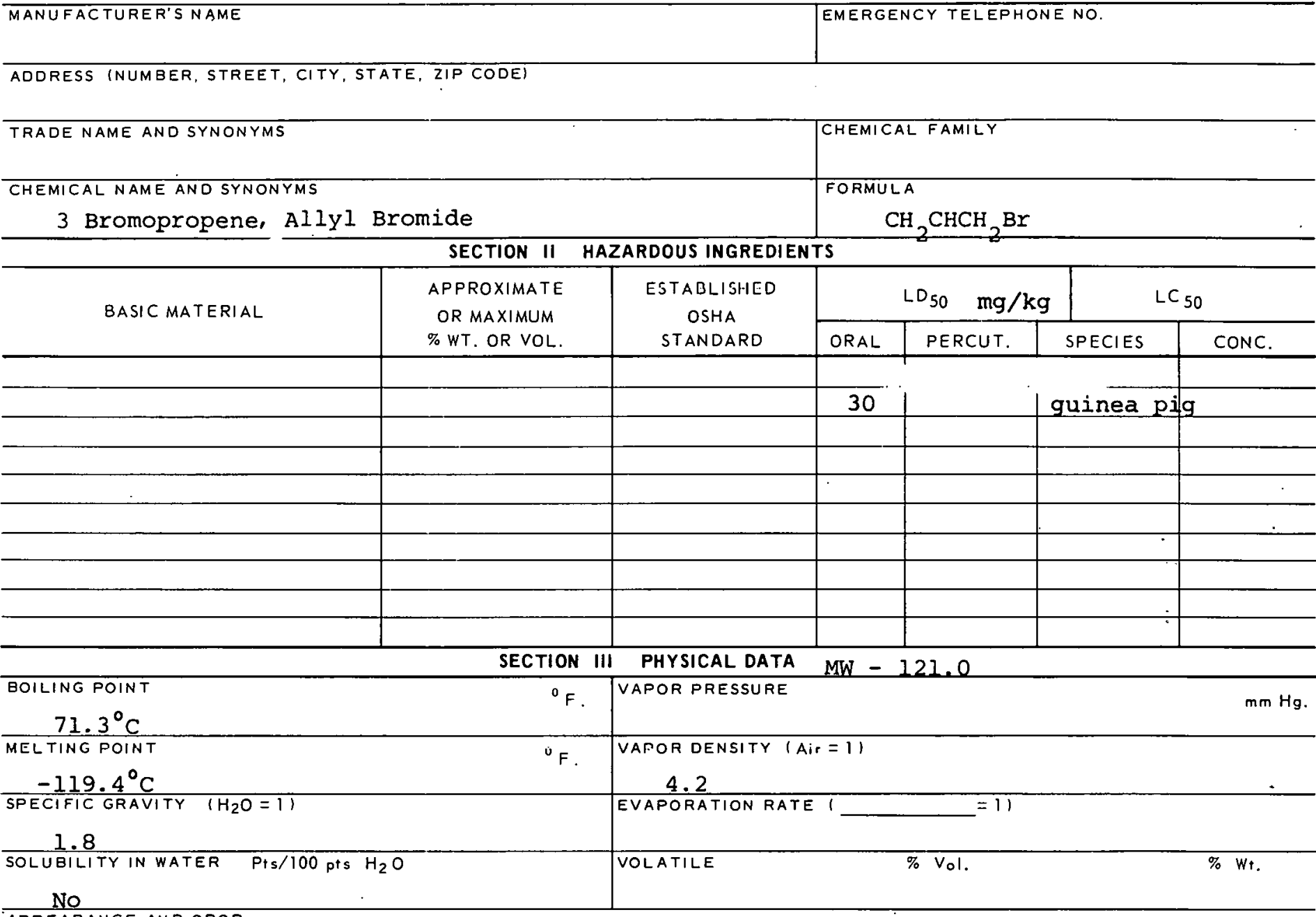

APPEARANCE AND OOOR

Colorless liquid, unpleasant odor

SECTION IV FIRE AND EXPLOSION HAZARD DATA

FLASH POINT

$300 \mathrm{~F}$

METHOO USED

EXTINGUISHING MEDIA

Alcohol foam

SPECIAL FIRE FIGHTING PROCEDURES

Water may be ineffective except as a blanket. UNUSUAL FIRE AND EXPLOSION HAZARDS 
Allyl bromide

SECTION $V$ HEALTH HAZARD DATA

TOXIC LEVEL

Not available PRINCIPLE ROUTES OF ABSORBTION

Inhalation, ingestion, skin

CARCINOGENIC No SKIN AND EYE IRRITATION Severe REVELANT SYMPTOMS OF EXPOSURE

Irritation of eyes, skin and respiratory tract EFFECTS OF CHRONIC EXPOSURE

Liver and kidney damage

EMERGENCY AND FIRST AIDPROCEDURES Wash skin \& eyes if swallowed - wash mouth, induce vomiting, get medical aid, inhalation - rest; keep warm.

CONDITIONS CONTRIBUTING TO INSTABILITY SECTION VI REACTIVITY DATA

CONDITIONS CONTRIBUTING TO HAZARDOUS POLYMERIZATION IACOMRATABILITY (MATERIALS TO AVOID) HAZARDOUS DECOMPOSITION PRODUCTS

SECTION VII SPILL OR LEAK PROCEDURES

STEPS TO BE TAKEN IN CASE MATERIAL IS RELEASED OR SPILLED

Absorb spills with paper towels. Burn paper towels in hood. WASTE DISPOSAL METHOD

SECTION VIII SPECIAL PROTECTION INFORMATION

\begin{tabular}{l|l}
\hline VENTILATION REQUIREMENTS LOCAL EXHAUST & $\begin{array}{c}\text { PROTECTIVE EQUIPMENT ISPECIFY TYPESI EYE } \\
\text { Safety goggles }\end{array}$ \\
\hline MECHANICAL IGENERAL) & $\begin{array}{c}\text { GLOVES } \\
\text { Rubber }\end{array}$ \\
\hline SPECIAL & $\begin{array}{c}\text { RESPIRATOR } \\
\text { WIth proper filtcr }\end{array}$ \\
\hline
\end{tabular}
OTHER PROTECTIVE EQUIPMENT

\section{SECTION IX SPECIAL PRECAUTIONS}

PRECAUTIONS TO BE TAKEN IN HANDLING AND STORAGE

OTHER PRECAUTIONS 
CAS : 000107051

\section{PRODUCT DESIGNATION}

Allyl Chloride

$03-211-3303$

\section{MATERIAL SAFETY \\ DATA SHEET}

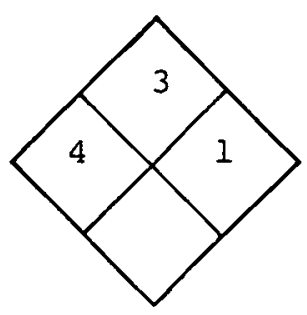

\section{SECTION I SOURCE AND NOMENCLATURE}

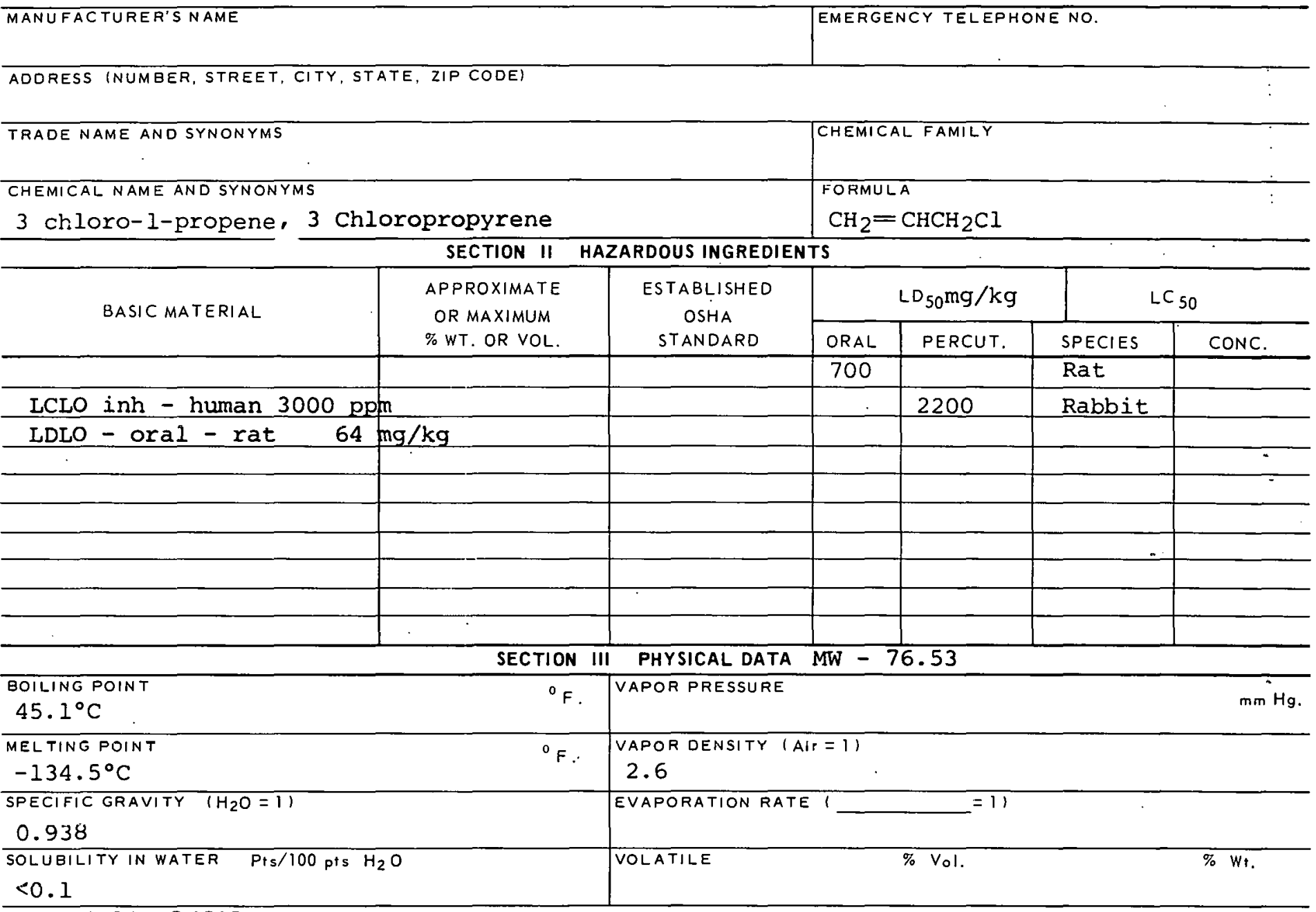

APPEARANCE AND ODOR

Colorless liquid, pungent odor

SECTION IV FIRE AND EXPLOSION HAZARD DATA

\begin{tabular}{l|c|c}
\hline FLASH POINT & FLAMMABLE & UPPER \\
$-25^{\circ} \mathrm{F}$ & (EXPLOSIVE) & 11.18 \\
METHOD USED & LOWER \\
& LIMITS & $3.3 \%$
\end{tabular}

EXTINGUISHING MEDIA

Alcohol foam, carbon dioxide or dry chemical

SPECIAL FIRE FIGHTING PROCEDURES

Water may be ineffective.

UNUSUAL FIRE AND EXPLOSION HAZARDS

$N / A$ 


\section{TOXIC LEVEL}

TLV $1 \mathrm{ppm}, 3 \mathrm{mg} / \mathrm{C} . \mathrm{M}$. PRINCIPLE ROUTES OF ABSORBTION

Inhalation, absorption through skin, ingestion

REVELANT SYMPTOMS OF EXPOSURE

Eye irritation, deep eye and bone aches

EFFECTS OF CHRONIC EXPOSURE

Liver and kidney damage

EMERGENCY AND FIRST AID PROCEOURES Wash from skin or eyes; inhalation exposiure (if severe) get medical attention; if swallowed - wash mouth with water, induce vomiting with mild emetic.

\begin{tabular}{l}
\hline SONDITIONS CONTRIBUTING TO INSTABILITY \\
\hline CONDITIONS CONTRIBUTING TO HAZARDOUS POLYMERIZATION \\
INCOMPATABILITY REACTIVITY DATA \\
\hline MATERIALS TO AVOIOI
\end{tabular}

\section{HAZARDOUS DECOMTOSITION FRODUCTS}

SECTION VII SPILL OR LEAK PROCEDURES

STEPS TO BE TAKEN IN CASE MATERIAL IS RELEASED OR SPILLED

WASTE DISPOSAL METHOD

SECTION VIII SPECIAL PROTECTION INFORMATION

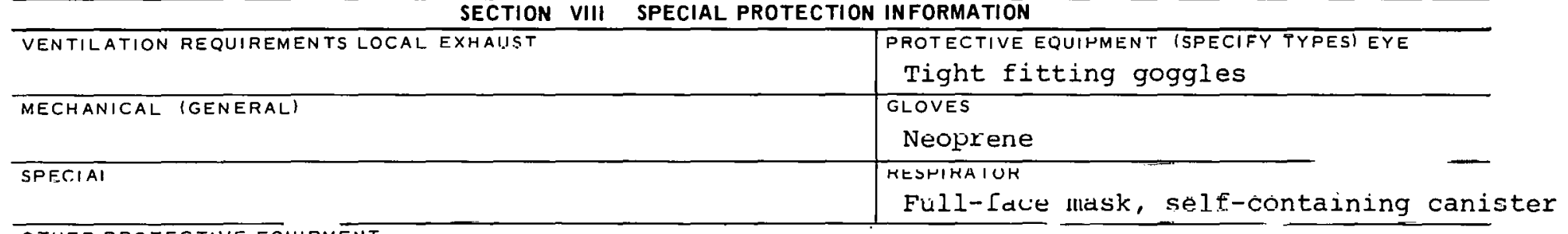

OTHER PROTECTIVE EQUIPMENT

Impervious clothing made of neoprene

SECTION IX SPECIAL PRECAUTIONS

PRECAUTIONS TO BE TAKEN IN HANDLING AND STORAGE

\section{Keep from sources of ignition.}

OTHER PRECAUTIONS

SIGNATURE DATE

$211-3303 B$ 


\section{MATERIAL SAFETY DATA SHEET}

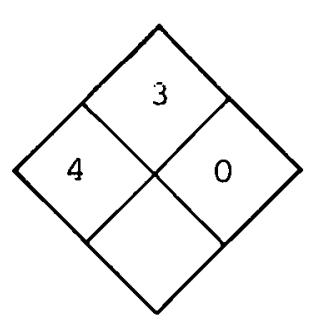

SECTION I SOURCE AND NOMENCLATURE

\section{MANUFACTURER'S.NAME}

ADDRESS (NUMBER, STREET, CITY, STATE, ZIP CODE)

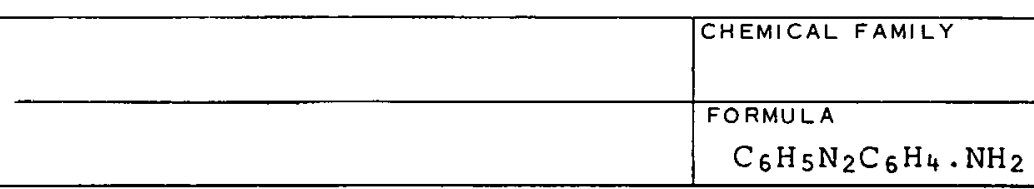

SECTION II HAZARDOUS INGREDIENTS \begin{tabular}{l|l} 
APPROXIMATE & ESTABLISHED
\end{tabular}

OR MAXIMUM OSHA $\%$ WT. OR VOL.

\section{NTS}

BASIC MATERIAL

Lowest published toxic dose

Oral - rat $128 \mathrm{~g} / \mathrm{Kg}$ over 2 years carcinggen ...skin - rat - $1550 \mathrm{mg} / \mathrm{kg} / 97$ weeks

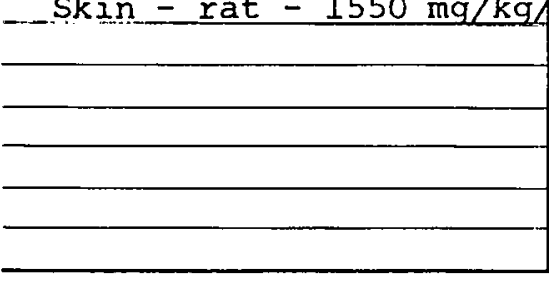

BOILING POINT

$>360^{\circ} \mathrm{C}$.

MELTING POINT

$124-126^{\circ} \mathrm{C}$.

SPECIFIC GRAVITY $\left(\mathrm{H}_{2} \mathrm{O}=1\right)$

SOLUBILITY IN WATER Pts/100 pts $\mathrm{H}_{2} \mathrm{O}$

Slightly soluble APPEARANCE AND ODOR

Brownish-yellow needles

SECTION IV FIRE AND EXPLOSION HAZARD DATA

\begin{tabular}{|c|c|c|}
\hline $\begin{array}{l}\text { FLASH HOINT } \\
\text { None available }\end{array}$ & $\begin{array}{l}\text { FLAMMABLE } \\
\text { (EXPLOSIVE) }\end{array}$ & UPPER \\
\hline METHOD USED & LIMITS & LOWER \\
\hline
\end{tabular}

EXTINGUISHING MEOIA

Water spray, foam, carbon dioxide.

SPECIAL FIRE FIGHTING PROCEDURES

UNUSUAL FIRE AND EXPLOSION HAZAROS

Vapur funlls explusive mixture in air. 


\section{TOXIC LEVEL \\ None available}

PRINCIPLE ROUTES OF ABSORBTION

Inhalation, ingestion, skin REVELANT SYMPTOMS OF EXPOSURE

jaundice and anemia.

EFFECTS OF CHRONIC EXPOSURE Weight loss, aremia, weakness, irritability, liver damage,

kidney irritation, liver cancer, kidney cancer.

EMERGENCY AND FIRST AID PROCEDURES Inhalation - rest; wash eyes and skin; ingestion - remove

by gastric lavage.

SECTION VI REACTIVITY DATA
SKIN AND EYE

IRRITATION SIIght
CARCINOGENIC

Yes

CONDITIONS CONTRIBUTING TO INSTABILITY

CONDITIONS CONTRIBUTING TO HAZARDOUS POLYMERIZATION

IN COMPATARII,I TY (MATF.RIAL.STO AVOIO)

HAZARUUUS UELUMRUSIIIUN RHUUUUT'T

SECTION VII SPILL OR LEAK PROCEDURES

STEPS TO BE TAKEN IN CASE MATERIAL IS RELEASED OR SPILLED

WASTE DISPOSAL METHOD

SECTION VIII SPECIAL PROTECTION INFORMATION

\begin{tabular}{l|l}
\hline VENTILATION REQUIREMENTS LOCAL EXHAUST & $\begin{array}{c}\text { PROTECTIVE EQUIPMENT (SPECIFY TYPES) EYE } \\
\text { Goggles }\end{array}$ \\
\hline MECHANICAL IGENERALI & $\begin{array}{c}\text { GLOVES } \\
\text { Rubber }\end{array}$ \\
\hline SPECIAL & $\begin{array}{c}\text { RESFIR̈ATUR } \\
\text { CheInical respirator }\end{array}$ \\
\hline
\end{tabular}

OTHER PROTECTIVE EQUIPMENT

\section{SECTION IX SPECIAL PRECAUTIONS}

PRECAUTIONS TO BE TAKEN IN HANDLING AND STORAGE 
BY82350

PRODUCT DESIGNATION

03-211-6703

P-Aminoazobenzene Hydrochloride
MATERIAL SAFETY

DATA SHEET

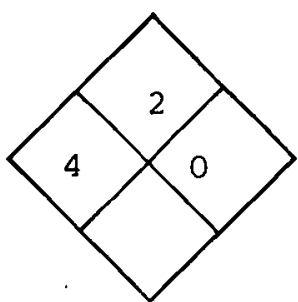

SECTION I SOURCE AND NOMENCLATURE

\begin{tabular}{l|l|l}
\hline MANUFACTURER'S NAME & EMERGENCY TELEPHONE NO \\
\hline ADDRESS INUMBER, STREET, CITY, STATE, ZIP CODEI & CHEMICAL FAMILY \\
\hline TRADE NAME AND SYNONYMS & \\
\hline CHEMICAL NAME AND SYNONYMS & $\mathrm{C}_{6} \mathrm{H}_{5} \mathrm{~N}_{\mathbf{N}} \mathrm{NC}_{6} \mathrm{H}_{4} \mathrm{NH}_{2} \mathrm{HCl}_{1}$ \\
\hline
\end{tabular}

P-Aminoazobenzene Hydrochloride

SECTION II HAZARDOUS INGREDIENTS

BASIC MATERIAL

\begin{tabular}{l|l} 
APPROXIMATE & ESTABLISHED
\end{tabular}

OR MAXIMUM OSHA

$\%$ WT. OR VOL.

\begin{tabular}{|l|}
\hline \\
\hline \\
\hline
\end{tabular}

BOILING POINT

MELTING POINT

$228^{\circ} \mathrm{C}$.

SPECIFIC GRAVITY $\left(\mathrm{H}_{2} \mathrm{O}=1\right)$

SOI IIAIIITY IN WATER Prs/100 pts $\mathrm{H}_{3} \mathrm{O}$

Soluble

APPEARANCE AND ODOR

steel blue crystals

SECTION IV FIRE AND EXPLOSION HAZARD DATA

FLASH POINT
None available

METHOD USED

None available

SECTION III PHYSICAL DATA MW-233.7

${ }^{0} \mathrm{~F}$ VAPOR PRESSURE

${ }^{\circ} \mathrm{F} . \quad$ VAPOR DENSITY $(A \mathrm{Air}=1)$

EVAPORATION RATE!

VOLATILE

\begin{tabular}{|c|c|c|c|}
\hline \multicolumn{2}{|c|}{$\operatorname{LD}_{50}$} & \multicolumn{2}{|c|}{$L^{L C}{ }_{50}$} \\
\hline ORAL & PERCUT. & SPECIES & CONC. \\
\hline 1250 & & Rat & \\
\hline & & & \\
\hline & & & \\
\hline & & & \\
\hline & & & \\
\hline & & & \\
\hline & & & \\
\hline & & & \\
\hline & & & \\
\hline & & & \\
\hline
\end{tabular}

$\mathrm{mm} \mathrm{Hg}$.

$=11$

$\%$ vol.

$\% W_{+}$.

EXTINGIISHING MEDIA

Water spray, foam, carbon dioxide

SPECIAL FIRE FIGHTING PROCEDURES

UNUSUAL FIRE AND EXPLOSION HAZARDS

Vapor forms explosive mixture in air.

FLAMMABLE (EXPLOSIVE)

LIMITS
UPPER

LOWER 


\section{PRODUCT DESIGNATION \\ $\mathrm{P}$-Aminoazobenzene Hyarochloride}

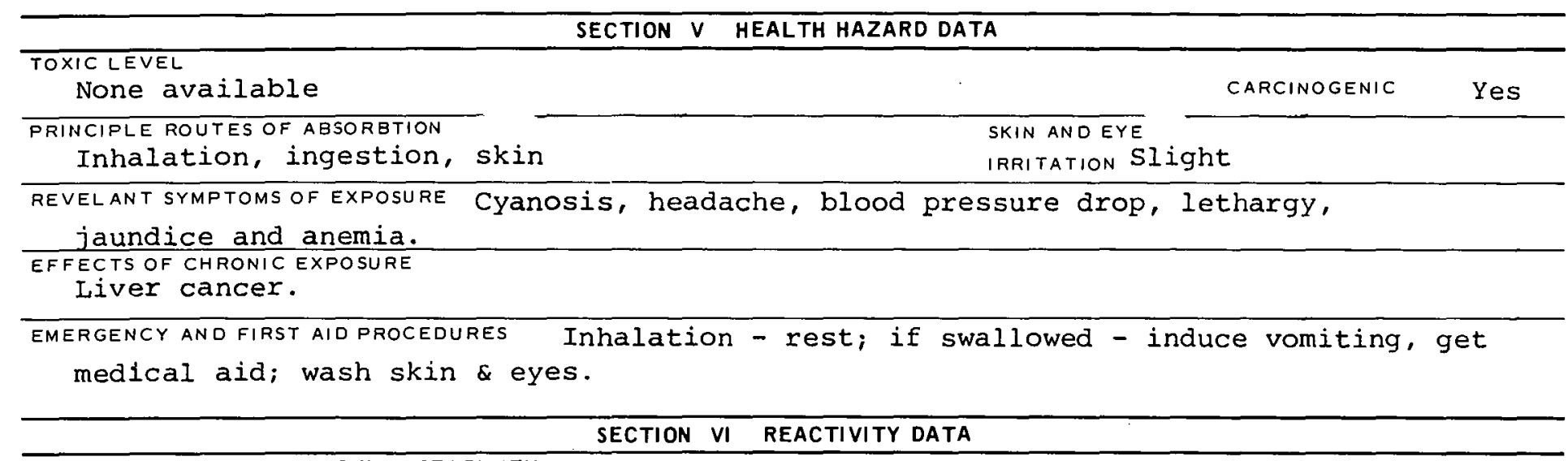

CONDITIONS CONTRIBUTING TO INSTABILITY

CONDITIONS CONTRIBU TING TO HAZARDOUS POLYMERIZATION

INCOMPATABILITY (MATERIALS TO AVOIO)

HAZARDOUS DECOMPOSITION PRODUCTS

SECTION VII SPILL OR LEAK PROCEDURES

STEPS TO BE TAKEN IN CASE MATERIAL IS RELEASED OR SPILLED

.

$-$

WASTE DISPOSAL METHOD

-

\begin{tabular}{|c|c|c|}
\hline SECTION & VIII & SPECIAL PROTECTION INFORMATION \\
\hline VENTILATION REQUIREMENTS LOCAL EXHAUST & & $\begin{array}{l}\text { PROTEC.TIVE EQUIPMENT (SPECIFY TYFES) EYE } \\
\text { Safety glasses }\end{array}$ \\
\hline MECHANICAL (GENERAL) & & $\begin{array}{l}\text { GLOVES } \\
\text { Rubber }\end{array}$ \\
\hline $\begin{array}{c}\text { SPECIAL } \\
.\end{array}$ & & RESPIRATOA \\
\hline
\end{tabular}

OTHER PROTECTIVE EQUIPMENT

SECTION IX SPECIAL PRECAUTIONS

PRECAUTIONS TO BE TAKEN IN HANDLING AND STORAGE

OTHER PRECAUTIONS

SIGNATURE

DATE

$211-6703 B$ 


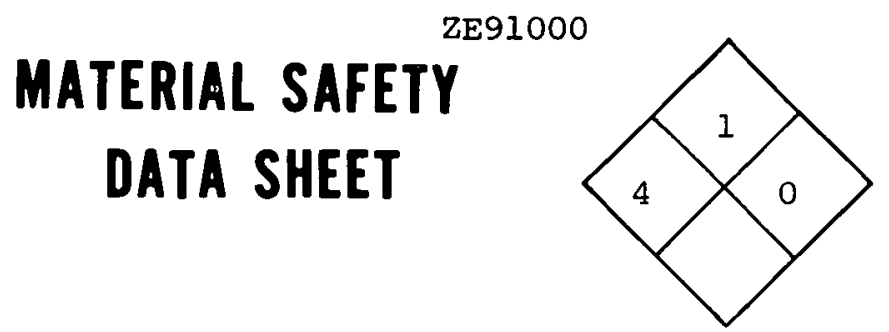

SECTION I SOURCE AND NOMENCLATURE

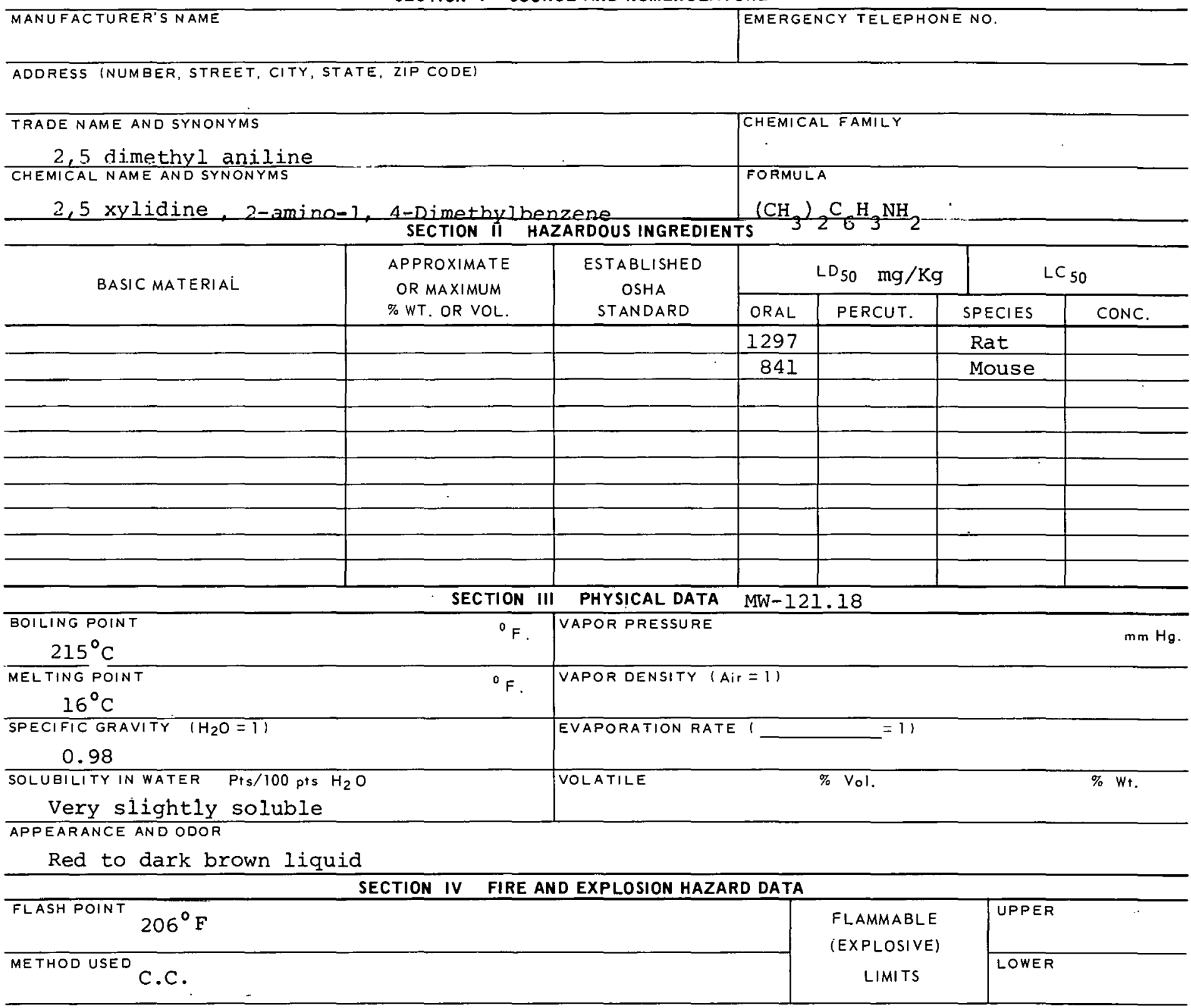

EXTINGUISHING MEDIA

Foam, carbon dioxide, dry chemical

SPECIAL FIRE FIGHTING PROCEDURES

UNUSUAL FIRE AND EXPLOSION HAZARDS 
2 amino-1, 4-Dimethylbenzene

\section{SECTION $V$ HEALTH HAZARD DATA}

\section{TOXIC LEVEL \\ TLV 5 PPM, $25 \mathrm{mg} / \mathrm{C} . \mathrm{M}$.}

PRINCIPLE ROUTES OF ABSORETION

Inhalation, ingestion, skin REVELANT SYMPTOMS OF EXPOSURE

convulsions, central nervous system depression

EFFECTS OF CHRONIC EXPOSURE Liver and kidney damage, fatigue, loss of appetite

EMERGENCY AND FIRST AID PROCEDURES

wash from skin \& eyes.
SKIN ANDEYE

IRRITATION
CARCINOGENIC

No

slight

If swallowed - give emetic, get medical aid; inhalation - rest;

\section{SECTION VI REACTIVITY DATA}

CONDITIONS CONTRIBUTING TO INSTABILITY

CONOITIONS CONTRIBUTING TO HAZARDOUS POLYMERIZATION

INCOMPATABILITY (MATERIALSTO AVOIDI

HAZAROQUE DECOMTOSITIOH FRODUCT

SECTION VII SPILL OR LEAK PROCEDURES

STEPS TO BE TAKEN IN CASE MATERIAL IS RELEASED OR SPILLED

WASTE DISPOSAL METHOD

SECTION VIII SPECIAL PROTECTION INFORMATION

VENTILATION REQUIREMENTS LOCAL EXHAUST

PROTECTIVE EQUIPMENT (SPECIFY TYFEG) EYE

MECHANICAL (GENERAL) GLoves

Rubber

SPECIAL

RESPIRATOR

with proper filter

OTHER PROTECTIVE EQUIPMENT

SECTION IX SPECIAL PRECAUTIONS

PRECAUTIONS TO BE TAKEN IN HANDLING AND STORAGE

OTHER PRECAUTIONS

SIGNATURE

DATE

212-0104B 


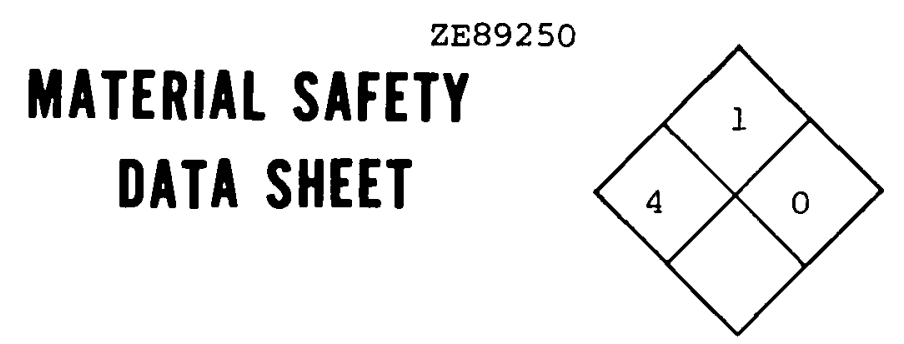

$03-212-0134$

4 amino - 1,3-Dimethylbenzene

SECTION I SOURCE AND NOMENCLATURE

ADDRESS (NUMBER, STREET, CITY, STATE, ZIP CODE)

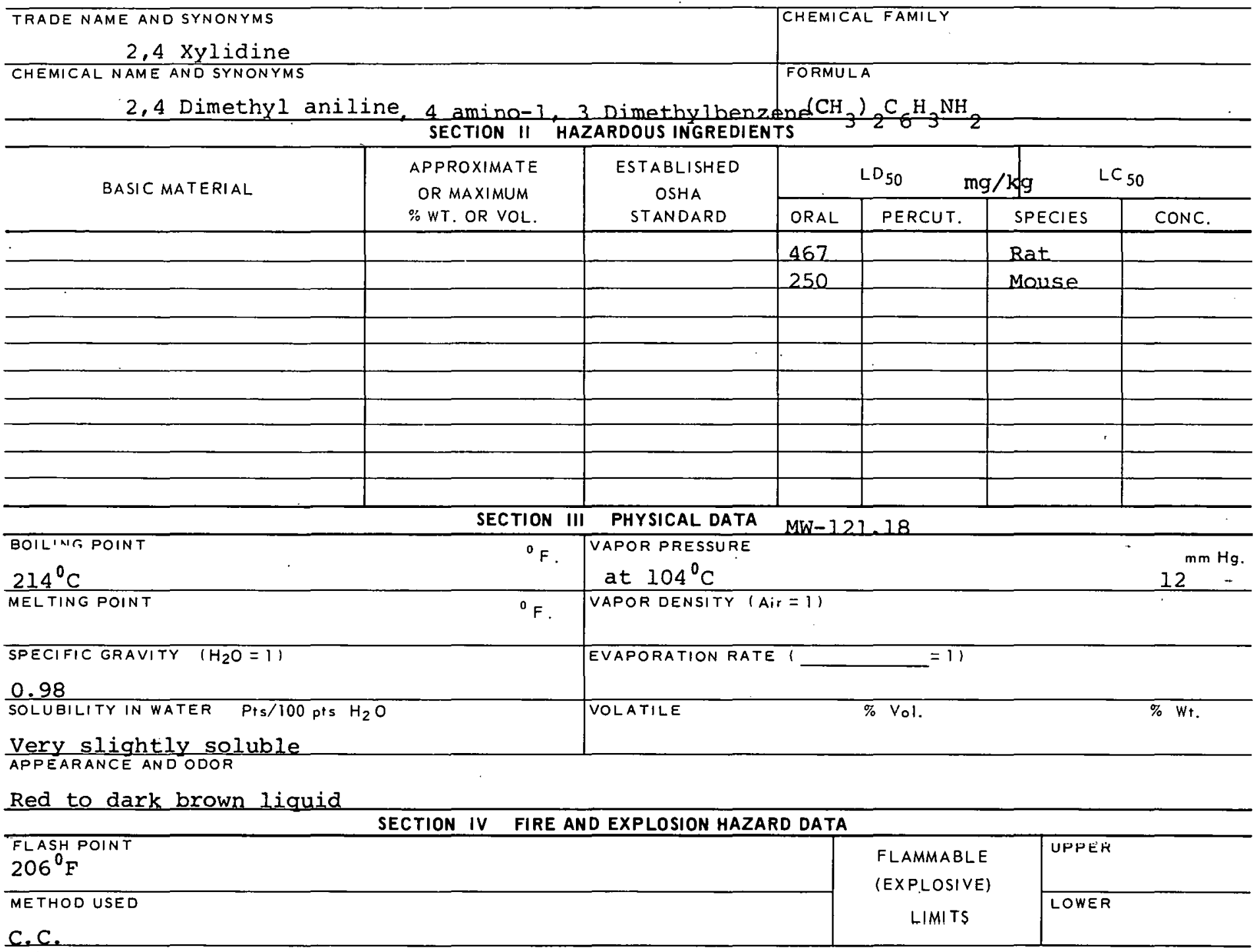

EXTINGUISHING MEDIA

Foam, carbon dioxide, dry chemical

SPECIAL FIRE FIGHTING PROCEDURES

UNUSUAL FIRE AND EXPLOSION HAZARDS 
4 amino, - 1,3 Dimethylbenzene

SECTION $V$ HEALTH HAZARD DATA

\section{TOXIC LEVEL}

TLV 5 PPM, $25 \mathrm{mg} / \mathrm{C} . \mathrm{M}$.

PRINCIPLE ROUTES OF ABSORBTION

Inhalation, ingestion, skin SKIN AND EYE

CARCINOGENIC

IRRITATION no

REVELANT SYMPTOMS OF EXPOSURE Headache, drowsiness, cyanosis, mental confusion, convulsions, central nervous system depression

EFFECTS OF CHRONIC EXPOSURE

Liver and kidney damage, fatigue, loss of appetite

EMERGENCY AND FIRST AID PROCEOURES If swallowed - give emetic, get medical aid;

inhalation - rest; wash from skin and eyes.

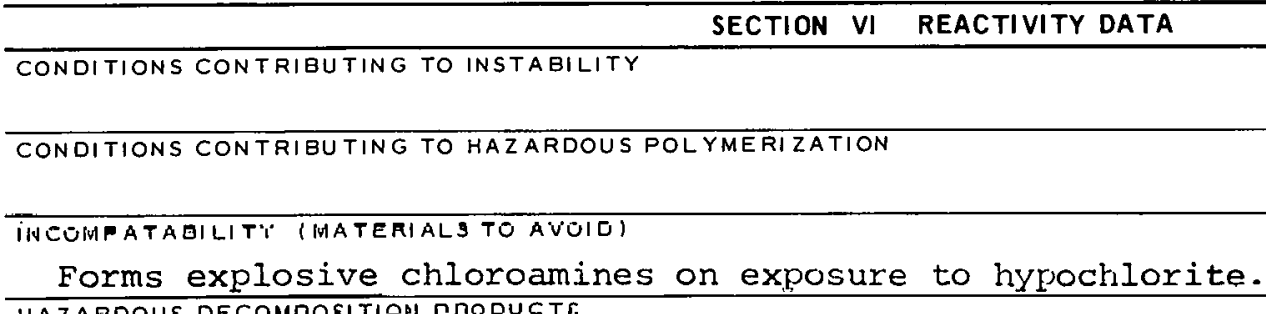

SECTION VII SPILL OR LEAK PROCEDURES

STEPS TO BE TAKEN IN CASE MATERIAL IS RELEASED OR SPILLED

WASTE DISPOSAL METHOD

SECTION VIII SPECIAL PROTECTION INFORMATION

\begin{tabular}{l|l}
\hline VENTILATION REOUIREMENTS LOCAL EXHALST & $\begin{array}{l}\text { PROTECTIVE CQUIPMENT (SPECIFY TYPES) EYE } \\
\text { GOGgleS }\end{array}$ \\
\hline MECHANICAL (GENERAL) & $\begin{array}{l}\text { GLOVES } \\
\text { Rubber }\end{array}$ \\
\hline SPECIAL & $\begin{array}{l}\text { RFSAIRATOE } \\
\text { WLth proper filter }\end{array}$ \\
\hline
\end{tabular}

OTHER PROTECTIVE EQUIPMENT

Full protective clothing

SECTION IX SPECIAL PRECAUTIONS

PRECAUTIONS TO BE TAKEN IN HANDLING AND STORAGE

Protect against physical damaqe, store in cool, dry place, OTHER PRECAUTIONS 


\section{PRODUCT DESIGNATION}

03-212-0164

5-amino-1,3-Dime thy lbenzene
MATERIAL SAFETY DATA SHEET

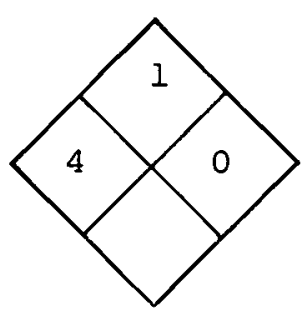

SECTION I SOURCE AND NOMENCLATURE

ADDRESS (NUMBER, STREET, CITY, STATE, ZIP CODE)

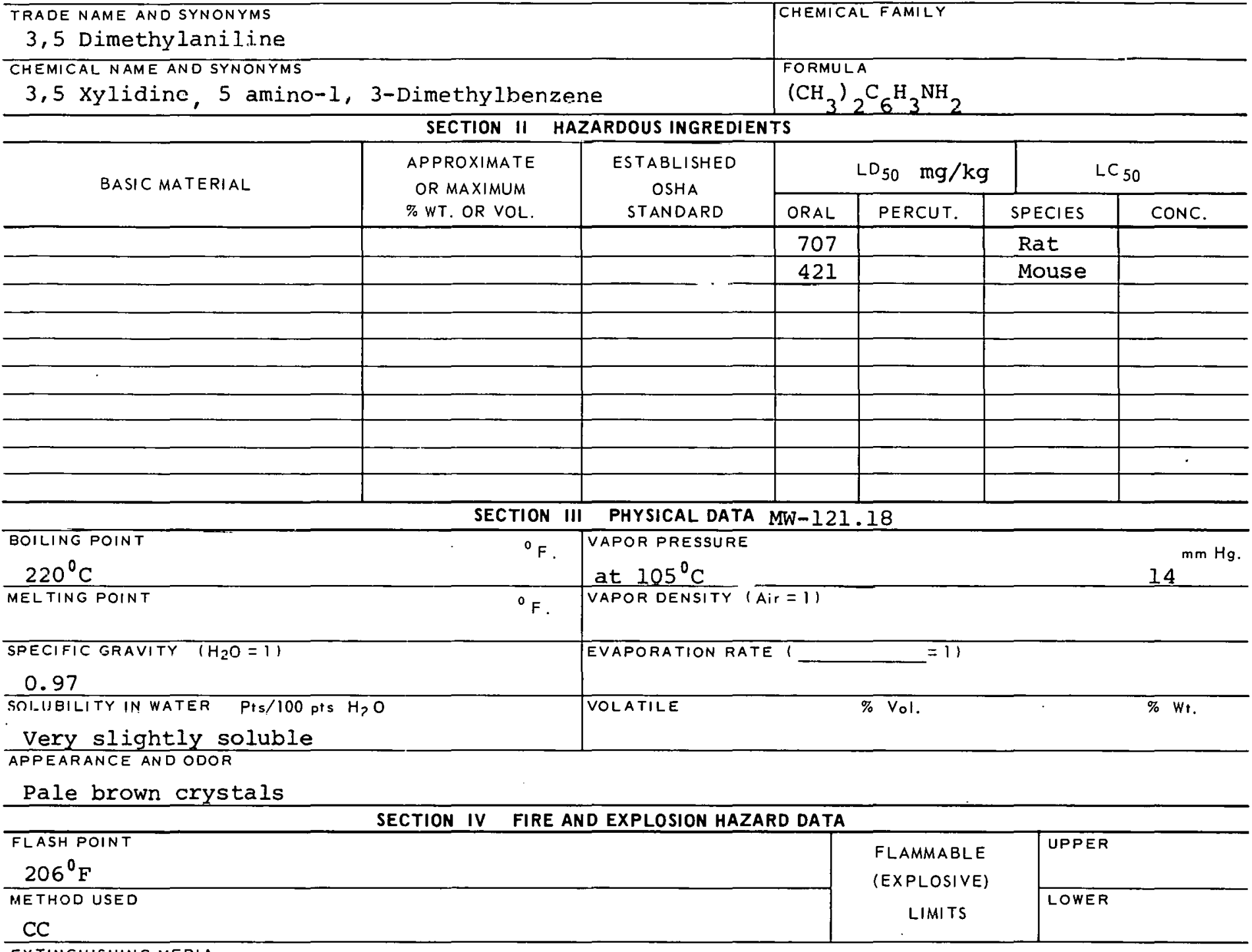

EXTINGIIISHING MEDIA

Foam, Carbon dioxide, dry chemical

SPECIAL FIRE FIGHTING PROCEDURES

UNUSUAL FIRE AND EXPLOSION HAZARDS 
5 amino - 1,3 Dimethylbenzene SECTION $V$ HEALTH HAZARD DATA

TOXIC LEVEL

TLV 5 PPM, $25 \mathrm{mg} / \mathrm{C} . \mathrm{M}$.

PRINCIPLE ROUTES OF ABSORBTION

CARCINOGENIC

Inhalation, ingestion, skin

SKIN AND EYE

IRRITATION

REVELANT SYMPTOMS OF EXPOSURE Headache, drowsiness, cyanosis, mental confusion, convulsions, central nervous system depression

EFFECTS OF CHRONIC EXPOSURE

Liver and kidney damage, fatigue, loss of appetite.

EMERGENCY AND FIRST AIDPROCEOURES If swallowed, give emetic, get medical aid;

inhalation - rest; wash from skin \& eyes.

\section{SECTION VI REACTIVITY DATA}

CONDITIONS CONTRIBUTING TO INSTABILITY

CONDITIONS CONTRIBUTING TO HAZARDOUS POLYMERI ZATION

INCOMPATÄUILITY (MATERIALS TOO AVOOOO)

Forms explosive chloroamines on exposure to hypochlorites.

HAZARDOUS DECOMPOSITION FRODUCTS

\section{SECTION VII SPILL OR LEAK PROCEDURES}

STEPS TO BE TAKEN IN CASE MATERIAL IS RELEASED OR SPILLED

WASTE DISPOSAL METHOD

SECTION VIII SPECIAL PROTECTION INFORMATION

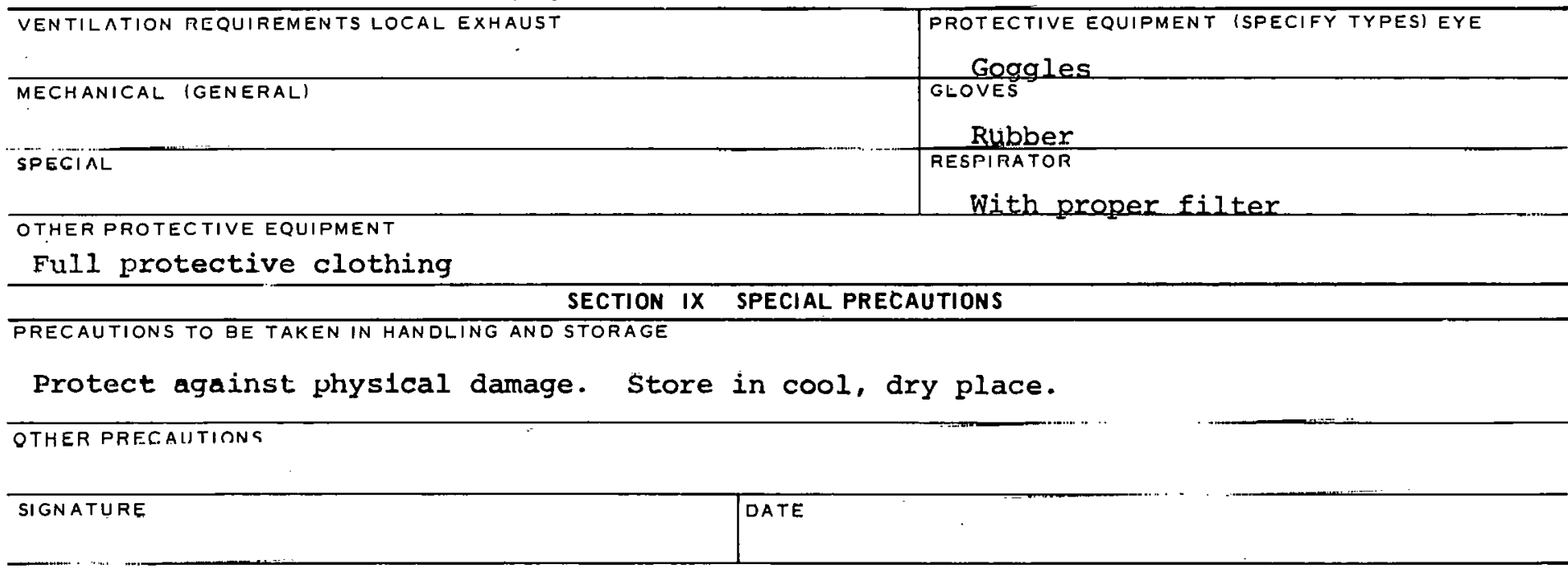


CAS : 000090415

\section{MATERIAL SAFETY \\ DATA SHEET}

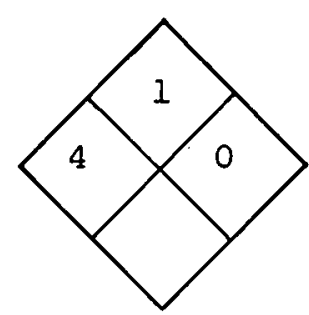

SECTION I SOURCE AND NOMENCLATURE

\begin{tabular}{l} 
MANUFACTURER'S NAME \\
ADORESS INUMBER, STREET, CITY \\
TRADE NAME AND SYNONYMS \\
O-aminobiphenY] \\
CHEMICAL NAME AT, SYNONYMS \\
O-aminodiphenYI \\
\hline
\end{tabular}

| EMERGENCY TELEPHONE NO.

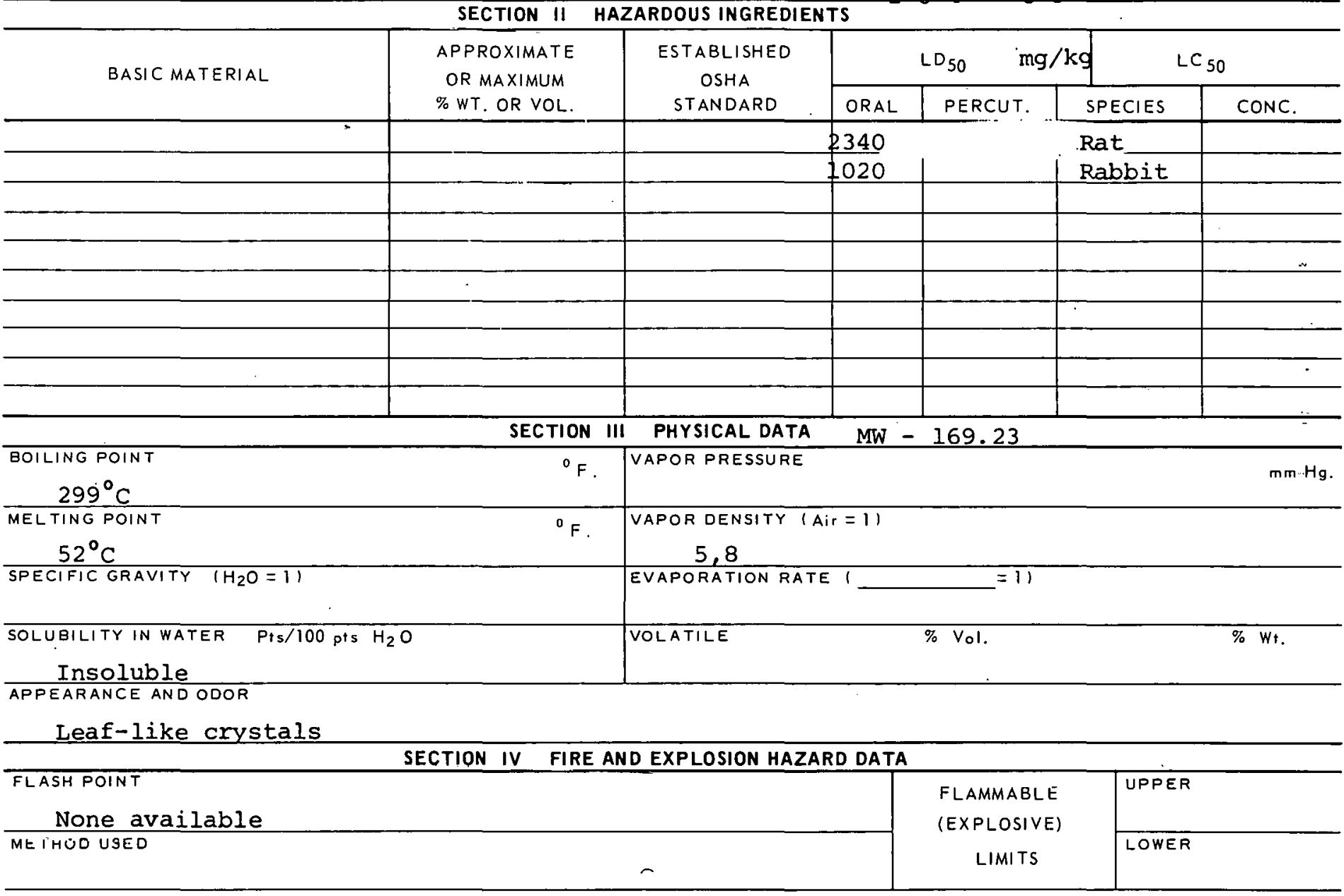

EXTINGUISHING MEDIA

Carbon-dioxide, dry chemical

SPECIAL FIRE FIGHTING PROCEDURES

IINIISUAL FIRE AND EXPLOSION HAZARDS 


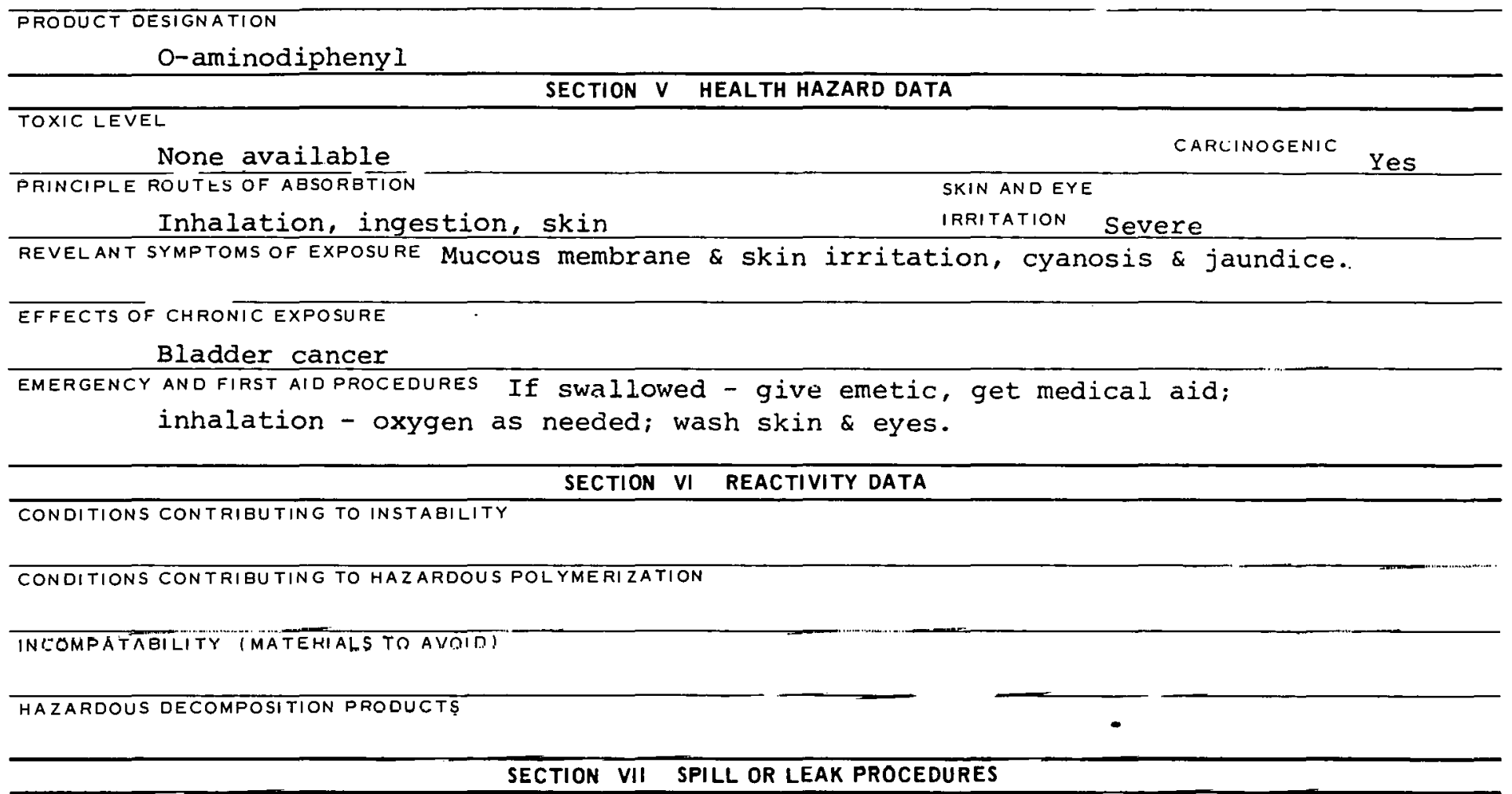

STEPS TO BE TAKEN IN CASE MATERIAL IS RELEASED OR SPILLED

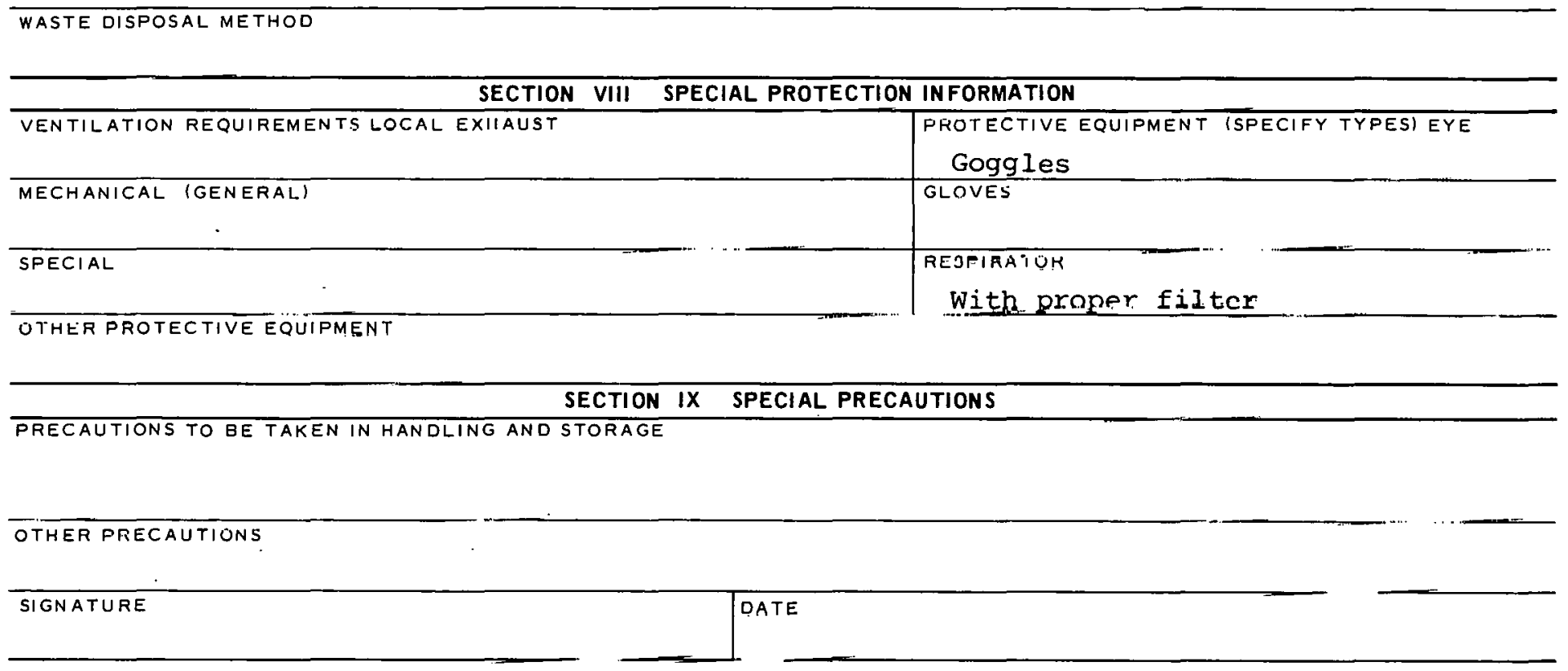




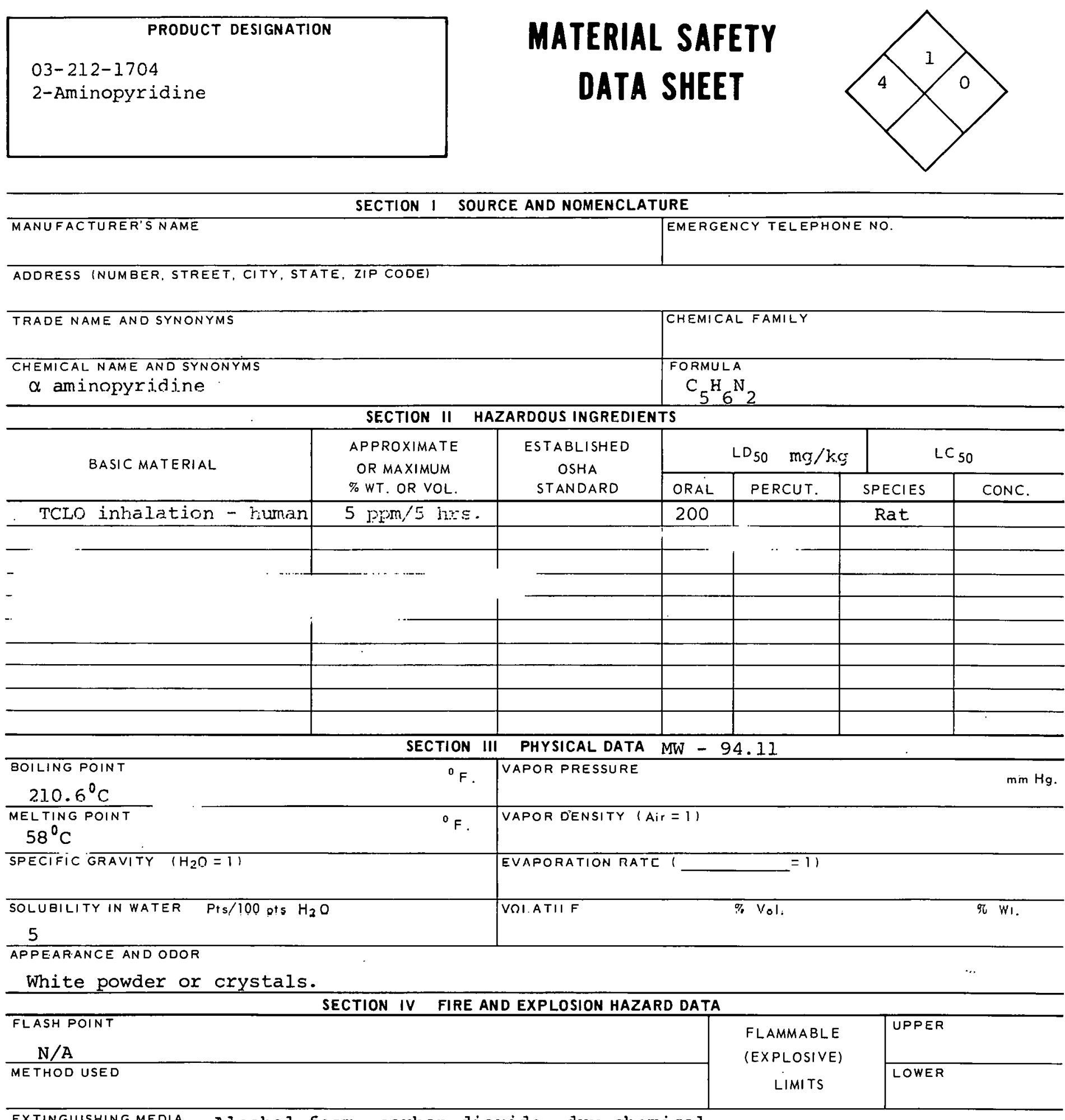

03-212-1704

2-Aminopyridine
DATA SHEET 
TOXIC LEVEL

TLV -0.5 PPM, $2 \mathrm{mg} / \mathrm{C} . \mathrm{M}$. PRINCIPLE ROUTES OF ABSORBTION

Skin, inhalation, ingestion
CARCINOGENIC No SKIN AND EYE IRRITATION

Moderate

REVELANT SYMPTOMS OF EXPOSURE Headache, increased blood pressure, nausea, convulsions, respiratory failure.

EFFECTS OF CHRONIC EXPOSURE

$--$

EMERGENCY AND FIRST AID PROCEDURES Inhalation - remove from exposure, rest, artificial respiration as needed, get medical aid; ingestion - induce vomiting, get medical aid; wash from skin \& eyes.

SECTION VI REACTIVITY DATA

CONDITIONS CONTRIBUTING TO INSTABILITY

CONDITIONS CONTRIBUTING TO HAZARDOUS POLYMERIZATION

INCOMPATABILITY (MAIERIALS IU AVUIOD)

HALARUUUS LELOBIFEMITIEId PRODUGTS

SECTION VII SPILL OR LEAK PROCEDURES

STEPS TO BE TAKEN IN CASE MATERIAL IS RELEASED OR SPILLED

Cover with a $9 / 1$ mixture of sand and soda ash; transfer into paper carton,

burn in furnace.

WASTE DISPOSAL METHOD

SECTION VIII SPECIAL PROTECTION INFORMATION

\begin{tabular}{l|l}
\hline VENTILATION REQUIREMENTS LOCAL EXHAUST & $\begin{array}{c}\text { PROTECTIVE EQUIPMENT (SPECIFY TYPES) EYE } \\
\text { GOggles }\end{array}$ \\
\hline MECHANICAL IGENERAL) & $\begin{array}{c}\text { GLOVES } \\
\text { Rubber (butyl) }\end{array}$ \\
\hline SPECIAL & RESPIRATOR \\
Self-contained respirator
\end{tabular}

PRECAUTIONS TO BE TAKEN IN HANDLING AND STORAGE 
PRODUCT DESIGNATION

03-212-5004

Iso amyl acetate
MATERIAL SAFETY

DATA SHEET

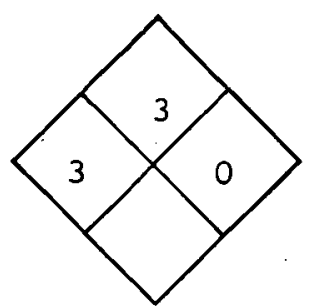

SECTION I SOURCE AND NOMENCLATURE

MANUFACTURER'S NAME

| EMERGENCY TELEPHONE NO.

ADDRESS (NUMBER, STREET, CITY, STATE, ZIP CODE)

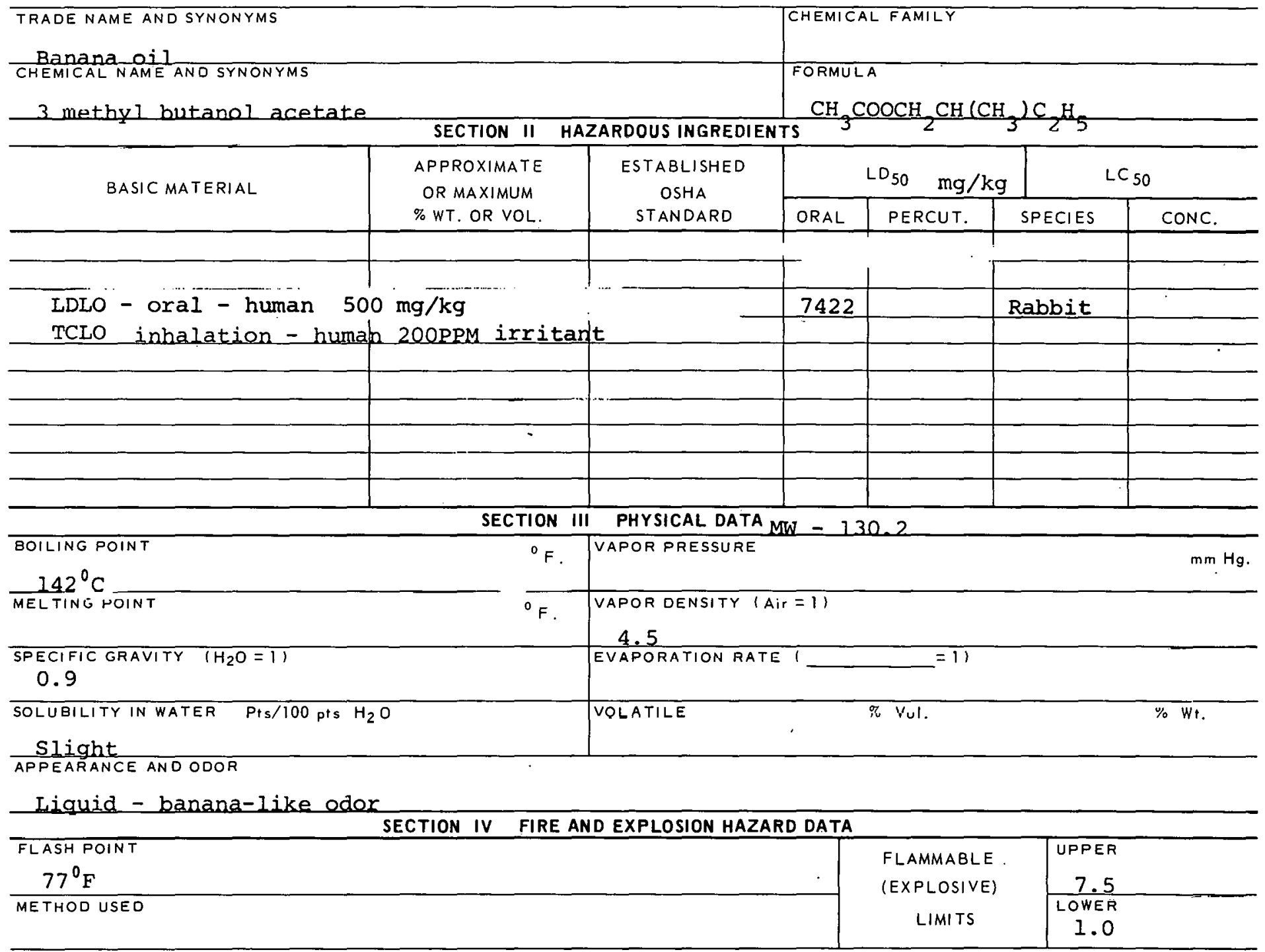

EXTINGUISHING MEDIA Alcohol foam, carbon dioxide, dry chemical water may be ineffective.

SPECIAL FIRE FIGHTING PROCEDURES

UNUSUAL FIRE AND EXPLOSION HAZARDS 


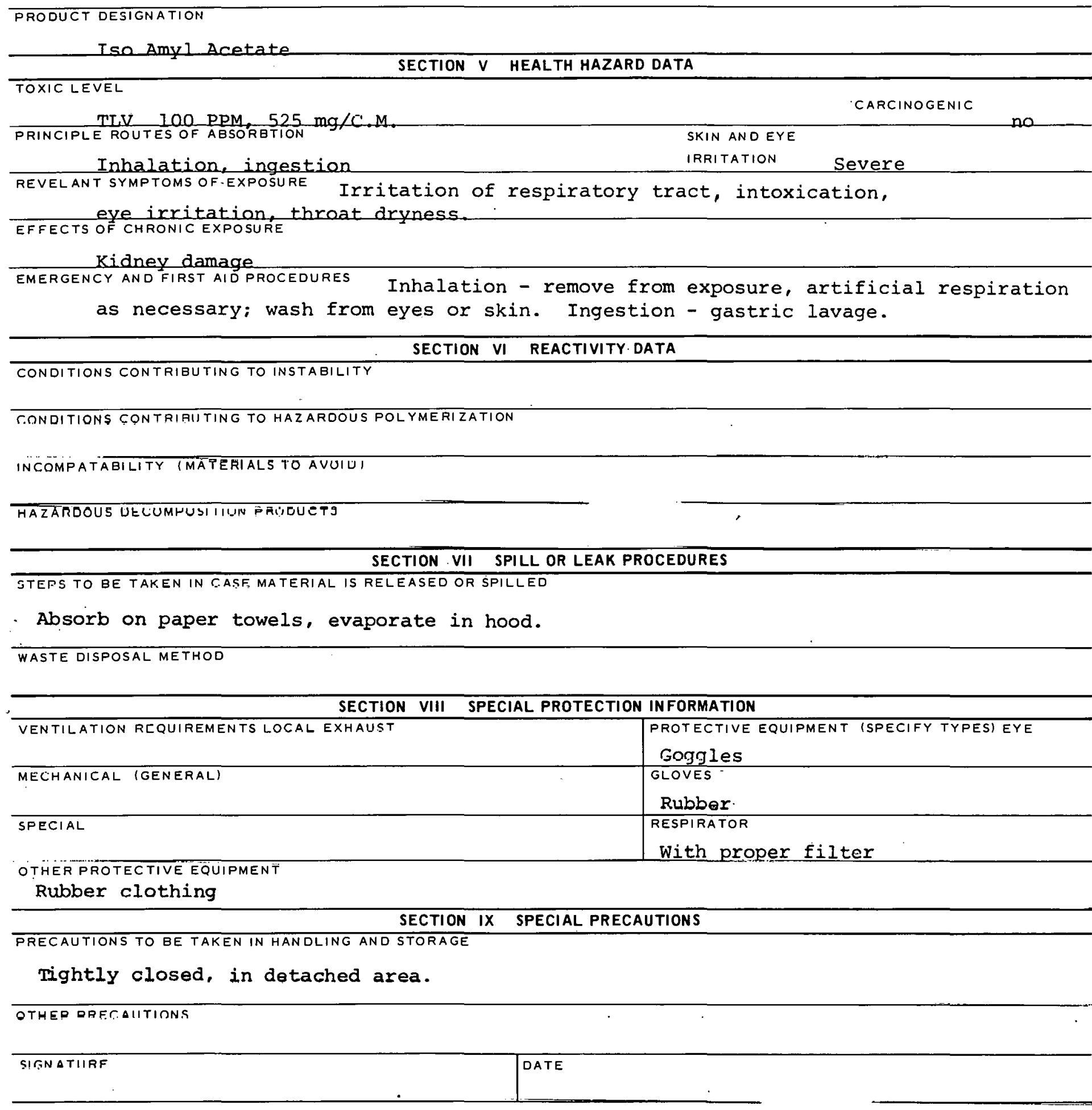




\begin{tabular}{|l} 
PRODUCT DESIGNATION \\
n-Amyl Acetate \\
$03-212-5104$
\end{tabular}

\section{MATERIAL SAFETY DATA SHEET}

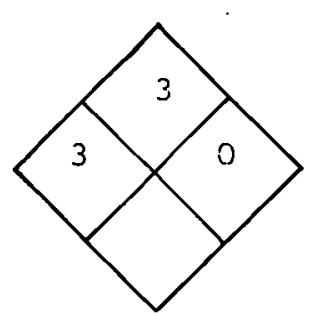

\section{SECTION I SOURCE AND NOMENCLATURE}

SECTION I SOURCE AND NOMENCLATURE
\begin{tabular}{l|l|l}
\hline MANUFACTURER'SNAME & EMERGENCY TELEPHONE NO. \\
\hline ADDRESS (NUMBER, STREET, CITY, STATE, ZIP CODE)
\end{tabular}

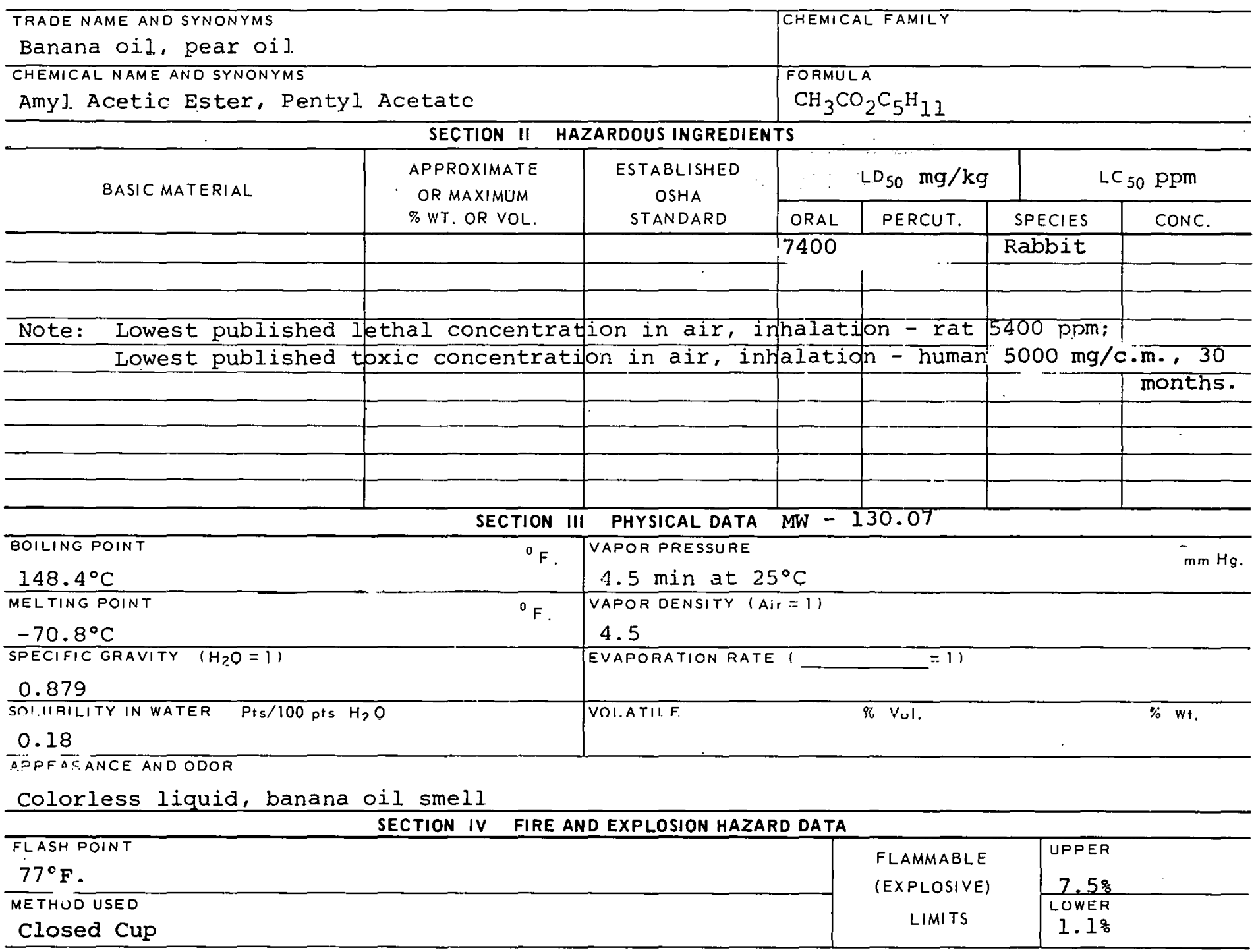

EXTINGUISHING MEDIA

Alcohol foam, carbon dioxide, dry chemical

SPECIAL FIRE FIGHTING PROCEDURES

Water may be ineffective

UNUSUAL FIRE AND EXPLOSION HAZARDS 


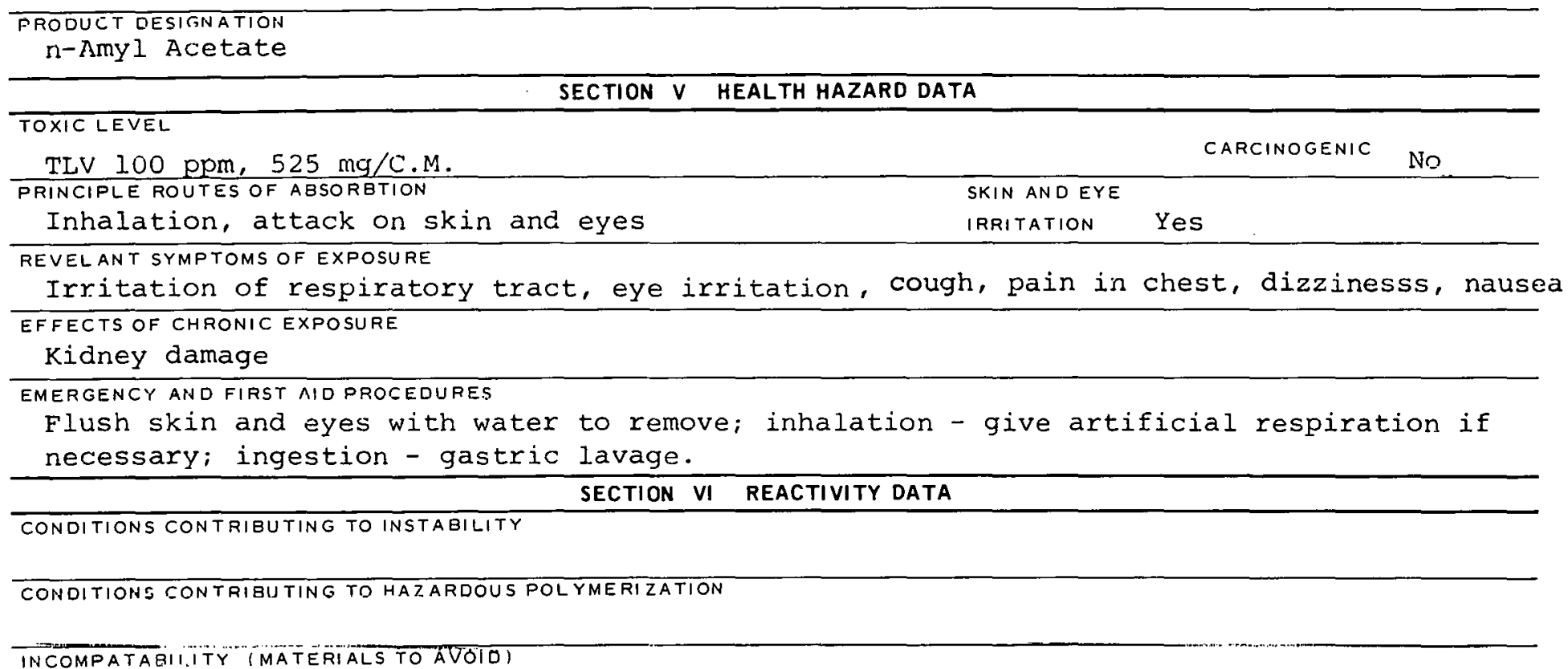
HALARDOUS DECOMPOSITION HRULULI'

SECTION VII SPILL OR LEAK PROCEDURES

STEPS TO BE. TAKEN IN CASE MATERIAL IS RELEASED OR SPILLED

Absorb on paper - burn paper in well-ventilated area. WASTE DISPOSAL METHOD $\rightarrow$

\begin{tabular}{l|l}
\hline \multicolumn{2}{|c}{ SECTION VIII SPECIAL PROTECTION INFORMATION } \\
\hline $\begin{array}{l}\text { VENTILATION REQUIREMENTS LOCAL EXHAUST } \\
\text { USE In WElI ventilated roOm }\end{array}$ & $\begin{array}{l}\text { PROTECTIVE EQUIPMENT (SPECIFY TYPESI EYE } \\
\text { Safety gOggles }\end{array}$ \\
\hline MECHANICAL IGENERAL) & $\begin{array}{c}\text { SI OVES } \\
\text { Rubber }\end{array}$ \\
\hline SFECIAL & $\begin{array}{c}\text { RESPIRATOR } \\
\text { Only in poor ventilation }\end{array}$ \\
\hline
\end{tabular}

OTHER PROTECTIVE EQUIPMENT

Rubber protective clothing

SECTION IX SPECIAL PRECAUTIONS

PRECAUTIONS TO BE TAKEN IN HANDLING AND STORAGE

Store large quantities outdoors. Store drums in a cool, well-ventilated area. OTHEK PRECAUTIONS 
Aniline

03-213-2005

\section{MATERIAL SAFETY DATA SHEET}

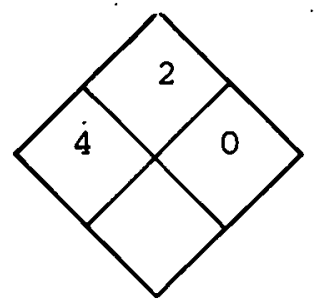

SECTION I SOURCE AND NOMENCLATURE

MANUFACTURER'S NAME
AODRESS (NUMBER, STREET, CITY, STA
TRADE NAME AND SYNONYMS
BEnZenamine
CHEMICAL NAME AND SYNONYMS
Aminobenzene, PhenYlamin

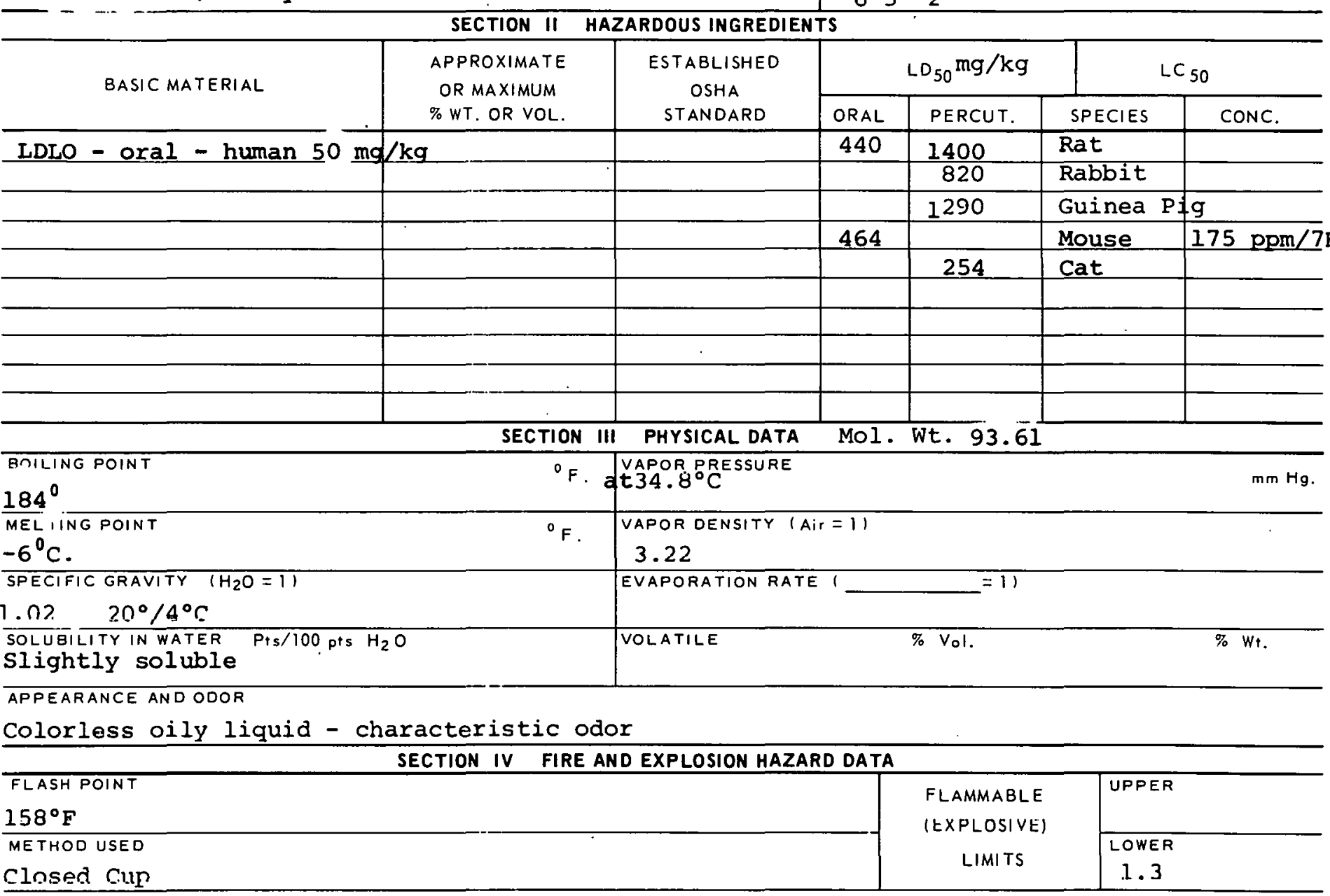

EXTINGUISHING MEDIA

Water spray, fog, carbon dioxide, alcohol foam

SPECIAL FIRE FIGHTING PROCEDURES

None.

UNUSUAL FIRE AND EXPLOSION HAZARDS

Vapor forms explosive mixtures in air. 


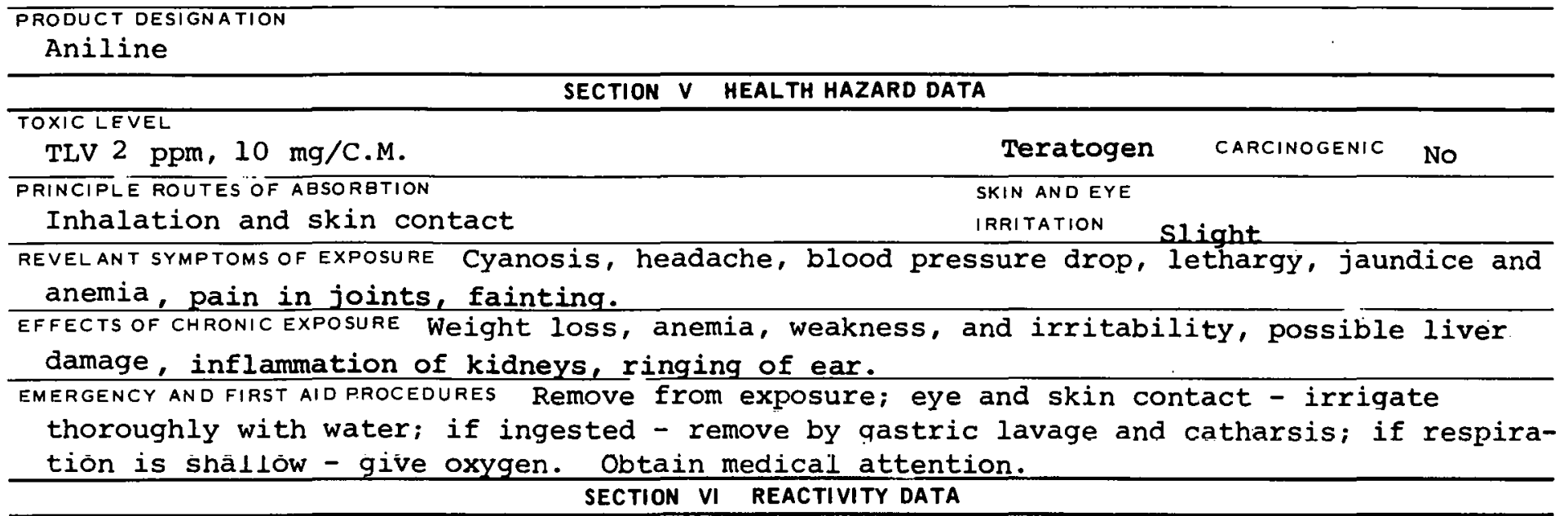

CONDITIONS CONTRIBUTING TO INSTABILITY

CONDITIONS CONTRIBUTING TO HAZARDOUS POLYMERIZATION

INCOMPATABILITY (MATERIALS TOAVOIO) SOdium or potassium peroxide, trichloromelamine, ozone. perchloric acid, formaldehyde, hexachloromelamine, nitric acid HAZARDOUS DECOMPOSITION PRODUCTS

SECTION VII SPILL OR LEAK PROCEDURES

STEPS TO BE TAKEN IN CASE MATERIAL IS RELEASED OR SPILLED

Wear protective clothing during cleanup operations; use water spray.

WASTE DISPOSAL METHOD

Burial - call Safety Department.

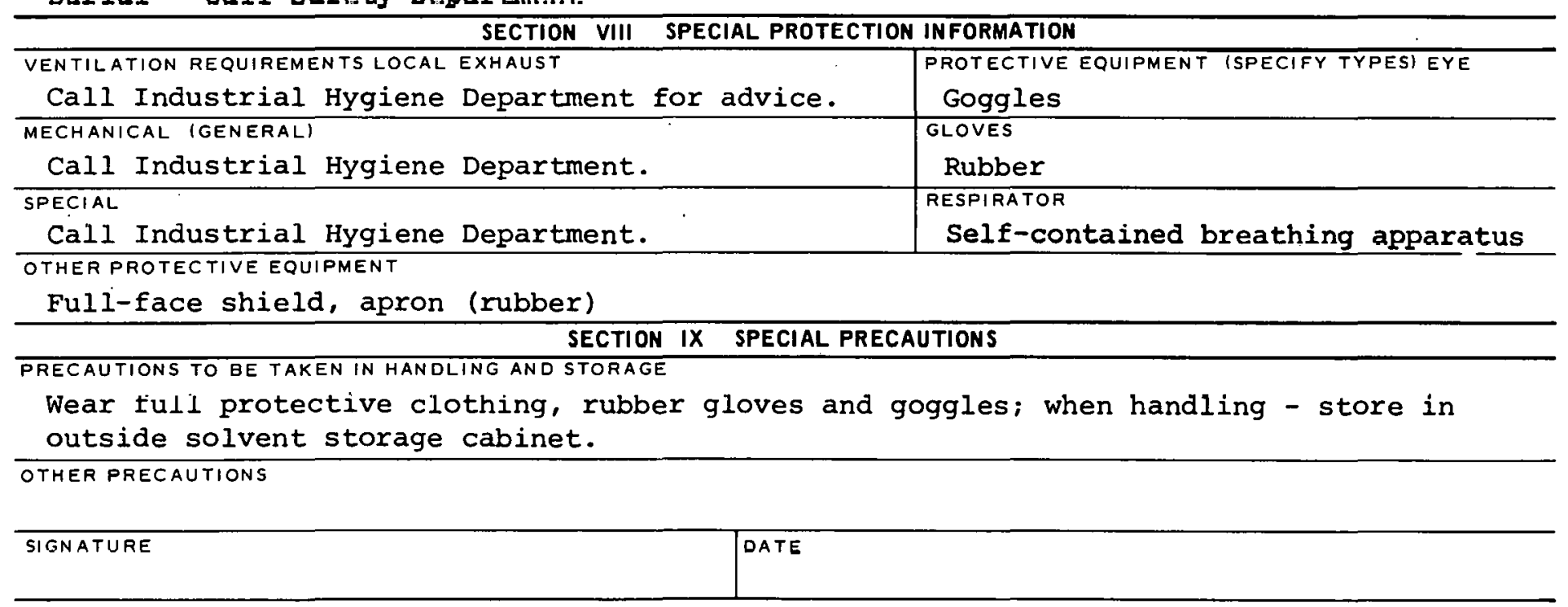


CAS. 000142041

PRODUCT DESIGNATION

03-213-2205

Aniline Hydrochloride

\section{MATERIAL SAFETY \\ DATA SHEET}

(Rev. Dec. 1979)

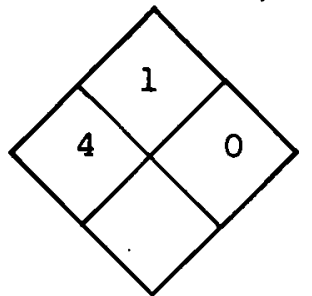

\section{SECTION I SOURCE AND NOMENCLATURE}

\begin{tabular}{l|l}
\hline MANUFACTURER'S NAME & EMERGENCY TELEPH \\
\hline ADORESS (NUMBER, STREET, CITY, STATE, ZIP CODE) & CHEMICAL FAMILY \\
\hline $\begin{array}{l}\text { TRADE NAME AND SYNONYMS } \\
\text { Aniline ChlOride, Benzenamine Hydrochloride }\end{array}$ & \\
\hline CHEMICAL NAME AND SYNONYMS & $\mathrm{C}_{6} \mathrm{H}_{5} \mathrm{NH}_{2} . \mathrm{H} \mathrm{Cl}$ \\
Aniline HYdrochloride
\end{tabular}

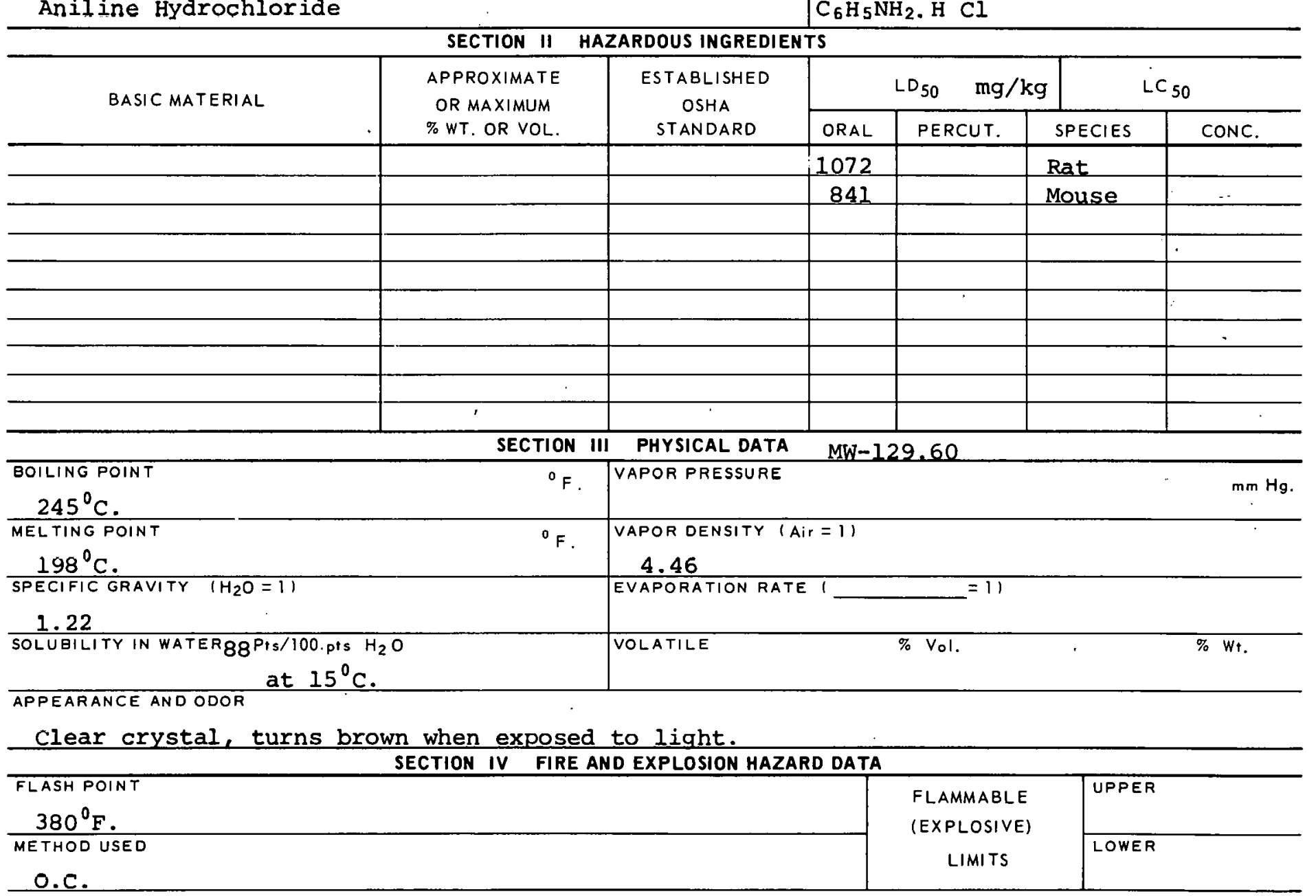

EXTINGUISHING MEDIA

Alcohol foam, dry chemical, carbon dioxide.

SPECIAL FIRE FIGHTING PROCEDURES

Self-contained gas mask, full protective clothing.

UNUSUAL FIRE AND EXPLOSION HAZARDS

Vapors of aniline and $\mathrm{H} \mathrm{Cl}$. 


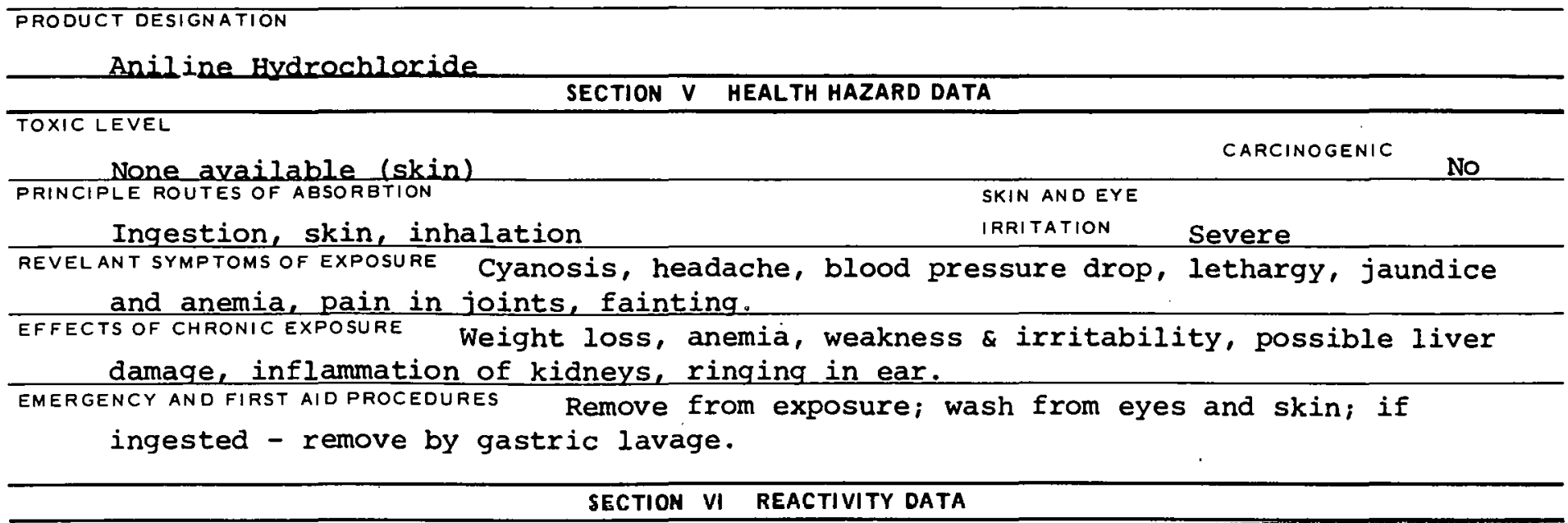

CUNUIIIUNS LUNIRIGUIING IU INSIABILIIY

CONDITIONS CONTRIBUTING TO HAZARDOUS POLYMERIZATION

INCOMPATAEILITY (MATERIALS TO AVOID)

Ozone, perchloric acid, sodium peroxide, potassium peroxide.

HAZARDOUS DECOMPOSITION PRODUCTS

SECTION VII SPILL OR LEAK PROCEDURES

STEPS TO BE TAKEN IN CASE MATERIAL IS RELEASED OR SPILLED

Wear protective clothing during clean ups.

WASTE DISPOSAL METHOD

SECTION VIII SPECIAL PROTECTION INFORMATION

\begin{tabular}{|c|c|}
\hline VENTILATION REQUIREMENTS LOCAL EXHAUST & $\begin{array}{l}\text { PROTECTIVE EQUIPMENT (SPECIFY TYPES) EYE } \\
\text { SaIECY GOYg Lēs }\end{array}$ \\
\hline MECHANICAL (GENERAL) & $\begin{array}{l}\text { GLOVES } \\
\text { Rubber }\end{array}$ \\
\hline SPECIAL & $\begin{array}{l}\text { RESPIRATOR } \\
\text { Self-contained }\end{array}$ \\
\hline
\end{tabular}

OTHER RROTECTIVE EQUITMENT

Full protective clothing.

SECTION IX SPECIAL PRECAUTIONS

PRECAUTIONS TO BE TAKEN IN HANDLING AND STOQRAGE

Preferred outside storage in solvent storage cabinet.

OTHER PRECAUTIONS

SIGNATURE

DATE

$213-2205 B$ 


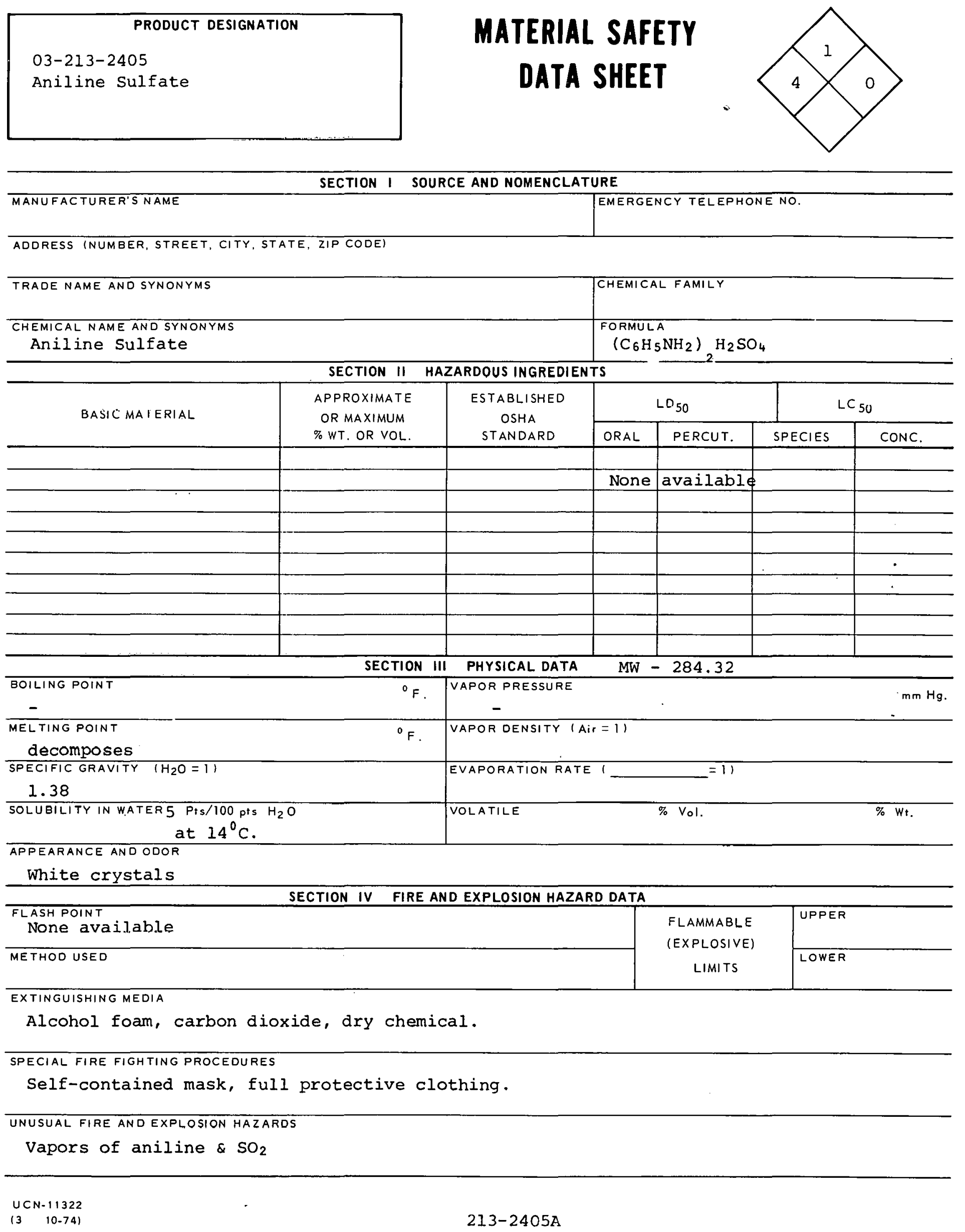




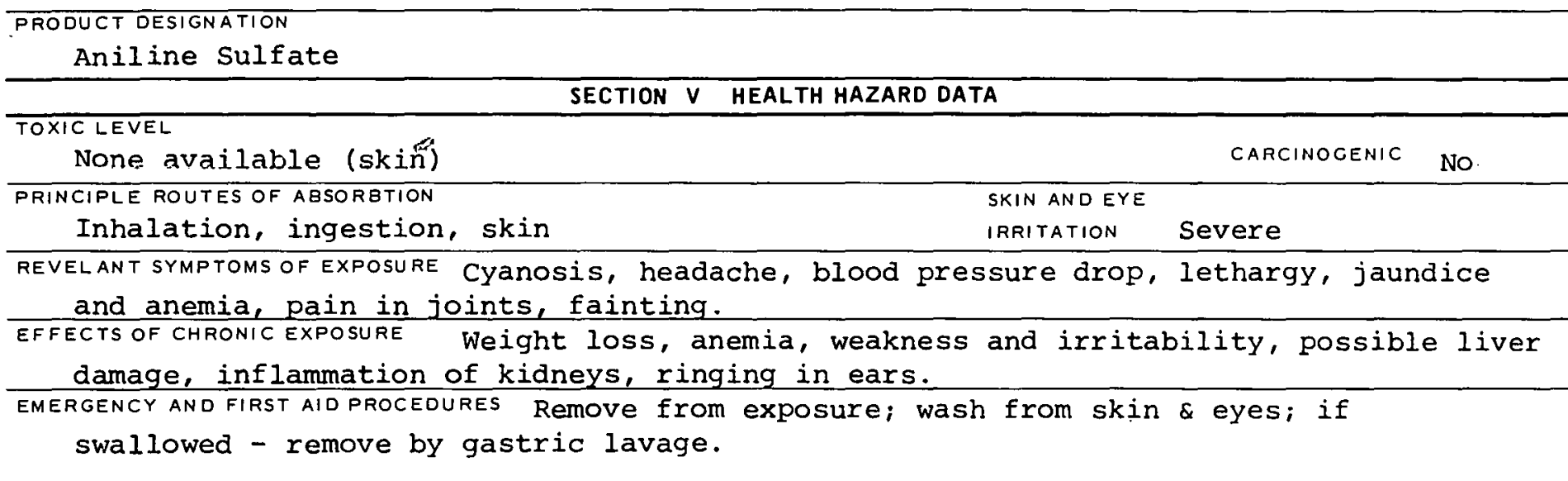

\begin{tabular}{ll}
\hline & SECTION VI REACTIVITY DATA \\
\hline CONDITIONS CONTRIBUTING TO INSTABILITY & \\
\hline CONDITIONS CONTRIBUTING TO HAZARDOUS POLYMERIZATION
\end{tabular}

CONDITIONS CONTRIBUTING TO HAZARDOUS POLYMERIZATION

INCOMPATABILITY (MATEREIALS TO AVOID) Ozone, perchloric acid, sodium peroxide, potassium peroxide.

SECTION VII SPILL OR LEAK PROCEDURES

STEPS TO BE TAKEN IN CASE MATERIAL IS RELEASED OR SPILLED

Wear full, protective clothing during cleanup. WASTE DISPOSAL METHOD

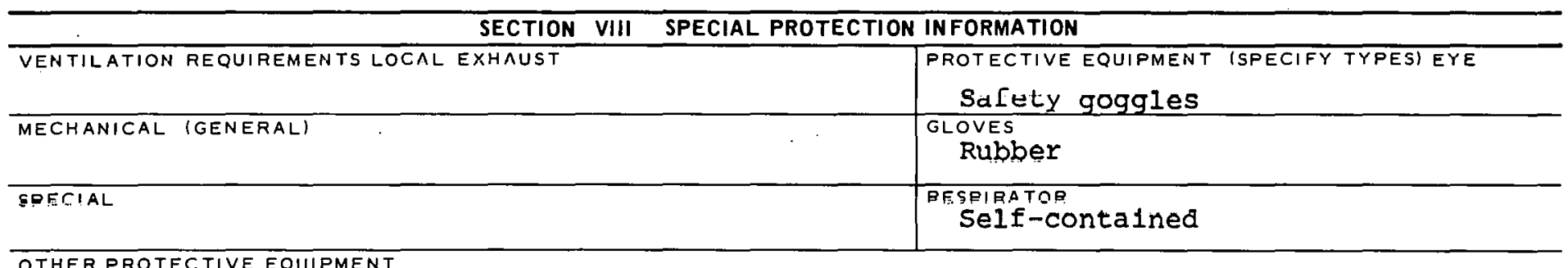
OTHERPROTECTIVE EQUIPMENT
Wear protective clothing.

\section{SECTION IX SPECIAL PRECAUTIONS}

PRECAUTIONS TO BE TAKEN IN HANDLING AND STORAGE
Preferred outside storage in solvent storage cabinet. OTHER PREG.AIJTIONS 


\begin{tabular}{|l|l|l|l|l}
\hline PRODUCT DESIGNATION \\
O-Anisidine \\
$0.3-213-3205$
\end{tabular}

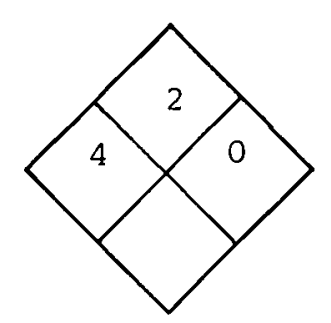

SECTION I SOURCE AND NOMENCLATURE

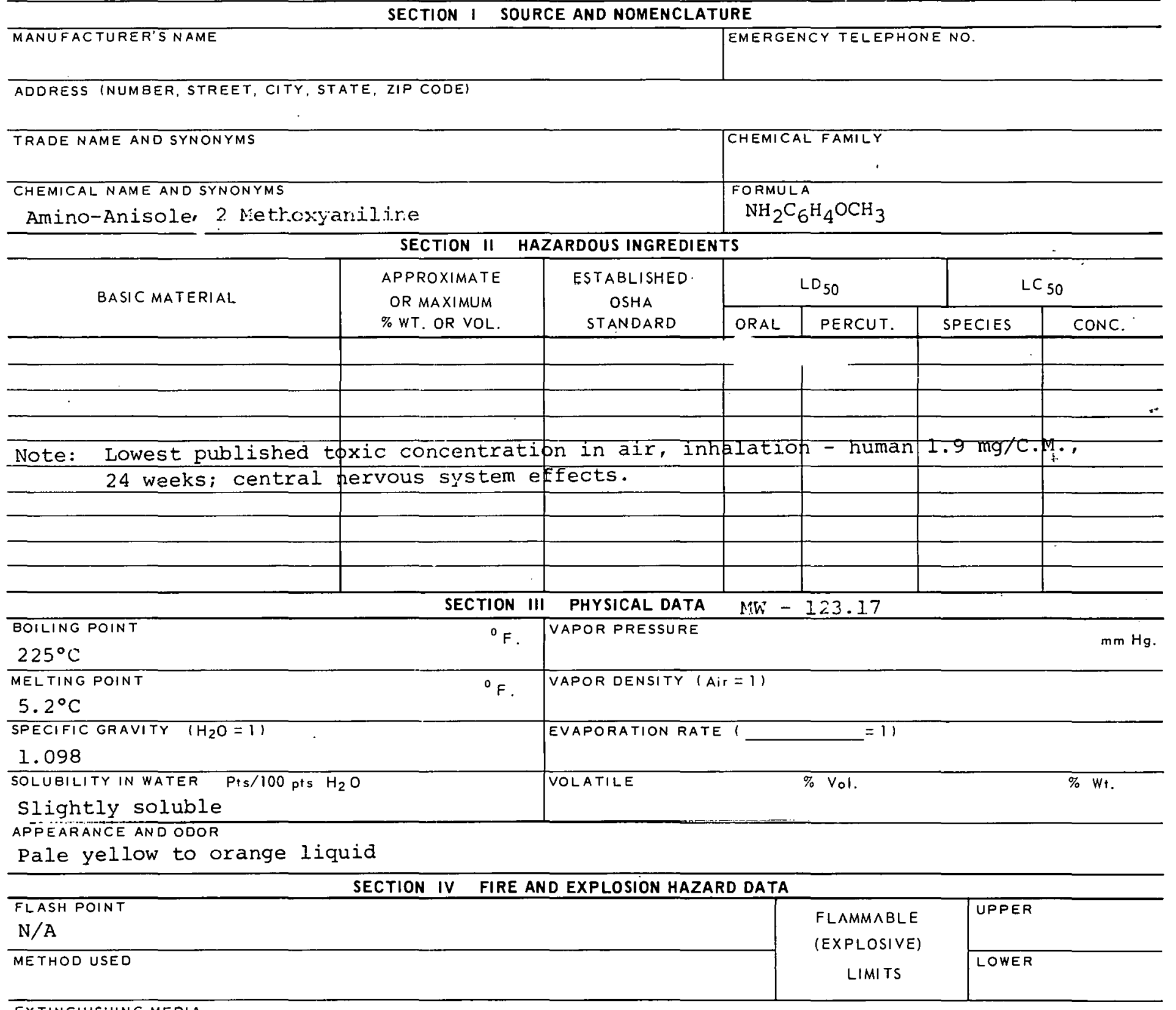

EXTINGUISHING MEOIA

Similar to aniline; water spray, alcohol foam, dry chemical

SPECIAL FIRE FIGHTING PROCEDURES

UNUSUAL FIRE ANO EXPLOSION HAZAROS

Emits toxic fumcs, when heated to the decomposition point. 


\section{TOXIC LEVEL}

TLV $0.1 \mathrm{pPm}, 0.5 \mathrm{mg} / \mathrm{C} . \mathrm{M}$. PRINCIPLE ROUTES OF ABSORBTION Inhalation, skin absorption REVELANT SYMPTOMS OF EXPOSURE Headache, drowsiness, cyanosis, stir irritant
CARCINOGENIC

No

\section{EFFECTS OF CHRONIC EXPOSURE}

EMERGENCY AND FIRST AID PROCEDURES

Wash skin with soap and water, flush eyes with water; if swallowed, wash mouth with water and get medical attention.

\section{SECTION VI REACTIVITY DATA}

CONDITIONS CONTRIBUTING TO INSTABILITY

CONDITIONS CONTRIBUTING TO HAZARDOUS POLYMERIZATION

INCOMPATABILITY IMAIERIALS IU AVUIU I

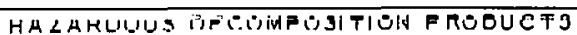

SECTION VII SPILL OR LEAK PROCEDURES

STEPS TO BE TAKEN IN CASE MATERIAL IS RELEASED OR SPILLED

WASTE DISPOSAL METHOD

SECTION VIII SPECIAL PROTECTION INFORMATION

VENTILATION REQUIREMENTS LOCAL EXHAUST

UJC in hood

MECHANICAL (GENERAL)

SOERIAI

OTHER PROTECTIVE EQUIPMENT

Neoprene protective clothing

SECTION IX SPECIAL PRECAUTIONS
PROTECTIVE EQUIPMENT (SPECIFY TYPES) EYE

Eafcty gogglco

GLOVES

Rubber gloves (butyl)

RESPIRATOR

SeLf-cortained breathjing apparetus

SECTION IX SPECIAL PRECAUTIONS


CAS : 000104949

B.5.5000

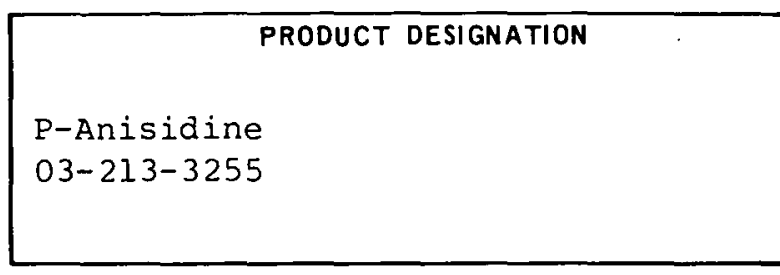

\section{MATERIAL SAFETY DATA SHEET}

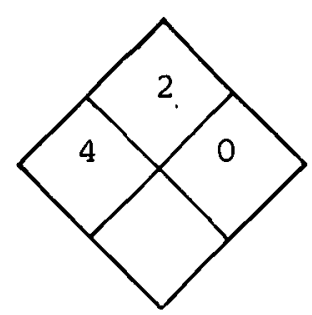

SECTION I SOURCE AND NOMENCLATURE

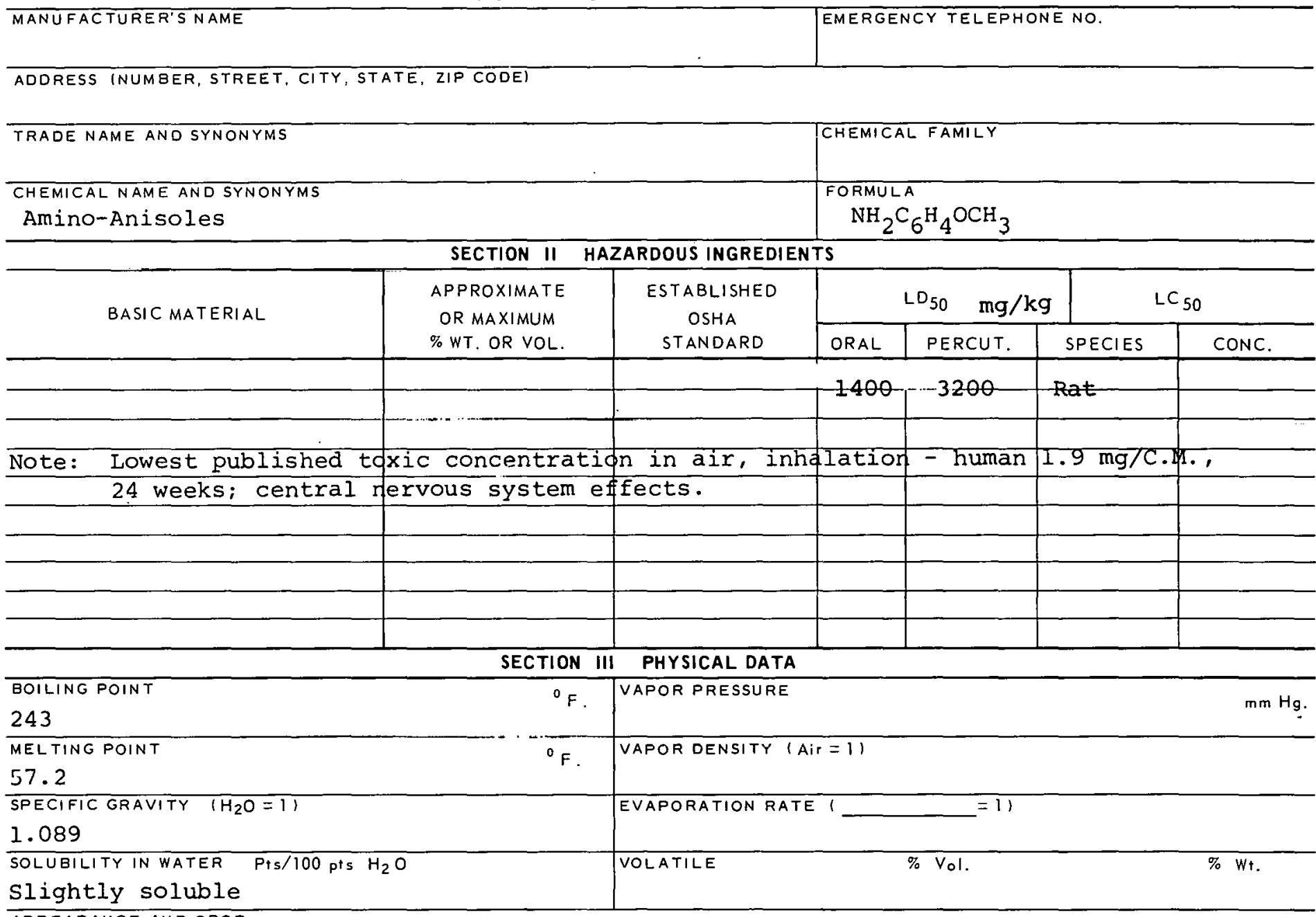

APPEARANCE AND ODOR

Pale yellow to brown solid

SECTION IV FIRE AND EXPLOSION HAZARD DATA

FLASH POINT

Not available

METHOD USED

\begin{tabular}{l|l}
\hline FLAMMABLE \\
\hline
\end{tabular}

(EXPLOSIVE)

LIMITS

UPPER

EXTINGUISHING MEDIA

Similar to aniline; water spray, alcohol foam, dry chemical

SPECIAL FIRE FIGHTING PROCEDURES

UNUSUAL FIRE ANDEXPLOSION HAZARDS

Emits toxic fumes when heated to decompositiun puinl. 


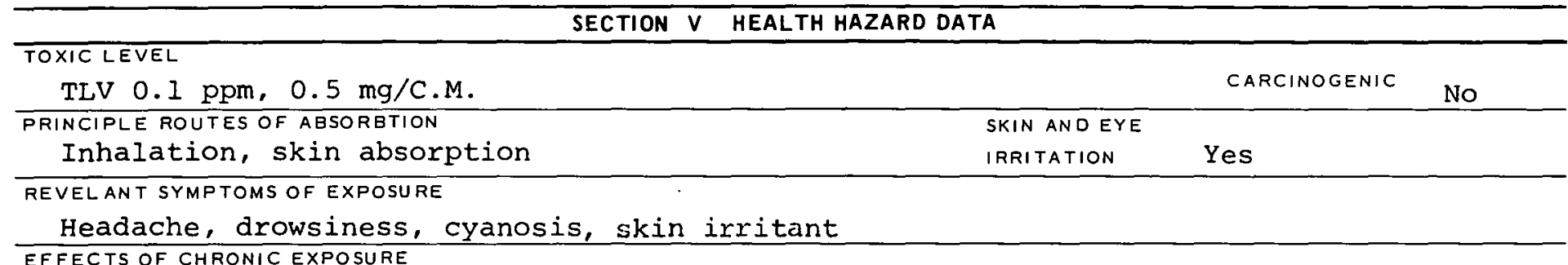
EFFECTS OF CHRONIC EXPOSURE

EMERGENCY AND FIRST AID PROCEDURES

Wash skin with soap and water, flush eyes with water, if swallowed, wash mouth with water and get medical attention.

\begin{tabular}{|c|c|}
\hline \multicolumn{2}{|c|}{ SECTION VI REACTIVITY'DATA } \\
\hline \multicolumn{2}{|l|}{ CONDITIONS CONTRIBUTING TO INSTABILITY } \\
\hline \multicolumn{2}{|l|}{. } \\
\hline \multicolumn{2}{|l|}{ CONDITIONS CONTRIBUTING TO HAZARDOUS POLYMERIZATION } \\
\hline \multicolumn{2}{|l|}{ IN COMPATARII ITY (MATFRIAI S TO $\triangle V O I \cap !$} \\
\hline \multicolumn{2}{|l|}{ HAZAROOUS DECOMPOSITION PRODUCTS } \\
\hline \multicolumn{2}{|c|}{ SECTION VII SPILL OR LEAK PROCEDURES } \\
\hline \multicolumn{2}{|l|}{$\begin{array}{l}\text { STEPS TO BE TAKEN IN CASE MA TERIAL IS RELEASED OR SPILLED } \\
\text { - }\end{array}$} \\
\hline \multicolumn{2}{|l|}{ WASTE DISPOSAL METHOD } \\
\hline SECTION VIII SPECI & IL PROTECTION IN FORMATION \\
\hline $\begin{array}{l}\text { VENTILATION REQUIREMENTS LOCAL EXHAUST } \\
\text { USe in hoOd }\end{array}$ & \begin{tabular}{l|l} 
& $\begin{array}{l}\text { PROTECTIVE EOUIPMENT (SPEC.IFY TYPES) EYE } \\
\text { Safety goggles }\end{array}$
\end{tabular} \\
\hline MECHANICAL (GENERAL) & $\begin{array}{l}\text { GLOVES } \\
\text { Rubber gloves }\end{array}$ \\
\hline SPECIAL & $\begin{array}{l}\text { RESPIRATOR } \\
\text { Selt-contained breathing apparatus }\end{array}$ \\
\hline
\end{tabular}

\section{SECTION IX SPECIAL PRECAUTIONS}

PRECAUTIONS TO BE TAKEN IN HANDLING AND STORAGE 


\begin{tabular}{|l|}
\hline \multicolumn{1}{|c|}{ PRODUCT DESIGNATION } \\
$03-213-4005$ \\
Anthracene
\end{tabular}

\section{MATERIAL SAFETY DATA SHEET}

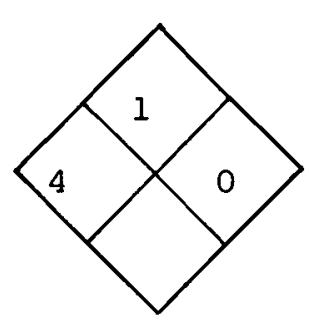

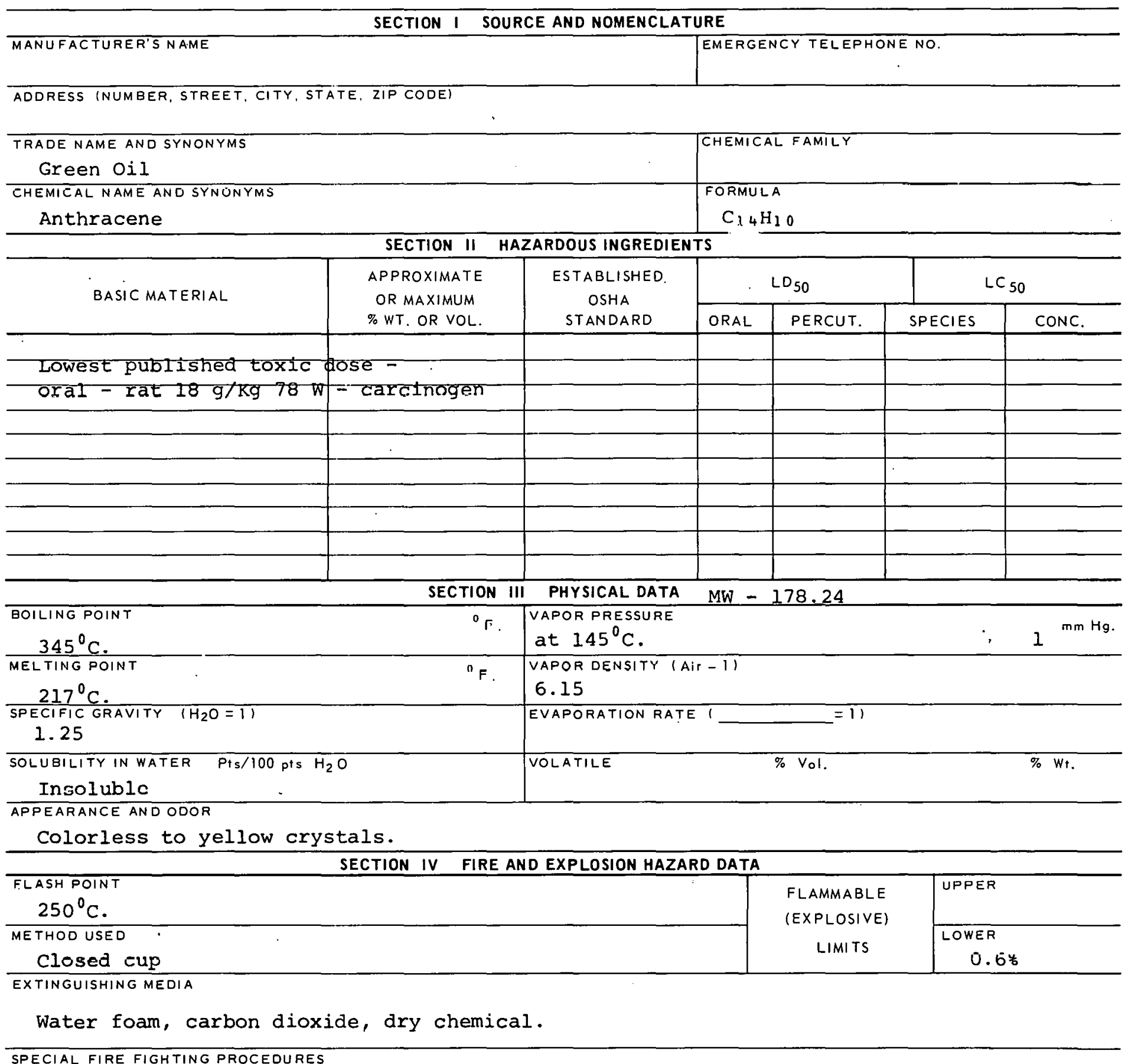

SPECIAL FIRE FIGHTING PROCEOURES 


\section{TOXIC LEVEL \\ None available}

PRINCIPLE ROUTES OF ABSORBTION Inhalation, ingestion
CARCINOGENIC YES

SKIN ANDEYE
IRRITATION YES

REVELANT SYMPTOMS OF EXPOSURE Irritation of eyes and respiratory tract - if ingested headache, gastrointestinal symptoms.

EFFECTS OF CHRONIC EXPOSURE

May give cancer

EMERGENCY AND FIRST AID PROCEDURES

Wash eyes and skin; if ingested - gastric lavage, get medical aid; inhalation - rest.

\section{SECTION VI REACTIVITY DATA}

CONDITIONS CONTRIBUTING TO INSTABILITY

CONDITIONS CONTRIBUTING TO HAZARDOUS POLYMERIZATION

INCUMHA IAUILII' IMATERIALSTO A'VOIDI

TAEAROOUS DECOHROCITIOH RTODUETE

\section{SECTION VII SPILL OR LEAK PROCEDURES}

STEPS TO BE TAKEN IN CASE MATERIAL IS RELEASEO OR SPILLED

WASTE DISPOSAL METHOD

SECTION VIII SPECIAL PROTECTION INFORMATION

\begin{tabular}{|c|c|}
\hline VENTILATION REQUIREMENTS LOCAL EXHAUST & $\begin{array}{l}\text { PROTECTIVE EQUIPMEN I (SHECIFY TYPES) EYE } \\
\text { GOqQleS }\end{array}$ \\
\hline MAFHANICAI (FIFNFRAI) & $\begin{array}{r}\text { GLQYES } \\
\text { Rubber }\end{array}$ \\
\hline GPFRIAI & $\begin{array}{l}\text { RESPIRATOR } \\
\text { Chemical cartridge }\end{array}$ \\
\hline
\end{tabular}

OTHER PROTECTIVE EQUIPMENT

SECTION IX SPECIAL PRECAUTIONS

PRECAUTIONS TO BE TAKEN IN HANDLING AND STORAGE 
CAS: 000103333

CN14000

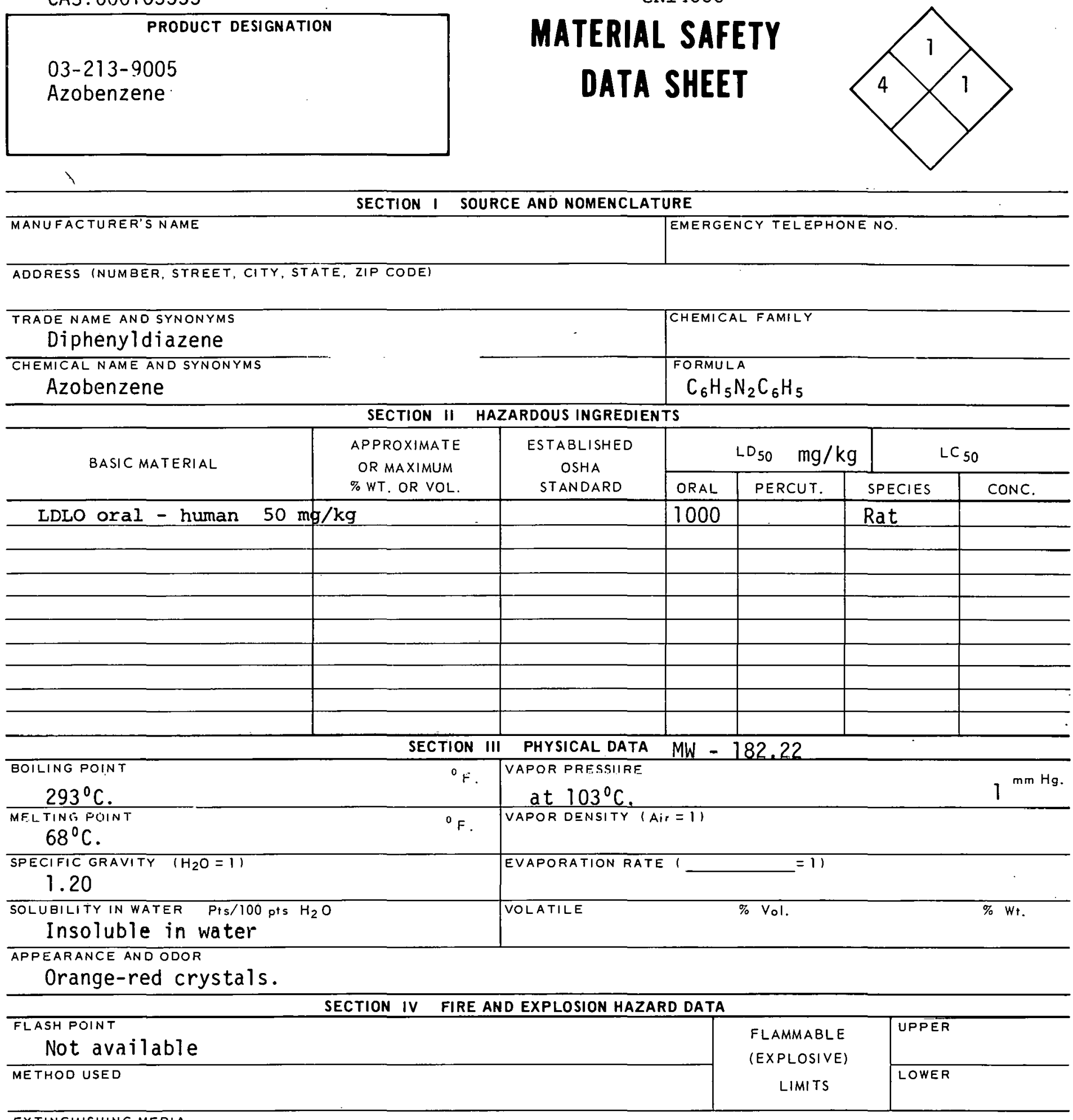

EXTINGUISHING MEDIA

Not specified.

SPECIAL FIRE FIGHTING PROCEDURES

UNUSUAL FIRE AND EXPLOSION HAZARDS 


\section{PROOUCT DESIGNATION \\ Azobenzene}

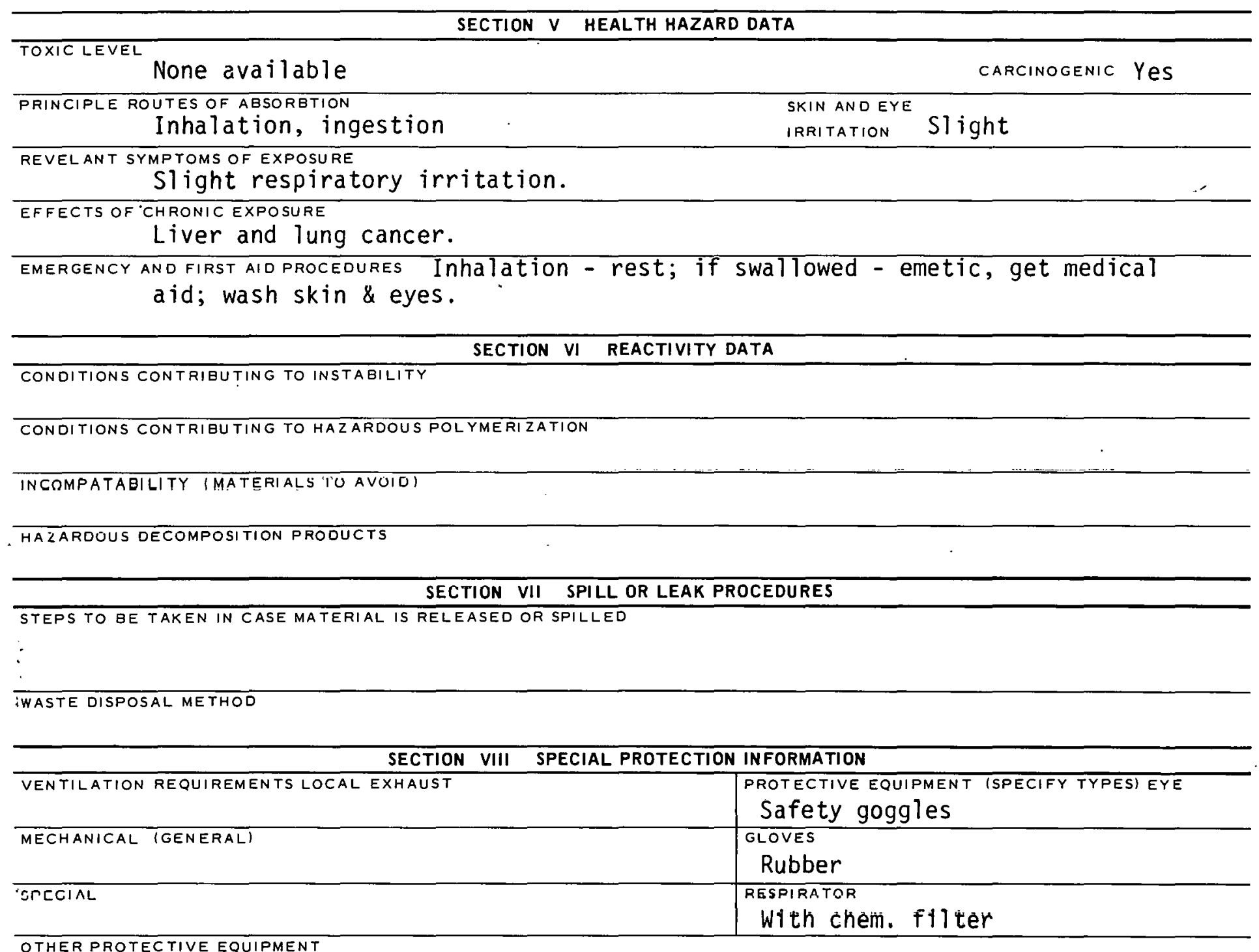

SECTION IX SPECIAL PRECAUTIONS

PRECAUTIONS TO BE TAKEN IN HANDLING AND STORAGE

OTHER PRECAUTIONS

\begin{tabular}{l|l}
\hline SIGNATURE & DATE \\
\hline
\end{tabular}

\begin{tabular}{|l|l}
\hline SIGNATURE & DATE \\
\hline
\end{tabular}

\section{3-9005B}


CAS - 000495487

PRODUCT DESIGNATION

03-213-9505

Azoxybenzene

\section{MATERIAL SAFETY \\ DATA SHEET}

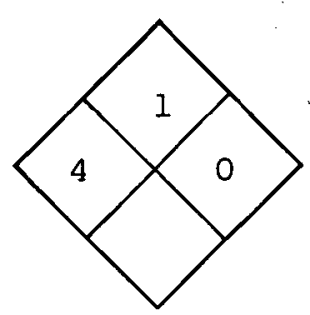

SECTION I SOURCE AND NOMENCLATURE

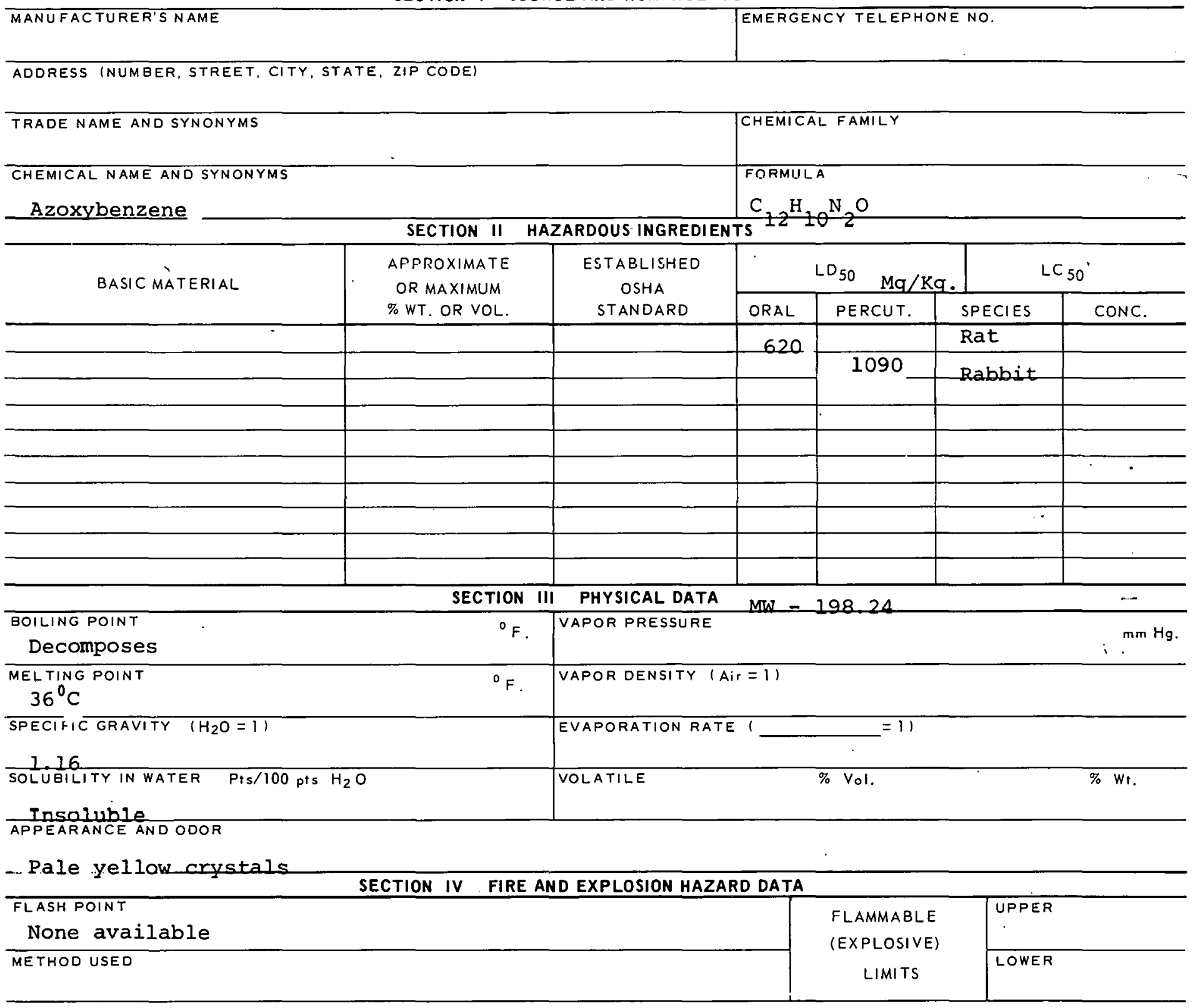

EXTINGUISHING MEDIA

Carbon dioxide, dry chemical

SPECIAL FIRE FIGHTING PROCEDURES

UNUSUAL FIRE AND EXPLOSION HAZARDS

on decomposition emits toxic fumes 


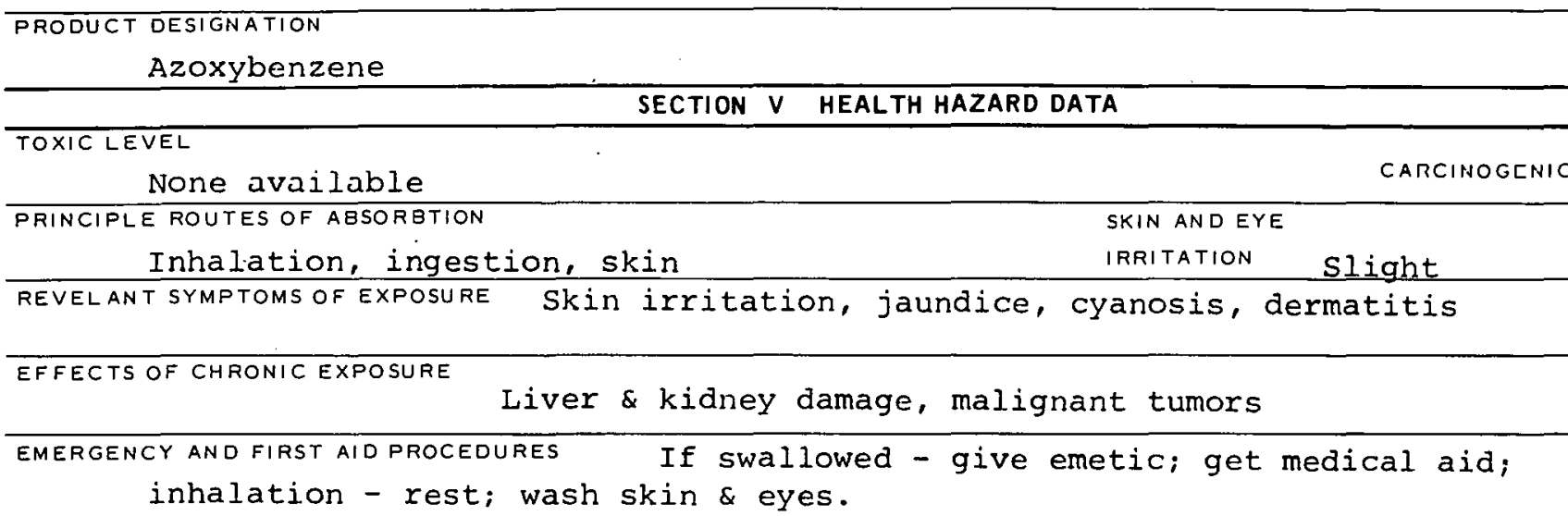




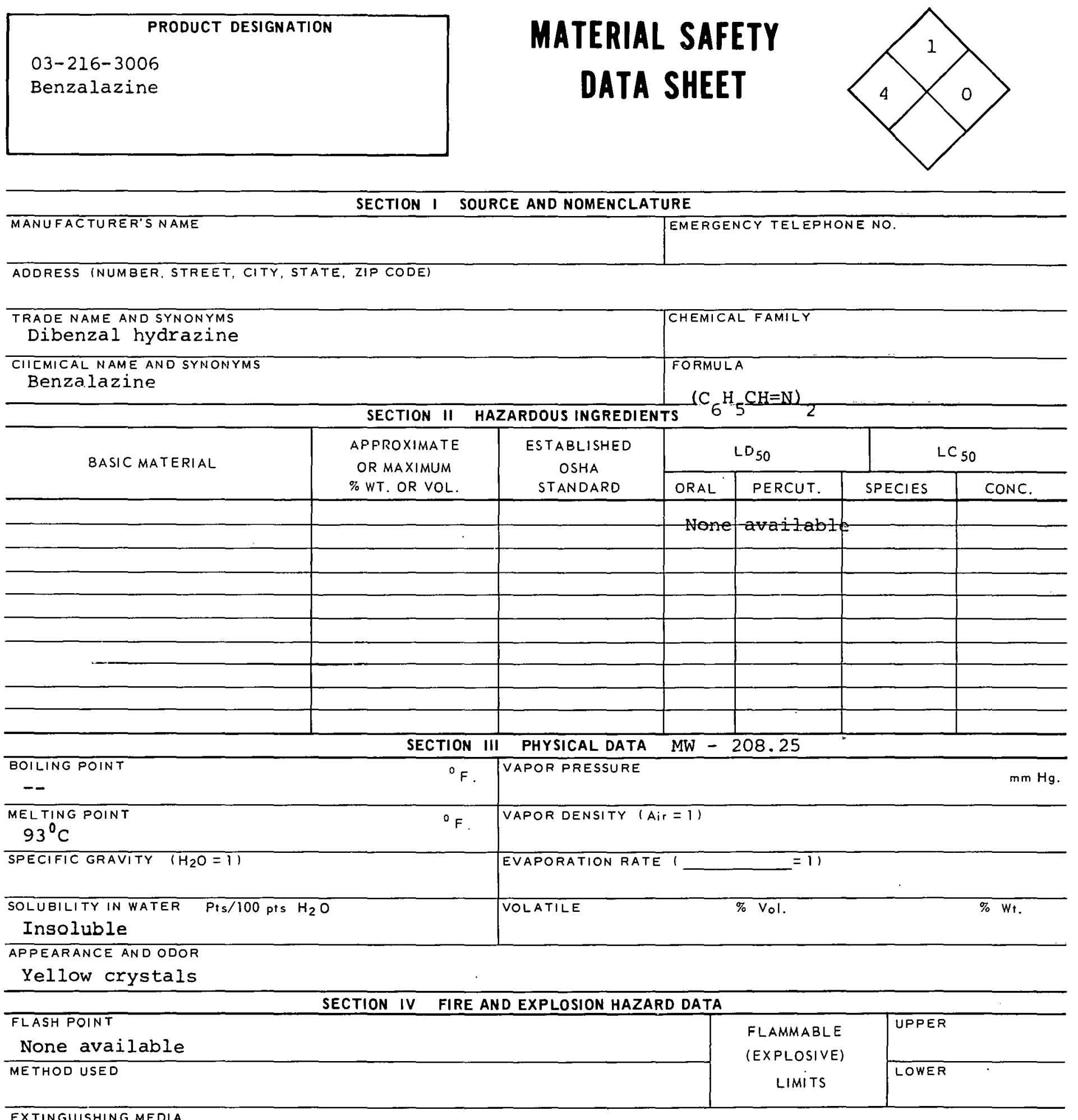

EXTINGUISHING MEDIA

Alcohol foam, carbon dioxide, dry chemical

SPECIAL FIRE FIGHTING PROCEDURES

UNUSUAL FIRE AND EXPLOSION HAZARDS

(3) $10-74) \quad 2.16-3006 \mathrm{~A}$ 


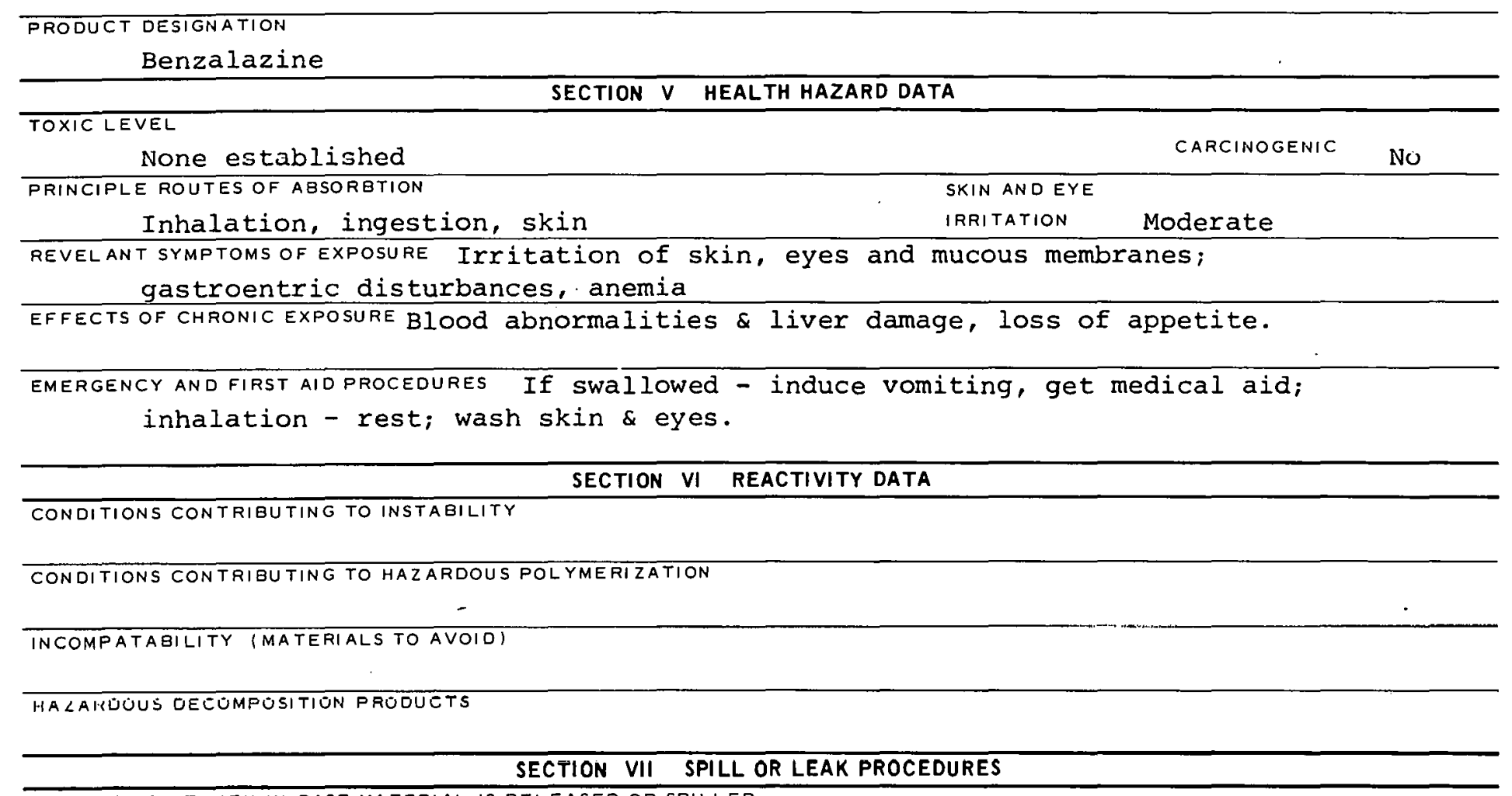

STEPS TO EE TAKEN IN CASE MATERIAL IS RELEASEO OR SPILLED

\section{WASTE DISPOSAL METHOD}

\section{SECTION VIII SPECIAL PROTECTION INFORMATION}

\begin{tabular}{|c|c|}
\hline VENTILATION REQUIREMENTS LOCAL EXHAUST & $\begin{array}{l}\text { PROTECTIVE EUUIPMEN I (SPECIFY TYPES) EYE } \\
\text { GÜYYlES }\end{array}$ \\
\hline MECHANICAL (GENERAL) & $\begin{array}{l}\text { GLOVES } \\
\text { Ruluber: } \\
\end{array}$ \\
\hline GCEGIAL & $\begin{array}{l}\text { RESTIRATOR } \\
\text { Self-contained }\end{array}$ \\
\hline
\end{tabular}

OTHER PROTECTIVE EQUIPMENT

\section{SECTION IX SPECIAL PRECAUTIONS}

PRECAUTIONS TO BE TAKEN IN HANDLING AND STORAGE

\begin{tabular}{l|l|l}
\hline OTHER DRETAUTIONS & \\
\hline SISANATIIRF & DATE \\
\hline
\end{tabular}




\begin{tabular}{|c|}
\hline $\begin{array}{l}\text { PRODUCT DESIGNATION } \\
03-216-6006 \\
1,2-\text { Benzanthracene }\end{array}$ \\
\hline
\end{tabular}

\section{SECTION I SOURCE AND NOMENCLATURE}

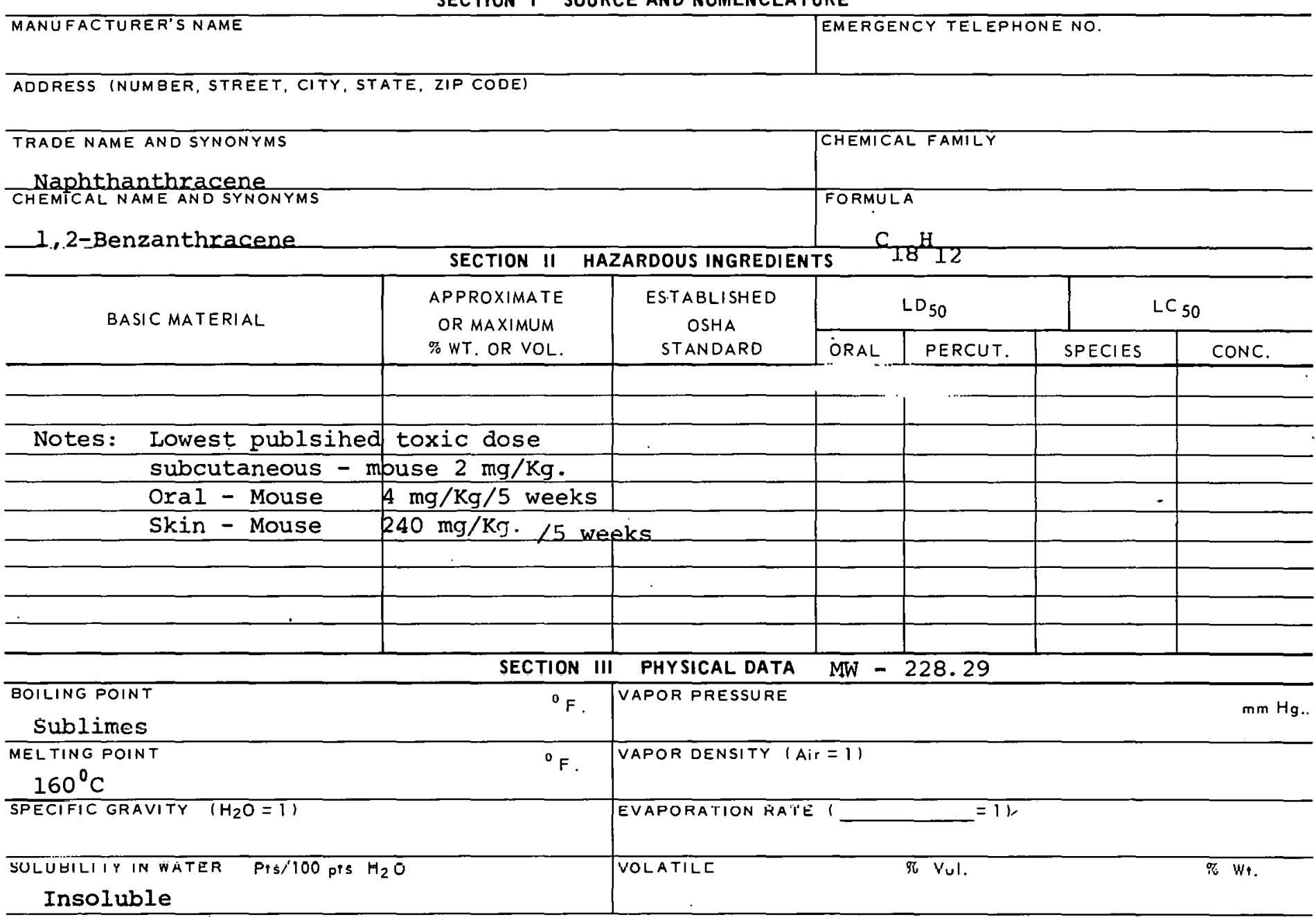

APPEARANCE ANO ODOR

SECTION IV FIRE AND EXPLOSION HAZARD DATA

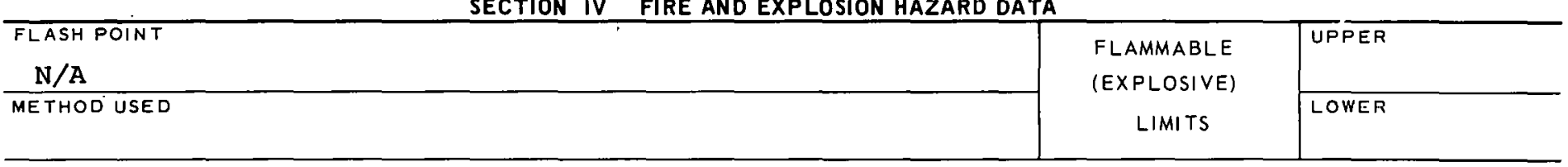

EXTINGUISHING MEUIA

Water, carbon dioxide, foam or dry chemical

SPECIAL FIRE FICHTING PROCEDURES

Wear goggles and self contained breathing apparatus. UNUSUAL FIRE AND EXPLOSION HATARTS 


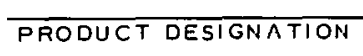




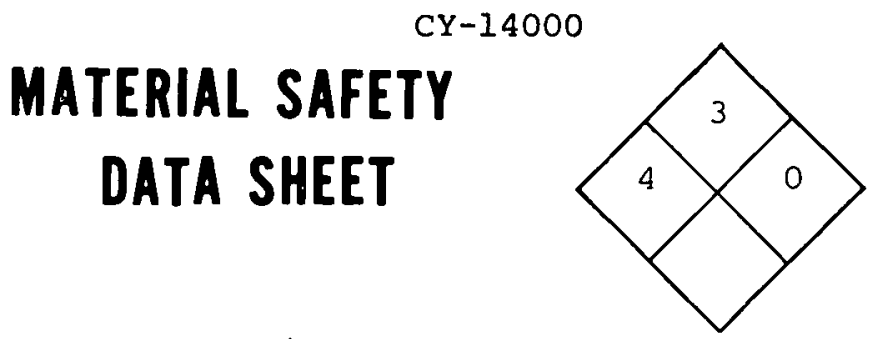

Benzene (Benzol)

03-216-7106

PRODUCT DESIGNATION

03-216-7006

SECTION I SOURCE AND NOMENCLATURE

\section{MANUFACTURER'S NAME

TRADE NAME AND SYNONYMS
BenzO1
CHEMICAL NAME AND SYNONYMS
PhenYl HYdride

ADDRESS (NUMBER, STREET, CITY, STATE, ZIP CODE)

EMERGENCY TELEPHONE NO.

BASIC MATERIAL

\begin{tabular}{l|l} 
& CHEMICAL FAMILY \\
& $\begin{array}{l}\text { FORMULA } \\
C_{6}{ }_{6}\end{array}$ \\
\hline SECTION II HAZARDOUS INGREDIENTS
\end{tabular}

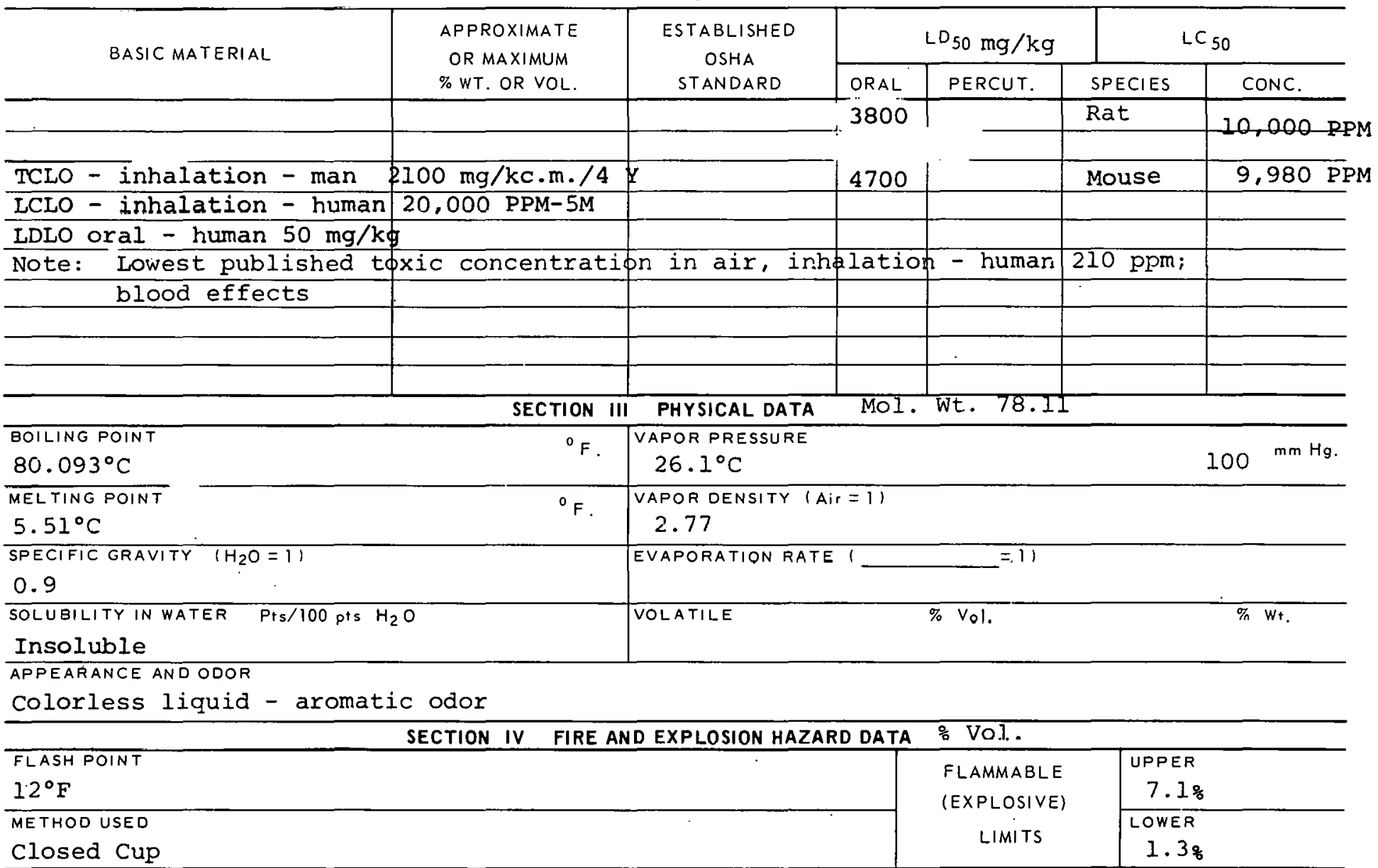

EXTINGUISHING MEDIA

Dry chemical, foam, or carbon dioxide
UNUSUAL FIRE AND EXPLOSION HAZARDS

Vapur forms explosive mistures with air. Flash back. 


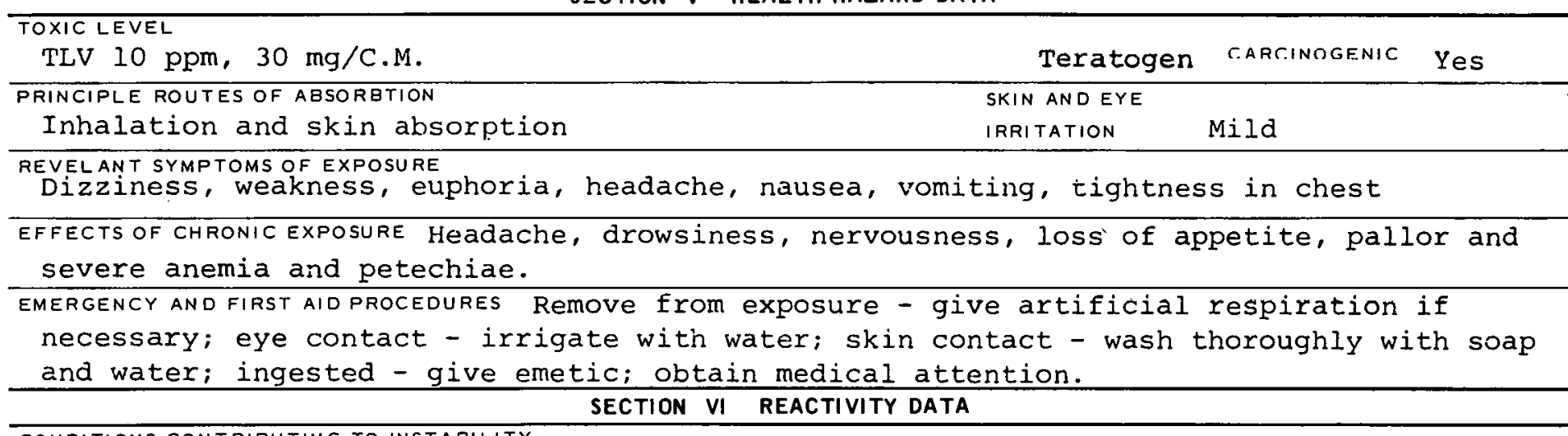

CONDITIONS CONTRIBUTING TO INSTABILITY

CONDITIONS CONTRIBUTING TO HAZARDOUS POLYMERIZATION

INCOMPATABILITY (MATERIALS TO AVOID) Chlorine, nitryl perchlorate, oxygen, ozone (perchloryl

fluoride and aluminum chloride), (permanganates and sulfuric acid), potassium peroxide, HAZARDOUS DECOMPOSITION PRODUCTS silver perchlorate, sodium peroxide.

\section{SECTION VII SPILL OR LEAK PROCEDURES}

STEPS TO BE TAKEN IN CASE MATERIAL IS RELEASED OR SPILLED

Uee water spray.

WASTE DISPOSAL METHOD

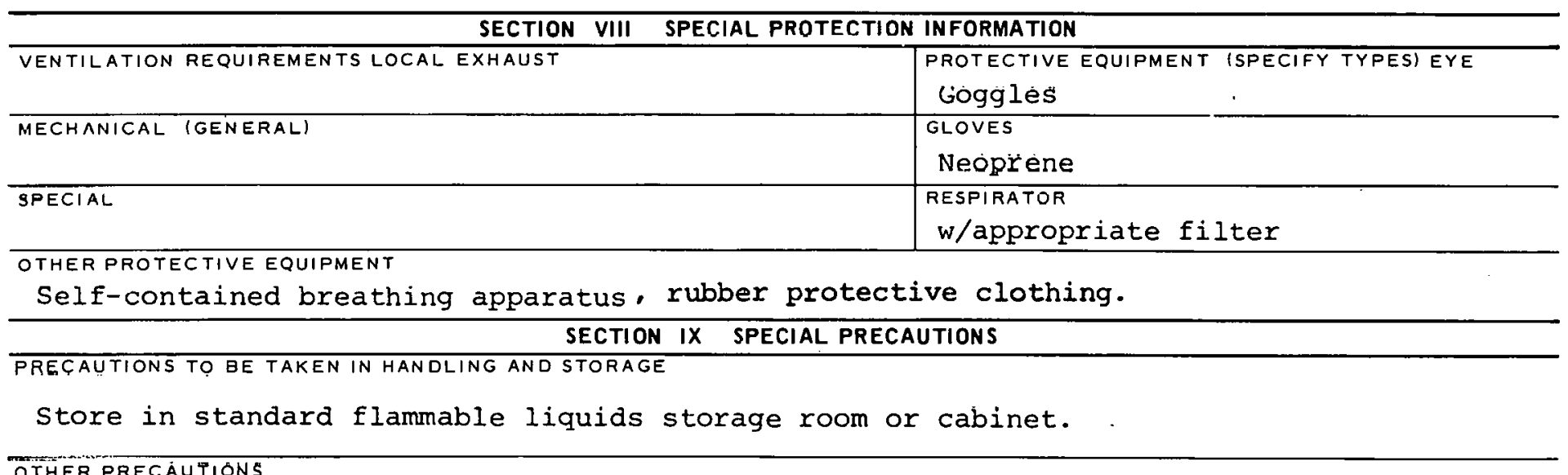
OTHER PRECAUTIONS 
CAS : 000531851

\section{PRODUCT DESIGNATION}

03-216-9146

Benzidine, Dihydrochloride

\section{MATERIAL SAFETY \\ DATA SHEET}

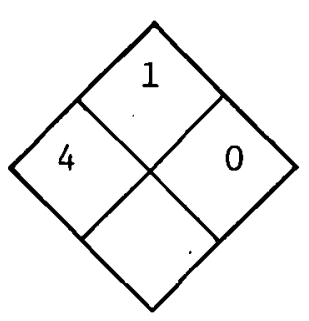

\section{SECTION I SOURCE AND NOMENCLATURE}

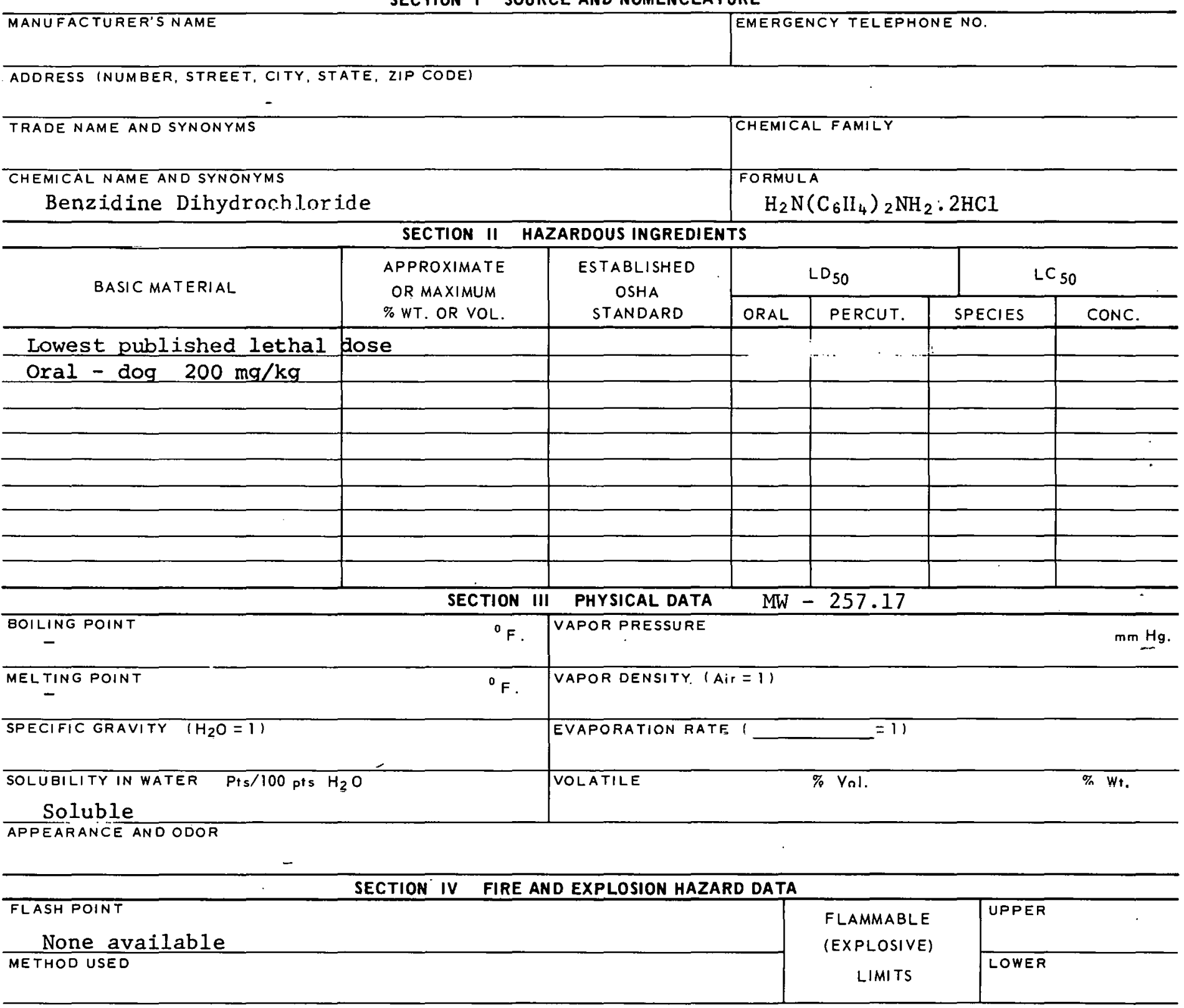

EXTINGUISHING MEDIA

Carbon dioxide, dry chemical.

SPECIAL FIRE FIGHTING PROCEDURES

UNUSUAL FIRE AND EXPLOSION HAZARDS 


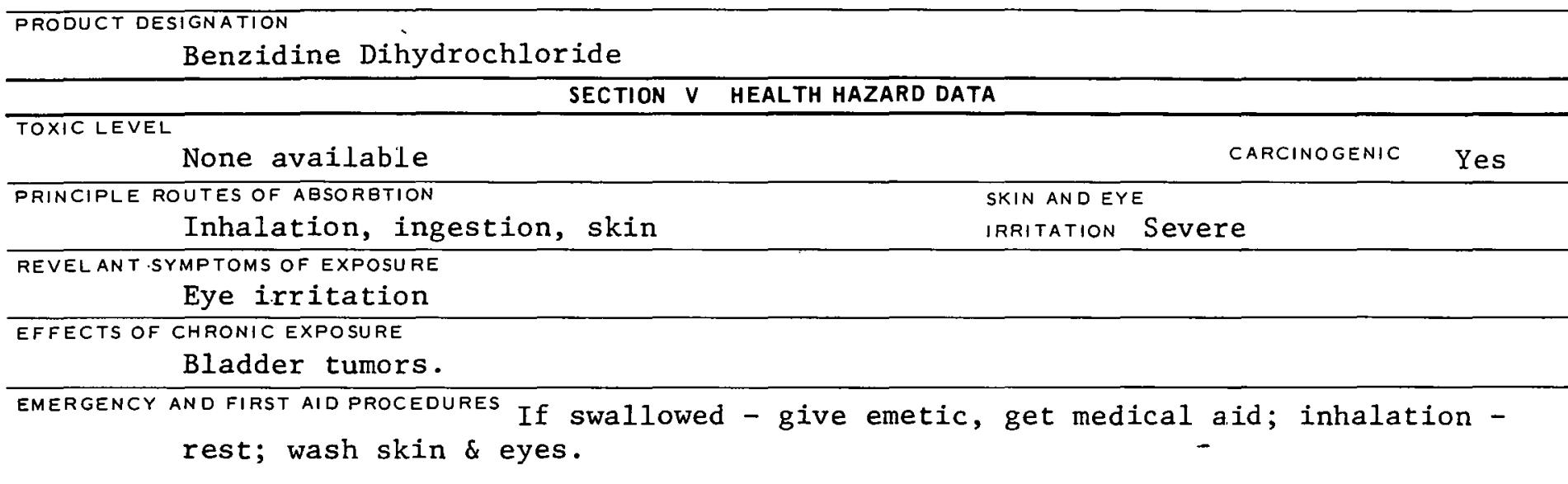

\begin{tabular}{l}
\hline SECTION VI REACTIVITY DATA \\
\hline CONDITIONS CONTRIBUTING TO INSTABILITY \\
\hline INCOMDITIONS CONTRIBUTING TO HAZARDOUS POLYMERIZATION \\
HAZARDOUS DECOMPOSITION PRODUCTS
\end{tabular}

SECTION VII SPILL OR LEAK PROCEDURES

STEPS TO BE TAKEN IN CASE MATERIAL IS RELEASED OR SPILLED

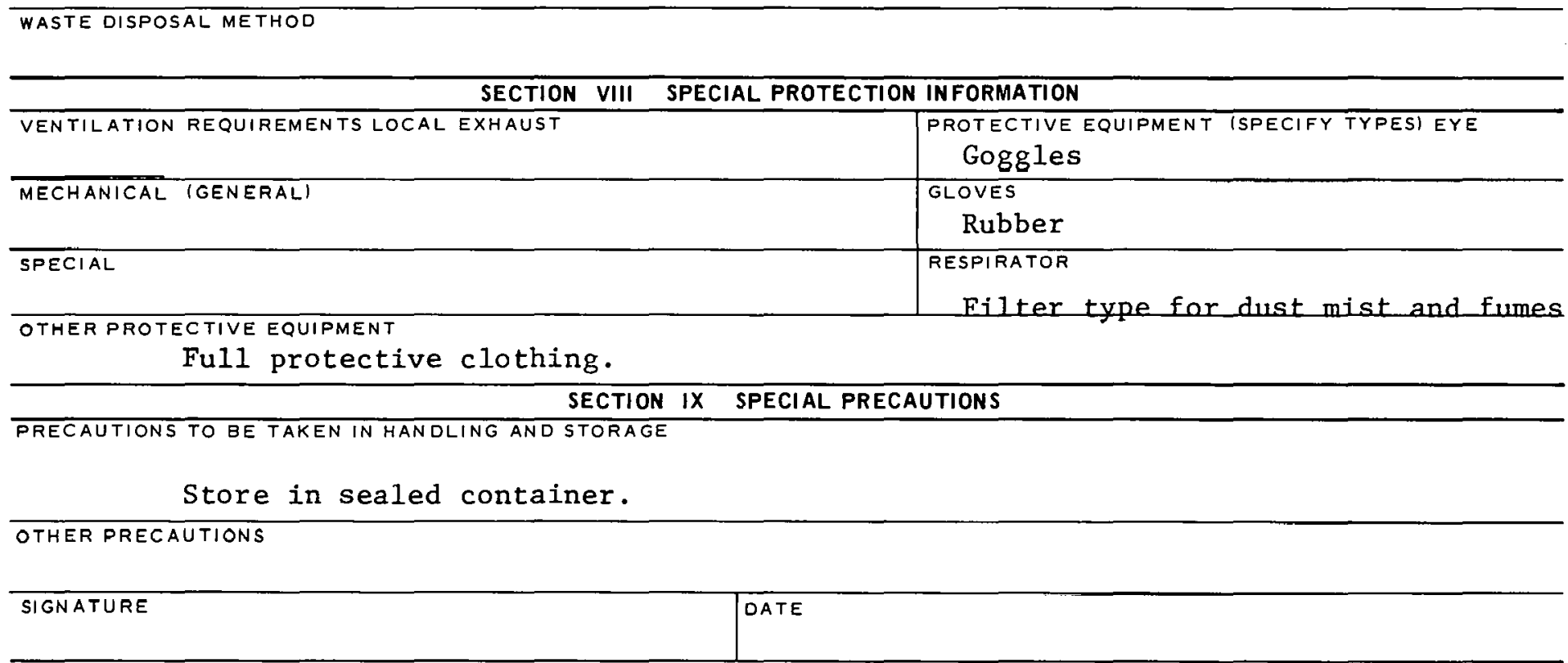



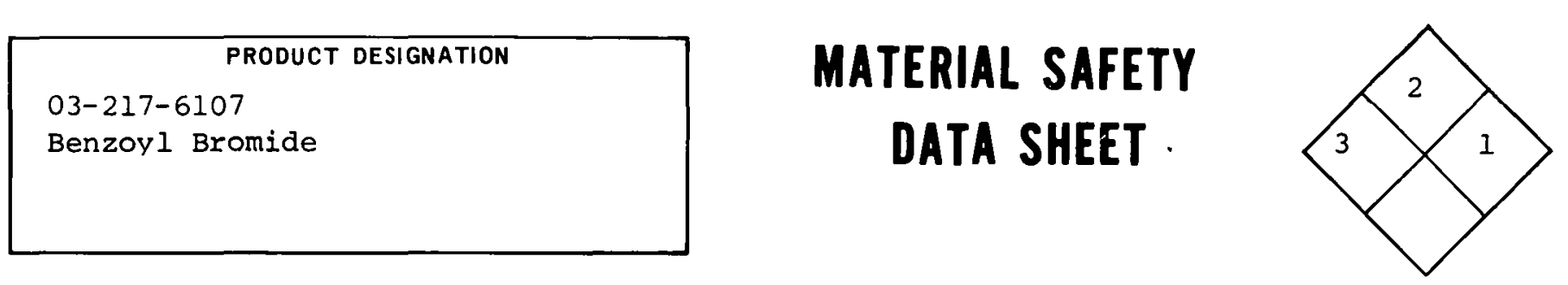

SECTION I SOURCE AND NOMENCLATURE

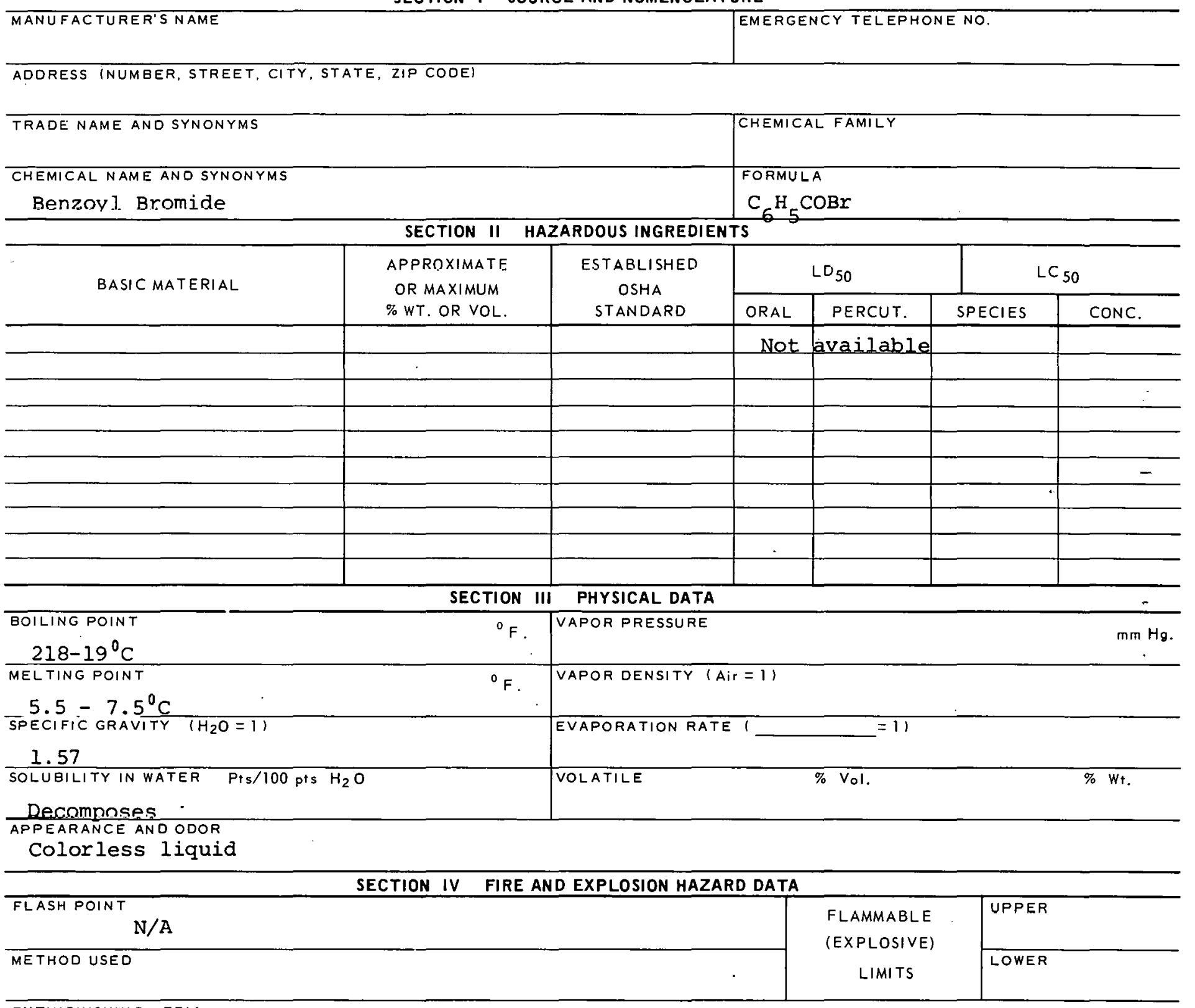

EXTINGUISHING MEDIA Use water spray sparingly, foam, carbon dioxide

or dry chemiral.

SPECIAL FIRE FIGHTING PROCEDURES

Wear full protective clothing.

UNUSUAL FIRE ANDEXPLOSION HAZARDS 


\section{TOXIC'LEVEL}

Not available PRINCIPLE ROUTES OF ABSORBTION

Inhalation, ingestion

REVELANT SYMPTOMS OF EXPOSURE Irritates respiratory system; burns skin, eyes, mouth and digestive system.

EFFECTS OF CHRONIC EXPOSURE

$--$

EMERGENCY AND FIRST AID PROCEDURES Inhalation - rest; get medical aid; if swallowed wash mouth, drink water, milk of magnesia; get medical. aid; wash skin \& eyes.

\section{SECTION VI REACTIVITY DATA}

CONDITIONS CONTRIBUTING TO INSTABILITY

Reacts with oxidizing materials

CONDITIONS CONTRIBU TING TO HAZARDOUS POLYMERIZATION

INCOMPATABILITY (MATERIALS IO AVUIU)

HALARLUUS BECOURFBSITIEN RUDUETg

SECTION VII SPILL OR LEAK PROCEDURES

STEPS TO BE TAKEN IN CASE MATERIAL IS RELEASED OR SPILLED

WASTE DISPOSAL METHOD

SECTION VIII SPECIAL PROTECTION INFORMATION

VENTILATION REQUIREMENTS LOCAL EXHAUST

MECHANICAL (GENERAL)

.

Sofrial

.

OTHER PROTECTIVE EQUIPMENT

SECTION IX SPECIAL PRECAUTIONS
PROTECTIVE EQUIPMENT (SPECIFY TYPES) EYE

safety goggles

GLOVES

Rubber

RESPIRATOR

SeIf centained

PRECAUTIONS TO BE TAKEN IN HANDLING AND STORAGE 
CAS : 000098884

DM66000

PRODUCT DESIGNATION

Benzoyl Chloride

03-217-7107

MATERIAL SAFETY

DATA SHEET

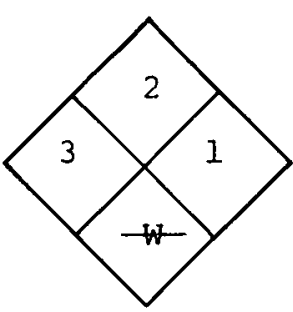

\section{SECTION I SOURCE AND NOMENCLATURE}

MANUFACTURER'S NAME
ADDRESS INUMBER, STREET, CITY. ST
TRADE NAME AND SYNONYMS
CHEMICAL NAME AND SYNONYMS
BENZYI CarbONYI ChlOride

EMERGENCY TELEPHONE NO.

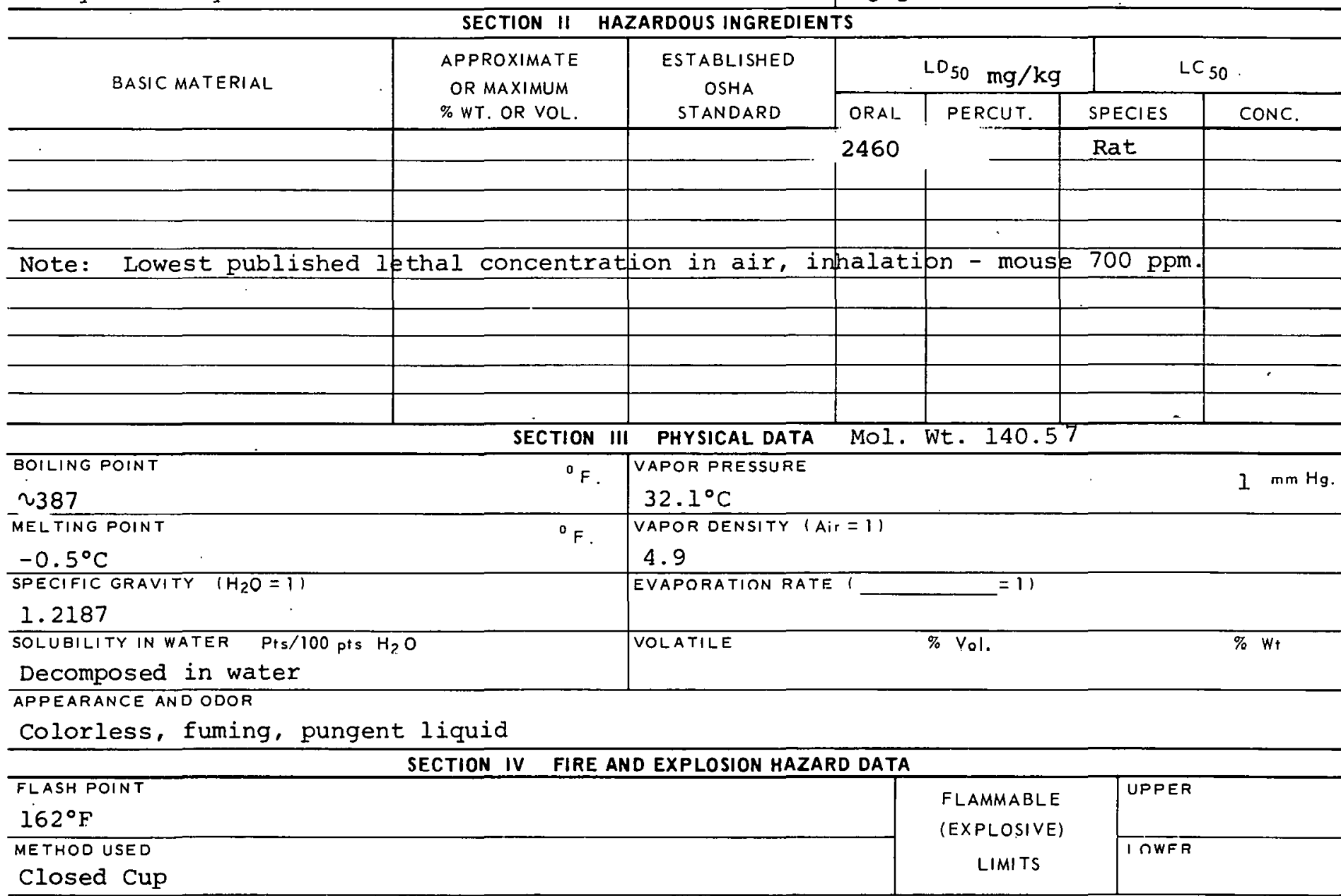

EXTINGUISHING MEDIA

Water spray sparingly, foam, carbon dioxide or dry chemical.

SPECIAL FIRE FIGHTING PROCEDURES

Full protective clothing

UNUSUAL FIRE AND EXPLOSION HAZARDS 


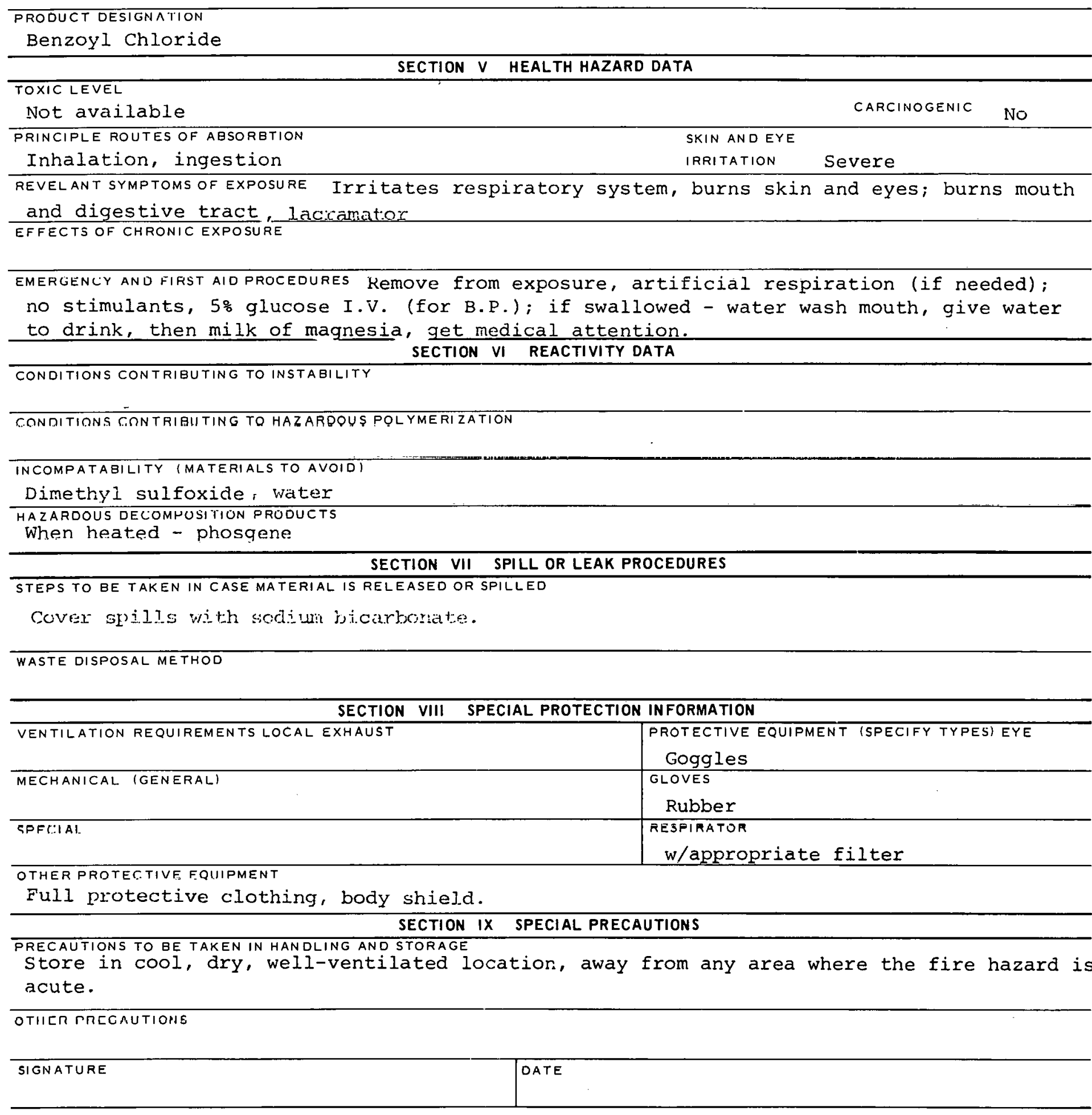




PRODUCT DESIGNATION
03-219-0708
Benzyl Bromide

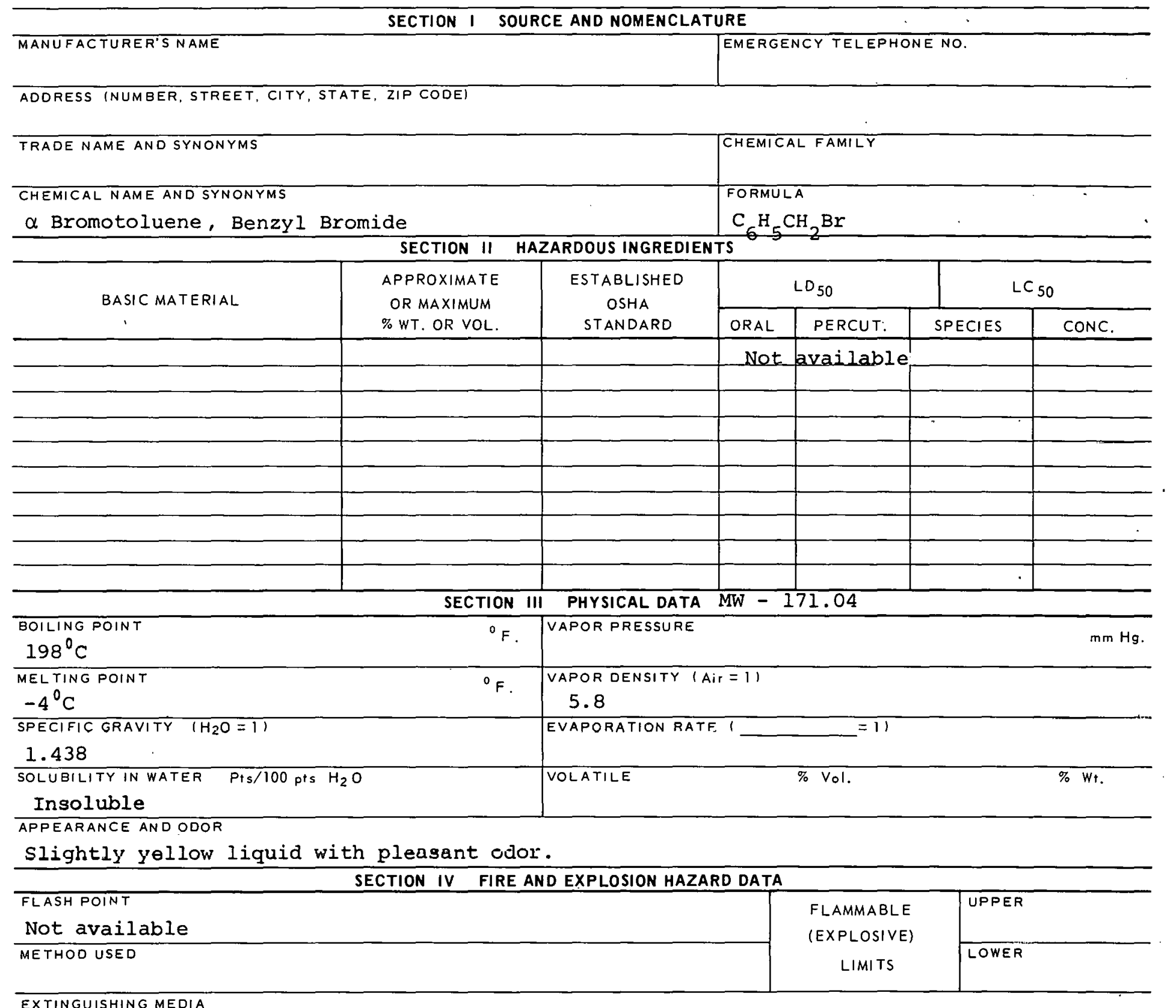

EXTINGUISHING MEDIA

Water spray, dry chemical, carbur dioxide

SPECIAL FIRE FIGHTIHC FROCEDURES

UNUSUAL FIRE AND EXPLOSION HAZARDS 
TOXIC LEVEL

Not available PRINCIPLE ROUTES OF ABSORBTION

Inhalation, ingestion

CARCINOGENIC SKIN AND EYE IRRITATION high

REVELANT SYMPTOMS OF EXPOSURE Irritation of eyes, nose and throat; severe burns to digestive tract if swallowed. EFFECTS OF CHRONIC EXPOSURE $--$

EMERGENCY AND FIRST AID PROCEDURES If inhaled - rest; if swallowed - wash mouth, give emetic; get medical aid; wash skin and eyes.

\section{SECTION VI REACTIVITY DATA}

CONDITIONS CONTRIBUTING TO INSTABILITY

CON IITIONS CONTRIBUTING TO HAZARDOUS POLYMERIZATION

INCOMPATABILITY (MATERIALS TO AVOID)

HAZARDOUS DECOMFIUSITION PRODUCTS

SECTION VII SPILL OR LEAK PROCEDURES

STEPS TO $B E$ TAKEN IN CASE MATERIAL IS RELEASED OR SPILLED

Absorb spill with paper towels. After material has evaporated, burn in hood.

WASTE DISPOSAL METHOD

Dissolve in combustible solvent and burn. SECTION VIII SPECIAL PROTECTION INFORMATION

\begin{tabular}{l|l}
\hline VENTILATION REQUIREMENTS LOCAL EXHAUST & $\begin{array}{l}\text { FROTECTIVE EQUIPMENT (SPECIFY TYPES) EYE } \\
\text { Goggleg }\end{array}$ \\
\hline MECHANICAL IGENERALI & $\begin{array}{l}\text { GLOVES } \\
\text { Rubber }\end{array}$ \\
\hline SHECIAL & RESPIRATOR \\
& Self-contained breathing apparatus \\
\hline
\end{tabular}

OTHER PROTECTIVE EQUIPMENT

SECTION IX SPECIAL PRECAUTIONS

PRECAUTIONS TO BE TAKEN IN HANDLING AND STORAGE

Store in cool, ary place.

OTHER RREGAUTIONG 
CAS : 000100447

\section{PRODUCT DESIGNATION}

$03-219-1708$

03-219-1758

Benzyl Chloride
XS89250

\section{MATERIAL SAFETY \\ DATA SHEET}

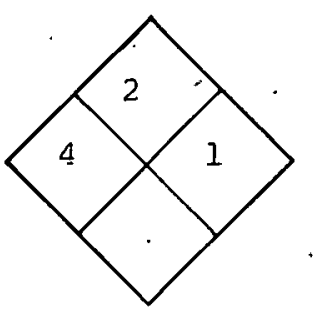

\section{SECTION I SOURCE AND NOMENCLATURE}

MANUFACTURER'S NAME

EMERGENCY TELEPHONE NO.

ADDRESS INUMBER, STREET, CITY, STATE, ZIP CODE

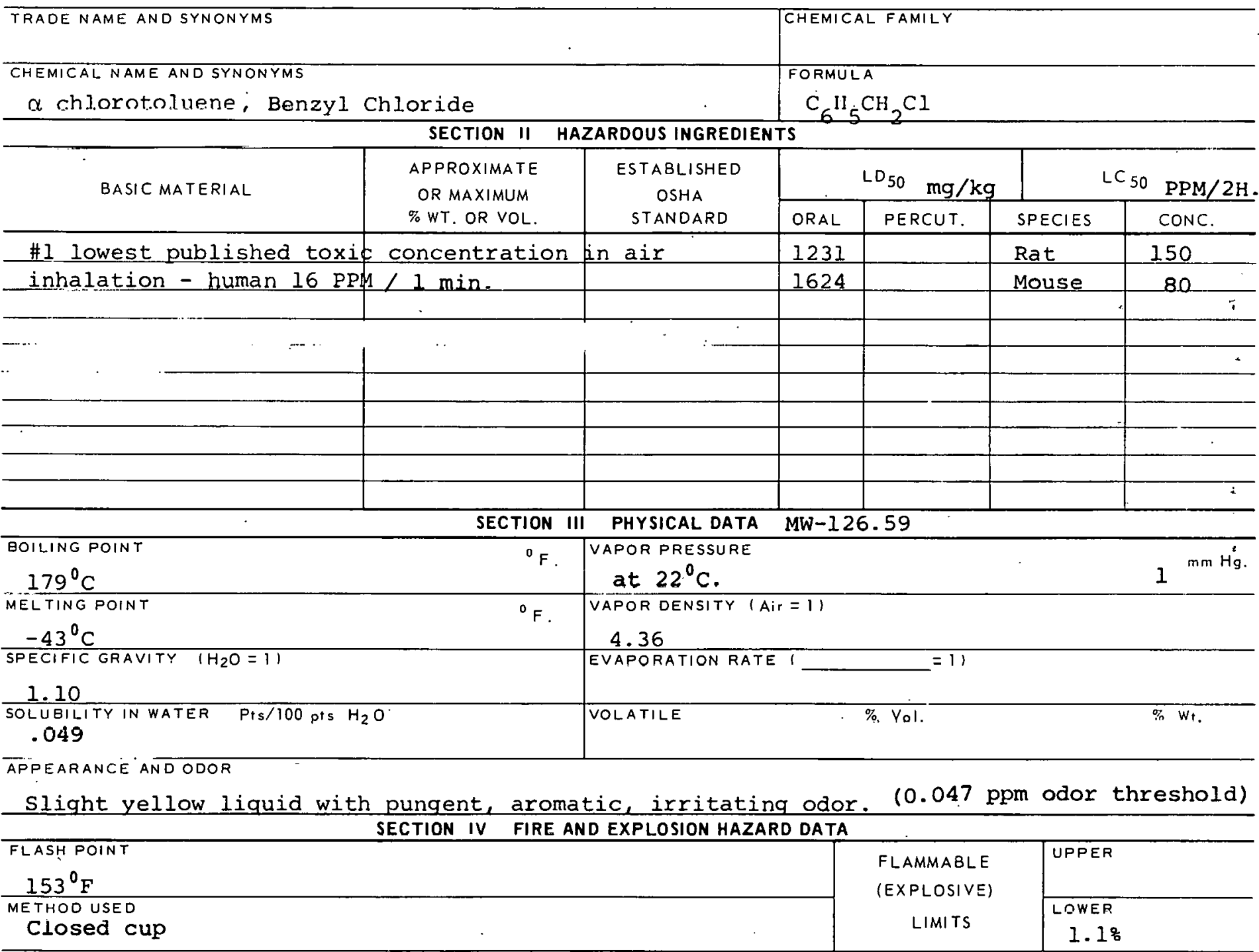

EXTINGUISHING MEDIA

Water spray, dry chemical, carbon dioxide, chemical foam.

SPECIAL FIRE FIGHTING PROCEDURES 


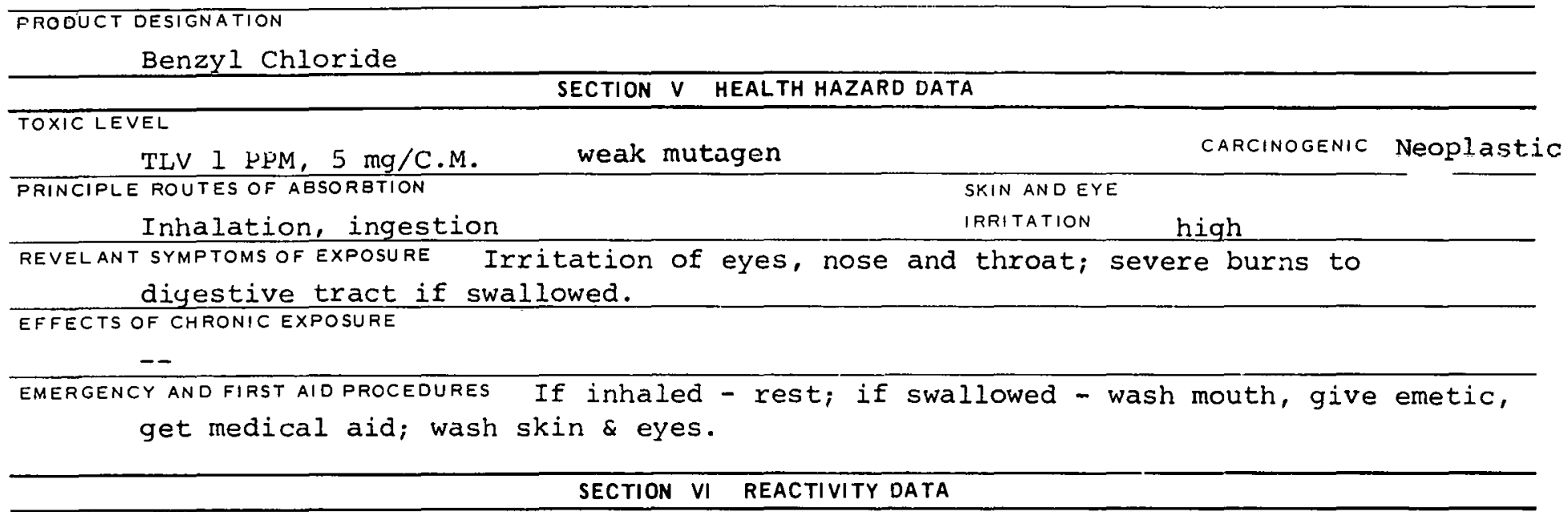

CONDITIONS CONTRIBUTING TO INSTABILITY
CONDITIONS CONTRIBUTING TO HAZARDOUS POLYMERIZATION MaY undergo cxothcrmic condensation in
presenCe Of COPpCr, aluminum, iron, zinc, magnesium, tin and various othcr metals,
INCOMPATABILITYMATERIALSTOAVOIU) as catalysts.

HAZARDOUS DECOMPUSITIOIN PHODUTETS

SECTION VII SPILL OR LEAK PROCEDURES

STEPS TO BE TAKEN IN CASE MATERIAL IS RELEASED OR SPILLED Flush spills with large quantities

of water and ventilate area to remove vapors.

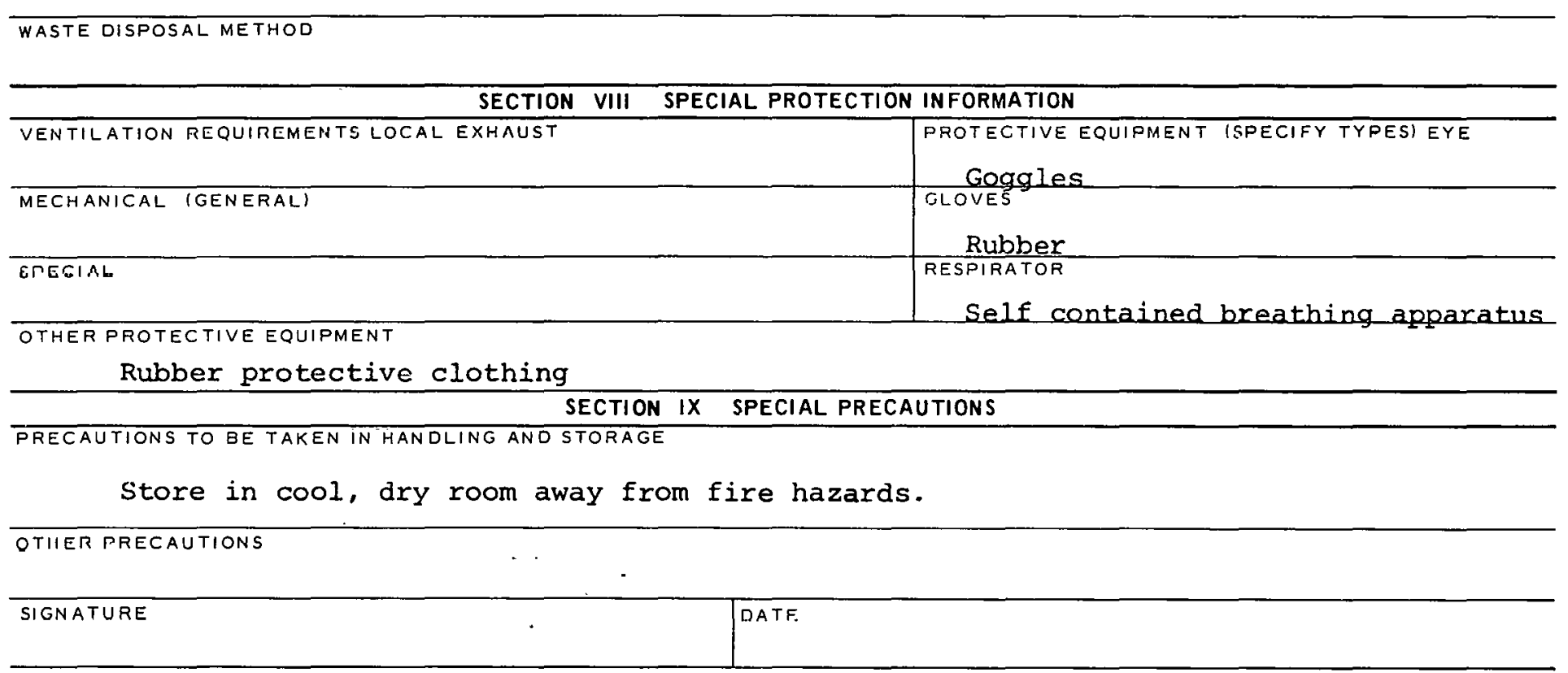




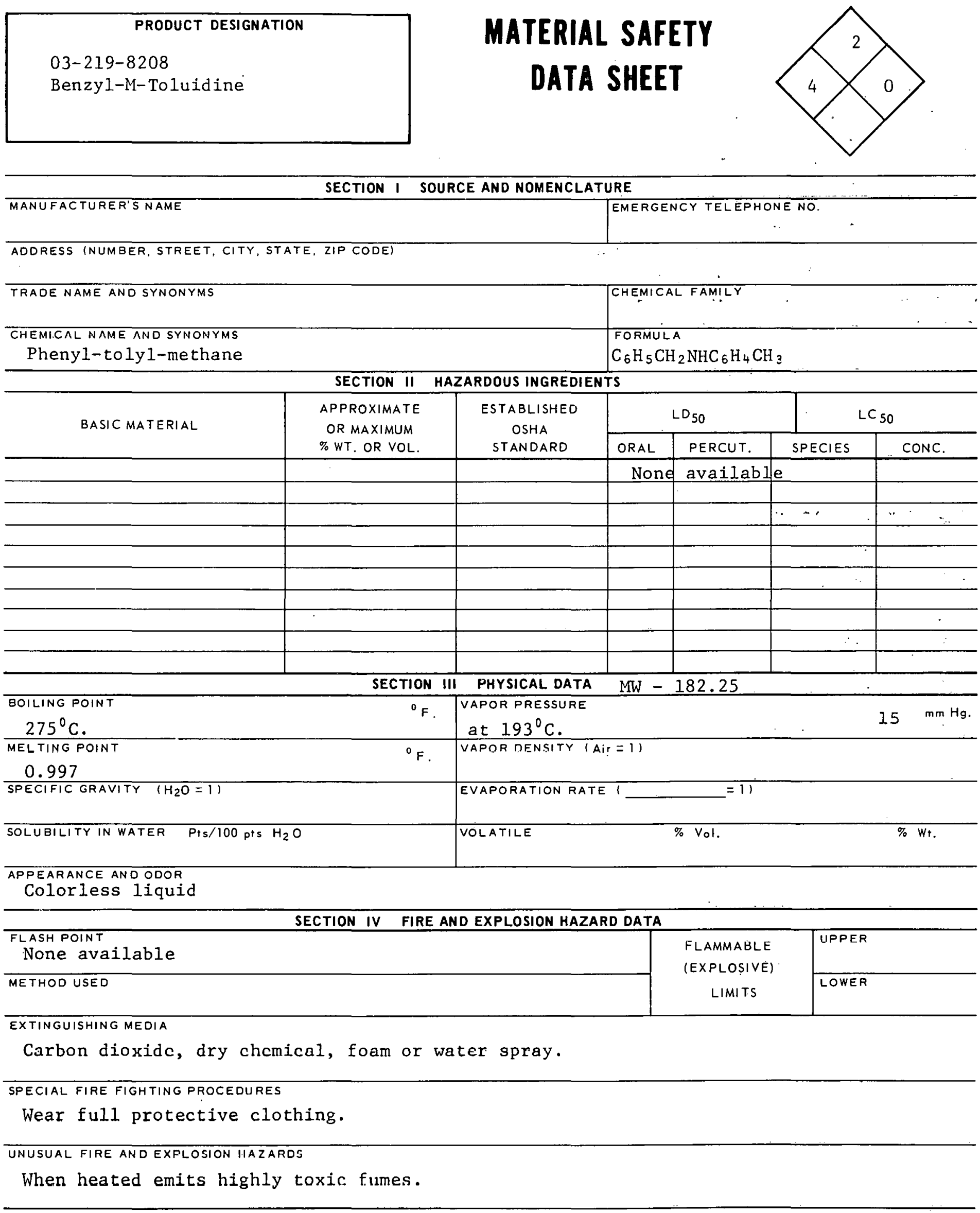


TOXIC LEVEL

Nonc available

PRINCIPLE ROUTES OF ABSORBTION

Inhalation, ingestion, skin
CARCINOGENIC NO SKIN AND EYE

IRRITATION MOderate

REVELANT SYMPTOMS OF EXPOSURE Cyanosis, air hunger, nausea, vomiting, lcw blood pressure, corvulsions.

EFFECTS OF CHRONIC EXPOSURE

Weight loss, anemia, weakness.

EMERGENCY AND FIRST AIDPROCEDURES Inhalation - rest; if swallowed - give emetic, get medical aid; wash from skin \& eyes.

\section{SECTION VI REACTIVITY DATA}

CONDITIONS CONTRIBUTING TO INSTABILITY

CONDITIONS CONTRIBUTING TO HAZARDOUS POLYMERIZATION

INCOOMPAIABILITY (MATEHIALS IO AVOIU)

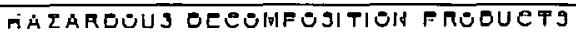

SECTION VII SFILL OR LEAK PROCEDURES

STEPS TO BE TAKEN IN CASE MATERIAL IS RELEASED OR SPILLED

WASTE DISPOSAL METHOD

\section{SECTION VIII SPECIAL PROTECTION INFORMATION}

\begin{tabular}{|c|c|}
\hline VENTILATION REQUIREMENTS LOCAL EXHAUST & $\begin{array}{l}\text { PROTECTIVE EQUIPMENT (GPECIFY TYPES) EYE } \\
\text { SafetY gOggles }\end{array}$ \\
\hline $\begin{array}{l}\text { MECHANICAL (GENERAL) } \\
\text {. }\end{array}$ & $\begin{array}{l}\text { GLOVES } \\
\text { Rubber gloves }\end{array}$ \\
\hline SPECIAL & $\begin{array}{l}\text { RFSPIRATOR } \\
\text { With proper filter }\end{array}$ \\
\hline
\end{tabular}

OTHER PROTECTIVE EQUIPMENT

SECTION IX SPECIAL PRECAUTIONS

PRECAUTIONS TO BE TAKEN IN HANDLING AND STORAGE

OTHER PRECAUTIONS

SIGNATURE

DATF

219-8208B 


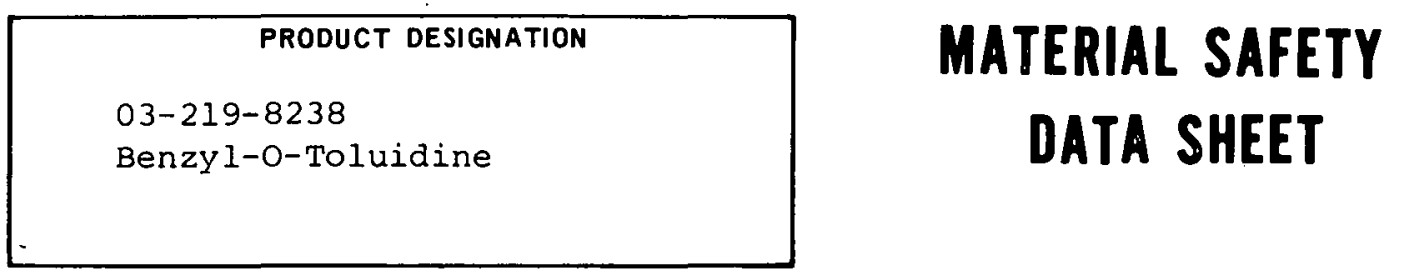

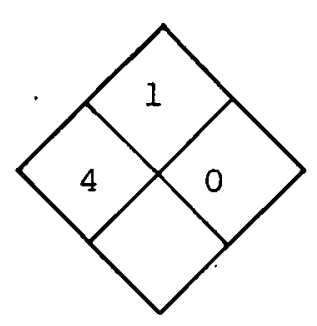

SECTION I SOURCE AND NOMENCLATURE

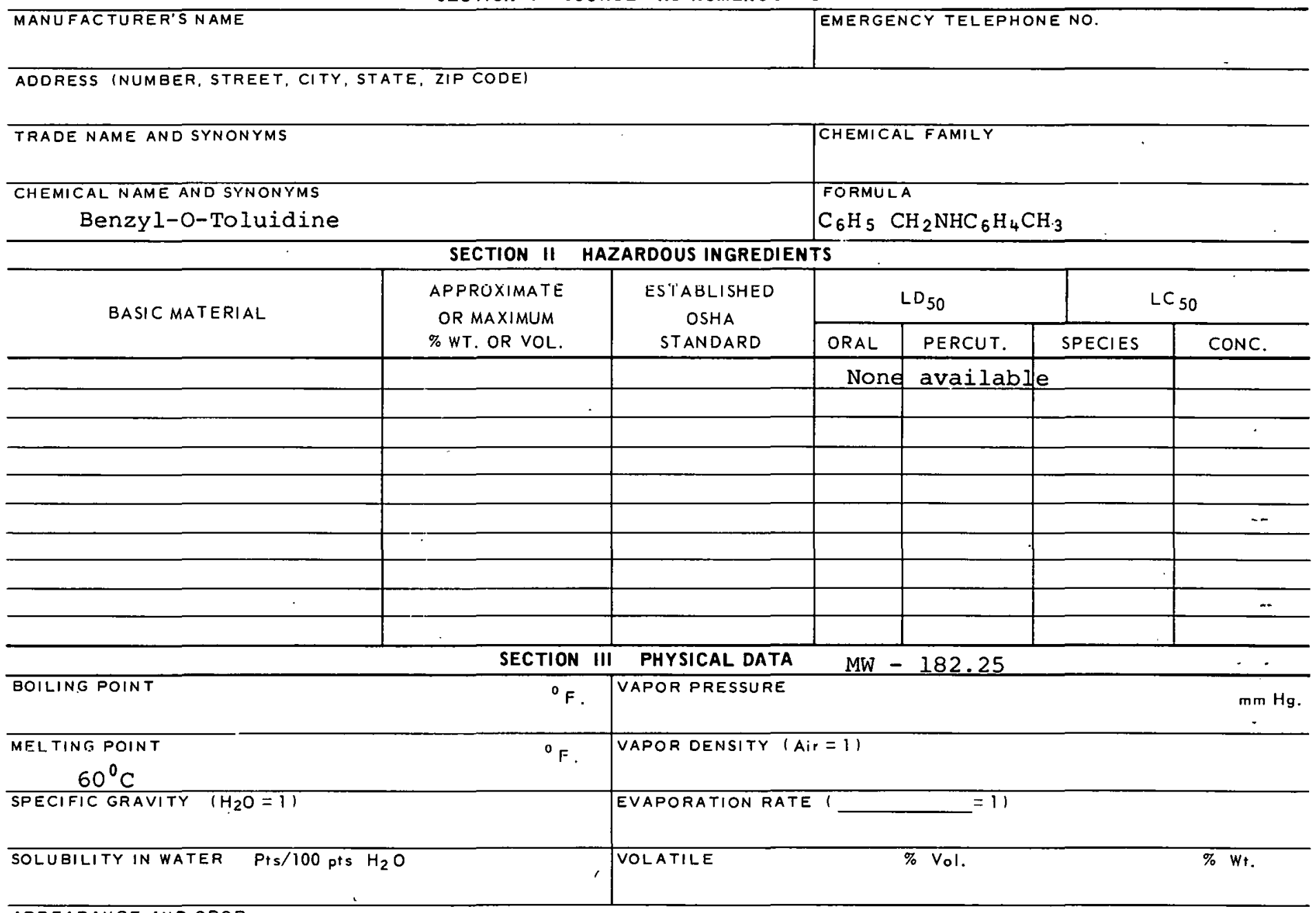

APPEARANCE AND ODOR

Crystaline solid

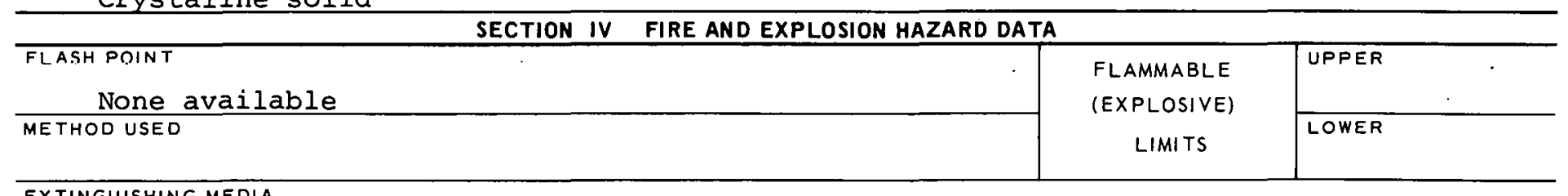

EXTINGUISHING MEDIA

Carbon dioxide, ary chemical, fuall or water spray.

SPECIAL FIRE FIGHTING PROCEDURES

$--$

UNUSUAL FIRE AND EXPLOSION HAZARDS

$--$ 


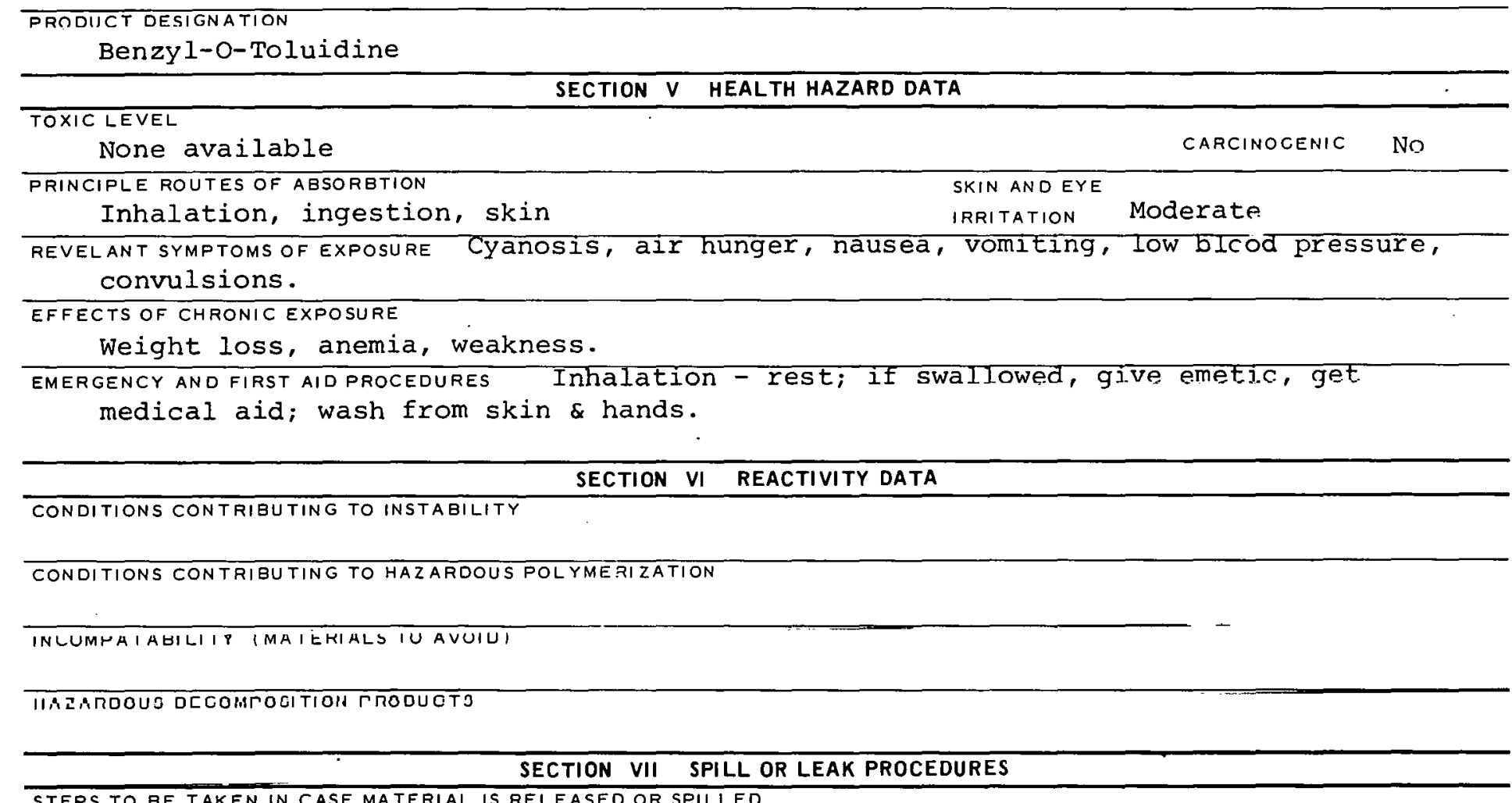
STEPS TO BE TAKEN IN CASE MATERIAL IS RELEASED OR SPILLED

WASTE DISPOSAL METHOD

SECTION VIII SPECIAL PROTECTION INFORMATION

\begin{tabular}{|c|c|}
\hline VENTILATION REQUIREMENTS I.NCAI_EXHAUST & $\begin{array}{l}\text { FROTCCTIVE EQUIPMENT (SPECIFY TYPES) EYE } \\
\text { Safety goggles }\end{array}$ \\
\hline MECHANICAL (GENERAL) & $\begin{array}{l}\text { GLOVES } \\
\text { Rubber gloves }\end{array}$ \\
\hline SPECIAL & RFCRIRATRR \\
\hline
\end{tabular}

OTHER PROTECTIVE EQUIPMENT

SECTION IX SPECIAL PRECAUTIONS

\begin{tabular}{l|l|l}
\hline SECTION IX SPECIAL PRECAUTIONS \\
\hline PRECAUTIONS TO BE TAKEN IN HANDLING AND STORAGE \\
\hline OTHER PRECAUTIONS & OATE \\
\hline SIGNATURE & \\
\hline
\end{tabular}



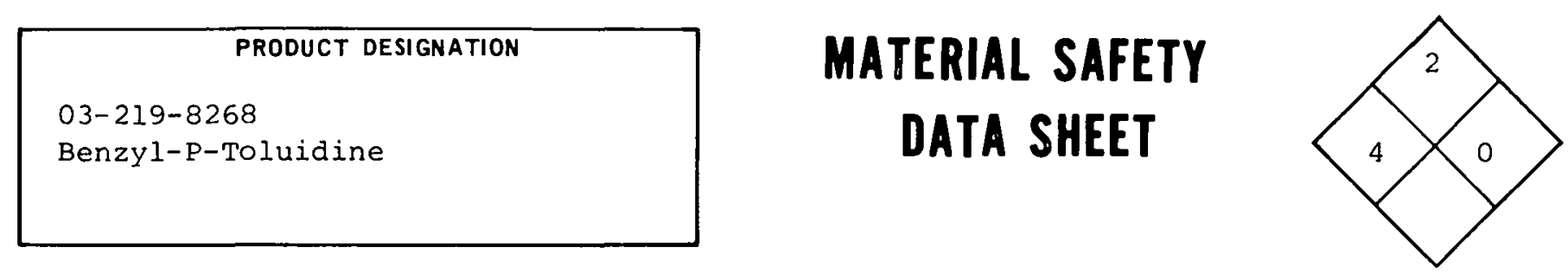

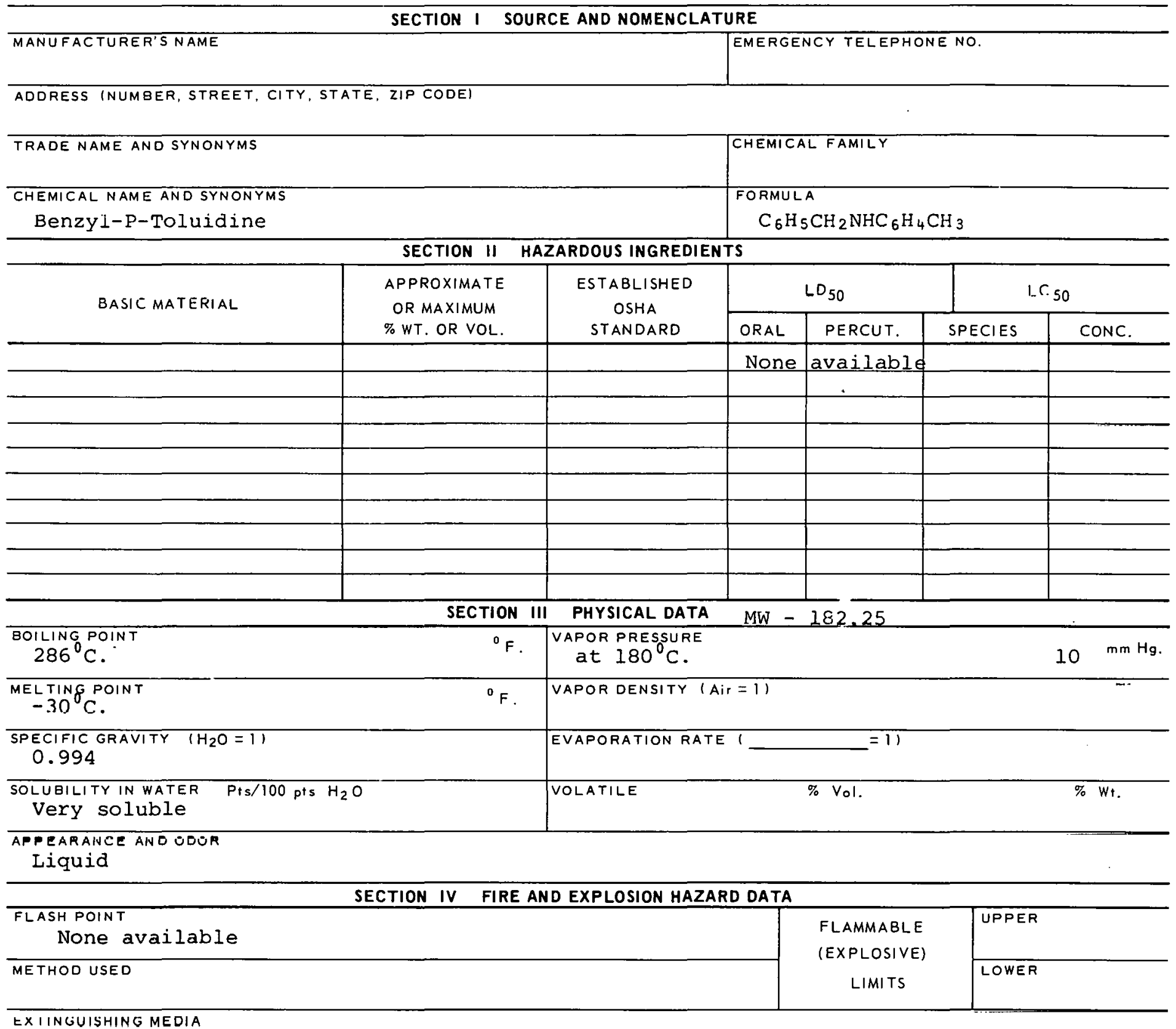

EXIINGUISHING MEDIA

Carbon dioxide, dry chemical, foam or water spray.

SPECIAL FIRE FIGHTING PROCEOURES

Wear full protective clothing.

UNUSUAL FIRE ANOEXPLOSION HAZARDS

When heated emits highly toxic fumes. 


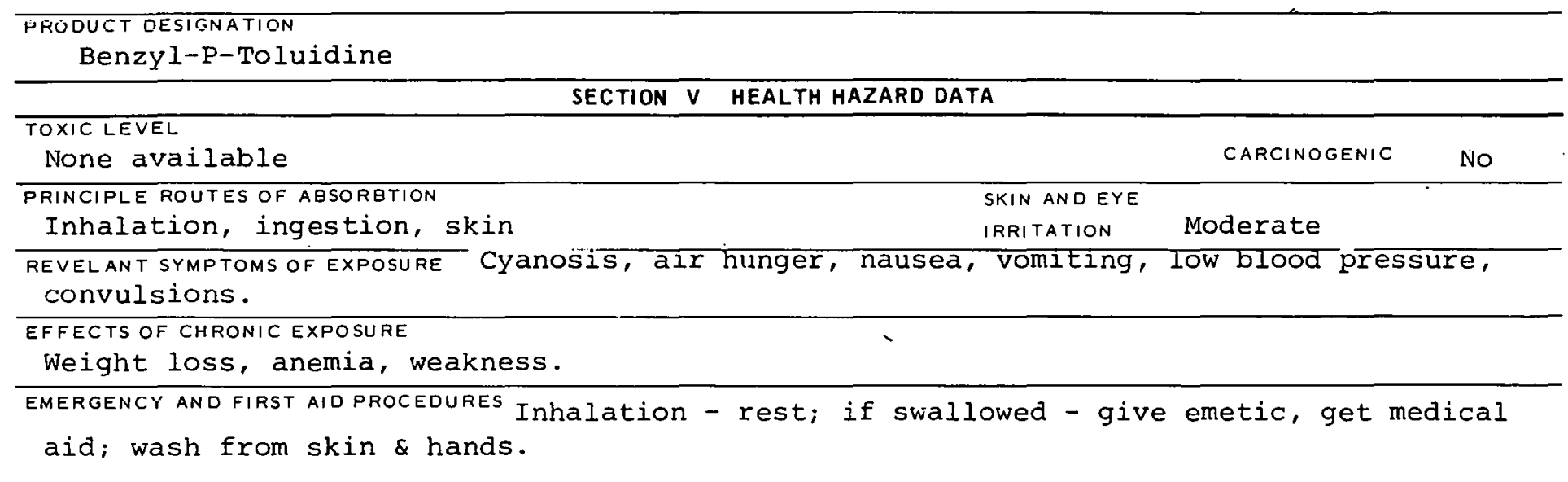

\section{SECTION VI REACTIVITY DATA}

CONDITIONS CONTRIBUTING TO INSTABILITY

CQONDITIONS CONTRIBU TING TO HAZARDOUS POLYM $\overline{E R I \overline{Z A T I O N ~}}$

INCOMPATABILITY (MATERIALS TO AVOID)

HAZARDOUS DECOMPOSITION PRODUCTS

SECTION VII SPILL OR LEAK PROCEDURES

STEPS TO BE TAKEN IN CASE MATERIAL IS RELEASED OR SPILLED

WASTE UISHOSAL METHOD

SECTION VIII SPECIAL PROTECTION INFORMATION

\begin{tabular}{l|l}
\hline VENTILAIION REQUIREMENTS LOCAL EXHAUST & $\begin{array}{l}\text { PROTECTIVE EQUIPMENT (SPECIFY TYPESIEYE } \\
\text { SafCtY gOggleg }\end{array}$ \\
\hline $\begin{array}{l}\text { MECHANICAL IGENERALI } \\
\text { SPECIAL }\end{array}$ & $\begin{array}{l}\text { GLOVES } \\
\text { Rubber glOVes }\end{array}$ \\
\hline
\end{tabular}

OTHER PROTECTIVE EQUIPMENT

SECTION IX SPECIAL PRECAUTIONS

PRECAUTIONS TO BE TAKEN IN HANDLING AND STORAGE

OTHER PRECAUTIONS

SIGNATURE

TOATL

$219-8268 B$ 
CAS:-000507426

PRODUCT DESIGNATION

03-220-0809

Bromal
$A B 33250$

\section{MATERIAL SAFETY \\ DATA SHEET}

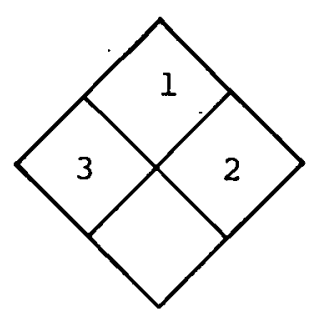

\section{SECTION I SOURCE AND NOMENCLATURE}

\begin{tabular}{l} 
MANUFACTURER'S NAME \\
\hline ADDRESS INUMBER, STREET, CITY \\
TRADE NAME AND SYNONYMS \\
TribrOMOaCE TaIdehYde \\
CHEMICAL NAME AND SYNONYMS \\
Bromal
\end{tabular}

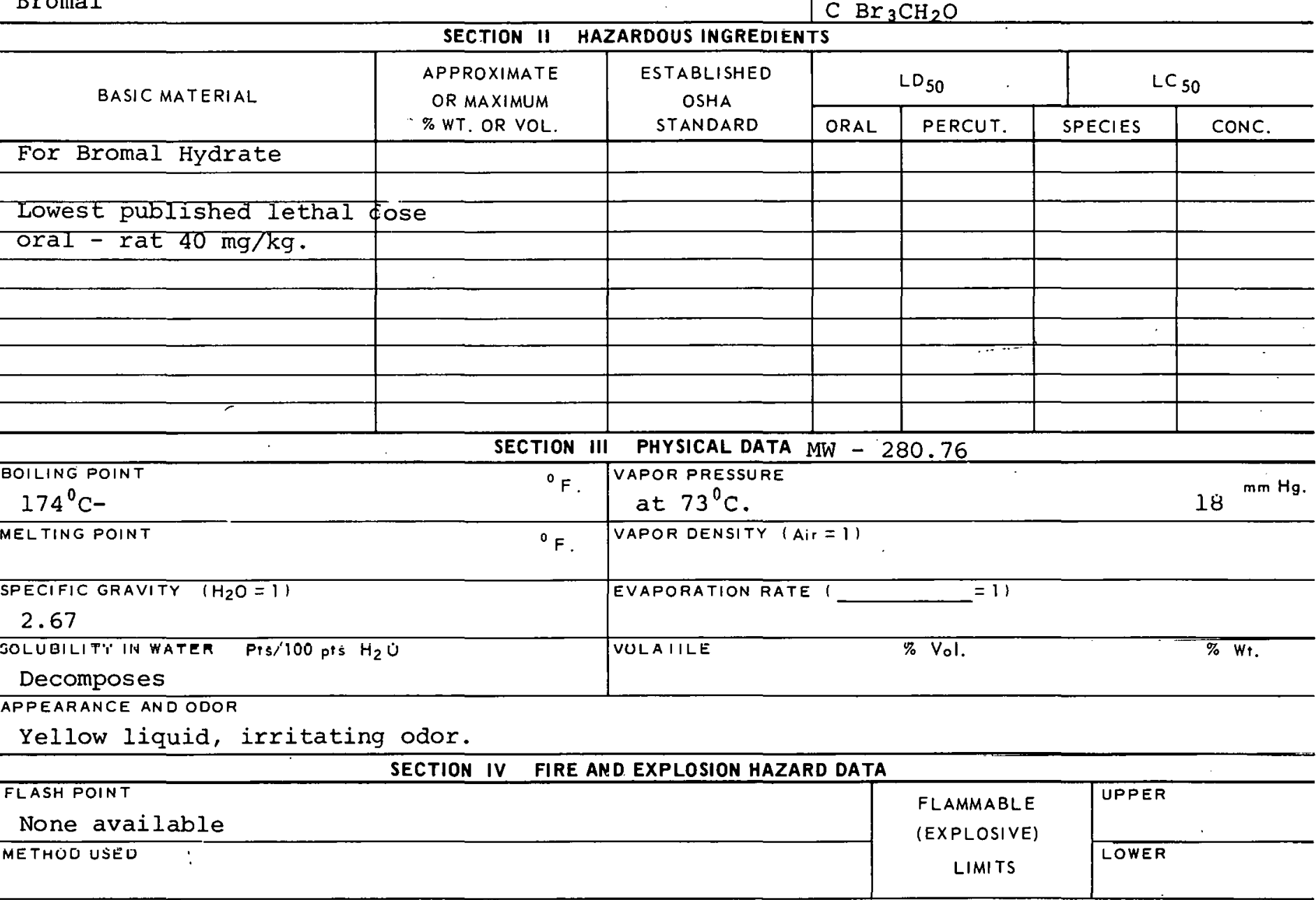

EXTINGUISHINE MEUIA

Alcohol foam, carbon dioxide, dry chemical.

SPECIAL FIRE FIGHTING PROCEDURES

IINIISIIAI FIRF ANI FXPLOSION HAZARDS

When heated to decomposition emits toxic fumes. 
TOXIC LEVEL

None availablc

PRINCIPLE ROUTES OF ABSORBTION Inhalation, ingestion
CARCINOGENIC NO

SKIN AND EYE

IRRITATION SEvere

REVELANT SYMPTOMS OF EXPOSURE Ingestion - nausea, vomiting, colaness of eXtremities, unconsciousness, skin \& eye irritation, hypnotic effects.

EFFECTS OF CHRONGC EXPOSURE irritation.

EMERGENCY AND FIRST AID PROCEDURES

Ingestion - give emetic, get medical aid, inhalation - rest; wash skin and eyes with tor. SECTION VI REACTIVITY DATA

CONDITIONS CONTRIBUTING TO INSTABILITY

CONDITIONS CONTRIRIITING TO HAZARDOUS POLYMERIZATION

INCOMPATABILITY (MATERIALSTOO AVOIO)

HARARDOUS UELUMPUSIIIUN RRULUU.-1S

SECTION VII SPILL OR LEAK PROCEDURES

STEPS TO BE TAKEN IN CASE MATERIAL IS RELEASED OR SPILLED

WASTE DISPOSAL METHOD

SECTION VIII SPECIAL PROTECTION INFORMATION

\begin{tabular}{l|l}
\hline VENTILATION REQUIREMENTS LOCAL EXHAUST & $\begin{array}{c}\text { PROTECTIVE EQUIPMENT (SPECIFY TYPES) EYE } \\
\text { GOGgleS }\end{array}$ \\
\hline MECHANICAL IGENERAL) & GLOVES \\
\hline CTEGIAL & $\begin{array}{c}\text { RFSPIRATnR } \\
\text { Self-contained breathing apparatus }\end{array}$ \\
\hline
\end{tabular}

OTHER PROTEC.TIVF_ EQIIIPMENT

SECTION IX SPECIAL PRECAUTIONS

PRECAUTIONS TO BE TAKEN IN HANDLING AND STORAGE

Protect against pinysical damage, detached storage preferred. Inside storage

in flammable liquids cabinet.

OTHER PRECAUTIONS

SIGNATURE

DATE

$2.20-0809 B$ 


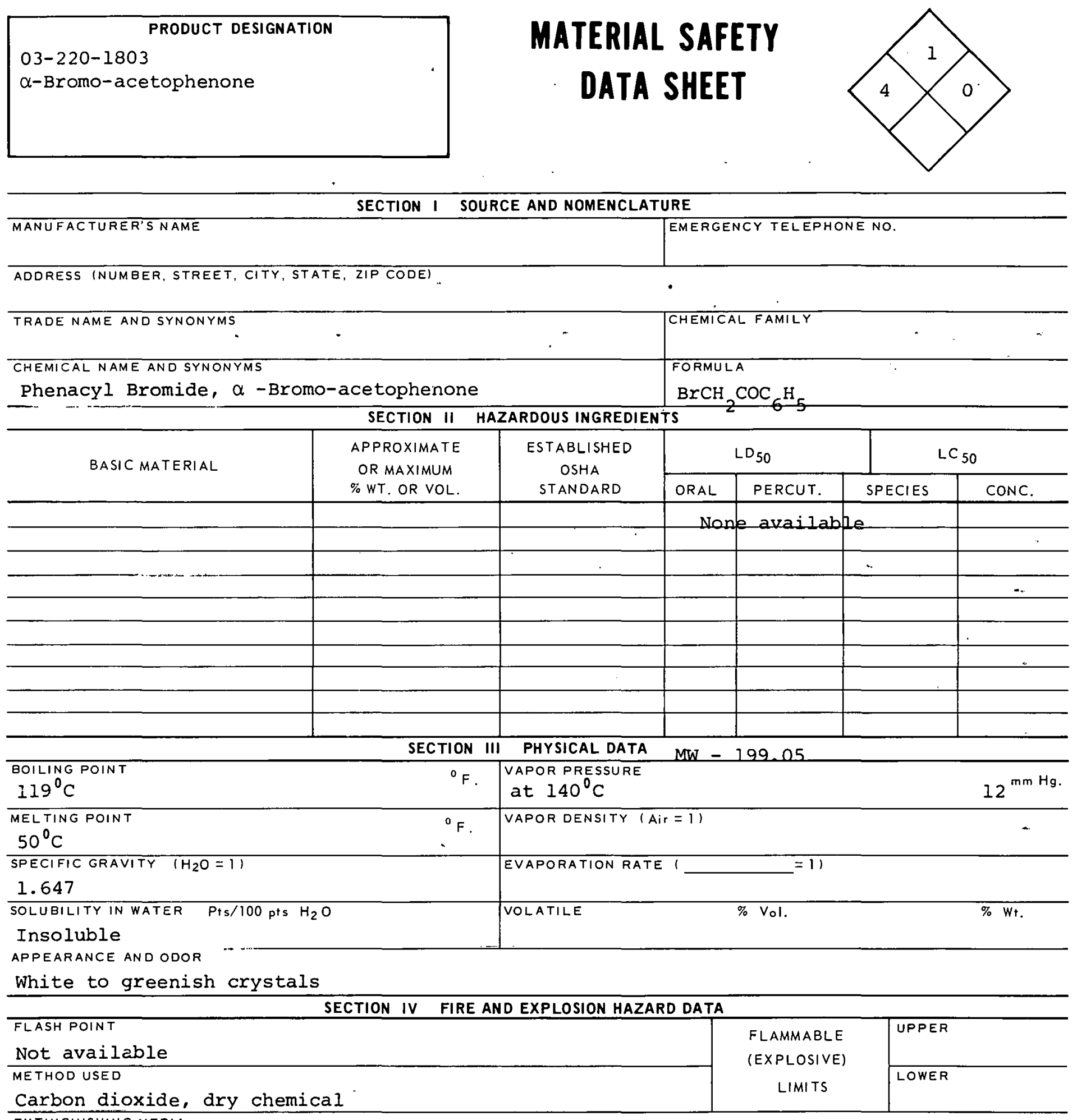




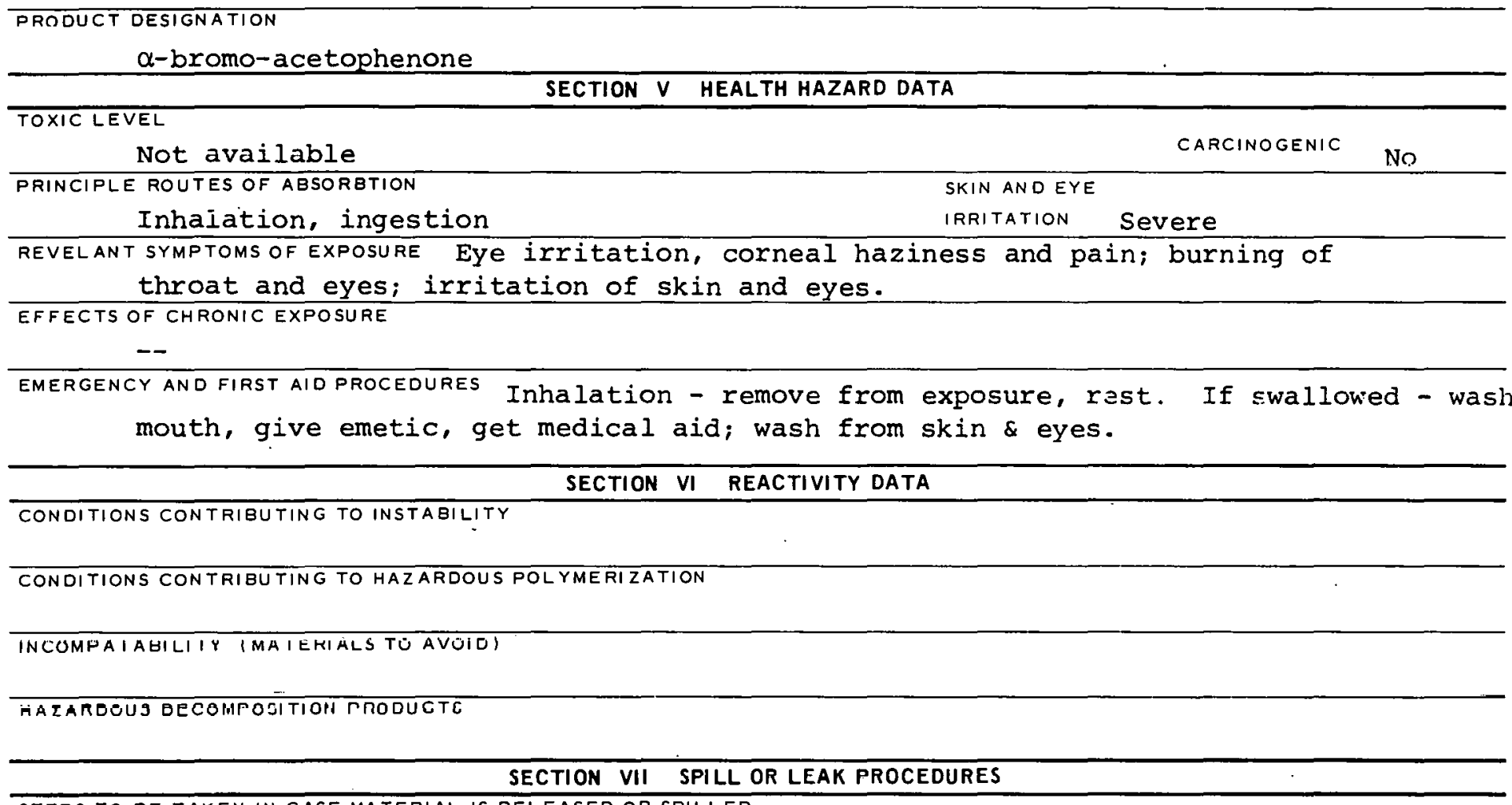
STEPS TO BE TAKEN IN CASE MATERIAL IS RELEASED OR SPILLED

WASTE DISPOSAL METHOD

SECTION VIII SPECIAL PROTECTION INFORMATION

\begin{tabular}{|c|c|}
\hline VENTILATION REQUIREMENTS LOCAL EXHAIIST & $\begin{array}{l}\text { PROTFCTIVE EQUIPMENT (SPECIFY TYPESI EYE } \\
\text { ringgles }\end{array}$ \\
\hline MECHANICAL (GENERAL) & $\begin{array}{l}\text { GLOVES } \\
\text { Rubber }\end{array}$ \\
\hline SPECIAL & RFSPIRATOR \\
\hline
\end{tabular}

OTHER PROTECTIVE EQUIPMENT

\section{SECTION IX SPECIAL PRECAUTIONS}

PRECAUTIONS TO BE TAKEN IN HANDLING AND STORAGE 


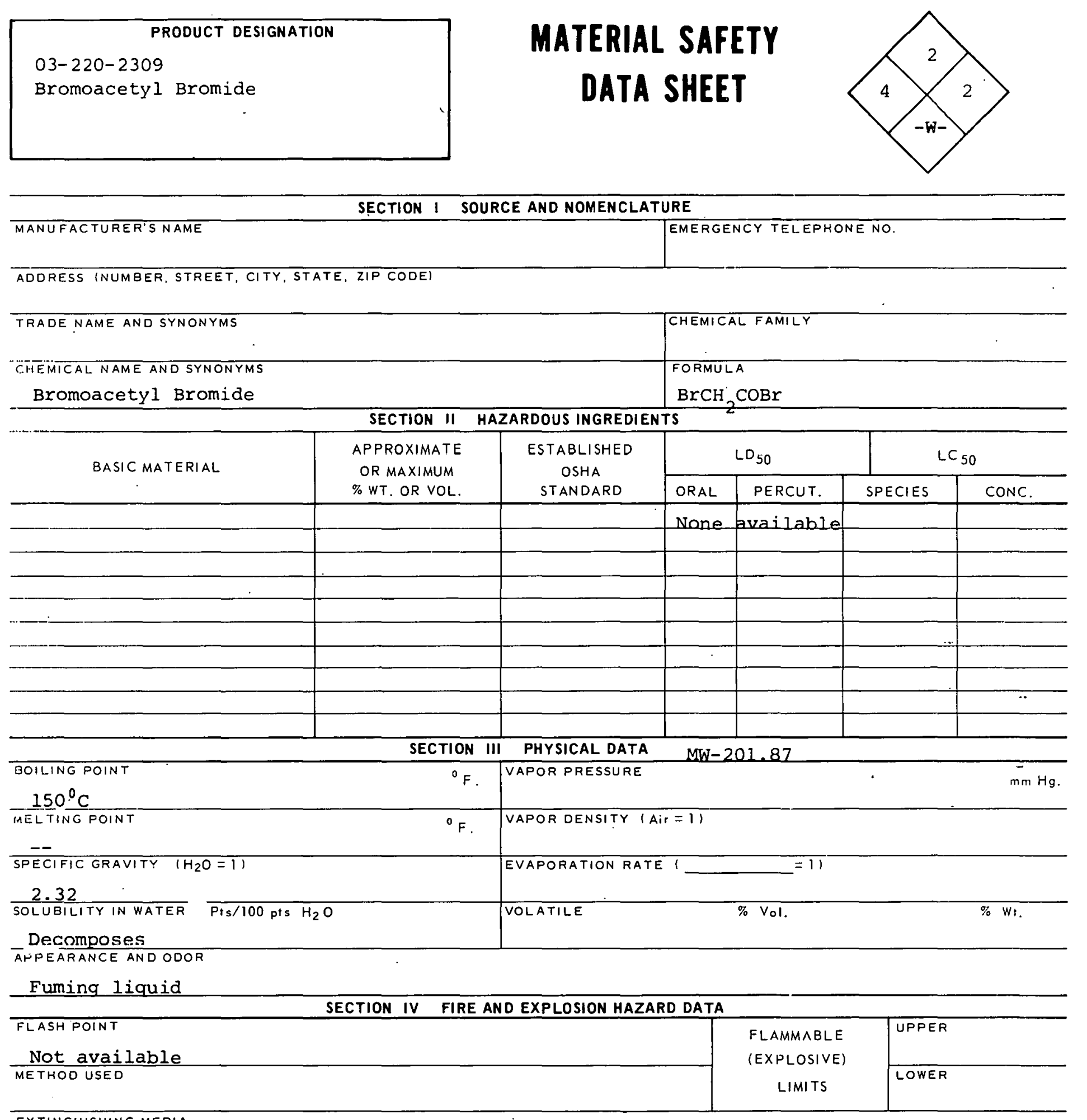

\section{EXTINGUISHING MEDIA Do not use water or foam, use carbon}

dioxide or dry chemical.

SPECIAL FIRE FIGHTING PROCEDURES

UNUSUAL FIRE AND EXPLOSION HAZARDS When heated to decomposition point gives off toxic HBr. 
Bromoacetyl Bromide

\section{SECTION $V$ HEALTH HAZARD DATA}

\section{TOXIC LEVEL}

None available

PRINCIPLE ROUTES OF ABSORBTION

Inhalation, ingestion

REVELANT SYMPTOMS OF EXPOSURE Irritation of eyes \& respiratory system; severe internal

damage iE swallowed; eye \& skin burns.

EFFECTS OF CHRONIC EXPOSURE

Bronchial irritation, errosion of teeth.

EMERGENCY AND FIRST AID PROCEDURES Inhalation - rest; get medical aid; if swallowed - drink water, milk of magnesia, get medical aid, wash eyes \& skin.

\section{SECTION VI REACTIVITY DATA}

CONDITIONS CONTRIBUTING TO INSTABILITY

CONDITIONS CONTRIBUTING TO HAZARDOUS POLYMERIZATION

INCOMPATABILITY (MATERIALS TO AVOID)

Reacts violently with water or alcohol. HAZARDOUS DECOMPOSITION PRODUCTS

SECTION VII SPILL OR LEAK PROCEDURES

STEPS TO BE TAKEN IN CASE MATERIAL IS RELEASED OR SPILLED

WASTE DISPOSAL METHOD

SECTION VIII SPECIAL PROTECTION INFORMATION

\begin{tabular}{l|l}
\hline VENTILATION REQUIREMENTS LOCAL EXHAUST & $\begin{array}{l}\text { PROTECTIVE EQUIPMENT (SPECIFY TYPES) EYE } \\
\text { GOggles }\end{array}$ \\
\hline MECHANICAL IGENERALI & $\begin{array}{c}\text { GLOVES } \\
\text { Rubber }\end{array}$ \\
\hline SFEEIAL & RESPIRATÜ \\
& Self COntained \\
\hline
\end{tabular}

OTHER PROTECTIVE EQUIPMENT

Rubber apron

SECTION IX SPECIAL PRECAUTIONS

PRECAUTIONS TO BE TAKEN IN HANDLING AND STORAGE

OTHER DRFTAIITIONS

SIGNATURE

DATE

$220-2309 \mathrm{P}$ 


\section{XU43750 \\ MATERIAL SAFETY \\ DATA SHEET}

PRODUCT DESIGNATION

03-220-2809

3 Bromo-4-Aminotoluene

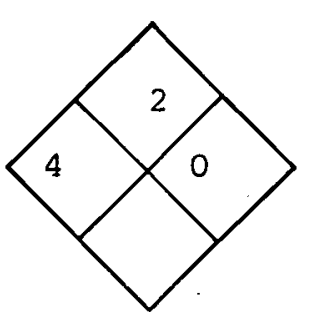

SECTION I SOURCE AND NOMENCLATURE

MANUFACTURER'SNAME

ADORESS (NUMBER, STREET, CITY, STATE, ZIP CODE)

TRADE NAME AND SYNONYMS

3 Bromo $P$ Toluidine

CHEMICAL NAME AND SYNONYMS

2 Bromo-4 Methyl Aniline

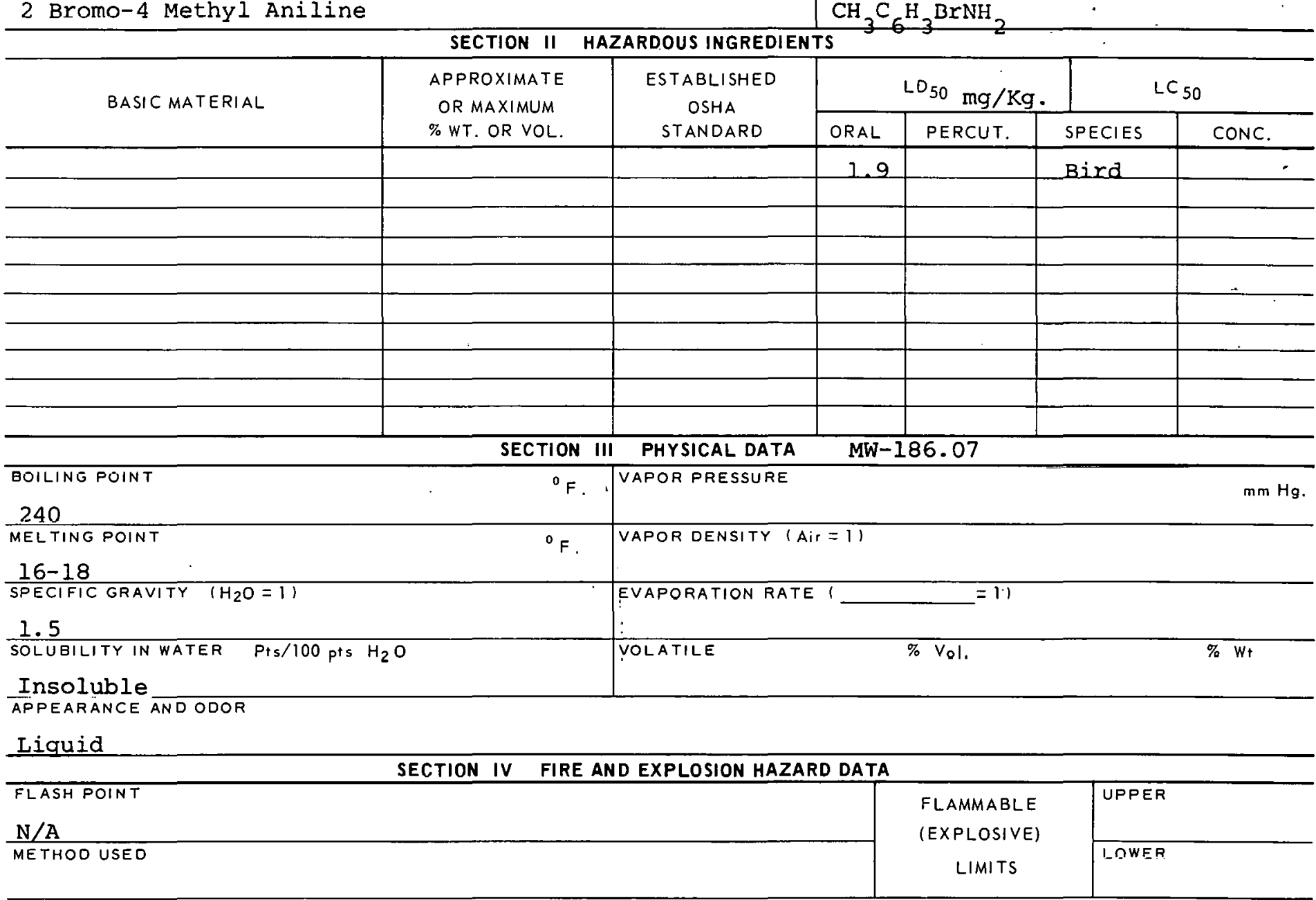

EXTINGUISHING MEDIA

Carbon dioxide, dry chemical, foam or water spray

SPECIAL FIRE FIGHTING PROCEDURES

Wear full protective clothing

UNUSUAL FIRE AND EXPLOSION HAZARDS

When lieated emits highly toxic fumes. 
TOXIC LEVEL

Not available PRINCIPLE ROUTES OF ABSORBTION

Inhalation, ingestion, skin REVELANT SYMPTOMS OF EXPOSURE. Cyanosis, air hunger, nausea, vomiting, low blo convulsions

EFFECTS OF CHRONIC EXPOSURE

Weight loss, anemia, weakness

EMERGENCY ANDFIRST AIDPROCEDURES Inhalation - rest. If swallowed - wash mouth, give emetic, get medical aid; wash from skin \& eyes.

\section{SECTION VI REACTIVITY DATA}

CONDITIONS CONTRIBUTING TO INSTABILITY

CONDITIONS CONTRIBUTING TO HAZARDOUS POLYMERIZATION

INCOMPATABILITY (MATERIALS TO AVOIO)

HALARUUUS UEGUMRUSIIIUN H.RUUUL IS

SECTION VII SPILL OR LEAK PROCEDURES

STEPS TO BE TAKEN IN CASE MATERIAL IS RELEASED OR SPILLED

WASTE DISPOSAL METHOP

SECTION VIII SPECIAL PROTECTION INFORMATION

\begin{tabular}{l|l}
\hline VENTILATION REQUIREMENTS LOCAL EXHAUST & $\begin{array}{l}\text { PROTECTIVE EQUIPMENT (SPECIFY TYPES) EYE } \\
\text { SafetY gOggLes }\end{array}$ \\
\hline MECHANICAL IGENERAL) & $\begin{array}{c}\text { RLOVES } \\
\text { Rubber }\end{array}$ \\
\hline GPEGIAL & $\begin{array}{c}\text { REGRIRATON } \\
\text { Wi.th proper filter }\end{array}$ \\
\hline
\end{tabular}

OTHER PROTECTIVE EQUIPMENT

SECTION IX SPECIAL PRECAUTIONS

PRECAUTIONS TO BE TAKEN IN HANDLING AND STORAGE

OTHFR PRFC.AIITIONS

SIGNATURE

DATE

$220-2809 B$ 
03-220-3309

5-Bromo-2-Aminotoluene
MATERIAL SAFETY

DATA SHEET

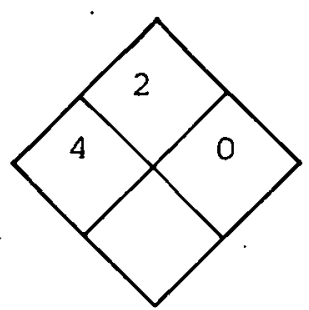

\section{SECTION I SOURCE AND NOMENCLATURE}

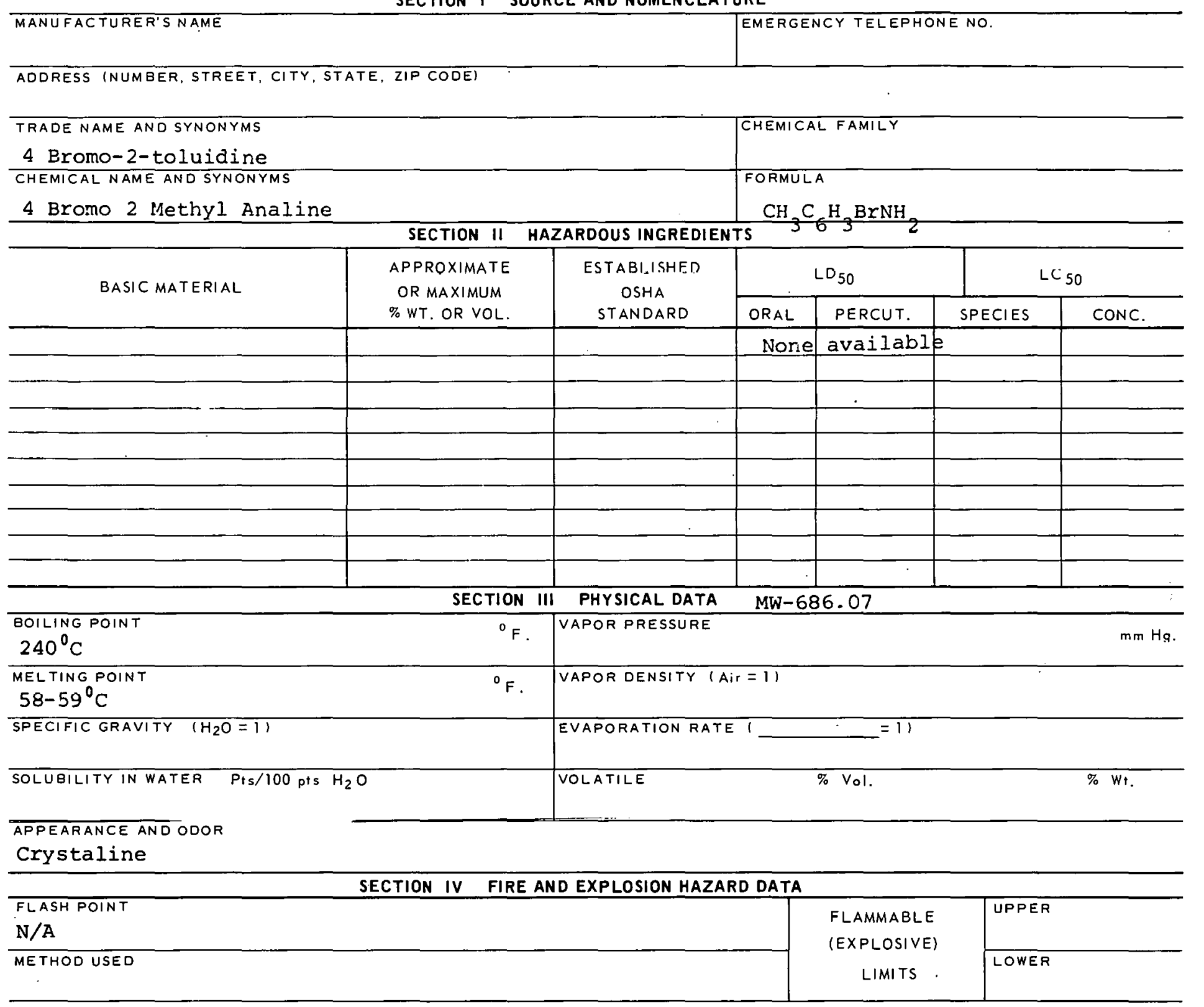

EXTINGUISHING MEDIA

Carbon dioxide, dry chemical, foam or water spray

SPECIAL FIRE FIGHTING PROCEDURES

Wear full protective clothing

UNUSUAL FIRE AND EXPLOSION HAZAROS

When heated emits highly toxic fumes. 


\section{TOXIC LEVEL}

Not available

PRINCIPLE ROUTES O.F ABSORBTION SKIN ANDEYE

CARCINOGENIC

Inhalation; ingestion, skin IRRITATION EYES

REVELANT SYMPTOMS OF EXPOSURE Cyanosis, air hunger, nausea, vomiting, low blood pressure, convulsions.

EFFECTS OF CHRONIC EXPOSURE

Weight loss, anemia, weakness

EMERGENCY AND FIRST AID PROCEDURES Inhalation - rest. If swallowed - wash mouth, give emetic, get medial aid; wash from skin \& eyes.

SECTION VI REACTIVITY DATA

CONOITIONS CONTRIBUTING TO INSTABILITY

CONDITIONS CONTRIBUTING TO HAZARDOUS POLYMERIZATION

INCOMPATAEILITY (MATEAINLE TO AVOIO)

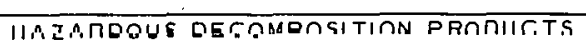

SECTION VII SPILL OR LEAK PROCEDURES

STEPS TO BE TAKEN IN CASE MATERIAL IS RELEASED OR SPILLED

WASTE DISPOSAL METHOD

SECTION VIII SPECIAL PROTECTION INFORMATION

VENTILATION REQUIREMENTS LOCAL EXHAUST

MFR.HANIS.AI (GENERAL)

SPECIAL

OTHER PROTECTIVE EQUIPMENT

$\begin{aligned} & \text { PROTECTIVE EQUIPMENT (SPECIFY TYPES) EYE } \\ & \text { Safety goggles }\end{aligned}$
$\begin{aligned} & \text { GLOVES } \\ & \text { Rubber }\end{aligned}$
RESPIRATOR
With proper filter

\section{SECTION IX SPECIAL PRECAUTIONS}

PRECAUTIONS TO BE TAKEN IN HANDLING AND STORAGE 


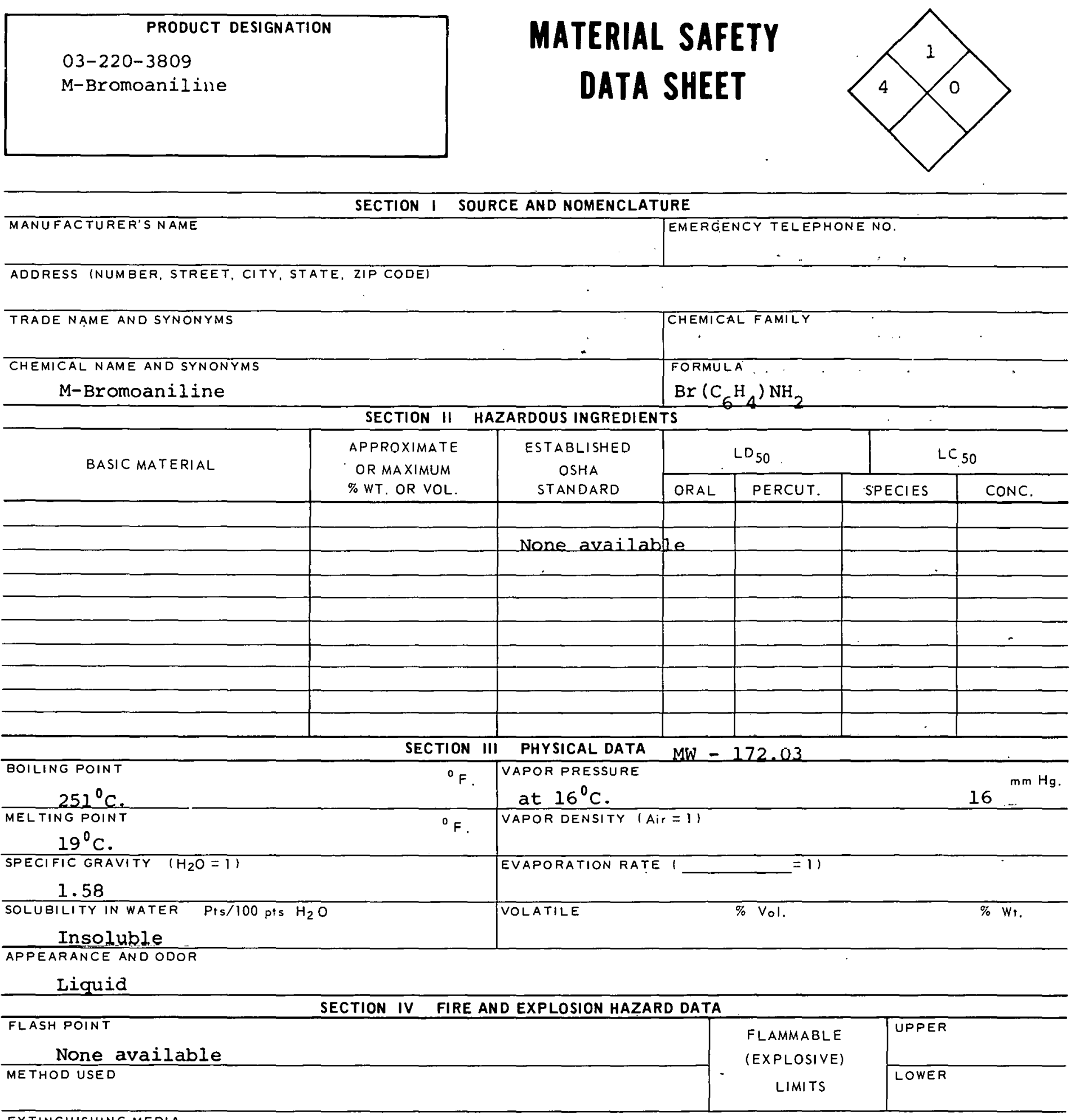

EXTINGUISHING MEDIA

Water spray, carbon dioxide, alcohol foam.

SPECIAL FIRE FIGHTING PROCEDURES

Self-contained breathing apparatus.

UNUSUAL FIRE AND EXPLOSION HAZARUS

Vapor may form explosive mixture in air.
(3) 10-74) 


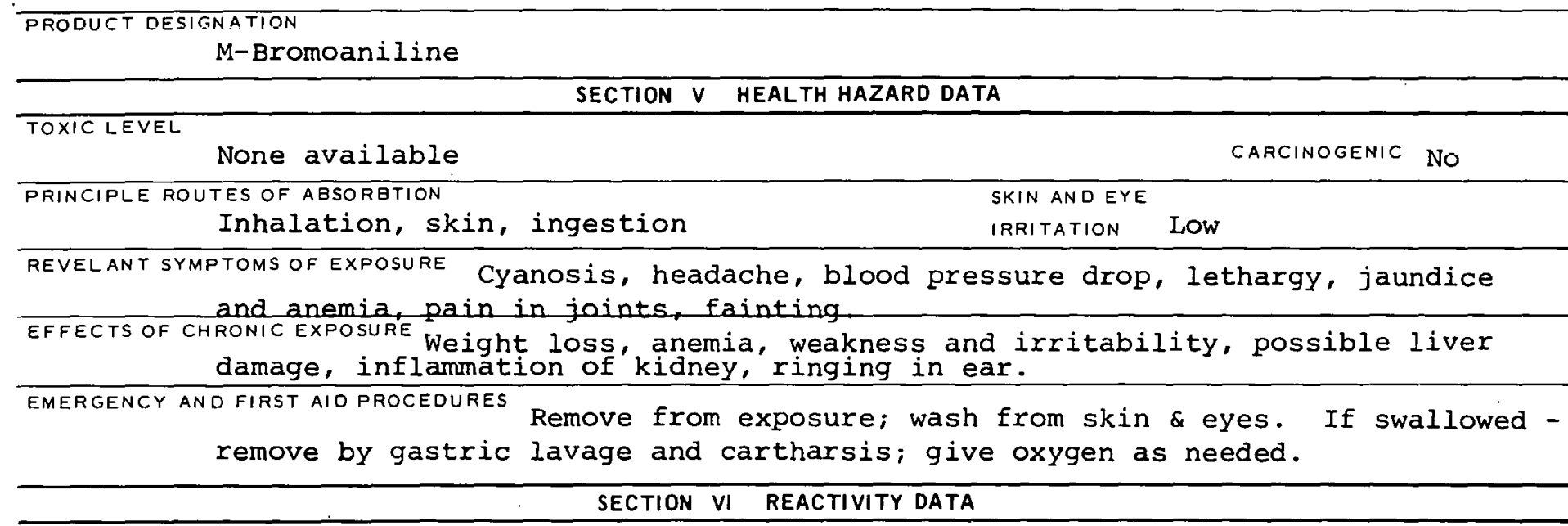

CONDITIONS CONTRIBUTING TO INSTABILITY

CONDITIONS CONTRIBUTING TO HAZARDOUS POLYMERIZATION

INCOMPATABILI TY IMA TERIALS IO AVUIUI

Sodium and potassium peroxide ozone, perchloric acid.

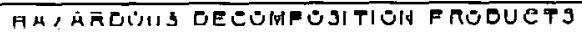

SECTION VII SPILL OR LEAK PROCEDURES

STEPS TO BE TAKEN IN CASE MATERIAL IS RELEASED OR SPILLED

Wear protective clothing during clean up.

WASTE DISPOSAL METHOD

SECTION VIII SPECIAL PROTECTION INFORMATION

\begin{tabular}{|c|c|c|}
\hline \multicolumn{2}{|l|}{ VENTILATION REQUIREMENTS LOCAL EXHAUST } & $\begin{array}{l}\text { PROTECTIVE EQUIFMENT (SPECIFY TYFLS) EYE } \\
\text { COggleg }\end{array}$ \\
\hline \multicolumn{2}{|l|}{ MECHANICAL (GENERAL) } & $\begin{array}{l}\text { GLOVES } \\
\text { Rubber }\end{array}$ \\
\hline \multicolumn{2}{|l|}{ SPECIAL. } & $\begin{array}{l}\text { RFSPIRATOR } \\
\text { Self-contained }\end{array}$ \\
\hline \multicolumn{3}{|l|}{ Protective clothing } \\
\hline \multicolumn{3}{|c|}{$\begin{array}{l}\text { PRECAUTIONS TO BE TAKEN IN HANDLING AND STORAGE } \\
\text { Store in flammable liquids cabinet. }\end{array}$} \\
\hline \multicolumn{3}{|l|}{ OTHER PRECLAUTIONS } \\
\hline SIGNATURE & DATE & \\
\hline
\end{tabular}



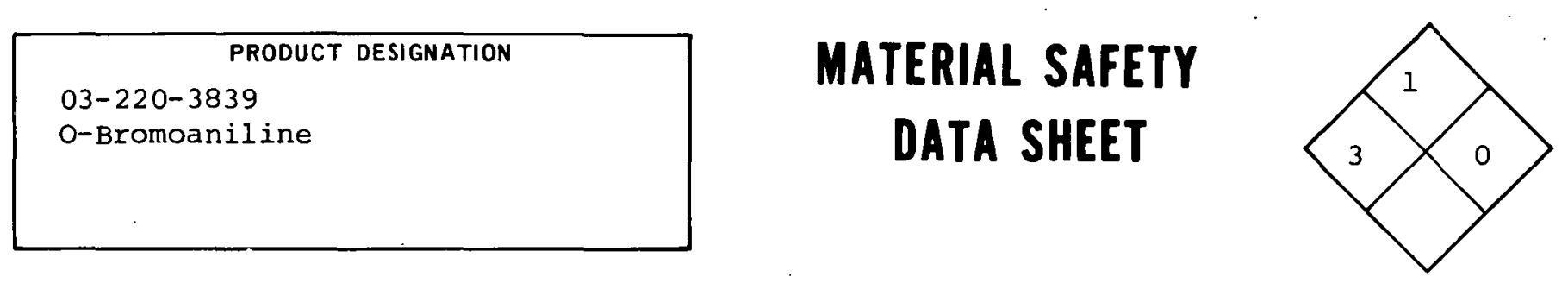

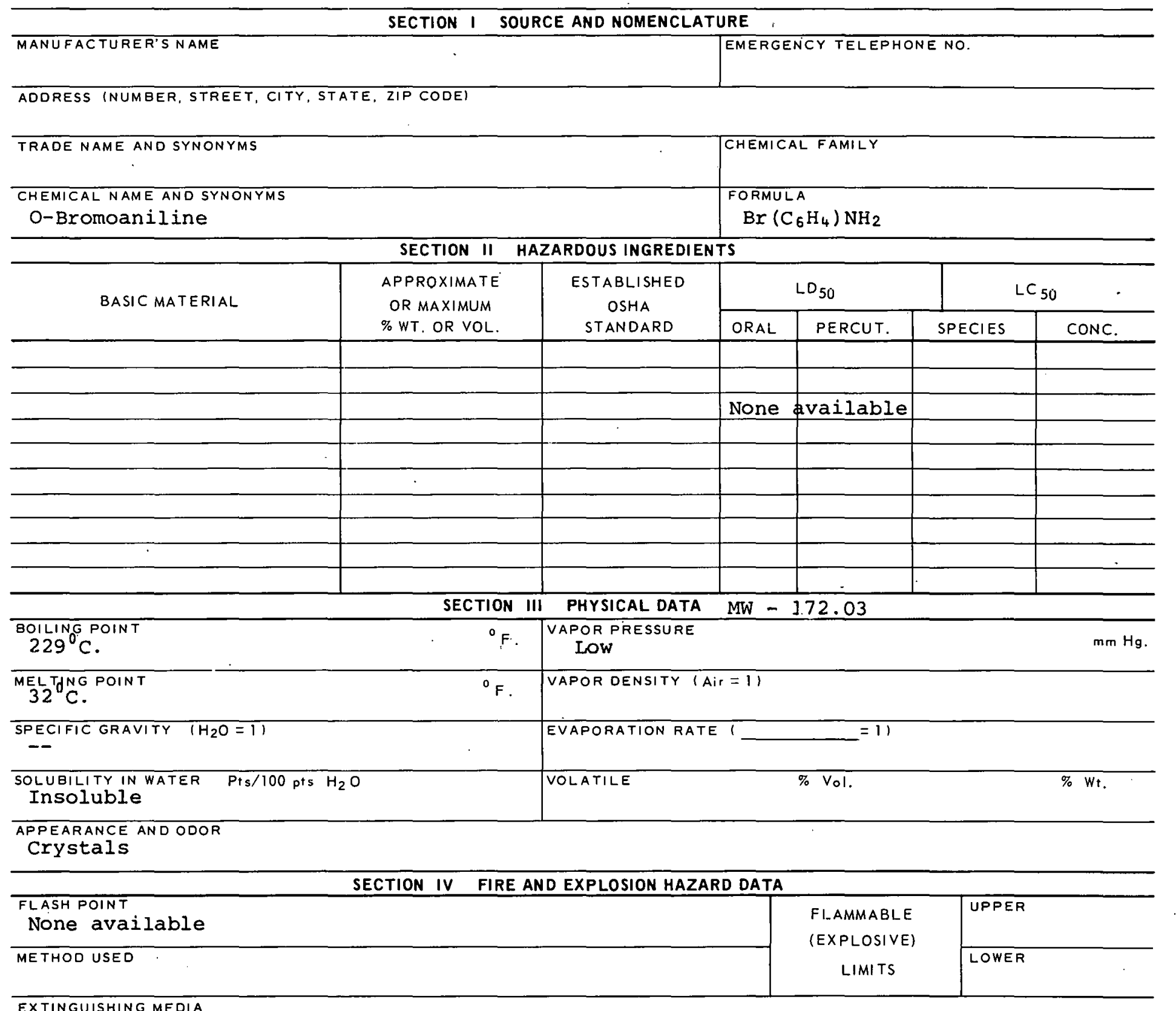

Water spray, carbon dioxide, alcohol foam.

SPECIAL FIRE FIGHTING PROCEDURES

Self-contained breathing apparatus.

UNUSUAL FIRE ANÚ EXPLUSION HAZAROS

Vapor may form explosive mixture in air. 


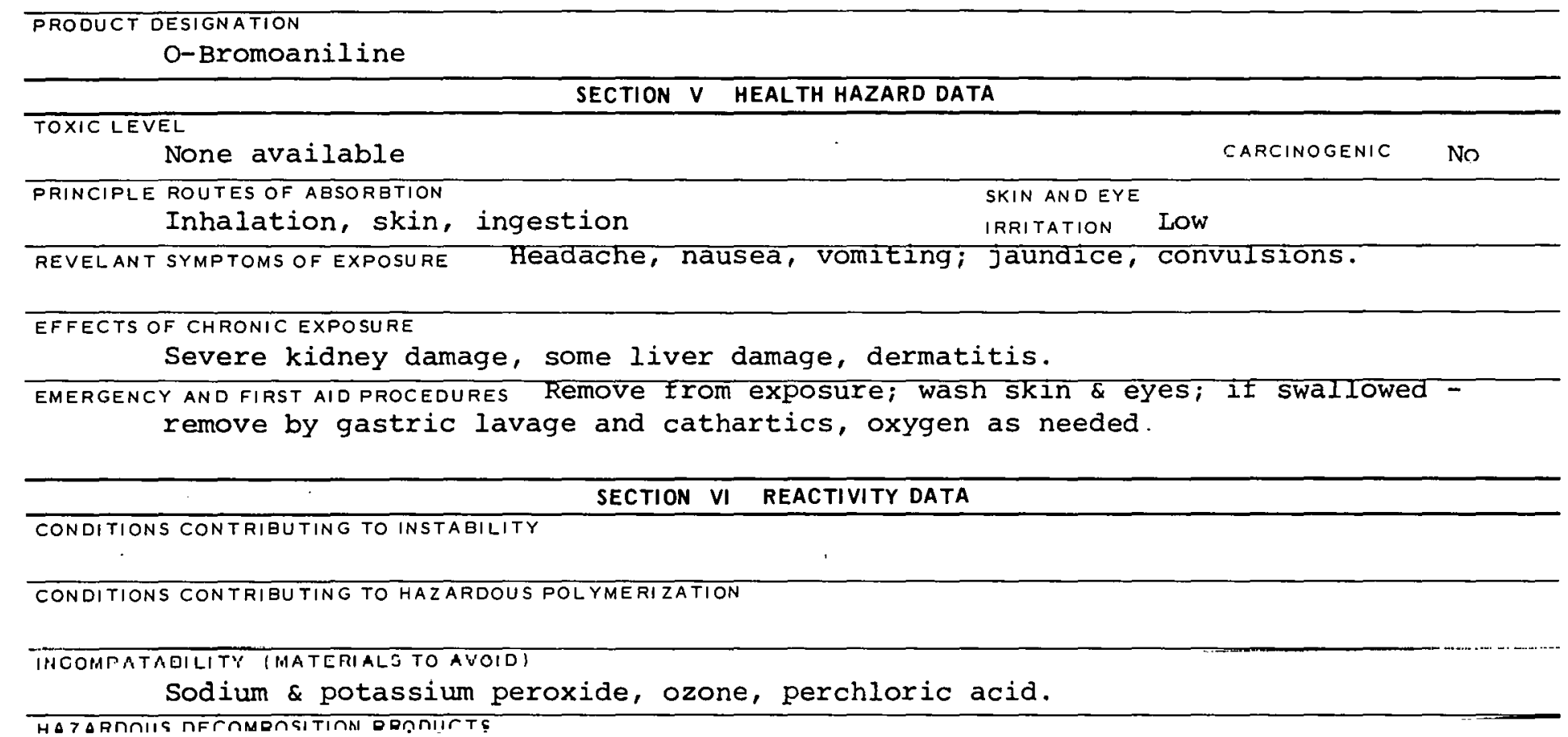

\section{SECTION VII SPILL OR LEAK PROCEDURES}

STEPS TO BE TAKEN IN CASE MATERIAL IS RELEASED OR SPILLED

Wear protective clothing during cleanup.

WASTE DISPOSAL METHOD

SECTION VIII SPECIAL PROTECTION INFORMATION

\begin{tabular}{|c|c|}
\hline VENTILATION REQUIREMENTS LOCAL EXHAUST & $\begin{array}{l}\text { PROTECTIVE EQUIPMENT (STECIFY TYRES) EYE } \\
\text { GOggleS }\end{array}$ \\
\hline MECHANICAL (GENERAL) & $\begin{array}{l}\text { G.oyss } \\
\text { Rubber }\end{array}$ \\
\hline SPECIAL & $\begin{array}{l}\text { RESPIRATRR } \\
\text { Self-contained }\end{array}$ \\
\hline \multicolumn{2}{|l|}{ OTHER PROTECTIVE EQUIPMENT } \\
\hline SECTION IX & SPECIAL ,PRECAUTIONS \\
\hline
\end{tabular}

PRECAUTIONS TO BE TAKEN IN HANDLING AND STORAGE

Store in flammable liquids cabinet. 


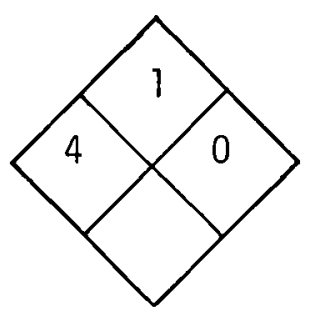

MANUFACTURER'S NAME
ADDRESS INUMBER, STREET, CITY,
TRADE NAME AND SYNONYMS
CHEMICAL NAME AND SYNONYMS
P-BROMOANII InE

\section{SECTION I SOURCE AND NOMENCLATURE}

moaniline

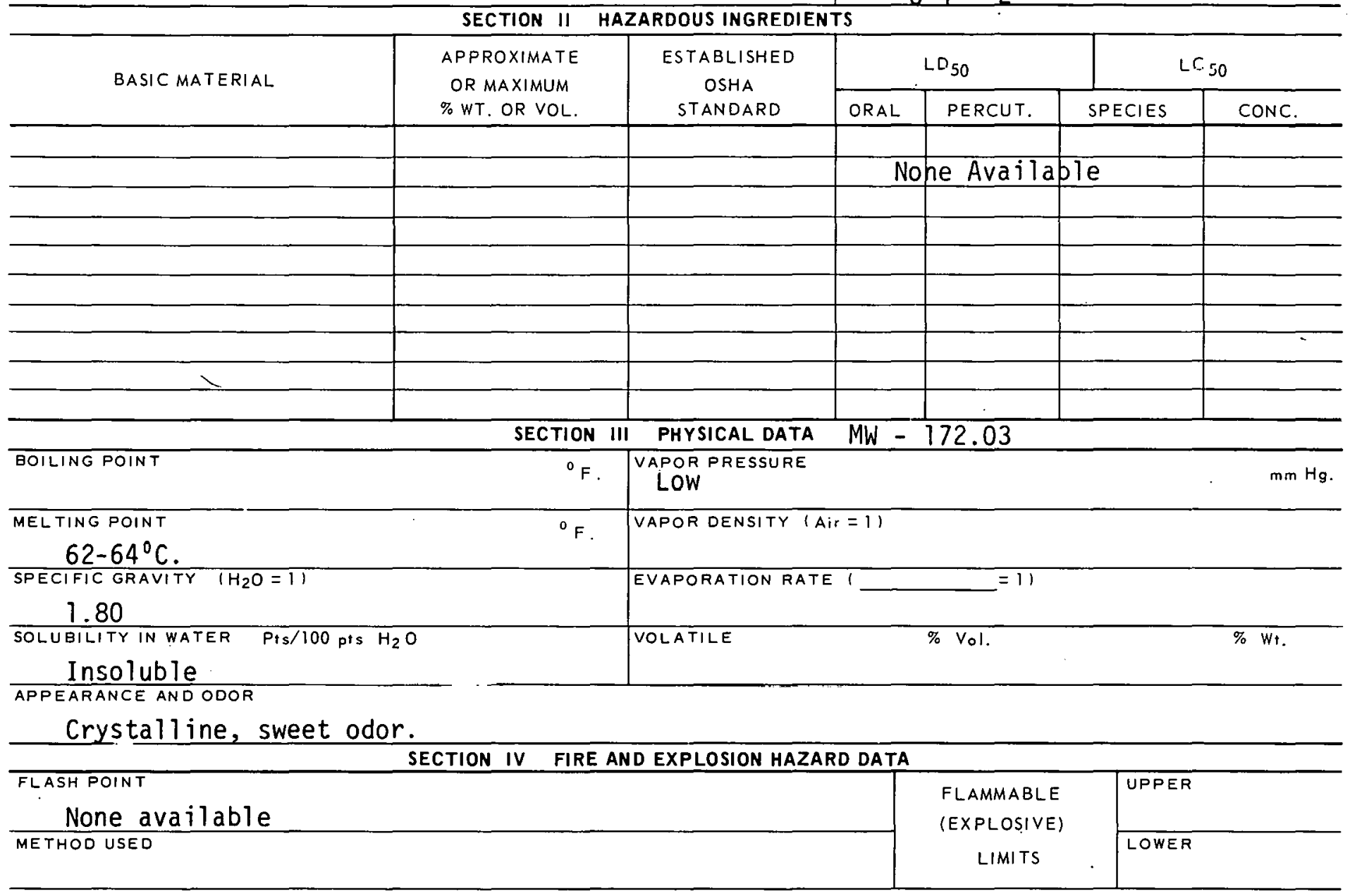

EXTINGUISHING MEDIA

Water spray, carbon dioxide, alcohol foam.

SPECIAL FIRE FIGHTING PROCEDURES

Self-contained breathing apparatus.

UNUSUAL FIRE AND EXPLOSION HAZARDS

Vapor may form explosive mixtures in air. 


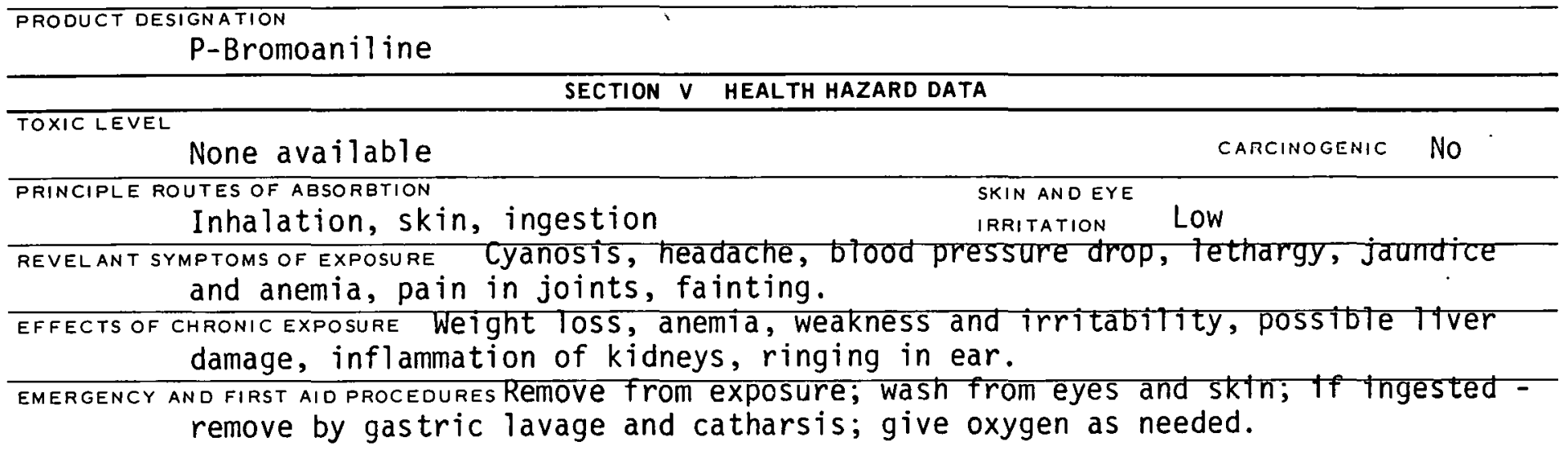

SECTION VI REACTIVITY DATA

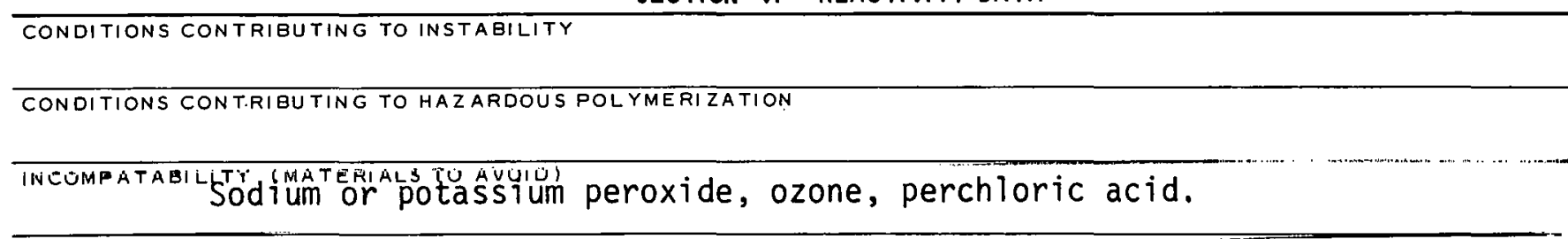

IIAZARDOUG DEGOMROGITIOH RMODUGTG

SECTION VII SPILL OR LEAK PROCEDURES

STEPS TO BE TAKEN IN CASE MA TERIAL IS RELEASED OR SPILLED

Wear protective clothing during clean up.

WASTE DISPOSAL METHOD

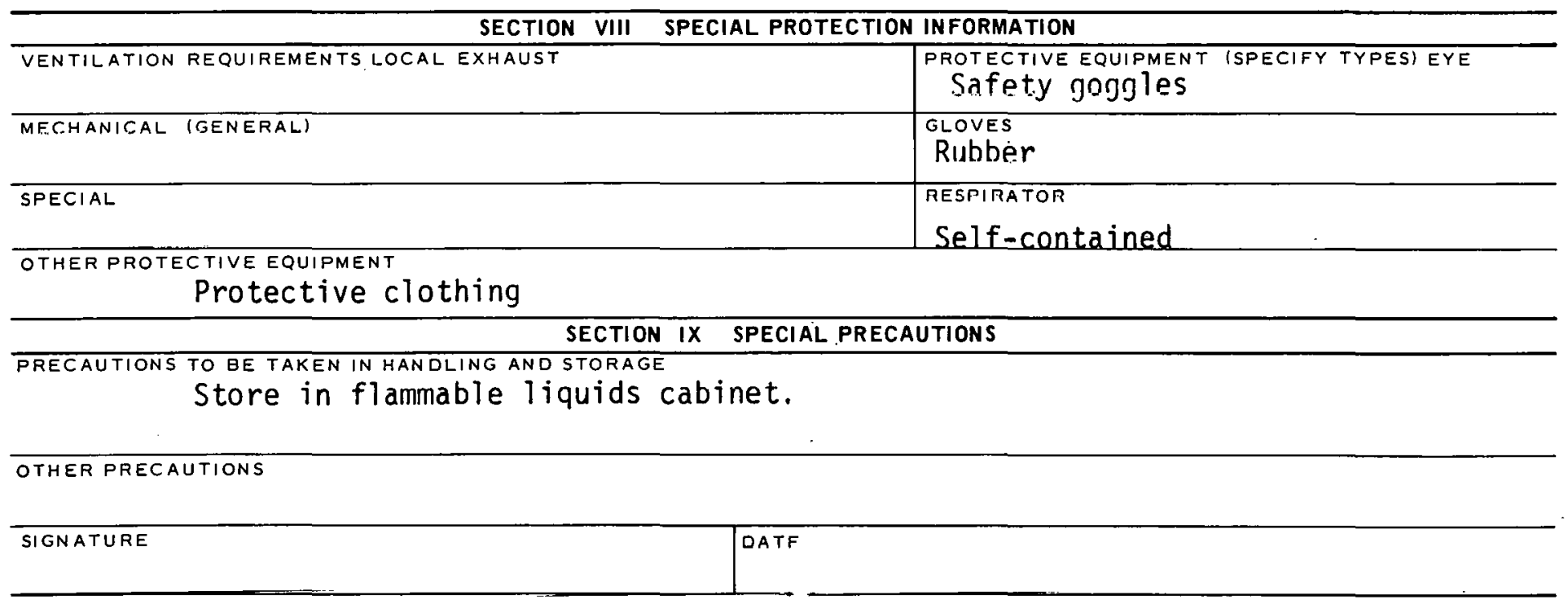


CAS : 000108861

CY90000

PRODUCT DESIGNATION
03-220-4859
Bromobenzene

\section{MATERIAL SAFETY \\ DATA SHEET}

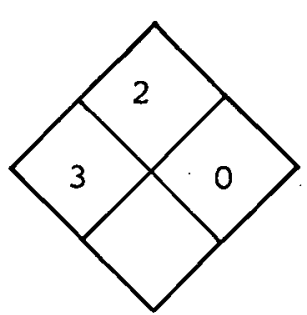

\section{SECTION $1 \cdot$ SOURCE AND NOMENCLATURE}

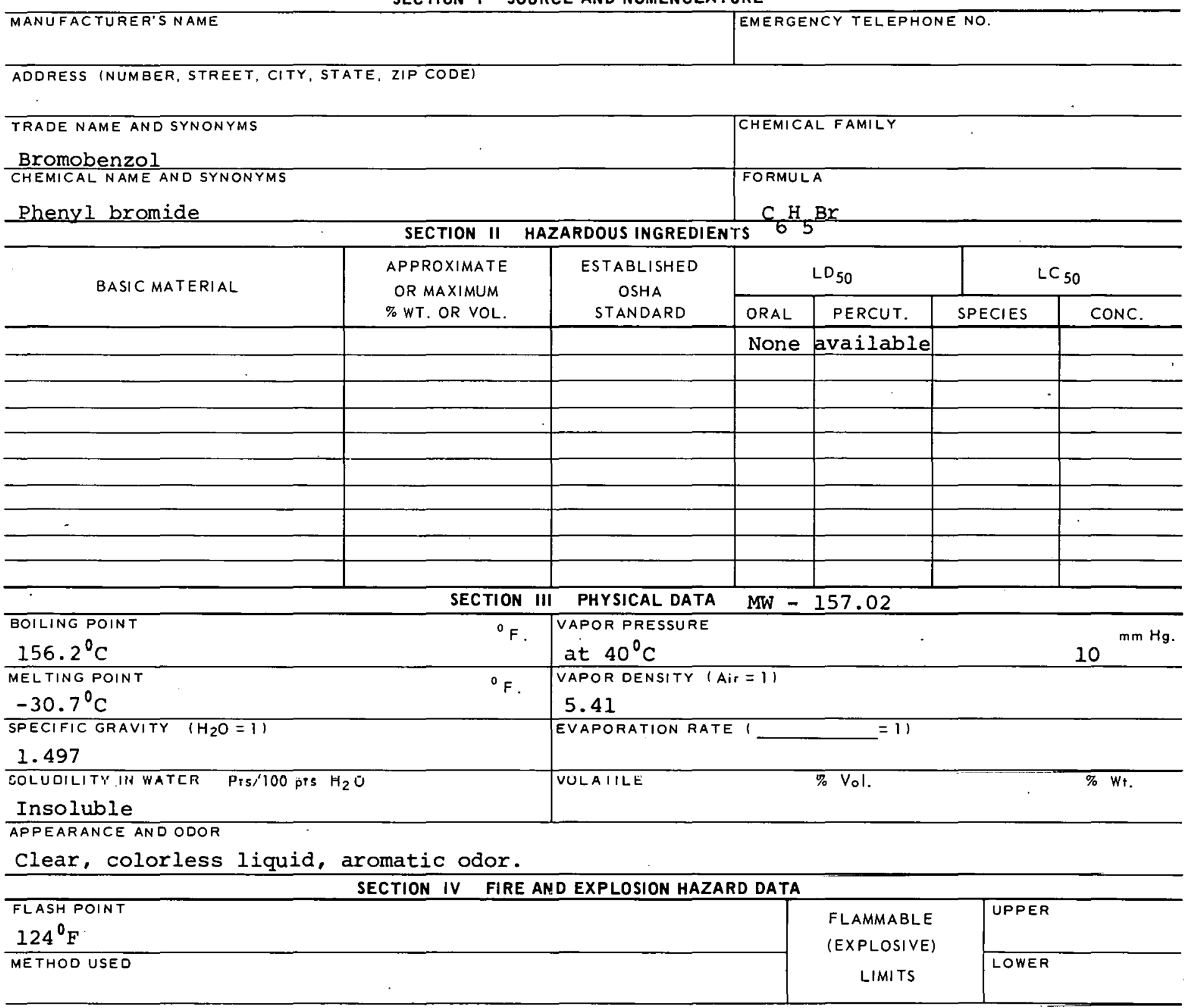

EXTINGUISHINE MEDIA

Water, foam, carbon dioxide, dry chemical

SPECIAL FIRE FIGHTING PROCEDURES Wear goggles and self contained breathing apparatus. 


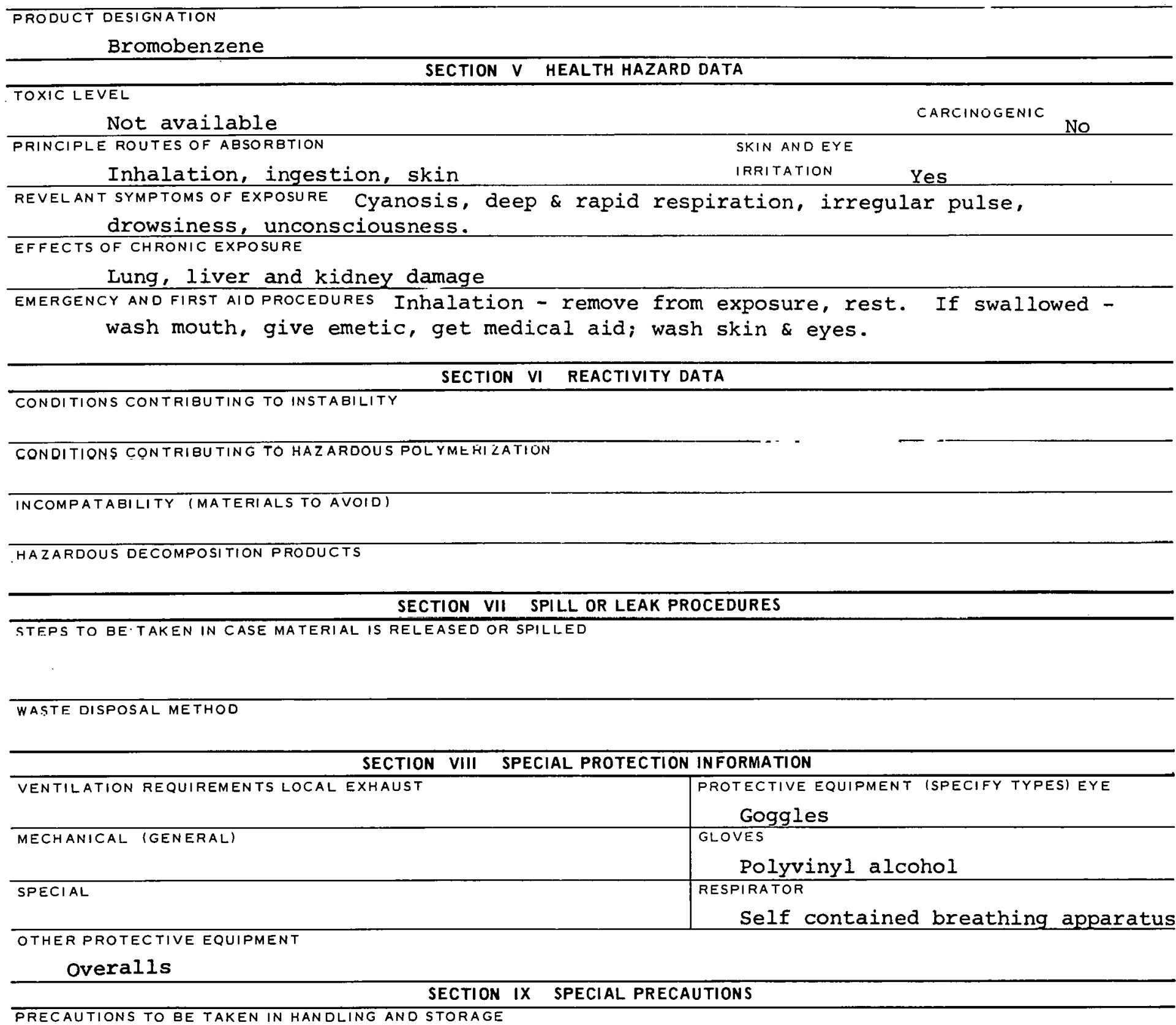

\begin{tabular}{l|l}
\hline OTHER PRECAUTIONS & DATE \\
\hline SIGNATURE & \\
\hline
\end{tabular}


Similar to benzoyl chloride

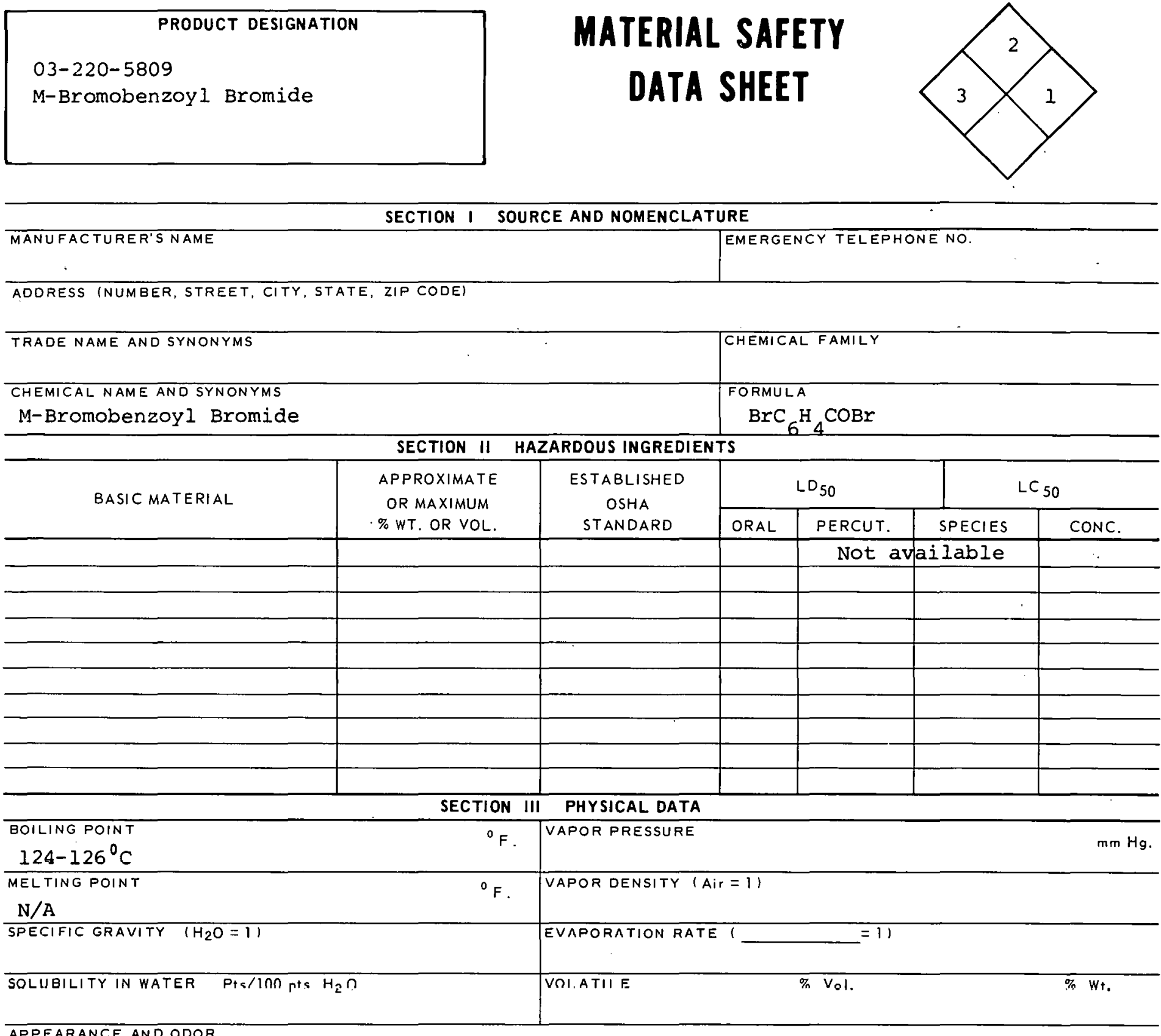

\section{AD AND ODOR}

Colorless liquid

\begin{tabular}{l|c|c}
\hline \multicolumn{2}{c}{ SECTION IV FIRE AND EXPLOSION HAZARD DATA } \\
\hline FLASH POINT & FLAMMABLE \\
N/A & UPPER & (EXPLOSIVE) \\
\cline { 2 - 3 } METHOD USED & LIMITS & \\
\hline
\end{tabular}

EXTINGU!SHING MEOIA Use water spray sparingly, foam, carbon dioxide or dry chemical.

\section{SPECIAL FlRE FIGHTING PROCEDUREShing.}




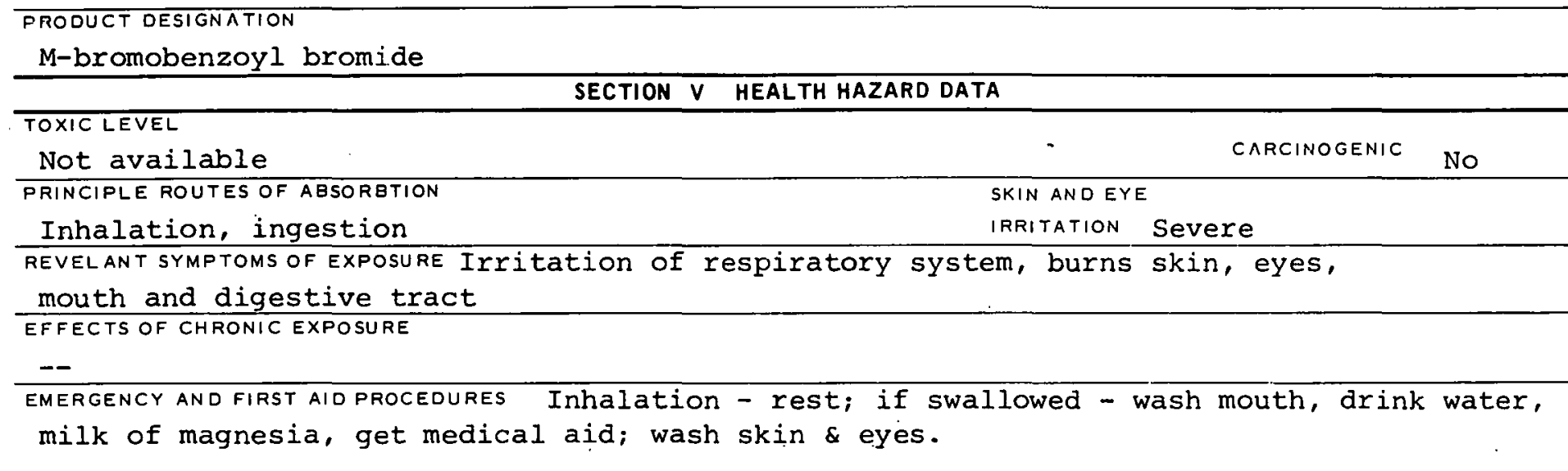

\section{SECTION VI REACTIVITY DATA}

CONDITIONS CONTRIBUTING TO INSTABILITY

Reacts with oxidizing materials

CONOITIONS CONTRIBUTING TO HAZARDOUS POLYMERIZATION

INCOMPATABILITY (MATERIALS TO AVOID)

HAZAHUOUS UELUMRUSIIIUN HRUUUL IS

SECTION VII SPILL OR LEAK PROCEDURES

STEPS TO BE TAKEN IN CASE MATERIAL IS RELEASED OR SPILLED

WASTE DISPOSAL METHOD

SECTION VIII SPECIAL PROTECTION INFORMATION

\begin{tabular}{l|l}
\hline VENTILATION REQUIREMENTS LOCAL EXHAUST & $\begin{array}{c}\text { SPOTECTIVE EQUIPMENT (SPECIFY TYPES) EYE } \\
\text { GOggles }\end{array}$ \\
\hline MECHANICAL (GENERAL) & $\begin{array}{c}\text { GLOVES } \\
\text { Rubber }\end{array}$ \\
\hline EREGIAL- & RESPIRATOR \\
& Self-contained \\
\hline OTHER PROTECTIVE EQUIPMENT.
\end{tabular}

OTHER PROTECTIVE EQUIPMENT.

SECTION IX SPECIAL PRECAUTIONS

PRECAUTIONS TO BE TAKEN IN HANDLING AND STORAGE

OTHER PRECAIITIONS

SISN $\triangle T I I R F$

DNTE

.

$220-5809 B$ 
CAS : 000074975

PA52500

PRODUCT DESIGNATION

03-220-6809

Bromochloromethane

MATERIAL SAFETY DATA SHEET

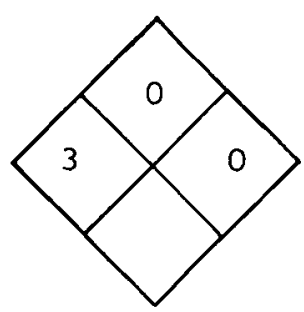

SECTION I SOURCE AND NOMENCLATURE

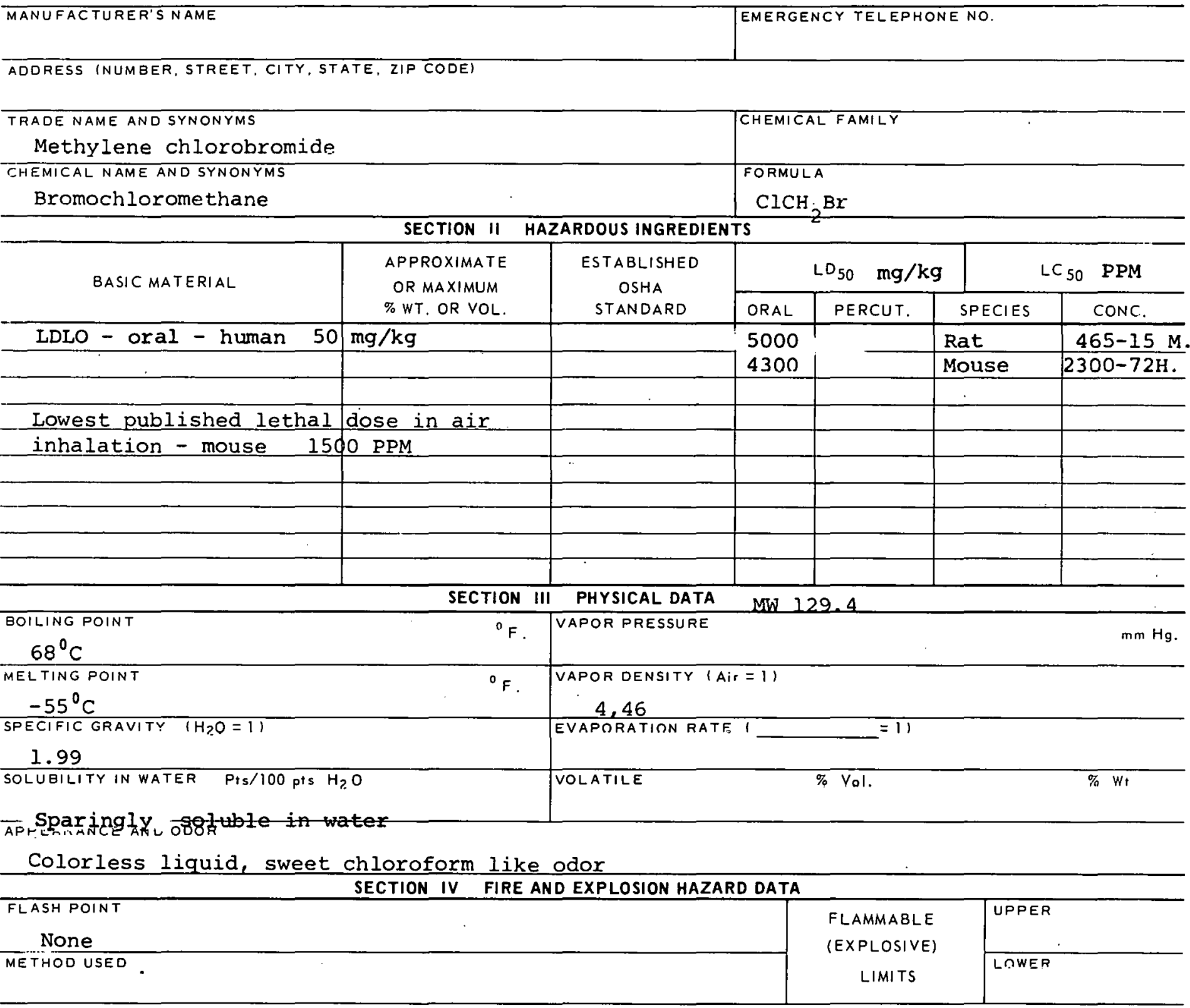

EXTINGUISHING MEDIA

SPECIAL FIRE FIGHTING PROCEDURES

UNUSUAL FIRE AND EXPLOSION HAZARDS

May emit toxic fumes when heated. 


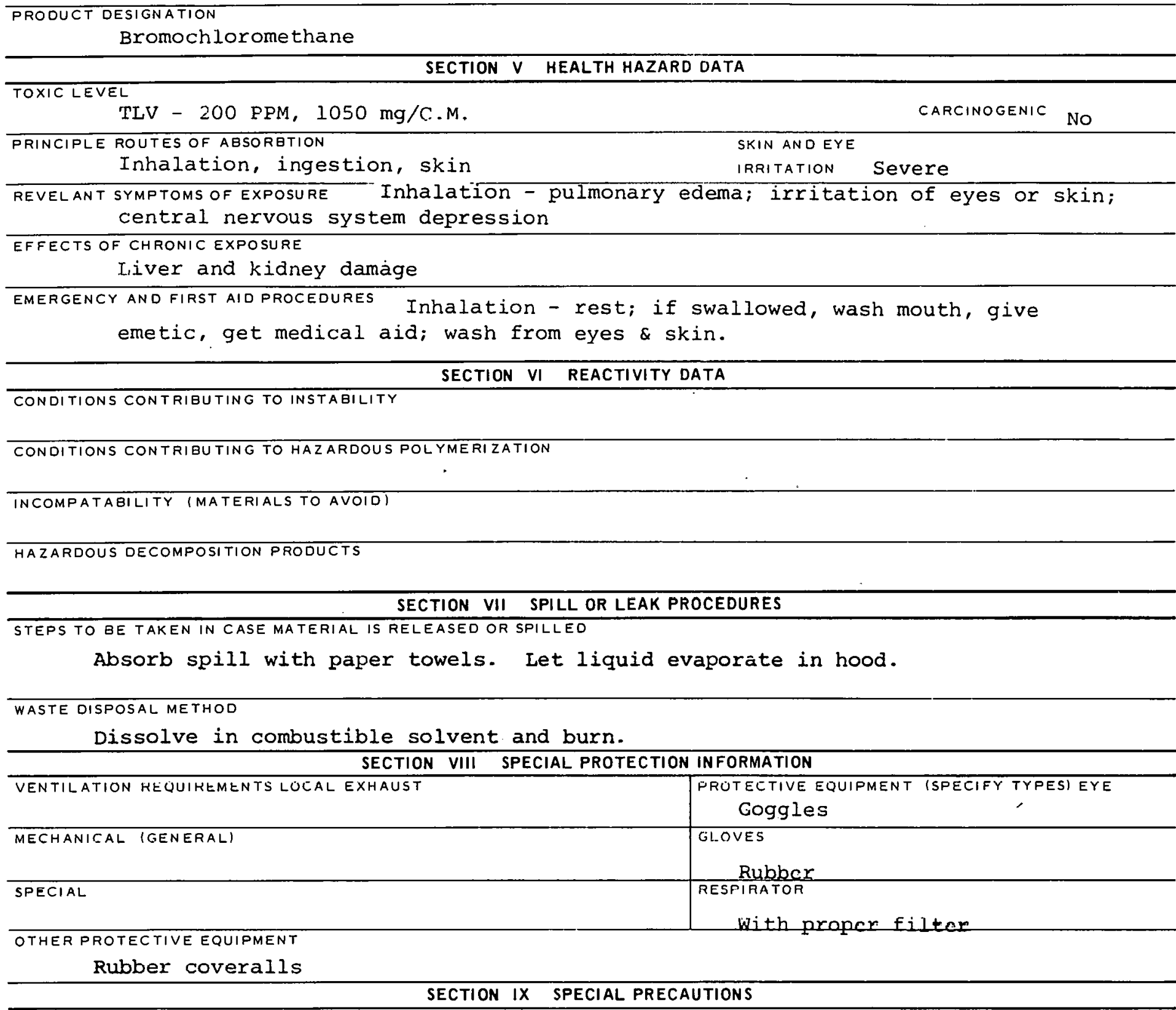
PRECAUTIONS TO BE TAKEN IN HANDLING AND STORAGE 


\begin{tabular}{|l|}
\hline \multicolumn{1}{|c|}{ PRODUCT DESIGNATION } \\
03-220-7809 \\
p-Bromodiethylaniline \\
\hline
\end{tabular}

\section{MATERIAL SAFETY DATA SHEET}

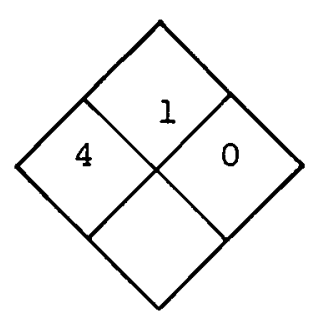

\section{SECTION I SOURCE AND NOMENCLATURE}

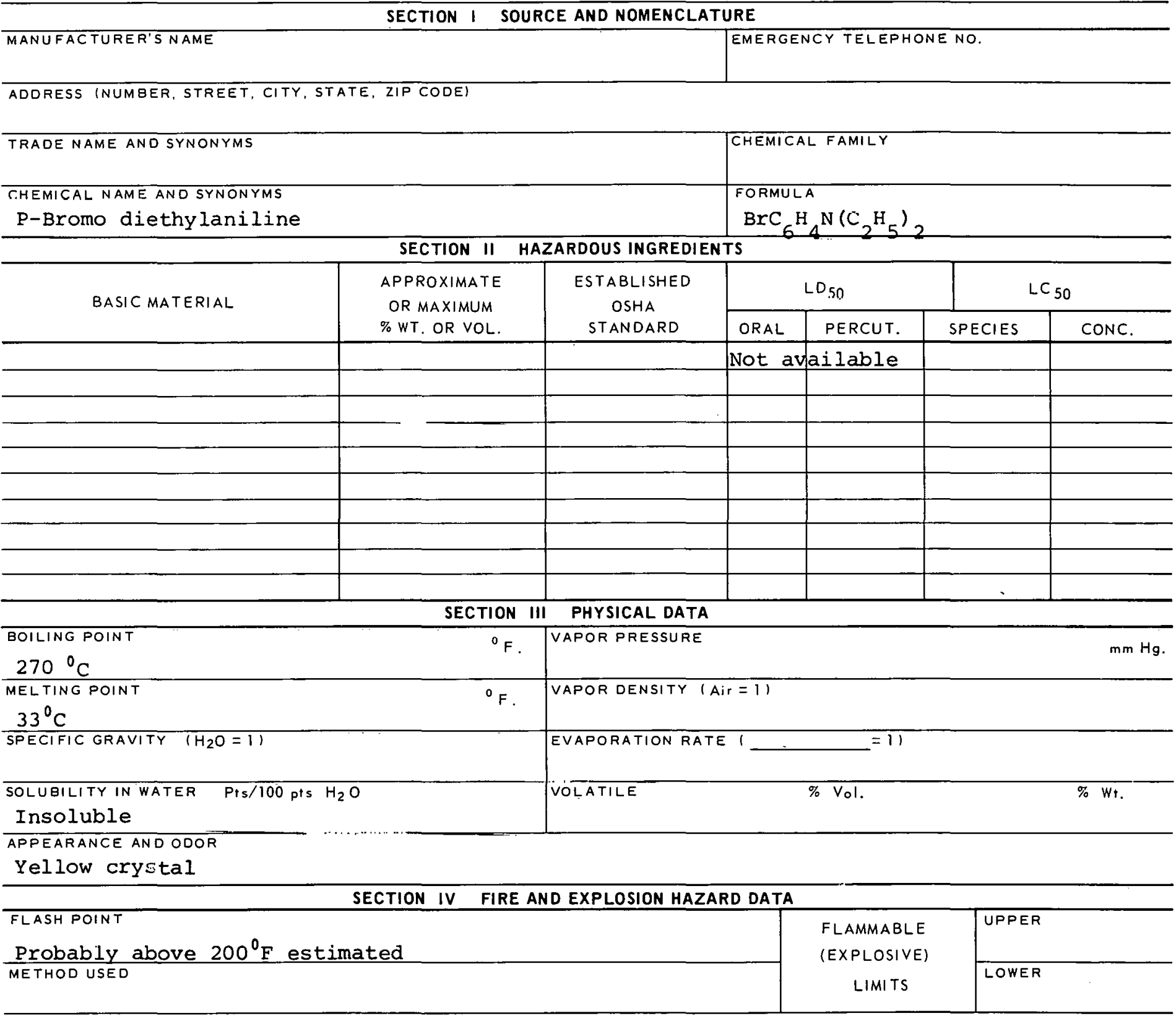

EXTINGUISHING MEDIA Alcohol foam, water spray, carbon dioxide, dry chemical

SPECIAL FIRE FIGHTING PROCEOURES

UNUSUAL FIRE AND EXPLOSION HAZARDS 


\section{TOXIC LEVEL}

Not available

CARTINOGENIC

No

\begin{tabular}{|c|c|c|c|c|}
\hline PRINCIPLE ROUTES OF ABSORBTION & & & SKIN AND EYE & \\
\hline Inhalation, ingestion, skin & & & IRRITATION & $y$ \\
\hline $\begin{array}{c}\text { REVELANT SYMPTOMS OF EXPOSURE Weakness, } \\
\text { nervous system depressant }\end{array}$ & tremors, & cyanosis, & drowsine & \\
\hline
\end{tabular}

EMERGENCY AND FIRST AID PROCEDURES Inhalation - rest, get medical aid; if swallowed -

wash mouth, give emetic; get medical aid; wash from skin \& eyes.

\section{SECTION VI REACTIVITY DATA}

CONDITIONS CONTRIBUTING TO INSTABILITY

CONDITIONS CONTRIBUTTING TO HAZARDOUS POLYMERIZATION

INCOMPATABILITY TMATERIALS TOO AVOOOI

HAZARDOUS DECOMPOSITION PRODUCTS

SECTION VII SPILL OR LEAK PROCEDURES

STEPS TO BE TAKEN IN CASE MATERIAL IS RELEASED OR SPILLED

WASTE DISPOSAL METHOD

SECTION VIII SPECIAL PROTECTION INFORMATION

\begin{tabular}{l|l}
\hline \multicolumn{1}{|c|}{ SECTION VIII SPECIAL PROTECTION INFORMATION } \\
\hline MECHANICAL IGENERALI & $\begin{array}{c}\text { PROTECTIVE EQUIPMENT (SPECIFY TYPESI EYE } \\
\text { Goggles }\end{array}$ \\
\hline SPECIAL & $\begin{array}{c}\text { GLOVES } \\
\text { Gloves }\end{array}$ \\
\hline
\end{tabular}

OTHER PROTECTIVE EQUIPMENT

SECTION IX SPECIAL PRECAUTIONS

PRECAUTIONS TO BE TAKEN IN HANDLING AND STORAGE

OTHER PRECAUTIONS

SIGNATURE

DATE

$220-7809 B$ 
03-220-8309

P-Bromodimethylaniline
MATERIAL SAFETY

DATA SHEET

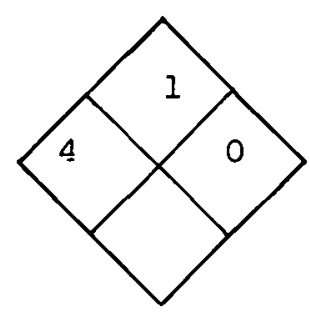

\section{SECTION I SOURCE AND NOMENCLATURE}

MANUFACTURER'S NAME

|EMERGENCY TELEPHONE NO.

ADDRESS (NUMBER, STREET, CITY, STATE, ZIP CODE)

TRADE NAME AND SYNONYMS

P-bromo, $N, N$ 'dimethyl aniline CHEMICAL NAME AND SYNONYMS

P-bromodimethylaniline

BASIC MATERIAL

LDLO oral - rat $500 \mathrm{mg} / \mathrm{k}$

\begin{tabular}{l|l} 
& CHEMICAL FAMILY \\
\hline & FORMULA \\
& $\operatorname{BrC}_{6} \mathrm{H}_{4} \mathrm{~N}\left(\mathrm{CH}_{3}\right)_{2}$
\end{tabular}

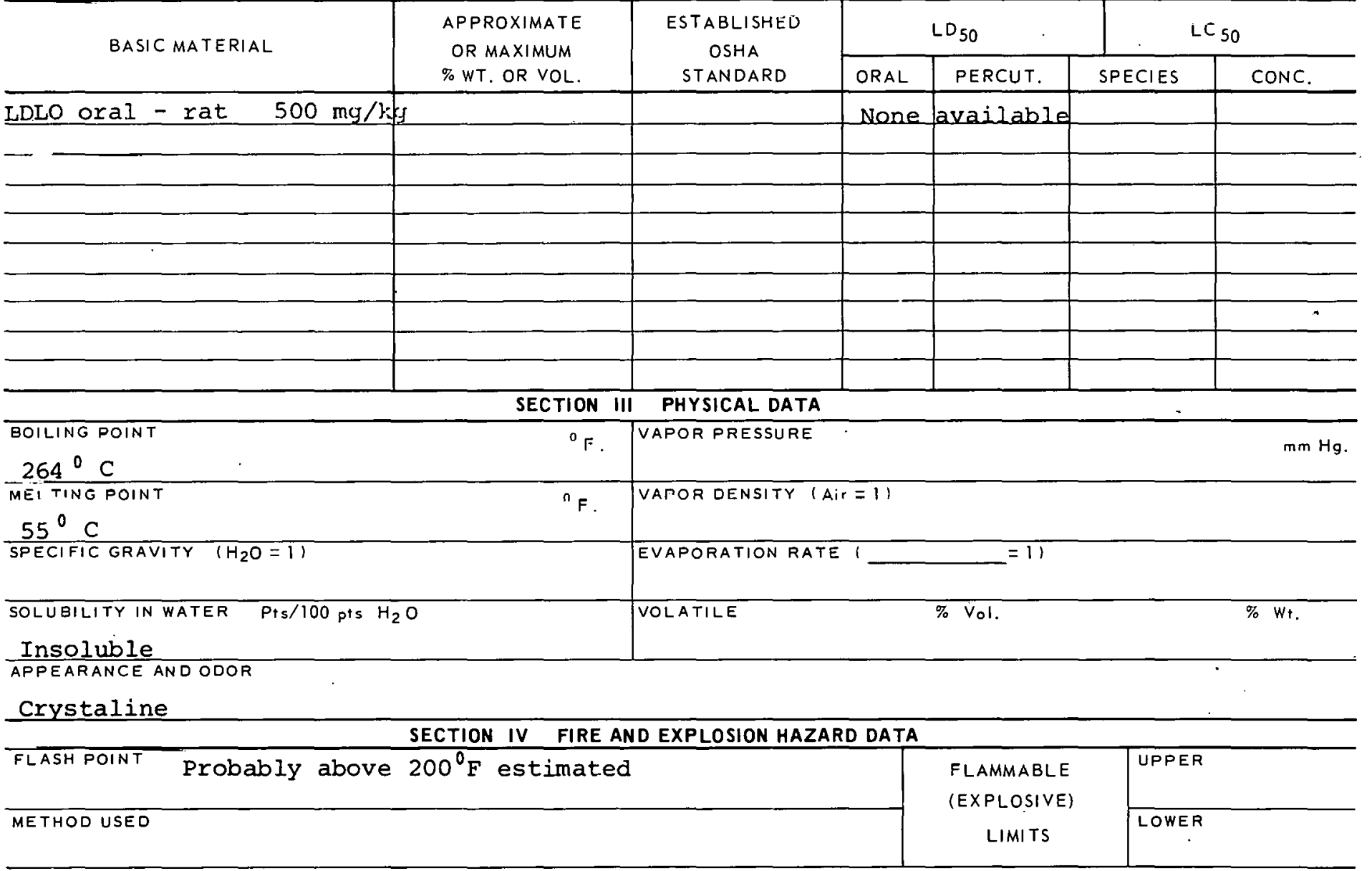

EXTINGUISHING MEDIA

Alcohol foam, water spray, carbon dioxide, ary chemical SPECIAL FIRE FIGHTING PROCEDURES 


\section{TOXICLEVEL}

Not available

PRINCIPLE ROUTES OF ABSORETION

Inhalation, ingestion, skin

REVELANT SYMPTOMS OF EXPOSURE Weakness, tremors, cyanosis, drowsiness, central nervous

system depressant

EFFECTS OF CHRONIC EXPOSURE Fatigue, loss of appetite, headache, dizziness

EMERGENCY AND FIRST AIO PROCEDURES Inhalation - rest, get medical aid; if swallowed - wash mouth, give emetic, get medical aid; wash from eyes \& skin.

\section{SECTION VI REACTIVITY DATA}

CONDITIONS CONTRIBUTING TO INSTABILITY

CONDITIONS CONTRIBUTING TO HAZARDOUS POLYMERIZATION

INCOMPATABILITY (MATERIALS TO AVQID)

HAZARDOUS DECOMPOSITION PRODUCTS

SECTION VII SPILL OR LEAK PROCEDURES

STEPS TO BE TAKEN IN CASE MATERIAL IS RELEASED OR SPILLED

WASTE DISPOSAL METHOD

SECTION VIII SPECIAL PROTECTION INFORMATION

\begin{tabular}{l|l}
\hline VENTILATION REQUIREMENTS IOOCAL EXHAUST & $\begin{array}{l}\text { PROTFC.TIV: EQUIPMENT (SPECIFY TYPES) EYE } \\
\text { Goggles }\end{array}$ \\
\hline MECHANICAL IGENERALI & $\begin{array}{r}\text { GLOVES } \\
\text { Rubber }\end{array}$ \\
\hline SPECIAL & $\begin{array}{r}\text { RESPIRATOR } \\
\text { With proper filter }\end{array}$ \\
\hline
\end{tabular}

OTHER PROTECTIVE EQUIPMENT

SECTION IX SPECIAL PRECAUTIONS

PRECAUTIONS TO BE TAKEN IN HANDLING AND STORAGE

OTHER PRECAUTIUNS

SIGNATURE

DATE

$220-8309 B$ 
03-220-8809

2 Bromo-1, 4-dimethybenzene
MATERIAL SAFETY DATA SHEET

Similar to xylere

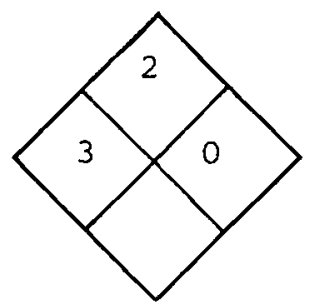

SECTION I SOURCE AND NOMENCLATURE

\begin{abstract}
MANUFACTURER'S NAME

\section{TRADE NAME AND SYNONYMS \\ CHFMIC $\triangle$ I. NAME AND SYNONYMS \\ 2-Bromo-P-XYlene}

ADDRESS (NUMBER, STREET, CITY, STATE, ZIP CODE)
Note:

Lowest published toxic concentration in air

inhalation - human 75 PPM 110 months \begin{tabular}{l|l} 
& CH \\
\hline & $F O R$
\end{tabular} SECTION $\|$ HAZARDOUS INGREDIENTS

EMERGENCY TELEPHONE NO.

$$
\mathrm{BrC}_{6} \mathrm{H}_{3}\left(\mathrm{CH}_{3}\right)_{2}
$$

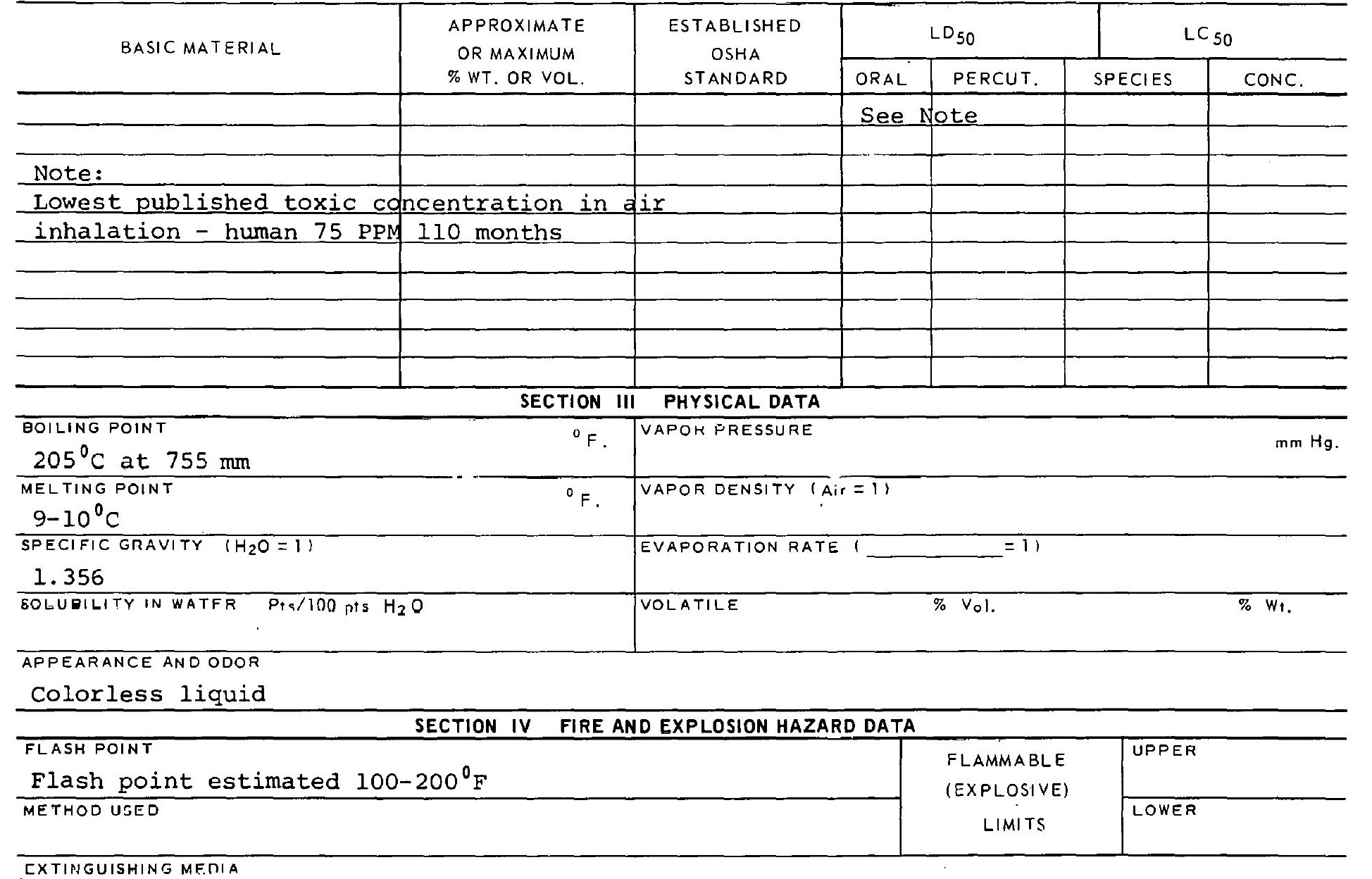

Foam, carbon dioxide, dry chemical

water spray may be used to reduce rate of burning

SPECIAL FIRE FIGHTING PROCEDURES

Wear self contained breathing apparatus

UNUSUAL FIRE AND EXPLOSION HAZARDS 
TOXIC LEVEL

Not available PRINCIPLE ROUTES OF ABSORBTION

Inhalation, ingestion REVELANT SYMPTOMS OF EXPOSURE Irritation of respiratory tract, disturbance of vision, dizziness, nausea, collapse, coma; burning of skin or eyes. EFFECTS OF CHRONIC EXPOSURE

Dermatitis, blood \& liver damage

EMERGENCY AND FIRST AID PROCEDURES Inhalation - rest, get medical aid; if swallowed - give saline cathartics and demulcents, get medical aid; wash skin \& eyes.

\section{SECTION VI REACTIVITY DATA}

CONDITIONS CONTRIBUTING TO INSTABILITY

CONDITIONS CONTRIBUTING TO HAZARDOUS POLYMERIZATION

INCOMPATABILITY IMATERIALS TO AVOIOI

HAZAROOIIS DFCOMPOSITION PRODUGTS

SECTION VII SPILL OR LEAK PROCEDURES

STEPS TO BE TAKEN IN CASE MATERIAL IS RELEASED OR SPILLED

WASTE DISPOSAL METHOD

SECTION VIII SPECIAL PROTECTION INFORMATION

VENTILATION.REQUIREMENTS LOCAL EXHAUST

MECHANICAL (GENERAL)

SPECIAL

OTHER PROTECTIVE EQUIPMENT
CARCINOGENIC No SKIN ANO EYE

IRRITATION 


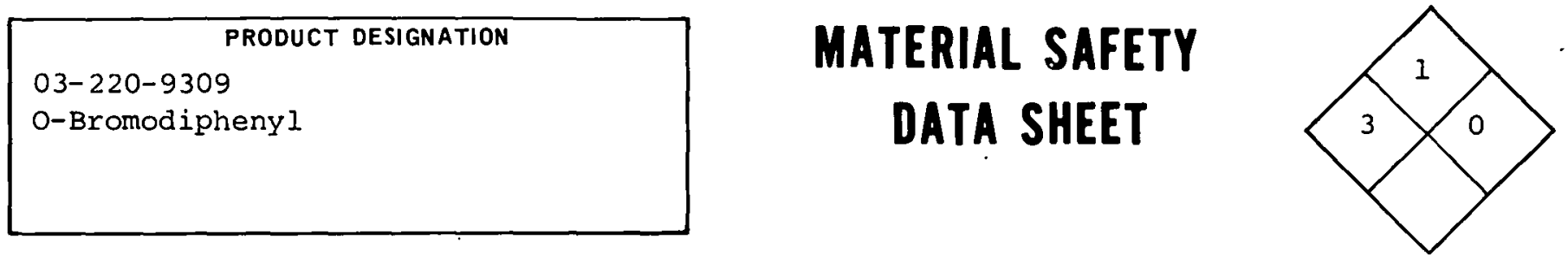

\section{SECTION I SOURCE AND NOMENCLATURE}

MANUFACTURER'S NAME
ADDRESS INUMBER, STREET, CITY
TRADE NAME AND SYNONYMS
CHEMICAL NAME AND SYNONYMS
O-bYOMOdIPhENYI

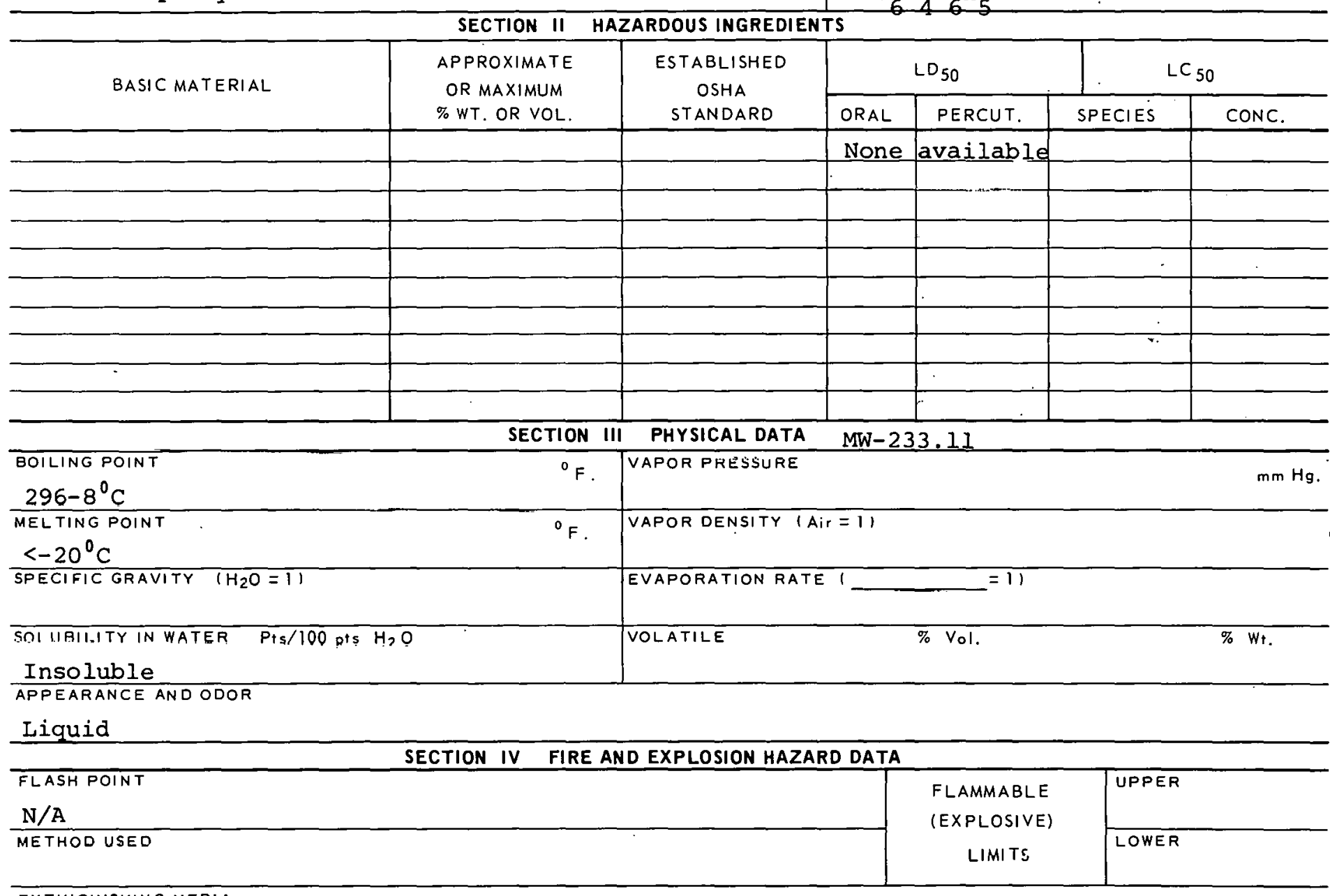

EXTINGIIISHING MEDIA

\begin{tabular}{l|l} 
& CHEMICAL FAMILY \\
\hline SECTION II HAZARDOUS INGREDIENTS & $\begin{array}{l}\text { FORMULA } \\
\text { BrC }\end{array} 6{ }_{4} \mathrm{C}_{6} \mathrm{H}_{5}$
\end{tabular}

Water, foam, carbon dioxide, dry chemical

SPECIAL FIRE FIGHTING PROCEDURES

UNUSUAL FIRE AND EXPLOSION HAZARDS 
O-Bromodiphenyl

\section{SECTION $V$ HEALTH HAZARD DATA}

TOXIC LEVEL

Not available

PRINCIPLE ROUTES OF ABSORETION

Inhalation, ingestion skin

REVELANT SYMPTOMS OF EXPOSURE

Acne, irritation of respiratory passages

EFFECTS OF CHRONIC EXPOSURE

\section{Liver damage}

EMERGENCY AND FIRST AID PROCEDURES Inhalation - rest, get medical aid; if swallowed, wash mouth, give emetic, get medical aid, wash skin \& eyes. SECTION VI REACTIVITY DATA

CONDITIONS CONTRIBUTING TO INSTABILITY

CONDITIONS CONTRIBUTING TO HAZARDOUS POLYMERIZ̈ATION

INCOMPATABILITY IMATERIALS TO AVOID)

HAZARDOUS DECOMPOSITION PRODUCTS

SECTION VII SPILL OR LEAK PROCEDURES

STEPS TO BE TAKEN IN CASE MATERIAL IS RELEASED OR SPILLED

WASTE DISPOSAL METHOD

SECTION VIII SPECIAL PROTECTION INFORMATION

\begin{tabular}{|c|c|}
\hline VENTILATION REQUIREMENTS LOCAL EXHAUST & $\begin{array}{l}\text { PROTECTIVE EQUIPMFNT (SPEGIFY TYPES) EYC } \\
\text { GOggles }\end{array}$ \\
\hline MECHANICAL (GENFRAL) & $\begin{array}{l}\text { GLOVES } \\
\text { Rubber }\end{array}$ \\
\hline$\overline{S P E C I} \overline{A L}$ & $\begin{array}{l}\text { RESPIRATOR } \\
\text { Solt oontained breatiling appa }\end{array}$ \\
\hline
\end{tabular}

OTHER PROTEC̈TIVE EQUIPMENT

\section{SECTION IX SPECIAL PRECAUTIONS}

PRECAUTIONS TO BE TAKEN IN HANDLING AND STORAGE

OTHER PRECAUTIONS

SIGNATURE

DATE

$220-9309 B$ 

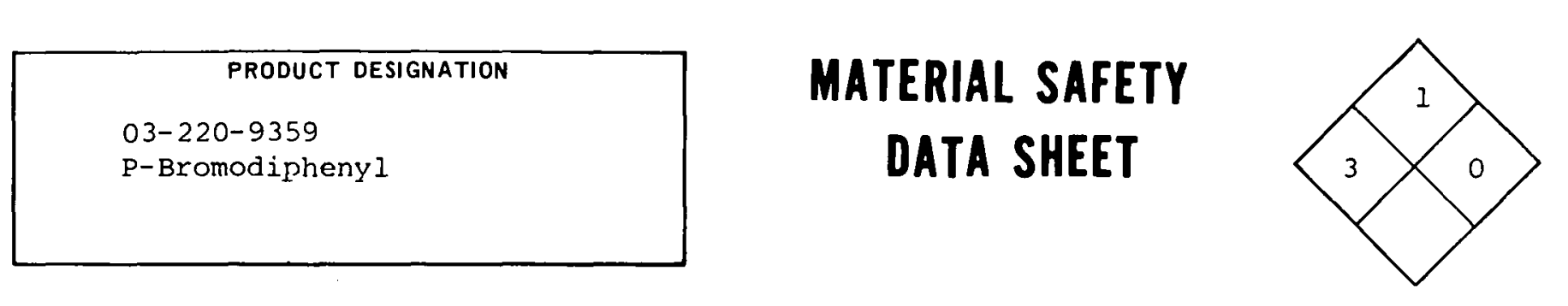

SECTION I SOURCE AND NOMENCLATURE

\begin{tabular}{l|l|l}
\hline MANUFACTURER'S NAME & EMERGENCY TELEPHONE NO. \\
\hline ADDRESS INUMBER, STREET, CITY, STATE, ZIP CODE)
\end{tabular}

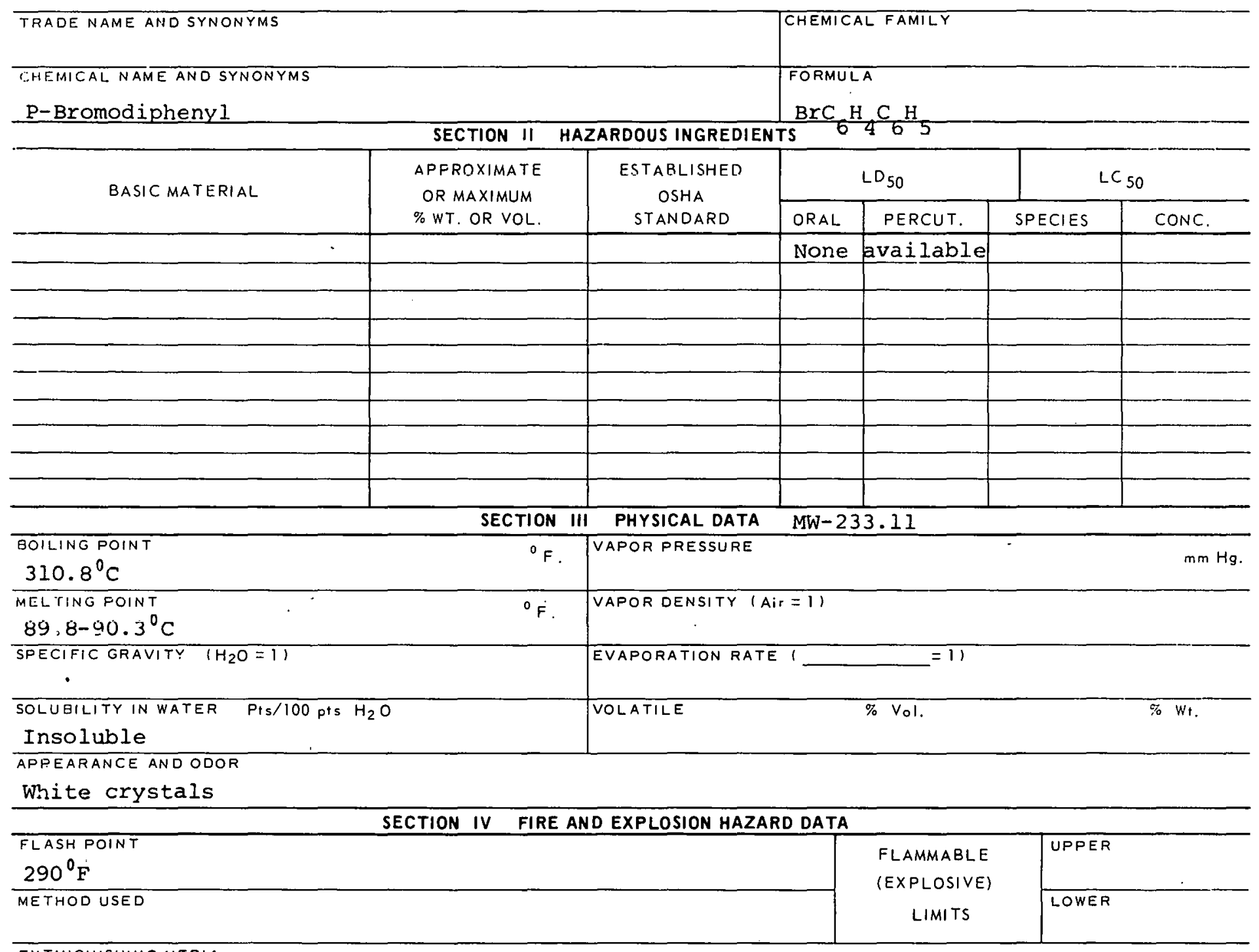

EXTINGUISHING MEDIA

Water, foam, carbon dioxide, dry chemical

SPECIAL FIRE FIGHTING PROCEDURES

UNUSUAL FIHE ANU EXPLOSION HAZARDS 


\section{PRODUCT DESIGNATION \\ P-Bromodiphenyl}

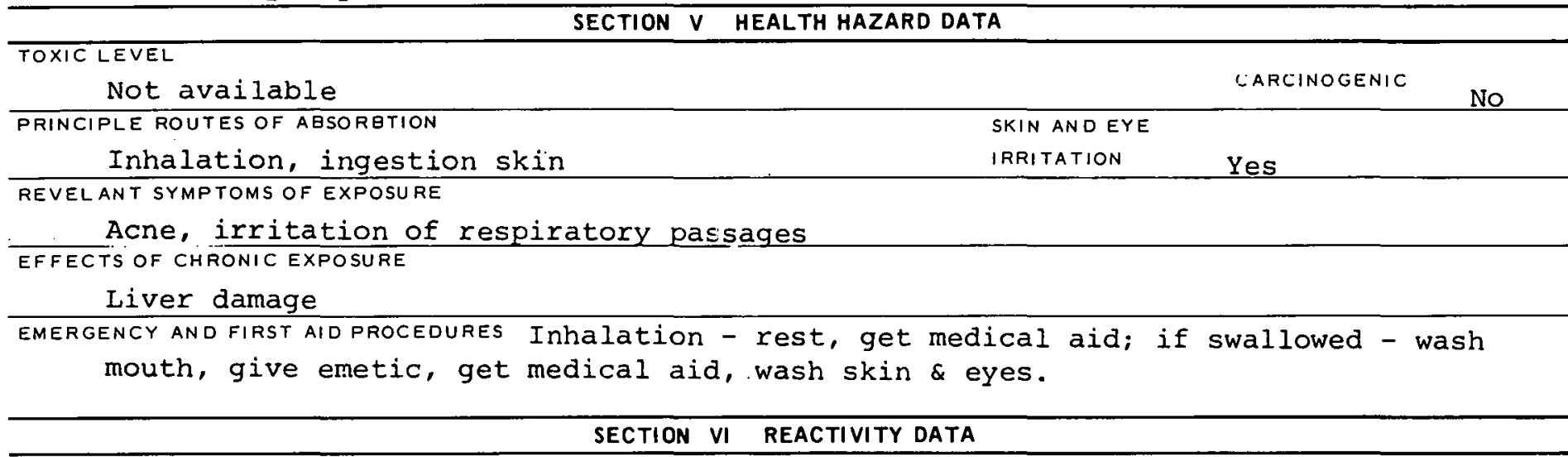

CONDITIONS CONTRIBUTING TO INSTABILITY

CONDITIONS CONTRIBUTING TO HAZARDOUS POLYMERIZATION

INCOMPATABILITY (MATERIALS TO AVOID)

HAZARDOUS DECOMPOSITION PRODUCTS

SECTION VII SPILL OR LEAK PROCEDURES

STEPS TO BE TAKEN IN CASE MATERIAL IS RELEASED OR SPILLED

WASTE DISPOSAL METHOD

SECTION VIII SPECIAL PROTECTION INFORMATION

\begin{tabular}{|c|c|}
\hline VENTILATION REQUIREMENTS LOCAL EXHAUST & $\begin{array}{l}\text { PROTECTIVE EQUIPMENT (SPECIFY TYPESI EYE } \\
\text { Goggles }\end{array}$ \\
\hline MECHANICAL (GENERAL) & GLOVES \\
\hline GPEGIAL & $\begin{array}{l}\text { FESPIRATOR } \\
\text { With proper filter }\end{array}$ \\
\hline
\end{tabular}

OTHER PROTECTIVE EQUIPMENT

SECTION IX SPECIAL PRECAUTIONS

PRECAUTIONS TO BE TAKEN IN HANDLING AND STORAGE

OTHER PRECAUTIONS

SIGNATURE

DATE

$220-9359 B$ 
CHEMICAL ABSTRACT SERVICE REGISTRY NUMBER

Inorganic

Chromic Acid

Hydriodic Acid

Hydrobromic Acid

Hydrochloric Acid

Hydrofluoric Acid

Hydrofluosilicic Acid

Hypophosphorous Acid (30\%)

Iodic Acid

Nitric Acid

Perchloric Acid

Periodic Acid

Phosphoric Anhydride

Phosphoric Acid

Phosphotungstic Acid

Sulfuric Acid

Sulfurous Acid

Aluminum Chloride

Ammonium Bichromate

Ammonium Bifluoride

Ammonium Fluoride

Ammonium Hydroxide

Ammonium Oxalate

Ammonium Sulfide Solution

Ammonium Vanadate

Antimony

Antimony Trichloride

Arsenic Metal

Barium Acetate

Barium Carbonate
CAS. NO.

007738945

010034852

010035106

$007647010^{\circ}$

007664393

016961834

006303215

007782685

007697372

0076,01903

013444718

001314563

007664382

012067991

007664939

007782992

007446700

007789095

001341497

012125018

001336216

014258492

012124991

007803556

007440360

010025919

007440382

000543806

000513779
PAGE NO.

1-0721

1-0922

1-1113

$1-1324$

$1-1500$

1-1716

1-1917

1-2128

1-2933

1-3703

1-3904

1-4506

1-4516

1-4807

$1-7212$

1-7262

6-1310

8-1102

8-1503

8-4401

8-5123

8-6527

8-7851

8-9336

10-0360

10-0600

13-0401

15-0102

15-0903 


\begin{tabular}{|c|c|c|}
\hline Barium Chloride & 010361372 & $15-1904$ \\
\hline Barium Hydroxide & 017194002 & $15-3706$ \\
\hline Barium Metal & 007740393 & $15-4607$ \\
\hline Barium Nitrate & 010022318 & $15-5538$ \\
\hline Beryllium Nitrate & 007787555 & $17-6661$ \\
\hline Bromine & 007726956 & $24-5204$ \\
\hline Cadmium Chloride & 010108642 & $26-2805$ \\
\hline Cadmium (Mctal) & 007440439 & $26-4805$ \\
\hline Calcium Fluorido & 007789755 & $38-3516$ \\
\hline Calcium oxide & 001305788 & $28-5926$ \\
\hline Chromium Metal & 007440473 & $40-3400$ \\
\hline Chromic Nitrate & 007789028 & $40-4550$ \\
\hline Chromium Potassium Sulfate & 007788990 & $40-5600$ \\
\hline Cobalt Metal & 007440484 & $42-4001$ \\
\hline Cupric Carbonate & 012069691 & $46-2903$ \\
\hline Cupric Chloride & 001344678 & $46-3433$ \\
\hline Cupric Nitrate & 010031433 & $46-4403$ \\
\hline Cupric Oxide & 001317380 & $46-4953$ \\
\hline Cupric Sulfate & 007758987 & $46-6403$ \\
\hline Cuprous Chloride & 007758896 & $46-8423$ \\
\hline Cuprous Cyanide & 000544923 & $46-8721$ \\
\hline Cuprous Oxide & 001317391 & $46-9303$ \\
\hline Ferrir Chloride & n1กก2.5771 & $53-2.524$ \\
\hline Ferric Nitrate & $00 \% 782618$ & $53-3804$ \\
\hline Iodine & 007553562 & $74-1009$ \\
\hline Lead Acetate & 000301042 & 82.-0102 \\
\hline Lead Chlus iclu & 007758954 & $82-1802$ \\
\hline Lead Dioxide & 001309600 & $82-3002$ \\
\hline Lead Meta]. & 007439921 & $82-4802$ \\
\hline Lead Nitrate & 010099748 & $82-5402$ \\
\hline Lead Oxide & 001317368 & $82-6002$ \\
\hline Lead Sulfate & 007446142 & $82-7202$ \\
\hline Lithium Aluminum Hydride & 016853853 & $84-1003$ \\
\hline Lithium Metal. & 007439932 & $84-8003$ \\
\hline
\end{tabular}


Lithium Fluoride

Lithium Hydroxide

Mercuric Chloride

Mercuric Iodide

Mercuric Nitrate

Mercurous Nitrate

Mercury Metal

Nickel Carbonyl

Nickel Metal

Nickelous Ammonium Sulfate

Nickelous Chloride

Nickelous Nitrate

Nickelous Sulfate

Phosphorous Pentachloride

Potassium Arsenate

Potassium Bichromate

Potassium Bifluoride

Potassium Chromate

Potassium Cyanide

Potassium Fluoride

Potassium Hydroxide

Potassium Metal

Potassium Oxalate

Potassium Periodate

Ruthenium Chloride

Selenium Metal

Sodium Arsenite

Sorium Azide

Sodium Calcium Hydrate

Sodium Chromate

Sodium Cyanide

Sodium Dichromate

Sodium Fluoride

Sodium Hydroxide

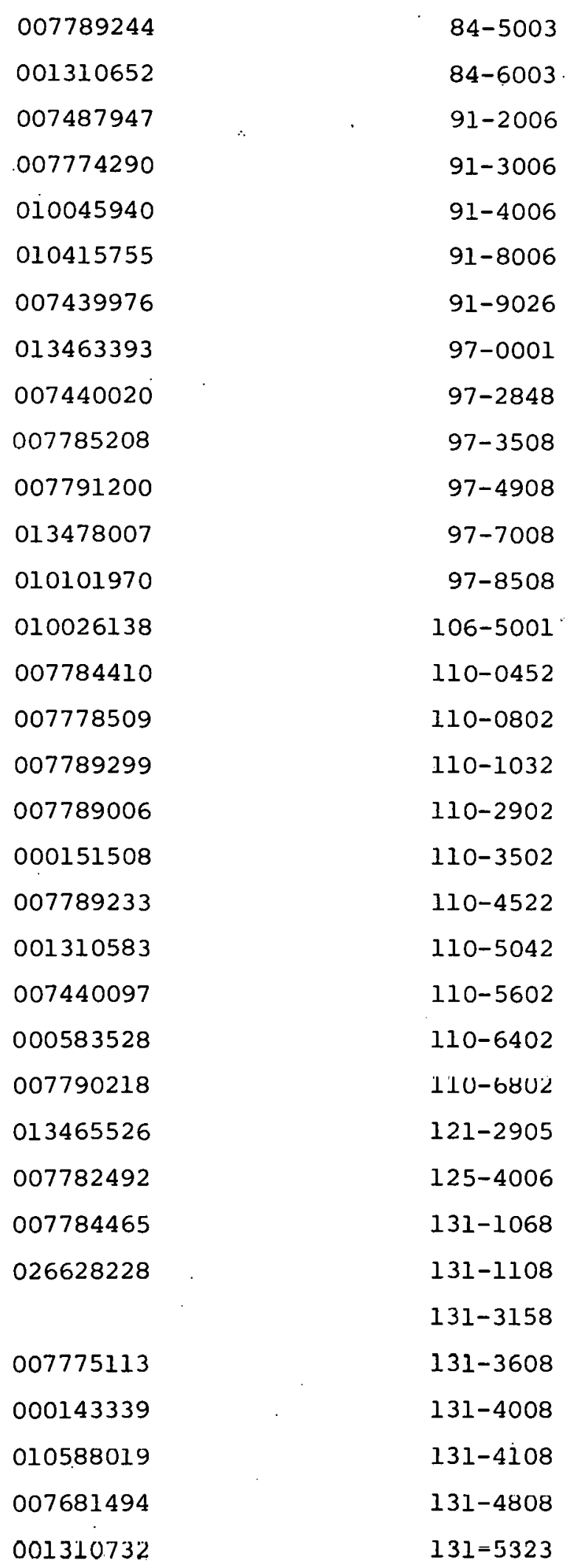


Sodium Hypochlorite

007681529

131-5508

Sodium Metal

007440235

131-6028

Sodium Oxalate

000062760

$131-6908$

Sodium Peroxide

001313606

131-7338

Sodium Silicate

006834920

131-8148

Sodium Tellurite

010102202

131-8758

Stannic Chloride

007646788

133-3008

Stannous Chloride

007772998

133-6008

Tell lus ilum Melarl.

ن1349่48نิ

141-5902

Titanium Chloride

007550450

150-4003

Vanadium Pentoxide

001314621

154-6035

Yttrium Metal

007440655

159-5006

Yttrium Oxide

001314369

159-8006

zinc Chloride

007646857

161-2007

\section{Gases}

Ammonia (anhydrous)

007664417

910-4030

Carbon Monoxide

000630080

927-5550

Chlorine, liquid, compressed

007782505

934-4000

Hydrogen Sulfide

007783064

950-9425

sulfur Dioxide

007446095

988-2020

ntherr

Asbestos Fiber

$609-2050$

\section{Precious Metals}

Gold Cyanide

000506650

5201-1400

Sel-Rex Bright Gold Bl

Sel-Rex Bright Gold $\mathrm{M}^{2}$

Gold(ous) Potassium Cyanide

020816120

5201-4000

$5201 .-4050$

Osmic Acid Anhydride

5201-6200

Chloroplatinic Acid

017083704

5401-0401

Platinum Chloride

013454961

5601-0801

5601-1120 


$$
A-1=5
$$

Diamino Platinum Nitrite

5601-1510

Rhodium Trichloride

010049077

5801-1105

Silver Carbonate

012537767

5901-1101

Silver Cyanide

000506649

5901-1251

Silver Nitrate

007761888

$5901-5621$

\section{Organic Acids}

Acetic Anhydride

000108247

201-0100

Acetic Acid

000064197

201-0200

P-Arsanilic Acid

000098500

201-2102

Bromoacetic Acid

000079083

201-4004

B-Bromopropionic Acid

000590921

$201-4454$

Chloroacetic Acid

000079.1 .18

201-5905

Chloromaleic Anhydride

000096026

201-6186

Chlorosulfonic Acid

007790945

201-6706

Formic Acid

000064186

202-1400

8 Hydroxy-5-Quinoline Sulfonic Acid

000084888

202-2903

Maleic Acid

000110167

202-4405

Maleic Anhydride

000108316

202-4505

Malonic Acid

000141822

202-4805

Methoxyacetic Acid

000625456

202-5206

Nitrophthalic Anhydride

000641703

202-9610

Oxalic Acid

000144627

203-0302

Phenylarsonic Acid

000098055

203-1303

Phthalic Anhydride

000085449

203-2024

Picramic Acid

000096913

203-2504

Picric Acid

000088891

203-2704

Pyrogallic Acid

000087661

203-3455

Tannic Acid

001401554

203-7309

Thioglycolic Acid

000068111

203-8310

Trichloro Acetic Acid

000076039

203-9202 


$$
\text { A-1-6 }
$$

Organic

Acetal
Acetaldehyde
Acetamide
Acetonitrile
Acetonylacetone
Acetyl Chloride
Acetyl Methyl Amine
Aretyl Methylurea
Acetyl Thiourea
Acetyl Urea
Acrylonitrile

$\begin{array}{ll}000105577 & 205-1550 \\ 000075070 & 205-2000 \\ 000060355 & 205-2500 \\ 000075058 & 205-5000 \\ 000110134 & 205-6000 \\ 000075365 & 206-3501 \\ 000079163 & 206-4501 \\ 000633596 & 206-6001 \\ 000531082 & 206-6501 \\ 000591071 & 206-7501 \\ 000107131 & 206-8801\end{array}$

\section{Alcohols}

$\begin{array}{lcc}\text { Allyl Alcohol } & 000107186 & 208-0202 \\ \text { Iso-Amyl Alcohol } & 000123513 & 208-0332 \\ \text { n-Butyl Alcohol } & 000071363 & 208-0902 \\ \text { Isobutyl Alcohol. } & 000078831 & 208-0942 \\ \text { Sec Butyl Alcohol } & 000078922 & 208-0952 \\ \text { Tertlary Butyl Alcohol } & 000075650 & 208-0982 \\ \text { Diacetone Alcohol } & 000123422 & 208-1602 \\ 2 \text { Di-N-Butylaminoethyl } & 000102818 & 208-2202 \\ \quad \text { Alcohol } & - & 208-2752 \\ \text { B-Di Ethylaminoethyl Alcohol } & 000100378 & 208-3102 \\ \text { B-Dimethylaminoethyl Alcohol } & 000108010 & 208-5002 \\ \text { Furfuryl Alcohol } & 000098000 & 208-5202 \\ \text { n-Heptyl Alcohol } & 000111706 & 208-6002 \\ \text { Methyl Alcohol } & 000067561 & 208-8702 \\ \text { N-Propyl Alcohol } & 000071238 & \end{array}$


Organic

Allylamine

Allyl Bromide

Allyl Chloride

P-Aminoazobenzene

P-Aminoazobenzene Hydrochloride

2-Amino-1, 4-Dimethylbenzene

4 amino-1, 3-Dimethylbenzene

5-amino-1, 3-Dimethylbenzene

o-aminodiphenyl

2-Aminopyridiṇe

Iso amyl acetate

n-Amyl Acetate

Aniline

Aniline Hydrochloride

Aniline sulfate

o-Anisidine

P-Anisidine

Anthracene

Azobenzene

Azoxybenzene

Benzalazine

1, 2-Benzarthracene

Benzene (Benzol)

Benzidine, Dihydrochloride

Benzoyl Bromide

Benzoyl Chloride

Benzyl Bromide

Benzyl Chloride

Benzyl-M-Toluidine

Benzyl-0-Toluidine

Benzyl-P-Toluidine

Dromal

\begin{tabular}{|c|c|}
\hline 000107119 & $211-3103$ \\
\hline 000106956 & $211-3203$ \\
\hline 000107051 & $211-3303$ \\
\hline 000060093 & $211-6503$ \\
\hline 003457985 & $211-6703$ \\
\hline 000095783 & $212-0104$ \\
\hline 00009568.1 & $212-0134$ \\
\hline 000108690 & $212-0164$ \\
\hline 000090415 & $212-0204$ \\
\hline 000504290 & $212-1704$ \\
\hline 000123922 & $212-5004$ \\
\hline 000628637 & $212-5104$ \\
\hline 000062533 & $213-2005$ \\
\hline 000142041 & $213-2205$ \\
\hline 020305504 & $213-2405$ \\
\hline 000090040 & $213-3205$ \\
\hline 000104949 & $213-3255$ \\
\hline 000120127 & $213-4005$ \\
\hline 000103333 & $213-9005$ \\
\hline 000495487 & $213-9505$ \\
\hline 000588681 & $216-3006$ \\
\hline 000056553 & $216-6006$ \\
\hline 000071432 & $216-7006$ \\
\hline 000531851 & al6-9146 \\
\hline 000618326 & $217-6107$ \\
\hline 000098884 & $217-7107$ \\
\hline 000100390 & $219-0700$ \\
\hline חถก1กก447 & $219-1708$ \\
\hline 005405174 & $219-8208$ \\
\hline 005405130 & $219-8238$ \\
\hline 005405152 & $219-8268$ \\
\hline 000115173 & $220-0809$ \\
\hline
\end{tabular}




$$
\text { A-1-8 }
$$

a-Bromo-acetophenone
Bromoacetyl Bromide
3 Bromo-4-Aminotoluene
5-Bromo-2-Aminotoluene
M-Bromoaniline
O-Bromoaniline
P-Bromoaniline
Bromobenzene
M-Bromobonzoyl Bromide
Bromochloromethane
F-BromuDiellyldulline
P-Bromodimethylaniline
2 Bromo-l, 4-dimethylbenzne
O-Bromodiphenyl
P-Bromodiphenyl

\begin{tabular}{ll}
000070111 & $220-1803$ \\
000598210 & $220-2309$ \\
000583686 & $220-2809$ \\
000583755 & $220-3309$ \\
000591195 & $220-3809$ \\
000615361 & $220-3839$ \\
000106401 & $220-3869$ \\
000108861 & $220-4859$ \\
000074975 & $220-5809$ \\
002052064 & $220-6809$ \\
000586776 & $220-7809$ \\
000553946 & $220-8309$ \\
002052075 & $220-8809$ \\
000092660 & $220-9309$ \\
\hline
\end{tabular}




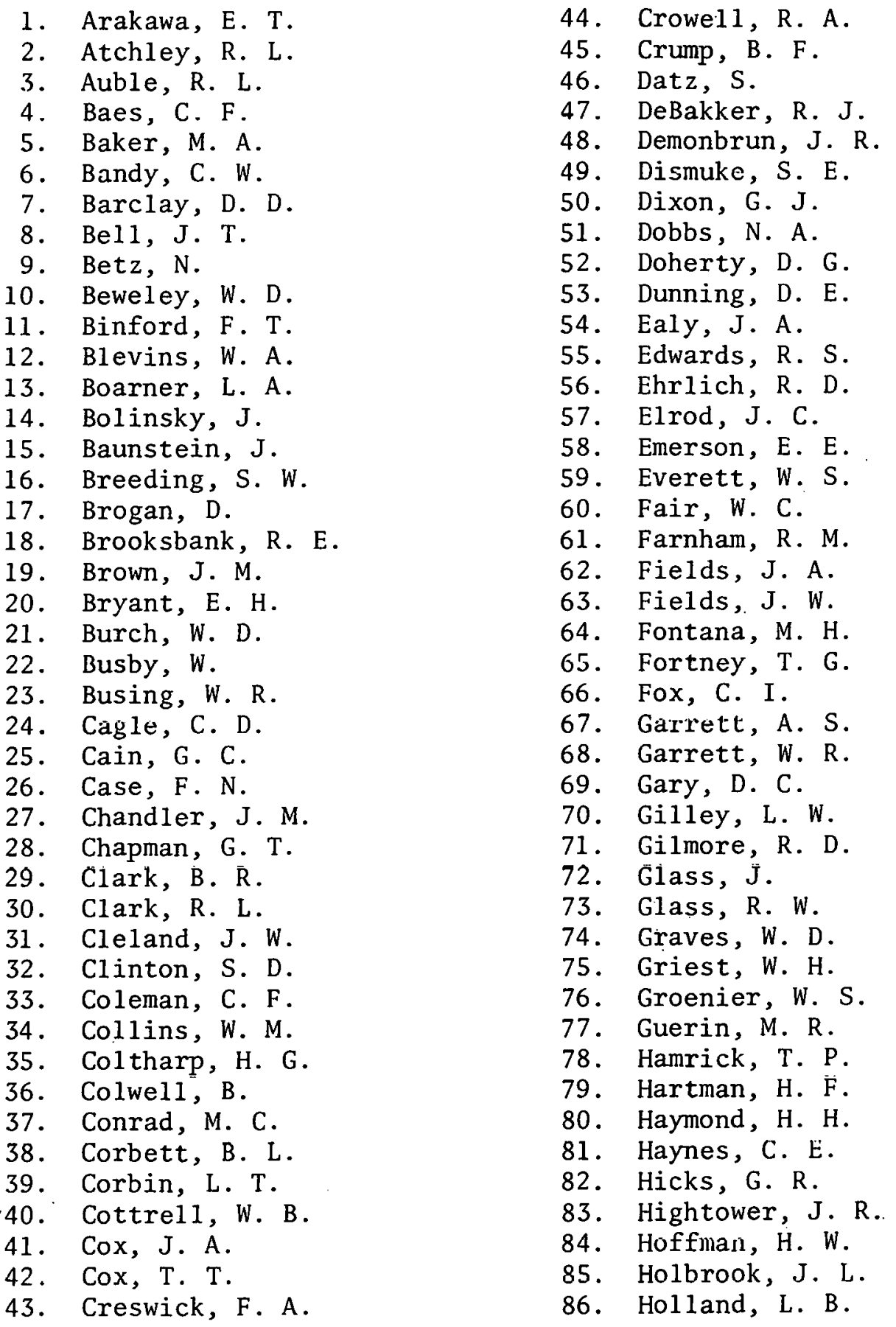


87. Hood, W. B.

88. Hul 1, G. T.

89. Hurt, S. S.

90. Hutchins, J. D.

91. Ind. Hyg. Library (K-25)

92-102. Ind. Hyg. Dept. (ORNL)

103. Jackson, J. W.

104. Jamison, K. E.

105. Jenkins, L. H.

106. Jenkins, R. A.

107. Jenness, R. G.

108. Jennings, R, M.

109. Juhnson, C. E.

110. Johnston, R. L.

111. Jones, J. F., Jr.

112. Jones, J. R.

113. Jones, R. S.

114. Judish, T. P.

115. Kappelindnn, F. A.

116. Keesee, H. F.

117-118. Ketchen, E. E.

119. King, E. M.

120. King, L. J.

121. Klaus, H. V.

122. Kobisk, E. H.

123. Kowhler, W. C.

124. Lake, M. M.

125. Lampton, R. E.

126. Lance, E. D.

127. Langford, L.

128. Lasher, L. C.

129. Lees, E. M.

130. Leinart, R. L .

131. Leuze, R. E.

132. Library (Paducah)

133. Lieberman, B.

134. Lines, R. L.

135. Livingston, $R$.

136. Lloyd, M. H.

137. Love, $\mathrm{G}$.

138. Lovelace, H. G.

139. Lyon, W. S.

140. MacPherson, R. E.

141. Macres, J.

142. Mailen, J. C.

143. Malinauskas, A. P.

144. Martin., J. R.

145. Massengi11, J.

146. McCord, R. V.
147. McDougal, B. E.

148. McDowe11, J. W.

149. McNabb, J. G.

150. Mesmer, R. E.

151. Michel, J.W.

152. Milan, D.

153. Millican, R.

154. Miller, J. M.

155. Miller, P. D.

156. Miller, W. H.

157. Mitche11, M. E.

158. Murray, J. A.

.159. N1col, R. G.

160. Noggle, T. S.

161. Oakes, T. W.

162. Odel1, 'I'. T'.

163. Oliphant, G. W.

164. Ottinger, C. L.

165. Parrott, J. R.

166. Parzyck, O. C.

167. Perry, T.P.A.

168. Peterson, C. H.

169. Pickel, T. W.

170. Pierce, E. E.

171. Pope, R. G.

172. Porter, W. E.

173. Posey, F. A.

174. Poteet, K. H.

175. Poutsma, M. L.

176. Pugh, L. P.

177. Pulley, H.

178. Ragland, W. R.

179. Rickard, C. R.

180. Rickard, R. R.

181. Robbins, $D$.

182. Rohwer, P. S.

183, Ross, R. G.

184. Ruch, J. B.

185. Ryon, A. D.

186. Schaich, R. W.

$18 \%$ Sichmett, $L$.

188. Scott, C. B.

189. Senkan, S. M.

190. Setaro, J. A.

191. Shanks, M. H.

192. Sherrod, J,

193. Shults, W. D.

194. Skidmore, M. R.

195. Smith, G. P. 
196. Snider, J. W.

197. Spiewak, I.

198. Stinton, L. H.

199. Stokely, J. R.

200. Swanks, J. H.

201. Taylor, W. C.

202. Testerman, G. E.

203. Thomas, I. L.

204. Thompson, W. E.

205. Tidwel1, B. L.

206. Tidwell, K. W.

207. Todd, W. D.

208. Tuck, H. H.

209. Tuholsky, A. M.

210. Turok, C. W.

211. Vaughen, $V$.

212. Viar, C. L.

213. Vollman, C. L.

214. Walden, J. M.

215. Walls, A. A.

216. Ward, R. H.

217. Weeren, W. H.

218. White, J. E.

219. Whitman, G. D.

220. Whitson, W. R.

221. Wicker, C. D.

222. Wilson, G. R.

223. Wilson, M. A.

224. Winget, R. H.

225. Wolfenbarger, J. D.

226. Wood, R. F.

227. Wright, J. A.

228. Wright, J. R.

229. Young, F. W.

230. Young, G. B.

231. Youngblood, E. L.

232-233. Centra1 Research Library

234. Document Reference Section

235. ORNL Patent Office

236-237. Laboratory Records

238. Laboratory Records (RC)

239-270. Industrial Hygiene Department (ORNL) 


\section{EXTERNAL DISTRIBUTION}

271-297. Technical Information Center

298. Office of Asst. Mgr. Energy Research \& Development, Department of Energy, Oak Ridge Operations Office, Oak Ridge, TN

299. N. E. Bolton, Union Carbide Corporation - NYO

300. John Janous, 222 Rainbow Blvd. N. - Box 728, Niagara Falls, Ny 14302 